\title{
SUPPORTING INFORMATION FOR: Semiempirical Quantum-Chemical Orthogonalization-Corrected Methods: Benchmarks of Electronically Excited States
}

Deniz Tuna, ${ }^{*}$ You Lu, Axel Koslowski, and Walter Thiel ${ }^{*}$

Max-Planck-Institut für Kohlenforschung, 45470 Mülheim an der Ruhr, Germany

*email: tuna@kofo.mpg.de, thiel@kofo.mpg.de 


\section{Contents}

1 28-Molecules Benchmark Set on Vertical Excitation Energies S3

2 32-Molecules Benchmark Set on Excited-State Equilibrium Ge$\begin{array}{ll}\text { ometries } & \text { S18 }\end{array}$

3 Comparisons of ZPVEs and $\triangle \mathrm{ZPVE} \quad \mathrm{S31}$

4 Presentation of Raw Data Necessary for Reproducibility $\quad$ S37

4.1 32-Molecules Benchmark Set on Excited-State Equilibrium Ge-

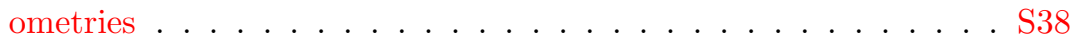

4.1.1 OM2/MRCI-Optimized Excited-State Minima . . . . . . S40

4.1.2 Structural Parameters of the OM2/MRCI-Optimized Excited-

State Minima . . . . . . . . . . . . . . . . S150

4.1.3 OM3/MRCI-Optimized Excited-State Minima . . . . . . S251

4.1.4 Structural Parameters of the OM3/MRCI-Optimized Excited-

State Minima . . . . . . . . . . . . . . . S362

4.1.5 TDDFT/B3LYP-Optimized Excited-State Minima . . . . S463

4.1.6 Structural Parameters of the TDDFT/B3LYP-Optimized

Excited-State Minima . . . . . . . . . . . . . S554

4.1.7 CC2-Optimized Excited-State Minima . . . . . . . . . . S645

4.1.8 Structural Parameters of the CC2-Optimized Excited-State

Minima ................... . . S744

4.1.9 OM2/SCF-Optimized Ground-State Minima . . . . . . . S843

4.1.10 DFT/B3LYP-Optimized Ground-State Minima . . . . . . S876

4.1.11 OM2/SCF-OM2/MRCI Results on Significant Changes Between Ground-State and Excited-State Minima . . . . S909

4.1.12 DFT-TDDFT Results on Significant Changes Between

Ground-State and Excited-State Minima . . . . . . . . . S995

4.2 OM2-Optimized Minima for the Computation of ZPVEs . . . . . S1081

4.2.1 OM2/RHF-Optimized Ground-State Minima . . . . . . . S1081

4.2.2 OM2/ROHF-Optimized Excited-State Minima . . . . . . S1142

4.2.3 OM2/MRCI-Optimized Ground- and Excited-State MinimaS1176

4.3 Send-Kühn-Furche Benchmark Set on 0-0 Transition Energies . S1177

4.3.1 OM2/MRCI-Optimized Ground- and Excited-State MinimaS1177

4.3.2 OM3/MRCI-Optimized Ground- and Excited-State MinimaS1324

4.4 Winter-Graf-Leutwyler-Hättig Benchmark Set on 0-0 Transi-

tion Energies . . . . . . . . . . . . . . . S1466

4.4.1 OM2/MRCI-Optimized Ground- and Excited-State MinimaS1468

4.4.2 OM3/MRCI-Optimized Ground- and Excited-State MinimaS1630 
1 28-Molecules Benchmark Set on Vertical Excitation Energies 

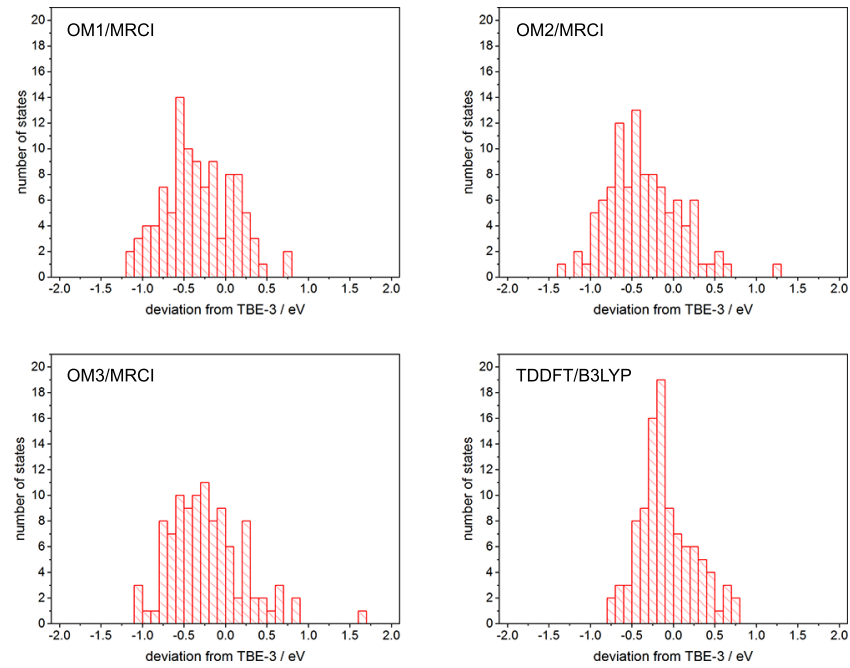

Figure S1 Histograms of the deviations of the computed singlet vertical excitation energies from the TBE-3 reference values. 
Table S1 Deviations of OMx/MRCI- and TDDFT/B3LYP-computed vertical singlet excitation energies (in eV) from Theoretical Best Estimates TBE-3 separated into molecule classes.

\begin{tabular}{|c|c|c|c|c|}
\hline & OM1/MRCI & OM2/MRCI & OM3/MRCI & TDDFT/B3LYP \\
\hline \multicolumn{5}{|c|}{ CH-containing molecules } \\
\hline count & 25 & 25 & 25 & 25 \\
\hline mean deviation & -0.23 & -0.25 & -0.21 & -0.09 \\
\hline mean absolute deviation & 0.36 & 0.35 & 0.35 & 0.36 \\
\hline standard deviation & 0.48 & 0.46 & 0.44 & 0.42 \\
\hline maximum deviation & -1.08 & -0.96 & -0.93 & +0.76 \\
\hline \multicolumn{5}{|c|}{ CHN-containing molecules } \\
\hline count & 43 & 43 & 43 & $42^{\mathrm{a}}$ \\
\hline mean deviation & -0.28 & -0.25 & -0.11 & -0.03 \\
\hline mean absolute deviation & 0.39 & 0.44 & 0.42 & 0.23 \\
\hline standard deviation & 0.49 & 0.53 & 0.54 & 0.27 \\
\hline maximum deviation & -1.16 & +1.28 & +1.62 & +0.64 \\
\hline \multicolumn{5}{|c|}{ CHO-containing molecules } \\
\hline count & 15 & 15 & 15 & 15 \\
\hline mean deviation & -0.25 & -0.42 & -0.19 & -0.21 \\
\hline mean absolute deviation & 0.44 & 0.52 & 0.40 & 0.26 \\
\hline standard deviation & 0.53 & 0.68 & 0.49 & 0.35 \\
\hline maximum deviation & -1.18 & -1.33 & -1.06 & -0.71 \\
\hline \multicolumn{5}{|c|}{ CHNO-containing molecules } \\
\hline count & 21 & 21 & 21 & 21 \\
\hline mean deviation & -0.63 & -0.65 & -0.48 & -0.13 \\
\hline mean absolute deviation & 0.63 & 0.65 & 0.48 & 0.30 \\
\hline standard deviation & 0.70 & 0.70 & 0.53 & 0.38 \\
\hline maximum deviation & -1.06 & -0.99 & -0.86 & +0.78 \\
\hline
\end{tabular}


Table S2 Deviations of OMx/MRCI- and TDDFT/B3LYP-computed vertical singlet excitation energies (in eV) from Theoretical Best Estimates TBE-3 separated into $n \pi^{*}$ and $\pi \pi^{*}$ states.

\begin{tabular}{lcccc}
\hline \hline & OM1/MRCI & OM2/MRCI & OM3/MRCI & TDDFT/B3LYP \\
\hline$n \pi^{*}$ states & & & & \\
count & 38 & 38 & 38 & $37^{\mathrm{a}}$ \\
mean deviation & -0.32 & -0.28 & -0.22 & -0.21 \\
mean absolute deviation & 0.44 & 0.49 & 0.45 & 0.23 \\
standard deviation & 0.55 & 0.58 & 0.56 & 0.27 \\
maximum deviation & -1.06 & +1.28 & +1.62 & -0.71 \\
& & & & \\
$\pi \pi^{*}$ states & & & 63 & 63 \\
count & -0.35 & -0.37 & -0.21 & -0.01 \\
mean deviation & 0.45 & 0.43 & 0.39 & 0.31 \\
mean absolute deviation & 0.53 & 0.52 & 0.47 & 0.38 \\
standard deviation & -1.18 & -1.14 & -1.08 & +0.78 \\
maximum deviation &
\end{tabular}

${ }^{a}$ The $1{ }^{1} \mathrm{~B}_{3 g}$ state of tetrazine is missing, since TDDFT/B3LYP cannot describe the double-excitation character of this state. 

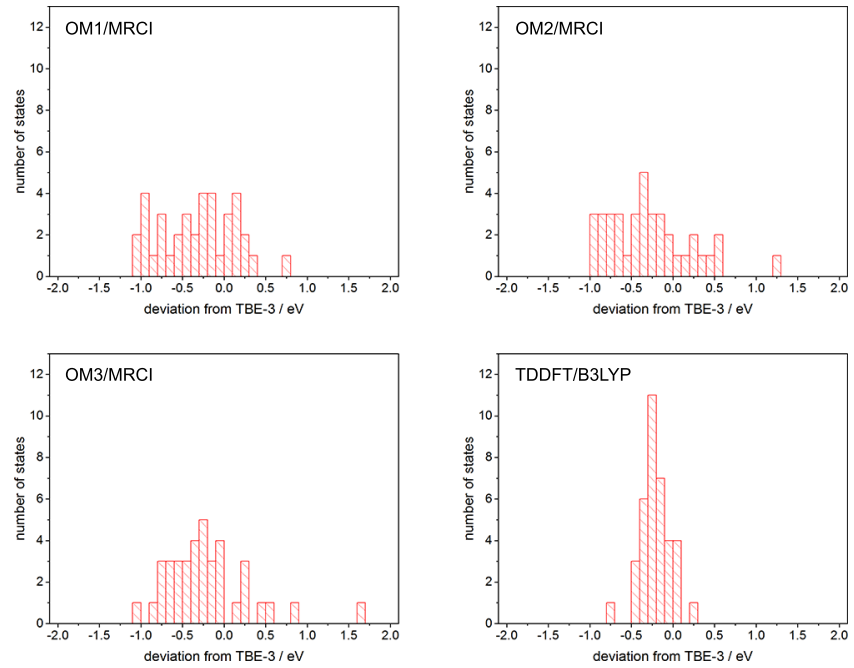

Figure S2 Histograms of the deviations of the computed $n \pi^{*}$ singlet vertical excitation energies from the TBE-3 reference values. 

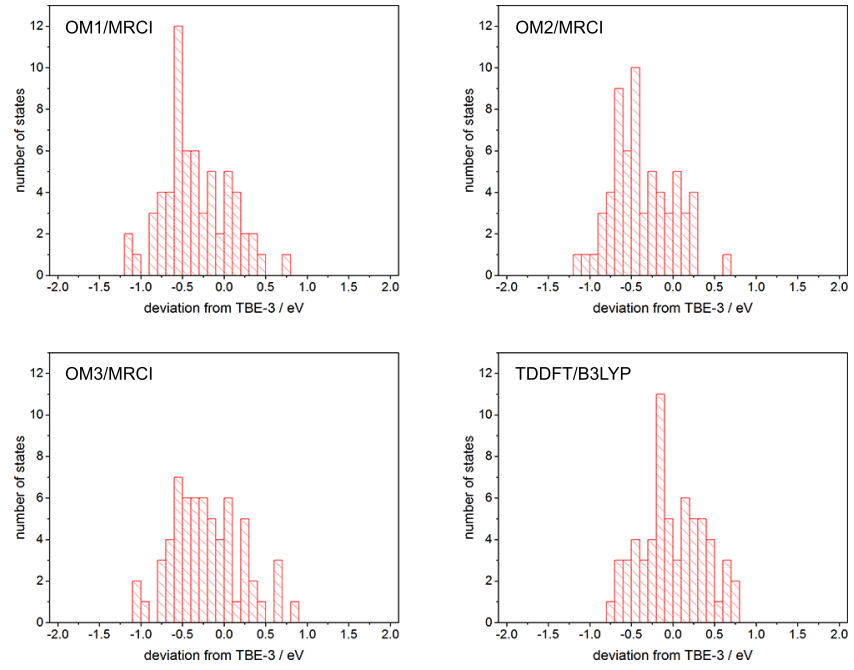

Figure S3 Histograms of the deviations of the computed $\pi \pi^{*}$ singlet vertical excitation energies from the TBE-3 reference values. 
Table S3 Deviations of OMx/MRCI- and TDDFT/B3LYP-computed vertical singlet excitation energies (in eV) from Theoretical Best Estimates TBE-3 of aldehydes, ketones, and amides separated into $n \pi^{*}$ and $\pi \pi^{*}$ states.

\begin{tabular}{lcccc}
\hline \hline & OM1/MRCI & OM2/MRCI & OM3/MRCI & TDDFT/B3LYP \\
\hline$n \pi^{*}$ states & 8 & & & 8 \\
count & -0.48 & -0.40 & -0.31 & -0.11 \\
mean deviation & 0.55 & 0.52 & 0.44 & 0.11 \\
mean absolute deviation & 0.70 & 0.65 & 0.51 & 0.17 \\
standard deviation & -1.03 & -0.99 & -0.73 & -0.31 \\
maximum deviation & & & & \\
& & & & \\
$\pi \pi^{*}$ states & 8 & -0.34 & -0.02 & -0.02 \\
count & -0.18 & 0.39 & 0.29 & 0.49 \\
mean deviation & 0.39 & 0.50 & 0.34 & 0.59 \\
mean absolute deviation & 0.44 & -0.82 & +0.60 & +0.78 \\
standard deviation & -0.58 & & & \\
maximum deviation & & &
\end{tabular}


Table S4 Deviations of OMx/MRCI- and TDDFT/B3LYP-computed vertical singlet excitation energies (in eV) from Theoretical Best Estimates TBE-3 of heterocycles separated into $n \pi^{*}$ and $\pi \pi^{*}$ states.

\begin{tabular}{lcccc}
\hline \hline & OM1/MRCI & OM2/MRCI & OM3/MRCI & TDDFT/B3LYP \\
\hline$n \pi^{*}$ states & 9 & & & \\
count & -0.75 & -0.69 & -0.56 & -0.38 \\
mean deviation & 0.75 & 0.69 & 0.56 & 0.38 \\
mean absolute deviation & 0.82 & 0.76 & 0.63 & 0.44 \\
standard deviation & -1.06 & -0.98 & -0.86 & -0.71 \\
maximum deviation & & & & \\
& & & & \\
$\pi \pi^{*}$ states & 10 & 10 & 10 & 10 \\
count & -0.48 & -0.59 & -0.49 & -0.15 \\
mean deviation & 0.48 & 0.59 & 0.49 & 0.19 \\
mean absolute deviation & 0.54 & 0.65 & 0.54 & 0.23 \\
standard deviation & -0.85 & -0.85 & -0.73 & -0.39 \\
maximum deviation & & & & \\
\hline \hline
\end{tabular}



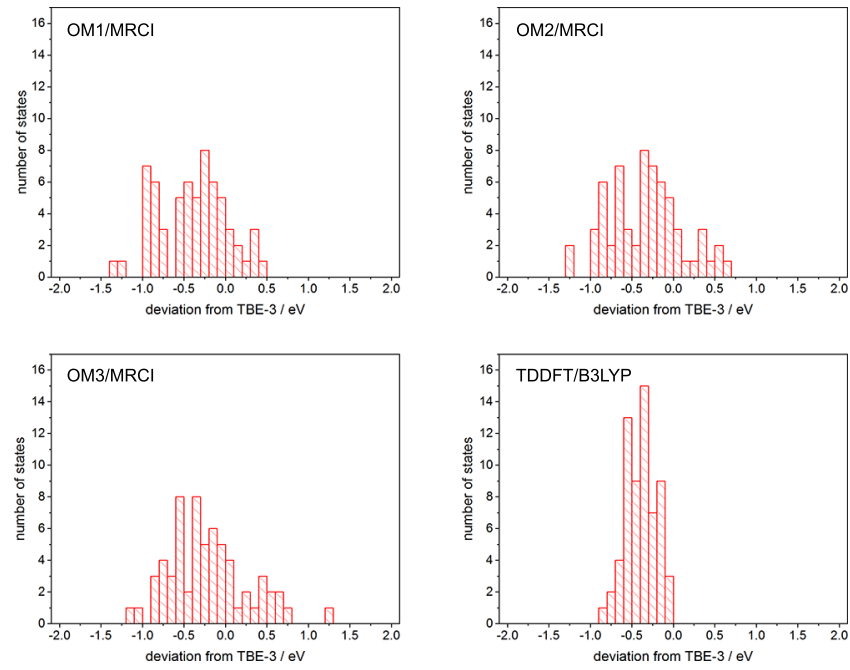

Figure S4 Histograms of the deviations of the computed triplet vertical excitation energies from the TBE-3 reference values. 
Table S5 Deviations of OMx/MRCI- and TDDFT/B3LYP-computed vertical triplet excitation energies (in eV) from Theoretical Best Estimates TBE-3 separated into molecule classes.

\begin{tabular}{lcccc}
\hline \hline & OM1/MRCI & OM2/MRCI & OM3/MRCI & TDDFT/B3LYP \\
\hline CH-containing molecules & & & & \\
count & 27 & 27 & 27 & 27 \\
mean deviation & -0.35 & -0.31 & -0.29 & -0.38 \\
mean absolute deviation & 0.44 & 0.41 & 0.40 & 0.38 \\
standard deviation & 0.54 & 0.52 & 0.50 & 0.43 \\
maximum deviation & -1.31 & -1.21 & -1.17 & -0.79 \\
& & & & \\
CHN-containing molecules & & & & 20 \\
count & 20 & 20 & 0.00 & -0.33 \\
mean deviation & -0.34 & -0.22 & 0.47 & 0.33 \\
mean absolute deviation & 0.40 & 0.41 & 0.56 & 0.37 \\
standard deviation & 0.52 & 0.49 & +1.23 & -0.55 \\
maximum deviation & -0.96 & -0.83 & & \\
& & & 10 & 10 \\
CHO-containing molecules & & & -0.16 & -0.54 \\
count & 10 & 10 & 0.33 & 0.54 \\
mean deviation & -0.31 & -0.26 & 0.43 & 0.60 \\
mean absolute deviation & 0.40 & 0.38 & -0.84 & -0.83 \\
standard deviation & 0.54 & 0.50 & & \\
maximum deviation & -0.99 & -0.89 & & \\
CHNO-containing molecules & & & 6 & -0.47 \\
count & 6 & 6 & -0.70 & 0.47 \\
mean deviation & -0.94 & -0.92 & 0.79 & -0.80 \\
mean absolute deviation & 0.94 & 0.92 & -1.01 & \\
standard deviation & 1.04 & 1.02 & & \\
maximum deviation & -1.21 & -1.21 & & \\
\hline \hline
\end{tabular}


Table S6 Deviations of OMx/MRCI- and TDDFT/B3LYP-computed vertical triplet excitation energies (in eV) from Theoretical Best Estimates TBE-3 separated into $n \pi^{*}$ and $\pi \pi^{*}$ states.

\begin{tabular}{lcccc}
\hline \hline & OM1/MRCI & OM2/MRCI & OM3/MRCI & TDDFT/B3LYP \\
\hline$n \pi^{*}$ states & & & & \\
count & 15 & 15 & 15 & 15 \\
mean deviation & -0.29 & -0.18 & -0.11 & -0.41 \\
mean absolute deviation & 0.39 & 0.46 & 0.47 & 0.41 \\
standard deviation & 0.53 & 0.55 & 0.53 & 0.44 \\
maximum deviation & -0.97 & -0.94 & +0.77 & -0.58 \\
& & & & \\
$\pi \pi^{*}$ states & 47 & 47 & 47 & 47 \\
count & -0.44 & -0.37 & -0.24 & -0.39 \\
mean deviation & 0.49 & 0.44 & 0.42 & 0.39 \\
mean absolute deviation & 0.60 & 0.55 & 0.53 & 0.45 \\
standard deviation & -1.31 & -1.21 & +1.23 & -0.83 \\
maximum deviation & & & & \\
\hline \hline
\end{tabular}



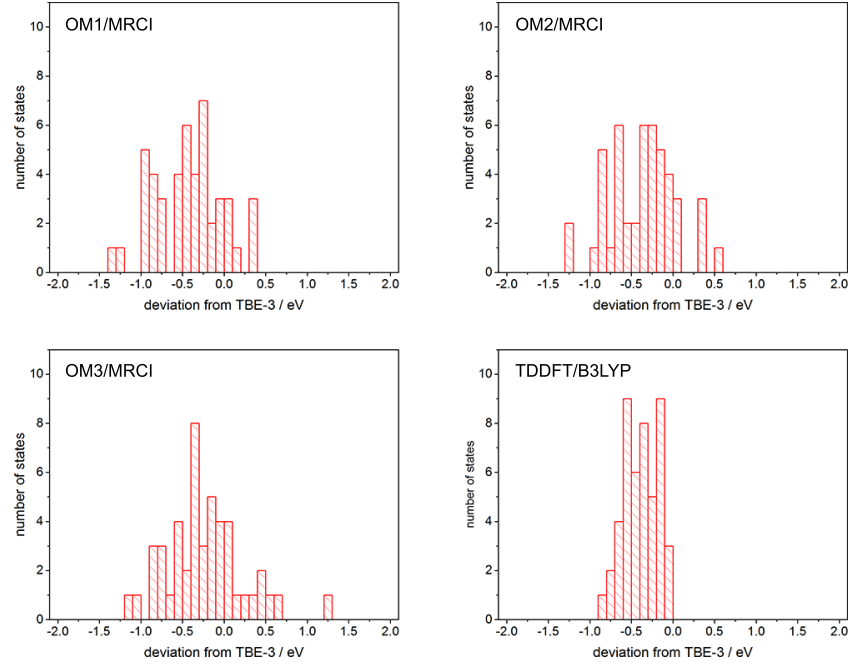

Figure S5 Histograms of the deviations of the computed $\pi \pi^{*}$ triplet vertical excitation energies from the TBE-3 reference values. 
Table S7 Deviations of OMx/MRCI- and TDDFT/B3LYP-computed vertical triplet excitation energies (in eV) from Theoretical Best Estimates TBE-3 of aldehydes, ketones, and amides separated into $n \pi^{*}$ and $\pi \pi^{*}$ states.

\begin{tabular}{lcccc}
\hline \hline & OM1/MRCI & OM2/MRCI & OM3/MRCI & TDDFT/B3LYP \\
\hline$n \pi^{*}$ states & 7 & & & 7 \\
count & -0.44 & -0.36 & -0.26 & -0.41 \\
mean deviation & 0.56 & 0.53 & 0.44 & 0.41 \\
mean absolute deviation & 0.72 & 0.67 & 0.53 & 0.46 \\
standard deviation & -0.94 & +0.94 & -0.70 & -0.58 \\
maximum deviation & & & & \\
& & & & \\
$\pi \pi^{*}$ states & 7 & -0.59 & -0.37 & -0.67 \\
count & -0.58 & 0.59 & 0.43 & 0.67 \\
mean deviation & 0.58 & 0.77 & 0.59 & 0.74 \\
mean absolute deviation & 0.76 & -1.21 & -1.01 & -0.83 \\
standard deviation & -1.21 & & & \\
maximum deviation & & &
\end{tabular}


Table S8 Deviations of OMx/MRCI- and TDDFT/B3LYP-computed vertical singlet excitation energies (in eV) from MS-CASPT2/aug-cc-pVTZ values separated into molecule classes.

\begin{tabular}{|c|c|c|c|c|}
\hline & OM1/MRCI & OM2/MRCI & OM3/MRCI & TDDFT/B3LYP \\
\hline \multicolumn{5}{|c|}{$\mathrm{CH}$-containing molecules } \\
\hline count & 30 & 30 & 30 & 30 \\
\hline mean deviation & -0.13 & -0.16 & -0.12 & -0.04 \\
\hline mean absolute deviation & 0.40 & 0.41 & 0.40 & 0.35 \\
\hline standard deviation & 0.51 & 0.50 & 0.49 & 0.50 \\
\hline maximum deviation & +1.12 & -1.02 & +1.08 & +1.96 \\
\hline \multicolumn{5}{|c|}{ CHN-containing molecules } \\
\hline count & 71 & 71 & 71 & $70^{\mathrm{a}}$ \\
\hline mean deviation & -0.31 & -0.26 & -0.05 & -0.04 \\
\hline mean absolute deviation & 0.45 & 0.48 & 0.48 & 0.25 \\
\hline standard deviation & 0.57 & 0.59 & 0.63 & 0.34 \\
\hline maximum deviation & -1.56 & -1.51 & +1.75 & +1.11 \\
\hline \multicolumn{5}{|c|}{ CHO-containing molecules } \\
\hline count & 16 & 16 & 16 & 16 \\
\hline mean deviation & -0.25 & -0.41 & -0.19 & -0.25 \\
\hline mean absolute deviation & 0.42 & 0.53 & 0.38 & 0.33 \\
\hline standard deviation & 0.50 & 0.69 & 0.47 & 0.40 \\
\hline maximum deviation & -0.96 & -1.54 & -0.91 & -0.65 \\
\hline \multicolumn{5}{|c|}{ CHNO-containing molecules } \\
\hline count & 31 & 31 & 31 & 31 \\
\hline mean deviation & -0.58 & -0.66 & -0.48 & -0.05 \\
\hline mean absolute deviation & 0.58 & 0.66 & 0.48 & 0.27 \\
\hline standard deviation & 0.67 & 0.74 & 0.56 & 0.35 \\
\hline maximum deviation & -1.13 & -1.51 & -1.35 & -0.85 \\
\hline \multicolumn{5}{|l|}{ all molecules } \\
\hline count & 148 & 148 & 148 & $147^{\mathrm{a}}$ \\
\hline mean deviation & -0.32 & -0.34 & -0.17 & -0.01 \\
\hline mean absolute deviation & 0.46 & 0.51 & 0.45 & 0.28 \\
\hline standard deviation & 0.57 & 0.61 & 0.57 & 0.38 \\
\hline maximum deviation & -1.56 & -1.54 & +1.75 & +1.96 \\
\hline
\end{tabular}

\footnotetext{
${ }^{a}$ The $1{ }^{1} \mathrm{~B}_{3 g}$ state of tetrazine is missing, since TDDFT/B3LYP cannot describe the double-excitation character of
} this state. 
Table S9 Deviations of OMx/MRCI- and TDDFT/B3LYP-computed vertical triplet excitation energies (in eV) from MS-CASPT2/aug-cc-pVTZ values separated into molecule classes.

\begin{tabular}{|c|c|c|c|c|}
\hline & "OM1/MRCI & "OM2/MRCI & OM3/MRCI & TDDFT/B3LYP \\
\hline \multicolumn{5}{|c|}{$\mathrm{CH}$-containing molecules } \\
\hline count & 27 & 27 & 27 & 27 \\
\hline mean deviation & -0.32 & -0.27 & -0.25 & -0.34 \\
\hline mean absolute deviation & 0.43 & 0.40 & 0.39 & 0.35 \\
\hline standard deviation & 0.49 & 0.48 & 0.46 & 0.41 \\
\hline maximum deviation & -1.12 & -1.02 & -0.98 & -0.63 \\
\hline \multicolumn{5}{|c|}{ CHN-containing molecules } \\
\hline count & 27 & 27 & 27 & 27 \\
\hline mean deviation & -0.36 & -0.22 & -0.01 & -0.22 \\
\hline mean absolute deviation & 0.47 & 0.51 & 0.58 & 0.35 \\
\hline standard deviation & 0.58 & 0.61 & 0.69 & 0.44 \\
\hline maximum deviation & -1.19 & -1.30 & -1.44 & +1.33 \\
\hline \multicolumn{5}{|c|}{ CHO-containing molecules } \\
\hline count & 10 & 10 & 10 & 10 \\
\hline mean deviation & -0.33 & -0.28 & -0.18 & -0.56 \\
\hline mean absolute deviation & 0.42 & 0.40 & 0.35 & 0.56 \\
\hline standard deviation & 0.53 & 0.49 & 0.43 & 0.62 \\
\hline maximum deviation & -0.90 & -0.80 & -0.75 & -0.81 \\
\hline \multicolumn{5}{|c|}{ CHNO-containing molecules } \\
\hline count & 6 & 6 & 6 & 6 \\
\hline mean deviation & -1.00 & -0.98 & -0.76 & -0.53 \\
\hline mean absolute deviation & 1.00 & 0.98 & 0.76 & 0.53 \\
\hline standard deviation & 1.11 & 1.08 & 0.84 & 0.59 \\
\hline maximum deviation & -1.16 & -1.16 & -0.96 & -0.75 \\
\hline \multicolumn{5}{|l|}{ all molecules } \\
\hline count & 70 & 70 & 70 & 70 \\
\hline mean deviation & -0.40 & -0.32 & -0.19 & -0.34 \\
\hline mean absolute deviation & 0.49 & 0.49 & 0.49 & 0.39 \\
\hline standard deviation & 0.59 & 0.58 & 0.58 & 0.46 \\
\hline maximum deviation & -1.19 & -1.30 & -1.44 & +1.33 \\
\hline
\end{tabular}




\section{32-Molecules Benchmark Set on Excited-State Equilibrium Geometries}


Table S10 Deviations between $\mathrm{C}-\mathrm{C}, \mathrm{C}-\mathrm{H}, \mathrm{C}-\mathrm{O}, \mathrm{C}-\mathrm{N}$, and $\mathrm{N}-\mathrm{H}$ bond lengths (in $\AA$ ) in the $n \pi^{*}$ excited-state equilibrium geometries of the 32 molecules benchmark set optimized at two different levels of theory.

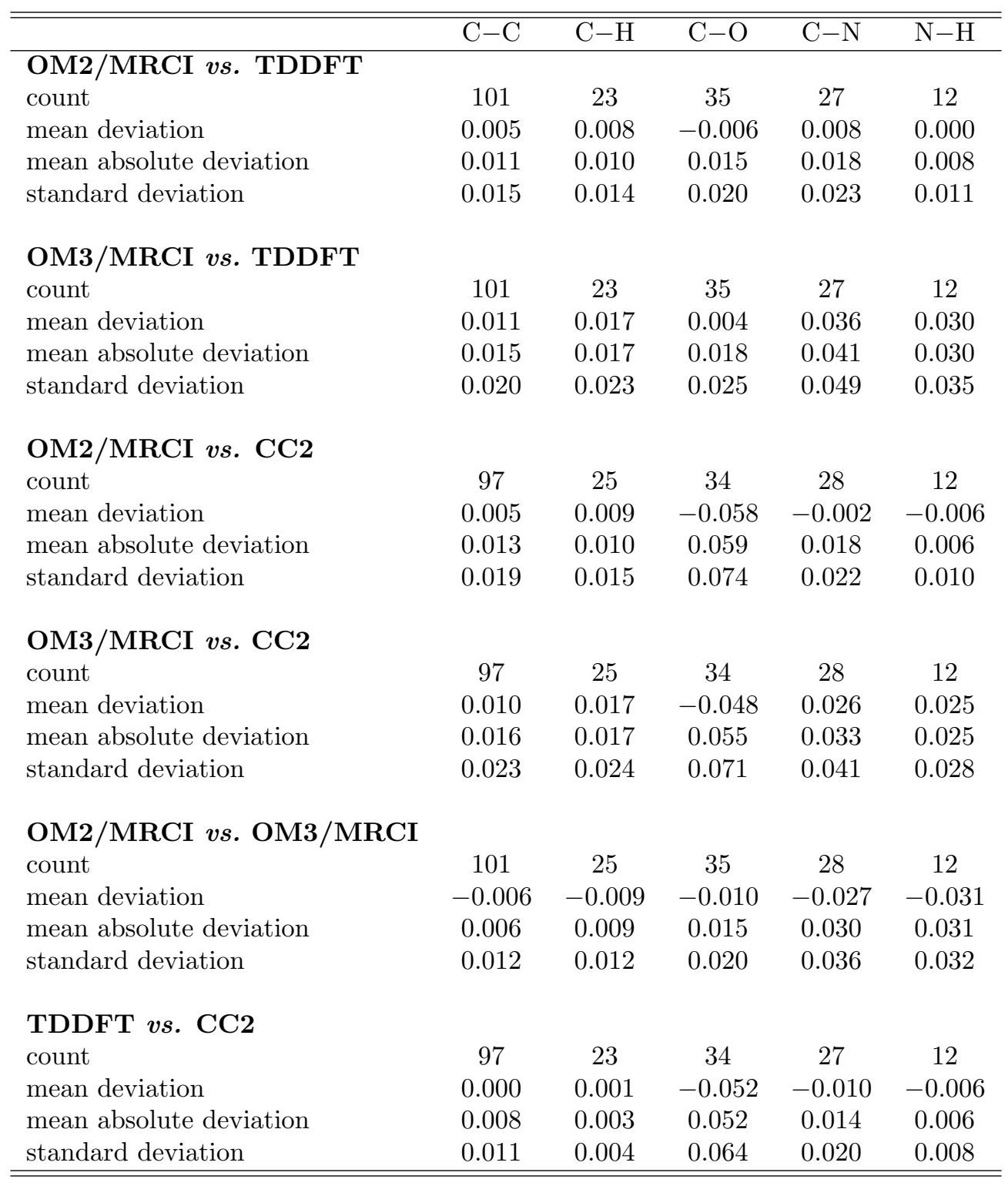


Table S11 Deviations between $\mathrm{C}-\mathrm{C}, \mathrm{C}-\mathrm{H}, \mathrm{C}-\mathrm{O}, \mathrm{C}-\mathrm{N}$, and $\mathrm{N}-\mathrm{H}$ bond lengths (in $\AA$ ) in the $\pi \pi^{*}$ excited-state equilibrium geometries of the 32 molecules benchmark set optimized at two different levels of theory.

\begin{tabular}{|c|c|c|c|c|c|}
\hline & $\overline{\mathrm{C}-\mathrm{C}}$ & $\overline{\mathrm{C}-\mathrm{H}}$ & $\overline{\mathrm{C}-\mathrm{O}}$ & $\overline{\mathrm{C}-\mathrm{N}}$ & $\overline{\mathrm{N}-\mathrm{H}}$ \\
\hline \multicolumn{6}{|c|}{ OM2/MRCI vs. TDDFT } \\
\hline count & 190 & 48 & 23 & 41 & 21 \\
\hline mean deviation & 0.002 & 0.008 & -0.006 & -0.001 & -0.004 \\
\hline mean absolute deviation & 0.018 & 0.011 & 0.020 & 0.030 & 0.010 \\
\hline standard deviation & 0.027 & 0.013 & 0.026 & 0.041 & 0.012 \\
\hline \multicolumn{6}{|c|}{ OM3/MRCI vs. TDDFT } \\
\hline count & 190 & 48 & 23 & 41 & 21 \\
\hline mean deviation & 0.009 & 0.016 & 0.005 & 0.025 & 0.029 \\
\hline mean absolute deviation & 0.020 & 0.017 & 0.024 & 0.039 & 0.029 \\
\hline standard deviation & 0.030 & 0.019 & 0.029 & 0.051 & 0.031 \\
\hline \multicolumn{6}{|l|}{ OM2/MRCI vs. CC2 } \\
\hline mean deviation & -0.002 & 0.008 & -0.022 & -0.011 & -0.007 \\
\hline mean absolute deviation & 0.013 & 0.011 & 0.029 & 0.027 & 0.010 \\
\hline standard deviation & 0.020 & 0.013 & 0.039 & 0.037 & 0.012 \\
\hline \multicolumn{6}{|l|}{ OM3/MRCI vs. CC2 } \\
\hline count & 201 & 50 & 28 & 40 & 21 \\
\hline mean deviation & 0.005 & 0.016 & -0.011 & 0.016 & 0.026 \\
\hline mean absolute deviation & 0.014 & 0.017 & 0.025 & 0.031 & 0.026 \\
\hline standard deviation & 0.022 & 0.019 & 0.035 & 0.043 & 0.028 \\
\hline \multicolumn{6}{|c|}{ OM2/MRCI vs. OM3/MRCI } \\
\hline $\begin{array}{l}\text { count } \\
\text { mean deviation }\end{array}$ & $\begin{array}{l}209 \\
-0.007\end{array}$ & $\begin{array}{c}56 \\
-0.008\end{array}$ & $\begin{array}{c}28 \\
-0.011\end{array}$ & $\begin{array}{c}42 \\
-0.026\end{array}$ & $\begin{array}{c}22 \\
-0.034\end{array}$ \\
\hline mean absolute deviation & 0.007 & 0.008 & 0.011 & 0.026 & 0.034 \\
\hline standard deviation & 0.007 & 0.009 & 0.015 & 0.028 & 0.035 \\
\hline \multicolumn{6}{|l|}{ TDDFT vs. CC2 } \\
\hline count & 182 & 42 & 23 & 39 & 20 \\
\hline mean deviation & -0.004 & 0.000 & -0.008 & -0.008 & -0.002 \\
\hline mean absolute deviation & 0.013 & 0.002 & 0.018 & 0.024 & 0.003 \\
\hline standard deviation & 0.021 & 0.003 & 0.028 & 0.038 & 0.005 \\
\hline
\end{tabular}


Table S12 Deviations between $\mathrm{C}-\mathrm{C}, \mathrm{C}-\mathrm{H}, \mathrm{C}-\mathrm{O}, \mathrm{C}-\mathrm{N}$, and $\mathrm{N}-\mathrm{H}$ bond lengths (in $\AA$ ) in the singlet excited-state equilibrium geometries of the 32molecules benchmark set optimized at two different levels of theory.

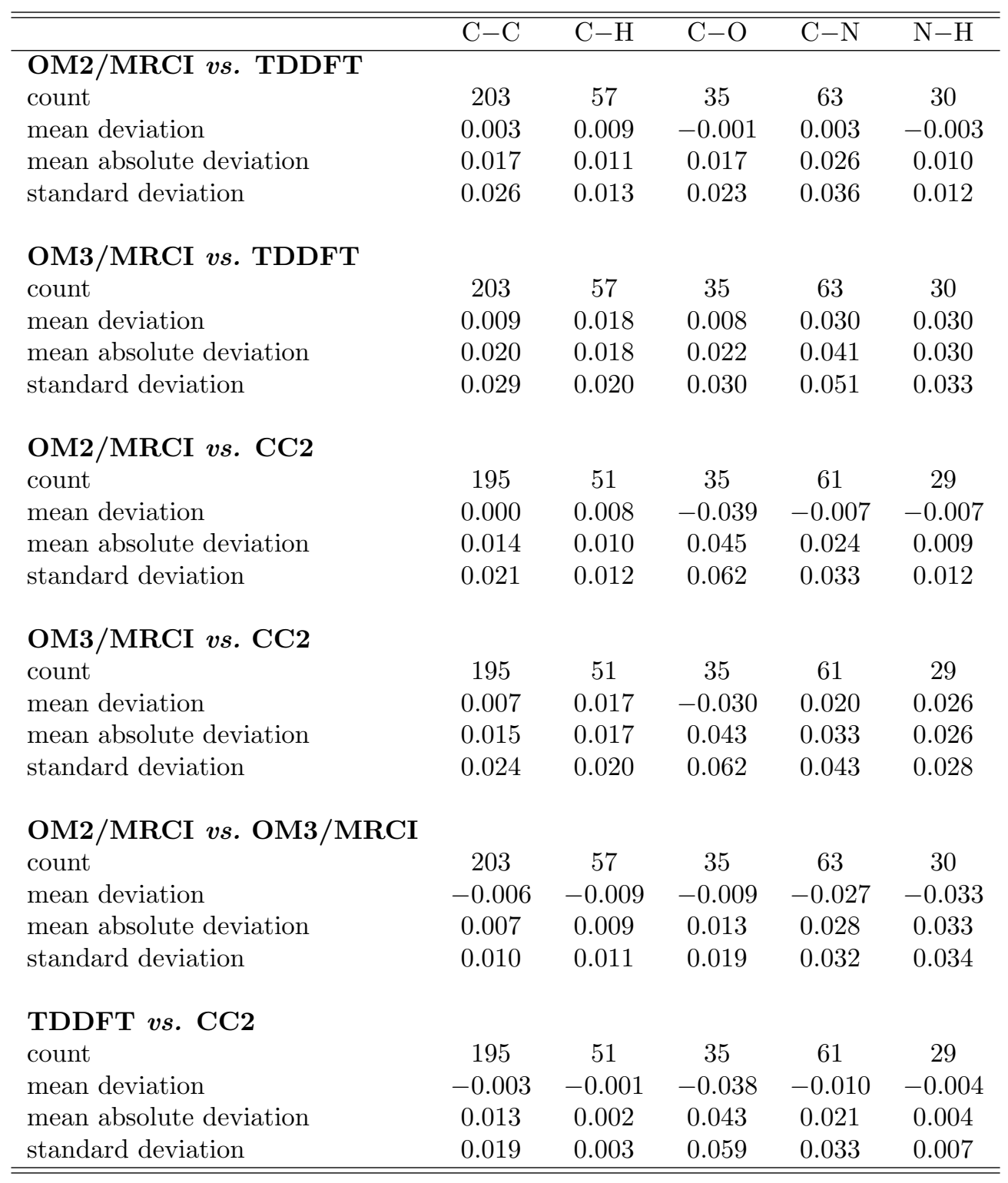


Table S13 Deviations between $\mathrm{C}-\mathrm{C}, \mathrm{C}-\mathrm{H}, \mathrm{C}-\mathrm{O}, \mathrm{C}-\mathrm{N}$, and $\mathrm{N}-\mathrm{H}$ bond lengths (in $\AA$ ) in the triplet excited-state equilibrium geometries of the 32molecules benchmark set optimized at two different levels of theory.

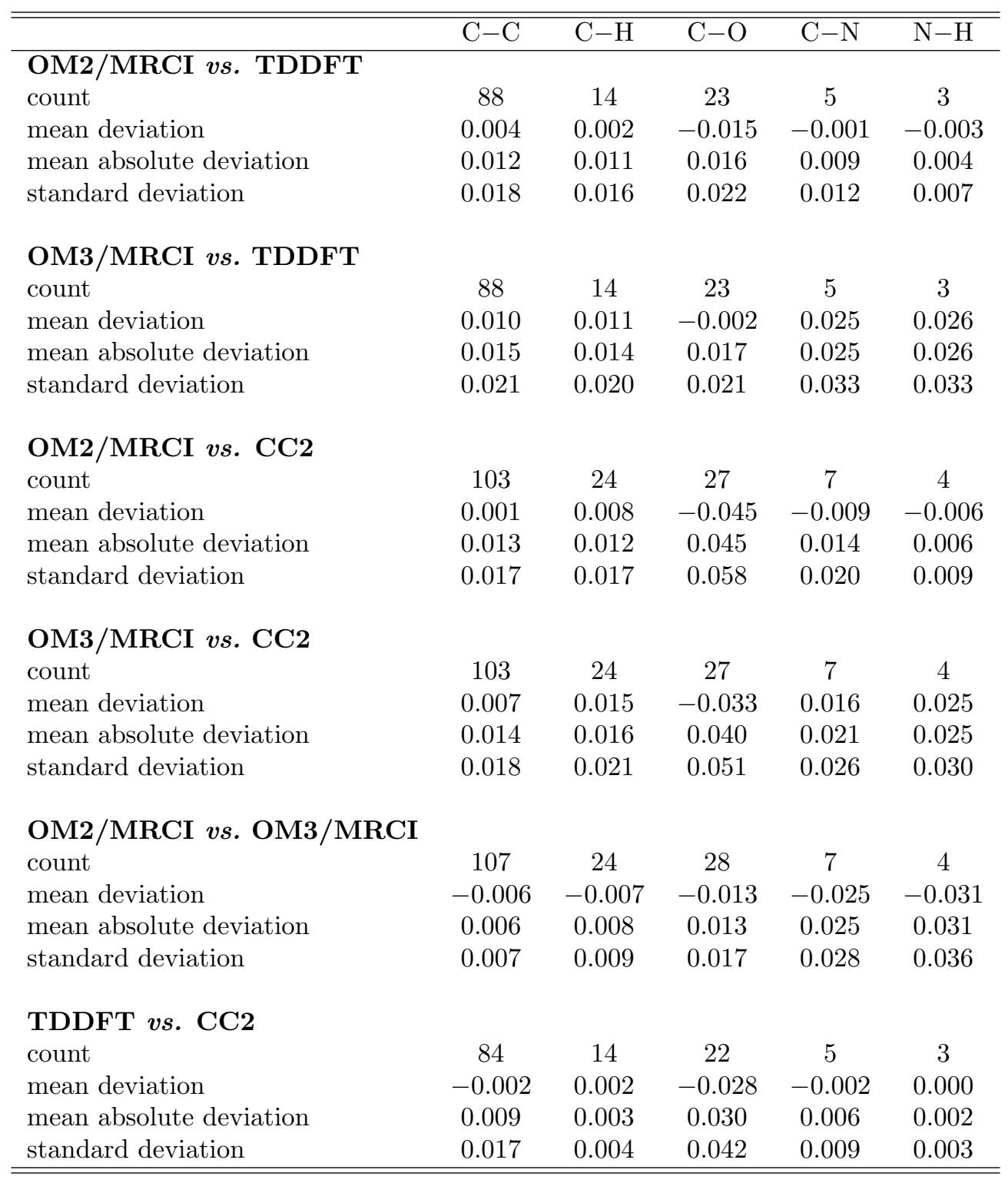



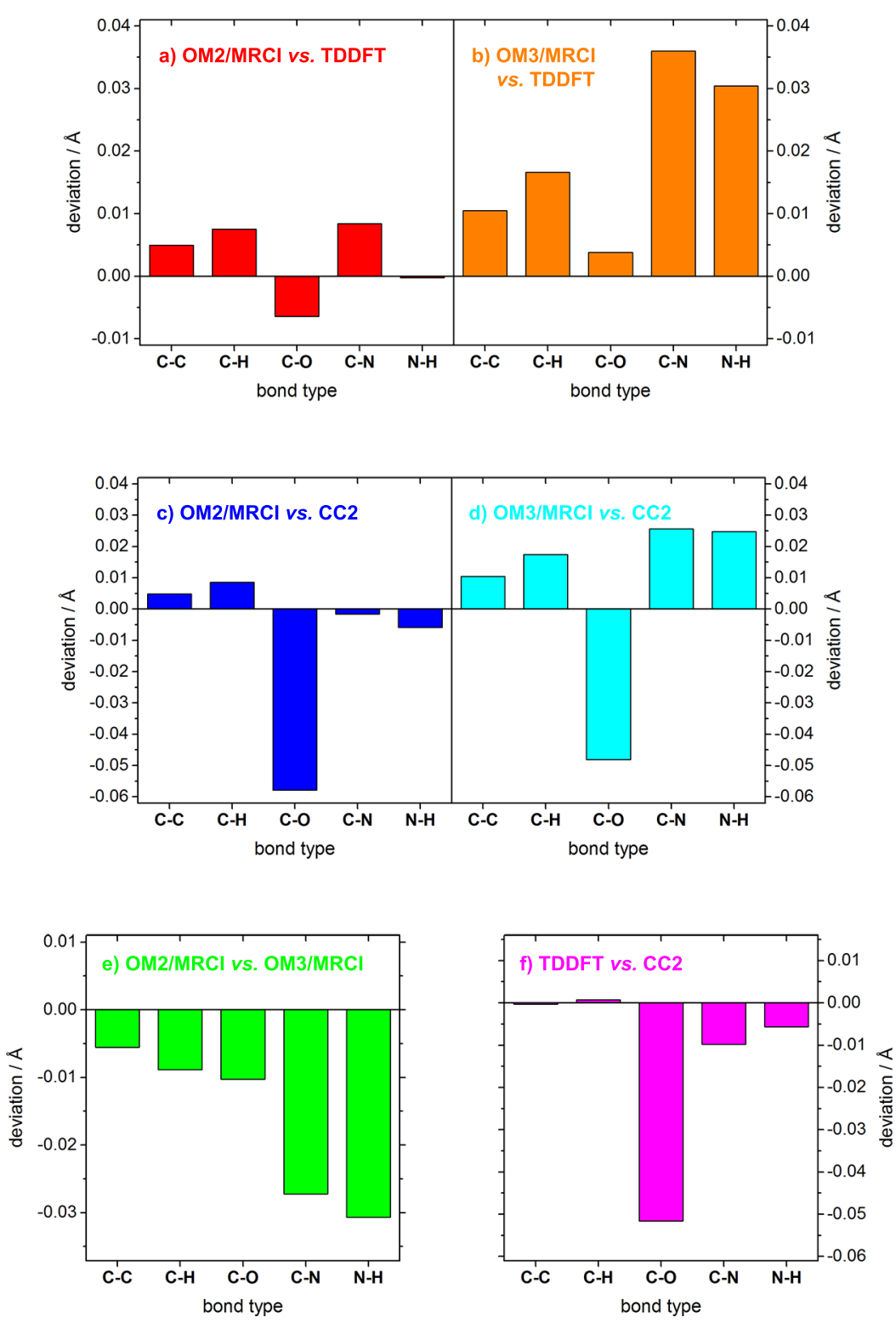

Figure S6 Comparison of $\mathrm{C}-\mathrm{C}, \mathrm{C}-\mathrm{H}, \mathrm{C}-\mathrm{O}, \mathrm{C}-\mathrm{N}$, and $\mathrm{N}-\mathrm{H}$ bond lengths in the optimized $n \pi^{*}$ excited-state equilibrium geometries of the 32-molecules benchmark set: mean deviations (in $\AA$ ) for all pairs of methods considered. 

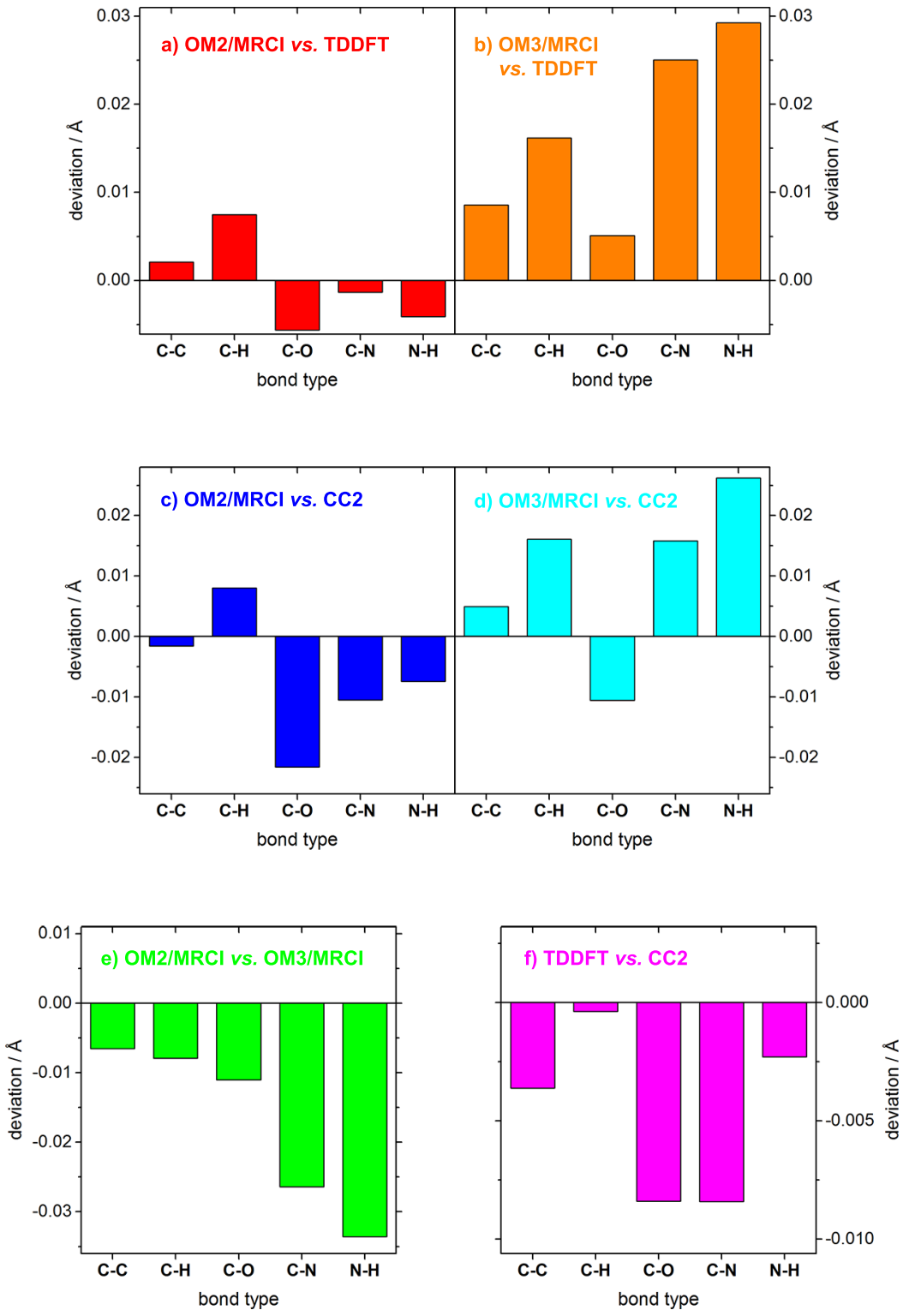

Figure S7 Comparison of $\mathrm{C}-\mathrm{C}, \mathrm{C}-\mathrm{H}, \mathrm{C}-\mathrm{O}, \mathrm{C}-\mathrm{N}$, and $\mathrm{N}-\mathrm{H}$ bond lengths in the optimized $\pi \pi^{*}$ excited-state equilibrium geometries of the 32-molecules benchmark set: mean deviations (in $\AA$ ) for all pairs of methods considered. 

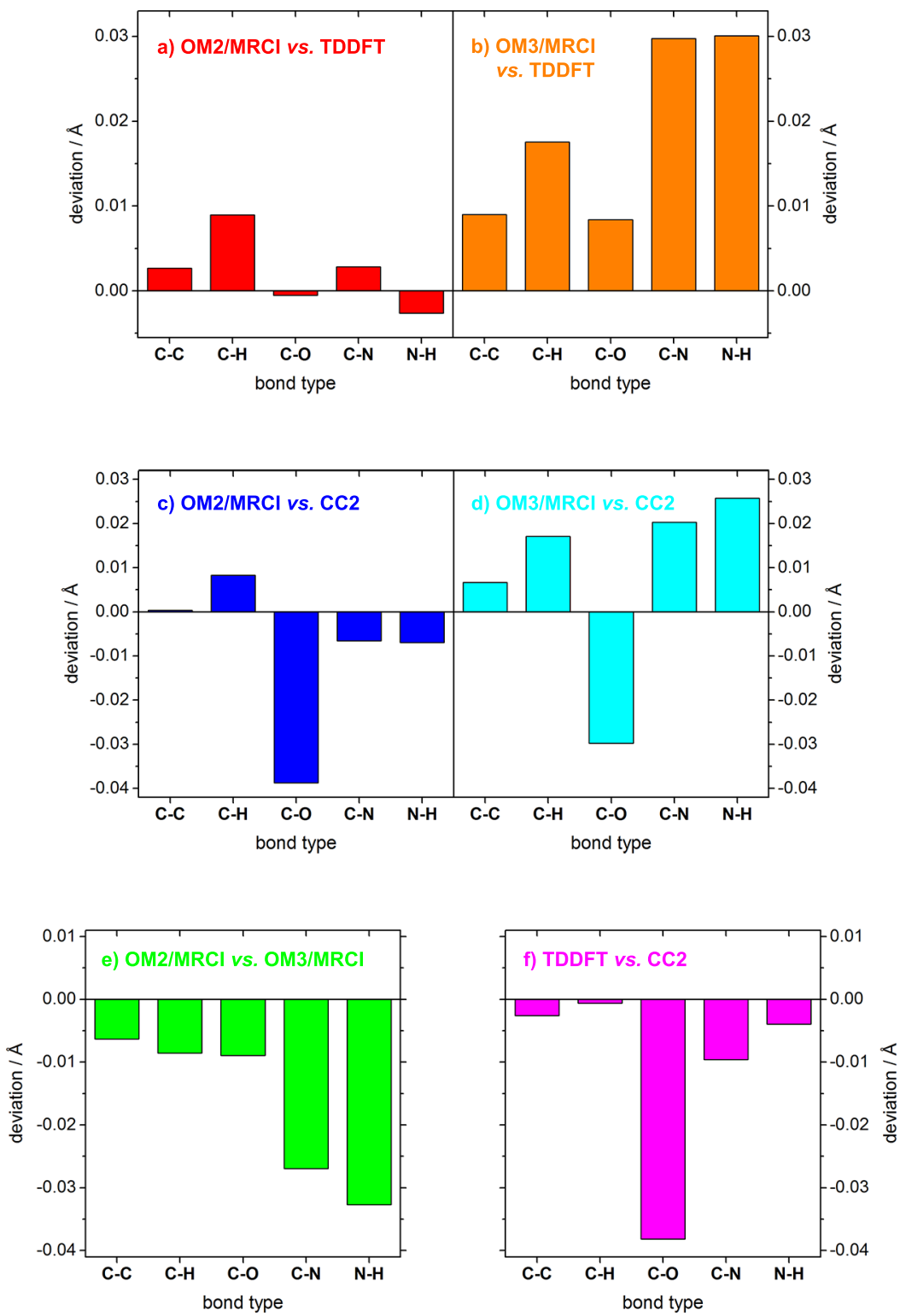

Figure S8 Comparison of $\mathrm{C}-\mathrm{C}, \mathrm{C}-\mathrm{H}, \mathrm{C}-\mathrm{O}, \mathrm{C}-\mathrm{N}$, and $\mathrm{N}-\mathrm{H}$ bond lengths in the optimized singlet excited-state equilibrium geometries of the 32-molecules benchmark set: mean deviations (in $\AA$ ) for all pairs of methods considered. 

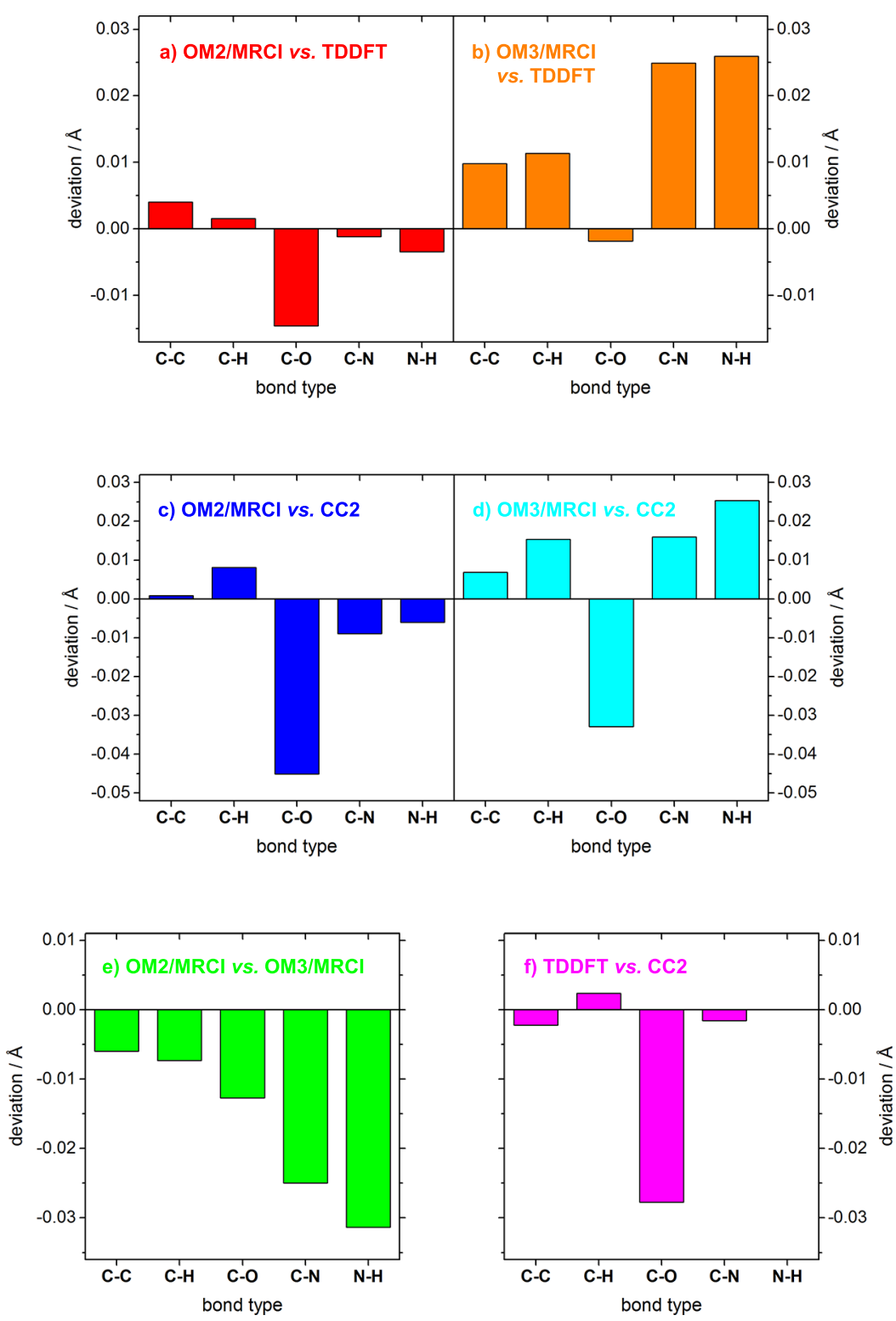

Figure S9 Comparison of $\mathrm{C}-\mathrm{C}, \mathrm{C}-\mathrm{H}, \mathrm{C}-\mathrm{O}, \mathrm{C}-\mathrm{N}$, and $\mathrm{N}-\mathrm{H}$ bond lengths in the optimized triplet excited-state equilibrium geometries of the 32-molecules benchmark set: mean deviations (in $\AA$ ) for all pairs of methods considered. 

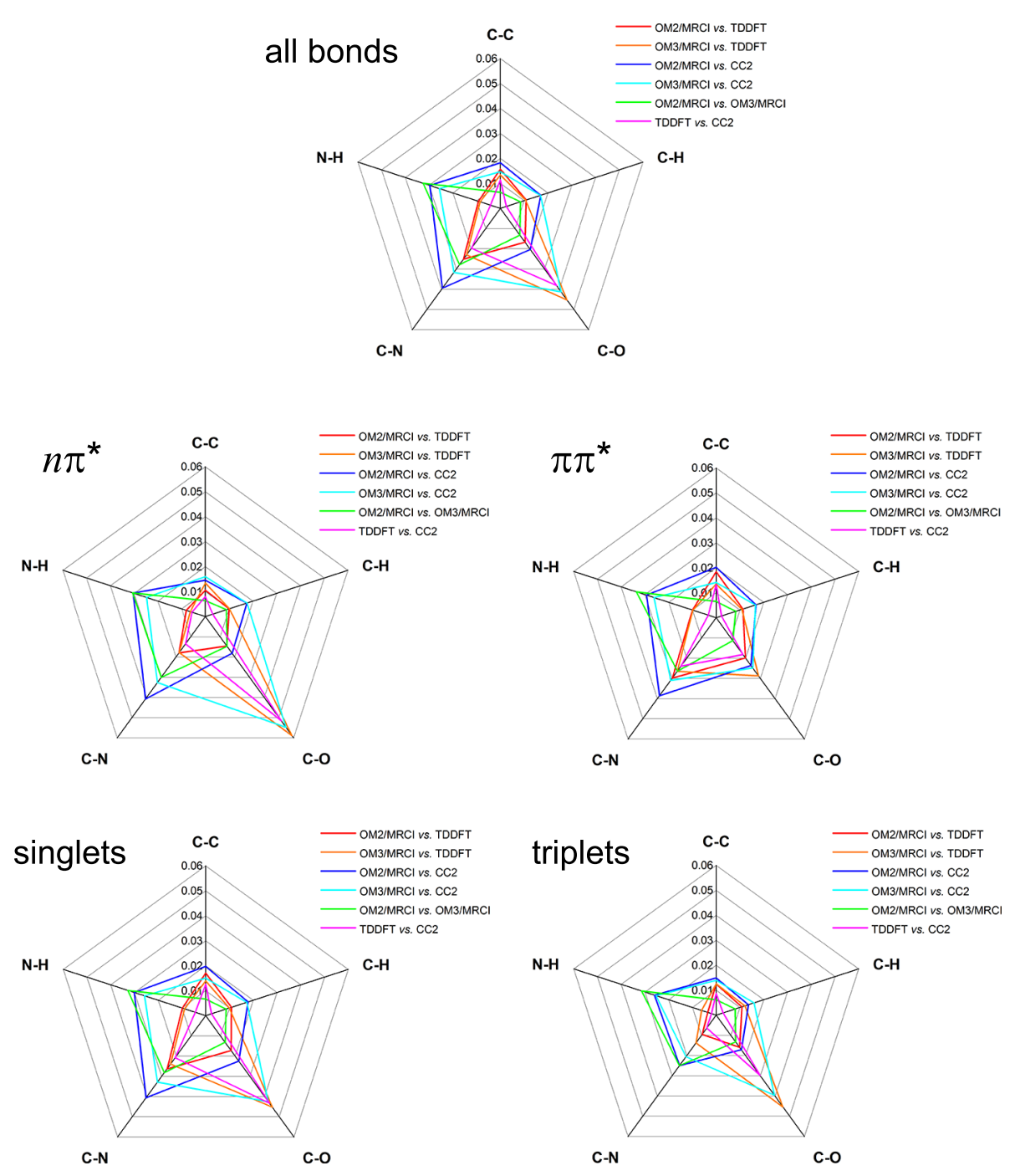

Figure S10 Comparison of $\mathrm{C}-\mathrm{C}, \mathrm{C}-\mathrm{H}, \mathrm{C}-\mathrm{O}, \mathrm{C}-\mathrm{N}$, and $\mathrm{N}-\mathrm{H}$ bond lengths in the optimized excited-state equilibrium geometries of the 32-molecules benchmark set (total set and subsets of $n \pi^{*}, \pi \pi^{*}$, singlet, and triplet states): mean absolute deviations (in $\AA$ ) for all pairs of methods considered. 
Table S14 Deviations between bond angles (in ${ }^{\circ}$ ) in the excited-state equilibrium geometries of the 32-molecules benchmark set optimized at two different levels of theory (subsets of $n \pi^{*}, \pi \pi^{*}$, singlet, and triplet states and total set).

\begin{tabular}{|c|c|c|c|c|c|}
\hline & $\overline{2 n \pi^{*}}$ & 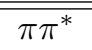 & singlets & triplets & $\overline{\text { total }}$ \\
\hline \multicolumn{6}{|c|}{ OM2/MRCI vs. TDDFT } \\
\hline mean deviation & -0.5 & -0.2 & -0.4 & -0.2 & -0.3 \\
\hline mean absolute deviation & 1.8 & 1.9 & 1.8 & 2.0 & 1.9 \\
\hline standard deviation & 2.7 & 3.0 & 2.7 & 3.2 & 2.9 \\
\hline \multicolumn{6}{|c|}{ OM3/MRCI vs. TDDFT } \\
\hline count & 104 & 174 & 201 & 77 & 278 \\
\hline mean deviation & -0.7 & -0.1 & -0.3 & -0.4 & -0.3 \\
\hline mean absolute deviation & 1.8 & 1.9 & 1.8 & 2.0 & 1.9 \\
\hline standard deviation & 2.8 & 3.1 & 2.9 & 3.2 & 2.9 \\
\hline \multicolumn{6}{|l|}{ OM2/MRCI vs. CC2 } \\
\hline mean deviation & -0.3 & 0.0 & -0.1 & 0.0 & -0.1 \\
\hline mean absolute deviation & 2.2 & 1.9 & 2.0 & 2.0 & 2.0 \\
\hline standard deviation & 3.5 & 3.0 & 3.2 & 3.3 & 3.2 \\
\hline \multicolumn{6}{|l|}{ OM3/MRCI vs. CC2 } \\
\hline $\begin{array}{l}\text { count } \\
\text { mean deviation }\end{array}$ & $\begin{array}{c}104 \\
-0.5\end{array}$ & $\begin{array}{l}183 \\
0.1\end{array}$ & $\begin{array}{c}187 \\
-0.1\end{array}$ & $\begin{array}{c}100 \\
-0.1\end{array}$ & $\begin{array}{c}287 \\
-0.1\end{array}$ \\
\hline mean absolute deviation & 2.3 & 1.8 & 2.1 & 1.9 & 2.0 \\
\hline standard deviation & 3.8 & 3.2 & 3.4 & 3.4 & 3.4 \\
\hline \multicolumn{6}{|c|}{ OM2/MRCI vs. OM3/MRCI } \\
\hline $\begin{array}{l}\text { count } \\
\text { mean deviation }\end{array}$ & $\begin{array}{l}108 \\
0.2\end{array}$ & $\begin{array}{c}197 \\
-0.1\end{array}$ & $\begin{array}{c}201 \\
-0.1\end{array}$ & $\begin{array}{l}104 \\
0.2\end{array}$ & $\begin{array}{l}305 \\
0.0\end{array}$ \\
\hline mean absolute deviation & 1.1 & 0.6 & 0.8 & 0.8 & 0.8 \\
\hline standard deviation & 1.4 & 1.1 & 1.3 & 1.2 & 1.2 \\
\hline \multicolumn{6}{|l|}{ TDDFT vs. CC2 } \\
\hline count & 100 & 160 & 187 & 73 & 260 \\
\hline mean deviation & 0.1 & 0.2 & 0.3 & -0.1 & 0.2 \\
\hline mean absolute deviation & 1.1 & 0.9 & 0.9 & 1.0 & 1.0 \\
\hline standard deviation & 1.8 & 1.4 & 1.5 & 1.8 & 1.6 \\
\hline
\end{tabular}



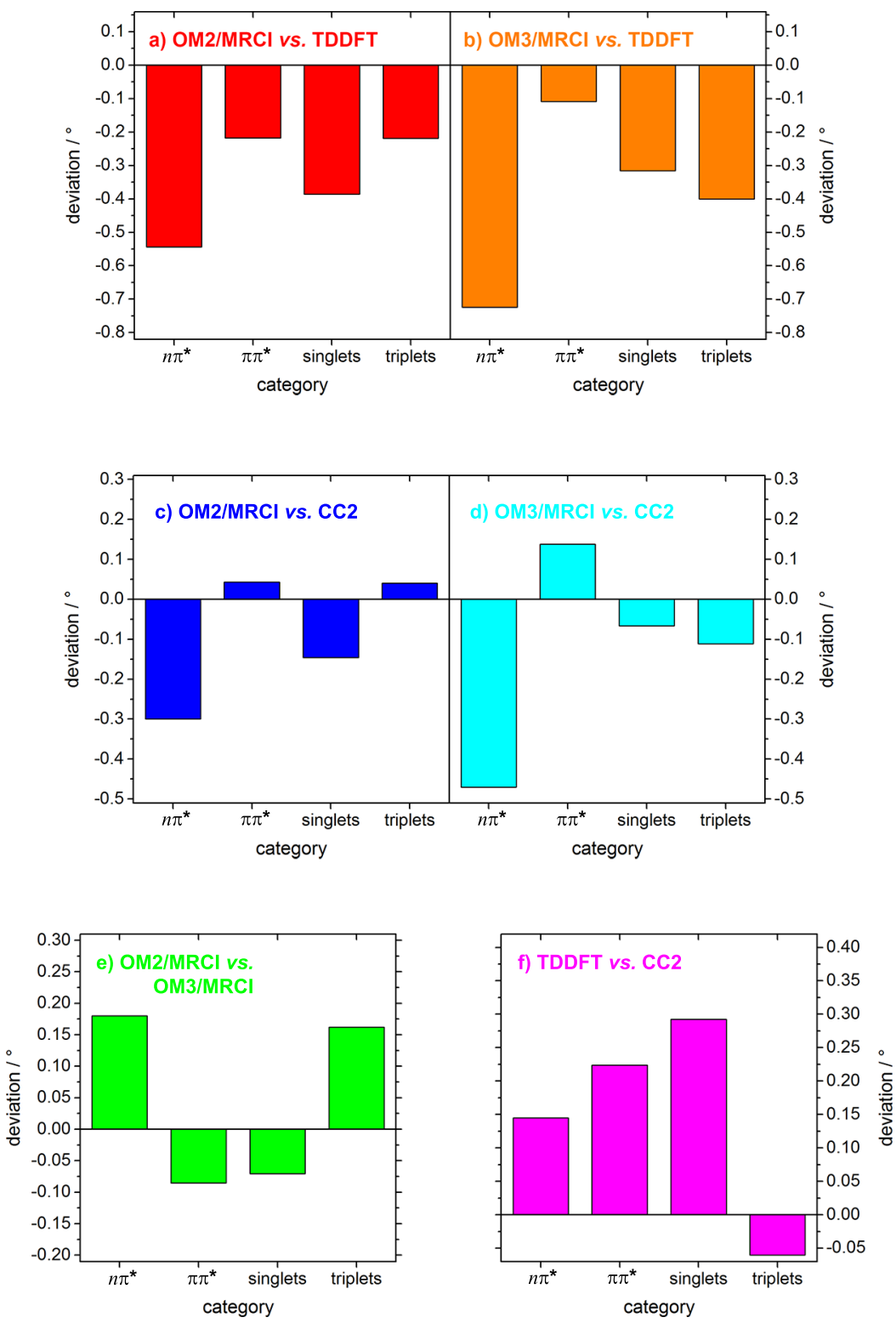

Figure S11 Comparison of bond angles in the optimized excited-state equilibrium geometries of the 32 -molecules benchmark set: Mean deviations (in ${ }^{\circ}$ ) for all pairs of methods considered. 


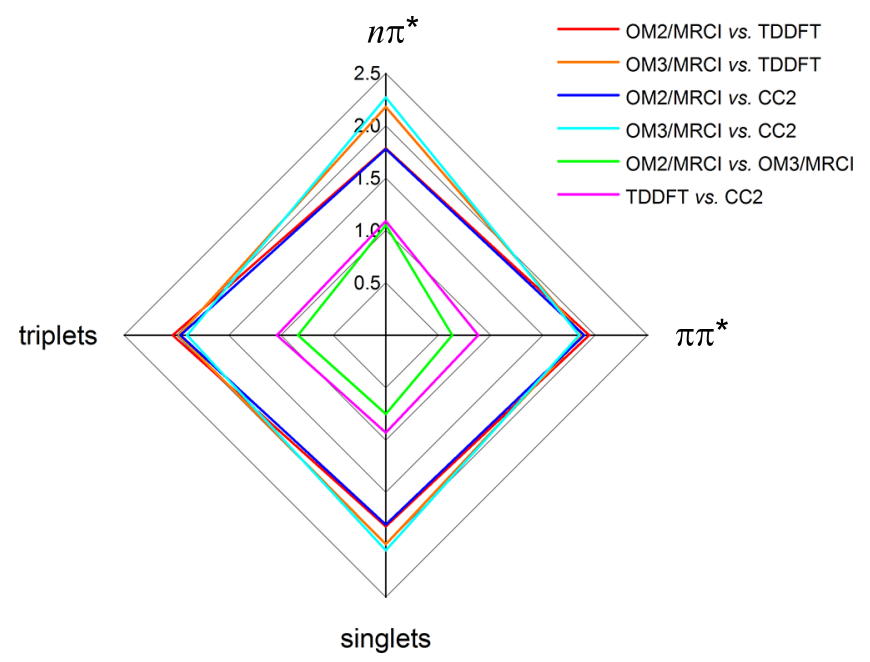

Figure S12 Comparison of bond angles in the optimized excited-state equilibrium geometries of the 32-molecules benchmark set: mean absolute deviations $\left(\right.$ in $\left.^{\circ}\right)$ for all pairs of methods considered. 
3 Comparisons of ZPVEs and $\triangle \mathrm{ZPVE}$ 


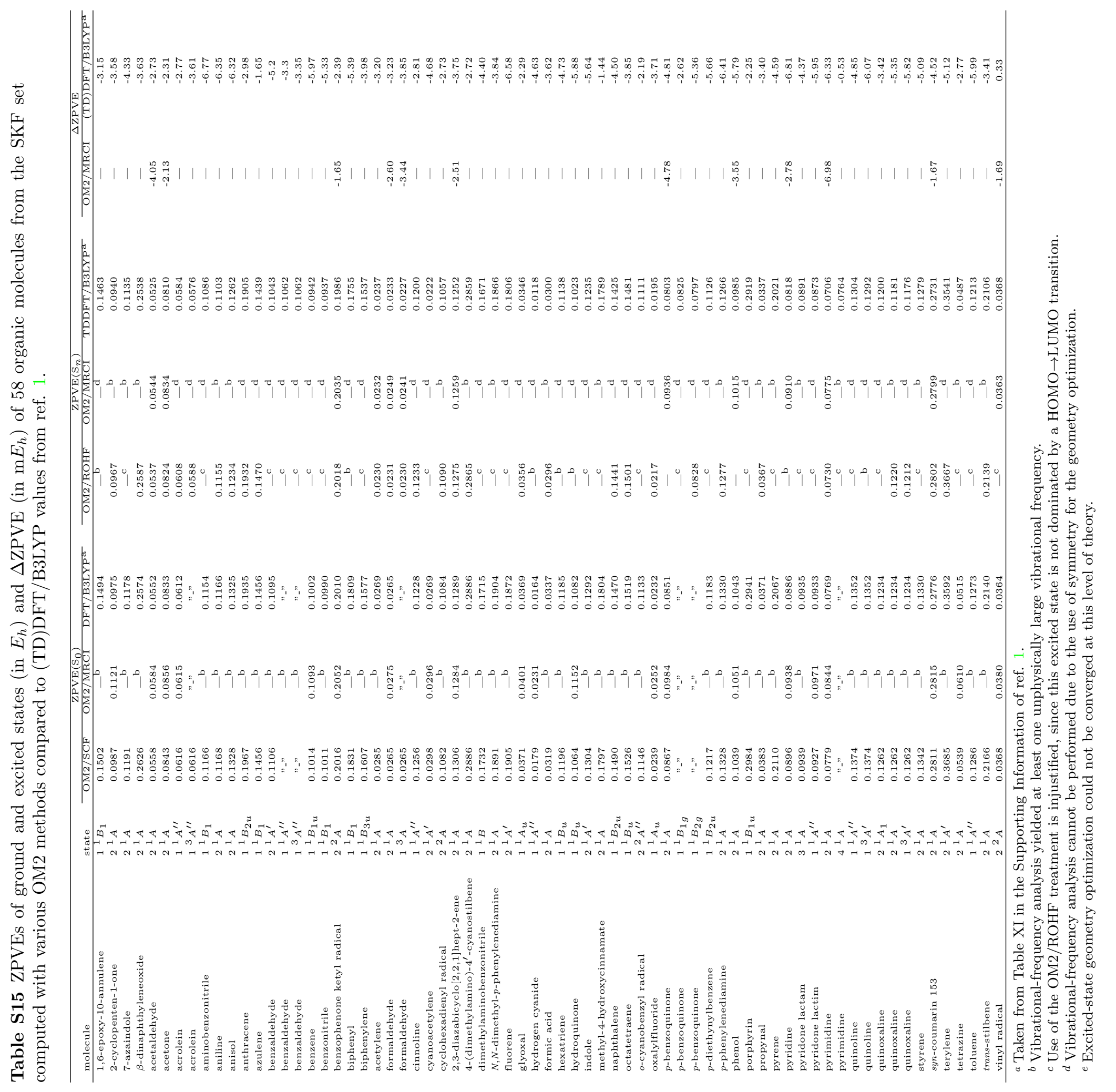


Table S16 Deviations of ground- and excited-state ZPVEs (in $\mathrm{E}_{h}$ ) and $\Delta$ ZPVE (in $\mathrm{mE}_{h}$ ) computed at various variants of the OM2 method against literature values obtained at the (TD)DFT/B3LYP for the SKF set.[1]

\begin{tabular}{|c|c|}
\hline & "OM2/SCF vs. DFT/B3LYP \\
\hline \multicolumn{2}{|l|}{ ZPVEs $\left(\mathbf{S}_{0}\right)$} \\
\hline count & 59 \\
\hline mean deviation & 0.00 \\
\hline mean absolute deviation & 0.00 \\
\hline standard deviation & 0.00 \\
\hline \multirow[t]{2}{*}{ maximum deviation } & +0.01 \\
\hline & OM2/ROHF vs. TDDFT/B3LYP \\
\hline \multicolumn{2}{|l|}{$\operatorname{ZPVEs}\left(\mathbf{S}_{n}\right)$} \\
\hline count & 32 \\
\hline mean deviation & 0.00 \\
\hline mean absolute deviation & 0.00 \\
\hline standard deviation & 0.00 \\
\hline \multirow[t]{2}{*}{ maximum deviation } & +0.01 \\
\hline & OM2/MRCI vs. DFT/B3LYP \\
\hline \multicolumn{2}{|l|}{$\operatorname{ZPVEs}\left(\mathbf{S}_{0}\right)$} \\
\hline count & 21 \\
\hline mean deviation & 0.00 \\
\hline mean absolute deviation & 0.00 \\
\hline standard deviation & 0.01 \\
\hline \multirow[t]{2}{*}{ maximum deviation } & +0.01 \\
\hline & OM2/MRCI vs. TDDFT/B3LYP \\
\hline \multicolumn{2}{|l|}{ ZPVEs $\left(\mathbf{S}_{n}\right)$} \\
\hline count & 13 \\
\hline mean deviation & 0.00 \\
\hline mean absolute deviation & 0.00 \\
\hline standard deviation & 0.06 \\
\hline \multirow[t]{2}{*}{ maximum deviation } & +0.01 \\
\hline & OM2/MRCI vs. (TD)DFT/B3LYP \\
\hline \multicolumn{2}{|l|}{$\triangle \mathrm{ZPVE}$} \\
\hline count & 12 \\
\hline mean deviation & 0.70 \\
\hline mean absolute deviation & 1.36 \\
\hline standard deviation & 1.87 \\
\hline maximum deviation & +4.03 \\
\hline
\end{tabular}


Table S17 Deviations of OM2/MRCI- and (TD)DFT/B3LYP-computed 0-0 transition energies (in $\mathrm{eV}$ ) from spectroscopically measured $0-0$ band origins for the SKF set[1]: subsets of $n \pi^{*}$ and $\pi \pi^{*}$ states. $^{a}$

\begin{tabular}{lccc}
\hline \hline & OM2/MRCI & OM3/MRCI $^{b}$ & TDDFT/B3LYP \\
\hline$n \pi^{*}$ states & & & \\
count & 23 & 22 & 23 \\
mean error & 0.07 & 0.06 & -0.17 \\
mean absolute error & 0.20 & 0.22 & 0.23 \\
standard error & 0.25 & 0.28 & 0.28 \\
maximum error & +0.51 & +0.59 & -0.57 \\
& & & \\
$\pi \pi^{*}$ states & & 43 & 44 \\
count & 44 & -0.08 & -0.08 \\
mean error & -0.04 & 0.30 & 0.25 \\
mean absolute error & 0.30 & 0.36 & 0.33 \\
standard error & 0.36 & +0.72 & -0.73 \\
maximum error & +0.91 & & \\
\hline \hline
\end{tabular}

${ }^{a}$ The excited state evaluated for the vinyl radical is a $\pi \sigma^{*}$ state and is thus not considered in this evaluation.

${ }^{b}$ For OM3/MRCI, the $\pi \pi^{*}$ state of the benzophenone ketyl radical and the $n \pi^{*}$ state of 2,3-diazabicyclo[2,2,1] hept-2-ene are missing due to convergence problems. 
Table S18 Deviations of 0-0 transition energies (in eV) computed at the full OM2/MRCI level, at the mixed OM2/MRCI//(TD)DFT level, and at the (TD)DFT level.

\begin{tabular}{lcc}
\hline \hline & OM2/MRCI vs. OM2/MRCI//(TD)DFT & OM2/MRCI vs. (TD)DFT \\
\hline & 13 & 13 \\
count & 0.02 & 0.10 \\
mean deviation & 0.04 & 0.24 \\
mean absolute deviation & 0.05 & 0.30 \\
standard deviation & +0.11 & +0.68 \\
maximum deviation &
\end{tabular}




\section{References}

[1] R. Send, M. Kühn, F. Furche, J. Chem. Theory Comput. 2011, 7, $2376-2386$. 


\section{Presentation of Raw Data Necessary for Re- producibility}

N.B.: According to the standard output format of the respective quantumchemical program, the total energies of the geometries obtained by the MNDO program are specified in $\mathrm{eV}$, whereas the total energies of the geometries obtained by Turbomole are specified in $E_{h}$. All Cartesian coordinates are given in $\AA$. All specified bond lengths (or changes thereof) are given in $\AA$, all specified bond angles (or changes thereof) are given in ${ }^{\circ}$.

In point groups with equivalent symmetry operations (i.e. $C_{2 v}, D_{2}$, and $D_{2 h}$ ) some of the irreducible representations depend on the definition of the coordinate axes within the molecule. Therefore, the specification of the excited states in such point groups can be ambiguous (e.g. $B_{1}$ and $B_{2}$ states in $C_{2 v}$-symmetric systems). Since Turbomole uses an automatic procedure for the definition of the coordinate axes within the molecule that differs from our standard way of defining the axes in MNDO, some of the excited states of molecules optimized in these three point groups are found to belong to different irreducible representations in MNDO and in Turbomole. In such cases, we specify which of the states found in Turbomole corresponds to the state found in MNDO. 
4.1 32-Molecules Benchmark Set on Excited-State Equilibrium Geometries 


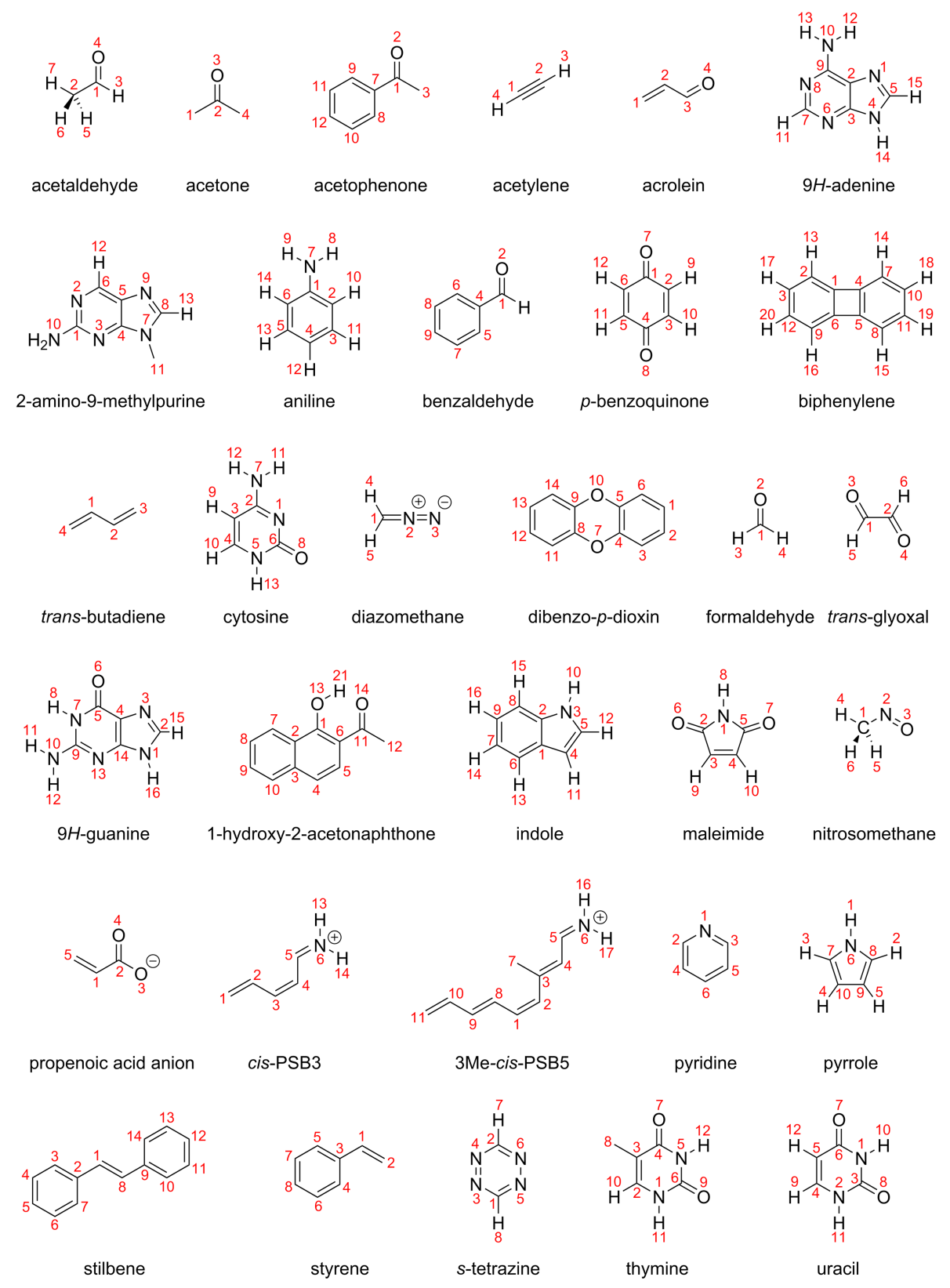

Figure S13 Structural formulas of the 32 molecules in the benchmark set on excited-state equilibrium geometries. Atom numbers referring to the structural parameters extracted for the statistical analyses (presented in the tables below) are given in red font. 
4.1.1 OM2/MRCI-Optimized Excited-State Minima 
Table S19 Employed active space, reference configurations, and Cartesian coordinates of the OM2/MRCISD-optimized excited-state equilibrium geometry of the $2{ }^{1} A\left({ }^{1} n \pi^{*}\right)$ state of $9 H$-adenine (excited-state energy: $\left.-1774.17179 \mathrm{eV}\right)$.

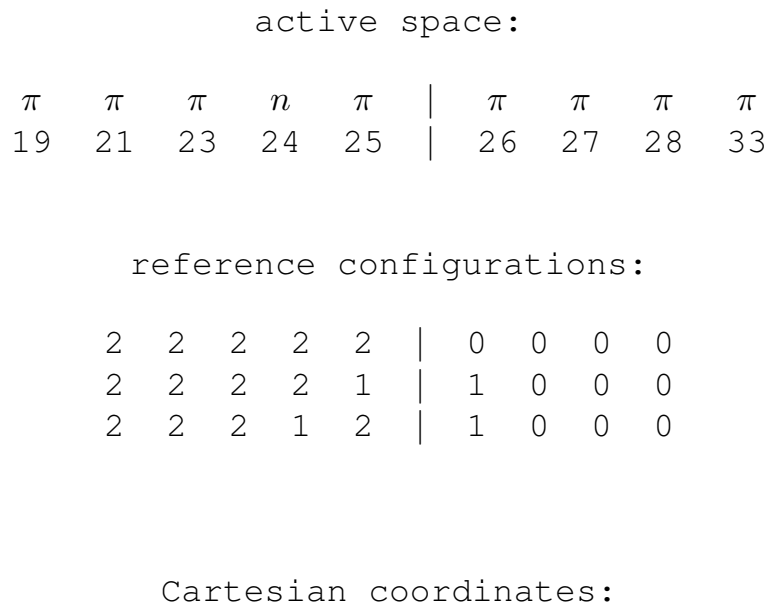


Table S20 Employed active space, reference configurations, and Cartesian coordinates of the OM2/MRCISD-optimized excited-state equilibrium geometry of the $2{ }^{1} A\left({ }^{1} n \pi^{*}\right)$ state of acetaldehyde (excited-state energy: $\left.-643.08094 \mathrm{eV}\right)$.

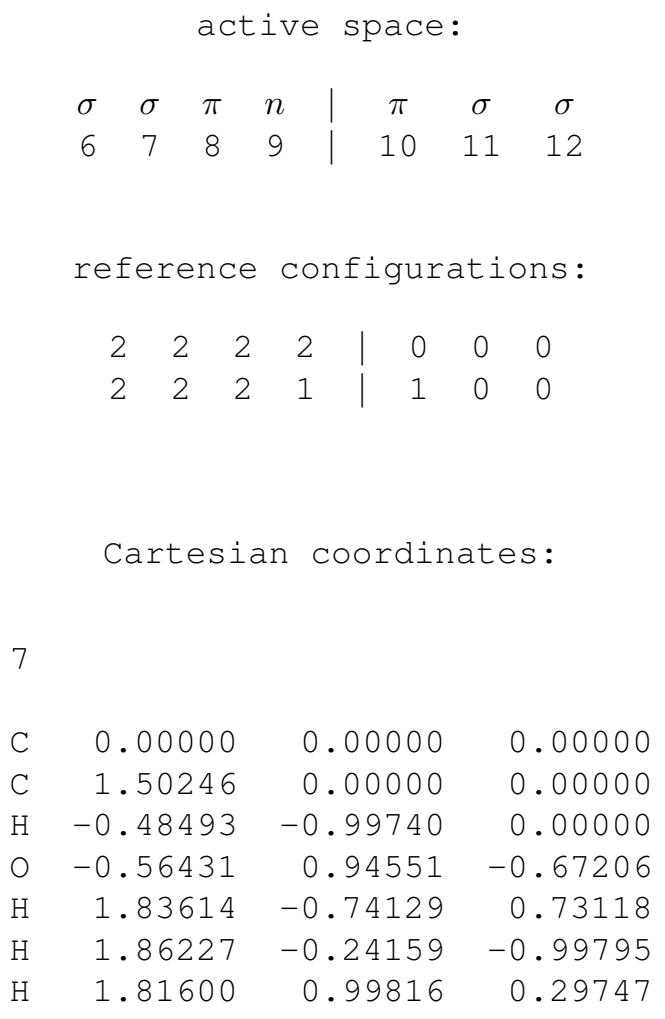


Table S21 Employed active space, reference configurations, and Cartesian coordinates of the OM2/MRCISD-optimized excited-state equilibrium geometry of the $1^{3} A\left({ }^{3} n \pi^{*}\right)$ state of acetaldehyde (excited-state energy: $\left.-643.45298 \mathrm{eV}\right)$.

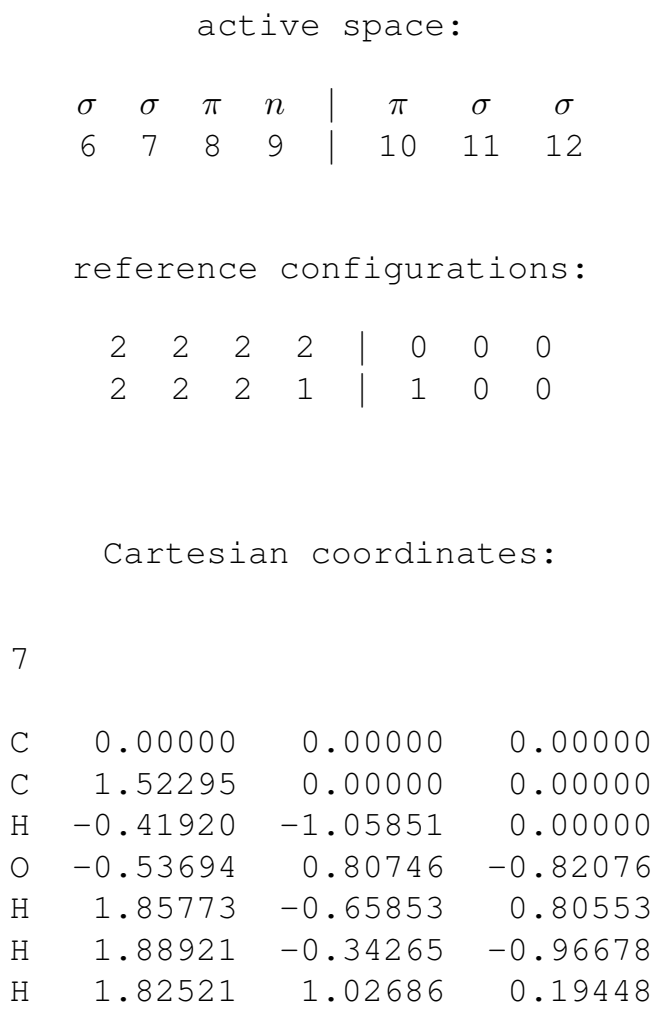


Table S22 Employed active space, reference configurations, and Cartesian coordinates of the OM2/MRCISD-optimized excited-state equilibrium geometry of the $2^{3} A\left({ }^{3} \pi \pi^{*}\right)$ state of acetaldehyde (excited-state energy: $\left.-641.76050 \mathrm{eV}\right)$.

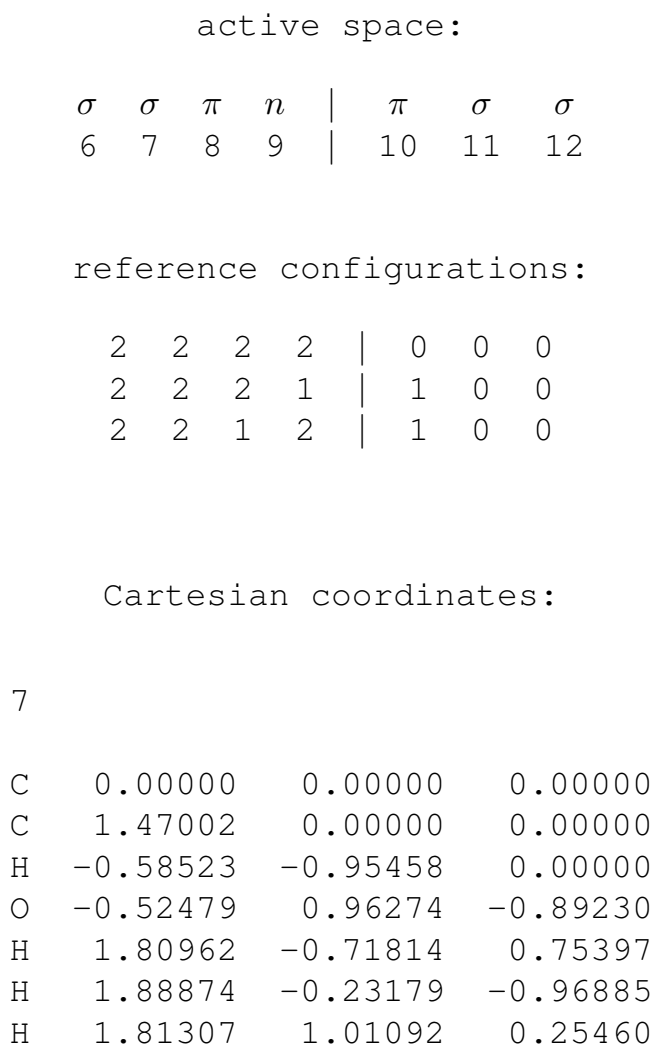


Table S23 Employed active space, reference configurations, and Cartesian coordinates of the OM2/MRCISD-optimized excited-state equilibrium geometry of the $1{ }^{1} A^{\prime \prime}\left({ }^{1} n \pi^{*}\right)$ state of acetone (excited-state energy: $\left.-800.82045 \mathrm{eV}\right)$.

\begin{tabular}{|c|c|c|c|c|}
\hline$\sigma$ & $\pi$ & $n$ & $\pi$ & $\sigma$ \\
\hline 10 & 11 & 12 & 13 & 14 \\
\hline \multicolumn{2}{|c|}{ reference } & \multicolumn{3}{|c|}{ configurations: } \\
\hline & 2 & 2 & 0 & 0 \\
\hline 2 & 2 & 1 & 1 & 0 \\
\hline \multicolumn{5}{|c|}{ Cartesian coordinates: } \\
\hline-1.29 & & & 5289 & 0.00000 \\
\hline 0.00 & & & 0000 & 0.00000 \\
\hline 0.00 & & -1 & 445 & -0.75911 \\
\hline 1.29 & & & 5289 & 0.00000 \\
\hline-2.12 & & & 5553 & 0.17506 \\
\hline-1.43 & & & 4542 & -0.97355 \\
\hline-1.27 & 17 & & 9955 & 0.80168 \\
\hline 2.12 & 04 & & 5553 & 0.17506 \\
\hline 1.27 & & & 9955 & 0.80168 \\
\hline 1.43 & & & 4542 & -0.97355 \\
\hline
\end{tabular}


Table S24 Employed active space, reference configurations, and Cartesian coordinates of the OM2/MRCISD-optimized excited-state equilibrium geometry of the $1^{3} A^{\prime \prime}\left({ }^{3} n \pi^{*}\right)$ state of acetone (excited-state energy: $\left.-801.23336 \mathrm{eV}\right)$.

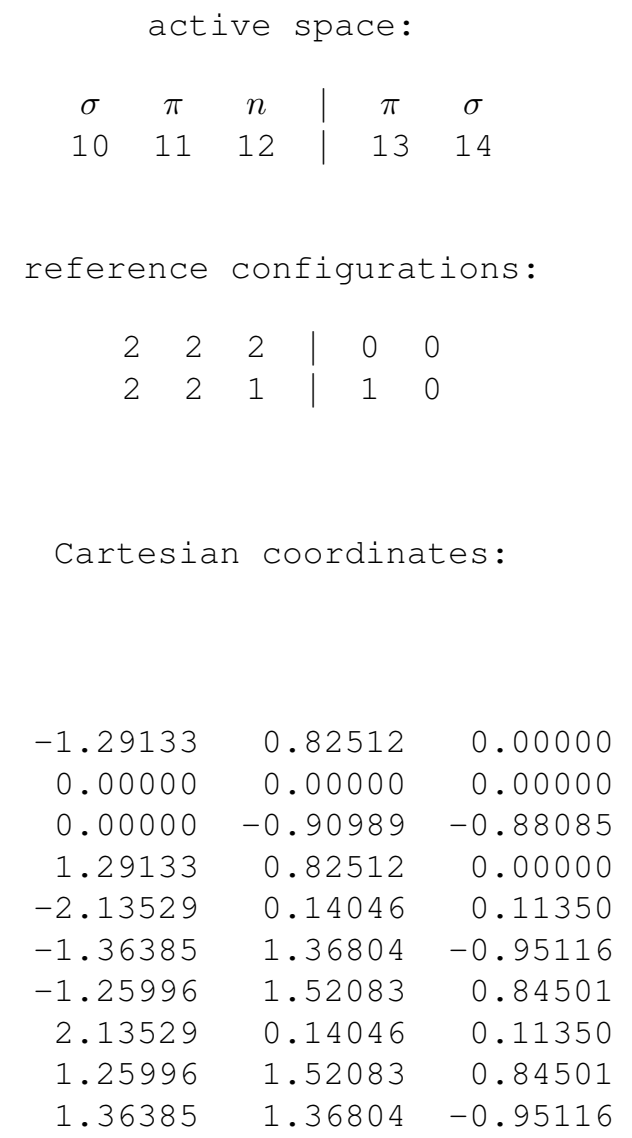


Table S25 Employed active space, reference configurations, and Cartesian coordinates of the OM2/MRCISD-optimized excited-state equilibrium geometry of the $1^{3} A^{\prime}\left({ }^{3} \pi \pi^{*}\right)$ state of acetone (excited-state energy: $\left.-799.89331 \mathrm{eV}\right)$.

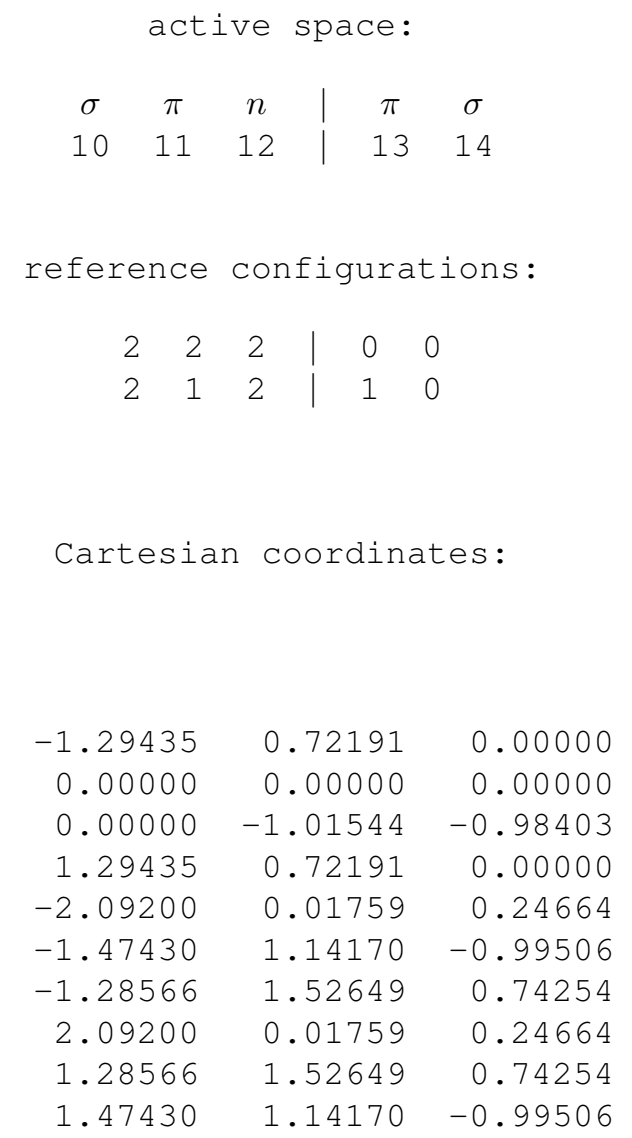


Table S26 Employed active space, reference configurations, and Cartesian coordinates of the OM2/MRCISDTQ-optimized excited-state equilibrium geometry of the $1^{1} A^{\prime \prime}\left({ }^{1} n \pi^{*}\right)$ state of acetophenone (excited-state energy: $-1468.66151 \mathrm{eV})$.

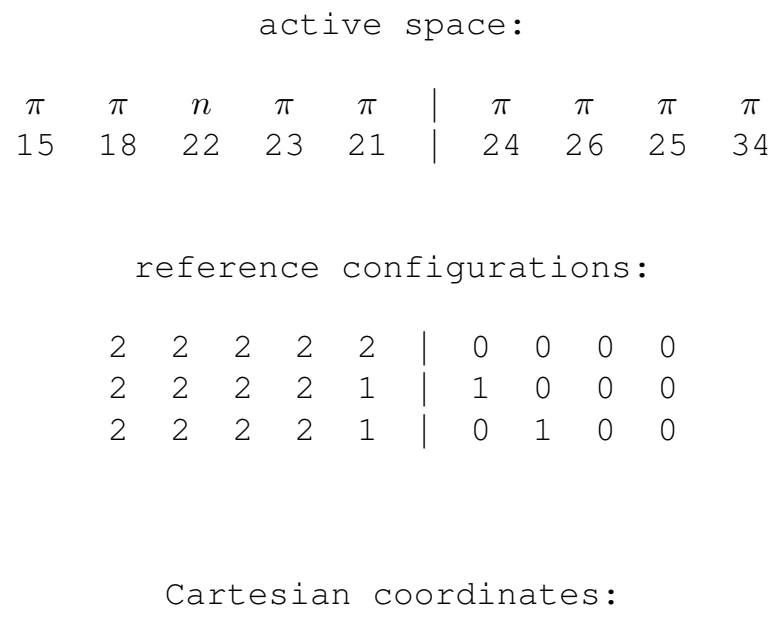


Table S27 Employed active space, reference configurations, and Cartesian coordinates of the OM2/MRCISD-optimized excited-state equilibrium geometry of the $2{ }^{1} A^{\prime}\left({ }^{1} \pi \pi^{*}\right)$ state of acetophenone (excited-state energy: $\left.-1468.18046 \mathrm{eV}\right)$.

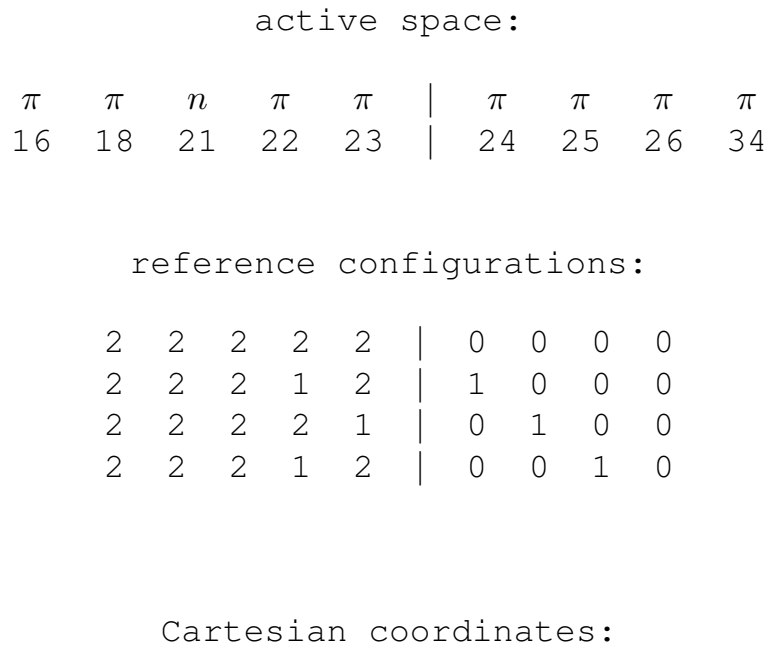


Table S28 Employed active space, reference configurations, and Cartesian coordinates of the OM2/MRCISDT-optimized excited-state equilibrium geometry of the $1^{3} A^{\prime \prime}\left({ }^{3} n \pi^{*}\right)$ state of acetophenone (excited-state energy: $\left.-1468.86614 \mathrm{eV}\right)$.

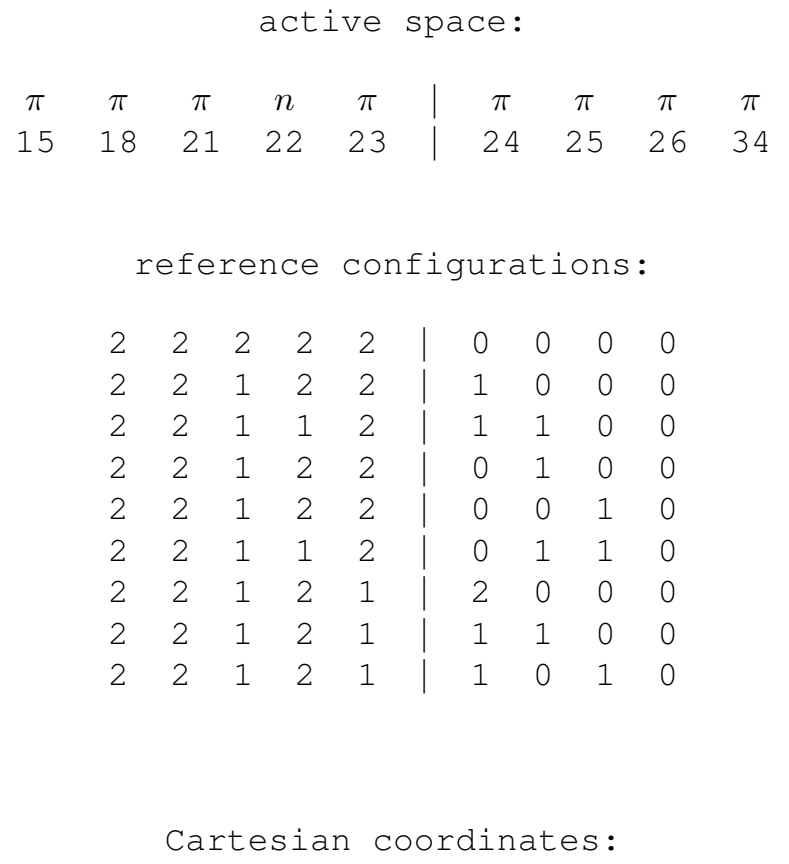

$\begin{array}{lrrr}17 & & & \\ & & & \\ \mathrm{C} & 0.00000 & 0.00000 & 0.00000 \\ \mathrm{O} & 1.28209 & 0.00000 & 0.00000 \\ \mathrm{C} & -0.36428 & -1.48297 & 0.00000 \\ \mathrm{H} & 0.54521 & -2.09708 & 0.00000 \\ \mathrm{H} & -0.92014 & -1.65912 & -0.90985 \\ \mathrm{H} & -0.92014 & -1.65912 & 0.90985 \\ \mathrm{C} & -0.90022 & 1.10329 & 0.00000 \\ \mathrm{C} & -2.30566 & 0.88728 & 0.00000 \\ \mathrm{C} & -0.36648 & 2.41449 & 0.00000 \\ \mathrm{C} & -3.13747 & 1.99948 & 0.00000 \\ \mathrm{C} & -1.22795 & 3.50504 & 0.00000 \\ \mathrm{C} & -2.61793 & 3.30057 & 0.00000 \\ \mathrm{H} & -2.71119 & -0.12714 & 0.00000 \\ \mathrm{H} & 0.71629 & 2.56171 & 0.00000 \\ \mathrm{H} & -4.22587 & 1.85949 & 0.00000 \\ \mathrm{H} & -0.83905 & 4.52632 & 0.00000 \\ \mathrm{H} & -3.29169 & 4.16216 & 0.00000\end{array}$


Table S29 Employed active space, reference configurations, and Cartesian coordinates of the OM2/MRCISD-optimized excited-state equilibrium geometry of the $1^{3} A^{\prime}\left({ }^{3} \pi \pi^{*}\right)$ state of acetophenone (excited-state energy: $-1469.14890 \mathrm{eV}$ ).

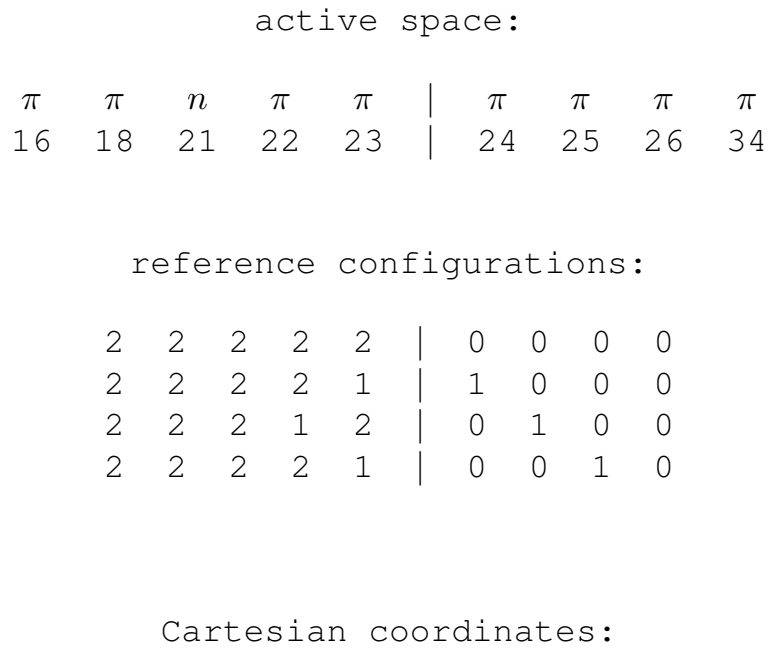


Table S30 Employed active space, reference configurations, and Cartesian coordinates of the OM2/MRCISD-optimized excited-state equilibrium geometry of the $1{ }^{1} A_{2}\left({ }^{1} \pi \pi^{*}\right)$ state of acetylene (excited-state energy: $-278.60450 \mathrm{eV}$ ).

\begin{tabular}{|c|c|c|c|c|c|c|c|c|c|}
\hline$\sigma$ & $\sigma$ & $\sigma$ & $\pi$ & $\pi$ & $\pi$ & $\sigma$ & $\pi$ & $\sigma$ & $\sigma$ \\
\hline \multirow[t]{2}{*}{1} & 2 & 3 & 5 & 4 & 6 & 9 & 7 & 8 & 10 \\
\hline & \multicolumn{4}{|c|}{ reference } & fic & Ira & ic & IS: & \\
\hline 2 & 2 & 2 & 2 & 2 & 0 & 0 & 0 & 0 & 0 \\
\hline 2 & 2 & 2 & 1 & 2 & 0 & 0 & 1 & 0 & 0 \\
\hline 2 & 2 & 2 & 2 & 1 & 0 & 1 & 0 & 0 & 0 \\
\hline
\end{tabular}

Cartesian coordinates:

4

$\begin{array}{rrrr}\mathrm{C} & -0.66303 & 0.00000 & 0.00000 \\ \mathrm{C} & 0.66303 & 0.00000 & 0.00000 \\ \mathrm{H} & -1.60696 & -0.60782 & 0.00000 \\ \mathrm{H} & 1.60696 & -0.60782 & 0.00000\end{array}$


Table S31 Employed active space, reference configurations, and Cartesian coordinates of the OM2/MRCISD-optimized excited-state equilibrium geometry of the $2{ }^{1} A_{2}\left({ }^{1} \pi \pi^{*}\right)$ state of acetylene (excited-state energy: $-277.50811 \mathrm{eV}$ ).

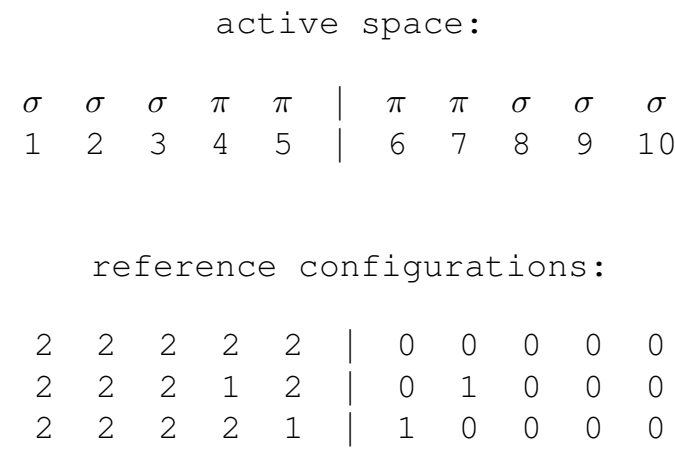

Cartesian coordinates:

4

$\begin{array}{rrrr}\mathrm{C} & -0.66103 & 0.00000 & 0.00000 \\ \mathrm{C} & 0.66103 & 0.00000 & 0.00000 \\ \mathrm{H} & -1.70750 & 0.00000 & 0.00000 \\ \mathrm{H} & 1.70750 & 0.00000 & 0.00000\end{array}$


Table S32 Employed active space, reference configurations, and Cartesian coordinates of the OM2/MRCISD-optimized excited-state equilibrium geometry of the $1{ }^{1} B_{2}\left({ }^{1} \pi \pi^{*}\right)$ state of acetylene (excited-state energy: $\left.-278.19473 \mathrm{eV}\right)$.

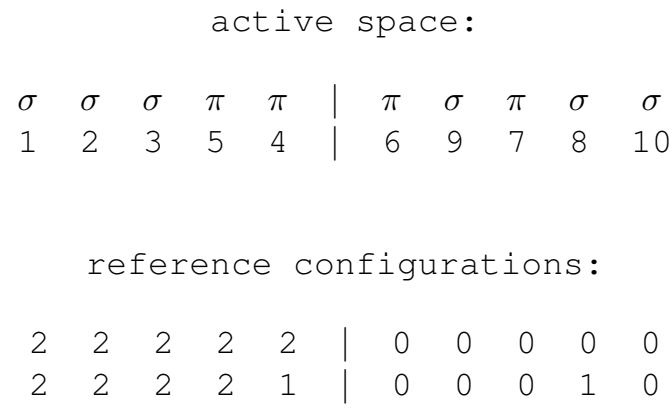

Cartesian coordinates:

4

$\begin{array}{rrrr}\mathrm{C} & -0.65142 & 0.00000 & 0.00000 \\ \mathrm{C} & 0.65142 & 0.00000 & 0.00000 \\ \mathrm{H} & -1.51618 & -0.73910 & 0.00000 \\ \mathrm{H} & 1.51618 & -0.73910 & 0.00000\end{array}$


Table S33 Employed active space, reference configurations, and Cartesian coordinates of the OM2/MRCISD-optimized excited-state equilibrium geometry of the $1^{3} A_{2}\left({ }^{3} \pi \pi^{*}\right)$ state of acetylene (excited-state energy: $-279.29413 \mathrm{eV}$ ).

\begin{tabular}{|c|c|c|c|c|c|c|c|c|c|}
\hline$\sigma$ & $\sigma$ & $\sigma$ & $\pi$ & $\pi$ & $\pi$ & $\sigma$ & $\pi$ & $\sigma$ & $\sigma$ \\
\hline \multirow[t]{2}{*}{1} & 2 & 3 & 5 & 4 & 6 & 9 & 7 & 8 & 10 \\
\hline & \multicolumn{4}{|c|}{ reference } & fic & Ira & ic & IS: & \\
\hline 2 & 2 & 2 & 2 & 2 & 0 & 0 & 0 & 0 & 0 \\
\hline 2 & 2 & 2 & 1 & 2 & 0 & 0 & 1 & 0 & 0 \\
\hline 2 & 2 & 2 & 2 & 1 & 0 & 1 & 0 & 0 & 0 \\
\hline
\end{tabular}

Cartesian coordinates:

4

$\begin{array}{rrrr}\mathrm{C} & -0.66748 & 0.00000 & 0.00000 \\ \mathrm{C} & 0.66748 & 0.00000 & 0.00000 \\ \mathrm{H} & -1.56877 & -0.64258 & 0.00000 \\ \mathrm{H} & 1.56877 & -0.64258 & 0.00000\end{array}$


Table S34 Employed active space, reference configurations, and Cartesian coordinates of the OM2/MRCISD-optimized excited-state equilibrium geometry of the $2{ }^{3} A_{2}\left({ }^{3} \pi \pi^{*}\right)$ state of acetylene (excited-state energy: $\left.-278.40810 \mathrm{eV}\right)$.

\begin{tabular}{|c|c|c|c|c|c|c|c|c|c|}
\hline$\sigma$ & $\sigma$ & $\sigma$ & $\pi$ & $\pi$ & $\sigma$ & $\pi$ & $\pi$ & $\sigma$ & $\sigma$ \\
\hline \multirow[t]{2}{*}{1} & 2 & 3 & 4 & 5 & 8 & 7 & 6 & 9 & 10 \\
\hline & \multicolumn{4}{|c|}{ reference } & fi & $\operatorname{lra}$ & Eio & is: & \\
\hline 2 & 2 & 2 & 2 & 2 & 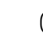 & 0 & 0 & 0 & 0 \\
\hline 2 & 2 & 2 & 2 & 1 & . & 0 & 0 & 0 & 0 \\
\hline 2 & 2 & 2 & 1 & 1 & & 0 & 1 & 1 & 0 \\
\hline 2 & 2 & 2 & 1 & 2 & & 1 & 0 & 0 & 0 \\
\hline
\end{tabular}

Cartesian coordinates:

4

$\begin{array}{rrrr}\mathrm{C} & -0.66243 & 0.00000 & 0.00000 \\ \mathrm{C} & 0.66243 & 0.00000 & 0.00000 \\ \mathrm{H} & -1.71051 & 0.00000 & 0.00000 \\ \mathrm{H} & 1.71051 & 0.00000 & 0.00000\end{array}$


Table S35 Employed active space, reference configurations, and Cartesian coordinates of the OM2/MRCISDT-optimized excited-state equilibrium geometry of the $1^{3} B_{2}\left({ }^{3} \pi \pi^{*}\right)$ state of acetylene (excited-state energy: $\left.-280.45133 \mathrm{eV}\right)$.

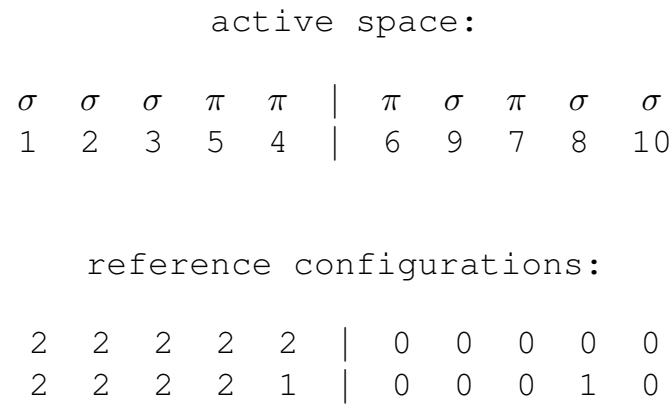

Cartesian coordinates:

4

$\begin{array}{rrrr}\mathrm{C} & -0.66015 & 0.00000 & 0.00000 \\ \mathrm{C} & 0.66015 & 0.00000 & 0.00000 \\ \mathrm{H} & -1.42428 & -0.79649 & 0.00000 \\ \mathrm{H} & 1.42428 & -0.79649 & 0.00000\end{array}$


Table S36 Employed active space, reference configurations, and Cartesian coordinates of the OM2/MRCISD-optimized excited-state equilibrium geometry of the $1{ }^{1} A_{u}\left({ }^{1} \pi \pi^{*}\right)$ state of acetylene (excited-state energy: $-278.67533 \mathrm{eV}$ ).

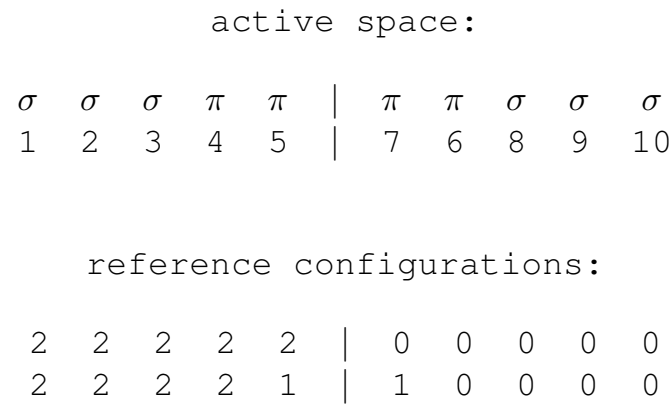

Cartesian coordinates:

4

$\begin{array}{rrrr}\mathrm{C} & -0.68047 & 0.00000 & 0.00000 \\ \mathrm{C} & 0.68047 & 0.00000 & 0.00000 \\ \mathrm{H} & -1.31692 & 0.90969 & 0.00000 \\ \mathrm{H} & 1.31692 & -0.90969 & 0.00000\end{array}$


Table S37 Employed active space, reference configurations, and Cartesian coordinates of the OM2/MRCISD-optimized excited-state equilibrium geometry of the $2{ }^{1} A_{u}\left({ }^{1} \pi \pi^{*}\right)$ state of acetylene (excited-state energy: $\left.-277.16372 \mathrm{eV}\right)$.

\begin{tabular}{|c|c|c|c|c|c|c|c|c|c|}
\hline$\sigma$ & $\sigma$ & $\sigma$ & $\pi$ & $\pi$ & $\pi$ & $\pi$ & $\sigma$ & $\sigma$ & $\sigma$ \\
\hline \multirow[t]{2}{*}{1} & 2 & 3 & 4 & 5 & 6 & 7 & 8 & 9 & 10 \\
\hline & \multicolumn{4}{|c|}{ reference } & fic & ura & io & is : & \\
\hline 2 & 2 & 2 & 2 & 2 & o & 0 & 0 & 0 & 0 \\
\hline 2 & 2 & 2 & 2 & 1 & 0 & 1 & 0 & 0 & 0 \\
\hline 2 & 2 & 2 & 1 & 1 & 1 & 1 & 0 & 0 & 0 \\
\hline 2 & 2 & 2 & 1 & 2 & C & 0 & 1 & 0 & 0 \\
\hline 2 & 2 & 2 & 2 & 1 & c & 0 & 0 & 1 & 0 \\
\hline 2 & 2 & 2 & 2 & 0 & 1 & 0 & 1 & 0 & 0 \\
\hline
\end{tabular}

Cartesian coordinates:

4

$\begin{array}{rrrr}\mathrm{C} & -0.65921 & 0.00000 & 0.00000 \\ \mathrm{C} & 0.65921 & 0.00000 & 0.00000 \\ \mathrm{H} & -1.69394 & 0.18239 & 0.00000 \\ \mathrm{H} & 1.69394 & -0.18239 & 0.00000\end{array}$


Table S38 Employed active space, reference configurations, and Cartesian coordinates of the OM2/MRCISD-optimized excited-state equilibrium geometry of the $1{ }^{1} B_{u}\left({ }^{1} \pi \pi^{*}\right)$ state of acetylene (excited-state energy: $\left.-277.39229 \mathrm{eV}\right)$.

\begin{tabular}{|c|c|c|c|c|c|c|c|c|c|}
\hline$\sigma$ & $\sigma$ & $\sigma$ & $\pi$ & $\pi$ & $\pi$ & $\sigma$ & $\pi$ & $\sigma$ & $\sigma$ \\
\hline \multirow[t]{2}{*}{1} & 2 & 3 & 4 & 5 & 7 & 8 & 6 & 9 & 10 \\
\hline & \multicolumn{4}{|c|}{ reference } & fic & dra & io & IS: & \\
\hline 2 & 2 & 2 & 2 & 2 & 0 & 0 & 0 & 0 & 0 \\
\hline 2 & 2 & 2 & 1 & 2 & 1 & 0 & 0 & 0 & 0 \\
\hline 2 & 2 & 2 & 2 & 1 & 0 & 1 & 0 & 0 & 0 \\
\hline
\end{tabular}

Cartesian coordinates:

4

$\begin{array}{rrrr}\mathrm{C} & -0.66335 & 0.00000 & 0.00000 \\ \mathrm{C} & 0.66335 & 0.00000 & 0.00000 \\ \mathrm{H} & -1.53328 & 0.65106 & 0.00000 \\ \mathrm{H} & 1.53328 & -0.65106 & 0.00000\end{array}$


Table S39 Employed active space, reference configurations, and Cartesian coordinates of the OM2/MRCISD-optimized excited-state equilibrium geometry of the $1^{3} A_{u}\left({ }^{3} \pi \pi^{*}\right)$ state of acetylene (excited-state energy: $-279.50483 \mathrm{eV}$ ).

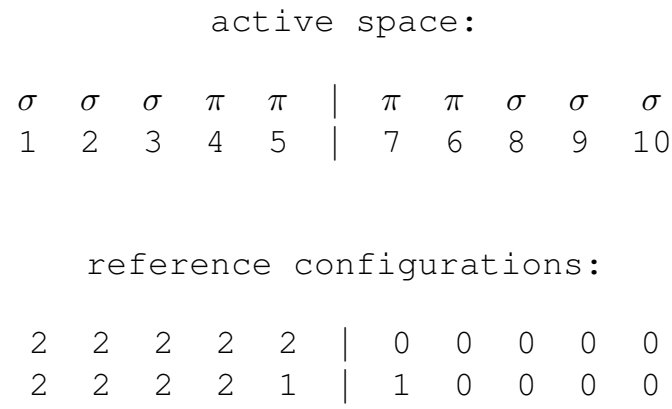

Cartesian coordinates:

4

$\begin{array}{rrrr}\mathrm{C} & -0.68515 & 0.00000 & 0.00000 \\ \mathrm{C} & 0.68515 & 0.00000 & 0.00000 \\ \mathrm{H} & -1.29978 & 0.92158 & 0.00000 \\ \mathrm{H} & 1.29978 & -0.92158 & 0.00000\end{array}$


Table S40 Employed active space, reference configurations, and Cartesian coordinates of the OM2/MRCISD-optimized excited-state equilibrium geometry of the $2{ }^{3} A_{u}\left({ }^{3} \pi \pi^{*}\right)$ state of acetylene (excited-state energy: $-277.86150 \mathrm{eV}$ ).

\begin{tabular}{|c|c|c|c|c|c|c|c|c|c|}
\hline$\sigma$ & $\sigma$ & $\sigma$ & $\pi$ & $\pi$ & $\pi$ & $\pi$ & $\sigma$ & $\sigma$ & $\sigma$ \\
\hline \multirow[t]{2}{*}{1} & 2 & 3 & 4 & 5 & 6 & 7 & 8 & 9 & 10 \\
\hline & \multicolumn{4}{|c|}{ reference } & fic & ura & io & IS : & \\
\hline 2 & 2 & 2 & 2 & 2 & o & 0 & 0 & 0 & 0 \\
\hline 2 & 2 & 2 & 2 & 1 & 0 & 1 & 0 & 0 & 0 \\
\hline 2 & 2 & 2 & 1 & 1 & 1 & 1 & 0 & 0 & 0 \\
\hline 2 & 2 & 2 & 2 & 1 & C & 0 & 0 & 1 & 0 \\
\hline 2 & 2 & 2 & 1 & 2 & c & 0 & 1 & 0 & 0 \\
\hline 2 & 2 & 2 & 2 & 0 & 1 & 0 & 1 & 0 & 0 \\
\hline
\end{tabular}

Cartesian coordinates:

4

$\begin{array}{rrrr}\mathrm{C} & -0.66390 & 0.00000 & 0.00000 \\ \mathrm{C} & 0.66390 & 0.00000 & 0.00000 \\ \mathrm{H} & -1.67677 & 0.29424 & 0.00000 \\ \mathrm{H} & 1.67677 & -0.29424 & 0.00000\end{array}$


Table S41 Employed active space, reference configurations, and Cartesian coordinates of the OM2/MRCISD-optimized excited-state equilibrium geometry of the $1{ }^{3} B_{u}\left({ }^{3} \pi \pi^{*}\right)$ state of acetylene (excited-state energy: $\left.-279.87021 \mathrm{eV}\right)$.

\begin{tabular}{|c|c|c|c|c|c|c|c|c|c|}
\hline$\sigma$ & $\sigma$ & $\sigma$ & $\pi$ & $\pi$ & $\pi$ & $\sigma$ & $\pi$ & $\sigma$ & $\sigma$ \\
\hline \multirow[t]{2}{*}{1} & 2 & 3 & 4 & 5 & 7 & 8 & 6 & 9 & 10 \\
\hline & \multicolumn{4}{|c|}{ reference } & fic & dra & io & IS: & \\
\hline 2 & 2 & 2 & 2 & 2 & 0 & 0 & 0 & 0 & 0 \\
\hline 2 & 2 & 2 & 1 & 2 & 1 & 0 & 0 & 0 & 0 \\
\hline 2 & 2 & 2 & 2 & 1 & 0 & 1 & 0 & 0 & 0 \\
\hline
\end{tabular}

Cartesian coordinates:

4

$\begin{array}{rrrr}\mathrm{C} & -0.66906 & 0.00000 & 0.00000 \\ \mathrm{C} & 0.66906 & 0.00000 & 0.00000 \\ \mathrm{H} & -1.47141 & 0.71552 & 0.00000 \\ \mathrm{H} & 1.47141 & -0.71552 & 0.00000\end{array}$


Table S42 Employed active space, reference configurations, and Cartesian coordinates of the OM2/MRCISD-optimized excited-state equilibrium geometry of the $2{ }^{3} B_{u}\left({ }^{3} \pi \pi^{*}\right)$ state of acetylene (excited-state energy: $\left.-278.56304 \mathrm{eV}\right)$.

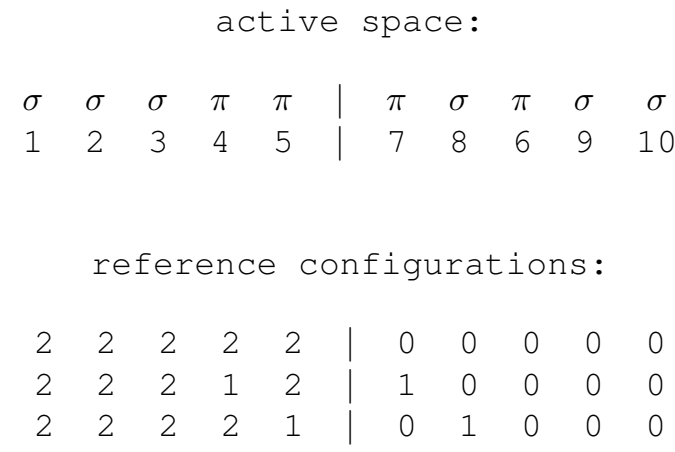

Cartesian coordinates:

4

$\begin{array}{rrrr}\mathrm{C} & -0.67058 & 0.00000 & 0.00000 \\ \mathrm{C} & 0.67058 & 0.00000 & 0.00000 \\ \mathrm{H} & -1.59567 & 0.52414 & 0.00000 \\ \mathrm{H} & 1.59567 & -0.52414 & 0.00000\end{array}$


Table S43 Employed active space, reference configurations, and Cartesian coordinates of the OM2/MRCISD-optimized excited-state equilibrium geometry of the $1^{1} A^{\prime \prime}\left({ }^{1} n \pi^{*}\right)$ state of acrolein (excited-state energy: $-770.18486 \mathrm{eV}$ ).

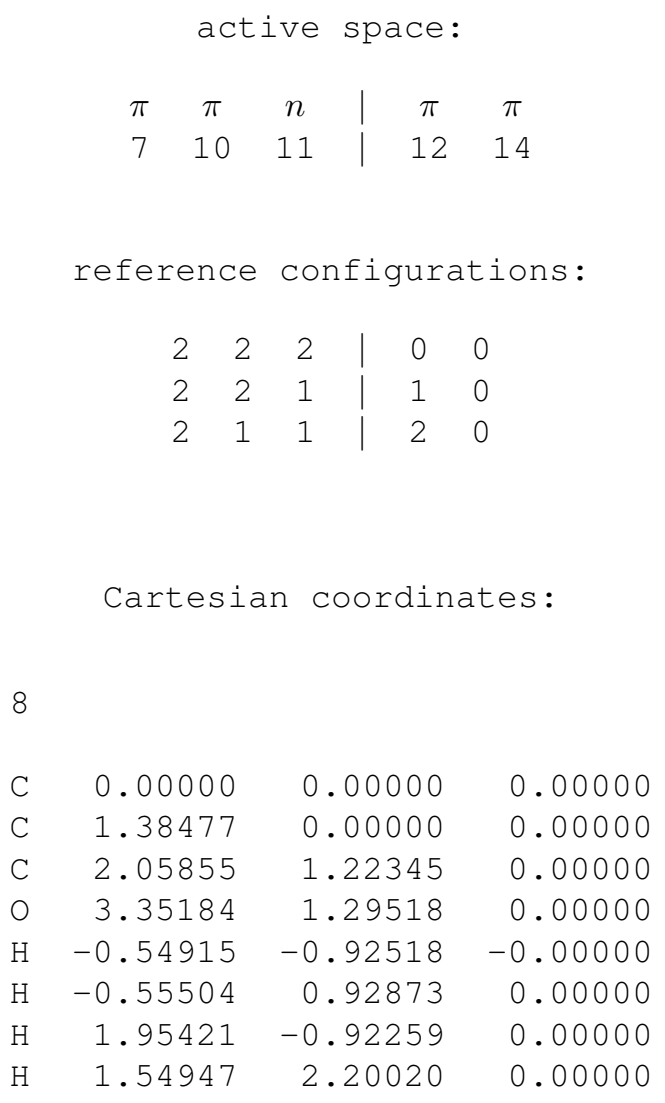


Table S44 Employed active space, reference configurations, and Cartesian coordinates of the OM2/MRCISD-optimized excited-state equilibrium geometry of the $1^{3} A^{\prime \prime}\left({ }^{3} n \pi^{*}\right)$ state of acrolein (excited-state energy: $-770.34705 \mathrm{eV}$ ).

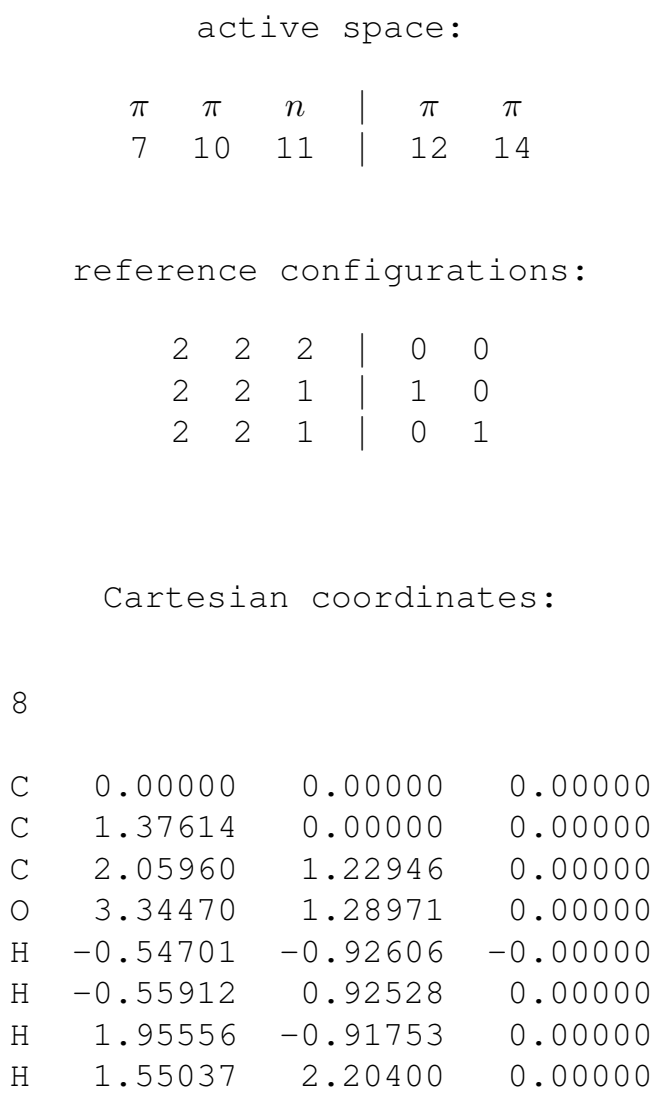


Table S45 Employed active space, reference configurations, and Cartesian coordinates of the OM2/MRCISD-optimized excited-state equilibrium geometry of the $1^{3} A^{\prime}\left({ }^{3} \pi \pi^{*}\right)$ state of acrolein (excited-state energy: $\left.-770.50094 \mathrm{eV}\right)$.

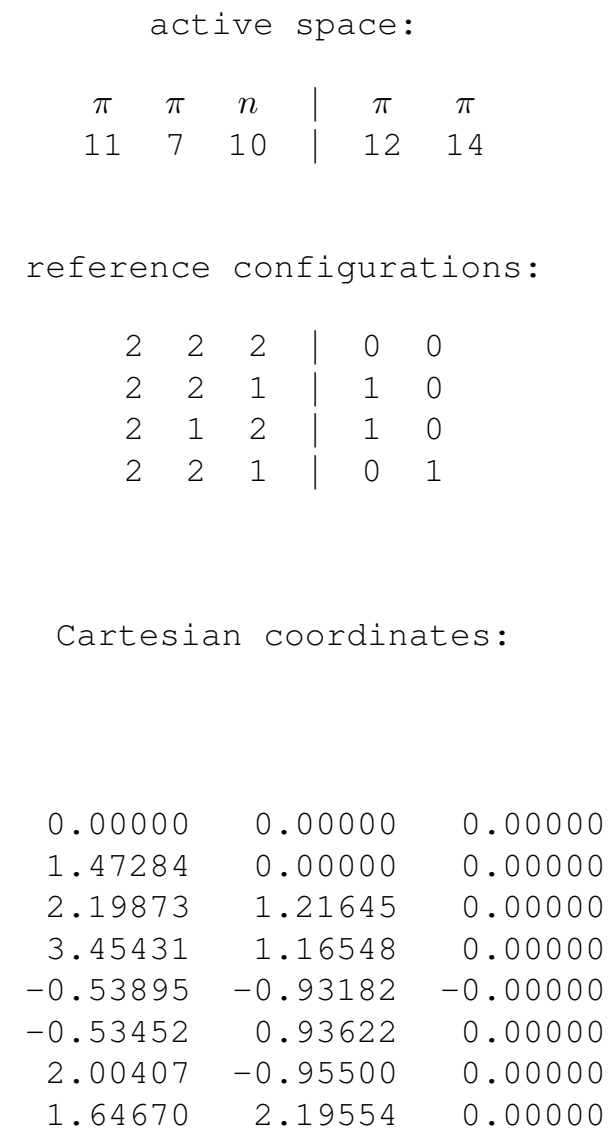


Table S46 Employed active space, reference configurations, and Cartesian coordinates of the OM2/MRCISD-optimized excited-state equilibrium geometry of the $2{ }^{1} A\left({ }^{1} \pi \pi^{*}\right)$ state of 2 -amino-9-methylpurine (excited-state energy: $-1931.77733 \mathrm{eV})$.

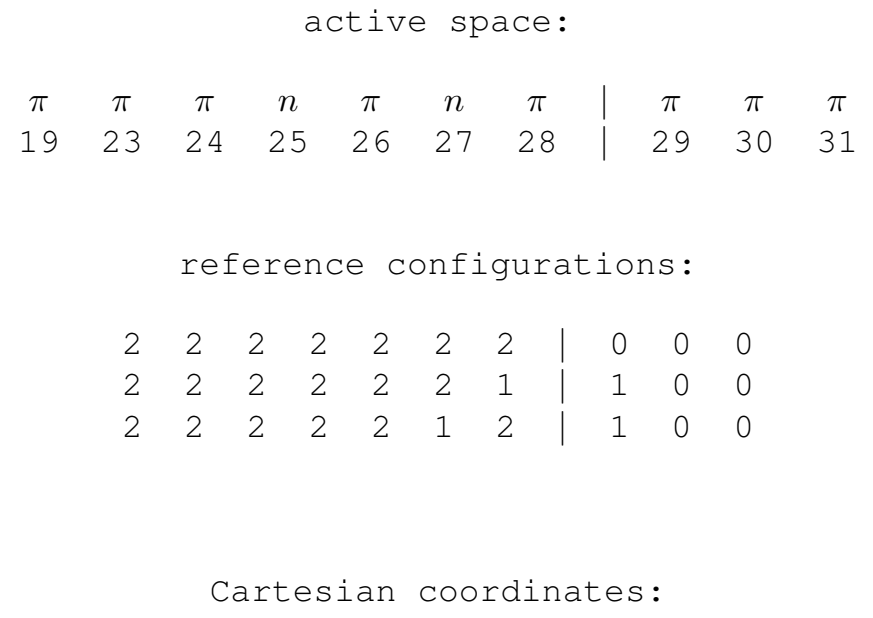

18

$\begin{array}{llll}\mathrm{C} & 0.00000 & 0.00000 & 0.00000\end{array}$

$\begin{array}{lrrr}\mathrm{N} & 1.34884 & 0.00000 & 0.00000\end{array}$

$\begin{array}{llll}\mathrm{N} & -0.75837 & -1.16285 & 0.00000\end{array}$

$\begin{array}{llll}\mathrm{C} & -0.08233 & -2.30597 & -0.03678\end{array}$

$\begin{array}{llll}\text { C } & 1.39426 & -2.38826 & 0.03429\end{array}$

$\begin{array}{llll}\mathrm{C} & 2.06358 & -1.11806 & -0.10708\end{array}$

$\begin{array}{llll}\mathrm{N} & -0.51588 & -3.58976 & 0.28395\end{array}$

$\begin{array}{llll}\mathrm{C} & 0.62553 & -4.39594 & 0.32147\end{array}$

$\begin{array}{llll}\mathrm{N} & 1.75644 & -3.65630 & 0.25182\end{array}$

$\begin{array}{llll}\mathrm{N} & -0.67085 & 1.18098 & 0.11864\end{array}$

$\begin{array}{llll}\mathrm{C} & -1.87341 & -4.05899 & 0.13958\end{array}$

$\mathrm{H} \quad 3.05000 \quad-1.12121 \quad-0.57829$

$\begin{array}{lrrr}\mathrm{H} & 0.59501 & -5.47811 & 0.40132\end{array}$

$\mathrm{H} \quad-1.63493 \quad 1.23553-0.12478$

$\begin{array}{llll}\mathrm{H} & -0.16477 & 2.03371 & 0.21271\end{array}$

$\mathrm{H} \quad-2.57463-3.23466 \quad 0.36712$

$\mathrm{H} \quad-2.06082-4.88245 \quad 0.85268$

$\mathrm{H} \quad-2.05417-4.41174 \quad-0.89231$ 
Table S47 Employed active space, reference configurations, and Cartesian coordinates of the OM2/MRCISD-optimized excited-state equilibrium geometry of the $1{ }^{1} B_{2}\left({ }^{1} \pi \pi^{*}\right)$ state of aniline (excited-state energy: $-1080.06598 \mathrm{eV}$ ).

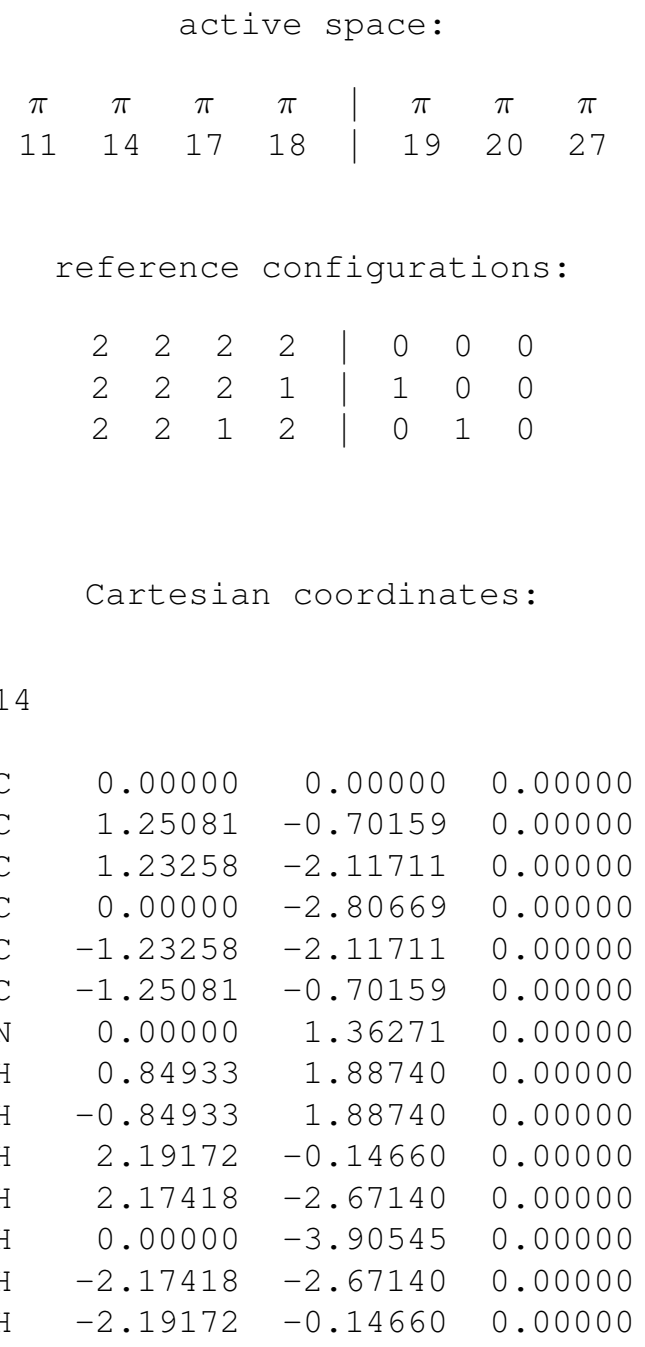


Table S48 Employed active space, reference configurations, and Cartesian coordinates of the OM2/MRCISD-optimized excited-state equilibrium geometry of the $1^{3} A^{\prime}\left({ }^{3} \pi \pi^{*}\right)$ state of aniline (excited-state energy: $-1080.41915 \mathrm{eV}$ ).

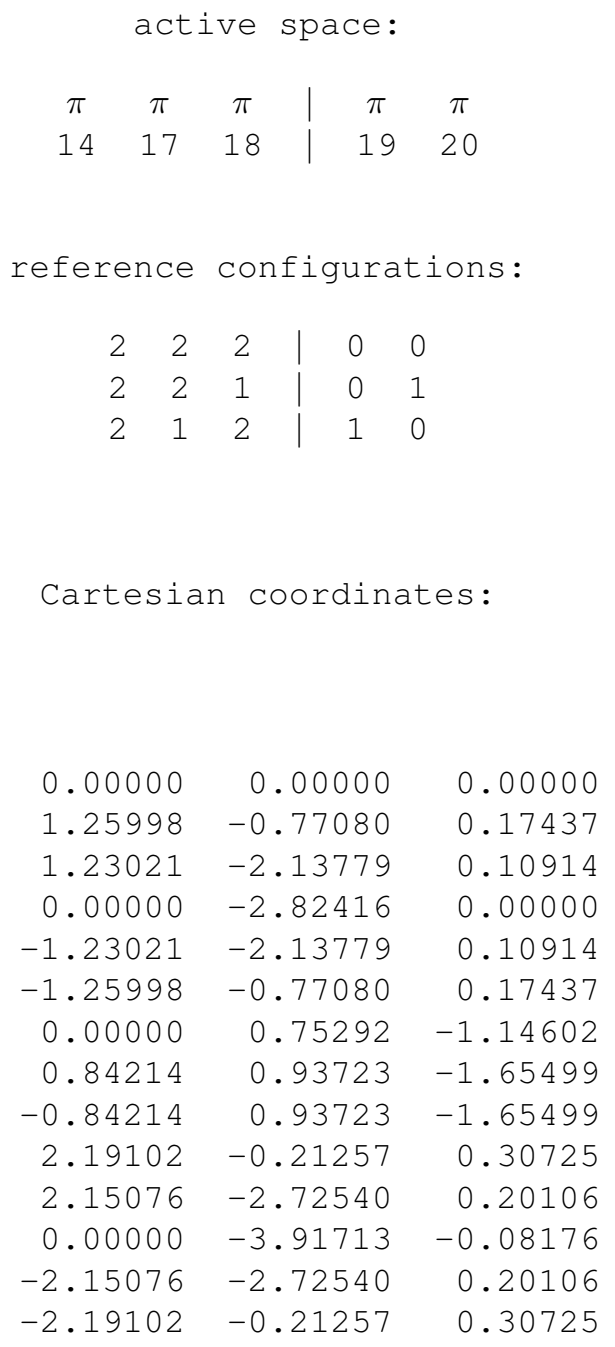


Table S49 Employed active space, reference configurations, and Cartesian coordinates of the OM2/MRCISD-optimized excited-state equilibrium geometry of the $1^{1} A^{\prime \prime}\left({ }^{1} n \pi^{*}\right)$ state of benzaldehyde (excited-state energy: $\left.-1310.77409 \mathrm{eV}\right)$.

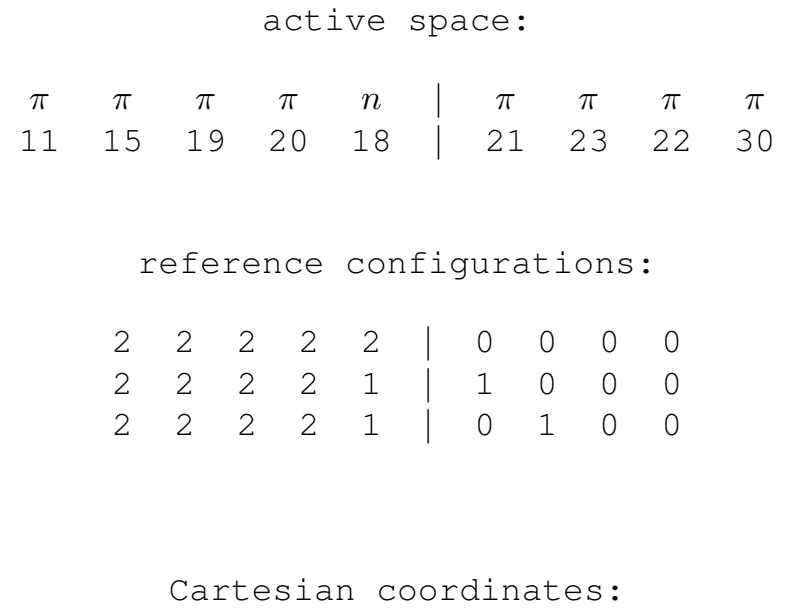


Table S50 Employed active space, reference configurations, and Cartesian coordinates of the OM2/MRCISD-optimized excited-state equilibrium geometry of the $2{ }^{1} A^{\prime}\left({ }^{1} \pi \pi^{*}\right)$ state of benzaldehyde (excited-state energy: $\left.-1310.33862 \mathrm{eV}\right)$.

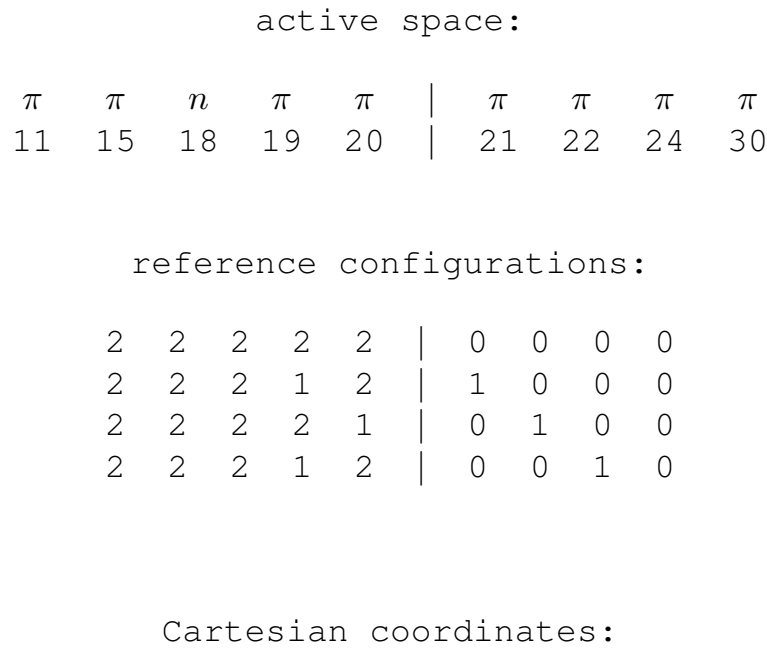


Table S51 Employed active space, reference configurations, and Cartesian coordinates of the OM2/MRCISD-optimized excited-state equilibrium geometry of the $1^{3} A^{\prime \prime}\left({ }^{3} n \pi^{*}\right)$ state of benzaldehyde (excited-state energy: $\left.-1310.98889 \mathrm{eV}\right)$.

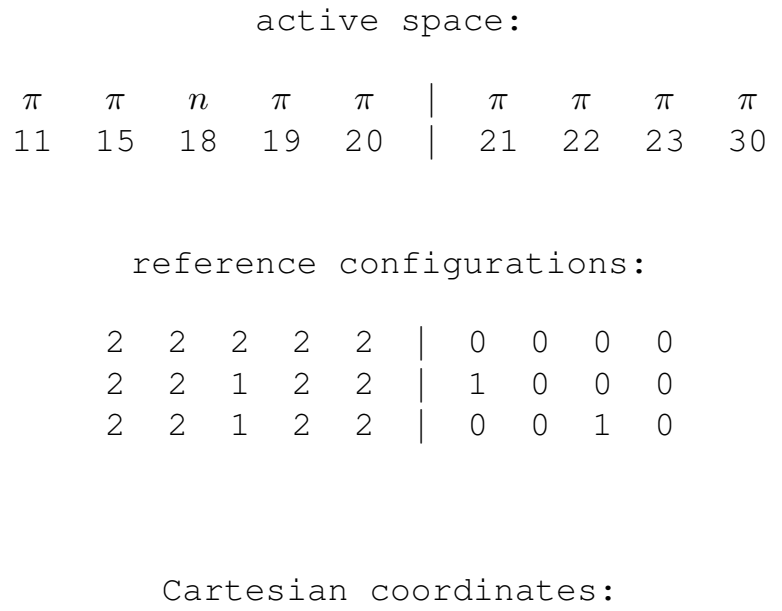


Table S52 Employed active space, reference configurations, and Cartesian coordinates of the OM2/MRCISD-optimized excited-state equilibrium geometry of the $1^{3} A^{\prime}\left({ }^{3} \pi \pi^{*}\right)$ state of benzaldehyde (excited-state energy: $-1311.32145 \mathrm{eV}$ ).

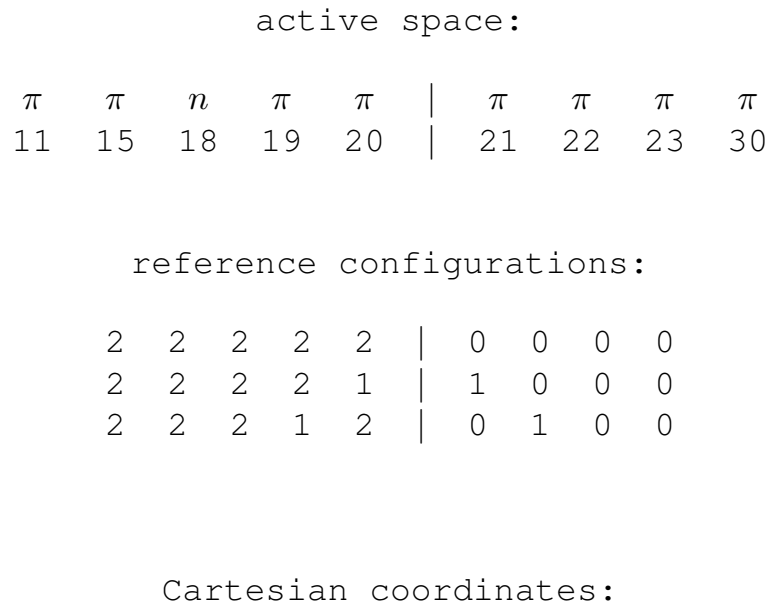


Table S53 Employed active space, reference configurations, and Cartesian coordinates of the OM2/MRCISD-optimized excited-state equilibrium geometry of the $1{ }^{1} A_{u}\left({ }^{1} n \pi^{*}\right)$ state of $p$-benzoquinone (excited-state energy: $-1484.10738 \mathrm{eV})$.

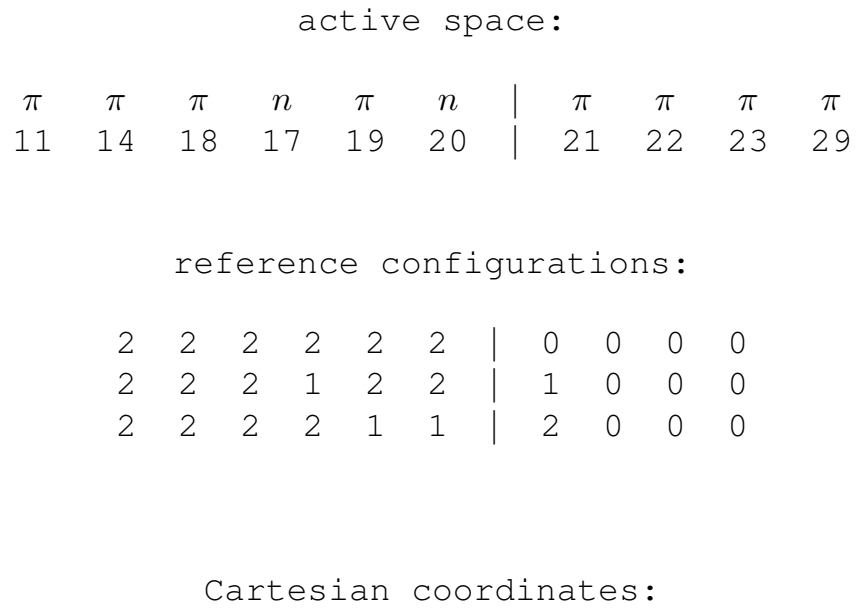


Table S54 Employed active space, reference configurations, and Cartesian coordinates of the OM2/MRCISD-optimized excited-state equilibrium geometry of the $1^{1} B_{1 g}\left({ }^{1} n \pi^{*}\right)$ state of $p$-benzoquinone (excited-state energy: $-1484.54231 \mathrm{eV})$.

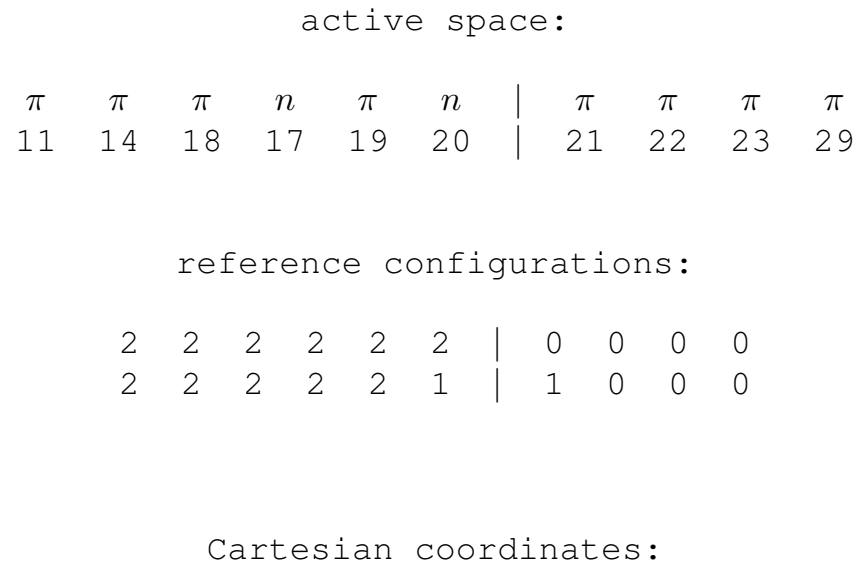


Table S55 Employed active space, reference configurations, and Cartesian coordinates of the OM2/MRCISD-optimized excited-state equilibrium geometry of the $1^{1} B_{1 u}\left({ }^{1} \pi \pi^{*}\right)$ state of $p$-benzoquinone (excited-state energy: $-1481.86753 \mathrm{eV})$.

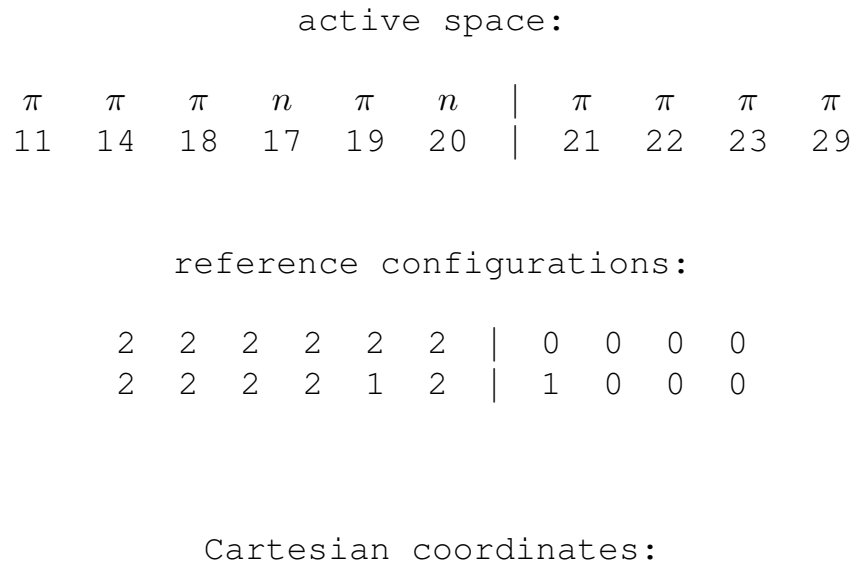


Table S56 Employed active space, reference configurations, and Cartesian coordinates of the OM2/MRCISD-optimized excited-state equilibrium geometry of the $1^{1} B_{3 g}\left({ }^{1} \pi \pi^{*}\right)$ state of $p$-benzoquinone (excited-state energy: $-1482.96859 \mathrm{eV})$.

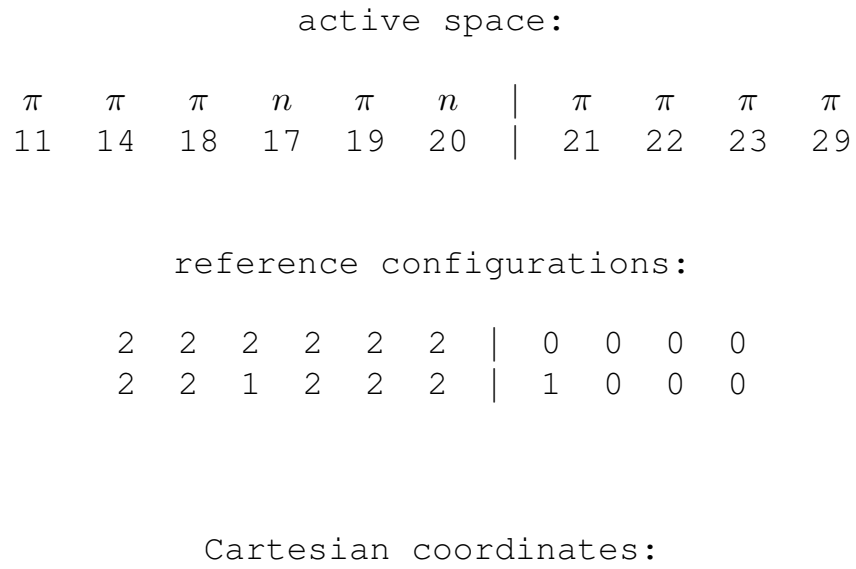


Table S57 Employed active space, reference configurations, and Cartesian coordinates of the OM2/MRCISD-optimized excited-state equilibrium geometry of the $1{ }^{3} A_{u}\left({ }^{3} n \pi^{*}\right)$ state of $p$-benzoquinone (excited-state energy: $-1484.20871 \mathrm{eV})$.

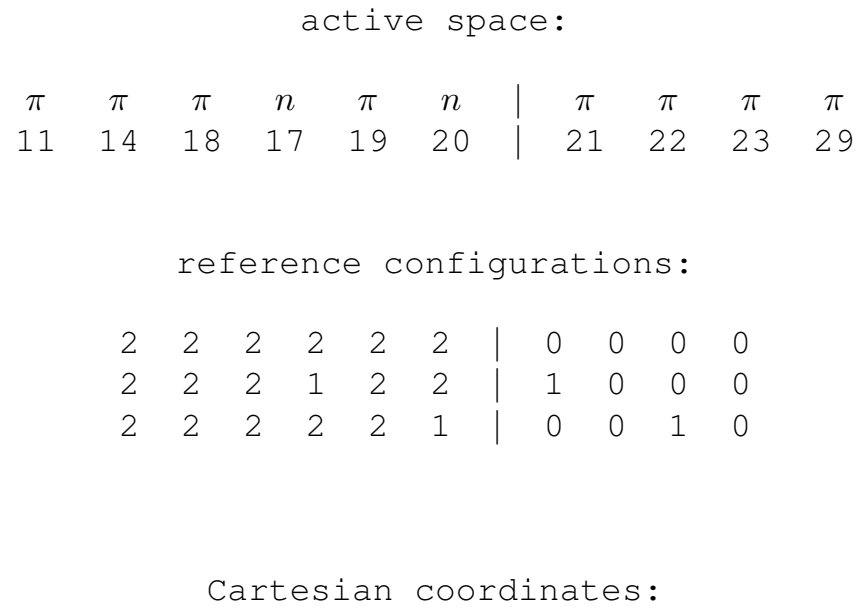


Table S58 Employed active space, reference configurations, and Cartesian coordinates of the OM2/MRCISD-optimized excited-state equilibrium geometry of the $1{ }^{3} B_{1 g}\left({ }^{3} n \pi^{*}\right)$ state of $p$-benzoquinone (excited-state energy: $-1484.67982 \mathrm{eV})$.

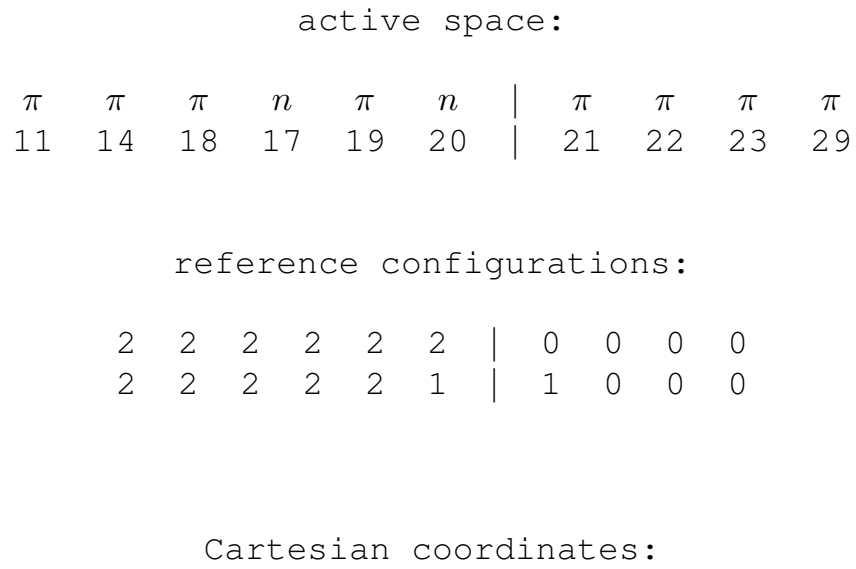


Table S59 Employed active space, reference configurations, and Cartesian coordinates of the OM2/MRCISD-optimized excited-state equilibrium geometry of the $1{ }^{3} B_{1 u}\left({ }^{3} \pi \pi^{*}\right)$ state of $p$-benzoquinone (excited-state energy: $-1484.81149 \mathrm{eV})$.

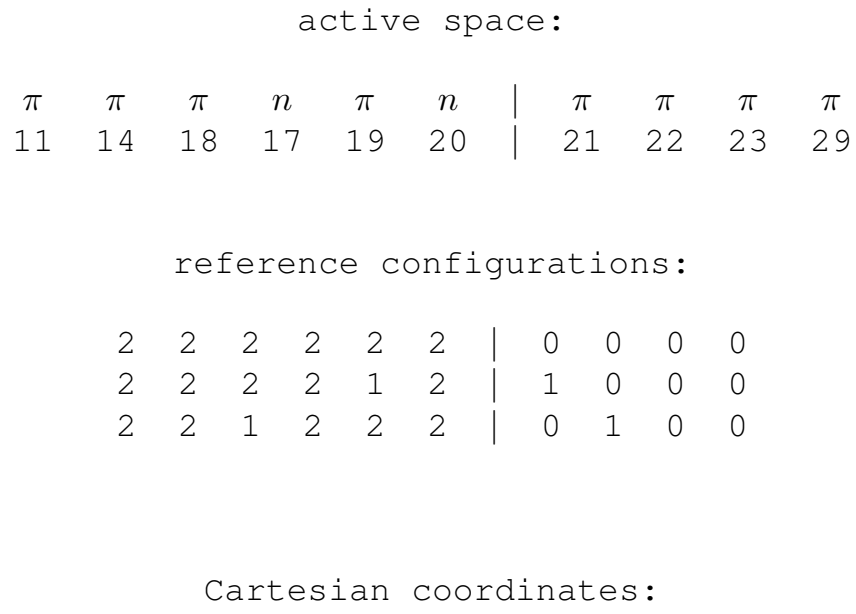


Table S60 Employed active space, reference configurations, and Cartesian coordinates of the OM2/MRCISD-optimized excited-state equilibrium geometry of the $1{ }^{3} B_{3 g}\left({ }^{3} \pi \pi^{*}\right)$ state of $p$-benzoquinone (excited-state energy: $-1484.20825 \mathrm{eV})$.

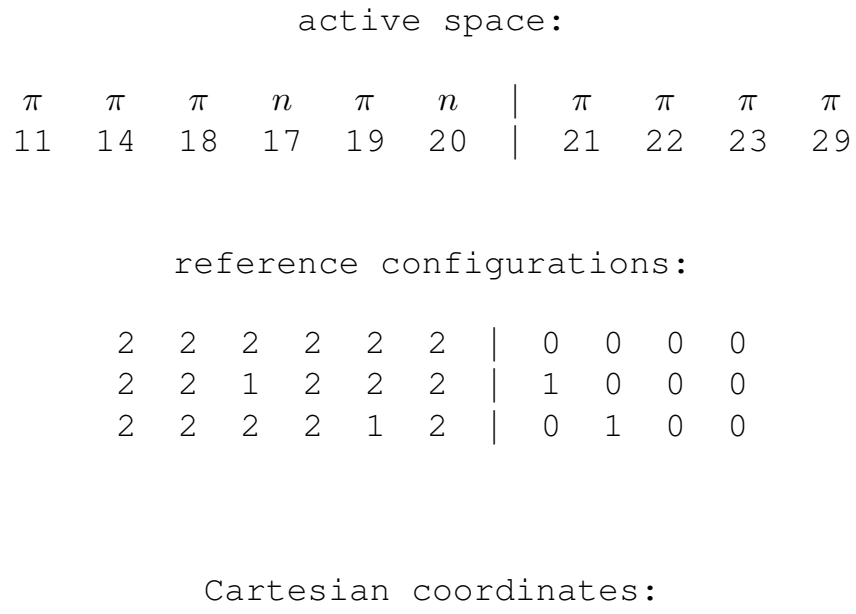


Table S61 Employed active space, reference configurations, and Cartesian coordinates of the OM2/MRCISD-optimized excited-state equilibrium geometry of the $1{ }^{1} B_{3 g}\left({ }^{1} \pi \pi^{*}\right)$ state of biphenylene (excited-state energy: $-1645.88808 \mathrm{eV}$ ).

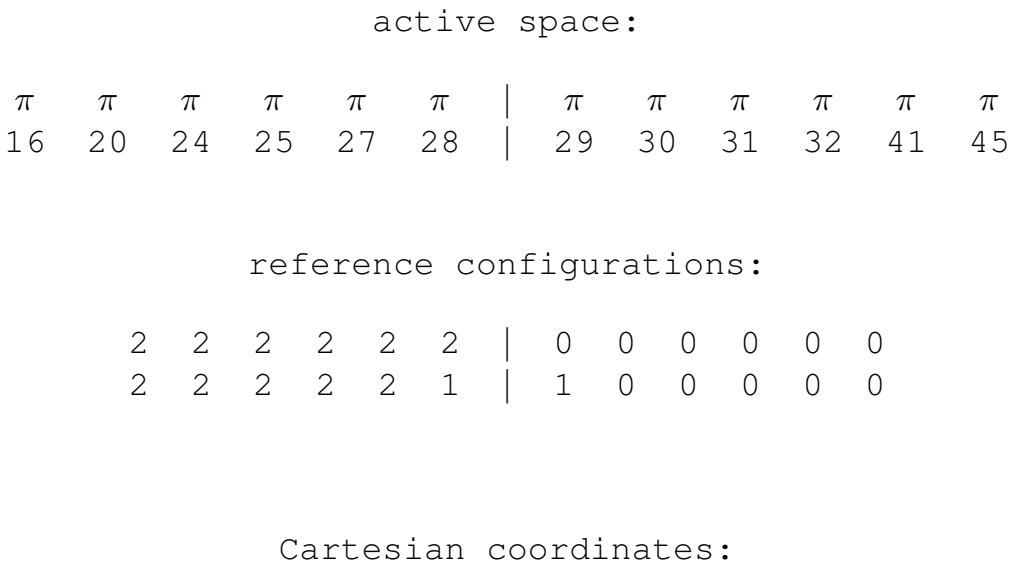

$\mathrm{H} \quad 0.00000 \quad-1.21932 \quad 4.03941$ 
Table S62 Employed active space, reference configurations, and Cartesian coordinates of the OM2/MRCISD-optimized excited-state equilibrium geometry of the $1{ }^{1} B_{1 u}\left({ }^{1} \pi \pi^{*}\right)$ state of biphenylene (excited-state energy: $\left.-1645.36751 \mathrm{eV}\right)$.

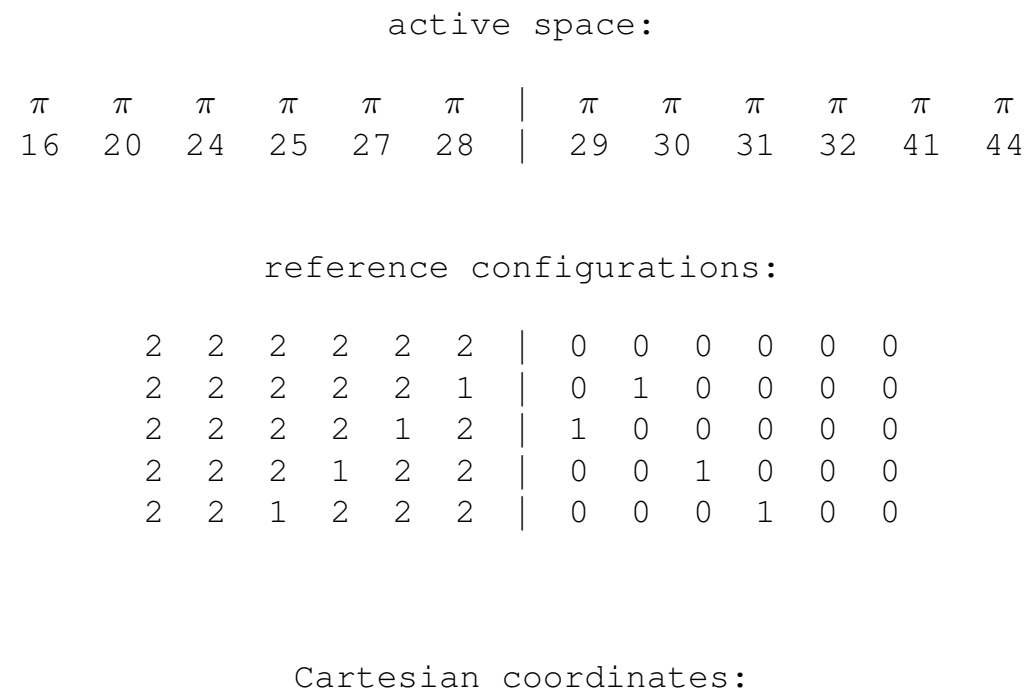

20
0.00000
0.72877
0.73252
1.47671
1.90974
0.00000
0.70239
3.09363
$\begin{array}{lll}0.72877 & -0.73252\end{array}$
$-0.72877-0.73252$
$-0.72877$
0.73252
0.00000
1.47671
$-1.90974$
$-1.47671$
$-1.90974$
0.00000
$-1.47671$
1.90974
0.70239
$-3.09363$
0.00000
$-0.70239$
$-3.09363$
0.00000
$-0.70239$
3.09363
0.00000
2.56463
1.92840
0.00000
2.56463
$-1.92840$
0.00000
$-2.56463$
$-1.92840$
0.00000
$-2.56463$
1.92840
0.00000
1.22605
4.05817
0.00000
1.22605
$-4.05817$
$-1.22605$
$-4.05817$

\begin{abstract}
0.00000
\end{abstract}
$-1.22605$
4.05817

C $\quad 0.00000$

C $\quad 0.00000$

C $\quad 0.00000$

C $\quad 0.00000$

C $\quad 0.00000$

C $\quad 0.00000$

H $\quad 0.00000$ 
Table S63 Employed active space, reference configurations, and Cartesian coordinates of the OM2/MRCISD-optimized excited-state equilibrium geometry of the $2{ }^{1} B_{1 u}\left({ }^{1} \pi \pi^{*}\right)$ state of biphenylene (excited-state energy: $\left.-1643.44270 \mathrm{eV}\right)$.

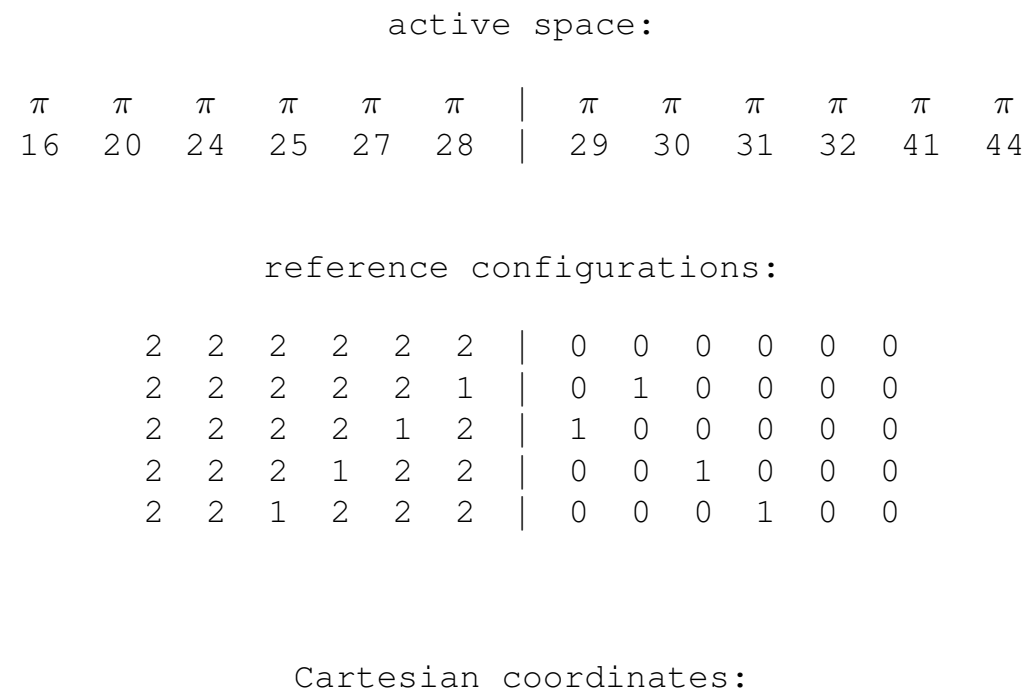

20

0.00000

0.72947

0.73474

C 0.00000

1.47289

1. 90897

C 0.00000

$0.69717 \quad 3.10001$

C 0.00000

$0.72947-0.73474$

C 0.00000

$-0.72947$

$-0.73474$

C 0.00000

$-0.72947$

0.73474

C 0.00000

$1.47289-1.90897$

C 0.00000

$-1.47289$

$-1.90897$

0.00000

$-1.47289$

1.90897

0.00000

0.69717

$-3.10001$

C 0.00000

$-0.69717$

$-3.10001$

0.00000

$-0.69717$

3.10001

0.00000

$2.56297 \quad 1.93177$

0.00000

2.56297

$-1.93177$

0.00000

$-2.56297$

$-1.93177$

$-2.56297 \quad 1.93177$

0.00000

1.22640

4.06049

0.00000

1.22640

$-4.06049$

0.00000

$-1.22640$

$-4.06049$

0.00000

$-1.22640$

4.06049 
Table S64 Employed active space, reference configurations, and Cartesian coordinates of the OM2/MRCISD-optimized excited-state equilibrium geometry of the $1{ }^{1} B_{2 u}\left({ }^{1} \pi \pi^{*}\right)$ state of biphenylene (excited-state energy: $\left.-1644.07334 \mathrm{eV}\right)$.

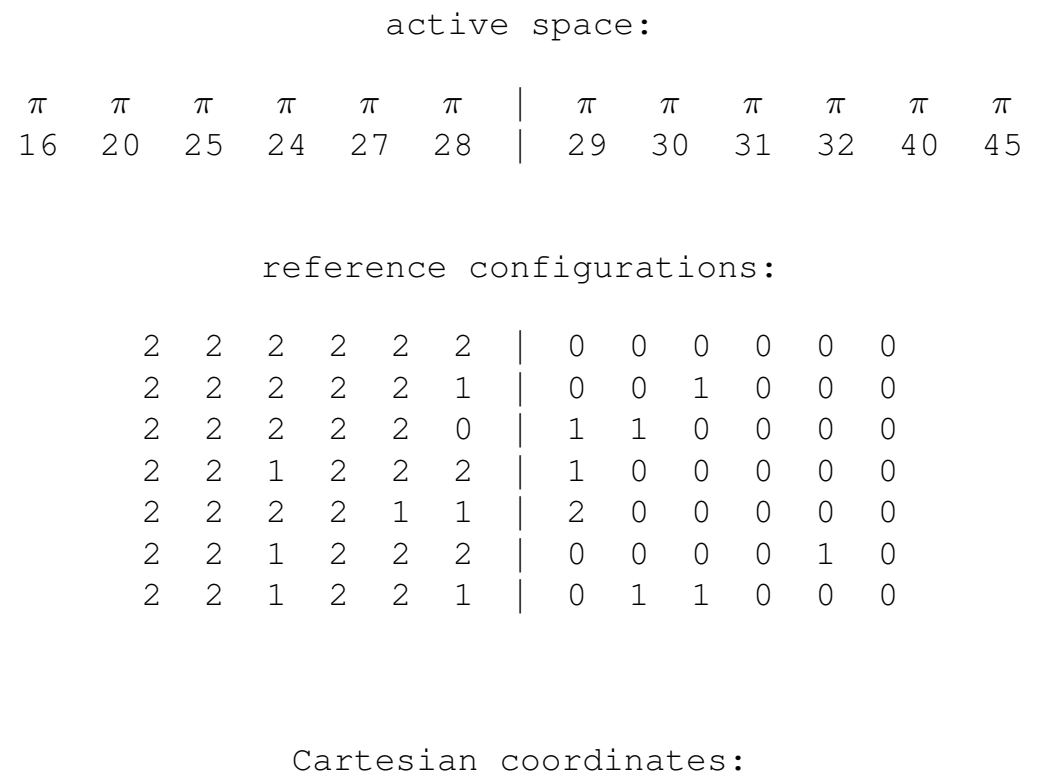

20

\begin{tabular}{lrrr}
$\mathrm{C}$ & 0.00000 & 0.74726 & 0.71917 \\
$\mathrm{C}$ & 0.00000 & 1.48585 & 1.89733 \\
$\mathrm{C}$ & 0.00000 & 0.72203 & 3.07366 \\
$\mathrm{C}$ & 0.00000 & 0.74726 & -0.71917 \\
$\mathrm{C}$ & 0.00000 & -0.74726 & -0.71917 \\
$\mathrm{C}$ & 0.00000 & -0.74726 & 0.71917 \\
$\mathrm{C}$ & 0.00000 & 1.48585 & -1.89733 \\
$\mathrm{C}$ & 0.00000 & -1.48585 & -1.89733 \\
$\mathrm{C}$ & 0.00000 & -1.48585 & 1.89733 \\
$\mathrm{C}$ & 0.00000 & 0.72203 & -3.07366 \\
$\mathrm{C}$ & 0.00000 & -0.72203 & -3.07366 \\
$\mathrm{C}$ & 0.00000 & -0.72203 & 3.07366 \\
$\mathrm{H}$ & 0.00000 & 2.57506 & 1.91412 \\
$\mathrm{H}$ & 0.00000 & 2.57506 & -1.91412 \\
$\mathrm{H}$ & 0.00000 & -2.57506 & -1.91412 \\
$\mathrm{H}$ & 0.00000 & -2.57506 & 1.91412 \\
$\mathrm{H}$ & 0.00000 & 1.22979 & 4.04451 \\
$\mathrm{H}$ & 0.00000 & 1.22979 & -4.04451 \\
$\mathrm{H}$ & 0.00000 & -1.22979 & -4.04451 \\
\hline & & \multicolumn{3}{c}{ Continued on next page }
\end{tabular}

S86 
Continued from previous page

$\mathrm{H} \quad 0.00000 \quad-1.22979 \quad 4.04451$ 
Table S65 Employed active space, reference configurations, and Cartesian coordinates of the OM2/MRCISD-optimized excited-state equilibrium geometry of the $1{ }^{1} B_{u}\left({ }^{1} \pi \pi^{*}\right)$ state of trans-butadiene (excited-state energy: $-593.74432 \mathrm{eV})$.

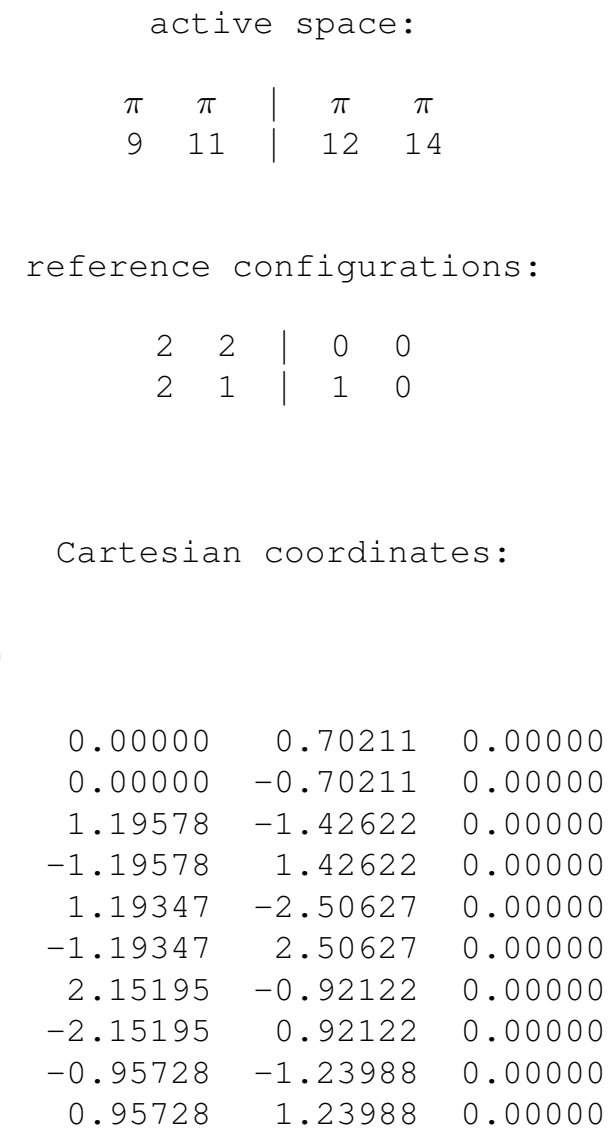


Table S66 Employed active space, reference configurations, and Cartesian coordinates of the OM2/MRCISD-optimized excited-state equilibrium geometry of the $2{ }^{1} A_{g}\left({ }^{1} \pi \pi^{*}\right)$ state of trans-butadiene (excited-state energy: $-594.73435 \mathrm{eV})$.

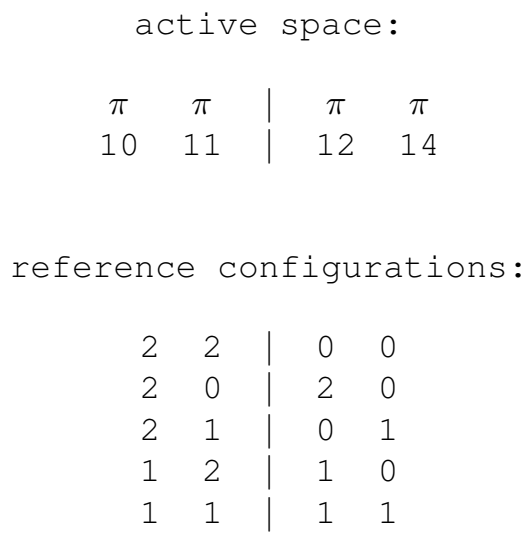

$\begin{array}{lrrr}\mathrm{C} & 0.00000 & 0.69741 & 0.00000 \\ \mathrm{C} & 0.00000 & -0.69741 & 0.00000 \\ \mathrm{C} & 1.26605 & -1.43233 & 0.00000 \\ \mathrm{C} & -1.26605 & 1.43233 & 0.00000 \\ \mathrm{H} & 1.26570 & -2.51095 & 0.00000 \\ \mathrm{H} & -1.26570 & 2.51095 & 0.00000 \\ \mathrm{H} & 2.20201 & -0.89435 & 0.00000 \\ \mathrm{H} & -2.20201 & 0.89435 & 0.00000 \\ \mathrm{H} & -0.93453 & -1.26471 & 0.00000 \\ \mathrm{H} & 0.93453 & 1.26471 & 0.00000\end{array}$


Table S67 Employed active space, reference configurations, and Cartesian coordinates of the OM2/MRCISD-optimized excited-state equilibrium geometry of the $3{ }^{1} A\left({ }^{1} n \pi^{*}\right)$ state of cytosine (excited-state energy: $-1551.71302 \mathrm{eV}$ ).

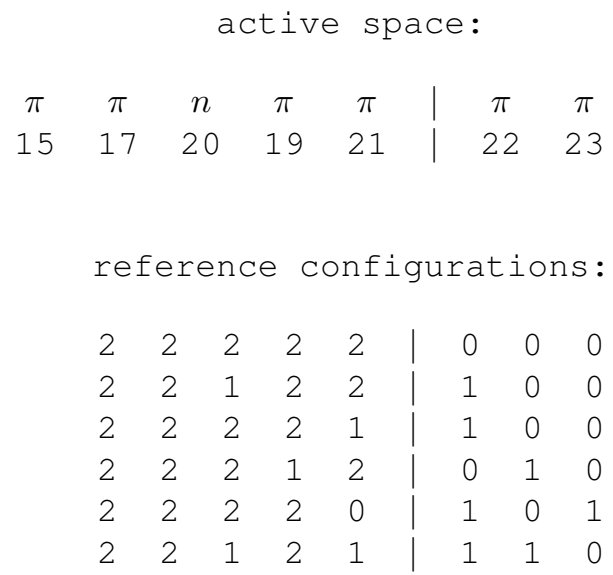

Cartesian coordinates:

13

$\begin{array}{lrrr}\mathrm{N} & 0.00000 & 0.00000 & 0.00000 \\ \mathrm{C} & 1.38352 & 0.00000 & 0.00000 \\ \mathrm{C} & 2.15147 & 1.18865 & 0.00000 \\ \mathrm{C} & 1.49589 & 2.41589 & 0.00001 \\ \mathrm{~N} & 0.09772 & 2.41842 & 0.00008 \\ \mathrm{C} & -0.54775 & 1.20015 & 0.00006 \\ \mathrm{~N} & 1.96062 & -1.24998 & -0.00002 \\ \mathrm{O} & -1.86480 & 1.29571 & 0.00011 \\ \mathrm{H} & 3.23977 & 1.14615 & -0.00002 \\ \mathrm{H} & 2.01613 & 3.37092 & -0.00002 \\ \mathrm{H} & 1.39117 & -2.06250 & 0.00007 \\ \mathrm{H} & 2.94594 & -1.36660 & -0.00006 \\ \mathrm{H} & -0.42210 & 3.28320 & 0.00010\end{array}$


Table S68 Employed active space, reference configurations, and Cartesian coordinates of the OM2/MRCISD-optimized excited-state equilibrium geometry of the $2{ }^{1} A\left({ }^{1} \pi \pi^{*}\right)$ state of cytosine (excited-state energy: $\left.-1552.10074 \mathrm{eV}\right)$.

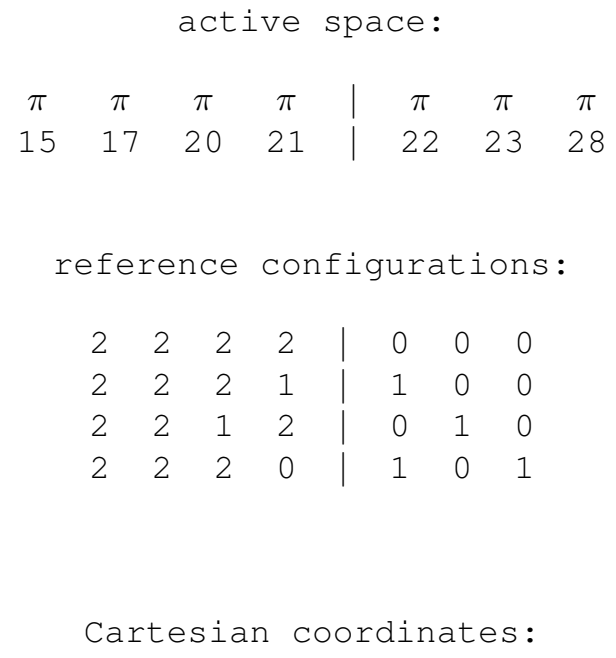

13

$\begin{array}{lrrr}\mathrm{N} & 0.00000 & 0.00000 & 0.00000 \\ \mathrm{C} & 1.39364 & 0.00000 & 0.00000 \\ \mathrm{C} & 2.16248 & 1.17631 & 0.00000 \\ \mathrm{C} & 1.49233 & 2.43519 & -0.00000 \\ \mathrm{~N} & 0.12965 & 2.42824 & -0.00000 \\ \mathrm{C} & -0.59621 & 1.20238 & -0.00000 \\ \mathrm{~N} & 1.96316 & -1.24514 & 0.00000 \\ \mathrm{O} & -1.85536 & 1.37832 & -0.00000 \\ \mathrm{H} & 3.24994 & 1.14493 & 0.00000 \\ \mathrm{H} & 2.04873 & 3.37494 & 0.00000 \\ \mathrm{H} & 1.38659 & -2.05505 & 0.00000 \\ \mathrm{H} & 2.94829 & -1.37347 & 0.00000 \\ \mathrm{H} & -0.38950 & 3.30254 & -0.00000\end{array}$


Table S69 Employed active space, reference configurations, and Cartesian coordinates of the OM2/MRCISD-optimized excited-state equilibrium geometry of the $1{ }^{1} A_{2}\left({ }^{1} n \pi^{*}\right)$ state of diazomethane (excited-state energy: $-581.16528 \mathrm{eV}$ ).

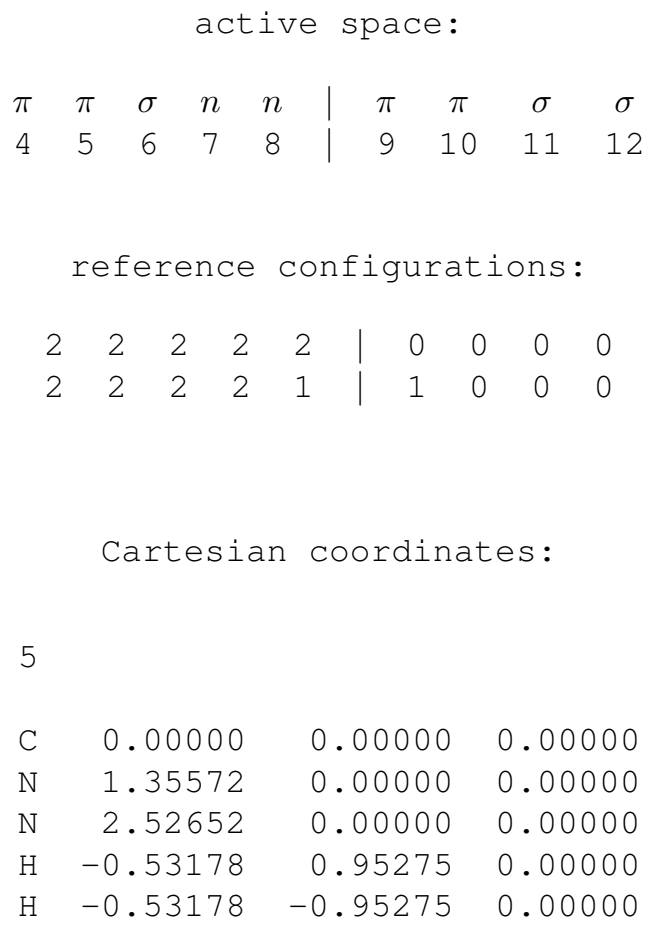


Table S70 Employed active space, reference configurations, and Cartesian coordinates of the OM2/MRCISD-optimized excited-state equilibrium geometry of the $1{ }^{1} B_{3 g}\left({ }^{1} \pi \pi^{*}\right)$ state of dibenzo- $p$-dioxin (excited-state energy: $-2308.52322 \mathrm{eV})$.

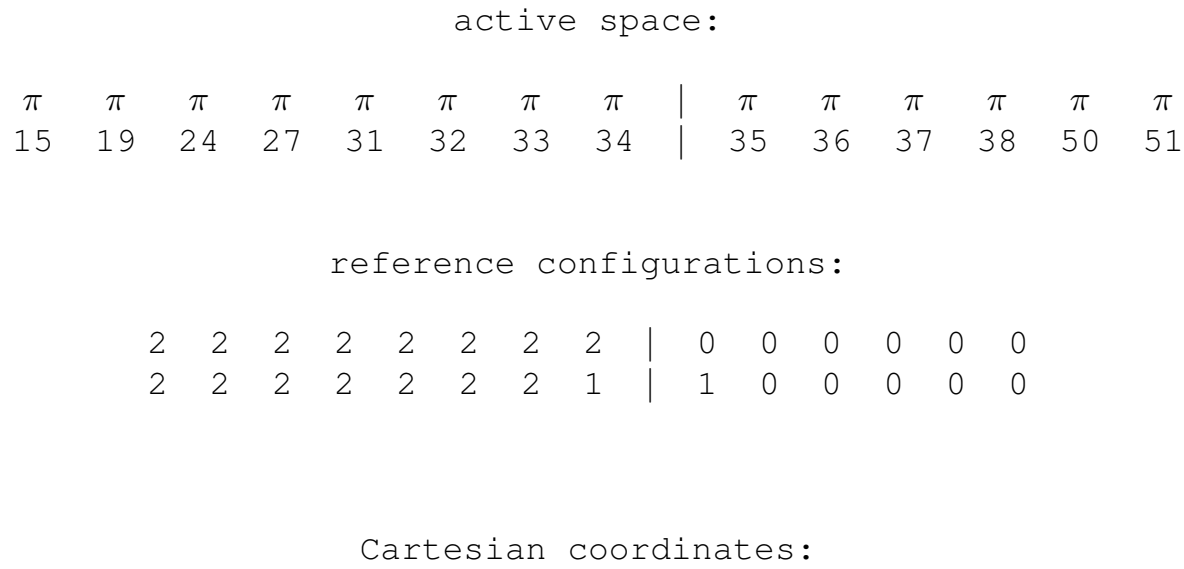


Table S71 Employed active space, reference configurations, and Cartesian coordinates of the OM2/MRCISD-optimized excited-state equilibrium geometry of the $1{ }^{1} B_{2 u}\left({ }^{1} \pi \pi^{*}\right)$ state of dibenzo- $p$-dioxin (excited-state energy: $-2308.66902 \mathrm{eV})$.

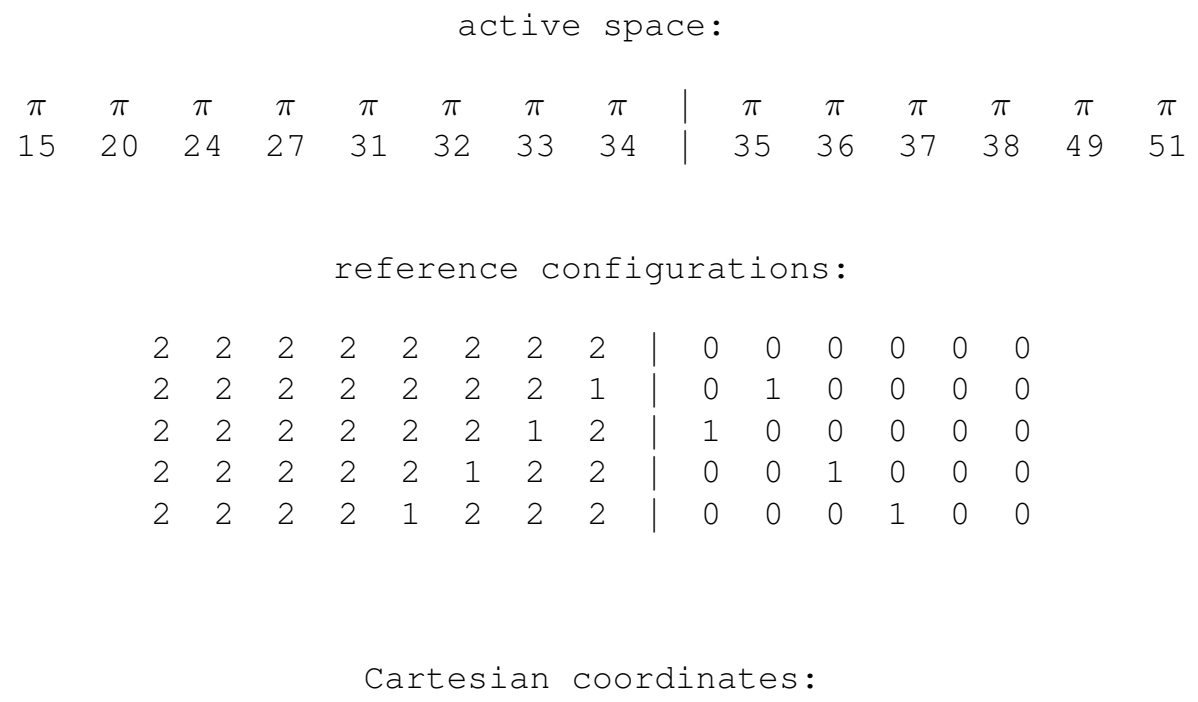

22

\begin{tabular}{lrrr}
$\mathrm{C}$ & 0.00000 & -3.55518 & 0.70215 \\
$\mathrm{C}$ & 0.00000 & -3.55518 & -0.70215 \\
$\mathrm{C}$ & 0.00000 & -2.36343 & -1.44074 \\
$\mathrm{C}$ & 0.00000 & -1.15092 & -0.70992 \\
$\mathrm{C}$ & 0.00000 & -1.15092 & 0.70992 \\
$\mathrm{C}$ & 0.00000 & -2.36343 & 1.44074 \\
$\mathrm{O}$ & 0.00000 & 0.00000 & -1.43522 \\
$\mathrm{C}$ & 0.00000 & 1.15092 & -0.70992 \\
$\mathrm{C}$ & 0.00000 & 1.15092 & 0.70992 \\
$\mathrm{O}$ & 0.00000 & 0.00000 & 1.43522 \\
$\mathrm{C}$ & 0.00000 & 2.36343 & -1.44074 \\
$\mathrm{C}$ & 0.00000 & 3.55518 & -0.70215 \\
$\mathrm{C}$ & 0.00000 & 3.55518 & 0.70215 \\
$\mathrm{C}$ & 0.00000 & 2.36343 & 1.44074 \\
$\mathrm{H}$ & 0.00000 & 4.50947 & 1.23952 \\
$\mathrm{H}$ & 0.00000 & -4.50947 & -1.23952 \\
$\mathrm{H}$ & 0.00000 & -2.34319 & -2.52591 \\
$\mathrm{H}$ & 0.00000 & -2.34319 & 2.52591 \\
$\mathrm{H}$ & 0.00000 & 2.34319 & -2.52591 \\
$\mathrm{H}$ & 0.00000 & 4.50947 & -1.23952 \\
\hline & & Continued & on next page
\end{tabular}

S94 
Continued from previous page

\begin{tabular}{rrrr}
\hline $\mathrm{H}$ & 0.00000 & -4.50947 & 1.23952 \\
$\mathrm{H}$ & 0.00000 & 2.34319 & 2.52591
\end{tabular}


Table S72 Employed active space, reference configurations, and Cartesian coordinates of the OM2/MRCISD-optimized excited-state equilibrium geometry of the $1^{1} B_{1 u}\left({ }^{1} \pi \pi^{*}\right)$ state of dibenzo- $p$-dioxin (excited-state energy: $-2307.19954 \mathrm{eV})$.

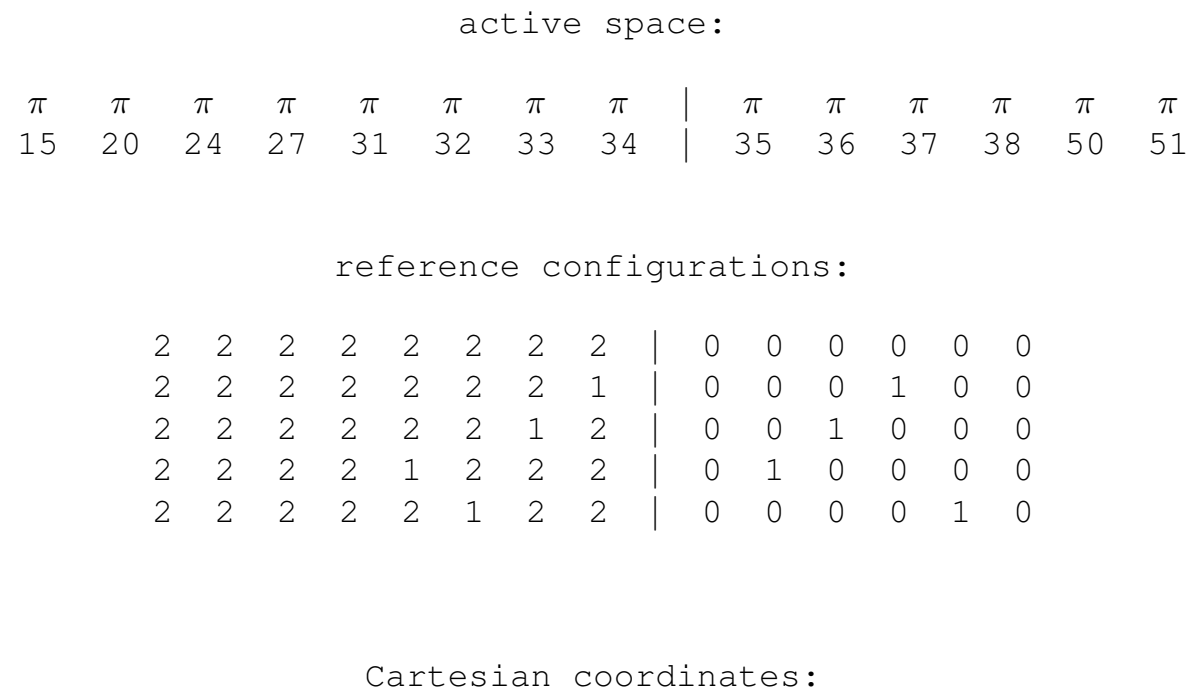

$$
22
$$$$
\text { C } \quad 0.00000-3.55295
$$$$
\text { C } \quad 0.00000-3.55295-0.70969
$$$$
\text { C } \quad 0.00000-2.36069-1.43382
$$$$
\text { C } \quad 0.00000-1.15236-0.71280
$$$$
\text { C } \quad 0.00000-1.15236 \quad 0.71280
$$$$
\text { C } \quad 0.00000-2.36069 \quad 1.43382
$$$$
0 \quad 0.00000 \quad 0.00000-1.44026
$$$$
\begin{array}{llll}
\text { C } & 0.00000 & 1.15236 & -0.71280
\end{array}
$$$$
\begin{array}{llll}
\text { C } & 0.00000 & 1.15236 & 0.71280
\end{array}
$$$$
\begin{array}{llll}
0 & 0.00000 & 0.00000 & 1.44026
\end{array}
$$$$
\begin{array}{llll}
\text { C } & 0.00000 & 2.36069 & -1.43382
\end{array}
$$$$
\begin{array}{llll}
\text { C } & 0.00000 & 3.55295 & -0.70969
\end{array}
$$$$
\begin{array}{llll}
\text { C } & 0.00000 & 3.55295 & 0.70969
\end{array}
$$$$
\text { C } \quad 0.00000 \quad 2.36069 \quad 1.43382
$$$$
\mathrm{H} \quad 0.00000 \quad 4.50605 \quad 1.24289
$$$$
\text { H } \quad 0.00000-4.50605-1.24289
$$$$
\mathrm{H} \quad 0.00000 \quad-2.33791-2.52255
$$$$
\begin{array}{llll}
\mathrm{H} & 0.00000 & -2.33791 & 2.52255
\end{array}
$$$$
\begin{array}{llll}
\mathrm{H} & 0.00000 & 2.33791 & -2.52255
\end{array}
$$$$
\mathrm{H} \quad 0.00000 \quad 4.50605 \quad-1.24289
$$

Continued on next page 
Continued from previous page

\begin{tabular}{rrrr}
\hline $\mathrm{H}$ & 0.00000 & -4.50605 & 1.24289 \\
$\mathrm{H}$ & 0.00000 & 2.33791 & 2.52255
\end{tabular}


Table S73 Employed active space, reference configurations, and Cartesian coordinates of the OM2/MRCISD-optimized excited-state equilibrium geometry of the $1^{3} A_{g}\left({ }^{3} \pi \pi^{*}\right)$ state of dibenzo- $p$-dioxin (excited-state energy: $-2308.66869 \mathrm{eV})$.

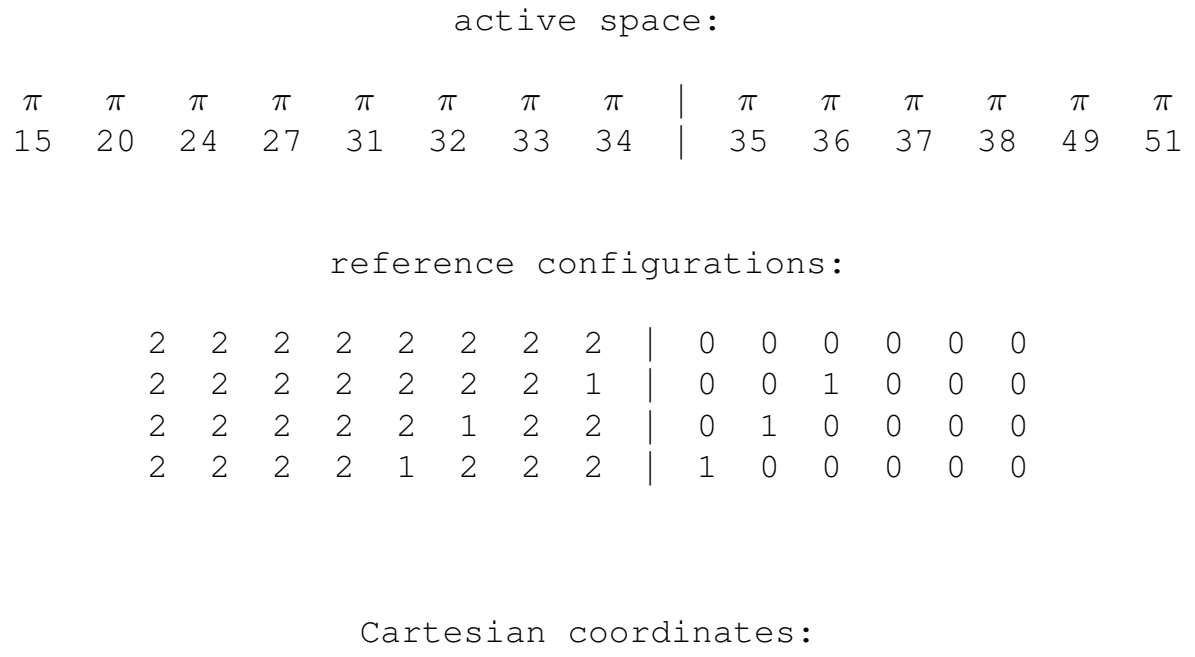


Continued from previous page

$\begin{array}{llll}\mathrm{H} & 0.00000 & 2.34171 & 2.52583\end{array}$ 
Table S74 Employed active space, reference configurations, and Cartesian coordinates of the OM2/MRCISD-optimized excited-state equilibrium geometry of the $1{ }^{3} B_{3 g}\left({ }^{3} \pi \pi^{*}\right)$ state of dibenzo-p-dioxin (excited-state energy: $-2309.62144 \mathrm{eV})$.

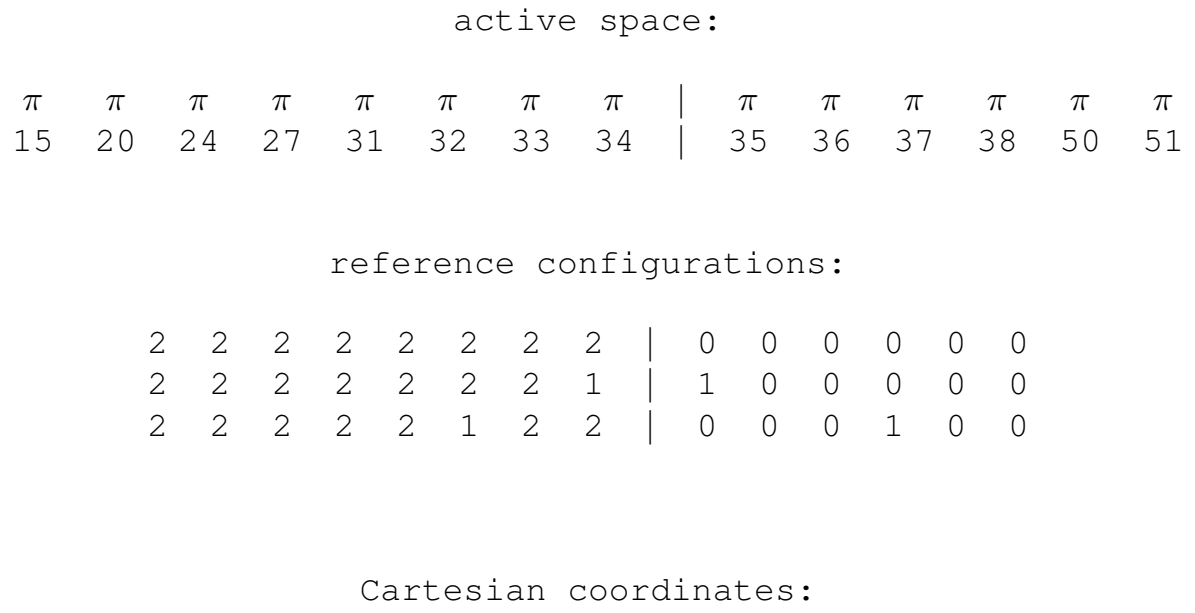


Table S75 Employed active space, reference configurations, and Cartesian coordinates of the OM2/MRCISD-optimized excited-state equilibrium geometry of the $1{ }^{3} B_{2 u}\left({ }^{3} \pi \pi^{*}\right)$ state of dibenzo- $p$-dioxin (excited-state energy: $-2308.80161 \mathrm{eV})$.

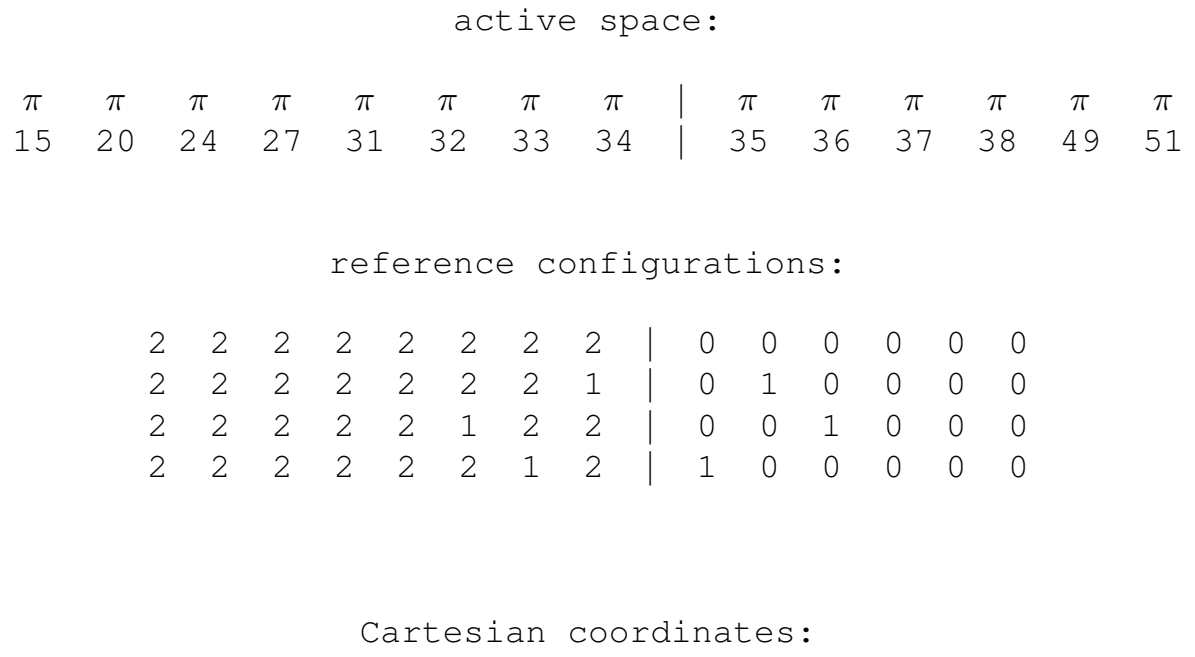

Continued on next page

S101 
Continued from previous page

$\begin{array}{llll}\mathrm{H} & 0.00000 & 2.34523 & 2.52707\end{array}$ 
Table S76 Employed active space, reference configurations, and Cartesian coordinates of the OM2/MRCISD-optimized excited-state equilibrium geometry of the $1{ }^{3} B_{1 u}\left({ }^{3} \pi \pi^{*}\right)$ state of dibenzo- $p$-dioxin (excited-state energy: $-2309.15632 \mathrm{eV})$.

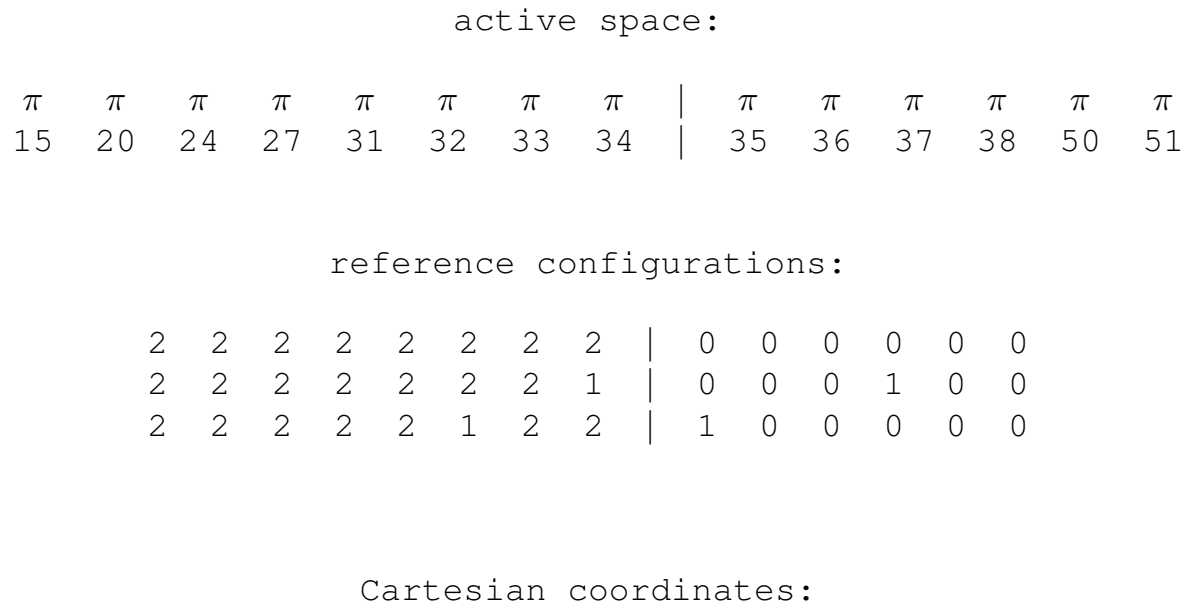


Table S77 Employed active space, reference configurations, and Cartesian coordinates of the OM2/MRCISD-optimized excited-state equilibrium geometry of the $1^{1} A_{u}\left({ }^{1} n \pi^{*}\right)$ state of dibenzo- $p$-dioxin (excited-state energy: $-2305.85396 \mathrm{eV})$.

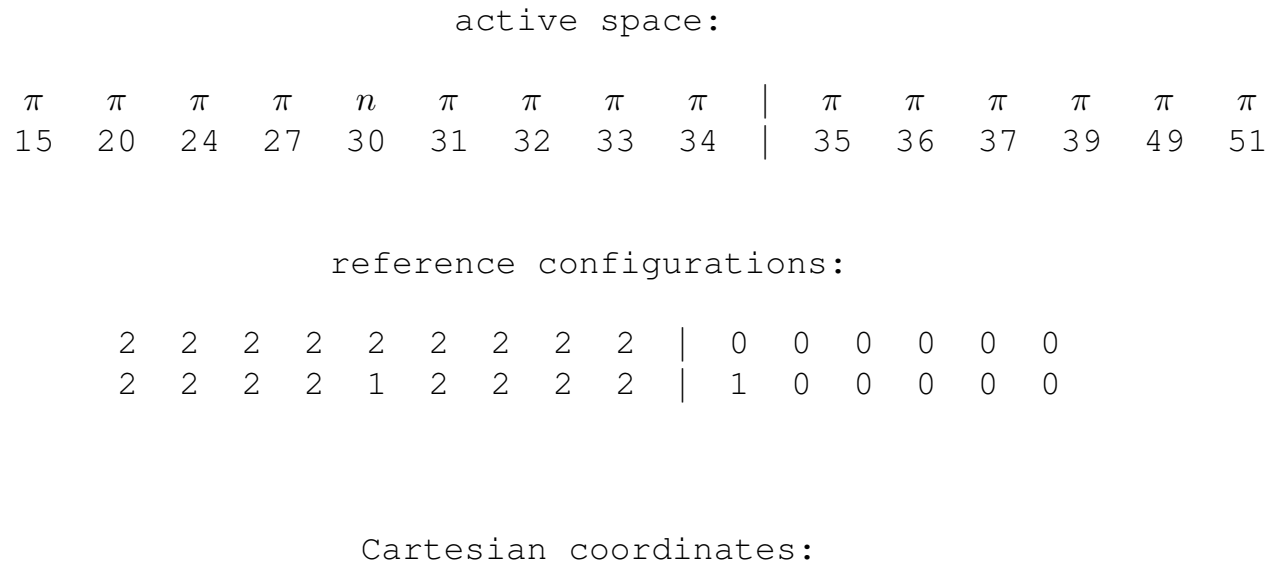


Table S78 Employed active space, reference configurations, and Cartesian coordinates of the OM2/MRCISD-optimized excited-state equilibrium geometry of the $1^{1} B_{1 g}\left({ }^{1} n \pi^{*}\right)$ state of dibenzo-p-dioxin (excited-state energy: $-2305.43859 \mathrm{eV})$.

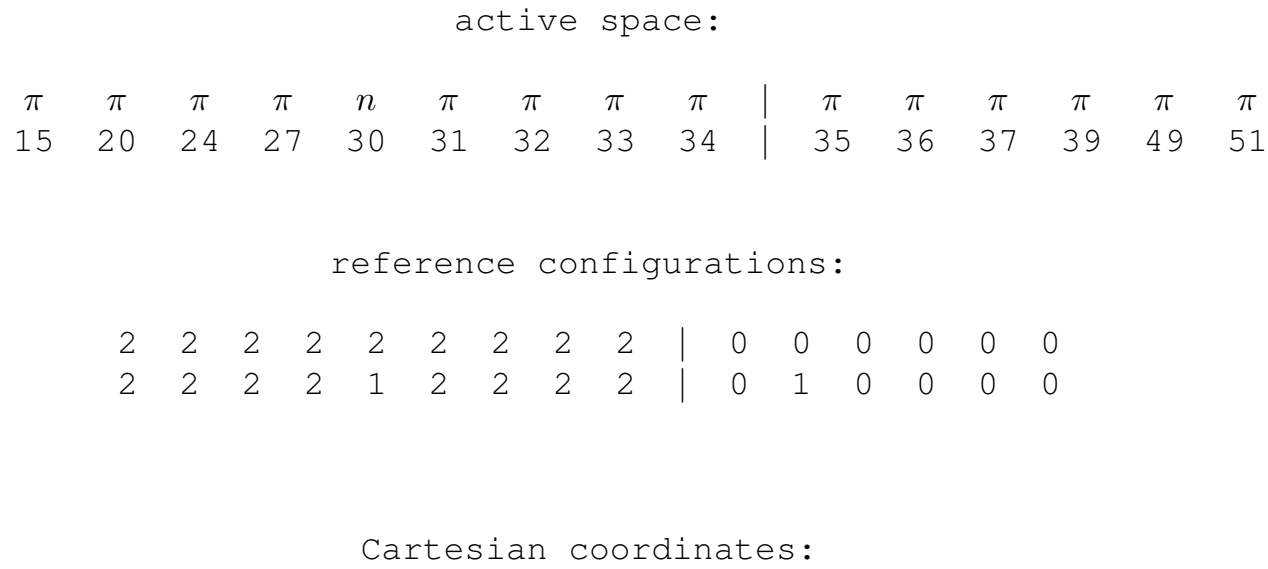


Table S79 Employed active space, reference configurations, and Cartesian coordinates of the OM2/MRCISD-optimized excited-state equilibrium geometry of the $1{ }^{1} B_{3 u}\left({ }^{1} n \pi^{*}\right)$ state of dibenzo- $p$-dioxin (excited-state energy: $-2305.11431 \mathrm{eV})$.

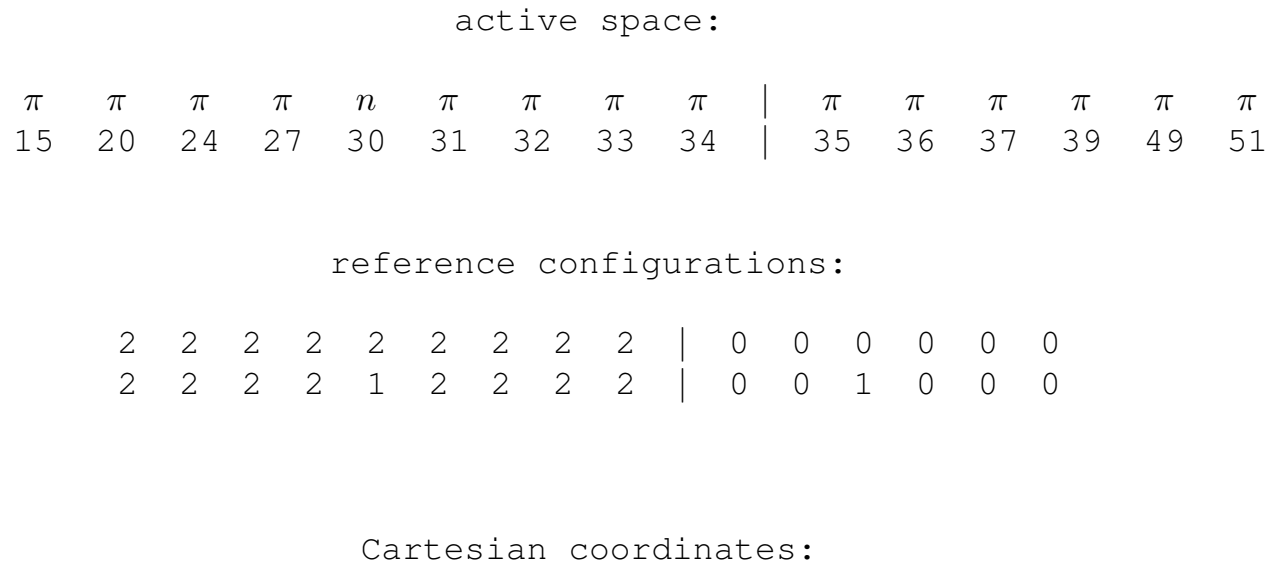


Table S80 Employed active space, reference configurations, and Cartesian coordinates of the OM2/MRCISD-optimized excited-state equilibrium geometry of the $1{ }^{1} B_{2 g}\left({ }^{1} n \pi^{*}\right)$ state of dibenzo- $p$-dioxin (excited-state energy: $-2304.90780 \mathrm{eV})$.

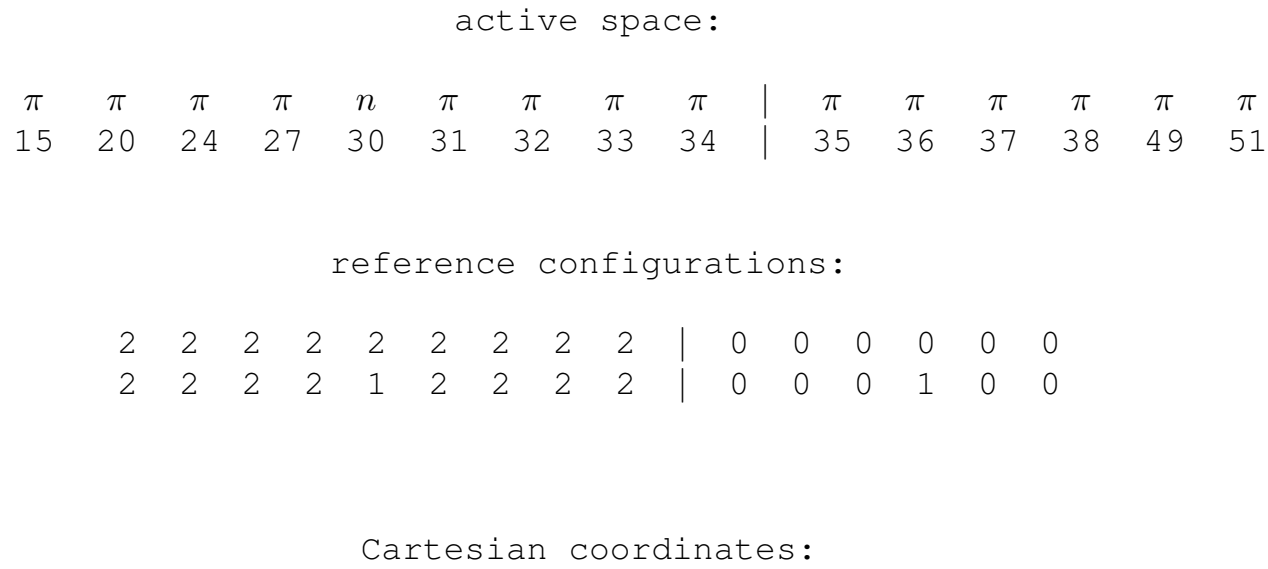


Table S81 Employed active space, reference configurations, and Cartesian coordinates of the OM2/MRCISD-optimized excited-state equilibrium geometry of the $1^{3} A_{u}\left({ }^{3} n \pi^{*}\right)$ state of dibenzo- $p$-dioxin (excited-state energy: $-2305.91522 \mathrm{eV})$.

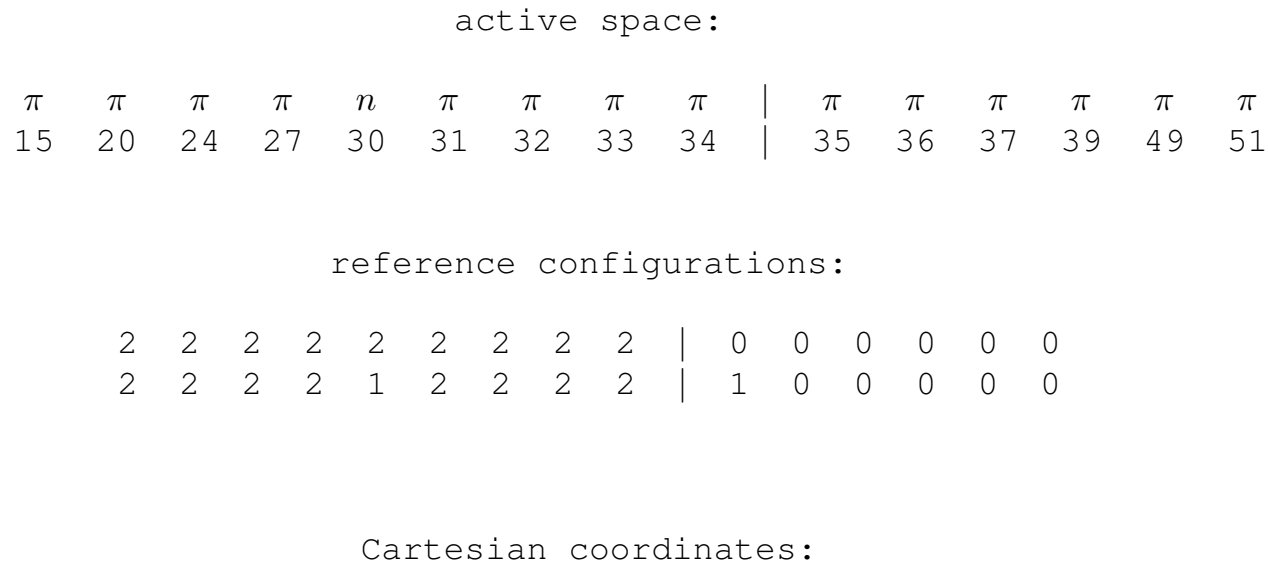


Table S82 Employed active space, reference configurations, and Cartesian coordinates of the OM2/MRCISD-optimized excited-state equilibrium geometry of the $1{ }^{3} B_{1 g}\left({ }^{3} n \pi^{*}\right)$ state of dibenzo-p-dioxin (excited-state energy: $-2305.49293 \mathrm{eV})$.

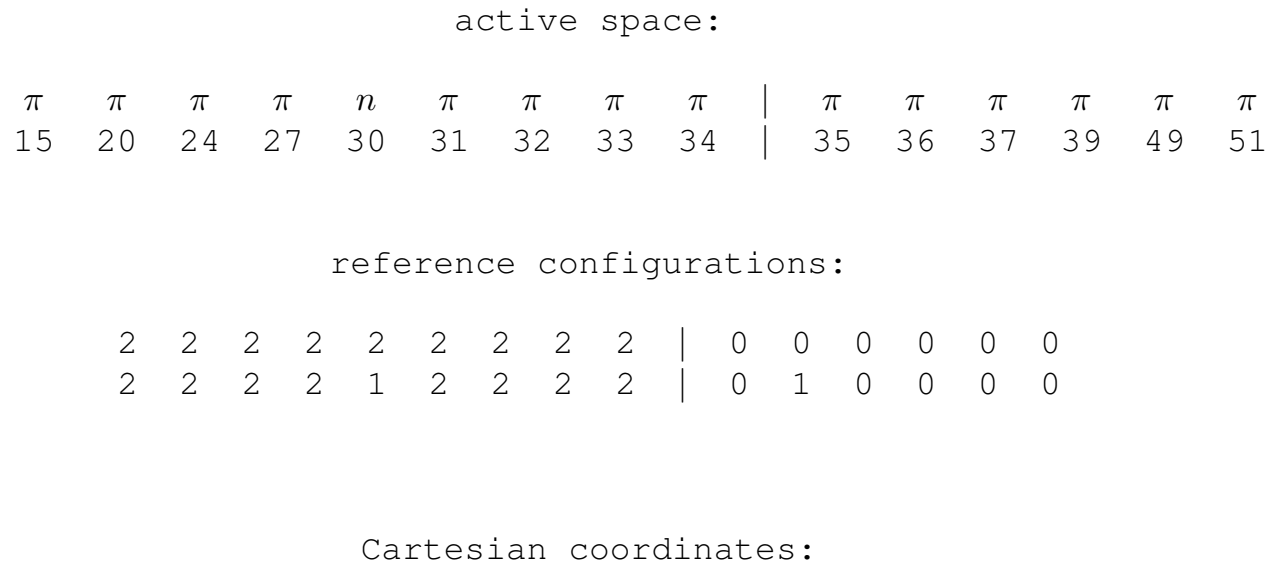


Table S83 Employed active space, reference configurations, and Cartesian coordinates of the OM2/MRCISD-optimized excited-state equilibrium geometry of the $1{ }^{3} B_{3 u}\left({ }^{3} n \pi^{*}\right)$ state of dibenzo- $p$-dioxin (excited-state energy: $-2305.18811 \mathrm{eV})$.

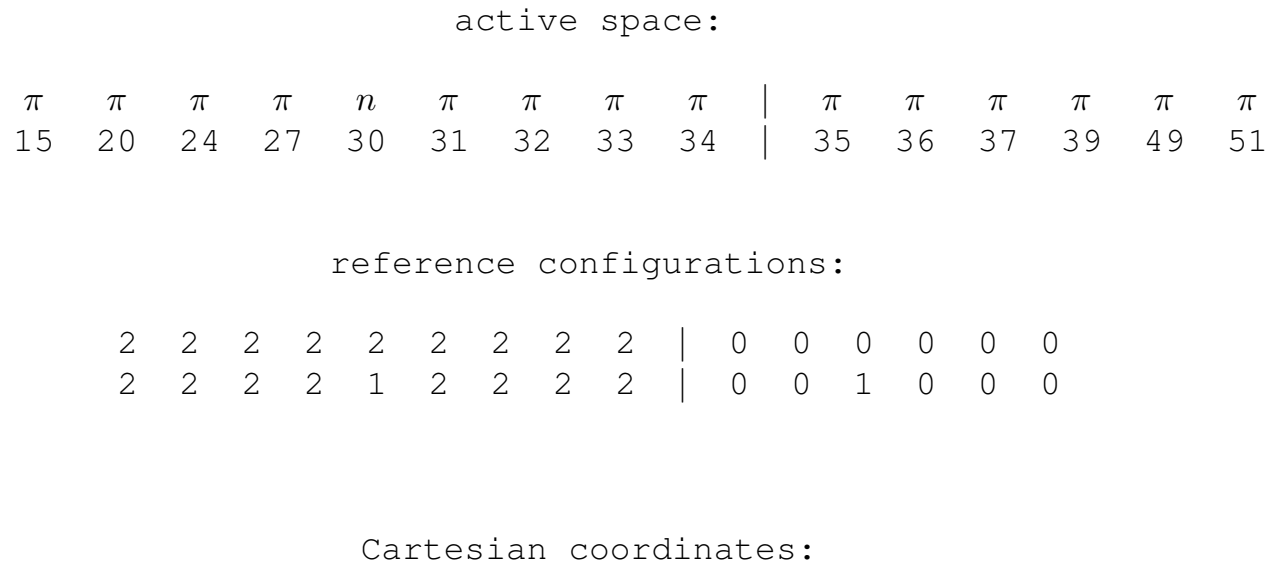


Table S84 Employed active space, reference configurations, and Cartesian coordinates of the OM2/MRCISD-optimized excited-state equilibrium geometry of the $1{ }^{3} B_{2 g}\left({ }^{3} n \pi^{*}\right)$ state of dibenzo-p-dioxin (excited-state energy: $-2304.97592 \mathrm{eV})$.

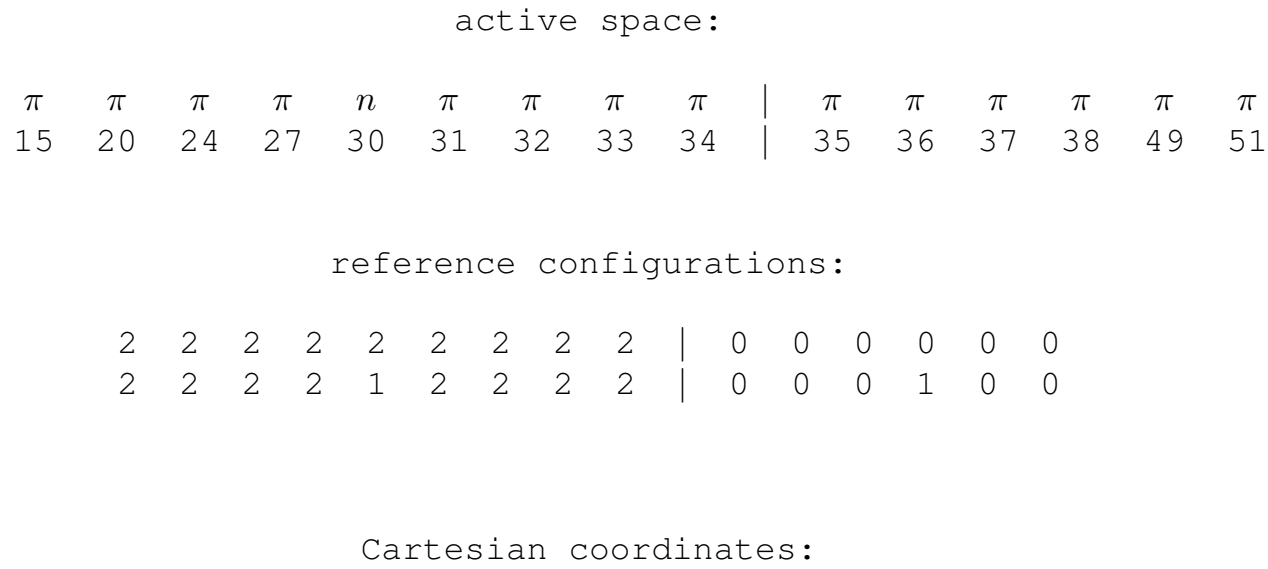


Table S85 Employed active space, reference configurations, and Cartesian coordinates of the OM2/MRCISD-optimized excited-state equilibrium geometry of the $1{ }^{1} A^{\prime \prime}\left({ }^{1} n \pi^{*}\right)$ state of formaldehyde (excited-state energy: $-485.90682 \mathrm{eV}$ ).

\begin{tabular}{|c|c|c|c|c|c|c|c|c|c|}
\hline$\sigma$ & $\sigma$ & $\sigma$ & $\sigma$ & $\pi$ & $n$ & $\pi$ & $\sigma$ & & \\
\hline \multirow[t]{2}{*}{1} & 2 & 3 & 4 & 5 & 6 & 7 & 8 & & \\
\hline & \multicolumn{4}{|c|}{ reference } & \multicolumn{5}{|c|}{ configurations: } \\
\hline 2 & 2 & 2 & 2 & 2 & 2 & 0 & 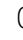 & & 0 \\
\hline 2 & 2 & 2 & 2 & 2 & 1 & 1 & & & 0 \\
\hline
\end{tabular}

Cartesian coordinates:

4

$\begin{array}{rrrr}\mathrm{C} & 0.00000 & 0.00000 & 0.00000 \\ \mathrm{O} & 0.00000 & 1.13653 & 0.58558 \\ \mathrm{H} & 0.93292 & -0.60696 & 0.00000 \\ \mathrm{H} & -0.93292 & -0.60696 & 0.00000\end{array}$


Table S86 Employed active space, reference configurations, and Cartesian coordinates of the OM2/MRCISD-optimized excited-state equilibrium geometry of the $1^{3} A^{\prime \prime}\left({ }^{3} n \pi^{*}\right)$ state of formaldehyde (excited-state energy: $\left.-486.17146 \mathrm{eV}\right)$.

\begin{tabular}{|c|c|c|c|c|c|c|c|c|c|}
\hline$\sigma$ & $\sigma$ & $\sigma$ & $\sigma$ & $\pi$ & $n$ & $\pi$ & $\sigma$ & & \\
\hline \multirow[t]{2}{*}{1} & 2 & 3 & 4 & 5 & 6 & 7 & 8 & & \\
\hline & \multicolumn{4}{|c|}{ reference } & \multicolumn{5}{|c|}{ configurations: } \\
\hline 2 & 2 & 2 & 2 & 2 & 2 & 0 & 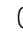 & & 0 \\
\hline 2 & 2 & 2 & 2 & 2 & 1 & 1 & & & 0 \\
\hline
\end{tabular}

Cartesian coordinates:

4

$\begin{array}{rrrr}\mathrm{C} & 0.00000 & 0.00000 & 0.00000 \\ \mathrm{O} & 0.00000 & 0.96356 & 0.81316 \\ \mathrm{H} & 0.92946 & -0.67446 & 0.00000 \\ \mathrm{H} & -0.92946 & -0.67446 & 0.00000\end{array}$

S113 
Table S87 Employed active space, reference configurations, and Cartesian coordinates of the OM2/MRCISD-optimized excited-state equilibrium geometry of the $1^{3} A^{\prime}\left({ }^{3} \pi \pi^{*}\right)$ state of formaldehyde (excited-state energy: $-484.34770 \mathrm{eV}$ ).

\begin{tabular}{|c|c|c|c|c|c|c|c|c|}
\hline$\sigma$ & $\sigma$ & $\sigma$ & $\sigma$ & $\pi$ & $n$ & $\pi$ & $\sigma$ & $\sigma$ \\
\hline \multirow[t]{2}{*}{1} & 2 & 3 & 4 & 5 & 6 & 7 & 8 & 9 \\
\hline & \multicolumn{4}{|c|}{ reference } & \multicolumn{4}{|c|}{ configurations } \\
\hline 2 & 2 & 2 & 2 & 2 & 2 & 0 & 0 & \\
\hline 2 & 2 & 2 & 2 & 1 & 2 & 1 & 0 & \\
\hline
\end{tabular}

Cartesian coordinates:

4

$\begin{array}{rrrr}\mathrm{C} & 0.00000 & 0.00000 & 0.00000 \\ \mathrm{O} & 0.00000 & 1.14870 & 0.79648 \\ \mathrm{H} & 0.95366 & -0.57806 & 0.00000 \\ \mathrm{H} & -0.95366 & -0.57806 & 0.00000\end{array}$

S114 
Table S88 Employed active space, reference configurations, and Cartesian coordinates of the OM2/MRCISD-optimized excited-state equilibrium geometry of the $1{ }^{1} A_{u}\left({ }^{1} n \pi^{*}\right)$ state of trans-glyoxal (excited-state energy: $-944.59295 \mathrm{eV}$ ).

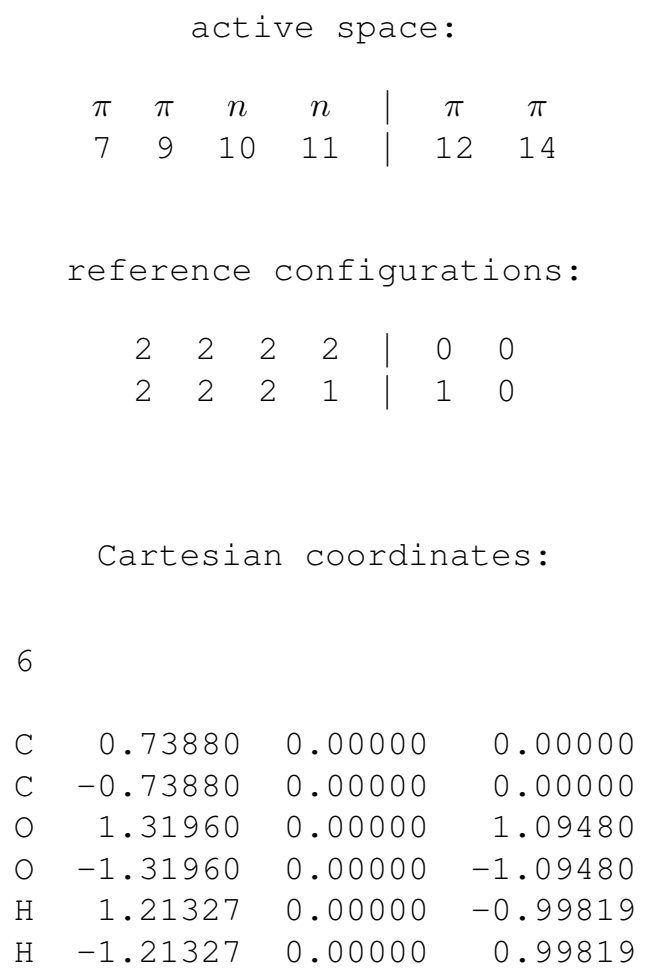


Table S89 Employed active space, reference configurations, and Cartesian coordinates of the OM2/MRCISD-optimized excited-state equilibrium geometry of the $1^{3} A_{u}\left({ }^{3} n \pi^{*}\right)$ state of trans-glyoxal (excited-state energy: $-944.86690 \mathrm{eV}$ ).

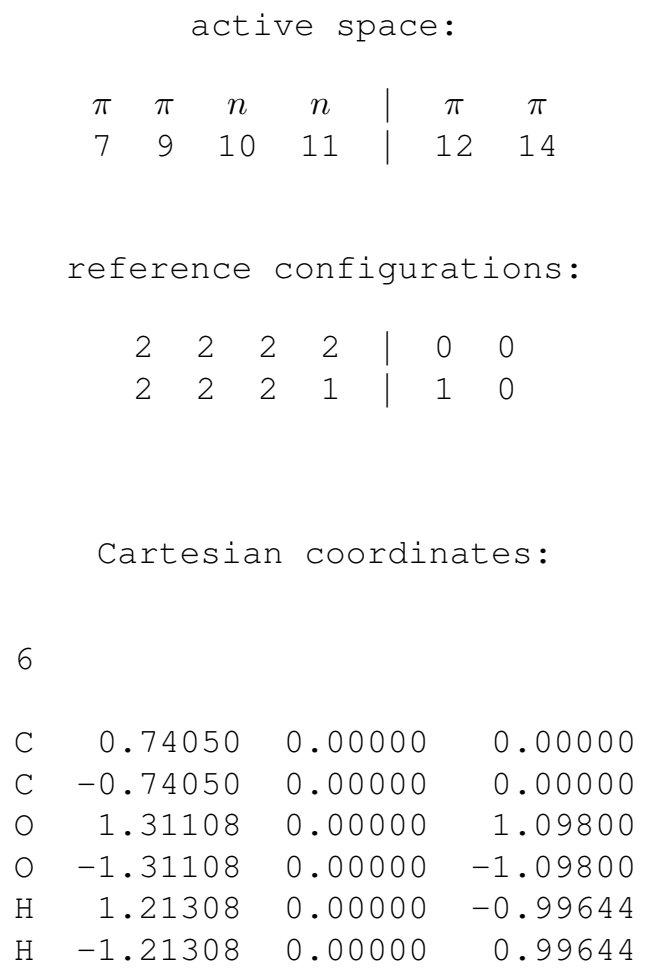


Table S90 Employed active space, reference configurations, and Cartesian coordinates of the OM2/MRCISD-optimized excited-state equilibrium geometry of the $2{ }^{1} A\left({ }^{1} \pi \pi^{*}\right)$ state of $9 H$-guanine (excited-state energy: $\left.-2105.89524 \mathrm{eV}\right)$.

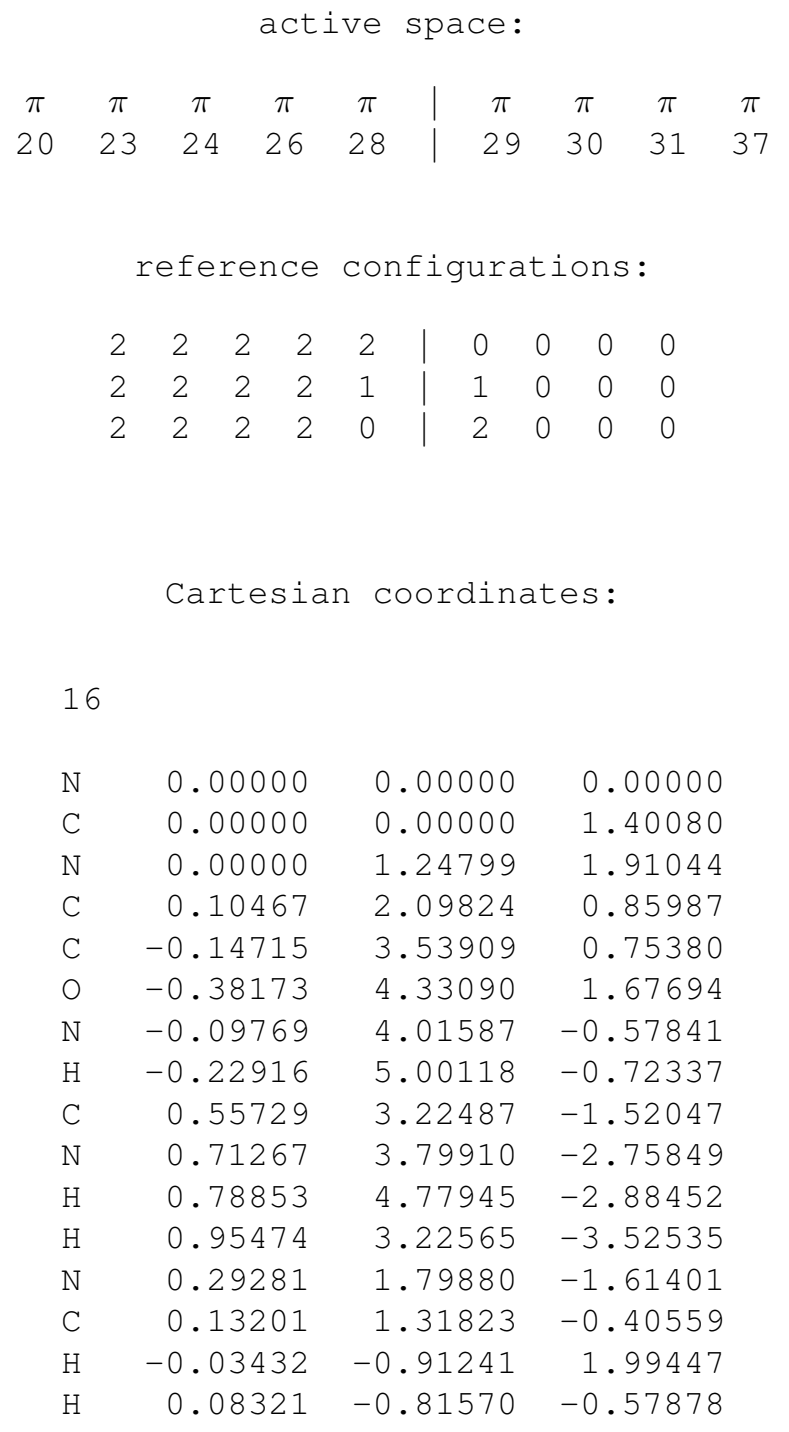


Table S91 Employed active space, reference configurations, and Cartesian coordinates of the OM2/MRCISD-optimized excited-state equilibrium geometry of the $2{ }^{1} A^{\prime}\left({ }^{1} \pi \pi^{*}\right)$ state of 1-hydroxy-2-acetonaphthone (excited-state energy: $-2340.17717 \mathrm{eV})$.

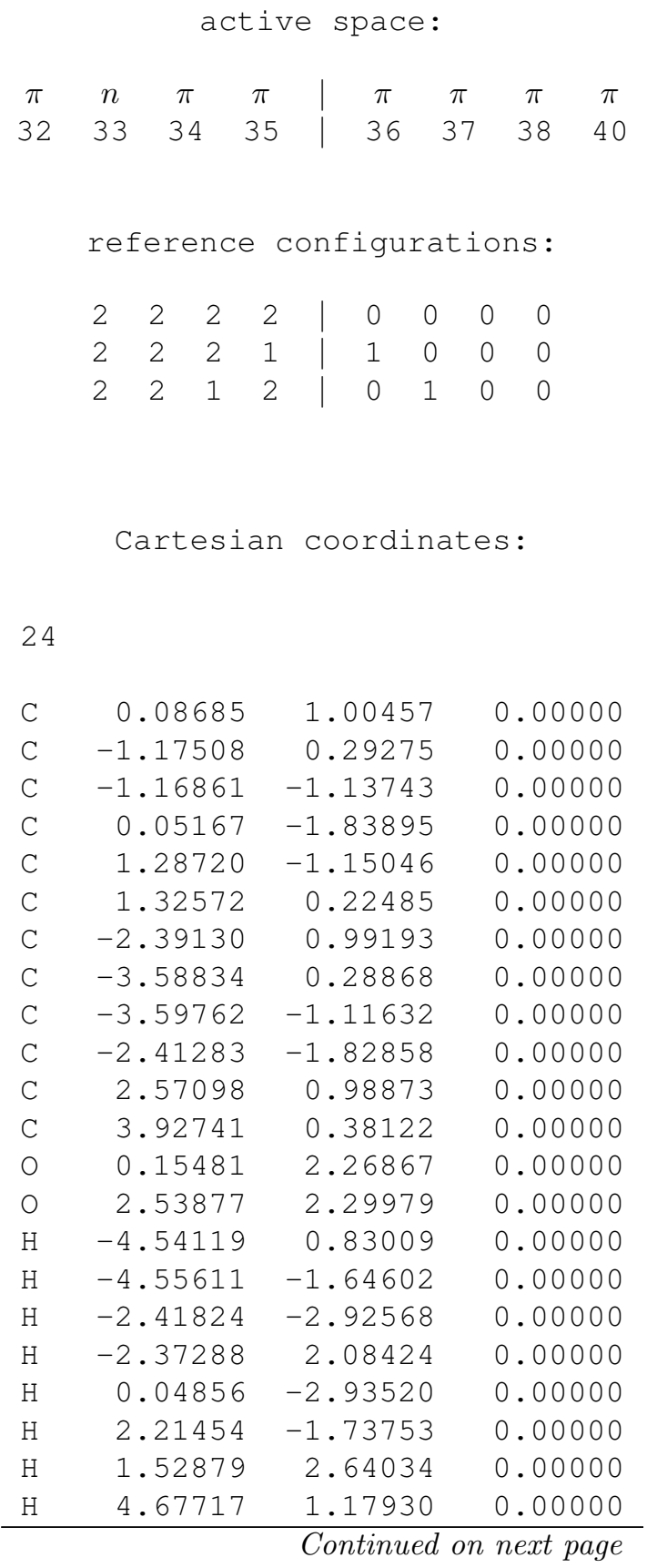

S118 
Continued from previous page

\begin{tabular}{lllr}
\hline $\mathrm{H}$ & 4.05547 & -0.24282 & -0.89819 \\
$\mathrm{H}$ & 4.05547 & -0.24282 & 0.89819
\end{tabular}

S119 
Table S92 Employed active space, reference configurations, and Cartesian coordinates of the OM2/MRCISD-optimized excited-state equilibrium geometry of the $2{ }^{1} A^{\prime}\left({ }^{1} \pi \pi^{*}\right)$ state of indole (excited-state energy: $\left.-1334.68423 \mathrm{eV}\right)$.

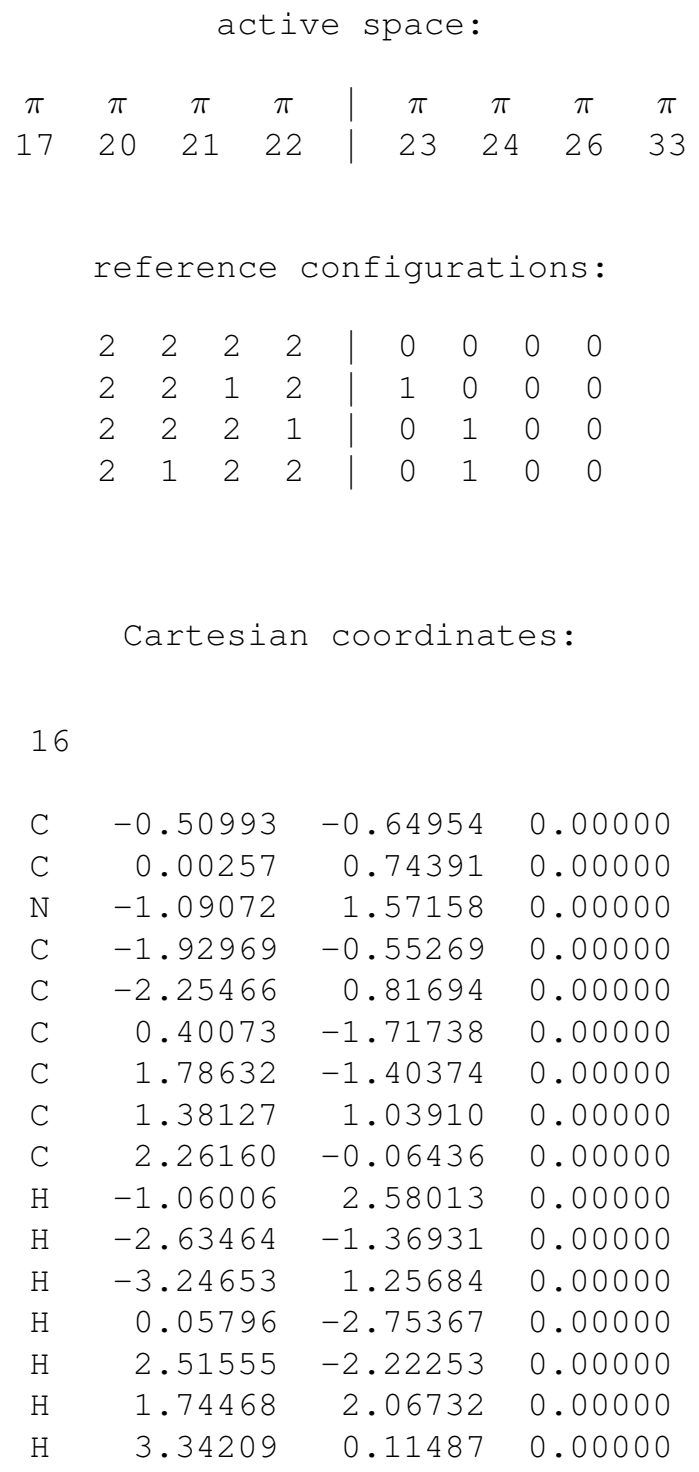


Table S93 Employed active space, reference configurations, and Cartesian coordinates of the OM2/MRCISD-optimized excited-state equilibrium geometry of the $3{ }^{1} A^{\prime}\left({ }^{1} \pi \pi^{*}\right)$ state of indole (excited-state energy: $\left.-1334.03255 \mathrm{eV}\right)$.

\begin{tabular}{|c|c|c|c|c|c|c|c|}
\hline \multirow{2}{*}{$\begin{array}{c}\pi \\
17\end{array}$} & $\pi$ & $\pi$ & $\pi$ & $\pi$ & \multirow{2}{*}{\multicolumn{2}{|c|}{$\begin{array}{c}\pi \\
24\end{array}$}} & \multirow{2}{*}{$\begin{array}{c}\pi \\
26\end{array}$} \\
\hline & 20 & 21 & 22 & 23 & & & \\
\hline \multicolumn{3}{|c|}{ reference } & \multicolumn{5}{|c|}{ configurations: } \\
\hline & 2 & 22 & 2 & 0 & 0 & 0 & 0 \\
\hline & 2 & 22 & 1 & 1 & 0 & 0 & 0 \\
\hline & 2 & $2 \quad 1$ & 2 & 1 & 0 & 0 & 0 \\
\hline & 2 & 22 & 1 & 0 & & 0 & 0 \\
\hline & 2 & 21 & 2 & 0 & 1 & 0 & 0 \\
\hline & 2 & 12 & 2 & 0 & 1 & 0 & 0 \\
\hline \multicolumn{8}{|c|}{ Cartesian coordinates: } \\
\hline 16 & & & & & & & \\
\hline $\mathrm{C}$ & -0 & .49145 & & -0.6477 & & & 00000 \\
\hline $\mathrm{C}$ & & .00962 & & 0.7137 & & & 00000 \\
\hline $\mathrm{N}$ & -1 & .10424 & & 1.5740 & & & 00000 \\
\hline C & -1 & .90105 & & -0.5560 & & & 00000 \\
\hline C & -2 & .25052 & & 0.8500 & & & 00000 \\
\hline C & & .42684 & & -1.7166 & & & 00000 \\
\hline C & & .79763 & & -1.3907 & & & 00000 \\
\hline $\mathrm{C}$ & & .36221 & & 1.0330 & & & 00000 \\
\hline $\mathrm{C}$ & & .25538 & & -0.0780 & & & 00000 \\
\hline $\mathrm{H}$ & -1 & .05270 & & 2.5873 & & & 00000 \\
\hline $\mathrm{H}$ & -2 & .61991 & & -1.3620 & & & 00000 \\
\hline $\mathrm{H}$ & -3 & .25220 & & 1.2674 & & & 00000 \\
\hline $\mathrm{H}$ & & .08162 & & -2.7498 & & & 00000 \\
\hline $\mathrm{H}$ & & .52900 & & -2.2102 & & & 00000 \\
\hline $\mathrm{H}$ & & .72506 & & 2.0597 & & & 00000 \\
\hline $\mathrm{H}$ & & .33219 & & 0.1277 & & & 00000 \\
\hline
\end{tabular}


Table S94 Employed active space, reference configurations, and Cartesian coordinates of the OM2/MRCISD-optimized excited-state equilibrium geometry of the $1{ }^{1} B_{1}\left({ }^{1} n \pi^{*}\right)$ state of maleimide (excited-state energy: $\left.-1426.33177 \mathrm{eV}\right)$.

\begin{tabular}{lcccc|cccc}
$\pi$ & \multicolumn{1}{c}{ active space: } \\
9 & $\pi$ & $\pi$ & $n$ & $\pi$ & $\pi$ & $\pi$ & $\pi$ \\
9 & 13 & 16 & 17 & 18 & 19 & 22 & 23 \\
& \\
reference configurations: \\
2 & 2 & 2 & 2 & 2 & 0 & 0 & 0 \\
2 & 2 & 2 & 1 & 2 & 1 & 0 & 0
\end{tabular}

Cartesian coordinates:

10

$\begin{array}{lrrr}\mathrm{N} & 0.00000 & 0.00000 & 0.00000 \\ \mathrm{C} & 1.15288 & 0.82484 & 0.00000 \\ \mathrm{C} & 0.68690 & 2.21204 & 0.00000 \\ \mathrm{C} & -0.68690 & 2.21204 & 0.00000 \\ \mathrm{C} & -1.15288 & 0.82484 & 0.00000 \\ \mathrm{O} & 2.28434 & 0.29821 & 0.00000 \\ \mathrm{O} & -2.28434 & 0.29821 & 0.00000 \\ \mathrm{H} & 0.00000 & -1.01209 & 0.00000 \\ \mathrm{H} & 1.38186 & 3.03201 & 0.00000 \\ \mathrm{H} & -1.38186 & 3.03201 & 0.00000\end{array}$


Table S95 Employed active space, reference configurations, and Cartesian coordinates of the OM2/MRCISD-optimized excited-state equilibrium geometry of the $1{ }^{1} A_{2}\left({ }^{1} n \pi^{*}\right)$ state of maleimide (excited-state energy: $-1426.75001 \mathrm{eV}$ ).

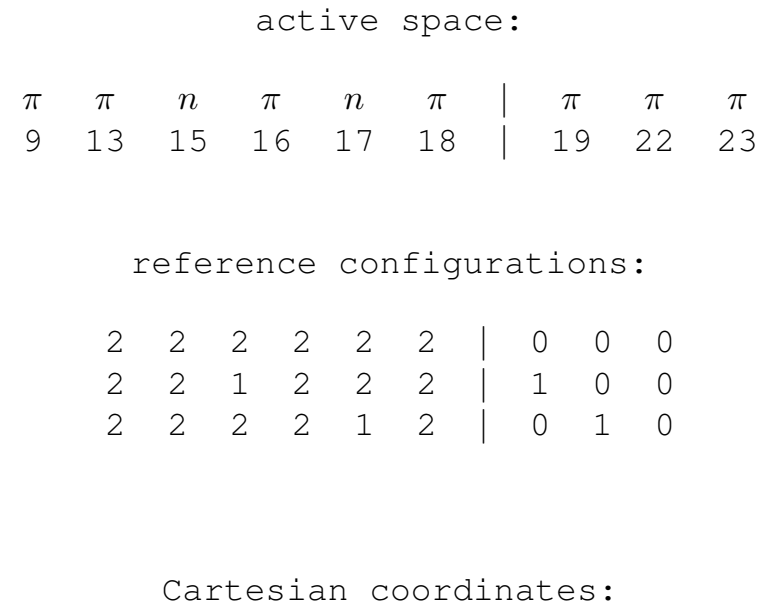


Table S96 Employed active space, reference configurations, and Cartesian coordinates of the OM2/MRCISD-optimized excited-state equilibrium geometry of the $1{ }^{1} B_{2}\left({ }^{1} \pi \pi^{*}\right)$ state of maleimide (excited-state energy: $\left.-1426.55619 \mathrm{eV}\right)$.

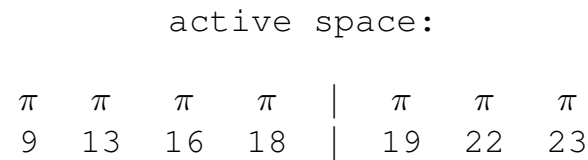

reference configurations:

\begin{tabular}{llll|lll}
2 & 2 & 2 & 2 & 0 & 0 & 0 \\
2 & 2 & 1 & 2 & 1 & 0 & 0 \\
2 & 2 & 2 & 1 & 1 & 0 & 0 \\
2 & 1 & 2 & 1 & 2 & 0 & 0
\end{tabular}

Cartesian coordinates:

10

$\begin{array}{lrrr}\mathrm{N} & 0.00000 & 0.00000 & 0.00000 \\ \mathrm{C} & 1.19694 & 0.84359 & 0.00000 \\ \mathrm{C} & 0.70340 & 2.18944 & 0.00000 \\ \mathrm{C} & -0.70340 & 2.18944 & 0.00000 \\ \mathrm{C} & -1.19694 & 0.84359 & 0.00000 \\ \mathrm{O} & 2.30721 & 0.27947 & 0.00000 \\ \mathrm{O} & -2.30721 & 0.27947 & 0.00000 \\ \mathrm{H} & 0.00000 & -1.02305 & 0.00000 \\ \mathrm{H} & 1.35320 & 3.04952 & 0.00000 \\ \mathrm{H} & -1.35320 & 3.04952 & 0.00000\end{array}$


Table S97 Employed active space, reference configurations, and Cartesian coordinates of the OM2/MRCISD-optimized excited-state equilibrium geometry of the $2{ }^{1} B_{2}\left({ }^{1} \pi \pi^{*}\right)$ state of maleimide (excited-state energy: $-1424.94822 \mathrm{eV}$ ).

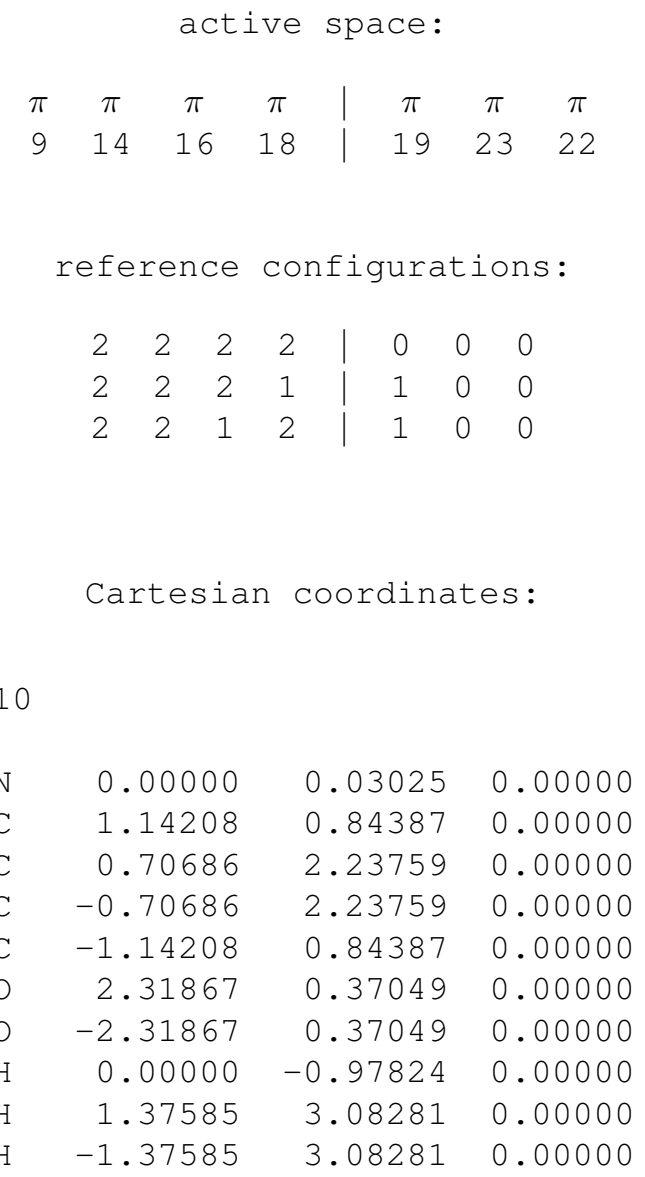


Table S98 Employed active space, reference configurations, and Cartesian coordinates of the OM2/MRCISD-optimized excited-state equilibrium geometry of the $1{ }^{3} B_{1}\left({ }^{3} n \pi^{*}\right)$ state of maleimide (excited-state energy: $\left.-1426.52987 \mathrm{eV}\right)$.

\begin{tabular}{lcccc|cccc}
$\pi$ & \multicolumn{1}{c}{ active space: } \\
9 & $\pi$ & $\pi$ & $n$ & $\pi$ & $\pi$ & $\pi$ & $\pi$ \\
9 & 13 & 16 & 17 & 18 & 19 & 22 & 23 \\
& \\
reference configurations: \\
2 & 2 & 2 & 2 & 2 & 0 & 0 & 0 \\
2 & 2 & 2 & 1 & 2 & 1 & 0 & 0
\end{tabular}

Cartesian coordinates:

10

$\begin{array}{lrrr}\mathrm{N} & 0.00000 & 0.00000 & 0.00000 \\ \mathrm{C} & 1.15154 & 0.82084 & 0.00000 \\ \mathrm{C} & 0.68618 & 2.21014 & 0.00000 \\ \mathrm{C} & -0.68618 & 2.21014 & 0.00000 \\ \mathrm{C} & -1.15154 & 0.82084 & 0.00000 \\ \mathrm{O} & 2.29008 & 0.30959 & 0.00000 \\ \mathrm{O} & -2.29008 & 0.30959 & 0.00000 \\ \mathrm{H} & 0.00000 & -1.01211 & 0.00000 \\ \mathrm{H} & 1.38388 & 3.02761 & 0.00000 \\ \mathrm{H} & -1.38388 & 3.02761 & 0.00000\end{array}$


Table S99 Employed active space, reference configurations, and Cartesian coordinates of the OM2/MRCISD-optimized excited-state equilibrium geometry of the $1^{3} A_{2}\left({ }^{3} n \pi^{*}\right)$ state of maleimide (excited-state energy: $-1426.87668 \mathrm{eV}$ ).

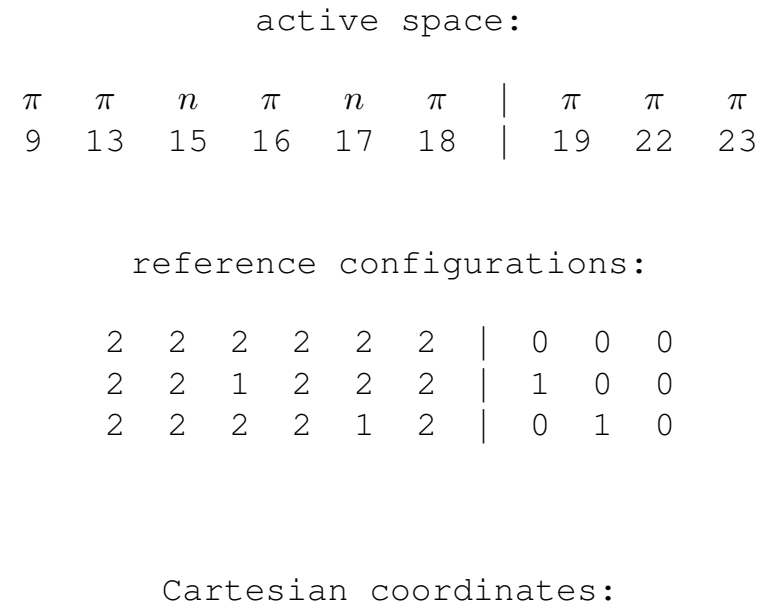


Table S100 Employed active space, reference configurations, and Cartesian coordinates of the OM2/MRCISD-optimized excited-state equilibrium geometry of the $1^{3} B_{2}\left({ }^{3} \pi \pi^{*}\right)$ state of maleimide (excited-state energy: $-1427.73600 \mathrm{eV}$ ).

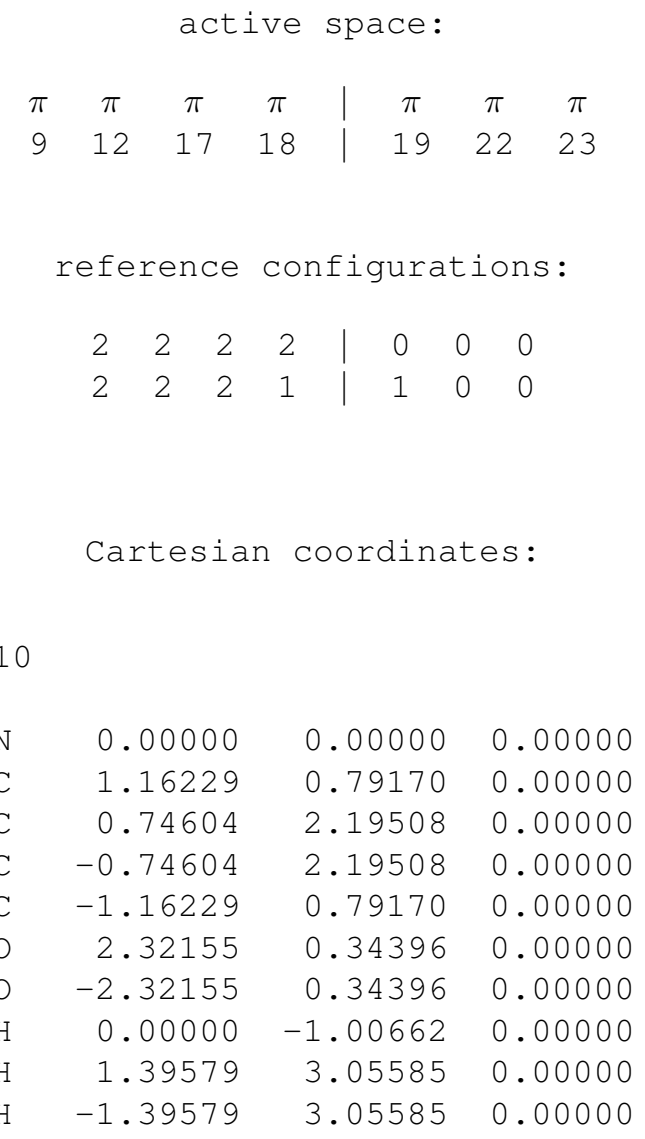


Table S101 Employed active space, reference configurations, and Cartesian coordinates of the OM2/MRCISD-optimized excited-state equilibrium geometry of the $1^{1} A^{\prime \prime}\left({ }^{1} n \pi^{*}\right)$ state of nitrosomethane (excited-state energy: $-713.44566 \mathrm{eV})$.

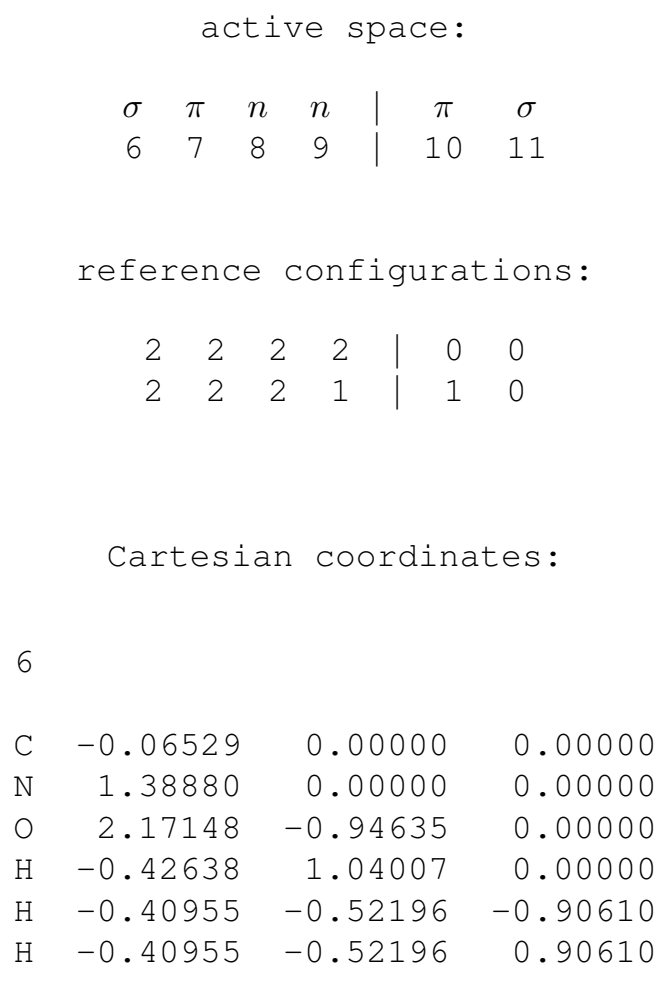


Table S102 Employed active space, reference configurations, and Cartesian coordinates of the OM2/MRCISD-optimized excited-state equilibrium geometry of the $1^{3} A^{\prime \prime}\left({ }^{3} n \pi^{*}\right)$ state of nitrosomethane (excited-state energy: $-714.16995 \mathrm{eV})$.

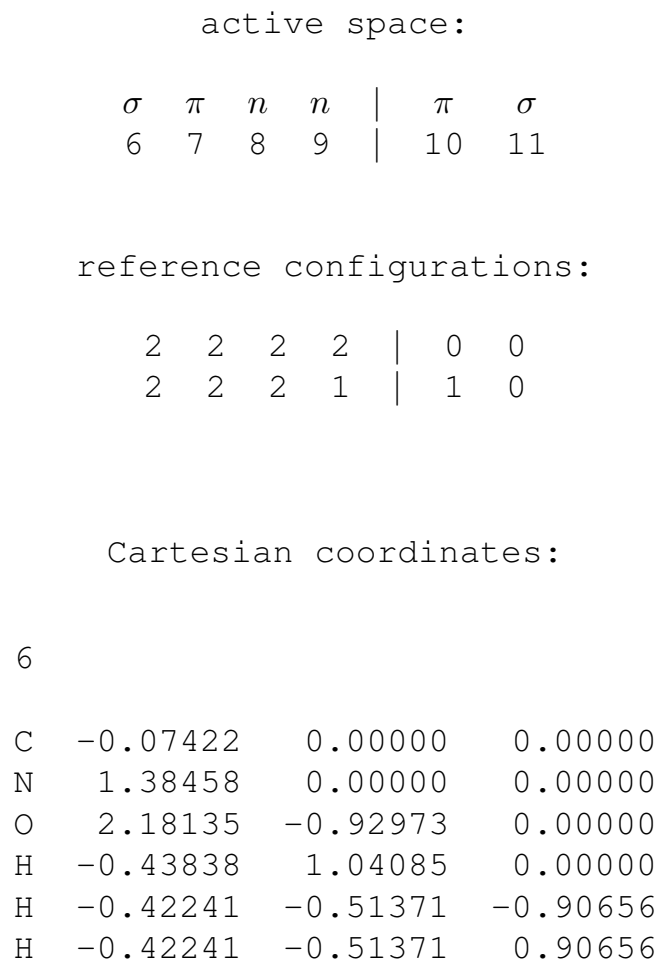


Table S103 Employed active space, reference configurations, and Cartesian coordinates of the OM2/MRCISD-optimized excited-state equilibrium geometry of the $1^{1} A^{\prime \prime}\left({ }^{1} n \pi^{*}\right)$ state of propenoic acid anion (excited-state energy: $-1087.33588 \mathrm{eV})$.

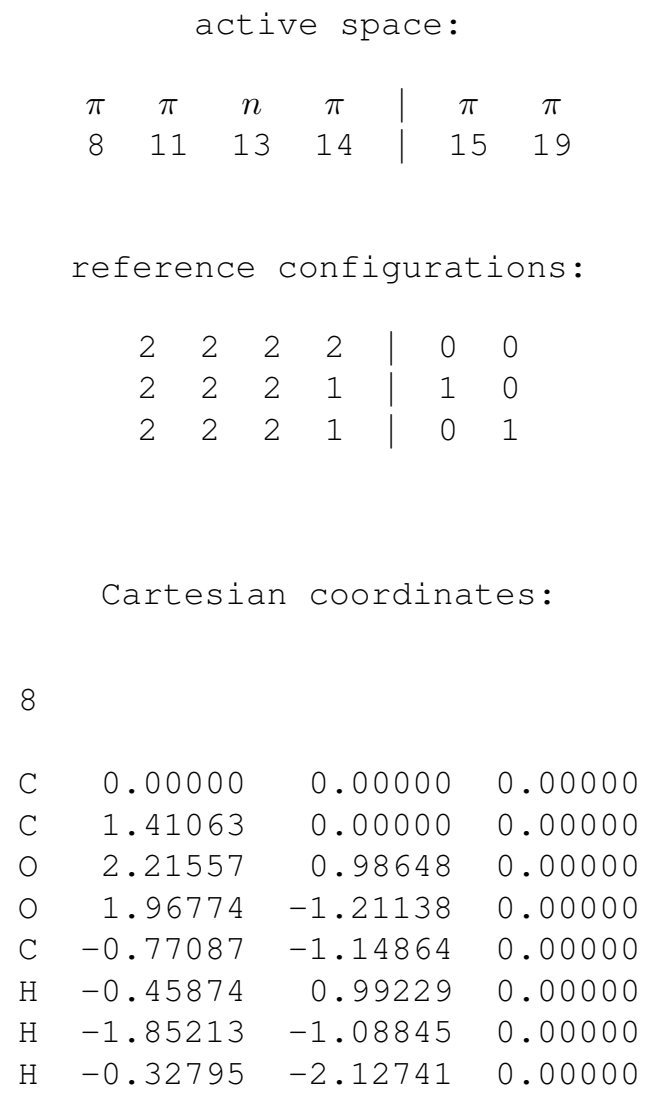


Table S104 Employed active space, reference configurations, and Cartesian coordinates of the OM2/MRCISD-optimized excited-state equilibrium geometry of the $1{ }^{1} A_{2}\left({ }^{1} n \pi^{*}\right)$ state of pyridine (excited-state energy: $-921.97652 \mathrm{eV}$ ).

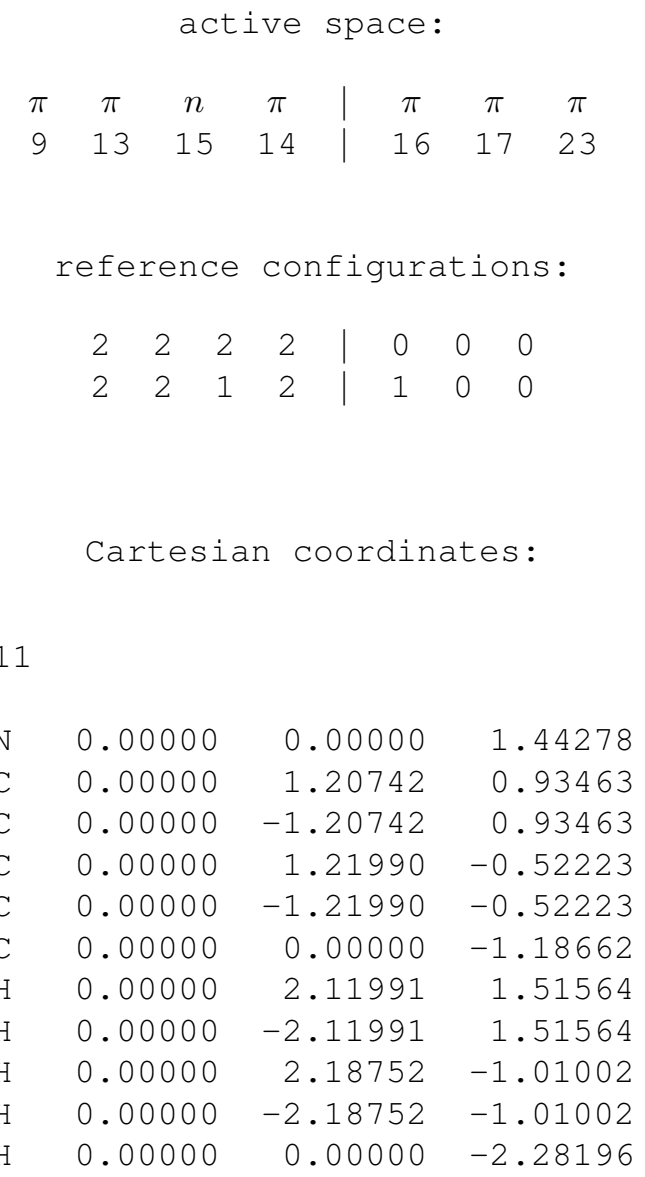


Table S105 Employed active space, reference configurations, and Cartesian coordinates of the OM2/MRCISD-optimized excited-state equilibrium geometry of the $2{ }^{1} A^{\prime}\left({ }^{1} n \pi^{*}\right)$ state of pyridine (excited-state energy: $-921.88873 \mathrm{eV}$ ).

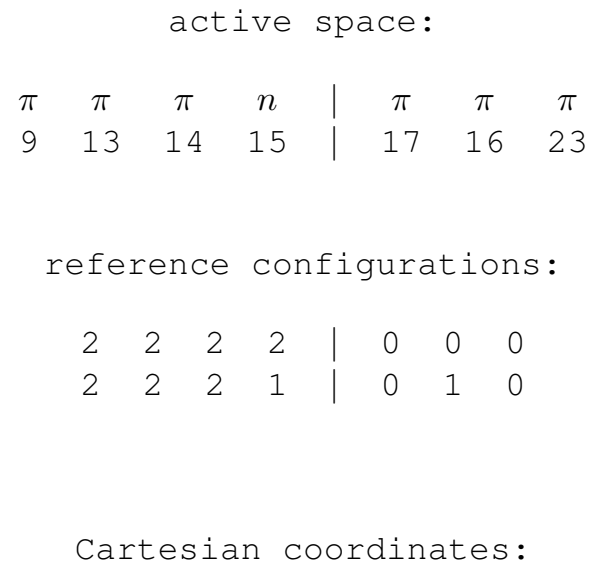


Table S106 Employed active space, reference configurations, and Cartesian coordinates of the OM2/MRCISD-optimized excited-state equilibrium geometry of the $1^{3} A_{1}\left({ }^{3} \pi \pi^{*}\right)$ state of pyridine (excited-state energy: $-922.77782 \mathrm{eV}$ ).

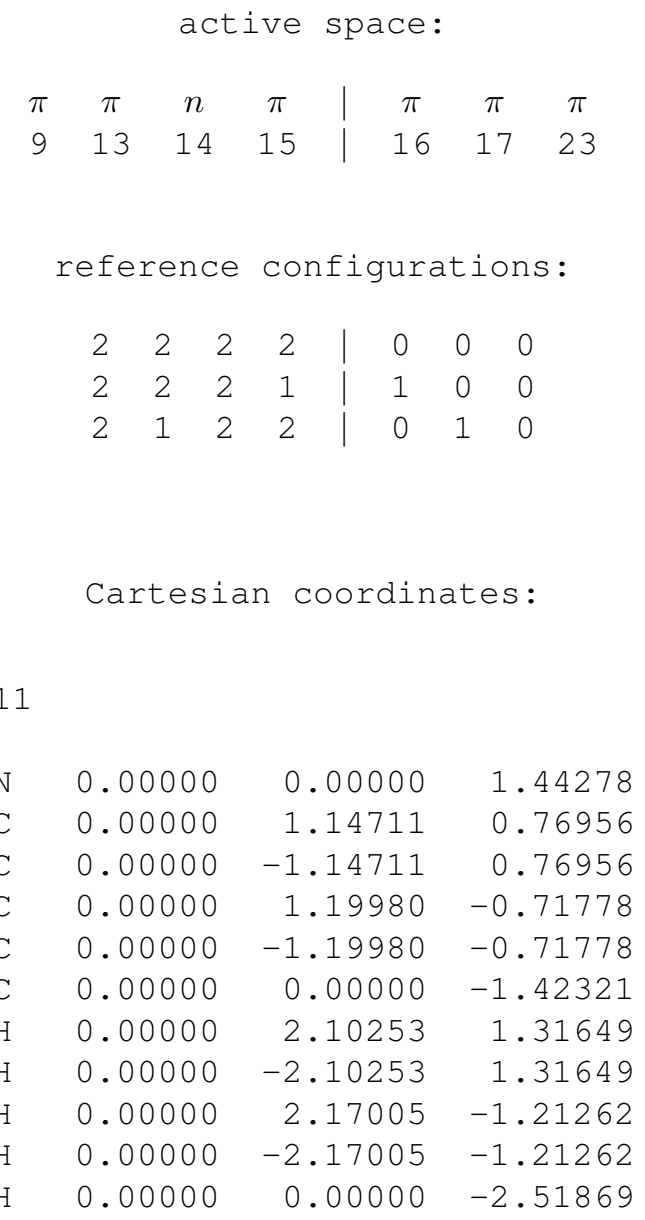

S134 
Table S107 Employed active space, reference configurations, and Cartesian coordinates of the OM2/MRCISD-optimized excited-state equilibrium geometry of the $2{ }^{3} A_{1}\left({ }^{3} \pi \pi^{*}\right)$ state of pyridine (excited-state energy: $-921.68221 \mathrm{eV}$ ).

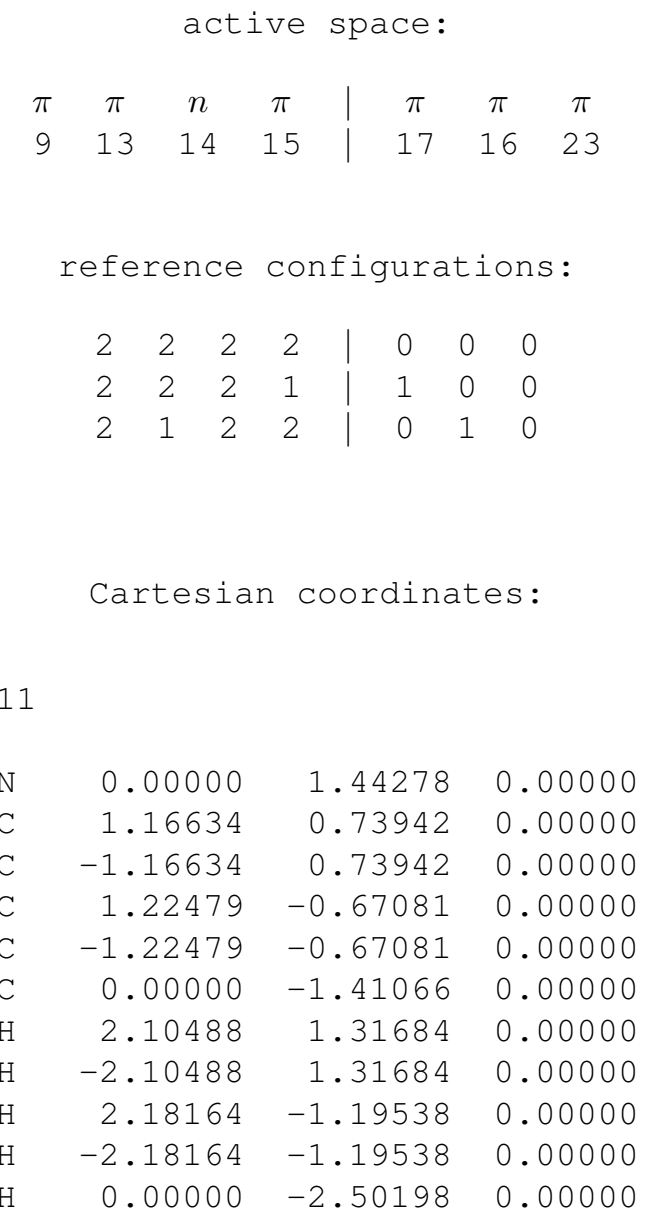


Table S108 Employed active space, reference configurations, and Cartesian coordinates of the OM2/MRCISD-optimized excited-state equilibrium geometry of the $2{ }^{1} A^{\prime}\left({ }^{1} \pi \pi^{*}\right)$ state of pyrrole (excited-state energy: $\left.-793.11022 \mathrm{eV}\right)$.

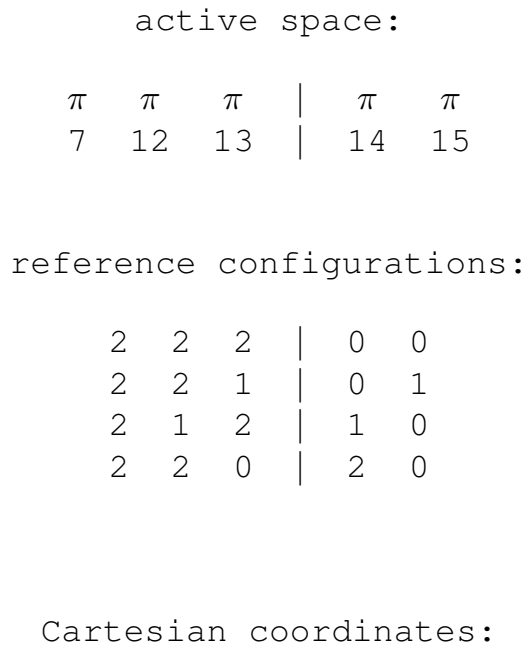

$\begin{array}{rrrr}\mathrm{H} & 0.00000 & 1.78904 & 0.10553 \\ \mathrm{H} & 2.13510 & 0.37438 & 0.12796 \\ \mathrm{H} & -2.13510 & 0.37438 & 0.12796 \\ \mathrm{H} & -1.35049 & -2.19284 & 0.41923 \\ \mathrm{H} & 1.35049 & -2.19284 & 0.41923 \\ \mathrm{~N} & 0.00000 & 0.77800 & 0.08173 \\ \mathrm{C} & -1.13020 & 0.00000 & 0.00000 \\ \mathrm{C} & 1.13020 & 0.00000 & 0.00000 \\ \mathrm{C} & 0.72022 & -1.41589 & 0.00000 \\ \mathrm{C} & -0.72022 & -1.41589 & 0.00000\end{array}$


Table S109 Employed active space, reference configurations, and Cartesian coordinates of the OM2/MRCISD-optimized excited-state equilibrium geometry of the $1{ }^{1} B_{2}\left({ }^{1} \pi \pi^{*}\right)$ state of pyrrole (excited-state energy: $-792.69505 \mathrm{eV}$ ).

active space:
\begin{tabular}{ccc|cc}
$\pi$ & $\pi$ & $\pi$ & $\pi$ & $\pi$ \\
7 & 13 & 12 & 14 & 16 \\
reference configurations: \\
2 \\
2 & 2 & 2 & 0 & 0 \\
2 & 2 & 1 & 0 & 1 \\
2 & 1 & 2 & 1 & 0
\end{tabular}

Cartesian coordinates:

10

$\begin{array}{lrrr}\mathrm{H} & 0.00000 & 0.00000 & 0.00000 \\ \mathrm{H} & 2.13921 & -1.46813 & 0.00000 \\ \mathrm{H} & -2.13921 & -1.46813 & 0.00000 \\ \mathrm{H} & -1.36808 & -4.06407 & 0.00000 \\ \mathrm{H} & 1.36808 & -4.06407 & 0.00000 \\ \mathrm{~N} & 0.00000 & -1.00984 & 0.00000 \\ \mathrm{C} & -1.11894 & -1.83654 & 0.00000 \\ \mathrm{C} & 1.11894 & -1.83654 & 0.00000 \\ \mathrm{C} & 0.69016 & -3.22679 & 0.00000 \\ \mathrm{C} & -0.69016 & -3.22679 & 0.00000\end{array}$


Table S110 Employed active space, reference configurations, and Cartesian coordinates of the OM2/MRCISD-optimized excited-state equilibrium geometry of the $2{ }^{1} A^{\prime}\left({ }^{1} \pi \pi^{*}\right)$ state of cis-PSB3 (excited-state energy: $\left.-960.28256 \mathrm{eV}\right)$.

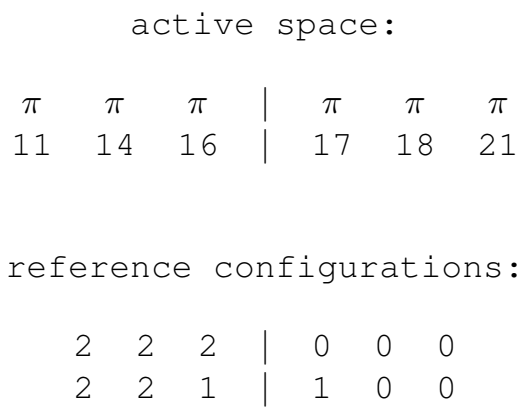

$\begin{array}{rrr}1.38575 & 0.00000 & 0.00000 \\ 0.00000 & 0.00000 & 0.00000 \\ -0.70244 & 1.22836 & 0.00000 \\ -2.11960 & 1.29556 & 0.00000 \\ -2.96132 & 0.13441 & 0.00000 \\ -4.29343 & 0.30906 & 0.00000 \\ 1.94701 & -0.93142 & 0.00000 \\ 1.95521 & 0.92823 & 0.00000 \\ -0.53760 & -0.95362 & 0.00000 \\ -0.14157 & 2.17014 & 0.00000 \\ -2.59181 & 2.28358 & 0.00000 \\ -2.55148 & -0.88060 & 0.00000 \\ -4.92776 & -0.46931 & 0.00000 \\ -4.71370 & 1.22131 & 0.00000\end{array}$


Table S111 Employed active space, reference configurations, and Cartesian coordinates of the OM2/MRCISD-optimized excited-state equilibrium geometry of the $2{ }^{1} A^{\prime}\left({ }^{1} \pi \pi^{*}\right)$ state of 3Me-cis-PSB5 (excited-state energy: $-1689.15130 \mathrm{eV}$ ).

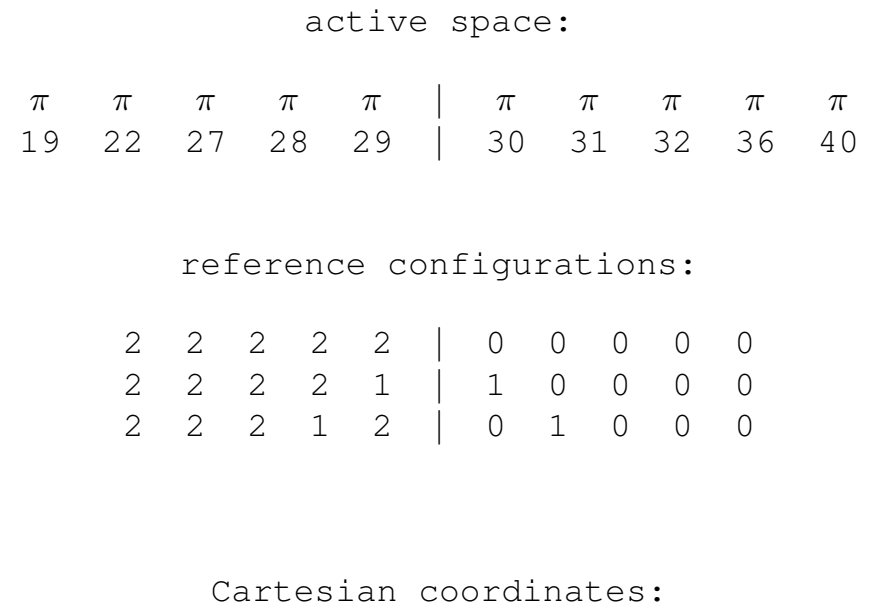

\begin{tabular}{lrrr}
25 & & & \\
& & & \\
$\mathrm{C}$ & 0.00000 & 0.00000 & 0.00000 \\
$\mathrm{C}$ & 1.45278 & 0.00000 & 0.00000 \\
$\mathrm{C}$ & 2.32367 & -1.07848 & 0.00000 \\
$\mathrm{C}$ & 3.73194 & -0.72612 & 0.00000 \\
$\mathrm{C}$ & 4.76888 & -1.66317 & 0.00000 \\
$\mathrm{~N}$ & 6.05222 & -1.25754 & 0.00000 \\
$\mathrm{C}$ & 1.89151 & -2.50623 & 0.00000 \\
$\mathrm{C}$ & -0.83754 & -1.09157 & 0.00000 \\
$\mathrm{C}$ & -2.24503 & -0.88044 & 0.00000 \\
$\mathrm{C}$ & -3.13969 & -1.97309 & 0.00000 \\
$\mathrm{C}$ & -4.49039 & -1.75893 & 0.00000 \\
$\mathrm{H}$ & -0.45408 & 1.00705 & 0.00000 \\
$\mathrm{H}$ & 1.89102 & 1.01061 & 0.00000 \\
$\mathrm{H}$ & 3.98971 & 0.34108 & 0.00000 \\
$\mathrm{H}$ & 4.56103 & -2.74605 & 0.00000 \\
$\mathrm{H}$ & 6.81538 & -1.90864 & 0.00000 \\
$\mathrm{H}$ & 6.30207 & -0.28515 & 0.00000 \\
$\mathrm{H}$ & 2.75416 & -3.19222 & 0.00000 \\
$\mathrm{H}$ & 1.32082 & -2.70120 & 0.90813 \\
$\mathrm{H}$ & 1.32082 & -2.70120 & -0.90813 \\
$\mathrm{H}$ & -0.47145 & -2.11640 & 0.00000 \\
$\mathrm{H}$ & -2.64190 & 0.14874 & 0.00000 \\
$\mathrm{H}$ & -2.73954 & -2.99051 & 0.00000 \\
\hline & & Continued \\
& & &
\end{tabular}

S139 
Continued from previous page

\begin{tabular}{llll}
\hline $\mathrm{H}$ & -5.19089 & -2.58704 & 0.00000 \\
$\mathrm{H}$ & -4.91220 & -0.75692 & 0.00000
\end{tabular}

S140 
Table S112 Employed active space, reference configurations, and Cartesian coordinates of the OM2/MRCISD-optimized excited-state equilibrium geometry of the $1{ }^{1} B_{u}\left({ }^{1} \pi \pi^{*}\right)$ state of trans-stilbene (excited-state energy: $\left.-1961.53124 \mathrm{eV}\right)$.

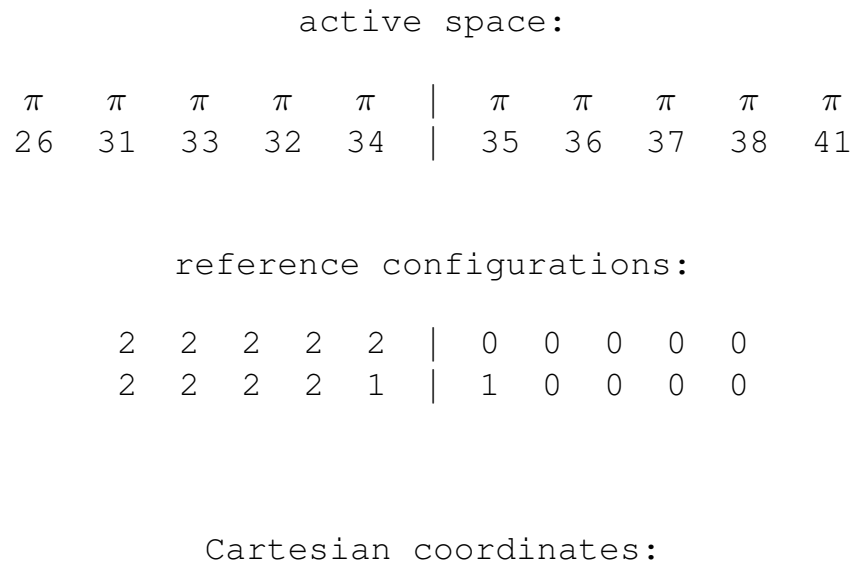


Continued from previous page

$\begin{array}{llll}\mathrm{H} & 4.60805 & -1.25802 & 0.00000 \\ \mathrm{H} & 2.60094 & 0.19603 & 0.00000\end{array}$

$\begin{array}{llll}\mathrm{H} & 2.60094 & 0.19603 & 0.00000\end{array}$

S142 
Table S113 Employed active space, reference configurations, and Cartesian coordinates of the OM2/MRCISD-optimized excited-state equilibrium geometry of the $1{ }^{1} B\left({ }^{1} \pi \pi^{*}\right)$ state of stilbene (excited-state energy: $-1961.11367 \mathrm{eV}$ ).

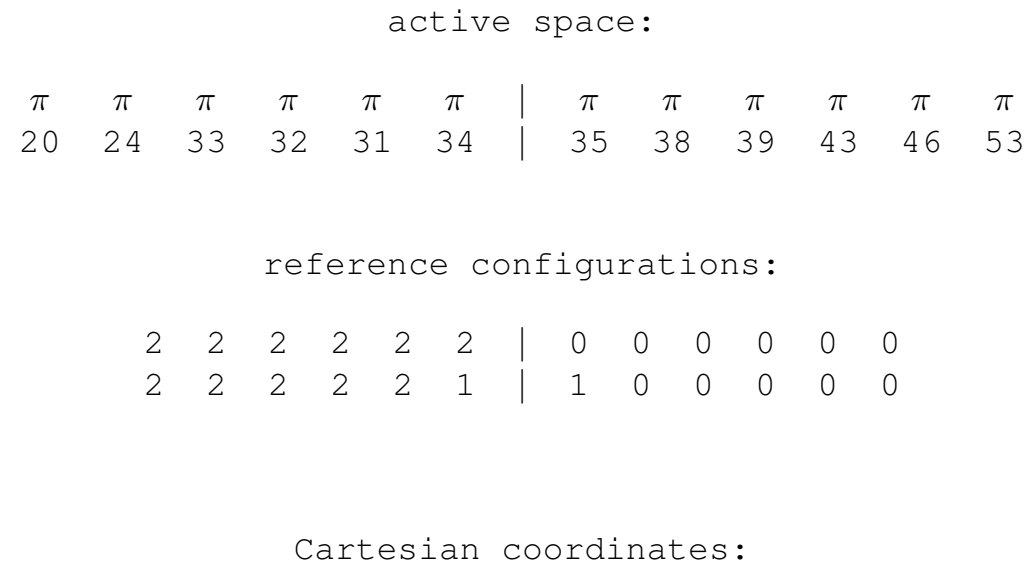

Continued on next page

S143 
Continued from previous page

$\begin{array}{rrrr}\mathrm{H} & 3.19379 & -0.99747 & 3.32192 \\ \mathrm{H} & 1.66990 & 0.31248 & 1.83647\end{array}$

S144 
Table S114 Employed active space, reference configurations, and Cartesian coordinates of the OM2/MRCISD-optimized excited-state equilibrium geometry of the $2{ }^{1} A^{\prime}\left({ }^{1} \pi \pi^{*}\right)$ state of styrene (excited-state energy: $-1136.51724 \mathrm{eV}$ ).

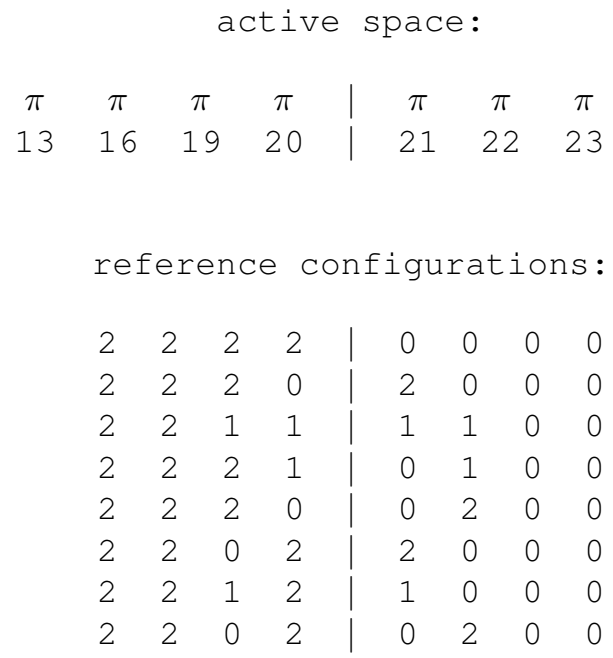

Cartesian coordinates:

16

$\begin{array}{lrrr}\mathrm{C} & 1.56483 & -0.04589 & 0.00000 \\ \mathrm{C} & 0.20637 & -0.07289 & 0.00000 \\ \mathrm{C} & 2.39130 & -1.21615 & 0.00000 \\ \mathrm{C} & 1.85087 & -2.54340 & 0.00000 \\ \mathrm{C} & 3.81697 & -1.04832 & 0.00000 \\ \mathrm{C} & 2.72152 & -3.66410 & 0.00000 \\ \mathrm{C} & 4.67119 & -2.18138 & 0.00000 \\ \mathrm{C} & 4.12296 & -3.48231 & 0.00000 \\ \mathrm{H} & 2.08513 & 0.92553 & 0.00000 \\ \mathrm{H} & -0.37361 & 0.84124 & 0.00000 \\ \mathrm{H} & -0.35562 & -0.99852 & 0.00000 \\ \mathrm{H} & 0.76763 & -2.69847 & 0.00000 \\ \mathrm{H} & 4.24479 & -0.03988 & 0.00000 \\ \mathrm{H} & 2.30187 & -4.67482 & 0.00000 \\ \mathrm{H} & 5.75655 & -2.04353 & 0.00000 \\ \mathrm{H} & 4.78544 & -4.35390 & 0.00000\end{array}$


Table S115 Employed active space, reference configurations, and Cartesian coordinates of the OM2/MRCISD-optimized excited-state equilibrium geometry of the $1^{3} A^{\prime}\left({ }^{3} \pi \pi^{*}\right)$ state of styrene (excited-state energy: $-1137.91479 \mathrm{eV}$ ).

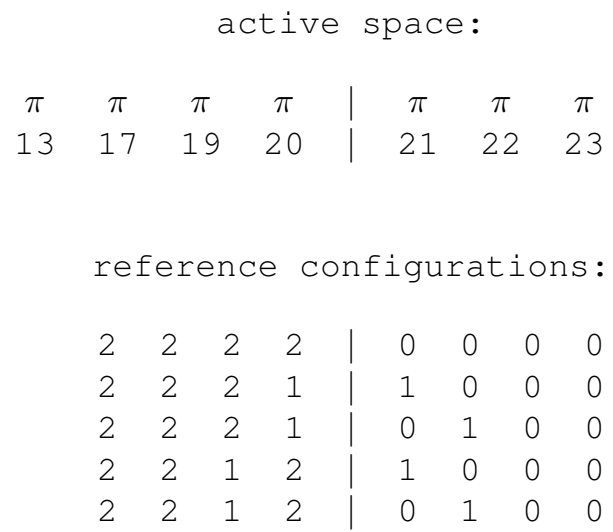

Cartesian coordinates:

16

$\begin{array}{lrrr}\mathrm{C} & 1.62147 & -0.07264 & 0.00000 \\ \mathrm{C} & 0.18486 & -0.09026 & 0.00000 \\ \mathrm{C} & 2.40436 & -1.22028 & 0.00000 \\ \mathrm{C} & 1.85336 & -2.55532 & 0.00000 \\ \mathrm{C} & 3.84597 & -1.08701 & 0.00000 \\ \mathrm{C} & 2.68808 & -3.65357 & 0.00000 \\ \mathrm{C} & 4.65456 & -2.20290 & 0.00000 \\ \mathrm{C} & 4.08936 & -3.49574 & 0.00000 \\ \mathrm{H} & 2.11120 & 0.91083 & 0.00000 \\ \mathrm{H} & -0.36497 & 0.83891 & 0.00000 \\ \mathrm{H} & -0.36811 & -1.01780 & 0.00000 \\ \mathrm{H} & 0.76519 & -2.68456 & 0.00000 \\ \mathrm{H} & 4.27876 & -0.07885 & 0.00000 \\ \mathrm{H} & 2.26646 & -4.66577 & 0.00000 \\ \mathrm{H} & 5.74559 & -2.09731 & 0.00000 \\ \mathrm{H} & 4.73919 & -4.37653 & 0.00000\end{array}$


Table S116 Employed active space, reference configurations, and Cartesian coordinates of the OM2/MRCISD-optimized excited-state equilibrium geometry of the $1{ }^{1} B_{3 u}\left({ }^{1} n \pi^{*}\right)$ state of $s$-tetrazine (excited-state energy: $\left.-1134.45754 \mathrm{eV}\right)$.

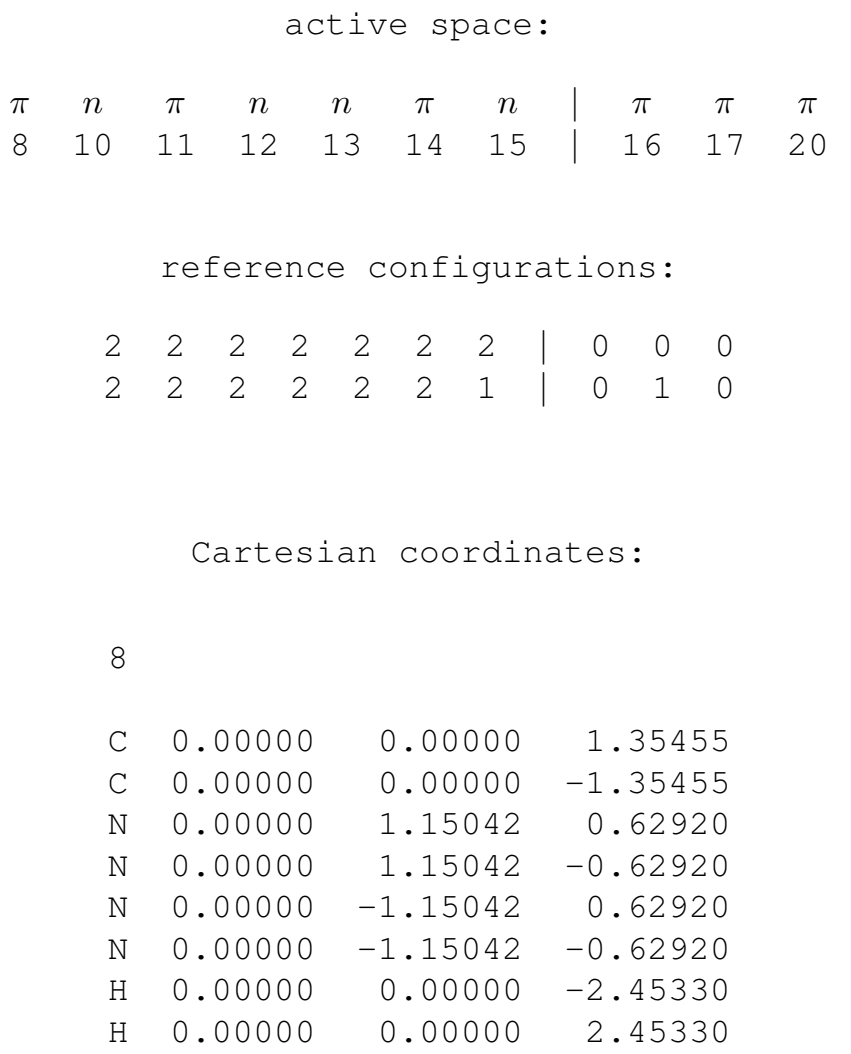


Table S117 Employed active space, reference configurations, and Cartesian coordinates of the OM2/MRCISD-optimized excited-state equilibrium geometry of the $2{ }^{1} A\left({ }^{1} n \pi^{*}\right)$ state of thymine (excited-state energy: $-1813.25713 \mathrm{eV}$ ).

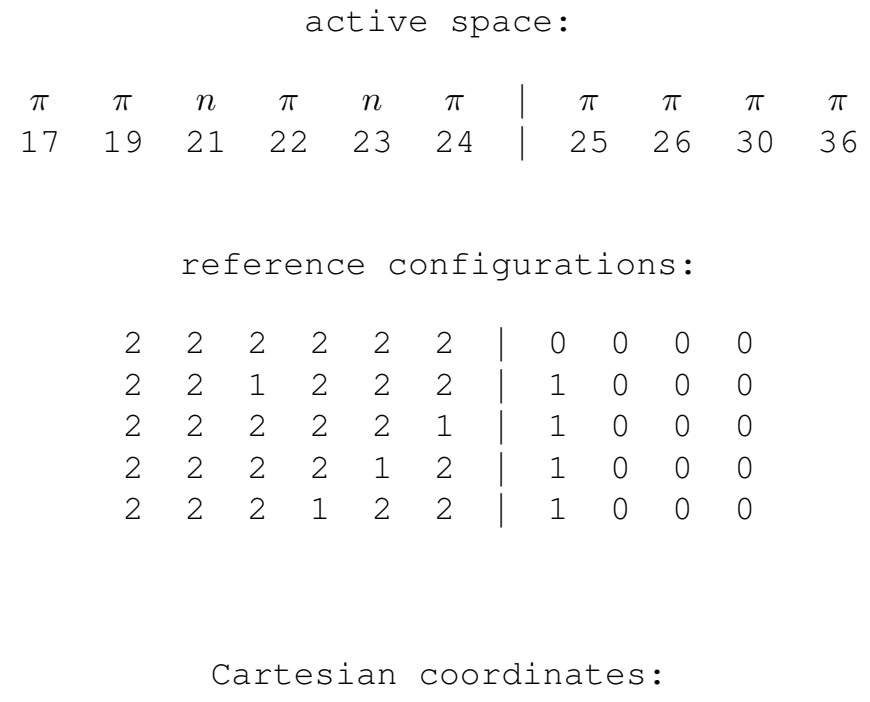

15

$\begin{array}{lrrr}\mathrm{N} & 0.00000 & 0.00000 & 0.00000 \\ \mathrm{C} & 1.38890 & 0.00000 & 0.00000 \\ \mathrm{C} & 2.07129 & 1.22987 & 0.00000 \\ \mathrm{C} & 1.28424 & 2.39135 & 0.00000 \\ \mathrm{~N} & -0.09639 & 2.36431 & 0.00001 \\ \mathrm{C} & -0.78396 & 1.14463 & 0.00000 \\ \mathrm{O} & 1.83879 & 3.59809 & 0.00000 \\ \mathrm{C} & 3.56005 & 1.29653 & -0.00000 \\ \mathrm{O} & -2.03189 & 1.10810 & 0.00000 \\ \mathrm{H} & 1.92619 & -0.94483 & -0.00000 \\ \mathrm{H} & -0.49516 & -0.88526 & -0.00000 \\ \mathrm{H} & -0.63013 & 3.22320 & 0.00001 \\ \mathrm{H} & 3.89531 & 2.34096 & -0.00002 \\ \mathrm{H} & 3.94601 & 0.79762 & -0.89667 \\ \mathrm{H} & 3.94602 & 0.79765 & 0.89668\end{array}$


Table S118 Employed active space, reference configurations, and Cartesian coordinates of the OM2/MRCISD-optimized excited-state equilibrium geometry of the $2{ }^{1} A^{\prime}\left({ }^{1} \pi \pi^{*}\right)$ state of uracil (excited-state energy: $-1655.03671 \mathrm{eV}$ ).

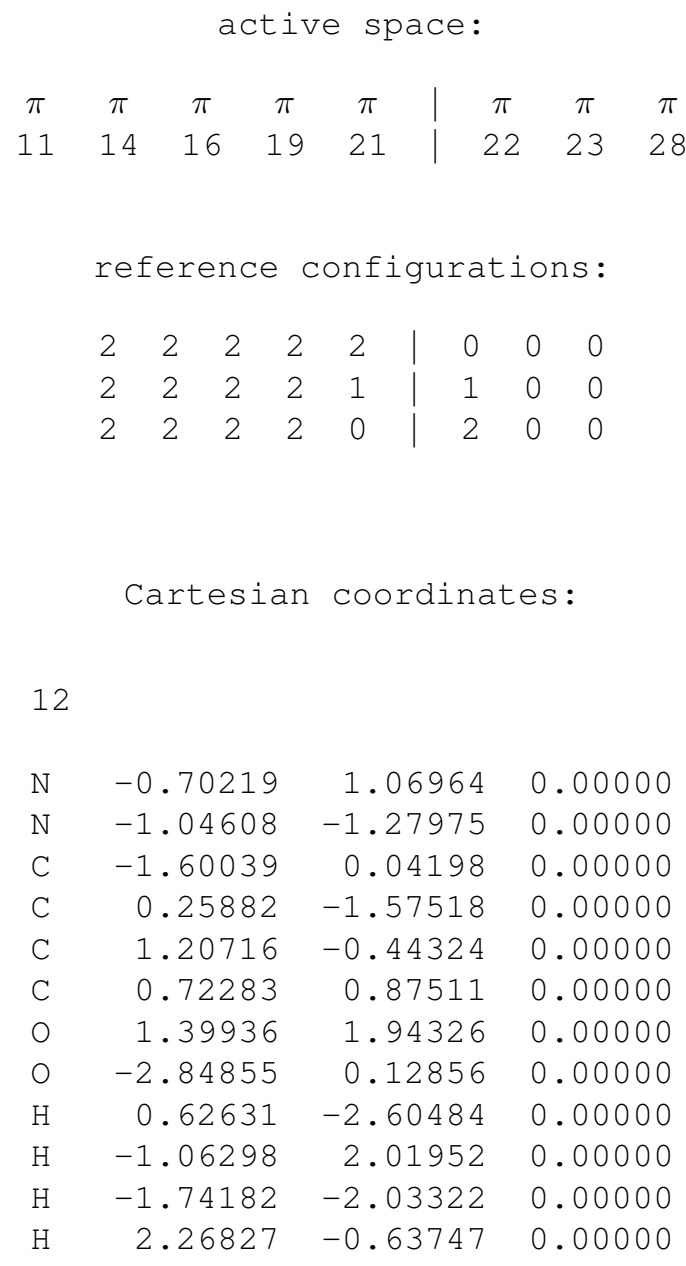


4.1.2 Structural Parameters of the OM2/MRCI-Optimized ExcitedState Minima 
Table S119 Structural parameters of the OM2/MRCISDSD-optimized excitedstate equilibrium geometry of the $2{ }^{1} A\left({ }^{1} n \pi^{*}\right)$ state of $9 H$-adenine used for the statistical evaluations.

\begin{tabular}{ll}
$\begin{array}{l}\text { bond } \\
\text { or angle }\end{array}$ & $\begin{array}{l}\text { length } / \AA \\
\text { or angle } /\end{array}$ \\
\hline N1-C2 & 1.352 \\
C2-C3 & 1.475 \\
C2-C9 & 1.446 \\
C3-N4 & 1.382 \\
N4-C5 & 1.408 \\
N1-C5 & 1.345 \\
C3-N6 & 1.313 \\
N6-C7 & 1.386 \\
C7-N8 & 1.371 \\
N8-C9 & 1.337 \\
C9-N10 & 1.349 \\
C7-H11 & 1.095 \\
C5-H15 & 1.088 \\
N4-H14 & 1.011 \\
N10-H12 & 1.001 \\
N10-H13 & 1.000 \\
&
\end{tabular}


Table S120 Structural parameters of the OM2/MRCISDSD-optimized excitedstate equilibrium geometry of the $2{ }^{1} A\left({ }^{1} n \pi^{*}\right)$ state of acetaldehyde used for the statistical evaluations.

\begin{tabular}{ll}
$\begin{array}{l}\text { bond } \\
\text { or angle }\end{array}$ & $\begin{array}{l}\text { length } / \AA \\
\text { or angle / } /\end{array}$ \\
\hline $\mathrm{C} 1-\mathrm{O} 4$ & 1.290 \\
$\mathrm{C} 1-\mathrm{C} 2$ & 1.502 \\
$\mathrm{C} 2-\mathrm{H} 7$ & 1.088 \\
$\mathrm{O} 4-\mathrm{C} 1-\mathrm{C} 2$ & 115.9 \\
$\mathrm{C} 2-\mathrm{C} 1-\mathrm{H} 3$ & 115.9
\end{tabular}


Table S121 Structural parameters of the OM2/MRCISDSD-optimized excitedstate equilibrium geometry of the $1^{3} A\left({ }^{3} n \pi^{*}\right)$ state of acetaldehyde used for the statistical evaluations.

\begin{tabular}{ll}
$\begin{array}{l}\text { bond } \\
\text { or angle }\end{array}$ & $\begin{array}{l}\text { length } / \AA \\
\text { or angle / } /\end{array}$ \\
\hline $\mathrm{C} 1-\mathrm{O} 4$ & 1.270 \\
$\mathrm{C} 1-\mathrm{C} 2$ & 1.523 \\
$\mathrm{C} 2-\mathrm{H} 7$ & 1.088 \\
$\mathrm{O} 4-\mathrm{C} 1-\mathrm{C} 2$ & 115.0 \\
$\mathrm{C} 2-\mathrm{C} 1-\mathrm{H} 3$ & 111.6
\end{tabular}


Table S122 Structural parameters of the OM2/MRCISD-optimized excitedstate equilibrium geometry of the $2{ }^{3} A\left({ }^{3} \pi \pi^{*}\right)$ state of acetaldehyde used for the statistical evaluations.

\begin{tabular}{ll}
$\begin{array}{l}\text { bond } \\
\text { or angle }\end{array}$ & $\begin{array}{l}\text { length } / \AA \\
\text { or angle / } /\end{array}$ \\
\hline $\mathrm{C} 1-\mathrm{O} 4$ & 1.414 \\
$\mathrm{C} 1-\mathrm{C} 2$ & 1.470 \\
$\mathrm{C} 2-\mathrm{H} 7$ & 1.097 \\
$\mathrm{O} 4-\mathrm{C} 1-\mathrm{C} 2$ & 111.8 \\
$\mathrm{C} 2-\mathrm{C} 1-\mathrm{H} 3$ & 121.5
\end{tabular}


Table S123 Structural parameters of the OM2/MRCISD-optimized excitedstate equilibrium geometry of the $1{ }^{1} A^{\prime \prime}\left({ }^{1} n \pi^{*}\right)$ state of acetone used for the statistical evaluations.

\begin{tabular}{ll} 
bond & $\begin{array}{l}\text { length } / \AA \\
\text { or angle } \\
\text { or angle / }\end{array}$ \\
\hline $\mathrm{C} 2-\mathrm{O} 3$ & 1.291 \\
$\mathrm{C} 1-\mathrm{C} 2$ & 1.499 \\
$\mathrm{C} 1-\mathrm{C} 2-\mathrm{C} 4$ & 119.7
\end{tabular}


Table S124 Structural parameters of the OM2/MRCISD-optimized excitedstate equilibrium geometry of the $1^{3} A^{\prime \prime}\left({ }^{3} n \pi^{*}\right)$ state of acetone used for the statistical evaluations.

\begin{tabular}{ll} 
bond & $\begin{array}{l}\text { length } / \AA \\
\text { or angle } \\
\text { or angle / }\end{array}$ \\
\hline $\mathrm{C} 2-\mathrm{O} 3$ & 1.266 \\
$\mathrm{C} 1-\mathrm{C} 2$ & 1.532 \\
$\mathrm{C} 1-\mathrm{C} 2-\mathrm{C} 4$ & 114.8
\end{tabular}


Table S125 Structural parameters of the OM2/MRCISD-optimized excitedstate equilibrium geometry of the $1^{3} A^{\prime}\left({ }^{3} \pi \pi^{*}\right)$ state of acetone used for the statistical evaluations.

\begin{tabular}{ll} 
bond & $\begin{array}{l}\text { length } / \AA \\
\text { or angle } \\
\text { or angle / }\end{array}$ \\
\hline $\mathrm{C} 2-\mathrm{O} 3$ & 1.414 \\
$\mathrm{C} 1-\mathrm{C} 2$ & 1.482 \\
$\mathrm{C} 1-\mathrm{C} 2-\mathrm{C} 4$ & 121.7
\end{tabular}


Table S126 Structural parameters of the OM2/MRCISDTQ-optimized excitedstate equilibrium geometry of the $1{ }^{1} A^{\prime \prime}\left({ }^{1} n \pi^{*}\right)$ state of acetophenone used for the statistical evaluations.

\begin{tabular}{ll}
$\begin{array}{l}\text { bond } \\
\text { or angle }\end{array}$ & $\begin{array}{l}\text { length } / \AA \\
\text { or angle / }\end{array}$ \\
\hline C7-C9 & 1.419 \\
C9-C11 & 1.388 \\
C11-C12 & 1.406 \\
C10-C12 & 1.402 \\
C $8-C 10$ & 1.388 \\
C7-C8 & 1.426 \\
C1-C7 & 1.418 \\
C1-C3 & 1.522 \\
C1-O2 & 1.289
\end{tabular}


Table S127 Structural parameters of the OM2/MRCISD-optimized excitedstate equilibrium geometry of the $2{ }^{1} A^{\prime}\left({ }^{1} \pi \pi^{*}\right)$ state of acetophenone used for the statistical evaluations.

\begin{tabular}{ll}
$\begin{array}{l}\text { bond } \\
\text { or angle }\end{array}$ & $\begin{array}{l}\text { length } / \AA \\
\text { or angle / }\end{array}$ \\
\hline C7-C9 & 1.430 \\
C9-C11 & 1.423 \\
C11-C12 & 1.420 \\
C10-C12 & 1.412 \\
C8-C10 & 1.424 \\
C7-C8 & 1.433 \\
C1-C7 & 1.469 \\
C1-C3 & 1.511 \\
C1-O2 & 1.241
\end{tabular}


Table S128 Structural parameters of the OM2/MRCISDT-optimized excitedstate equilibrium geometry of the $1^{3} A^{\prime \prime}\left({ }^{3} n \pi^{*}\right)$ state of acetophenone used for the statistical evaluations.

\begin{tabular}{ll}
$\begin{array}{l}\text { bond } \\
\text { or angle }\end{array}$ & $\begin{array}{l}\text { length } / \AA \\
\text { or angle / }\end{array}$ \\
\hline C7-C9 & 1.416 \\
C9-C11 & 1.390 \\
C11-C12 & 1.405 \\
C10-C12 & 1.401 \\
C $8-C 10$ & 1.389 \\
C7-C8 & 1.422 \\
C1-C7 & 1.424 \\
C1-C3 & 1.527 \\
C1-O2 & 1.282
\end{tabular}


Table S129 Structural parameters of the OM2/MRCISD-optimized excitedstate equilibrium geometry of the $1^{3} A^{\prime}\left({ }^{3} \pi \pi^{*}\right)$ state of acetophenone used for the statistical evaluations.

\begin{tabular}{ll}
$\begin{array}{l}\text { bond } \\
\text { or angle }\end{array}$ & $\begin{array}{c}\text { length } / \AA \\
\text { or angle / }\end{array}$ \\
\hline C7-C9 & 1.466 \\
C9-C11 & 1.368 \\
C11-C12 & 1.437 \\
C10-C12 & 1.439 \\
C8-C10 & 1.368 \\
C7-C8 & 1.462 \\
C1-C7 & 1.448 \\
C1-C3 & 1.508 \\
C1-O2 & 1.252
\end{tabular}


Table S130 Structural parameters of the OM2/MRCISD-optimized excitedstate equilibrium geometry of the $1{ }^{1} A_{2}\left({ }^{1} \pi \pi^{*}\right)$ state of acetylene used for the statistical evaluations.

\begin{tabular}{ll} 
bond & $\begin{array}{l}\text { length } / \AA \\
\text { or angle } \\
\text { or angle / }\end{array}$ \\
\hline $\mathrm{C} 1-\mathrm{C} 2$ & 1.326 \\
$\mathrm{C} 1-\mathrm{H} 3$ & 1.123 \\
$\mathrm{C} 1-\mathrm{C} 2-\mathrm{H} 4$ & 147.2
\end{tabular}


Table S131 Structural parameters of the OM2/MRCISD-optimized excitedstate equilibrium geometry of the $2{ }^{1} A_{2}\left({ }^{1} \pi \pi^{*}\right)$ state of acetylene used for the statistical evaluations.

\begin{tabular}{ll} 
bond & $\begin{array}{l}\text { length } / \AA \\
\text { or angle } \\
\text { or angle / }\end{array}$ \\
\hline $\mathrm{C} 1-\mathrm{C} 2$ & 1.322 \\
$\mathrm{C} 1-\mathrm{H} 3$ & 1.046 \\
$\mathrm{C} 1-\mathrm{C} 2-\mathrm{H} 4$ & 180.0
\end{tabular}


Table S132 Structural parameters of the OM2/MRCISD-optimized excitedstate equilibrium geometry of the $1{ }^{1} B_{2}\left({ }^{1} \pi \pi^{*}\right)$ state of acetylene used for the statistical evaluations.

\begin{tabular}{ll} 
bond & $\begin{array}{l}\text { length } / \AA \\
\text { or angle } \\
\text { or angle / }\end{array}$ \\
\hline $\mathrm{C} 1-\mathrm{C} 2$ & 1.303 \\
$\mathrm{C} 1-\mathrm{H} 3$ & 1.138 \\
$\mathrm{C} 1-\mathrm{C} 2-\mathrm{H} 4$ & 139.5
\end{tabular}


Table S133 Structural parameters of the OM2/MRCISD-optimized excitedstate equilibrium geometry of the $1^{3} A_{2}\left({ }^{3} \pi \pi^{*}\right)$ state of acetylene used for the statistical evaluations.

\begin{tabular}{ll} 
bond & $\begin{array}{l}\text { length } / \AA \\
\text { or angle } \\
\text { or angle / }\end{array}$ \\
\hline $\mathrm{C} 1-\mathrm{C} 2$ & 1.335 \\
$\mathrm{C} 1-\mathrm{H} 3$ & 1.107 \\
$\mathrm{C} 1-\mathrm{C} 2-\mathrm{H} 4$ & 144.5
\end{tabular}


Table S134 Structural parameters of the OM2/MRCISD-optimized excitedstate equilibrium geometry of the $2{ }^{3} A_{2}\left({ }^{3} \pi \pi^{*}\right)$ state of acetylene used for the statistical evaluations.

\begin{tabular}{ll} 
bond & $\begin{array}{l}\text { length } / \AA \\
\text { or angle } \\
\text { or angle / }\end{array}$ \\
\hline $\mathrm{C} 1-\mathrm{C} 2$ & 1.325 \\
$\mathrm{C} 1-\mathrm{H} 3$ & 1.048 \\
$\mathrm{C} 1-\mathrm{C} 2-\mathrm{H} 4$ & 180.0
\end{tabular}


Table S135 Structural parameters of the OM2/MRCISDT-optimized excitedstate equilibrium geometry of the $1{ }^{3} B_{2}\left({ }^{3} \pi \pi^{*}\right)$ state of acetylene used for the statistical evaluations.

\begin{tabular}{ll} 
bond & $\begin{array}{l}\text { length } / \AA \\
\text { or angle } \\
\text { or angle / }\end{array}$ \\
\hline $\mathrm{C} 1-\mathrm{C} 2$ & 1.320 \\
$\mathrm{C} 1-\mathrm{H} 3$ & 1.104 \\
$\mathrm{C} 1-\mathrm{C} 2-\mathrm{H} 4$ & 133.8
\end{tabular}


Table S136 Structural parameters of the OM2/MRCISD-optimized excitedstate equilibrium geometry of the $1{ }^{1} A_{u}\left({ }^{1} \pi \pi^{*}\right)$ state of acetylene used for the statistical evaluations.

\begin{tabular}{ll} 
bond & $\begin{array}{l}\text { length } / \AA \\
\text { or angle } \\
\text { or angle / }\end{array}$ \\
\hline $\mathrm{C} 1-\mathrm{C} 2$ & 1.361 \\
$\mathrm{C} 1-\mathrm{H} 3$ & 1.110 \\
$\mathrm{C} 1-\mathrm{C} 2-\mathrm{H} 4$ & 125.0
\end{tabular}


Table S137 Structural parameters of the OM2/MRCISD-optimized excitedstate equilibrium geometry of the $2{ }^{1} A_{u}\left({ }^{1} \pi \pi^{*}\right)$ state of acetylene used for the statistical evaluations.

\begin{tabular}{ll} 
bond & $\begin{array}{l}\text { length } / \AA \\
\text { or angle } \\
\text { or angle / }\end{array}$ \\
\hline $\mathrm{C} 1-\mathrm{C} 2$ & 1.318 \\
$\mathrm{C} 1-\mathrm{H} 3$ & 1.051 \\
$\mathrm{C} 1-\mathrm{C} 2-\mathrm{H} 4$ & 170.0
\end{tabular}


Table S138 Structural parameters of the OM2/MRCISD-optimized excitedstate equilibrium geometry of the $1{ }^{1} B_{u}\left({ }^{1} \pi \pi^{*}\right)$ state of acetylene used for the statistical evaluations.

\begin{tabular}{ll} 
bond & $\begin{array}{l}\text { length } / \AA \\
\text { or angle } \\
\text { or angle / }\end{array}$ \\
\hline $\mathrm{C} 1-\mathrm{C} 2$ & 1.327 \\
$\mathrm{C} 1-\mathrm{H} 3$ & 1.087 \\
$\mathrm{C} 1-\mathrm{C} 2-\mathrm{H} 4$ & 143.2
\end{tabular}


Table S139 Structural parameters of the OM2/MRCISD-optimized excitedstate equilibrium geometry of the $1^{3} A_{u}\left({ }^{3} \pi \pi^{*}\right)$ state of acetylene used for the statistical evaluations.

\begin{tabular}{ll} 
bond & $\begin{array}{l}\text { length } / \AA \\
\text { or angle } \\
\text { or angle / }\end{array}$ \\
\hline $\mathrm{C} 1-\mathrm{C} 2$ & 1.370 \\
$\mathrm{C} 1-\mathrm{H} 3$ & 1.108 \\
$\mathrm{C} 1-\mathrm{C} 2-\mathrm{H} 4$ & 123.7
\end{tabular}


Table S140 Structural parameters of the OM2/MRCISD-optimized excitedstate equilibrium geometry of the $2{ }^{3} A_{u}\left({ }^{3} \pi \pi^{*}\right)$ state of acetylene used for the statistical evaluations.

\begin{tabular}{ll} 
bond & $\begin{array}{l}\text { length } / \AA \\
\text { or angle } \\
\text { or angle / }\end{array}$ \\
\hline $\mathrm{C} 1-\mathrm{C} 2$ & 1.328 \\
$\mathrm{C} 1-\mathrm{H} 3$ & 1.055 \\
$\mathrm{C} 1-\mathrm{C} 2-\mathrm{H} 4$ & 163.8
\end{tabular}


Table S141 Structural parameters of the OM2/MRCISD-optimized excitedstate equilibrium geometry of the $1{ }^{3} B_{u}\left({ }^{3} \pi \pi^{*}\right)$ state of acetylene used for the statistical evaluations.

\begin{tabular}{ll} 
bond & $\begin{array}{l}\text { length } / \AA \\
\text { or angle } \\
\text { or angle / }\end{array}$ \\
\hline $\mathrm{C} 1-\mathrm{C} 2$ & 1.338 \\
$\mathrm{C} 1-\mathrm{H} 3$ & 1.075 \\
$\mathrm{C} 1-\mathrm{C} 2-\mathrm{H} 4$ & 138.3
\end{tabular}


Table S142 Structural parameters of the OM2/MRCISD-optimized excitedstate equilibrium geometry of the $2{ }^{3} B_{u}\left({ }^{3} \pi \pi^{*}\right)$ state of acetylene used for the statistical evaluations.

\begin{tabular}{ll} 
bond & $\begin{array}{l}\text { length } / \AA \\
\text { or angle } \\
\text { or angle / }\end{array}$ \\
\hline $\mathrm{C} 1-\mathrm{C} 2$ & 1.341 \\
$\mathrm{C} 1-\mathrm{H} 3$ & 1.063 \\
$\mathrm{C} 1-\mathrm{C} 2-\mathrm{H} 4$ & 150.5
\end{tabular}


Table S143 Structural parameters of the OM2/MRCISD-optimized excitedstate equilibrium geometry of the $1^{1} A^{\prime \prime}\left({ }^{1} n \pi^{*}\right)$ state of acrolein used for the statistical evaluations.

\begin{tabular}{ll} 
bond & $\begin{array}{l}\text { length / } \AA \\
\text { or angle } \\
\text { or angle / }\end{array}$ \\
\hline $\mathrm{C} 3-\mathrm{O} 4$ & 1.295 \\
$\mathrm{C} 2-\mathrm{C} 3$ & 1.397 \\
$\mathrm{C} 1-\mathrm{C} 2$ & 1.385 \\
$\mathrm{O} 4-\mathrm{C} 3-\mathrm{C} 2$ & 122.0 \\
$\mathrm{C} 3-\mathrm{C} 2-\mathrm{C} 1$ & 118.8
\end{tabular}


Table S144 Structural parameters of the OM2/MRCISD-optimized excitedstate equilibrium geometry of the $1^{3} A^{\prime \prime}\left({ }^{3} n \pi^{*}\right)$ state of acrolein used for the statistical evaluations.

\begin{tabular}{ll} 
bond & $\begin{array}{l}\text { length / } \AA \\
\text { or angle } \\
\text { or angle / }\end{array}$ \\
\hline $\mathrm{C} 3-\mathrm{O} 4$ & 1.287 \\
$\mathrm{C} 2-\mathrm{C} 3$ & 1.407 \\
$\mathrm{C} 1-\mathrm{C} 2$ & 1.376 \\
$\mathrm{O} 4-\mathrm{C} 3-\mathrm{C} 2$ & 121.8 \\
$\mathrm{C} 3-\mathrm{C} 2-\mathrm{C} 1$ & 119.1
\end{tabular}


Table S145 Structural parameters of the OM2/MRCISD-optimized excitedstate equilibrium geometry of the $1^{3} A^{\prime}\left({ }^{3} \pi \pi^{*}\right)$ state of acrolein used for the statistical evaluations.

\begin{tabular}{ll} 
bond & $\begin{array}{l}\text { length / } \AA \\
\text { or angle } \\
\text { or angle / }\end{array}$ \\
\hline $\mathrm{C} 3-\mathrm{O} 4$ & 1.257 \\
$\mathrm{C} 2-\mathrm{C} 3$ & 1.417 \\
$\mathrm{C} 1-\mathrm{C} 2$ & 1.473 \\
$\mathrm{O} 4-\mathrm{C} 3-\mathrm{C} 2$ & 118.5 \\
$\mathrm{C} 3-\mathrm{C} 2-\mathrm{C} 1$ & 120.8
\end{tabular}


Table S146 Structural parameters of the OM2/MRCISD-optimized excitedstate equilibrium geometry of the $2{ }^{1} A\left({ }^{1} \pi \pi^{*}\right)$ state of 2-amino-9-methylpurine used for the statistical evaluations.

$\begin{array}{ll}\text { bond } & \begin{array}{c}\text { length } / \AA \\ \text { or angle }\end{array} \\ \text { or angle / } /\end{array}$


Table S147 Structural parameters of the OM2/MRCISD-optimized excitedstate equilibrium geometry of the $1{ }^{1} B_{2}\left({ }^{1} \pi \pi^{*}\right)$ state of aniline used for the statistical evaluations.

\begin{tabular}{ll}
$\begin{array}{l}\text { bond } \\
\text { or angle }\end{array}$ & $\begin{array}{c}\text { length } / \AA \\
\text { or angle } /\end{array}$ \\
\hline C1-C2 & 1.434 \\
C2-C3 & 1.416 \\
C3-C4 & 1.412 \\
C1-N7 & 1.363 \\
N7-H8 & 0.998 \\
C2-H10 & 1.092 \\
C3-H11 & 1.093 \\
C4-H12 & 1.099 \\
C1-C2-C3 & 118.6 \\
C2-C1-C6 & 121.4 \\
C2-C3-C4 & 120.0 \\
C3-C4-C5 & 121.5 \\
N7-C1-C6 & 119.3 \\
H8-N7-H9 & 116.6 \\
H8-N7-C1 & 121.7 \\
H14-C6-C1 & 120.2 \\
H14-C6-C5 & 121.3 \\
H13-C5-C4 & 120.3 \\
H13-C5-C6 & 119.7 \\
H12-C4-C3 & 119.2
\end{tabular}


Table S148 Structural parameters of the OM2/MRCISD-optimized excitedstate equilibrium geometry of the $1^{3} A^{\prime}\left({ }^{3} \pi \pi^{*}\right)$ state of aniline used for the statistical evaluations.

\begin{tabular}{ll}
$\begin{array}{l}\text { bond } \\
\text { or angle }\end{array}$ & $\begin{array}{c}\text { length } / \AA \\
\text { or angle } /\end{array}$ \\
\hline C1-C2 & 1.487 \\
C2-C3 & 1.369 \\
C3-C4 & 1.413 \\
C1-N7 & 1.371 \\
N7-H8 & 1.001 \\
C2-H10 & 1.094 \\
C3-H11 & 1.096 \\
C4-H12 & 1.096 \\
C1-C2-C3 & 119.6 \\
C2-C1-C6 & 115.8 \\
C2-C3-C4 & 120.5 \\
C3-C4-C5 & 121.1 \\
N7-C1-C6 & 112.5 \\
H8-N7-H9 & 114.5 \\
H8-N7-C1 & 121.7 \\
H14-C6-C1 & 118.1 \\
H14-C6-C5 & 122.3 \\
H13-C5-C4 & 118.5 \\
H13-C5-C6 & 120.9 \\
H12-C4-C3 & 119.4
\end{tabular}


Table S149 Structural parameters of the OM2/MRCISD-optimized excitedstate equilibrium geometry of the $1{ }^{1} A^{\prime \prime}\left({ }^{1} n \pi^{*}\right)$ state of benzaldehyde used for the statistical evaluations.

\begin{tabular}{ll}
$\begin{array}{l}\text { bond } \\
\text { or angle }\end{array}$ & $\begin{array}{l}\text { length } / \AA \\
\text { or angle } /{ }^{\circ}\end{array}$ \\
\hline C4-C6 & 1.419 \\
C6-C8 & 1.383 \\
C $8-C 9$ & 1.408 \\
C7-C9 & 1.404 \\
C5-C7 & 1.381 \\
C4-C5 & 1.430 \\
C1-C4 & 1.400 \\
C1-O2 & 1.288
\end{tabular}


Table S150 Structural parameters of the OM2/MRCISD-optimized excitedstate equilibrium geometry of the $2{ }^{1} A^{\prime}\left({ }^{1} \pi \pi^{*}\right)$ state of benzaldehyde used for the statistical evaluations.

\begin{tabular}{ll}
$\begin{array}{l}\text { bond } \\
\text { or angle }\end{array}$ & $\begin{array}{l}\text { length } / \AA \\
\text { or angle } /{ }^{\circ}\end{array}$ \\
\hline C4-C6 & 1.425 \\
C6-C8 & 1.420 \\
C $8-C 9$ & 1.417 \\
C7-C9 & 1.410 \\
C5-C7 & 1.421 \\
C4-C5 & 1.428 \\
C1-C4 & 1.457 \\
C1-O2 & 1.236
\end{tabular}


Table S151 Structural parameters of the OM2/MRCISD-optimized excitedstate equilibrium geometry of the $1^{3} A^{\prime \prime}\left({ }^{3} n \pi^{*}\right)$ state of benzaldehyde used for the statistical evaluations.

\begin{tabular}{ll}
$\begin{array}{l}\text { bond } \\
\text { or angle }\end{array}$ & $\begin{array}{l}\text { length } / \AA \\
\text { or angle / }\end{array}$ \\
\hline C4-C6 & 1.416 \\
C6-C8 & 1.384 \\
C $8-C 9$ & 1.407 \\
C7-C9 & 1.404 \\
C5-C7 & 1.382 \\
C4-C5 & 1.427 \\
C1-C4 & 1.406 \\
C1-O2 & 1.282
\end{tabular}


Table S152 Structural parameters of the OM2/MRCISD-optimized excitedstate equilibrium geometry of the $1^{3} A^{\prime}\left({ }^{3} \pi \pi^{*}\right)$ state of benzaldehyde used for the statistical evaluations.

\begin{tabular}{ll}
$\begin{array}{l}\text { bond } \\
\text { or angle }\end{array}$ & $\begin{array}{l}\text { length } / \AA \\
\text { or angle } /{ }^{\circ}\end{array}$ \\
\hline C4-C6 & 1.461 \\
C6-C8 & 1.368 \\
C $8-C 9$ & 1.433 \\
C7-C9 & 1.433 \\
C5-C7 & 1.368 \\
C4-C5 & 1.460 \\
C1-C4 & 1.432 \\
C1-O2 & 1.250
\end{tabular}


Table S153 Structural parameters of the OM2/MRCISD-optimized excitedstate equilibrium geometry of the $1^{1} A_{u}\left({ }^{1} n \pi^{*}\right)$ state of $p$-benzoquinone used for the statistical evaluations.

\begin{tabular}{ll}
$\begin{array}{l}\text { bond } \\
\text { or angle }\end{array}$ & $\begin{array}{l}\text { length } / \AA \\
\text { or angle / }\end{array}$ \\
\hline $\mathrm{C} 1-\mathrm{O} 7$ & 1.275 \\
$\mathrm{C} 1-\mathrm{C} 2$ & 1.435 \\
$\mathrm{C} 2-\mathrm{C} 3$ & 1.382 \\
$\mathrm{C} 2-\mathrm{H} 9$ & 1.087 \\
$\mathrm{O} 7-\mathrm{C} 1-\mathrm{C} 2$ & 119.0 \\
$\mathrm{C} 6-\mathrm{C} 1-\mathrm{C} 2$ & 122.0 \\
$\mathrm{H} 9-\mathrm{C} 2-\mathrm{C} 3$ & 121.9
\end{tabular}


Table S154 Structural parameters of the OM2/MRCISD-optimized excitedstate equilibrium geometry of the $1{ }^{1} B_{1 g}\left({ }^{1} n \pi^{*}\right)$ state of $p$-benzoquinone used for the statistical evaluations.

\begin{tabular}{ll}
$\begin{array}{l}\text { bond } \\
\text { or angle }\end{array}$ & $\begin{array}{l}\text { length } / \AA \\
\text { or angle / } /\end{array}$ \\
\hline $\mathrm{C} 1-\mathrm{O} 7$ & 1.247 \\
$\mathrm{C} 1-\mathrm{C} 2$ & 1.458 \\
$\mathrm{C} 2-\mathrm{C} 3$ & 1.356 \\
$\mathrm{C} 2-\mathrm{H} 9$ & 1.088 \\
$\mathrm{O} 7-\mathrm{C} 1-\mathrm{C} 2$ & 121.5 \\
$\mathrm{C} 6-\mathrm{C} 1-\mathrm{C} 2$ & 117.0 \\
$\mathrm{H} 9-\mathrm{C} 2-\mathrm{C} 3$ & 123.7
\end{tabular}


Table S155 Structural parameters of the OM2/MRCISD-optimized excitedstate equilibrium geometry of the $1{ }^{1} B_{1 u}\left({ }^{1} \pi \pi^{*}\right)$ state of $p$-benzoquinone used for the statistical evaluations.

\begin{tabular}{ll}
$\begin{array}{l}\text { bond } \\
\text { or angle }\end{array}$ & $\begin{array}{l}\text { length } / \AA \\
\text { or angle / } /\end{array}$ \\
\hline $\mathrm{C} 1-\mathrm{O} 7$ & 1.267 \\
$\mathrm{C} 1-\mathrm{C} 2$ & 1.453 \\
$\mathrm{C} 2-\mathrm{C} 3$ & 1.381 \\
$\mathrm{C} 2-\mathrm{H} 9$ & 1.092 \\
$\mathrm{O} 7-\mathrm{C} 1-\mathrm{C} 2$ & 120.0 \\
$\mathrm{C} 6-\mathrm{C} 1-\mathrm{C} 2$ & 120.0 \\
$\mathrm{H} 9-\mathrm{C} 2-\mathrm{C} 3$ & 122.3
\end{tabular}


Table S156 Structural parameters of the OM2/MRCISD-optimized excitedstate equilibrium geometry of the $1{ }^{1} B_{3 g}\left({ }^{1} \pi \pi^{*}\right)$ state of $p$-benzoquinone used for the statistical evaluations.

\begin{tabular}{ll}
$\begin{array}{l}\text { bond } \\
\text { or angle }\end{array}$ & $\begin{array}{l}\text { length } / \AA \\
\text { or angle / } /\end{array}$ \\
\hline $\mathrm{C} 1-\mathrm{O} 7$ & 1.261 \\
$\mathrm{C} 1-\mathrm{C} 2$ & 1.440 \\
$\mathrm{C} 2-\mathrm{C} 3$ & 1.408 \\
$\mathrm{C} 2-\mathrm{H} 9$ & 1.097 \\
$\mathrm{O} 7-\mathrm{C} 1-\mathrm{C} 2$ & 121.5 \\
$\mathrm{C} 6-\mathrm{C} 1-\mathrm{C} 2$ & 117.0 \\
$\mathrm{H} 9-\mathrm{C} 2-\mathrm{C} 3$ & 120.7
\end{tabular}


Table S157 Structural parameters of the OM2/MRCISD-optimized excitedstate equilibrium geometry of the $1^{3} A_{u}\left({ }^{3} n \pi^{*}\right)$ state of $p$-benzoquinone used for the statistical evaluations.

\begin{tabular}{ll}
$\begin{array}{l}\text { bond } \\
\text { or angle }\end{array}$ & $\begin{array}{l}\text { length } / \AA \\
\text { or angle / } /\end{array}$ \\
\hline $\mathrm{C} 1-\mathrm{O} 7$ & 1.269 \\
$\mathrm{C} 1-\mathrm{C} 2$ & 1.441 \\
$\mathrm{C} 2-\mathrm{C} 3$ & 1.376 \\
$\mathrm{C} 2-\mathrm{H} 9$ & 1.088 \\
$\mathrm{O} 7-\mathrm{C} 1-\mathrm{C} 2$ & 119.2 \\
$\mathrm{C} 6-\mathrm{C} 1-\mathrm{C} 2$ & 121.7 \\
$\mathrm{H} 9-\mathrm{C} 2-\mathrm{C} 3$ & 122.0
\end{tabular}


Table S158 Structural parameters of the OM2/MRCISD-optimized excitedstate equilibrium geometry of the $1{ }^{3} B_{1 g}\left({ }^{3} n \pi^{*}\right)$ state of $p$-benzoquinone used for the statistical evaluations.

\begin{tabular}{ll}
$\begin{array}{l}\text { bond } \\
\text { or angle }\end{array}$ & $\begin{array}{l}\text { length } / \AA \\
\text { or angle / } /\end{array}$ \\
\hline $\mathrm{C} 1-\mathrm{O} 7$ & 1.246 \\
$\mathrm{C} 1-\mathrm{C} 2$ & 1.460 \\
$\mathrm{C} 2-\mathrm{C} 3$ & 1.355 \\
$\mathrm{C} 2-\mathrm{H} 9$ & 1.087 \\
$\mathrm{O} 7-\mathrm{C} 1-\mathrm{C} 2$ & 121.5 \\
$\mathrm{C} 6-\mathrm{C} 1-\mathrm{C} 2$ & 117.0 \\
$\mathrm{H} 9-\mathrm{C} 2-\mathrm{C} 3$ & 123.8
\end{tabular}


Table S159 Structural parameters of the OM2/MRCISD-optimized excitedstate equilibrium geometry of the $1^{3} B_{1 u}\left({ }^{3} \pi \pi^{*}\right)$ state of $p$-benzoquinone used for the statistical evaluations.

\begin{tabular}{ll}
$\begin{array}{l}\text { bond } \\
\text { or angle }\end{array}$ & $\begin{array}{l}\text { length } / \AA \\
\text { or angle / }\end{array}$ \\
\hline $\mathrm{C} 1-\mathrm{O} 7$ & 1.275 \\
$\mathrm{C} 1-\mathrm{C} 2$ & 1.436 \\
$\mathrm{C} 2-\mathrm{C} 3$ & 1.395 \\
$\mathrm{C} 2-\mathrm{H} 9$ & 1.091 \\
$\mathrm{O} 7-\mathrm{C} 1-\mathrm{C} 2$ & 119.4 \\
$\mathrm{C} 6-\mathrm{C} 1-\mathrm{C} 2$ & 121.2 \\
$\mathrm{H} 9-\mathrm{C} 2-\mathrm{C} 3$ & 121.9
\end{tabular}


Table S160 Structural parameters of the OM2/MRCISD-optimized excitedstate equilibrium geometry of the $1^{3} B_{3 g}\left({ }^{3} \pi \pi^{*}\right)$ state of $p$-benzoquinone used for the statistical evaluations.

\begin{tabular}{ll}
$\begin{array}{l}\text { bond } \\
\text { or angle }\end{array}$ & $\begin{array}{l}\text { length } / \AA \\
\text { or angle / }\end{array}$ \\
\hline $\mathrm{C} 1-\mathrm{O} 7$ & 1.244 \\
$\mathrm{C} 1-\mathrm{C} 2$ & 1.459 \\
$\mathrm{C} 2-\mathrm{C} 3$ & 1.414 \\
$\mathrm{C} 2-\mathrm{H} 9$ & 1.093 \\
$\mathrm{O} 7-\mathrm{C} 1-\mathrm{C} 2$ & 121.0 \\
$\mathrm{C} 6-\mathrm{C} 1-\mathrm{C} 2$ & 117.9 \\
$\mathrm{H} 9-\mathrm{C} 2-\mathrm{C} 3$ & 121.0
\end{tabular}


Table S161 Structural parameters of the OM2/MRCISD-optimized excitedstate equilibrium geometry of the $1^{1} B_{3 g}\left({ }^{1} \pi \pi^{*}\right)$ state of biphenylene used for the statistical evaluations.

\begin{tabular}{ll}
$\begin{array}{l}\text { bond } \\
\text { or angle }\end{array}$ & $\begin{array}{c}\text { length } / \AA \\
\text { or angle / } /\end{array}$ \\
\hline C1-C2 & 1.399 \\
C2-C3 & 1.387 \\
C3-C12 & 1.425 \\
C1-C6 & 1.496 \\
C1-C4 & 1.425 \\
C2-H13 & 1.091 \\
C2-H13 & 1.098 \\
C1-C2-C3 & 115.3 \\
C2-C3-C12 & 123.4 \\
C4-C1-C2 & 148.7 \\
C1-C6-C9 & 121.3 \\
\end{tabular}


Table S162 Structural parameters of the OM2/MRCISD-optimized excitedstate equilibrium geometry of the $1{ }^{1} B_{1 u}\left({ }^{1} \pi \pi^{*}\right)$ state of biphenylene used for the statistical evaluations.

\begin{tabular}{ll}
$\begin{array}{l}\text { bond } \\
\text { or angle }\end{array}$ & $\begin{array}{c}\text { length } / \AA \\
\text { or angle / } /\end{array}$ \\
\hline C1-C2 & 1.395 \\
C2-C3 & 1.415 \\
C3-C12 & 1.405 \\
C1-C6 & 1.458 \\
C1-C4 & 1.465 \\
C2-H13 & 1.088 \\
C2-H13 & 1.098 \\
C1-C2-C3 & 114.4 \\
C2-C3-C12 & 123.2 \\
C4-C1-C2 & 147.6 \\
C1-C6-C9 & 122.4
\end{tabular}


Table S163 Structural parameters of the OM2/MRCISD-optimized excitedstate equilibrium geometry of the $2{ }^{1} B_{1 u}\left({ }^{1} \pi \pi^{*}\right)$ state of biphenylene used for the statistical evaluations.

\begin{tabular}{ll}
$\begin{array}{l}\text { bond } \\
\text { or angle }\end{array}$ & $\begin{array}{c}\text { length } / \AA \\
\text { or angle / } /\end{array}$ \\
\hline C1-C2 & 1.390 \\
C2-C3 & 1.421 \\
C3-C12 & 1.394 \\
C1-C6 & 1.459 \\
C1-C4 & 1.469 \\
C2-H13 & 1.090 \\
C2-H13 & 1.097 \\
C1-C2-C3 & 114.6 \\
C2-C3-C12 & 123.1 \\
C4-C1-C2 & 147.7 \\
C1-C6-C9 & 122.3 \\
\end{tabular}


Table S164 Structural parameters of the OM2/MRCISD-optimized excitedstate equilibrium geometry of the $1{ }^{1} B_{2 u}\left({ }^{1} \pi \pi^{*}\right)$ state of biphenylene used for the statistical evaluations.

\begin{tabular}{ll}
$\begin{array}{l}\text { bond } \\
\text { or angle }\end{array}$ & $\begin{array}{c}\text { length } / \AA \\
\text { or angle / } /\end{array}$ \\
\hline C1-C2 & 1.391 \\
C2-C3 & 1.403 \\
C3-C12 & 1.444 \\
C1-C6 & 1.495 \\
C1-C4 & 1.438 \\
C2-H13 & 1.089 \\
C2-H13 & 1.096 \\
C1-C2-C3 & 114.9 \\
C2-C3-C12 & 123.0 \\
C4-C1-C2 & 147.9 \\
C1-C6-C9 & 122.1 \\
\end{tabular}


Table S165 Structural parameters of the OM2/MRCISD-optimized excitedstate equilibrium geometry of the $1{ }^{1} B_{u}\left({ }^{1} \pi \pi^{*}\right)$ state of trans-butadiene used for the statistical evaluations.

\begin{tabular}{ll}
$\begin{array}{l}\text { bond } \\
\text { or angle }\end{array}$ & $\begin{array}{l}\text { length } / \AA \\
\text { or angle } / \circ\end{array}$ \\
\hline C2-C3 & 1.398 \\
C1-C2 & 1.404
\end{tabular}


Table S166 Structural parameters of the OM2/MRCISD-optimized excitedstate equilibrium geometry of the $2{ }^{1} A_{g}\left({ }^{1} \pi \pi^{*}\right)$ state of trans-butadiene used for the statistical evaluations.

\begin{tabular}{ll}
$\begin{array}{l}\text { bond } \\
\text { or angle }\end{array}$ & $\begin{array}{l}\text { length } / \AA \\
\text { or angle } /\end{array}$ \\
\hline C2-C3 & 1.464 \\
C1-C2 & 1.395
\end{tabular}


Table S167 Structural parameters of the OM2/MRCISD-optimized excitedstate equilibrium geometry of the $3{ }^{1} A\left({ }^{1} n \pi^{*}\right)$ state of cytosine used for the statistical evaluations.

\begin{tabular}{ll}
$\begin{array}{l}\text { bond } \\
\text { or angle }\end{array}$ & $\begin{array}{c}\text { length } / \AA \\
\text { or angle } /\end{array}$ \\
\hline N5-C6 & 1.379 \\
N1-C6 & 1.319 \\
N1-C2 & 1.384 \\
C2-C3 & 1.415 \\
C3-C4 & 1.391 \\
C4-N5 & 1.398 \\
C6-O8 & 1.321 \\
C2-N7 & 1.377 \\
C4-H10 & 1.088 \\
C3-H9 & 1.089 \\
N5-H13 & 1.009 \\
N7-H11 & 0.992 \\
N7-H12 & 0.992 \\
N5-C6-N1 & 127.6 \\
C6-N1-C2 & 114.5 \\
N1-C2-C3 & 122.9 \\
C2-C3-C4 & 119.0 \\
C3-C4-N5 & 118.2 \\
N1-C2-N7 & 114.8 \\
N5-C6-O8 & 113.8
\end{tabular}


Table S168 Structural parameters of the OM2/MRCISD-optimized excitedstate equilibrium geometry of the $2{ }^{1} A\left({ }^{1} \pi \pi^{*}\right)$ state of cytosine used for the statistical evaluations.

\begin{tabular}{ll}
$\begin{array}{l}\text { bond } \\
\text { or angle }\end{array}$ & $\begin{array}{c}\text { length } / \AA \\
\text { or angle } /\end{array}$ \\
\hline N5-C6 & 1.425 \\
N1-C6 & 1.342 \\
N1-C2 & 1.394 \\
C2-C3 & 1.405 \\
C3-C4 & 1.426 \\
C4-N5 & 1.363 \\
C6-O8 & 1.271 \\
C2-N7 & 1.369 \\
C4-H10 & 1.092 \\
C3-H9 & 1.088 \\
N5-H13 & 1.017 \\
N7-H11 & 0.994 \\
N7-H12 & 0.993 \\
N5-C6-N1 & 123.0 \\
C6-N1-C2 & 116.4 \\
N1-C2-C3 & 123.2 \\
C2-C3-C4 & 118.8 \\
C3-C4-N5 & 117.7 \\
N1-C2-N7 & 114.6 \\
N5-C6-O8 & 112.7
\end{tabular}


Table S169 Structural parameters of the OM2/MRCISD-optimized excitedstate equilibrium geometry of the $1^{1} A_{2}\left({ }^{1} n \pi^{*}\right)$ state of diazomethane used for the statistical evaluations.

\begin{tabular}{ll} 
bond & $\begin{array}{l}\text { length / } \AA \\
\text { or angle } \\
\text { or angle / }\end{array}$ \\
\hline N2-N3 & 1.171 \\
C1-N2 & 1.356 \\
C1-H4 & 1.091 \\
H4-C1-H5 & 121.7
\end{tabular}


Table S170 Structural parameters of the OM2/MRCISD-optimized excitedstate equilibrium geometry of the $1{ }^{1} B_{3 g}\left({ }^{1} \pi \pi^{*}\right)$ state of dibenzo- $p$-dioxin used for the statistical evaluations.

\begin{tabular}{ll} 
bond & $\begin{array}{l}\text { length / } \AA \\
\text { or angle } \\
\text { or angle / }\end{array}$ \\
\hline C4-07 & 1.346 \\
C4-C5 & 1.442 \\
C5-C6 & 1.416 \\
C1-C6 & 1.379 \\
C1-C2 & 1.425 \\
C4-O7-C8 & 116.4 \\
C4-C5-C6 & 120.3 \\
C2-C3-C4 & 118.1 \\
C1-C2-C3 & 121.6
\end{tabular}


Table S171 Structural parameters of the OM2/MRCISD-optimized excitedstate equilibrium geometry of the $1{ }^{1} B_{2 u}\left({ }^{1} \pi \pi^{*}\right)$ state of dibenzo- $p$-dioxin used for the statistical evaluations.

\begin{tabular}{|c|c|}
\hline $\begin{array}{l}\text { bond } \\
\text { or angle }\end{array}$ & $\begin{array}{l}\text { length / } \AA \\
\text { or angle / }\end{array}$ \\
\hline C4-07 & 1.360 \\
\hline $\mathrm{C} 4-\mathrm{C} 5$ & 1.420 \\
\hline $\mathrm{C} 5-\mathrm{C} 6$ & 1.416 \\
\hline $\mathrm{C} 1-\mathrm{C} 6$ & 1.402 \\
\hline $\mathrm{C} 1-\mathrm{C} 2$ & 1.404 \\
\hline $\mathrm{C} 4-07-\mathrm{C} 8$ & 115.6 \\
\hline $\mathrm{C} 4-\mathrm{C} 5-\mathrm{C} 6$ & 121.1 \\
\hline $\mathrm{C} 2-\mathrm{C} 3-\mathrm{C} 4$ & 117.1 \\
\hline $\mathrm{C} 1-\mathrm{C} 2-\mathrm{C} 3$ & 121.8 \\
\hline
\end{tabular}


Table S172 Structural parameters of the OM2/MRCISD-optimized excitedstate equilibrium geometry of the $1{ }^{1} B_{1 u}\left({ }^{1} \pi \pi^{*}\right)$ state of dibenzo- $p$-dioxin used for the statistical evaluations.

\begin{tabular}{ll} 
bond & $\begin{array}{l}\text { length / } \AA \\
\text { or angle }\end{array}$ or angle / \\
\hline C4-O7 & 1.363 \\
C4-C5 & 1.426 \\
C5-C6 & 1.407 \\
C1-C6 & 1.395 \\
C1-C2 & 1.419 \\
C4-O7-C8 & 115.5 \\
C4-C5-C6 & 120.8 \\
C2-C3-C4 & 117.9 \\
C1-C2-C3 & 121.3
\end{tabular}


Table S173 Structural parameters of the OM2/MRCISD-optimized excitedstate equilibrium geometry of the $1^{3} A_{g}\left({ }^{3} \pi \pi^{*}\right)$ state of dibenzo- $p$-dioxin used for the statistical evaluations.

\begin{tabular}{ll} 
bond & $\begin{array}{l}\text { length / } \AA \\
\text { or angle }\end{array}$ or angle / \\
\hline C4-O7 & 1.360 \\
C4-C5 & 1.425 \\
C5-C6 & 1.413 \\
C1-C6 & 1.403 \\
C1-C2 & 1.400 \\
C4-O7-C8 & 115.5 \\
C4-C5-C6 & 121.1 \\
C2-C3-C4 & 116.9 \\
C1-C2-C3 & 122.0
\end{tabular}


Table S174 Structural parameters of the OM2/MRCISD-optimized excitedstate equilibrium geometry of the $1^{3} B_{3 g}\left({ }^{3} \pi \pi^{*}\right)$ state of dibenzo- $p$-dioxin used for the statistical evaluations.

\begin{tabular}{ll} 
bond & $\begin{array}{l}\text { length / } \AA \\
\text { or angle }\end{array}$ or angle / \\
\hline C4-O7 & 1.354 \\
C4-C5 & 1.445 \\
C5-C6 & 1.413 \\
C1-C6 & 1.381 \\
C1-C2 & 1.432 \\
C4-O7-C8 & 115.9 \\
C4-C5-C6 & 120.4 \\
C2-C3-C4 & 118.1 \\
C1-C2-C3 & 121.5
\end{tabular}


Table S175 Structural parameters of the OM2/MRCISD-optimized excitedstate equilibrium geometry of the $1^{3} B_{2 u}\left({ }^{3} \pi \pi^{*}\right)$ state of dibenzo- $p$-dioxin used for the statistical evaluations.

\begin{tabular}{ll} 
bond & $\begin{array}{l}\text { length / } \AA \\
\text { or angle }\end{array}$ or angle / \\
\hline C4-O7 & 1.357 \\
C4-C5 & 1.429 \\
C5-C6 & 1.411 \\
C1-C6 & 1.405 \\
C1-C2 & 1.397 \\
C4-O7-C8 & 116.4 \\
C4-C5-C6 & 121.1 \\
C2-C3-C4 & 116.8 \\
C1-C2-C3 & 122.0
\end{tabular}


Table S176 Structural parameters of the OM2/MRCISD-optimized excitedstate equilibrium geometry of the $1^{3} B_{1 u}\left({ }^{3} \pi \pi^{*}\right)$ state of dibenzo- $p$-dioxin used for the statistical evaluations.

\begin{tabular}{ll} 
bond & $\begin{array}{l}\text { length / } \AA \\
\text { or angle }\end{array}$ or angle / \\
\hline C4-O7 & 1.365 \\
C4-C5 & 1.444 \\
C5-C6 & 1.405 \\
C1-C6 & 1.388 \\
C1-C2 & 1.432 \\
C4-O7-C8 & 114.6 \\
C4-C5-C6 & 120.5 \\
C2-C3-C4 & 118.4 \\
C1-C2-C3 & 121.2
\end{tabular}


Table S177 Structural parameters of the OM2/MRCISD-optimized excitedstate equilibrium geometry of the $1{ }^{1} A_{u}\left({ }^{1} n \pi^{*}\right)$ state of dibenzo- $p$-dioxin used for the statistical evaluations.

\begin{tabular}{ll} 
bond & $\begin{array}{l}\text { length / } \AA \\
\text { or angle }\end{array}$ or angle / \\
\hline C4-O7 & 1.353 \\
C4-C5 & 1.455 \\
C5-C6 & 1.400 \\
C1-C6 & 1.387 \\
C1-C2 & 1.412 \\
C4-O7-C8 & 122.5 \\
C4-C5-C6 & 119.3 \\
C2-C3-C4 & 120.0 \\
C1-C2-C3 & 120.7
\end{tabular}


Table S178 Structural parameters of the OM2/MRCISD-optimized excitedstate equilibrium geometry of the $1{ }^{1} B_{1 g}\left({ }^{1} n \pi^{*}\right)$ state of dibenzo- $p$-dioxin used for the statistical evaluations.

\begin{tabular}{ll} 
bond & $\begin{array}{l}\text { length / } \AA \\
\text { or angle }\end{array}$ or angle / \\
\hline C4-O7 & 1.359 \\
C4-C5 & 1.429 \\
C5-C6 & 1.400 \\
C1-C6 & 1.412 \\
C1-C2 & 1.383 \\
C4-O7-C8 & 122.8 \\
C4-C5-C6 & 120.4 \\
C2-C3-C4 & 118.4 \\
C1-C2-C3 & 121.2
\end{tabular}


Table S179 Structural parameters of the OM2/MRCISD-optimized excitedstate equilibrium geometry of the $1{ }^{1} B_{3 u}\left({ }^{1} n \pi^{*}\right)$ state of dibenzo- $p$-dioxin used for the statistical evaluations.

\begin{tabular}{ll} 
bond & $\begin{array}{l}\text { length / } \AA \\
\text { or angle }\end{array}$ or angle / \\
\hline C4-O7 & 1.365 \\
C4-C5 & 1.420 \\
C5-C6 & 1.405 \\
C1-C6 & 1.406 \\
C1-C2 & 1.387 \\
C4-O7-C8 & 121.4 \\
C4-C5-C6 & 120.4 \\
C2-C3-C4 & 118.5 \\
C1-C2-C3 & 121.1
\end{tabular}


Table S180 Structural parameters of the OM2/MRCISD-optimized excitedstate equilibrium geometry of the $1{ }^{1} B_{2 g}\left({ }^{1} n \pi^{*}\right)$ state of dibenzo- $p$-dioxin used for the statistical evaluations.

\begin{tabular}{ll} 
bond & $\begin{array}{l}\text { length / } \AA \\
\text { or angle }\end{array}$ or angle / \\
\hline C4-O7 & 1.366 \\
C4-C5 & 1.448 \\
C5-C6 & 1.393 \\
C1-C6 & 1.392 \\
C1-C2 & 1.413 \\
C4-O7-C8 & 121.0 \\
C4-C5-C6 & 119.4 \\
C2-C3-C4 & 120.3 \\
C1-C2-C3 & 120.3
\end{tabular}


Table S181 Structural parameters of the OM2/MRCISD-optimized excitedstate equilibrium geometry of the $1{ }^{3} A_{u}\left({ }^{3} n \pi^{*}\right)$ state of dibenzo- $p$-dioxin used for the statistical evaluations.

\begin{tabular}{ll} 
bond & $\begin{array}{l}\text { length / } \AA \\
\text { or angle }\end{array}$ or angle / \\
\hline C4-O7 & 1.353 \\
C4-C5 & 1.456 \\
C5-C6 & 1.400 \\
C1-C6 & 1.387 \\
C1-C2 & 1.412 \\
C4-O7-C8 & 122.5 \\
C4-C5-C6 & 119.3 \\
C2-C3-C4 & 120.0 \\
C1-C2-C3 & 120.7
\end{tabular}


Table S182 Structural parameters of the OM2/MRCISD-optimized excitedstate equilibrium geometry of the $1^{3} B_{1 g}\left({ }^{3} n \pi^{*}\right)$ state of dibenzo- $p$-dioxin used for the statistical evaluations.

\begin{tabular}{ll} 
bond & $\begin{array}{l}\text { length / } \AA \\
\text { or angle }\end{array}$ or angle / \\
\hline C4-O7 & 1.359 \\
C4-C5 & 1.429 \\
C5-C6 & 1.400 \\
C1-C6 & 1.412 \\
C1-C2 & 1.383 \\
C4-O7-C8 & 122.8 \\
C4-C5-C6 & 120.4 \\
C2-C3-C4 & 118.4 \\
C1-C2-C3 & 121.2
\end{tabular}


Table S183 Structural parameters of the OM2/MRCISD-optimized excitedstate equilibrium geometry of the $1^{3} B_{3 u}\left({ }^{3} n \pi^{*}\right)$ state of dibenzo- $p$-dioxin used for the statistical evaluations.

\begin{tabular}{ll} 
bond & $\begin{array}{l}\text { length / } \AA \\
\text { or angle }\end{array}$ or angle / \\
\hline C4-O7 & 1.364 \\
C4-C5 & 1.421 \\
C5-C6 & 1.404 \\
C1-C6 & 1.406 \\
C1-C2 & 1.387 \\
C4-O7-C8 & 121.4 \\
C4-C5-C6 & 120.4 \\
C2-C3-C4 & 118.5 \\
C1-C2-C3 & 121.1
\end{tabular}


Table S184 Structural parameters of the OM2/MRCISD-optimized excitedstate equilibrium geometry of the $1^{3} B_{2 g}\left({ }^{3} n \pi^{*}\right)$ state of dibenzo- $p$-dioxin used for the statistical evaluations.

\begin{tabular}{ll} 
bond & $\begin{array}{l}\text { length / } \AA \\
\text { or angle }\end{array}$ or angle / \\
\hline C4-O7 & 1.366 \\
C4-C5 & 1.449 \\
C5-C6 & 1.393 \\
C1-C6 & 1.392 \\
C1-C2 & 1.413 \\
C4-O7-C8 & 121.1 \\
C4-C5-C6 & 119.4 \\
C2-C3-C4 & 120.3 \\
C1-C2-C3 & 120.3
\end{tabular}


Table S185 Structural parameters of the OM2/MRCISD-optimized excitedstate equilibrium geometry of the $1{ }^{1} A^{\prime \prime}\left({ }^{1} n \pi^{*}\right)$ state of formaldehyde used for the statistical evaluations.

\begin{tabular}{ll}
$\begin{array}{l}\text { bond } \\
\text { or angle }\end{array}$ & $\begin{array}{l}\text { length } / \AA \\
\text { or angle / }\end{array}$ \\
\hline $\mathrm{C} 1-\mathrm{O} 2$ & 1.279 \\
$\mathrm{C} 1-\mathrm{H} 3$ & 1.113 \\
$\mathrm{H} 3-\mathrm{C} 1-\mathrm{H} 4$ & 113.9
\end{tabular}


Table S186 Structural parameters of the OM2/MRCISD-optimized excitedstate equilibrium geometry of the $1^{3} A^{\prime \prime}\left({ }^{3} n \pi^{*}\right)$ state of formaldehyde used for the statistical evaluations.

\begin{tabular}{ll}
$\begin{array}{l}\text { bond } \\
\text { or angle }\end{array}$ & $\begin{array}{l}\text { length } / \AA \\
\text { or angle / }\end{array}$ \\
\hline $\mathrm{C} 1-\mathrm{O} 2$ & 1.261 \\
$\mathrm{C} 1-\mathrm{H} 3$ & 1.148 \\
$\mathrm{H} 3-\mathrm{C} 1-\mathrm{H} 4$ & 108.1
\end{tabular}


Table S187 Structural parameters of the OM2/MRCISD-optimized excitedstate equilibrium geometry of the $1^{3} A^{\prime}\left({ }^{3} \pi \pi^{*}\right)$ state of formaldehyde used for the statistical evaluations.

\begin{tabular}{ll}
$\begin{array}{l}\text { bond } \\
\text { or angle }\end{array}$ & $\begin{array}{l}\text { length } / \AA \\
\text { or angle } /\end{array}$ \\
\hline $\mathrm{C} 1-\mathrm{O} 2$ & 1.398 \\
$\mathrm{C} 1-\mathrm{H} 3$ & 1.115 \\
$\mathrm{H} 3-\mathrm{C} 1-\mathrm{H} 4$ & 117.6
\end{tabular}


Table S188 Structural parameters of the OM2/MRCISD-optimized excitedstate equilibrium geometry of the $1{ }^{1} A_{u}\left({ }^{1} n \pi^{*}\right)$ state of trans-glyoxal used for the statistical evaluations.

\begin{tabular}{ll}
$\begin{array}{l}\text { bond } \\
\text { or angle }\end{array}$ & $\begin{array}{l}\text { length } / \AA \\
\text { or angle } /\end{array}$ \\
\hline $\mathrm{C} 1-\mathrm{H} 5$ & 1.105 \\
$\mathrm{C} 1-\mathrm{C} 2$ & 1.478 \\
$\mathrm{C} 1-\mathrm{O} 3$ & 1.239 \\
$\mathrm{C} 1-\mathrm{C} 2-\mathrm{H} 6$ & 115.4 \\
$\mathrm{C} 1-\mathrm{C} 2-\mathrm{O} 4$ & 117.9
\end{tabular}


Table S189 Structural parameters of the OM2/MRCISD-optimized excitedstate equilibrium geometry of the $1{ }^{3} A_{u}\left({ }^{3} n \pi^{*}\right)$ state of trans-glyoxal used for the statistical evaluations.

\begin{tabular}{ll}
$\begin{array}{l}\text { bond } \\
\text { or angle }\end{array}$ & $\begin{array}{l}\text { length } / \AA \\
\text { or angle / } /\end{array}$ \\
\hline $\mathrm{C} 1-\mathrm{H} 5$ & 1.103 \\
$\mathrm{C} 1-\mathrm{C} 2$ & 1.481 \\
$\mathrm{C} 1-\mathrm{O} 3$ & 1.237 \\
$\mathrm{C} 1-\mathrm{C} 2-\mathrm{H} 6$ & 115.4 \\
$\mathrm{C} 1-\mathrm{C} 2-\mathrm{O} 4$ & 117.5
\end{tabular}


Table S190 Structural parameters of the OM2/MRCISD-optimized excitedstate equilibrium geometry of the $2{ }^{1} A\left({ }^{1} \pi \pi^{*}\right)$ state of $9 H$-guanine used for the statistical evaluations.

\begin{tabular}{ll}
$\begin{array}{l}\text { bond } \\
\text { or angle }\end{array}$ & $\begin{array}{c}\text { length } / \AA \\
\text { or angle } /\end{array}$ \\
\hline N7-C9 & 1.394 \\
C9-N13 & 1.453 \\
N13-C14 & 1.310 \\
C4-C14 & 1.487 \\
C4-C5 & 1.467 \\
C5-N7 & 1.416 \\
N3-C4 & 1.356 \\
C2-N3 & 1.348 \\
N1-C2 & 1.401 \\
N1-C14 & 1.386 \\
C9-N10 & 1.374 \\
C5-O6 & 1.239 \\
C2-H15 & 1.089 \\
N10-H11 & 0.991 \\
N10-H12 & 0.988 \\
N7-H8 & 1.005 \\
N1-H16 & 1.004
\end{tabular}


Table S191 Structural parameters of the OM2/MRCISD-optimized excitedstate equilibrium geometry of the $2{ }^{1} A^{\prime}\left({ }^{1} \pi \pi^{*}\right)$ state of 1-hydroxy-2acetonaphthone used for the statistical evaluations.

\begin{tabular}{ll}
$\begin{array}{l}\text { bond } \\
\text { or angle }\end{array}$ & $\begin{array}{c}\text { length } / \AA \\
\text { or angle } /\end{array}$ \\
\hline C1-C6 & 1.464 \\
C5-C6 & 1.376 \\
C4-C5 & 1.414 \\
C3-C4 & 1.408 \\
C3-C10 & 1.423 \\
C9-C10 & 1.382 \\
C8-C9 & 1.405 \\
C7-C8 & 1.388 \\
C2-C7 & 1.403 \\
C2-C3 & 1.430 \\
C1-C2 & 1.449 \\
C6-C11 & 1.461 \\
C11-C12 & 1.486 \\
C1-O13 & 1.266 \\
C11-O14 & 1.311 \\
O13-H21 & 1.423 \\
O14-H21 & 1.066 \\
O13-O14 & 2.384
\end{tabular}


Table S192 Structural parameters of the OM2/MRCISD-optimized excitedstate equilibrium geometry of the $2{ }^{1} A^{\prime}\left({ }^{1} \pi \pi^{*}\right)$ state of indole used for the statistical evaluations.

$\begin{array}{ll}\text { bond } & \begin{array}{c}\text { length } / \AA \\ \text { or angle }\end{array} \\ \text { or angle / } /\end{array}$


Table S193 Structural parameters of the OM2/MRCISD-optimized excitedstate equilibrium geometry of the $3{ }^{1} A^{\prime}\left({ }^{1} \pi \pi^{*}\right)$ state of indole used for the statistical evaluations.

\begin{tabular}{|c|c|}
\hline $\begin{array}{l}\text { bond } \\
\text { or angle }\end{array}$ & $\begin{array}{l}\text { length / } \AA \\
\text { or angle / }\end{array}$ \\
\hline N3-C5 & 1.356 \\
\hline $\mathrm{C} 4-\mathrm{C} 5$ & 1.449 \\
\hline $\mathrm{C} 1-\mathrm{C} 4$ & 1.413 \\
\hline $\mathrm{C} 1-\mathrm{C} 6$ & 1.409 \\
\hline $\mathrm{C} 6-\mathrm{C} 7$ & 1.409 \\
\hline $\mathrm{C} 7-\mathrm{C} 9$ & 1.390 \\
\hline $\mathrm{C} 8-\mathrm{C} 9$ & 1.426 \\
\hline $\mathrm{C} 2-\mathrm{C} 8$ & 1.390 \\
\hline $\mathrm{N} 3-\mathrm{H} 10$ & 1.015 \\
\hline $\mathrm{C} 5-\mathrm{H} 12$ & 1.085 \\
\hline $\mathrm{C} 4-\mathrm{H} 11$ & 1.080 \\
\hline $\mathrm{C} 6-\mathrm{H} 13$ & 1.089 \\
\hline $\mathrm{C} 7-\mathrm{H} 14$ & 1.098 \\
\hline $\mathrm{C} 9-\mathrm{H} 16$ & 1.096 \\
\hline $\mathrm{C} 8-\mathrm{H} 15$ & 1.089 \\
\hline $\mathrm{C} 1-\mathrm{C} 2$ & 1.451 \\
\hline $\mathrm{C} 2-\mathrm{N} 3$ & 1.407 \\
\hline $\mathrm{N} 3-\mathrm{C} 5-\mathrm{C} 4$ & 108.3 \\
\hline $\mathrm{C} 5-\mathrm{C} 4-\mathrm{C} 1$ & 107.7 \\
\hline $\mathrm{C} 4-\mathrm{C} 1-\mathrm{C} 6$ & 134.4 \\
\hline $\mathrm{C} 1-\mathrm{C} 6-\mathrm{C} 7$ & 117.3 \\
\hline $\mathrm{C} 6-\mathrm{C} 7-\mathrm{C} 9$ & 122.6 \\
\hline $\mathrm{C} 7-\mathrm{C} 9-\mathrm{C} 8$ & 122.0 \\
\hline $\mathrm{C} 9-\mathrm{C} 8-\mathrm{C} 2$ & 115.5 \\
\hline $\mathrm{C} 2-\mathrm{N} 3-\mathrm{H} 10$ & 124.8 \\
\hline $\mathrm{N} 3-\mathrm{C} 5-\mathrm{H} 12$ & 125.1 \\
\hline $\mathrm{C} 5-\mathrm{C} 4-\mathrm{H} 11$ & 124.3 \\
\hline $\mathrm{C} 1-\mathrm{C} 6-\mathrm{H} 13$ & 120.9 \\
\hline $\mathrm{C} 6-\mathrm{C} 7-\mathrm{H} 14$ & 118.4 \\
\hline $\mathrm{C} 7-\mathrm{C} 9-\mathrm{H} 16$ & 120.0 \\
\hline $\mathrm{C} 9-\mathrm{C} 8-\mathrm{H} 15$ & 121.7 \\
\hline
\end{tabular}


Table S194 Structural parameters of the OM2/MRCISD-optimized excitedstate equilibrium geometry of the $1{ }^{1} B_{1}\left({ }^{1} n \pi^{*}\right)$ state of maleimide used for the statistical evaluations.

\begin{tabular}{ll}
$\begin{array}{l}\text { bond } \\
\text { or angle }\end{array}$ & $\begin{array}{l}\text { length } / \AA \\
\text { or angle } /\end{array}$ \\
\hline $\mathrm{N} 1-\mathrm{C} 2$ & 1.418 \\
$\mathrm{C} 2-\mathrm{C} 3$ & 1.463 \\
$\mathrm{C} 3-\mathrm{C} 4$ & 1.374 \\
$\mathrm{C} 2-\mathrm{O} 6$ & 1.248 \\
$\mathrm{C} 3-\mathrm{H} 9$ & 1.075 \\
$\mathrm{C} 2-\mathrm{N} 1-\mathrm{C} 5$ & 108.8 \\
$\mathrm{~N} 1-\mathrm{C} 2-\mathrm{O} 6$ & 119.5 \\
O6-C2-C4 & 133.5 \\
$\mathrm{C} 2-\mathrm{C} 3-\mathrm{C} 4$ & 108.6 \\
$\mathrm{C} 2-\mathrm{C} 3-\mathrm{H} 9$ & 121.1 \\
$\mathrm{~N} 1-\mathrm{H} 8$ & 1.012
\end{tabular}


Table S195 Structural parameters of the OM2/MRCISD-optimized excitedstate equilibrium geometry of the $1^{1} A_{2}\left({ }^{1} n \pi^{*}\right)$ state of maleimide used for the statistical evaluations.

\begin{tabular}{ll}
$\begin{array}{l}\text { bond } \\
\text { or angle }\end{array}$ & $\begin{array}{l}\text { length } / \AA \\
\text { or angle } /\end{array}$ \\
\hline $\mathrm{N} 1-\mathrm{C} 2$ & 1.391 \\
$\mathrm{C} 2-\mathrm{C} 3$ & 1.454 \\
$\mathrm{C} 3-\mathrm{C} 4$ & 1.393 \\
$\mathrm{C} 2-\mathrm{O} 6$ & 1.271 \\
$\mathrm{C} 3-\mathrm{H} 9$ & 1.075 \\
$\mathrm{C} 2-\mathrm{N} 1-\mathrm{C} 5$ & 108.2 \\
$\mathrm{~N} 1-\mathrm{C} 2-\mathrm{O} 6$ & 125.2 \\
O6-C2-C4 & 126.1 \\
$\mathrm{C} 2-\mathrm{C} 3-\mathrm{C} 4$ & 107.2 \\
$\mathrm{C} 2-\mathrm{C} 3-\mathrm{H} 9$ & 124.0 \\
$\mathrm{~N} 1-\mathrm{H} 8$ & 1.006
\end{tabular}


Table S196 Structural parameters of the OM2/MRCISD-optimized excitedstate equilibrium geometry of the $1^{1} B_{2}\left({ }^{1} \pi \pi^{*}\right)$ state of maleimide used for the statistical evaluations.

\begin{tabular}{ll}
$\begin{array}{l}\text { bond } \\
\text { or angle }\end{array}$ & $\begin{array}{l}\text { length } / \AA \\
\text { or angle } /\end{array}$ \\
\hline N1-C2 & 1.464 \\
C2-C3 & 1.433 \\
C3-C4 & 1.407 \\
C2-O6 & 1.245 \\
C3-H9 & 1.078 \\
C2-N1-C5 & 109.6 \\
N1-C2-O6 & 117.9 \\
O6-C2-C4 & 137.1 \\
C2-C3-C4 & 110.1 \\
C2-C3-H9 & 122.8 \\
N1-H8 & 1.023
\end{tabular}


Table S197 Structural parameters of the OM2/MRCISD-optimized excitedstate equilibrium geometry of the $2{ }^{1} B_{2}\left({ }^{1} \pi \pi^{*}\right)$ state of maleimide used for the statistical evaluations.

\begin{tabular}{ll}
$\begin{array}{l}\text { bond } \\
\text { or angle }\end{array}$ & $\begin{array}{l}\text { length } / \AA \\
\text { or angle } /\end{array}$ \\
\hline $\mathrm{N} 1-\mathrm{C} 2$ & 1.402 \\
$\mathrm{C} 2-\mathrm{C} 3$ & 1.460 \\
$\mathrm{C} 3-\mathrm{C} 4$ & 1.414 \\
$\mathrm{C} 2-\mathrm{O} 6$ & 1.268 \\
$\mathrm{C} 3-\mathrm{H} 9$ & 1.078 \\
$\mathrm{C} 2-\mathrm{N} 1-\mathrm{C} 5$ & 109.1 \\
$\mathrm{~N} 1-\mathrm{C} 2-\mathrm{O} 6$ & 122.6 \\
O6-C2-C4 & 129.3 \\
$\mathrm{C} 2-\mathrm{C} 3-\mathrm{C} 4$ & 107.3 \\
$\mathrm{C} 2-\mathrm{C} 3-\mathrm{H} 9$ & 124.3 \\
$\mathrm{~N} 1-\mathrm{H} 8$ & 1.008
\end{tabular}


Table S198 Structural parameters of the OM2/MRCISD-optimized excitedstate equilibrium geometry of the $1^{3} B_{1}\left({ }^{3} \pi \pi^{*}\right)$ state of maleimide used for the statistical evaluations.

\begin{tabular}{ll}
$\begin{array}{l}\text { bond } \\
\text { or angle }\end{array}$ & $\begin{array}{l}\text { length } / \AA \\
\text { or angle } /\end{array}$ \\
\hline $\mathrm{N} 1-\mathrm{C} 2$ & 1.414 \\
$\mathrm{C} 2-\mathrm{C} 3$ & 1.465 \\
$\mathrm{C} 3-\mathrm{C} 4$ & 1.372 \\
$\mathrm{C} 2-\mathrm{O} 6$ & 1.248 \\
$\mathrm{C} 3-\mathrm{H} 9$ & 1.075 \\
$\mathrm{C} 2-\mathrm{N} 1-\mathrm{C} 5$ & 109.0 \\
$\mathrm{~N} 1-\mathrm{C} 2-\mathrm{O} 6$ & 120.3 \\
O6-C2-C4 & 132.7 \\
$\mathrm{C} 2-\mathrm{C} 3-\mathrm{C} 4$ & 108.5 \\
$\mathrm{C} 2-\mathrm{C} 3-\mathrm{H} 9$ & 121.0 \\
$\mathrm{~N} 1-\mathrm{H} 8$ & 1.012 \\
\end{tabular}


Table S199 Structural parameters of the OM2/MRCISD-optimized excitedstate equilibrium geometry of the $1^{3} A_{2}\left({ }^{3} n \pi^{*}\right)$ state of maleimide used for the statistical evaluations.

\begin{tabular}{ll}
$\begin{array}{l}\text { bond } \\
\text { or angle }\end{array}$ & $\begin{array}{l}\text { length } / \AA \\
\text { or angle } /\end{array}$ \\
\hline N1-C2 & 1.391 \\
C2-C3 & 1.457 \\
C3-C4 & 1.391 \\
C2-O6 & 1.269 \\
C3-H9 & 1.075 \\
C2-N1-C5 & 108.2 \\
N1-C2-O6 & 125.5 \\
O6-C2-C4 & 125.8 \\
C2-C3-C4 & 107.3 \\
C2-C3-H9 & 124.0 \\
N1-H8 & 1.006
\end{tabular}


Table S200 Structural parameters of the OM2/MRCISD-optimized excitedstate equilibrium geometry of the $1^{3} B_{2}\left({ }^{3} \pi \pi^{*}\right)$ state of maleimide used for the statistical evaluations.

\begin{tabular}{ll}
$\begin{array}{l}\text { bond } \\
\text { or angle }\end{array}$ & $\begin{array}{l}\text { length } / \AA \\
\text { or angle } /\end{array}$ \\
\hline N1-C2 & 1.406 \\
C2-C3 & 1.464 \\
C3-C4 & 1.492 \\
C2-O6 & 1.243 \\
C3-H9 & 1.078 \\
C2-N1-C5 & 111.5 \\
N1-C2-O6 & 124.6 \\
O6-C2-C4 & 127.6 \\
C2-C3-C4 & 106.5 \\
C2-C3-H9 & 126.4 \\
N1-H8 & 1.007
\end{tabular}


Table S201 Structural parameters of the OM2/MRCISD-optimized excitedstate equilibrium geometry of the $1^{1} A^{\prime \prime}\left({ }^{1} n \pi^{*}\right)$ state of nitrosomethane used for the statistical evaluations.

\begin{tabular}{ll} 
bond & $\begin{array}{l}\text { length / } \AA \\
\text { or angle } \\
\text { or angle / }\end{array}$ \\
\hline $\mathrm{N} 2-\mathrm{O} 3$ & 1.228 \\
$\mathrm{C} 1-\mathrm{N} 2$ & 1.454 \\
$\mathrm{C} 1-\mathrm{H} 4$ & 1.101 \\
$\mathrm{C} 1-\mathrm{H} 5$ & 1.101 \\
$\mathrm{C} 1-\mathrm{N} 2-\mathrm{O} 3$ & 129.6 \\
$\mathrm{~N} 2-\mathrm{C} 1-\mathrm{H} 4$ & 109.1 \\
$\mathrm{~N} 2-\mathrm{C} 1-\mathrm{H} 5$ & 108.2 \\
$\mathrm{H} 4-\mathrm{C} 1-\mathrm{H} 5$ & 110.2
\end{tabular}


Table S202 Structural parameters of the OM2/MRCISD-optimized excitedstate equilibrium geometry of the $1{ }^{3} A^{\prime \prime}\left({ }^{3} n \pi^{*}\right)$ state of nitrosomethane used for the statistical evaluations.

\begin{tabular}{ll} 
bond & $\begin{array}{l}\text { length / } \AA \\
\text { or angle } \\
\text { or angle / }\end{array}$ \\
\hline $\mathrm{N} 2-\mathrm{O} 3$ & 1.224 \\
$\mathrm{C} 1-\mathrm{N} 2$ & 1.459 \\
$\mathrm{C} 1-\mathrm{H} 4$ & 1.103 \\
$\mathrm{C} 1-\mathrm{H} 5$ & 1.099 \\
$\mathrm{C} 1-\mathrm{N} 2-\mathrm{O} 3$ & 130.6 \\
$\mathrm{~N} 2-\mathrm{C} 1-\mathrm{H} 4$ & 109.3 \\
$\mathrm{~N} 2-\mathrm{C} 1-\mathrm{H} 5$ & 108.5 \\
$\mathrm{H} 4-\mathrm{C} 1-\mathrm{H} 5$ & 109.7
\end{tabular}


Table S203 Structural parameters of the OM2/MRCISD-optimized excitedstate equilibrium geometry of the $1^{1} A^{\prime \prime}\left({ }^{1} n \pi^{*}\right)$ state of propenoic acid anion used for the statistical evaluations.

\begin{tabular}{ll}
$\begin{array}{l}\text { bond } \\
\text { or angle }\end{array}$ & $\begin{array}{l}\text { length } / \AA \\
\text { or angle / } /\end{array}$ \\
\hline $\mathrm{C} 2-\mathrm{O} 3$ & 1.273 \\
$\mathrm{C} 2-\mathrm{O} 4$ & 1.333 \\
$\mathrm{C} 1-\mathrm{C} 2$ & 1.411 \\
$\mathrm{C} 1-\mathrm{C} 5$ & 1.383 \\
$\mathrm{O} 4-\mathrm{C} 2-\mathrm{C} 1$ & 114.7 \\
$\mathrm{C} 2-\mathrm{C} 1-\mathrm{C} 5$ & 123.9 \\
$\mathrm{O} 3-\mathrm{C} 2-\mathrm{O} 4$ & 116.1
\end{tabular}


Table S204 Structural parameters of the OM2/MRCISD-optimized excitedstate equilibrium geometry of the $1{ }^{1} A_{2}\left({ }^{1} n \pi^{*}\right)$ state of pyridine used for the statistical evaluations.

\begin{tabular}{ll} 
bond & $\begin{array}{l}\text { length / } \AA \\
\text { or angle } \\
\text { or angle / }\end{array}$ \\
\hline $\mathrm{N} 1-\mathrm{C} 2$ & 1.310 \\
$\mathrm{C} 2-\mathrm{C} 4$ & 1.457 \\
$\mathrm{C} 4-\mathrm{C} 6$ & 1.389 \\
$\mathrm{C} 2-\mathrm{N} 1-\mathrm{C} 3$ & 134.4 \\
$\mathrm{~N} 1-\mathrm{C} 2-\mathrm{C} 4$ & 113.3 \\
$\mathrm{C} 2-\mathrm{C} 4-\mathrm{C} 6$ & 118.1 \\
$\mathrm{C} 4-\mathrm{C} 6-\mathrm{C} 5$ & 122.9
\end{tabular}


Table S205 Structural parameters of the OM2/MRCISD-optimized excitedstate equilibrium geometry of the $2{ }^{1} A^{\prime}\left({ }^{1} n \pi^{*}\right)$ state of pyridine used for the statistical evaluations.

\begin{tabular}{ll}
$\begin{array}{l}\text { bond } \\
\text { or angle }\end{array}$ & $\begin{array}{l}\text { length angle } / \\
\text { or } /\end{array}$ \\
\hline $\mathrm{N} 1-\mathrm{C} 2$ & 1.351 \\
$\mathrm{C} 2-\mathrm{C} 4$ & 1.391 \\
$\mathrm{C} 4-\mathrm{C} 6$ & 1.417 \\
$\mathrm{C} 2-\mathrm{N} 1-\mathrm{C} 3$ & 131.8 \\
$\mathrm{~N} 1-\mathrm{C} 2-\mathrm{C} 4$ & 113.8 \\
$\mathrm{C} 2-\mathrm{C} 4-\mathrm{C} 6$ & 120.4 \\
$\mathrm{C} 4-\mathrm{C} 6-\mathrm{C} 5$ & 119.8
\end{tabular}


Table S206 Structural parameters of the OM2/MRCISD-optimized excitedstate equilibrium geometry of the $1{ }^{3} A_{1}\left({ }^{3} \pi \pi^{*}\right)$ state of pyridine used for the statistical evaluations.

\begin{tabular}{ll}
$\begin{array}{l}\text { bond } \\
\text { or angle }\end{array}$ & $\begin{array}{l}\text { length } / \AA \\
\text { or angle / } /\end{array}$ \\
\hline $\mathrm{N} 1-\mathrm{C} 2$ & 1.330 \\
$\mathrm{C} 2-\mathrm{C} 4$ & 1.488 \\
$\mathrm{C} 4-\mathrm{C} 6$ & 1.392 \\
$\mathrm{C} 2-\mathrm{N} 1-\mathrm{C} 3$ & 119.2 \\
$\mathrm{~N} 1-\mathrm{C} 2-\mathrm{C} 4$ & 122.4 \\
$\mathrm{C} 2-\mathrm{C} 4-\mathrm{C} 6$ & 118.4 \\
$\mathrm{C} 4-\mathrm{C} 6-\mathrm{C} 5$ & 119.1
\end{tabular}


Table S207 Structural parameters of the OM2/MRCISD-optimized excitedstate equilibrium geometry of the $2{ }^{3} A_{1}\left({ }^{3} \pi \pi^{*}\right)$ state of pyridine used for the statistical evaluations.

\begin{tabular}{ll} 
bond & $\begin{array}{l}\text { length / } \AA \\
\text { or angle } \\
\text { or angle / }\end{array}$ \\
\hline $\mathrm{N} 1-\mathrm{C} 2$ & 1.362 \\
$\mathrm{C} 2-\mathrm{C} 4$ & 1.411 \\
$\mathrm{C} 4-\mathrm{C} 6$ & 1.431 \\
$\mathrm{C} 2-\mathrm{N} 1-\mathrm{C} 3$ & 117.8 \\
$\mathrm{~N} 1-\mathrm{C} 2-\mathrm{C} 4$ & 123.5 \\
$\mathrm{C} 2-\mathrm{C} 4-\mathrm{C} 6$ & 118.8 \\
$\mathrm{C} 4-\mathrm{C} 6-\mathrm{C} 5$ & 117.7
\end{tabular}


Table S208 Structural parameters of the OM2/MRCISD-optimized excitedstate equilibrium geometry of the $2{ }^{1} A^{\prime}\left({ }^{1} \pi \pi^{*}\right)$ state of pyrrole used for the statistical evaluations.

\begin{tabular}{ll}
$\begin{array}{l}\text { bond } \\
\text { or angle }\end{array}$ & $\begin{array}{c}\text { length } / \AA \\
\text { or angle / }\end{array}$ \\
\hline N6-C7 & 1.375 \\
C7-C10 & 1.474 \\
C9-C10 & 1.440 \\
C7-N6-C8 & 110.6 \\
C10-C7-N6 & 108.4 \\
C7-C10-C9 & 106.1 \\
H1-N6 & 1.011
\end{tabular}


Table S209 Structural parameters of the OM2/MRCISD-optimized excitedstate equilibrium geometry of the $1{ }^{1} B_{2}\left({ }^{1} \pi \pi^{*}\right)$ state of pyrrole used for the statistical evaluations.

\begin{tabular}{ll}
$\begin{array}{l}\text { bond } \\
\text { or angle }\end{array}$ & $\begin{array}{l}\text { length } / \AA \\
\text { or angle / }\end{array}$ \\
\hline N6-C7 & 1.391 \\
C7-C10 & 1.455 \\
C9-C10 & 1.380 \\
C7-N6-C8 & 107.1 \\
C10-C7-N6 & 109.3 \\
C7-C10-C9 & 107.1 \\
H1-N6 & 1.010
\end{tabular}


Table S210 Structural parameters of the OM2/MRCISD-optimized excitedstate equilibrium geometry of the $2{ }^{1} A^{\prime}\left({ }^{1} \pi \pi^{*}\right)$ state of cis-PSB3 used for the statistical evaluations.

\begin{tabular}{ll}
$\begin{array}{l}\text { bond } \\
\text { or angle }\end{array}$ & $\begin{array}{l}\text { length } / \AA \\
\text { or angle } /\end{array}$ \\
\hline C1-C2 & 1.386 \\
C2-C3 & 1.415 \\
C3-C4 & 1.419 \\
C4-C5 & 1.434 \\
C5-N6 & 1.344 \\
C1-C2-C3 & 119.8 \\
C2-C3-C4 & 122.5 \\
C3-C4-C5 & 123.2 \\
C4-C5-N6 & 118.5 \\
N6-H13 & 1.004 \\
N6-H14 & 1.004
\end{tabular}


Table S211 Structural parameters of the OM2/MRCISD-optimized excitedstate equilibrium geometry of the $2{ }^{1} A^{\prime}\left({ }^{1} \pi \pi^{*}\right)$ state of 3Me-cis-PSB5 used for the statistical evaluations.

\begin{tabular}{ll}
$\begin{array}{l}\text { bond } \\
\text { or angle }\end{array}$ & $\begin{array}{c}\text { length } / \AA \\
\text { or angle / }\end{array}$ \\
\hline $\mathrm{C} 10-\mathrm{C} 11$ & 1.368 \\
$\mathrm{C} 9-\mathrm{C} 10$ & 1.412 \\
$\mathrm{C} 8-\mathrm{C} 9$ & 1.423 \\
$\mathrm{C} 1-\mathrm{C} 8$ & 1.376 \\
$\mathrm{C} 1-\mathrm{C} 2$ & 1.453 \\
$\mathrm{C} 2-\mathrm{C} 3$ & 1.386 \\
$\mathrm{C} 4-\mathrm{C} 4$ & 1.452 \\
$\mathrm{C} 4-\mathrm{C} 5$ & 1.398 \\
$\mathrm{C} 5-\mathrm{N} 6$ & 1.346 \\
$\mathrm{C} 3-\mathrm{C} 7$ & 1.492 \\
$\mathrm{C} 11-\mathrm{C} 10-\mathrm{C} 9$ & 120.3 \\
$\mathrm{C} 10-\mathrm{C} 9-\mathrm{C} 8$ & 120.8 \\
$\mathrm{C} 9-\mathrm{C} 8-\mathrm{C} 1$ & 119.0 \\
$\mathrm{C} 8-\mathrm{C} 1-\mathrm{C} 2$ & 127.5 \\
$\mathrm{C} 1-\mathrm{C} 2-\mathrm{C} 3$ & 128.9 \\
$\mathrm{C} 2-\mathrm{C} 3-\mathrm{C} 4$ & 114.9 \\
$\mathrm{C} 3-\mathrm{C} 4-\mathrm{C} 5$ & 123.8 \\
$\mathrm{C} 4-\mathrm{C} 5-\mathrm{N} 6$ & 120.4 \\
$\mathrm{C} 2-\mathrm{C} 3-\mathrm{C} 7$ & 124.2 \\
$\mathrm{~N} 6-\mathrm{H} 16$ & 1.003 \\
$\mathrm{~N} 6-\mathrm{H} 17$ & 1.004
\end{tabular}


Table S212 Structural parameters of the OM2/MRCISD-optimized excitedstate equilibrium geometry of the $1{ }^{1} B_{u}\left({ }^{1} \pi \pi^{*}\right)$ state of stilbene used for the statistical evaluations.

\begin{tabular}{ll}
$\begin{array}{l}\text { bond } \\
\text { or angle }\end{array}$ & $\begin{array}{l}\text { length } / \AA \\
\text { or angle } /\end{array}$ \\
\hline $\mathrm{C} 1-\mathrm{C} 8$ & 1.404 \\
$\mathrm{C} 1-\mathrm{C} 2$ & 1.411 \\
$\mathrm{C} 2-\mathrm{C} 3$ & 1.431 \\
$\mathrm{C} 3-\mathrm{C} 4$ & 1.380 \\
$\mathrm{C} 4-\mathrm{C} 5$ & 1.404 \\
$\mathrm{C} 5-\mathrm{C} 6$ & 1.398 \\
$\mathrm{C} 6-\mathrm{C} 7$ & 1.385 \\
$\mathrm{C} 2-\mathrm{C} 7$ & 1.429 \\
$\mathrm{C} 8-\mathrm{C} 1-\mathrm{C} 2$ & 123.2 \\
$\mathrm{C} 1-\mathrm{C} 2-\mathrm{C} 3$ & 118.9 \\
$\mathrm{C} 1-\mathrm{C} 2-\mathrm{C} 7$ & 122.8
\end{tabular}


Table S213 Structural parameters of the OM2/MRCISD-optimized excitedstate equilibrium geometry of the $1^{1} B\left({ }^{1} \pi \pi^{*}\right)$ state of stilbene used for the statistical evaluations.

\begin{tabular}{ll}
$\begin{array}{l}\text { bond } \\
\text { or angle }\end{array}$ & $\begin{array}{l}\text { length } / \AA \\
\text { or angle } /\end{array}$ \\
\hline $\mathrm{C} 1-\mathrm{C} 8$ & 1.383 \\
$\mathrm{C} 1-\mathrm{C} 2$ & 1.436 \\
$\mathrm{C} 2-\mathrm{C} 3$ & 1.412 \\
$\mathrm{C} 3-\mathrm{C} 4$ & 1.384 \\
$\mathrm{C} 4-\mathrm{C} 5$ & 1.398 \\
$\mathrm{C} 5-\mathrm{C} 6$ & 1.391 \\
$\mathrm{C} 6-\mathrm{C} 7$ & 1.389 \\
$\mathrm{C} 2-\mathrm{C} 7$ & 1.412 \\
$\mathrm{C} 8-\mathrm{C} 1-\mathrm{C} 2$ & 121.8 \\
$\mathrm{C} 1-\mathrm{C} 2-\mathrm{C} 3$ & 120.6 \\
$\mathrm{C} 1-\mathrm{C} 2-\mathrm{C} 7$ & 119.8
\end{tabular}


Table S214 Structural parameters of the OM2/MRCISD-optimized excitedstate equilibrium geometry of the $2{ }^{1} A^{\prime}\left({ }^{1} \pi \pi^{*}\right)$ state of styrene used for the statistical evaluations.

\begin{tabular}{ll}
$\begin{array}{l}\text { bond } \\
\text { or angle }\end{array}$ & $\begin{array}{l}\text { length } / \AA \\
\text { or angle } /{ }^{\circ}\end{array}$ \\
\hline C1-C2 & 1.359 \\
C1-C3 & 1.433 \\
C3-C4 & 1.433 \\
C3-C5 & 1.436 \\
C4-C6 & 1.419 \\
C5-C7 & 1.419 \\
C6-C8 & 1.413 \\
C7-C8 & 1.412
\end{tabular}


Table S215 Structural parameters of the OM2/MRCISD-optimized excitedstate equilibrium geometry of the $1^{3} A^{\prime}\left({ }^{3} \pi \pi^{*}\right)$ state of styrene used for the statistical evaluations.

\begin{tabular}{ll}
$\begin{array}{l}\text { bond } \\
\text { or angle }\end{array}$ & $\begin{array}{l}\text { length } / \AA \\
\text { or angle } /{ }^{\circ}\end{array}$ \\
\hline C1-C2 & 1.437 \\
C1-C3 & 1.389 \\
C3-C4 & 1.444 \\
C3-C5 & 1.448 \\
C4-C6 & 1.379 \\
C5-C7 & 1.378 \\
C6-C8 & 1.410 \\
C7-C8 & 1.411
\end{tabular}


Table S216 Structural parameters of the OM2/MRCISD-optimized excitedstate equilibrium geometry of the $1{ }^{1} B_{3 u}\left({ }^{1} n \pi^{*}\right)$ state of $s$-tetrazine used for the statistical evaluations.

\begin{tabular}{ll} 
bond & $\begin{array}{l}\text { length / } \AA \\
\text { or angle } \\
\text { or angle / }\end{array}$ \\
\hline N3-N4 & 1.258 \\
C2-N4 & 1.360 \\
C2-H7 & 1.099 \\
N3-C1-N5 & 115.5
\end{tabular}


Table S217 Structural parameters of the OM2/MRCISD-optimized excitedstate equilibrium geometry of the $2{ }^{1} A\left({ }^{1} n \pi^{*}\right)$ state of thymine used for the statistical evaluations.

\begin{tabular}{ll}
$\begin{array}{l}\text { bond } \\
\text { or angle }\end{array}$ & $\begin{array}{l}\text { length } / \AA \\
\text { or angle } /\end{array}$ \\
\hline N1-C6 & 1.387 \\
C5-N6 & 1.400 \\
N4-C5 & 1.381 \\
C3-C4 & 1.403 \\
C2-C3 & 1.407 \\
N1-C2 & 1.389 \\
C6-09 & 1.248 \\
C4-07 & 1.328 \\
C3-C8 & 1.490 \\
C2-H10 & 1.087 \\
N1-H11 & 1.014 \\
N5-H12 & 1.011
\end{tabular}


Table S218 Structural parameters of the OM2/MRCISD-optimized excitedstate equilibrium geometry of the $2{ }^{1} A^{\prime}\left({ }^{1} \pi \pi^{*}\right)$ state of uracil used for the statistical evaluations.

\begin{tabular}{ll}
$\begin{array}{l}\text { bond } \\
\text { or angle }\end{array}$ & $\begin{array}{l}\text { length } / \AA \\
\text { or angle } /\end{array}$ \\
\hline N1-C3 & 1.365 \\
N2-C3 & 1.433 \\
N2-C4 & 1.338 \\
C4-C5 & 1.477 \\
C5-C6 & 1.405 \\
N1-C6 & 1.438 \\
C3-O8 & 1.251 \\
C6-O7 & 1.264 \\
C4-H9 & 1.093 \\
C5-H12 & 1.079 \\
N2-H11 & 1.026 \\
N1-H10 & 1.016
\end{tabular}


4.1.3 OM3/MRCI-Optimized Excited-State Minima 
Table S219 Employed active space, reference configurations, and Cartesian coordinates of the OM3/MRCISD-optimized excited-state equilibrium geometry of the $2{ }^{1} A\left({ }^{1} n \pi^{*}\right)$ state of $9 H$-adenine (excited-state energy: $\left.-1773.98650 \mathrm{eV}\right)$.

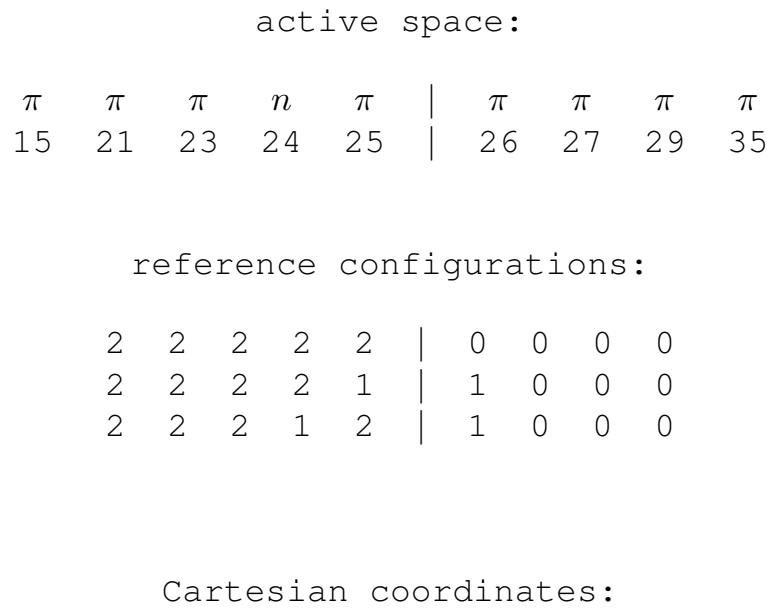


Table S220 Employed active space, reference configurations, and Cartesian coordinates of the OM3/MRCISD-optimized excited-state equilibrium geometry of the $2{ }^{1} A\left({ }^{1} n \pi^{*}\right)$ state of acetaldehyde (excited-state energy: $-645.27681 \mathrm{eV}$ ).

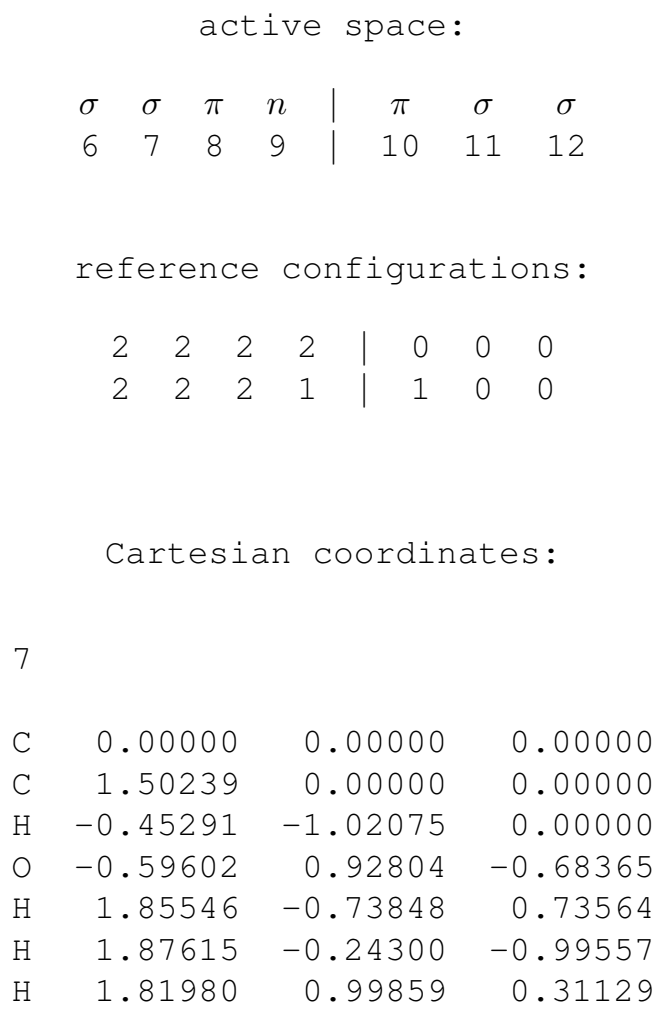


Table S221 Employed active space, reference configurations, and Cartesian coordinates of the OM3/MRCISD-optimized excited-state equilibrium geometry of the $1^{3} A\left({ }^{3} n \pi^{*}\right)$ state of acetaldehyde (excited-state energy: $\left.-645.63170 \mathrm{eV}\right)$.

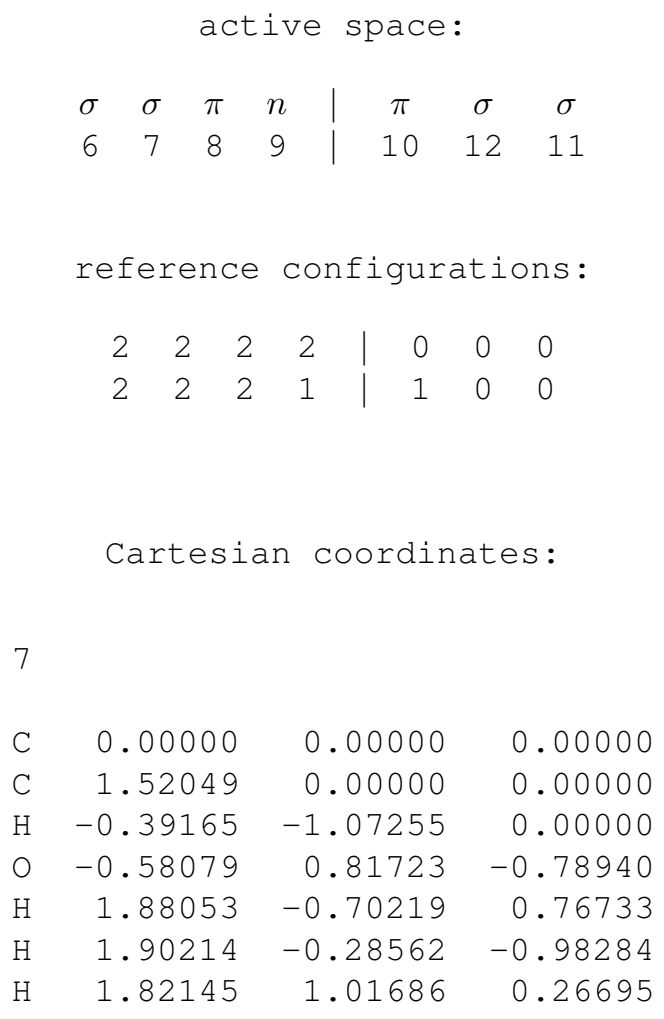


Table S222 Employed active space, reference configurations, and Cartesian coordinates of the OM3/MRCISD-optimized excited-state equilibrium geometry of the $2{ }^{3} A\left({ }^{3} \pi \pi^{*}\right)$ state of acetaldehyde (excited-state energy: $-644.14080 \mathrm{eV}$ ).

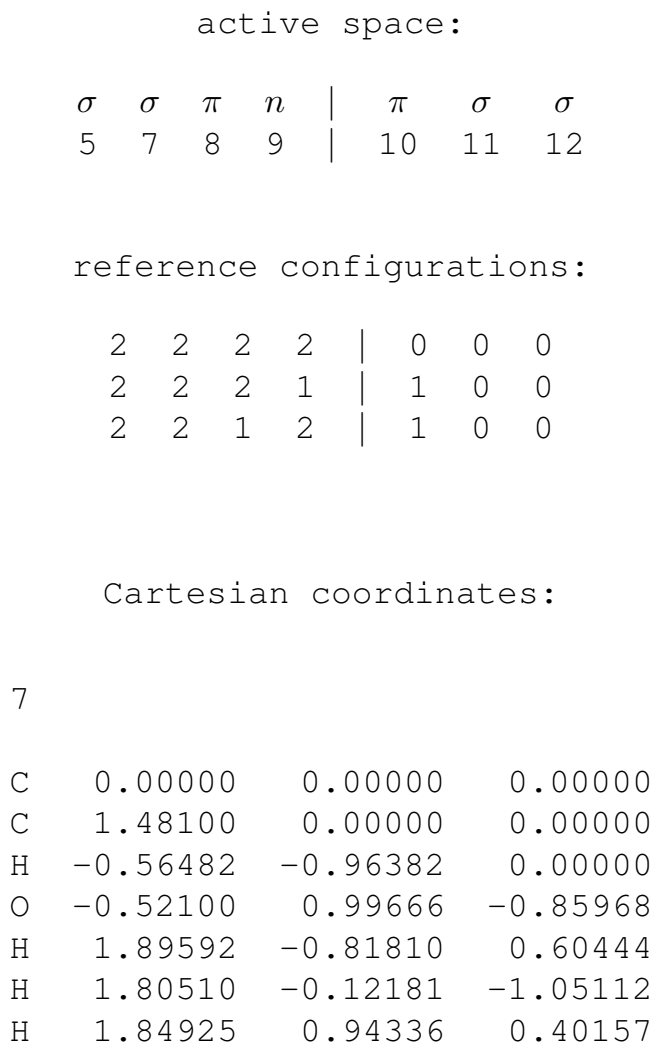


Table S223 Employed active space, reference configurations, and Cartesian coordinates of the OM3/MRCISD-optimized excited-state equilibrium geometry of the $1{ }^{1} A^{\prime \prime}\left({ }^{1} n \pi^{*}\right)$ state of acetone (excited-state energy: $-800.14014 \mathrm{eV}$ ).

\begin{tabular}{|c|c|c|c|c|}
\hline$\sigma$ & $\pi$ & $n$ & $\pi$ & $\sigma$ \\
\hline 10 & 11 & 12 & 13 & 14 \\
\hline \multicolumn{2}{|c|}{ reference } & \multicolumn{3}{|c|}{ configurations: } \\
\hline & 2 & 2 & 0 & 0 \\
\hline 2 & 2 & 1 & 1 & 0 \\
\hline \multicolumn{5}{|c|}{ Cartesian coordinates: } \\
\hline-1.29 & & & 4858 & 0.00000 \\
\hline 0.00 & & & 0000 & 0.00000 \\
\hline 0.00 & & -1 & 3104 & -0.73972 \\
\hline 1.29 & & & 4858 & 0.00000 \\
\hline-2.12 & & & 5879 & 0.21665 \\
\hline-1.46 & & & 281 & -0.98551 \\
\hline-1.27 & 74 & & 2720 & 0.77769 \\
\hline 2.12 & & & 5879 & 0.21665 \\
\hline 1.27 & & & 2720 & 0.77769 \\
\hline 1.46 & & & 281 & -0.98551 \\
\hline
\end{tabular}


Table S224 Employed active space, reference configurations, and Cartesian coordinates of the OM3/MRCISD-optimized excited-state equilibrium geometry of the $1^{3} A^{\prime \prime}\left({ }^{3} n \pi^{*}\right)$ state of acetone (excited-state energy: $-800.48311 \mathrm{eV}$ ).

\begin{tabular}{|c|c|c|c|c|}
\hline$\sigma$ & $\pi$ & $n$ & $\pi$ & $\sigma$ \\
\hline 10 & 11 & 12 & 13 & 14 \\
\hline \multicolumn{2}{|c|}{ reference } & \multicolumn{3}{|c|}{ configurations: } \\
\hline & 2 & 2 & 0 & 0 \\
\hline 2 & 2 & 1 & 1 & 0 \\
\hline \multicolumn{5}{|c|}{ Cartesian coordinates: } \\
\hline-1.29 & & & 713 & 0.00000 \\
\hline 0.00 & & & 0000 & 0.00000 \\
\hline 0.00 & & -0 & 3008 & -0.82939 \\
\hline 1.29 & & & 713 & 0.00000 \\
\hline-2.13 & & & 2629 & 0.17553 \\
\hline-1.41 & & & 959 & -0.97321 \\
\hline-1.25 & & & 4974 & 0.81142 \\
\hline 2.13 & & & 2629 & 0.17553 \\
\hline 1.25 & & & 9974 & 0.81142 \\
\hline 1.41 & & & 959 & -0.97321 \\
\hline
\end{tabular}


Table S225 Employed active space, reference configurations, and Cartesian coordinates of the OM3/MRCISD-optimized excited-state equilibrium geometry of the $1^{3} A^{\prime}\left({ }^{3} \pi \pi^{*}\right)$ state of acetone (excited-state energy: $\left.-799.19731 \mathrm{eV}\right)$.

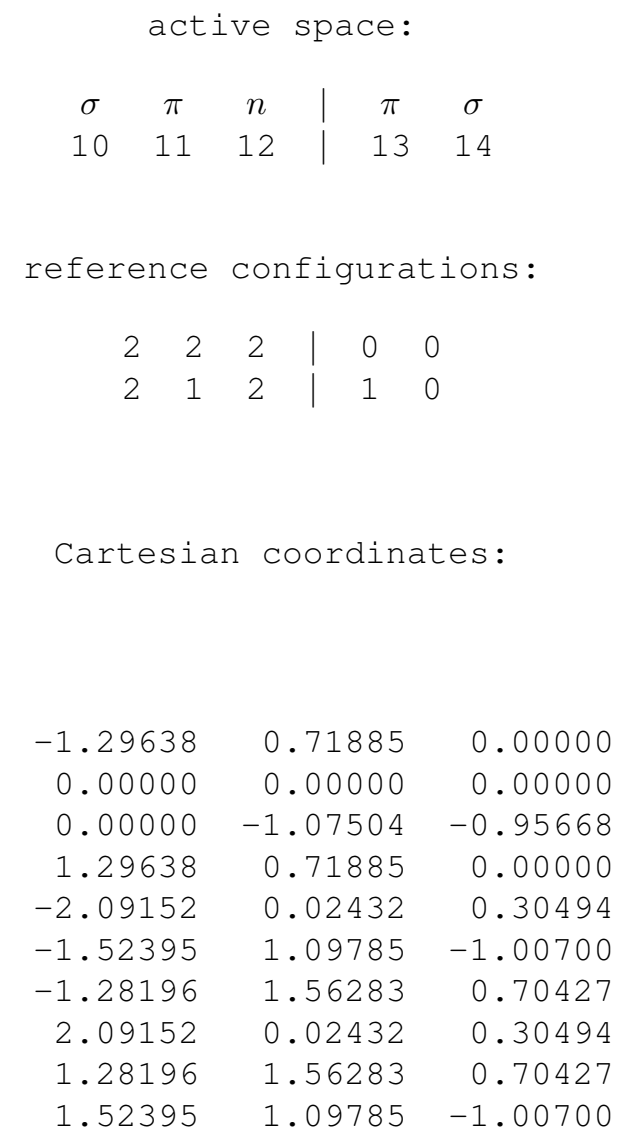


Table S226 Employed active space, reference configurations, and Cartesian coordinates of the OM3/MRCISDTQ-optimized excited-state equilibrium geometry of the $1^{1} A^{\prime \prime}\left({ }^{1} n \pi^{*}\right)$ state of acetophenone (excited-state energy: $-1455.24983 \mathrm{eV})$.

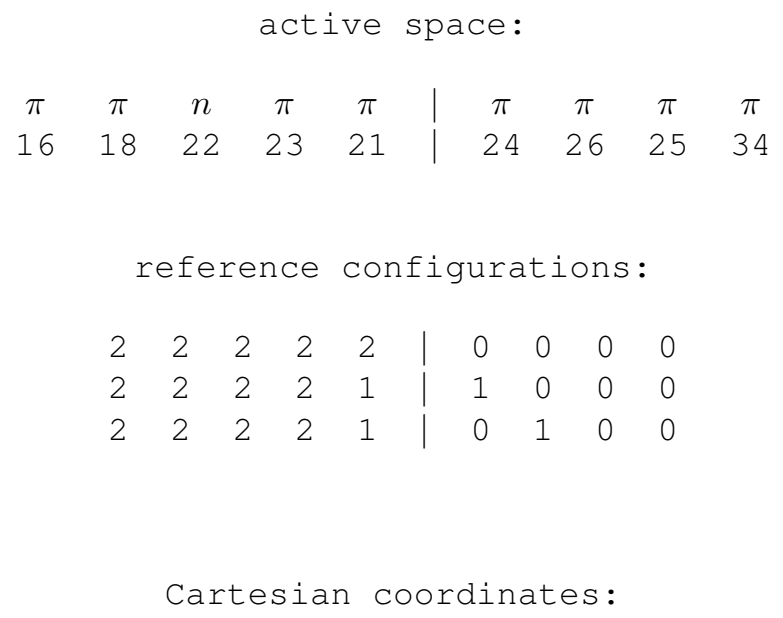


Table S227 Employed active space, reference configurations, and Cartesian coordinates of the OM3/MRCISD-optimized excited-state equilibrium geometry of the $2{ }^{1} A^{\prime}\left({ }^{1} \pi \pi^{*}\right)$ state of acetophenone (excited-state energy: $\left.-1454.72236 \mathrm{eV}\right)$.

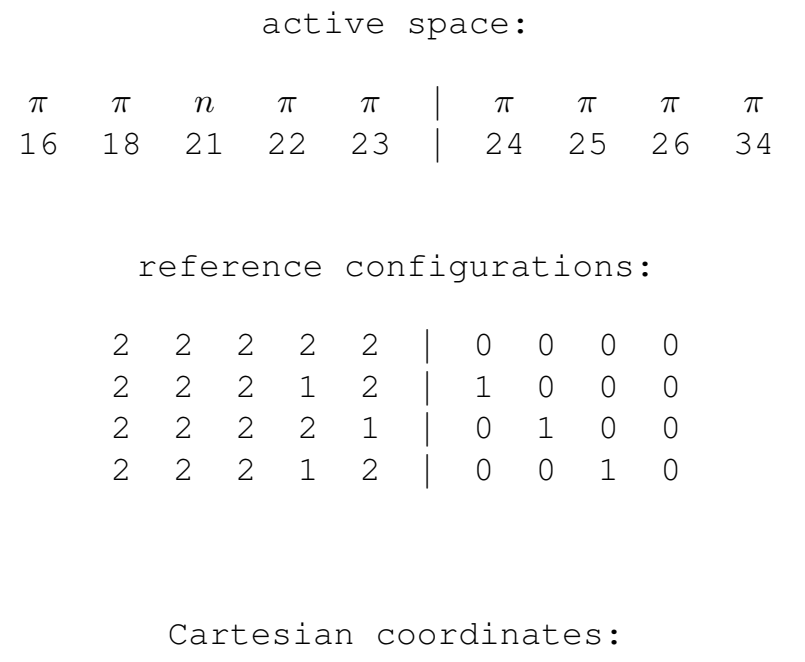

17

$\begin{array}{rrrr}\mathrm{C} & 0.00000 & 0.00000 & 0.00000 \\ \mathrm{O} & 1.24559 & 0.00000 & 0.00000 \\ \mathrm{C} & -0.73938 & -1.32978 & 0.00000 \\ \mathrm{H} & 0.01219 & -2.13751 & 0.00000 \\ \mathrm{H} & -1.31283 & -1.41998 & -0.90245 \\ \mathrm{H} & -1.31283 & -1.41998 & 0.90245 \\ \mathrm{C} & -0.77523 & 1.25912 & 0.00000 \\ \mathrm{C} & -2.21461 & 1.30596 & 0.00000 \\ \mathrm{C} & -0.03494 & 2.49163 & 0.00000 \\ \mathrm{C} & -2.89017 & 2.56703 & 0.00000 \\ \mathrm{C} & -0.72734 & 3.74282 & 0.00000 \\ \mathrm{C} & -2.15282 & 3.77992 & 0.00000 \\ \mathrm{H} & -2.80682 & 0.37735 & 0.00000 \\ \mathrm{H} & 1.06216 & 2.45797 & 0.00000 \\ \mathrm{H} & -3.99085 & 2.59152 & 0.00000 \\ \mathrm{H} & -0.15310 & 4.68241 & 0.00000 \\ \mathrm{H} & -2.67845 & 4.74496 & 0.00000\end{array}$


Table S228 Employed active space, reference configurations, and Cartesian coordinates of the OM3/MRCISDT-optimized excited-state equilibrium geometry of the $1^{3} A^{\prime \prime}\left({ }^{3} n \pi^{*}\right)$ state of acetophenone (excited-state energy: $-1455.44971 \mathrm{eV})$.

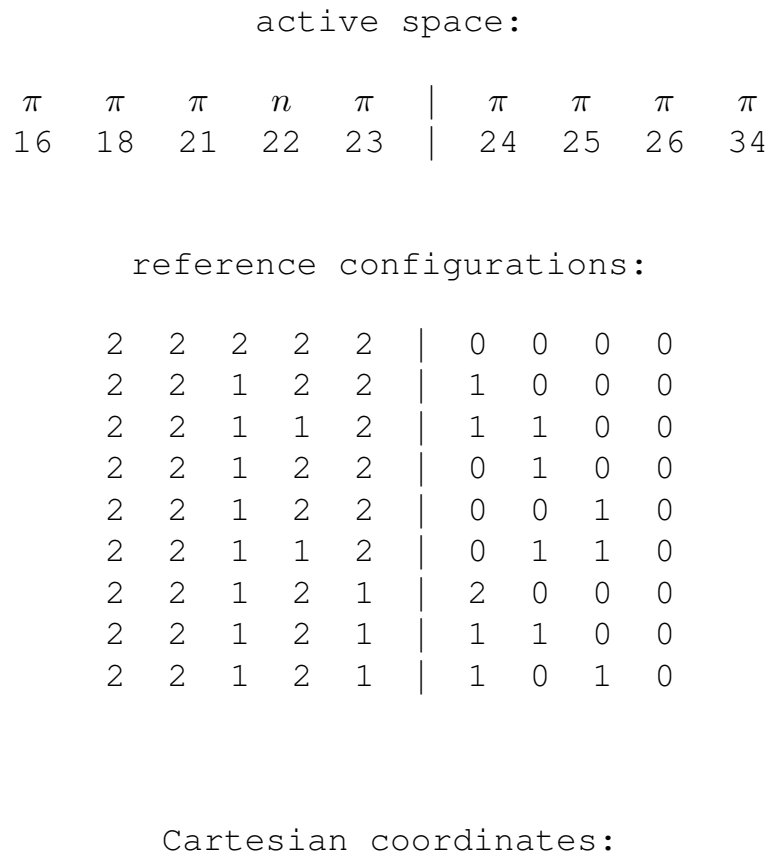

\begin{tabular}{lrrr}
17 & & \\
& & & \\
$\mathrm{C}$ & 0.00000 & 0.00000 & 0.00000 \\
$\mathrm{O}$ & 1.29934 & 0.00000 & 0.00000 \\
$\mathrm{C}$ & -0.42069 & -1.46837 & 0.00000 \\
$\mathrm{H}$ & 0.46584 & -2.12743 & 0.00000 \\
$\mathrm{H}$ & -0.97834 & -1.64185 & -0.91146 \\
$\mathrm{H}$ & -0.97834 & -1.64185 & 0.91146 \\
$\mathrm{C}$ & -0.87999 & 1.12949 & 0.00000 \\
$\mathrm{C}$ & -2.29973 & 0.96028 & 0.00000 \\
$\mathrm{C}$ & -0.32021 & 2.43742 & 0.00000 \\
$\mathrm{C}$ & -3.10774 & 2.09637 & 0.00000 \\
$\mathrm{C}$ & -1.15402 & 3.55683 & 0.00000 \\
$\mathrm{C}$ & -2.55392 & 3.38952 & 0.00000 \\
$\mathrm{H}$ & -2.73862 & -0.04741 & 0.00000 \\
$\mathrm{H}$ & 0.77192 & 2.56583 & 0.00000 \\
$\mathrm{H}$ & -4.20537 & 1.98305 & 0.00000 \\
$\mathrm{H}$ & -0.73417 & 4.57274 & 0.00000 \\
\hline & \multicolumn{3}{c}{ Continued on next page }
\end{tabular}

S261 
Continued from previous page

\begin{tabular}{llll}
\hline $\mathrm{H}$ & -3.20932 & 4.27335 & 0.00000
\end{tabular} 
Table S229 Employed active space, reference configurations, and Cartesian coordinates of the OM3/MRCISD-optimized excited-state equilibrium geometry of the $1^{3} A^{\prime}\left({ }^{3} \pi \pi^{*}\right)$ state of acetophenone (excited-state energy: $-1455.67375 \mathrm{eV}$ ).

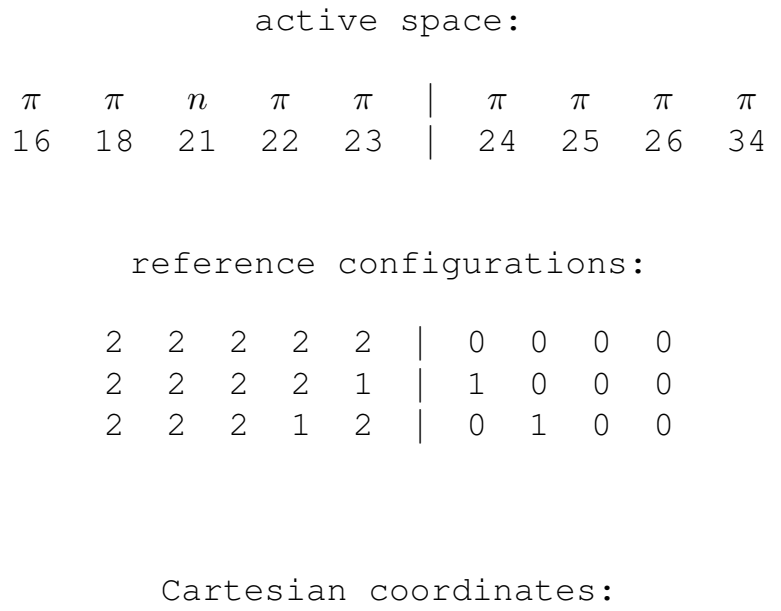


Table S230 Employed active space, reference configurations, and Cartesian coordinates of the OM3/MRCISD-optimized excited-state equilibrium geometry of the $1{ }^{1} A_{2}\left({ }^{1} \pi \pi^{*}\right)$ state of acetylene (excited-state energy: $-273.25664 \mathrm{eV}$ ).

\begin{tabular}{|c|c|c|c|c|c|c|c|c|c|}
\hline$\sigma$ & $\sigma$ & $\sigma$ & $\pi$ & $\pi$ & $\pi$ & $\sigma$ & $\pi$ & $\sigma$ & $\sigma$ \\
\hline \multirow[t]{2}{*}{1} & 2 & 3 & 5 & 4 & 6 & 9 & 7 & 8 & 10 \\
\hline & \multicolumn{4}{|c|}{ reference } & fic & Ira & io & IS: & \\
\hline 2 & 2 & 2 & 2 & 2 & 0 & 0 & 0 & 0 & 0 \\
\hline 2 & 2 & 2 & 2 & 1 & 0 & 1 & 0 & 0 & 0 \\
\hline 2 & 2 & 2 & 1 & 2 & 0 & 0 & 1 & 0 & 0 \\
\hline
\end{tabular}

Cartesian coordinates:

4

$\begin{array}{rrrr}\mathrm{C} & -0.66684 & 0.00000 & 0.00000 \\ \mathrm{C} & 0.66684 & 0.00000 & 0.00000 \\ \mathrm{H} & -1.63084 & -0.57761 & 0.00000 \\ \mathrm{H} & 1.63084 & -0.57761 & 0.00000\end{array}$


Table S231 Employed active space, reference configurations, and Cartesian coordinates of the OM3/MRCISDT-optimized excited-state equilibrium geometry of the $2{ }^{1} A_{2}\left({ }^{1} \pi \pi^{*}\right)$ state of acetylene (excited-state energy: $-272.18178 \mathrm{eV}$ ).

\begin{tabular}{lllll|lllll}
\multicolumn{11}{c}{ active space: } \\
\begin{tabular}{ccccc|ccccc}
$\sigma$ & $\sigma$ & $\sigma$ & $\pi$ & $\pi$ & $\pi$ & $\pi$ & $\sigma$ & $\sigma$ & $\sigma$ \\
1 & 2 & 3 & 4 & 5 & 6 & 7 & 8 & 9 & 10 \\
10 \\
2 & 2 & 2 & 2 & 2 & 0 & 0 & 0 & 0 & 0 \\
2 & 2 & 2 & 2 & 1 & 0 & 0 & 1 & 0 & 0 \\
2 & 2 & 2 & 1 & 2 & 0 & 1 & 0 & 0 & 0 \\
2 & 2 & 2 & 2 & 1 & 1 & 0 & 0 & 0 & 0
\end{tabular}
\end{tabular}

Cartesian coordinates:

4

$\begin{array}{rrrr}\mathrm{C} & -0.66388 & 0.00000 & 0.00000 \\ \mathrm{C} & 0.66388 & 0.00000 & 0.00000 \\ \mathrm{H} & -1.72273 & -0.00000 & 0.00000 \\ \mathrm{H} & 1.72273 & -0.00000 & 0.00000\end{array}$


Table S232 Employed active space, reference configurations, and Cartesian coordinates of the OM3/MRCISD-optimized excited-state equilibrium geometry of the $1{ }^{1} B_{2}\left({ }^{1} \pi \pi^{*}\right)$ state of acetylene (excited-state energy: $-272.75558 \mathrm{eV}$ ).

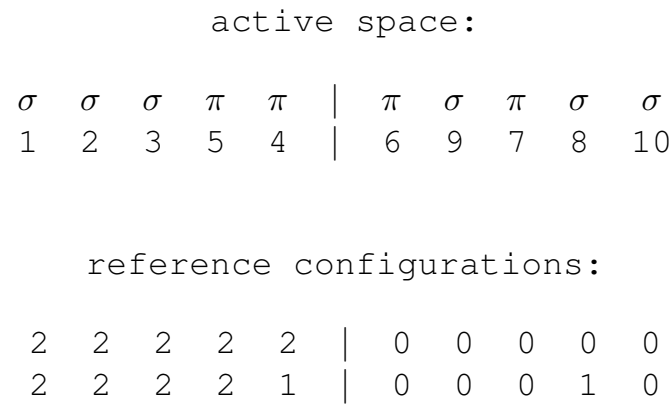

Cartesian coordinates:

4

$\begin{array}{rrrr}\mathrm{C} & -0.65829 & 0.00000 & 0.00000 \\ \mathrm{C} & 0.65829 & 0.00000 & 0.00000 \\ \mathrm{H} & -1.64958 & -0.55552 & 0.00000 \\ \mathrm{H} & 1.64958 & -0.55552 & 0.00000\end{array}$


Table S233 Employed active space, reference configurations, and Cartesian coordinates of the OM3/MRCISD-optimized excited-state equilibrium geometry of the $1^{3} A_{2}\left({ }^{3} \pi \pi^{*}\right)$ state of acetylene (excited-state energy: $\left.-274.02333 \mathrm{eV}\right)$.

\begin{tabular}{|c|c|c|c|c|c|c|c|c|c|}
\hline$\sigma$ & $\sigma$ & $\sigma$ & $\pi$ & $\pi$ & $\pi$ & $\sigma$ & $\pi$ & $\sigma$ & $\sigma$ \\
\hline \multirow[t]{2}{*}{1} & 2 & 3 & 5 & 4 & 6 & 9 & 7 & 8 & 10 \\
\hline & \multicolumn{4}{|c|}{ reference } & fic & Ira & io & IS: & \\
\hline 2 & 2 & 2 & 2 & 2 & 0 & 0 & 0 & 0 & 0 \\
\hline 2 & 2 & 2 & 2 & 1 & 0 & 1 & 0 & 0 & 0 \\
\hline 2 & 2 & 2 & 1 & 2 & 0 & 0 & 1 & 0 & 0 \\
\hline
\end{tabular}

Cartesian coordinates:

4

$\begin{array}{rrrr}\mathrm{C} & -0.67190 & 0.00000 & 0.00000 \\ \mathrm{C} & 0.67190 & 0.00000 & 0.00000 \\ \mathrm{H} & -1.57651 & -0.64311 & 0.00000 \\ \mathrm{H} & 1.57651 & -0.64311 & 0.00000\end{array}$


Table S234 Employed active space, reference configurations, and Cartesian coordinates of the OM3/MRCISD-optimized excited-state equilibrium geometry of the $2{ }^{3} A_{2}\left({ }^{3} \pi \pi^{*}\right)$ state of acetylene (excited-state energy: $-273.13568 \mathrm{eV}$ ).

\begin{tabular}{lllll|lllll}
\multicolumn{11}{c}{ active space: } \\
\begin{tabular}{ccccc|ccccc}
$\sigma$ & $\sigma$ & $\sigma$ & $\pi$ & $\pi$ & $\sigma$ & $\pi$ & $\pi$ & $\sigma$ & $\sigma$ \\
1 & 2 & 3 & 4 & 5 & 7 & 8 & 6 & 9 & 10 \\
10 \\
2 & 2 & 2 & 2 & 2 & 0 & 0 & 0 & 0 & 0 \\
2 & 2 & 2 & 2 & 1 & 0 & 0 & 1 & 0 & 0 \\
2 & 2 & 2 & 1 & 2 & 0 & 1 & 0 & 0 & 0 \\
2 & 2 & 2 & 2 & 1 & 1 & 0 & 0 & 0 & 0
\end{tabular}
\end{tabular}

Cartesian coordinates:

4

$\begin{array}{rrrr}\mathrm{C} & -0.66524 & 0.00000 & 0.00000 \\ \mathrm{C} & 0.66524 & 0.00000 & 0.00000 \\ \mathrm{H} & -1.72584 & 0.00000 & 0.00000 \\ \mathrm{H} & 1.72584 & 0.00000 & 0.00000\end{array}$


Table S235 Employed active space, reference configurations, and Cartesian coordinates of the OM3/MRCISDT-optimized excited-state equilibrium geometry of the $1^{3} B_{2}\left({ }^{3} \pi \pi^{*}\right)$ state of acetylene (excited-state energy: $\left.-275.25001 \mathrm{eV}\right)$.

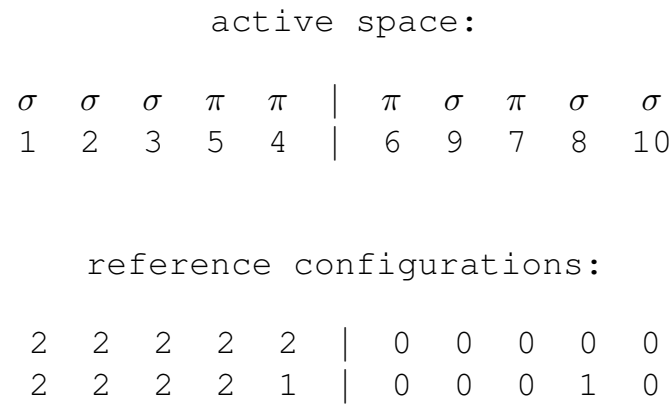

Cartesian coordinates:

4

$\begin{array}{rrrr}\mathrm{C} & -0.66484 & 0.00000 & 0.00000 \\ \mathrm{C} & 0.66484 & 0.00000 & 0.00000 \\ \mathrm{H} & -1.43206 & -0.79665 & 0.00000 \\ \mathrm{H} & 1.43206 & -0.79665 & 0.00000\end{array}$


Table S236 Employed active space, reference configurations, and Cartesian coordinates of the OM3/MRCISD-optimized excited-state equilibrium geometry of the $1{ }^{1} A_{u}\left({ }^{1} \pi \pi^{*}\right)$ state of acetylene (excited-state energy: $\left.-273.47269 \mathrm{eV}\right)$.

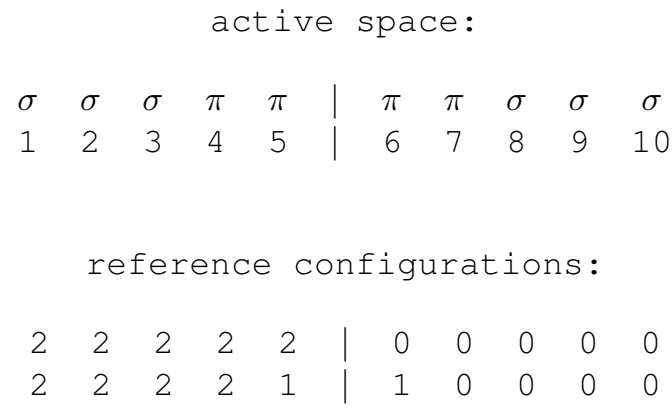

Cartesian coordinates:

4

$\begin{array}{rrrr}\mathrm{C} & -0.68498 & 0.00000 & 0.00000 \\ \mathrm{C} & 0.68498 & 0.00000 & 0.00000 \\ \mathrm{H} & -1.33241 & 0.92004 & 0.00000 \\ \mathrm{H} & 1.33241 & -0.92004 & 0.00000\end{array}$


Table S237 Employed active space, reference configurations, and Cartesian coordinates of the OM3/MRCISD-optimized excited-state equilibrium geometry of the $2{ }^{1} A_{u}\left({ }^{1} \pi \pi^{*}\right)$ state of acetylene (excited-state energy: $\left.-271.89895 \mathrm{eV}\right)$.

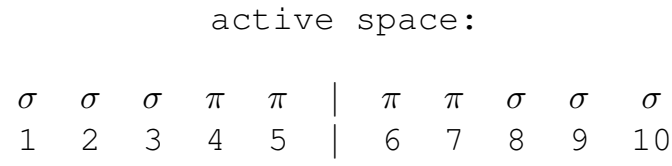

\begin{tabular}{lllll|lllll}
\multicolumn{1}{c}{ reference configurations: } \\
2 & 2 & 2 & 2 & 2 & 0 & 0 & 0 & 0 & 0 \\
2 & 2 & 2 & 2 & 1 & 1 & 0 & 0 & 0 & 0 \\
2 & 2 & 2 & 1 & 1 & 1 & 1 & 0 & 0 & 0 \\
2 & 2 & 2 & 2 & 1 & 0 & 0 & 0 & 1 & 0 \\
2 & 2 & 2 & 1 & 2 & 0 & 0 & 1 & 0 & 0 \\
2 & 2 & 2 & 2 & 0 & 0 & 1 & 1 & 0 & 0 \\
2 & 2 & 2 & 1 & 1 & 0 & 1 & 0 & 1 & 0
\end{tabular}

Cartesian coordinates:

4

$\begin{array}{rrrr}\mathrm{C} & -0.66089 & 0.00000 & 0.00000 \\ \mathrm{C} & 0.66089 & 0.00000 & 0.00000 \\ \mathrm{H} & -1.71548 & 0.15325 & 0.00000 \\ \mathrm{H} & 1.71548 & -0.15325 & 0.00000\end{array}$


Table S238 Employed active space, reference configurations, and Cartesian coordinates of the OM3/MRCISD-optimized excited-state equilibrium geometry of the $1{ }^{1} B_{u}\left({ }^{1} \pi \pi^{*}\right)$ state of acetylene (excited-state energy: $-272.21641 \mathrm{eV}$ ).

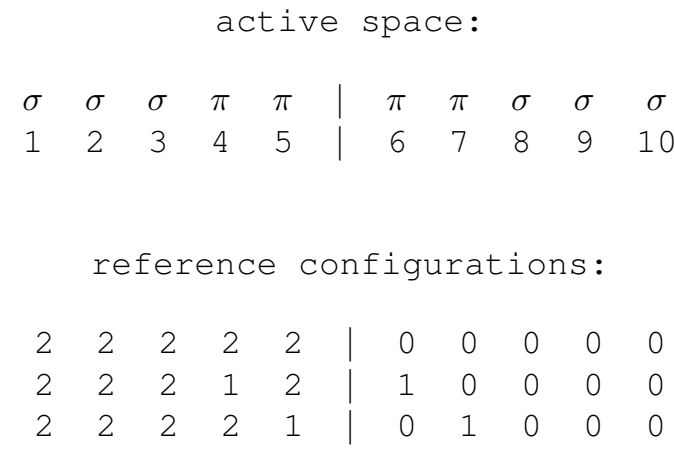

Cartesian coordinates:

4

$\begin{array}{rrrr}\mathrm{C} & -0.66364 & 0.00000 & 0.00000 \\ \mathrm{C} & 0.66364 & 0.00000 & 0.00000 \\ \mathrm{H} & -1.57094 & 0.62660 & 0.00000 \\ \mathrm{H} & 1.57094 & -0.62660 & 0.00000\end{array}$


Table S239 Employed active space, reference configurations, and Cartesian coordinates of the OM3/MRCISD-optimized excited-state equilibrium geometry of the $1^{3} A_{u}\left({ }^{3} \pi \pi^{*}\right)$ state of acetylene (excited-state energy: $\left.-274.36864 \mathrm{eV}\right)$.

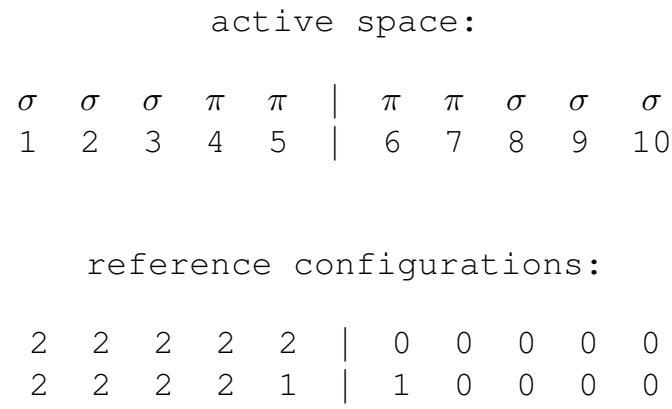

Cartesian coordinates:

4

$\begin{array}{rrrr}\mathrm{C} & -0.69156 & 0.00000 & 0.00000 \\ \mathrm{C} & 0.69156 & 0.00000 & 0.00000 \\ \mathrm{H} & -1.29692 & 0.94480 & 0.00000 \\ \mathrm{H} & 1.29692 & -0.94480 & 0.00000\end{array}$


Table S240 Employed active space, reference configurations, and Cartesian coordinates of the OM3/MRCISD-optimized excited-state equilibrium geometry of the $2{ }^{3} A_{u}\left({ }^{3} \pi \pi^{*}\right)$ state of acetylene (excited-state energy: $-272.58975 \mathrm{eV}$ ).

\begin{tabular}{|c|c|c|c|c|c|c|c|c|c|}
\hline$\sigma$ & $\sigma$ & $\sigma$ & $\pi$ & $\pi$ & $\pi$ & $\pi$ & $\sigma$ & $\sigma$ & $\sigma$ \\
\hline \multirow[t]{2}{*}{1} & 2 & 3 & 4 & 5 & 6 & 7 & 8 & 9 & 10 \\
\hline & \multicolumn{3}{|c|}{ reference } & \multicolumn{6}{|c|}{ configurations: } \\
\hline 2 & 2 & 2 & 2 & 2 & 0 & 0 & 0 & 0 & 0 \\
\hline 2 & 2 & 2 & 2 & 1 & 1 & 0 & 0 & 0 & 0 \\
\hline 2 & 2 & 2 & 1 & 1 & 1 & 0 & 1 & 0 & 0 \\
\hline 2 & 2 & 2 & 2 & 1 & 0 & 0 & 0 & 1 & 0 \\
\hline 2 & 2 & 2 & 1 & 2 & 0 & 1 & 0 & 0 & 0 \\
\hline 2 & 2 & 2 & 2 & 0 & 0 & 1 & 1 & 0 & 0 \\
\hline
\end{tabular}

Cartesian coordinates:

4

$\begin{array}{rrrr}\mathrm{C} & -0.66620 & 0.00000 & 0.00000 \\ \mathrm{C} & 0.66620 & 0.00000 & 0.00000 \\ \mathrm{H} & -1.69448 & 0.29202 & 0.00000 \\ \mathrm{H} & 1.69448 & -0.29202 & 0.00000\end{array}$


Table S241 Employed active space, reference configurations, and Cartesian coordinates of the OM3/MRCISD-optimized excited-state equilibrium geometry of the $1{ }^{3} B_{u}\left({ }^{3} \pi \pi^{*}\right)$ state of acetylene (excited-state energy: $-274.84451 \mathrm{eV}$ ).

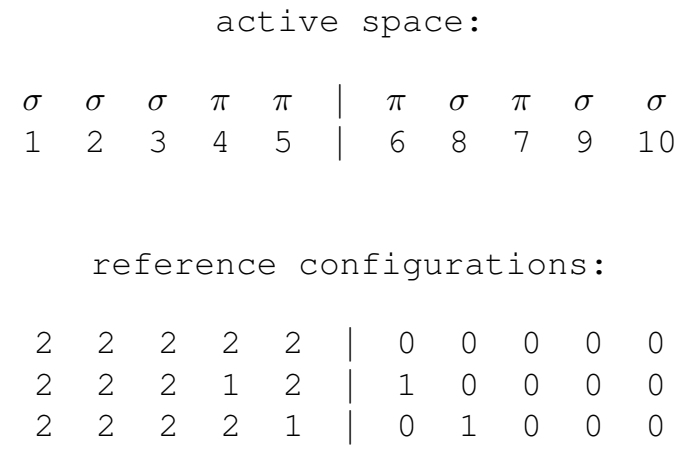

Cartesian coordinates:

4

$\begin{array}{rrrr}\mathrm{C} & -0.67006 & 0.00000 & 0.00000 \\ \mathrm{C} & 0.67006 & 0.00000 & 0.00000 \\ \mathrm{H} & -1.45202 & 0.75794 & 0.00000 \\ \mathrm{H} & 1.45202 & -0.75794 & 0.00000\end{array}$


Table S242 Employed active space, reference configurations, and Cartesian coordinates of the OM3/MRCISD-optimized excited-state equilibrium geometry of the $2{ }^{3} B_{u}\left({ }^{3} \pi \pi^{*}\right)$ state of acetylene (excited-state energy: $-273.33260 \mathrm{eV}$ ).

\begin{tabular}{|c|c|c|c|c|c|c|c|c|c|}
\hline$\sigma$ & $\sigma$ & $\sigma$ & $\pi$ & $\pi$ & $\pi$ & $\pi$ & $\sigma$ & $\sigma$ & $\sigma$ \\
\hline \multirow[t]{2}{*}{1} & 2 & 3 & 4 & 5 & 6 & 7 & 8 & 9 & 10 \\
\hline & \multicolumn{4}{|c|}{ reference } & fic & Ira & io & IS: & \\
\hline 2 & 2 & 2 & 2 & 2 & 0 & 0 & 0 & 0 & 0 \\
\hline 2 & 2 & 2 & 1 & 2 & 1 & 0 & 0 & 0 & 0 \\
\hline 2 & 2 & 2 & 2 & 1 & 0 & 1 & 0 & 0 & 0 \\
\hline
\end{tabular}

Cartesian coordinates:

4

$\begin{array}{rrrr}\mathrm{C} & -0.67239 & 0.00000 & 0.00000 \\ \mathrm{C} & 0.67239 & 0.00000 & 0.00000 \\ \mathrm{H} & -1.62851 & 0.49198 & 0.00000 \\ \mathrm{H} & 1.62851 & -0.49198 & 0.00000\end{array}$


Table S243 Employed active space, reference configurations, and Cartesian coordinates of the OM3/MRCISD-optimized excited-state equilibrium geometry of the $1{ }^{1} A^{\prime \prime}\left({ }^{1} n \pi^{*}\right)$ state of acrolein (excited-state energy: $-769.89773 \mathrm{eV}$ ).

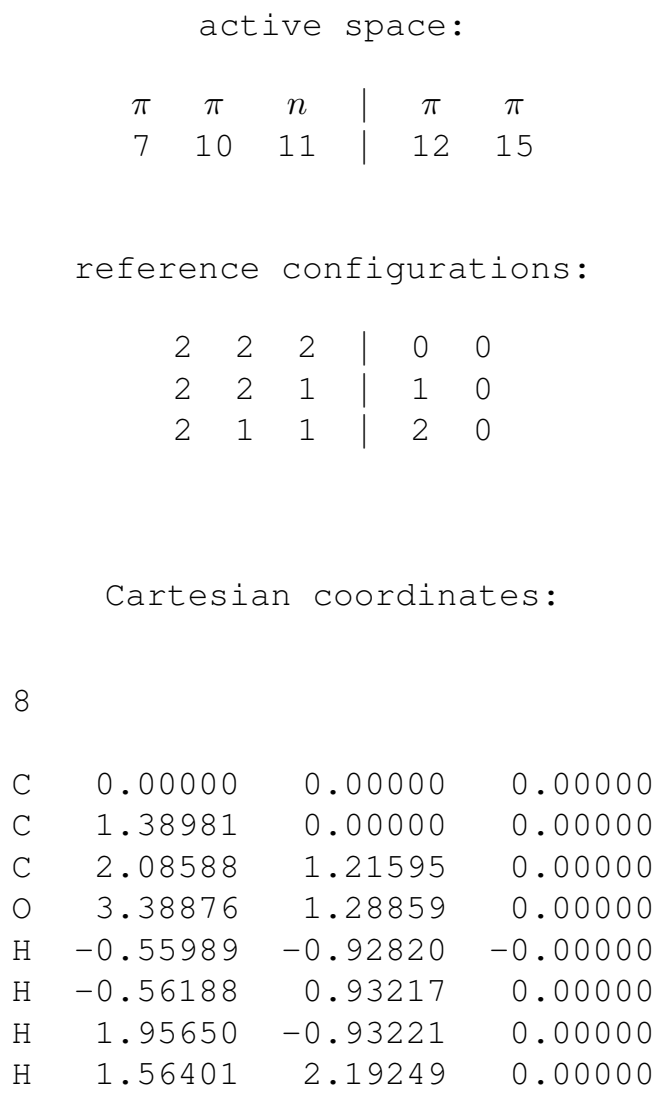


Table S244 Employed active space, reference configurations, and Cartesian coordinates of the OM3/MRCISD-optimized excited-state equilibrium geometry of the $1^{3} A^{\prime \prime}\left({ }^{3} n \pi^{*}\right)$ state of acrolein (excited-state energy: $-770.06702 \mathrm{eV}$ ).

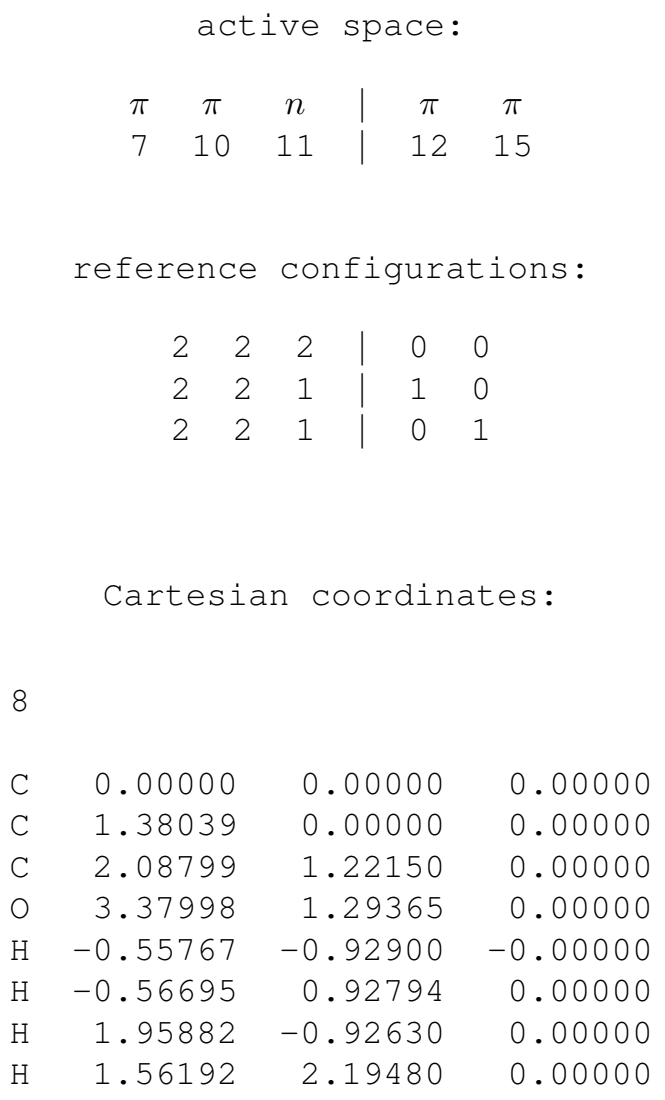


Table S245 Employed active space, reference configurations, and Cartesian coordinates of the OM3/MRCISD-optimized excited-state equilibrium geometry of the $1^{3} A^{\prime}\left({ }^{3} \pi \pi^{*}\right)$ state of acrolein (excited-state energy: $\left.-770.17476 \mathrm{eV}\right)$.

\begin{tabular}{|c|c|c|c|c|}
\hline$\pi$ & $\pi$ & $n$ & $\pi$ & $\pi$ \\
\hline 11 & 7 & 10 & 12 & 15 \\
\hline \multicolumn{2}{|c|}{ reference } & \multicolumn{3}{|c|}{ configurations: } \\
\hline 2 & 2 & 2 & 0 & 0 \\
\hline 2 & 2 & 1 & 1 & 0 \\
\hline 2 & 1 & 2 & 1 & 0 \\
\hline 2 & 2 & 1 & 0 & 1 \\
\hline \multicolumn{5}{|c|}{ Cartesian coordinates: } \\
\hline 0.000 & & & 000 & 0.00000 \\
\hline 1.482 & & & & 0.00000 \\
\hline 2.230 & & & 904 & 0.00000 \\
\hline 3.487 & & & 9482 & 0.00000 \\
\hline-0.549 & & -0 . & 3461 & -0.00000 \\
\hline-0.540 & & & 163 & 0.00000 \\
\hline 2.011 & & -0 . & 5520 & 0.00000 \\
\hline 1.651 & & & 0031 & 0.00000 \\
\hline
\end{tabular}


Table S246 Employed active space, reference configurations, and Cartesian coordinates of the OM3/MRCISD-optimized excited-state equilibrium geometry of the $2{ }^{1} A\left({ }^{1} \pi \pi^{*}\right)$ state of 2-amino-9-methylpurine (excited-state energy: $-1928.61573 \mathrm{eV})$.

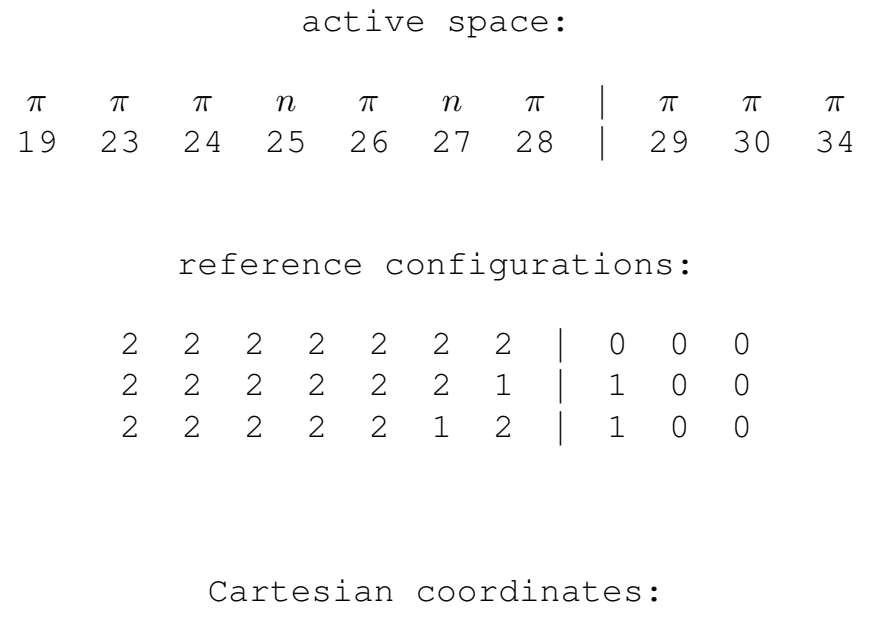

18

$\begin{array}{llll}\text { C } & 0.00000 & 0.00000 & 0.00000\end{array}$

$\mathrm{N} \quad 1.37749 \quad 0.00000 \quad 0.00000$

$\mathrm{N} \quad-0.73980-1.19482 \quad 0.00000$

$\begin{array}{llll}\text { C } & -0.05590 & -2.35594 & 0.03558\end{array}$

$\begin{array}{lrrr}\text { C } & 1.44941 & -2.41403 & 0.13791\end{array}$

C $\quad 2.11235-1.13149-0.10673$

$\begin{array}{llll}\mathrm{N} & -0.49947 & -3.57688 & 0.66764\end{array}$

$\begin{array}{llll}\text { C } & 0.66601 & -4.36909 & 0.82950\end{array}$

$\begin{array}{llll}\mathrm{N} & 1.81510 & -3.62272 & 0.62686\end{array}$

$\begin{array}{llll}\mathrm{N} & -0.69735 & 1.19990 & 0.00747\end{array}$

$\begin{array}{llll}\text { C } & -1.88167 & -4.03567 & 0.74958\end{array}$

$\mathrm{H} \quad 2.94023 \quad-1.15913 \quad-0.84731$

$\begin{array}{llll}\mathrm{H} & 0.64887 & -5.43603 & 1.08303\end{array}$

$\mathrm{H} \quad-1.69836 \quad 1.24163 \quad-0.22786$

$\begin{array}{llll}\mathrm{H} & -0.19921 & 2.10006 & 0.03612\end{array}$

$\begin{array}{llll}\mathrm{H} & -2.56958 & -3.16275 & 0.67959\end{array}$

$\mathrm{H} \quad \begin{array}{rrr}-2.04880 & -4.54346 & 1.72313\end{array}$

$\begin{array}{llll}\mathrm{H} & -2.12307 & -4.73666 & -0.08438\end{array}$ 
Table S247 Employed active space, reference configurations, and Cartesian coordinates of the OM3/MRCISD-optimized excited-state equilibrium geometry of the $1{ }^{1} B_{2}\left({ }^{1} \pi \pi^{*}\right)$ state of aniline (excited-state energy: $-1066.73378 \mathrm{eV}$ ).

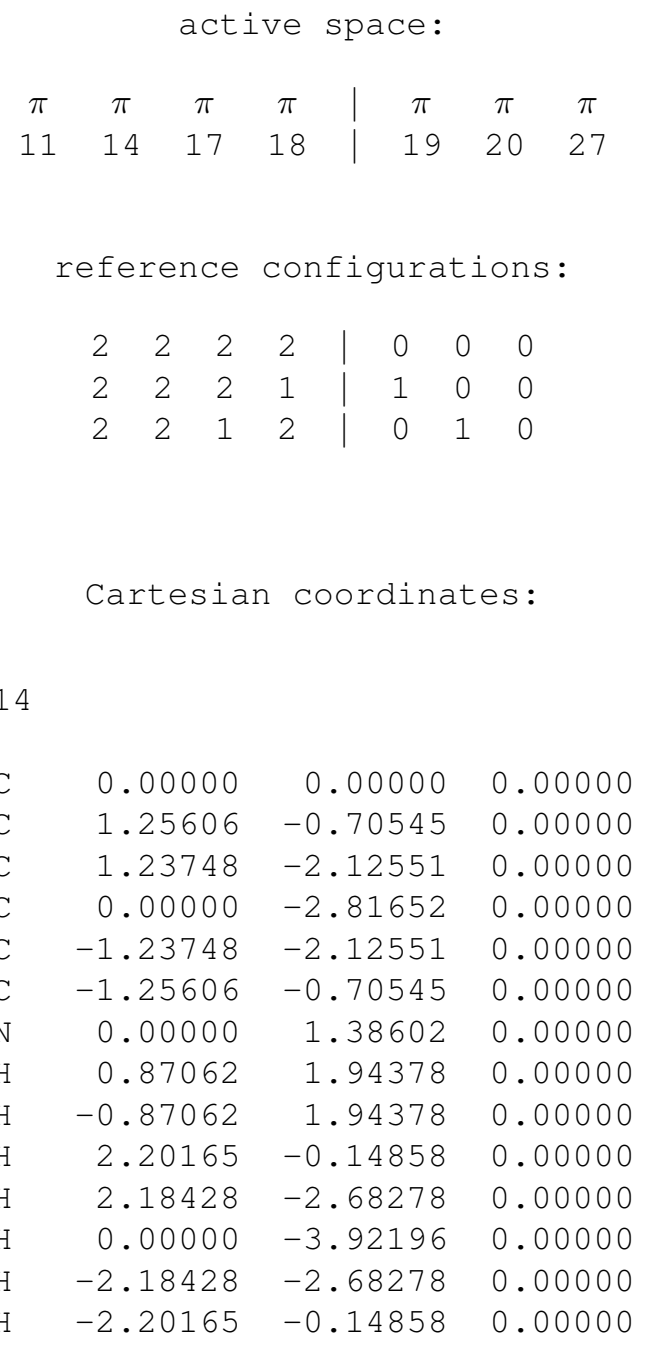


Table S248 Employed active space, reference configurations, and Cartesian coordinates of the OM3/MRCISD-optimized excited-state equilibrium geometry of the $1^{3} A^{\prime}\left({ }^{3} \pi \pi^{*}\right)$ state of aniline (excited-state energy: $\left.-1067.09304 \mathrm{eV}\right)$.

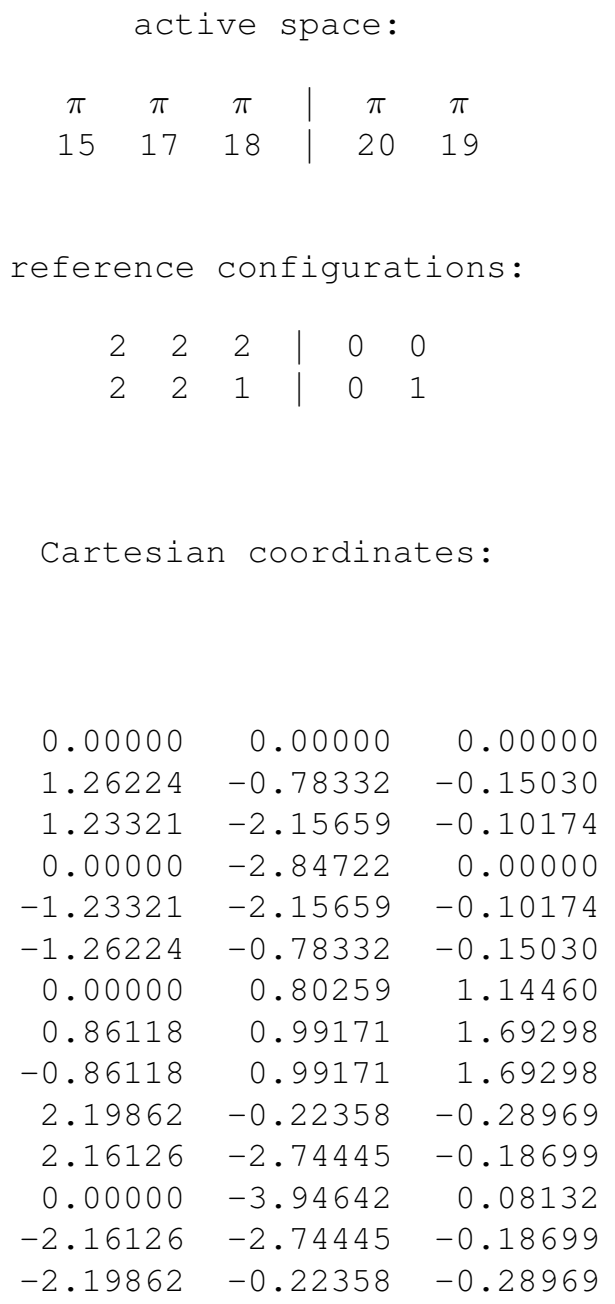


Table S249 Employed active space, reference configurations, and Cartesian coordinates of the OM3/MRCISD-optimized excited-state equilibrium geometry of the $1^{1} A^{\prime \prime}\left({ }^{1} n \pi^{*}\right)$ state of benzaldehyde (excited-state energy: $\left.-1300.30699 \mathrm{eV}\right)$.

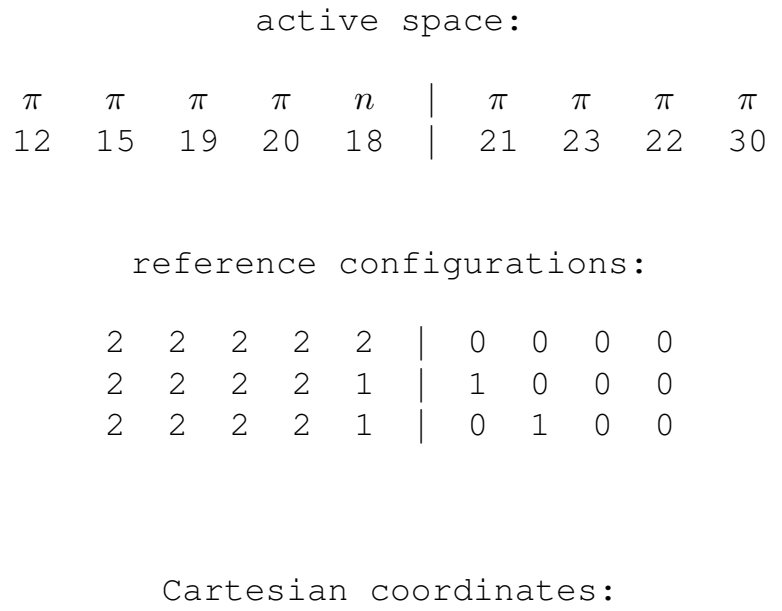


Table S250 Employed active space, reference configurations, and Cartesian coordinates of the OM3/MRCISD-optimized excited-state equilibrium geometry of the $2{ }^{1} A^{\prime}\left({ }^{1} \pi \pi^{*}\right)$ state of benzaldehyde (excited-state energy: $\left.-1299.81519 \mathrm{eV}\right)$.

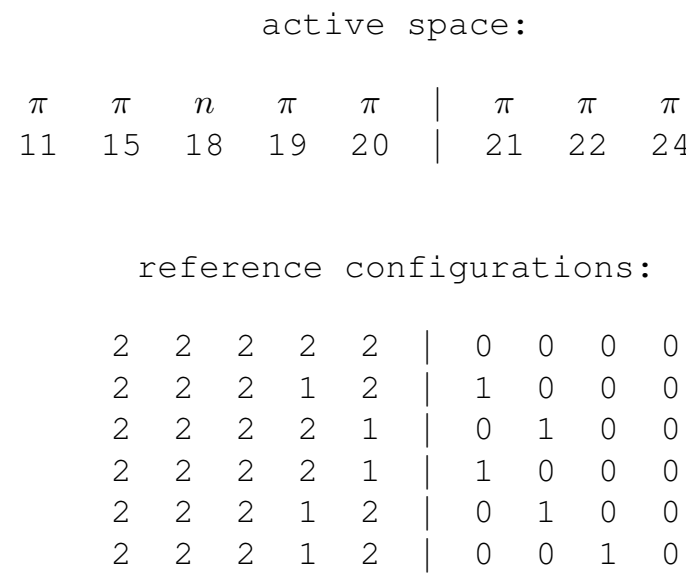

Cartesian coordinates:

14

$\begin{array}{rrrr}\mathrm{C} & -0.31955 & 2.04445 & 0.00000 \\ \mathrm{O} & -1.49419 & 2.43918 & 0.00000 \\ \mathrm{H} & 0.56761 & 2.75704 & 0.00000 \\ \mathrm{C} & 0.00923 & 0.61339 & 0.00000 \\ \mathrm{C} & 1.36781 & 0.16307 & 0.00000 \\ \mathrm{C} & -1.04795 & -0.35697 & 0.00000 \\ \mathrm{C} & 1.66123 & -1.23285 & 0.00000 \\ \mathrm{C} & -0.74141 & -1.74927 & 0.00000 \\ \mathrm{C} & 0.60970 & -2.18720 & 0.00000 \\ \mathrm{H} & 2.18601 & 0.89991 & 0.00000 \\ \mathrm{H} & -2.09053 & -0.01023 & 0.00000 \\ \mathrm{H} & 2.70979 & -1.56889 & 0.00000 \\ \mathrm{H} & -1.55862 & -2.48654 & 0.00000 \\ \mathrm{H} & 0.84156 & -3.26118 & 0.00000\end{array}$


Table S251 Employed active space, reference configurations, and Cartesian coordinates of the OM3/MRCISD-optimized excited-state equilibrium geometry of the $1^{3} A^{\prime \prime}\left({ }^{3} n \pi^{*}\right)$ state of benzaldehyde (excited-state energy: $\left.-1300.53484 \mathrm{eV}\right)$.

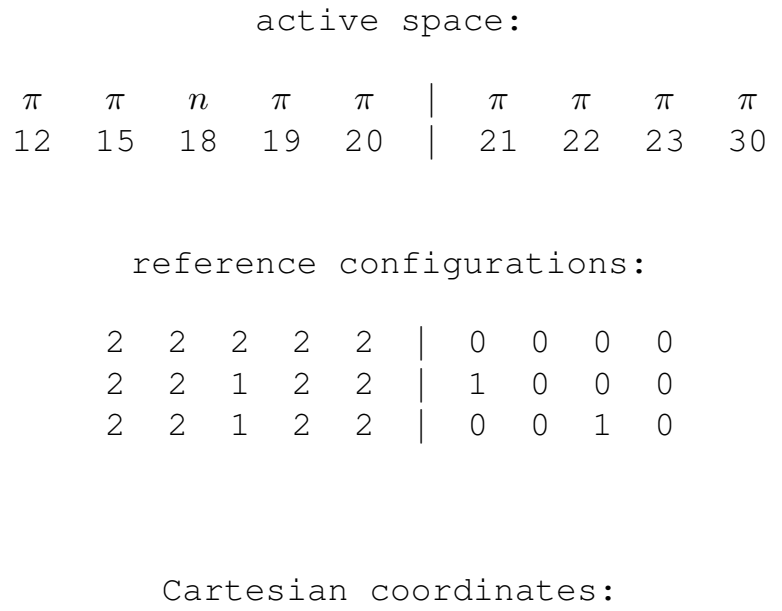


Table S252 Employed active space, reference configurations, and Cartesian coordinates of the OM3/MRCISD-optimized excited-state equilibrium geometry of the $1^{3} A^{\prime}\left({ }^{3} \pi \pi^{*}\right)$ state of benzaldehyde (excited-state energy: $-1300.76538 \mathrm{eV}$ ).

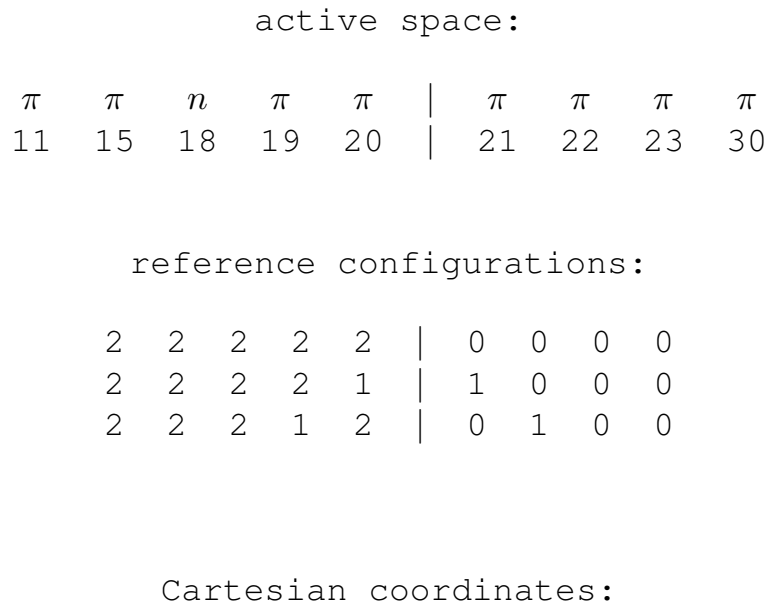


Table S253 Employed active space, reference configurations, and Cartesian coordinates of the OM3/MRCISD-optimized excited-state equilibrium geometry of the $1{ }^{1} A_{u}\left({ }^{1} n \pi^{*}\right)$ state of $p$-benzoquinone (excited-state energy: $-1484.22488 \mathrm{eV})$.

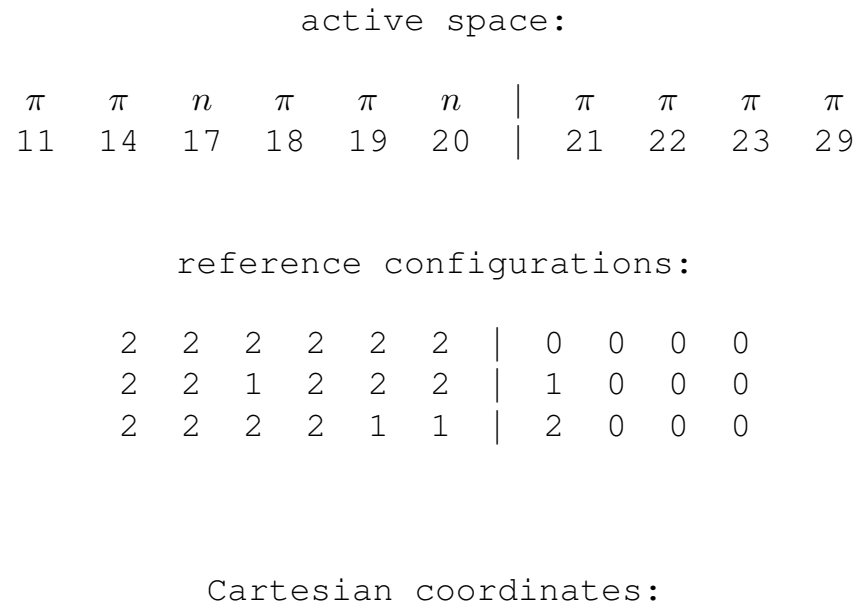


Table S254 Employed active space, reference configurations, and Cartesian coordinates of the OM3/MRCISD-optimized excited-state equilibrium geometry of the $1{ }^{1} B_{1 g}\left({ }^{1} n \pi^{*}\right)$ state of $p$-benzoquinone (excited-state energy: $-1484.74681 \mathrm{eV})$.

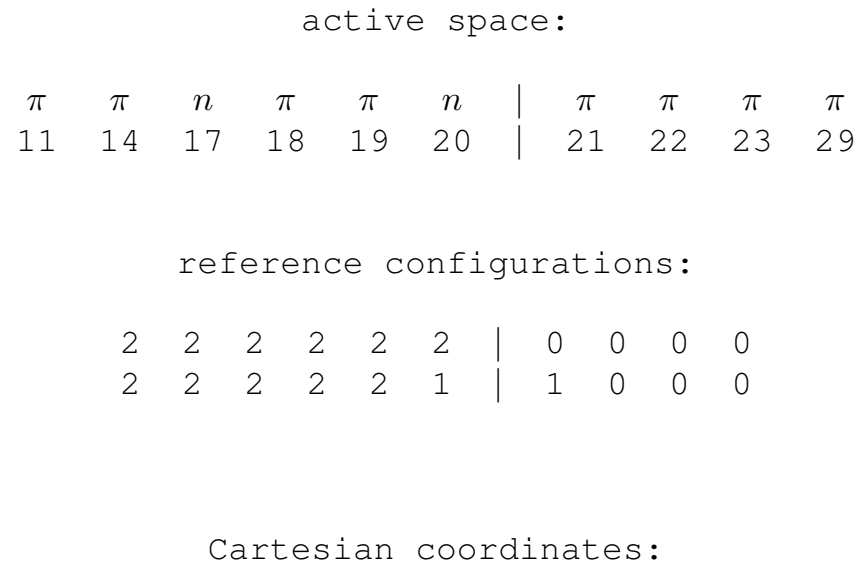


Table S255 Employed active space, reference configurations, and Cartesian coordinates of the OM3/MRCISD-optimized excited-state equilibrium geometry of the $1^{1} B_{1 u}\left({ }^{1} \pi \pi^{*}\right)$ state of $p$-benzoquinone (excited-state energy: $-1481.84750 \mathrm{eV})$.

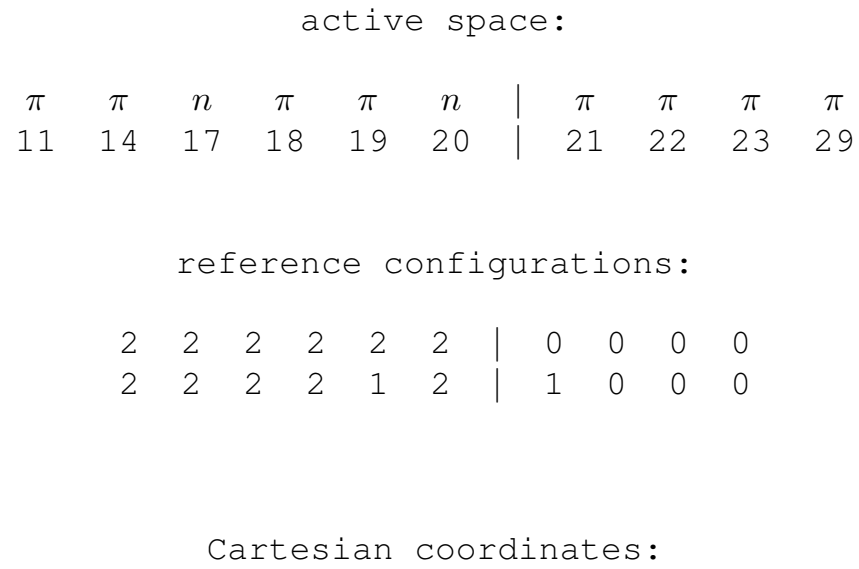


Table S256 Employed active space, reference configurations, and Cartesian coordinates of the OM3/MRCISD-optimized excited-state equilibrium geometry of the $1{ }^{1} B_{3 g}\left({ }^{1} \pi \pi^{*}\right)$ state of $p$-benzoquinone (excited-state energy: $-1483.09168 \mathrm{eV})$.

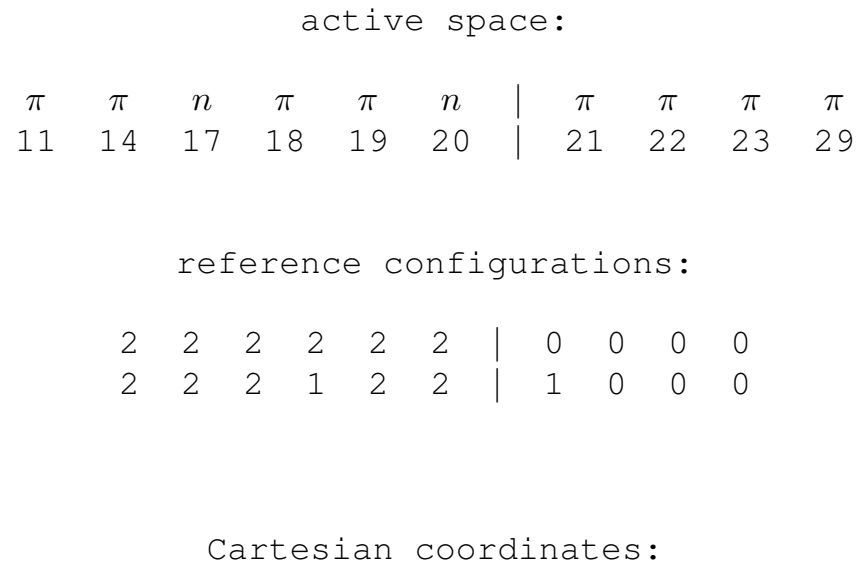


Table S257 Employed active space, reference configurations, and Cartesian coordinates of the OM3/MRCISD-optimized excited-state equilibrium geometry of the $1{ }^{3} A_{u}\left({ }^{3} n \pi^{*}\right)$ state of $p$-benzoquinone (excited-state energy: $-1484.32694 \mathrm{eV})$.

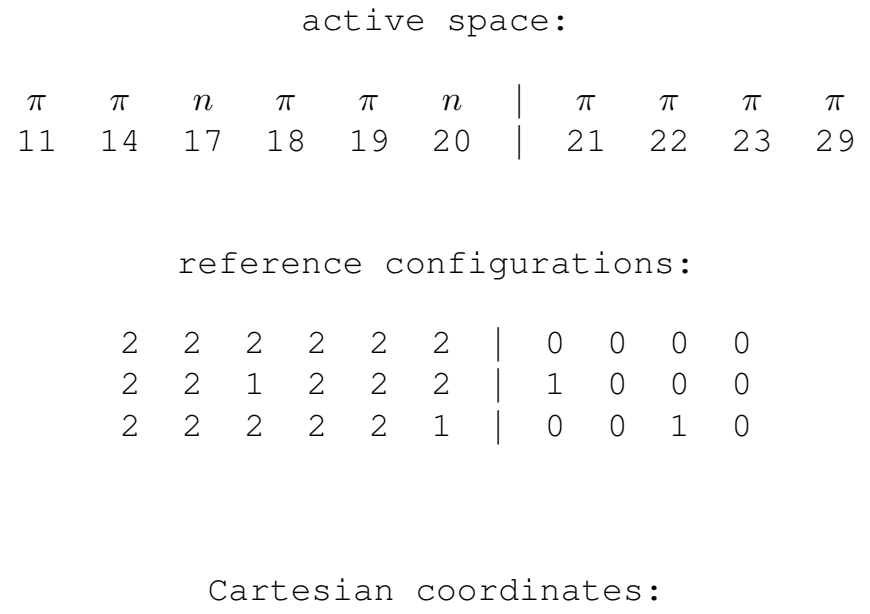


Table S258 Employed active space, reference configurations, and Cartesian coordinates of the OM3/MRCISD-optimized excited-state equilibrium geometry of the $1{ }^{3} B_{1 g}\left({ }^{3} n \pi^{*}\right)$ state of $p$-benzoquinone (excited-state energy: $-1484.89791 \mathrm{eV})$.

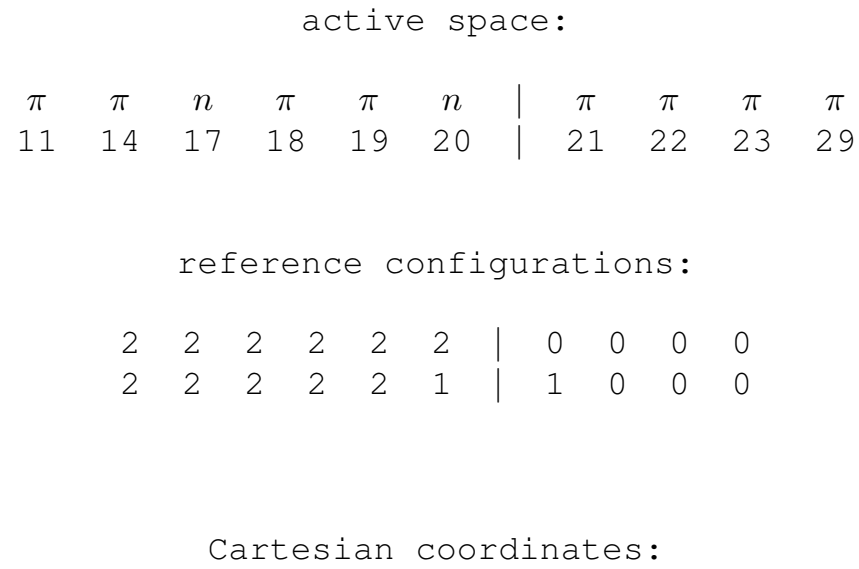


Table S259 Employed active space, reference configurations, and Cartesian coordinates of the OM3/MRCISD-optimized excited-state equilibrium geometry of the $1^{3} B_{1 u}\left({ }^{3} \pi \pi^{*}\right)$ state of $p$-benzoquinone (excited-state energy: $-1484.83269 \mathrm{eV})$.

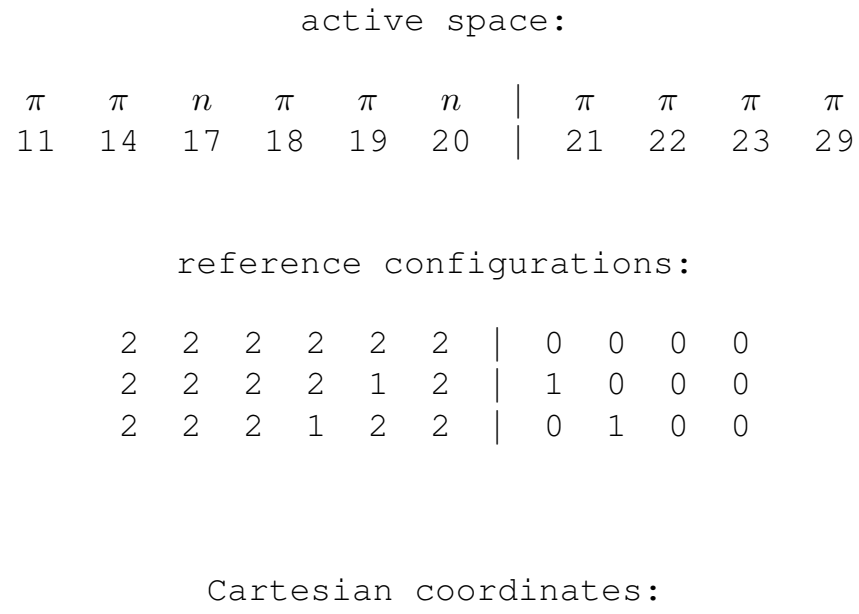

H $\quad 0.00000 \quad 2.18659 \quad 1.27490$ 
Table S260 Employed active space, reference configurations, and Cartesian coordinates of the OM3/MRCISD-optimized excited-state equilibrium geometry of the $1{ }^{3} B_{3 g}\left({ }^{3} \pi \pi^{*}\right)$ state of $p$-benzoquinone (excited-state energy: $-1484.36844 \mathrm{eV})$.

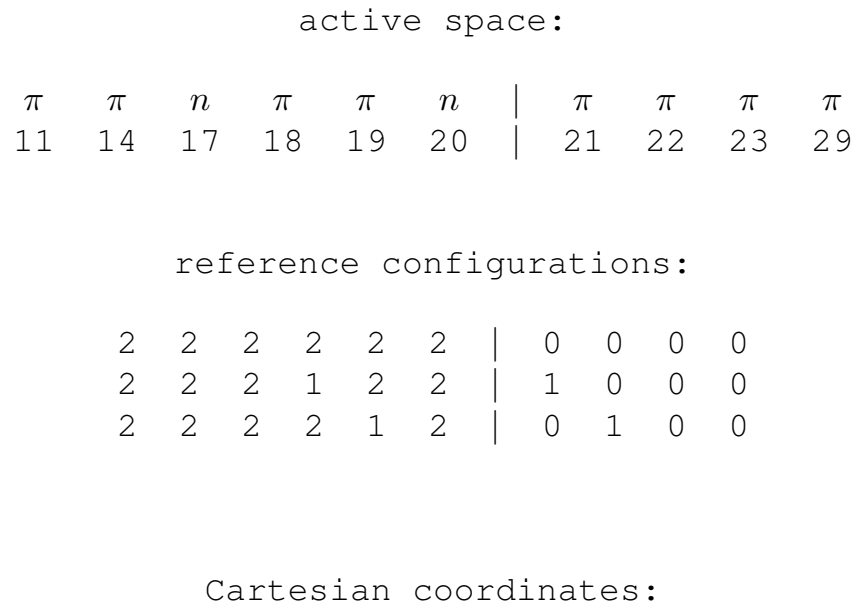

H $0.00000 \quad 2.19422 \quad 1.26910$ 
Table S261 Employed active space, reference configurations, and Cartesian coordinates of the OM3/MRCISD-optimized excited-state equilibrium geometry of the $1{ }^{1} B_{3 g}\left({ }^{1} \pi \pi^{*}\right)$ state of biphenylene (excited-state energy: $-1614.78799 \mathrm{eV}$ ).

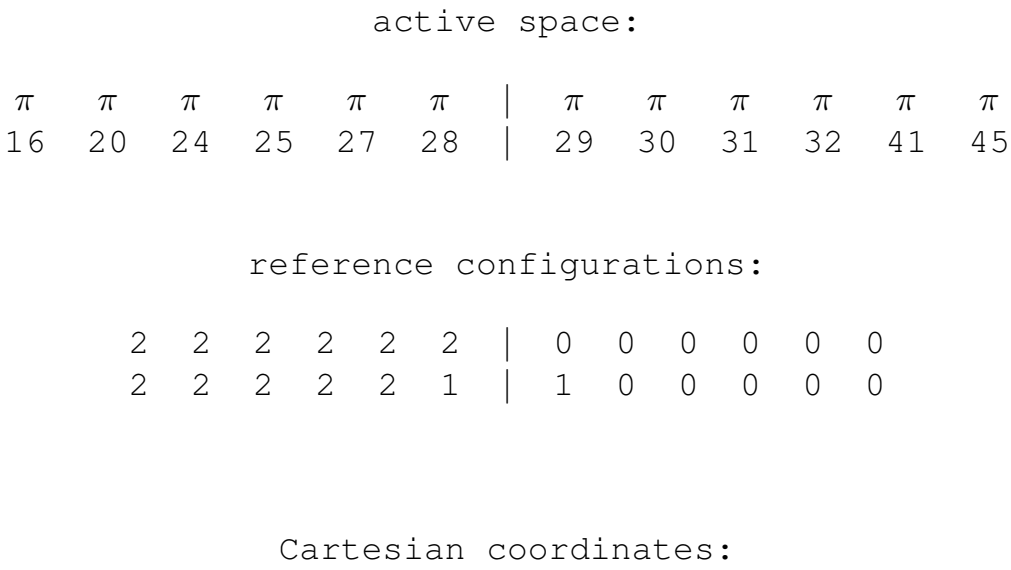


Table S262 Employed active space, reference configurations, and Cartesian coordinates of the OM3/MRCISD-optimized excited-state equilibrium geometry of the $1{ }^{1} B_{1 u}\left({ }^{1} \pi \pi^{*}\right)$ state of biphenylene (excited-state energy: $\left.-1614.29451 \mathrm{eV}\right)$.

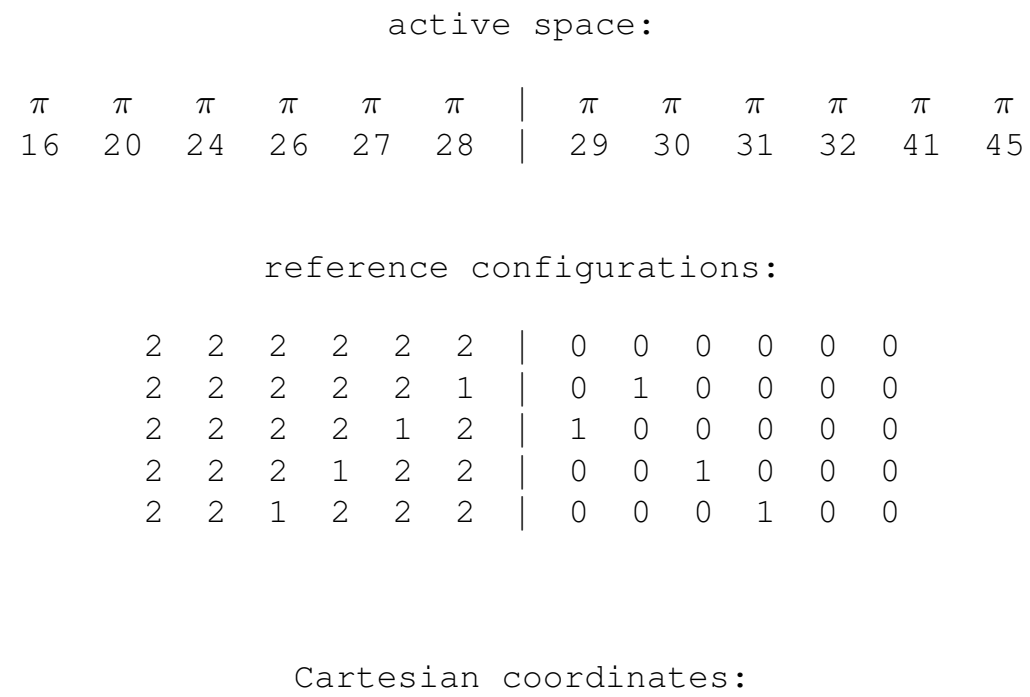

20
0.00000
0.73153
0.73459
1.48023
1.91915
0.70517
3.10817
$0.73153-0.73459$
$-0.73153$
$-0.73459$
$-0.73153$
0.73459
1.48023
$-1.91915$
$-1.48023$
$-1.91915$
$-1.48023$
1.91915
0.70517
$-3.10817$
$-0.70517$
$-3.10817$
$-0.70517$
3.10817
0.00000
2.57497
1.93459
0.00000
2.57497
$-1.93459$
0.00000
$-2.57497$
$-1.93459$
0.00000
$-2.57497$
1.93459
0.00000
1. 23219
4.07784
0.00000
1.23219
$-1.23219$
$-4.07784$
$-1.23219$
$-4.07784$
4.07784

C $\quad 0.00000$

C $\quad 0.00000$

C $\quad 0.00000$

C $\quad 0.00000$

C $\quad 0.00000$

C $\quad 0.00000$

C $\quad 0.00000$

C $\quad 0.00000$

C $\quad 0.00000$

C $\quad 0.00000$

C $\quad 0.00000$

$\begin{array}{ll}\mathrm{H} & 0.00000 \\ \mathrm{H} & 0.00000\end{array}$ 
Table S263 Employed active space, reference configurations, and Cartesian coordinates of the OM3/MRCISD-optimized excited-state equilibrium geometry of the $2{ }^{1} B_{1 u}\left({ }^{1} \pi \pi^{*}\right)$ state of biphenylene (excited-state energy: $-1612.34555 \mathrm{eV}$ ).

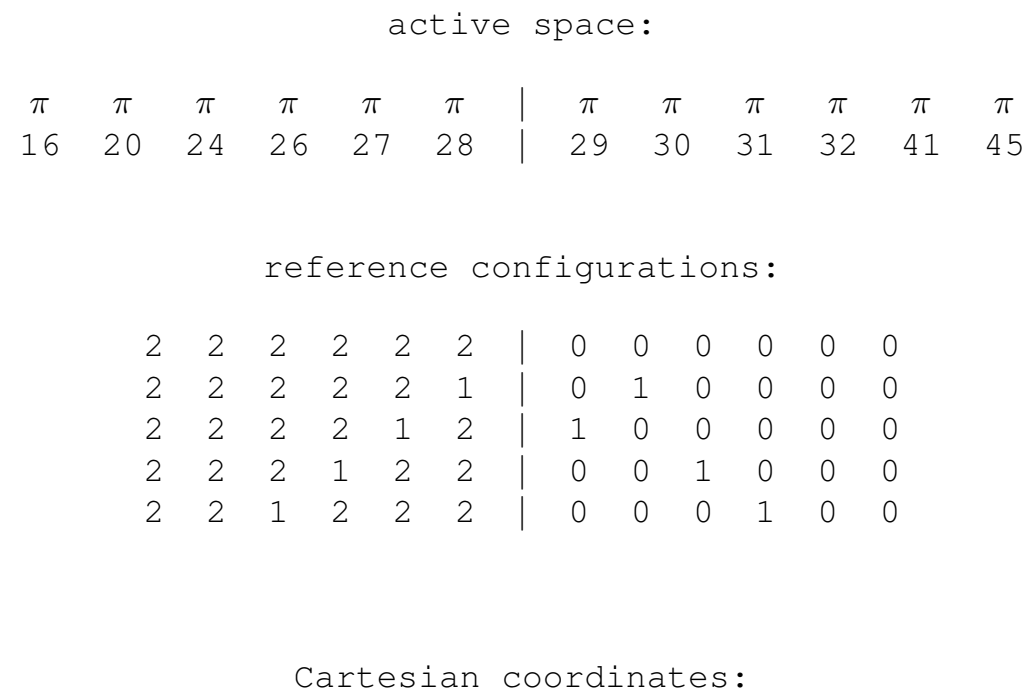

20

0.00000

0.73187

0.73682

C 0.00000

1. 47618

1. 91839

C $\quad 0.00000$

0.69972

3.11514

C 0.00000

$0.73187-0.73682$

C 0.00000

$-0.73187$

$-0.73682$

C 0.00000

$-0.73187$

0.73682

C 0.00000

1.47618

$-1.91839$

0.00000

$-1.47618$

$-1.91839$

0.00000

$-1.47618$

1.91839

0.00000

0.69972

$-3.11514$

C 0.00000

$-0.69972$

$-3.11514$

0.00000

$-0.69972$

3. 11514

0.00000

2.57332

1.93789

0.00000

2.57332

$-1.93789$

0.00000

$-2.57332$

$-1.93789$

0.00000

$-2.57332$

1. 93789

0.00000

1. 23259

4.08046

0.00000

1.23259

$-4.08046$

$\mathrm{H} \quad 0.00000$

$-1.23259$

$-4.08046$

$\mathrm{H}$

\begin{abstract}
0.00000
\end{abstract}
$-1.23259$

4.08046 
Table S264 Employed active space, reference configurations, and Cartesian coordinates of the OM3/MRCISD-optimized excited-state equilibrium geometry of the $1{ }^{1} B_{2 u}\left({ }^{1} \pi \pi^{*}\right)$ state of biphenylene (excited-state energy: $\left.-1613.01017 \mathrm{eV}\right)$.

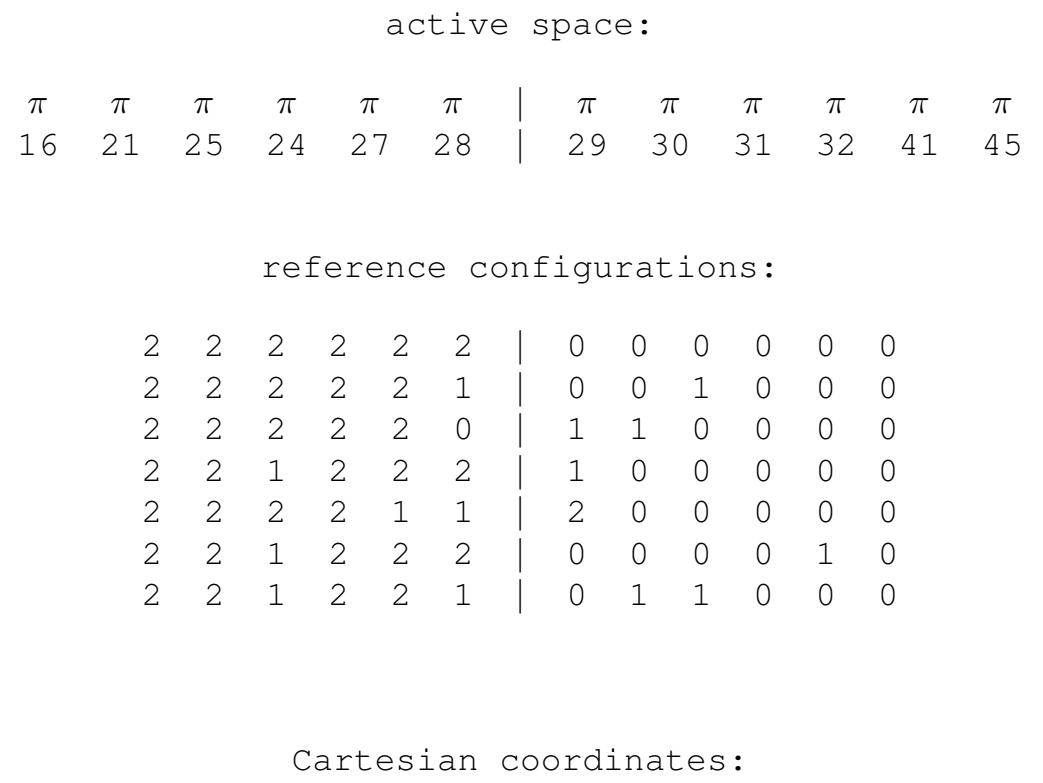

20

\begin{tabular}{lrrr}
$\mathrm{C}$ & 0.00000 & 0.75022 & 0.72146 \\
$\mathrm{C}$ & 0.00000 & 1.48943 & 1.90679 \\
$\mathrm{C}$ & 0.00000 & 0.72504 & 3.08803 \\
$\mathrm{C}$ & 0.00000 & 0.75022 & -0.72146 \\
$\mathrm{C}$ & 0.00000 & -0.75022 & -0.72146 \\
$\mathrm{C}$ & 0.00000 & -0.75022 & 0.72146 \\
$\mathrm{C}$ & 0.00000 & 1.48943 & -1.90679 \\
$\mathrm{C}$ & 0.00000 & -1.48943 & -1.90679 \\
$\mathrm{C}$ & 0.00000 & -1.48943 & 1.90679 \\
$\mathrm{C}$ & 0.00000 & 0.72504 & -3.08803 \\
$\mathrm{C}$ & 0.00000 & -0.72504 & -3.08803 \\
$\mathrm{C}$ & 0.00000 & -0.72504 & 3.08803 \\
$\mathrm{H}$ & 0.00000 & 2.58521 & 1.92053 \\
$\mathrm{H}$ & 0.00000 & 2.58521 & -1.92053 \\
$\mathrm{H}$ & 0.00000 & -2.58521 & -1.92053 \\
$\mathrm{H}$ & 0.00000 & -2.58521 & 1.92053 \\
$\mathrm{H}$ & 0.00000 & 1.23573 & 4.06425 \\
$\mathrm{H}$ & 0.00000 & 1.23573 & -4.06425 \\
$\mathrm{H}$ & 0.00000 & -1.23573 & -4.06425 \\
\hline & & \multicolumn{3}{c}{ Continued on next page }
\end{tabular}

S298 
Continued from previous page

$\begin{array}{llll}\mathrm{H} & 0.00000 & -1.23573 & 4.06425\end{array}$

S299 
Table S265 Employed active space, reference configurations, and Cartesian coordinates of the OM3/MRCISD-optimized excited-state equilibrium geometry of the $1{ }^{1} B_{u}\left({ }^{1} \pi \pi^{*}\right)$ state of trans-butadiene (excited-state energy: $-582.66518 \mathrm{eV})$.

\begin{tabular}{|c|c|c|c|}
\hline$\pi \quad \pi$ & $\pi$ & \multicolumn{2}{|l|}{$\pi$} \\
\hline $\begin{array}{ll}9 & 11\end{array}$ & \begin{tabular}{l|l}
-12 & 12
\end{tabular} & \multicolumn{2}{|l|}{16} \\
\hline reference & \multicolumn{3}{|c|}{ configurations: } \\
\hline 2 & 0 & 0 & \\
\hline 2 & 11 & 0 & \\
\hline \multicolumn{4}{|c|}{ Cartesian coordinates: } \\
\hline 0.00000 & 0.7045 & & 0.00000 \\
\hline 0.00000 & -0.7045 & 55 & 0.00000 \\
\hline 1.18700 & -1.4505 & 52 & 0.00000 \\
\hline-1.18700 & 1.4505 & & 0.00000 \\
\hline 1.17765 & -2.5379 & & 0.00000 \\
\hline-1.17765 & 2.5379 & & 0.00000 \\
\hline 2.15939 & -0.9609 & & 0.00000 \\
\hline-2.15939 & 0.9609 & & 0.00000 \\
\hline-0.96867 & -1.2364 & & 0.00000 \\
\hline 0.96867 & 1.2364 & 49 & 0.00000 \\
\hline
\end{tabular}


Table S266 Employed active space, reference configurations, and Cartesian coordinates of the OM3/MRCISD-optimized excited-state equilibrium geometry of the $2{ }^{1} A_{g}\left({ }^{1} \pi \pi^{*}\right)$ state of trans-butadiene (excited-state energy: $-583.69131 \mathrm{eV})$.

active space:
\begin{tabular}{rc|cc}
$\pi$ & $\pi$ & $\pi$ & $\pi$ \\
10 & 11 & 12 & 16 \\
reference configurations: \\
2 & 2 & 0 & 0 \\
2 & 0 & 2 & 0 \\
2 & 1 & 0 & 1 \\
1 & 2 & 1 & 0 \\
2 & 0 & 0 & 2
\end{tabular}

Cartesian coordinates:

10

$\begin{array}{lrrr}\mathrm{C} & 0.00000 & 0.69989 & 0.00000 \\ \mathrm{C} & 0.00000 & -0.69989 & 0.00000 \\ \mathrm{C} & 1.25980 & -1.45756 & 0.00000 \\ \mathrm{C} & -1.25980 & 1.45756 & 0.00000 \\ \mathrm{H} & 1.25210 & -2.54400 & 0.00000 \\ \mathrm{H} & -1.25210 & 2.54400 & 0.00000 \\ \mathrm{H} & 2.21256 & -0.93349 & 0.00000 \\ \mathrm{H} & -2.21256 & 0.93349 & 0.00000 \\ \mathrm{H} & -0.94539 & -1.26288 & 0.00000 \\ \mathrm{H} & 0.94539 & 1.26288 & 0.00000\end{array}$


Table S267 Employed active space, reference configurations, and Cartesian coordinates of the OM3/MRCISD-optimized excited-state equilibrium geometry of the $1^{1} A^{\prime \prime}\left({ }^{1} n \pi^{*}\right)$ state of cytosine (excited-state energy: $-1556.60699 \mathrm{eV}$ ).

\begin{tabular}{|c|c|c|c|c|c|c|c|}
\hline \multirow{2}{*}{$\begin{array}{c}\pi \\
15\end{array}$} & $\pi$ & $\pi$ & $n$ & \multirow{2}{*}{$\begin{array}{c}\pi \\
21\end{array}$} & \multicolumn{2}{|c|}{$\pi$} & $\pi$ \\
\hline & 17 & 19 & 20 & & \multicolumn{2}{|c|}{22} & 23 \\
\hline \multicolumn{8}{|c|}{ reference configurations: } \\
\hline & 22 & 2 & 2 & 2 & 0 & 0 & 0 \\
\hline & 22 & 2 & 1 & 2 & 1 & 0 & 0 \\
\hline & 22 & 2 & 2 & 1 & 1 & 0 & 0 \\
\hline & 2 & 2 & 1 & 1 & 1 & 1 & 0 \\
\hline & 22 & 2 & 2 & 0 & 1 & 1 & 0 \\
\hline \multicolumn{4}{|c|}{ Cartesian } & \multicolumn{4}{|c|}{ coordinates: } \\
\hline 13 & & & & & & & \\
\hline $\mathrm{N}$ & & 0000 & & 0.0 & 000 & & 00000 \\
\hline C & & 0939 & & 0.0 & 000 & & 00000 \\
\hline $\mathrm{C}$ & & 6058 & & 1.2 & 99 & & 00000 \\
\hline C & & 0452 & & 2.4 & 344 & & 00000 \\
\hline $\mathrm{N}$ & & 8084 & & 2.4 & & & 00000 \\
\hline $\mathrm{C}$ & -0 . & 8858 & & 1.2 & 940 & & 00000 \\
\hline $\mathrm{N}$ & & 9272 & & $-1 \cdot 2$ & 542 & & 00000 \\
\hline 0 & -1 & 2131 & & 1.3 & 265 & & 00000 \\
\hline $\mathrm{H}$ & & 5451 & & 1.1 & 256 & & 00000 \\
\hline $\mathrm{H}$ & & $197 \varepsilon$ & & 3.4 & 360 & & 00000 \\
\hline $\mathrm{H}$ & & 1413 & & -2.1 & 217 & & 00000 \\
\hline $\mathrm{H}$ & & 0906 & & -1.4 & 648 & & 00000 \\
\hline $\mathrm{H}$ & -0 . & 5311 & & 3.3 & & & 00000 \\
\hline
\end{tabular}


Table S268 Employed active space, reference configurations, and Cartesian coordinates of the OM3/MRCISD-optimized excited-state equilibrium geometry of the $2{ }^{1} A\left({ }^{1} \pi \pi^{*}\right)$ state of cytosine (excited-state energy: $\left.-1557.05181 \mathrm{eV}\right)$.

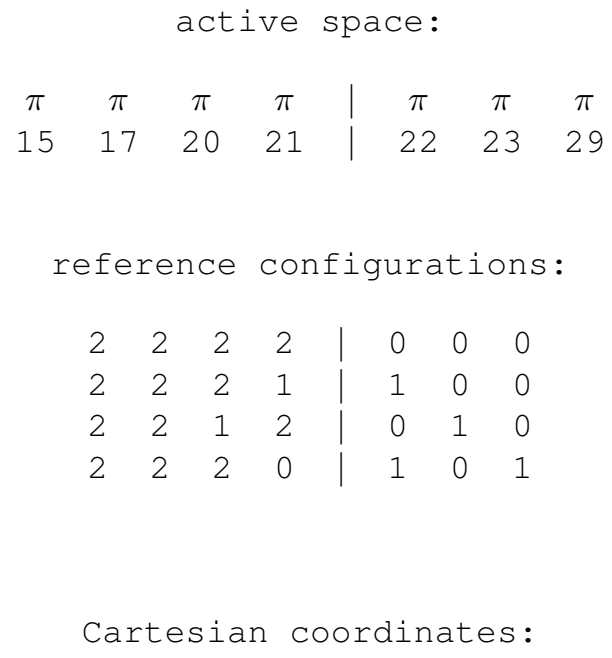

13

$\begin{array}{rrrr}\mathrm{N} & 0.00000 & 0.00000 & 0.00000 \\ \mathrm{C} & 1.40550 & 0.00000 & 0.00000 \\ \mathrm{C} & 2.16540 & 1.19890 & 0.00000 \\ \mathrm{C} & 1.49280 & 2.47432 & -0.00000 \\ \mathrm{~N} & 0.11330 & 2.46521 & -0.00002 \\ \mathrm{C} & -0.66052 & 1.19762 & -0.00000 \\ \mathrm{~N} & 2.00135 & -1.26202 & -0.00001 \\ \mathrm{O} & -1.91300 & 1.39261 & 0.00001 \\ \mathrm{H} & 3.25939 & 1.17765 & 0.00003 \\ \mathrm{H} & 2.05604 & 3.42087 & 0.00003 \\ \mathrm{H} & 1.42579 & -2.11330 & -0.00000 \\ \mathrm{H} & 3.01881 & -1.40366 & -0.00002 \\ \mathrm{H} & -0.42400 & 3.36553 & -0.00000\end{array}$


Table S269 Employed active space, reference configurations, and Cartesian coordinates of the OM3/MRCISD-optimized excited-state equilibrium geometry of the $1{ }^{1} A_{2}\left({ }^{1} n \pi^{*}\right)$ state of diazomethane (excited-state energy: $-583.53510 \mathrm{eV}$ ).

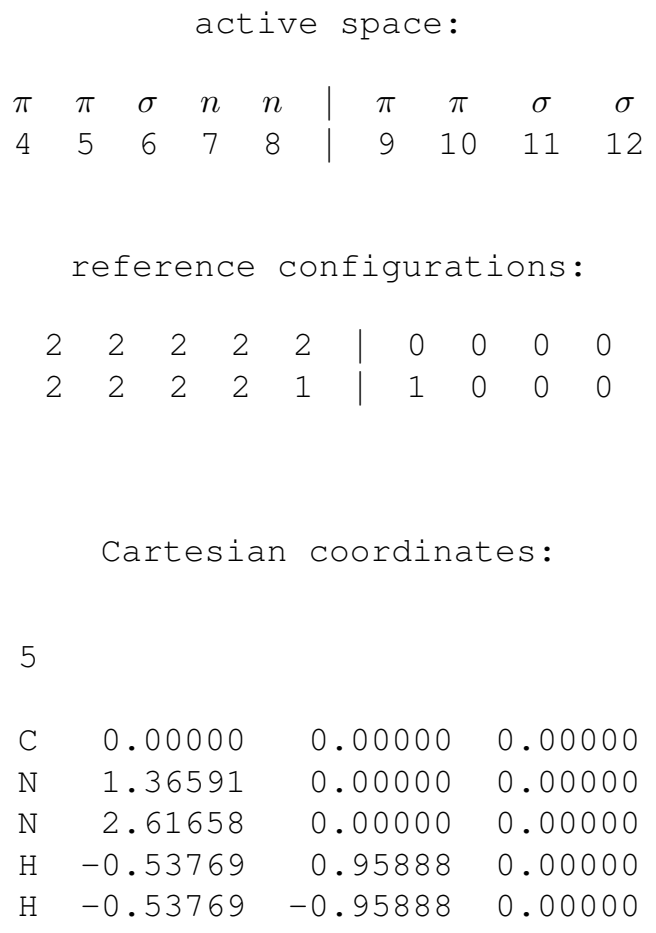


Table S270 Employed active space, reference configurations, and Cartesian coordinates of the OM3/MRCISD-optimized excited-state equilibrium geometry of the $1{ }^{1} B_{3 g}\left({ }^{1} \pi \pi^{*}\right)$ state of dibenzo- $p$-dioxin (excited-state energy: $-2292.83741 \mathrm{eV})$.

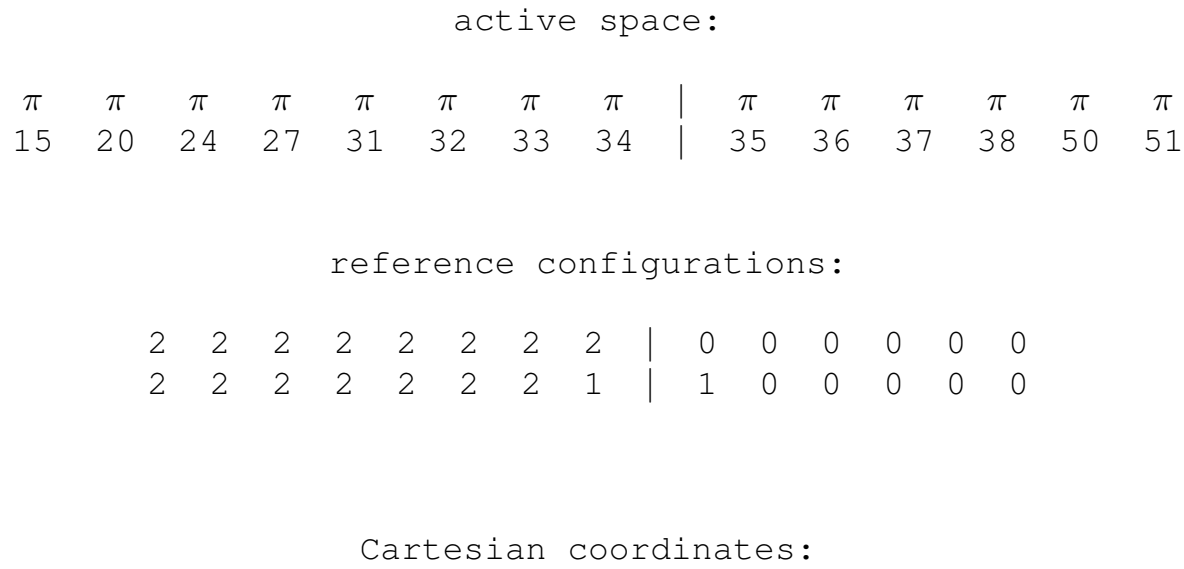


Table S271 Employed active space, reference configurations, and Cartesian coordinates of the OM3/MRCISD-optimized excited-state equilibrium geometry of the $1{ }^{1} B_{2 u}\left({ }^{1} \pi \pi^{*}\right)$ state of dibenzo- $p$-dioxin (excited-state energy: $-2293.05608 \mathrm{eV})$.

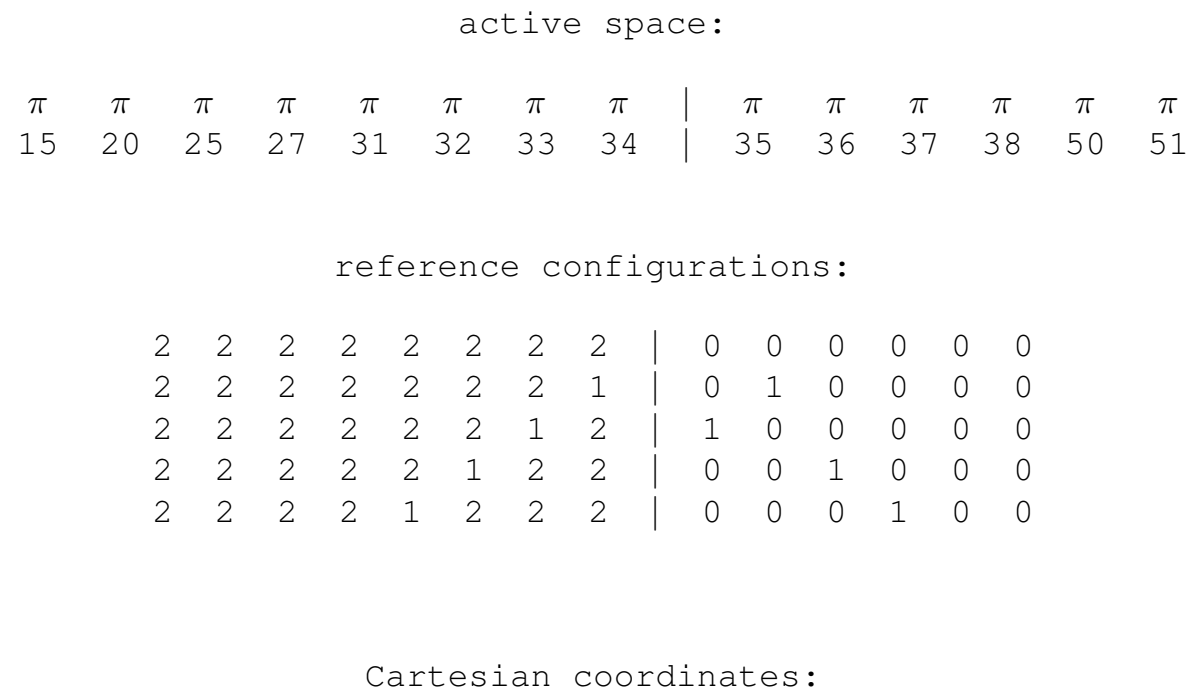

$$
22
$$$$
\text { C } \quad 0.00000 \quad-3.58541
$$$$
\begin{array}{llll}
\text { C } & 0.00000 & -3.58541 & -0.70506
\end{array}
$$$$
\text { C } \quad 0.00000 \quad-2.38296-1.43684
$$$$
\text { C } \quad 0.00000 \quad-1.16295 \quad-0.71314
$$$$
\begin{array}{llll}
\text { C } & 0.00000 & -1.16295 & 0.71314
\end{array}
$$$$
\text { C } \quad 0.00000 \quad-2.38296 \quad 1.43684
$$$$
\begin{array}{llll}
0 & 0.00000 & 0.00000 & -1.46644
\end{array}
$$$$
\begin{array}{llll}
\text { C } & 0.00000 & 1.16295 & -0.71314
\end{array}
$$$$
\text { C } \quad 0.00000 \quad 1.16295 \quad 0.71314
$$$$
\begin{array}{llll}
0 & 0.00000 & 0.00000 & 1.46644
\end{array}
$$$$
\begin{array}{llll}
\text { C } & 0.00000 & 2.38296 & -1.43684
\end{array}
$$$$
\begin{array}{llll}
\text { C } & 0.00000 & 3.58541 & -0.70506
\end{array}
$$$$
\begin{array}{llll}
\text { C } & 0.00000 & 3.58541 & 0.70506
\end{array}
$$$$
\text { C } \quad 0.00000 \quad 2.38296 \quad 1.43684
$$$$
\mathrm{H} \quad 0.00000 \quad 4.54270 \quad 1.24938
$$$$
\mathrm{H} \quad 0.00000 \quad-4.54270-1.24938
$$$$
\mathrm{H} \quad 0.00000 \quad-2.36179 \quad-2.52970
$$$$
\mathrm{H} \quad 0.00000 \quad-2.36179 \quad 2.52970
$$$$
\begin{array}{llll}
\mathrm{H} & 0.00000 & 2.36179 & -2.52970
\end{array}
$$$$
\mathrm{H} \quad 0.00000 \quad 4.54270 \quad-1.24938
$$

Continued on next page

S306 
Continued from previous page

\begin{tabular}{rrrr}
\hline $\mathrm{H}$ & 0.00000 & -4.54270 & 1.24938 \\
$\mathrm{H}$ & 0.00000 & 2.36179 & 2.52970
\end{tabular}

S307 
Table S272 Employed active space, reference configurations, and Cartesian coordinates of the OM3/MRCISD-optimized excited-state equilibrium geometry of the $1{ }^{1} B_{1 u}\left({ }^{1} \pi \pi^{*}\right)$ state of dibenzo- $p$-dioxin (excited-state energy: $-2291.56209 \mathrm{eV})$.

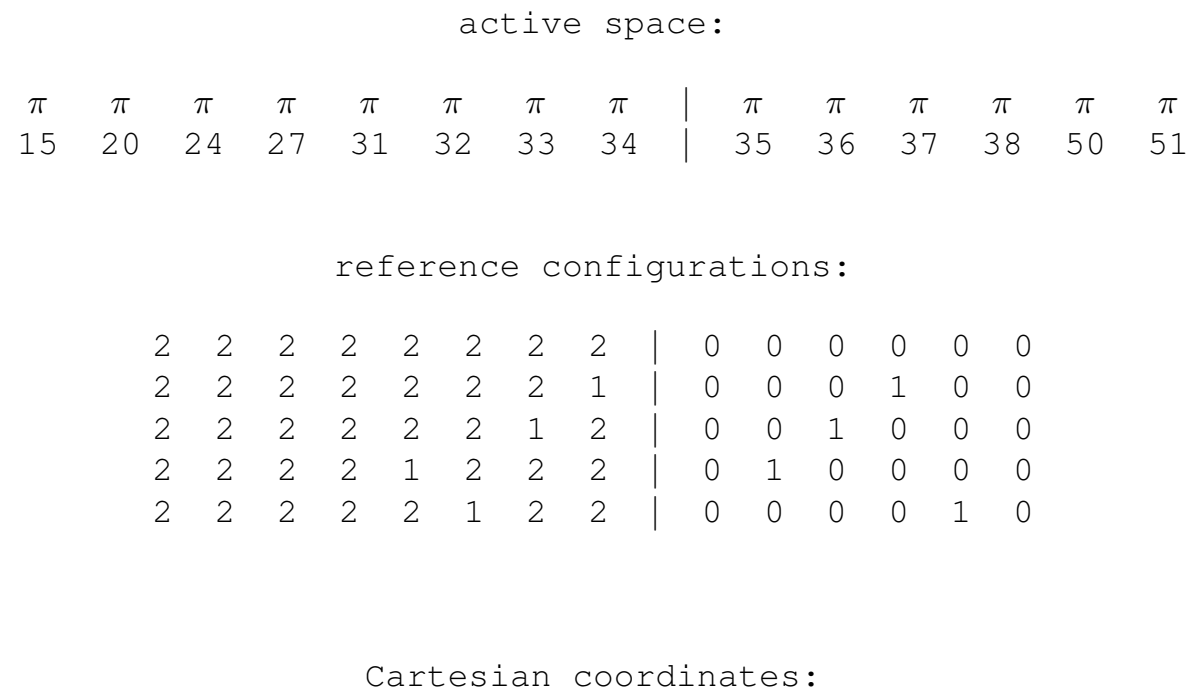

$$
22
$$$$
\text { C } \quad 0.00000 \quad-3.58130
$$$$
\text { C } \quad 0.00000 \quad-3.58130-0.71134
$$$$
\text { C } \quad 0.00000 \quad-2.38052 \quad-1.43102
$$$$
\text { C } \quad 0.00000 \quad-1.16433 \quad-0.71537
$$$$
\begin{array}{llll}
\text { C } & 0.00000 & -1.16433 & 0.71537
\end{array}
$$$$
\text { C } 0.00000 \quad-2.38052 \quad 1.43102
$$$$
\begin{array}{llll}
0 & 0.00000 & 0.00000 & -1.47138
\end{array}
$$$$
\begin{array}{llll}
\text { C } & 0.00000 & 1.16433 & -0.71537
\end{array}
$$$$
\begin{array}{llll}
\text { C } & 0.00000 & 1.16433 & 0.71537
\end{array}
$$$$
\begin{array}{llll}
0 & 0.00000 & 0.00000 & 1.47138
\end{array}
$$$$
\begin{array}{llll}
\text { C } & 0.00000 & 2.38052 & -1.43102
\end{array}
$$$$
\begin{array}{llll}
\text { C } & 0.00000 & 3.58130 & -0.71134
\end{array}
$$$$
\begin{array}{llll}
\text { C } & 0.00000 & 3.58130 & 0.71134
\end{array}
$$$$
\text { C } \quad 0.00000 \quad 2.38052 \quad 1.43102
$$$$
\text { H } \quad 0.00000 \quad 4.53818 \quad 1.25091
$$$$
\mathrm{H} \quad 0.00000 \quad-4.53818 \quad-1.25091
$$$$
\text { H } \quad 0.00000 \quad-2.35721 \quad-2.52694
$$$$
\mathrm{H} \quad 0.00000 \quad-2.35721 \quad 2.52694
$$$$
\text { H } \quad 0.00000 \quad 2.35721 \quad-2.52694
$$$$
\mathrm{H} \quad 0.00000 \quad 4.53818 \quad-1.25091
$$

Continued on next page

\section{S308}


Continued from previous page

\begin{tabular}{rrrr}
\hline $\mathrm{H}$ & 0.00000 & -4.53818 & 1.25091 \\
$\mathrm{H}$ & 0.00000 & 2.35721 & 2.52694
\end{tabular}

S309 
Table S273 Employed active space, reference configurations, and Cartesian coordinates of the OM3/MRCISD-optimized excited-state equilibrium geometry of the $1^{3} A_{g}\left({ }^{3} \pi \pi^{*}\right)$ state of dibenzo- $p$-dioxin (excited-state energy: $-2293.02192 \mathrm{eV})$.

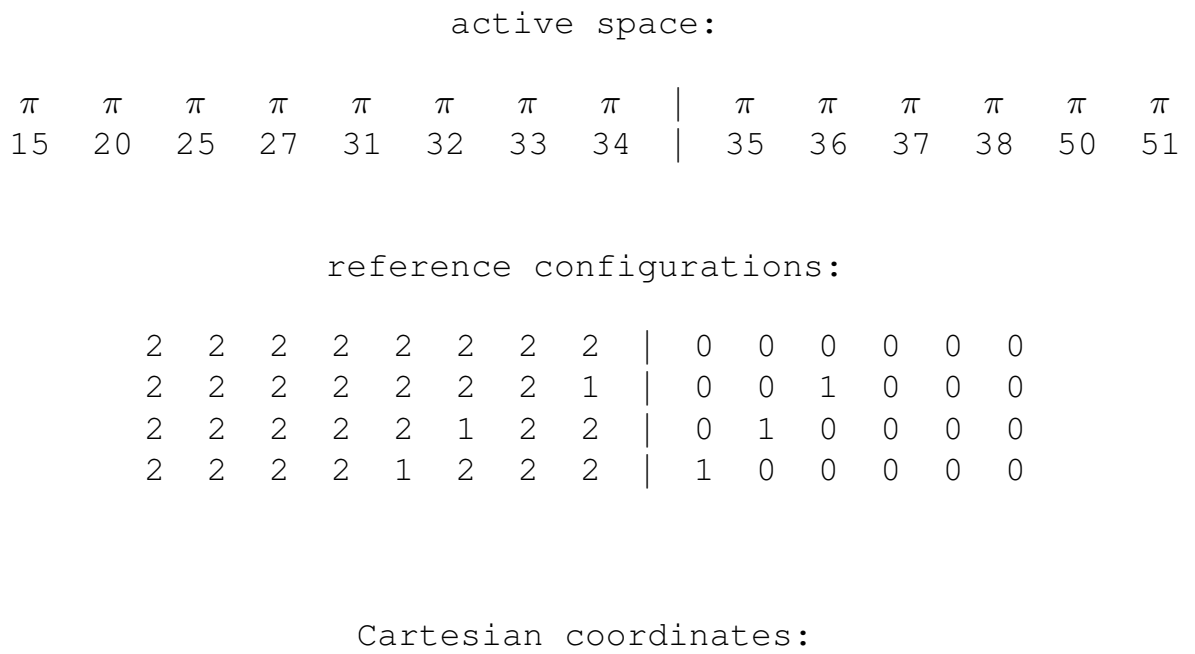

$$
22
$$$$
\begin{array}{llll}
\text { C } & 0.00000 & -3.57963 & 0.70311
\end{array}
$$$$
\begin{array}{llll}
\text { C } & 0.00000 & -3.57963 & -0.70311
\end{array}
$$$$
\begin{array}{llll}
\text { C } & 0.00000 & -2.37959 & -1.43897
\end{array}
$$$$
\text { C } \quad 0.00000 \quad-1.16296 \quad-0.71604
$$$$
\text { C } \quad 0.00000-1.16296 \quad 0.71604
$$$$
\text { C } \quad 0.00000 \quad-2.37959 \quad 1.43897
$$$$
\text { O } 0.00000 \quad 0.00000-1.46955
$$$$
\text { C } \quad 0.00000 \quad 1.16296-0.71604
$$$$
\begin{array}{llll}
\text { C } & 0.00000 & 1.16296 & 0.71604
\end{array}
$$$$
\begin{array}{llll}
0 & 0.00000 & 0.00000 & 1.46955
\end{array}
$$$$
\begin{array}{llll}
\text { C } & 0.00000 & 2.37959 & -1.43897
\end{array}
$$$$
\begin{array}{llll}
\text { C } & 0.00000 & 3.57963 & -0.70311
\end{array}
$$$$
\begin{array}{llll}
\text { C } & 0.00000 & 3.57963 & 0.70311
\end{array}
$$$$
\begin{array}{llll}
\text { C } & 0.00000 & 2.37959 & 1.43897
\end{array}
$$$$
\mathrm{H} \quad 0.00000 \quad 4.53750 \quad 1.24833
$$$$
\mathrm{H} \quad 0.00000 \quad-4.53750 \quad-1.24833
$$$$
\mathrm{H} \quad 0.00000 \quad-2.36066 \quad-2.52958
$$$$
\text { H } \quad 0.00000 \quad-2.36066 \quad 2.52958
$$$$
\mathrm{H} \quad 0.00000 \quad 2.36066 \quad-2.52958
$$$$
\mathrm{H} \quad 0.00000 \quad 4.53750 \quad-1.24833
$$$$
\begin{array}{llll}
\mathrm{H} & 0.00000 & -4.53750 & 1.24833 \\
\hline
\end{array}
$$

Continued on next page

S310 
Continued from previous page

$\begin{array}{llll}\mathrm{H} & 0.00000 & 2.36066 & 2.52958\end{array}$

S311 
Table S274 Employed active space, reference configurations, and Cartesian coordinates of the OM3/MRCISD-optimized excited-state equilibrium geometry of the $1{ }^{3} B_{3 g}\left({ }^{3} \pi \pi^{*}\right)$ state of dibenzo-p-dioxin (excited-state energy: $-2294.01829 \mathrm{eV})$.

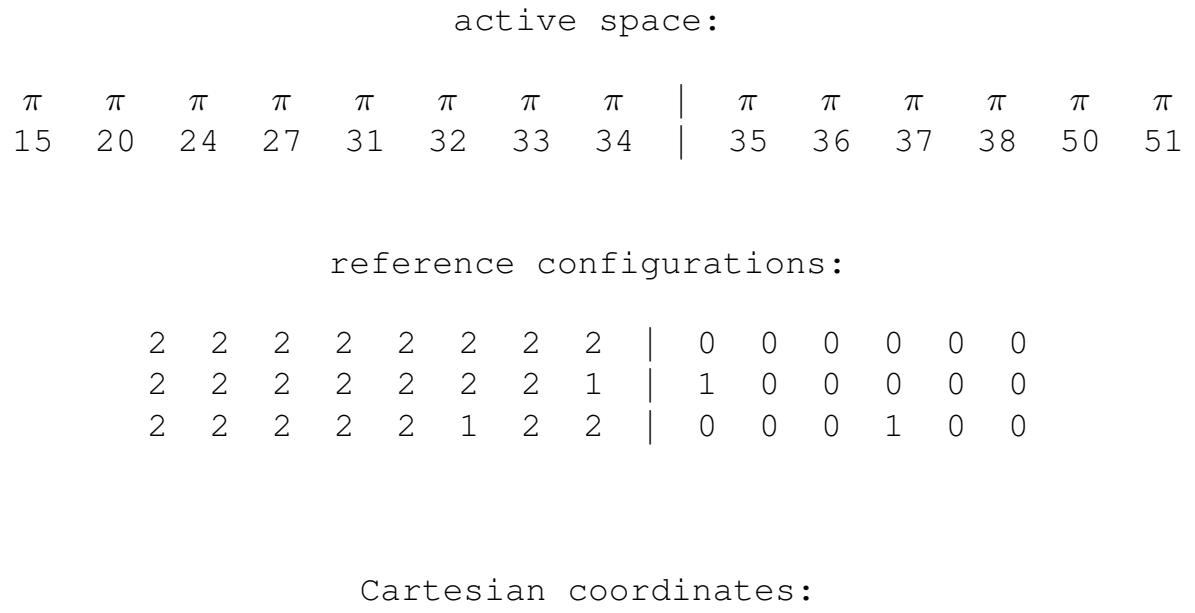


Table S275 Employed active space, reference configurations, and Cartesian coordinates of the OM3/MRCISD-optimized excited-state equilibrium geometry of the $1^{3} B_{2 u}\left({ }^{3} \pi \pi^{*}\right)$ state of dibenzo- $p$-dioxin (excited-state energy: $-2293.13631 \mathrm{eV})$.

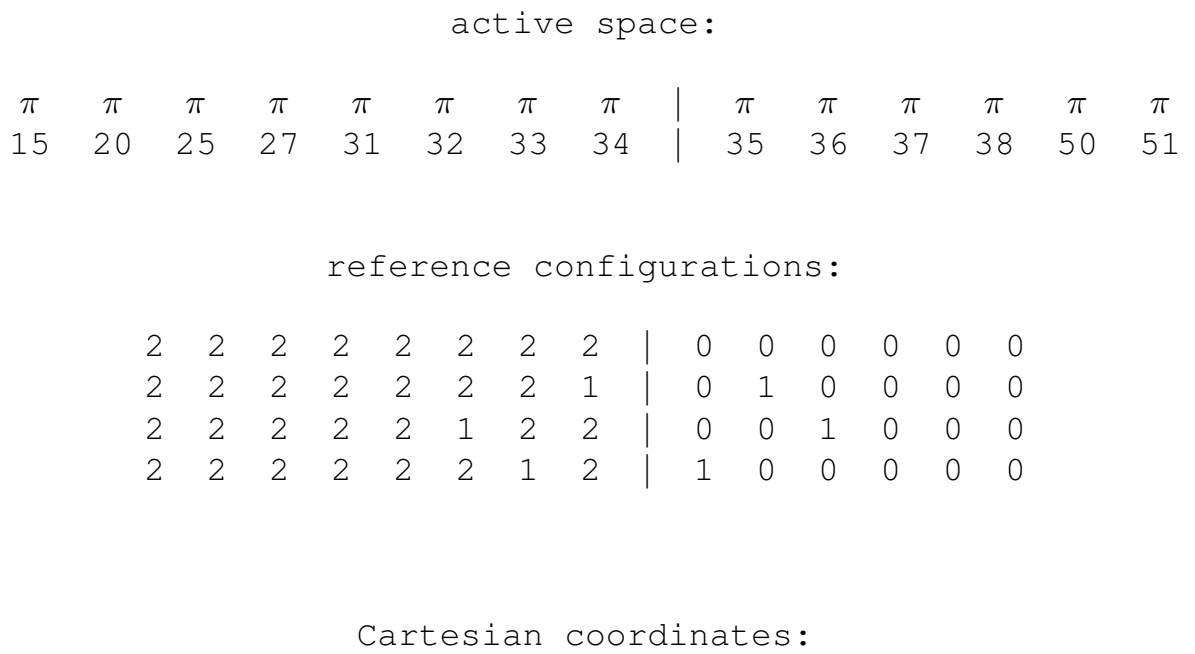

$$
22
$$$$
\begin{array}{llll}
\text { C } & 0.00000 & -3.58103 & 0.70173
\end{array}
$$$$
\text { C } \quad 0.00000 \quad-3.58103 \quad-0.70173
$$$$
\text { C } \quad 0.00000 \quad-2.37976 \quad-1.44011
$$$$
\text { C } \quad 0.00000 \quad-1.16498 \quad-0.71794
$$$$
\text { C } \quad 0.00000 \quad-1.16498 \quad 0.71794
$$$$
\begin{array}{llll}
\text { C } & 0.00000 & -2.37976 & 1.44011
\end{array}
$$$$
\text { O } 0.00000 \quad 0.00000-1.46246
$$$$
\begin{array}{llll}
\text { C } & 0.00000 & 1.16498 & -0.71794
\end{array}
$$$$
\begin{array}{llll}
\text { C } & 0.00000 & 1.16498 & 0.71794
\end{array}
$$$$
\begin{array}{llll}
0 & 0.00000 & 0.00000 & 1.46246
\end{array}
$$$$
\begin{array}{llll}
\text { C } & 0.00000 & 2.37976 & -1.44011
\end{array}
$$$$
\begin{array}{llll}
\text { C } & 0.00000 & 3.58103 & -0.70173
\end{array}
$$$$
\begin{array}{llll}
\text { C } & 0.00000 & 3.58103 & 0.70173
\end{array}
$$$$
\begin{array}{llll}
\text { C } & 0.00000 & 2.37976 & 1.44011
\end{array}
$$$$
\mathrm{H} \quad 0.00000 \quad 4.53808 \quad 1.24829
$$$$
\text { H } \quad 0.00000 \quad-4.53808-1.24829
$$$$
\mathrm{H} \quad 0.00000 \quad-2.36324 \quad-2.53058
$$$$
\text { H } \quad 0.00000 \quad-2.36324 \quad 2.53058
$$$$
\text { H } \quad 0.00000 \quad 2.36324 \quad-2.53058
$$$$
\mathrm{H} \quad 0.00000 \quad 4.53808 \quad-1.24829
$$$$
\mathrm{H} \quad 0.00000 \quad-4.53808 \quad 1.24829
$$

Continued on next page

S313 
Continued from previous page

$\begin{array}{llll}\mathrm{H} & 0.00000 & 2.36324 & 2.53058\end{array}$

S314 
Table S276 Employed active space, reference configurations, and Cartesian coordinates of the OM3/MRCISD-optimized excited-state equilibrium geometry of the $1^{3} B_{1 u}\left({ }^{3} \pi \pi^{*}\right)$ state of dibenzo- $p$-dioxin (excited-state energy: $-2293.61247 \mathrm{eV})$.

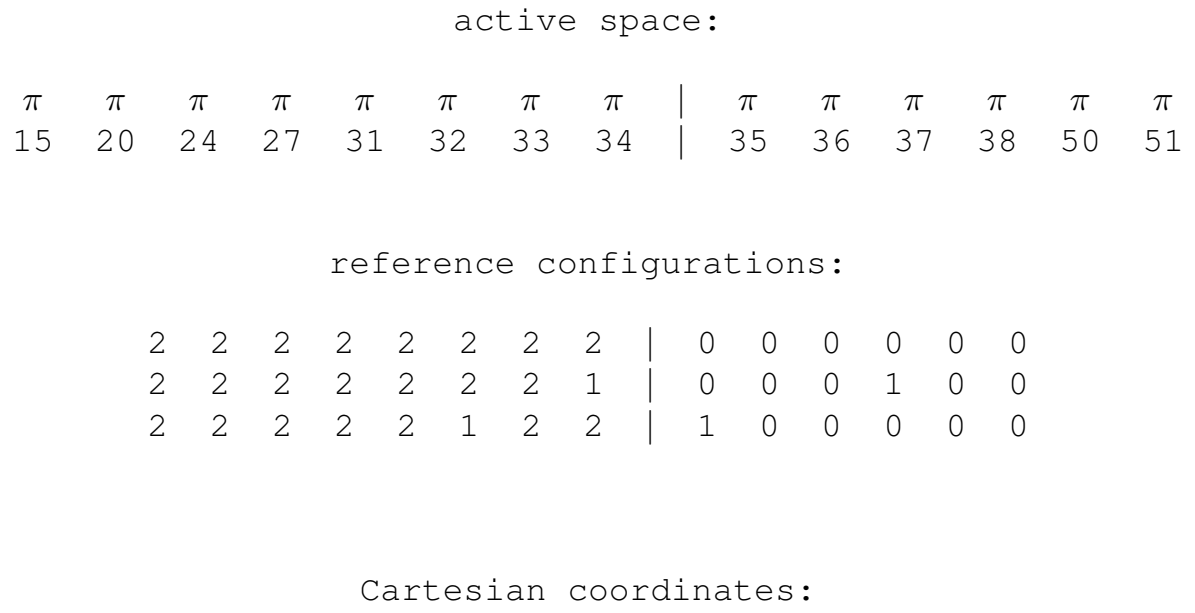


Table S277 Employed active space, reference configurations, and Cartesian coordinates of the OM3/MRCISD-optimized excited-state equilibrium geometry of the $1^{1} A_{u}\left({ }^{1} n \pi^{*}\right)$ state of dibenzo- $p$-dioxin (excited-state energy: $-2290.03485 \mathrm{eV})$.

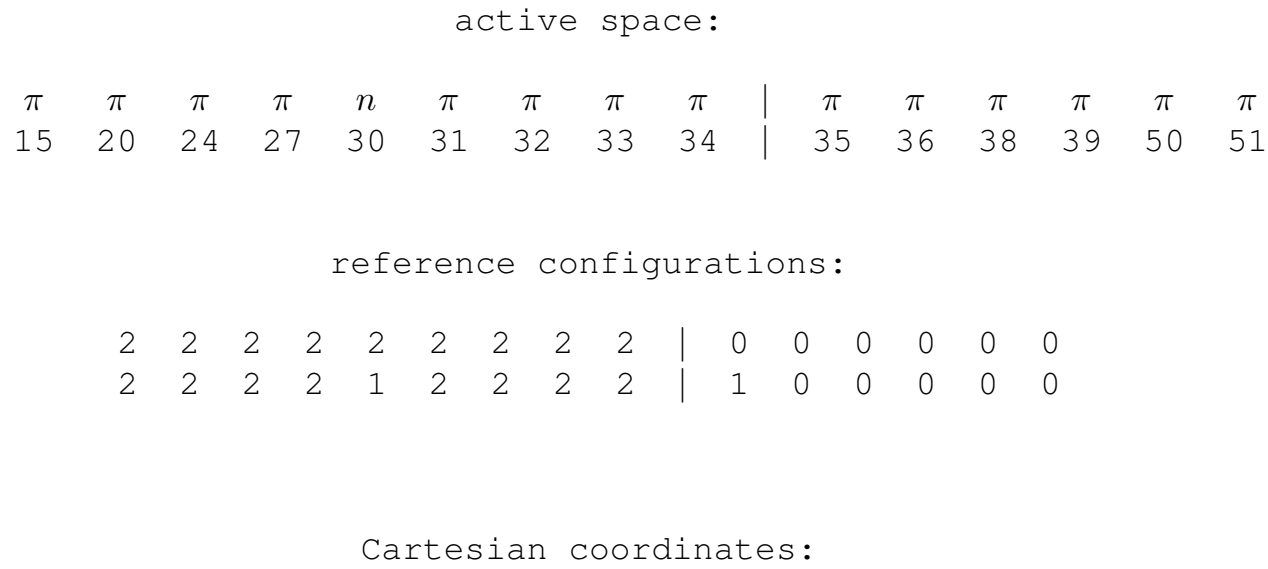


Table S278 Employed active space, reference configurations, and Cartesian coordinates of the OM3/MRCISD-optimized excited-state equilibrium geometry of the $1{ }^{1} B_{1 g}\left({ }^{1} n \pi^{*}\right)$ state of dibenzo- $p$-dioxin (excited-state energy: $-2289.55156 \mathrm{eV})$.

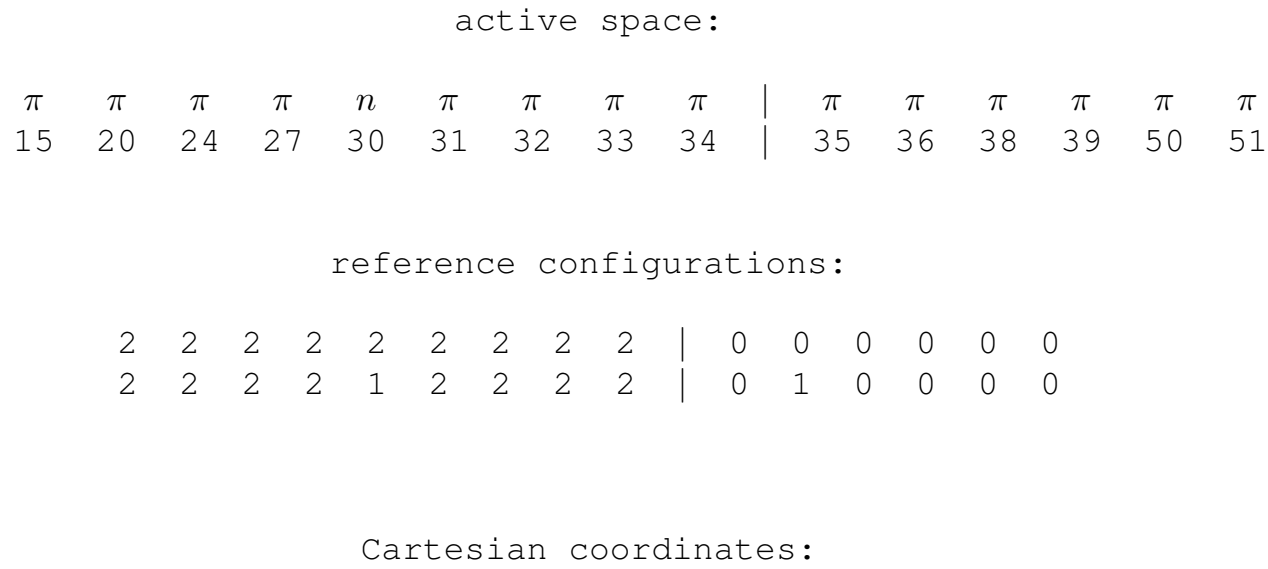


Table S279 Employed active space, reference configurations, and Cartesian coordinates of the OM3/MRCISD-optimized excited-state equilibrium geometry of the $1{ }^{1} B_{3 u}\left({ }^{1} n \pi^{*}\right)$ state of dibenzo- $p$-dioxin (excited-state energy: $-2289.25671 \mathrm{eV})$.

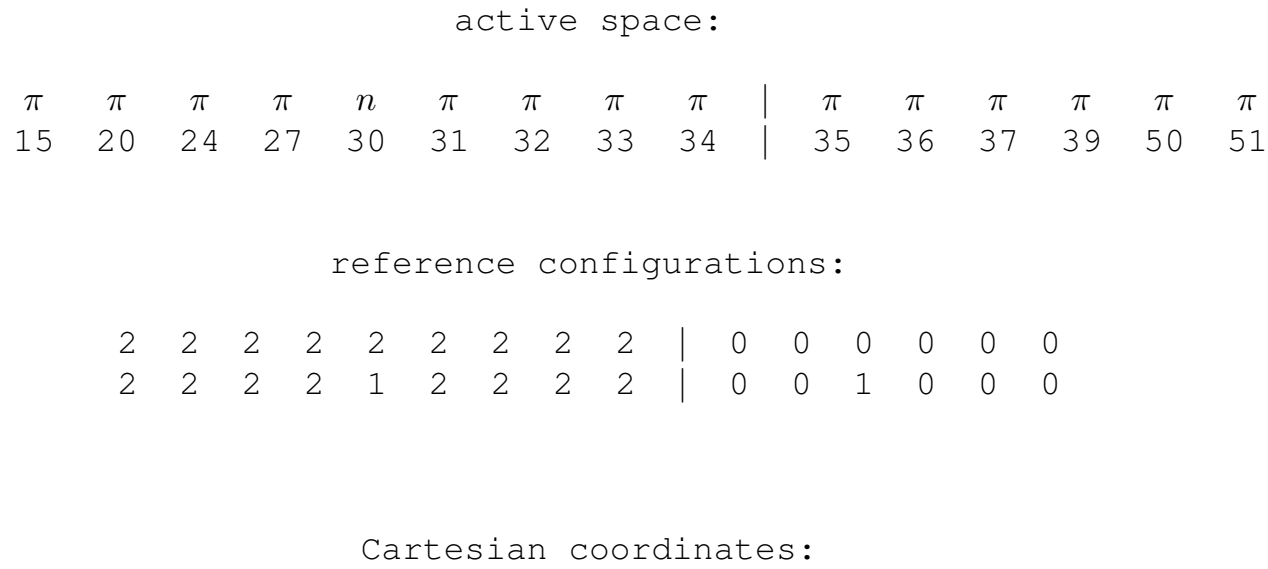


Table S280 Employed active space, reference configurations, and Cartesian coordinates of the OM3/MRCISD-optimized excited-state equilibrium geometry of the $1{ }^{1} B_{2 g}\left({ }^{1} n \pi^{*}\right)$ state of dibenzo- $p$-dioxin (excited-state energy: $-2289.12754 \mathrm{eV})$.

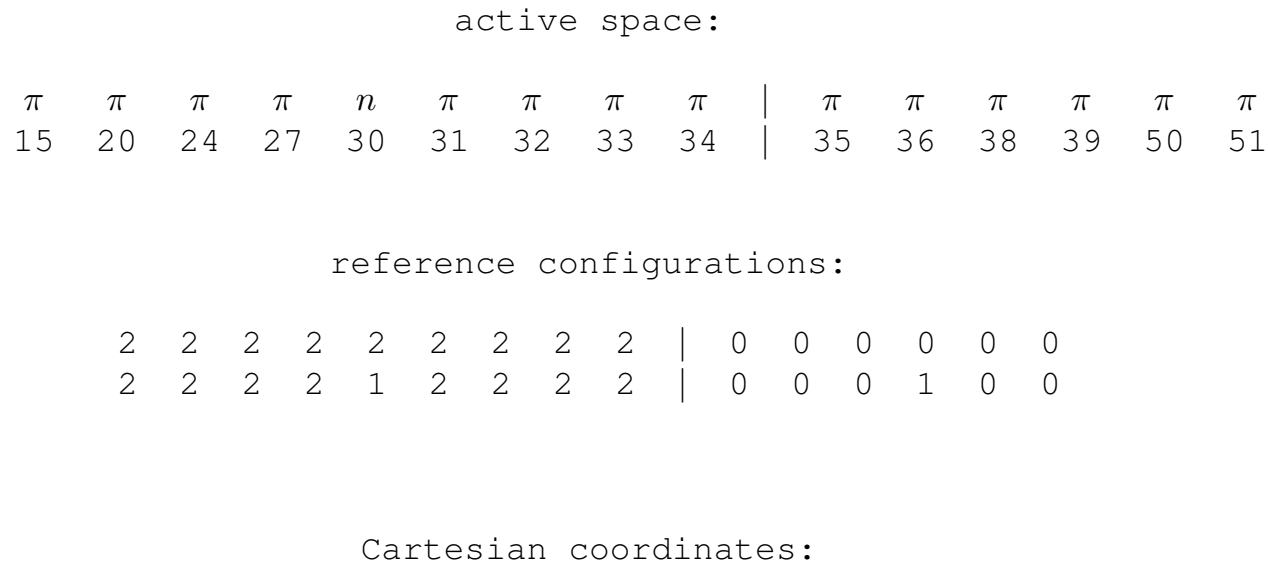


Table S281 Employed active space, reference configurations, and Cartesian coordinates of the OM3/MRCISD-optimized excited-state equilibrium geometry of the $1^{3} A_{u}\left({ }^{3} n \pi^{*}\right)$ state of dibenzo- $p$-dioxin (excited-state energy: $-2290.09394 \mathrm{eV})$.

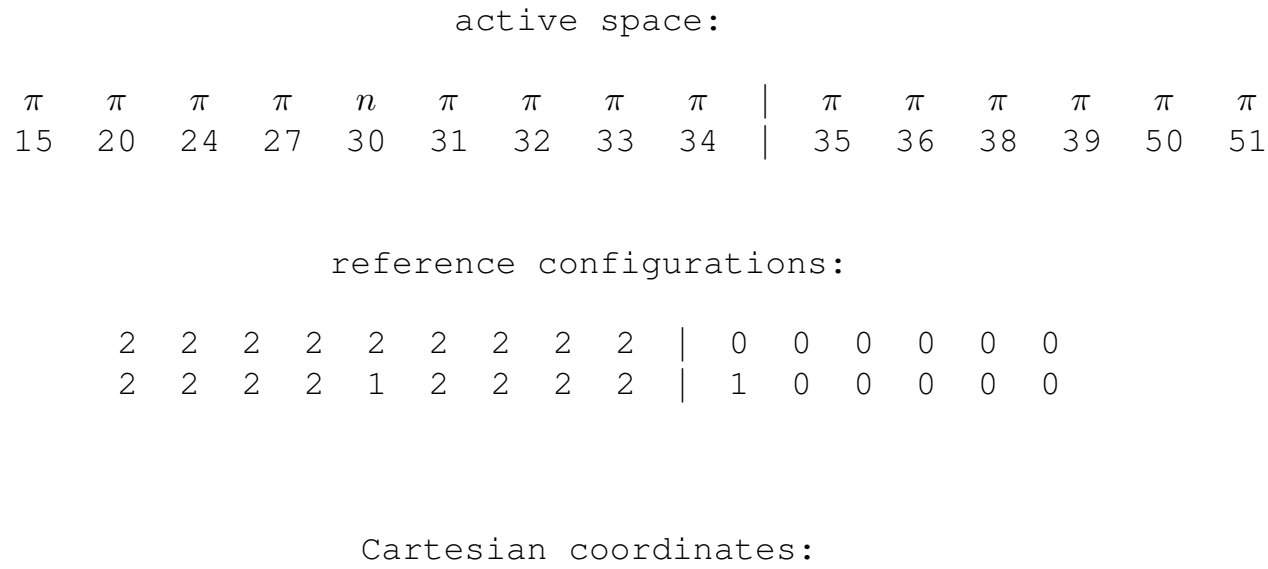


Table S282 Employed active space, reference configurations, and Cartesian coordinates of the OM3/MRCISD-optimized excited-state equilibrium geometry of the $1^{3} B_{1 g}\left({ }^{3} n \pi^{*}\right)$ state of dibenzo- $p$-dioxin (excited-state energy: $-2289.60833 \mathrm{eV})$.

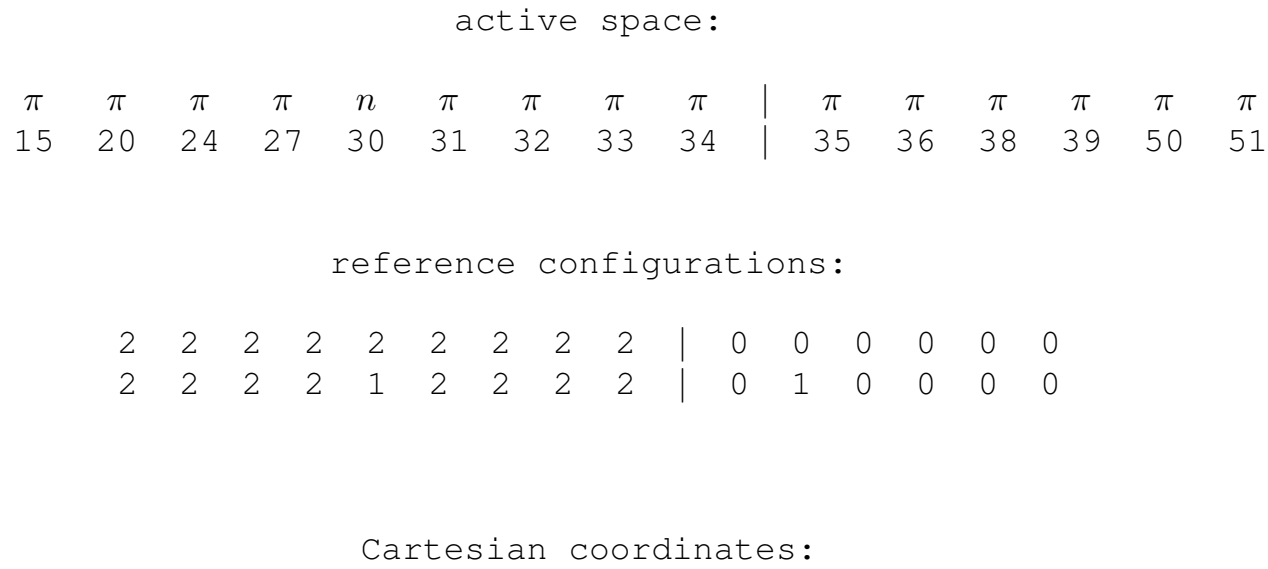


Table S283 Employed active space, reference configurations, and Cartesian coordinates of the OM3/MRCISD-optimized excited-state equilibrium geometry of the $1^{3} B_{3 u}\left({ }^{3} n \pi^{*}\right)$ state of dibenzo- $p$-dioxin (excited-state energy: $-2289.33065 \mathrm{eV})$.

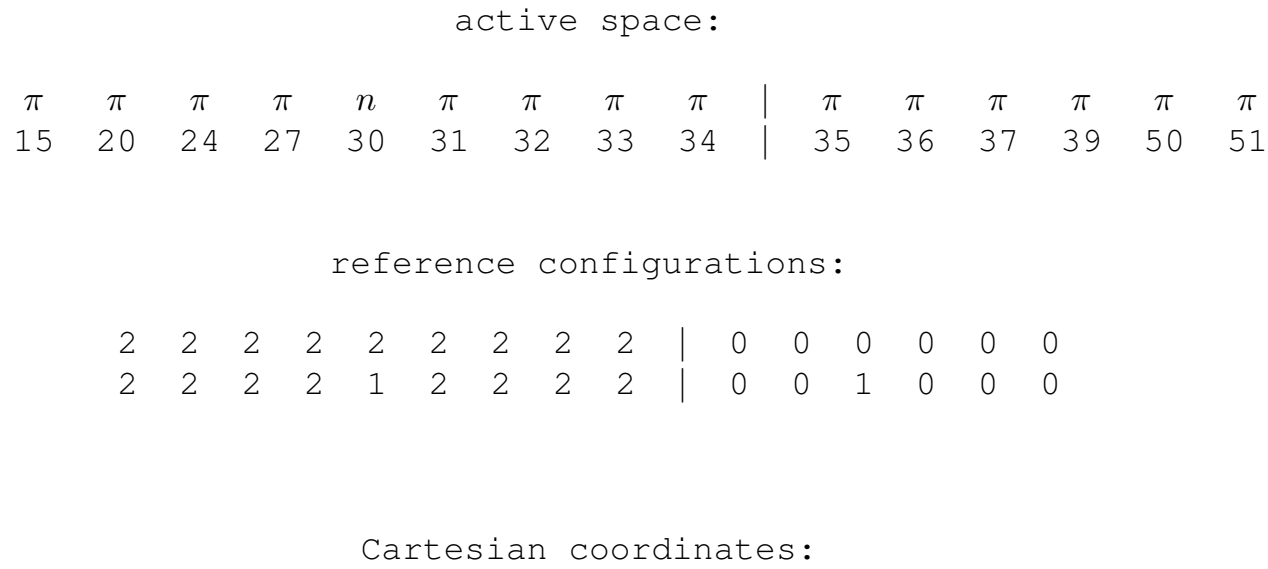


Table S284 Employed active space, reference configurations, and Cartesian coordinates of the OM3/MRCISD-optimized excited-state equilibrium geometry of the $1{ }^{3} B_{2 g}\left({ }^{3} n \pi^{*}\right)$ state of dibenzo- $p$-dioxin (excited-state energy: $-2289.19539 \mathrm{eV})$.

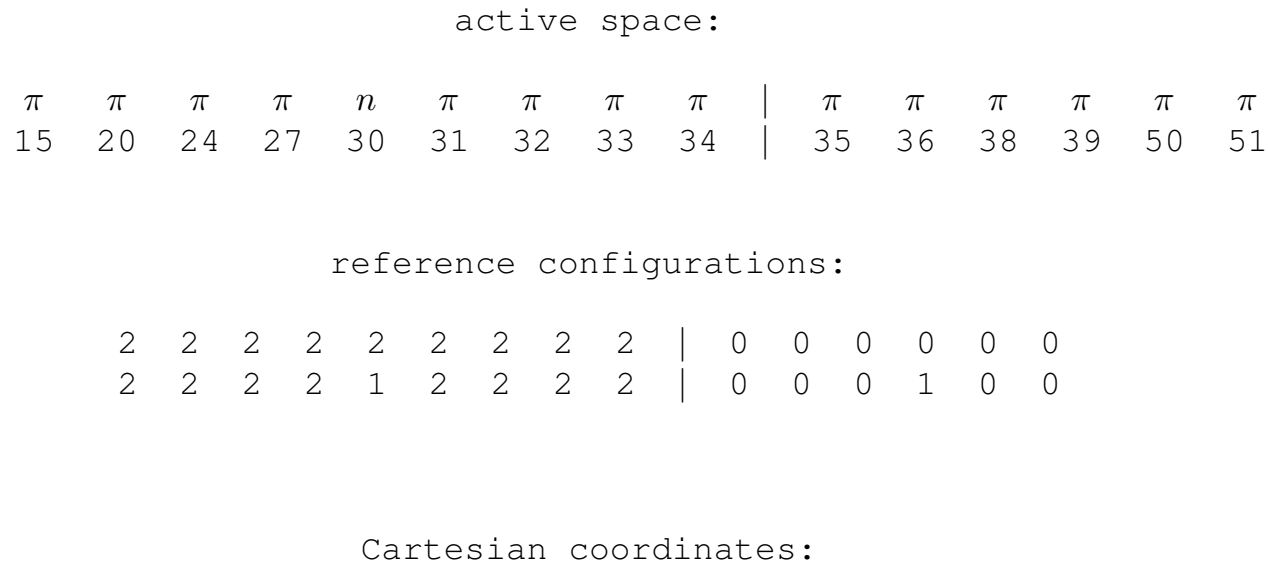


Table S285 Employed active space, reference configurations, and Cartesian coordinates of the OM3/MRCISD-optimized excited-state equilibrium geometry of the $1{ }^{1} A^{\prime \prime}\left({ }^{1} n \pi^{*}\right)$ state of formaldehyde (excited-state energy: $\left.-490.99034 \mathrm{eV}\right)$.

\begin{tabular}{|c|c|c|c|c|c|c|c|c|c|}
\hline$\sigma$ & $\sigma$ & $\sigma$ & $\sigma$ & $\pi$ & $n$ & $\pi$ & $\sigma$ & $\sigma$ & $\sigma$ \\
\hline \multirow[t]{2}{*}{1} & 2 & 3 & 4 & 5 & 6 & 7 & 8 & 9 & 10 \\
\hline & \multicolumn{4}{|c|}{ reference } & \multicolumn{4}{|c|}{ configurations: } & \\
\hline 2 & 2 & 2 & 2 & 2 & 2 & 0 & 0 & 0 & 0 \\
\hline 2 & 2 & 2 & 2 & 2 & 1 & 1 & 0 & 0 & 0 \\
\hline
\end{tabular}

Cartesian coordinates:

4

$\begin{array}{rrrr}\mathrm{C} & 0.00000 & 0.00000 & 0.00000 \\ \mathrm{O} & 0.00000 & 1.09140 & 0.66138 \\ \mathrm{H} & 0.90996 & -0.66243 & 0.00000 \\ \mathrm{H} & -0.90996 & -0.66243 & 0.00000\end{array}$


Table S286 Employed active space, reference configurations, and Cartesian coordinates of the OM3/MRCISD-optimized excited-state equilibrium geometry of the $1^{3} A^{\prime \prime}\left({ }^{3} n \pi^{*}\right)$ state of formaldehyde (excited-state energy: $-491.27605 \mathrm{eV}$ ).

\begin{tabular}{|c|c|c|c|c|c|c|c|c|c|}
\hline$\sigma$ & $\sigma$ & $\sigma$ & $\sigma$ & $\pi$ & $n$ & $\pi$ & $\sigma$ & & \\
\hline \multirow[t]{2}{*}{1} & 2 & 3 & 4 & 5 & 6 & 7 & 8 & & \\
\hline & \multicolumn{4}{|c|}{ reference } & \multicolumn{5}{|c|}{ configurations: } \\
\hline 2 & 2 & 2 & 2 & 2 & 2 & 0 & 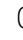 & & 0 \\
\hline 2 & 2 & 2 & 2 & 2 & 1 & 1 & & & 0 \\
\hline
\end{tabular}

Cartesian coordinates:

4

$\begin{array}{rrrr}\mathrm{C} & 0.00000 & 0.00000 & 0.00000 \\ \mathrm{O} & 0.00000 & 0.97574 & 0.78697 \\ \mathrm{H} & 0.89946 & -0.73212 & 0.00000 \\ \mathrm{H} & -0.89946 & -0.73212 & 0.00000\end{array}$


Table S287 Employed active space, reference configurations, and Cartesian coordinates of the OM3/MRCISD-optimized excited-state equilibrium geometry of the $1^{3} A^{\prime}\left({ }^{3} \pi \pi^{*}\right)$ state of formaldehyde (excited-state energy: $-489.35910 \mathrm{eV}$ ).

\begin{tabular}{|c|c|c|c|c|c|c|c|c|}
\hline$\sigma$ & $\sigma$ & $\sigma$ & $\sigma$ & $\pi$ & $n$ & $\pi$ & $\sigma$ & $\sigma$ \\
\hline \multirow[t]{2}{*}{1} & 2 & 3 & 4 & 5 & 6 & 7 & 8 & 9 \\
\hline & \multicolumn{4}{|c|}{ reference } & \multicolumn{4}{|c|}{ configurations } \\
\hline 2 & 2 & 2 & 2 & 2 & 2 & 0 & 0 & \\
\hline 2 & 2 & 2 & 2 & 1 & 2 & 1 & 0 & \\
\hline
\end{tabular}

Cartesian coordinates:

4

$\begin{array}{rrrr}\mathrm{C} & 0.00000 & 0.00000 & 0.00000 \\ \mathrm{O} & 0.00000 & 1.15089 & 0.83838 \\ \mathrm{H} & 0.95241 & -0.58696 & 0.00000 \\ \mathrm{H} & -0.95241 & -0.58696 & 0.00000\end{array}$


Table S288 Employed active space, reference configurations, and Cartesian coordinates of the OM3/MRCISD-optimized excited-state equilibrium geometry of the $1{ }^{1} A_{u}\left({ }^{1} n \pi^{*}\right)$ state of trans-glyoxal (excited-state energy: $-955.08180 \mathrm{eV}$ ).

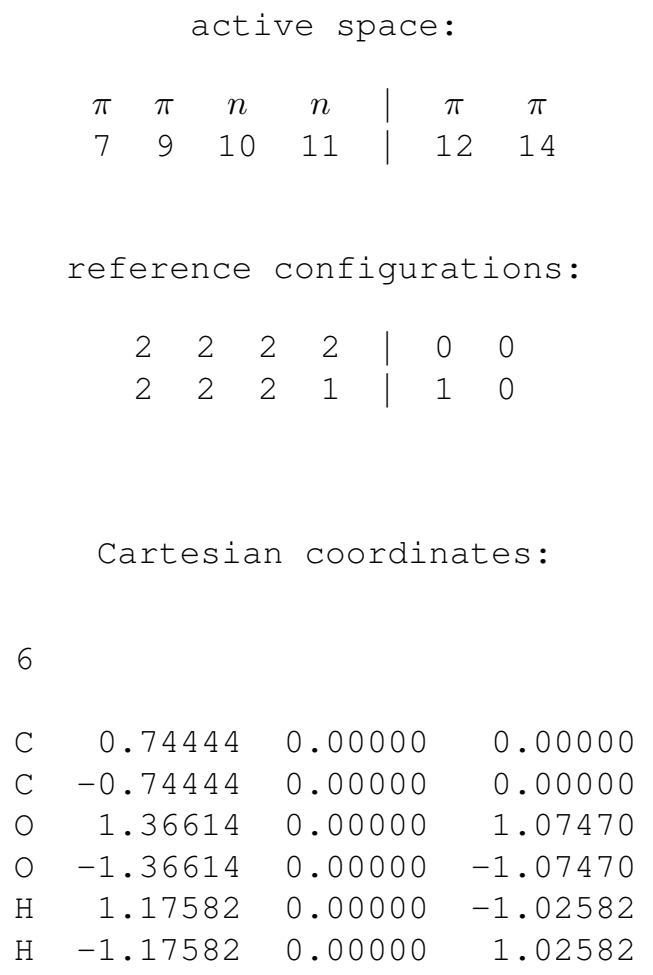


Table S289 Employed active space, reference configurations, and Cartesian coordinates of the OM3/MRCISD-optimized excited-state equilibrium geometry of the $1^{3} A_{u}\left({ }^{3} n \pi^{*}\right)$ state of trans-glyoxal (excited-state energy: $-955.37525 \mathrm{eV}$ ).

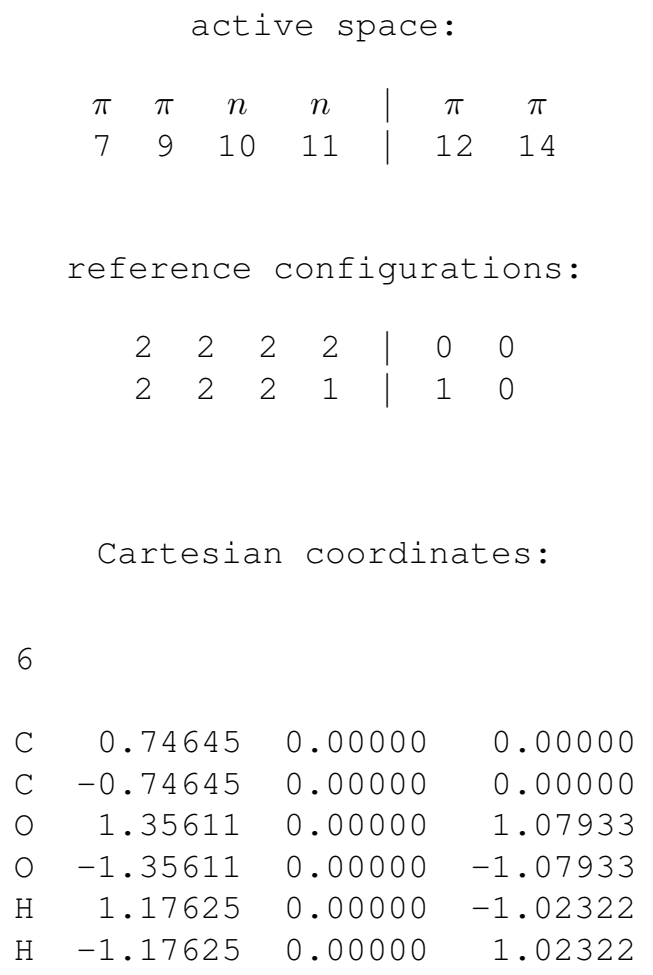


Table S290 Employed active space, reference configurations, and Cartesian coordinates of the OM3/MRCISD-optimized excited-state equilibrium geometry of the $2{ }^{1} A\left({ }^{1} \pi \pi^{*}\right)$ state of $9 H$-guanine (excited-state energy: $\left.-2113.67059 \mathrm{eV}\right)$.

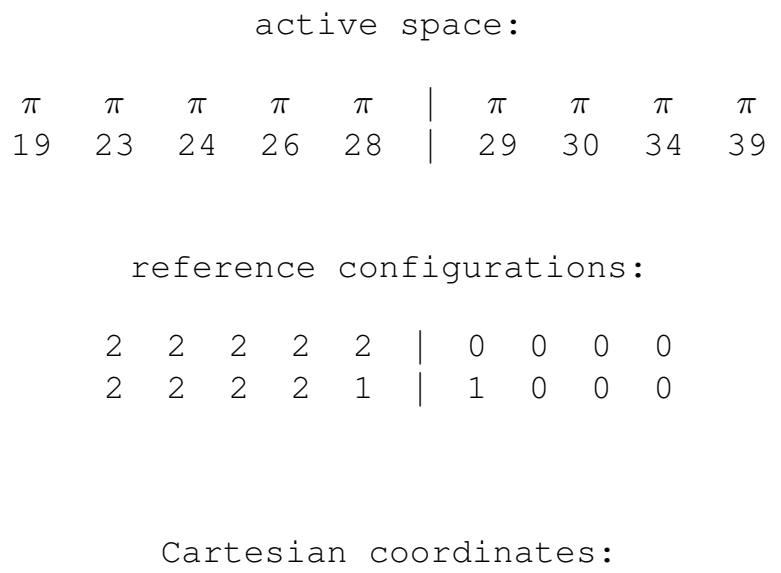


Table S291 Employed active space, reference configurations, and Cartesian coordinates of the OM3/MRCISD-optimized excited-state equilibrium geometry of the $2{ }^{1} A^{\prime}\left({ }^{1} \pi \pi^{*}\right)$ state of 1-hydroxy-2-acetonaphthone (excited-state energy: $-2324.37177 \mathrm{eV})$.

\begin{tabular}{|c|c|c|c|c|c|c|c|}
\hline \multirow{2}{*}{$\begin{array}{c}\pi \\
32\end{array}$} & $n$ & $\pi$ & $\pi$ & $\pi$ & \multicolumn{2}{|l|}{$\pi$} & 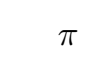 \\
\hline & 33 & 34 & 35 & 36 & 37 & & 38 \\
\hline & ref & erenc & e c & onfigura & cati & ions & \\
\hline & 2 & 22 & 2 & 0 & 0 & 0 & 0 \\
\hline & 2 & 22 & 1 & 10 & 0 & 0 & 0 \\
\hline & 2 & 21 & 2 & 0 & 1 & 0 & 0 \\
\hline & & artesi & an & coordin & nate & es: & \\
\hline 24 & & & & & & & \\
\hline $\mathrm{C}$ & & 08048 & & 0.99869 & & & 00000 \\
\hline C & -1 & 18821 & & 0.28804 & & & 00000 \\
\hline C & -1 & 18633 & & -1.15006 & & & 00000 \\
\hline C & & 04440 & & -1.85083 & & & 00000 \\
\hline C & & 28375 & & -1.16350 & & & 00000 \\
\hline C & & 33439 & & 0.21725 & & & 00000 \\
\hline C & -2 & 41126 & & 0.98656 & & & 00000 \\
\hline C & -3 & 61681 & & 0.28392 & & & 00000 \\
\hline C & -3 & 62888 & & -1.12384 & & & 00000 \\
\hline C & -2 & 43618 & & -1.83812 & & & 00000 \\
\hline C & & 57118 & & 1.00499 & & & 00000 \\
\hline C & & 93158 & & 0.40101 & & & 00000 \\
\hline 0 & & 14129 & & 2.28160 & & & 00000 \\
\hline 0 & & 49523 & & 2.32386 & & & 00000 \\
\hline $\mathrm{H}$ & -4 & 57249 & & 0.83323 & & & 00000 \\
\hline $\mathrm{H}$ & -4 & 59170 & & -1.65805 & & & 00000 \\
\hline $\mathrm{H}$ & -2 & 44166 & & -2.94156 & & & 00000 \\
\hline $\mathrm{H}$ & -2 & 39401 & & 2.08649 & & & 00000 \\
\hline $\mathrm{H}$ & & 03807 & & -2.95405 & & & 00000 \\
\hline $\mathrm{H}$ & & 21505 & & -1.75524 & & & 00000 \\
\hline $\mathrm{H}$ & & 40925 & & 2.62232 & & & 00000 \\
\hline $\mathrm{H}$ & & 68625 & & 1.20211 & & & 00000 \\
\hline
\end{tabular}

S330 
Continued from previous page

\begin{tabular}{llrr}
\hline $\mathrm{H}$ & 4.07768 & -0.22514 & -0.89901 \\
$\mathrm{H}$ & 4.07768 & -0.22514 & 0.89901
\end{tabular}

S331 
Table S292 Employed active space, reference configurations, and Cartesian coordinates of the OM3/MRCISD-optimized excited-state equilibrium geometry of the $2{ }^{1} A^{\prime}\left({ }^{1} \pi \pi^{*}\right)$ state of indole (excited-state energy: $-1316.30612 \mathrm{eV}$ ).

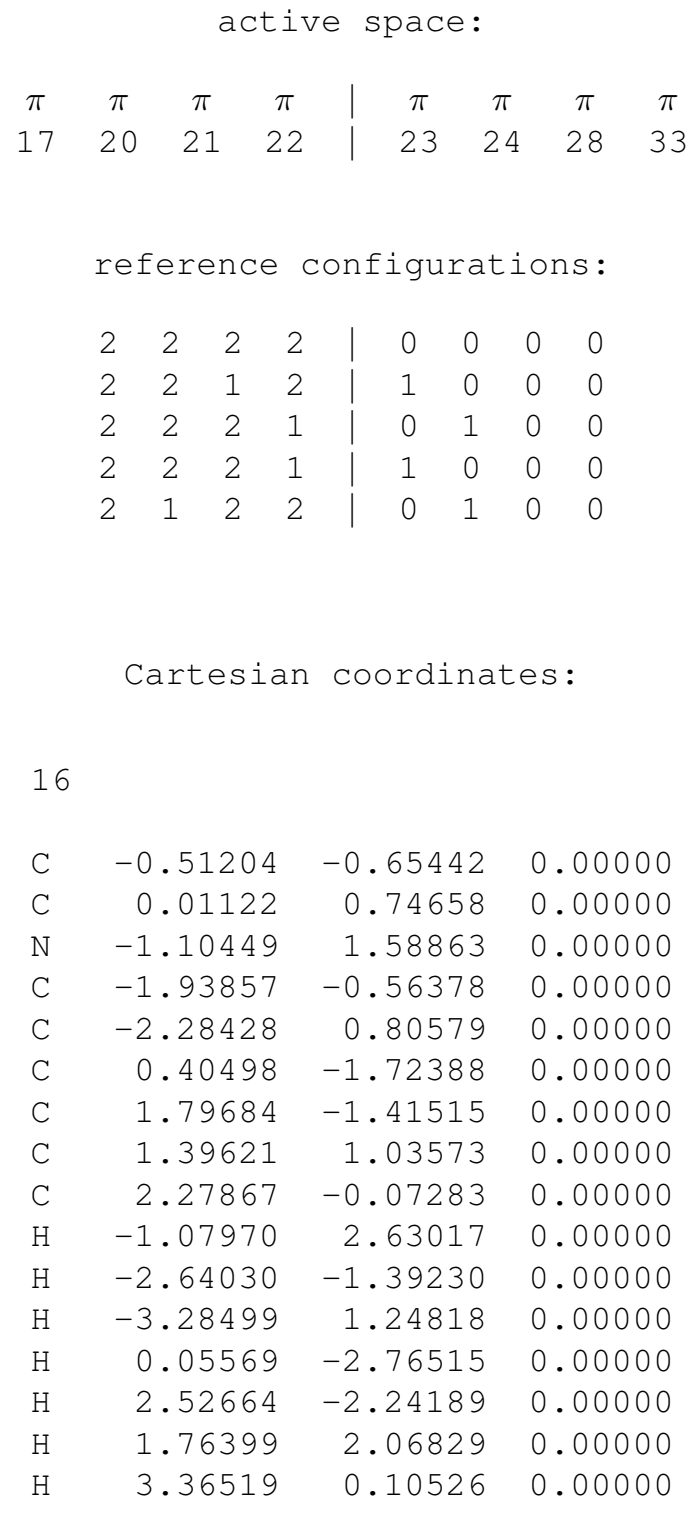


Table S293 Employed active space, reference configurations, and Cartesian coordinates of the OM3/MRCISD-optimized excited-state equilibrium geometry of the $3{ }^{1} A^{\prime}\left({ }^{1} \pi \pi^{*}\right)$ state of indole (excited-state energy: $-1315.69723 \mathrm{eV}$ ).

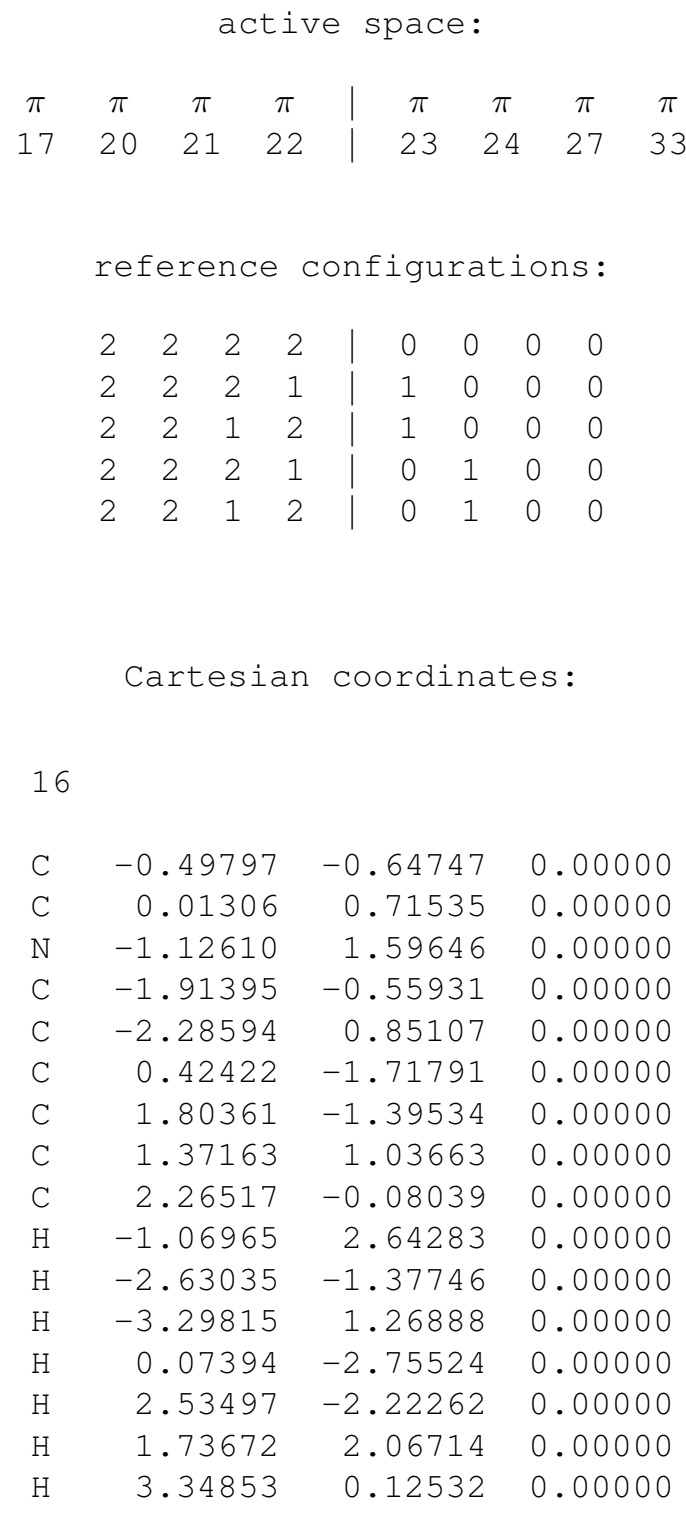


Table S294 Employed active space, reference configurations, and Cartesian coordinates of the OM3/MRCISD-optimized excited-state equilibrium geometry of the $1{ }^{1} B_{1}\left({ }^{1} n \pi^{*}\right)$ state of maleimide (excited-state energy: $\left.-1434.50387 \mathrm{eV}\right)$.

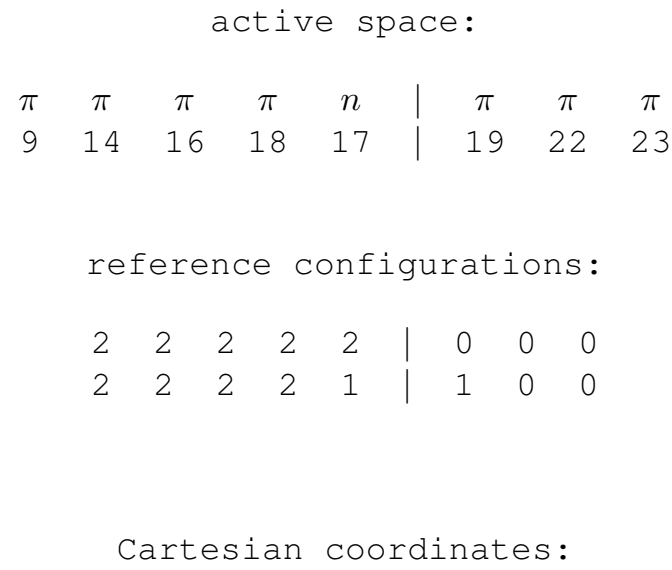

$\begin{array}{lrrr}\mathrm{N} & 0.00000 & 0.00000 & 0.00000 \\ \mathrm{C} & 1.18136 & 0.84987 & 0.00000 \\ \mathrm{C} & 0.68810 & 2.23065 & 0.00000 \\ \mathrm{C} & -0.68810 & 2.23065 & 0.00000 \\ \mathrm{C} & -1.18136 & 0.84987 & 0.00000 \\ \mathrm{O} & 2.30465 & 0.29861 & 0.00000 \\ \mathrm{O} & -2.30465 & 0.29861 & 0.00000 \\ \mathrm{H} & 0.00000 & -1.04198 & 0.00000 \\ \mathrm{H} & 1.38202 & 3.06243 & 0.00000 \\ \mathrm{H} & -1.38202 & 3.06243 & 0.00000\end{array}$


Table S295 Employed active space, reference configurations, and Cartesian coordinates of the OM3/MRCISD-optimized excited-state equilibrium geometry of the $1^{1} A_{2}\left({ }^{1} n \pi^{*}\right)$ state of maleimide (excited-state energy: $-1434.71245 \mathrm{eV}$ ).

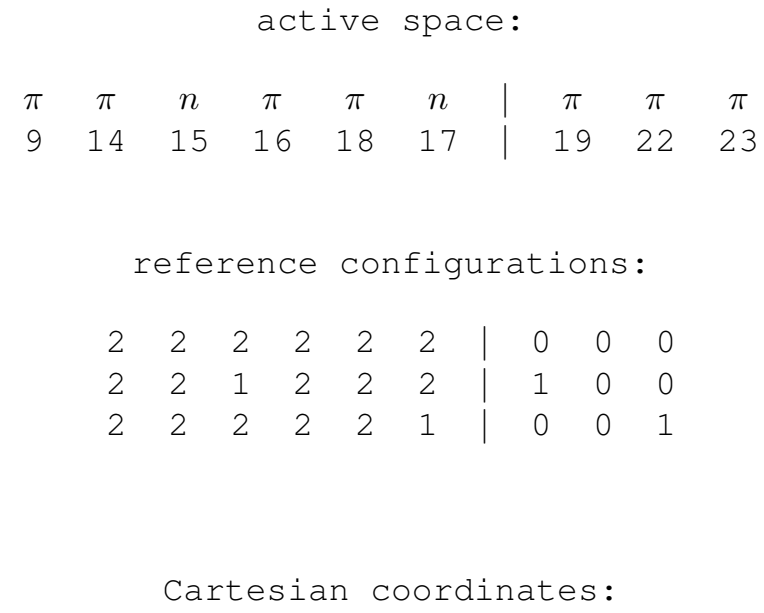


Table S296 Employed active space, reference configurations, and Cartesian coordinates of the OM3/MRCISD-optimized excited-state equilibrium geometry of the $1^{1} B_{2}\left({ }^{1} \pi \pi^{*}\right)$ state of maleimide (excited-state energy: $-1434.82444 \mathrm{eV}$ ).

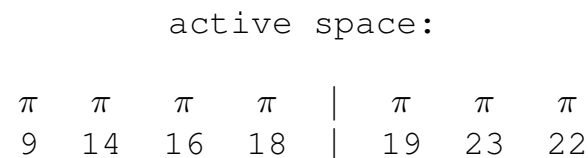

reference configurations:

\begin{tabular}{llll|lll}
2 & 2 & 2 & 2 & 0 & 0 & 0 \\
2 & 2 & 1 & 2 & 1 & 0 & 0 \\
2 & 2 & 2 & 1 & 1 & 0 & 0 \\
2 & 2 & 1 & 1 & $\mid$ & 1 & 0
\end{tabular}

Cartesian coordinates:

10

$\begin{array}{lrrr}\mathrm{N} & 0.00000 & 0.00000 & 0.00000 \\ \mathrm{C} & 1.22822 & 0.85935 & 0.00000 \\ \mathrm{C} & 0.70449 & 2.19740 & 0.00000 \\ \mathrm{C} & -0.70449 & 2.19740 & 0.00000 \\ \mathrm{C} & -1.22822 & 0.85935 & 0.00000 \\ \mathrm{O} & 2.34585 & 0.30614 & 0.00000 \\ \mathrm{O} & -2.34585 & 0.30614 & 0.00000 \\ \mathrm{H} & 0.00000 & -1.05164 & 0.00000 \\ \mathrm{H} & 1.35297 & 3.06882 & 0.00000 \\ \mathrm{H} & -1.35297 & 3.06882 & 0.00000\end{array}$


Table S297 Employed active space, reference configurations, and Cartesian coordinates of the OM3/MRCISD-optimized excited-state equilibrium geometry of the $2{ }^{1} B_{2}\left({ }^{1} \pi \pi^{*}\right)$ state of maleimide (excited-state energy: $-1432.91200 \mathrm{eV}$ ).

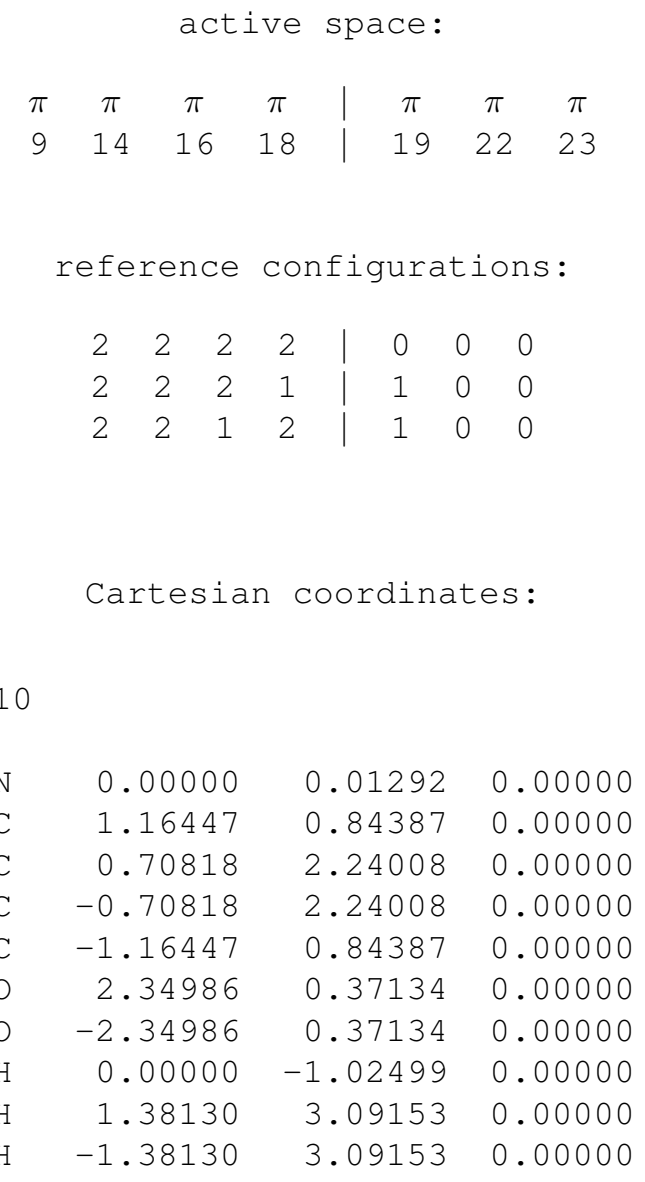


Table S298 Employed active space, reference configurations, and Cartesian coordinates of the OM3/MRCISD-optimized excited-state equilibrium geometry of the $1{ }^{3} B_{1}\left({ }^{3} n \pi^{*}\right)$ state of maleimide (excited-state energy: $\left.-1434.70020 \mathrm{eV}\right)$.

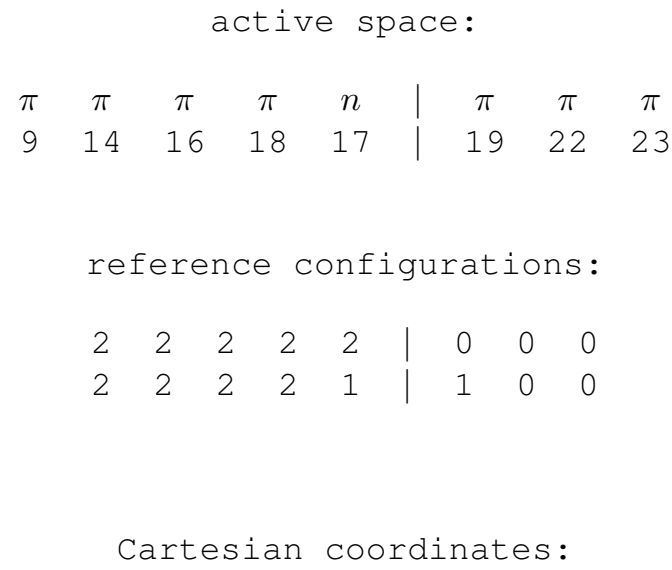

$\begin{array}{lrrr}\mathrm{N} & 0.00000 & 0.00000 & 0.00000 \\ \mathrm{C} & 1.17978 & 0.84432 & 0.00000 \\ \mathrm{C} & 0.68740 & 2.22766 & 0.00000 \\ \mathrm{C} & -0.68740 & 2.22766 & 0.00000 \\ \mathrm{C} & -1.17978 & 0.84432 & 0.00000 \\ \mathrm{O} & 2.31156 & 0.30939 & 0.00000 \\ \mathrm{O} & -2.31156 & 0.30939 & 0.00000 \\ \mathrm{H} & 0.00000 & -1.04226 & 0.00000 \\ \mathrm{H} & 1.38388 & 3.05722 & 0.00000 \\ \mathrm{H} & -1.38388 & 3.05722 & 0.00000\end{array}$


Table S299 Employed active space, reference configurations, and Cartesian coordinates of the OM3/MRCISD-optimized excited-state equilibrium geometry of the $1^{3} A_{2}\left({ }^{3} n \pi^{*}\right)$ state of maleimide (excited-state energy: $-1434.84215 \mathrm{eV}$ ).

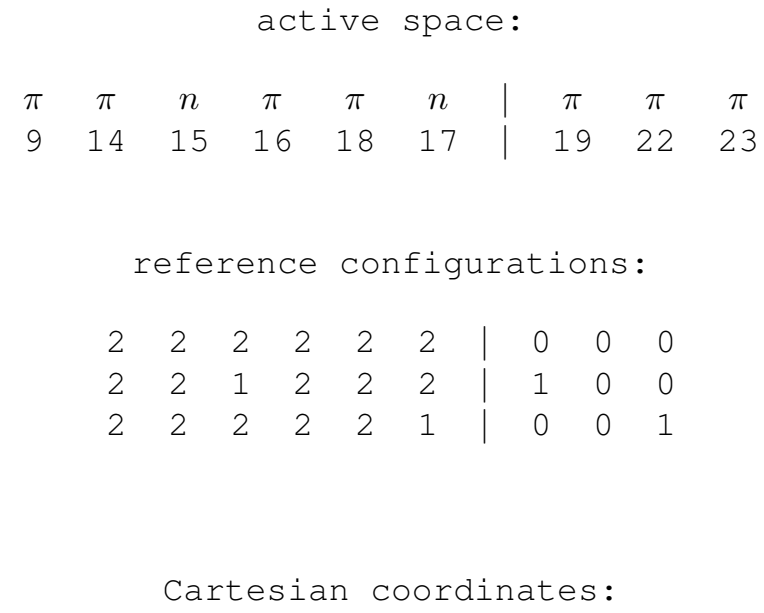


Table S300 Employed active space, reference configurations, and Cartesian coordinates of the OM3/MRCISD-optimized excited-state equilibrium geometry of the $1{ }^{3} B_{2}\left({ }^{3} \pi \pi^{*}\right)$ state of maleimide (excited-state energy: $-1435.83935 \mathrm{eV}$ ).

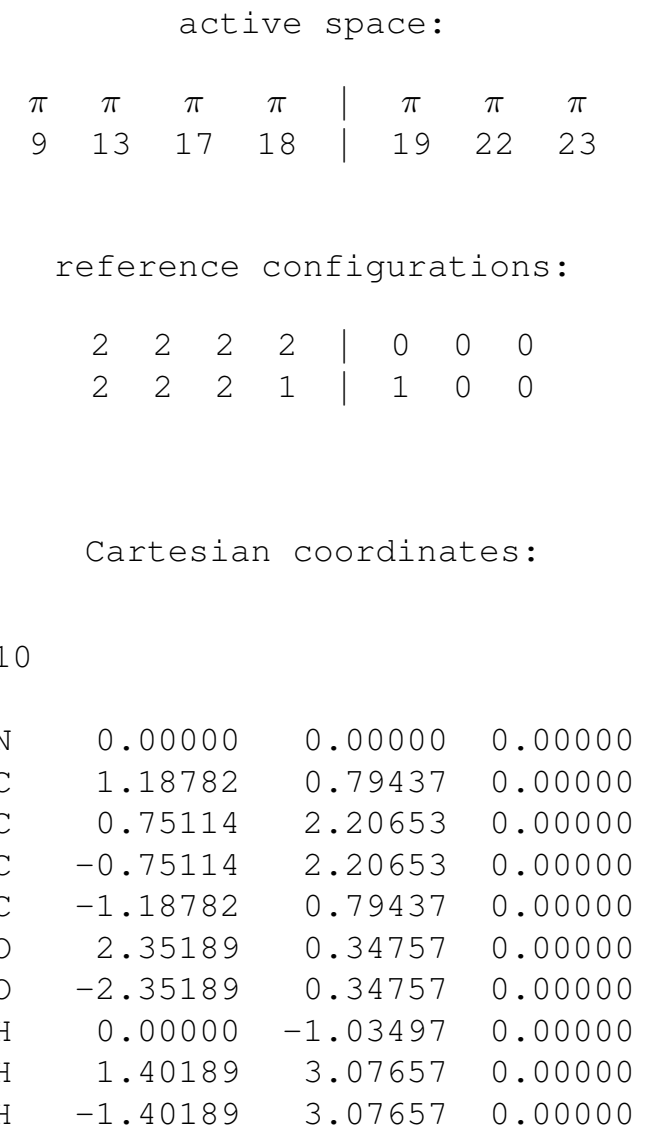


Table S301 Employed active space, reference configurations, and Cartesian coordinates of the OM3/MRCISD-optimized excited-state equilibrium geometry of the $1^{1} A^{\prime \prime}\left({ }^{1} n \pi^{*}\right)$ state of nitrosomethane (excited-state energy: $-720.94906 \mathrm{eV})$.

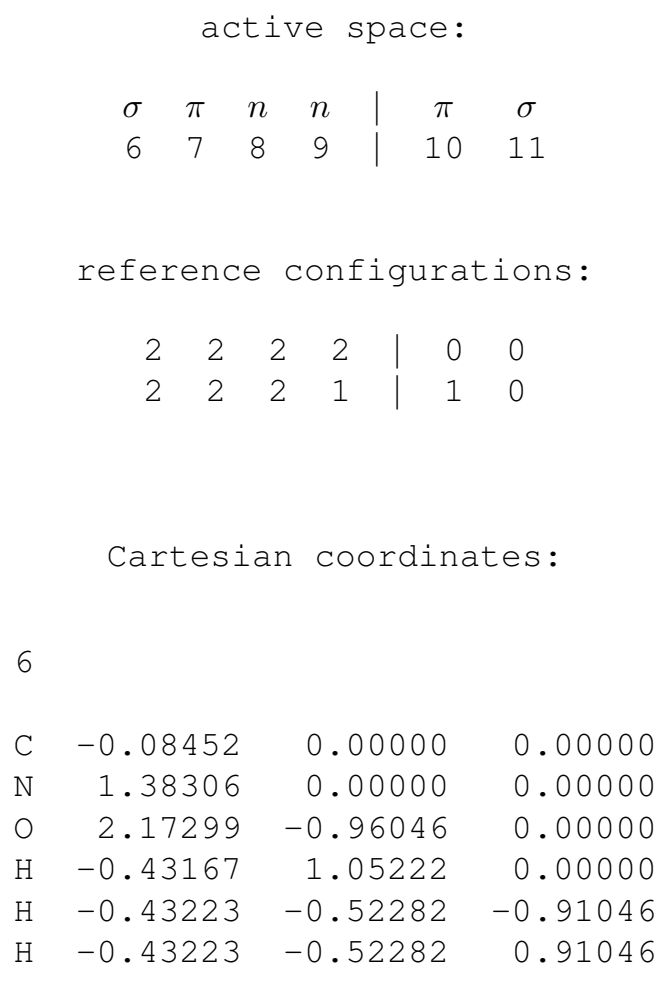


Table S302 Employed active space, reference configurations, and Cartesian coordinates of the OM3/MRCISD-optimized excited-state equilibrium geometry of the $1^{3} A^{\prime \prime}\left({ }^{3} n \pi^{*}\right)$ state of nitrosomethane (excited-state energy: $-721.64639 \mathrm{eV})$.

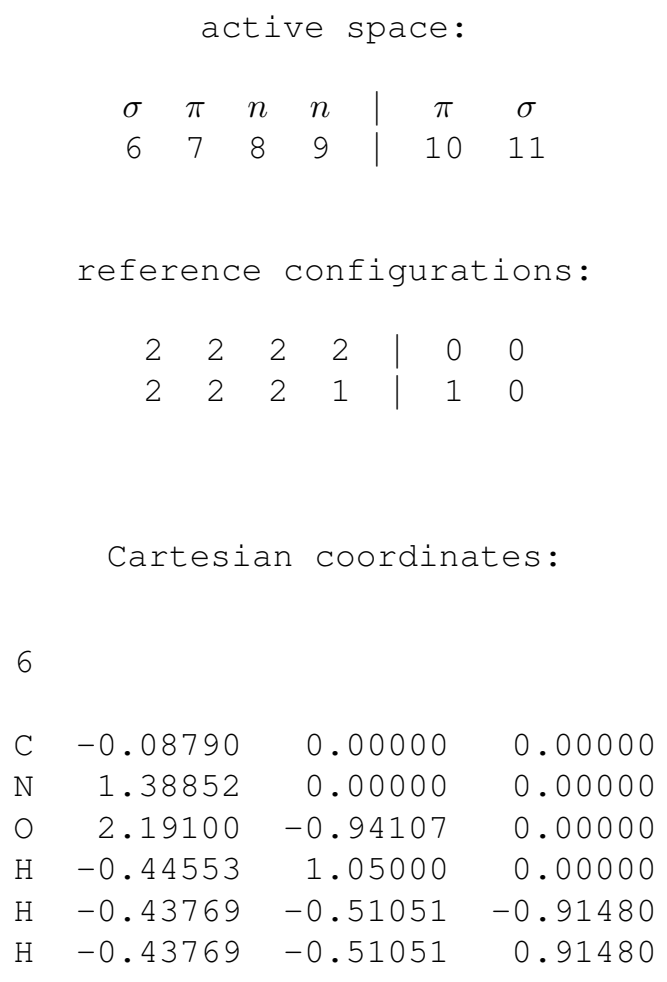


Table S303 Employed active space, reference configurations, and Cartesian coordinates of the OM3/MRCISD-optimized excited-state equilibrium geometry of the $1{ }^{1} A^{\prime \prime}\left({ }^{1} n \pi^{*}\right)$ state of propenoic acid anion (excited-state energy: $-1095.33119 \mathrm{eV})$.

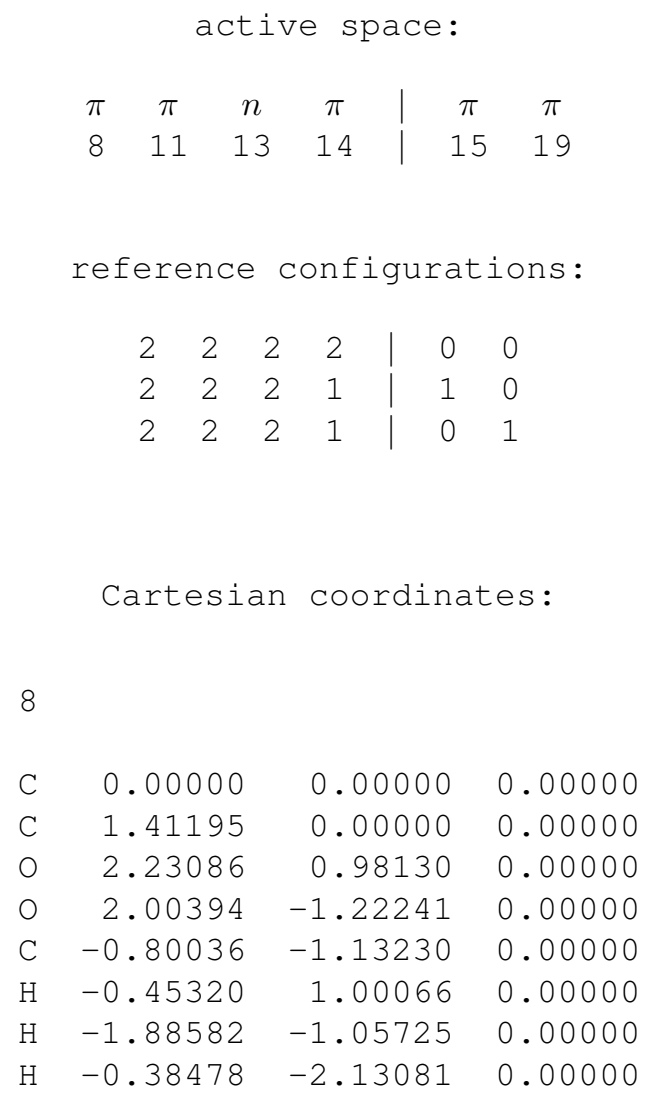


Table S304 Employed active space, reference configurations, and Cartesian coordinates of the OM3/MRCISD-optimized excited-state equilibrium geometry of the $1{ }^{1} A_{2}\left({ }^{1} n \pi^{*}\right)$ state of pyridine (excited-state energy: $-911.55433 \mathrm{eV}$ ).

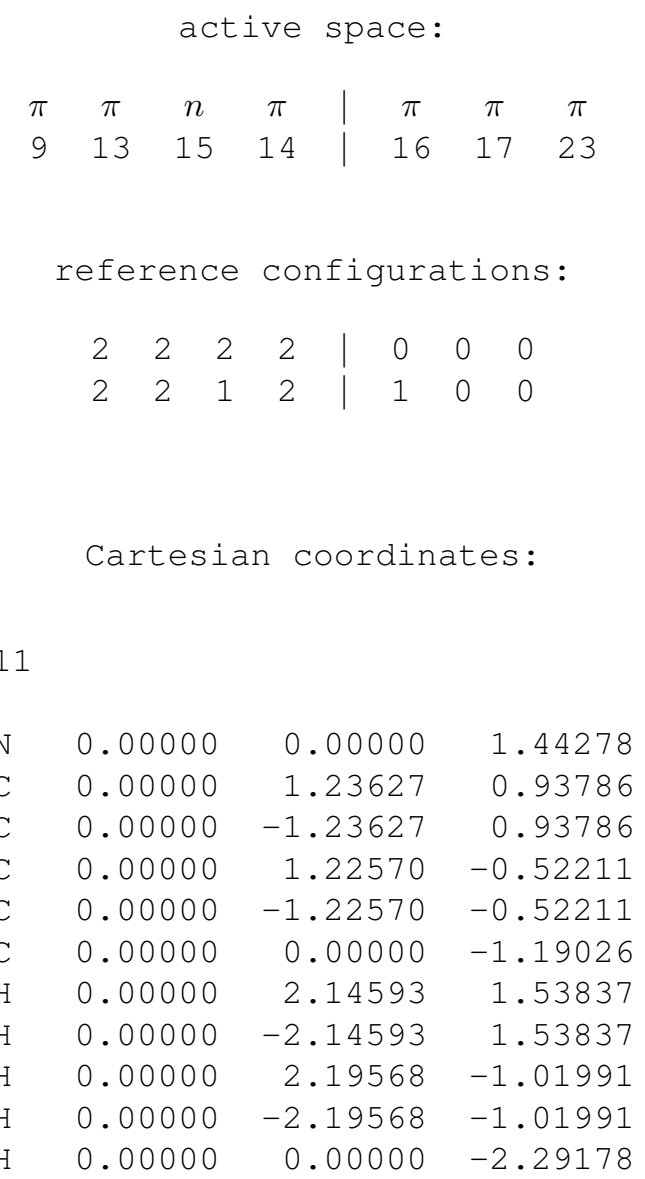


Table S305 Employed active space, reference configurations, and Cartesian coordinates of the OM3/MRCISD-optimized excited-state equilibrium geometry of the $2{ }^{1} A^{\prime}\left({ }^{1} n \pi^{*}\right)$ state of pyridine (excited-state energy: $-911.33804 \mathrm{eV}$ ).

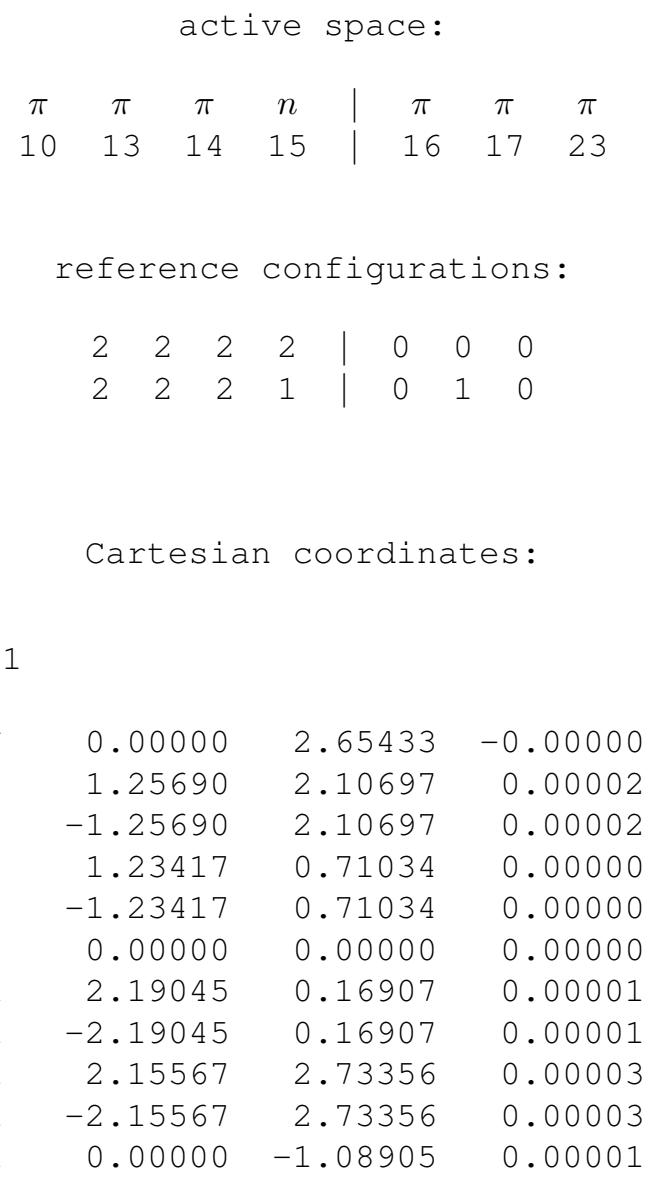


Table S306 Employed active space, reference configurations, and Cartesian coordinates of the OM3/MRCISD-optimized excited-state equilibrium geometry of the $1{ }^{3} A_{1}\left({ }^{3} \pi \pi^{*}\right)$ state of pyridine (excited-state energy: $-912.13922 \mathrm{eV}$ ).

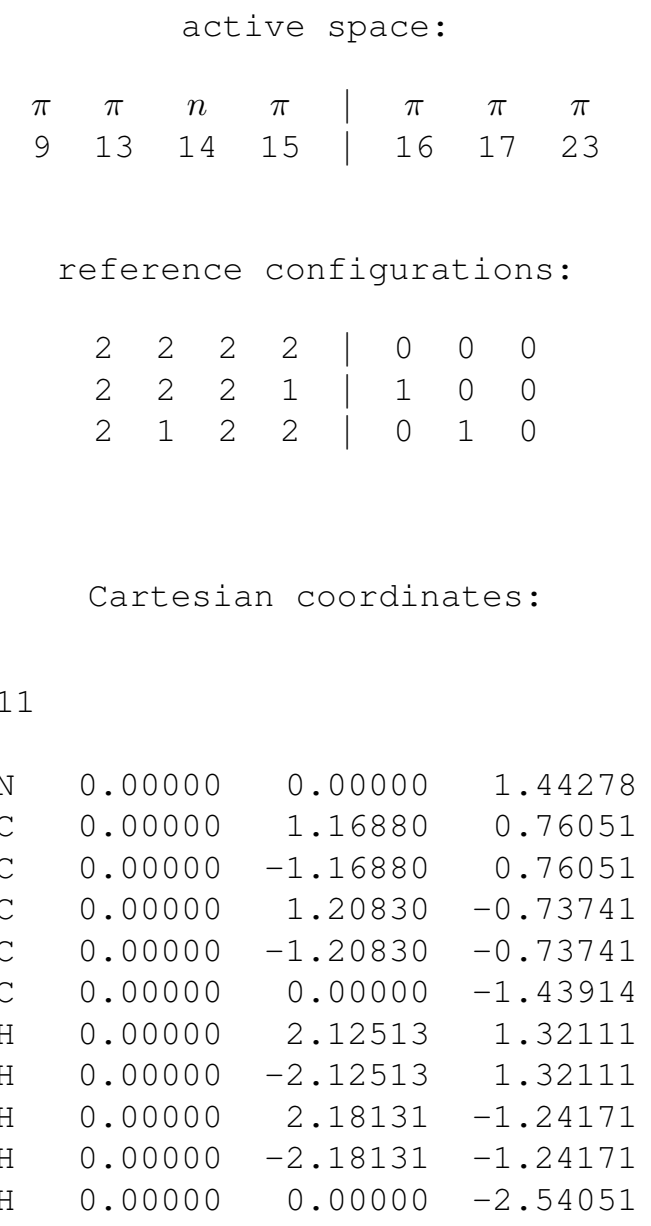


Table S307 Employed active space, reference configurations, and Cartesian coordinates of the OM3/MRCISD-optimized excited-state equilibrium geometry of the $2{ }^{3} A_{1}\left({ }^{3} \pi \pi^{*}\right)$ state of pyridine (excited-state energy: $-910.94488 \mathrm{eV}$ ).

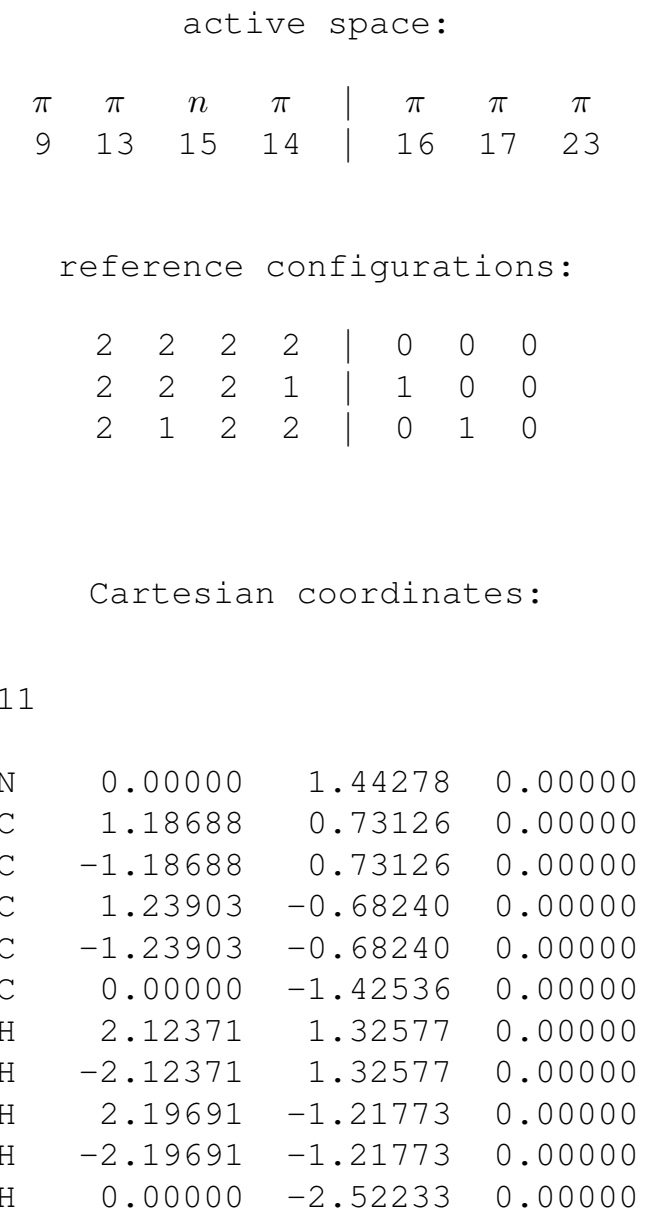


Table S308 Employed active space, reference configurations, and Cartesian coordinates of the OM3/MRCISD-optimized excited-state equilibrium geometry of the $2{ }^{1} A^{\prime}\left({ }^{1} \pi \pi^{*}\right)$ state of pyrrole (excited-state energy: $\left.-784.96373 \mathrm{eV}\right)$.

active space:
$\pi$\begin{tabular}{ccc|cc}
$\pi$ & $\pi$ & $\pi$ & $\pi$ & $\pi$ \\
7 & 13 & 12 & 14 & 15 \\
reference configurations: \\
2 & 2 & 2 & 0 & 0 \\
2 & 2 & 1 & 1 & 0
\end{tabular}

Cartesian coordinates:

10

$\begin{array}{lrrr}\mathrm{H} & 0.00000 & 1.84797 & 0.08460 \\ \mathrm{H} & 2.15928 & 0.38269 & 0.09511 \\ \mathrm{H} & -2.15928 & 0.38269 & 0.09511 \\ \mathrm{H} & -1.34824 & -2.18453 & 0.47071 \\ \mathrm{H} & 1.34824 & -2.18453 & 0.47071 \\ \mathrm{~N} & 0.00000 & 0.80356 & 0.07160 \\ \mathrm{C} & -1.14559 & 0.00000 & 0.00000 \\ \mathrm{C} & 1.14559 & 0.00000 & 0.00000 \\ \mathrm{C} & 0.72485 & -1.41831 & 0.00000 \\ \mathrm{C} & -0.72485 & -1.41831 & 0.00000\end{array}$


Table S309 Employed active space, reference configurations, and Cartesian coordinates of the OM3/MRCISD-optimized excited-state equilibrium geometry of the $1{ }^{1} B_{2}\left({ }^{1} \pi \pi^{*}\right)$ state of pyrrole (excited-state energy: $-784.49299 \mathrm{eV}$ ).

active space:
\begin{tabular}{ccc|cc}
$\pi$ & $\pi$ & $\pi$ & $\pi$ & $\pi$ \\
7 & 13 & 12 & 14 & 18 \\
reference configurations: \\
2 \\
2 & 2 & 2 & 0 & 0 \\
2 & 2 & 1 & 0 & 1 \\
2 & 1 & 2 & 1 & 0
\end{tabular}

Cartesian coordinates:

10

$\begin{array}{lrrr}\mathrm{H} & 0.00000 & 0.00000 & 0.00000 \\ \mathrm{H} & 2.16285 & -1.51922 & 0.00000 \\ \mathrm{H} & -2.16285 & -1.51922 & 0.00000 \\ \mathrm{H} & -1.37169 & -4.12956 & 0.00000 \\ \mathrm{H} & 1.37169 & -4.12956 & 0.00000 \\ \mathrm{~N} & 0.00000 & -1.04240 & 0.00000 \\ \mathrm{C} & -1.13519 & -1.89400 & 0.00000 \\ \mathrm{C} & 1.13519 & -1.89400 & 0.00000 \\ \mathrm{C} & 0.69205 & -3.28495 & 0.00000 \\ \mathrm{C} & -0.69205 & -3.28495 & 0.00000\end{array}$


Table S310 Employed active space, reference configurations, and Cartesian coordinates of the OM3/MRCISD-optimized excited-state equilibrium geometry of the $2{ }^{1} A^{\prime}\left({ }^{1} \pi \pi^{*}\right)$ state of cis-PSB3 (excited-state energy: $\left.-948.90450 \mathrm{eV}\right)$.

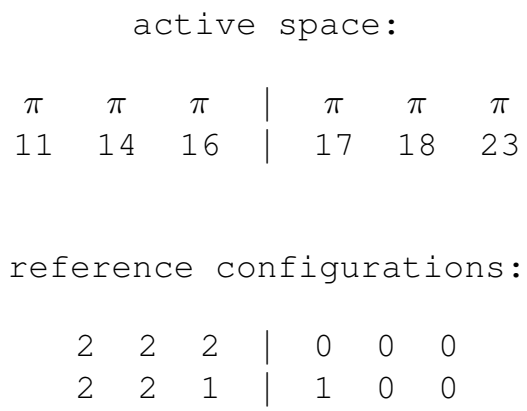


Table S311 Employed active space, reference configurations, and Cartesian coordinates of the OM3/MRCISD-optimized excited-state equilibrium geometry of the $2{ }^{1} A^{\prime}\left({ }^{1} \pi \pi^{*}\right)$ state of 3Me-cis-PSB5 (excited-state energy: $-1664.27080 \mathrm{eV}$ ).

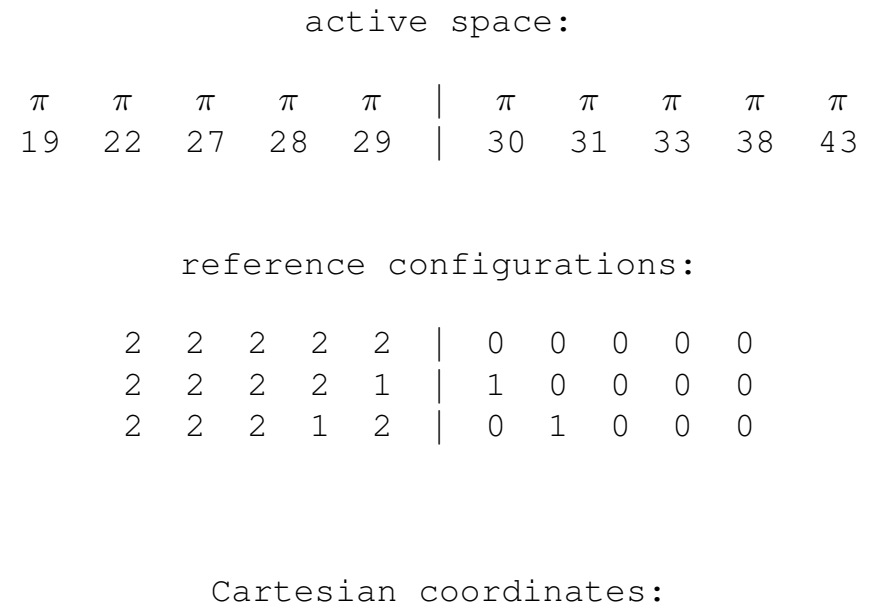

\begin{tabular}{lrrr}
25 & & & \\
& & & \\
$\mathrm{C}$ & 0.00000 & 0.00000 & 0.00000 \\
$\mathrm{C}$ & 1.46009 & 0.00000 & 0.00000 \\
$\mathrm{C}$ & 2.34816 & -1.06823 & 0.00000 \\
$\mathrm{C}$ & 3.76487 & -0.70793 & 0.00000 \\
$\mathrm{C}$ & 4.82420 & -1.62755 & 0.00000 \\
$\mathrm{~N}$ & 6.13141 & -1.21356 & 0.00000 \\
$\mathrm{C}$ & 1.93532 & -2.50642 & 0.00000 \\
$\mathrm{C}$ & -0.85588 & -1.08279 & 0.00000 \\
$\mathrm{C}$ & -2.26951 & -0.87824 & 0.00000 \\
$\mathrm{C}$ & -3.18330 & -1.96259 & 0.00000 \\
$\mathrm{C}$ & -4.53841 & -1.75031 & 0.00000 \\
$\mathrm{H}$ & -0.45354 & 1.01500 & 0.00000 \\
$\mathrm{H}$ & 1.89553 & 1.01729 & 0.00000 \\
$\mathrm{H}$ & 4.00946 & 0.36685 & 0.00000 \\
$\mathrm{H}$ & 4.63944 & -2.72457 & 0.00000 \\
$\mathrm{H}$ & 6.93303 & -1.87166 & 0.00000 \\
$\mathrm{H}$ & 6.40330 & -0.21191 & 0.00000 \\
$\mathrm{H}$ & 2.80769 & -3.18753 & 0.00000 \\
$\mathrm{H}$ & 1.36691 & -2.72214 & 0.90927 \\
$\mathrm{H}$ & 1.36691 & -2.72214 & -0.90927 \\
$\mathrm{H}$ & -0.49298 & -2.11634 & 0.00000 \\
$\mathrm{H}$ & -2.66378 & 0.16039 & 0.00000 \\
$\mathrm{H}$ & -2.78383 & -2.98814 & 0.00000 \\
\hline & & Continued \\
& & &
\end{tabular}

S351 
Continued from previous page

\begin{tabular}{llll}
\hline $\mathrm{H}$ & -5.25115 & -2.57930 & 0.00000 \\
$\mathrm{H}$ & -4.96851 & -0.74287 & 0.00000
\end{tabular}

S352 
Table S312 Employed active space, reference configurations, and Cartesian coordinates of the OM3/MRCISD-optimized excited-state equilibrium geometry of the $1{ }^{1} B_{u}\left({ }^{1} \pi \pi^{*}\right)$ state of trans-stilbene (excited-state energy: $-1924.63216 \mathrm{eV}$ ).

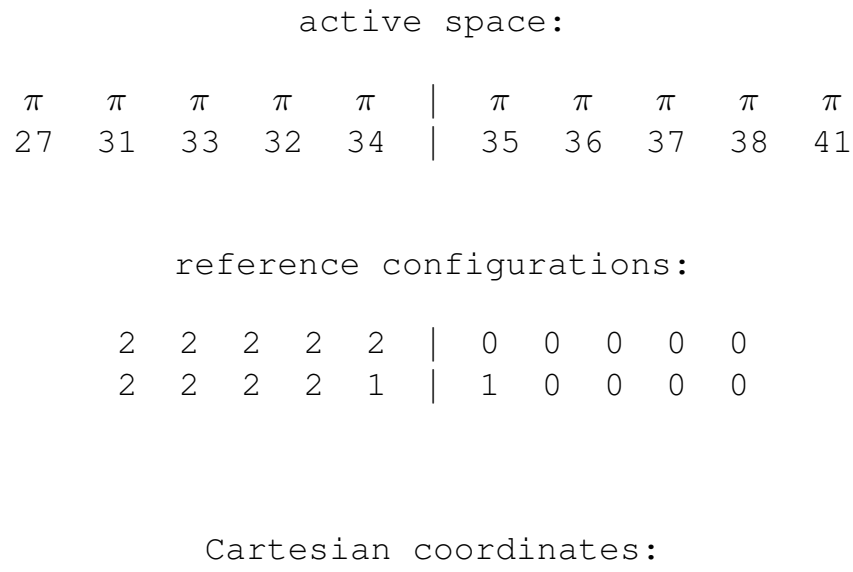


Continued from previous page

$\begin{array}{llll}\mathrm{H} & 4.62035 & -1.32865 & 0.00000 \\ \mathrm{H} & 2.62083 & 0.15549 & 0.00000\end{array}$

$\begin{array}{llll}\mathrm{H} & 2.62083 & 0.15549 & 0.00000\end{array}$

S354 
Table S313 Employed active space, reference configurations, and Cartesian coordinates of the OM3/MRCISD-optimized excited-state equilibrium geometry of the $1{ }^{1} B\left({ }^{1} \pi \pi^{*}\right)$ state of stilbene (excited-state energy: $-1924.02427 \mathrm{eV}$ ).

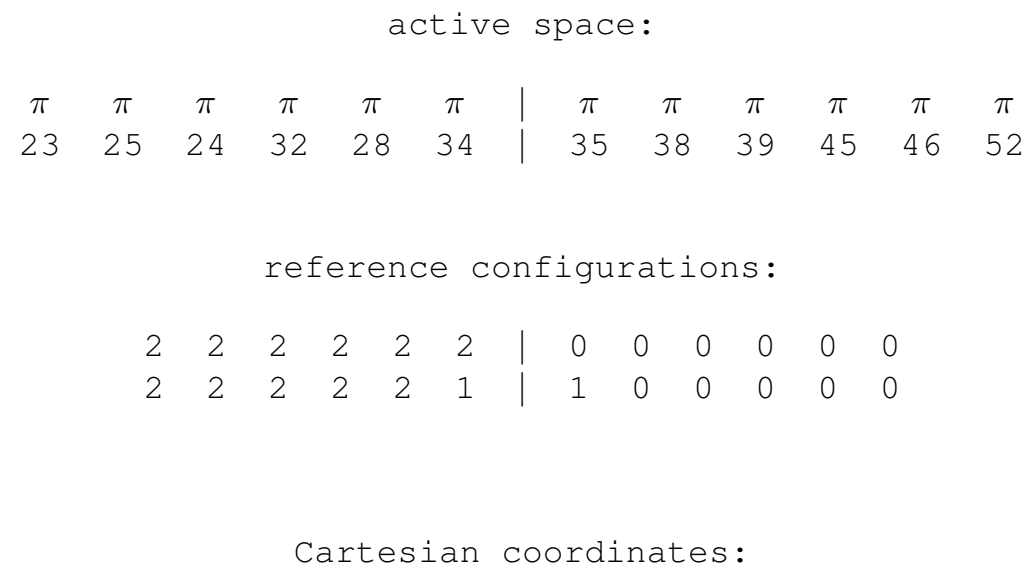

Continued on next page

S355 
Continued from previous page

\begin{tabular}{rrrr}
\hline $\mathrm{H}$ & 3.17738 & -1.11261 & 3.35658 \\
$\mathrm{H}$ & 1.69341 & 0.25346 & 1.87641
\end{tabular}

S356 
Table S314 Employed active space, reference configurations, and Cartesian coordinates of the OM3/MRCISD-optimized excited-state equilibrium geometry of the $2{ }^{1} A^{\prime}\left({ }^{1} \pi \pi^{*}\right)$ state of styrene (excited-state energy: $-1115.24224 \mathrm{eV}$ ).

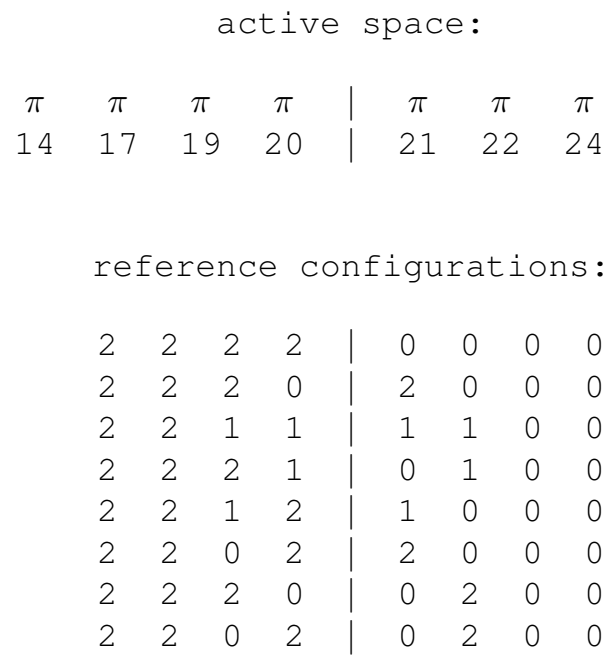

Cartesian coordinates:

16

$\begin{array}{lrrr}\mathrm{C} & 1.52962 & -0.06238 & 0.00000 \\ \mathrm{C} & 0.16700 & -0.08592 & 0.00000 \\ \mathrm{C} & 2.37480 & -1.22693 & 0.00000 \\ \mathrm{C} & 1.85152 & -2.56862 & 0.00000 \\ \mathrm{C} & 3.80670 & -1.05075 & 0.00000 \\ \mathrm{C} & 2.73529 & -3.68642 & 0.00000 \\ \mathrm{C} & 4.67692 & -2.17882 & 0.00000 \\ \mathrm{C} & 4.14054 & -3.49071 & 0.00000 \\ \mathrm{H} & 2.04979 & 0.91664 & 0.00000 \\ \mathrm{H} & -0.42380 & 0.82955 & 0.00000 \\ \mathrm{H} & -0.40344 & -1.01449 & 0.00000 \\ \mathrm{H} & 0.76372 & -2.73588 & 0.00000 \\ \mathrm{H} & 4.22682 & -0.03245 & 0.00000 \\ \mathrm{H} & 2.32197 & -4.70651 & 0.00000 \\ \mathrm{H} & 5.76684 & -2.02769 & 0.00000 \\ \mathrm{H} & 4.81566 & -4.36062 & 0.00000\end{array}$


Table S315 Employed active space, reference configurations, and Cartesian coordinates of the OM3/MRCISD-optimized excited-state equilibrium geometry of the $1^{3} A^{\prime}\left({ }^{3} \pi \pi^{*}\right)$ state of styrene (excited-state energy: $-1116.63554 \mathrm{eV}$ ).

\begin{tabular}{|c|c|c|c|c|c|c|c|c|c|}
\hline \multirow{2}{*}{$\begin{array}{c}\pi \\
13\end{array}$} & $\pi$ & \multicolumn{2}{|c|}{$\pi$} & $\pi$ & \multicolumn{2}{|c|}{$\pi$} & \multicolumn{2}{|c|}{$\pi$} & $\pi$ \\
\hline & 17 & 1 & & 20 & & 21 & & 2 & \\
\hline \multicolumn{5}{|c|}{ reference } & \multicolumn{5}{|c|}{ configurations: } \\
\hline & 2 & 2 & 2 & 2 & & 0 & 0 & 0 & 0 \\
\hline & 2 & 2 & 2 & 1 & & 1 & 0 & 0 & 0 \\
\hline & 2 & 2 & 1 & 2 & & 1 & 0 & 0 & 0 \\
\hline & 2 & 2 & 2 & 1 & & 0 & 1 & 0 & 0 \\
\hline & 2 & 2 & 1 & 2 & 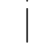 & 0 & 1 & 0 & 0 \\
\hline \multicolumn{5}{|c|}{ Cartesian } & \multicolumn{5}{|c|}{ coordinates: } \\
\hline \multicolumn{10}{|l|}{16} \\
\hline C & & .57 & 912 & & & .087 & & 0 & .00000 \\
\hline C & & .13 & 858 & & & .099 & & & .00000 \\
\hline C & & .37 & 875 & & & .230 & & & .00000 \\
\hline $\mathrm{C}$ & & .84 & 273 & & & .579 & & & .00000 \\
\hline $\mathrm{C}$ & & .82 & 775 & & & 090 & & & .00000 \\
\hline $\mathrm{C}$ & & .68 & 856 & & & .676 & & & .00000 \\
\hline $\mathrm{C}$ & & .64 & 998 & & & 202 & & & .00000 \\
\hline $\mathrm{C}$ & & $.0 s$ & 445 & & & .505 & & & .00000 \\
\hline $\mathrm{H}$ & & .06 & 970 & & & .903 & & & .00000 \\
\hline $\mathrm{H}$ & & .42 & 047 & & & .832 & & & .00000 \\
\hline $\mathrm{H}$ & & .42 & 484 & & & .029 & & & .00000 \\
\hline $\mathrm{H}$ & & .72 & 952 & & & .718 & & & .00000 \\
\hline $\mathrm{H}$ & & .25 & 348 & & & .072 & & & .00000 \\
\hline $\mathrm{H}$ & & .27 & 259 & & & 697 & & & .00000 \\
\hline $\mathrm{H}$ & & .72 & 604 & & & .085 & & & .00000 \\
\hline $\mathrm{H}$ & & .75 & 589 & & & .386 & & & .00000 \\
\hline
\end{tabular}


Table S316 Employed active space, reference configurations, and Cartesian coordinates of the OM3/MRCISD-optimized excited-state equilibrium geometry of the $1{ }^{1} B_{3 u}\left({ }^{1} n \pi^{*}\right)$ state of $s$-tetrazine (excited-state energy: $\left.-1139.20679 \mathrm{eV}\right)$.

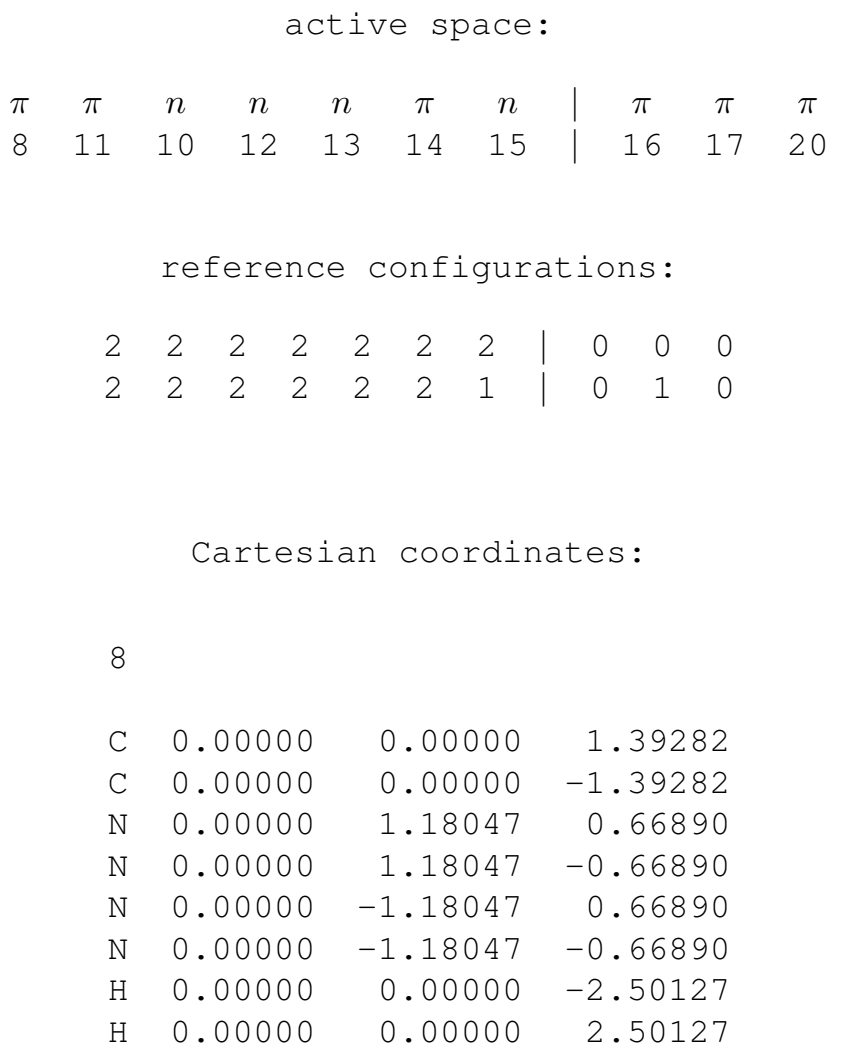


Table S317 Employed active space, reference configurations, and Cartesian coordinates of the OM3/MRCISD-optimized excited-state equilibrium geometry of the $2{ }^{1} A\left({ }^{1} n \pi^{*}\right)$ state of thymine (excited-state energy: $-1820.78577 \mathrm{eV}$ ).

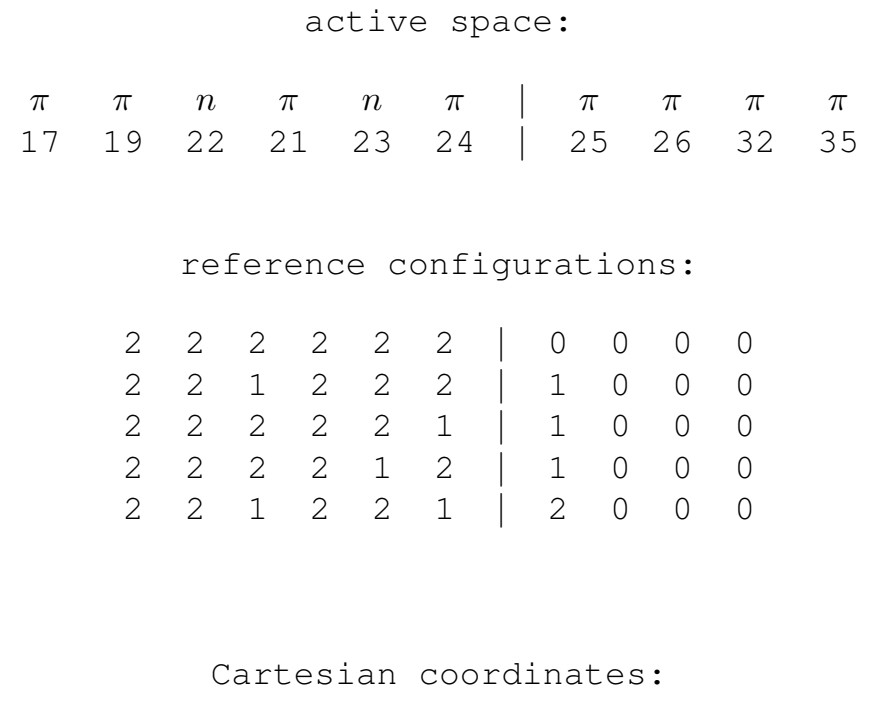

15

$\begin{array}{lrrr}\mathrm{N} & 0.00000 & 0.00000 & 0.00000 \\ \mathrm{C} & 1.37011 & 0.00000 & 0.00000 \\ \mathrm{C} & 2.00596 & 1.35970 & 0.00000 \\ \mathrm{C} & 1.24334 & 2.53156 & -0.28378 \\ \mathrm{~N} & -0.20331 & 2.34149 & -0.45404 \\ \mathrm{C} & -0.87837 & 1.14653 & -0.31548 \\ \mathrm{O} & 1.65654 & 3.71265 & -0.42765 \\ \mathrm{C} & 3.45443 & 1.42715 & 0.21222 \\ \mathrm{O} & -2.10434 & 0.92247 & -0.41460 \\ \mathrm{H} & 1.91203 & -0.84422 & 0.53039 \\ \mathrm{H} & -0.52964 & -0.89406 & 0.17795 \\ \mathrm{H} & -0.77473 & 3.19044 & -0.66868 \\ \mathrm{H} & 3.80574 & 2.47162 & 0.24064 \\ \mathrm{H} & 3.98978 & 0.89482 & -0.60405 \\ \mathrm{H} & 3.71887 & 0.94070 & 1.17166\end{array}$


Table S318 Employed active space, reference configurations, and Cartesian coordinates of the OM3/MRCISD-optimized excited-state equilibrium geometry of the $2{ }^{1} A^{\prime}\left({ }^{1} \pi \pi^{*}\right)$ state of uracil (excited-state energy: $-1665.66688 \mathrm{eV}$ ).

\begin{tabular}{|c|c|c|c|c|c|c|c|}
\hline \multirow{2}{*}{$\begin{array}{c}\pi \\
11\end{array}$} & $\pi$ & $\pi$ & $\pi$ & $\pi$ & \multicolumn{2}{|c|}{$\pi$} & $\pi$ \\
\hline & 14 & 16 & 19 & 21 & & 2 & 3 \\
\hline \multicolumn{4}{|c|}{ reference } & \multicolumn{4}{|c|}{ configurations: } \\
\hline & 2 & 22 & 2 & 2 & 0 & 0 & 0 \\
\hline & 2 & 22 & 2 & 1 & 1 & 0 & 0 \\
\hline & 2 & 22 & 2 & 0 & 2 & 0 & 0 \\
\hline \multicolumn{4}{|c|}{ Cartesian } & \multicolumn{4}{|c|}{ coordinates: } \\
\hline 12 & & & & & & & \\
\hline $\mathrm{N}$ & & .67542 & & 1.0 & & 0 & .00000 \\
\hline $\mathrm{N}$ & & .0394 & & -1.2 & & & .00000 \\
\hline C & & .6175 & & 0.0 & & & .00000 \\
\hline C & & .2885 & & -1.5 & & & .00000 \\
\hline C & & .2313 & & -0.4 & & & .00000 \\
\hline C & & .7823 & & 0.8 & & & .00000 \\
\hline O & & .4731 & & 1.9 & & & .00000 \\
\hline 0 & & .8684 & & 0.1 & & & .00000 \\
\hline $\mathrm{H}$ & & .6455 & & -2.6 & & & .00000 \\
\hline $\mathrm{H}$ & & $.0319^{\circ}$ & & 2.0 & 36 & & .00000 \\
\hline $\mathrm{H}$ & & .7628 & & -2.0 & 17 & & .00000 \\
\hline $\mathrm{H}$ & & $.2992^{\circ}$ & & -0.6 & 240 & & .00000 \\
\hline
\end{tabular}


4.1.4 Structural Parameters of the OM3/MRCI-Optimized ExcitedState Minima 
Table S319 Structural parameters of the OM3/MRCISDSD-optimized excitedstate equilibrium geometry of the $2{ }^{1} A\left({ }^{1} n \pi^{*}\right)$ state of $9 H$-adenine used for the statistical evaluations.

\begin{tabular}{ll}
$\begin{array}{l}\text { bond } \\
\text { or angle }\end{array}$ & $\begin{array}{l}\text { length } / \AA \\
\text { or angle } /\end{array}$ \\
\hline N1-C2 & 1.384 \\
C2-C3 & 1.477 \\
C2-C9 & 1.460 \\
C3-N4 & 1.400 \\
N4-C5 & 1.447 \\
N1-C5 & 1.367 \\
C3-N6 & 1.352 \\
N6-C7 & 1.415 \\
C7-N8 & 1.407 \\
N8-C9 & 1.355 \\
C9-N10 & 1.371 \\
C7-H11 & 1.098 \\
C5-H15 & 1.094 \\
N4-H14 & 1.037 \\
N10-H12 & 1.025 \\
N10-H13 & 1.025
\end{tabular}


Table S320 Structural parameters of the OM3/MRCISDSD-optimized excitedstate equilibrium geometry of the $2{ }^{1} A\left({ }^{1} n \pi^{*}\right)$ state of acetaldehyde used for the statistical evaluations.

\begin{tabular}{ll}
$\begin{array}{l}\text { bond } \\
\text { or angle }\end{array}$ & $\begin{array}{l}\text { length } / \AA \\
\text { or angle / } /\end{array}$ \\
\hline $\mathrm{C} 1-\mathrm{O} 4$ & 1.298 \\
$\mathrm{C} 1-\mathrm{C} 2$ & 1.502 \\
$\mathrm{C} 2-\mathrm{H} 7$ & 1.093 \\
$\mathrm{O} 4-\mathrm{C} 1-\mathrm{C} 2$ & 117.3 \\
$\mathrm{C} 2-\mathrm{C} 1-\mathrm{H} 3$ & 113.9
\end{tabular}


Table S321 Structural parameters of the OM3/MRCISD-optimized excitedstate equilibrium geometry of the $1^{3} A\left({ }^{3} n \pi^{*}\right)$ state of acetaldehyde used for the statistical evaluations.

\begin{tabular}{ll}
$\begin{array}{l}\text { bond } \\
\text { or angle }\end{array}$ & $\begin{array}{l}\text { length } / \AA \\
\text { or angle / } /\end{array}$ \\
\hline $\mathrm{C} 1-\mathrm{O} 4$ & 1.276 \\
$\mathrm{C} 1-\mathrm{C} 2$ & 1.520 \\
$\mathrm{C} 2-\mathrm{H} 7$ & 1.094 \\
$\mathrm{O} 4-\mathrm{C} 1-\mathrm{C} 2$ & 117.1 \\
$\mathrm{C} 2-\mathrm{C} 1-\mathrm{H} 3$ & 110.1
\end{tabular}


Table S322 Structural parameters of the OM3/MRCISD-optimized excitedstate equilibrium geometry of the $2{ }^{3} A\left({ }^{3} \pi \pi^{*}\right)$ state of acetaldehyde used for the statistical evaluations.

\begin{tabular}{ll}
$\begin{array}{l}\text { bond } \\
\text { or angle }\end{array}$ & $\begin{array}{l}\text { length } / \AA \\
\text { or angle / } /\end{array}$ \\
\hline $\mathrm{C} 1-\mathrm{O} 4$ & 1.416 \\
$\mathrm{C} 1-\mathrm{C} 2$ & 1.481 \\
$\mathrm{C} 2-\mathrm{H} 7$ & 1.089 \\
$\mathrm{O} 4-\mathrm{C} 1-\mathrm{C} 2$ & 111.6 \\
$\mathrm{C} 2-\mathrm{C} 1-\mathrm{H} 3$ & 120.4
\end{tabular}


Table S323 Structural parameters of the OM3/MRCISD-optimized excitedstate equilibrium geometry of the $1^{1} A^{\prime \prime}\left({ }^{1} n \pi^{*}\right)$ state of acetone used for the statistical evaluations.

\begin{tabular}{ll} 
bond & $\begin{array}{l}\text { length } / \AA \\
\text { or angle } \\
\text { or angle / }\end{array}$ \\
\hline $\mathrm{C} 2-\mathrm{O} 3$ & 1.310 \\
$\mathrm{C} 1-\mathrm{C} 2$ & 1.498 \\
$\mathrm{C} 1-\mathrm{C} 2-\mathrm{C} 4$ & 120.0
\end{tabular}


Table S324 Structural parameters of the OM3/MRCISD-optimized excitedstate equilibrium geometry of the $1{ }^{3} A^{\prime \prime}\left({ }^{3} n \pi^{*}\right)$ state of acetone used for the statistical evaluations.

\begin{tabular}{ll} 
bond & $\begin{array}{l}\text { length } / \AA \\
\text { or angle } \\
\text { or angle / }\end{array}$ \\
\hline $\mathrm{C} 2-\mathrm{O} 3$ & 1.284 \\
$\mathrm{C} 1-\mathrm{C} 2$ & 1.524 \\
$\mathrm{C} 1-\mathrm{C} 2-\mathrm{C} 4$ & 116.0
\end{tabular}


Table S325 Structural parameters of the OM3/MRCISD-optimized excitedstate equilibrium geometry of the $1^{3} A^{\prime}\left({ }^{3} \pi \pi^{*}\right)$ state of acetone used for the statistical evaluations.

\begin{tabular}{ll} 
bond & $\begin{array}{l}\text { length } / \AA \\
\text { or angle } \\
\text { or angle / }\end{array}$ \\
\hline $\mathrm{C} 2-\mathrm{O} 3$ & 1.439 \\
$\mathrm{C} 1-\mathrm{C} 2$ & 1.482 \\
$\mathrm{C} 1-\mathrm{C} 2-\mathrm{C} 4$ & 122.0
\end{tabular}


Table S326 Structural parameters of the OM3/MRCISDTQ-optimized excitedstate equilibrium geometry of the $1{ }^{1} A^{\prime \prime}\left({ }^{1} n \pi^{*}\right)$ state of acetophenone used for the statistical evaluations.

\begin{tabular}{ll}
$\begin{array}{l}\text { bond } \\
\text { or angle }\end{array}$ & $\begin{array}{l}\text { length } / \AA \\
\text { or angle / }\end{array}$ \\
\hline $\mathrm{C} 7-\mathrm{C} 9$ & 1.426 \\
$\mathrm{C} 9-\mathrm{C} 11$ & 1.394 \\
$\mathrm{C} 11-\mathrm{C} 12$ & 1.411 \\
$\mathrm{C} 10-\mathrm{C} 12$ & 1.408 \\
$\mathrm{C} 8-\mathrm{C} 10$ & 1.393 \\
$\mathrm{C} 7-\mathrm{C} 8$ & 1.434 \\
$\mathrm{C} 1-\mathrm{C} 7$ & 1.424 \\
$\mathrm{C} 1-\mathrm{C} 3$ & 1.517 \\
$\mathrm{C} 1-\mathrm{O} 2$ & 1.313 \\
\end{tabular}


Table S327 Structural parameters of the OM3/MRCISD-optimized excitedstate equilibrium geometry of the $2{ }^{1} A^{\prime}\left({ }^{1} \pi \pi^{*}\right)$ state of acetophenone used for the statistical evaluations.

\begin{tabular}{ll}
$\begin{array}{l}\text { bond } \\
\text { or angle }\end{array}$ & $\begin{array}{l}\text { length } / \AA \\
\text { or angle / }\end{array}$ \\
\hline C7-C9 & 1.438 \\
C9-C11 & 1.430 \\
C11-C12 & 1.426 \\
C10-C12 & 1.419 \\
C $8-C 10$ & 1.431 \\
C7-C8 & 1.440 \\
C1-C7 & 1.479 \\
C1-C3 & 1.522 \\
C1-O2 & 1.246
\end{tabular}


Table S328 Structural parameters of the OM3/MRCISDT-optimized excitedstate equilibrium geometry of the $1^{3} A^{\prime \prime}\left({ }^{3} n \pi^{*}\right)$ state of acetophenone used for the statistical evaluations.

\begin{tabular}{ll}
$\begin{array}{l}\text { bond } \\
\text { or angle }\end{array}$ & $\begin{array}{l}\text { length } / \AA \\
\text { or angle / }\end{array}$ \\
\hline C7-C9 & 1.423 \\
C9-C11 & 1.396 \\
C11-C12 & 1.410 \\
C10-C12 & 1.407 \\
C $8-C 10$ & 1.394 \\
C7-C8 & 1.430 \\
C1-C7 & 1.432 \\
C1-C3 & 1.527 \\
C1-O2 & 1.299 \\
\end{tabular}


Table S329 Structural parameters of the OM3/MRCISD-optimized excitedstate equilibrium geometry of the $1^{3} A^{\prime}\left({ }^{3} \pi \pi^{*}\right)$ state of acetophenone used for the statistical evaluations.

\begin{tabular}{ll}
$\begin{array}{l}\text { bond } \\
\text { or angle }\end{array}$ & $\begin{array}{l}\text { length } / \AA \\
\text { or angle / }\end{array}$ \\
\hline C7-C9 & 1.474 \\
C9-C11 & 1.373 \\
C11-C12 & 1.445 \\
C10-C12 & 1.447 \\
C $8-C 10 ~$ & 1.373 \\
C7-C8 & 1.472 \\
C1-C7 & 1.460 \\
C1-C3 & 1.517 \\
C1-O2 & 1.255
\end{tabular}


Table S330 Structural parameters of the OM3/MRCISD-optimized excitedstate equilibrium geometry of the $1{ }^{1} A_{2}\left({ }^{1} \pi \pi^{*}\right)$ state of acetylene used for the statistical evaluations.

\begin{tabular}{ll} 
bond & $\begin{array}{l}\text { length } / \AA \\
\text { or angle } \\
\text { or angle / }\end{array}$ \\
\hline $\mathrm{C} 1-\mathrm{C} 2$ & 1.334 \\
$\mathrm{C} 1-\mathrm{H} 3$ & 1.124 \\
$\mathrm{C} 1-\mathrm{C} 2-\mathrm{H} 4$ & 149.1
\end{tabular}


Table S331 Structural parameters of the OM3/MRCISDT-optimized excitedstate equilibrium geometry of the $2{ }^{1} A_{2}\left({ }^{1} \pi \pi^{*}\right)$ state of acetylene used for the statistical evaluations.

\begin{tabular}{ll} 
bond & $\begin{array}{l}\text { length } / \AA \\
\text { or angle } \\
\text { or angle / }\end{array}$ \\
\hline $\mathrm{C} 1-\mathrm{C} 2$ & 1.328 \\
$\mathrm{C} 1-\mathrm{H} 3$ & 1.059 \\
$\mathrm{C} 1-\mathrm{C} 2-\mathrm{H} 4$ & 180.0
\end{tabular}


Table S332 Structural parameters of the OM3/MRCISD-optimized excitedstate equilibrium geometry of the $1{ }^{1} B_{2}\left({ }^{1} \pi \pi^{*}\right)$ state of acetylene used for the statistical evaluations.

\begin{tabular}{ll} 
bond & $\begin{array}{l}\text { length / } \AA \\
\text { or angle } \\
\text { or angle / }\end{array}$ \\
\hline $\mathrm{C} 1-\mathrm{C} 2$ & 1.317 \\
$\mathrm{C} 1-\mathrm{H} 3$ & 1.136 \\
$\mathrm{C} 1-\mathrm{C} 2-\mathrm{H} 4$ & 150.7
\end{tabular}


Table S333 Structural parameters of the OM3/MRCISD-optimized excitedstate equilibrium geometry of the $1^{3} A_{2}\left({ }^{3} \pi \pi^{*}\right)$ state of acetylene used for the statistical evaluations.

\begin{tabular}{ll} 
bond & $\begin{array}{l}\text { length } / \AA \\
\text { or angle } \\
\text { or angle / }\end{array}$ \\
\hline $\mathrm{C} 1-\mathrm{C} 2$ & 1.344 \\
$\mathrm{C} 1-\mathrm{H} 3$ & 1.110 \\
$\mathrm{C} 1-\mathrm{C} 2-\mathrm{H} 4$ & 144.6
\end{tabular}


Table S334 Structural parameters of the OM3/MRCISD-optimized excitedstate equilibrium geometry of the $2{ }^{3} A_{2}\left({ }^{3} \pi \pi^{*}\right)$ state of acetylene used for the statistical evaluations.

\begin{tabular}{ll} 
bond & $\begin{array}{l}\text { length } / \AA \\
\text { or angle } \\
\text { or angle / }\end{array}$ \\
\hline $\mathrm{C} 1-\mathrm{C} 2$ & 1.330 \\
$\mathrm{C} 1-\mathrm{H} 3$ & 1.061 \\
$\mathrm{C} 1-\mathrm{C} 2-\mathrm{H} 4$ & 180.0
\end{tabular}


Table S335 Structural parameters of the OM3/MRCISDT-optimized excitedstate equilibrium geometry of the $1{ }^{3} B_{2}\left({ }^{3} \pi \pi^{*}\right)$ state of acetylene used for the statistical evaluations.

\begin{tabular}{ll} 
bond & $\begin{array}{l}\text { length } / \AA \\
\text { or angle } \\
\text { or angle / }\end{array}$ \\
\hline $\mathrm{C} 1-\mathrm{C} 2$ & 1.330 \\
$\mathrm{C} 1-\mathrm{H} 3$ & 1.106 \\
$\mathrm{C} 1-\mathrm{C} 2-\mathrm{H} 4$ & 133.9
\end{tabular}


Table S336 Structural parameters of the OM3/MRCISD-optimized excitedstate equilibrium geometry of the $1{ }^{1} A_{u}\left({ }^{1} \pi \pi^{*}\right)$ state of acetylene used for the statistical evaluations.

\begin{tabular}{ll} 
bond & $\begin{array}{l}\text { length } / \AA \\
\text { or angle } \\
\text { or angle / }\end{array}$ \\
\hline $\mathrm{C} 1-\mathrm{C} 2$ & 1.370 \\
$\mathrm{C} 1-\mathrm{H} 3$ & 1.125 \\
$\mathrm{C} 1-\mathrm{C} 2-\mathrm{H} 4$ & 125.1
\end{tabular}


Table S337 Structural parameters of the OM3/MRCISD-optimized excitedstate equilibrium geometry of the $2{ }^{1} A_{u}\left({ }^{1} \pi \pi^{*}\right)$ state of acetylene used for the statistical evaluations.

\begin{tabular}{ll} 
bond & $\begin{array}{l}\text { length } / \AA \\
\text { or angle } \\
\text { or angle / }\end{array}$ \\
\hline $\mathrm{C} 1-\mathrm{C} 2$ & 1.322 \\
$\mathrm{C} 1-\mathrm{H} 3$ & 1.066 \\
$\mathrm{C} 1-\mathrm{C} 2-\mathrm{H} 4$ & 171.7
\end{tabular}


Table S338 Structural parameters of the OM3/MRCISD-optimized excitedstate equilibrium geometry of the $1{ }^{1} B_{u}\left({ }^{1} \pi \pi^{*}\right)$ state of acetylene used for the statistical evaluations.

\begin{tabular}{ll} 
bond & $\begin{array}{l}\text { length } / \AA \\
\text { or angle } \\
\text { or angle / }\end{array}$ \\
\hline $\mathrm{C} 1-\mathrm{C} 2$ & 1.327 \\
$\mathrm{C} 1-\mathrm{H} 3$ & 1.103 \\
$\mathrm{C} 1-\mathrm{C} 2-\mathrm{H} 4$ & 145.4
\end{tabular}


Table S339 Structural parameters of the OM3/MRCISD-optimized excitedstate equilibrium geometry of the $1^{3} A_{u}\left({ }^{3} \pi \pi^{*}\right)$ state of acetylene used for the statistical evaluations.

\begin{tabular}{ll} 
bond & $\begin{array}{l}\text { length } / \AA \\
\text { or angle } \\
\text { or angle / }\end{array}$ \\
\hline $\mathrm{C} 1-\mathrm{C} 2$ & 1.383 \\
$\mathrm{C} 1-\mathrm{H} 3$ & 1.122 \\
$\mathrm{C} 1-\mathrm{C} 2-\mathrm{H} 4$ & 122.6
\end{tabular}


Table S340 Structural parameters of the OM3/MRCISD-optimized excitedstate equilibrium geometry of the $2{ }^{3} A_{u}\left({ }^{3} \pi \pi^{*}\right)$ state of acetylene used for the statistical evaluations.

\begin{tabular}{ll} 
bond & $\begin{array}{l}\text { length } / \AA \\
\text { or angle } \\
\text { or angle / }\end{array}$ \\
\hline $\mathrm{C} 1-\mathrm{C} 2$ & 1.332 \\
$\mathrm{C} 1-\mathrm{H} 3$ & 1.069 \\
$\mathrm{C} 1-\mathrm{C} 2-\mathrm{H} 4$ & 164.1
\end{tabular}


Table S341 Structural parameters of the OM3/MRCISD-optimized excitedstate equilibrium geometry of the $1{ }^{3} B_{u}\left({ }^{3} \pi \pi^{*}\right)$ state of acetylene used for the statistical evaluations.

\begin{tabular}{ll} 
bond & $\begin{array}{l}\text { length } / \AA \\
\text { or angle } \\
\text { or angle / }\end{array}$ \\
\hline $\mathrm{C} 1-\mathrm{C} 2$ & 1.340 \\
$\mathrm{C} 1-\mathrm{H} 3$ & 1.089 \\
$\mathrm{C} 1-\mathrm{C} 2-\mathrm{H} 4$ & 135.9
\end{tabular}


Table S342 Structural parameters of the OM3/MRCISD-optimized excitedstate equilibrium geometry of the $2{ }^{3} B_{u}\left({ }^{3} \pi \pi^{*}\right)$ state of acetylene used for the statistical evaluations.

\begin{tabular}{ll} 
bond & $\begin{array}{l}\text { length } / \AA \\
\text { or angle } \\
\text { or angle / }\end{array}$ \\
\hline $\mathrm{C} 1-\mathrm{C} 2$ & 1.345 \\
$\mathrm{C} 1-\mathrm{H} 3$ & 1.075 \\
$\mathrm{C} 1-\mathrm{C} 2-\mathrm{H} 4$ & 152.8
\end{tabular}


Table S343 Structural parameters of the OM3/MRCISD-optimized excitedstate equilibrium geometry of the $1^{1} A^{\prime \prime}\left({ }^{1} n \pi^{*}\right)$ state of acrolein used for the statistical evaluations.

\begin{tabular}{ll} 
bond & $\begin{array}{l}\text { length / } \AA \\
\text { or angle } \\
\text { or angle / }\end{array}$ \\
\hline $\mathrm{C} 3-\mathrm{O} 4$ & 1.305 \\
$\mathrm{C} 2-\mathrm{C} 3$ & 1.401 \\
$\mathrm{C} 1-\mathrm{C} 2$ & 1.390 \\
$\mathrm{O} 4-\mathrm{C} 3-\mathrm{C} 2$ & 123.0 \\
$\mathrm{C} 3-\mathrm{C} 2-\mathrm{C} 1$ & 119.8
\end{tabular}


Table S344 Structural parameters of the OM3/MRCISD-optimized excitedstate equilibrium geometry of the $1^{3} A^{\prime \prime}\left({ }^{3} n \pi^{*}\right)$ state of acrolein used for the statistical evaluations.

\begin{tabular}{ll} 
bond & $\begin{array}{l}\text { length / } \AA \\
\text { or angle } \\
\text { or angle / }\end{array}$ \\
\hline $\mathrm{C} 3-\mathrm{O} 4$ & 1.294 \\
$\mathrm{C} 2-\mathrm{C} 3$ & 1.412 \\
$\mathrm{C} 1-\mathrm{C} 2$ & 1.380 \\
$\mathrm{O} 4-\mathrm{C} 3-\mathrm{C} 2$ & 123.3 \\
$\mathrm{C} 3-\mathrm{C} 2-\mathrm{C} 1$ & 120.1
\end{tabular}


Table S345 Structural parameters of the OM3/MRCISD-optimized excitedstate equilibrium geometry of the $1^{3} A^{\prime}\left({ }^{3} \pi \pi^{*}\right)$ state of acrolein used for the statistical evaluations.

\begin{tabular}{ll} 
bond & $\begin{array}{l}\text { length / } \AA \\
\text { or angle } \\
\text { or angle / }\end{array}$ \\
\hline $\mathrm{C} 3-\mathrm{O} 4$ & 1.258 \\
$\mathrm{C} 2-\mathrm{C} 3$ & 1.430 \\
$\mathrm{C} 1-\mathrm{C} 2$ & 1.483 \\
$\mathrm{O} 4-\mathrm{C} 3-\mathrm{C} 2$ & 120.4 \\
$\mathrm{C} 3-\mathrm{C} 2-\mathrm{C} 1$ & 121.5
\end{tabular}


Table S346 Structural parameters of the OM3/MRCISD-optimized excitedstate equilibrium geometry of the $2{ }^{1} A\left({ }^{1} \pi \pi^{*}\right)$ state of 2-amino-9-methylpurine used for the statistical evaluations.

\begin{tabular}{ll} 
bond & $\begin{array}{c}\text { length } / \AA \\
\text { or angle }\end{array}$ \\
or angle / $/$ \\
\hline C1-N2 & 1.377 \\
C1-N3 & 1.405 \\
N3-C4 & 1.348 \\
C4-C5 & 1.510 \\
C5-C6 & 1.464 \\
N2-C6 & 1.353 \\
C5-N9 & 1.354 \\
C8-N9 & 1.385 \\
N7-C8 & 1.419 \\
C4-N7 & 1.445 \\
C8-H13 & 1.097 \\
C1-H10 & 1.388 \\
N7-C11 & 1.459 \\
C6-H12 & 1.111 \\
N2-C1-N3 & 121.8 \\
C1-N3-C4 & 117.7 \\
N3-C4-N5 & 122.7 \\
C4-C5-C6 & 114.0 \\
C5-C6-N2 & 118.2 \\
C6-N2-C1 & 122.9 \\
C5-C4-N7 & 104.1 \\
C4-N7-C8 & 105.6 \\
N7-C8-N9 & 111.3 \\
C8-N9-C5 & 108.0 \\
N9-C5-C4 & 109.2 \\
N2-C1-N10 & 120.2 \\
C8-N7-C11 & 126.6 \\
C5-C6-H12 & 115.3 \\
N7-C8-H13 & 123.8 \\
&
\end{tabular}


Table S347 Structural parameters of the OM3/MRCISD-optimized excitedstate equilibrium geometry of the $1{ }^{1} B_{2}\left({ }^{1} \pi \pi^{*}\right)$ state of aniline used for the statistical evaluations.

\begin{tabular}{ll}
$\begin{array}{l}\text { bond } \\
\text { or angle }\end{array}$ & $\begin{array}{c}\text { length } / \AA \\
\text { or angle } /\end{array}$ \\
\hline C1-C2 & 1.441 \\
C2-C3 & 1.420 \\
C3-C4 & 1.417 \\
C1-N7 & 1.386 \\
N7-H8 & 1.034 \\
C2-H10 & 1.097 \\
C3-H11 & 1.099 \\
C4-H12 & 1.105 \\
C1-C2-C3 & 118.6 \\
C2-C1-C6 & 121.4 \\
C2-C3-C4 & 119.9 \\
C3-C4-C5 & 121.6 \\
N7-C1-C6 & 119.3 \\
H8-N7-H9 & 114.7 \\
H8-N7-C1 & 122.6 \\
H14-C6-C1 & 120.2 \\
H14-C6-C5 & 121.2 \\
H13-C5-C4 & 120.3 \\
H13-C5-C6 & 119.7 \\
H12-C4-C3 & 119.2
\end{tabular}


Table S348 Structural parameters of the OM3/MRCISD-optimized excitedstate equilibrium geometry of the $1^{3} A^{\prime}\left({ }^{3} \pi \pi^{*}\right)$ state of aniline used for the statistical evaluations.

\begin{tabular}{ll}
$\begin{array}{l}\text { bond } \\
\text { or angle }\end{array}$ & $\begin{array}{c}\text { length } / \AA \\
\text { or angle } /\end{array}$ \\
\hline C1-C2 & 1.493 \\
C2-C3 & 1.374 \\
C3-C4 & 1.417 \\
C1-N7 & 1.398 \\
N7-H8 & 1.038 \\
C2-H10 & 1.100 \\
C3-H11 & 1.102 \\
C4-H12 & 1.102 \\
C1-C2-C3 & 120.2 \\
C2-C1-C6 & 115.4 \\
C2-C3-C4 & 120.5 \\
C3-C4-C5 & 121.0 \\
N7-C1-C6 & 112.6 \\
H8-N7-H9 & 112.1 \\
H8-N7-C1 & 122.5 \\
H14-C6-C1 & 117.7 \\
H14-C6-C5 & 122.1 \\
H13-C5-C4 & 118.6 \\
H13-C5-C6 & 120.8 \\
H12-C4-C3 & 119.4
\end{tabular}


Table S349 Structural parameters of the OM3/MRCISD-optimized excitedstate equilibrium geometry of the $1{ }^{1} A^{\prime \prime}\left({ }^{1} n \pi^{*}\right)$ state of benzaldehyde used for the statistical evaluations.

\begin{tabular}{ll} 
bond & $\begin{array}{l}\text { length / } \AA \\
\text { or angle } \\
\text { or angle / }\end{array}$ \\
\hline C4-C6 & 1.423 \\
C6-C8 & 1.390 \\
C $8-C 9$ & 1.413 \\
C7-C9 & 1.409 \\
C5-C7 & 1.387 \\
C4-C5 & 1.435 \\
C1-C4 & 1.410 \\
C1-O2 & 1.299
\end{tabular}


Table S350 Structural parameters of the OM3/MRCISD-optimized excitedstate equilibrium geometry of the $2{ }^{1} A^{\prime}\left({ }^{1} \pi \pi^{*}\right)$ state of benzaldehyde used for the statistical evaluations.

\begin{tabular}{ll}
$\begin{array}{l}\text { bond } \\
\text { or angle }\end{array}$ & $\begin{array}{l}\text { length } / \AA \\
\text { or angle } /{ }^{\circ}\end{array}$ \\
\hline C4-C6 & 1.435 \\
C6-C8 & 1.426 \\
C $8-C 9$ & 1.420 \\
C7-C9 & 1.420 \\
C5-C7 & 1.426 \\
C4-C5 & 1.431 \\
C1-C4 & 1.468 \\
C1-O2 & 1.239
\end{tabular}


Table S351 Structural parameters of the OM3/MRCISD-optimized excitedstate equilibrium geometry of the $1^{3} A^{\prime \prime}\left({ }^{3} n \pi^{*}\right)$ state of benzaldehyde used for the statistical evaluations.

\begin{tabular}{ll}
$\begin{array}{l}\text { bond } \\
\text { or angle }\end{array}$ & $\begin{array}{l}\text { length } / \AA \\
\text { or angle / }\end{array}$ \\
\hline C4-C6 & 1.420 \\
C6-C8 & 1.391 \\
C $8-C 9$ & 1.411 \\
C7-C9 & 1.408 \\
C5-C7 & 1.388 \\
C4-C5 & 1.432 \\
C1-C4 & 1.417 \\
C1-O2 & 1.291
\end{tabular}


Table S352 Structural parameters of the OM3/MRCISD-optimized excitedstate equilibrium geometry of the $1^{3} A^{\prime}\left({ }^{3} \pi \pi^{*}\right)$ state of benzaldehyde used for the statistical evaluations.

\begin{tabular}{ll}
$\begin{array}{l}\text { bond } \\
\text { or angle }\end{array}$ & $\begin{array}{l}\text { length } / \AA \\
\text { or angle } /{ }^{\circ}\end{array}$ \\
\hline C4-C6 & 1.467 \\
C6-C8 & 1.373 \\
C $8-C 9$ & 1.443 \\
C7-C9 & 1.441 \\
C5-C7 & 1.372 \\
C4-C5 & 1.468 \\
C1-C4 & 1.447 \\
C1-O2 & 1.251
\end{tabular}


Table S353 Structural parameters of the OM3/MRCISD-optimized excitedstate equilibrium geometry of the $1^{1} A_{u}\left({ }^{1} n \pi^{*}\right)$ state of $p$-benzoquinone used for the statistical evaluations.

\begin{tabular}{ll}
$\begin{array}{l}\text { bond } \\
\text { or angle }\end{array}$ & $\begin{array}{l}\text { length } / \AA \\
\text { or angle / }\end{array}$ \\
\hline $\mathrm{C} 1-\mathrm{O} 7$ & 1.286 \\
$\mathrm{C} 1-\mathrm{C} 2$ & 1.442 \\
$\mathrm{C} 2-\mathrm{C} 3$ & 1.387 \\
$\mathrm{C} 2-\mathrm{H} 9$ & 1.094 \\
$\mathrm{O} 7-\mathrm{C} 1-\mathrm{C} 2$ & 119.6 \\
$\mathrm{C} 6-\mathrm{C} 1-\mathrm{C} 2$ & 120.8 \\
$\mathrm{H} 9-\mathrm{C} 2-\mathrm{C} 3$ & 121.6
\end{tabular}


Table S354 Structural parameters of the OM3/MRCISD-optimized excitedstate equilibrium geometry of the $1{ }^{1} B_{1 g}\left({ }^{1} n \pi^{*}\right)$ state of $p$-benzoquinone used for the statistical evaluations.

\begin{tabular}{ll} 
bond & $\begin{array}{l}\text { length / } \AA \\
\text { or angle } \\
\text { or angle / }\end{array}$ \\
\hline $\mathrm{C} 1-\mathrm{O} 7$ & 1.251 \\
$\mathrm{C} 1-\mathrm{C} 2$ & 1.467 \\
$\mathrm{C} 2-\mathrm{C} 3$ & 1.359 \\
$\mathrm{C} 2-\mathrm{H} 9$ & 1.095 \\
$\mathrm{O} 7-\mathrm{C} 1-\mathrm{C} 2$ & 122.3 \\
$\mathrm{C} 6-\mathrm{C} 1-\mathrm{C} 2$ & 115.4 \\
$\mathrm{H} 9-\mathrm{C} 2-\mathrm{C} 3$ & 123.4
\end{tabular}


Table S355 Structural parameters of the OM3/MRCISD-optimized excitedstate equilibrium geometry of the $1{ }^{1} B_{1 u}\left({ }^{1} \pi \pi^{*}\right)$ state of $p$-benzoquinone used for the statistical evaluations.

\begin{tabular}{ll}
$\begin{array}{l}\text { bond } \\
\text { or angle }\end{array}$ & $\begin{array}{l}\text { length } / \AA \\
\text { or angle / }\end{array}$ \\
\hline $\mathrm{C} 1-\mathrm{O} 7$ & 1.272 \\
$\mathrm{C} 1-\mathrm{C} 2$ & 1.463 \\
$\mathrm{C} 2-\mathrm{C} 3$ & 1.388 \\
$\mathrm{C} 2-\mathrm{H} 9$ & 1.099 \\
$\mathrm{O} 7-\mathrm{C} 1-\mathrm{C} 2$ & 120.7 \\
$\mathrm{C} 6-\mathrm{C} 1-\mathrm{C} 2$ & 118.6 \\
$\mathrm{H} 9-\mathrm{C} 2-\mathrm{C} 3$ & 121.9
\end{tabular}


Table S356 Structural parameters of the OM3/MRCISD-optimized excitedstate equilibrium geometry of the $1{ }^{1} B_{3 g}\left({ }^{1} \pi \pi^{*}\right)$ state of $p$-benzoquinone used for the statistical evaluations.

\begin{tabular}{ll}
$\begin{array}{l}\text { bond } \\
\text { or angle }\end{array}$ & $\begin{array}{l}\text { length } / \AA \\
\text { or angle / }\end{array}$ \\
\hline $\mathrm{C} 1-\mathrm{O} 7$ & 1.265 \\
$\mathrm{C} 1-\mathrm{C} 2$ & 1.449 \\
$\mathrm{C} 2-\mathrm{C} 3$ & 1.414 \\
$\mathrm{C} 2-\mathrm{H} 9$ & 1.104 \\
$\mathrm{O} 7-\mathrm{C} 1-\mathrm{C} 2$ & 122.2 \\
$\mathrm{C} 6-\mathrm{C} 1-\mathrm{C} 2$ & 115.5 \\
$\mathrm{H} 9-\mathrm{C} 2-\mathrm{C} 3$ & 120.3
\end{tabular}


Table S357 Structural parameters of the OM3/MRCISD-optimized excitedstate equilibrium geometry of the $1^{3} A_{u}\left({ }^{3} n \pi^{*}\right)$ state of $p$-benzoquinone used for the statistical evaluations.

\begin{tabular}{ll}
$\begin{array}{l}\text { bond } \\
\text { or angle }\end{array}$ & $\begin{array}{l}\text { length } / \AA \\
\text { or angle / }\end{array}$ \\
\hline $\mathrm{C} 1-\mathrm{O} 7$ & 1.278 \\
$\mathrm{C} 1-\mathrm{C} 2$ & 1.449 \\
$\mathrm{C} 2-\mathrm{C} 3$ & 1.380 \\
$\mathrm{C} 2-\mathrm{H} 9$ & 1.095 \\
$\mathrm{O} 7-\mathrm{C} 1-\mathrm{C} 2$ & 119.8 \\
$\mathrm{C} 6-\mathrm{C} 1-\mathrm{C} 2$ & 120.5 \\
$\mathrm{H} 9-\mathrm{C} 2-\mathrm{C} 3$ & 121.8
\end{tabular}


Table S358 Structural parameters of the OM3/MRCISD-optimized excitedstate equilibrium geometry of the $1{ }^{3} B_{1 g}\left({ }^{3} n \pi^{*}\right)$ state of $p$-benzoquinone used for the statistical evaluations.

\begin{tabular}{ll}
$\begin{array}{l}\text { bond } \\
\text { or angle }\end{array}$ & $\begin{array}{l}\text { length } / \AA \\
\text { or angle / }\end{array}$ \\
\hline $\mathrm{C} 1-\mathrm{O} 7$ & 1.250 \\
$\mathrm{C} 1-\mathrm{C} 2$ & 1.468 \\
$\mathrm{C} 2-\mathrm{C} 3$ & 1.358 \\
$\mathrm{C} 2-\mathrm{H} 9$ & 1.095 \\
$\mathrm{O} 7-\mathrm{C} 1-\mathrm{C} 2$ & 122.3 \\
$\mathrm{C} 6-\mathrm{C} 1-\mathrm{C} 2$ & 115.5 \\
$\mathrm{H} 9-\mathrm{C} 2-\mathrm{C} 3$ & 123.5
\end{tabular}


Table S359 Structural parameters of the OM3/MRCISD-optimized excitedstate equilibrium geometry of the $1^{3} B_{1 u}\left({ }^{3} \pi \pi^{*}\right)$ state of $p$-benzoquinone used for the statistical evaluations.

\begin{tabular}{ll}
$\begin{array}{l}\text { bond } \\
\text { or angle }\end{array}$ & $\begin{array}{l}\text { length } / \AA \\
\text { or angle / }\end{array}$ \\
\hline $\mathrm{C} 1-\mathrm{O} 7$ & 1.283 \\
$\mathrm{C} 1-\mathrm{C} 2$ & 1.446 \\
$\mathrm{C} 2-\mathrm{C} 3$ & 1.401 \\
$\mathrm{C} 2-\mathrm{H} 9$ & 1.098 \\
$\mathrm{O} 7-\mathrm{C} 1-\mathrm{C} 2$ & 120.1 \\
$\mathrm{C} 6-\mathrm{C} 1-\mathrm{C} 2$ & 119.7 \\
$\mathrm{H} 9-\mathrm{C} 2-\mathrm{C} 3$ & 121.5
\end{tabular}


Table S360 Structural parameters of the OM3/MRCISD-optimized excitedstate equilibrium geometry of the $1^{3} B_{3 g}\left({ }^{3} \pi \pi^{*}\right)$ state of $p$-benzoquinone used for the statistical evaluations.

\begin{tabular}{ll}
$\begin{array}{l}\text { bond } \\
\text { or angle }\end{array}$ & $\begin{array}{l}\text { length } / \AA \\
\text { or angle / }\end{array}$ \\
\hline $\mathrm{C} 1-\mathrm{O} 7$ & 1.248 \\
$\mathrm{C} 1-\mathrm{C} 2$ & 1.468 \\
$\mathrm{C} 2-\mathrm{C} 3$ & 1.419 \\
$\mathrm{C} 2-\mathrm{H} 9$ & 1.100 \\
$\mathrm{O} 7-\mathrm{C} 1-\mathrm{C} 2$ & 121.8 \\
$\mathrm{C} 6-\mathrm{C} 1-\mathrm{C} 2$ & 116.4 \\
$\mathrm{H} 9-\mathrm{C} 2-\mathrm{C} 3$ & 120.6
\end{tabular}

S404 
Table S361 Structural parameters of the OM3/MRCISD-optimized excitedstate equilibrium geometry of the $1^{1} B_{3 g}\left({ }^{1} \pi \pi^{*}\right)$ state of biphenylene used for the statistical evaluations.

\begin{tabular}{ll}
$\begin{array}{l}\text { bond } \\
\text { or angle }\end{array}$ & $\begin{array}{c}\text { length } / \AA \\
\text { or angle / } /\end{array}$ \\
\hline C1-C2 & 1.406 \\
C2-C3 & 1.392 \\
C3-C12 & 1.431 \\
C1-C6 & 1.501 \\
C1-C4 & 1.429 \\
C2-H13 & 1.098 \\
C2-H13 & 1.104 \\
C1-C2-C3 & 115.5 \\
C2-C3-C12 & 123.3 \\
C4-C1-C2 & 148.8 \\
C1-C6-C9 & 121.2 \\
\end{tabular}


Table S362 Structural parameters of the OM3/MRCISD-optimized excitedstate equilibrium geometry of the $1{ }^{1} B_{1 u}\left({ }^{1} \pi \pi^{*}\right)$ state of biphenylene used for the statistical evaluations.

\begin{tabular}{ll}
$\begin{array}{l}\text { bond } \\
\text { or angle }\end{array}$ & $\begin{array}{c}\text { length } / \AA \\
\text { or angle / } /\end{array}$ \\
\hline C1-C2 & 1.401 \\
C2-C3 & 1.419 \\
C3-C12 & 1.410 \\
C1-C6 & 1.463 \\
C1-C4 & 1.469 \\
C2-H13 & 1.095 \\
C2-H13 & 1.104 \\
C1-C2-C3 & 114.6 \\
C2-C3-C12 & 123.1 \\
C4-C1-C2 & 147.7 \\
C1-C6-C9 & 122.3 \\
\end{tabular}


Table S363 Structural parameters of the OM3/MRCISD-optimized excitedstate equilibrium geometry of the $2{ }^{1} B_{1 u}\left({ }^{1} \pi \pi^{*}\right)$ state of biphenylene used for the statistical evaluations.

\begin{tabular}{ll}
$\begin{array}{l}\text { bond } \\
\text { or angle }\end{array}$ & $\begin{array}{c}\text { length } / \AA \\
\text { or angle / } /\end{array}$ \\
\hline C1-C2 & 1.396 \\
C2-C3 & 1.427 \\
C3-C12 & 1.399 \\
C1-C6 & 1.464 \\
C1-C4 & 1.474 \\
C2-H13 & 1.097 \\
C2-H13 & 1.103 \\
C1-C2-C3 & 114.8 \\
C2-C3-C12 & 123.0 \\
C4-C1-C2 & 147.8 \\
C1-C6-C9 & 122.2 \\
\end{tabular}


Table S364 Structural parameters of the OM3/MRCISD-optimized excitedstate equilibrium geometry of the $1{ }^{1} B_{2 u}\left({ }^{1} \pi \pi^{*}\right)$ state of biphenylene used for the statistical evaluations.

\begin{tabular}{ll}
$\begin{array}{l}\text { bond } \\
\text { or angle }\end{array}$ & $\begin{array}{c}\text { length } / \AA \\
\text { or angle / } /\end{array}$ \\
\hline C1-C2 & 1.397 \\
C2-C3 & 1.407 \\
C3-C12 & 1.450 \\
C1-C6 & 1.500 \\
C1-C4 & 1.443 \\
C2-H13 & 1.096 \\
C2-H13 & 1.102 \\
C1-C2-C3 & 115.1 \\
C2-C3-C12 & 122.9 \\
C4-C1-C2 & 148.1 \\
C1-C6-C9 & 121.9
\end{tabular}


Table S365 Structural parameters of the OM3/MRCISD-optimized excitedstate equilibrium geometry of the $1{ }^{1} B_{u}\left({ }^{1} \pi \pi^{*}\right)$ state of trans-butadiene used for the statistical evaluations.

\begin{tabular}{ll}
$\begin{array}{l}\text { bond } \\
\text { or angle }\end{array}$ & $\begin{array}{l}\text { length } / \AA \\
\text { or angle } /\end{array}$ \\
\hline C2-C3 & 1.402 \\
C1-C2 & 1.409
\end{tabular}


Table S366 Structural parameters of the OM3/MRCISD-optimized excitedstate equilibrium geometry of the $2{ }^{1} A_{g}\left({ }^{1} \pi \pi^{*}\right)$ state of trans-butadiene used for the statistical evaluations.

\begin{tabular}{ll}
$\begin{array}{l}\text { bond } \\
\text { or angle }\end{array}$ & $\begin{array}{l}\text { length } / \AA \\
\text { or angle } / \circ\end{array}$ \\
\hline C2-C3 & 1.470 \\
C1-C2 & 1.400
\end{tabular}


Table S367 Structural parameters of the OM3/MRCISD-optimized excitedstate equilibrium geometry of the $1^{1} A^{\prime \prime}\left({ }^{1} n \pi^{*}\right)$ state of cytosine used for the statistical evaluations.

\begin{tabular}{ll}
$\begin{array}{l}\text { bond } \\
\text { or angle }\end{array}$ & $\begin{array}{c}\text { length } / \AA \\
\text { or angle } /\end{array}$ \\
\hline N5-C6 & 1.409 \\
N1-C6 & 1.345 \\
N1-C2 & 1.409 \\
C2-C3 & 1.417 \\
C3-C4 & 1.401 \\
C4-N5 & 1.424 \\
C6-O8 & 1.336 \\
C2-N7 & 1.402 \\
C4-H10 & 1.094 \\
C3-H9 & 1.094 \\
N5-H13 & 1.040 \\
N7-H11 & 1.026 \\
N7-H12 & 1.026 \\
N5-C6-N1 & 125.7 \\
C6-N1-C2 & 116.0 \\
N1-C2-C3 & 122.0 \\
C2-C3-C4 & 120.0 \\
C3-C4-N5 & 118.4 \\
N1-C2-N7 & 114.6 \\
N5-C6-O8 & 114.4
\end{tabular}


Table S368 Structural parameters of the OM3/MRCISD-optimized excitedstate equilibrium geometry of the $2{ }^{1} A\left({ }^{1} \pi \pi^{*}\right)$ state of cytosine used for the statistical evaluations.

\begin{tabular}{ll}
$\begin{array}{l}\text { bond } \\
\text { or angle }\end{array}$ & $\begin{array}{c}\text { length } / \AA \\
\text { or angle } /\end{array}$ \\
\hline N5-C6 & 1.485 \\
N1-C6 & 1.368 \\
N1-C2 & 1.405 \\
C2-C3 & 1.419 \\
C3-C4 & 1.442 \\
C4-N5 & 1.380 \\
C6-O8 & 1.268 \\
C2-N7 & 1.396 \\
C4-H10 & 1.101 \\
C3-H9 & 1.094 \\
N5-H13 & 1.048 \\
N7-H11 & 1.028 \\
N7-H12 & 1.027 \\
N5-C6-N1 & 119.7 \\
C6-N1-C2 & 118.9 \\
N1-C2-C3 & 122.4 \\
C2-C3-C4 & 119.8 \\
C3-C4-N5 & 117.4 \\
N1-C2-N7 & 115.3 \\
N5-C6-O8 & 112.6
\end{tabular}


Table S369 Structural parameters of the OM3/MRCISD-optimized excitedstate equilibrium geometry of the $1^{1} A_{2}\left({ }^{1} n \pi^{*}\right)$ state of diazomethane used for the statistical evaluations.

\begin{tabular}{ll} 
bond & $\begin{array}{l}\text { length / } \AA \\
\text { or angle } \\
\text { or angle / }\end{array}$ \\
\hline N2-N3 & 1.251 \\
C1-N2 & 1.366 \\
C1-H4 & 1.099 \\
H4-C1-H5 & 121.4
\end{tabular}

S413 
Table S370 Structural parameters of the OM3/MRCISD-optimized excitedstate equilibrium geometry of the $1{ }^{1} B_{3 g}\left({ }^{1} \pi \pi^{*}\right)$ state of dibenzo- $p$-dioxin used for the statistical evaluations.

\begin{tabular}{|c|c|}
\hline $\begin{array}{l}\text { bond } \\
\text { or angle }\end{array}$ & $\begin{array}{l}\text { length / } \AA \\
\text { or angle / }\end{array}$ \\
\hline C4-07 & 1.369 \\
\hline $\mathrm{C} 4-\mathrm{C} 5$ & 1.447 \\
\hline $\mathrm{C} 5-\mathrm{C} 6$ & 1.420 \\
\hline $\mathrm{C} 1-\mathrm{C} 6$ & 1.384 \\
\hline $\mathrm{C} 1-\mathrm{C} 2$ & 1.430 \\
\hline $\mathrm{C} 4-07-\mathrm{C} 8$ & 115.1 \\
\hline $\mathrm{C} 4-\mathrm{C} 5-\mathrm{C} 6$ & 120.0 \\
\hline $\mathrm{C} 2-\mathrm{C} 3-\mathrm{C} 4$ & 118.7 \\
\hline $\mathrm{C} 1-\mathrm{C} 2-\mathrm{C} 3$ & 121.3 \\
\hline
\end{tabular}


Table S371 Structural parameters of the OM3/MRCISD-optimized excitedstate equilibrium geometry of the $1{ }^{1} B_{2 u}\left({ }^{1} \pi \pi^{*}\right)$ state of dibenzo- $p$-dioxin used for the statistical evaluations.

\begin{tabular}{|c|c|}
\hline $\begin{array}{l}\text { bond } \\
\text { or angle }\end{array}$ & $\begin{array}{l}\text { length / } \AA \\
\text { or angle / }\end{array}$ \\
\hline C4-07 & 1.386 \\
\hline $\mathrm{C} 4-\mathrm{C} 5$ & 1.426 \\
\hline $\mathrm{C} 5-\mathrm{C} 6$ & 1.419 \\
\hline $\mathrm{C} 1-\mathrm{C} 6$ & 1.408 \\
\hline $\mathrm{C} 1-\mathrm{C} 2$ & 1.410 \\
\hline $\mathrm{C} 4-07-\mathrm{C} 8$ & 114.1 \\
\hline $\mathrm{C} 4-\mathrm{C} 5-\mathrm{C} 6$ & 120.7 \\
\hline $\mathrm{C} 2-\mathrm{C} 3-\mathrm{C} 4$ & 118.0 \\
\hline $\mathrm{C} 1-\mathrm{C} 2-\mathrm{C} 3$ & 121.3 \\
\hline
\end{tabular}


Table S372 Structural parameters of the OM3/MRCISD-optimized excitedstate equilibrium geometry of the $1{ }^{1} B_{1 u}\left({ }^{1} \pi \pi^{*}\right)$ state of dibenzo- $p$-dioxin used for the statistical evaluations.

\begin{tabular}{|c|c|}
\hline $\begin{array}{l}\text { bond } \\
\text { or angle }\end{array}$ & $\begin{array}{l}\text { length / } \AA \\
\text { or angle / }\end{array}$ \\
\hline C4-07 & 1.388 \\
\hline $\mathrm{C} 4-\mathrm{C} 5$ & 1.431 \\
\hline $\mathrm{C} 5-\mathrm{C} 6$ & 1.411 \\
\hline $\mathrm{C} 1-\mathrm{C} 6$ & 1.400 \\
\hline $\mathrm{C} 1-\mathrm{C} 2$ & 1.423 \\
\hline $\mathrm{C} 4-07-\mathrm{C} 8$ & 114.0 \\
\hline $\mathrm{C} 4-\mathrm{C} 5-\mathrm{C} 6$ & 120.5 \\
\hline $\mathrm{C} 2-\mathrm{C} 3-\mathrm{C} 4$ & 118.6 \\
\hline $\mathrm{C} 1-\mathrm{C} 2-\mathrm{C} 3$ & 120.9 \\
\hline
\end{tabular}


Table S373 Structural parameters of the OM3/MRCISD-optimized excitedstate equilibrium geometry of the $1^{3} A_{g}\left({ }^{3} \pi \pi^{*}\right)$ state of dibenzo- $p$-dioxin used for the statistical evaluations.

\begin{tabular}{|c|c|}
\hline $\begin{array}{l}\text { bond } \\
\text { or angle }\end{array}$ & $\begin{array}{l}\text { length / } \AA \\
\text { or angle / }\end{array}$ \\
\hline C4-07 & 1.386 \\
\hline $\mathrm{C} 4-\mathrm{C} 5$ & 1.432 \\
\hline $\mathrm{C} 5-\mathrm{C} 6$ & 1.415 \\
\hline $\mathrm{C} 1-\mathrm{C} 6$ & 1.408 \\
\hline $\mathrm{C} 1-\mathrm{C} 2$ & 1.406 \\
\hline $\mathrm{C} 4-07-\mathrm{C} 8$ & 114.1 \\
\hline $\mathrm{C} 4-\mathrm{C} 5-\mathrm{C} 6$ & 120.7 \\
\hline $\mathrm{C} 2-\mathrm{C} 3-\mathrm{C} 4$ & 117.8 \\
\hline $\mathrm{C} 1-\mathrm{C} 2-\mathrm{C} 3$ & 121.5 \\
\hline
\end{tabular}


Table S374 Structural parameters of the OM3/MRCISD-optimized excitedstate equilibrium geometry of the $1^{3} B_{3 g}\left({ }^{3} \pi \pi^{*}\right)$ state of dibenzo- $p$-dioxin used for the statistical evaluations.

\begin{tabular}{|c|c|}
\hline $\begin{array}{l}\text { bond } \\
\text { or angle }\end{array}$ & $\begin{array}{l}\text { length / } \AA \\
\text { or angle / }\end{array}$ \\
\hline C4-07 & 1.379 \\
\hline $\mathrm{C} 4-\mathrm{C} 5$ & 1.451 \\
\hline $\mathrm{C} 5-\mathrm{C} 6$ & 1.416 \\
\hline $\mathrm{C} 1-\mathrm{C} 6$ & 1.387 \\
\hline $\mathrm{C} 1-\mathrm{C} 2$ & 1.438 \\
\hline $\mathrm{C} 4-07-\mathrm{C} 8$ & 114.6 \\
\hline $\mathrm{C} 4-\mathrm{C} 5-\mathrm{C} 6$ & 120.1 \\
\hline $\mathrm{C} 2-\mathrm{C} 3-\mathrm{C} 4$ & 118.8 \\
\hline $\mathrm{C} 1-\mathrm{C} 2-\mathrm{C} 3$ & 121.1 \\
\hline
\end{tabular}


Table S375 Structural parameters of the OM3/MRCISD-optimized excitedstate equilibrium geometry of the $1^{3} B_{2 u}\left({ }^{3} \pi \pi^{*}\right)$ state of dibenzo- $p$-dioxin used for the statistical evaluations.

\begin{tabular}{ll} 
bond & $\begin{array}{l}\text { length / } \AA \\
\text { or angle } \\
\text { or angle / }\end{array}$ \\
\hline C4-07 & 1.383 \\
C4-C5 & 1.436 \\
C5-C6 & 1.413 \\
C1-C6 & 1.410 \\
C1-C2 & 1.403 \\
C4-O7-C8 & 114.8 \\
C4-C5-C6 & 120.7 \\
C2-C3-C4 & 117.7 \\
C1-C2-C3 & 121.6
\end{tabular}


Table S376 Structural parameters of the OM3/MRCISD-optimized excitedstate equilibrium geometry of the $1^{3} B_{1 u}\left({ }^{3} \pi \pi^{*}\right)$ state of dibenzo- $p$-dioxin used for the statistical evaluations.

\begin{tabular}{|c|c|}
\hline $\begin{array}{l}\text { bond } \\
\text { or angle }\end{array}$ & $\begin{array}{l}\text { length / } \AA \\
\text { or angle / }\end{array}$ \\
\hline C4-07 & 1.391 \\
\hline $\mathrm{C} 4-\mathrm{C} 5$ & 1.450 \\
\hline $\mathrm{C} 5-\mathrm{C} 6$ & 1.408 \\
\hline $\mathrm{C} 1-\mathrm{C} 6$ & 1.394 \\
\hline $\mathrm{C} 1-\mathrm{C} 2$ & 1.438 \\
\hline $\mathrm{C} 4-07-\mathrm{C} 8$ & 113.4 \\
\hline $\mathrm{C} 4-\mathrm{C} 5-\mathrm{C} 6$ & 120.2 \\
\hline $\mathrm{C} 2-\mathrm{C} 3-\mathrm{C} 4$ & 119.0 \\
\hline $\mathrm{C} 1-\mathrm{C} 2-\mathrm{C} 3$ & 120.8 \\
\hline
\end{tabular}


Table S377 Structural parameters of the OM3/MRCISD-optimized excitedstate equilibrium geometry of the $1{ }^{1} A_{u}\left({ }^{1} n \pi^{*}\right)$ state of dibenzo- $p$-dioxin used for the statistical evaluations.

\begin{tabular}{|c|c|}
\hline $\begin{array}{l}\text { bond } \\
\text { or angle }\end{array}$ & $\begin{array}{l}\text { length / } \AA \\
\text { or angle / }\end{array}$ \\
\hline C4-07 & 1.378 \\
\hline $\mathrm{C} 4-\mathrm{C} 5$ & 1.460 \\
\hline $\mathrm{C} 5-\mathrm{C} 6$ & 1.401 \\
\hline $\mathrm{C} 1-\mathrm{C} 6$ & 1.391 \\
\hline $\mathrm{C} 1-\mathrm{C} 2$ & 1.418 \\
\hline $\mathrm{C} 4-07-\mathrm{C} 8$ & 120.9 \\
\hline $\mathrm{C} 4-\mathrm{C} 5-\mathrm{C} 6$ & 118.8 \\
\hline $\mathrm{C} 2-\mathrm{C} 3-\mathrm{C} 4$ & 121.2 \\
\hline $\mathrm{C} 1-\mathrm{C} 2-\mathrm{C} 3$ & 120.0 \\
\hline
\end{tabular}


Table S378 Structural parameters of the OM3/MRCISD-optimized excitedstate equilibrium geometry of the $1{ }^{1} B_{1 g}\left({ }^{1} n \pi^{*}\right)$ state of dibenzo- $p$-dioxin used for the statistical evaluations.

\begin{tabular}{ll} 
bond & $\begin{array}{l}\text { length / } \AA \\
\text { or angle }\end{array}$ or angle / \\
\hline C4-O7 & 1.385 \\
C4-C5 & 1.436 \\
C5-C6 & 1.401 \\
C1-C6 & 1.415 \\
C1-C2 & 1.389 \\
C4-O7-C8 & 121.0 \\
C4-C5-C6 & 119.8 \\
C2-C3-C4 & 119.7 \\
C1-C2-C3 & 120.5
\end{tabular}


Table S379 Structural parameters of the OM3/MRCISD-optimized excitedstate equilibrium geometry of the $1{ }^{1} B_{3 u}\left({ }^{1} n \pi^{*}\right)$ state of dibenzo- $p$-dioxin used for the statistical evaluations.

\begin{tabular}{ll} 
bond & $\begin{array}{l}\text { length / } \AA \\
\text { or angle }\end{array}$ or angle / \\
\hline C4-O7 & 1.390 \\
C4-C5 & 1.427 \\
C5-C6 & 1.406 \\
C1-C6 & 1.410 \\
C1-C2 & 1.394 \\
C4-O7-C8 & 119.8 \\
C4-C5-C6 & 119.8 \\
C2-C3-C4 & 119.7 \\
C1-C2-C3 & 120.5
\end{tabular}


Table S380 Structural parameters of the OM3/MRCISD-optimized excitedstate equilibrium geometry of the $1{ }^{1} B_{2 g}\left({ }^{1} n \pi^{*}\right)$ state of dibenzo- $p$-dioxin used for the statistical evaluations.

\begin{tabular}{ll} 
bond & $\begin{array}{l}\text { length / } \AA \\
\text { or angle }\end{array}$ or angle / \\
\hline C4-O7 & 1.393 \\
C4-C5 & 1.454 \\
C5-C6 & 1.395 \\
C1-C6 & 1.396 \\
C1-C2 & 1.419 \\
C4-O7-C8 & 119.3 \\
C4-C5-C6 & 118.9 \\
C2-C3-C4 & 121.5 \\
C1-C2-C3 & 119.7
\end{tabular}


Table S381 Structural parameters of the OM3/MRCISD-optimized excitedstate equilibrium geometry of the $1^{3} A_{u}\left({ }^{3} n \pi^{*}\right)$ state of dibenzo- $p$-dioxin used for the statistical evaluations.

\begin{tabular}{|c|c|}
\hline $\begin{array}{l}\text { bond } \\
\text { or angle }\end{array}$ & $\begin{array}{l}\text { length / } \AA \\
\text { or angle / }\end{array}$ \\
\hline C4-07 & 1.378 \\
\hline $\mathrm{C} 4-\mathrm{C} 5$ & 1.461 \\
\hline $\mathrm{C} 5-\mathrm{C} 6$ & 1.402 \\
\hline $\mathrm{C} 1-\mathrm{C} 6$ & 1.391 \\
\hline $\mathrm{C} 1-\mathrm{C} 2$ & 1.418 \\
\hline $\mathrm{C} 4-07-\mathrm{C} 8$ & 120.9 \\
\hline $\mathrm{C} 4-\mathrm{C} 5-\mathrm{C} 6$ & 118.8 \\
\hline $\mathrm{C} 2-\mathrm{C} 3-\mathrm{C} 4$ & 121.2 \\
\hline $\mathrm{C} 1-\mathrm{C} 2-\mathrm{C} 3$ & 120.0 \\
\hline
\end{tabular}


Table S382 Structural parameters of the OM3/MRCISD-optimized excitedstate equilibrium geometry of the $1^{3} B_{1 g}\left({ }^{3} n \pi^{*}\right)$ state of dibenzo- $p$-dioxin used for the statistical evaluations.

\begin{tabular}{ll} 
bond & $\begin{array}{l}\text { length / } \AA \\
\text { or angle }\end{array}$ or angle / \\
\hline C4-O7 & 1.385 \\
C4-C5 & 1.435 \\
C5-C6 & 1.401 \\
C1-C6 & 1.415 \\
C1-C2 & 1.390 \\
C4-O7-C8 & 120.9 \\
C4-C5-C6 & 119.8 \\
C2-C3-C4 & 119.7 \\
C1-C2-C3 & 120.5
\end{tabular}


Table S383 Structural parameters of the OM3/MRCISD-optimized excitedstate equilibrium geometry of the $1^{3} B_{3 u}\left({ }^{3} n \pi^{*}\right)$ state of dibenzo- $p$-dioxin used for the statistical evaluations.

\begin{tabular}{ll} 
bond & $\begin{array}{l}\text { length / } \AA \\
\text { or angle }\end{array}$ or angle / \\
\hline C4-O7 & 1.390 \\
C4-C5 & 1.428 \\
C5-C6 & 1.405 \\
C1-C6 & 1.410 \\
C1-C2 & 1.394 \\
C4-O7-C8 & 119.8 \\
C4-C5-C6 & 119.8 \\
C2-C3-C4 & 119.7 \\
C1-C2-C3 & 120.5
\end{tabular}


Table S384 Structural parameters of the OM3/MRCISD-optimized excitedstate equilibrium geometry of the $1^{3} B_{2 g}\left({ }^{3} n \pi^{*}\right)$ state of dibenzo- $p$-dioxin used for the statistical evaluations.

\begin{tabular}{ll} 
bond & $\begin{array}{l}\text { length / } \AA \\
\text { or angle }\end{array}$ or angle / \\
\hline C4-O7 & 1.392 \\
C4-C5 & 1.455 \\
C5-C6 & 1.395 \\
C1-C6 & 1.395 \\
C1-C2 & 1.419 \\
C4-O7-C8 & 119.4 \\
C4-C5-C6 & 118.9 \\
C2-C3-C4 & 121.4 \\
C1-C2-C3 & 119.7
\end{tabular}


Table S385 Structural parameters of the OM3/MRCISD-optimized excitedstate equilibrium geometry of the $1{ }^{1} A^{\prime \prime}\left({ }^{1} n \pi^{*}\right)$ state of formaldehyde used for the statistical evaluations.

\begin{tabular}{ll}
$\begin{array}{l}\text { bond } \\
\text { or angle }\end{array}$ & $\begin{array}{l}\text { length } / \AA \\
\text { or angle / }\end{array}$ \\
\hline $\mathrm{C} 1-\mathrm{O} 2$ & 1.276 \\
$\mathrm{C} 1-\mathrm{H} 3$ & 1.126 \\
$\mathrm{H} 3-\mathrm{C} 1-\mathrm{H} 4$ & 107.9
\end{tabular}


Table S386 Structural parameters of the OM3/MRCISD-optimized excitedstate equilibrium geometry of the $1^{3} A^{\prime \prime}\left({ }^{3} n \pi^{*}\right)$ state of formaldehyde used for the statistical evaluations.

\begin{tabular}{ll}
$\begin{array}{l}\text { bond } \\
\text { or angle }\end{array}$ & $\begin{array}{l}\text { length } / \AA \\
\text { or angle } /\end{array}$ \\
\hline $\mathrm{C} 1-\mathrm{O} 2$ & 1.254 \\
$\mathrm{C} 1-\mathrm{H} 3$ & 1.160 \\
$\mathrm{H} 3-\mathrm{C} 1-\mathrm{H} 4$ & 101.7
\end{tabular}


Table S387 Structural parameters of the OM3/MRCISD-optimized excitedstate equilibrium geometry of the $1^{3} A^{\prime}\left({ }^{3} \pi \pi^{*}\right)$ state of formaldehyde used for the statistical evaluations.

\begin{tabular}{ll}
$\begin{array}{l}\text { bond } \\
\text { or angle }\end{array}$ & $\begin{array}{l}\text { length } / \AA \\
\text { or angle / }\end{array}$ \\
\hline $\mathrm{C} 1-\mathrm{O} 2$ & 1.424 \\
$\mathrm{C} 1-\mathrm{H} 3$ & 1.119 \\
$\mathrm{H} 3-\mathrm{C} 1-\mathrm{H} 4$ & 116.7
\end{tabular}


Table S388 Structural parameters of the OM3/MRCISD-optimized excitedstate equilibrium geometry of the $1{ }^{1} A_{u}\left({ }^{1} n \pi^{*}\right)$ state of trans-glyoxal used for the statistical evaluations.

\begin{tabular}{ll}
$\begin{array}{l}\text { bond } \\
\text { or angle }\end{array}$ & $\begin{array}{l}\text { length } / \AA \\
\text { or angle } /\end{array}$ \\
\hline $\mathrm{C} 1-\mathrm{H} 5$ & 1.113 \\
$\mathrm{C} 1-\mathrm{C} 2$ & 1.489 \\
$\mathrm{C} 1-\mathrm{O} 3$ & 1.242 \\
$\mathrm{C} 1-\mathrm{C} 2-\mathrm{H} 6$ & 112.8 \\
$\mathrm{C} 1-\mathrm{C} 2-\mathrm{O} 4$ & 120.0
\end{tabular}


Table S389 Structural parameters of the OM3/MRCISD-optimized excitedstate equilibrium geometry of the $1{ }^{3} A_{u}\left({ }^{3} n \pi^{*}\right)$ state of trans-glyoxal used for the statistical evaluations.

\begin{tabular}{ll}
$\begin{array}{l}\text { bond } \\
\text { or angle }\end{array}$ & $\begin{array}{l}\text { length } / \AA \\
\text { or angle } /\end{array}$ \\
\hline $\mathrm{C} 1-\mathrm{H} 5$ & 1.110 \\
$\mathrm{C} 1-\mathrm{C} 2$ & 1.493 \\
$\mathrm{C} 1-\mathrm{O} 3$ & 1.240 \\
$\mathrm{C} 1-\mathrm{C} 2-\mathrm{H} 6$ & 112.8 \\
$\mathrm{C} 1-\mathrm{C} 2-\mathrm{O} 4$ & 119.5
\end{tabular}


Table S390 Structural parameters of the OM3/MRCISD-optimized excitedstate equilibrium geometry of the $2{ }^{1} A\left({ }^{1} \pi \pi^{*}\right)$ state of $9 H$-guanine used for the statistical evaluations.

\begin{tabular}{ll}
$\begin{array}{l}\text { bond } \\
\text { or angle }\end{array}$ & $\begin{array}{c}\text { length } / \AA \\
\text { or angle } /\end{array}$ \\
\hline N7-C9 & 1.424 \\
C9-N13 & 1.480 \\
N13-C14 & 1.337 \\
C4-C14 & 1.506 \\
C4-C5 & 1.484 \\
C5-N7 & 1.434 \\
N3-C4 & 1.374 \\
C2-N3 & 1.372 \\
N1-C2 & 1.427 \\
N1-C14 & 1.423 \\
C9-N10 & 1.391 \\
C5-O6 & 1.242 \\
C2-H15 & 1.101 \\
N10-H11 & 1.036 \\
N10-H12 & 1.029 \\
N7-H8 & 1.038 \\
N1-H16 & 1.039
\end{tabular}


Table S391 Structural parameters of the OM3/MRCISD-optimized excitedstate equilibrium geometry of the $2{ }^{1} A^{\prime}\left({ }^{1} \pi \pi^{*}\right)$ state of 1-hydroxy-2acetonaphthone used for the statistical evaluations.

\begin{tabular}{ll}
$\begin{array}{l}\text { bond } \\
\text { or angle }\end{array}$ & $\begin{array}{c}\text { length } / \AA \\
\text { or angle } /\end{array}$ \\
\hline C1-C6 & 1.477 \\
C5-C6 & 1.382 \\
C4-C5 & 1.417 \\
C3-C4 & 1.416 \\
C3-C10 & 1.427 \\
C9-C10 & 1.390 \\
C8-C9 & 1.408 \\
C7-C8 & 1.395 \\
C2-C7 & 1.408 \\
C2-C3 & 1.438 \\
C1-C2 & 1.454 \\
C6-C11 & 1.466 \\
C11-C12 & 1.488 \\
C1-O13 & 1.284 \\
C11-O14 & 1.321 \\
O13-H21 & 1.313 \\
O14-H21 & 1.126 \\
O13-O14 & 2.354
\end{tabular}


Table S392 Structural parameters of the OM3/MRCISD-optimized excitedstate equilibrium geometry of the $2{ }^{1} A^{\prime}\left({ }^{1} \pi \pi^{*}\right)$ state of indole used for the statistical evaluations.

$\begin{array}{ll}\text { bond } & \begin{array}{c}\text { length } / \AA \\ \text { or angle }\end{array} \\ \text { or angle / } /\end{array}$


Table S393 Structural parameters of the OM3/MRCISD-optimized excitedstate equilibrium geometry of the $3{ }^{1} A^{\prime}\left({ }^{1} \pi \pi^{*}\right)$ state of indole used for the statistical evaluations.

\begin{tabular}{|c|c|}
\hline $\begin{array}{l}\text { bond } \\
\text { or angle }\end{array}$ & $\begin{array}{l}\text { length / } \AA \\
\text { or angle / }\end{array}$ \\
\hline N3-C5 & 1.379 \\
\hline $\mathrm{C} 4-\mathrm{C} 5$ & 1.459 \\
\hline $\mathrm{C} 1-\mathrm{C} 4$ & 1.419 \\
\hline $\mathrm{C} 1-\mathrm{C} 6$ & 1.413 \\
\hline $\mathrm{C} 6-\mathrm{C} 7$ & 1.417 \\
\hline $\mathrm{C} 7-\mathrm{C} 9$ & 1.394 \\
\hline $\mathrm{C} 8-\mathrm{C} 9$ & 1.430 \\
\hline $\mathrm{C} 2-\mathrm{C} 8$ & 1.396 \\
\hline $\mathrm{N} 3-\mathrm{H} 10$ & 1.048 \\
\hline $\mathrm{C} 5-\mathrm{H} 12$ & 1.095 \\
\hline $\mathrm{C} 4-\mathrm{H} 11$ & 1.087 \\
\hline $\mathrm{C} 6-\mathrm{H} 13$ & 1.095 \\
\hline $\mathrm{C} 7-\mathrm{H} 14$ & 1.104 \\
\hline $\mathrm{C} 9-\mathrm{H} 16$ & 1.103 \\
\hline $\mathrm{C} 8-\mathrm{H} 15$ & 1.093 \\
\hline $\mathrm{C} 1-\mathrm{C} 2$ & 1.455 \\
\hline $\mathrm{C} 2-\mathrm{N} 3$ & 1.440 \\
\hline $\mathrm{N} 3-\mathrm{C} 5-\mathrm{C} 4$ & 108.0 \\
\hline $\mathrm{C} 5-\mathrm{C} 4-\mathrm{C} 1$ & 108.3 \\
\hline $\mathrm{C} 4-\mathrm{C} 1-\mathrm{C} 6$ & 134.3 \\
\hline $\mathrm{C} 1-\mathrm{C} 6-\mathrm{C} 7$ & 117.6 \\
\hline $\mathrm{C} 6-\mathrm{C} 7-\mathrm{C} 9$ & 122.5 \\
\hline $\mathrm{C} 7-\mathrm{C} 9-\mathrm{C} 8$ & 122.0 \\
\hline $\mathrm{C} 9-\mathrm{C} 8-\mathrm{C} 2$ & 115.4 \\
\hline $\mathrm{C} 2-\mathrm{N} 3-\mathrm{H} 10$ & 124.6 \\
\hline $\mathrm{N} 3-\mathrm{C} 5-\mathrm{H} 12$ & 124.8 \\
\hline $\mathrm{C} 5-\mathrm{C} 4-\mathrm{H} 11$ & 124.0 \\
\hline $\mathrm{C} 1-\mathrm{C} 6-\mathrm{H} 13$ & 120.6 \\
\hline $\mathrm{C} 6-\mathrm{C} 7-\mathrm{H} 14$ & 118.3 \\
\hline $\mathrm{C} 7-\mathrm{C} 9-\mathrm{H} 16$ & 120.1 \\
\hline $\mathrm{C} 9-\mathrm{C} 8-\mathrm{H} 15$ & 121.8 \\
\hline
\end{tabular}


Table S394 Structural parameters of the OM3/MRCISD-optimized excitedstate equilibrium geometry of the $1{ }^{1} B_{1}\left({ }^{1} n \pi^{*}\right)$ state of maleimide used for the statistical evaluations.

\begin{tabular}{ll}
$\begin{array}{l}\text { bond } \\
\text { or angle }\end{array}$ & $\begin{array}{l}\text { length } / \AA \\
\text { or angle } /\end{array}$ \\
\hline $\mathrm{N} 1-\mathrm{C} 2$ & 1.455 \\
$\mathrm{C} 2-\mathrm{C} 3$ & 1.466 \\
$\mathrm{C} 3-\mathrm{C} 4$ & 1.376 \\
$\mathrm{C} 2-\mathrm{O} 6$ & 1.251 \\
$\mathrm{C} 3-\mathrm{H} 9$ & 1.083 \\
$\mathrm{C} 2-\mathrm{N} 1-\mathrm{C} 5$ & 108.5 \\
$\mathrm{~N} 1-\mathrm{C} 2-\mathrm{O} 6$ & 118.1 \\
O6-C2-C4 & 135.8 \\
$\mathrm{C} 2-\mathrm{C} 3-\mathrm{C} 4$ & 109.7 \\
$\mathrm{C} 2-\mathrm{C} 3-\mathrm{H} 9$ & 120.5 \\
$\mathrm{~N} 1-\mathrm{H} 8$ & 1.042 \\
\end{tabular}


Table S395 Structural parameters of the OM3/MRCISD-optimized excitedstate equilibrium geometry of the $1^{1} A_{2}\left({ }^{1} n \pi^{*}\right)$ state of maleimide used for the statistical evaluations.

\begin{tabular}{ll}
$\begin{array}{l}\text { bond } \\
\text { or angle }\end{array}$ & $\begin{array}{l}\text { length } / \AA \\
\text { or angle } /\end{array}$ \\
\hline $\mathrm{N} 1-\mathrm{C} 2$ & 1.417 \\
$\mathrm{C} 2-\mathrm{C} 3$ & 1.463 \\
$\mathrm{C} 3-\mathrm{C} 4$ & 1.394 \\
$\mathrm{C} 2-\mathrm{O} 6$ & 1.282 \\
$\mathrm{C} 3-\mathrm{H} 9$ & 1.083 \\
$\mathrm{C} 2-\mathrm{N} 1-\mathrm{C} 5$ & 108.0 \\
$\mathrm{~N} 1-\mathrm{C} 2-\mathrm{O} 6$ & 125.6 \\
O6-C2-C4 & 126.3 \\
$\mathrm{C} 2-\mathrm{C} 3-\mathrm{C} 4$ & 107.9 \\
$\mathrm{C} 2-\mathrm{C} 3-\mathrm{H} 9$ & 123.4 \\
$\mathrm{~N} 1-\mathrm{H} 8$ & 1.036
\end{tabular}


Table S396 Structural parameters of the OM3/MRCISD-optimized excitedstate equilibrium geometry of the $1{ }^{1} B_{2}\left({ }^{1} \pi \pi^{*}\right)$ state of maleimide used for the statistical evaluations.

\begin{tabular}{ll}
$\begin{array}{l}\text { bond } \\
\text { or angle }\end{array}$ & $\begin{array}{l}\text { length } / \AA \\
\text { or angle } /\end{array}$ \\
\hline $\mathrm{N} 1-\mathrm{C} 2$ & 1.499 \\
$\mathrm{C} 2-\mathrm{C} 3$ & 1.437 \\
$\mathrm{C} 3-\mathrm{C} 4$ & 1.409 \\
$\mathrm{C} 2-\mathrm{O} 6$ & 1.247 \\
$\mathrm{C} 3-\mathrm{H} 9$ & 1.086 \\
$\mathrm{C} 2-\mathrm{N} 1-\mathrm{C} 5$ & 110.0 \\
$\mathrm{~N} 1-\mathrm{C} 2-\mathrm{O} 6$ & 118.7 \\
O6-C2-C4 & 137.7 \\
$\mathrm{C} 2-\mathrm{C} 3-\mathrm{C} 4$ & 111.4 \\
$\mathrm{C} 2-\mathrm{C} 3-\mathrm{H} 9$ & 122.0 \\
$\mathrm{~N} 1-\mathrm{H} 8$ & 1.052 \\
\end{tabular}


Table S397 Structural parameters of the OM3/MRCISD-optimized excitedstate equilibrium geometry of the $2{ }^{1} B_{2}\left({ }^{1} \pi \pi^{*}\right)$ state of maleimide used for the statistical evaluations.

\begin{tabular}{ll}
$\begin{array}{l}\text { bond } \\
\text { or angle }\end{array}$ & $\begin{array}{l}\text { length } / \AA \\
\text { or angle } /\end{array}$ \\
\hline $\mathrm{N} 1-\mathrm{C} 2$ & 1.431 \\
$\mathrm{C} 2-\mathrm{C} 3$ & 1.469 \\
$\mathrm{C} 3-\mathrm{C} 4$ & 1.416 \\
$\mathrm{C} 2-\mathrm{O} 6$ & 1.276 \\
$\mathrm{C} 3-\mathrm{H} 9$ & 1.085 \\
$\mathrm{C} 2-\mathrm{N} 1-\mathrm{C} 5$ & 109.0 \\
$\mathrm{~N} 1-\mathrm{C} 2-\mathrm{O} 6$ & 122.8 \\
O6-C2-C4 & 129.8 \\
$\mathrm{C} 2-\mathrm{C} 3-\mathrm{C} 4$ & 108.1 \\
$\mathrm{C} 2-\mathrm{C} 3-\mathrm{H} 9$ & 123.6 \\
$\mathrm{~N} 1-\mathrm{H} 8$ & 1.038 \\
\end{tabular}


Table S398 Structural parameters of the OM3/MRCISD-optimized excitedstate equilibrium geometry of the $1^{3} B_{1}\left({ }^{3} n \pi^{*}\right)$ state of maleimide used for the statistical evaluations.

\begin{tabular}{ll}
$\begin{array}{l}\text { bond } \\
\text { or angle }\end{array}$ & $\begin{array}{l}\text { length } / \AA \\
\text { or angle } /\end{array}$ \\
\hline N1-C2 & 1.451 \\
C2-C3 & 1.468 \\
C3-C4 & 1.375 \\
C2-O6 & 1.252 \\
C3-H9 & 1.083 \\
C2-N1-C5 & 108.8 \\
N1-C2-O6 & 119.1 \\
O6-C2-C4 & 134.9 \\
C2-C3-C4 & 109.6 \\
C2-C3-H9 & 120.4 \\
N1-H8 & 1.042
\end{tabular}


Table S399 Structural parameters of the OM3/MRCISD-optimized excitedstate equilibrium geometry of the $1^{3} A_{2}\left({ }^{3} n \pi^{*}\right)$ state of maleimide used for the statistical evaluations.

\begin{tabular}{ll}
$\begin{array}{l}\text { bond } \\
\text { or angle }\end{array}$ & $\begin{array}{l}\text { length } / \AA \\
\text { or angle } /\end{array}$ \\
\hline $\mathrm{N} 1-\mathrm{C} 2$ & 1.418 \\
$\mathrm{C} 2-\mathrm{C} 3$ & 1.467 \\
$\mathrm{C} 3-\mathrm{C} 4$ & 1.391 \\
$\mathrm{C} 2-\mathrm{O} 6$ & 1.279 \\
$\mathrm{C} 3-\mathrm{H} 9$ & 1.083 \\
$\mathrm{C} 2-\mathrm{N} 1-\mathrm{C} 5$ & 108.1 \\
$\mathrm{~N} 1-\mathrm{C} 2-\mathrm{O} 6$ & 126.0 \\
O6-C2-C4 & 126.0 \\
$\mathrm{C} 2-\mathrm{C} 3-\mathrm{C} 4$ & 107.9 \\
$\mathrm{C} 2-\mathrm{C} 3-\mathrm{H} 9$ & 123.4 \\
$\mathrm{~N} 1-\mathrm{H} 8$ & 1.036
\end{tabular}


Table S400 Structural parameters of the OM3/MRCISD-optimized excitedstate equilibrium geometry of the $1^{3} B_{2}\left({ }^{3} \pi \pi^{*}\right)$ state of maleimide used for the statistical evaluations.

\begin{tabular}{ll}
$\begin{array}{l}\text { bond } \\
\text { or angle }\end{array}$ & $\begin{array}{l}\text { length } / \AA \\
\text { or angle } /\end{array}$ \\
\hline $\mathrm{N} 1-\mathrm{C} 2$ & 1.429 \\
$\mathrm{C} 2-\mathrm{C} 3$ & 1.478 \\
$\mathrm{C} 3-\mathrm{C} 4$ & 1.502 \\
$\mathrm{C} 2-\mathrm{O} 6$ & 1.247 \\
$\mathrm{C} 3-\mathrm{H} 9$ & 1.086 \\
$\mathrm{C} 2-\mathrm{N} 1-\mathrm{C} 5$ & 112.5 \\
$\mathrm{~N} 1-\mathrm{C} 2-\mathrm{O} 6$ & 125.2 \\
O6-C2-C4 & 128.2 \\
$\mathrm{C} 2-\mathrm{C} 3-\mathrm{C} 4$ & 107.2 \\
$\mathrm{C} 2-\mathrm{C} 3-\mathrm{H} 9$ & 126.0 \\
$\mathrm{~N} 1-\mathrm{H} 8$ & 1.035
\end{tabular}


Table S401 Structural parameters of the OM3/MRCISD-optimized excitedstate equilibrium geometry of the $1^{1} A^{\prime \prime}\left({ }^{1} n \pi^{*}\right)$ state of nitrosomethane used for the statistical evaluations.

\begin{tabular}{ll} 
bond & $\begin{array}{l}\text { length / } \AA \\
\text { or angle } \\
\text { or angle / }\end{array}$ \\
\hline $\mathrm{N} 2-\mathrm{O} 3$ & 1.244 \\
$\mathrm{C} 1-\mathrm{N} 2$ & 1.468 \\
$\mathrm{C} 1-\mathrm{H} 4$ & 1.108 \\
$\mathrm{C} 1-\mathrm{H} 5$ & 1.106 \\
$\mathrm{C} 1-\mathrm{N} 2-\mathrm{O} 3$ & 129.4 \\
$\mathrm{~N} 2-\mathrm{C} 1-\mathrm{H} 4$ & 108.3 \\
$\mathrm{~N} 2-\mathrm{C} 1-\mathrm{H} 5$ & 108.3 \\
$\mathrm{H} 4-\mathrm{C} 1-\mathrm{H} 5$ & 110.5
\end{tabular}


Table S402 Structural parameters of the OM3/MRCISD-optimized excitedstate equilibrium geometry of the $1{ }^{3} A^{\prime \prime}\left({ }^{3} n \pi^{*}\right)$ state of nitrosomethane used for the statistical evaluations.

\begin{tabular}{ll} 
bond & $\begin{array}{l}\text { length / } \AA \\
\text { or angle } \\
\text { or angle / }\end{array}$ \\
\hline $\mathrm{N} 2-\mathrm{O} 3$ & 1.237 \\
$\mathrm{C} 1-\mathrm{N} 2$ & 1.476 \\
$\mathrm{C} 1-\mathrm{H} 4$ & 1.109 \\
$\mathrm{C} 1-\mathrm{H} 5$ & 1.104 \\
$\mathrm{C} 1-\mathrm{N} 2-\mathrm{O} 3$ & 130.5 \\
$\mathrm{~N} 2-\mathrm{C} 1-\mathrm{H} 4$ & 108.8 \\
$\mathrm{~N} 2-\mathrm{C} 1-\mathrm{H} 5$ & 108.5 \\
$\mathrm{H} 4-\mathrm{C} 1-\mathrm{H} 5$ & 109.6
\end{tabular}


Table S403 Structural parameters of the OM3/MRCISD-optimized excitedstate equilibrium geometry of the $1{ }^{1} A^{\prime \prime}\left({ }^{1} n \pi^{*}\right)$ state of propenoic acid anion used for the statistical evaluations.

\begin{tabular}{ll}
$\begin{array}{l}\text { bond } \\
\text { or angle }\end{array}$ & $\begin{array}{l}\text { length } / \AA \\
\text { or angle / } /\end{array}$ \\
\hline $\mathrm{C} 2-\mathrm{O} 3$ & 1.278 \\
$\mathrm{C} 2-\mathrm{O} 4$ & 1.358 \\
$\mathrm{C} 1-\mathrm{C} 2$ & 1.412 \\
$\mathrm{C} 1-\mathrm{C} 5$ & 1.387 \\
$\mathrm{O} 4-\mathrm{C} 2-\mathrm{C} 1$ & 115.8 \\
$\mathrm{C} 2-\mathrm{C} 1-\mathrm{C} 5$ & 125.3 \\
$\mathrm{O} 3-\mathrm{C} 2-\mathrm{O} 4$ & 114.3
\end{tabular}


Table S404 Structural parameters of the OM3/MRCISD-optimized excitedstate equilibrium geometry of the $1^{1} A_{2}\left({ }^{1} n \pi^{*}\right)$ state of pyridine used for the statistical evaluations.

\begin{tabular}{ll} 
bond & $\begin{array}{l}\text { length / } \AA \\
\text { or angle } \\
\text { or angle / }\end{array}$ \\
\hline $\mathrm{N} 1-\mathrm{C} 2$ & 1.335 \\
$\mathrm{C} 2-\mathrm{C} 4$ & 1.460 \\
$\mathrm{C} 4-\mathrm{C} 6$ & 1.396 \\
$\mathrm{C} 2-\mathrm{N} 1-\mathrm{C} 3$ & 135.6 \\
$\mathrm{~N} 1-\mathrm{C} 2-\mathrm{C} 4$ & 111.8 \\
$\mathrm{C} 2-\mathrm{C} 4-\mathrm{C} 6$ & 119.0 \\
$\mathrm{C} 4-\mathrm{C} 6-\mathrm{C} 5$ & 122.8
\end{tabular}


Table S405 Structural parameters of the OM3/MRCISD-optimized excitedstate equilibrium geometry of the $2{ }^{1} A^{\prime}\left({ }^{1} n \pi^{*}\right)$ state of pyridine used for the statistical evaluations.

\begin{tabular}{ll} 
bond & $\begin{array}{l}\text { length / } \AA \\
\text { or angle } \\
\text { or angle / }\end{array}$ \\
\hline N1-C2 & 1.371 \\
C2-C4 & 1.397 \\
C4-C6 & 1.424 \\
C2-N1-C3 & 132.9 \\
N1-C2-C4 & 112.6 \\
C2-C4-C6 & 120.9 \\
C4-C6-C5 & 120.2
\end{tabular}


Table S406 Structural parameters of the OM3/MRCISD-optimized excitedstate equilibrium geometry of the $1{ }^{3} A_{1}\left({ }^{3} \pi \pi^{*}\right)$ state of pyridine used for the statistical evaluations.

\begin{tabular}{ll} 
bond & $\begin{array}{l}\text { length / } \AA \\
\text { or angle } \\
\text { or angle / }\end{array}$ \\
\hline $\mathrm{N} 1-\mathrm{C} 2$ & 1.353 \\
$\mathrm{C} 2-\mathrm{C} 4$ & 1.498 \\
$\mathrm{C} 4-\mathrm{C} 6$ & 1.397 \\
$\mathrm{C} 2-\mathrm{N} 1-\mathrm{C} 3$ & 119.5 \\
$\mathrm{~N} 1-\mathrm{C} 2-\mathrm{C} 4$ & 121.8 \\
$\mathrm{C} 2-\mathrm{C} 4-\mathrm{C} 6$ & 118.6 \\
$\mathrm{C} 4-\mathrm{C} 6-\mathrm{C} 5$ & 119.7
\end{tabular}


Table S407 Structural parameters of the OM3/MRCISD-optimized excitedstate equilibrium geometry of the $2{ }^{3} A_{1}\left({ }^{3} \pi \pi^{*}\right)$ state of pyridine used for the statistical evaluations.

\begin{tabular}{ll}
$\begin{array}{l}\text { bond } \\
\text { or angle }\end{array}$ & $\begin{array}{l}\text { length angle } / \\
\text { or } /\end{array}$ \\
\hline $\mathrm{N} 1-\mathrm{C} 2$ & 1.384 \\
$\mathrm{C} 2-\mathrm{C} 4$ & 1.415 \\
$\mathrm{C} 4-\mathrm{C} 6$ & 1.445 \\
$\mathrm{C} 2-\mathrm{N} 1-\mathrm{C} 3$ & 118.1 \\
$\mathrm{~N} 1-\mathrm{C} 2-\mathrm{C} 4$ & 123.1 \\
$\mathrm{C} 2-\mathrm{C} 4-\mathrm{C} 6$ & 118.8 \\
$\mathrm{C} 4-\mathrm{C} 6-\mathrm{C} 5$ & 118.1
\end{tabular}


Table S408 Structural parameters of the OM3/MRCISD-optimized excitedstate equilibrium geometry of the $2{ }^{1} A^{\prime}\left({ }^{1} \pi \pi^{*}\right)$ state of pyrrole used for the statistical evaluations.

\begin{tabular}{ll}
$\begin{array}{l}\text { bond } \\
\text { or angle }\end{array}$ & $\begin{array}{c}\text { length } / \AA \\
\text { or angle / }\end{array}$ \\
\hline N6-C7 & 1.401 \\
C7-C10 & 1.479 \\
C9-C10 & 1.450 \\
C7-N6-C8 & 109.7 \\
C10-C7-N6 & 108.5 \\
C7-C10-C9 & 106.5 \\
H1-N6 & 1.044
\end{tabular}


Table S409 Structural parameters of the OM3/MRCISD-optimized excitedstate equilibrium geometry of the $1{ }^{1} B_{2}\left({ }^{1} \pi \pi^{*}\right)$ state of pyrrole used for the statistical evaluations.

\begin{tabular}{ll}
$\begin{array}{l}\text { bond } \\
\text { or angle }\end{array}$ & $\begin{array}{c}\text { length } / \AA \\
\text { or angle / }\end{array}$ \\
\hline N6-C7 & 1.419 \\
C7-C10 & 1.460 \\
C9-C10 & 1.384 \\
C7-N6-C8 & 106.2 \\
C10-C7-N6 & 109.2 \\
C7-C10-C9 & 107.7 \\
H1-N6 & 1.042
\end{tabular}


Table S410 Structural parameters of the OM3/MRCISD-optimized excitedstate equilibrium geometry of the $2{ }^{1} A^{\prime}\left({ }^{1} \pi \pi^{*}\right)$ state of cis-PSB3 used for the statistical evaluations.

\begin{tabular}{ll}
$\begin{array}{l}\text { bond } \\
\text { or angle }\end{array}$ & $\begin{array}{l}\text { length } / \AA \\
\text { or angle } /\end{array}$ \\
\hline C1-C2 & 1.392 \\
C2-C3 & 1.419 \\
C3-C4 & 1.425 \\
C4-C5 & 1.440 \\
C5-N6 & 1.368 \\
C1-C2-C3 & 120.7 \\
C2-C3-C4 & 123.2 \\
C3-C4-C5 & 124.1 \\
C4-C5-N6 & 119.4 \\
N6-H13 & 1.038 \\
N6-H14 & 1.038
\end{tabular}


Table S411 Structural parameters of the OM3/MRCISD-optimized excitedstate equilibrium geometry of the $2{ }^{1} A^{\prime}\left({ }^{1} \pi \pi^{*}\right)$ state of 3Me-cis-PSB5 used for the statistical evaluations.

\begin{tabular}{ll}
$\begin{array}{l}\text { bond } \\
\text { or angle }\end{array}$ & $\begin{array}{c}\text { length } / \AA \\
\text { or angle / }\end{array}$ \\
\hline $\mathrm{C} 10-\mathrm{C} 11$ & 1.372 \\
$\mathrm{C} 9-\mathrm{C} 10$ & 1.418 \\
$\mathrm{C} 8-\mathrm{C} 9$ & 1.428 \\
$\mathrm{C} 1-\mathrm{C} 8$ & 1.380 \\
$\mathrm{C} 1-\mathrm{C} 2$ & 1.460 \\
$\mathrm{C} 2-\mathrm{C} 3$ & 1.389 \\
$\mathrm{C} 4-\mathrm{C} 4$ & 1.462 \\
$\mathrm{C} 4-\mathrm{C} 5$ & 1.403 \\
$\mathrm{C} 5-\mathrm{N} 6$ & 1.371 \\
$\mathrm{C} 3-\mathrm{C} 7$ & 1.496 \\
$\mathrm{C} 11-\mathrm{C} 10-\mathrm{C} 9$ & 121.2 \\
$\mathrm{C} 10-\mathrm{C} 9-\mathrm{C} 8$ & 121.9 \\
$\mathrm{C} 9-\mathrm{C} 8-\mathrm{C} 1$ & 120.1 \\
$\mathrm{C} 8-\mathrm{C} 1-\mathrm{C} 2$ & 128.3 \\
$\mathrm{C} 1-\mathrm{C} 2-\mathrm{C} 3$ & 129.7 \\
$\mathrm{C} 2-\mathrm{C} 3-\mathrm{C} 4$ & 115.5 \\
$\mathrm{C} 3-\mathrm{C} 4-\mathrm{C} 5$ & 124.8 \\
$\mathrm{C} 4-\mathrm{C} 5-\mathrm{N} 6$ & 121.5 \\
$\mathrm{C} 2-\mathrm{C} 3-\mathrm{C} 7$ & 124.2 \\
$\mathrm{~N} 6-\mathrm{H} 16$ & 1.037 \\
$\mathrm{~N} 6-\mathrm{H} 17$ & 1.038
\end{tabular}


Table S412 Structural parameters of the OM3/MRCISD-optimized excitedstate equilibrium geometry of the $1{ }^{1} B_{u}\left({ }^{1} \pi \pi^{*}\right)$ state of stilbene used for the statistical evaluations.

\begin{tabular}{ll}
$\begin{array}{l}\text { bond } \\
\text { or angle }\end{array}$ & $\begin{array}{l}\text { length } / \AA \\
\text { or angle } /\end{array}$ \\
\hline $\mathrm{C} 1-\mathrm{C} 8$ & 1.408 \\
$\mathrm{C} 1-\mathrm{C} 2$ & 1.417 \\
$\mathrm{C} 2-\mathrm{C} 3$ & 1.438 \\
$\mathrm{C} 3-\mathrm{C} 4$ & 1.385 \\
$\mathrm{C} 4-\mathrm{C} 5$ & 1.410 \\
$\mathrm{C} 5-\mathrm{C} 6$ & 1.404 \\
$\mathrm{C} 6-\mathrm{C} 7$ & 1.389 \\
$\mathrm{C} 2-\mathrm{C} 7$ & 1.436 \\
$\mathrm{C} 8-\mathrm{C} 1-\mathrm{C} 2$ & 124.2 \\
$\mathrm{C} 1-\mathrm{C} 2-\mathrm{C} 3$ & 119.4 \\
$\mathrm{C} 1-\mathrm{C} 2-\mathrm{C} 7$ & 122.8
\end{tabular}


Table S413 Structural parameters of the OM3/MRCISD-optimized excitedstate equilibrium geometry of the $1^{1} B\left({ }^{1} \pi \pi^{*}\right)$ state of stilbene used for the statistical evaluations.

\begin{tabular}{ll}
$\begin{array}{l}\text { bond } \\
\text { or angle }\end{array}$ & $\begin{array}{l}\text { length } / \AA \\
\text { or angle } /\end{array}$ \\
\hline $\mathrm{C} 1-\mathrm{C} 8$ & 1.382 \\
$\mathrm{C} 1-\mathrm{C} 2$ & 1.445 \\
$\mathrm{C} 2-\mathrm{C} 3$ & 1.418 \\
$\mathrm{C} 3-\mathrm{C} 4$ & 1.391 \\
$\mathrm{C} 4-\mathrm{C} 5$ & 1.403 \\
$\mathrm{C} 5-\mathrm{C} 6$ & 1.396 \\
$\mathrm{C} 6-\mathrm{C} 7$ & 1.397 \\
$\mathrm{C} 2-\mathrm{C} 7$ & 1.415 \\
$\mathrm{C} 8-\mathrm{C} 1-\mathrm{C} 2$ & 123.1 \\
$\mathrm{C} 1-\mathrm{C} 2-\mathrm{C} 3$ & 120.7 \\
$\mathrm{C} 1-\mathrm{C} 2-\mathrm{C} 7$ & 120.0
\end{tabular}


Table S414 Structural parameters of the OM3/MRCISD-optimized excitedstate equilibrium geometry of the $2{ }^{1} A^{\prime}\left({ }^{1} \pi \pi^{*}\right)$ state of styrene used for the statistical evaluations.

\begin{tabular}{ll}
$\begin{array}{l}\text { bond } \\
\text { or angle }\end{array}$ & $\begin{array}{l}\text { length } / \AA \\
\text { or angle } /{ }^{\circ}\end{array}$ \\
\hline C1-C2 & 1.363 \\
C1-C3 & 1.439 \\
C3-C4 & 1.440 \\
C3-C5 & 1.443 \\
C4-C6 & 1.425 \\
C5-C7 & 1.425 \\
C6-C8 & 1.419 \\
C7-C8 & 1.417
\end{tabular}


Table S415 Structural parameters of the OM3/MRCISD-optimized excitedstate equilibrium geometry of the $1^{3} A^{\prime}\left({ }^{3} \pi \pi^{*}\right)$ state of styrene used for the statistical evaluations.

\begin{tabular}{ll}
$\begin{array}{l}\text { bond } \\
\text { or angle }\end{array}$ & $\begin{array}{l}\text { length } / \AA \\
\text { or angle } /{ }^{\circ}\end{array}$ \\
\hline C1-C2 & 1.441 \\
C1-C3 & 1.395 \\
C3-C4 & 1.452 \\
C3-C5 & 1.456 \\
C4-C6 & 1.385 \\
C5-C7 & 1.383 \\
C6-C8 & 1.416 \\
C7-C8 & 1.417
\end{tabular}


Table S416 Structural parameters of the OM3/MRCISD-optimized excitedstate equilibrium geometry of the $1{ }^{1} B_{3 u}\left({ }^{1} n \pi^{*}\right)$ state of $s$-tetrazine used for the statistical evaluations.

\begin{tabular}{ll}
$\begin{array}{l}\text { bond } \\
\text { or angle }\end{array}$ & $\begin{array}{l}\text { length } / \AA \\
\text { or angle / }\end{array}$ \\
\hline N3-N4 & 1.338 \\
C2-N4 & 1.385 \\
C2-H7 & 1.108 \\
N3-C1-N5 & 117.0
\end{tabular}


Table S417 Structural parameters of the OM3/MRCISD-optimized excitedstate equilibrium geometry of the $2{ }^{1} A\left({ }^{1} n \pi^{*}\right)$ state of thymine used for the statistical evaluations.

\begin{tabular}{ll}
$\begin{array}{l}\text { bond } \\
\text { or angle }\end{array}$ & $\begin{array}{l}\text { length } / \AA \\
\text { or angle } /\end{array}$ \\
\hline N1-C6 & 1.478 \\
C5-N6 & 1.379 \\
N4-C5 & 1.469 \\
C3-C4 & 1.427 \\
C2-C3 & 1.501 \\
N1-C2 & 1.370 \\
C6-09 & 1.250 \\
C4-07 & 1.260 \\
C3-C8 & 1.465 \\
C2-H10 & 1.135 \\
N1-H11 & 1.054 \\
N5-H12 & 1.046
\end{tabular}


Table S418 Structural parameters of the OM3/MRCISD-optimized excitedstate equilibrium geometry of the $2{ }^{1} A^{\prime}\left({ }^{1} \pi \pi^{*}\right)$ state of uracil used for the statistical evaluations.

\begin{tabular}{ll}
$\begin{array}{l}\text { bond } \\
\text { or angle }\end{array}$ & $\begin{array}{l}\text { length } / \AA \\
\text { or angle } /\end{array}$ \\
\hline N1-C3 & 1.388 \\
N2-C3 & 1.471 \\
N2-C4 & 1.364 \\
C4-C5 & 1.481 \\
C5-C6 & 1.414 \\
N1-C6 & 1.472 \\
C3-O8 & 1.254 \\
C6-O7 & 1.265 \\
C4-H9 & 1.101 \\
C5-H12 & 1.086 \\
N2-H11 & 1.056 \\
N1-H10 & 1.045
\end{tabular}




\subsubsection{TDDFT/B3LYP-Optimized Excited-State Minima}

For the TDDFT/B3LYP excited-state geometry optimizations, we normally employed the same point-group symmetry as we did for the OMx/MRCI geometry optimizations. Only in rare cases did we diverge from this practice by imposing a lower level of symmetry constraints, for example when the use of the same point-group symmetry would restrict the molecule in such a way that key structural properties would be distorted. The pyramidalization of exocyclic amino groups that is found for all TDDFT-optimized excited-state structures (see Table 8 in the article) is one example for which the point group of certain molecules was lowered (e.g. the ${ }^{1} \pi \pi^{*}$ state of aniline for which $C_{s}$ symmetry was used at the TDDFT level but $C_{2 v}$ at the OMx/MRCI level).

In other cases, we were unable to converge the geometry in a to-be-preferred lower symmetry. Exemplary of this, we highlight the case of the ${ }^{1} \pi \pi^{*}$ state of 2-amino-9-methylpurine here, which possibly constitutes an artefact of the TDDFT method. Geometry optimization of the ${ }^{1} \pi \pi^{*}$ state in $C_{s}$ symmetry $\left(2^{1} A^{\prime}\right)$ results in a completely planar geometry. This geometry exhibits an imaginary vibrational mode that displaces the planar amino group toward a nonplanar arrangement. Geometry optimization of the same state in $C_{1}$ symmetry $\left(2{ }^{1} A\right)$ starting from a geometry with a strongly bent structure (i.e. a nonplanar aromatic system and out-of-plane-bent amino group) again converges to a completely planar structure that exhibits the same imaginary vibrational mode. 
Table S419 Cartesian coordinates of the TDDFT/TZVP-optimized excitedstate equilibrium geometry of the $2{ }^{1} A\left({ }^{1} n \pi^{*}\right)$ state of $9 H$-adenine (excited-state energy: $\left.-467.05578 E_{h}\right)$.

$\begin{array}{lrrr}15 & & & \\ & & & \\ \mathrm{C} & -1.2019380 & -0.6945958 & 0.0012563 \\ \mathrm{C} & 0.2383382 & -0.5419738 & -0.0026802 \\ \mathrm{C} & 0.6792336 & 0.8018929 & -0.0064495 \\ \mathrm{~N} & -0.1188283 & 1.8523845 & -0.0101704 \\ \mathrm{C} & -1.4901674 & 1.6754085 & 0.0118038 \\ \mathrm{~N} & -1.9085522 & 0.4115074 & 0.0103515 \\ \mathrm{~N} & 2.0399150 & 0.7086293 & -0.0039892 \\ \mathrm{C} & 2.3684495 & -0.6645597 & 0.0079684 \\ \mathrm{~N} & 1.2650912 & -1.3971725 & 0.0086854 \\ \mathrm{~N} & -1.7861367 & -1.9455389 & -0.0567420 \\ \mathrm{H} & -2.1645204 & 2.5104414 & 0.0221401 \\ \mathrm{H} & -1.1814522 & -2.6924501 & 0.2549239 \\ \mathrm{H} & -2.7301339 & -2.0067888 & 0.2949440 \\ \mathrm{H} & 2.6817477 & 1.4841052 & -0.0046566 \\ \mathrm{H} & 3.3833946 & -1.0169329 & 0.0115678\end{array}$

S464 
Table S420 Cartesian coordinates of the TDDFT/TZVP-optimized excitedstate equilibrium geometry of the $2{ }^{1} A\left({ }^{1} n \pi^{*}\right)$ state of acetaldehyde (excitedstate energy: $\left.-153.66193 E_{h}\right)$.

7

$\begin{array}{rrrr}\mathrm{C} & -0.0937654 & -0.4951126 & -0.0748689 \\ \mathrm{C} & 1.2483153 & 0.1867561 & 0.0132414 \\ \mathrm{H} & -0.2056025 & -1.4878971 & 0.3737271 \\ \mathrm{O} & -1.1540493 & 0.2578323 & 0.0165172 \\ \mathrm{H} & 2.0287690 & -0.5545459 & -0.1553071 \\ \mathrm{H} & 1.3303115 & 0.9701233 & -0.7434010 \\ \mathrm{H} & 1.4068013 & 0.6542049 & 0.9971696\end{array}$


Table S421 Cartesian coordinates of the TDDFT/TZVP-optimized excitedstate equilibrium geometry of the $1^{3} A\left({ }^{3} n \pi^{*}\right)$ state of acetaldehyde (excitedstate energy: $-153.69000 E_{h}$ ).

7

C $\quad 1.2486086$

C -0.0997970

O -1.1495669

0.1874670

0.0172206

$\mathrm{H} \quad 2.0296393$

$-0.5051384$

$-0.0953445$

H 1.3301218

0.2613336

0.0202299

$\mathrm{H} \quad 1.4088814$

$-0.5481223$

$-0.1715096$

$\mathrm{H} \quad-0.2111329$

0.9796693

$-0.7282712$

0.6298089

1.0092830

$-1.4240472$

0.5003293 
Table S422 Cartesian coordinates of the TDDFT/TZVP-optimized excitedstate equilibrium geometry of the $1^{1} A^{\prime \prime}\left({ }^{1} n \pi^{*}\right)$ state of acetone (excited-state energy: $-192.96943 E_{h}$ ).

$\begin{array}{lrrr}10 & & & \\ \text { C } & 0.6725847 & -0.0461752 & -1.3048556 \\ \mathrm{C} & -0.0302835 & 0.2249833 & 0.0000000 \\ \mathrm{O} & -1.3100305 & -0.0553674 & 0.0000000 \\ \mathrm{C} & 0.6725847 & -0.0461752 & 1.3048556 \\ \mathrm{H} & 0.1422383 & 0.4160440 & -2.1389457 \\ \mathrm{H} & 0.7379455 & -1.1286299 & -1.5084265 \\ \mathrm{H} & 1.6825957 & 0.3617669 & -1.2551110 \\ \mathrm{H} & 0.1422383 & 0.4160440 & 2.1389457 \\ \mathrm{H} & 1.6825957 & 0.3617669 & 1.2551110 \\ \mathrm{H} & 0.7379455 & -1.1286299 & 1.5084265\end{array}$


Table S423 Cartesian coordinates of the TDDFT/TZVP-optimized excitedstate equilibrium geometry of the $1^{3} A^{\prime \prime}\left({ }^{3} n \pi^{*}\right)$ state of acetone (excited-state energy: $\left.-192.99462 E_{h}\right)$.

$\begin{array}{lrrr}10 & & & \\ \text { C } & 0.6734107 & -0.0559048 & -1.2986608 \\ \mathrm{C} & -0.0388727 & 0.2688775 & 0.0000000 \\ \mathrm{O} & -1.3070564 & -0.0677622 & 0.0000000 \\ \mathrm{C} & 0.6734107 & -0.0559048 & 1.2986608 \\ \mathrm{H} & 0.1105357 & 0.3125886 & -2.1567188 \\ \mathrm{H} & 0.8200766 & -1.1398351 & -1.4234655 \\ \mathrm{H} & 1.6498961 & 0.4292135 & -1.2888710 \\ \mathrm{H} & 0.1105357 & 0.3125886 & 2.1567188 \\ \mathrm{H} & 1.6498961 & 0.4292135 & 1.2888710 \\ \mathrm{H} & 0.8200766 & -1.1398351 & 1.4234655\end{array}$


Table S424 Cartesian coordinates of the TDDFT/TZVP-optimized excitedstate equilibrium geometry of the $1^{1} A^{\prime \prime}\left({ }^{1} n \pi^{*}\right)$ state of acetophenone (excitedstate energy: $-384.66846 E_{h}$ ).

$\begin{array}{lrrr}17 & & & \\ & & & \\ \mathrm{C} & 0.0737527 & -1.6176214 & 0.0000000 \\ \mathrm{O} & 1.1642574 & -2.3190643 & 0.0000000 \\ \mathrm{C} & -1.1447374 & -2.5353880 & 0.0000000 \\ \mathrm{H} & -0.8394966 & -3.5804180 & 0.0000000 \\ \mathrm{H} & -1.7421699 & -2.3424337 & -0.8929222 \\ \mathrm{H} & -1.7421699 & -2.3424337 & 0.8929222 \\ \mathrm{C} & 0.0229386 & -0.2210033 & 0.0000000 \\ \mathrm{C} & -1.2222614 & 0.4837410 & 0.0000000 \\ \mathrm{C} & 1.2272416 & 0.5557978 & 0.0000000 \\ \mathrm{C} & -1.2447498 & 1.8614377 & 0.0000000 \\ \mathrm{C} & 1.1709896 & 1.9320587 & 0.0000000 \\ \mathrm{C} & -0.0572209 & 2.6060604 & 0.0000000 \\ \mathrm{H} & -2.1567349 & -0.0622716 & 0.0000000 \\ \mathrm{H} & 2.1852027 & 0.0522160 & 0.0000000 \\ \mathrm{H} & -2.1998097 & 2.3742989 & 0.0000000 \\ \mathrm{H} & 2.0946333 & 2.4995714 & 0.0000000 \\ \mathrm{H} & -0.0894348 & 3.6876581 & 0.0000000\end{array}$

S469 
Table S425 Cartesian coordinates of the TDDFT/TZVP-optimized excitedstate equilibrium geometry of the $2{ }^{1} A^{\prime}\left({ }^{1} \pi \pi^{*}\right)$ state of acetophenone (excitedstate energy: $\left.-384.62427 E_{h}\right)$.

$\begin{array}{lrrr}17 & & & \\ & & & \\ \mathrm{C} & 0.0873474 & -1.7212088 & 0.0000000 \\ \mathrm{O} & 1.2237146 & -2.2562318 & 0.0000000 \\ \mathrm{C} & -1.1815948 & -2.5435603 & 0.0000000 \\ \mathrm{H} & -0.9053932 & -3.5967525 & 0.0000000 \\ \mathrm{H} & -1.8067891 & -2.3526019 & -0.8819095 \\ \mathrm{H} & -1.8067891 & -2.3526019 & 0.8819095 \\ \mathrm{C} & -0.0148631 & -0.2626845 & 0.0000000 \\ \mathrm{C} & -1.2117158 & 0.4784718 & 0.0000000 \\ \mathrm{C} & 1.1768762 & 0.5146950 & 0.0000000 \\ \mathrm{C} & -1.2190016 & 1.9149635 & 0.0000000 \\ \mathrm{C} & 1.1635945 & 1.9422340 & 0.0000000 \\ \mathrm{C} & -0.0373633 & 2.6600808 & 0.0000000 \\ \mathrm{H} & -2.1662785 & -0.0311646 & 0.0000000 \\ \mathrm{H} & 2.1160646 & -0.0239346 & 0.0000000 \\ \mathrm{H} & -2.1779718 & 2.4192158 & 0.0000000 \\ \mathrm{H} & 2.1123830 & 2.4648724 & 0.0000000 \\ \mathrm{H} & -0.0521356 & 3.7400368 & 0.0000000\end{array}$


Table S426 Cartesian coordinates of the TDDFT/TZVP-optimized excitedstate equilibrium geometry of the $1^{3} A^{\prime \prime}\left({ }^{3} n \pi^{*}\right)$ state of acetophenone (excitedstate energy: $-384.68733 E_{h}$ ).

$\begin{array}{lrrr}17 & & \\ & & & \\ \mathrm{C} & 0.0610607 & -1.6257012 & 0.0000000 \\ \mathrm{O} & 1.1786945 & -2.3079552 & 0.0000000 \\ \mathrm{C} & -1.1452397 & -2.5407317 & 0.0000000 \\ \mathrm{H} & -0.8358031 & -3.5852933 & 0.0000000 \\ \mathrm{H} & -1.7535029 & -2.3618818 & -0.8900574 \\ \mathrm{H} & -1.7535029 & -2.3618818 & 0.8900574 \\ \mathrm{C} & 0.0250703 & -0.2203607 & 0.0000000 \\ \mathrm{C} & -1.2156914 & 0.4805485 & 0.0000000 \\ \mathrm{C} & 1.2223397 & 0.5564315 & 0.0000000 \\ \mathrm{C} & -1.2432603 & 1.8616205 & 0.0000000 \\ \mathrm{C} & 1.1655738 & 1.9349077 & 0.0000000 \\ \mathrm{C} & -0.0611643 & 2.6071658 & 0.0000000 \\ \mathrm{H} & -2.1484773 & -0.0682947 & 0.0000000 \\ \mathrm{H} & 2.1836333 & 0.0592182 & 0.0000000 \\ \mathrm{H} & -2.2004833 & 2.3704930 & 0.0000000 \\ \mathrm{H} & 2.0893758 & 2.5020891 & 0.0000000 \\ \mathrm{H} & -0.0946555 & 3.6888942 & 0.0000000\end{array}$


Table S427 Cartesian coordinates of the TDDFT/TZVP-optimized excitedstate equilibrium geometry of the $1^{3} A^{\prime}\left({ }^{3} \pi \pi^{*}\right)$ state of acetophenone (excitedstate energy: $-384.68399 E_{h}$ ).

$\begin{array}{lrrr}17 & & & \\ & & & \\ \mathrm{C} & 0.0776055 & -1.6641783 & 0.0000000 \\ \mathrm{O} & 1.2322123 & -2.2163591 & 0.0000000 \\ \mathrm{C} & -1.1644874 & -2.5315201 & 0.0000000 \\ \mathrm{H} & -0.8566712 & -3.5755817 & 0.0000000 \\ \mathrm{H} & -1.7868365 & -2.3524524 & -0.8817550 \\ \mathrm{H} & -1.7868365 & -2.3524524 & 0.8817550 \\ \mathrm{C} & -0.0027443 & -0.2539595 & 0.0000000 \\ \mathrm{C} & -1.2719277 & 0.4832755 & 0.0000000 \\ \mathrm{C} & 1.2469300 & 0.5515966 & 0.0000000 \\ \mathrm{C} & -1.2824206 & 1.8384277 & 0.0000000 \\ \mathrm{C} & 1.1962562 & 1.9109114 & 0.0000000 \\ \mathrm{C} & -0.0507453 & 2.6012082 & 0.0000000 \\ \mathrm{H} & -2.2009660 & -0.0704451 & 0.0000000 \\ \mathrm{H} & 2.1803598 & 0.0085744 & 0.0000000 \\ \mathrm{H} & -2.2253941 & 2.3728131 & 0.0000000 \\ \mathrm{H} & 2.1157355 & 2.4844782 & 0.0000000 \\ \mathrm{H} & -0.0846671 & 3.6820417 & 0.0000000\end{array}$


Table S428 Cartesian coordinates of the TDDFT/TZVP-optimized excitedstate equilibrium geometry of the $1^{1} A_{2}\left({ }^{1} \pi \pi^{*}\right)$ state of acetylene (excited-state energy: $\left.-77.12173 E_{h}\right)$.

4

$\begin{array}{lrrr}\mathrm{C} & -0.6696185 & 0.0000000 & -0.0628488 \\ \mathrm{C} & 0.6696185 & 0.0000000 & -0.0628488 \\ \mathrm{H} & -1.4077720 & 0.0000000 & 0.7489177 \\ \mathrm{H} & 1.4077720 & 0.0000000 & 0.7489177\end{array}$


Table S429 Cartesian coordinates of the TDDFT/TZVP-optimized excitedstate equilibrium geometry of the $1{ }^{1} B_{2}\left(\equiv{ }^{1} B_{1}\right.$ in Turbomole) $\left({ }^{1} \pi \pi^{*}\right)$ state of acetylene (excited-state energy: $-77.08556 E_{h}$ ).

4

C $\quad-0.6640136$

$0.0000000-0.0500504$

C $\quad 0.6640136 \quad 0.0000000 \quad-0.0500504$

$\mathrm{H} \quad-1.5456536 \quad 0.0000000 \quad 0.5964098$

$\mathrm{H} \quad 1.5456536 \quad 0.0000000 \quad 0.5964098$ 
Table S430 Cartesian coordinates of the TDDFT/TZVP-optimized excitedstate equilibrium geometry of the $2{ }^{1} A_{2}\left({ }^{1} \pi \pi^{*}\right)$ state of acetylene (excited-state energy: $\left.-77.07752 E_{h}\right)$.

4

$\begin{array}{rrrr}\mathrm{C} & -0.6614451 & 0.0000000 & -0.0081591 \\ \mathrm{C} & 0.6614451 & 0.0000000 & -0.0081591 \\ \mathrm{H} & -1.7197247 & 0.0000000 & 0.0972258 \\ \mathrm{H} & 1.7197247 & 0.0000000 & 0.0972258\end{array}$


Table S431 Cartesian coordinates of the TDDFT/TZVP-optimized excitedstate equilibrium geometry of the $2{ }^{3} A_{2}\left({ }^{3} \pi \pi^{*}\right)$ state of acetylene (excited-state energy: $\left.-77.08894 E_{h}\right)$.

4

$\begin{array}{lrrr}\mathrm{C} & -0.6650442 & 0.0000000 & -0.0200889 \\ \mathrm{C} & 0.6650442 & 0.0000000 & -0.0200889 \\ \mathrm{H} & -1.7018944 & 0.0000000 & 0.2393824 \\ \mathrm{H} & 1.7018944 & 0.0000000 & 0.2393824\end{array}$


Table S432 Cartesian coordinates of the TDDFT/TZVP-optimized excitedstate equilibrium geometry of the $1^{1} A_{u}\left({ }^{1} \pi \pi^{*}\right)$ state of acetylene (excited-state energy: $\left.-77.13377 E_{h}\right)$.

4

$\begin{array}{lrrr}\mathrm{C} & -0.6720251 & -0.1208914 & 0.0000000 \\ \mathrm{C} & 0.6720251 & 0.1208914 & 0.0000000 \\ \mathrm{H} & -1.4167137 & 0.6833383 & 0.0000000 \\ \mathrm{H} & 1.4167137 & -0.6833383 & 0.0000000\end{array}$


Table S433 Cartesian coordinates of the TDDFT/TZVP-optimized excitedstate equilibrium geometry of the $1{ }^{1} B_{u}\left({ }^{1} \pi \pi^{*}\right)$ state of acetylene (excited-state energy: $\left.-77.08012 E_{h}\right)$.

4

$\begin{array}{rrrr}\mathrm{C} & -0.6609998 & -0.0739095 & 0.0000000 \\ \mathrm{C} & 0.6609998 & 0.0739095 & 0.0000000 \\ \mathrm{H} & -1.6469862 & 0.3534668 & 0.0000000 \\ \mathrm{H} & 1.6469862 & -0.3534668 & 0.0000000\end{array}$


Table S434 Cartesian coordinates of the TDDFT/TZVP-optimized excitedstate equilibrium geometry of the $2{ }^{1} A_{u}\left({ }^{1} \pi \pi^{*}\right)$ state of acetylene (excited-state energy: $\left.-77.07731 E_{h}\right)$.

4

$\begin{array}{lrrr}\mathrm{C} & -0.6613325 & -0.0123616 & 0.0000000 \\ \mathrm{C} & 0.6613325 & 0.0123616 & 0.0000000 \\ \mathrm{H} & -1.7219757 & 0.0565726 & 0.0000000 \\ \mathrm{H} & 1.7219757 & -0.0565726 & 0.0000000\end{array}$


Table S435 Cartesian coordinates of the TDDFT/TZVP-optimized excitedstate equilibrium geometry of the $1^{3} B_{u}\left({ }^{3} \pi \pi^{*}\right)$ state of acetylene (excited-state energy: $\left.-77.23126 E_{h}\right)$.

4

$\begin{array}{lrrr}\mathrm{C} & -0.6780969 & -0.1121376 & 0.0000000 \\ \mathrm{C} & 0.6780969 & 0.1121376 & 0.0000000 \\ \mathrm{H} & -1.4967804 & 0.6053716 & 0.0000000 \\ \mathrm{H} & 1.4967804 & -0.6053716 & 0.0000000\end{array}$


Table S436 Cartesian coordinates of the TDDFT/TZVP-optimized excitedstate equilibrium geometry of the $1^{3} A_{u}\left({ }^{3} \pi \pi^{*}\right)$ state of acetylene (excited-state energy: $\left.-77.17163 E_{h}\right)$.

4

$\begin{array}{lrrr}\mathrm{C} & -0.7003548 & -0.1227031 & 0.0000000 \\ \mathrm{C} & 0.7003548 & 0.1227031 & 0.0000000 \\ \mathrm{H} & -1.3571174 & 0.7545591 & 0.0000000 \\ \mathrm{H} & 1.3571174 & -0.7545591 & 0.0000000\end{array}$


Table S437 Cartesian coordinates of the TDDFT/TZVP-optimized excitedstate equilibrium geometry of the $2{ }^{3} B_{u}\left({ }^{3} \pi \pi^{*}\right)$ state of acetylene (excited-state energy: $\left.-77.12935 E_{h}\right)$.

4

$\begin{array}{lrrr}\mathrm{C} & -0.6770300 & -0.0587800 & 0.0000000 \\ \mathrm{C} & 0.6770300 & 0.0587800 & 0.0000000 \\ \mathrm{H} & -1.6887751 & 0.2808035 & 0.0000000 \\ \mathrm{H} & 1.6887751 & -0.2808035 & 0.0000000\end{array}$


Table S438 Cartesian coordinates of the TDDFT/TZVP-optimized excitedstate equilibrium geometry of the $2{ }^{3} A_{u}\left({ }^{3} \pi \pi^{*}\right)$ state of acetylene (excited-state energy: $\left.-77.08784 E_{h}\right)$.

4

$\begin{array}{lrrr}\mathrm{C} & -0.6645356 & -0.0434597 & 0.0000000 \\ \mathrm{C} & 0.6645356 & 0.0434597 & 0.0000000 \\ \mathrm{H} & -1.7012931 & 0.2022846 & 0.0000000 \\ \mathrm{H} & 1.7012931 & -0.2022846 & 0.0000000\end{array}$

S483 
Table S439 Cartesian coordinates of the TDDFT/TZVP-optimized excitedstate equilibrium geometry of the $2{ }^{1} A\left({ }^{1} n \pi^{*}\right)$ state of acrolein (excited-state energy: $-191.75745 E_{h}$ ).

8

$\begin{array}{rrrr}\text { C } & -1.8871014 & 0.1454905 & 0.0000016 \\ \mathrm{C} & -0.6238984 & -0.4140335 & -0.0000016 \\ \mathrm{C} & 0.5519297 & 0.3197792 & -0.0000031 \\ \mathrm{O} & 1.7736080 & -0.0803063 & 0.0000022 \\ \mathrm{H} & -2.7638254 & -0.4844473 & 0.0000053 \\ \mathrm{H} & -2.0424407 & 1.2162219 & 0.0000015 \\ \mathrm{H} & -0.5135291 & -1.4926392 & -0.0000035 \\ \mathrm{H} & 0.5121329 & 1.4250177 & -0.0000004\end{array}$


Table S440 Cartesian coordinates of the TDDFT/TZVP-optimized excitedstate equilibrium geometry of the $1^{3} A\left({ }^{3} n \pi^{*}\right)$ state of acrolein (excited-state energy: $\left.-191.77914 E_{h}\right)$.

8

$\begin{array}{rrrr}\text { C } & -1.8875625 & 0.1255500 & 0.0000046 \\ \mathrm{C} & -0.6198137 & -0.3962153 & -0.0000046 \\ \mathrm{C} & 0.5508316 & 0.3560888 & -0.0000050 \\ \mathrm{O} & 1.7691708 & -0.1045369 & 0.0000028 \\ \mathrm{H} & -2.7472960 & -0.5276552 & -0.0000165 \\ \mathrm{H} & -2.0713648 & 1.1920341 & 0.0000074 \\ \mathrm{H} & -0.4926728 & -1.4738193 & 0.0000185 \\ \mathrm{H} & 0.5440090 & 1.4508222 & 0.0000049\end{array}$


Table S441 Cartesian coordinates of the TDDFT/TZVP-optimized excitedstate equilibrium geometry of the $2{ }^{1} A^{\prime}\left({ }^{1} \pi \pi^{*}\right)$ state of 2-amino-9-methylpurine (excited-state energy: $-506.35446 E_{h}$ ).

$\begin{array}{lrrr}18 & & \\ & & & \\ \mathrm{~N} & 1.8363617 & -1.5891373 & 0.0000000 \\ \mathrm{C} & 1.4492267 & -0.2897243 & 0.0000000 \\ \mathrm{C} & 0.0361314 & -0.2084185 & 0.0000000 \\ \mathrm{~N} & -0.4156101 & -1.5195673 & 0.0000000 \\ \mathrm{C} & 0.6935697 & -2.3029399 & 0.0000000 \\ \mathrm{C} & 2.1780323 & 0.9362308 & 0.0000000 \\ \mathrm{~N} & 1.4409699 & 2.1015000 & 0.0000000 \\ \mathrm{C} & 0.1205129 & 1.9966352 & 0.0000000 \\ \mathrm{~N} & -0.7068013 & 0.9067187 & 0.0000000 \\ \mathrm{~N} & -0.5769167 & 3.1757437 & 0.0000000 \\ \mathrm{C} & -1.8083762 & -1.9363583 & 0.0000000 \\ \mathrm{H} & 3.2539965 & 1.0030068 & 0.0000000 \\ \mathrm{H} & 0.6402234 & -3.3799783 & 0.0000000 \\ \mathrm{H} & -1.5845360 & 3.1502225 & 0.0000000 \\ \mathrm{H} & -0.0693011 & 4.0470699 & 0.0000000 \\ \mathrm{H} & -2.4241034 & -1.0394740 & 0.0000000 \\ \mathrm{H} & -2.0316899 & -2.5257648 & 0.8904831 \\ \mathrm{H} & -2.0316899 & -2.5257648 & -0.8904831\end{array}$


Table S442 Cartesian coordinates of the TDDFT/TZVP-optimized excitedstate equilibrium geometry of the $1^{1} B_{2}\left(\equiv 1^{1} A^{\prime \prime}\right.$ in Turbomole) $\left({ }^{1} \pi \pi^{*}\right)$ state of aniline (excited-state energy: $-287.35581 E_{h}$ ).

$\begin{array}{lrrr}14 & & \\ & & & \\ \mathrm{C} & 0.2255846 & -0.0111203 & -1.2462100 \\ \mathrm{C} & 0.8905402 & -0.0553517 & 0.0000000 \\ \mathrm{C} & 0.2255846 & -0.0111203 & 1.2462100 \\ \mathrm{C} & -1.1997288 & 0.0802707 & 1.2367025 \\ \mathrm{C} & -1.8690183 & 0.1241509 & 0.0000000 \\ \mathrm{C} & -1.1997288 & 0.0802707 & -1.2367025 \\ \mathrm{~N} & 2.2612556 & -0.1306335 & 0.0000000 \\ \mathrm{H} & 2.7733090 & -0.2117898 & 0.8640581 \\ \mathrm{H} & 2.7733090 & -0.2117898 & -0.8640581 \\ \mathrm{H} & 0.7916256 & -0.0424776 & 2.1685262 \\ \mathrm{H} & -1.7564833 & 0.1178630 & 2.1619331 \\ \mathrm{H} & -2.9513917 & 0.1963424 & 0.0000000 \\ \mathrm{H} & -1.7564833 & 0.1178630 & -2.1619331 \\ \mathrm{H} & 0.7916256 & -0.0424776 & -2.1685262\end{array}$


Table S443 Cartesian coordinates of the TDDFT/TZVP-optimized excitedstate equilibrium geometry of the $1^{1} A^{\prime \prime}\left({ }^{1} n \pi^{*}\right)$ state of benzaldehyde (excitedstate energy: $-345.36306 E_{h}$ ).

$\begin{array}{lrrr}14 & & & \\ \text { C } & 0.4999089 & 1.8797664 & 0.0000000 \\ \mathrm{O} & -0.3362704 & 2.8636379 & 0.0000000 \\ \mathrm{H} & 1.5396309 & 2.2418148 & 0.0000000 \\ \mathrm{C} & 0.2298690 & 0.5126238 & 0.0000000 \\ \mathrm{C} & 1.3050609 & -0.4298420 & 0.0000000 \\ \mathrm{C} & -1.1094079 & 0.0086171 & 0.0000000 \\ \mathrm{C} & 1.0466382 & -1.7810137 & 0.0000000 \\ \mathrm{C} & -1.3346960 & -1.3517884 & 0.0000000 \\ \mathrm{C} & -0.2718112 & -2.2621728 & 0.0000000 \\ \mathrm{H} & 2.3279006 & -0.0713557 & 0.0000000 \\ \mathrm{H} & -1.9416332 & 0.7001541 & 0.0000000 \\ \mathrm{H} & 1.8744195 & -2.4804296 & 0.0000000 \\ \mathrm{H} & -2.3546963 & -1.7185854 & 0.0000000 \\ \mathrm{H} & -0.4641398 & -3.3270919 & 0.0000000\end{array}$


Table S444 Cartesian coordinates of the TDDFT/TZVP-optimized excitedstate equilibrium geometry of the $2{ }^{1} A^{\prime}\left({ }^{1} \pi \pi^{*}\right)$ state of benzaldehyde (excitedstate energy: $-345.32084 E_{h}$ ).

$\begin{array}{lrrr}14 & & \\ \text { C } & 0.5329601 & 1.9843799 & 0.0000000 \\ \mathrm{O} & -0.4101372 & 2.8076369 & 0.0000000 \\ \mathrm{H} & 1.5868215 & 2.3043268 & 0.0000000 \\ \mathrm{C} & 0.2938923 & 0.5451470 & 0.0000000 \\ \mathrm{C} & 1.3009373 & -0.4305209 & 0.0000000 \\ \mathrm{C} & -1.0340078 & 0.0467641 & 0.0000000 \\ \mathrm{C} & 1.0043415 & -1.8347278 & 0.0000000 \\ \mathrm{C} & -1.3275053 & -1.3538361 & 0.0000000 \\ \mathrm{C} & -0.3106877 & -2.3103019 & 0.0000000 \\ \mathrm{H} & 2.3413962 & -0.1253873 & 0.0000000 \\ \mathrm{H} & -1.8380809 & 0.7724735 & 0.0000000 \\ \mathrm{H} & 1.8331917 & -2.5323716 & 0.0000000 \\ \mathrm{H} & -2.3665585 & -1.6598056 & 0.0000000 \\ \mathrm{H} & -0.5273188 & -3.3684694 & 0.0000000\end{array}$


Table S445 Cartesian coordinates of the TDDFT/TZVP-optimized excitedstate equilibrium geometry of the $1^{3} A^{\prime \prime}\left({ }^{3} n \pi^{*}\right)$ state of benzaldehyde (excitedstate energy: $-345.38337 E_{h}$ ).

$\begin{array}{lrrr}14 & & \\ \text { C } & 0.5186108 & 1.8859710 & 0.0000000 \\ \mathrm{O} & -0.3505705 & 2.8571293 & 0.0000000 \\ \mathrm{H} & 1.5434481 & 2.2644840 & 0.0000000 \\ \mathrm{C} & 0.2334211 & 0.5124846 & 0.0000000 \\ \mathrm{C} & 1.3005429 & -0.4303991 & 0.0000000 \\ \mathrm{C} & -1.1000546 & 0.0120978 & 0.0000000 \\ \mathrm{C} & 1.0400931 & -1.7846814 & 0.0000000 \\ \mathrm{C} & -1.3325289 & -1.3497243 & 0.0000000 \\ \mathrm{C} & -0.2751284 & -2.2629101 & 0.0000000 \\ \mathrm{H} & 2.3246288 & -0.0756296 & 0.0000000 \\ \mathrm{H} & -1.9337570 & 0.7019802 & 0.0000000 \\ \mathrm{H} & 1.8680481 & -2.4839796 & 0.0000000 \\ \mathrm{H} & -2.3544870 & -1.7108336 & 0.0000000 \\ \mathrm{H} & -0.4705176 & -3.3274252 & 0.0000000\end{array}$


Table S446 Cartesian coordinates of the TDDFT/TZVP-optimized excitedstate equilibrium geometry of the $1^{3} A^{\prime}\left({ }^{3} \pi \pi^{*}\right)$ state of benzaldehyde (excitedstate energy: $-345.38096 E_{h}$ ).

$\begin{array}{lrrr}14 & & \\ \text { C } & 0.5605825 & 1.8997248 & 0.0000000 \\ \mathrm{O} & -0.3780226 & 2.7842681 & 0.0000000 \\ \mathrm{H} & 1.6063725 & 2.2275166 & 0.0000000 \\ \mathrm{C} & 0.2827627 & 0.5327290 & 0.0000000 \\ \mathrm{C} & 1.3474949 & -0.4701787 & 0.0000000 \\ \mathrm{C} & -1.1155329 & 0.0451353 & 0.0000000 \\ \mathrm{C} & 1.0485753 & -1.7917322 & 0.0000000 \\ \mathrm{C} & -1.3775485 & -1.2921186 & 0.0000000 \\ \mathrm{C} & -0.3250077 & -2.2472421 & 0.0000000 \\ \mathrm{H} & 2.3761838 & -0.1298496 & 0.0000000 \\ \mathrm{H} & -1.8995940 & 0.7884233 & 0.0000000 \\ \mathrm{H} & 1.8429285 & -2.5288139 & 0.0000000 \\ \mathrm{H} & -2.4041125 & -1.6393192 & 0.0000000 \\ \mathrm{H} & -0.5420653 & -3.3067545 & 0.0000000\end{array}$


Table S447 Cartesian coordinates of the TDDFT/TZVP-optimized excitedstate equilibrium geometry of the $1^{1} A_{u}\left({ }^{1} n \pi^{*}\right)$ state of $p$-benzoquinone (excitedstate energy: $-381.28879 E_{h}$ ).

$\begin{array}{lrrr}12 & & \\ & & & \\ \mathrm{C} & 0.0000000 & 1.4169856 & 0.0000000 \\ \mathrm{C} & -1.2382539 & 0.6838014 & 0.0000000 \\ \mathrm{C} & -1.2382539 & -0.6838014 & 0.0000000 \\ \mathrm{C} & 0.0000000 & -1.4169856 & 0.0000000 \\ \mathrm{C} & 1.2382539 & -0.6838014 & 0.0000000 \\ \mathrm{C} & 1.2382539 & 0.6838014 & 0.0000000 \\ \mathrm{O} & 0.0000000 & 2.6816403 & 0.0000000 \\ \mathrm{O} & 0.0000000 & -2.6816403 & 0.0000000 \\ \mathrm{H} & -2.1641261 & 1.2463227 & 0.0000000 \\ \mathrm{H} & -2.1641261 & -1.2463227 & 0.0000000 \\ \mathrm{H} & 2.1641261 & -1.2463227 & 0.0000000 \\ \mathrm{H} & 2.1641261 & 1.2463227 & 0.0000000\end{array}$


Table S448 Cartesian coordinates of the TDDFT/TZVP-optimized excitedstate equilibrium geometry of the $1{ }^{1} B_{1 g}$ ( $\equiv 1{ }^{1} B_{2 g}$ in Turbomole) $\left({ }^{1} n \pi^{*}\right)$ state of $p$-benzoquinone (excited-state energy: $-381.29078 E_{h}$ ).

$\begin{array}{lrrr}12 & & \\ & & & \\ \mathrm{C} & 0.0000000 & 1.4522818 & 0.0000000 \\ \mathrm{C} & -1.2333539 & 0.6743924 & 0.0000000 \\ \mathrm{C} & -1.2333539 & -0.6743924 & 0.0000000 \\ \mathrm{C} & 0.0000000 & -1.4522818 & 0.0000000 \\ \mathrm{C} & 1.2333539 & -0.6743924 & 0.0000000 \\ \mathrm{C} & 1.2333539 & 0.6743924 & 0.0000000 \\ \mathrm{O} & 0.0000000 & 2.7001474 & 0.0000000 \\ \mathrm{O} & 0.0000000 & -2.7001474 & 0.0000000 \\ \mathrm{H} & -2.1539611 & 1.2425027 & 0.0000000 \\ \mathrm{H} & -2.1539611 & -1.2425027 & 0.0000000 \\ \mathrm{H} & 2.1539611 & -1.2425027 & 0.0000000 \\ \mathrm{H} & 2.1539611 & 1.2425027 & 0.0000000\end{array}$


Table S449 Cartesian coordinates of the TDDFT/TZVP-optimized excitedstate equilibrium geometry of the $1{ }^{1} B_{1 u}\left(\equiv 1^{1} B_{2 u}\right.$ in Turbomole) $\left({ }^{1} \pi \pi^{*}\right)$ state of $p$-benzoquinone (excited-state energy: $-381.20505 E_{h}$ ).

12

C $\quad 0.0000000$

1.4605670

0.0000000

C -1.2503230

$0.6972838 \quad 0.0000000$

C $\quad-1.2503230$

$-0.6972838$

0.0000000

C $\quad 0.0000000$

$-1.4605670 \quad 0.0000000$

C $\quad 1.2503230$

$-0.6972838$

0.0000000

C $\quad 1.2503230$

0.6972838

0.0000000

0.0000000

2.7081661

0.0000000

O 0.0000000

$-2.7081661$

$-2.1708394$

1.2675069

$-2.1708394-1.2675069$

H 2.1708394

$-1.2675069$

0.0000000

0.0000000

1. 2675069

0.0000000

0.0000000

2.1708394

0.0000000 
Table S450 Cartesian coordinates of the TDDFT/TZVP-optimized excitedstate equilibrium geometry of the $1{ }^{1} B_{3 g}\left(\equiv 1{ }^{1} B_{1 g}\right.$ in Turbomole) $\left({ }^{1} \pi \pi^{*}\right)$ state of $p$-benzoquinone (excited-state energy: $-381.25386 E_{h}$ ).

$\begin{array}{lrrr}12 & & \\ & & & \\ \mathrm{C} & 0.0000000 & 1.5005055 & 0.0000000 \\ \mathrm{C} & -1.2067858 & 0.7060195 & 0.0000000 \\ \mathrm{C} & -1.2067858 & -0.7060195 & 0.0000000 \\ \mathrm{C} & 0.0000000 & -1.5005055 & 0.0000000 \\ \mathrm{C} & 1.2067858 & -0.7060195 & 0.0000000 \\ \mathrm{C} & 1.2067858 & 0.7060195 & 0.0000000 \\ \mathrm{O} & 0.0000000 & 2.7493380 & 0.0000000 \\ \mathrm{O} & 0.0000000 & -2.7493380 & 0.0000000 \\ \mathrm{H} & -2.1501024 & 1.2416997 & 0.0000000 \\ \mathrm{H} & -2.1501024 & -1.2416997 & 0.0000000 \\ \mathrm{H} & 2.1501024 & -1.2416997 & 0.0000000 \\ \mathrm{H} & 2.1501024 & 1.2416997 & 0.0000000\end{array}$


Table S451 Cartesian coordinates of the TDDFT/TZVP-optimized excitedstate equilibrium geometry of the $1^{3} A_{u}\left({ }^{3} n \pi^{*}\right)$ state of $p$-benzoquinone (excitedstate energy: $\left.-381.30924 E_{h}\right)$.

$\begin{array}{lrrr}12 & & \\ & & & \\ \mathrm{C} & 0.0000000 & 1.4125394 & 0.0000000 \\ \mathrm{C} & -1.2364170 & 0.6836603 & 0.0000000 \\ \mathrm{C} & -1.2364170 & -0.6836603 & 0.0000000 \\ \mathrm{C} & 0.0000000 & -1.4125394 & 0.0000000 \\ \mathrm{C} & 1.2364170 & -0.6836603 & 0.0000000 \\ \mathrm{C} & 1.2364170 & 0.6836603 & 0.0000000 \\ \mathrm{O} & 0.0000000 & 2.6832594 & 0.0000000 \\ \mathrm{O} & 0.0000000 & -2.6832594 & 0.0000000 \\ \mathrm{H} & -2.1630534 & 1.2460101 & 0.0000000 \\ \mathrm{H} & -2.1630534 & -1.2460101 & 0.0000000 \\ \mathrm{H} & 2.1630534 & -1.2460101 & 0.0000000 \\ \mathrm{H} & 2.1630534 & 1.2460101 & 0.0000000\end{array}$


Table S452 Cartesian coordinates of the TDDFT/TZVP-optimized excitedstate equilibrium geometry of the $1^{3} B_{1 g}\left(\equiv 1^{3} B_{2 g}\right.$ in Turbomole) $\left({ }^{3} n \pi^{*}\right)$ state of $p$-benzoquinone (excited-state energy: $-381.31003 E_{h}$ ).

$\begin{array}{lrrr}12 & & & \\ & & & \\ \mathrm{C} & 0.0000000 & 1.4423632 & 0.0000000 \\ \mathrm{C} & -1.2335027 & 0.6751570 & 0.0000000 \\ \mathrm{C} & -1.2335027 & -0.6751570 & 0.0000000 \\ \mathrm{C} & 0.0000000 & -1.4423632 & 0.0000000 \\ \mathrm{C} & 1.2335027 & -0.6751570 & 0.0000000 \\ \mathrm{C} & 1.2335027 & 0.6751570 & 0.0000000 \\ \mathrm{O} & 0.0000000 & 2.6971878 & 0.0000000 \\ \mathrm{O} & 0.0000000 & -2.6971878 & 0.0000000 \\ \mathrm{H} & -2.1551019 & 1.2407019 & 0.0000000 \\ \mathrm{H} & -2.1551019 & -1.2407019 & 0.0000000 \\ \mathrm{H} & 2.1551019 & -1.2407019 & 0.0000000 \\ \mathrm{H} & 2.1551019 & 1.2407019 & 0.0000000\end{array}$


Table S453 Cartesian coordinates of the TDDFT/TZVP-optimized excitedstate equilibrium geometry of the $1^{3} B_{3 g}$ ( $\equiv 1^{3} B_{1 g}$ in Turbomole) $\left({ }^{3} \pi \pi^{*}\right)$ state of $p$-benzoquinone (excited-state energy: $-381.29434 E_{h}$ ).

$\begin{array}{lrrr}12 & & \\ & & & \\ \mathrm{C} & 0.0000000 & 1.5131801 & 0.0000000 \\ \mathrm{C} & -1.2134513 & 0.7149779 & 0.0000000 \\ \mathrm{C} & -1.2134513 & -0.7149779 & 0.0000000 \\ \mathrm{C} & 0.0000000 & -1.5131801 & 0.0000000 \\ \mathrm{C} & 1.2134513 & -0.7149779 & 0.0000000 \\ \mathrm{C} & 1.2134513 & 0.7149779 & 0.0000000 \\ \mathrm{O} & 0.0000000 & 2.7485122 & 0.0000000 \\ \mathrm{O} & 0.0000000 & -2.7485122 & 0.0000000 \\ \mathrm{H} & -2.1560164 & 1.2510324 & 0.0000000 \\ \mathrm{H} & -2.1560164 & -1.2510324 & 0.0000000 \\ \mathrm{H} & 2.1560164 & -1.2510324 & 0.0000000 \\ \mathrm{H} & 2.1560164 & 1.2510324 & 0.0000000\end{array}$


Table S454 Cartesian coordinates of the TDDFT/TZVP-optimized excitedstate equilibrium geometry of the $1{ }^{1} B_{3 g}\left(\equiv 1{ }^{1} B_{1 g}\right.$ in Turbomole) $\left({ }^{1} \pi \pi^{*}\right)$ state of biphenylene (excited-state energy: $-461.78203 E_{h}$ ).

$\begin{array}{lrrr}20 & & & \\ & & & \\ \mathrm{C} & 0.7483916 & 0.7079755 & 0.0000000 \\ \mathrm{C} & 1.4697197 & 1.9065618 & 0.0000000 \\ \mathrm{C} & 0.7171781 & 3.0697846 & 0.0000000 \\ \mathrm{C} & 0.7483916 & -0.7079755 & 0.0000000 \\ \mathrm{C} & -0.7483916 & -0.7079755 & 0.0000000 \\ \mathrm{C} & -0.7483916 & 0.7079755 & 0.0000000 \\ \mathrm{C} & 1.4697197 & -1.9065618 & 0.0000000 \\ \mathrm{C} & -1.4697197 & -1.9065618 & 0.0000000 \\ \mathrm{C} & -1.4697197 & 1.9065618 & 0.0000000 \\ \mathrm{C} & 0.7171781 & -3.0697846 & 0.0000000 \\ \mathrm{C} & -0.7171781 & -3.0697846 & 0.0000000 \\ \mathrm{C} & -0.7171781 & 3.0697846 & 0.0000000 \\ \mathrm{H} & 2.5524243 & 1.9424556 & 0.0000000 \\ \mathrm{H} & 2.5524243 & -1.9424556 & 0.0000000 \\ \mathrm{H} & -2.5524243 & -1.9424556 & 0.0000000 \\ \mathrm{H} & -2.5524243 & 1.9424556 & 0.0000000 \\ \mathrm{H} & 1.2226444 & 4.0280826 & 0.0000000 \\ \mathrm{H} & 1.2226444 & -4.0280826 & 0.0000000 \\ \mathrm{H} & -1.2226444 & -4.0280826 & 0.0000000 \\ \mathrm{H} & -1.2226444 & 4.0280826 & 0.0000000\end{array}$


Table S455 Cartesian coordinates of the TDDFT/TZVP-optimized excitedstate equilibrium geometry of the $1{ }^{1} B_{1 u}\left(\equiv 1^{1} B_{2 u}\right.$ in Turbomole) $\left({ }^{1} \pi \pi^{*}\right)$ state of biphenylene (excited-state energy: $-461.74163 E_{h}$ ).

$\begin{array}{lrrr}20 & & & \\ & & & \\ \mathrm{C} & 0.7218125 & 0.7285369 & 0.0000000 \\ \mathrm{C} & 1.4687345 & 1.9026644 & 0.0000000 \\ \mathrm{C} & 0.6988071 & 3.0999840 & 0.0000000 \\ \mathrm{C} & 0.7218125 & -0.7285369 & 0.0000000 \\ \mathrm{C} & -0.7218125 & -0.7285369 & 0.0000000 \\ \mathrm{C} & -0.7218125 & 0.7285369 & 0.0000000 \\ \mathrm{C} & 1.4687345 & -1.9026644 & 0.0000000 \\ \mathrm{C} & -1.4687345 & -1.9026644 & 0.0000000 \\ \mathrm{C} & -1.4687345 & 1.9026644 & 0.0000000 \\ \mathrm{C} & 0.6988071 & -3.0999840 & 0.0000000 \\ \mathrm{C} & -0.6988071 & -3.0999840 & 0.0000000 \\ \mathrm{C} & -0.6988071 & 3.0999840 & 0.0000000 \\ \mathrm{H} & 2.5496445 & 1.9269564 & 0.0000000 \\ \mathrm{H} & 2.5496445 & -1.9269564 & 0.0000000 \\ \mathrm{H} & -2.5496445 & -1.9269564 & 0.0000000 \\ \mathrm{H} & -2.5496445 & 1.9269564 & 0.0000000 \\ \mathrm{H} & 1.2138665 & 4.0531029 & 0.0000000 \\ \mathrm{H} & 1.2138665 & -4.0531029 & 0.0000000 \\ \mathrm{H} & -1.2138665 & -4.0531029 & 0.0000000 \\ \mathrm{H} & -1.2138665 & 4.0531029 & 0.0000000\end{array}$


Table S456 Cartesian coordinates of the TDDFT/TZVP-optimized excitedstate equilibrium geometry of the $2{ }^{1} B_{1 u}\left(\equiv 2{ }^{1} B_{2 u}\right.$ in Turbomole) $\left({ }^{1} \pi \pi^{*}\right)$ state of biphenylene (excited-state energy: $-461.69040 E_{h}$ ).

$\begin{array}{lrrr}20 & & & \\ & & & \\ \mathrm{C} & 0.7185533 & 0.7381604 & 0.0000000 \\ \mathrm{C} & 1.4529966 & 1.9224470 & 0.0000000 \\ \mathrm{C} & 0.6983051 & 3.1287525 & 0.0000000 \\ \mathrm{C} & 0.7185533 & -0.7381604 & 0.0000000 \\ \mathrm{C} & -0.7185533 & -0.7381604 & 0.0000000 \\ \mathrm{C} & -0.7185533 & 0.7381604 & 0.0000000 \\ \mathrm{C} & 1.4529966 & -1.9224470 & 0.0000000 \\ \mathrm{C} & -1.4529966 & -1.9224470 & 0.0000000 \\ \mathrm{C} & -1.4529966 & 1.9224470 & 0.0000000 \\ \mathrm{C} & 0.6983051 & -3.1287525 & 0.0000000 \\ \mathrm{C} & -0.6983051 & -3.1287525 & 0.0000000 \\ \mathrm{C} & -0.6983051 & 3.1287525 & 0.0000000 \\ \mathrm{H} & 2.5359049 & 1.9466827 & 0.0000000 \\ \mathrm{H} & 2.5359049 & -1.9466827 & 0.0000000 \\ \mathrm{H} & -2.5359049 & -1.9466827 & 0.0000000 \\ \mathrm{H} & -2.5359049 & 1.9466827 & 0.0000000 \\ \mathrm{H} & 1.2250779 & 4.0753644 & 0.0000000 \\ \mathrm{H} & 1.2250779 & -4.0753644 & 0.0000000 \\ \mathrm{H} & -1.2250779 & -4.0753644 & 0.0000000 \\ \mathrm{H} & -1.2250779 & 4.0753644 & 0.0000000\end{array}$


Table S457 Cartesian coordinates of the TDDFT/TZVP-optimized excitedstate equilibrium geometry of the $1{ }^{1} B_{2 u}\left(\equiv 1^{1} B_{3 u}\right.$ in Turbomole) $\left({ }^{1} \pi \pi^{*}\right)$ state of biphenylene (excited-state energy: $-461.68629 E_{h}$ ).

$\begin{array}{lrrr}20 & & & \\ & & & \\ \mathrm{C} & 0.7355424 & 0.7408589 & 0.0000000 \\ \mathrm{C} & 1.4648690 & 1.9048943 & 0.0000000 \\ \mathrm{C} & 0.7269692 & 3.1051744 & 0.0000000 \\ \mathrm{C} & 0.7355424 & -0.7408589 & 0.0000000 \\ \mathrm{C} & -0.7355424 & -0.7408589 & 0.0000000 \\ \mathrm{C} & -0.7355424 & 0.7408589 & 0.0000000 \\ \mathrm{C} & 1.4648690 & -1.9048943 & 0.0000000 \\ \mathrm{C} & -1.4648690 & -1.9048943 & 0.0000000 \\ \mathrm{C} & -1.4648690 & 1.9048943 & 0.0000000 \\ \mathrm{C} & 0.7269692 & -3.1051744 & 0.0000000 \\ \mathrm{C} & -0.7269692 & -3.1051744 & 0.0000000 \\ \mathrm{C} & -0.7269692 & 3.1051744 & 0.0000000 \\ \mathrm{H} & 2.5479464 & 1.9232427 & 0.0000000 \\ \mathrm{H} & 2.5479464 & -1.9232427 & 0.0000000 \\ \mathrm{H} & -2.5479464 & -1.9232427 & 0.0000000 \\ \mathrm{H} & -2.5479464 & 1.9232427 & 0.0000000 \\ \mathrm{H} & 1.2450203 & 4.0546701 & 0.0000000 \\ \mathrm{H} & 1.2450203 & -4.0546701 & 0.0000000 \\ \mathrm{H} & -1.2450203 & -4.0546701 & 0.0000000 \\ \mathrm{H} & -1.2450203 & 4.0546701 & 0.0000000\end{array}$


Table S458 Cartesian coordinates of the TDDFT/TZVP-optimized excitedstate equilibrium geometry of the $1^{1} B_{u}\left({ }^{1} \pi \pi^{*}\right)$ state of trans-butadiene (excitedstate energy: $-155.74319 E_{h}$ ).

$\begin{array}{lrrr}10 & & & \\ & -0.3953823 & -0.5785643 & 0.0000000 \\ \mathrm{C} & -0.3953823 & 0.5785643 & 0.0000000 \\ \mathrm{C} & 0.1087298 & 1.9087735 & 0.0000000 \\ \mathrm{C} & -0.10800000 \\ \mathrm{C} & 0.1087298 & -1.9087735 & 0.0000000 \\ \mathrm{H} & -1.4754406 & -0.4532812 & 0.0000000 \\ \mathrm{H} & 1.4754406 & 0.4532812 & 0.0000000 \\ \mathrm{H} & 0.5616928 & 2.7573721 & 0.0000000 \\ \mathrm{H} & -1.1758292 & 2.1009596 & 0.0000000 \\ \mathrm{H} & -0.5616928 & -2.7573721 & 0.0000000 \\ \mathrm{H} & 1.1758292 & -2.1009596 & 0.0000000\end{array}$


Table S459 Cartesian coordinates of the TDDFT/TZVP-optimized excitedstate equilibrium geometry of the $2{ }^{1} A_{g}\left({ }^{1} \pi \pi^{*}\right)$ state of trans-butadiene (excitedstate energy: $-155.70425 E_{h}$ ).

$\begin{array}{lrrr}10 & & & \\ & & & \\ \mathrm{C} & -0.4529884 & -0.5822620 & 0.0000000 \\ \mathrm{C} & 0.4529884 & 0.5822620 & 0.0000000 \\ \mathrm{C} & -0.1223825 & 1.8830337 & 0.0000000 \\ \mathrm{C} & 0.1223825 & -1.8830337 & 0.0000000 \\ \mathrm{H} & -1.5225833 & -0.4304776 & 0.0000000 \\ \mathrm{H} & 1.5225833 & 0.4304776 & 0.0000000 \\ \mathrm{H} & 0.4856488 & 2.7767963 & 0.0000000 \\ \mathrm{H} & -1.1967034 & 2.0062493 & 0.0000000 \\ \mathrm{H} & -0.4856488 & -2.7767963 & 0.0000000 \\ \mathrm{H} & 1.1967034 & -2.0062493 & 0.0000000\end{array}$


Table S460 Cartesian coordinates of the TDDFT/TZVP-optimized excitedstate equilibrium geometry of the $2{ }^{1} A\left({ }^{1} n \pi^{*}\right)$ state of cytosine (excited-state energy: $\left.-394.72216 E_{h}\right)$.

$\begin{array}{lrrr}13 & & & \\ & & & \\ \mathrm{~N} & 0.0518024 & -1.0131296 & 0.0669354 \\ \mathrm{C} & 1.2044194 & -0.2042225 & 0.0146504 \\ \mathrm{C} & 1.1304309 & 1.1622787 & 0.0283828 \\ \mathrm{C} & -0.1178826 & 1.7979935 & -0.0146346 \\ \mathrm{~N} & -1.2497597 & 0.9384360 & -0.0253933 \\ \mathrm{C} & -1.0663754 & -0.3970214 & 0.0097014 \\ \mathrm{~N} & 2.3584793 & -0.9397426 & -0.0857492 \\ \mathrm{O} & -2.1995968 & -1.1049743 & -0.0132462 \\ \mathrm{H} & 2.0296586 & 1.7649011 & 0.0384387 \\ \mathrm{H} & -0.3029222 & 2.8321423 & 0.2186406 \\ \mathrm{H} & 2.2828604 & -1.8973711 & 0.2199778 \\ \mathrm{H} & 3.2325583 & -0.4823949 & 0.1156285 \\ \mathrm{H} & -2.1654066 & 1.3078139 & -0.2221372\end{array}$


Table S461 Cartesian coordinates of the TDDFT/TZVP-optimized excitedstate equilibrium geometry of the $3^{1} A\left({ }^{1} \pi \pi^{*}\right)$ state of cytosine (excited-state energy: $\left.-394.71478 E_{h}\right)$.

$\begin{array}{lrrr}13 & & & \\ & & & \\ \mathrm{~N} & 0.0564932 & -1.0243155 & 0.0050190 \\ \mathrm{C} & 1.2181897 & -0.1990123 & 0.0084551 \\ \mathrm{C} & 1.1391456 & 1.1657228 & 0.0109384 \\ \mathrm{C} & -0.1248654 & 1.7938132 & -0.0013118 \\ \mathrm{~N} & -1.2351012 & 0.9373287 & -0.0055249 \\ \mathrm{C} & -1.0753550 & -0.4144043 & -0.0006169 \\ \mathrm{~N} & 2.3654500 & -0.9405368 & -0.0433236 \\ \mathrm{O} & -2.2266282 & -1.0824528 & 0.0015704 \\ \mathrm{H} & 2.0327653 & 1.7761546 & 0.0142206 \\ \mathrm{H} & -0.3026204 & 2.8532824 & -0.0088040 \\ \mathrm{H} & 2.2659687 & -1.9114872 & 0.2083302 \\ \mathrm{H} & 3.2451508 & -0.5034477 & 0.1750787 \\ \mathrm{H} & -2.1789183 & 1.2887956 & -0.0128136\end{array}$


Table S462 Cartesian coordinates of the TDDFT/TZVP-optimized excitedstate equilibrium geometry of the $1{ }^{1} A_{2}\left({ }^{1} n \pi^{*}\right)$ state of diazomethane (excitedstate energy: $-148.61082 E_{h}$ ).

\section{5}

C $\quad 0.0000000$

0.0000000

$-1.2395249$

$\mathrm{N} \quad 0.0000000$

0.0000000

0.0631515

$\mathrm{N} \quad 0.0000000$

$0.0000000 \quad 1.2585078$

$\mathrm{H} \quad 0.9396945 \quad 0.0000000 \quad-1.7976557$

$\mathrm{H} \quad-0.9396945 \quad 0.0000000 \quad-1.7976557$ 
Table S463 Cartesian coordinates of the TDDFT/TZVP-optimized excitedstate equilibrium geometry of the $1{ }^{1} B_{3 g}\left(\equiv 1{ }^{1} B_{1 g}\right.$ in Turbomole) $\left({ }^{1} \pi \pi^{*}\right)$ state of dibenzo- $p$-dioxin (excited-state energy: $-612.22984 E_{h}$ ).

$\begin{array}{lrrr}22 & & & \\ & & & \\ \mathrm{C} & -3.5681529 & 0.7180807 & 0.0000000 \\ \mathrm{C} & -3.5681529 & -0.7180807 & 0.0000000 \\ \mathrm{C} & -2.3813824 & -1.4163614 & 0.0000000 \\ \mathrm{C} & -1.1679392 & -0.7242569 & 0.0000000 \\ \mathrm{C} & -1.1679392 & 0.7242569 & 0.0000000 \\ \mathrm{C} & -2.3813824 & 1.4163614 & 0.0000000 \\ \mathrm{O} & 0.0000000 & -1.4045568 & 0.0000000 \\ \mathrm{C} & 1.1679392 & -0.7242569 & 0.0000000 \\ \mathrm{C} & 1.1679392 & 0.7242569 & 0.0000000 \\ \mathrm{O} & 0.0000000 & 1.4045568 & 0.0000000 \\ \mathrm{C} & 2.3813824 & -1.4163614 & 0.0000000 \\ \mathrm{C} & 3.5681529 & -0.7180807 & 0.0000000 \\ \mathrm{C} & 3.5681529 & 0.7180807 & 0.0000000 \\ \mathrm{C} & 2.3813824 & 1.4163614 & 0.0000000 \\ \mathrm{H} & 4.5075066 & 1.2543139 & 0.0000000 \\ \mathrm{H} & -4.5075066 & -1.2543139 & 0.0000000 \\ \mathrm{H} & -2.3595917 & -2.4992754 & 0.0000000 \\ \mathrm{H} & -2.3595917 & 2.4992754 & 0.0000000 \\ \mathrm{H} & 2.3595917 & -2.4992754 & 0.0000000 \\ \mathrm{H} & 4.5075066 & -1.2543139 & 0.0000000 \\ \mathrm{H} & -4.5075066 & 1.2543139 & 0.0000000 \\ \mathrm{H} & 2.3595917 & 2.4992754 & 0.0000000\end{array}$


Table S464 Cartesian coordinates of the TDDFT/TZVP-optimized excitedstate equilibrium geometry of the $1{ }^{1} B_{2 u}\left(\equiv 1{ }^{1} B_{3 u}\right.$ in Turbomole) $\left({ }^{1} \pi \pi^{*}\right)$ state of dibenzo- $p$-dioxin (excited-state energy: $-612.21360 E_{h}$ ).

$\begin{array}{lrrr}22 & & & \\ & & & \\ \mathrm{C} & -3.5770793 & 0.7002731 & 0.0000000 \\ \mathrm{C} & -3.5770793 & -0.7002731 & 0.0000000 \\ \mathrm{C} & -2.3834779 & -1.4308528 & 0.0000000 \\ \mathrm{C} & -1.1760119 & -0.6985204 & 0.0000000 \\ \mathrm{C} & -1.1760119 & 0.6985204 & 0.0000000 \\ \mathrm{C} & -2.3834779 & 1.4308528 & 0.0000000 \\ \mathrm{O} & 0.0000000 & -1.3903711 & 0.0000000 \\ \mathrm{C} & 1.1760119 & -0.6985204 & 0.0000000 \\ \mathrm{C} & 1.1760119 & 0.6985204 & 0.0000000 \\ \mathrm{O} & 0.0000000 & 1.3903711 & 0.0000000 \\ \mathrm{C} & 2.3834779 & -1.4308528 & 0.0000000 \\ \mathrm{C} & 3.5770793 & -0.7002731 & 0.0000000 \\ \mathrm{C} & 3.5770793 & 0.7002731 & 0.0000000 \\ \mathrm{C} & 2.3834779 & 1.4308528 & 0.0000000 \\ \mathrm{H} & 4.5216992 & 1.2294579 & 0.0000000 \\ \mathrm{H} & -4.5216992 & -1.2294579 & 0.0000000 \\ \mathrm{H} & -2.3545883 & -2.5103117 & 0.0000000 \\ \mathrm{H} & -2.3545883 & 2.5103117 & 0.0000000 \\ \mathrm{H} & 2.3545883 & -2.5103117 & 0.0000000 \\ \mathrm{H} & 4.5216992 & -1.2294579 & 0.0000000 \\ \mathrm{H} & -4.5216992 & 1.2294579 & 0.0000000 \\ \mathrm{H} & 2.3545883 & 2.5103117 & 0.0000000\end{array}$


Table S465 Cartesian coordinates of the TDDFT/TZVP-optimized excitedstate equilibrium geometry of the $1{ }^{1} B_{1 u}\left(\equiv 1^{1} B_{2 u}\right.$ in Turbomole) $\left({ }^{1} \pi \pi^{*}\right)$ state of dibenzo- $p$-dioxin (excited-state energy: $-612.17403 E_{h}$ ).

$\begin{array}{lrrr}22 & & & \\ & & & \\ \mathrm{C} & -3.5843660 & 0.7235462 & 0.0000000 \\ \mathrm{C} & -3.5843660 & -0.7235462 & 0.0000000 \\ \mathrm{C} & -2.3780872 & -1.4129684 & 0.0000000 \\ \mathrm{C} & -1.1795408 & -0.7186574 & 0.0000000 \\ \mathrm{C} & -1.1795408 & 0.7186574 & 0.0000000 \\ \mathrm{C} & -2.3780872 & 1.4129684 & 0.0000000 \\ \mathrm{O} & 0.0000000 & -1.4222201 & 0.0000000 \\ \mathrm{C} & 1.1795408 & -0.7186574 & 0.0000000 \\ \mathrm{C} & 1.1795408 & 0.7186574 & 0.0000000 \\ \mathrm{O} & 0.0000000 & 1.4222201 & 0.0000000 \\ \mathrm{C} & 2.3780872 & -1.4129684 & 0.0000000 \\ \mathrm{C} & 3.5843660 & -0.7235462 & 0.0000000 \\ \mathrm{C} & 3.5843660 & 0.7235462 & 0.0000000 \\ \mathrm{C} & 2.3780872 & 1.4129684 & 0.0000000 \\ \mathrm{H} & 4.5184432 & 1.2668828 & 0.0000000 \\ \mathrm{H} & -4.5184432 & -1.2668828 & 0.0000000 \\ \mathrm{H} & -2.3453463 & -2.4953978 & 0.0000000 \\ \mathrm{H} & -2.3453463 & 2.4953978 & 0.0000000 \\ \mathrm{H} & 2.3453463 & -2.4953978 & 0.0000000 \\ \mathrm{H} & 4.5184432 & -1.2668828 & 0.0000000 \\ \mathrm{H} & -4.5184432 & 1.2668828 & 0.0000000 \\ \mathrm{H} & 2.3453463 & 2.4953978 & 0.0000000\end{array}$


Table S466 Cartesian coordinates of the TDDFT/TZVP-optimized excitedstate equilibrium geometry of the $1{ }^{3} A_{g}\left({ }^{3} \pi \pi^{*}\right)$ state of dibenzo- $p$-dioxin (excited-state energy: $-612.23853 E_{h}$ ).

$\begin{array}{lrrr}22 & & & \\ & & & \\ \text { C } & -3.5641337 & 0.6987433 & 0.0000000 \\ \text { C } & -3.5641337 & -0.6987433 & 0.0000000 \\ \text { C } & -2.3710444 & -1.4307600 & 0.0000000 \\ \text { C } & -1.1657638 & -0.7003143 & 0.0000000 \\ \text { C } & -1.1657638 & 0.7003143 & 0.0000000 \\ \text { C } & -2.3710444 & 1.4307600 & 0.0000000 \\ \text { O } & 0.0000000 & -1.4092013 & 0.0000000 \\ \text { C } & 1.1657638 & -0.7003143 & 0.0000000 \\ \text { C } & 1.1657638 & 0.7003143 & 0.0000000 \\ \text { O } & 0.0000000 & 1.4092013 & 0.0000000 \\ \text { C } & 2.3710444 & -1.4307600 & 0.0000000 \\ \text { C } & 3.5641337 & -0.6987433 & 0.0000000 \\ \text { C } & 3.5641337 & 0.6987433 & 0.0000000 \\ \text { C } & 2.3710444 & 1.4307600 & 0.0000000 \\ \text { H } & 4.5093119 & 1.2272899 & 0.0000000 \\ \text { H } & -4.5093119 & -1.2272899 & 0.0000000 \\ \text { H } & -2.3399049 & -2.5097243 & 0.0000000 \\ \text { H } & -2.3399049 & 2.5097243 & 0.0000000 \\ \text { H } & 2.3399049 & -2.5097243 & 0.0000000 \\ \text { H } & 4.5093119 & -1.2272899 & 0.0000000 \\ \text { H } & -4.5093119 & 1.2272899 & 0.0000000 \\ \text { H } & 2.3399049 & 2.5097243 & 0.0000000\end{array}$


Table S467 Cartesian coordinates of the TDDFT/TZVP-optimized excitedstate equilibrium geometry of the $1^{3} B_{3 g}\left(\equiv 1^{3} B_{1 g}\right.$ in Turbomole) $\left({ }^{3} \pi \pi^{*}\right)$ state of dibenzo- $p$-dioxin (excited-state energy: $-612.26450 E_{h}$ ).

$\begin{array}{lrrr}22 & & & \\ & & & \\ \text { C } & -3.5730359 & 0.7213999 & 0.0000000 \\ \text { C } & -3.5730359 & -0.7213999 & 0.0000000 \\ \text { C } & -2.3828814 & -1.4187811 & 0.0000000 \\ \text { C } & -1.1713556 & -0.7247380 & 0.0000000 \\ \text { C } & -1.1713556 & 0.7247380 & 0.0000000 \\ \text { C } & -2.3828814 & 1.4187811 & 0.0000000 \\ \text { O } & 0.0000000 & -1.4081666 & 0.0000000 \\ \text { C } & 1.1713556 & -0.7247380 & 0.0000000 \\ \mathrm{C} & 1.1713556 & 0.7247380 & 0.0000000 \\ \text { O } & 0.0000000 & 1.4081666 & 0.0000000 \\ \mathrm{C} & 2.3828814 & -1.4187811 & 0.0000000 \\ \mathrm{C} & 3.5730359 & -0.7213999 & 0.0000000 \\ \mathrm{C} & 3.5730359 & 0.7213999 & 0.0000000 \\ \mathrm{C} & 2.3828814 & 1.4187811 & 0.0000000 \\ \mathrm{H} & 4.5126900 & 1.2572101 & 0.0000000 \\ \mathrm{H} & -4.5126900 & -1.2572101 & 0.0000000 \\ \mathrm{H} & -2.3594707 & -2.5015911 & 0.0000000 \\ \mathrm{H} & -2.3594707 & 2.5015911 & 0.0000000 \\ \mathrm{H} & 2.3594707 & -2.5015911 & 0.0000000 \\ \mathrm{H} & 4.5126900 & -1.2572101 & 0.0000000 \\ \mathrm{H} & -4.5126900 & 1.2572101 & 0.0000000 \\ \mathrm{H} & 2.3594707 & 2.5015911 & 0.0000000\end{array}$


Table S468 Cartesian coordinates of the TDDFT/TZVP-optimized excitedstate equilibrium geometry of the $1^{3} B_{2 u}\left(\equiv 1^{3} B_{3 u}\right.$ in Turbomole) $\left({ }^{3} \pi \pi^{*}\right)$ state of dibenzo- $p$-dioxin (excited-state energy: $-612.23592 E_{h}$ ).

$\begin{array}{lrrr}22 & & & \\ & & & \\ \mathrm{C} & -3.5768957 & 0.6977310 & 0.0000000 \\ \mathrm{C} & -3.5768957 & -0.6977310 & 0.0000000 \\ \mathrm{C} & -2.3827256 & -1.4343352 & 0.0000000 \\ \mathrm{C} & -1.1784296 & -0.7010384 & 0.0000000 \\ \mathrm{C} & -1.1784296 & 0.7010384 & 0.0000000 \\ \mathrm{C} & -2.3827256 & 1.4343352 & 0.0000000 \\ \mathrm{O} & 0.0000000 & -1.3819979 & 0.0000000 \\ \mathrm{C} & 1.1784296 & -0.7010384 & 0.0000000 \\ \mathrm{C} & 1.1784296 & 0.7010384 & 0.0000000 \\ \mathrm{O} & 0.0000000 & 1.3819979 & 0.0000000 \\ \mathrm{C} & 2.3827256 & -1.4343352 & 0.0000000 \\ \mathrm{C} & 3.5768957 & -0.6977310 & 0.0000000 \\ \mathrm{C} & 3.5768957 & 0.6977310 & 0.0000000 \\ \mathrm{C} & 2.3827256 & 1.4343352 & 0.0000000 \\ \mathrm{H} & 4.5222226 & 1.2260346 & 0.0000000 \\ \mathrm{H} & -4.5222226 & -1.2260346 & 0.0000000 \\ \mathrm{H} & -2.3546675 & -2.5132658 & 0.0000000 \\ \mathrm{H} & -2.3546675 & 2.5132658 & 0.0000000 \\ \mathrm{H} & 2.3546675 & -2.5132658 & 0.0000000 \\ \mathrm{H} & 4.5222226 & -1.2260346 & 0.0000000 \\ \mathrm{H} & -4.5222226 & 1.2260346 & 0.0000000 \\ \mathrm{H} & 2.3546675 & 2.5132658 & 0.0000000\end{array}$


Table S469 Cartesian coordinates of the TDDFT/TZVP-optimized excitedstate equilibrium geometry of the $1{ }^{3} B_{1 u}$ ( $\equiv 1^{3} B_{2 u}$ in Turbomole) $\left({ }^{3} \pi \pi^{*}\right)$ state of dibenzo- $p$-dioxin (excited-state energy: $-612.23712 E_{h}$ ).

$\begin{array}{lrrr}22 & & & \\ & & & \\ \text { C } & -3.5930005 & 0.7160248 & 0.0000000 \\ \text { C } & -3.5930005 & -0.7160248 & 0.0000000 \\ \text { C } & -2.3798380 & -1.4129035 & 0.0000000 \\ \text { C } & -1.1757197 & -0.7154261 & 0.0000000 \\ \text { C } & -1.1757197 & 0.7154261 & 0.0000000 \\ \text { C } & -2.3798380 & 1.4129035 & 0.0000000 \\ \text { O } & 0.0000000 & -1.4370087 & 0.0000000 \\ \text { C } & 1.1757197 & -0.7154261 & 0.0000000 \\ \mathrm{C} & 1.1757197 & 0.7154261 & 0.0000000 \\ \text { O } & 0.0000000 & 1.4370087 & 0.0000000 \\ \mathrm{C} & 2.3798380 & -1.4129035 & 0.0000000 \\ \mathrm{C} & 3.5930005 & -0.7160248 & 0.0000000 \\ \mathrm{C} & 3.5930005 & 0.7160248 & 0.0000000 \\ \mathrm{C} & 2.3798380 & 1.4129035 & 0.0000000 \\ \mathrm{H} & 4.5278143 & 1.2598376 & 0.0000000 \\ \mathrm{H} & -4.5278143 & -1.2598376 & 0.0000000 \\ \mathrm{H} & -2.3498070 & -2.4949937 & 0.0000000 \\ \mathrm{H} & -2.3498070 & 2.4949937 & 0.0000000 \\ \mathrm{H} & 2.3498070 & -2.4949937 & 0.0000000 \\ \mathrm{H} & 4.5278143 & -1.2598376 & 0.0000000 \\ \mathrm{H} & -4.5278143 & 1.2598376 & 0.0000000 \\ \mathrm{H} & 2.3498070 & 2.4949937 & 0.0000000\end{array}$


Table S470 Cartesian coordinates of the TDDFT/TZVP-optimized excitedstate equilibrium geometry of the $1{ }^{1} A_{u}\left({ }^{1} n \pi^{*}\right)$ state of dibenzo- $p$-dioxin (excited-state energy: $-612.11978 E_{h}$ ).

$\begin{array}{lrrr}22 & & & \\ & & & \\ \text { C } & -3.6268806 & 0.7087712 & 0.0000000 \\ \text { C } & -3.6268806 & -0.7087712 & 0.0000000 \\ \text { C } & -2.4242058 & -1.3896253 & 0.0000000 \\ \text { C } & -1.2035741 & -0.7337537 & 0.0000000 \\ \text { C } & -1.2035741 & 0.7337537 & 0.0000000 \\ \text { C } & -2.4242058 & 1.3896253 & 0.0000000 \\ \text { O } & 0.0000000 & -1.3567094 & 0.0000000 \\ \text { C } & 1.2035741 & -0.7337537 & 0.0000000 \\ \text { C } & 1.2035741 & 0.7337537 & 0.0000000 \\ \text { O } & 0.0000000 & 1.3567094 & 0.0000000 \\ \text { C } & 2.4242058 & -1.3896253 & 0.0000000 \\ \text { C } & 3.6268806 & -0.7087712 & 0.0000000 \\ \text { C } & 3.6268806 & 0.7087712 & 0.0000000 \\ \text { C } & 2.4242058 & 1.3896253 & 0.0000000 \\ \text { H } & 4.5582460 & 1.2581464 & 0.0000000 \\ \text { H } & -4.5582460 & -1.2581464 & 0.0000000 \\ \text { H } & -2.3920832 & -2.4756572 & 0.0000000 \\ \text { H } & -2.3920832 & 2.4756572 & 0.0000000 \\ \text { H } & 2.3920832 & -2.4756572 & 0.0000000 \\ \text { H } & 4.5582460 & -1.2581464 & 0.0000000 \\ \text { H } & -4.5582460 & 1.2581464 & 0.0000000 \\ \text { H } & 2.3920832 & 2.4756572 & 0.0000000\end{array}$


Table S471 Cartesian coordinates of the TDDFT/TZVP-optimized excitedstate equilibrium geometry of the $1{ }^{1} B_{1 g}\left(\equiv 2{ }^{1} B_{2 g}\right.$ in Turbomole) $\left({ }^{1} n \pi^{*}\right)$ state of dibenzo- $p$-dioxin (excited-state energy: $-612.09999 E_{h}$ ).

$\begin{array}{lrrr}22 & & & \\ & & & \\ \mathrm{C} & -3.6355619 & 0.6891528 & 0.0000000 \\ \mathrm{C} & -3.6355619 & -0.6891528 & 0.0000000 \\ \mathrm{C} & -2.4248331 & -1.4080616 & 0.0000000 \\ \mathrm{C} & -1.2112835 & -0.7045023 & 0.0000000 \\ \mathrm{C} & -1.2112835 & 0.7045023 & 0.0000000 \\ \mathrm{C} & -2.4248331 & 1.4080616 & 0.0000000 \\ \mathrm{O} & 0.0000000 & -1.3356870 & 0.0000000 \\ \mathrm{C} & 1.2112835 & -0.7045023 & 0.0000000 \\ \mathrm{C} & 1.2112835 & 0.7045023 & 0.0000000 \\ \mathrm{O} & 0.0000000 & 1.3356870 & 0.0000000 \\ \mathrm{C} & 2.4248331 & -1.4080616 & 0.0000000 \\ \mathrm{C} & 3.6355619 & -0.6891528 & 0.0000000 \\ \mathrm{C} & 3.6355619 & 0.6891528 & 0.0000000 \\ \mathrm{C} & 2.4248331 & 1.4080616 & 0.0000000 \\ \mathrm{H} & 4.5730098 & 1.2311158 & 0.0000000 \\ \mathrm{H} & -4.5730098 & -1.2311158 & 0.0000000 \\ \mathrm{H} & -2.3818580 & -2.4900495 & 0.0000000 \\ \mathrm{H} & -2.3818580 & 2.4900495 & 0.0000000 \\ \mathrm{H} & 2.3818580 & -2.4900495 & 0.0000000 \\ \mathrm{H} & 4.5730098 & -1.2311158 & 0.0000000 \\ \mathrm{H} & -4.5730098 & 1.2311158 & 0.0000000 \\ \mathrm{H} & 2.3818580 & 2.4900495 & 0.0000000\end{array}$


Table S472 Cartesian coordinates of the TDDFT/TZVP-optimized excitedstate equilibrium geometry of the $1{ }^{1} B_{3 u}\left(\equiv 3{ }^{1} B_{1 u}\right.$ in Turbomole) $\left({ }^{1} n \pi^{*}\right)$ state of dibenzo- $p$-dioxin (excited-state energy: $-612.09695 E_{h}$ ).

$\begin{array}{lrrr}22 & & & \\ & & & \\ \mathrm{C} & -3.6218127 & 0.6918243 & 0.0000000 \\ \mathrm{C} & -3.6218127 & -0.6918243 & 0.0000000 \\ \mathrm{C} & -2.4147049 & -1.4041332 & 0.0000000 \\ \mathrm{C} & -1.1989667 & -0.7015498 & 0.0000000 \\ \mathrm{C} & -1.1989667 & 0.7015498 & 0.0000000 \\ \mathrm{C} & -2.4147049 & 1.4041332 & 0.0000000 \\ \mathrm{O} & 0.0000000 & -1.3715400 & 0.0000000 \\ \mathrm{C} & 1.1989667 & -0.7015498 & 0.0000000 \\ \mathrm{C} & 1.1989667 & 0.7015498 & 0.0000000 \\ \mathrm{O} & 0.0000000 & 1.3715400 & 0.0000000 \\ \mathrm{C} & 2.4147049 & -1.4041332 & 0.0000000 \\ \mathrm{C} & 3.6218127 & -0.6918243 & 0.0000000 \\ \mathrm{C} & 3.6218127 & 0.6918243 & 0.0000000 \\ \mathrm{C} & 2.4147049 & 1.4041332 & 0.0000000 \\ \mathrm{H} & 4.5610760 & 1.2310592 & 0.0000000 \\ \mathrm{H} & -4.5610760 & -1.2310592 & 0.0000000 \\ \mathrm{H} & -2.3689073 & -2.4865256 & 0.0000000 \\ \mathrm{H} & -2.3689073 & 2.4865256 & 0.0000000 \\ \mathrm{H} & 2.3689073 & -2.4865256 & 0.0000000 \\ \mathrm{H} & 4.5610760 & -1.2310592 & 0.0000000 \\ \mathrm{H} & -4.5610760 & 1.2310592 & 0.0000000 \\ \mathrm{H} & 2.3689073 & 2.4865256 & 0.0000000\end{array}$


Table S473 Cartesian coordinates of the TDDFT/TZVP-optimized excitedstate equilibrium geometry of the $1{ }^{1} B_{2 g}\left(\equiv 8{ }^{1} B_{3 g}\right.$ in Turbomole) $\left({ }^{1} n \pi^{*}\right)$ state of dibenzo- $p$-dioxin (excited-state energy: $-612.05531 E_{h}$ ).

$\begin{array}{lrrr}22 & & & \\ & & & \\ \text { C } & -3.6078186 & 0.7003752 & 0.0000000 \\ \text { C } & -3.6078186 & -0.7003752 & 0.0000000 \\ \text { C } & -2.3956933 & -1.4020970 & 0.0000000 \\ \text { C } & -1.1909983 & -0.7097087 & 0.0000000 \\ \text { C } & -1.1909983 & 0.7097087 & 0.0000000 \\ \text { C } & -2.3956933 & 1.4020970 & 0.0000000 \\ \text { O } & 0.0000000 & -1.4072148 & 0.0000000 \\ \text { C } & 1.1909983 & -0.7097087 & 0.0000000 \\ \mathrm{C} & 1.1909983 & 0.7097087 & 0.0000000 \\ \text { O } & 0.0000000 & 1.4072148 & 0.0000000 \\ \mathrm{C} & 2.3956933 & -1.4020970 & 0.0000000 \\ \mathrm{C} & 3.6078186 & -0.7003752 & 0.0000000 \\ \mathrm{C} & 3.6078186 & 0.7003752 & 0.0000000 \\ \mathrm{C} & 2.3956933 & 1.4020970 & 0.0000000 \\ \mathrm{H} & 4.5458797 & 1.2547267 & 0.0000000 \\ \mathrm{H} & -4.5458797 & -1.2547267 & 0.0000000 \\ \mathrm{H} & -2.3773441 & -2.4882803 & 0.0000000 \\ \mathrm{H} & -2.3773441 & 2.4882803 & 0.0000000 \\ \mathrm{H} & 2.3773441 & -2.4882803 & 0.0000000 \\ \mathrm{H} & 4.5458797 & -1.2547267 & 0.0000000 \\ \mathrm{H} & -4.5458797 & 1.2547267 & 0.0000000 \\ \mathrm{H} & 2.3773441 & 2.4882803 & 0.0000000\end{array}$


Table S474 Cartesian coordinates of the TDDFT/TZVP-optimized excitedstate equilibrium geometry of the $1{ }^{3} A_{u}\left({ }^{3} n \pi^{*}\right)$ state of dibenzo- $p$-dioxin (excited-state energy: $-612.12398 E_{h}$ ).

$\begin{array}{lrrr}22 & & & \\ & & & \\ \text { C } & -3.6264792 & 0.7091147 & 0.0000000 \\ \text { C } & -3.6264792 & -0.7091147 & 0.0000000 \\ \text { C } & -2.4249469 & -1.3895799 & 0.0000000 \\ \text { C } & -1.2027810 & -0.7339351 & 0.0000000 \\ \text { C } & -1.2027810 & 0.7339351 & 0.0000000 \\ \text { C } & -2.4249469 & 1.3895799 & 0.0000000 \\ \text { O } & 0.0000000 & -1.3587623 & 0.0000000 \\ \text { C } & 1.2027810 & -0.7339351 & 0.0000000 \\ \text { C } & 1.2027810 & 0.7339351 & 0.0000000 \\ \text { O } & 0.0000000 & 1.3587623 & 0.0000000 \\ \text { C } & 2.4249469 & -1.3895799 & 0.0000000 \\ \text { C } & 3.6264792 & -0.7091147 & 0.0000000 \\ \text { C } & 3.6264792 & 0.7091147 & 0.0000000 \\ \text { C } & 2.4249469 & 1.3895799 & 0.0000000 \\ \text { H } & 4.5586838 & 1.2572322 & 0.0000000 \\ \text { H } & -4.5586838 & -1.2572322 & 0.0000000 \\ \text { H } & -2.3922580 & -2.4754691 & 0.0000000 \\ \text { H } & -2.3922580 & 2.4754691 & 0.0000000 \\ \text { H } & 2.3922580 & -2.4754691 & 0.0000000 \\ \text { H } & 4.5586838 & -1.2572322 & 0.0000000 \\ \text { H } & -4.5586838 & 1.2572322 & 0.0000000 \\ \text { H } & 2.3922580 & 2.4754691 & 0.0000000\end{array}$


Table S475 Cartesian coordinates of the TDDFT/TZVP-optimized excitedstate equilibrium geometry of the $1{ }^{3} B_{1 g}$ ( $\equiv 3{ }^{3} B_{2 g}$ in Turbomole) $\left({ }^{3} n \pi^{*}\right)$ state of dibenzo- $p$-dioxin (excited-state energy: $-612.10071 E_{h}$ ).

$\begin{array}{lrrr}22 & & & \\ & & & \\ \text { C } & -3.6217885 & 0.6988971 & 0.0000000 \\ \text { C } & -3.6217885 & -0.6988971 & 0.0000000 \\ \text { C } & -2.4155503 & -1.4020004 & 0.0000000 \\ \text { C } & -1.2097242 & -0.7033153 & 0.0000000 \\ \text { C } & -1.2097242 & 0.7033153 & 0.0000000 \\ \text { C } & -2.4155503 & 1.4020004 & 0.0000000 \\ \text { O } & 0.0000000 & -1.4012919 & 0.0000000 \\ \text { C } & 1.2097242 & -0.7033153 & 0.0000000 \\ \mathrm{C} & 1.2097242 & 0.7033153 & 0.0000000 \\ \text { O } & 0.0000000 & 1.4012919 & 0.0000000 \\ \mathrm{C} & 2.4155503 & -1.4020004 & 0.0000000 \\ \mathrm{C} & 3.6217885 & -0.6988971 & 0.0000000 \\ \mathrm{C} & 3.6217885 & 0.6988971 & 0.0000000 \\ \mathrm{C} & 2.4155503 & 1.4020004 & 0.0000000 \\ \mathrm{H} & 4.5670040 & 1.2343767 & 0.0000000 \\ \mathrm{H} & -4.5670040 & -1.2343767 & 0.0000000 \\ \mathrm{H} & -2.3971128 & -2.4894596 & 0.0000000 \\ \mathrm{H} & -2.3971128 & 2.4894596 & 0.0000000 \\ \mathrm{H} & 2.3971128 & -2.4894596 & 0.0000000 \\ \mathrm{H} & 4.5670040 & -1.2343767 & 0.0000000 \\ \mathrm{H} & -4.5670040 & 1.2343767 & 0.0000000 \\ \mathrm{H} & 2.3971128 & 2.4894596 & 0.0000000\end{array}$


Table S476 Cartesian coordinates of the TDDFT/TZVP-optimized excitedstate equilibrium geometry of the $1{ }^{3} B_{3 u}\left(\equiv 3{ }^{3} B_{1 u}\right.$ in Turbomole) $\left({ }^{3} n \pi^{*}\right)$ state of dibenzo- $p$-dioxin (excited-state energy: $-612.10486 E_{h}$ ).

$\begin{array}{lrrr}22 & & & \\ & & & \\ \mathrm{C} & -3.6226281 & 0.6916808 & 0.0000000 \\ \mathrm{C} & -3.6226281 & -0.6916808 & 0.0000000 \\ \mathrm{C} & -2.4135331 & -1.4020503 & 0.0000000 \\ \mathrm{C} & -1.1974007 & -0.7021846 & 0.0000000 \\ \mathrm{C} & -1.1974007 & 0.7021846 & 0.0000000 \\ \mathrm{C} & -2.4135331 & 1.4020503 & 0.0000000 \\ \mathrm{O} & 0.0000000 & -1.3724157 & 0.0000000 \\ \mathrm{C} & 1.1974007 & -0.7021846 & 0.0000000 \\ \mathrm{C} & 1.1974007 & 0.7021846 & 0.0000000 \\ \mathrm{O} & 0.0000000 & 1.3724157 & 0.0000000 \\ \mathrm{C} & 2.4135331 & -1.4020503 & 0.0000000 \\ \mathrm{C} & 3.6226281 & -0.6916808 & 0.0000000 \\ \mathrm{C} & 3.6226281 & 0.6916808 & 0.0000000 \\ \mathrm{C} & 2.4135331 & 1.4020503 & 0.0000000 \\ \mathrm{H} & 4.5622999 & 1.2301550 & 0.0000000 \\ \mathrm{H} & -4.5622999 & -1.2301550 & 0.0000000 \\ \mathrm{H} & -2.3675237 & -2.4850989 & 0.0000000 \\ \mathrm{H} & -2.3675237 & 2.4850989 & 0.0000000 \\ \mathrm{H} & 2.3675237 & -2.4850989 & 0.0000000 \\ \mathrm{H} & 4.5622999 & -1.2301550 & 0.0000000 \\ \mathrm{H} & -4.5622999 & 1.2301550 & 0.0000000 \\ \mathrm{H} & 2.3675237 & 2.4850989 & 0.0000000\end{array}$


Table S477 Cartesian coordinates of the TDDFT/TZVP-optimized excitedstate equilibrium geometry of the $1{ }^{3} B_{2 g}$ ( $\equiv 6{ }^{3} B_{3 g}$ in Turbomole) $\left({ }^{3} n \pi^{*}\right)$ state of dibenzo- $p$-dioxin (excited-state energy: $-612.06588 E_{h}$ ).

$\begin{array}{lrrr}22 & & & \\ & & & \\ \mathrm{C} & -3.6276358 & 0.7174670 & 0.0000000 \\ \mathrm{C} & -3.6276358 & -0.7174670 & 0.0000000 \\ \mathrm{C} & -2.4108989 & -1.3838152 & 0.0000000 \\ \mathrm{C} & -1.2021708 & -0.7240530 & 0.0000000 \\ \mathrm{C} & -1.2021708 & 0.7240530 & 0.0000000 \\ \mathrm{C} & -2.4108989 & 1.3838152 & 0.0000000 \\ \mathrm{O} & 0.0000000 & -1.3961935 & 0.0000000 \\ \mathrm{C} & 1.2021708 & -0.7240530 & 0.0000000 \\ \mathrm{C} & 1.2021708 & 0.7240530 & 0.0000000 \\ \mathrm{O} & 0.0000000 & 1.3961935 & 0.0000000 \\ \mathrm{C} & 2.4108989 & -1.3838152 & 0.0000000 \\ \mathrm{C} & 3.6276358 & -0.7174670 & 0.0000000 \\ \mathrm{C} & 3.6276358 & 0.7174670 & 0.0000000 \\ \mathrm{C} & 2.4108989 & 1.3838152 & 0.0000000 \\ \mathrm{H} & 4.5689095 & 1.2512459 & 0.0000000 \\ \mathrm{H} & -4.5689095 & -1.2512459 & 0.0000000 \\ \mathrm{H} & -2.3830210 & -2.4737716 & 0.0000000 \\ \mathrm{H} & -2.3830210 & 2.4737716 & 0.0000000 \\ \mathrm{H} & 2.3830210 & -2.4737716 & 0.0000000 \\ \mathrm{H} & 4.5689095 & -1.2512459 & 0.0000000 \\ \mathrm{H} & -4.5689095 & 1.2512459 & 0.0000000 \\ \mathrm{H} & 2.3830210 & 2.4737716 & 0.0000000\end{array}$


Table S478 Cartesian coordinates of the TDDFT/TZVP-optimized excitedstate equilibrium geometry of the $1^{1} A^{\prime \prime}\left({ }^{1} n \pi^{*}\right)$ state of formaldehyde (excitedstate energy: $-114.35466 E_{h}$ ).

4

$\begin{array}{rrrr}\mathrm{C} & -0.0352022 & 0.1263645 & 0.0000000 \\ \mathrm{O} & 1.2223955 & 0.4405342 & 0.0000000 \\ \mathrm{H} & -0.4384848 & -0.2775765 & 0.9343572 \\ \mathrm{H} & -0.4384848 & -0.2775765 & -0.9343572\end{array}$


Table S479 Cartesian coordinates of the TDDFT/TZVP-optimized excitedstate equilibrium geometry of the $1^{3} A^{\prime \prime}\left({ }^{3} n \pi^{*}\right)$ state of formaldehyde (excitedstate energy: $\left.-114.38644 E_{h}\right)$.

4

$\begin{array}{rrrr}\mathrm{C} & -0.1029729 & 0.2394782 & 0.0000000 \\ \mathrm{O} & 1.1848601 & 0.4042534 & 0.0000000 \\ \mathrm{H} & -0.4720517 & -0.2529247 & 0.9138264 \\ \mathrm{H} & -0.4720517 & -0.2529247 & -0.9138264\end{array}$


Table S480 Cartesian coordinates of the TDDFT/TZVP-optimized excitedstate equilibrium geometry of the $1^{1} A_{u}\left({ }^{1} n \pi^{*}\right)$ state of trans-glyoxal (excitedstate energy: $-227.70590 E_{h}$ ).

$\begin{array}{lrrr}\mathrm{C} & 0.6360711 & 0.3780369 & 0.0000000 \\ \mathrm{C} & -0.6360711 & -0.3780369 & 0.0000000 \\ \mathrm{O} & 1.7568084 & -0.1291747 & 0.0000000 \\ \mathrm{O} & -1.7568084 & 0.1291747 & 0.0000000 \\ \mathrm{H} & 0.5012758 & 1.4697980 & 0.0000000 \\ \mathrm{H} & -0.5012758 & -1.4697980 & 0.0000000\end{array}$


Table S481 Cartesian coordinates of the TDDFT/TZVP-optimized excitedstate equilibrium geometry of the $1^{3} A_{u}\left({ }^{3} n \pi^{*}\right)$ state of trans-glyoxal (excitedstate energy: $-227.72970 E_{h}$ ).

0.6266858

0.3849322

0.0000000

C -0.6266858

O 1.7495700

$-0.3849322 \quad 0.0000000$

0.1299116

H $\quad-0.4980768-1.4720267 \quad 0.0000000$ 
Table S482 Cartesian coordinates of the TDDFT/TZVP-optimized excitedstate equilibrium geometry of the $2{ }^{1} A\left({ }^{1} \pi \pi^{*}\right)$ state of $9 H$-guanine (excited-state energy: $\left.-542.31115 E_{h}\right)$.

$\begin{array}{lrrr}16 & & & \\ & & & \\ \mathrm{~N} & 1.8113965 & -0.3307743 & -1.3892251 \\ \mathrm{C} & 2.6014398 & 0.2276036 & -0.3999081 \\ \mathrm{H} & 3.6664073 & 0.3472624 & -0.5332973 \\ \mathrm{~N} & 1.9462296 & 0.5754050 & 0.6820385 \\ \mathrm{C} & 0.6426600 & 0.2930577 & 0.4240596 \\ \mathrm{C} & -0.3954010 & -0.0776032 & 1.4140382 \\ \mathrm{O} & -0.2229358 & -0.2180474 & 2.6032154 \\ \mathrm{~N} & -1.6291088 & -0.3218002 & 0.7681957 \\ \mathrm{H} & -2.1742831 & -1.1151203 & 1.1126572 \\ \mathrm{C} & -1.5591164 & 0.0796345 & -0.5717445 \\ \mathrm{~N} & -2.6472534 & 0.6374179 & -1.1760622 \\ \mathrm{H} & -3.1367288 & 1.3545060 & -0.6567618 \\ \mathrm{H} & -2.5490350 & 0.8071964 & -2.1660034 \\ \mathrm{~N} & -0.6094606 & -0.6174700 & -1.4587858 \\ \mathrm{C} & 0.4983026 & -0.2316330 & -0.9502882 \\ \mathrm{H} & 2.1048843 & -0.6059703 & -2.3121307\end{array}$


Table S483 Cartesian coordinates of the TDDFT/TZVP-optimized excitedstate equilibrium geometry of the $2{ }^{1} A^{\prime}\left({ }^{1} \pi \pi^{*}\right)$ state of 1-hydroxy-2acetonaphthone (excited-state energy: $-613.49895 E_{h}$ ).

$\begin{array}{lrrr}24 & & & \\ & & & \\ \text { C } & -2.9913182 & -0.3733307 & 0.0000000 \\ \text { C } & -1.5675434 & -0.3772130 & 0.0000000 \\ \text { C } & -0.8555765 & 0.8826349 & 0.0000000 \\ \text { C } & -1.6036834 & 2.0666345 & 0.0000000 \\ \text { C } & -2.9916866 & 2.0331056 & 0.0000000 \\ \text { C } & -3.6952112 & 0.8078101 & 0.0000000 \\ \text { C } & -0.8277497 & -1.5596052 & 0.0000000 \\ \text { C } & 0.5837442 & -1.5177347 & 0.0000000 \\ \text { C } & 1.3184857 & -0.3281661 & 0.0000000 \\ \text { C } & 0.5928141 & 0.9255206 & 0.0000000 \\ \text { O } & 1.2198358 & 2.0388423 & 0.0000000 \\ \text { C } & 2.7518131 & -0.3264896 & 0.0000000 \\ \text { O } & 3.3928879 & 0.8314274 & 0.0000000 \\ \text { C } & 3.6107030 & -1.5445698 & 0.0000000 \\ \text { H } & -3.5462278 & 2.9641077 & 0.0000000 \\ \text { H } & -4.7774662 & 0.8038807 & 0.0000000 \\ \text { H } & -3.5106825 & -1.3251234 & 0.0000000 \\ \text { H } & -1.0698916 & 3.0079209 & 0.0000000 \\ \text { H } & -1.3305690 & -2.5188818 & 0.0000000 \\ \text { H } & 1.1124604 & -2.4643418 & 0.0000000 \\ \text { H } & 2.6466790 & 1.5577536 & 0.0000000 \\ \text { H } & 4.6550363 & -1.2357761 & 0.0000000 \\ \text { H } & 3.4415733 & -2.1742031 & -0.8816944 \\ \text { H } & 3.4415733 & -2.1742031 & 0.8816944 \\ & & & \end{array}$


Table S484 Cartesian coordinates of the TDDFT/TZVP-optimized excitedstate equilibrium geometry of the $2{ }^{1} A^{\prime}\left({ }^{1} \pi \pi^{*}\right)$ state of indole (excited-state energy: $\left.-363.54419 E_{h}\right)$.

$\begin{array}{lrrr}16 & & & \\ & & & \\ \mathrm{C} & -0.5251096 & -0.5881328 & 0.0000000 \\ \mathrm{C} & -0.0444663 & 0.7205887 & 0.0000000 \\ \mathrm{~N} & -1.1774103 & 1.5749040 & 0.0000000 \\ \mathrm{C} & -1.9539199 & -0.5022941 & 0.0000000 \\ \mathrm{C} & -2.3215271 & 0.8774802 & 0.0000000 \\ \mathrm{C} & 0.3751209 & -1.6920448 & 0.0000000 \\ \mathrm{C} & 1.7643365 & -1.3672825 & 0.0000000 \\ \mathrm{C} & 1.3167664 & 1.0671682 & 0.0000000 \\ \mathrm{C} & 2.2134465 & -0.0628048 & 0.0000000 \\ \mathrm{H} & -1.1204740 & 2.5826319 & 0.0000000 \\ \mathrm{H} & -2.6561092 & -1.3215605 & 0.0000000 \\ \mathrm{H} & -3.2911160 & 1.3447053 & 0.0000000 \\ \mathrm{H} & 0.0241829 & -2.7136660 & 0.0000000 \\ \mathrm{H} & 2.4911224 & -2.1709451 & 0.0000000 \\ \mathrm{H} & 1.6726426 & 2.0865222 & 0.0000000 \\ \mathrm{H} & 3.2802703 & 0.1281456 & 0.0000000\end{array}$


Table S485 Cartesian coordinates of the TDDFT/TZVP-optimized excitedstate equilibrium geometry of the $3^{1} A^{\prime}\left({ }^{1} \pi \pi^{*}\right)$ state of indole (excited-state energy: $\left.-363.53333 E_{h}\right)$.

$\begin{array}{lrrr}16 & & & \\ & & & \\ \mathrm{C} & -0.5582865 & -0.6069175 & 0.0000000 \\ \mathrm{C} & -0.0458267 & 0.7604745 & 0.0000000 \\ \mathrm{~N} & -1.1409141 & 1.5927950 & 0.0000000 \\ \mathrm{C} & -1.9683399 & -0.5071075 & 0.0000000 \\ \mathrm{C} & -2.3116245 & 0.8397829 & 0.0000000 \\ \mathrm{C} & 0.3540699 & -1.6867930 & 0.0000000 \\ \mathrm{C} & 1.7385030 & -1.3753117 & 0.0000000 \\ \mathrm{C} & 1.3336618 & 1.0712957 & 0.0000000 \\ \mathrm{C} & 2.2133891 & -0.0265671 & 0.0000000 \\ \mathrm{H} & -1.1034770 & 2.5977031 & 0.0000000 \\ \mathrm{H} & -2.6716467 & -1.3245861 & 0.0000000 \\ \mathrm{H} & -3.2772769 & 1.3163784 & 0.0000000 \\ \mathrm{H} & 0.0117103 & -2.7119516 & 0.0000000 \\ \mathrm{H} & 2.4651085 & -2.1773439 & 0.0000000 \\ \mathrm{H} & 1.6933803 & 2.0909419 & 0.0000000 \\ \mathrm{H} & 3.2815737 & 0.1480746 & 0.0000000\end{array}$


Table S486 Cartesian coordinates of the TDDFT/TZVP-optimized excitedstate equilibrium geometry of the $1{ }^{1} B_{1}\left(\equiv{ }^{1}{ }^{1} B_{2}\right.$ in Turbomole) $\left({ }^{1} n \pi^{*}\right)$ state of maleimide (excited-state energy: $-359.26204 E_{h}$ ).

$\begin{array}{lrlr}10 & & & \\ & & & \\ \mathrm{~N} & 0.0000000 & 0.0000000 & -0.8801492 \\ \mathrm{C} & -1.1497307 & 0.0000000 & -0.0686134 \\ \mathrm{C} & -0.6921383 & 0.0000000 & 1.2880249 \\ \mathrm{C} & 0.6921383 & 0.0000000 & 1.2880249 \\ \mathrm{C} & 1.1497307 & 0.0000000 & -0.0686134 \\ \mathrm{O} & -2.2687303 & 0.0000000 & -0.6048025 \\ \mathrm{O} & 2.2687303 & 0.0000000 & -0.6048025 \\ \mathrm{H} & 0.0000000 & 0.0000000 & -1.8890755 \\ \mathrm{H} & -1.3632896 & 0.0000000 & 2.1290327 \\ \mathrm{H} & 1.3632896 & 0.0000000 & 2.1290327\end{array}$


Table S487 Cartesian coordinates of the TDDFT/TZVP-optimized excitedstate equilibrium geometry of the $1^{1} A_{2}\left({ }^{1} n \pi^{*}\right)$ state of maleimide (excited-state energy: $\left.-359.23701 E_{h}\right)$.

$\begin{array}{lrrr}10 & & & \\ & & & \\ \mathrm{~N} & 0.0000000 & 0.0000000 & -0.9013351 \\ \mathrm{C} & -1.1320778 & 0.0000000 & -0.1067318 \\ \mathrm{C} & -0.6993382 & 0.0000000 & 1.2535882 \\ \mathrm{C} & 0.6993382 & 0.0000000 & 1.2535882 \\ \mathrm{C} & 1.1320778 & 0.0000000 & -0.1067318 \\ \mathrm{O} & -2.3196389 & 0.0000000 & -0.5388385 \\ \mathrm{O} & 2.3196389 & 0.0000000 & -0.5388385 \\ \mathrm{H} & 0.0000000 & 0.0000000 & -1.9079612 \\ \mathrm{H} & -1.3612238 & 0.0000000 & 2.1032155 \\ \mathrm{H} & 1.3612238 & 0.0000000 & 2.1032155\end{array}$


Table S488 Cartesian coordinates of the TDDFT/TZVP-optimized excitedstate equilibrium geometry of the $1{ }^{1} B_{2}\left(\equiv{ }^{1}{ }^{1} B_{1}\right.$ in Turbomole) $\left({ }^{1} \pi \pi^{*}\right)$ state of maleimide (excited-state energy: $-359.23989 E_{h}$ ).

$\begin{array}{lrrr}10 & & & \\ & & & \\ \mathrm{~N} & 0.0000000 & 0.0000000 & -0.9090115 \\ \mathrm{C} & -1.2552293 & 0.0000000 & -0.0533218 \\ \mathrm{C} & -0.7109334 & 0.0000000 & 1.2437588 \\ \mathrm{C} & 0.7109334 & 0.0000000 & 1.2437588 \\ \mathrm{C} & 1.2552293 & 0.0000000 & -0.0533218 \\ \mathrm{O} & -2.3499456 & 0.0000000 & -0.5688590 \\ \mathrm{O} & 2.3499456 & 0.0000000 & -0.5688590 \\ \mathrm{H} & 0.0000000 & 0.0000000 & -1.9277296 \\ \mathrm{H} & -1.3346616 & 0.0000000 & 2.1236331 \\ \mathrm{H} & 1.3346616 & 0.0000000 & 2.1236331\end{array}$


Table S489 Cartesian coordinates of the TDDFT/TZVP-optimized excitedstate equilibrium geometry of the $2{ }^{1} B_{2}\left(\equiv 2{ }^{1} B_{1}\right.$ in Turbomole $)\left({ }^{1} \pi \pi^{*}\right)$ state of maleimide (excited-state energy: $-359.17735 E_{h}$ ).

$\begin{array}{lrlr}10 & & & \\ & & & \\ \mathrm{~N} & 0.0000000 & 0.0000000 & -0.8898642 \\ \mathrm{C} & -1.1575249 & 0.0000000 & -0.1018609 \\ \mathrm{C} & -0.7169537 & 0.0000000 & 1.2848932 \\ \mathrm{C} & 0.7169537 & 0.0000000 & 1.2848932 \\ \mathrm{C} & 1.1575249 & 0.0000000 & -0.1018609 \\ \mathrm{O} & -2.3107926 & 0.0000000 & -0.5727222 \\ \mathrm{O} & 2.3107926 & 0.0000000 & -0.5727222 \\ \mathrm{H} & 0.0000000 & 0.0000000 & -1.9001627 \\ \mathrm{H} & -1.3894007 & 0.0000000 & 2.1263705 \\ \mathrm{H} & 1.3894007 & 0.0000000 & 2.1263705\end{array}$


Table S490 Cartesian coordinates of the TDDFT/TZVP-optimized excitedstate equilibrium geometry of the $1{ }^{3} B_{1}\left(\equiv 1{ }^{3} B_{2}\right.$ in Turbomole) $\left({ }^{3} n \pi^{*}\right)$ state of maleimide (excited-state energy: $-359.27758 E_{h}$ ).

$\begin{array}{lrrr}10 & & \\ & & & \\ \mathrm{~N} & 0.0000000 & 0.0000000 & -0.8803062 \\ \mathrm{C} & -1.1471245 & 0.0000000 & -0.0712258 \\ \mathrm{C} & -0.6908750 & 0.0000000 & 1.2838029 \\ \mathrm{C} & 0.6908750 & 0.0000000 & 1.2838029 \\ \mathrm{C} & 1.1471245 & 0.0000000 & -0.0712258 \\ \mathrm{O} & -2.2752412 & 0.0000000 & -0.5994402 \\ \mathrm{O} & 2.2752412 & 0.0000000 & -0.5994402 \\ \mathrm{H} & 0.0000000 & 0.0000000 & -1.8890958 \\ \mathrm{H} & -1.3600460 & 0.0000000 & 2.1264583 \\ \mathrm{H} & 1.3600460 & 0.0000000 & 2.1264583\end{array}$


Table S491 Cartesian coordinates of the TDDFT/TZVP-optimized excitedstate equilibrium geometry of the $1^{3} A_{2}\left({ }^{3} n \pi^{*}\right)$ state of maleimide (excited-state energy: $\left.-359.25392 E_{h}\right)$.

10

$\mathrm{N} \quad 0.0000000$

C $\quad-1.1314102$

C $\quad-0.6981758$

0.0000000

$-0.9030882$

C $\quad 0.6981758$

0.0000000

$-0.1076297$

C $\quad 1.1314102$

0.0000000

1.2495707

$-2.3242174$

0.0000000

1.2495707

O

$\mathrm{O} \quad 2.3242174$

0.0000000

$-0.1076297$

0.0000000

$-0.5343139$

$\begin{array}{rr}\mathrm{H} & 0.0000000 \\ \mathrm{H} & -1.3557747\end{array}$

0.0000000

$-0.5343139$

0.0000000

$-1.9093342$

$\mathrm{H}$

1. 3557747

0.0000000

2. 1028364

0.0000000

2. 1028364 
Table S492 Cartesian coordinates of the TDDFT/TZVP-optimized excitedstate equilibrium geometry of the $1{ }^{3} B_{2}\left(\equiv 2{ }^{3} B_{1}\right.$ in Turbomole) $\left({ }^{3} \pi \pi^{*}\right)$ state of maleimide (excited-state energy: $-359.26784 E_{h}$ ).

$\begin{array}{lrlr}10 & & & \\ & & & \\ \mathrm{~N} & 0.0000000 & 0.0000000 & -1.1283976 \\ \mathrm{C} & -1.1818770 & 0.0000000 & -0.3317392 \\ \mathrm{C} & -0.7033165 & 0.0000000 & 1.0026854 \\ \mathrm{C} & 0.7033165 & 0.0000000 & 1.0026854 \\ \mathrm{C} & 1.1818770 & 0.0000000 & -0.3317392 \\ \mathrm{O} & -2.2979373 & 0.0000000 & -0.8966062 \\ \mathrm{O} & 2.2979373 & 0.0000000 & -0.8966062 \\ \mathrm{H} & 0.0000000 & 0.0000000 & -2.1453265 \\ \mathrm{H} & -1.3521499 & 0.0000000 & 1.8625220 \\ \mathrm{H} & 1.3521499 & 0.0000000 & 1.8625220\end{array}$


Table S493 Cartesian coordinates of the TDDFT/TZVP-optimized excitedstate equilibrium geometry of the $1^{1} A^{\prime \prime}\left({ }^{1} n \pi^{*}\right)$ state of nitrosomethane (excitedstate energy: $-169.71209 E_{h}$ ).

6

$\begin{array}{lrrr}\mathrm{C} & -1.2419082 & -0.1566106 & 0.0000000 \\ \mathrm{~N} & 0.0982203 & 0.4343441 & 0.0000000 \\ \mathrm{O} & 1.1433456 & -0.2067047 & 0.0000000 \\ \mathrm{H} & -1.9587145 & 0.6596189 & 0.0000000 \\ \mathrm{H} & -1.3777763 & -0.7740204 & -0.8955339 \\ \mathrm{H} & -1.3777763 & -0.7740204 & 0.8955339\end{array}$


Table S494 Cartesian coordinates of the TDDFT/TZVP-optimized excitedstate equilibrium geometry of the $1^{1} A^{\prime \prime}\left({ }^{1} n \pi^{*}\right)$ state of propenoic acid anion (excited-state energy: $-266.47376 E_{h}$ ).

$\begin{array}{rrrr}8 & & & \\ \text { C } & -0.2707905 & 0.4015229 & 0.0000000 \\ \mathrm{C} & 1.1505101 & 0.4192593 & 0.0000000 \\ \mathrm{O} & 1.9008637 & 1.4592932 & 0.0000000 \\ \mathrm{O} & 1.9280033 & -0.6105994 & 0.0000000 \\ \mathrm{C} & -1.0718406 & -0.7616554 & 0.0000000 \\ \mathrm{H} & -0.7352853 & 1.3808482 & 0.0000000 \\ \mathrm{H} & -2.1510162 & -0.6864373 & 0.0000000 \\ \mathrm{H} & -0.6506158 & -1.7561723 & 0.0000000\end{array}$


Table S495 Cartesian coordinates of the TDDFT/TZVP-optimized excitedstate equilibrium geometry of the $1^{1} A_{2}\left({ }^{1} n \pi^{*}\right)$ state of pyridine (excited-state energy: $\left.-248.05406 E_{h}\right)$.

11

$\begin{array}{lrrr}\mathrm{N} & 0.0000000 & 0.0000000 & 1.2575841 \\ \mathrm{C} & -1.1983900 & 0.0000000 & 0.7544394 \\ \mathrm{C} & 1.1983900 & 0.0000000 & 0.7544394 \\ \mathrm{C} & -1.2228125 & 0.0000000 & -0.7158153 \\ \mathrm{C} & 1.2228125 & 0.0000000 & -0.7158153 \\ \mathrm{C} & 0.0000000 & 0.0000000 & -1.3678963 \\ \mathrm{H} & -2.0651437 & 0.0000000 & 1.3970869 \\ \mathrm{H} & 2.0651437 & 0.0000000 & 1.3970869 \\ \mathrm{H} & -2.1772459 & 0.0000000 & -1.2178963 \\ \mathrm{H} & 2.1772459 & 0.0000000 & -1.2178963 \\ \mathrm{H} & 0.0000000 & 0.0000000 & -2.4541128\end{array}$


Table S496 Cartesian coordinates of the TDDFT/TZVP-optimized excitedstate equilibrium geometry of the $2{ }^{1} A^{\prime}\left({ }^{1} n \pi^{*}\right)$ state of pyridine (excited-state energy: $-248.05208 E_{h}$ ).

$\begin{array}{lrrr}11 & & & \\ & & & \\ \mathrm{~N} & 1.2786048 & 0.0880306 & 0.0000000 \\ \mathrm{C} & 0.7066890 & -0.0393803 & 1.2188361 \\ \mathrm{C} & 0.7066890 & -0.0393803 & -1.2188361 \\ \mathrm{C} & -0.6672710 & -0.0218363 & 1.2256793 \\ \mathrm{C} & -0.6672710 & -0.0218363 & -1.2256793 \\ \mathrm{C} & -1.3899016 & 0.0598206 & 0.0000000 \\ \mathrm{H} & -1.1697462 & -0.1177359 & 2.1803675 \\ \mathrm{H} & -1.1697462 & -0.1177359 & -2.1803675 \\ \mathrm{H} & 1.3321239 & -0.1485736 & 2.0933662 \\ \mathrm{H} & 1.3321239 & -0.1485736 & -2.0933662 \\ \mathrm{H} & -2.4692926 & 0.0554514 & 0.0000000\end{array}$


Table S497 Cartesian coordinates of the TDDFT/TZVP-optimized excitedstate equilibrium geometry of the $1^{3} A_{1}\left({ }^{3} \pi \pi^{*}\right)$ state of pyridine (excited-state energy: $\left.-248.08279 E_{h}\right)$.

11

$\begin{array}{lrrr}\mathrm{N} & 0.0000000 & 0.0000000 & 1.4374469 \\ \mathrm{C} & -1.1423249 & 0.0000000 & 0.7595349 \\ \mathrm{C} & 1.1423249 & 0.0000000 & 0.7595349 \\ \mathrm{C} & -1.1989448 & 0.0000000 & -0.7645093 \\ \mathrm{C} & 1.1989448 & 0.0000000 & -0.7645093 \\ \mathrm{C} & 0.0000000 & 0.0000000 & -1.4640870 \\ \mathrm{H} & -2.0644369 & 0.0000000 & 1.3283066 \\ \mathrm{H} & 2.0644369 & 0.0000000 & 1.3283066 \\ \mathrm{H} & -2.1604811 & 0.0000000 & -1.2589323 \\ \mathrm{H} & 2.1604811 & 0.0000000 & -1.2589323 \\ \mathrm{H} & 0.0000000 & 0.0000000 & -2.5485644\end{array}$


Table S498 Cartesian coordinates of the TDDFT/TZVP-optimized excitedstate equilibrium geometry of the $2{ }^{3} A_{1}\left({ }^{3} \pi \pi^{*}\right)$ state of pyridine (excited-state energy: $\left.-248.03867 E_{h}\right)$.

11

$\begin{array}{lrrr}\mathrm{N} & 0.0000000 & 0.0000000 & 1.6544960 \\ \mathrm{C} & -1.1645893 & 0.0000000 & 0.9331339 \\ \mathrm{C} & 1.1645893 & 0.0000000 & 0.9331339 \\ \mathrm{C} & -1.2214967 & 0.0000000 & -0.4814042 \\ \mathrm{C} & 1.2214967 & 0.0000000 & -0.4814042 \\ \mathrm{C} & 0.0000000 & 0.0000000 & -1.2221600 \\ \mathrm{H} & -2.0769349 & 0.0000000 & 1.5164076 \\ \mathrm{H} & 2.0769349 & 0.0000000 & 1.5164076 \\ \mathrm{H} & -2.1778377 & 0.0000000 & -0.9865806 \\ \mathrm{H} & 2.1778377 & 0.0000000 & -0.9865806 \\ \mathrm{H} & 0.0000000 & 0.0000000 & -2.3033958\end{array}$


Table S499 Cartesian coordinates of the TDDFT/TZVP-optimized excitedstate equilibrium geometry of the $2{ }^{1} A^{\prime}\left({ }^{1} \pi \pi^{*}\right)$ state of pyrrole (excited-state energy: $\left.-209.88067 E_{h}\right)$.

$\begin{array}{lrrr}10 & & & \\ & & & \\ \mathrm{H} & 2.1137901 & -0.2136490 & 0.0000000 \\ \mathrm{H} & 0.7674864 & 0.3081605 & 2.1151843 \\ \mathrm{H} & 0.7674864 & 0.3081605 & -2.1151843 \\ \mathrm{H} & -1.8740597 & -0.0984880 & -1.3609441 \\ \mathrm{H} & -1.8740597 & -0.0984880 & 1.3609441 \\ \mathrm{~N} & 1.1254219 & 0.0161764 & 0.0000000 \\ \mathrm{C} & 0.3510445 & -0.0333213 & -1.1715896 \\ \mathrm{C} & 0.3510445 & -0.0333213 & 1.1715896 \\ \mathrm{C} & -1.0030761 & 0.0152583 & 0.7346603 \\ \mathrm{C} & -1.0030761 & 0.0152583 & -0.7346603\end{array}$

S 544 
Table S500 Cartesian coordinates of the TDDFT/TZVP-optimized excitedstate equilibrium geometry of the $1{ }^{1} B_{2}\left(\equiv{ }^{1}{ }^{1} B_{1}\right.$ in Turbomole) $\left({ }^{1} \pi \pi^{*}\right)$ state of pyrrole (excited-state energy: $-209.88853 E_{h}$ ).

$\begin{array}{lrlr}10 & & & \\ & & & \\ \mathrm{H} & 0.0000000 & 0.0000000 & 2.1909954 \\ \mathrm{H} & -2.1393051 & 0.0000000 & 0.7456252 \\ \mathrm{H} & 2.1393051 & 0.0000000 & 0.7456252 \\ \mathrm{H} & 1.3440856 & 0.0000000 & -1.8854261 \\ \mathrm{H} & -1.3440856 & 0.0000000 & -1.8854261 \\ \mathrm{~N} & 0.0000000 & 0.0000000 & 1.1895601 \\ \mathrm{C} & 1.1393458 & 0.0000000 & 0.3443120 \\ \mathrm{C} & -1.1393458 & 0.0000000 & 0.3443120 \\ \mathrm{C} & -0.6835926 & 0.0000000 & -1.0341918 \\ \mathrm{C} & 0.6835926 & 0.0000000 & -1.0341918\end{array}$


Table S501 Cartesian coordinates of the TDDFT/TZVP-optimized excitedstate equilibrium geometry of the $2{ }^{1} A^{\prime}\left({ }^{1} \pi \pi^{*}\right)$ state of cis-PSB3 (excited-state energy: $\left.-249.60279 E_{h}\right)$.

$\begin{array}{lrrr}14 & & & \\ & & & \\ \text { C } & 2.8432062 & -0.4991456 & 0.0000000 \\ \text { C } & 1.4692940 & -0.4062670 & 0.0000000 \\ \text { C } & 0.7039985 & 0.8670264 & 0.0000000 \\ \text { C } & -0.6440904 & 0.8731732 & 0.0000000 \\ \mathrm{C} & -1.5091530 & -0.3195324 & 0.0000000 \\ \mathrm{~N} & -2.8289238 & -0.1714167 & 0.0000000 \\ \mathrm{H} & 3.3323246 & -1.4652761 & 0.0000000 \\ \mathrm{H} & 3.4719725 & 0.3846805 & 0.0000000 \\ \mathrm{H} & 0.9116331 & -1.3350901 & 0.0000000 \\ \mathrm{H} & 1.2524266 & 1.7995879 & 0.0000000 \\ \mathrm{H} & -1.1584092 & 1.8295814 & 0.0000000 \\ \mathrm{H} & -1.1281977 & -1.3290519 & 0.0000000 \\ \mathrm{H} & -3.4496829 & -0.9695646 & 0.0000000 \\ \mathrm{H} & -3.2663986 & 0.7412950 & 0.0000000\end{array}$


Table S502 Cartesian coordinates of the TDDFT/TZVP-optimized excitedstate equilibrium geometry of the $2{ }^{1} A^{\prime}\left({ }^{1} \pi \pi^{*}\right)$ state of 3Me-cis-PSB5 (excitedstate energy: $-443.72087 E_{h}$ ).

$\begin{array}{lrrr}25 & & & \\ & & & \\ \mathrm{C} & -0.8468129 & 1.2429200 & 0.0000000 \\ \mathrm{C} & 0.5043760 & 1.1617410 & 0.0000000 \\ \mathrm{C} & 1.4459364 & 0.0166127 & 0.0000000 \\ \mathrm{C} & 2.7822414 & 0.3582136 & 0.0000000 \\ \mathrm{C} & 3.8813722 & -0.5225080 & 0.0000000 \\ \mathrm{~N} & 5.1448185 & -0.1016687 & 0.0000000 \\ \mathrm{C} & 0.9744867 & -1.4043815 & 0.0000000 \\ \mathrm{C} & -1.8423629 & 0.1750825 & 0.0000000 \\ \mathrm{C} & -3.1857586 & 0.4088014 & 0.0000000 \\ \mathrm{C} & -4.1591684 & -0.6448684 & 0.0000000 \\ \mathrm{C} & -5.5024618 & -0.4362455 & 0.0000000 \\ \mathrm{H} & -1.2565372 & 2.2481007 & 0.0000000 \\ \mathrm{H} & 1.0131263 & 2.1206714 & 0.0000000 \\ \mathrm{H} & 3.0272490 & 1.4171436 & 0.0000000 \\ \mathrm{H} & 3.7538741 & -1.5970881 & 0.0000000 \\ \mathrm{H} & 5.9152131 & -0.7527259 & 0.0000000 \\ \mathrm{H} & 5.3778488 & 0.8816771 & 0.0000000 \\ \mathrm{H} & 1.7953256 & -2.1179602 & 0.0000000 \\ \mathrm{H} & 0.3651344 & -1.6168933 & 0.8830740 \\ \mathrm{H} & 0.3651344 & -1.6168933 & -0.8830740 \\ \mathrm{H} & -1.5112536 & -0.8549382 & 0.0000000 \\ \mathrm{H} & -3.5492299 & 1.4331199 & 0.0000000 \\ \mathrm{H} & -3.7887352 & -1.6665219 & 0.0000000 \\ \mathrm{H} & -6.1997804 & -1.2640593 & 0.0000000 \\ \mathrm{H} & -5.9202704 & 0.5645236 & 0.0000000 \\ & & & \end{array}$


Table S503 Cartesian coordinates of the TDDFT/TZVP-optimized excitedstate equilibrium geometry of the $1{ }^{1} B_{u}\left({ }^{1} \pi \pi^{*}\right)$ state of stilbene (excited-state energy: $\left.-540.39967 E_{h}\right)$.

$\begin{array}{lrrr}26 & & & \\ & & & \\ \mathrm{C} & -0.5096258 & 0.4899977 & 0.0000000 \\ \mathrm{C} & -1.8956248 & 0.2232710 & 0.0000000 \\ \mathrm{C} & -2.8335608 & 1.3016444 & 0.0000000 \\ \mathrm{C} & -4.1899460 & 1.0756027 & 0.0000000 \\ \mathrm{C} & -4.6916279 & -0.2412793 & 0.0000000 \\ \mathrm{C} & -3.8007112 & -1.3176921 & 0.0000000 \\ \mathrm{C} & -2.4350073 & -1.1106189 & 0.0000000 \\ \mathrm{C} & 0.5096258 & -0.4899977 & 0.0000000 \\ \mathrm{C} & 1.8956248 & -0.2232710 & 0.0000000 \\ \mathrm{C} & 2.8335608 & -1.3016444 & 0.0000000 \\ \mathrm{C} & 4.1899460 & -1.0756027 & 0.0000000 \\ \mathrm{C} & 4.6916279 & 0.2412793 & 0.0000000 \\ \mathrm{C} & 3.8007112 & 1.3176921 & 0.0000000 \\ \mathrm{C} & 2.4350073 & 1.1106189 & 0.0000000 \\ \mathrm{H} & -4.1871133 & -2.3306305 & 0.0000000 \\ \mathrm{H} & -5.7606199 & -0.4171254 & 0.0000000 \\ \mathrm{H} & -4.8780580 & 1.9125867 & 0.0000000 \\ \mathrm{H} & -2.4519286 & 2.3169502 & 0.0000000 \\ \mathrm{H} & -1.7670790 & -1.9615039 & 0.0000000 \\ \mathrm{H} & -0.2201047 & 1.5342436 & 0.0000000 \\ \mathrm{H} & 0.2201047 & -1.5342436 & 0.0000000 \\ \mathrm{H} & 2.4519286 & -2.3169502 & 0.0000000 \\ \mathrm{H} & 4.8780580 & -1.9125867 & 0.0000000 \\ \mathrm{H} & 5.7606199 & 0.4171254 & 0.0000000 \\ \mathrm{H} & 4.1871133 & 2.3306305 & 0.0000000 \\ \mathrm{H} & 1.7670790 & 1.9615039 & 0.0000000\end{array}$


Table S504 Cartesian coordinates of the TDDFT/TZVP-optimized excitedstate equilibrium geometry of the $1^{1} B\left({ }^{1} \pi \pi^{*}\right)$ state of stilbene (excited-state energy: $\left.-540.40210 E_{h}\right)$.

$\begin{array}{lrrr}26 & & & \\ \text { C } & 0.7463941 & -1.1035543 & 0.3585204 \\ \mathrm{C} & 0.0629860 & -1.5461621 & -0.8411283 \\ \mathrm{C} & -0.4551859 & -2.8741290 & -0.8428317 \\ \mathrm{C} & -0.3937204 & -3.6651591 & 0.2779078 \\ \mathrm{C} & 0.2195224 & -3.1894114 & 1.4637289 \\ \mathrm{C} & 0.7965139 & -1.9319666 & 1.4814782 \\ \mathrm{C} & -0.1128976 & -0.6972332 & -1.9523142 \\ \mathrm{C} & 0.1128976 & 0.6972332 & -1.9523142 \\ \mathrm{C} & -0.0629860 & 1.5461621 & -0.8411283 \\ \mathrm{C} & 0.4551859 & 2.8741290 & -0.8428317 \\ \mathrm{C} & 0.3937204 & 3.6651591 & 0.2779078 \\ \mathrm{C} & -0.2195224 & 3.1894114 & 1.4637289 \\ \mathrm{C} & -0.7965139 & 1.9319666 & 1.4814782 \\ \mathrm{C} & -0.7463941 & 1.1035543 & 0.3585204 \\ \mathrm{H} & -1.3197923 & 1.5895640 & 2.3669978 \\ \mathrm{H} & -0.2657953 & 3.8259556 & 2.3388287 \\ \mathrm{H} & 0.8200026 & 4.6612480 & 0.2594813 \\ \mathrm{H} & 0.9434870 & 3.2368760 & -1.7407945 \\ \mathrm{H} & -1.3652726 & 0.2204878 & 0.3164711 \\ \mathrm{H} & 0.4058492 & 1.1639055 & -2.8898982 \\ \mathrm{H} & -0.4058492 & -1.1639055 & -2.8898982 \\ \mathrm{H} & -0.9434870 & -3.2368760 & -1.7407945 \\ \mathrm{H} & -0.8200026 & -4.6612480 & 0.2594813 \\ \mathrm{H} & 0.2657953 & -3.8259556 & 2.3388287 \\ \mathrm{H} & 1.3197923 & -1.5895640 & 2.3669978 \\ \mathrm{H} & 1.3652726 & -0.2204878 & 0.3164711\end{array}$


Table S505 Cartesian coordinates of the TDDFT/TZVP-optimized excitedstate equilibrium geometry of the $2{ }^{1} A^{\prime}\left({ }^{1} \pi \pi^{*}\right)$ state of styrene (excited-state energy: $-309.38010 E_{h}$ ).

$\begin{array}{lrrr}16 & & & \\ & & & \\ \mathrm{C} & 0.5889598 & 1.9255485 & 0.0000000 \\ \mathrm{C} & -0.3863240 & 2.9278180 & 0.0000000 \\ \mathrm{C} & 0.2916016 & 0.5517089 & 0.0000000 \\ \mathrm{C} & -1.1069615 & 0.0906899 & 0.0000000 \\ \mathrm{C} & 1.3239767 & -0.4337067 & 0.0000000 \\ \mathrm{C} & -1.3665657 & -1.2692854 & 0.0000000 \\ \mathrm{C} & 1.0361592 & -1.7755487 & 0.0000000 \\ \mathrm{C} & -0.3310560 & -2.2033974 & 0.0000000 \\ \mathrm{H} & 1.6336635 & 2.2195207 & 0.0000000 \\ \mathrm{H} & -0.1033404 & 3.9722209 & 0.0000000 \\ \mathrm{H} & -1.4435252 & 2.7038946 & 0.0000000 \\ \mathrm{H} & -1.9127234 & 0.8086883 & 0.0000000 \\ \mathrm{H} & 2.3542243 & -0.0940122 & 0.0000000 \\ \mathrm{H} & -2.3926332 & -1.6189600 & 0.0000000 \\ \mathrm{H} & 1.8303646 & -2.5105340 & 0.0000000 \\ \mathrm{H} & -0.5593395 & -3.2623494 & 0.0000000\end{array}$


Table S506 Cartesian coordinates of the TDDFT/TZVP-optimized excitedstate equilibrium geometry of the $1{ }^{1} B_{3 u}\left(\equiv 1^{1} B_{1 u}\right.$ in Turbomole) $\left({ }^{1} n \pi^{*}\right)$ state of tetrazine (excited-state energy: $-296.18095 E_{h}$ ).

8

$\begin{array}{lrrr}\mathrm{C} & 0.0000000 & 1.3126305 & 0.0000000 \\ \mathrm{C} & 0.0000000 & -1.3126305 & 0.0000000 \\ \mathrm{~N} & 1.1554242 & 0.6577880 & 0.0000000 \\ \mathrm{~N} & 1.1554242 & -0.6577880 & 0.0000000 \\ \mathrm{~N} & -1.1554242 & 0.6577880 & 0.0000000 \\ \mathrm{~N} & -1.1554242 & -0.6577880 & 0.0000000 \\ \mathrm{H} & 0.0000000 & -2.3928871 & 0.0000000 \\ \mathrm{H} & 0.0000000 & 2.3928871 & 0.0000000\end{array}$


Table S507 Cartesian coordinates of the TDDFT/TZVP-optimized excitedstate equilibrium geometry of the $2{ }^{1} A\left({ }^{1} n \pi^{*}\right)$ state of thymine (excited-state energy: $\left.-453.90454 E_{h}\right)$.

$\begin{array}{lrrr}15 & & & \\ & & & \\ \mathrm{~N} & -1.1107051 & -1.2479604 & 0.0007375 \\ \mathrm{C} & 0.2548814 & -1.5705390 & 0.0007585 \\ \mathrm{C} & 1.2036390 & -0.5362732 & 0.0004945 \\ \mathrm{C} & 0.7298548 & 0.7496557 & 0.0006149 \\ \mathrm{~N} & -0.6374404 & 1.0158036 & 0.0022844 \\ \mathrm{C} & -1.6186532 & 0.0170358 & -0.0000811 \\ \mathrm{O} & 1.4531546 & 1.8655187 & -0.0003574 \\ \mathrm{C} & 2.6830171 & -0.8202432 & -0.0016996 \\ \mathrm{O} & -2.8018267 & 0.2955639 & -0.0020484 \\ \mathrm{H} & 0.5056436 & -2.6167777 & 0.0035687 \\ \mathrm{H} & -1.8042244 & -1.9794209 & -0.0010674 \\ \mathrm{H} & -0.9738318 & 1.9665510 & -0.0010478 \\ \mathrm{H} & 3.1800328 & -0.3890883 & 0.8709890 \\ \mathrm{H} & 3.1720578 & -0.4188347 & -0.8930334 \\ \mathrm{H} & 2.8595733 & -1.8957415 & 0.0157454\end{array}$


Table S508 Cartesian coordinates of the TDDFT/TZVP-optimized excitedstate equilibrium geometry of the $2{ }^{1} A^{\prime}\left({ }^{1} \pi \pi^{*}\right)$ state of uracil (excited-state energy: $\left.-414.57831 E_{h}\right)$.

$\begin{array}{lrrr}12 & & & \\ & & & \\ \mathrm{~N} & -1.2259972 & -0.9888252 & 0.0000000 \\ \mathrm{C} & -1.1675870 & 0.3363773 & 0.0000000 \\ \mathrm{~N} & -0.0058990 & 0.9504604 & 0.0000000 \\ \mathrm{C} & 1.3659362 & 0.2519485 & 0.0000000 \\ \mathrm{C} & 1.2016318 & -1.1372352 & 0.0000000 \\ \mathrm{C} & -0.0269490 & -1.7989326 & 0.0000000 \\ \mathrm{O} & -2.2702385 & 1.0435488 & 0.0000000 \\ \mathrm{O} & 2.3314300 & 1.0067213 & 0.0000000 \\ \mathrm{H} & -2.1415435 & -1.4077622 & 0.0000000 \\ \mathrm{H} & -0.0049326 & 1.9634777 & 0.0000000 \\ \mathrm{H} & 2.1075883 & -1.7272237 & 0.0000000 \\ \mathrm{H} & -0.1753238 & -2.8617899 & 0.0000000\end{array}$


4.1.6 Structural Parameters of the TDDFT/B3LYP-Optimized ExcitedState Minima 
Table S509 Structural parameters of the TDDFT/TZVP/TZVP-optimized excited-state equilibrium geometry of the $2{ }^{1} A\left({ }^{1} n \pi^{*}\right)$ state of $9 H$-adenine used for the statistical evaluations.

\begin{tabular}{ll}
$\begin{array}{l}\text { bond } \\
\text { or angle }\end{array}$ & $\begin{array}{l}\text { length } / \AA \\
\text { or angle } /\end{array}$ \\
\hline N1-C2 & 1.336 \\
C2-C3 & 1.414 \\
C2-C9 & 1.448 \\
C3-N4 & 1.364 \\
N4-C5 & 1.412 \\
N1-C5 & 1.324 \\
C3-N6 & 1.319 \\
N6-C7 & 1.383 \\
C7-N8 & 1.331 \\
N8-C9 & 1.313 \\
C9-N10 & 1.382 \\
C7-H11 & 1.073 \\
C5-H15 & 1.074 \\
N4-H14 & 1.007 \\
N10-H12 & 1.009 \\
N10-H13 & 1.010
\end{tabular}


Table S510 Structural parameters of the TDDFT/TZVP/TZVP-optimized excited-state equilibrium geometry of the $2{ }^{1} A\left({ }^{1} n \pi^{*}\right)$ state of acetaldehyde used for the statistical evaluations.

\begin{tabular}{ll} 
bond & $\begin{array}{l}\text { length / } \AA \\
\text { or angle } \\
\text { or angle / }\end{array}$ \\
\hline $\mathrm{C} 1-\mathrm{O} 4$ & 1.304 \\
$\mathrm{C} 1-\mathrm{C} 2$ & 1.508 \\
$\mathrm{C} 2-\mathrm{H} 7$ & 1.095 \\
$\mathrm{O} 4-\mathrm{C} 1-\mathrm{C} 2$ & 117.3 \\
$\mathrm{C} 2-\mathrm{C} 1-\mathrm{H} 3$ & 118.5
\end{tabular}


Table S511 Structural parameters of the TDDFT/TZVP/TZVP-optimized excited-state equilibrium geometry of the $1{ }^{3} A\left({ }^{3} n \pi^{*}\right)$ state of acetaldehyde used for the statistical evaluations.

\begin{tabular}{ll} 
bond & $\begin{array}{l}\text { length / } \AA \\
\text { or angle } \\
\text { or angle / }\end{array}$ \\
\hline $\mathrm{C} 1-\mathrm{O} 4$ & 1.305 \\
$\mathrm{C} 1-\mathrm{C} 2$ & 1.520 \\
$\mathrm{C} 2-\mathrm{H} 7$ & 1.101 \\
$\mathrm{O} 4-\mathrm{C} 1-\mathrm{C} 2$ & 116.1 \\
$\mathrm{C} 2-\mathrm{C} 1-\mathrm{H} 3$ & 115.5
\end{tabular}


Table S512 Structural parameters of the TDDFT/TZVP-optimized excitedstate equilibrium geometry of the $1^{1} A^{\prime \prime}\left({ }^{1} n \pi^{*}\right)$ state of acetone used for the statistical evaluations.

\begin{tabular}{ll} 
bond & $\begin{array}{l}\text { length } / \AA \\
\text { or angle } \\
\text { or angle / }\end{array}$ \\
\hline $\mathrm{C} 2-\mathrm{O} 3$ & 1.310 \\
$\mathrm{C} 1-\mathrm{C} 2$ & 1.507 \\
$\mathrm{C} 1-\mathrm{C} 2-\mathrm{C} 4$ & 120.0
\end{tabular}


Table S513 Structural parameters of the TDDFT/TZVP-optimized excitedstate equilibrium geometry of the $1^{3} A^{\prime \prime}\left({ }^{3} n \pi^{*}\right)$ state of acetone used for the statistical evaluations.

\begin{tabular}{ll} 
bond & $\begin{array}{l}\text { length } / \AA \\
\text { or angle } \\
\text { or angle / }\end{array}$ \\
\hline $\mathrm{C} 2-\mathrm{O} 3$ & 1.312 \\
$\mathrm{C} 1-\mathrm{C} 2$ & 1.516 \\
$\mathrm{C} 1-\mathrm{C} 2-\mathrm{C} 4$ & 117.8
\end{tabular}


Table S514 Structural parameters of the TDDFT/TZVP-optimized excitedstate equilibrium geometry of the $1^{1} A^{\prime \prime}\left({ }^{1} n \pi^{*}\right)$ state of acetophenone used for the statistical evaluations.

\begin{tabular}{ll}
$\begin{array}{l}\text { bond } \\
\text { or angle }\end{array}$ & $\begin{array}{l}\text { length } / \AA \\
\text { or angle / }\end{array}$ \\
\hline C7-C9 & 1.433 \\
C9-C11 & 1.377 \\
C11-C12 & 1.401 \\
C10-C12 & 1.402 \\
C $8-C 10$ & 1.378 \\
C7-C8 & 1.431 \\
C1-C7 & 1.398 \\
C1-C3 & 1.525 \\
C1-O2 & 1.297 \\
\end{tabular}


Table S515 Structural parameters of the TDDFT/TZVP-optimized excitedstate equilibrium geometry of the $2{ }^{1} A^{\prime}\left({ }^{1} \pi \pi^{*}\right)$ state of acetophenone used for the statistical evaluations.

\begin{tabular}{ll}
$\begin{array}{l}\text { bond } \\
\text { or angle }\end{array}$ & $\begin{array}{l}\text { length } / \AA \\
\text { or angle / }\end{array}$ \\
\hline C7-C9 & 1.423 \\
C9-C11 & 1.428 \\
C11-C12 & 1.399 \\
C10-C12 & 1.397 \\
C $8-C 10$ & 1.437 \\
C7-C8 & 1.408 \\
C1-C7 & 1.462 \\
C1-C3 & 1.512 \\
C1-O2 & 1.256
\end{tabular}


Table S516 Structural parameters of the TDDFT/TZVP-optimized excitedstate equilibrium geometry of the $1^{3} A^{\prime \prime}\left({ }^{3} n \pi^{*}\right)$ state of acetophenone used for the statistical evaluations.

\begin{tabular}{ll}
$\begin{array}{l}\text { bond } \\
\text { or angle }\end{array}$ & $\begin{array}{l}\text { length } / \AA \\
\text { or angle / }\end{array}$ \\
\hline C7-C9 & 1.427 \\
C9-C11 & 1.380 \\
C11-C12 & 1.399 \\
C10-C12 & 1.398 \\
C $8-C 10$ & 1.381 \\
C7-C8 & 1.425 \\
C1-C7 & 1.406 \\
C1-C3 & 1.514 \\
C1-O2 & 1.309
\end{tabular}


Table S517 Structural parameters of the TDDFT/TZVP-optimized excitedstate equilibrium geometry of the $1^{3} A^{\prime}\left({ }^{3} \pi \pi^{*}\right)$ state of acetophenone used for the statistical evaluations.

\begin{tabular}{ll}
$\begin{array}{l}\text { bond } \\
\text { or angle }\end{array}$ & $\begin{array}{l}\text { length } / \AA \\
\text { or angle / }\end{array}$ \\
\hline C7-C9 & 1.487 \\
C9-C11 & 1.360 \\
C11-C12 & 1.425 \\
C10-C12 & 1.449 \\
C $8-C 10$ & 1.355 \\
C7-C8 & 1.468 \\
C1-C7 & 1.413 \\
C1-C3 & 1.515 \\
C1-O2 & 1.280
\end{tabular}


Table S518 Structural parameters of the TDDFT/TZVP-optimized excitedstate equilibrium geometry of the $1{ }^{1} A_{2}\left({ }^{1} \pi \pi^{*}\right)$ state of acetylene used for the statistical evaluations.

\begin{tabular}{ll} 
bond & $\begin{array}{l}\text { length } / \AA \\
\text { or angle } \\
\text { or angle / }\end{array}$ \\
\hline $\mathrm{C} 1-\mathrm{C} 2$ & 1.339 \\
$\mathrm{C} 1-\mathrm{H} 3$ & 1.097 \\
$\mathrm{C} 1-\mathrm{C} 2-\mathrm{H} 4$ & 132.3
\end{tabular}


Table S519 Structural parameters of the TDDFT/TZVP-optimized excitedstate equilibrium geometry of the $2{ }^{1} A_{2}\left({ }^{1} \pi \pi^{*}\right)$ state of acetylene used for the statistical evaluations.

\begin{tabular}{ll} 
bond & $\begin{array}{l}\text { length } / \AA \\
\text { or angle } \\
\text { or angle / }\end{array}$ \\
\hline $\mathrm{C} 1-\mathrm{C} 2$ & 1.323 \\
$\mathrm{C} 1-\mathrm{H} 3$ & 1.064 \\
$\mathrm{C} 1-\mathrm{C} 2-\mathrm{H} 4$ & 174.3
\end{tabular}


Table S520 Structural parameters of the TDDFT/TZVP-optimized excitedstate equilibrium geometry of the $1{ }^{1} B_{2}\left({ }^{1} \pi \pi^{*}\right)$ state of acetylene used for the statistical evaluations.

\begin{tabular}{ll} 
bond & $\begin{array}{l}\text { length } / \AA \\
\text { or angle } \\
\text { or angle / }\end{array}$ \\
\hline $\mathrm{C} 1-\mathrm{C} 2$ & 1.328 \\
$\mathrm{C} 1-\mathrm{H} 3$ & 1.093 \\
$\mathrm{C} 1-\mathrm{C} 2-\mathrm{H} 4$ & 143.7
\end{tabular}


Table S521 Structural parameters of the TDDFT/TZVP-optimized excitedstate equilibrium geometry of the $2{ }^{3} A_{2}\left({ }^{3} \pi \pi^{*}\right)$ state of acetylene used for the statistical evaluations.

\begin{tabular}{ll} 
bond & $\begin{array}{l}\text { length } / \AA \\
\text { or angle } \\
\text { or angle / }\end{array}$ \\
\hline $\mathrm{C} 1-\mathrm{C} 2$ & 1.330 \\
$\mathrm{C} 1-\mathrm{H} 3$ & 1.069 \\
$\mathrm{C} 1-\mathrm{C} 2-\mathrm{H} 4$ & 166.0
\end{tabular}


Table S522 Structural parameters of the TDDFT/TZVP-optimized excitedstate equilibrium geometry of the $1{ }^{1} A_{u}\left({ }^{1} \pi \pi^{*}\right)$ state of acetylene used for the statistical evaluations.

\begin{tabular}{ll} 
bond & $\begin{array}{l}\text { length } / \AA \\
\text { or angle } \\
\text { or angle / }\end{array}$ \\
\hline $\mathrm{C} 1-\mathrm{C} 2$ & 1.366 \\
$\mathrm{C} 1-\mathrm{H} 3$ & 1.096 \\
$\mathrm{C} 1-\mathrm{C} 2-\mathrm{H} 4$ & 122.6
\end{tabular}


Table S523 Structural parameters of the TDDFT/TZVP-optimized excitedstate equilibrium geometry of the $2{ }^{1} A_{u}\left({ }^{1} \pi \pi^{*}\right)$ state of acetylene used for the statistical evaluations.

\begin{tabular}{ll} 
bond & $\begin{array}{l}\text { length } / \AA \\
\text { or angle } \\
\text { or angle / }\end{array}$ \\
\hline $\mathrm{C} 1-\mathrm{C} 2$ & 1.323 \\
$\mathrm{C} 1-\mathrm{H} 3$ & 1.063 \\
$\mathrm{C} 1-\mathrm{C} 2-\mathrm{H} 4$ & 175.2
\end{tabular}


Table S524 Structural parameters of the TDDFT/TZVP-optimized excitedstate equilibrium geometry of the $1{ }^{1} B_{u}\left({ }^{1} \pi \pi^{*}\right)$ state of acetylene used for the statistical evaluations.

\begin{tabular}{ll} 
bond & $\begin{array}{l}\text { length / } \AA \\
\text { or angle } \\
\text { or angle / }\end{array}$ \\
\hline $\mathrm{C} 1-\mathrm{C} 2$ & 1.330 \\
$\mathrm{C} 1-\mathrm{H} 3$ & 1.075 \\
$\mathrm{C} 1-\mathrm{C} 2-\mathrm{H} 4$ & 150.2
\end{tabular}


Table S525 Structural parameters of the TDDFT/TZVP-optimized excitedstate equilibrium geometry of the $1{ }^{3} A_{u}\left({ }^{3} \pi \pi^{*}\right)$ state of acetylene used for the statistical evaluations.

\begin{tabular}{ll} 
bond & $\begin{array}{l}\text { length } / \AA \\
\text { or angle } \\
\text { or angle / }\end{array}$ \\
\hline $\mathrm{C} 1-\mathrm{C} 2$ & 1.422 \\
$\mathrm{C} 1-\mathrm{H} 3$ & 1.096 \\
$\mathrm{C} 1-\mathrm{C} 2-\mathrm{H} 4$ & 116.9
\end{tabular}


Table S526 Structural parameters of the TDDFT/TZVP-optimized excitedstate equilibrium geometry of the $2{ }^{3} A_{u}\left({ }^{3} \pi \pi^{*}\right)$ state of acetylene used for the statistical evaluations.

\begin{tabular}{ll} 
bond & $\begin{array}{l}\text { length } / \AA \\
\text { or angle } \\
\text { or angle / }\end{array}$ \\
\hline $\mathrm{C} 1-\mathrm{C} 2$ & 1.332 \\
$\mathrm{C} 1-\mathrm{H} 3$ & 1.065 \\
$\mathrm{C} 1-\mathrm{C} 2-\mathrm{H} 4$ & 162.9
\end{tabular}


Table S527 Structural parameters of the TDDFT/TZVP-optimized excitedstate equilibrium geometry of the $1{ }^{3} B_{u}\left({ }^{3} \pi \pi^{*}\right)$ state of acetylene used for the statistical evaluations.

\begin{tabular}{ll} 
bond & $\begin{array}{l}\text { length } / \AA \\
\text { or angle } \\
\text { or angle / }\end{array}$ \\
\hline $\mathrm{C} 1-\mathrm{C} 2$ & 1.375 \\
$\mathrm{C} 1-\mathrm{H} 3$ & 1.089 \\
$\mathrm{C} 1-\mathrm{C} 2-\mathrm{H} 4$ & 129.4
\end{tabular}


Table S528 Structural parameters of the TDDFT/TZVP-optimized excitedstate equilibrium geometry of the $2{ }^{3} B_{u}\left({ }^{3} \pi \pi^{*}\right)$ state of acetylene used for the statistical evaluations.

\begin{tabular}{ll} 
bond & $\begin{array}{l}\text { length } / \AA \\
\text { or angle } \\
\text { or angle / }\end{array}$ \\
\hline $\mathrm{C} 1-\mathrm{C} 2$ & 1.359 \\
$\mathrm{C} 1-\mathrm{H} 3$ & 1.067 \\
$\mathrm{C} 1-\mathrm{C} 2-\mathrm{H} 4$ & 156.5
\end{tabular}


Table S529 Structural parameters of the TDDFT/TZVP-optimized excitedstate equilibrium geometry of the $2{ }^{1} A\left({ }^{1} n \pi^{*}\right)$ state of acrolein used for the statistical evaluations.

\begin{tabular}{|c|c|}
\hline $\begin{array}{l}\text { bond } \\
\text { or angle }\end{array}$ & $\begin{array}{l}\text { length / } \AA \\
\text { or angle / }\end{array}$ \\
\hline C3-04 & 1.286 \\
\hline $\mathrm{C} 2-\mathrm{C} 3$ & 1.386 \\
\hline $\mathrm{C} 1-\mathrm{C} 2$ & 1.382 \\
\hline $\mathrm{O} 4-\mathrm{C} 3-\mathrm{C} 2$ & 129.9 \\
\hline $\mathrm{C} 3-\mathrm{C} 2-\mathrm{C} 1$ & 124.1 \\
\hline
\end{tabular}


Table S530 Structural parameters of the TDDFT/TZVP-optimized excitedstate equilibrium geometry of the $1{ }^{3} A\left({ }^{3} n \pi^{*}\right)$ state of acrolein used for the statistical evaluations.

\begin{tabular}{ll} 
bond & $\begin{array}{l}\text { length / } \AA \\
\text { or angle } \\
\text { or angle / }\end{array}$ \\
\hline $\mathrm{C} 3-\mathrm{O} 4$ & 1.303 \\
$\mathrm{C} 2-\mathrm{C} 3$ & 1.392 \\
$\mathrm{C} 1-\mathrm{C} 2$ & 1.371 \\
$\mathrm{O} 4-\mathrm{C} 3-\mathrm{C} 2$ & 126.6 \\
$\mathrm{C} 3-\mathrm{C} 2-\mathrm{C} 1$ & 124.9
\end{tabular}


Table S531 Structural parameters of the TDDFT/TZVP-optimized excitedstate equilibrium geometry of the $2{ }^{1} A^{\prime}\left({ }^{1} \pi \pi^{*}\right)$ state of 2-amino-9-methylpurine used for the statistical evaluations.

$\begin{array}{ll}\text { bond } & \begin{array}{c}\text { length } / \AA \\ \text { or angle }\end{array} \\ \text { or angle / } /\end{array}$


Table S532 Structural parameters of the TDDFT/TZVP-optimized excitedstate equilibrium geometry of the $1^{1} A^{\prime \prime}\left({ }^{1} \pi \pi^{*}\right)$ state of aniline used for the statistical evaluations.

\begin{tabular}{ll}
$\begin{array}{l}\text { bond } \\
\text { or angle }\end{array}$ & $\begin{array}{c}\text { length } / \AA \\
\text { or angle } /\end{array}$ \\
\hline C1-C2 & 1.413 \\
C2-C3 & 1.428 \\
C3-C4 & 1.407 \\
C1-N7 & 1.373 \\
N7-H8 & 1.008 \\
C2-H10 & 1.083 \\
C3-H11 & 1.080 \\
C4-H12 & 1.085 \\
C1-C2-C3 & 117.8 \\
C2-C1-C6 & 123.7 \\
C2-C3-C4 & 118.9 \\
C3-C4-C5 & 123.1 \\
N7-C1-C6 & 118.1 \\
H8-N7-H9 & 118.1 \\
H8-N7-C1 & 120.8 \\
H14-C6-C1 & 120.3 \\
H14-C6-C5 & 122.0 \\
H13-C5-C4 & 120.4 \\
H13-C5-C6 & 120.7 \\
H12-C4-C3 & 118.5
\end{tabular}


Table S533 Structural parameters of the TDDFT/TZVP-optimized excitedstate equilibrium geometry of the $1^{1} A^{\prime \prime}\left({ }^{1} n \pi^{*}\right)$ state of benzaldehyde used for the statistical evaluations.

\begin{tabular}{ll} 
bond & $\begin{array}{l}\text { length / } \AA \\
\text { or angle } \\
\text { or angle / }\end{array}$ \\
\hline C4-C6 & 1.431 \\
C6-C8 & 1.379 \\
C $8-C 9$ & 1.399 \\
C7-C9 & 1.404 \\
C5-C7 & 1.376 \\
C4-C5 & 1.430 \\
C1-C4 & 1.394 \\
C1-O2 & 1.291
\end{tabular}


Table S534 Structural parameters of the TDDFT/TZVP-optimized excitedstate equilibrium geometry of the $2{ }^{1} A^{\prime}\left({ }^{1} \pi \pi^{*}\right)$ state of benzaldehyde used for the statistical evaluations.

\begin{tabular}{ll}
$\begin{array}{l}\text { bond } \\
\text { or angle }\end{array}$ & $\begin{array}{l}\text { length } / \AA \\
\text { or angle } /{ }^{\circ}\end{array}$ \\
\hline C4-C6 & 1.418 \\
C6-C8 & 1.431 \\
C $8-C 9$ & 1.396 \\
C7-C9 & 1.398 \\
C5-C7 & 1.435 \\
C4-C5 & 1.402 \\
C1-C4 & 1.459 \\
C1-O2 & 1.252
\end{tabular}


Table S535 Structural parameters of the TDDFT/TZVP-optimized excitedstate equilibrium geometry of the $1^{3} A^{\prime \prime}\left({ }^{3} n \pi^{*}\right)$ state of benzaldehyde used for the statistical evaluations.

\begin{tabular}{ll} 
bond & $\begin{array}{l}\text { length / } \AA \\
\text { or angle } \\
\text { or angle / }\end{array}$ \\
\hline C4-C6 & 1.424 \\
C6-C8 & 1.382 \\
C $8-C 9$ & 1.397 \\
C7-C9 & 1.399 \\
C5-C7 & 1.379 \\
C4-C5 & 1.424 \\
C1-C4 & 1.403 \\
C1-O2 & 1.303
\end{tabular}


Table S536 Structural parameters of the TDDFT/TZVP-optimized excitedstate equilibrium geometry of the $1^{3} A^{\prime}\left({ }^{3} \pi \pi^{*}\right)$ state of benzaldehyde used for the statistical evaluations.

\begin{tabular}{ll}
$\begin{array}{l}\text { bond } \\
\text { or angle }\end{array}$ & $\begin{array}{l}\text { length } / \AA \\
\text { or angle / }\end{array}$ \\
\hline C4-C6 & 1.481 \\
C6-C8 & 1.363 \\
C $8-C 9$ & 1.421 \\
C7-C9 & 1.447 \\
C5-C7 & 1.355 \\
C4-C5 & 1.463 \\
C1-C4 & 1.395 \\
C1-O2 & 1.290
\end{tabular}


Table S537 Structural parameters of the TDDFT/TZVP-optimized excitedstate equilibrium geometry of the $1^{1} A_{u}\left({ }^{1} n \pi^{*}\right)$ state of $p$-benzoquinone used for the statistical evaluations.

\begin{tabular}{ll}
$\begin{array}{l}\text { bond } \\
\text { or angle }\end{array}$ & $\begin{array}{l}\text { length } / \AA \\
\text { or angle / } /\end{array}$ \\
\hline $\mathrm{C} 1-\mathrm{O} 7$ & 1.265 \\
$\mathrm{C} 1-\mathrm{C} 2$ & 1.439 \\
$\mathrm{C} 2-\mathrm{C} 3$ & 1.368 \\
$\mathrm{C} 2-\mathrm{H} 9$ & 1.083 \\
$\mathrm{O} 7-\mathrm{C} 1-\mathrm{C} 2$ & 120.6 \\
$\mathrm{C} 6-\mathrm{C} 1-\mathrm{C} 2$ & 118.7 \\
$\mathrm{H} 9-\mathrm{C} 2-\mathrm{C} 3$ & 121.3
\end{tabular}


Table S538 Structural parameters of the TDDFT/TZVP-optimized excitedstate equilibrium geometry of the $1{ }^{1} B_{1 g}\left({ }^{1} n \pi^{*}\right)$ state of $p$-benzoquinone used for the statistical evaluations.

\begin{tabular}{ll}
$\begin{array}{l}\text { bond } \\
\text { or angle }\end{array}$ & $\begin{array}{l}\text { length } / \AA \\
\text { or angle / }\end{array}$ \\
\hline $\mathrm{C} 1-\mathrm{O} 7$ & 1.248 \\
$\mathrm{C} 1-\mathrm{C} 2$ & 1.458 \\
$\mathrm{C} 2-\mathrm{C} 3$ & 1.349 \\
$\mathrm{C} 2-\mathrm{H} 9$ & 1.082 \\
$\mathrm{O} 7-\mathrm{C} 1-\mathrm{C} 2$ & 122.2 \\
$\mathrm{C} 6-\mathrm{C} 1-\mathrm{C} 2$ & 115.5 \\
$\mathrm{H} 9-\mathrm{C} 2-\mathrm{C} 3$ & 121.7
\end{tabular}


Table S539 Structural parameters of the TDDFT/TZVP-optimized excitedstate equilibrium geometry of the $1{ }^{1} B_{1 u}\left({ }^{1} \pi \pi^{*}\right)$ state of $p$-benzoquinone used for the statistical evaluations.

\begin{tabular}{ll}
$\begin{array}{l}\text { bond } \\
\text { or angle }\end{array}$ & $\begin{array}{l}\text { length } / \AA \\
\text { or angle / }\end{array}$ \\
\hline $\mathrm{C} 1-\mathrm{O} 7$ & 1.248 \\
$\mathrm{C} 1-\mathrm{C} 2$ & 1.465 \\
$\mathrm{C} 2-\mathrm{C} 3$ & 1.395 \\
$\mathrm{C} 2-\mathrm{H} 9$ & 1.083 \\
$\mathrm{O} 7-\mathrm{C} 1-\mathrm{C} 2$ & 121.4 \\
$\mathrm{C} 6-\mathrm{C} 1-\mathrm{C} 2$ & 117.2 \\
$\mathrm{H} 9-\mathrm{C} 2-\mathrm{C} 3$ & 121.8
\end{tabular}


Table S540 Structural parameters of the TDDFT/TZVP-optimized excitedstate equilibrium geometry of the $1{ }^{1} B_{3 g}\left({ }^{1} \pi \pi^{*}\right)$ state of $p$-benzoquinone used for the statistical evaluations.

\begin{tabular}{ll}
$\begin{array}{l}\text { bond } \\
\text { or angle }\end{array}$ & $\begin{array}{l}\text { length } / \AA \\
\text { or angle / }\end{array}$ \\
\hline $\mathrm{C} 1-\mathrm{O} 7$ & 1.249 \\
$\mathrm{C} 1-\mathrm{C} 2$ & 1.445 \\
$\mathrm{C} 2-\mathrm{C} 3$ & 1.412 \\
$\mathrm{C} 2-\mathrm{H} 9$ & 1.085 \\
$\mathrm{O} 7-\mathrm{C} 1-\mathrm{C} 2$ & 123.4 \\
$\mathrm{C} 6-\mathrm{C} 1-\mathrm{C} 2$ & 113.3 \\
$\mathrm{H} 9-\mathrm{C} 2-\mathrm{C} 3$ & 119.6
\end{tabular}


Table S541 Structural parameters of the TDDFT/TZVP-optimized excitedstate equilibrium geometry of the $1^{3} A_{u}\left({ }^{3} n \pi^{*}\right)$ state of $p$-benzoquinone used for the statistical evaluations.

\begin{tabular}{ll}
$\begin{array}{l}\text { bond } \\
\text { or angle }\end{array}$ & $\begin{array}{l}\text { length } / \AA \\
\text { or angle / }\end{array}$ \\
\hline $\mathrm{C} 1-\mathrm{O} 7$ & 1.271 \\
$\mathrm{C} 1-\mathrm{C} 2$ & 1.435 \\
$\mathrm{C} 2-\mathrm{C} 3$ & 1.367 \\
$\mathrm{C} 2-\mathrm{H} 9$ & 1.084 \\
$\mathrm{O} 7-\mathrm{C} 1-\mathrm{C} 2$ & 120.5 \\
$\mathrm{C} 6-\mathrm{C} 1-\mathrm{C} 2$ & 119.0 \\
$\mathrm{H} 9-\mathrm{C} 2-\mathrm{C} 3$ & 121.3
\end{tabular}


Table S542 Structural parameters of the TDDFT/TZVP-optimized excitedstate equilibrium geometry of the $1{ }^{3} B_{1 g}\left({ }^{3} n \pi^{*}\right)$ state of $p$-benzoquinone used for the statistical evaluations.

\begin{tabular}{ll}
$\begin{array}{l}\text { bond } \\
\text { or angle }\end{array}$ & $\begin{array}{l}\text { length } / \AA \\
\text { or angle / }\end{array}$ \\
\hline $\mathrm{C} 1-\mathrm{O} 7$ & 1.255 \\
$\mathrm{C} 1-\mathrm{C} 2$ & 1.453 \\
$\mathrm{C} 2-\mathrm{C} 3$ & 1.350 \\
$\mathrm{C} 2-\mathrm{H} 9$ & 1.081 \\
$\mathrm{O} 7-\mathrm{C} 1-\mathrm{C} 2$ & 121.9 \\
$\mathrm{C} 6-\mathrm{C} 1-\mathrm{C} 2$ & 116.2 \\
$\mathrm{H} 9-\mathrm{C} 2-\mathrm{C} 3$ & 121.5
\end{tabular}


Table S543 Structural parameters of the TDDFT/TZVP-optimized excitedstate equilibrium geometry of the $1^{3} B_{3 g}\left({ }^{3} \pi \pi^{*}\right)$ state of $p$-benzoquinone used for the statistical evaluations.

\begin{tabular}{ll}
$\begin{array}{l}\text { bond } \\
\text { or angle }\end{array}$ & $\begin{array}{l}\text { length } / \AA \\
\text { or angle / } /\end{array}$ \\
\hline $\mathrm{C} 1-\mathrm{O} 7$ & 1.235 \\
$\mathrm{C} 1-\mathrm{C} 2$ & 1.452 \\
$\mathrm{C} 2-\mathrm{C} 3$ & 1.430 \\
$\mathrm{C} 2-\mathrm{H} 9$ & 1.084 \\
$\mathrm{O} 7-\mathrm{C} 1-\mathrm{C} 2$ & 123.3 \\
$\mathrm{C} 6-\mathrm{C} 1-\mathrm{C} 2$ & 113.3 \\
$\mathrm{H} 9-\mathrm{C} 2-\mathrm{C} 3$ & 119.6
\end{tabular}


Table S544 Structural parameters of the TDDFT/TZVP-optimized excitedstate equilibrium geometry of the $1{ }^{1} B_{3 g}\left({ }^{1} \pi \pi^{*}\right)$ state of biphenylene used for the statistical evaluations.

\begin{tabular}{ll}
$\begin{array}{l}\text { bond } \\
\text { or angle }\end{array}$ & $\begin{array}{c}\text { length } / \AA \\
\text { or angle / } /\end{array}$ \\
\hline C1-C2 & 1.399 \\
C2-C3 & 1.385 \\
C $3-C 12$ & 1.434 \\
C1-C6 & 1.497 \\
C1-C4 & 1.416 \\
C2-H13 & 1.083 \\
C2-H13 & 1.083 \\
C1-C2-C3 & 116.1 \\
C2-C3-C12 & 122.9 \\
C4-C1-C2 & 149.0 \\
C1-C6-C9 & 121.0
\end{tabular}


Table S545 Structural parameters of the TDDFT/TZVP-optimized excitedstate equilibrium geometry of the $1{ }^{1} B_{1 u}\left({ }^{1} \pi \pi^{*}\right)$ state of biphenylene used for the statistical evaluations.

\begin{tabular}{ll}
$\begin{array}{l}\text { bond } \\
\text { or angle }\end{array}$ & $\begin{array}{c}\text { length } / \AA \\
\text { or angle / } /\end{array}$ \\
\hline C1-C2 & 1.392 \\
C2-C3 & 1.423 \\
C3-C12 & 1.398 \\
C1-C6 & 1.444 \\
C1-C4 & 1.457 \\
C2-H13 & 1.081 \\
C2-H13 & 1.083 \\
C1-C2-C3 & 114.8 \\
C2-C3-C12 & 122.7 \\
C4-C1-C2 & 147.5 \\
C1-C6-C9 & 122.5
\end{tabular}


Table S546 Structural parameters of the TDDFT/TZVP-optimized excitedstate equilibrium geometry of the $2{ }^{1} B_{1 u}\left({ }^{1} \pi \pi^{*}\right)$ state of biphenylene used for the statistical evaluations.

\begin{tabular}{ll}
$\begin{array}{l}\text { bond } \\
\text { or angle }\end{array}$ & $\begin{array}{c}\text { length } / \AA \\
\text { or angle / } /\end{array}$ \\
\hline $\mathrm{C} 1-\mathrm{C} 2$ & 1.394 \\
$\mathrm{C} 2-\mathrm{C} 3$ & 1.423 \\
$\mathrm{C} 3-\mathrm{C} 12$ & 1.397 \\
$\mathrm{C} 1-\mathrm{C} 6$ & 1.437 \\
$\mathrm{C} 1-\mathrm{C} 4$ & 1.476 \\
$\mathrm{C} 2-\mathrm{H} 13$ & 1.083 \\
$\mathrm{C} 2-\mathrm{H} 13$ & 1.083 \\
$\mathrm{C} 1-\mathrm{C} 2-\mathrm{C} 3$ & 116.2 \\
$\mathrm{C} 2-\mathrm{C} 3-\mathrm{C} 12$ & 122.0 \\
$\mathrm{C} 4-\mathrm{C} 1-\mathrm{C} 2$ & 148.2 \\
$\mathrm{C} 1-\mathrm{C} 6-\mathrm{C} 9$ & 121.8 \\
\end{tabular}


Table S547 Structural parameters of the TDDFT/TZVP-optimized excitedstate equilibrium geometry of the $1{ }^{1} B_{2 u}\left({ }^{1} \pi \pi^{*}\right)$ state of biphenylene used for the statistical evaluations.

\begin{tabular}{ll}
$\begin{array}{l}\text { bond } \\
\text { or angle }\end{array}$ & $\begin{array}{c}\text { length } / \AA \\
\text { or angle / } /\end{array}$ \\
\hline C1-C2 & 1.374 \\
C2-C3 & 1.409 \\
C3-C12 & 1.454 \\
C1-C6 & 1.471 \\
C1-C4 & 1.482 \\
C2-H13 & 1.083 \\
C2-H13 & 1.082 \\
C1-C2-C3 & 116.3 \\
C2-C3-C12 & 121.6 \\
C4-C1-C2 & 147.9 \\
C1-C6-C9 & 122.1 \\
\end{tabular}


Table S548 Structural parameters of the TDDFT/TZVP-optimized excitedstate equilibrium geometry of the $1^{1} B_{u}\left({ }^{1} \pi \pi^{*}\right)$ state of trans-butadiene used for the statistical evaluations.

\begin{tabular}{ll}
$\begin{array}{l}\text { bond } \\
\text { or angle }\end{array}$ & $\begin{array}{l}\text { length } / \AA \\
\text { or angle } /\end{array}$ \\
\hline C2-C3 & 1.423 \\
C1-C2 & 1.402
\end{tabular}


Table S549 Structural parameters of the TDDFT/TZVP-optimized excitedstate equilibrium geometry of the $2{ }^{1} A_{g}\left({ }^{1} \pi \pi^{*}\right)$ state of trans-butadiene used for the statistical evaluations.

\begin{tabular}{ll}
$\begin{array}{l}\text { bond } \\
\text { or angle }\end{array}$ & $\begin{array}{l}\text { length } / \AA \\
\text { or angle } /\end{array}$ \\
\hline C2-C3 & 1.422 \\
C1-C2 & 1.475
\end{tabular}


Table S550 Structural parameters of the TDDFT/TZVP-optimized excitedstate equilibrium geometry of the $2{ }^{1} A\left({ }^{1} n \pi^{*}\right)$ state of cytosine used for the statistical evaluations.

\begin{tabular}{ll}
$\begin{array}{l}\text { bond } \\
\text { or angle }\end{array}$ & $\begin{array}{c}\text { length } / \AA \\
\text { or angle } /\end{array}$ \\
\hline N5-C6 & 1.348 \\
N1-C6 & 1.278 \\
N1-C2 & 1.409 \\
C2-C3 & 1.369 \\
C3-C4 & 1.402 \\
C4-N5 & 1.421 \\
C6-O8 & 1.336 \\
C2-N7 & 1.372 \\
C4-H10 & 1.076 \\
C3-H9 & 1.083 \\
N5-H13 & 1.007 \\
N7-H11 & 1.007 \\
N7-H12 & 1.008 \\
N5-C6-N1 & 126.7 \\
C6-N1-C2 & 115.9 \\
N1-C2-C3 & 122.7 \\
C2-C3-C4 & 120.0 \\
C3-C4-N5 & 115.8 \\
N1-C2-N7 & 112.5 \\
N5-C6-O8 & 114.1
\end{tabular}


Table S551 Structural parameters of the TDDFT/TZVP-optimized excitedstate equilibrium geometry of the $3{ }^{1} A\left({ }^{1} \pi \pi^{*}\right)$ state of cytosine used for the statistical evaluations.

\begin{tabular}{ll}
$\begin{array}{l}\text { bond } \\
\text { or angle }\end{array}$ & $\begin{array}{c}\text { length } / \AA \\
\text { or angle } /\end{array}$ \\
\hline N5-C6 & 1.361 \\
N1-C6 & 1.286 \\
N1-C2 & 1.425 \\
C2-C3 & 1.367 \\
C3-C4 & 1.412 \\
C4-N5 & 1.402 \\
C6-O8 & 1.331 \\
C2-N7 & 1.367 \\
C4-H10 & 1.074 \\
C3-H9 & 1.082 \\
N5-H13 & 1.007 \\
N7-H11 & 1.006 \\
N7-H12 & 1.008 \\
N5-C6-N1 & 125.1 \\
C6-N1-C2 & 116.3 \\
N1-C2-C3 & 121.6 \\
C2-C3-C4 & 119.7 \\
C3-C4-N5 & 115.9 \\
N1-C2-N7 & 111.7 \\
N5-C6-O8 & 113.4
\end{tabular}


Table S552 Structural parameters of the TDDFT/TZVP-optimized excitedstate equilibrium geometry of the $1^{1} A_{2}\left({ }^{1} n \pi^{*}\right)$ state of diazomethane used for the statistical evaluations.

\begin{tabular}{ll} 
bond & $\begin{array}{l}\text { length / } \AA \\
\text { or angle } \\
\text { or angle / }\end{array}$ \\
\hline N2-N3 & 1.195 \\
C1-N2 & 1.303 \\
C1-H4 & 1.093 \\
H4-C1-H5 & 118.6
\end{tabular}


Table S553 Structural parameters of the TDDFT/TZVP-optimized excitedstate equilibrium geometry of the $1{ }^{1} B_{3 g}\left({ }^{1} \pi \pi^{*}\right)$ state of dibenzo- $p$-dioxin used for the statistical evaluations.

\begin{tabular}{ll} 
bond & $\begin{array}{l}\text { length / } \AA \\
\text { or angle }\end{array}$ or angle / \\
\hline C4-O7 & 1.352 \\
C4-C5 & 1.449 \\
C5-C6 & 1.397 \\
C1-C6 & 1.377 \\
C1-C2 & 1.436 \\
C4-O7-C8 & 119.6 \\
C4-C5-C6 & 119.7 \\
C2-C3-C4 & 119.8 \\
C1-C2-C3 & 120.5
\end{tabular}


Table S554 Structural parameters of the TDDFT/TZVP-optimized excitedstate equilibrium geometry of the $1{ }^{1} B_{2 u}\left({ }^{1} \pi \pi^{*}\right)$ state of dibenzo- $p$-dioxin used for the statistical evaluations.

\begin{tabular}{ll} 
bond & $\begin{array}{l}\text { length / } \AA \\
\text { or angle }\end{array}$ or angle / \\
\hline C4-O7 & 1.364 \\
C4-C5 & 1.397 \\
C5-C6 & 1.412 \\
C1-C6 & 1.399 \\
C1-C2 & 1.401 \\
C4-O7-C8 & 119.1 \\
C4-C5-C6 & 121.2 \\
C2-C3-C4 & 117.3 \\
C1-C2-C3 & 121.5
\end{tabular}


Table S555 Structural parameters of the TDDFT/TZVP-optimized excitedstate equilibrium geometry of the $1{ }^{1} B_{1 u}\left({ }^{1} \pi \pi^{*}\right)$ state of dibenzo- $p$-dioxin used for the statistical evaluations.

\begin{tabular}{ll} 
bond & $\begin{array}{l}\text { length / } \AA \\
\text { or angle }\end{array}$ or angle / \\
\hline C4-O7 & 1.373 \\
C4-C5 & 1.437 \\
C5-C6 & 1.385 \\
C1-C6 & 1.389 \\
C1-C2 & 1.447 \\
C4-O7-C8 & 118.4 \\
C4-C5-C6 & 120.1 \\
C2-C3-C4 & 120.2 \\
C1-C2-C3 & 119.7
\end{tabular}


Table S556 Structural parameters of the TDDFT/TZVP-optimized excitedstate equilibrium geometry of the $1^{3} A_{g}\left({ }^{3} \pi \pi^{*}\right)$ state of dibenzo- $p$-dioxin used for the statistical evaluations.

\begin{tabular}{ll} 
bond & $\begin{array}{l}\text { length / } \AA \\
\text { or angle }\end{array}$ or angle / \\
\hline C4-O7 & 1.364 \\
C4-C5 & 1.401 \\
C5-C6 & 1.409 \\
C1-C6 & 1.400 \\
C1-C2 & 1.397 \\
C4-O7-C8 & 117.4 \\
C4-C5-C6 & 121.2 \\
C2-C3-C4 & 117.3 \\
C1-C2-C3 & 121.5
\end{tabular}


Table S557 Structural parameters of the TDDFT/TZVP-optimized excitedstate equilibrium geometry of the $1^{3} B_{3 g}\left({ }^{3} \pi \pi^{*}\right)$ state of dibenzo- $p$-dioxin used for the statistical evaluations.

\begin{tabular}{ll}
$\begin{array}{l}\text { bond } \\
\text { or angle }\end{array}$ & $\begin{array}{l}\text { length } / \AA \\
\text { or angle / }\end{array}$ \\
\hline C4-07 & 1.356 \\
C4-C5 & 1.449 \\
C5-C6 & 1.396 \\
C1-C6 & 1.379 \\
C1-C2 & 1.443 \\
C4-O7-C8 & 119.5 \\
C4-C5-C6 & 119.8 \\
C2-C3-C4 & 119.8 \\
C1-C2-C3 & 120.4
\end{tabular}


Table S558 Structural parameters of the TDDFT/TZVP-optimized excitedstate equilibrium geometry of the $1^{3} B_{2 u}\left({ }^{3} \pi \pi^{*}\right)$ state of dibenzo- $p$-dioxin used for the statistical evaluations.

\begin{tabular}{ll} 
bond & $\begin{array}{l}\text { length / } \AA \\
\text { or angle }\end{array}$ or angle / \\
\hline C4-O7 & 1.361 \\
C4-C5 & 1.402 \\
C5-C6 & 1.410 \\
C1-C6 & 1.403 \\
C1-C2 & 1.395 \\
C4-O7-C8 & 120.0 \\
C4-C5-C6 & 121.3 \\
C2-C3-C4 & 117.0 \\
C1-C2-C3 & 121.7
\end{tabular}


Table S559 Structural parameters of the TDDFT/TZVP-optimized excitedstate equilibrium geometry of the $1^{3} B_{1 u}\left({ }^{3} \pi \pi^{*}\right)$ state of dibenzo- $p$-dioxin used for the statistical evaluations.

\begin{tabular}{ll} 
bond & $\begin{array}{l}\text { length / } \AA \\
\text { or angle }\end{array}$ or angle / \\
\hline C4-O7 & 1.379 \\
C4-C5 & 1.431 \\
C5-C6 & 1.392 \\
C1-C6 & 1.399 \\
C1-C2 & 1.432 \\
C4-O7-C8 & 116.9 \\
C4-C5-C6 & 120.1 \\
C2-C3-C4 & 120.0 \\
C1-C2-C3 & 119.9
\end{tabular}


Table S560 Structural parameters of the TDDFT/TZVP-optimized excitedstate equilibrium geometry of the $1{ }^{1} A_{u}\left({ }^{1} n \pi^{*}\right)$ state of dibenzo- $p$-dioxin used for the statistical evaluations.

\begin{tabular}{ll} 
bond & $\begin{array}{l}\text { length / } \AA \\
\text { or angle }\end{array}$ or angle / \\
\hline C4-O7 & 1.335 \\
C4-C5 & 1.468 \\
C5-C6 & 1.386 \\
C1-C6 & 1.382 \\
C1-C2 & 1.418 \\
C4-O7-C8 & 125.3 \\
C4-C5-C6 & 118.3 \\
C2-C3-C4 & 122.2 \\
C1-C2-C3 & 119.5
\end{tabular}


Table S561 Structural parameters of the TDDFT/TZVP-optimized excitedstate equilibrium geometry of the $1{ }^{1} B_{1 g}\left({ }^{1} n \pi^{*}\right)$ state of dibenzo- $p$-dioxin used for the statistical evaluations.

\begin{tabular}{ll} 
bond & $\begin{array}{l}\text { length / } \AA \\
\text { or angle }\end{array}$ or angle / \\
\hline C4-O7 & 1.366 \\
C4-C5 & 1.409 \\
C5-C6 & 1.403 \\
C1-C6 & 1.408 \\
C1-C2 & 1.378 \\
C4-O7-C8 & 125.0 \\
C4-C5-C6 & 120.1 \\
C2-C3-C4 & 119.2 \\
C1-C2-C3 & 120.7
\end{tabular}


Table S562 Structural parameters of the TDDFT/TZVP-optimized excitedstate equilibrium geometry of the $1{ }^{1} B_{3 u}\left({ }^{1} n \pi^{*}\right)$ state of dibenzo- $p$-dioxin used for the statistical evaluations.

\begin{tabular}{|c|c|}
\hline $\begin{array}{l}\text { bond } \\
\text { or angle }\end{array}$ & $\begin{array}{l}\text { length / } \AA \\
\text { or angle / }\end{array}$ \\
\hline C4-07 & 1.373 \\
\hline $\mathrm{C} 4-\mathrm{C} 5$ & 1.403 \\
\hline $\mathrm{C} 5-\mathrm{C} 6$ & 1.404 \\
\hline $\mathrm{C} 1-\mathrm{C} 6$ & 1.402 \\
\hline $\mathrm{C} 1-\mathrm{C} 2$ & 1.384 \\
\hline $\mathrm{C} 4-07-\mathrm{C} 8$ & 121.6 \\
\hline $\mathrm{C} 4-\mathrm{C} 5-\mathrm{C} 6$ & 120.0 \\
\hline $\mathrm{C} 2-\mathrm{C} 3-\mathrm{C} 4$ & 119.4 \\
\hline $\mathrm{C} 1-\mathrm{C} 2-\mathrm{C} 3$ & 120.5 \\
\hline
\end{tabular}


Table S563 Structural parameters of the TDDFT/TZVP-optimized excitedstate equilibrium geometry of the $1{ }^{1} B_{2 g}\left({ }^{1} n \pi^{*}\right)$ state of dibenzo- $p$-dioxin used for the statistical evaluations.

\begin{tabular}{ll}
$\begin{array}{l}\text { bond } \\
\text { or angle }\end{array}$ & $\begin{array}{l}\text { length } / \AA \\
\text { or angle / }\end{array}$ \\
\hline C4-07 & 1.380 \\
C4-C5 & 1.419 \\
C5-C6 & 1.389 \\
C1-C6 & 1.401 \\
C1-C2 & 1.401 \\
C4-O7-C8 & 119.3 \\
C4-C5-C6 & 119.9 \\
C2-C3-C4 & 120.0 \\
C1-C2-C3 & 120.1
\end{tabular}


Table S564 Structural parameters of the TDDFT/TZVP-optimized excitedstate equilibrium geometry of the $1^{3} A_{u}\left({ }^{3} n \pi^{*}\right)$ state of dibenzo- $p$-dioxin used for the statistical evaluations.

\begin{tabular}{ll} 
bond & $\begin{array}{l}\text { length / } \AA \\
\text { or angle }\end{array}$ or angle / \\
\hline C4-O7 & 1.355 \\
C4-C5 & 1.468 \\
C5-C6 & 1.387 \\
C1-C6 & 1.381 \\
C1-C2 & 1.418 \\
C4-O7-C8 & 125.1 \\
C4-C5-C6 & 118.2 \\
C2-C3-C4 & 122.3 \\
C1-C2-C3 & 119.5
\end{tabular}


Table S565 Structural parameters of the TDDFT/TZVP-optimized excitedstate equilibrium geometry of the $1^{3} B_{1 g}\left({ }^{3} n \pi^{*}\right)$ state of dibenzo- $p$-dioxin used for the statistical evaluations.

\begin{tabular}{ll} 
bond & $\begin{array}{l}\text { length / } \AA \\
\text { or angle }\end{array}$ or angle / \\
\hline C4-O7 & 1.397 \\
C4-C5 & 1.407 \\
C5-C6 & 1.394 \\
C1-C6 & 1.396 \\
C1-C2 & 1.398 \\
C4-O7-C8 & 120.0 \\
C4-C5-C6 & 120.1 \\
C2-C3-C4 & 119.7 \\
C1-C2-C3 & 120.2
\end{tabular}


Table S566 Structural parameters of the TDDFT/TZVP-optimized excitedstate equilibrium geometry of the $1^{3} B_{3 u}\left({ }^{3} n \pi^{*}\right)$ state of dibenzo- $p$-dioxin used for the statistical evaluations.

\begin{tabular}{ll}
$\begin{array}{l}\text { bond } \\
\text { or angle }\end{array}$ & $\begin{array}{l}\text { length } / \AA \\
\text { or angle / }\end{array}$ \\
\hline C4-07 & 1.372 \\
C4-C5 & 1.404 \\
C5-C6 & 1.403 \\
C1-C6 & 1.402 \\
C1-C2 & 1.383 \\
C4-O7-C8 & 121.5 \\
C4-C5-C6 & 119.9 \\
C2-C3-C4 & 119.6 \\
C1-C2-C3 & 120.4
\end{tabular}


Table S567 Structural parameters of the TDDFT/TZVP-optimized excitedstate equilibrium geometry of the $1^{3} B_{2 g}\left({ }^{3} n \pi^{*}\right)$ state of dibenzo- $p$-dioxin used for the statistical evaluations.

\begin{tabular}{ll} 
bond & $\begin{array}{l}\text { length / } \AA \\
\text { or angle }\end{array}$ or angle / \\
\hline C4-O7 & 1.377 \\
C4-C5 & 1.448 \\
C5-C6 & 1.377 \\
C1-C6 & 1.387 \\
C1-C2 & 1.435 \\
C4-O7-C8 & 121.6 \\
C4-C5-C6 & 118.6 \\
C2-C3-C4 & 122.7 \\
C1-C2-C3 & 118.7
\end{tabular}


Table S568 Structural parameters of the TDDFT/TZVP-optimized excitedstate equilibrium geometry of the $1^{1} A^{\prime \prime}\left({ }^{1} n \pi^{*}\right)$ state of formaldehyde used for the statistical evaluations.

\begin{tabular}{ll}
$\begin{array}{l}\text { bond } \\
\text { or angle }\end{array}$ & $\begin{array}{l}\text { length } / \AA \\
\text { or angle / }\end{array}$ \\
\hline $\mathrm{C} 1-\mathrm{O} 2$ & 1.296 \\
$\mathrm{C} 1-\mathrm{H} 3$ & 1.095 \\
$\mathrm{H} 3-\mathrm{C} 1-\mathrm{H} 4$ & 117.2
\end{tabular}


Table S569 Structural parameters of the TDDFT/TZVP-optimized excitedstate equilibrium geometry of the $1^{3} A^{\prime \prime}\left({ }^{3} n \pi^{*}\right)$ state of formaldehyde used for the statistical evaluations.

\begin{tabular}{ll}
$\begin{array}{l}\text { bond } \\
\text { or angle }\end{array}$ & $\begin{array}{l}\text { length } / \AA \\
\text { or angle / }\end{array}$ \\
\hline $\mathrm{C} 1-\mathrm{O} 2$ & 1.298 \\
$\mathrm{C} 1-\mathrm{H} 3$ & 1.102 \\
$\mathrm{H} 3-\mathrm{C} 1-\mathrm{H} 4$ & 112.1
\end{tabular}


Table S570 Structural parameters of the TDDFT/TZVP-optimized excitedstate equilibrium geometry of the $1{ }^{1} A_{u}\left({ }^{1} n \pi^{*}\right)$ state of trans-glyoxal used for the statistical evaluations.

\begin{tabular}{ll}
$\begin{array}{l}\text { bond } \\
\text { or angle }\end{array}$ & $\begin{array}{l}\text { length } / \AA \\
\text { or angle } /\end{array}$ \\
\hline $\mathrm{C} 1-\mathrm{H} 5$ & 1.100 \\
$\mathrm{C} 1-\mathrm{C} 2$ & 1.480 \\
$\mathrm{C} 1-\mathrm{O} 3$ & 1.230 \\
$\mathrm{C} 1-\mathrm{C} 2-\mathrm{H} 6$ & 113.7 \\
$\mathrm{C} 1-\mathrm{C} 2-\mathrm{O} 4$ & 124.9
\end{tabular}


Table S571 Structural parameters of the TDDFT/TZVP-optimized excitedstate equilibrium geometry of the $1{ }^{3} A_{u}\left({ }^{3} n \pi^{*}\right)$ state of trans-glyoxal used for the statistical evaluations.

\begin{tabular}{ll}
$\begin{array}{l}\text { bond } \\
\text { or angle }\end{array}$ & $\begin{array}{l}\text { length } / \AA \\
\text { or angle } /\end{array}$ \\
\hline $\mathrm{C} 1-\mathrm{H} 5$ & 1.095 \\
$\mathrm{C} 1-\mathrm{C} 2$ & 1.471 \\
$\mathrm{C} 1-\mathrm{O} 3$ & 1.235 \\
$\mathrm{C} 1-\mathrm{C} 2-\mathrm{H} 6$ & 114.8 \\
$\mathrm{C} 1-\mathrm{C} 2-\mathrm{O} 4$ & 123.8
\end{tabular}


Table S572 Structural parameters of the TDDFT/TZVP-optimized excitedstate equilibrium geometry of the $2{ }^{1} A\left({ }^{1} \pi \pi^{*}\right)$ state of $9 H$-guanine used for the statistical evaluations.

\begin{tabular}{ll}
$\begin{array}{l}\text { bond } \\
\text { or angle }\end{array}$ & $\begin{array}{c}\text { length } / \AA \\
\text { or angle } /\end{array}$ \\
\hline N7-C9 & 1.401 \\
C9-N13 & 1.475 \\
N13-C14 & 1.279 \\
C4-C14 & 1.478 \\
C4-C5 & 1.482 \\
C5-N7 & 1.414 \\
N3-C4 & 1.359 \\
C2-N3 & 1.312 \\
N1-C2 & 1.384 \\
N1-C14 & 1.388 \\
C9-N10 & 1.364 \\
C5-O6 & 1.210 \\
C2-H15 & 1.080 \\
N10-H11 & 1.012 \\
N10-H12 & 1.009 \\
N7-H8 & 1.022 \\
N1-H16 & 1.007
\end{tabular}


Table S573 Structural parameters of the TDDFT/TZVP-optimized excitedstate equilibrium geometry of the $2{ }^{1} A^{\prime}\left({ }^{1} \pi \pi^{*}\right)$ state of 1-hydroxy-2acetonaphthone used for the statistical evaluations.

\begin{tabular}{ll}
$\begin{array}{l}\text { bond } \\
\text { or angle }\end{array}$ & $\begin{array}{c}\text { length } / \AA \\
\text { or angle } /\end{array}$ \\
\hline C1-C6 & 1.449 \\
C5-C6 & 1.398 \\
C4-C5 & 1.412 \\
C3-C4 & 1.395 \\
C3-C10 & 1.424 \\
C9-C10 & 1.375 \\
C8-C9 & 1.413 \\
C7-C8 & 1.388 \\
C2-C7 & 1.401 \\
C2-C3 & 1.447 \\
C1-C2 & 1.449 \\
C6-C11 & 1.433 \\
C11-C12 & 1.490 \\
C1-O13 & 1.278 \\
C11-O14 & 1.324 \\
O13-H21 & 1.506 \\
O14-H21 & 1.041 \\
O13-O14 & 2.486
\end{tabular}


Table S574 Structural parameters of the TDDFT/TZVP-optimized excitedstate equilibrium geometry of the $2{ }^{1} A^{\prime}\left({ }^{1} \pi \pi^{*}\right)$ state of indole used for the statistical evaluations.

\begin{tabular}{|c|c|}
\hline $\begin{array}{l}\text { bond } \\
\text { or angle }\end{array}$ & $\begin{array}{l}\text { length / } \AA \\
\text { or angle / }\end{array}$ \\
\hline N3-C5 & 1.340 \\
\hline $\mathrm{C} 4-\mathrm{C} 5$ & 1.428 \\
\hline $\mathrm{C} 1-\mathrm{C} 4$ & 1.431 \\
\hline $\mathrm{C} 1-\mathrm{C} 6$ & 1.424 \\
\hline $\mathrm{C} 6-\mathrm{C} 7$ & 1.380 \\
\hline $\mathrm{C} 7-\mathrm{C} 9$ & 1.380 \\
\hline $\mathrm{C} 8-\mathrm{C} 9$ & 1.443 \\
\hline $\mathrm{C} 2-\mathrm{C} 8$ & 1.405 \\
\hline $\mathrm{N} 3-\mathrm{H} 10$ & 1.009 \\
\hline $\mathrm{C} 5-\mathrm{H} 12$ & 1.076 \\
\hline $\mathrm{C} 4-\mathrm{H} 11$ & 1.079 \\
\hline $\mathrm{C} 6-\mathrm{H} 13$ & 1.080 \\
\hline $\mathrm{C} 7-\mathrm{H} 14$ & 1.084 \\
\hline $\mathrm{C} 9-\mathrm{H} 16$ & 1.084 \\
\hline $\mathrm{C} 8-\mathrm{H} 15$ & 1.080 \\
\hline $\mathrm{C} 1-\mathrm{C} 2$ & 1.394 \\
\hline $\mathrm{C} 2-\mathrm{N} 3$ & 1.419 \\
\hline $\mathrm{N} 3-\mathrm{C} 5-\mathrm{C} 4$ & 106.4 \\
\hline $\mathrm{C} 5-\mathrm{C} 4-\mathrm{C} 1$ & 108.4 \\
\hline $\mathrm{C} 4-\mathrm{C} 1-\mathrm{C} 6$ & 132.6 \\
\hline $\mathrm{C} 1-\mathrm{C} 6-\mathrm{C} 7$ & 119.2 \\
\hline $\mathrm{C} 6-\mathrm{C} 7-\mathrm{C} 9$ & 125.8 \\
\hline $\mathrm{C} 7-\mathrm{C} 9-\mathrm{C} 8$ & 122.6 \\
\hline $\mathrm{C} 9-\mathrm{C} 8-\mathrm{C} 2$ & 114.1 \\
\hline $\mathrm{C} 2-\mathrm{N} 3-\mathrm{H} 10$ & 123.8 \\
\hline $\mathrm{N} 3-\mathrm{C} 5-\mathrm{H} 12$ & 122.9 \\
\hline $\mathrm{C} 5-\mathrm{C} 4-\mathrm{H} 11$ & 124.5 \\
\hline $\mathrm{C} 1-\mathrm{C} 6-\mathrm{H} 13$ & 121.8 \\
\hline $\mathrm{C} 6-\mathrm{C} 7-\mathrm{H} 14$ & 123.0 \\
\hline $\mathrm{C} 7-\mathrm{C} 9-\mathrm{H} 16$ & 119.1 \\
\hline $\mathrm{C} 9-\mathrm{C} 8-\mathrm{H} 15$ & 122.3 \\
\hline
\end{tabular}


Table S575 Structural parameters of the TDDFT/TZVP-optimized excitedstate equilibrium geometry of the $3^{1} A^{\prime}\left({ }^{1} \pi \pi^{*}\right)$ state of indole used for the statistical evaluations.

\begin{tabular}{|c|c|}
\hline $\begin{array}{l}\text { bond } \\
\text { or angle }\end{array}$ & $\begin{array}{l}\text { length / } \AA \\
\text { or angle / }\end{array}$ \\
\hline N3-C5 & 1.392 \\
\hline $\mathrm{C} 4-\mathrm{C} 5$ & 1.390 \\
\hline $\mathrm{C} 1-\mathrm{C} 4$ & 1.414 \\
\hline $\mathrm{C} 1-\mathrm{C} 6$ & 1.414 \\
\hline $\mathrm{C} 6-\mathrm{C} 7$ & 1.430 \\
\hline $\mathrm{C} 7-\mathrm{C} 9$ & 1.430 \\
\hline $\mathrm{C} 8-\mathrm{C} 9$ & 1.407 \\
\hline $\mathrm{C} 2-\mathrm{C} 8$ & 1.414 \\
\hline $\mathrm{N} 3-\mathrm{H} 10$ & 1.006 \\
\hline $\mathrm{C} 5-\mathrm{H} 12$ & 1.077 \\
\hline $\mathrm{C} 4-\mathrm{H} 11$ & 1.078 \\
\hline $\mathrm{C} 6-\mathrm{H} 13$ & 1.081 \\
\hline $\mathrm{C} 7-\mathrm{H} 14$ & 1.082 \\
\hline $\mathrm{C} 9-\mathrm{H} 16$ & 1.082 \\
\hline $\mathrm{C} 8-\mathrm{H} 15$ & 1.081 \\
\hline $\mathrm{C} 1-\mathrm{C} 2$ & 1.460 \\
\hline $\mathrm{C} 2-\mathrm{N} 3$ & 1.376 \\
\hline $\mathrm{N} 3-\mathrm{C} 5-\mathrm{C} 4$ & 108.5 \\
\hline $\mathrm{C} 5-\mathrm{C} 4-\mathrm{C} 1$ & 108.3 \\
\hline $\mathrm{C} 4-\mathrm{C} 1-\mathrm{C} 6$ & 134.2 \\
\hline $\mathrm{C} 1-\mathrm{C} 6-\mathrm{C} 7$ & 116.8 \\
\hline $\mathrm{C} 6-\mathrm{C} 7-\mathrm{C} 9$ & 121.3 \\
\hline $\mathrm{C} 7-\mathrm{C} 9-\mathrm{C} 8$ & 121.9 \\
\hline $\mathrm{C} 9-\mathrm{C} 8-\mathrm{C} 2$ & 116.0 \\
\hline $\mathrm{C} 2-\mathrm{N} 3-\mathrm{H} 10$ & 125.1 \\
\hline $\mathrm{N} 3-\mathrm{C} 5-\mathrm{H} 12$ & 121.0 \\
\hline $\mathrm{C} 5-\mathrm{C} 4-\mathrm{H} 11$ & 125.0 \\
\hline $\mathrm{C} 1-\mathrm{C} 6-\mathrm{H} 13$ & 121.3 \\
\hline $\mathrm{C} 6-\mathrm{C} 7-\mathrm{H} 14$ & 118.6 \\
\hline $\mathrm{C} 7-\mathrm{C} 9-\mathrm{H} 16$ & 118.7 \\
\hline $\mathrm{C} 9-\mathrm{C} 8-\mathrm{H} 15$ & 121.9 \\
\hline
\end{tabular}


Table S576 Structural parameters of the TDDFT/TZVP-optimized excitedstate equilibrium geometry of the $1{ }^{1} B_{1}\left({ }^{1} n \pi^{*}\right)$ state of maleimide used for the statistical evaluations.

\begin{tabular}{ll}
$\begin{array}{l}\text { bond } \\
\text { or angle }\end{array}$ & $\begin{array}{l}\text { length } / \AA \\
\text { or angle } /\end{array}$ \\
\hline N1-C2 & 1.407 \\
C2-C3 & 1.432 \\
C3-C4 & 1.384 \\
C2-O6 & 1.241 \\
C3-H9 & 1.076 \\
C2-N1-C5 & 109.6 \\
N1-C2-O6 & 119.2 \\
O6-C2-C4 & 134.2 \\
C2-C3-C4 & 108.6 \\
C2-C3-H9 & 122.8 \\
N1-H8 & 1.009
\end{tabular}


Table S577 Structural parameters of the TDDFT/TZVP-optimized excitedstate equilibrium geometry of the $1^{1} A_{2}\left({ }^{1} n \pi^{*}\right)$ state of maleimide used for the statistical evaluations.

\begin{tabular}{ll}
$\begin{array}{l}\text { bond } \\
\text { or angle }\end{array}$ & $\begin{array}{l}\text { length } / \AA \\
\text { or angle } /\end{array}$ \\
\hline N1-C2 & 1.383 \\
C2-C3 & 1.427 \\
C3-C4 & 1.399 \\
C2-O6 & 1.264 \\
C3-H9 & 1.077 \\
C2-N1-C5 & 109.9 \\
N1-C2-O6 & 124.9 \\
O6-C2-C4 & 127.6 \\
C2-C3-C4 & 107.6 \\
C2-C3-H9 & 124.4 \\
N1-H8 & 1.007
\end{tabular}


Table S578 Structural parameters of the TDDFT/TZVP-optimized excitedstate equilibrium geometry of the $1{ }^{1} B_{2}\left({ }^{1} \pi \pi^{*}\right)$ state of maleimide used for the statistical evaluations.

\begin{tabular}{ll}
$\begin{array}{l}\text { bond } \\
\text { or angle }\end{array}$ & $\begin{array}{l}\text { length } / \AA \\
\text { or angle } /\end{array}$ \\
\hline N1-C2 & 1.519 \\
C2-C3 & 1.407 \\
C3-C4 & 1.422 \\
C2-O6 & 1.210 \\
C3-H9 & 1.079 \\
C2-N1-C5 & 111.4 \\
N1-C2-O6 & 120.5 \\
O6-C2-C4 & 138.0 \\
C2-C3-C4 & 112.8 \\
C2-C3-H9 & 121.9 \\
N1-H8 & 1.029
\end{tabular}


Table S579 Structural parameters of the TDDFT/TZVP-optimized excitedstate equilibrium geometry of the $2{ }^{1} B_{2}\left({ }^{1} \pi \pi^{*}\right)$ state of maleimide used for the statistical evaluations.

\begin{tabular}{ll}
$\begin{array}{l}\text { bond } \\
\text { or angle }\end{array}$ & $\begin{array}{l}\text { length } / \AA \\
\text { or angle } /\end{array}$ \\
\hline $\mathrm{N} 1-\mathrm{C} 2$ & 1.400 \\
$\mathrm{C} 2-\mathrm{C} 3$ & 1.455 \\
$\mathrm{C} 3-\mathrm{C} 4$ & 1.434 \\
$\mathrm{C} 2-\mathrm{O} 6$ & 1.246 \\
$\mathrm{C} 3-\mathrm{H} 9$ & 1.077 \\
$\mathrm{C} 2-\mathrm{N} 1-\mathrm{C} 5$ & 111.5 \\
$\mathrm{~N} 1-\mathrm{C} 2-\mathrm{O} 6$ & 123.5 \\
O6-C2-C4 & 129.8 \\
$\mathrm{C} 2-\mathrm{C} 3-\mathrm{C} 4$ & 107.6 \\
$\mathrm{C} 2-\mathrm{C} 3-\mathrm{H} 9$ & 123.7 \\
$\mathrm{~N} 1-\mathrm{H} 8$ & 1.010 \\
\end{tabular}


Table S580 Structural parameters of the TDDFT/TZVP-optimized excitedstate equilibrium geometry of the $1^{3} B_{1}\left({ }^{3} n \pi^{*}\right)$ state of maleimide used for the statistical evaluations.

\begin{tabular}{ll}
$\begin{array}{l}\text { bond } \\
\text { or angle }\end{array}$ & $\begin{array}{l}\text { length } / \AA \\
\text { or angle } /\end{array}$ \\
\hline $\mathrm{N} 1-\mathrm{C} 2$ & 1.404 \\
$\mathrm{C} 2-\mathrm{C} 3$ & 1.430 \\
$\mathrm{C} 3-\mathrm{C} 4$ & 1.382 \\
$\mathrm{C} 2-\mathrm{O} 6$ & 1.246 \\
$\mathrm{C} 3-\mathrm{H} 9$ & 1.076 \\
$\mathrm{C} 2-\mathrm{N} 1-\mathrm{C} 5$ & 109.6 \\
$\mathrm{~N} 1-\mathrm{C} 2-\mathrm{O} 6$ & 119.7 \\
O6-C2-C4 & 133.7 \\
$\mathrm{C} 2-\mathrm{C} 3-\mathrm{C} 4$ & 108.6 \\
$\mathrm{C} 2-\mathrm{C} 3-\mathrm{H} 9$ & 122.9 \\
$\mathrm{~N} 1-\mathrm{H} 8$ & 1.012 \\
\end{tabular}


Table S581 Structural parameters of the TDDFT/TZVP-optimized excitedstate equilibrium geometry of the $1^{3} A_{2}\left({ }^{3} n \pi^{*}\right)$ state of maleimide used for the statistical evaluations.

\begin{tabular}{ll}
$\begin{array}{l}\text { bond } \\
\text { or angle }\end{array}$ & $\begin{array}{l}\text { length } / \AA \\
\text { or angle } /\end{array}$ \\
\hline $\mathrm{N} 1-\mathrm{C} 2$ & 1.383 \\
$\mathrm{C} 2-\mathrm{C} 3$ & 1.425 \\
$\mathrm{C} 3-\mathrm{C} 4$ & 1.396 \\
$\mathrm{C} 2-\mathrm{O} 6$ & 1.267 \\
$\mathrm{C} 3-\mathrm{H} 9$ & 1.077 \\
$\mathrm{C} 2-\mathrm{N} 1-\mathrm{C} 5$ & 109.8 \\
$\mathrm{~N} 1-\mathrm{C} 2-\mathrm{O} 6$ & 125.2 \\
O6-C2-C4 & 127.4 \\
$\mathrm{C} 2-\mathrm{C} 3-\mathrm{C} 4$ & 107.7 \\
$\mathrm{C} 2-\mathrm{C} 3-\mathrm{H} 9$ & 124.7 \\
$\mathrm{~N} 1-\mathrm{H} 8$ & 1.006 \\
\end{tabular}


Table S582 Structural parameters of the TDDFT/TZVP-optimized excitedstate equilibrium geometry of the $1^{3} B_{2}\left({ }^{3} \pi \pi^{*}\right)$ state of maleimide used for the statistical evaluations.

\begin{tabular}{ll}
$\begin{array}{l}\text { bond } \\
\text { or angle }\end{array}$ & $\begin{array}{l}\text { length } / \AA \\
\text { or angle } /\end{array}$ \\
\hline N1-C2 & 1.425 \\
C2-C3 & 1.418 \\
C3-C4 & 1.407 \\
C2-O6 & 1.251 \\
C3-H9 & 1.077 \\
C2-N1-C5 & 112.0 \\
N1-C2-O6 & 119.2 \\
O6-C2-C4 & 136.6 \\
C2-C3-C4 & 109.7 \\
C2-C3-H9 & 123.2 \\
N1-H8 & 1.017
\end{tabular}


Table S583 Structural parameters of the TDDFT/TZVP-optimized excitedstate equilibrium geometry of the $1^{1} A^{\prime \prime}\left({ }^{1} n \pi^{*}\right)$ state of nitrosomethane used for the statistical evaluations.

\begin{tabular}{ll} 
bond & $\begin{array}{l}\text { length / } \AA \\
\text { or angle } \\
\text { or angle / }\end{array}$ \\
\hline $\mathrm{N} 2-\mathrm{O} 3$ & 1.226 \\
$\mathrm{C} 1-\mathrm{N} 2$ & 1.465 \\
$\mathrm{C} 1-\mathrm{H} 4$ & 1.086 \\
$\mathrm{C} 1-\mathrm{H} 5$ & 1.096 \\
$\mathrm{C} 1-\mathrm{N} 2-\mathrm{O} 3$ & 124.7 \\
$\mathrm{~N} 2-\mathrm{C} 1-\mathrm{H} 4$ & 107.5 \\
$\mathrm{~N} 2-\mathrm{C} 1-\mathrm{H} 5$ & 109.9 \\
$\mathrm{H} 4-\mathrm{C} 1-\mathrm{H} 5$ & 109.6
\end{tabular}


Table S584 Structural parameters of the TDDFT/TZVP-optimized excitedstate equilibrium geometry of the $1{ }^{1} A^{\prime \prime}\left({ }^{1} n \pi^{*}\right)$ state of propenoic acid anion used for the statistical evaluations.

\begin{tabular}{ll}
$\begin{array}{l}\text { bond } \\
\text { or angle }\end{array}$ & $\begin{array}{l}\text { length } / \AA \\
\text { or angle / } /\end{array}$ \\
\hline $\mathrm{C} 2-\mathrm{O} 3$ & 1.282 \\
$\mathrm{C} 2-\mathrm{O} 4$ & 1.290 \\
$\mathrm{C} 1-\mathrm{C} 2$ & 1.421 \\
$\mathrm{C} 1-\mathrm{C} 5$ & 1.412 \\
$\mathrm{O} 4-\mathrm{C} 2-\mathrm{C} 1$ & 126.3 \\
$\mathrm{C} 2-\mathrm{C} 1-\mathrm{C} 5$ & 125.3 \\
$\mathrm{O} 3-\mathrm{C} 2-\mathrm{O} 4$ & 107.1
\end{tabular}


Table S585 Structural parameters of the TDDFT/TZVP-optimized excitedstate equilibrium geometry of the $1{ }^{1} A_{2}\left({ }^{1} n \pi^{*}\right)$ state of pyridine used for the statistical evaluations.

\begin{tabular}{ll} 
bond & $\begin{array}{l}\text { length / } \AA \\
\text { or angle } \\
\text { or angle / }\end{array}$ \\
\hline N1-C2 & 1.300 \\
C2 $-\mathrm{C} 4$ & 1.470 \\
C4-C6 & 1.386 \\
C2-N1-C3 & 134.4 \\
N1-C2-C4 & 113.7 \\
C2-C4-C6 & 117.1 \\
C4-C6-C5 & 123.9
\end{tabular}


Table S586 Structural parameters of the TDDFT/TZVP-optimized excitedstate equilibrium geometry of the $2{ }^{1} A^{\prime}\left({ }^{1} n \pi^{*}\right)$ state of pyridine used for the statistical evaluations.

\begin{tabular}{ll} 
bond & $\begin{array}{l}\text { length / } \AA \\
\text { or angle } \\
\text { or angle / }\end{array}$ \\
\hline $\mathrm{N} 1-\mathrm{C} 2$ & 1.352 \\
$\mathrm{C} 2-\mathrm{C} 4$ & 1.374 \\
$\mathrm{C} 4-\mathrm{C} 6$ & 1.425 \\
$\mathrm{C} 2-\mathrm{N} 1-\mathrm{C} 3$ & 128.6 \\
$\mathrm{~N} 1-\mathrm{C} 2-\mathrm{C} 4$ & 115.2 \\
$\mathrm{C} 2-\mathrm{C} 4-\mathrm{C} 6$ & 120.2 \\
$\mathrm{C} 4-\mathrm{C} 6-\mathrm{C} 5$ & 118.6
\end{tabular}


Table S587 Structural parameters of the TDDFT/TZVP-optimized excitedstate equilibrium geometry of the $1^{3} A_{1}\left({ }^{3} \pi \pi^{*}\right)$ state of pyridine used for the statistical evaluations.

\begin{tabular}{ll}
$\begin{array}{l}\text { bond } \\
\text { or angle }\end{array}$ & $\begin{array}{l}\text { length } / \AA \\
\text { or angle / } /\end{array}$ \\
\hline $\mathrm{N} 1-\mathrm{C} 2$ & 1.328 \\
$\mathrm{C} 2-\mathrm{C} 4$ & 1.525 \\
$\mathrm{C} 4-\mathrm{C} 6$ & 1.388 \\
$\mathrm{C} 2-\mathrm{N} 1-\mathrm{C} 3$ & 118.6 \\
$\mathrm{~N} 1-\mathrm{C} 2-\mathrm{C} 4$ & 122.8 \\
$\mathrm{C} 2-\mathrm{C} 4-\mathrm{C} 6$ & 118.1 \\
$\mathrm{C} 4-\mathrm{C} 6-\mathrm{C} 5$ & 119.5
\end{tabular}


Table S588 Structural parameters of the TDDFT/TZVP-optimized excitedstate equilibrium geometry of the $2{ }^{3} A_{1}\left({ }^{3} \pi \pi^{*}\right)$ state of pyridine used for the statistical evaluations.

\begin{tabular}{ll} 
bond & $\begin{array}{l}\text { length / } \AA \\
\text { or angle } \\
\text { or angle / }\end{array}$ \\
\hline N1-C2 & 1.370 \\
C2-C4 & 1.416 \\
C4-C6 & 1.429 \\
C2-N1-C3 & 116.5 \\
N1-C2-C4 & 124.1 \\
C2-C4-C6 & 118.9 \\
C4-C6-C5 & 117.5
\end{tabular}


Table S589 Structural parameters of the TDDFT/TZVP-optimized excitedstate equilibrium geometry of the $2{ }^{1} A^{\prime}\left({ }^{1} \pi \pi^{*}\right)$ state of pyrrole used for the statistical evaluations.

\begin{tabular}{ll}
$\begin{array}{l}\text { bond } \\
\text { or angle }\end{array}$ & $\begin{array}{c}\text { length } / \AA \\
\text { or angle / }\end{array}$ \\
\hline N6-C7 & 1.405 \\
C7-C10 & 1.424 \\
C9-C10 & 1.469 \\
C7-N6-C8 & 113.0 \\
C10-C7-N6 & 105.5 \\
C7-C10-C9 & 107.9 \\
H1-N6 & 1.015
\end{tabular}


Table S590 Structural parameters of the TDDFT/TZVP-optimized excitedstate equilibrium geometry of the $1{ }^{1} B_{2}\left({ }^{1} \pi \pi^{*}\right)$ state of pyrrole used for the statistical evaluations.

\begin{tabular}{ll}
$\begin{array}{l}\text { bond } \\
\text { or angle }\end{array}$ & $\begin{array}{l}\text { length } / \AA \\
\text { or angle / }\end{array}$ \\
\hline N6-C7 & 1.419 \\
C7-C10 & 1.452 \\
C9-C10 & 1.367 \\
C7-N6-C8 & 106.9 \\
C10-C7-N6 & 108.3 \\
C7-C10-C9 & 108.3 \\
H1-N6 & 1.001
\end{tabular}


Table S591 Structural parameters of the TDDFT/TZVP-optimized excitedstate equilibrium geometry of the $2{ }^{1} A^{\prime}\left({ }^{1} \pi \pi^{*}\right)$ state of cis-PSB3 used for the statistical evaluations.

\begin{tabular}{ll}
$\begin{array}{l}\text { bond } \\
\text { or angle }\end{array}$ & $\begin{array}{l}\text { length } / \AA \\
\text { or angle } /\end{array}$ \\
\hline C1-C2 & 1.378 \\
C2-C3 & 1.486 \\
C3-C4 & 1.348 \\
C4-C5 & 1.473 \\
C5-N6 & 1.328 \\
C1-C2-C3 & 124.9 \\
C2-C3-C4 & 121.3 \\
C3-C4-C5 & 125.7 \\
C4-C5-N6 & 119.6 \\
N6-H13 & 1.012 \\
N6-H14 & 1.011
\end{tabular}


Table S592 Structural parameters of the TDDFT/TZVP-optimized excitedstate equilibrium geometry of the $2{ }^{1} A^{\prime}\left({ }^{1} \pi \pi^{*}\right)$ state of 3Me-cis-PSB5 used for the statistical evaluations.

\begin{tabular}{ll}
$\begin{array}{l}\text { bond } \\
\text { or angle }\end{array}$ & $\begin{array}{c}\text { length } / \AA \\
\text { or angle } /\end{array}$ \\
\hline $\mathrm{C} 10-\mathrm{C} 11$ & 1.359 \\
$\mathrm{C} 9-\mathrm{C} 10$ & 1.434 \\
$\mathrm{C} 8-\mathrm{C} 9$ & 1.364 \\
$\mathrm{C} 1-\mathrm{C} 8$ & 1.460 \\
$\mathrm{C} 1-\mathrm{C} 2$ & 1.354 \\
$\mathrm{C} 2-\mathrm{C} 3$ & 1.483 \\
$\mathrm{C} 4-\mathrm{C} 4$ & 1.379 \\
$\mathrm{C} 4-\mathrm{C} 5$ & 1.408 \\
$\mathrm{C} 5-\mathrm{N} 6$ & 1.332 \\
$\mathrm{C} 3-\mathrm{C} 7$ & 1.497 \\
$\mathrm{C} 11-\mathrm{C} 10-\mathrm{C} 9$ & 123.9 \\
$\mathrm{C} 10-\mathrm{C} 9-\mathrm{C} 8$ & 122.9 \\
$\mathrm{C} 9-\mathrm{C} 8-\mathrm{C} 1$ & 123.1 \\
$\mathrm{C} 8-\mathrm{C} 1-\mathrm{C} 2$ & 129.6 \\
$\mathrm{C} 1-\mathrm{C} 2-\mathrm{C} 3$ & 132.9 \\
$\mathrm{C} 2-\mathrm{C} 3-\mathrm{C} 4$ & 115.1 \\
$\mathrm{C} 3-\mathrm{C} 4-\mathrm{C} 5$ & 127.0 \\
$\mathrm{C} 4-\mathrm{C} 5-\mathrm{N} 6$ & 122.9 \\
$\mathrm{C} 2-\mathrm{C} 3-\mathrm{C} 7$ & 122.2 \\
$\mathrm{~N} 6-\mathrm{H} 16$ & 1.011 \\
$\mathrm{~N} 6-\mathrm{H} 17$ & 1.009
\end{tabular}


Table S593 Structural parameters of the TDDFT/TZVP-optimized excitedstate equilibrium geometry of the $1{ }^{1} B_{u}\left({ }^{1} \pi \pi^{*}\right)$ state of stilbene used for the statistical evaluations.

\begin{tabular}{ll}
$\begin{array}{l}\text { bond } \\
\text { or angle }\end{array}$ & $\begin{array}{l}\text { length } / \AA \\
\text { or angle } /\end{array}$ \\
\hline C1-C8 & 1.414 \\
C1-C2 & 1.411 \\
C2-C3 & 1.429 \\
C3-C4 & 1.375 \\
C4-C5 & 1.409 \\
C5-C6 & 1.397 \\
C6-C7 & 1.381 \\
C2-C7 & 1.439 \\
C8-C1-C2 & 125.2 \\
C1-C2-C3 & 120.1 \\
C1-C2-C7 & 122.9
\end{tabular}


Table S594 Structural parameters of the TDDFT/TZVP-optimized excitedstate equilibrium geometry of the $1^{1} B\left({ }^{1} \pi \pi^{*}\right)$ state of stilbene used for the statistical evaluations.

\begin{tabular}{ll}
$\begin{array}{l}\text { bond } \\
\text { or angle }\end{array}$ & $\begin{array}{l}\text { length } / \AA \\
\text { or angle } /\end{array}$ \\
\hline $\mathrm{C} 1-\mathrm{C} 8$ & 1.413 \\
$\mathrm{C} 1-\mathrm{C} 2$ & 1.409 \\
$\mathrm{C} 2-\mathrm{C} 3$ & 1.425 \\
$\mathrm{C} 3-\mathrm{C} 4$ & 1.373 \\
$\mathrm{C} 4-\mathrm{C} 5$ & 1.417 \\
$\mathrm{C} 5-\mathrm{C} 6$ & 1.384 \\
$\mathrm{C} 6-\mathrm{C} 7$ & 1.396 \\
$\mathrm{C} 2-\mathrm{C} 7$ & 1.450 \\
$\mathrm{C} 8-\mathrm{C} 1-\mathrm{C} 2$ & 125.1 \\
$\mathrm{C} 1-\mathrm{C} 2-\mathrm{C} 3$ & 121.0 \\
$\mathrm{C} 1-\mathrm{C} 2-\mathrm{C} 7$ & 121.8
\end{tabular}


Table S595 Structural parameters of the TDDFT/TZVP-optimized excitedstate equilibrium geometry of the $2{ }^{1} A^{\prime}\left({ }^{1} \pi \pi^{*}\right)$ state of styrene used for the statistical evaluations.

\begin{tabular}{ll}
$\begin{array}{l}\text { bond } \\
\text { or angle }\end{array}$ & $\begin{array}{l}\text { length } / \AA \\
\text { or angle } /{ }^{\circ}\end{array}$ \\
\hline C1-C2 & 1.398 \\
C1-C3 & 1.406 \\
C3-C4 & 1.473 \\
C3-C5 & 1.427 \\
C4-C6 & 1.385 \\
C5-C7 & 1.372 \\
C6-C8 & 1.395 \\
C7-C8 & 1.433
\end{tabular}


Table S596 Structural parameters of the TDDFT/TZVP-optimized excitedstate equilibrium geometry of the $1{ }^{1} B_{3 u}\left({ }^{1} n \pi^{*}\right)$ state of $s$-tetrazine used for the statistical evaluations.

\begin{tabular}{ll} 
bond & $\begin{array}{l}\text { length / } \AA \\
\text { or angle } \\
\text { or angle / }\end{array}$ \\
\hline N3-N4 & 1.316 \\
C2-N4 & 1.328 \\
C2-H7 & 1.080 \\
N3-C1-N5 & 120.9
\end{tabular}


Table S597 Structural parameters of the TDDFT/TZVP-optimized excitedstate equilibrium geometry of the $2{ }^{1} A\left({ }^{1} n \pi^{*}\right)$ state of thymine used for the statistical evaluations.

\begin{tabular}{ll}
$\begin{array}{l}\text { bond } \\
\text { or angle }\end{array}$ & $\begin{array}{l}\text { length } / \AA \\
\text { or angle } /\end{array}$ \\
\hline N1-C6 & 1.363 \\
C5-N6 & 1.400 \\
N4-C5 & 1.393 \\
C3-C4 & 1.370 \\
C2-C3 & 1.404 \\
N1-C2 & 1.403 \\
C6-O9 & 1.216 \\
C4-O7 & 1.330 \\
C3-C8 & 1.506 \\
C2-H10 & 1.076 \\
N1-H11 & 0.993 \\
N5-H12 & 0.995
\end{tabular}


Table S598 Structural parameters of the TDDFT/TZVP-optimized excitedstate equilibrium geometry of the $2{ }^{1} A^{\prime}\left({ }^{1} \pi \pi^{*}\right)$ state of uracil used for the statistical evaluations.

\begin{tabular}{ll}
$\begin{array}{l}\text { bond } \\
\text { or angle }\end{array}$ & $\begin{array}{l}\text { length } / \AA \\
\text { or angle } /\end{array}$ \\
\hline N1-C3 & 1.314 \\
N2-C3 & 1.326 \\
N2-C4 & 1.447 \\
C4-C5 & 1.395 \\
C5-C6 & 1.399 \\
N1-C6 & 1.539 \\
C3-O8 & 1.310 \\
C6-O7 & 1.226 \\
C4-H9 & 1.073 \\
C5-H12 & 1.081 \\
N2-H11 & 1.007 \\
N1-H10 & 1.001
\end{tabular}




\subsubsection{CC2-Optimized Excited-State Minima}

Similar to what has been pointed out above for the TDDFT geometry optimizations, also for the $\mathrm{CC} 2$ optimizations we normally employed the same pointgroup symmetry as for the OMx/MRCI optimizations. Only in rare cases did we diverge from this practice by imposing a lower level of symmetry constraints, for example when the use of the same point-group symmetry would restrict the molecule in such a way that key structural properties would be distorted. The pyramidalization of exocyclic amino groups that is found for all CC2-optimized excited-state structures (see Table 8 in the article) is one example for which the point group of certain molecules was lowered (e.g. the ${ }^{1} \pi \pi^{*}$ state of aniline for which $C_{s}$ symmetry was used at the CC2 level but $C_{2 v}$ at the OMx/MRCI level).

In other cases, we were unable to converge the geometry in a to-be-preferred lower symmetry. For example, we failed to optimize the ${ }^{1} \pi \pi^{*}$ state of 2 -amino9-methylpurine in $C_{1}$ symmetry. 
Table S599 Cartesian coordinates of the CC2/TZVP-optimized excited-state equilibrium geometry of the $2{ }^{1} A\left({ }^{1} n \pi^{*}\right)$ state of $9 H$-adenine (excited-state energy: $-466.04524 E_{h}$ ).

$\begin{array}{lrrr}15 & & \\ & & & \\ \mathrm{~N} & -0.0186751 & -0.0084849 & 0.0675265 \\ \mathrm{C} & 1.3412352 & 0.0120884 & -0.0355806 \\ \mathrm{C} & 1.8572643 & 1.3315754 & -0.0804595 \\ \mathrm{~N} & 0.7330492 & 2.1183048 & -0.1094552 \\ \mathrm{C} & -0.3732493 & 1.2654310 & 0.0235477 \\ \mathrm{~N} & 3.1382510 & 1.6673315 & -0.1150221 \\ \mathrm{C} & 4.0748624 & 0.6939195 & 0.3483410 \\ \mathrm{~N} & 3.6117101 & -0.6034644 & 0.1313237 \\ \mathrm{C} & 2.3479398 & -0.9894572 & 0.1557286 \\ \mathrm{~N} & 2.0170118 & -2.3192428 & 0.2402333 \\ \mathrm{H} & 5.1139304 & 0.8793542 & 0.1152744 \\ \mathrm{H} & 1.0495596 & -2.4800313 & 0.4901506 \\ \mathrm{H} & 2.6862236 & -2.9053754 & 0.7211687 \\ \mathrm{H} & 0.7271303 & 3.1268832 & -0.0459736 \\ \mathrm{H} & -1.3816029 & 1.6446110 & 0.0072479\end{array}$


Table S600 Cartesian coordinates of the CC2/TZVP-optimized excited-state equilibrium geometry of the $2{ }^{1} A\left({ }^{1} n \pi^{*}\right)$ state of acetaldehyde (excited-state energy: $-153.32953 E_{h}$ ).

7

C $\quad 1.5062271 \quad 0.0003230$

$\mathrm{H}-0.5592937-0.9338496$

$0 \quad-0.5658679 \quad 0.8882506$

$1.8729811-0.7100459$

$\mathrm{H} \quad 1.9039577-0.2640977-0.9651882$

$\mathrm{H} \quad 1.8719804 \quad 0.9980116 \quad 0.2698867$ 
Table S601 Cartesian coordinates of the CC2/TZVP-optimized excited-state equilibrium geometry of the $1^{3} A\left({ }^{3} n \pi^{*}\right)$ state of acetaldehyde (excited-state energy: $-153.34640 E_{h}$ ).

7

C

C $\quad 1.5211860$

$\mathrm{H} \quad-0.4958280$

O -0.5595903

$\mathrm{H} \quad 1.8846025$

$\mathrm{H} \quad 1.9353012$

$-0.0354035$

0.0027540

$-0.0026506$

$-0.9964646$

0.0140382

.0779831

$-0.6254705$

$-0.9379245$

H 1.8738634

$-0.3581853$

0.8250788

$-0.9342519$

$1.0217527 \quad 0.1720792$ 
Table S602 Cartesian coordinates of the CC2/TZVP-optimized excited-state equilibrium geometry of the $2{ }^{3} A\left({ }^{3} \pi \pi^{*}\right)$ state of acetaldehyde (excited-state energy: $-153.29518 E_{h}$ ).

7

$\begin{array}{rrrr}\mathrm{C} & -0.0031331 & -0.0158255 & -0.0255997 \\ \mathrm{C} & 1.4652248 & -0.0039252 & 0.0366650 \\ \mathrm{H} & -0.5679309 & -0.9408719 & 0.0258080 \\ \mathrm{O} & -0.5801273 & 0.9672733 & -0.9656717 \\ \mathrm{H} & 1.8388378 & -0.7696524 & 0.7164245 \\ \mathrm{H} & 1.8846158 & -0.1949494 & -0.9638534 \\ \mathrm{H} & 1.8332574 & 0.9780252 & 0.3413096\end{array}$


Table S603 Cartesian coordinates of the CC2/TZVP-optimized excited-state equilibrium geometry of the $1^{1} A^{\prime \prime}\left({ }^{1} n \pi^{*}\right)$ state of acetone (excited-state energy: $\left.-192.54094 E_{h}\right)$.

$\begin{array}{lrrr}10 & & & \\ \text { C } & 0.1428751 & 0.0992791 & -1.3114290 \\ \mathrm{C} & -0.5523327 & 0.0564547 & 0.0000000 \\ \mathrm{O} & -1.6545816 & -0.8387376 & 0.0000000 \\ \mathrm{C} & 0.1428751 & 0.0992791 & 1.3114290 \\ \mathrm{H} & -0.5561523 & 0.3261584 & -2.1175458 \\ \mathrm{H} & 0.5850740 & -0.8851687 & -1.5253430 \\ \mathrm{H} & 0.9316603 & 0.8508727 & -1.2949468 \\ \mathrm{H} & -0.5561523 & 0.3261584 & 2.1175458 \\ \mathrm{H} & 0.9316603 & 0.8508727 & 1.2949468 \\ \mathrm{H} & 0.5850740 & -0.8851687 & 1.5253430\end{array}$


Table S604 Cartesian coordinates of the CC2/TZVP-optimized excited-state equilibrium geometry of the $1^{3} A^{\prime \prime}\left({ }^{3} n \pi^{*}\right)$ state of acetone (excited-state energy: $\left.-192.55473 E_{h}\right)$.

$\begin{array}{lrrr}10 & & & \\ & & & \\ \mathrm{C} & 0.1368676 & 0.0950518 & -1.3020422 \\ \mathrm{C} & -0.6015920 & 0.0728263 & 0.0000000 \\ \mathrm{O} & -1.6378999 & -0.8493297 & 0.0000000 \\ \mathrm{C} & 0.1368676 & 0.0950518 & 1.3020422 \\ \mathrm{H} & -0.5478855 & 0.2247762 & -2.1400661 \\ \mathrm{H} & 0.6791650 & -0.8491333 & -1.4493773 \\ \mathrm{H} & 0.8515988 & 0.9175571 & -1.2982450 \\ \mathrm{H} & -0.5478855 & 0.2247762 & 2.1400661 \\ \mathrm{H} & 0.8515988 & 0.9175571 & 1.2982450 \\ \mathrm{H} & 0.6791650 & -0.8491333 & 1.4493773\end{array}$


Table S605 Cartesian coordinates of the CC2/TZVP-optimized excited-state equilibrium geometry of the $1^{1} A^{\prime}\left({ }^{3} \pi \pi^{*}\right)$ state of acetone (excited-state energy: $\left.-192.50638 E_{h}\right)$.

$\begin{array}{lrrr}10 & & & \\ \text { C } & 0.1423419 & 0.1167773 & -1.2946106 \\ \mathrm{C} & -0.5656501 & 0.0730795 & 0.0000000 \\ \mathrm{O} & -1.6693235 & -0.9267203 & 0.0000000 \\ \mathrm{C} & 0.1423419 & 0.1167773 & 1.2946106 \\ \mathrm{H} & -0.5472761 & 0.3510016 & -2.1069209 \\ \mathrm{H} & 0.5617085 & -0.8781911 & -1.5186525 \\ \mathrm{H} & 0.9607125 & 0.8372326 & -1.2815458 \\ \mathrm{H} & -0.5472761 & 0.3510016 & 2.1069209 \\ \mathrm{H} & 0.9607125 & 0.8372326 & 1.2815458 \\ \mathrm{H} & 0.5617085 & -0.8781911 & 1.5186525\end{array}$


Table S606 Cartesian coordinates of the CC2/TZVP-optimized excited-state equilibrium geometry of the $1^{1} A^{\prime \prime}\left({ }^{1} n \pi^{*}\right)$ state of acetophenone (excited-state energy: $-383.78494 E_{h}$ ).

17

C $\quad 1.1884229-1.1800627$

0.0000000

O 2.6089761

$-1.0172896$

0.0000000

C $\quad 0.8197500-2.6256931$

0.0000000

$\mathrm{H} \quad 1.7171011$

$-3.2449593$

0.0000000

$\mathrm{H} \quad 0.2300084$

$-2.8655123$

$-0.8876834$

$\mathrm{H} \quad 0.2300084$

$-2.8655123$

0.8876834

0.3527837

$-0.0741849$

0.0000000

$-1.0699470$

$-0.2102283$

0.0000000

0.9147409

1.2399421

0.0000000

$-1.8715693$

0.9169921

0.0000000

0.0865466

2.3489611

0.0000000

$-1.3073928$

2.2028068

0.0000000

$-1.5190698$

$-1.1973329$

0.0000000

1.9946960

1.3495837

0.0000000

$-2.9499439$

0.8037408

0.0000000

0.5223617

3.3417430

0.0000000

$-1.9474731$

3.0770059

0.0000000

S653 
Table S607 Cartesian coordinates of the CC2/TZVP-optimized excited-state equilibrium geometry of the $2{ }^{1} A^{\prime}\left({ }^{1} \pi \pi^{*}\right)$ state of acetophenone (excited-state energy: $-383.73203 E_{h}$ ).

17

C $\quad 1.1376078-1.4156230$

0.0000000

O 2.3922721

$-1.4158622$

0.0000000

C $\quad 0.3571362-2.7140803$

0.0000000

$\mathrm{H} \quad 1.0625026$

$-3.5423298$

0.0000000

$-0.2837256$

$-2.7884465$

$-0.8829237$

$-0.2837256$

$-2.7884465$

0.8829237

0.3956632

$-0.1677114$

0.0000000

$-1.0371246$

$-0.0735720$

0.0000000

1.1213549

1.0736250

0.0000000

$-1.6954125$

1. 2008080

0.0000000

0.4492130

2. 3355543

0.0000000

$-0.9668690$

2. 4148756

0.0000000

$-1.6424918$

2.2035907

$-0.9712576$

0.0000000

$-2.7788957$

1.0215012

0.0000000

1.0437177

1. 2207188

0.0000000

$-1.4748134$

3.2412583

0.0000000

3. 3689879

0.0000000

S654 
Table S608 Cartesian coordinates of the CC2/TZVP-optimized excited-state equilibrium geometry of the $1^{3} A^{\prime \prime}\left({ }^{3} n \pi^{*}\right)$ state of acetophenone (excited-state energy: $-383.79488 E_{h}$ ).

17

C $\quad 1.2316125-1.1554742$

0.0000000

O 2.6200760

$-0.9666058$

0.0000000

C $\quad 0.8762181$

$-2.6071061$

0.0000000

$\mathrm{H} \quad 1.7788482$

$-3.2189185$

0.0000000

$\mathrm{H}$

0.2900454

$-2.8561481$

$-0.8878059$

$\mathrm{H}$

0.2900454

$-2.8561481$

0.8878059

0.3671910

$-0.0566165$

0.0000000

$-1.0462760$

$-0.2346568$

0.0000000

0.8852543

1. 2706704

0.0000000

$-1.8853157$

0.8682902

0.0000000

0.0230478

2.3552707

0.0000000

$-1.3650870$

2.1695155

0.0000000

$-1.4662203$

$-1.2343132$

0.0000000

1.9601280

1.4204913

0.0000000

$-2.9592533$

0.7182101

0.0000000

0.4311086

3.3598381

0.0000000

$-2.0314232$

3. 0237011

0.0000000 
Table S609 Cartesian coordinates of the CC2/TZVP-optimized excited-state equilibrium geometry of the $1^{3} A^{\prime}\left({ }^{3} \pi \pi^{*}\right)$ state of acetophenone (excited-state energy: $-383.77468 E_{h}$ ).

17

C $\quad 1.0769347-1.3956030$

0.0000000

O 2.3587171

$-1.3995274$

0.0000000

C $\quad 0.3165092$

$-2.7038174$

0.0000000

$\mathrm{H} \quad 1.0354408$

$-3.5203906$

0.0000000

$-0.3211733$

$-2.7919268$

$-0.8829142$

$-0.3211733$

$-2.7919268$

0.8829142

0.3802119

$-0.1584654$

0.0000000

$-1.0837989$

$-0.0585731$

0.0000000

1.1598939

1.1069084

0.0000000

$-1.6867712$

1.1590317

0.0000000

0.5180595

2. 3100376

0.0000000

$-0.9086092$

2.3867499

0.0000000

$-1.6760375$

2.2376956

$-0.9645104$

0.0000000

$-2.7683448$

1.0218394

0.0000000

1.0934141

1.2273738

0.0000000

$-1.4109687$

3. 2283747

0.0000000

3. 3444257

0.0000000 
Table S610 Cartesian coordinates of the CC2/TZVP-optimized excited-state equilibrium geometry of the $1{ }^{1} A_{2}\left({ }^{1} \pi \pi^{*}\right)$ state of acetylene (excited-state energy: $-76.90551 E_{h}$ ).

4

$\begin{array}{lrrr}\mathrm{C} & 0.6744358 & 0.0000000 & 0.4027572 \\ \mathrm{C} & -0.6744358 & 0.0000000 & 0.4027572 \\ \mathrm{H} & 1.4165227 & 0.0000000 & -0.4027572 \\ \mathrm{H} & -1.4165227 & 0.0000000 & -0.4027572\end{array}$


Table S611 Cartesian coordinates of the CC2/TZVP-optimized excited-state equilibrium geometry of the $1{ }^{1} B_{2}$ ( $\equiv 1{ }^{1} B_{1}$ in Turbomole) $\left({ }^{1} \pi \pi^{*}\right)$ state of acetylene (excited-state energy: $-76.86520 E_{h}$ ).

4

$\begin{array}{lrrr}\mathrm{C} & 0.6676687 & 0.0000000 & 0.3373574 \\ \mathrm{C} & -0.6676687 & 0.0000000 & 0.3373574 \\ \mathrm{H} & 1.5312092 & 0.0000000 & -0.3373574 \\ \mathrm{H} & -1.5312092 & 0.0000000 & -0.3373574\end{array}$


Table S612 Cartesian coordinates of the CC2/TZVP-optimized excited-state equilibrium geometry of the $2{ }^{1} A_{2}\left({ }^{1} \pi \pi^{*}\right)$ state of acetylene (excited-state energy: $-76.85391 E_{h}$ ).

4

$\begin{array}{lrrr}\mathrm{C} & 0.6638151 & 0.0000000 & -0.0775577 \\ \mathrm{C} & -0.6638151 & 0.0000000 & -0.0775577 \\ \mathrm{H} & 1.7161742 & 0.0000000 & 0.0775577 \\ \mathrm{H} & -1.7161742 & 0.0000000 & 0.0775577\end{array}$


Table S613 Cartesian coordinates of the CC2/TZVP-optimized excited-state equilibrium geometry of the $1{ }^{3} B_{2}\left({ }^{3} \pi \pi^{*}\right)$ state of acetylene (excited-state energy: $-76.97888 E_{h}$ ).

4

$\begin{array}{lrrr}\mathrm{C} & 0.6710872 & 0.0000000 & 0.4357549 \\ \mathrm{C} & -0.6710872 & 0.0000000 & 0.4357549 \\ \mathrm{H} & 1.3247086 & 0.0000000 & -0.4357549 \\ \mathrm{H} & -1.3247086 & 0.0000000 & -0.4357549\end{array}$


Table S614 Cartesian coordinates of the CC2/TZVP-optimized excited-state equilibrium geometry of the $1{ }^{3} A_{2}\left({ }^{3} \pi \pi^{*}\right)$ state of acetylene (excited-state energy: $-76.94052 E_{h}$ ).

4

$\begin{array}{lrrr}\mathrm{C} & 0.6818629 & 0.0000000 & 0.4163712 \\ \mathrm{C} & -0.6818629 & 0.0000000 & 0.4163712 \\ \mathrm{H} & 1.3894058 & 0.0000000 & -0.4163712 \\ \mathrm{H} & -1.3894058 & 0.0000000 & -0.4163712\end{array}$


Table S615 Cartesian coordinates of the CC2/TZVP-optimized excited-state equilibrium geometry of the $2{ }^{3} A_{2}\left({ }^{3} \pi \pi^{*}\right)$ state of acetylene (excited-state energy: $-76.87353 E_{h}$ ).

4

$\begin{array}{lrrr}\mathrm{C} & -0.6697416 & 0.0000000 & -0.1167784 \\ \mathrm{C} & 0.6697416 & 0.0000000 & -0.1167784 \\ \mathrm{H} & -1.7100595 & 0.0000000 & 0.1167784 \\ \mathrm{H} & 1.7100595 & 0.0000000 & 0.1167784\end{array}$


Table S616 Cartesian coordinates of the CC2/TZVP-optimized excited-state equilibrium geometry of the $1{ }^{1} A_{u}\left({ }^{1} \pi \pi^{*}\right)$ state of acetylene (excited-state energy: $\left.-76.92057 E_{h}\right)$.

4

$\begin{array}{lrrr}\mathrm{C} & -0.6903857 & -0.0054000 & 0.0000000 \\ \mathrm{C} & 0.6903857 & 0.0054000 & 0.0000000 \\ \mathrm{H} & -1.2778028 & 0.9162672 & 0.0000000 \\ \mathrm{H} & 1.2778028 & -0.9162672 & 0.0000000\end{array}$

S663 
Table S617 Cartesian coordinates of the CC2/TZVP-optimized excited-state equilibrium geometry of the $1{ }^{1} B_{u}\left({ }^{1} \pi \pi^{*}\right)$ state of acetylene (excited-state energy: $-76.85972 E_{h}$ ).

4

$\begin{array}{lrrr}\mathrm{C} & -0.6686525 & 0.0323952 & 0.0000000 \\ \mathrm{C} & 0.6686525 & -0.0323952 & 0.0000000 \\ \mathrm{H} & -1.5410254 & 0.6620945 & 0.0000000 \\ \mathrm{H} & 1.5410254 & -0.6620945 & 0.0000000\end{array}$


Table S618 Cartesian coordinates of the CC2/TZVP-optimized excited-state equilibrium geometry of the $2{ }^{1} A_{u}\left({ }^{1} \pi \pi^{*}\right)$ state of acetylene (excited-state energy: $-76.85393 E_{h}$ ).

4

$\begin{array}{lrrr}\mathrm{C} & -0.5975295 & 0.2895674 & 0.0000000 \\ \mathrm{C} & 0.5975295 & -0.2895674 & 0.0000000 \\ \mathrm{H} & -1.4709397 & 0.8947406 & 0.0000000 \\ \mathrm{H} & 1.4709397 & -0.8947406 & 0.0000000\end{array}$


Table S619 Cartesian coordinates of the CC2/TZVP-optimized excited-state equilibrium geometry of the $1{ }^{3} B_{u}\left({ }^{3} \pi \pi^{*}\right)$ state of acetylene (excited-state energy: $-76.96688 E_{h}$ ).

4

$\begin{array}{lrrr}\mathrm{C} & -0.6726054 & -0.0446414 & 0.0000000 \\ \mathrm{C} & 0.6726054 & 0.0446414 & 0.0000000 \\ \mathrm{H} & -1.4362088 & 0.7194988 & 0.0000000 \\ \mathrm{H} & 1.4362088 & -0.7194988 & 0.0000000\end{array}$


Table S620 Cartesian coordinates of the CC2/TZVP-optimized excited-state equilibrium geometry of the $1{ }^{3} A_{u}\left({ }^{3} \pi \pi^{*}\right)$ state of acetylene (excited-state energy: $-76.95240 E_{h}$ ).

4

$\begin{array}{lrrr}\mathrm{C} & -0.6945002 & -0.0074069 & 0.0000000 \\ \mathrm{C} & 0.6945002 & 0.0074069 & 0.0000000 \\ \mathrm{H} & -1.2577213 & 0.9270492 & 0.0000000 \\ \mathrm{H} & 1.2577213 & -0.9270492 & 0.0000000\end{array}$


Table S621 Cartesian coordinates of the CC2/TZVP-optimized excited-state equilibrium geometry of the $2{ }^{3} B_{u}\left({ }^{3} \pi \pi^{*}\right)$ state of acetylene (excited-state energy: $-76.90527 E_{h}$ ).

4

$\begin{array}{rrrr}\mathrm{C} & -0.6793108 & 0.0281099 & 0.0000000 \\ \mathrm{C} & 0.6793108 & -0.0281099 & 0.0000000 \\ \mathrm{H} & -1.6161248 & 0.5380299 & 0.0000000 \\ \mathrm{H} & 1.6161248 & -0.5380299 & 0.0000000\end{array}$


Table S622 Cartesian coordinates of the CC2/TZVP-optimized excited-state equilibrium geometry of the $2{ }^{3} A_{u}\left({ }^{3} \pi \pi^{*}\right)$ state of acetylene (excited-state energy: $-76.87328 E_{h}$ ).

4

$\begin{array}{lrrr}\mathrm{C} & -0.6173530 & 0.2617354 & 0.0000000 \\ \mathrm{C} & 0.6173530 & -0.2617354 & 0.0000000 \\ \mathrm{H} & -1.4522068 & 0.9211847 & 0.0000000 \\ \mathrm{H} & 1.4522068 & -0.9211847 & 0.0000000\end{array}$


Table S623 Cartesian coordinates of the CC2/TZVP-optimized excited-state equilibrium geometry of the $2{ }^{1} A\left({ }^{1} n \pi^{*}\right)$ state of acrolein (excited-state energy: $\left.-191.32661 E_{h}\right)$.

C $\quad 1.3663372 \quad 0.0199922$

C $2.0591072 \quad 1.2065865$

O $3.4399260 \quad 1.3491821$

$\mathrm{H} \quad-0.5446016 \quad-0.9683524$

$-0.0000082$

$\mathrm{H} \quad-0.6093853$

0.8869181

0.0000530

$\mathrm{H} \quad 1.9371431$

$-0.9025558$

0.0000185

0.0000737

0.0000314

$\mathrm{H} \quad 1.5717728$

$2.1856221-0.0000557$ 
Table S624 Cartesian coordinates of the CC2/TZVP-optimized excited-state equilibrium geometry of the $1^{3} A\left({ }^{3} n \pi^{*}\right)$ state of acrolein (excited-state energy: $\left.-191.33923 E_{h}\right)$.

C $\quad-0.0234862$

$-0.0286026$

$-0.0000102$

C $\quad 1.3511370$

0.0339641

0.0000075

C 2.0556042

1. 2298657

0.0000119

O 3.4241734

1. 3250117

$-0.0000575$

$\mathrm{H} \quad-0.5297762$

$-0.9829547$

0.0000012

$\mathrm{H} \quad-0.6283597$

0.8698713

$-0.0000372$

$\mathrm{H} \quad 1.9307442$

$-0.8835694$

0.0000316

$\mathrm{H}$

1.5911857

2. 2165007

0.0000616 
Table S625 Cartesian coordinates of the CC2/TZVP-optimized excited-state equilibrium geometry of the $1^{3} A^{\prime}\left({ }^{3} \pi \pi^{*}\right)$ state of acrolein (excited-state energy: $\left.-191.32835 E_{h}\right)$.$$
\text { C }-1.2330296
$$$$
\text { C } 0.2744825
$$$$
\text { C } 0.9846233
$$

O 2.2612329

$\mathrm{H} \quad-1.7249824$

$\mathrm{H} \quad-1.8216908$

$\mathrm{H} \quad 0.8492006$

$\mathrm{H} \quad 0.4101636$

$-0.5018541$

0.0000000

$-0.4221698 \quad 0.0000000$

$0.7801346 \quad 0.0000000$

0.82280620 .0000000

$-1.4599946 \quad 0.0000000$

0.40238390 .0000000

$-1.34064810 .0000000$

1.71934180 .0000000 
Table S626 Cartesian coordinates of the CC2/TZVP-optimized excited-state equilibrium geometry of the $2{ }^{1} A^{\prime}\left({ }^{1} \pi \pi^{*}\right)$ state of 2-amino-9-methylpurine (excited-state energy: $-505.24649 E_{h}$ ).

$\begin{array}{lrrr}18 & & \\ & & & \\ \mathrm{~N} & 1.8477487 & -1.5896962 & 0.0000000 \\ \mathrm{C} & 1.4459399 & -0.2922531 & 0.0000000 \\ \mathrm{C} & 0.0278562 & -0.2101931 & 0.0000000 \\ \mathrm{~N} & -0.4180943 & -1.5245649 & 0.0000000 \\ \mathrm{C} & 0.6968061 & -2.3015621 & 0.0000000 \\ \mathrm{C} & 2.1931067 & 0.9421243 & 0.0000000 \\ \mathrm{~N} & 1.4431304 & 2.1141073 & 0.0000000 \\ \mathrm{C} & 0.0976113 & 1.9900100 & 0.0000000 \\ \mathrm{~N} & -0.7511416 & 0.8992146 & 0.0000000 \\ \mathrm{~N} & -0.5732991 & 3.1707539 & 0.0000000 \\ \mathrm{C} & -1.8126502 & -1.9396147 & 0.0000000 \\ \mathrm{H} & 3.2702579 & 1.0128025 & 0.0000000 \\ \mathrm{H} & 0.6430075 & -3.3799410 & 0.0000000 \\ \mathrm{H} & -1.5840358 & 3.1594881 & 0.0000000 \\ \mathrm{H} & -0.0432618 & 4.0319005 & 0.0000000 \\ \mathrm{H} & -2.4174638 & -1.0347111 & 0.0000000 \\ \mathrm{H} & -2.0327590 & -2.5239324 & 0.8926881 \\ \mathrm{H} & -2.0327590 & -2.5239324 & -0.8926881\end{array}$


Table S627 Cartesian coordinates of the CC2/TZVP-optimized excited-state equilibrium geometry of the $1^{1} A^{\prime \prime}\left({ }^{1} \pi \pi^{*}\right)$ state of aniline (excited-state energy: $\left.-286.67044 E_{h}\right)$.

$\begin{array}{lrrr}14 & & & \\ & & & \\ \mathrm{C} & 0.2305890 & 0.0297138 & -1.2534576 \\ \mathrm{C} & 0.9048641 & -0.0568428 & 0.0000000 \\ \mathrm{C} & 0.2305890 & 0.0297138 & 1.2534576 \\ \mathrm{C} & -1.2001863 & 0.0407979 & 1.2462379 \\ \mathrm{C} & -1.8750437 & 0.1223358 & 0.0000000 \\ \mathrm{C} & -1.2001863 & 0.0407979 & -1.2462379 \\ \mathrm{~N} & 2.2747016 & -0.0425309 & 0.0000000 \\ \mathrm{H} & 2.7531036 & -0.2803256 & 0.8589506 \\ \mathrm{H} & 2.7531036 & -0.2803256 & -0.8589506 \\ \mathrm{H} & 0.7989085 & -0.0079703 & 2.1752190 \\ \mathrm{H} & -1.7561002 & 0.1084657 & 2.1716649 \\ \mathrm{H} & -2.9571509 & 0.1956752 & 0.0000000 \\ \mathrm{H} & -1.7561002 & 0.1084657 & -2.1716649 \\ \mathrm{H} & 0.7989085 & -0.0079703 & -2.1752190\end{array}$


Table S628 Cartesian coordinates of the CC2/TZVP-optimized excited-state equilibrium geometry of the $1^{3} A^{\prime}\left({ }^{3} \pi \pi^{*}\right)$ state of aniline (excited-state energy: $\left.-286.70544 E_{h}\right)$.

$\begin{array}{lrrr}14 & & & \\ & & & \\ \mathrm{C} & 1.1475191 & -0.2386229 & 0.0000000 \\ \mathrm{C} & 0.3812103 & -0.4356729 & 1.2529772 \\ \mathrm{C} & -0.9783514 & -0.2894807 & 1.2348744 \\ \mathrm{C} & -1.6744324 & -0.1403328 & 0.0000000 \\ \mathrm{C} & -0.9783514 & -0.2894807 & -1.2348744 \\ \mathrm{C} & 0.3812103 & -0.4356729 & -1.2529772 \\ \mathrm{~N} & 1.9396346 & 0.9322156 & 0.0000000 \\ \mathrm{H} & 1.9098883 & 1.4913872 & 0.8468681 \\ \mathrm{H} & 1.9098883 & 1.4913872 & -0.8468681 \\ \mathrm{H} & 0.9056448 & -0.6521784 & 2.1783748 \\ \mathrm{H} & -1.5488308 & -0.3734157 & 2.1546446 \\ \mathrm{H} & -2.7518436 & -0.0345390 & 0.0000000 \\ \mathrm{H} & -1.5488308 & -0.3734157 & -2.1546446 \\ \mathrm{H} & 0.9056448 & -0.6521784 & -2.1783748\end{array}$


Table S629 Cartesian coordinates of the CC2/TZVP-optimized excited-state equilibrium geometry of the $1{ }^{1} A^{\prime \prime}\left({ }^{1} n \pi^{*}\right)$ state of benzaldehyde (excited-state energy: $-344.57519 E_{h}$ ).

$\begin{array}{lrrr}14 & & & \\ & & & \\ \mathrm{C} & -0.4568739 & 2.2325450 & 0.0000000 \\ \mathrm{O} & -1.7643268 & 2.7378750 & 0.0000000 \\ \mathrm{H} & 0.2837069 & 3.0327113 & 0.0000000 \\ \mathrm{C} & -0.1650547 & 0.8760629 & 0.0000000 \\ \mathrm{C} & 1.1877703 & 0.4220675 & 0.0000000 \\ \mathrm{C} & -1.2172293 & -0.0875657 & 0.0000000 \\ \mathrm{C} & 1.4606710 & -0.9325651 & 0.0000000 \\ \mathrm{C} & -0.9128687 & -1.4388461 & 0.0000000 \\ \mathrm{C} & 0.4181752 & -1.8740662 & 0.0000000 \\ \mathrm{H} & 1.9937782 & 1.1488787 & 0.0000000 \\ \mathrm{H} & -2.2470025 & 0.2547132 & 0.0000000 \\ \mathrm{H} & 2.4905996 & -1.2706949 & 0.0000000 \\ \mathrm{H} & -1.7155567 & -2.1674671 & 0.0000000 \\ \mathrm{H} & 0.6442112 & -2.9336485 & 0.0000000\end{array}$


Table S630 Cartesian coordinates of the CC2/TZVP-optimized excited-state equilibrium geometry of the $2{ }^{1} A^{\prime}\left({ }^{1} \pi \pi^{*}\right)$ state of benzaldehyde (excited-state energy: $-344.52526 E_{h}$ ).

$\begin{array}{lrrr}14 & & & \\ & & & \\ \mathrm{C} & -0.1035334 & 2.3728989 & 0.0000000 \\ \mathrm{O} & -1.1744240 & 3.0156470 & 0.0000000 \\ \mathrm{H} & 0.8724811 & 2.8973609 & 0.0000000 \\ \mathrm{C} & -0.0292642 & 0.9339973 & 0.0000000 \\ \mathrm{C} & 1.2218486 & 0.2319995 & 0.0000000 \\ \mathrm{C} & -1.2235220 & 0.1423554 & 0.0000000 \\ \mathrm{C} & 1.2690036 & -1.1978602 & 0.0000000 \\ \mathrm{C} & -1.1595944 & -1.2881305 & 0.0000000 \\ \mathrm{C} & 0.0830688 & -1.9735949 & 0.0000000 \\ \mathrm{H} & 2.1475569 & 0.7981185 & 0.0000000 \\ \mathrm{H} & -2.1773315 & 0.6568495 & 0.0000000 \\ \mathrm{H} & 2.2359143 & -1.6864974 & 0.0000000 \\ \mathrm{H} & -2.0862301 & -1.8494860 & 0.0000000 \\ \mathrm{H} & 0.1240264 & -3.0536578 & 0.0000000\end{array}$


Table S631 Cartesian coordinates of the CC2/TZVP-optimized excited-state equilibrium geometry of the $1^{3} A^{\prime \prime}\left({ }^{3} n \pi^{*}\right)$ state of benzaldehyde (excited-state energy: $\left.-344.55978 E_{h}\right)$.

$\begin{array}{lrrr}14 & & & \\ & & & \\ \mathrm{C} & -0.4669397 & 2.2426685 & 0.0000000 \\ \mathrm{O} & -1.7544860 & 2.7375947 & 0.0000000 \\ \mathrm{H} & 0.2727300 & 3.0411670 & 0.0000000 \\ \mathrm{C} & -0.1760919 & 0.8720760 & 0.0000000 \\ \mathrm{C} & 1.1747116 & 0.4284856 & 0.0000000 \\ \mathrm{C} & -1.2160594 & -0.0964692 & 0.0000000 \\ \mathrm{C} & 1.4601073 & -0.9261571 & 0.0000000 \\ \mathrm{C} & -0.9026555 & -1.4478646 & 0.0000000 \\ \mathrm{C} & 0.4289056 & -1.8761751 & 0.0000000 \\ \mathrm{H} & 1.9765260 & 1.1597904 & 0.0000000 \\ \mathrm{H} & -2.2502962 & 0.2313041 & 0.0000000 \\ \mathrm{H} & 2.4937628 & -1.2529764 & 0.0000000 \\ \mathrm{H} & -1.7026893 & -2.1794685 & 0.0000000 \\ \mathrm{H} & 0.6624748 & -2.9339752 & 0.0000000\end{array}$


Table S632 Cartesian coordinates of the CC2/TZVP-optimized excited-state equilibrium geometry of the $1^{3} A^{\prime}\left({ }^{3} \pi \pi^{*}\right)$ state of benzaldehyde (excited-state energy: $-344.57378 E_{h}$ ).

14

$-1.6795095$

2.2729031

0.0000000

0.3650900

2.7131500

0.0000000

$-0.1712634$

2.9816919

0.0000000

1.2113513

0.8997552

0.0000000

$-1.2621861$

0.4239743

0.0000000

1.4742307

$-0.1056000$

0.0000000

$-0.9580830$

$-0.9080088$

0.0000000

0.3956672

$-1.4364841$

0.0000000

2. 0111628

$-1.8849501$

0.0000000

$-2.2792219$

1.1561892

0.0000000

2.4983049

0.2625849

0.0000000

$-1.7541749$

$-1.2619436$

0.0000000

0.6261329

$-2.1716864$

0.0000000

$-2.94157550 .0000000$ 
Table S633 Cartesian coordinates of the CC2/TZVP-optimized excited-state equilibrium geometry of the $1^{1} A_{u}\left({ }^{1} n \pi^{*}\right)$ state of $p$-benzoquinone (excited-state energy: $\left.-380.46517 E_{h}\right)$.

12

C $\quad 0.0000000$

1.4062621

0.0000000

C -1.2330838

$0.6922298 \quad 0.0000000$

C -1.2330838

C $\quad 0.0000000$

$-0.6922298 \quad 0.0000000$

C $\quad 1.2330838$

$\begin{array}{ll}-1.4062621 & 0.0000000\end{array}$

C $\quad 1.2330838$

$-0.6922298 \quad 0.0000000$

$0 \quad 0.0000000$

$0.6922298 \quad 0.0000000$

0.0000000

2.7172127

0.0000000

$-2.1598132$

$-2.7172127$

0.0000000

$-2.1598132$

1.2554898

0.0000000

$-1.2554898 \quad 0.0000000$

$\mathrm{H} \quad 2.1598132$

$-1.2554898$

0.0000000

$\mathrm{H}$

2.1598132

1. 2554898

0.0000000 
Table S634 Cartesian coordinates of the CC2/TZVP-optimized excited-state equilibrium geometry of the $1{ }^{1} B_{1 g}\left(\equiv 1^{1} B_{2 g}\right.$ in Turbomole) $\left({ }^{1} n \pi^{*}\right)$ state of p-benzoquinone (excited-state energy: $-380.46206 E_{h}$ ).

12

C $\quad 0.0000000$

1.4348914

0.0000000

C $\quad-1.2299950$

$0.6837110 \quad 0.0000000$

C $\quad-1.2299950$

$-0.6837110 \quad 0.0000000$

C $\quad 0.0000000$

$-1.43489140 .0000000$

C 1.2299950

$-0.6837110$

0.0000000

C $\quad 1.2299950$

0.6837110

0.0000000

0.0000000

2.7286146

0.0000000

O 0.0000000

$-2.7286146$

$-2.1543560$

1.2467969

$\mathrm{H} \quad-2.1543560-1.2467969$

0.0000000

$\mathrm{H} \quad 2.1543560$

$-1.2467969$

0.0000000

2.1543560

1.2467969

.0000000

0.0000000

0.0000000 
Table S635 Cartesian coordinates of the CC2/TZVP-optimized excited-state equilibrium geometry of the $1{ }^{1} B_{1 u}$ ( $\equiv 1^{1} B_{2 u}$ in Turbomole) $\left({ }^{1} \pi \pi^{*}\right)$ state of p-benzoquinone (excited-state energy: $-380.36222 E_{h}$ ).

12

C $\quad 0.0000000$

1.4445496

0.0000000

C $\quad-1.2449586$

$0.6966206 \quad 0.0000000$

C $\quad-1.2449586$

$-0.6966206$

0.0000000

C $\quad 0.0000000$

$-1.4445496 \quad 0.0000000$

C $\quad 1.2449586$

$-0.6966206$

0.0000000

C $\quad 1.2449586$

0.6966206

0.0000000

0.0000000

2.7352346

0.0000000

O 0.0000000

$-2.7352346$

0.0000000

$-2.1646394$

1.2713506

$\mathrm{H} \quad-2.1646394-1.2713506$

0.0000000

$-1.2713506$

0.0000000

H 2.1646394

1.2713506

0.0000000

H $\quad 2.1646394$

0.0000000 
Table S636 Cartesian coordinates of the CC2/TZVP-optimized excited-state equilibrium geometry of the $1{ }^{1} B_{3 g}$ ( $\equiv 1^{1} B_{1 g}$ in Turbomole) $\left({ }^{1} \pi \pi^{*}\right)$ state of p-benzoquinone (excited-state energy: $-380.40434 E_{h}$ ).

12

C $\quad 0.0000000$

1.4971683

0.0000000

C $\quad-1.1993658$

0.71132290 .0000000

C -1.1993658

$-0.71132290 .0000000$

C $\quad 0.0000000$

$-1.49716830 .0000000$

C

1.1993658

$-0.7113229$

0.0000000

$\mathrm{C}$

1.1993658

0.7113229

0.0000000

0.0000000

2.7761399

0.0000000

O 0.0000000

$-2.7761399$

$-2.1463813$

1. 2434352

$\mathrm{H} \quad-2.1463813-1.2434352$

0.0000000

$\mathrm{H} \quad 2.1463813$

$-1.2434352$

0.0000000

$\mathrm{H}$

2.1463813

1. 2434352

.0000000

0.0000000

0.0000000 
Table S637 Cartesian coordinates of the CC2/TZVP-optimized excited-state equilibrium geometry of the $1^{3} A_{u}\left({ }^{3} n \pi^{*}\right)$ state of $p$-benzoquinone (excited-state energy: $\left.-380.47636 E_{h}\right)$.

12

C $\quad 0.0000000$

1. 4066207

0.0000000

C -1.2339078

$0.6909601 \quad 0.0000000$

C -1.2339078

$-0.6909601 \quad 0.0000000$

C $\quad 0.0000000$

$-1.4066207 \quad 0.0000000$

C $\quad 1.2339078$

$\begin{array}{ll}-0.6909601 & 0.0000000\end{array}$

C 1.2339078

0.6909601

0.0000000

0.0000000

2.7134805

0.0000000

0.0000000

$-2.7134805$

0.0000000

$-2.1614289$

1.2529163

$-2.1614289-1.2529163$

0.0000000

$\begin{array}{ll}\mathrm{H} & 2.1614289 \\ \mathrm{H} & 2.1614289\end{array}$

$-1.2529163$

0.0000000

1.2529163

0.0000000

0.0000000 
Table S638 Cartesian coordinates of the CC2/TZVP-optimized excited-state equilibrium geometry of the $1{ }^{3} B_{1 g}$ ( $\equiv 1{ }^{3} B_{2 g}$ in Turbomole) $\left({ }^{3} n \pi^{*}\right)$ state of p-benzoquinone (excited-state energy: $-380.47379 E_{h}$ ).

12

C $\quad 0.0000000$

1.43381490 .0000000

C $\quad-1.2314092$

0.68256310 .0000000

C $\quad-1.2314092-0.6825631 \quad 0.0000000$

$\begin{array}{llll}\text { C } & 0.0000000 & -1.4338149 & 0.0000000\end{array}$

$\begin{array}{lllll}\text { C } & 1.2314092 & -0.6825631 & 0.0000000\end{array}$

$\begin{array}{llll}\text { C } & 1.2314092 & 0.6825631 & 0.0000000\end{array}$

$\begin{array}{lllll}0 & 0.0000000 & 2.7241907 & 0.0000000\end{array}$

$\begin{array}{lllll}0 & 0.0000000 & -2.7241907 & 0.0000000\end{array}$

$\mathrm{H} \quad-2.1558549 \quad 1.2451904 \quad 0.0000000$

$\mathrm{H} \quad-2.1558549-1.2451904 \quad 0.0000000$

$\mathrm{H} \quad 2.1558549 \quad-1.2451904 \quad 0.0000000$

$\mathrm{H} \quad 2.1558549 \quad 1.2451904 \quad 0.0000000$ 
Table S639 Cartesian coordinates of the CC2/TZVP-optimized excited-state equilibrium geometry of the $1{ }^{3} B_{1 u}$ ( $\equiv 1^{3} B_{2 u}$ in Turbomole) $\left({ }^{3} \pi \pi^{*}\right)$ state of p-benzoquinone (excited-state energy: $-380.46304 E_{h}$ ).

12

C $\quad 0.0000000$

1.4101175

0.0000000

C $\quad-1.2277881$

$0.7014377 \quad 0.0000000$

C $\quad-1.2277881$

$-0.7014377$

0.0000000

C $\quad 0.0000000$

$-1.41011750 .0000000$

C $\quad 1.2277881$

$-0.7014377$

0.0000000

C 1.2277881

0.7014377

0.0000000

o 0.0000000

2.7365112

0.0000000

0.0000000

$-2.7365112$

$-2.1487711 \quad 1.2713859$

0.0000000

$\mathrm{H} \quad-2.1487711-1.2713859$

0.0000000

$\mathrm{H} \quad 2.1487711$

$-1.2713859$

0.0000000

$\mathrm{H}$

2.1487711

1.2713859

0.0000000 
Table S640 Cartesian coordinates of the CC2/TZVP-optimized excited-state equilibrium geometry of the $1^{3} B_{3 g}$ ( $\equiv 1^{3} B_{1 g}$ in Turbomole) $\left({ }^{3} \pi \pi^{*}\right)$ state of p-benzoquinone (excited-state energy: $-380.44506 E_{h}$ ).

12

C $\quad 0.0000000$

1.5082769

0.0000000

C $\quad-1.2156737$

0.7154902

0.0000000

C $\quad-1.2156737$

$-0.7154902$

0.0000000

C $\quad 0.0000000$

$-1.50827690 .0000000$

C

1.2156737

$-0.7154902$

0.0000000

C

1.2156737

0.7154902

0.0000000

0.0000000

2.7619707

0.0000000

O 0.0000000

$-2.7619707$

0.0000000

$-2.1577416$

1.2525848

0.0000000

$-2.1577416$

$-1.2525848$

$\mathrm{H} \quad 2.1577416 \quad-1.2525848$

0.0000000

0.0000000

$\mathrm{H}$

2.1577416

1. 2525848

0.0000000 
Table S641 Cartesian coordinates of the CC2/TZVP-optimized excited-state equilibrium geometry of the $1^{1} B_{3 g}$ ( $\equiv 1^{1} B_{1 g}$ in Turbomole) $\left({ }^{1} \pi \pi^{*}\right)$ state of biphenylene (excited-state energy: $-460.66385 E_{h}$ ).

$\begin{array}{lrrr}20 & & & \\ & & & \\ \mathrm{C} & 0.7494025 & 0.7067727 & 0.0000000 \\ \mathrm{C} & 1.4768386 & 1.9097737 & 0.0000000 \\ \mathrm{C} & 0.7192553 & 3.0714881 & 0.0000000 \\ \mathrm{C} & 0.7494025 & -0.7067727 & 0.0000000 \\ \mathrm{C} & -0.7494025 & -0.7067727 & 0.0000000 \\ \mathrm{C} & -0.7494025 & 0.7067727 & 0.0000000 \\ \mathrm{C} & 1.4768386 & -1.9097737 & 0.0000000 \\ \mathrm{C} & -1.4768386 & -1.9097737 & 0.0000000 \\ \mathrm{C} & -1.4768386 & 1.9097737 & 0.0000000 \\ \mathrm{C} & 0.7192553 & -3.0714881 & 0.0000000 \\ \mathrm{C} & -0.7192553 & -3.0714881 & 0.0000000 \\ \mathrm{C} & -0.7192553 & 3.0714881 & 0.0000000 \\ \mathrm{H} & 2.5607946 & 1.9453623 & 0.0000000 \\ \mathrm{H} & 2.5607946 & -1.9453623 & 0.0000000 \\ \mathrm{H} & -2.5607946 & -1.9453623 & 0.0000000 \\ \mathrm{H} & -2.5607946 & 1.9453623 & 0.0000000 \\ \mathrm{H} & 1.2221607 & 4.0323711 & 0.0000000 \\ \mathrm{H} & 1.2221607 & -4.0323711 & 0.0000000 \\ \mathrm{H} & -1.2221607 & -4.0323711 & 0.0000000 \\ \mathrm{H} & -1.2221607 & 4.0323711 & 0.0000000\end{array}$


Table S642 Cartesian coordinates of the CC2/TZVP-optimized excited-state equilibrium geometry of the $1^{1} B_{1 u}$ ( $\equiv 1^{1} B_{2 u}$ in Turbomole) $\left({ }^{1} \pi \pi^{*}\right)$ state of biphenylene (excited-state energy: $-460.63970 E_{h}$ ).

$\begin{array}{lrrr}20 & & & \\ & & & \\ \mathrm{C} & 0.7229241 & 0.7268494 & 0.0000000 \\ \mathrm{C} & 1.4785684 & 1.9104627 & 0.0000000 \\ \mathrm{C} & 0.7046756 & 3.1033673 & 0.0000000 \\ \mathrm{C} & 0.7229241 & -0.7268494 & 0.0000000 \\ \mathrm{C} & -0.7229241 & -0.7268494 & 0.0000000 \\ \mathrm{C} & -0.7229241 & 0.7268494 & 0.0000000 \\ \mathrm{C} & 1.4785684 & -1.9104627 & 0.0000000 \\ \mathrm{C} & -1.4785684 & -1.9104627 & 0.0000000 \\ \mathrm{C} & -1.4785684 & 1.9104627 & 0.0000000 \\ \mathrm{C} & 0.7046756 & -3.1033673 & 0.0000000 \\ \mathrm{C} & -0.7046756 & -3.1033673 & 0.0000000 \\ \mathrm{C} & -0.7046756 & 3.1033673 & 0.0000000 \\ \mathrm{H} & 2.5611774 & 1.9339508 & 0.0000000 \\ \mathrm{H} & 2.5611774 & -1.9339508 & 0.0000000 \\ \mathrm{H} & -2.5611774 & -1.9339508 & 0.0000000 \\ \mathrm{H} & -2.5611774 & 1.9339508 & 0.0000000 \\ \mathrm{H} & 1.2147389 & 4.0602783 & 0.0000000 \\ \mathrm{H} & 1.2147389 & -4.0602783 & 0.0000000 \\ \mathrm{H} & -1.2147389 & -4.0602783 & 0.0000000 \\ \mathrm{H} & -1.2147389 & 4.0602783 & 0.0000000\end{array}$


Table S643 Cartesian coordinates of the CC2/TZVP-optimized excited-state equilibrium geometry of the $2{ }^{1} B_{1 u}$ ( $\equiv 2{ }^{1} B_{2 u}$ in Turbomole) $\left({ }^{1} \pi \pi^{*}\right)$ state of biphenylene (excited-state energy: $-460.57553 E_{h}$ ).

$\begin{array}{lrrr}20 & & & \\ & & & \\ \mathrm{C} & 0.7202922 & 0.7331338 & 0.0000000 \\ \mathrm{C} & 1.4628753 & 1.9253172 & 0.0000000 \\ \mathrm{C} & 0.7027750 & 3.1261463 & 0.0000000 \\ \mathrm{C} & 0.7202922 & -0.7331338 & 0.0000000 \\ \mathrm{C} & -0.7202922 & -0.7331338 & 0.0000000 \\ \mathrm{C} & -0.7202922 & 0.7331338 & 0.0000000 \\ \mathrm{C} & 1.4628753 & -1.9253172 & 0.0000000 \\ \mathrm{C} & -1.4628753 & -1.9253172 & 0.0000000 \\ \mathrm{C} & -1.4628753 & 1.9253172 & 0.0000000 \\ \mathrm{C} & 0.7027750 & -3.1261463 & 0.0000000 \\ \mathrm{C} & -0.7027750 & -3.1261463 & 0.0000000 \\ \mathrm{C} & -0.7027750 & 3.1261463 & 0.0000000 \\ \mathrm{H} & 2.5475334 & 1.9477006 & 0.0000000 \\ \mathrm{H} & 2.5475334 & -1.9477006 & 0.0000000 \\ \mathrm{H} & -2.5475334 & -1.9477006 & 0.0000000 \\ \mathrm{H} & -2.5475334 & 1.9477006 & 0.0000000 \\ \mathrm{H} & 1.2243260 & 4.0771830 & 0.0000000 \\ \mathrm{H} & 1.2243260 & -4.0771830 & 0.0000000 \\ \mathrm{H} & -1.2243260 & -4.0771830 & 0.0000000 \\ \mathrm{H} & -1.2243260 & 4.0771830 & 0.0000000\end{array}$


Table S644 Cartesian coordinates of the CC2/TZVP-optimized excited-state equilibrium geometry of the $1{ }^{1} B_{2 u}$ ( $\equiv 1^{1} B_{3 u}$ in Turbomole) $\left({ }^{1} \pi \pi^{*}\right)$ state of biphenylene (excited-state energy: $-460.56250 E_{h}$ ).

$\begin{array}{lrrr}20 & & & \\ & & & \\ \mathrm{C} & 0.7377570 & 0.7309417 & 0.0000000 \\ \mathrm{C} & 1.4755502 & 1.9087966 & 0.0000000 \\ \mathrm{C} & 0.7327415 & 3.1048131 & 0.0000000 \\ \mathrm{C} & 0.7377570 & -0.7309417 & 0.0000000 \\ \mathrm{C} & -0.7377570 & -0.7309417 & 0.0000000 \\ \mathrm{C} & -0.7377570 & 0.7309417 & 0.0000000 \\ \mathrm{C} & 1.4755502 & -1.9087966 & 0.0000000 \\ \mathrm{C} & -1.4755502 & -1.9087966 & 0.0000000 \\ \mathrm{C} & -1.4755502 & 1.9087966 & 0.0000000 \\ \mathrm{C} & 0.7327415 & -3.1048131 & 0.0000000 \\ \mathrm{C} & -0.7327415 & -3.1048131 & 0.0000000 \\ \mathrm{C} & -0.7327415 & 3.1048131 & 0.0000000 \\ \mathrm{H} & 2.5605505 & 1.9265092 & 0.0000000 \\ \mathrm{H} & 2.5605505 & -1.9265092 & 0.0000000 \\ \mathrm{H} & -2.5605505 & -1.9265092 & 0.0000000 \\ \mathrm{H} & -2.5605505 & 1.9265092 & 0.0000000 \\ \mathrm{H} & 1.2474762 & 4.0595144 & 0.0000000 \\ \mathrm{H} & 1.2474762 & -4.0595144 & 0.0000000 \\ \mathrm{H} & -1.2474762 & -4.0595144 & 0.0000000 \\ \mathrm{H} & -1.2474762 & 4.0595144 & 0.0000000\end{array}$


Table S645 Cartesian coordinates of the CC2/TZVP-optimized excited-state equilibrium geometry of the $1^{1} B_{u}\left({ }^{1} \pi \pi^{*}\right)$ state of trans-butadiene (excited-state energy: $-155.31642 E_{h}$ ).

$\begin{array}{lrrr}10 & & & \\ & & & \\ \mathrm{C} & -0.0253682 & 0.6963287 & 0.0000000 \\ \mathrm{C} & 0.0253682 & -0.6963287 & 0.0000000 \\ \mathrm{C} & 1.2279481 & -1.4579153 & 0.0000000 \\ \mathrm{C} & -1.2279481 & 1.4579153 & 0.0000000 \\ \mathrm{H} & 1.1992445 & -2.5386532 & 0.0000000 \\ \mathrm{H} & -1.1992445 & 2.5386532 & 0.0000000 \\ \mathrm{H} & 2.1919308 & -0.9615490 & 0.0000000 \\ \mathrm{H} & -2.1919308 & 0.9615490 & 0.0000000 \\ \mathrm{H} & -0.9173736 & -1.2389326 & 0.0000000 \\ \mathrm{H} & 0.9173736 & 1.2389326 & 0.0000000\end{array}$


Table S646 Cartesian coordinates of the CC2/TZVP-optimized excited-state equilibrium geometry of the $2{ }^{1} A_{g}\left({ }^{1} \pi \pi^{*}\right)$ state of trans-butadiene (excited-state energy: $-155.28573 E_{h}$ ).

$\begin{array}{lrrr}10 & & & \\ & & & \\ \mathrm{C} & 0.0204683 & 0.6989444 & 0.0000000 \\ \mathrm{C} & -0.0204683 & -0.6989444 & 0.0000000 \\ \mathrm{C} & 1.2620334 & -1.4394297 & 0.0000000 \\ \mathrm{C} & -1.2620334 & 1.4394297 & 0.0000000 \\ \mathrm{H} & 1.3009896 & -2.5205924 & 0.0000000 \\ \mathrm{H} & -1.3009896 & 2.5205924 & 0.0000000 \\ \mathrm{H} & 2.1883161 & -0.8761626 & 0.0000000 \\ \mathrm{H} & -2.1883161 & 0.8761626 & 0.0000000 \\ \mathrm{H} & -0.9666516 & -1.2273260 & 0.0000000 \\ \mathrm{H} & 0.9666516 & 1.2273260 & 0.0000000\end{array}$


Table S647 Cartesian coordinates of the CC2/TZVP-optimized excited-state equilibrium geometry of the $2{ }^{1} A\left({ }^{1} n \pi^{*}\right)$ state of cytosine (excited-state energy: $\left.-393.89756 E_{h}\right)$.

$\begin{array}{lrrr}13 & & & \\ & & & \\ \mathrm{~N} & -0.0196698 & 0.0388157 & -0.1699130 \\ \mathrm{C} & 1.4048060 & 0.0356974 & -0.1025187 \\ \mathrm{C} & 2.1209855 & 1.1923339 & 0.0536212 \\ \mathrm{C} & 1.4593572 & 2.4296754 & 0.2200283 \\ \mathrm{~N} & 0.0617642 & 2.4181868 & -0.1098487 \\ \mathrm{C} & -0.5274418 & 1.2003347 & -0.1278004 \\ \mathrm{~N} & 1.9486029 & -1.2290820 & -0.3502928 \\ \mathrm{O} & -1.9558937 & 1.2724980 & -0.1760310 \\ \mathrm{H} & 3.2017423 & 1.1666208 & 0.1341437 \\ \mathrm{H} & 1.9440300 & 3.3901104 & 0.1212124 \\ \mathrm{H} & 1.3152755 & -1.9639337 & -0.0585552 \\ \mathrm{H} & 2.8683444 & -1.3571541 & 0.0528677 \\ \mathrm{H} & -0.5025285 & 3.2139684 & 0.1617217\end{array}$


Table S648 Cartesian coordinates of the CC2/TZVP-optimized excited-state equilibrium geometry of the $3{ }^{1} A\left({ }^{1} \pi \pi^{*}\right)$ state of cytosine (excited-state energy: $\left.-393.87650 E_{h}\right)$.

$\begin{array}{lrrr}13 & & & \\ & & & \\ \mathrm{~N} & 0.0032996 & 0.0142465 & 0.0114051 \\ \mathrm{C} & 1.4638917 & 0.0201699 & 0.0198187 \\ \mathrm{C} & 2.1789525 & 1.1776862 & 0.0120731 \\ \mathrm{C} & 1.5207524 & 2.4325946 & -0.1069837 \\ \mathrm{~N} & 0.1161655 & 2.3794870 & 0.0375191 \\ \mathrm{C} & -0.5230686 & 1.1765575 & 0.0158878 \\ \mathrm{~N} & 1.9599763 & -1.2616241 & 0.2164274 \\ \mathrm{O} & -1.9043833 & 1.3236088 & 0.0317610 \\ \mathrm{H} & 3.2629052 & 1.1610512 & 0.0208892 \\ \mathrm{H} & 1.9955568 & 3.3901572 & 0.0448438 \\ \mathrm{H} & 1.3293256 & -1.9678955 & -0.1424909 \\ \mathrm{H} & 2.9143620 & -1.4006821 & -0.0886385 \\ \mathrm{H} & -0.4692274 & 3.1983155 & -0.0722243\end{array}$


Table S649 Cartesian coordinates of the CC2/TZVP-optimized excited-state equilibrium geometry of the $1^{1} A_{2}\left({ }^{1} n \pi^{*}\right)$ state of diazomethane (excited-state energy: $-148.28306 E_{h}$ ).

\section{5}

C $\quad 0.0000000$

0.0000000

$-0.5604132$

$\mathrm{N} \quad 0.0000000$

0.0000000

0.7783410

N $\quad 0.0000000$

$0.0000000 \quad 1.9884548$

$\mathrm{H} \quad 0.9437116 \quad 0.0000000 \quad-1.1031913$

$\mathrm{H} \quad-0.9437116 \quad 0.0000000 \quad-1.1031913$ 
Table S650 Cartesian coordinates of the CC2/TZVP-optimized excited-state equilibrium geometry of the $1{ }^{1} B_{3 g}$ ( $\equiv 1^{1} B_{1 g}$ in Turbomole) $\left({ }^{1} \pi \pi^{*}\right)$ state of dibenzo- $p$-dioxin (excited-state energy: $-610.84116 E_{h}$ ).

$\begin{array}{lrrr}22 & & & \\ & & & \\ \text { C } & -3.5713427 & 0.7197064 & 0.0000000 \\ \text { C } & -3.5713427 & -0.7197064 & 0.0000000 \\ \text { C } & -2.3836285 & -1.4197206 & 0.0000000 \\ \text { C } & -1.1645850 & -0.7235418 & 0.0000000 \\ \text { C } & -1.1645850 & 0.7235418 & 0.0000000 \\ \text { C } & -2.3836285 & 1.4197206 & 0.0000000 \\ \text { O } & 0.0000000 & -1.4220600 & 0.0000000 \\ \text { C } & 1.1645850 & -0.7235418 & 0.0000000 \\ \text { C } & 1.1645850 & 0.7235418 & 0.0000000 \\ \text { O } & 0.0000000 & 1.4220600 & 0.0000000 \\ \text { C } & 2.3836285 & -1.4197206 & 0.0000000 \\ \text { C } & 3.5713427 & -0.7197064 & 0.0000000 \\ \text { C } & 3.5713427 & 0.7197064 & 0.0000000 \\ \text { C } & 2.3836285 & 1.4197206 & 0.0000000 \\ \text { H } & 4.5107420 & 1.2576573 & 0.0000000 \\ \text { H } & -4.5107420 & -1.2576573 & 0.0000000 \\ \text { H } & -2.3576550 & -2.5038700 & 0.0000000 \\ \text { H } & -2.3576550 & 2.5038700 & 0.0000000 \\ \text { H } & 2.3576550 & -2.5038700 & 0.0000000 \\ \text { H } & 4.5107420 & -1.2576573 & 0.0000000 \\ \text { H } & -4.5107420 & 1.2576573 & 0.0000000 \\ \text { H } & 2.3576550 & 2.5038700 & 0.0000000\end{array}$


Table S651 Cartesian coordinates of the CC2/TZVP-optimized excited-state equilibrium geometry of the $1^{1} B_{2 u}$ ( $\equiv 1^{1} B_{3 u}$ in Turbomole) $\left({ }^{1} \pi \pi^{*}\right)$ state of dibenzo- $p$-dioxin (excited-state energy: $-610.83472 E_{h}$ ).

$\begin{array}{lrrr}22 & & & \\ & & & \\ \text { C } & -3.5836059 & 0.7031219 & 0.0000000 \\ \text { C } & -3.5836059 & -0.7031219 & 0.0000000 \\ \text { C } & -2.3844828 & -1.4362181 & 0.0000000 \\ \text { C } & -1.1734951 & -0.7020813 & 0.0000000 \\ \text { C } & -1.1734951 & 0.7020813 & 0.0000000 \\ \text { C } & -2.3844828 & 1.4362181 & 0.0000000 \\ \text { O } & 0.0000000 & -1.4093597 & 0.0000000 \\ \text { C } & 1.1734951 & -0.7020813 & 0.0000000 \\ \text { C } & 1.1734951 & 0.7020813 & 0.0000000 \\ \text { O } & 0.0000000 & 1.4093597 & 0.0000000 \\ \text { C } & 2.3844828 & -1.4362181 & 0.0000000 \\ \text { C } & 3.5836059 & -0.7031219 & 0.0000000 \\ \text { C } & 3.5836059 & 0.7031219 & 0.0000000 \\ \text { C } & 2.3844828 & 1.4362181 & 0.0000000 \\ \text { H } & 4.5282487 & 1.2332368 & 0.0000000 \\ \text { H } & -4.5282487 & -1.2332368 & 0.0000000 \\ \text { H } & -2.3513507 & -2.5172826 & 0.0000000 \\ \text { H } & -2.3513507 & 2.5172826 & 0.0000000 \\ \text { H } & 2.3513507 & -2.5172826 & 0.0000000 \\ \text { H } & 4.5282487 & -1.2332368 & 0.0000000 \\ \text { H } & -4.5282487 & 1.2332368 & 0.0000000 \\ \text { H } & 2.3513507 & 2.5172826 & 0.0000000\end{array}$


Table S652 Cartesian coordinates of the CC2/TZVP-optimized excited-state equilibrium geometry of the $1^{1} B_{1 u}$ ( $\equiv 1^{1} B_{2 u}$ in Turbomole) $\left({ }^{1} \pi \pi^{*}\right)$ state of dibenzo- $p$-dioxin (excited-state energy: $-610.77423 E_{h}$ ).

$\begin{array}{lrrr}22 & & & \\ & & & \\ \text { C } & -3.5925569 & 0.7222369 & 0.0000000 \\ \text { C } & -3.5925569 & -0.7222369 & 0.0000000 \\ \text { C } & -2.3798290 & -1.4162634 & 0.0000000 \\ \text { C } & -1.1775960 & -0.7122852 & 0.0000000 \\ \text { C } & -1.1775960 & 0.7122852 & 0.0000000 \\ \text { C } & -2.3798290 & 1.4162634 & 0.0000000 \\ \text { O } & 0.0000000 & -1.4336470 & 0.0000000 \\ \text { C } & 1.1775960 & -0.7122852 & 0.0000000 \\ \text { C } & 1.1775960 & 0.7122852 & 0.0000000 \\ \text { O } & 0.0000000 & 1.4336470 & 0.0000000 \\ \text { C } & 2.3798290 & -1.4162634 & 0.0000000 \\ \text { C } & 3.5925569 & -0.7222369 & 0.0000000 \\ \text { C } & 3.5925569 & 0.7222369 & 0.0000000 \\ \text { C } & 2.3798290 & 1.4162634 & 0.0000000 \\ \text { H } & 4.5259201 & 1.2696957 & 0.0000000 \\ \text { H } & -4.5259201 & -1.2696957 & 0.0000000 \\ \text { H } & -2.3379846 & -2.4997245 & 0.0000000 \\ \text { H } & -2.3379846 & 2.4997245 & 0.0000000 \\ \text { H } & 2.3379846 & -2.4997245 & 0.0000000 \\ \text { H } & 4.5259201 & -1.2696957 & 0.0000000 \\ \text { H } & -4.5259201 & 1.2696957 & 0.0000000 \\ \text { H } & 2.3379846 & 2.4997245 & 0.0000000\end{array}$


Table S653 Cartesian coordinates of the CC2/TZVP-optimized excited-state equilibrium geometry of the $1{ }^{3} A_{g}\left({ }^{3} \pi \pi^{*}\right)$ state of dibenzo- $p$-dioxin (excitedstate energy: $-610.84432 E_{h}$ ).

$\begin{array}{lrrr}22 & & & \\ & & & \\ \text { C } & -3.5640964 & 0.7005106 & 0.0000000 \\ \text { C } & -3.5640964 & -0.7005106 & 0.0000000 \\ \text { C } & -2.3678663 & -1.4349425 & 0.0000000 \\ \text { C } & -1.1620248 & -0.7026946 & 0.0000000 \\ \text { C } & -1.1620248 & 0.7026946 & 0.0000000 \\ \text { C } & -2.3678663 & 1.4349425 & 0.0000000 \\ \text { O } & 0.0000000 & -1.4290202 & 0.0000000 \\ \text { C } & 1.1620248 & -0.7026946 & 0.0000000 \\ \mathrm{C} & 1.1620248 & 0.7026946 & 0.0000000 \\ \text { O } & 0.0000000 & 1.4290202 & 0.0000000 \\ \mathrm{C} & 2.3678663 & -1.4349425 & 0.0000000 \\ \mathrm{C} & 3.5640964 & -0.7005106 & 0.0000000 \\ \mathrm{C} & 3.5640964 & 0.7005106 & 0.0000000 \\ \mathrm{C} & 2.3678663 & 1.4349425 & 0.0000000 \\ \mathrm{H} & 4.5097953 & 1.2290175 & 0.0000000 \\ \mathrm{H} & -4.5097953 & -1.2290175 & 0.0000000 \\ \mathrm{H} & -2.3315170 & -2.5153683 & 0.0000000 \\ \mathrm{H} & -2.3315170 & 2.5153683 & 0.0000000 \\ \mathrm{H} & 2.3315170 & -2.5153683 & 0.0000000 \\ \mathrm{H} & 4.5097953 & -1.2290175 & 0.0000000 \\ \mathrm{H} & -4.5097953 & 1.2290175 & 0.0000000 \\ \mathrm{H} & 2.3315170 & 2.5153683 & 0.0000000\end{array}$


Table S654 Cartesian coordinates of the CC2/TZVP-optimized excited-state equilibrium geometry of the $1{ }^{3} B_{3 g}$ ( $\equiv 1^{3} B_{1 g}$ in Turbomole) $\left({ }^{3} \pi \pi^{*}\right)$ state of dibenzo- $p$-dioxin (excited-state energy: $-610.87202 E_{h}$ ).

$\begin{array}{lrrr}22 & & & \\ & & & \\ \text { C } & -3.5740249 & 0.7224145 & 0.0000000 \\ \text { C } & -3.5740249 & -0.7224145 & 0.0000000 \\ \text { C } & -2.3827861 & -1.4210340 & 0.0000000 \\ \text { C } & -1.1671695 & -0.7246080 & 0.0000000 \\ \text { C } & -1.1671695 & 0.7246080 & 0.0000000 \\ \text { C } & -2.3827861 & 1.4210340 & 0.0000000 \\ \text { O } & 0.0000000 & -1.4257274 & 0.0000000 \\ \text { C } & 1.1671695 & -0.7246080 & 0.0000000 \\ \text { C } & 1.1671695 & 0.7246080 & 0.0000000 \\ \text { O } & 0.0000000 & 1.4257274 & 0.0000000 \\ \text { C } & 2.3827861 & -1.4210340 & 0.0000000 \\ \text { C } & 3.5740249 & -0.7224145 & 0.0000000 \\ \text { C } & 3.5740249 & 0.7224145 & 0.0000000 \\ \text { C } & 2.3827861 & 1.4210340 & 0.0000000 \\ \text { H } & 4.5134376 & 1.2595700 & 0.0000000 \\ \text { H } & -4.5134376 & -1.2595700 & 0.0000000 \\ \text { H } & -2.3557248 & -2.5046810 & 0.0000000 \\ \text { H } & -2.3557248 & 2.5046810 & 0.0000000 \\ \text { H } & 2.3557248 & -2.5046810 & 0.0000000 \\ \text { H } & 4.5134376 & -1.2595700 & 0.0000000 \\ \text { H } & -4.5134376 & 1.2595700 & 0.0000000 \\ \text { H } & 2.3557248 & 2.5046810 & 0.0000000\end{array}$


Table S655 Cartesian coordinates of the CC2/TZVP-optimized excited-state equilibrium geometry of the $1{ }^{3} B_{2 u}$ ( $\equiv 1^{3} B_{3 u}$ in Turbomole) $\left({ }^{3} \pi \pi^{*}\right)$ state of dibenzo- $p$-dioxin (excited-state energy: $-610.84208 E_{h}$ ).

$\begin{array}{lrrr}22 & & & \\ & & & \\ \text { C } & -3.5784180 & 0.6995532 & 0.0000000 \\ \text { C } & -3.5784180 & -0.6995532 & 0.0000000 \\ \text { C } & -2.3809343 & -1.4385095 & 0.0000000 \\ \text { C } & -1.1757772 & -0.7032384 & 0.0000000 \\ \text { C } & -1.1757772 & 0.7032384 & 0.0000000 \\ \text { C } & -2.3809343 & 1.4385095 & 0.0000000 \\ \text { O } & 0.0000000 & -1.4013277 & 0.0000000 \\ \text { C } & 1.1757772 & -0.7032384 & 0.0000000 \\ \text { C } & 1.1757772 & 0.7032384 & 0.0000000 \\ \text { O } & 0.0000000 & 1.4013277 & 0.0000000 \\ \text { C } & 2.3809343 & -1.4385095 & 0.0000000 \\ \text { C } & 3.5784180 & -0.6995532 & 0.0000000 \\ \text { C } & 3.5784180 & 0.6995532 & 0.0000000 \\ \text { C } & 2.3809343 & 1.4385095 & 0.0000000 \\ \text { H } & 4.5240273 & 1.2281305 & 0.0000000 \\ \text { H } & -4.5240273 & -1.2281305 & 0.0000000 \\ \text { H } & -2.3477003 & -2.5189741 & 0.0000000 \\ \text { H } & -2.3477003 & 2.5189741 & 0.0000000 \\ \text { H } & 2.3477003 & -2.5189741 & 0.0000000 \\ \text { H } & 4.5240273 & -1.2281305 & 0.0000000 \\ \text { H } & -4.5240273 & 1.2281305 & 0.0000000 \\ \text { H } & 2.3477003 & 2.5189741 & 0.0000000\end{array}$


Table S656 Cartesian coordinates of the CC2/TZVP-optimized excited-state equilibrium geometry of the $1{ }^{3} B_{1 u}$ ( $\equiv 1{ }^{3} B_{2 u}$ in Turbomole) $\left({ }^{3} \pi \pi^{*}\right)$ state of dibenzo- $p$-dioxin (excited-state energy: $-610.84416 E_{h}$ ).

$\begin{array}{lrrr}22 & & & \\ & & & \\ \text { C } & -3.6090951 & 0.6961811 & 0.0000000 \\ \text { C } & -3.6090951 & -0.6961811 & 0.0000000 \\ \text { C } & -2.3806476 & -1.4114321 & 0.0000000 \\ \text { C } & -1.1606847 & -0.6965762 & 0.0000000 \\ \text { C } & -1.1606847 & 0.6965762 & 0.0000000 \\ \text { C } & -2.3806476 & 1.4114321 & 0.0000000 \\ \text { O } & 0.0000000 & -1.4541737 & 0.0000000 \\ \text { C } & 1.1606847 & -0.6965762 & 0.0000000 \\ \text { C } & 1.1606847 & 0.6965762 & 0.0000000 \\ \text { O } & 0.0000000 & 1.4541737 & 0.0000000 \\ \text { C } & 2.3806476 & -1.4114321 & 0.0000000 \\ \text { C } & 3.6090951 & -0.6961811 & 0.0000000 \\ \text { C } & 3.6090951 & 0.6961811 & 0.0000000 \\ \text { C } & 2.3806476 & 1.4114321 & 0.0000000 \\ \text { H } & 4.5419191 & 1.2449004 & 0.0000000 \\ \text { H } & -4.5419191 & -1.2449004 & 0.0000000 \\ \text { H } & -2.3461165 & -2.4928352 & 0.0000000 \\ \text { H } & -2.3461165 & 2.4928352 & 0.0000000 \\ \text { H } & 2.3461165 & -2.4928352 & 0.0000000 \\ \text { H } & 4.5419191 & -1.2449004 & 0.0000000 \\ \text { H } & -4.5419191 & 1.2449004 & 0.0000000 \\ \text { H } & 2.3461165 & 2.4928352 & 0.0000000\end{array}$


Table S657 Cartesian coordinates of the CC2/TZVP-optimized excited-state equilibrium geometry of the $1{ }^{1} A_{u}\left({ }^{1} n \pi^{*}\right)$ state of dibenzo- $p$-dioxin (excitedstate energy: $\left.-610.72572 E_{h}\right)$.

$\begin{array}{lrrr}22 & & & \\ & & & \\ \text { C } & -3.6347244 & 0.7111715 & 0.0000000 \\ \text { C } & -3.6347244 & -0.7111715 & 0.0000000 \\ \text { C } & -2.4308272 & -1.3960990 & 0.0000000 \\ \text { C } & -1.2050387 & -0.7363366 & 0.0000000 \\ \text { C } & -1.2050387 & 0.7363366 & 0.0000000 \\ \text { C } & -2.4308272 & 1.3960990 & 0.0000000 \\ \text { O } & -0.0000000 & -1.3669034 & 0.0000000 \\ \text { C } & 1.2050387 & -0.7363366 & 0.0000000 \\ \mathrm{C} & 1.2050387 & 0.7363366 & 0.0000000 \\ \text { O } & 0.0000000 & 1.3669034 & 0.0000000 \\ \mathrm{C} & 2.4308272 & -1.3960990 & 0.0000000 \\ \mathrm{C} & 3.6347244 & -0.7111715 & 0.0000000 \\ \mathrm{C} & 3.6347244 & 0.7111715 & 0.0000000 \\ \mathrm{C} & 2.4308272 & 1.3960990 & 0.0000000 \\ \mathrm{H} & 4.5649423 & 1.2637700 & 0.0000000 \\ \mathrm{H} & -4.5649423 & -1.2637700 & 0.0000000 \\ \mathrm{H} & -2.3942142 & -2.4818488 & 0.0000000 \\ \mathrm{H} & -2.3942142 & 2.4818488 & 0.0000000 \\ \mathrm{H} & 2.3942142 & -2.4818488 & 0.0000000 \\ \mathrm{H} & 4.5649423 & -1.2637700 & 0.0000000 \\ \mathrm{H} & -4.5649423 & 1.2637700 & 0.0000000 \\ \mathrm{H} & 2.3942142 & 2.4818488 & 0.0000000\end{array}$

S704 
Table S658 Cartesian coordinates of the CC2/TZVP-optimized excited-state equilibrium geometry of the $1{ }^{1} B_{1 g}\left(\equiv 2{ }^{1} B_{2 g}\right.$ in Turbomole) $\left({ }^{1} n \pi^{*}\right)$ state of dibenzo- $p$-dioxin (excited-state energy: $-610.70605 E_{h}$ ).

$\begin{array}{lrrr}22 & & & \\ & & & \\ \text { C } & -3.6396890 & 0.6918555 & 0.0000000 \\ \text { C } & -3.6396890 & -0.6918555 & 0.0000000 \\ \text { C } & -2.4293415 & -1.4174350 & 0.0000000 \\ \text { C } & -1.2128406 & -0.7075261 & 0.0000000 \\ \text { C } & -1.2128406 & 0.7075261 & 0.0000000 \\ \text { C } & -2.4293415 & 1.4174350 & 0.0000000 \\ \text { O } & 0.0000000 & -1.3446513 & 0.0000000 \\ \text { C } & 1.2128406 & -0.7075261 & 0.0000000 \\ \text { C } & 1.2128406 & 0.7075261 & 0.0000000 \\ \text { O } & 0.0000000 & 1.3446513 & 0.0000000 \\ \text { C } & 2.4293415 & -1.4174350 & 0.0000000 \\ \text { C } & 3.6396890 & -0.6918555 & 0.0000000 \\ \text { C } & 3.6396890 & 0.6918555 & 0.0000000 \\ \text { C } & 2.4293415 & 1.4174350 & 0.0000000 \\ \text { H } & 4.5781956 & 1.2328755 & 0.0000000 \\ \text { H } & -4.5781956 & -1.2328755 & 0.0000000 \\ \text { H } & -2.3786617 & -2.4999566 & 0.0000000 \\ \text { H } & -2.3786617 & 2.4999566 & 0.0000000 \\ \text { H } & 2.3786617 & -2.4999566 & 0.0000000 \\ \text { H } & 4.5781956 & -1.2328755 & 0.0000000 \\ \text { H } & -4.5781956 & 1.2328755 & 0.0000000 \\ \text { H } & 2.3786617 & 2.4999566 & 0.0000000\end{array}$


Table S659 Cartesian coordinates of the CC2/TZVP-optimized excited-state equilibrium geometry of the $1{ }^{1} B_{3 u}$ ( $\equiv 3{ }^{1} B_{1 u}$ in Turbomole) $\left({ }^{1} n \pi^{*}\right)$ state of dibenzo- $p$-dioxin (excited-state energy: $-610.70260 E_{h}$ ).

$\begin{array}{lrrr}22 & & & \\ & & & \\ \text { C } & -3.6235027 & 0.6947716 & 0.0000000 \\ \text { C } & -3.6235027 & -0.6947716 & 0.0000000 \\ \text { C } & -2.4160022 & -1.4110897 & 0.0000000 \\ \text { C } & -1.1967731 & -0.7044922 & 0.0000000 \\ \text { C } & -1.1967731 & 0.7044922 & 0.0000000 \\ \text { C } & -2.4160022 & 1.4110897 & 0.0000000 \\ \text { O } & 0.0000000 & -1.3878908 & 0.0000000 \\ \text { C } & 1.1967731 & -0.7044922 & 0.0000000 \\ \text { C } & 1.1967731 & 0.7044922 & 0.0000000 \\ \text { O } & 0.0000000 & 1.3878908 & 0.0000000 \\ \text { C } & 2.4160022 & -1.4110897 & 0.0000000 \\ \text { C } & 3.6235027 & -0.6947716 & 0.0000000 \\ \text { C } & 3.6235027 & 0.6947716 & 0.0000000 \\ \text { C } & 2.4160022 & 1.4110897 & 0.0000000 \\ \text { H } & 4.5640177 & 1.2327194 & 0.0000000 \\ \text { H } & -4.5640177 & -1.2327194 & 0.0000000 \\ \text { H } & -2.3623069 & -2.4942689 & 0.0000000 \\ \text { H } & -2.3623069 & 2.4942689 & 0.0000000 \\ \text { H } & 2.3623069 & -2.4942689 & 0.0000000 \\ \text { H } & 4.5640177 & -1.2327194 & 0.0000000 \\ \text { H } & -4.5640177 & 1.2327194 & 0.0000000 \\ \text { H } & 2.3623069 & 2.4942689 & 0.0000000\end{array}$


Table S660 Cartesian coordinates of the CC2/TZVP-optimized excited-state equilibrium geometry of the $1{ }^{1} B_{2 g}$ ( $\equiv 9{ }^{1} B_{3 g}$ in Turbomole) $\left({ }^{1} n \pi^{*}\right)$ state of dibenzo- $p$-dioxin (excited-state energy: $-610.64658 E_{h}$ ).

$\begin{array}{lrrr}22 & & & \\ & & & \\ \text { C } & -3.6147054 & 0.7102494 & 0.0000000 \\ \text { C } & -3.6147054 & -0.7102494 & 0.0000000 \\ \text { C } & -2.3962057 & -1.4013888 & 0.0000000 \\ \text { C } & -1.1878313 & -0.7108771 & 0.0000000 \\ \text { C } & -1.1878313 & 0.7108771 & 0.0000000 \\ \text { C } & -2.3962057 & 1.4013888 & 0.0000000 \\ \text { O } & 0.0000000 & -1.4242581 & 0.0000000 \\ \text { C } & 1.1878313 & -0.7108771 & 0.0000000 \\ \text { C } & 1.1878313 & 0.7108771 & 0.0000000 \\ \text { O } & 0.0000000 & 1.4242581 & 0.0000000 \\ \text { C } & 2.3962057 & -1.4013888 & 0.0000000 \\ \text { C } & 3.6147054 & -0.7102494 & 0.0000000 \\ \text { C } & 3.6147054 & 0.7102494 & 0.0000000 \\ \text { C } & 2.3962057 & 1.4013888 & 0.0000000 \\ \text { H } & 4.5665259 & 1.2329129 & 0.0000000 \\ \text { H } & -4.5665259 & -1.2329129 & 0.0000000 \\ \text { H } & -2.3614980 & -2.4924090 & 0.0000000 \\ \text { H } & -2.3614980 & 2.4924090 & 0.0000000 \\ \text { H } & 2.3614980 & -2.4924090 & 0.0000000 \\ \text { H } & 4.5665259 & -1.2329129 & 0.0000000 \\ \text { H } & -4.5665259 & 1.2329129 & 0.0000000 \\ \text { H } & 2.3614980 & 2.4924090 & 0.0000000\end{array}$


Table S661 Cartesian coordinates of the CC2/TZVP-optimized excited-state equilibrium geometry of the $1{ }^{3} A_{u}\left({ }^{3} n \pi^{*}\right)$ state of dibenzo- $p$-dioxin (excitedstate energy: $-610.72819 E_{h}$ ).

$\begin{array}{lrrr}22 & & & \\ & & & \\ \text { C } & -3.6342606 & 0.7112501 & 0.0000000 \\ \text { C } & -3.6342606 & -0.7112501 & 0.0000000 \\ \text { C } & -2.4310739 & -1.3955935 & 0.0000000 \\ \text { C } & -1.2040593 & -0.7365559 & 0.0000000 \\ \text { C } & -1.2040593 & 0.7365559 & 0.0000000 \\ \text { C } & -2.4310739 & 1.3955935 & 0.0000000 \\ \text { O } & 0.0000000 & -1.3685076 & 0.0000000 \\ \text { C } & 1.2040593 & -0.7365559 & 0.0000000 \\ \mathrm{C} & 1.2040593 & 0.7365559 & 0.0000000 \\ \text { O } & 0.0000000 & 1.3685076 & 0.0000000 \\ \mathrm{C} & 2.4310739 & -1.3955935 & 0.0000000 \\ \mathrm{C} & 3.6342606 & -0.7112501 & 0.0000000 \\ \mathrm{C} & 3.6342606 & 0.7112501 & 0.0000000 \\ \mathrm{C} & 2.4310739 & 1.3955935 & 0.0000000 \\ \mathrm{H} & 4.5648707 & 1.2632021 & 0.0000000 \\ \mathrm{H} & -4.5648707 & -1.2632021 & 0.0000000 \\ \mathrm{H} & -2.3938534 & -2.4812671 & 0.0000000 \\ \mathrm{H} & -2.3938534 & 2.4812671 & 0.0000000 \\ \mathrm{H} & 2.3938534 & -2.4812671 & 0.0000000 \\ \mathrm{H} & 4.5648707 & -1.2632021 & 0.0000000 \\ \mathrm{H} & -4.5648707 & 1.2632021 & 0.0000000 \\ \mathrm{H} & 2.3938534 & 2.4812671 & 0.0000000\end{array}$


Table S662 Cartesian coordinates of the CC2/TZVP-optimized excited-state equilibrium geometry of the $1{ }^{3} B_{1 g}$ ( $\equiv 3{ }^{3} B_{2 g}$ in Turbomole) $\left({ }^{3} n \pi^{*}\right)$ state of dibenzo- $p$-dioxin (excited-state energy: $-610.70480 E_{h}$ ).

$\begin{array}{lrrr}22 & & & \\ & & & \\ \text { C } & -3.6249297 & 0.6998328 & 0.0000000 \\ \text { C } & -3.6249297 & -0.6998328 & 0.0000000 \\ \text { C } & -2.4181558 & -1.4088355 & 0.0000000 \\ \text { C } & -1.2060517 & -0.7058749 & 0.0000000 \\ \text { C } & -1.2060517 & 0.7058749 & 0.0000000 \\ \text { C } & -2.4181558 & 1.4088355 & 0.0000000 \\ \text { O } & 0.0000000 & -1.4107028 & 0.0000000 \\ \text { C } & 1.2060517 & -0.7058749 & 0.0000000 \\ \text { C } & 1.2060517 & 0.7058749 & 0.0000000 \\ \text { O } & 0.0000000 & 1.4107028 & 0.0000000 \\ \text { C } & 2.4181558 & -1.4088355 & 0.0000000 \\ \text { C } & 3.6249297 & -0.6998328 & 0.0000000 \\ \text { C } & 3.6249297 & 0.6998328 & 0.0000000 \\ \text { C } & 2.4181558 & 1.4088355 & 0.0000000 \\ \text { H } & 4.5669805 & 1.2365495 & 0.0000000 \\ \text { H } & -4.5669805 & -1.2365495 & 0.0000000 \\ \text { H } & -2.3872760 & -2.4934563 & 0.0000000 \\ \text { H } & -2.3872760 & 2.4934563 & 0.0000000 \\ \text { H } & 2.3872760 & -2.4934563 & 0.0000000 \\ \text { H } & 4.5669805 & -1.2365495 & 0.0000000 \\ \text { H } & -4.5669805 & 1.2365495 & 0.0000000 \\ \text { H } & 2.3872760 & 2.4934563 & 0.0000000\end{array}$


Table S663 Cartesian coordinates of the CC2/TZVP-optimized excited-state equilibrium geometry of the $1{ }^{3} B_{3 u}$ ( $\equiv 3{ }^{3} B_{1 u}$ in Turbomole) $\left({ }^{3} n \pi^{*}\right)$ state of dibenzo- $p$-dioxin (excited-state energy: $-610.70696 E_{h}$ ).

$\begin{array}{lrrr}22 & & & \\ & & & \\ \text { C } & -3.6239131 & 0.6947495 & 0.0000000 \\ \text { C } & -3.6239131 & -0.6947495 & 0.0000000 \\ \text { C } & -2.4148902 & -1.4085337 & 0.0000000 \\ \text { C } & -1.1948928 & -0.7046325 & 0.0000000 \\ \text { C } & -1.1948928 & 0.7046325 & 0.0000000 \\ \text { C } & -2.4148902 & 1.4085337 & 0.0000000 \\ \text { O } & 0.0000000 & -1.3887528 & 0.0000000 \\ \text { C } & 1.1948928 & -0.7046325 & 0.0000000 \\ \text { C } & 1.1948928 & 0.7046325 & 0.0000000 \\ \text { O } & 0.0000000 & 1.3887528 & 0.0000000 \\ \text { C } & 2.4148902 & -1.4085337 & 0.0000000 \\ \text { C } & 3.6239131 & -0.6947495 & 0.0000000 \\ \text { C } & 3.6239131 & 0.6947495 & 0.0000000 \\ \text { C } & 2.4148902 & 1.4085337 & 0.0000000 \\ \text { H } & 4.5646826 & 1.2321328 & 0.0000000 \\ \text { H } & -4.5646826 & -1.2321328 & 0.0000000 \\ \text { H } & -2.3594693 & -2.4919591 & 0.0000000 \\ \text { H } & -2.3594693 & 2.4919591 & 0.0000000 \\ \text { H } & 2.3594693 & -2.4919591 & 0.0000000 \\ \text { H } & 4.5646826 & -1.2321328 & 0.0000000 \\ \text { H } & -4.5646826 & 1.2321328 & 0.0000000 \\ \text { H } & 2.3594693 & 2.4919591 & 0.0000000\end{array}$


Table S664 Cartesian coordinates of the CC2/TZVP-optimized excited-state equilibrium geometry of the $1^{1} A^{\prime \prime}\left({ }^{1} n \pi^{*}\right)$ state of formaldehyde (excited-state energy: $-114.12084 E_{h}$ ).

4

$\begin{array}{rrrr}\mathrm{C} & -0.0336812 & -0.1584161 & 0.0000000 \\ \mathrm{O} & 1.1736617 & 0.4687697 & 0.0000000 \\ \mathrm{H} & -0.5699902 & -0.1551768 & 0.9480337 \\ \mathrm{H} & -0.5699902 & -0.1551768 & -0.9480337\end{array}$


Table S665 Cartesian coordinates of the CC2/TZVP-optimized excited-state equilibrium geometry of the $1^{3} A^{\prime \prime}\left({ }^{3} n \pi^{*}\right)$ state of formaldehyde (excited-state energy: $-114.14093 E_{h}$ ).

4

O $1.0690823 \quad 0.6272604$

$\mathrm{H} \quad-0.5450246-0.2075740$

$\mathrm{H} \quad-0.5450246-0.2075740 \quad-0.9353170$ 
Table S666 Cartesian coordinates of the CC2/TZVP-optimized excited-state equilibrium geometry of the $1^{3} A^{\prime}\left({ }^{3} \pi \pi^{*}\right)$ state of formaldehyde (excited-state energy: $\left.-114.08427 E_{h}\right)$.

4

$\begin{array}{rrrr}\mathrm{C} & -0.0451507 & -0.1790467 & 0.0000000 \\ \mathrm{O} & 1.1890007 & 0.6183601 & 0.0000000 \\ \mathrm{H} & -0.5719250 & -0.2196567 & 0.9430279 \\ \mathrm{H} & -0.5719250 & -0.2196567 & -0.9430279\end{array}$


Table S667 Cartesian coordinates of the CC2/TZVP-optimized excited-state equilibrium geometry of the $1{ }^{1} A_{u}\left({ }^{1} n \pi^{*}\right)$ state of trans-glyoxal (excited-state energy: $-227.23912 E_{h}$ ).

c

C -0.7396353

O 1.4023088

$0 \quad-1.4023088$

$\mathrm{H} \quad 1.2342563$

$\mathrm{H} \quad-1.2342563$

$-0.0267840$

0.0000000

$0.0267840 \quad 0.0000000$

$-1.0978896 \quad 0.0000000$

$1.0978896 \quad 0.0000000$

$0.9507603 \quad 0.0000000$

$-0.9507603 \quad 0.0000000$ 
Table S668 Cartesian coordinates of the CC2/TZVP-optimized excited-state equilibrium geometry of the $1{ }^{3} A_{u}\left({ }^{3} n \pi^{*}\right)$ state of trans-glyoxal (excited-state energy: $-227.25593 E_{h}$ ).

6

$\begin{array}{lrrr}\mathrm{C} & 0.7367022 & -0.0226760 & 0.0000000 \\ \mathrm{C} & -0.7367022 & 0.0226760 & 0.0000000 \\ \mathrm{O} & 1.3908693 & -1.0984914 & 0.0000000 \\ \mathrm{O} & -1.3908693 & 1.0984914 & 0.0000000 \\ \mathrm{H} & 1.2356963 & 0.9491152 & 0.0000000 \\ \mathrm{H} & -1.2356963 & -0.9491152 & 0.0000000\end{array}$


Table S669 Cartesian coordinates of the CC2/TZVP-optimized excited-state equilibrium geometry of the $2{ }^{1} A\left({ }^{1} \pi \pi^{*}\right)$ state of $9 H$-guanine (excited-state energy: $-541.17710 E_{h}$ ).

$\begin{array}{lrrr}16 & & & \\ & & & \\ \mathrm{~N} & -0.4905307 & 0.0094558 & -0.1014400 \\ \mathrm{C} & -0.2398801 & 0.1376831 & 1.1727415 \\ \mathrm{H} & -0.1240421 & -0.6845953 & 1.8642717 \\ \mathrm{~N} & -0.0896924 & 1.4577308 & 1.6200125 \\ \mathrm{C} & -0.2854750 & 2.2632386 & 0.5339801 \\ \mathrm{C} & -1.0516552 & 3.5302246 & 0.5366195 \\ \mathrm{O} & -1.6310659 & 3.9961610 & 1.5183398 \\ \mathrm{~N} & -1.0721079 & 4.0259106 & -0.7669033 \\ \mathrm{H} & -1.9613761 & 4.4011964 & -1.1004610 \\ \mathrm{C} & -0.2263913 & 3.1948054 & -1.5600588 \\ \mathrm{~N} & 0.3490280 & 3.7608961 & -2.6934132 \\ \mathrm{H} & 0.9668957 & 4.5386450 & -2.4875002 \\ \mathrm{H} & 0.7813698 & 3.0615029 & -3.2871836 \\ \mathrm{~N} & -0.6967121 & 1.7962117 & -1.7823456 \\ \mathrm{C} & -0.4837417 & 1.3292967 & -0.5829391 \\ \mathrm{H} & -0.1404184 & 1.7635368 & 2.5840209\end{array}$


Table S670 Cartesian coordinates of the CC2/TZVP-optimized excited-state equilibrium geometry of the $2{ }^{1} A^{\prime}\left({ }^{1} \pi \pi^{*}\right)$ state of 1-hydroxy-2-acetonaphthone (excited-state energy: $-612.11990 E_{h}$ ).

$\begin{array}{lrrr}24 & & & \\ & & & \\ \mathrm{C} & 0.0625298 & 1.0695885 & 0.0000000 \\ \mathrm{C} & -1.1697681 & 0.3599330 & 0.0000000 \\ \mathrm{C} & -1.1805572 & -1.0925581 & 0.0000000 \\ \mathrm{C} & 0.0400306 & -1.7677784 & 0.0000000 \\ \mathrm{C} & 1.2710404 & -1.0507042 & 0.0000000 \\ \mathrm{C} & 1.3125246 & 0.3285015 & 0.0000000 \\ \mathrm{C} & -2.4069282 & 1.0446253 & 0.0000000 \\ \mathrm{C} & -3.5999492 & 0.3404912 & 0.0000000 \\ \mathrm{C} & -3.6180783 & -1.0778172 & 0.0000000 \\ \mathrm{C} & -2.4259720 & -1.7760291 & 0.0000000 \\ \mathrm{C} & 2.5785972 & 1.0641762 & 0.0000000 \\ \mathrm{C} & 3.9129927 & 0.4047266 & 0.0000000 \\ \mathrm{O} & 0.1065094 & 2.3849344 & 0.0000000 \\ \mathrm{O} & 2.5431362 & 2.3861996 & 0.0000000 \\ \mathrm{H} & -4.5388018 & 0.8833800 & 0.0000000 \\ \mathrm{H} & -4.5633306 & -1.6072739 & 0.0000000 \\ \mathrm{H} & -2.4219511 & -2.8621771 & 0.0000000 \\ \mathrm{H} & -2.3955499 & 2.1286117 & 0.0000000 \\ \mathrm{H} & 0.0613701 & -2.8532864 & 0.0000000 \\ \mathrm{H} & 2.1958788 & -1.6198918 & 0.0000000 \\ \mathrm{H} & 1.4525091 & 2.5938037 & 0.0000000 \\ \mathrm{H} & 4.6832813 & 1.1745970 & 0.0000000 \\ \mathrm{H} & 4.0502430 & -0.2280264 & -0.8831332 \\ \mathrm{H} & 4.0502430 & -0.2280264 & 0.8831332\end{array}$


Table S671 Cartesian coordinates of the CC2/TZVP-optimized excited-state equilibrium geometry of the $2{ }^{1} A^{\prime}\left({ }^{1} \pi \pi^{*}\right)$ state of indole (excited-state energy: $\left.-362.68086 E_{h}\right)$.

$\begin{array}{lrrr}16 & & & \\ & & & \\ \mathrm{C} & -0.5671885 & -0.6058416 & 0.0000000 \\ \mathrm{C} & -0.0386336 & 0.7586385 & 0.0000000 \\ \mathrm{~N} & -1.1297513 & 1.5744924 & 0.0000000 \\ \mathrm{C} & -1.9934427 & -0.5080076 & 0.0000000 \\ \mathrm{C} & -2.3395597 & 0.8277198 & 0.0000000 \\ \mathrm{C} & 0.3477927 & -1.6817876 & 0.0000000 \\ \mathrm{C} & 1.7453569 & -1.3617660 & 0.0000000 \\ \mathrm{C} & 1.3341376 & 1.0795133 & 0.0000000 \\ \mathrm{C} & 2.2390858 & -0.0246145 & 0.0000000 \\ \mathrm{H} & -1.0848569 & 2.5842496 & 0.0000000 \\ \mathrm{H} & -2.6884230 & -1.3339654 & 0.0000000 \\ \mathrm{H} & -3.2920415 & 1.3293308 & 0.0000000 \\ \mathrm{H} & 0.0173463 & -2.7127932 & 0.0000000 \\ \mathrm{H} & 2.4606525 & -2.1766706 & 0.0000000 \\ \mathrm{H} & 1.6821180 & 2.1051498 & 0.0000000 \\ \mathrm{H} & 3.3074075 & 0.1463525 & 0.0000000\end{array}$


Table S672 Cartesian coordinates of the CC2/TZVP-optimized excited-state equilibrium geometry of the $1^{1} B_{1}\left({ }^{1} n \pi^{*}\right)$ state of maleimide (excited-state energy: $-358.51400 E_{h}$ ).

10

$\mathrm{N} \quad 0.0000000$

0.0000000

$-1.1435040$

C -1.1462324

C $\quad-0.6995977$

$0.0000000-0.3292193$

C $\quad 0.6995977$

$0.0000000 \quad 1.0194969$

C $\quad 1.1462324$

$0.0000000 \quad 1.0194969$

$-2.2869558$

0.0000000

$-0.3292193$

o 2.2869558

0.0000000

$-0.9052283$

0.0000000

0.0000000

$-0.9052283$

$-1.3682320$

0.0000000

$-2.1554412$

1.3682320

0.0000000

1.8644233

$0.0000000 \quad 1.8644233$ 
Table S673 Cartesian coordinates of the CC2/TZVP-optimized excited-state equilibrium geometry of the $1{ }^{1} A_{2}\left({ }^{1} n \pi^{*}\right)$ state of maleimide (excited-state energy: $-358.48882 E_{h}$ ).

10

$\mathrm{N} \quad 0.0000000$

C -1.1268783

C $\quad-0.7064408$

0.0000000

$-1.1547565$

C $\quad 0.7064408$

0.0000000

$-0.3561039$

C $\quad 1.1268783$

0.0000000

0.9949323

$-2.3481349$

0.0000000

0.9949323

O

O $\quad 2.3481349$

0.0000000

$-0.3561039$

0.0000000

$-0.8249564$

$\begin{array}{rr}\mathrm{H} & 0.0000000 \\ \mathrm{H} & -1.3692709\end{array}$

0.0000000

$-0.8249564$

0.0000000

$-2.1642395$

1.3692709

0.0000000

1.8456260

0.0000000

1.8456260 
Table S674 Cartesian coordinates of the CC2/TZVP-optimized excited-state equilibrium geometry of the $1^{1} B_{2}\left({ }^{1} \pi \pi^{*}\right)$ state of maleimide (excited-state energy: $-358.49169 E_{h}$ ).

10

$\mathrm{N} \quad 0.0000000$

C $\quad-1.3023246$

C $\quad-0.7077081$

0.0000000

$-1.2191754$

0.0000000

$-0.2924166$

C $\quad 0.7077081$

0.0000000

0.9759572

1.3023246

0.0000000

0.9759572

$-2.4045346$

0.0000000

$-0.2924166$

2. 4045346

0.0000000

$-0.8207829$

0.0000000

0.0000000

$-0.8207829$

$-1.3136865$

0.0000000

$-2.2479068$

1. 3136865

0.0000000

1.8707834

$0.0000000 \quad 1.8707834$ 
Table S675 Cartesian coordinates of the CC2/TZVP-optimized excited-state equilibrium geometry of the $2{ }^{1} B_{2}\left({ }^{1} \pi \pi^{*}\right)$ state of maleimide (excited-state energy: $-358.41767 E_{h}$ ).

10

$\mathrm{N} \quad 0.0000000$

C $\quad-1.1560203$

C $\quad-0.7197518$

C $\quad 0.7197518$

0.0000000

$-1.1443066$

C $\quad 1.1560203$

0.0000000

$-0.3655392$

$-2.3488677$

0.0000000

1.0039000

2.3488677

0.0000000

1.0039000

0

0.0000000

$-0.3655392$

$\mathrm{H} \quad 0.0000000$

0.0000000

$-0.8398766$

$\begin{array}{rr}\mathrm{H} & -1.3874187 \\ \mathrm{H} & 1.3874187\end{array}$

0.0000000

$-0.8398766$

.0000000

$-2.1568781$

0.0000000

1. 8521082

0.0000000

1. 8521082 
Table S676 Cartesian coordinates of the CC2/TZVP-optimized excited-state equilibrium geometry of the $1{ }^{3} B_{1}\left({ }^{3} n \pi^{*}\right)$ state of maleimide (excited-state energy: $-358.52218 E_{h}$ ).

10

$\mathrm{N} \quad 0.0000000$

C $\quad-1.1464861$

C $\quad-0.6976126$

0.0000000

$-1.1449675$

C $\quad 0.6976126$

0.0000000

$-0.3318651$

C $\quad 1.1464861$

0.0000000

1.0174332

$-2.2904571$

0.0000000

1.0174332

O

$\begin{array}{ll}\mathrm{O} & 2.2904571 \\ \mathrm{H} & 0.0000000\end{array}$

0.0000000

$-0.3318651$

0.0000000

$-0.8975296$

$\mathrm{H} \quad-1.3656250$

0.0000000

$-0.8975296$

$\mathrm{H} \quad 1.3656250$

0.0000000

$-2.1567265$

1. 8628085

$0.0000000 \quad 1.8628085$ 
Table S677 Cartesian coordinates of the CC2/TZVP-optimized excited-state equilibrium geometry of the $1{ }^{3} A_{2}\left({ }^{3} n \pi^{*}\right)$ state of maleimide (excited-state energy: $-358.49730 E_{h}$ ).

10

$\mathrm{N} \quad 0.0000000$

C $\quad-1.1285340$

C $\quad-0.7044848$

0.0000000

$-1.1588684$

C $\quad 0.7044848$

0.0000000

$-0.3597549$

C $\quad 1.1285340$

0.0000000

0.9919723

$-2.3498841$

0.0000000

0.9919723

O

2. 3498841

0.0000000

$-0.3597549$

O

$\begin{array}{rr}\mathrm{H} & 0.0000000 \\ \mathrm{H} & -1.3635630\end{array}$

0.0000000

$-0.8145253$

0.0000000

$-0.8145253$

$-1.3635630$

0.0000000

$-2.1679055$

$\mathrm{H}$

1. 3635630

0.0000000

1.8456949

$0.0000000 \quad 1.8456949$ 
Table S678 Cartesian coordinates of the CC2/TZVP-optimized excited-state equilibrium geometry of the $1{ }^{3} B_{2}\left({ }^{3} \pi \pi^{*}\right)$ state of maleimide (excited-state energy: $-358.51868 E_{h}$ ).

10

$\mathrm{N} \quad 0.0000000$

C $\quad-1.1953442$

C $\quad-0.7587937$

C $\quad 0.7587937$

0.0000000

$-1.1307438$

C $\quad 1.1953442$

0.0000000

$-0.3765524$

$-2.3372985$

0.0000000

1.0016475

2.3372985

0.0000000

1.0016475

0.0000000

0.0000000

$-0.3765524$

$-1.4149360$

0.0000000

$-0.8437667$

0.0000000

$-0.8437667$

1.4149360

0.0000000

$-2.1442811$

$0.0000000 \quad 1.8561841$

$\mathrm{H}$

0.0000000

1.8561841 
Table S679 Cartesian coordinates of the CC2/TZVP-optimized excited-state equilibrium geometry of the $1{ }^{1} A^{\prime \prime}\left({ }^{1} n \pi^{*}\right)$ state of nitrosomethane (excited-state energy: $-169.35535 E_{h}$ ).

6

$\begin{array}{rrrr}\mathrm{C} & -0.4256626 & 0.1625498 & 0.0000000 \\ \mathrm{~N} & 1.0496820 & 0.2715909 & 0.0000000 \\ \mathrm{O} & 1.6849186 & -0.8459705 & 0.0000000 \\ \mathrm{H} & -0.8200111 & 1.1721936 & 0.0000000 \\ \mathrm{H} & -0.7444634 & -0.3801819 & -0.8922179 \\ \mathrm{H} & -0.7444634 & -0.3801819 & 0.8922179\end{array}$


Table S680 Cartesian coordinates of the CC2/TZVP-optimized excited-state equilibrium geometry of the $1^{3} A^{\prime \prime}\left({ }^{3} n \pi^{*}\right)$ state of nitrosomethane (excited-state energy: $-169.38849 E_{h}$ ).

6

$\begin{array}{lrrr}\mathrm{C} & -0.4286276 & 0.1570338 & 0.0000000 \\ \mathrm{~N} & 1.0342447 & 0.2324472 & 0.0000000 \\ \mathrm{O} & 1.7362079 & -0.8145184 & 0.0000000 \\ \mathrm{H} & -0.8099040 & 1.1727133 & 0.0000000 \\ \mathrm{H} & -0.7659605 & -0.3738380 & -0.8921300 \\ \mathrm{H} & -0.7659605 & -0.3738380 & 0.8921300\end{array}$


Table S681 Cartesian coordinates of the CC2/TZVP-optimized excited-state equilibrium geometry of the $1{ }^{1} A^{\prime \prime}\left({ }^{1} n \pi^{*}\right)$ state of propenoic acid anion (excitedstate energy: $\left.-265.91106 E_{h}\right)$.

C $\quad-0.2855886$

0.45220650 .0000000

C $\quad 1.1038293 \quad 0.4172657 \quad 0.0000000$

$\begin{array}{llll}0 & 1.9745364 & 1.4028304 & 0.0000000\end{array}$

$\begin{array}{llll}0 & 1.8875352 & -0.6787823 & 0.0000000\end{array}$

C $\quad-1.1022496-0.7089346 \quad 0.0000000$

$\mathrm{H} \quad-0.7354900 \quad 1.4423280 \quad 0.0000000$

$\mathrm{H} \quad-2.1829766 \quad-0.6285367 \quad 0.0000000$

$\mathrm{H} \quad-0.6595962-1.6983770 \quad 0.0000000$ 
Table S682 Cartesian coordinates of the CC2/TZVP-optimized excited-state equilibrium geometry of the $1{ }^{1} A_{2}\left({ }^{1} n \pi^{*}\right)$ state of pyridine (excited-state energy: $\left.-247.46013 E_{h}\right)$.

11

$\mathrm{N} \quad 0.0000000$

C -1.2175333

C 1.2175333

C $\quad-1.2300305$

0.0000000

1.4474704

$0.0000000 \quad 0.9475539$

$-1.2300305$

0.0000000

0.9475539

$\mathrm{C}$

1.2300305

0.0000000

$-0.5219681$

C $\quad 0.0000000$

0.0000000

$-0.5219681$

$-2.0790863$

0.0000000

$-1.1733621$

2.0790863

0.0000000

1. 5985822

$-2.1823738$

0.0000000

1.5985822

$\begin{array}{ll}\mathrm{H} & 2.1823738 \\ \mathrm{H} & 0.0000000\end{array}$

0.0000000

$-1.0316892$

0.0000000

$-1.0316892$

$0.0000000-2.2590659$ 
Table S683 Cartesian coordinates of the CC2/TZVP-optimized excited-state equilibrium geometry of the $2{ }^{1} A^{\prime}\left({ }^{1} n \pi^{*}\right)$ state of pyridine (excited-state energy: $\left.-247.46040 E_{h}\right)$.

11

$\begin{array}{lrrr}\mathrm{N} & 1.3808066 & 0.5378304 & 0.0000000 \\ \mathrm{C} & 0.8957379 & 0.1404922 & 1.2142697 \\ \mathrm{C} & 0.8957379 & 0.1404922 & -1.2142697 \\ \mathrm{C} & -0.4698292 & -0.0376265 & 1.2287643 \\ \mathrm{C} & -0.4698292 & -0.0376265 & -1.2287643 \\ \mathrm{C} & -1.1946131 & 0.0565416 & 0.0000000 \\ \mathrm{H} & -0.9488421 & -0.3300097 & 2.1569525 \\ \mathrm{H} & -0.9488421 & -0.3300097 & -2.1569525 \\ \mathrm{H} & 1.5606424 & -0.0090982 & 2.0565524 \\ \mathrm{H} & 1.5606424 & -0.0090982 & -2.0565524 \\ \mathrm{H} & -2.2616113 & -0.1218878 & 0.0000000\end{array}$


Table S684 Cartesian coordinates of the CC2/TZVP-optimized excited-state equilibrium geometry of the $1^{3} A_{1}\left({ }^{3} \pi \pi^{*}\right)$ state of pyridine (excited-state energy: $\left.-247.47811 E_{h}\right)$.

11

$\mathrm{N} \quad 0.0000000$

C $\quad-1.1440066$

C 1.1440066

C -1.2003931

0.0000000

1.6669908

$0.0000000 \quad 0.9748029$

0.0000000

0.9748029

$\mathrm{C}$

1. 2003931

0.0000000

$-0.5374260$

C $\quad 0.0000000$

0.0000000

$-0.5374260$

$-2.0645285$

0.0000000

$-1.2401206$

2.0645285

0.0000000

1.5438689

$-2.1610838$

0.0000000

1.5438689

2. 1610838

0.0000000

$-1.0326978$

0.0000000

0.0000000

$-1.0326978$

$\mathrm{H}$

0.0000000

$-2.3239663$ 
Table S685 Cartesian coordinates of the CC2/TZVP-optimized excited-state equilibrium geometry of the $2{ }^{3} A_{1}\left({ }^{3} \pi \pi^{*}\right)$ state of pyridine (excited-state energy: $\left.-247.43932 E_{h}\right)$.

11

$\begin{array}{lrlr}\mathrm{N} & 0.0000000 & 0.0000000 & 1.6615621 \\ \mathrm{C} & -1.1664377 & 0.0000000 & 0.9257114 \\ \mathrm{C} & 1.1664377 & 0.0000000 & 0.9257114 \\ \mathrm{C} & -1.2229451 & 0.0000000 & -0.4918777 \\ \mathrm{C} & 1.2229451 & 0.0000000 & -0.4918777 \\ \mathrm{C} & 0.0000000 & 0.0000000 & -1.2350966 \\ \mathrm{H} & -2.0801066 & 0.0000000 & 1.5065063 \\ \mathrm{H} & 2.0801066 & 0.0000000 & 1.5065063 \\ \mathrm{H} & -2.1805577 & 0.0000000 & -0.9954778 \\ \mathrm{H} & 2.1805577 & 0.0000000 & -0.9954778 \\ \mathrm{H} & 0.0000000 & 0.0000000 & -2.3161899\end{array}$


Table S686 Cartesian coordinates of the CC2/TZVP-optimized excited-state equilibrium geometry of the $2{ }^{1} A^{\prime}\left({ }^{1} \pi \pi^{*}\right)$ state of pyrrole (excited-state energy: $\left.-209.39331 E_{h}\right)$.

$\begin{array}{lrrr}10 & & & \\ & & & \\ \mathrm{H} & 2.1305608 & -0.3173898 & 0.0000000 \\ \mathrm{H} & 0.8125457 & 0.2056184 & 2.1076005 \\ \mathrm{H} & 0.8125457 & 0.2056184 & -2.1076005 \\ \mathrm{H} & -1.8562884 & 0.0737160 & -1.3568275 \\ \mathrm{H} & -1.8562884 & 0.0737160 & 1.3568275 \\ \mathrm{~N} & 1.1490145 & -0.0674076 & 0.0000000 \\ \mathrm{C} & 0.3797054 & -0.1725174 & -1.1862507 \\ \mathrm{C} & 0.3797054 & -0.1725174 & 1.1862507 \\ \mathrm{C} & -0.9757504 & 0.0855816 & 0.7324424 \\ \mathrm{C} & -0.9757504 & 0.0855816 & -0.7324424\end{array}$


Table S687 Cartesian coordinates of the CC2/TZVP-optimized excited-state equilibrium geometry of the $1{ }^{1} B_{2}\left({ }^{1} \pi \pi^{*}\right)$ state of pyrrole (excited-state energy: $\left.-209.37821 E_{h}\right)$.

10

$\mathrm{H} \quad 0.0000000$

$-2.1358051$

0.0000000

2.2156370

$\mathrm{H}$

2.1358051

0.0000000

0.7863496

1. 3463282

0.0000000

0.7863496

$-1.3463282$

0.0000000

$-1.8608868$

0.0000000

0.0000000

$-1.8608868$

1.1401255

0.0000000

1. 2101430

$-1.1401255$

0.0000000

0.3743816

$\begin{array}{lr}\text { C } & -0.6819718 \\ \text { C } & 0.6819718\end{array}$

0.0000000

0.3743816

0.0000000

$-1.0127344$

0.0000000

$-1.0127344$ 
Table S688 Cartesian coordinates of the CC2/TZVP-optimized excited-state equilibrium geometry of the $2{ }^{1} A^{\prime}\left({ }^{1} \pi \pi^{*}\right)$ state of cis-PSB3 (excited-state energy: $-248.99386 E_{h}$ ).

$\begin{array}{lrrr}14 & & & \\ \text { C } & 2.8641433 & -0.4715010 & 0.0000000 \\ \mathrm{C} & 1.4788975 & -0.4174491 & 0.0000000 \\ \mathrm{C} & 0.7455386 & 0.8165042 & 0.0000000 \\ \mathrm{C} & -0.6557138 & 0.8323983 & 0.0000000 \\ \mathrm{C} & -1.5277241 & -0.3225355 & 0.0000000 \\ \mathrm{~N} & -2.8708285 & -0.1477856 & 0.0000000 \\ \mathrm{H} & 3.3855275 & -1.4213948 & 0.0000000 \\ \mathrm{H} & 3.4555466 & 0.4386557 & 0.0000000 \\ \mathrm{H} & 0.9363448 & -1.3574176 & 0.0000000 \\ \mathrm{H} & 1.2861518 & 1.7555861 & 0.0000000 \\ \mathrm{H} & -1.1434976 & 1.8030087 & 0.0000000 \\ \mathrm{H} & -1.1702045 & -1.3433969 & 0.0000000 \\ \mathrm{H} & -3.4985257 & -0.9399273 & 0.0000000 \\ \mathrm{H} & -3.2856558 & 0.7752548 & 0.0000000\end{array}$


Table S689 Cartesian coordinates of the CC2/TZVP-optimized excited-state equilibrium geometry of the $2{ }^{1} A^{\prime}\left({ }^{1} \pi \pi^{*}\right)$ state of 3Me-cis-PSB5 (excited-state energy: $-442.63452 E_{h}$ ).

$\begin{array}{lrrr}25 & & & \\ & & & \\ \mathrm{C} & -0.8448487 & 1.3168386 & 0.0000000 \\ \mathrm{C} & 0.5421591 & 1.2689093 & 0.0000000 \\ \mathrm{C} & 1.4825932 & 0.1581080 & 0.0000000 \\ \mathrm{C} & 2.8363837 & 0.5282965 & 0.0000000 \\ \mathrm{C} & 3.9392783 & -0.3368918 & 0.0000000 \\ \mathrm{~N} & 5.2104847 & 0.1072644 & 0.0000000 \\ \mathrm{C} & 1.0387909 & -1.2746383 & 0.0000000 \\ \mathrm{C} & -1.7691372 & 0.2305605 & 0.0000000 \\ \mathrm{C} & -3.1443171 & 0.4283734 & 0.0000000 \\ \mathrm{C} & -4.0744436 & -0.6420554 & 0.0000000 \\ \mathrm{C} & -5.4245598 & -0.4310369 & 0.0000000 \\ \mathrm{H} & -1.2839591 & 2.3114232 & 0.0000000 \\ \mathrm{H} & 1.0190669 & 2.2463370 & 0.0000000 \\ \mathrm{H} & 3.0612469 & 1.5936066 & 0.0000000 \\ \mathrm{H} & 3.8290104 & -1.4144078 & 0.0000000 \\ \mathrm{H} & 5.9869920 & -0.5372877 & 0.0000000 \\ \mathrm{H} & 5.4236002 & 1.0956615 & 0.0000000 \\ \mathrm{H} & 1.8801108 & -1.9649666 & 0.0000000 \\ \mathrm{H} & 0.4398183 & -1.4984962 & 0.8866514 \\ \mathrm{H} & 0.4398183 & -1.4984962 & -0.8866514 \\ \mathrm{H} & -1.4115626 & -0.7919745 & 0.0000000 \\ \mathrm{H} & -3.5288169 & 1.4471998 & 0.0000000 \\ \mathrm{H} & -3.6927502 & -1.6591798 & 0.0000000 \\ \mathrm{H} & -6.1238784 & -1.2577606 & 0.0000000 \\ \mathrm{H} & -5.8310802 & 0.5746130 & 0.0000000 \\ & & & \end{array}$


Table S690 Cartesian coordinates of the CC2/TZVP-optimized excited-state equilibrium geometry of the $1{ }^{1} B_{u}\left({ }^{1} \pi \pi^{*}\right)$ state of stilbene (excited-state energy: $\left.-539.07219 E_{h}\right)$.

$\begin{array}{lrrr}26 & & & \\ & & & \\ \mathrm{C} & -0.0140746 & 0.7094978 & 0.0000000 \\ \mathrm{C} & -1.1830740 & 1.4810376 & 0.0000000 \\ \mathrm{C} & -1.0888791 & 2.9131647 & 0.0000000 \\ \mathrm{C} & -2.2147293 & 3.7091476 & 0.0000000 \\ \mathrm{C} & -3.4987338 & 3.1217603 & 0.0000000 \\ \mathrm{C} & -3.6222915 & 1.7251782 & 0.0000000 \\ \mathrm{C} & -2.5051337 & 0.9053114 & 0.0000000 \\ \mathrm{C} & 0.0140746 & -0.7094978 & 0.0000000 \\ \mathrm{C} & 1.1830740 & -1.4810376 & 0.0000000 \\ \mathrm{C} & 1.0888791 & -2.9131647 & 0.0000000 \\ \mathrm{C} & 2.2147293 & -3.7091476 & 0.0000000 \\ \mathrm{C} & 3.4987338 & -3.1217603 & 0.0000000 \\ \mathrm{C} & 3.6222915 & -1.7251782 & 0.0000000 \\ \mathrm{C} & 2.5051337 & -0.9053114 & 0.0000000 \\ \mathrm{H} & -4.6106900 & 1.2781180 & 0.0000000 \\ \mathrm{H} & -4.3842463 & 3.7469527 & 0.0000000 \\ \mathrm{H} & -2.1142669 & 4.7886047 & 0.0000000 \\ \mathrm{H} & -0.0994626 & 3.3617034 & 0.0000000 \\ \mathrm{H} & -2.6276497 & -0.1711331 & 0.0000000 \\ \mathrm{H} & 0.9298161 & 1.2470509 & 0.0000000 \\ \mathrm{H} & -0.9298161 & -1.2470509 & 0.0000000 \\ \mathrm{H} & 0.0994626 & -3.3617034 & 0.0000000 \\ \mathrm{H} & 2.1142669 & -4.7886047 & 0.0000000 \\ \mathrm{H} & 4.3842463 & -3.7469527 & 0.0000000 \\ \mathrm{H} & 4.6106900 & -1.2781180 & 0.0000000 \\ \mathrm{H} & 2.6276497 & 0.1711331 & 0.0000000\end{array}$


Table S691 Cartesian coordinates of the CC2/TZVP-optimized excited-state equilibrium geometry of the $1^{1} B\left({ }^{1} \pi \pi^{*}\right)$ state of stilbene (excited-state energy: $\left.-539.09777 E_{h}\right)$.

$\begin{array}{lrrr}26 & & & \\ \text { C } & -0.2698476 & 0.6488698 & -2.0785327 \\ \text { C } & -0.8373304 & 1.2485305 & -0.9276885 \\ \text { C } & -1.0419343 & 2.6511241 & -0.8514513 \\ \text { C } & -1.4798482 & 3.2441124 & 0.3188677 \\ \text { C } & -1.7036030 & 2.4657573 & 1.4805495 \\ \text { C } & -1.6142862 & 1.0783801 & 1.4070021 \\ \text { C } & -1.1432241 & 0.4545729 & 0.2493670 \\ \text { C } & 0.2698476 & -0.6488698 & -2.0785327 \\ \text { C } & 0.8373304 & -1.2485305 & -0.9276885 \\ \text { C } & 1.0419343 & -2.6511241 & -0.8514513 \\ \text { C } & 1.4798482 & -3.2441124 & 0.3188677 \\ \text { C } & 1.7036030 & -2.4657573 & 1.4805495 \\ \text { C } & 1.6142862 & -1.0783801 & 1.4070021 \\ \text { C } & 1.1432241 & -0.4545729 & 0.2493670 \\ \text { H } & -1.8711680 & 0.4738168 & 2.2707755 \\ \text { H } & -2.0552792 & 2.9416628 & 2.3891746 \\ \text { H } & -1.5935777 & 4.3216121 & 0.3630372 \\ \text { H } & -0.8417501 & 3.2550600 & -1.7320362 \\ \text { H } & -1.2920851 & -0.6125991 & 0.1180908 \\ \text { H } & -0.2939647 & 1.2173454 & -3.0071558 \\ \text { H } & 0.2939647 & -1.2173454 & -3.0071558 \\ \text { H } & 0.8417501 & -3.2550600 & -1.7320362 \\ \text { H } & 1.5935777 & -4.3216121 & 0.3630372 \\ \text { H } & 2.0552792 & -2.9416628 & 2.3891746 \\ \text { H } & 1.8711680 & -0.4738168 & 2.2707755 \\ \text { H } & 1.2920851 & 0.6125991 & 0.1180908\end{array}$


Table S692 Cartesian coordinates of the CC2/TZVP-optimized excited-state equilibrium geometry of the $2{ }^{1} A^{\prime}\left({ }^{1} \pi \pi^{*}\right)$ state of styrene (excited-state energy: $\left.-308.61396 E_{h}\right)$.

$\begin{array}{lrrr}16 & & & \\ & & & \\ \mathrm{C} & -0.9826565 & 1.6494984 & 0.0000000 \\ \mathrm{C} & -2.3438607 & 1.6704520 & 0.0000000 \\ \mathrm{C} & -0.1620652 & 0.4820132 & 0.0000000 \\ \mathrm{C} & -0.6918005 & -0.8550585 & 0.0000000 \\ \mathrm{C} & 1.2747308 & 0.6455309 & 0.0000000 \\ \mathrm{C} & 0.2031000 & -1.9738496 & 0.0000000 \\ \mathrm{C} & 2.1534195 & -0.4791898 & 0.0000000 \\ \mathrm{C} & 1.6118687 & -1.7889026 & 0.0000000 \\ \mathrm{H} & -0.4570634 & 2.6017133 & 0.0000000 \\ \mathrm{H} & -2.8796128 & 2.6103171 & 0.0000000 \\ \mathrm{H} & -2.9347762 & 0.7633277 & 0.0000000 \\ \mathrm{H} & -1.7604794 & -1.0227370 & 0.0000000 \\ \mathrm{H} & 1.6799349 & 1.6515890 & 0.0000000 \\ \mathrm{H} & -0.2036000 & -2.9778215 & 0.0000000 \\ \mathrm{H} & 3.2249947 & -0.3267240 & 0.0000000 \\ \mathrm{H} & 2.2678662 & -2.6501586 & 0.0000000\end{array}$


Table S693 Cartesian coordinates of the CC2/TZVP-optimized excited-state equilibrium geometry of the $1^{3} A^{\prime}\left({ }^{3} \pi \pi^{*}\right)$ state of styrene (excited-state energy: $\left.-308.68046 E_{h}\right)$.

$\begin{array}{lrrr}16 & & & \\ & & & \\ \mathrm{C} & -0.9114118 & 1.6378985 & 0.0000000 \\ \mathrm{C} & -2.3802880 & 1.6447410 & 0.0000000 \\ \mathrm{C} & -0.1210524 & 0.5099777 & 0.0000000 \\ \mathrm{C} & -0.6674164 & -0.8363316 & 0.0000000 \\ \mathrm{C} & 1.3254832 & 0.6281290 & 0.0000000 \\ \mathrm{C} & 0.1681925 & -1.9337331 & 0.0000000 \\ \mathrm{C} & 2.1300062 & -0.4810625 & 0.0000000 \\ \mathrm{C} & 1.5672705 & -1.7848618 & 0.0000000 \\ \mathrm{H} & -0.4180710 & 2.6050357 & 0.0000000 \\ \mathrm{H} & -2.9125602 & 2.5837059 & 0.0000000 \\ \mathrm{H} & -2.9550426 & 0.7320741 & 0.0000000 \\ \mathrm{H} & -1.7402450 & -0.9780107 & 0.0000000 \\ \mathrm{H} & 1.7573973 & 1.6237092 & 0.0000000 \\ \mathrm{H} & -0.2604348 & -2.9296770 & 0.0000000 \\ \mathrm{H} & 3.2078859 & -0.3656422 & 0.0000000 \\ \mathrm{H} & 2.2102866 & -2.6559521 & 0.0000000\end{array}$


Table S694 Cartesian coordinates of the CC2/TZVP-optimized excited-state equilibrium geometry of the $1{ }^{1} B_{3 u}\left({ }^{1} n \pi^{*}\right)$ state of tetrazine (excited-state energy: $-295.55207 E_{h}$ ).

8

$\begin{array}{lrrr}\mathrm{C} & 0.0000000 & 1.3145747 & 0.0000000 \\ \mathrm{C} & 0.0000000 & -1.3145747 & 0.0000000 \\ \mathrm{~N} & 1.1722609 & 0.6682372 & 0.0000000 \\ \mathrm{~N} & 1.1722609 & -0.6682372 & 0.0000000 \\ \mathrm{~N} & -1.1722609 & 0.6682372 & 0.0000000 \\ \mathrm{~N} & -1.1722609 & -0.6682372 & 0.0000000 \\ \mathrm{H} & 0.0000000 & -2.3944231 & 0.0000000 \\ \mathrm{H} & 0.0000000 & 2.3944231 & 0.0000000\end{array}$


Table S695 Cartesian coordinates of the CC2/TZVP-optimized excited-state equilibrium geometry of the $2{ }^{1} A\left({ }^{1} n \pi^{*}\right)$ state of thymine (excited-state energy: $\left.-452.95586 E_{h}\right)$.

$\begin{array}{lrrr}15 & & & \\ & & & \\ \mathrm{~N} & -0.0118196 & 0.0102880 & 0.0000934 \\ \mathrm{C} & 1.3810004 & -0.0105487 & 0.0000459 \\ \mathrm{C} & 2.0798822 & 1.2460886 & -0.0001792 \\ \mathrm{C} & 1.3132664 & 2.3394055 & -0.0000155 \\ \mathrm{~N} & -0.0724087 & 2.3202154 & 0.0000352 \\ \mathrm{C} & -0.8100073 & 1.1312818 & 0.0000406 \\ \mathrm{O} & 1.7499601 & 3.7230187 & 0.0001303 \\ \mathrm{C} & 3.5792638 & 1.2981603 & 0.0000041 \\ \mathrm{O} & -2.0401835 & 1.1167957 & 0.0000383 \\ \mathrm{H} & 1.8634126 & -0.9764252 & -0.0003155 \\ \mathrm{H} & -0.5317489 & -0.8574951 & 0.0001263 \\ \mathrm{H} & -0.6067201 & 3.1793562 & -0.0001667 \\ \mathrm{H} & 3.9291150 & 2.3325181 & -0.0000691 \\ \mathrm{H} & 3.9896764 & 0.8074855 & -0.8848102 \\ \mathrm{H} & 3.9894696 & 0.8076810 & 0.8850223\end{array}$


Table S696 Cartesian coordinates of the CC2/TZVP-optimized excited-state equilibrium geometry of the $2{ }^{1} A^{\prime}\left({ }^{1} \pi \pi^{*}\right)$ state of uracil (excited-state energy: $\left.-413.69011 E_{h}\right)$.

12

$\begin{array}{lrrr}\mathrm{N} & -0.4793972 & 1.2189037 & 0.0000000 \\ \mathrm{~N} & -0.8476915 & -1.0618550 & 0.0000000 \\ \mathrm{C} & -1.4261511 & 0.2059223 & 0.0000000 \\ \mathrm{C} & 0.5091042 & -1.2825252 & 0.0000000 \\ \mathrm{C} & 1.3993042 & -0.2640024 & 0.0000000 \\ \mathrm{C} & 0.9310672 & 1.1120790 & 0.0000000 \\ \mathrm{O} & 1.6318946 & 2.1221640 & 0.0000000 \\ \mathrm{O} & -2.6367311 & 0.3883948 & 0.0000000 \\ \mathrm{H} & 0.8097757 & -2.3219004 & 0.0000000 \\ \mathrm{H} & -0.8527711 & 2.1623626 & 0.0000000 \\ \mathrm{H} & -1.5008297 & -1.8324924 & 0.0000000 \\ \mathrm{H} & 2.4624257 & -0.4470509 & 0.0000000\end{array}$


4.1.8 Structural Parameters of the CC2-Optimized Excited-State Minima 
Table S697 Structural parameters of the CC2/TZVP/TZVP-optimized excited-state equilibrium geometry of the $2{ }^{1} A\left({ }^{1} n \pi^{*}\right)$ state of $9 H$-adenine used for the statistical evaluations.

\begin{tabular}{ll}
$\begin{array}{l}\text { bond } \\
\text { or angle }\end{array}$ & $\begin{array}{l}\text { length } / \AA \\
\text { or angle } /\end{array}$ \\
\hline N1-C2 & 1.364 \\
C2-C3 & 1.418 \\
C2-C9 & 1.433 \\
C3-N4 & 1.372 \\
N4-C5 & 1.403 \\
N1-C5 & 1.323 \\
C3-N6 & 1.325 \\
N6-C7 & 1.428 \\
C7-N8 & 1.395 \\
N8-C9 & 1.322 \\
C9-N10 & 1.373 \\
C7-H11 & 1.081 \\
C5-H15 & 1.077 \\
N4-H14 & 1.011 \\
N10-H12 & 1.011 \\
N10-H13 & 1.012
\end{tabular}


Table S698 Structural parameters of the CC2/TZVP/TZVP-optimized excited-state equilibrium geometry of the $2{ }^{1} A\left({ }^{1} n \pi^{*}\right)$ state of acetaldehyde used for the statistical evaluations.

\begin{tabular}{ll} 
bond & $\begin{array}{l}\text { length / } \AA \\
\text { or angle } \\
\text { or angle / }\end{array}$ \\
\hline $\mathrm{C} 1-\mathrm{O} 4$ & 1.387 \\
$\mathrm{C} 1-\mathrm{C} 2$ & 1.489 \\
$\mathrm{C} 2-\mathrm{H} 7$ & 1.090 \\
$\mathrm{O} 4-\mathrm{C} 1-\mathrm{C} 2$ & 115.0 \\
$\mathrm{C} 2-\mathrm{C} 1-\mathrm{H} 3$ & 122.5
\end{tabular}


Table S699 Structural parameters of the CC2/TZVP/TZVP-optimized excited-state equilibrium geometry of the $1{ }^{3} A\left({ }^{3} n \pi^{*}\right)$ state of acetaldehyde used for the statistical evaluations.

\begin{tabular}{ll} 
bond & $\begin{array}{l}\text { length / } \AA \\
\text { or angle } \\
\text { or angle / }\end{array}$ \\
\hline $\mathrm{C} 1-\mathrm{O} 4$ & 1.364 \\
$\mathrm{C} 1-\mathrm{C} 2$ & 1.501 \\
$\mathrm{C} 2-\mathrm{H} 7$ & 1.094 \\
$\mathrm{O} 4-\mathrm{C} 1-\mathrm{C} 2$ & 114.7 \\
$\mathrm{C} 2-\mathrm{C} 1-\mathrm{H} 3$ & 119.6
\end{tabular}


Table S700 Structural parameters of the CC2/TZVP-optimized excited-state equilibrium geometry of the $2{ }^{3} A\left({ }^{3} \pi \pi^{*}\right)$ state of acetaldehyde used for the statistical evaluations.

\begin{tabular}{ll} 
bond & $\begin{array}{l}\text { length / } \AA \\
\text { or angle } \\
\text { or angle / }\end{array}$ \\
\hline $\mathrm{C} 1-\mathrm{O} 4$ & 1.478 \\
$\mathrm{C} 1-\mathrm{C} 2$ & 1.470 \\
$\mathrm{C} 2-\mathrm{H} 7$ & 1.085 \\
$\mathrm{O} 4-\mathrm{C} 1-\mathrm{C} 2$ & 114.3 \\
$\mathrm{C} 2-\mathrm{C} 1-\mathrm{H} 3$ & 121.7
\end{tabular}


Table S701 Structural parameters of the CC2/TZVP-optimized excited-state equilibrium geometry of the $1^{1} A^{\prime \prime}\left({ }^{1} n \pi^{*}\right)$ state of acetone used for the statistical evaluations.

\begin{tabular}{ll} 
bond & $\begin{array}{l}\text { length } / \AA \\
\text { or angle } \\
\text { or angle / }\end{array}$ \\
\hline $\mathrm{C} 2-\mathrm{O} 3$ & 1.420 \\
$\mathrm{C} 1-\mathrm{C} 2$ & 1.485 \\
$\mathrm{C} 1-\mathrm{C} 2-\mathrm{C} 4$ & 124.1
\end{tabular}


Table S702 Structural parameters of the CC2/TZVP-optimized excited-state equilibrium geometry of the $1^{3} A^{\prime \prime}\left({ }^{3} n \pi^{*}\right)$ state of acetone used for the statistical evaluations.

\begin{tabular}{ll} 
bond & $\begin{array}{l}\text { length } / \AA \\
\text { or angle } \\
\text { or angle / }\end{array}$ \\
\hline $\mathrm{C} 2-\mathrm{O} 3$ & 1.387 \\
$\mathrm{C} 1-\mathrm{C} 2$ & 1.497 \\
$\mathrm{C} 1-\mathrm{C} 2-\mathrm{C} 4$ & 120.9
\end{tabular}


Table S703 Structural parameters of the CC2/TZVP-optimized excited-state equilibrium geometry of the $1^{3} A^{\prime}\left({ }^{3} \pi \pi^{*}\right)$ state of acetone used for the statistical evaluations.

\begin{tabular}{ll} 
bond & $\begin{array}{l}\text { length } / \AA \\
\text { or angle } \\
\text { or angle / }\end{array}$ \\
\hline $\mathrm{C} 2-\mathrm{O} 3$ & 1.489 \\
$\mathrm{C} 1-\mathrm{C} 2$ & 1.476 \\
$\mathrm{C} 1-\mathrm{C} 2-\mathrm{C} 4$ & 122.6
\end{tabular}


Table S704 Structural parameters of the CC2/TZVP-optimized excited-state equilibrium geometry of the $1^{1} A^{\prime \prime}\left({ }^{1} n \pi^{*}\right)$ state of acetophenone used for the statistical evaluations.

\begin{tabular}{ll}
$\begin{array}{l}\text { bond } \\
\text { or angle }\end{array}$ & $\begin{array}{l}\text { length } / \AA \\
\text { or angle / }\end{array}$ \\
\hline C7-C9 & 1.429 \\
C9-C11 & 1.384 \\
C11-C12 & 1.402 \\
C10-C12 & 1.404 \\
C $8-C 10$ & 1.383 \\
C7-C8 & 1.429 \\
C1-C7 & 1.386 \\
C1-C3 & 1.492 \\
C1-O2 & 1.430
\end{tabular}


Table S705 Structural parameters of the CC2/TZVP-optimized excited-state equilibrium geometry of the $2{ }^{1} A^{\prime}\left({ }^{1} \pi \pi^{*}\right)$ state of acetophenone used for the statistical evaluations.

\begin{tabular}{ll}
$\begin{array}{l}\text { bond } \\
\text { or angle }\end{array}$ & $\begin{array}{l}\text { length } / \AA \\
\text { or angle / }\end{array}$ \\
\hline C7-C9 & 1.438 \\
C9-C11 & 1.430 \\
C11-C12 & 1.418 \\
C10-C12 & 1.416 \\
C $8-C 10$ & 1.434 \\
C7-C8 & 1.436 \\
C1-C7 & 1.452 \\
C1-C3 & 1.515 \\
C1-O2 & 1.255
\end{tabular}


Table S706 Structural parameters of the CC2/TZVP-optimized excited-state equilibrium geometry of the $1^{3} A^{\prime \prime}\left({ }^{3} n \pi^{*}\right)$ state of acetophenone used for the statistical evaluations.

\begin{tabular}{ll}
$\begin{array}{l}\text { bond } \\
\text { or angle }\end{array}$ & $\begin{array}{l}\text { length } / \AA \\
\text { or angle / }\end{array}$ \\
\hline C7-C9 & 1.425 \\
C9-C11 & 1.386 \\
C11-C12 & 1.401 \\
C10-C12 & 1.401 \\
C $8-C 10$ & 1.386 \\
C7-C8 & 1.425 \\
C1-C7 & 1.398 \\
C1-C3 & 1.495 \\
C1-O2 & 1.401
\end{tabular}


Table S707 Structural parameters of the CC2/TZVP-optimized excited-state equilibrium geometry of the $1^{3} A^{\prime}\left({ }^{3} \pi \pi^{*}\right)$ state of acetophenone used for the statistical evaluations.

\begin{tabular}{ll}
$\begin{array}{l}\text { bond } \\
\text { or angle }\end{array}$ & $\begin{array}{l}\text { length } / \AA \\
\text { or angle / }\end{array}$ \\
\hline C7-C9 & 1.486 \\
C9-C11 & 1.364 \\
C11-C12 & 1.429 \\
C10-C12 & 1.454 \\
C $8-C 10$ & 1.359 \\
C7-C8 & 1.467 \\
C1-C7 & 1.420 \\
C1-C3 & 1.513 \\
C1-O2 & 1.282
\end{tabular}


Table S708 Structural parameters of the CC2/TZVP-optimized excited-state equilibrium geometry of the $1{ }^{1} A_{2}\left({ }^{1} \pi \pi^{*}\right)$ state of acetylene used for the statistical evaluations.

\begin{tabular}{ll} 
bond & $\begin{array}{l}\text { length / } \AA \\
\text { or angle } \\
\text { or angle / }\end{array}$ \\
\hline $\mathrm{C} 1-\mathrm{C} 2$ & 1.349 \\
$\mathrm{C} 1-\mathrm{H} 3$ & 1.095 \\
$\mathrm{C} 1-\mathrm{C} 2-\mathrm{H} 4$ & 132.7
\end{tabular}


Table S709 Structural parameters of the CC2/TZVP-optimized excited-state equilibrium geometry of the $2{ }^{1} A_{2}\left({ }^{1} \pi \pi^{*}\right)$ state of acetylene used for the statistical evaluations.

\begin{tabular}{ll} 
bond & $\begin{array}{l}\text { length } / \AA \\
\text { or angle } \\
\text { or angle / }\end{array}$ \\
\hline $\mathrm{C} 1-\mathrm{C} 2$ & 1.328 \\
$\mathrm{C} 1-\mathrm{H} 3$ & 1.064 \\
$\mathrm{C} 1-\mathrm{C} 2-\mathrm{H} 4$ & 171.6
\end{tabular}


Table S710 Structural parameters of the CC2/TZVP-optimized excited-state equilibrium geometry of the $1{ }^{1} B_{2}\left({ }^{1} \pi \pi^{*}\right)$ state of acetylene used for the statistical evaluations.

\begin{tabular}{ll} 
bond & $\begin{array}{l}\text { length / } \AA \\
\text { or angle } \\
\text { or angle / }\end{array}$ \\
\hline $\mathrm{C} 1-\mathrm{C} 2$ & 1.335 \\
$\mathrm{C} 1-\mathrm{H} 3$ & 1.096 \\
$\mathrm{C} 1-\mathrm{C} 2-\mathrm{H} 4$ & 142.0
\end{tabular}


Table S711 Structural parameters of the CC2/TZVP-optimized excited-state equilibrium geometry of the $1{ }^{3} A_{2}\left({ }^{3} \pi \pi^{*}\right)$ state of acetylene used for the statistical evaluations.

\begin{tabular}{ll} 
bond & $\begin{array}{l}\text { length / } \AA \\
\text { or angle } \\
\text { or angle / }\end{array}$ \\
\hline $\mathrm{C} 1-\mathrm{C} 2$ & 1.364 \\
$\mathrm{C} 1-\mathrm{H} 3$ & 1.093 \\
$\mathrm{C} 1-\mathrm{C} 2-\mathrm{H} 4$ & 130.4
\end{tabular}


Table S712 Structural parameters of the CC2/TZVP-optimized excited-state equilibrium geometry of the $2{ }^{3} A_{2}\left({ }^{3} \pi \pi^{*}\right)$ state of acetylene used for the statistical evaluations.

\begin{tabular}{ll} 
bond & $\begin{array}{l}\text { length / } \AA \\
\text { or angle } \\
\text { or angle / }\end{array}$ \\
\hline $\mathrm{C} 1-\mathrm{C} 2$ & 1.339 \\
$\mathrm{C} 1-\mathrm{H} 3$ & 1.066 \\
$\mathrm{C} 1-\mathrm{C} 2-\mathrm{H} 4$ & 167.3
\end{tabular}


Table S713 Structural parameters of the CC2/TZVP-optimized excited-state equilibrium geometry of the $1^{3} B_{2}\left({ }^{3} \pi \pi^{*}\right)$ state of acetylene used for the statistical evaluations.

\begin{tabular}{ll} 
bond & $\begin{array}{l}\text { length / } \AA \\
\text { or angle } \\
\text { or angle / }\end{array}$ \\
\hline $\mathrm{C} 1-\mathrm{C} 2$ & 1.342 \\
$\mathrm{C} 1-\mathrm{H} 3$ & 1.089 \\
$\mathrm{C} 1-\mathrm{C} 2-\mathrm{H} 4$ & 126.9
\end{tabular}


Table S714 Structural parameters of the CC2/TZVP-optimized excited-state equilibrium geometry of the $1^{1} A_{u}\left({ }^{1} \pi \pi^{*}\right)$ state of acetylene used for the statistical evaluations.

\begin{tabular}{ll} 
bond & $\begin{array}{l}\text { length / } \AA \\
\text { or angle } \\
\text { or angle / }\end{array}$ \\
\hline $\mathrm{C} 1-\mathrm{C} 2$ & 1.381 \\
$\mathrm{C} 1-\mathrm{H} 3$ & 1.093 \\
$\mathrm{C} 1-\mathrm{C} 2-\mathrm{H} 4$ & 122.1
\end{tabular}


Table S715 Structural parameters of the CC2/TZVP-optimized excited-state equilibrium geometry of the $2{ }^{1} A_{u}\left({ }^{1} \pi \pi^{*}\right)$ state of acetylene used for the statistical evaluations.

\begin{tabular}{ll} 
bond & $\begin{array}{l}\text { length / } \AA \\
\text { or angle } \\
\text { or angle / }\end{array}$ \\
\hline $\mathrm{C} 1-\mathrm{C} 2$ & 1.328 \\
$\mathrm{C} 1-\mathrm{H} 3$ & 1.063 \\
$\mathrm{C} 1-\mathrm{C} 2-\mathrm{H} 4$ & 171.1
\end{tabular}


Table S716 Structural parameters of the CC2/TZVP-optimized excited-state equilibrium geometry of the $1{ }^{1} B_{u}\left({ }^{1} \pi \pi^{*}\right)$ state of acetylene used for the statistical evaluations.

\begin{tabular}{ll} 
bond & $\begin{array}{l}\text { length } / \AA \\
\text { or angle } \\
\text { or angle / }\end{array}$ \\
\hline $\mathrm{C} 1-\mathrm{C} 2$ & 1.339 \\
$\mathrm{C} 1-\mathrm{H} 3$ & 1.076 \\
$\mathrm{C} 1-\mathrm{C} 2-\mathrm{H} 4$ & 147.0
\end{tabular}


Table S717 Structural parameters of the CC2/TZVP-optimized excited-state equilibrium geometry of the $1^{3} A_{u}\left({ }^{3} \pi \pi^{*}\right)$ state of acetylene used for the statistical evaluations.

\begin{tabular}{ll} 
bond & $\begin{array}{l}\text { length / } \AA \\
\text { or angle } \\
\text { or angle / }\end{array}$ \\
\hline $\mathrm{C} 1-\mathrm{C} 2$ & 1.389 \\
$\mathrm{C} 1-\mathrm{H} 3$ & 1.091 \\
$\mathrm{C} 1-\mathrm{C} 2-\mathrm{H} 4$ & 120.5
\end{tabular}


Table S718 Structural parameters of the CC2/TZVP-optimized excited-state equilibrium geometry of the $2{ }^{3} A_{u}\left({ }^{3} \pi \pi^{*}\right)$ state of acetylene used for the statistical evaluations.

\begin{tabular}{ll} 
bond & $\begin{array}{l}\text { length / } \AA \\
\text { or angle } \\
\text { or angle / }\end{array}$ \\
\hline $\mathrm{C} 1-\mathrm{C} 2$ & 1.341 \\
$\mathrm{C} 1-\mathrm{H} 3$ & 1.064 \\
$\mathrm{C} 1-\mathrm{C} 2-\mathrm{H} 4$ & 164.7
\end{tabular}


Table S719 Structural parameters of the CC2/TZVP-optimized excited-state equilibrium geometry of the $1^{3} B_{u}\left({ }^{3} \pi \pi^{*}\right)$ state of acetylene used for the statistical evaluations.

\begin{tabular}{ll} 
bond & $\begin{array}{l}\text { length / } \AA \\
\text { or angle } \\
\text { or angle / }\end{array}$ \\
\hline $\mathrm{C} 1-\mathrm{C} 2$ & 1.348 \\
$\mathrm{C} 1-\mathrm{H} 3$ & 1.080 \\
$\mathrm{C} 1-\mathrm{C} 2-\mathrm{H} 4$ & 131.2
\end{tabular}


Table S720 Structural parameters of the CC2/TZVP-optimized excited-state equilibrium geometry of the $2{ }^{3} B_{u}\left({ }^{3} \pi \pi^{*}\right)$ state of acetylene used for the statistical evaluations.

\begin{tabular}{ll} 
bond & $\begin{array}{l}\text { length } / \AA \\
\text { or angle } \\
\text { or angle / }\end{array}$ \\
\hline $\mathrm{C} 1-\mathrm{C} 2$ & 1.360 \\
$\mathrm{C} 1-\mathrm{H} 3$ & 1.067 \\
$\mathrm{C} 1-\mathrm{C} 2-\mathrm{H} 4$ & 153.8
\end{tabular}


Table S721 Structural parameters of the CC2/TZVP-optimized excited-state equilibrium geometry of the $1^{1} A\left({ }^{1} n \pi^{*}\right)$ state of acrolein used for the statistical evaluations.

\begin{tabular}{ll} 
bond & $\begin{array}{l}\text { length / } \AA \\
\text { or angle } \\
\text { or angle / }\end{array}$ \\
\hline $\mathrm{C} 3-\mathrm{O} 4$ & 1.388 \\
$\mathrm{C} 2-\mathrm{C} 3$ & 1.374 \\
$\mathrm{C} 1-\mathrm{C} 2$ & 1.388 \\
$\mathrm{O} 4-\mathrm{C} 3-\mathrm{C} 2$ & 126.2 \\
$\mathrm{C} 3-\mathrm{C} 2-\mathrm{C} 1$ & 122.1
\end{tabular}


Table S722 Structural parameters of the CC2/TZVP-optimized excited-state equilibrium geometry of the $1^{3} A\left({ }^{3} n \pi^{*}\right)$ state of acrolein used for the statistical evaluations.

\begin{tabular}{ll} 
bond & $\begin{array}{l}\text { length / } \AA \\
\text { or angle } \\
\text { or angle / }\end{array}$ \\
\hline $\mathrm{C} 3-\mathrm{O} 4$ & 1.372 \\
$\mathrm{C} 2-\mathrm{C} 3$ & 1.388 \\
$\mathrm{C} 1-\mathrm{C} 2$ & 1.376 \\
$\mathrm{O} 4-\mathrm{C} 3-\mathrm{C} 2$ & 124.5 \\
$\mathrm{C} 3-\mathrm{C} 2-\mathrm{C} 1$ & 123.1
\end{tabular}


Table S723 Structural parameters of the CC2/TZVP-optimized excited-state equilibrium geometry of the $1^{3} A^{\prime}\left({ }^{3} \pi \pi^{*}\right)$ state of acrolein used for the statistical evaluations.

\begin{tabular}{ll} 
bond & $\begin{array}{l}\text { length / } \AA \\
\text { or angle } \\
\text { or angle / }\end{array}$ \\
\hline $\mathrm{C} 3-\mathrm{O} 4$ & 1.277 \\
$\mathrm{C} 2-\mathrm{C} 3$ & 1.396 \\
$\mathrm{C} 1-\mathrm{C} 2$ & 1.510 \\
$\mathrm{O} 4-\mathrm{C} 3-\mathrm{C} 2$ & 122.5 \\
$\mathrm{C} 3-\mathrm{C} 2-\mathrm{C} 1$ & 123.6
\end{tabular}


Table S724 Structural parameters of the CC2/TZVP-optimized excited-state equilibrium geometry of the $2{ }^{1} A^{\prime}\left({ }^{1} \pi \pi^{*}\right)$ state of 2-amino-9-methylpurine used for the statistical evaluations.

\begin{tabular}{ll} 
bond & $\begin{array}{c}\text { length / } \AA \\
\text { or angle } \\
\text { or angle / }\end{array}$ \\
\hline C1-N2 & 1.351 \\
C1-N3 & 1.382 \\
N3-C4 & 1.356 \\
C4-C5 & 1.420 \\
C5-C6 & 1.443 \\
N2-C6 & 1.391 \\
C5-N9 & 1.358 \\
C8-N9 & 1.353 \\
N7-C8 & 1.359 \\
C4-N7 & 1.388 \\
C8-H13 & 1.080 \\
C1-H10 & 1.358 \\
N7-C11 & 1.455 \\
C6-H12 & 1.079 \\
N2-C1-N3 & 133.2 \\
C1-N3-C4 & 107.0 \\
N3-C4-N5 & 128.4 \\
C4-C5-C6 & 117.9 \\
C5-C6-N2 & 116.2 \\
C6-N2-C1 & 117.3 \\
C5-C4-N7 & 105.4 \\
C4-N7-C8 & 106.1 \\
N7-C8-N9 & 113.4 \\
C8-N9-C5 & 104.5 \\
N9-C5-C4 & 110.5 \\
N2-C1-N10 & 114.3 \\
C8-N7-C11 & 128.6 \\
C5-C6-H12 & 124.9 \\
N7-C8-H13 & 122.0 \\
\end{tabular}


Table S725 Structural parameters of the CC2/TZVP-optimized excited-state equilibrium geometry of the $1^{1} A^{\prime \prime}\left({ }^{1} \pi \pi^{*}\right)$ state of aniline used for the statistical evaluations.

\begin{tabular}{ll}
$\begin{array}{l}\text { bond } \\
\text { or angle }\end{array}$ & $\begin{array}{c}\text { length } / \AA \\
\text { or angle } /\end{array}$ \\
\hline C1-C2 & 1.426 \\
C2-C3 & 1.431 \\
C3-C4 & 1.420 \\
C1-N7 & 1.370 \\
N7-H8 & 1.012 \\
C2-H10 & 1.084 \\
C3-H11 & 1.082 \\
C4-H12 & 1.085 \\
C1-C2-C3 & 118.0 \\
C2-C1-C6 & 123.1 \\
C2-C3-C4 & 118.7 \\
C3-C4-C5 & 122.8 \\
N7-C1-C6 & 118.2 \\
H8-N7-H9 & 116.2 \\
H8-N7-C1 & 118.1 \\
H14-C6-C1 & 119.8 \\
H14-C6-C5 & 121.9 \\
H13-C5-C4 & 120.2 \\
H13-C5-C6 & 120.7 \\
H12-C4-C3 & 118.6
\end{tabular}


Table S726 Structural parameters of the CC2/TZVP-optimized excited-state equilibrium geometry of the $1^{3} A^{\prime}\left({ }^{3} \pi \pi^{*}\right)$ state of aniline used for the statistical evaluations.

\begin{tabular}{ll}
$\begin{array}{l}\text { bond } \\
\text { or angle }\end{array}$ & $\begin{array}{c}\text { length } / \AA \\
\text { or angle } /\end{array}$ \\
\hline C1-C2 & 1.482 \\
C2-C3 & 1.368 \\
C3-C4 & 1.425 \\
C1-N7 & 1.414 \\
N7-H8 & 1.015 \\
C2-H10 & 1.085 \\
C3-H11 & 1.086 \\
C4-H12 & 1.083 \\
C1-C2-C3 & 119.3 \\
C2-C1-C6 & 115.5 \\
C2-C3-C4 & 120.5 \\
C3-C4-C5 & 120.1 \\
N7-C1-C6 & 113.6 \\
H8-N7-H9 & 113.1 \\
H8-N7-C1 & 116.1 \\
H14-C6-C1 & 119.8 \\
H14-C6-C5 & 120.9 \\
H13-C5-C4 & 119.0 \\
H13-C5-C6 & 120.2 \\
H12-C4-C3 & 119.8
\end{tabular}


Table S727 Structural parameters of the CC2/TZVP-optimized excited-state equilibrium geometry of the $1^{1} A^{\prime \prime}\left({ }^{1} n \pi^{*}\right)$ state of benzaldehyde used for the statistical evaluations.

\begin{tabular}{ll}
$\begin{array}{l}\text { bond } \\
\text { or angle }\end{array}$ & $\begin{array}{l}\text { length } / \AA \\
\text { or angle / }\end{array}$ \\
\hline C4-C6 & 1.427 \\
C6-C8 & 1.385 \\
C $8-C 9$ & 1.400 \\
C7-C9 & 1.405 \\
C5-C7 & 1.382 \\
C4-C5 & 1.427 \\
C1-C4 & 1.388 \\
C1-O2 & 1.402
\end{tabular}


Table S728 Structural parameters of the CC2/TZVP-optimized excited-state equilibrium geometry of the $2{ }^{1} A^{\prime}\left({ }^{1} \pi \pi^{*}\right)$ state of benzaldehyde used for the statistical evaluations.

\begin{tabular}{ll}
$\begin{array}{l}\text { bond } \\
\text { or angle }\end{array}$ & $\begin{array}{l}\text { length } / \AA \\
\text { or angle } /{ }^{\circ}\end{array}$ \\
\hline C4-C6 & 1.433 \\
C6-C8 & 1.432 \\
C8-C9 & 1.419 \\
C7-C9 & 1.417 \\
C5-C7 & 1.431 \\
C4-C5 & 1.435 \\
C1-C4 & 1.441 \\
C1-O2 & 1.249
\end{tabular}


Table S729 Structural parameters of the CC2/TZVP-optimized excited-state equilibrium geometry of the $1^{3} A^{\prime \prime}\left({ }^{3} n \pi^{*}\right)$ state of benzaldehyde used for the statistical evaluations.

\begin{tabular}{ll} 
bond & $\begin{array}{l}\text { length / } \AA \\
\text { or angle } \\
\text { or angle / }\end{array}$ \\
\hline C4-C6 & 1.421 \\
C6-C8 & 1.387 \\
C $8-$ C9 & 1.399 \\
C7-C9 & 1.402 \\
C5-C7 & 1.384 \\
C4-C5 & 1.421 \\
C1-C4 & 1.401 \\
C1-O2 & 1.379
\end{tabular}


Table S730 Structural parameters of the CC2/TZVP-optimized excited-state equilibrium geometry of the $1^{3} A^{\prime}\left({ }^{3} \pi \pi^{*}\right)$ state of benzaldehyde used for the statistical evaluations.

\begin{tabular}{ll} 
bond & $\begin{array}{l}\text { length / } \AA \\
\text { or angle } \\
\text { or angle / }\end{array}$ \\
\hline C4-C6 & 1.484 \\
C6-C8 & 1.365 \\
C $8-$ C9 & 1.426 \\
C7-C9 & 1.455 \\
C5-C7 & 1.358 \\
C4-C5 & 1.462 \\
C1-C4 & 1.407 \\
C1-O2 & 1.280
\end{tabular}


Table S731 Structural parameters of the CC2/TZVP-optimized excited-state equilibrium geometry of the $1{ }^{1} A_{u}\left({ }^{1} n \pi^{*}\right)$ state of $p$-benzoquinone used for the statistical evaluations.

\begin{tabular}{ll}
$\begin{array}{l}\text { bond } \\
\text { or angle }\end{array}$ & $\begin{array}{l}\text { length } / \AA \\
\text { or angle / } /\end{array}$ \\
\hline $\mathrm{C} 1-\mathrm{O} 7$ & 1.311 \\
$\mathrm{C} 1-\mathrm{C} 2$ & 1.425 \\
$\mathrm{C} 2-\mathrm{C} 3$ & 1.384 \\
$\mathrm{C} 2-\mathrm{H} 9$ & 1.084 \\
$\mathrm{O} 7-\mathrm{C} 1-\mathrm{C} 2$ & 120.1 \\
$\mathrm{C} 6-\mathrm{C} 1-\mathrm{C} 2$ & 119.9 \\
$\mathrm{H} 9-\mathrm{C} 2-\mathrm{C} 3$ & 121.3
\end{tabular}


Table S732 Structural parameters of the CC2/TZVP-optimized excited-state equilibrium geometry of the $1{ }^{1} B_{1 g}\left({ }^{1} n \pi^{*}\right)$ state of $p$-benzoquinone used for the statistical evaluations.

\begin{tabular}{ll}
$\begin{array}{l}\text { bond } \\
\text { or angle }\end{array}$ & $\begin{array}{l}\text { length } / \AA \\
\text { or angle / }\end{array}$ \\
\hline $\mathrm{C} 1-\mathrm{O} 7$ & 1.294 \\
$\mathrm{C} 1-\mathrm{C} 2$ & 1.441 \\
$\mathrm{C} 2-\mathrm{C} 3$ & 1.367 \\
$\mathrm{C} 2-\mathrm{H} 9$ & 1.082 \\
$\mathrm{O} 7-\mathrm{C} 1-\mathrm{C} 2$ & 121.4 \\
$\mathrm{C} 6-\mathrm{C} 1-\mathrm{C} 2$ & 117.2 \\
$\mathrm{H} 9-\mathrm{C} 2-\mathrm{C} 3$ & 121.3
\end{tabular}


Table S733 Structural parameters of the CC2/TZVP-optimized excited-state equilibrium geometry of the $1{ }^{1} B_{1 u}\left({ }^{1} \pi \pi^{*}\right)$ state of $p$-benzoquinone used for the statistical evaluations.

\begin{tabular}{ll}
$\begin{array}{l}\text { bond } \\
\text { or angle }\end{array}$ & $\begin{array}{l}\text { length } / \AA \\
\text { or angle / } /\end{array}$ \\
\hline $\mathrm{C} 1-\mathrm{O} 7$ & 1.291 \\
$\mathrm{C} 1-\mathrm{C} 2$ & 1.452 \\
$\mathrm{C} 2-\mathrm{C} 3$ & 1.393 \\
$\mathrm{C} 2-\mathrm{H} 9$ & 1.084 \\
$\mathrm{O} 7-\mathrm{C} 1-\mathrm{C} 2$ & 121.0 \\
$\mathrm{C} 6-\mathrm{C} 1-\mathrm{C} 2$ & 118.0 \\
$\mathrm{H} 9-\mathrm{C} 2-\mathrm{C} 3$ & 122.0
\end{tabular}


Table S734 Structural parameters of the CC2/TZVP-optimized excited-state equilibrium geometry of the $1{ }^{1} B_{3 g}\left({ }^{1} \pi \pi^{*}\right)$ state of $p$-benzoquinone used for the statistical evaluations.

\begin{tabular}{ll}
$\begin{array}{l}\text { bond } \\
\text { or angle }\end{array}$ & $\begin{array}{l}\text { length } / \AA \\
\text { or angle / }\end{array}$ \\
\hline $\mathrm{C} 1-\mathrm{O} 7$ & 1.279 \\
$\mathrm{C} 1-\mathrm{C} 2$ & 1.434 \\
$\mathrm{C} 2-\mathrm{C} 3$ & 1.423 \\
$\mathrm{C} 2-\mathrm{H} 9$ & 1.086 \\
$\mathrm{O} 7-\mathrm{C} 1-\mathrm{C} 2$ & 123.2 \\
$\mathrm{C} 6-\mathrm{C} 1-\mathrm{C} 2$ & 113.5 \\
$\mathrm{H} 9-\mathrm{C} 2-\mathrm{C} 3$ & 119.3
\end{tabular}


Table S735 Structural parameters of the CC2/TZVP-optimized excited-state equilibrium geometry of the $1{ }^{3} A_{u}\left({ }^{3} n \pi^{*}\right)$ state of $p$-benzoquinone used for the statistical evaluations.

\begin{tabular}{ll}
$\begin{array}{l}\text { bond } \\
\text { or angle }\end{array}$ & $\begin{array}{l}\text { length } / \AA \\
\text { or angle / } /\end{array}$ \\
\hline $\mathrm{C} 1-\mathrm{O} 7$ & 1.307 \\
$\mathrm{C} 1-\mathrm{C} 2$ & 1.426 \\
$\mathrm{C} 2-\mathrm{C} 3$ & 1.382 \\
$\mathrm{C} 2-\mathrm{H} 9$ & 1.084 \\
$\mathrm{O} 7-\mathrm{C} 1-\mathrm{C} 2$ & 120.1 \\
$\mathrm{C} 6-\mathrm{C} 1-\mathrm{C} 2$ & 119.8 \\
$\mathrm{H} 9-\mathrm{C} 2-\mathrm{C} 3$ & 121.2
\end{tabular}


Table S736 Structural parameters of the CC2/TZVP-optimized excited-state equilibrium geometry of the $1{ }^{3} B_{1 g}\left({ }^{3} n \pi^{*}\right)$ state of $p$-benzoquinone used for the statistical evaluations.

\begin{tabular}{ll}
$\begin{array}{l}\text { bond } \\
\text { or angle }\end{array}$ & $\begin{array}{l}\text { length } / \AA \\
\text { or angle / } /\end{array}$ \\
\hline $\mathrm{C} 1-\mathrm{O} 7$ & 1.290 \\
$\mathrm{C} 1-\mathrm{C} 2$ & 1.442 \\
$\mathrm{C} 2-\mathrm{C} 3$ & 1.365 \\
$\mathrm{C} 2-\mathrm{H} 9$ & 1.082 \\
$\mathrm{O} 7-\mathrm{C} 1-\mathrm{C} 2$ & 121.4 \\
$\mathrm{C} 6-\mathrm{C} 1-\mathrm{C} 2$ & 117.2 \\
$\mathrm{H} 9-\mathrm{C} 2-\mathrm{C} 3$ & 121.3
\end{tabular}


Table S737 Structural parameters of the CC2/TZVP-optimized excited-state equilibrium geometry of the $1^{3} B_{1 u}\left({ }^{3} \pi \pi^{*}\right)$ state of $p$-benzoquinone used for the statistical evaluations.

\begin{tabular}{ll}
$\begin{array}{l}\text { bond } \\
\text { or angle }\end{array}$ & $\begin{array}{l}\text { length } / \AA \\
\text { or angle / } /\end{array}$ \\
\hline $\mathrm{C} 1-\mathrm{O} 7$ & 1.326 \\
$\mathrm{C} 1-\mathrm{C} 2$ & 1.418 \\
$\mathrm{C} 2-\mathrm{C} 3$ & 1.403 \\
$\mathrm{C} 2-\mathrm{H} 9$ & 1.083 \\
$\mathrm{O} 7-\mathrm{C} 1-\mathrm{C} 2$ & 120.0 \\
$\mathrm{C} 6-\mathrm{C} 1-\mathrm{C} 2$ & 120.0 \\
$\mathrm{H} 9-\mathrm{C} 2-\mathrm{C} 3$ & 121.8
\end{tabular}


Table S738 Structural parameters of the CC2/TZVP-optimized excited-state equilibrium geometry of the $1{ }^{3} B_{3 g}\left({ }^{3} \pi \pi^{*}\right)$ state of $p$-benzoquinone used for the statistical evaluations.

\begin{tabular}{ll}
$\begin{array}{l}\text { bond } \\
\text { or angle }\end{array}$ & $\begin{array}{l}\text { length } / \AA \\
\text { or angle / } /\end{array}$ \\
\hline $\mathrm{C} 1-\mathrm{O} 7$ & 1.254 \\
$\mathrm{C} 1-\mathrm{C} 2$ & 1.451 \\
$\mathrm{C} 2-\mathrm{C} 3$ & 1.431 \\
$\mathrm{C} 2-\mathrm{H} 9$ & 1.084 \\
$\mathrm{O} 7-\mathrm{C} 1-\mathrm{C} 2$ & 123.1 \\
$\mathrm{C} 6-\mathrm{C} 1-\mathrm{C} 2$ & 113.8 \\
$\mathrm{H} 9-\mathrm{C} 2-\mathrm{C} 3$ & 119.7
\end{tabular}


Table S739 Structural parameters of the CC2/TZVP-optimized excited-state equilibrium geometry of the $1{ }^{1} B_{3 g}\left({ }^{1} \pi \pi^{*}\right)$ state of biphenylene used for the statistical evaluations.

\begin{tabular}{ll}
$\begin{array}{l}\text { bond } \\
\text { or angle }\end{array}$ & $\begin{array}{c}\text { length } / \AA \\
\text { or angle / }\end{array}$ \\
\hline C1-C2 & 1.406 \\
C2-C3 & 1.387 \\
C3-C12 & 1.439 \\
C1-C6 & 1.499 \\
C1-C4 & 1.414 \\
C2-H13 & 1.085 \\
C2-H13 & 1.085 \\
C1-C2-C3 & 115.7 \\
C2-C3-C12 & 123.1 \\
C4-C1-C2 & 148.8 \\
C1-C6-C9 & 121.2
\end{tabular}


Table S740 Structural parameters of the CC2/TZVP-optimized excited-state equilibrium geometry of the $1{ }^{1} B_{1 u}\left({ }^{1} \pi \pi^{*}\right)$ state of biphenylene used for the statistical evaluations.

\begin{tabular}{ll}
$\begin{array}{l}\text { bond } \\
\text { or angle }\end{array}$ & $\begin{array}{c}\text { length } / \AA \\
\text { or angle / }\end{array}$ \\
\hline $\mathrm{C} 1-\mathrm{C} 2$ & 1.404 \\
$\mathrm{C} 2-\mathrm{C} 3$ & 1.422 \\
$\mathrm{C} 3-\mathrm{C} 12$ & 1.409 \\
$\mathrm{C} 1-\mathrm{C} 6$ & 1.446 \\
$\mathrm{C} 1-\mathrm{C} 4$ & 1.454 \\
$\mathrm{C} 2-\mathrm{H} 13$ & 1.083 \\
$\mathrm{C} 2-\mathrm{H} 13$ & 1.084 \\
$\mathrm{C} 1-\mathrm{C} 2-\mathrm{C} 3$ & 114.5 \\
$\mathrm{C} 2-\mathrm{C} 3-\mathrm{C} 12$ & 123.0 \\
$\mathrm{C} 4-\mathrm{C} 1-\mathrm{C} 2$ & 147.4 \\
$\mathrm{C} 1-\mathrm{C} 6-\mathrm{C} 9$ & 122.6
\end{tabular}


Table S741 Structural parameters of the CC2/TZVP-optimized excited-state equilibrium geometry of the $2{ }^{1} B_{1 u}\left({ }^{1} \pi \pi^{*}\right)$ state of biphenylene used for the statistical evaluations.

\begin{tabular}{ll}
$\begin{array}{l}\text { bond } \\
\text { or angle }\end{array}$ & $\begin{array}{c}\text { length } / \AA \\
\text { or angle / } /\end{array}$ \\
\hline C1-C2 & 1.405 \\
C2-C3 & 1.421 \\
C3-C12 & 1.406 \\
C1-C6 & 1.441 \\
C1-C4 & 1.466 \\
C2-H13 & 1.085 \\
C2-H13 & 1.085 \\
C1-C2-C3 & 115.7 \\
C2-C3-C12 & 122.3 \\
C4-C1-C2 & 148.1 \\
C1-C6-C9 & 121.9
\end{tabular}


Table S742 Structural parameters of the CC2/TZVP-optimized excited-state equilibrium geometry of the $1{ }^{1} B_{2 u}\left({ }^{1} \pi \pi^{*}\right)$ state of biphenylene used for the statistical evaluations.

\begin{tabular}{ll}
$\begin{array}{l}\text { bond } \\
\text { or angle }\end{array}$ & $\begin{array}{c}\text { length } / \AA \\
\text { or angle / } /\end{array}$ \\
\hline C1-C2 & 1.390 \\
C2-C3 & 1.408 \\
C3-C12 & 1.465 \\
C1-C6 & 1.476 \\
C1-C4 & 1.462 \\
C2-H13 & 1.085 \\
C2-H13 & 1.085 \\
C1-C2-C3 & 116.1 \\
C2-C3-C12 & 121.8 \\
C4-C1-C2 & 147.9 \\
C1-C6-C9 & 122.1
\end{tabular}


Table S743 Structural parameters of the CC2/TZVP-optimized excited-state equilibrium geometry of the $1{ }^{1} B_{u}\left({ }^{1} \pi \pi^{*}\right)$ state of trans-butadiene used for the statistical evaluations.

\begin{tabular}{ll}
$\begin{array}{l}\text { bond } \\
\text { or angle }\end{array}$ & $\begin{array}{l}\text { length } / \AA \\
\text { or angle } /\end{array}$ \\
\hline C2-C3 & 1.423 \\
C1-C2 & 1.394
\end{tabular}


Table S744 Structural parameters of the CC2/TZVP-optimized excited-state equilibrium geometry of the $2{ }^{1} A_{g}\left({ }^{1} \pi \pi^{*}\right)$ state of trans-butadiene used for the statistical evaluations.

\begin{tabular}{ll}
$\begin{array}{l}\text { bond } \\
\text { or angle }\end{array}$ & $\begin{array}{l}\text { length } / \AA \\
\text { or angle } /\end{array}$ \\
\hline C2-C3 & 1.481 \\
C1-C2 & 1.398
\end{tabular}


Table S745 Structural parameters of the CC2/TZVP-optimized excited-state equilibrium geometry of the $2{ }^{1} A\left({ }^{1} n \pi^{*}\right)$ state of cytosine used for the statistical evaluations.

\begin{tabular}{ll}
$\begin{array}{l}\text { bond } \\
\text { or angle }\end{array}$ & $\begin{array}{c}\text { length } / \AA \\
\text { or angle } /\end{array}$ \\
\hline N5-C6 & 1.353 \\
N1-C6 & 1.268 \\
N1-C2 & 1.426 \\
C2-C3 & 1.369 \\
C3-C4 & 1.413 \\
C4-N5 & 1.436 \\
C6-O8 & 1.431 \\
C2-N7 & 1.399 \\
C4-H10 & 1.080 \\
C3-H9 & 1.084 \\
N5-H13 & 1.013 \\
N7-H11 & 1.013 \\
N7-H12 & 1.012 \\
N5-C6-N1 & 130.6 \\
C6-N1-C2 & 113.6 \\
N1-C2-C3 & 121.7 \\
C2-C3-C4 & 120.5 \\
C3-C4-N5 & 114.9 \\
N1-C2-N7 & 112.5 \\
N5-C6-O8 & 112.9
\end{tabular}


Table S746 Structural parameters of the CC2/TZVP-optimized excited-state equilibrium geometry of the $3^{1} A\left({ }^{1} \pi \pi^{*}\right)$ state of cytosine used for the statistical evaluations.

\begin{tabular}{ll}
$\begin{array}{l}\text { bond } \\
\text { or angle }\end{array}$ & $\begin{array}{c}\text { length } / \AA \\
\text { or angle } /\end{array}$ \\
\hline N5-C6 & 1.362 \\
N1-C6 & 1.276 \\
N1-C2 & 1.461 \\
C2-C3 & 1.361 \\
C3-C4 & 1.422 \\
C4-N5 & 1.413 \\
C6-O8 & 1.389 \\
C2-N7 & 1.388 \\
C4-H10 & 1.080 \\
C3-H9 & 1.084 \\
N5-H13 & 1.013 \\
N7-H11 & 1.013 \\
N7-H12 & 1.012 \\
N5-C6-N1 & 127.6 \\
C6-N1-C2 & 114.1 \\
N1-C2-C3 & 121.9 \\
C2-C3-C4 & 120.5 \\
C3-C4-N5 & 114.7 \\
N1-C2-N7 & 110.8 \\
N5-C6-O8 & 111.9
\end{tabular}


Table S747 Structural parameters of the CC2/TZVP-optimized excited-state equilibrium geometry of the $1^{1} A_{2}\left({ }^{1} n \pi^{*}\right)$ state of diazomethane used for the statistical evaluations.

\begin{tabular}{ll} 
bond & $\begin{array}{l}\text { length / } \AA \\
\text { or angle } \\
\text { or angle / }\end{array}$ \\
\hline N2-N3 & 1.210 \\
C1-N2 & 1.339 \\
C1-H4 & 1.089 \\
H4-C1-H5 & 120.2
\end{tabular}


Table S748 Structural parameters of the CC2/TZVP-optimized excited-state equilibrium geometry of the $1{ }^{1} B_{3 g}\left({ }^{1} \pi \pi^{*}\right)$ state of dibenzo- $p$-dioxin used for the statistical evaluations.

\begin{tabular}{|c|c|}
\hline $\begin{array}{l}\text { bond } \\
\text { or angle }\end{array}$ & $\begin{array}{l}\text { length / } \AA \\
\text { or angle / }\end{array}$ \\
\hline C4-07 & 1.358 \\
\hline $\mathrm{C} 4-\mathrm{C} 5$ & 1.447 \\
\hline $\mathrm{C} 5-\mathrm{C} 6$ & 1.404 \\
\hline $\mathrm{C} 1-\mathrm{C} 6$ & 1.379 \\
\hline $\mathrm{C} 1-\mathrm{C} 2$ & 1.439 \\
\hline $\mathrm{C} 4-07-\mathrm{C} 8$ & 118.1 \\
\hline $\mathrm{C} 4-\mathrm{C} 5-\mathrm{C} 6$ & 119.7 \\
\hline $\mathrm{C} 2-\mathrm{C} 3-\mathrm{C} 4$ & 119.8 \\
\hline $\mathrm{C} 1-\mathrm{C} 2-\mathrm{C} 3$ & 120.5 \\
\hline
\end{tabular}


Table S749 Structural parameters of the CC2/TZVP-optimized excited-state equilibrium geometry of the $1{ }^{1} B_{2 u}\left({ }^{1} \pi \pi^{*}\right)$ state of dibenzo- $p$-dioxin used for the statistical evaluations.

\begin{tabular}{|c|c|}
\hline $\begin{array}{l}\text { bond } \\
\text { or angle }\end{array}$ & $\begin{array}{l}\text { length / } \AA \\
\text { or angle / }\end{array}$ \\
\hline C4-07 & 1.370 \\
\hline $\mathrm{C} 4-\mathrm{C} 5$ & 1.404 \\
\hline $\mathrm{C} 5-\mathrm{C} 6$ & 1.416 \\
\hline $\mathrm{C} 1-\mathrm{C} 6$ & 1.405 \\
\hline $\mathrm{C} 1-\mathrm{C} 2$ & 1.406 \\
\hline $\mathrm{C} 4-07-\mathrm{C} 8$ & 117.8 \\
\hline $\mathrm{C} 4-\mathrm{C} 5-\mathrm{C} 6$ & 121.2 \\
\hline $\mathrm{C} 2-\mathrm{C} 3-\mathrm{C} 4$ & 117.3 \\
\hline $\mathrm{C} 1-\mathrm{C} 2-\mathrm{C} 3$ & 121.4 \\
\hline
\end{tabular}


Table S750 Structural parameters of the CC2/TZVP-optimized excited-state equilibrium geometry of the $1{ }^{1} B_{1 u}\left({ }^{1} \pi \pi^{*}\right)$ state of dibenzo- $p$-dioxin used for the statistical evaluations.

\begin{tabular}{ll} 
bond & $\begin{array}{l}\text { length / } \AA \\
\text { or angle }\end{array}$ or angle / \\
\hline C4-O7 & 1.381 \\
C4-C5 & 1.425 \\
C5-C6 & 1.393 \\
C1-C6 & 1.397 \\
C1-C2 & 1.444 \\
C4-O7-C8 & 117.0 \\
C4-C5-C6 & 120.4 \\
C2-C3-C4 & 119.9 \\
C1-C2-C3 & 119.8
\end{tabular}


Table S751 Structural parameters of the CC2/TZVP-optimized excited-state equilibrium geometry of the $1{ }^{3} A_{g}\left({ }^{3} \pi \pi^{*}\right)$ state of dibenzo- $p$-dioxin used for the statistical evaluations.

\begin{tabular}{ll}
$\begin{array}{l}\text { bond } \\
\text { or angle }\end{array}$ & $\begin{array}{l}\text { length } / \AA \\
\text { or angle / }\end{array}$ \\
\hline C4-07 & 1.370 \\
C4-C5 & 1.405 \\
C5-C6 & 1.411 \\
C1-C6 & 1.404 \\
C1-C2 & 1.401 \\
C4-O7-C8 & 116.0 \\
C4-C5-C6 & 121.3 \\
C2-C3-C4 & 117.2 \\
C1-C2-C3 & 121.5
\end{tabular}


Table S752 Structural parameters of the CC2/TZVP-optimized excited-state equilibrium geometry of the $1{ }^{3} B_{3 g}\left({ }^{3} \pi \pi^{*}\right)$ state of dibenzo- $p$-dioxin used for the statistical evaluations.

\begin{tabular}{ll}
$\begin{array}{l}\text { bond } \\
\text { or angle }\end{array}$ & $\begin{array}{l}\text { length } / \AA \\
\text { or angle / }\end{array}$ \\
\hline C4-O7 & 1.362 \\
C4-C5 & 1.449 \\
C5-C6 & 1.401 \\
C1-C6 & 1.381 \\
C1-C2 & 1.445 \\
C4-O7-C8 & 118.0 \\
C4-C5-C6 & 119.8 \\
C2-C3-C4 & 119.8 \\
C1-C2-C3 & 120.4
\end{tabular}


Table S753 Structural parameters of the CC2/TZVP-optimized excited-state equilibrium geometry of the $1{ }^{3} B_{2 u}\left({ }^{3} \pi \pi^{*}\right)$ state of dibenzo- $p$-dioxin used for the statistical evaluations.

\begin{tabular}{ll} 
bond & $\begin{array}{l}\text { length / } \AA \\
\text { or angle }\end{array}$ or angle / \\
\hline C4-O7 & 1.367 \\
C4-C5 & 1.406 \\
C5-C6 & 1.412 \\
C1-C6 & 1.407 \\
C1-C2 & 1.399 \\
C4-O7-C8 & 118.6 \\
C4-C5-C6 & 121.4 \\
C2-C3-C4 & 116.9 \\
C1-C2-C3 & 121.7
\end{tabular}


Table S754 Structural parameters of the CC2/TZVP-optimized excited-state equilibrium geometry of the $1{ }^{3} B_{1 u}\left({ }^{3} \pi \pi^{*}\right)$ state of dibenzo- $p$-dioxin used for the statistical evaluations.

\begin{tabular}{ll} 
bond & $\begin{array}{l}\text { length / } \AA \\
\text { or angle }\end{array}$ or angle / \\
\hline C4-O7 & 1.386 \\
C4-C5 & 1.393 \\
C5-C6 & 1.414 \\
C1-C6 & 1.422 \\
C1-C2 & 1.392 \\
C4-O7-C8 & 113.7 \\
C4-C5-C6 & 120.4 \\
C2-C3-C4 & 119.4 \\
C1-C2-C3 & 120.2
\end{tabular}


Table S755 Structural parameters of the CC2/TZVP-optimized excited-state equilibrium geometry of the $1{ }^{1} A_{u}\left({ }^{1} n \pi^{*}\right)$ state of dibenzo- $p$-dioxin used for the statistical evaluations.

\begin{tabular}{ll} 
bond & $\begin{array}{l}\text { length / } \AA \\
\text { or angle } \\
\text { or angle / }\end{array}$ \\
\hline C4-07 & 1.360 \\
C4-C5 & 1.473 \\
C5-C6 & 1.392 \\
C1-C6 & 1.385 \\
C1-C2 & 1.422 \\
C4-O7-C8 & 124.8 \\
C4-C5-C6 & 118.3 \\
C2-C3-C4 & 122.1 \\
C1-C2-C3 & 119.6
\end{tabular}


Table S756 Structural parameters of the CC2/TZVP-optimized excited-state equilibrium geometry of the $1{ }^{1} B_{1 g}\left({ }^{1} n \pi^{*}\right)$ state of dibenzo- $p$-dioxin used for the statistical evaluations.

\begin{tabular}{|c|c|}
\hline $\begin{array}{l}\text { bond } \\
\text { or angle }\end{array}$ & $\begin{array}{l}\text { length / } \AA \\
\text { or angle / }\end{array}$ \\
\hline C4-07 & 1.370 \\
\hline $\mathrm{C} 4-\mathrm{C} 5$ & 1.415 \\
\hline $\mathrm{C} 5-\mathrm{C} 6$ & 1.408 \\
\hline $\mathrm{C} 1-\mathrm{C} 6$ & 1.411 \\
\hline $\mathrm{C} 1-\mathrm{C} 2$ & 1.384 \\
\hline $\mathrm{C} 4-07-\mathrm{C} 8$ & 124.6 \\
\hline $\mathrm{C} 4-\mathrm{C} 5-\mathrm{C} 6$ & 120.3 \\
\hline $\mathrm{C} 2-\mathrm{C} 3-\mathrm{C} 4$ & 118.8 \\
\hline $\mathrm{C} 1-\mathrm{C} 2-\mathrm{C} 3$ & 120.9 \\
\hline
\end{tabular}


Table S757 Structural parameters of the CC2/TZVP-optimized excited-state equilibrium geometry of the $1{ }^{1} B_{3 u}\left({ }^{1} n \pi^{*}\right)$ state of dibenzo- $p$-dioxin used for the statistical evaluations.

\begin{tabular}{ll} 
bond & $\begin{array}{l}\text { length / } \AA \\
\text { or angle }\end{array}$ or angle / \\
\hline C4-O7 & 1.378 \\
C4-C5 & 1.409 \\
C5-C6 & 1.409 \\
C1-C6 & 1.404 \\
C1-C2 & 1.390 \\
C4-O7-C8 & 120.5 \\
C4-C5-C6 & 120.1 \\
C2-C3-C4 & 119.2 \\
C1-C2-C3 & 120.7
\end{tabular}


Table S758 Structural parameters of the CC2/TZVP-optimized excited-state equilibrium geometry of the $1{ }^{1} B_{2 g}\left({ }^{1} n \pi^{*}\right)$ state of dibenzo- $p$-dioxin used for the statistical evaluations.

\begin{tabular}{ll} 
bond & $\begin{array}{l}\text { length / } \AA \\
\text { or angle }\end{array}$ or angle / \\
\hline C4-O7 & 1.386 \\
C4-C5 & 1.422 \\
C5-C6 & 1.392 \\
C1-C6 & 1.401 \\
C1-C2 & 1.421 \\
C4-O7-C8 & 118.0 \\
C4-C5-C6 & 119.7 \\
C2-C3-C4 & 120.7 \\
C1-C2-C3 & 119.6
\end{tabular}


Table S759 Structural parameters of the CC2/TZVP-optimized excited-state equilibrium geometry of the $1{ }^{3} A_{u}\left({ }^{3} n \pi^{*}\right)$ state of dibenzo- $p$-dioxin used for the statistical evaluations.

\begin{tabular}{ll} 
bond & $\begin{array}{l}\text { length / } \AA \\
\text { or angle } \\
\text { or angle / }\end{array}$ \\
\hline C4-07 & 1.360 \\
C4-C5 & 1.473 \\
C5-C6 & 1.393 \\
C1-C6 & 1.384 \\
C1-C2 & 1.423 \\
C4-O7-C8 & 124.6 \\
C4-C5-C6 & 118.2 \\
C2-C3-C4 & 122.1 \\
C1-C2-C3 & 119.6
\end{tabular}


Table S760 Structural parameters of the CC2/TZVP-optimized excited-state equilibrium geometry of the $1{ }^{3} B_{1 g}\left({ }^{3} n \pi^{*}\right)$ state of dibenzo- $p$-dioxin used for the statistical evaluations.

\begin{tabular}{ll}
$\begin{array}{l}\text { bond } \\
\text { or angle }\end{array}$ & $\begin{array}{l}\text { length } / \AA \\
\text { or angle / }\end{array}$ \\
\hline C4-07 & 1.397 \\
C4-C5 & 1.412 \\
C5-C6 & 1.401 \\
C1-C6 & 1.400 \\
C1-C2 & 1.400 \\
C4-O7-C8 & 119.4 \\
C4-C5-C6 & 120.1 \\
C2-C3-C4 & 119.5 \\
C1-C2-C3 & 120.4
\end{tabular}


Table S761 Structural parameters of the CC2/TZVP-optimized excited-state equilibrium geometry of the $1{ }^{3} B_{3 u}\left({ }^{3} n \pi^{*}\right)$ state of dibenzo- $p$-dioxin used for the statistical evaluations.

\begin{tabular}{ll}
$\begin{array}{l}\text { bond } \\
\text { or angle }\end{array}$ & $\begin{array}{l}\text { length } / \AA \\
\text { or angle / }\end{array}$ \\
\hline C4-07 & 1.377 \\
C4-C5 & 1.409 \\
C5-C6 & 1.409 \\
C1-C6 & 1.404 \\
C1-C2 & 1.390 \\
C4-O7-C8 & 120.4 \\
C4-C5-C6 & 120.0 \\
C2-C3-C4 & 119.5 \\
C1-C2-C3 & 120.6
\end{tabular}


Table S762 Structural parameters of the CC2/TZVP-optimized excited-state equilibrium geometry of the $1^{1} A^{\prime \prime}\left({ }^{1} n \pi^{*}\right)$ state of formaldehyde used for the statistical evaluations.

\begin{tabular}{ll} 
bond & $\begin{array}{l}\text { length } / \AA \\
\text { or angle } \\
\text { or angle / }\end{array}$ \\
\hline $\mathrm{C} 1-\mathrm{O} 2$ & 1.361 \\
$\mathrm{C} 1-\mathrm{H} 3$ & 1.089 \\
$\mathrm{H} 3-\mathrm{C} 1-\mathrm{H} 4$ & 121.0
\end{tabular}


Table S763 Structural parameters of the CC2/TZVP-optimized excited-state equilibrium geometry of the $1^{3} A^{\prime \prime}\left({ }^{3} n \pi^{*}\right)$ state of formaldehyde used for the statistical evaluations.

\begin{tabular}{ll} 
bond & $\begin{array}{l}\text { length } / \AA \\
\text { or angle } \\
\text { or angle / }\end{array}$ \\
\hline $\mathrm{C} 1-\mathrm{O} 2$ & 1.343 \\
$\mathrm{C} 1-\mathrm{H} 3$ & 1.093 \\
$\mathrm{H} 3-\mathrm{C} 1-\mathrm{H} 4$ & 117.6
\end{tabular}


Table S764 Structural parameters of the CC2/TZVP-optimized excited-state equilibrium geometry of the $1{ }^{3} A^{\prime}\left({ }^{3} \pi \pi^{*}\right)$ state of formaldehyde used for the statistical evaluations.

\begin{tabular}{ll} 
bond & $\begin{array}{l}\text { length } / \AA \\
\text { or angle } \\
\text { or angle / }\end{array}$ \\
\hline $\mathrm{C} 1-\mathrm{O} 2$ & 1.469 \\
$\mathrm{C} 1-\mathrm{H} 3$ & 1.081 \\
$\mathrm{H} 3-\mathrm{C} 1-\mathrm{H} 4$ & 121.5
\end{tabular}


Table S765 Structural parameters of the CC2/TZVP-optimized excited-state equilibrium geometry of the $1{ }^{1} A_{u}\left({ }^{1} n \pi^{*}\right)$ state of trans-glyoxal used for the statistical evaluations.

\begin{tabular}{ll} 
bond & $\begin{array}{l}\text { length / } \AA \\
\text { or angle } \\
\text { or angle / }\end{array}$ \\
\hline $\mathrm{C} 1-\mathrm{H} 5$ & 1.096 \\
$\mathrm{C} 1-\mathrm{C} 2$ & 1.480 \\
$\mathrm{C} 1-\mathrm{O} 3$ & 1.260 \\
$\mathrm{C} 1-\mathrm{C} 2-\mathrm{H} 6$ & 114.8 \\
$\mathrm{C} 1-\mathrm{C} 2-\mathrm{O} 4$ & 123.8
\end{tabular}


Table S766 Structural parameters of the CC2/TZVP-optimized excited-state equilibrium geometry of the $1{ }^{3} A_{u}\left({ }^{3} n \pi^{*}\right)$ state of trans-glyoxal used for the statistical evaluations.

\begin{tabular}{ll} 
bond & $\begin{array}{l}\text { length / } \AA \\
\text { or angle } \\
\text { or angle / }\end{array}$ \\
\hline $\mathrm{C} 1-\mathrm{H} 5$ & 1.092 \\
$\mathrm{C} 1-\mathrm{C} 2$ & 1.474 \\
$\mathrm{C} 1-\mathrm{O} 3$ & 1.259 \\
$\mathrm{C} 1-\mathrm{C} 2-\mathrm{H} 6$ & 115.4 \\
$\mathrm{C} 1-\mathrm{C} 2-\mathrm{O} 4$ & 123.1
\end{tabular}


Table S767 Structural parameters of the CC2/TZVP-optimized excited-state equilibrium geometry of the $2{ }^{1} A\left({ }^{1} \pi \pi^{*}\right)$ state of $9 H$-guanine used for the statistical evaluations.

\begin{tabular}{ll}
$\begin{array}{l}\text { bond } \\
\text { or angle }\end{array}$ & $\begin{array}{l}\text { length } / \AA \\
\text { or angle } /\end{array}$ \\
\hline N7-C9 & 1.401 \\
C9-N13 & 1.484 \\
N13-C14 & 1.297 \\
C4-C14 & 1.458 \\
C4-C5 & 1.462 \\
C5-N7 & 1.446 \\
N3-C4 & 1.370 \\
C2-N3 & 1.325 \\
N1-C2 & 1.376 \\
N1-C14 & 1.392 \\
C9-N10 & 1.386 \\
C5-O6 & 1.228 \\
C2-H15 & 1.080 \\
N10-H11 & 1.015 \\
N10-H12 & 1.014 \\
N7-H8 & 1.017 \\
N1-H16 & 1.011
\end{tabular}


Table S768 Structural parameters of the CC2/TZVP-optimized excited-state equilibrium geometry of the $2{ }^{1} A^{\prime}\left({ }^{1} \pi \pi^{*}\right)$ state of 1-hydroxy-2-acetonaphthone used for the statistical evaluations.

\begin{tabular}{ll} 
bond & $\begin{array}{l}\text { length } / \AA \\
\text { or angle }\end{array}$ or angle / ${ }^{\circ}$ \\
\hline C1-C6 & 1.453 \\
C5-C6 & 1.380 \\
C4-C5 & 1.425 \\
C3-C 4 & 1.395 \\
C3-C10 & 1.421 \\
C9-C10 & 1.382 \\
C8-C9 & 1.418 \\
C7-C8 & 1.385 \\
C2-C7 & 1.414 \\
C2-C3 & 1.453 \\
C1-C2 & 1.422 \\
C6-C11 & 1.464 \\
C11-C12 & 1.488 \\
C1-013 & 1.316 \\
C11-O14 & 1.323 \\
O13-H21 & 1.362 \\
O14-H21 & 1.110 \\
O13-O14 & 2.437
\end{tabular}


Table S769 Structural parameters of the CC2/TZVP-optimized excited-state equilibrium geometry of the $2{ }^{1} A^{\prime}\left({ }^{1} \pi \pi^{*}\right)$ state of indole used for the statistical evaluations.

$\begin{array}{ll}\text { bond } & \begin{array}{c}\text { length } / \AA \\ \text { or angle }\end{array} \\ \text { or angle / } /\end{array}$


Table S770 Structural parameters of the CC2/TZVP-optimized excited-state equilibrium geometry of the $1{ }^{1} B_{1}\left({ }^{1} n \pi^{*}\right)$ state of maleimide used for the statistical evaluations.

\begin{tabular}{ll}
$\begin{array}{l}\text { bond } \\
\text { or angle }\end{array}$ & $\begin{array}{l}\text { length } / \AA \\
\text { or angle } /\end{array}$ \\
\hline N1-C2 & 1.406 \\
C2-C3 & 1.421 \\
C3-C4 & 1.399 \\
C2-O6 & 1.278 \\
C3-H9 & 1.077 \\
C2-N1-C5 & 109.2 \\
N1-C2-O6 & 117.8 \\
O6-C2-C4 & 135.1 \\
C2-C3-C4 & 108.3 \\
C2-C3-H9 & 123.3 \\
N1-H8 & 1.012
\end{tabular}


Table S771 Structural parameters of the CC2/TZVP-optimized excited-state equilibrium geometry of the $1^{1} A_{2}\left({ }^{1} n \pi^{*}\right)$ state of maleimide used for the statistical evaluations.

\begin{tabular}{ll}
$\begin{array}{l}\text { bond } \\
\text { or angle }\end{array}$ & $\begin{array}{l}\text { length } / \AA \\
\text { or angle } /\end{array}$ \\
\hline N1-C2 & 1.381 \\
C2-C3 & 1.415 \\
C3-C4 & 1.413 \\
C2-O6 & 1.308 \\
C3-H9 & 1.078 \\
C2-N1-C5 & 109.3 \\
N1-C2-O6 & 123.7 \\
O6-C2-C4 & 128.3 \\
C2-C3-C4 & 107.3 \\
C2-C3-H9 & 124.8 \\
N1-H8 & 1.009
\end{tabular}


Table S772 Structural parameters of the CC2/TZVP-optimized excited-state equilibrium geometry of the $1{ }^{1} B_{2}\left({ }^{1} \pi \pi^{*}\right)$ state of maleimide used for the statistical evaluations.

\begin{tabular}{ll}
$\begin{array}{l}\text { bond } \\
\text { or angle }\end{array}$ & $\begin{array}{l}\text { length } / \AA \\
\text { or angle } /\end{array}$ \\
\hline N1-C2 & 1.598 \\
C2-C3 & 1.401 \\
C3-C4 & 1.415 \\
C2-O6 & 1.222 \\
C3-H9 & 1.081 \\
C2-N1-C5 & 109.1 \\
N1-C2-O6 & 119.0 \\
O6-C2-C4 & 140.7 \\
C2-C3-C4 & 115.1 \\
C2-C3-H9 & 120.8 \\
N1-H8 & 1.029
\end{tabular}


Table S773 Structural parameters of the CC2/TZVP-optimized excited-state equilibrium geometry of the $2{ }^{1} B_{2}\left({ }^{1} \pi \pi^{*}\right)$ state of maleimide used for the statistical evaluations.

\begin{tabular}{ll}
$\begin{array}{l}\text { bond } \\
\text { or angle }\end{array}$ & $\begin{array}{l}\text { length } / \AA \\
\text { or angle } /\end{array}$ \\
\hline N1-C2 & 1.394 \\
C2-C3 & 1.437 \\
C3-C4 & 1.440 \\
C2-O6 & 1.284 \\
C3-H9 & 1.079 \\
C2-N1-C5 & 112.1 \\
N1-C2-O6 & 124.3 \\
O6-C2-C4 & 129.4 \\
C2-C3-C4 & 107.7 \\
C2-C3-H9 & 124.1 \\
N1-H8 & 1.013
\end{tabular}


Table S774 Structural parameters of the CC2/TZVP-optimized excited-state equilibrium geometry of the $1{ }^{3} B_{1}\left({ }^{3} n \pi^{*}\right)$ state of maleimide used for the statistical evaluations.

\begin{tabular}{ll}
$\begin{array}{l}\text { bond } \\
\text { or angle }\end{array}$ & $\begin{array}{l}\text { length } / \AA \\
\text { or angle } /\end{array}$ \\
\hline N1-C2 & 1.406 \\
C2-C3 & 1.422 \\
C3-C4 & 1.395 \\
C2-O6 & 1.276 \\
C3-H9 & 1.077 \\
C2-N1-C5 & 109.3 \\
N1-C2-O6 & 118.3 \\
O6-C2-C4 & 134.7 \\
C2-C3-C4 & 108.4 \\
C2-C3-H9 & 123.3 \\
N1-H8 & 1.012
\end{tabular}


Table S775 Structural parameters of the CC2/TZVP-optimized excited-state equilibrium geometry of the $1{ }^{3} A_{2}\left({ }^{3} n \pi^{*}\right)$ state of maleimide used for the statistical evaluations.

\begin{tabular}{ll}
$\begin{array}{l}\text { bond } \\
\text { or angle }\end{array}$ & $\begin{array}{l}\text { length } / \AA \\
\text { or angle } /\end{array}$ \\
\hline N1-C2 & 1.383 \\
C2-C3 & 1.417 \\
C3-C4 & 1.409 \\
C2-O6 & 1.303 \\
C3-H9 & 1.079 \\
C2-N1-C5 & 109.4 \\
N1-C2-O6 & 124.3 \\
O6-C2-C4 & 127.8 \\
C2-C3-C4 & 107.4 \\
C2-C3-H9 & 124.9 \\
N1-H8 & 1.009
\end{tabular}


Table S776 Structural parameters of the CC2/TZVP-optimized excited-state equilibrium geometry of the $1{ }^{3} B_{2}\left({ }^{3} \pi \pi^{*}\right)$ state of maleimide used for the statistical evaluations.

\begin{tabular}{ll}
$\begin{array}{l}\text { bond } \\
\text { or angle }\end{array}$ & $\begin{array}{l}\text { length } / \AA \\
\text { or angle } /\end{array}$ \\
\hline N1-C2 & 1.413 \\
C2-C3 & 1.446 \\
C3-C4 & 1.518 \\
C2-O6 & 1.239 \\
C3-H9 & 1.077 \\
C2-N1-C5 & 115.5 \\
N1-C2-O6 & 125.5 \\
O6-C2-C4 & 129.8 \\
C2-C3-C4 & 107.6 \\
C2-C3-H9 & 124.9 \\
N1-H8 & 1.014
\end{tabular}


Table S777 Structural parameters of the CC2/TZVP-optimized excited-state equilibrium geometry of the $1^{1} A^{\prime \prime}\left({ }^{1} n \pi^{*}\right)$ state of nitrosomethane used for the statistical evaluations.

\begin{tabular}{ll}
$\begin{array}{l}\text { bond } \\
\text { or angle }\end{array}$ & $\begin{array}{l}\text { length } / \AA \\
\text { or angle / }\end{array}$ \\
\hline $\mathrm{N} 2-\mathrm{O} 3$ & 1.285 \\
$\mathrm{C} 1-\mathrm{N} 2$ & 1.479 \\
$\mathrm{C} 1-\mathrm{H} 4$ & 1.084 \\
$\mathrm{C} 1-\mathrm{H} 5$ & 1.092 \\
$\mathrm{C} 1-\mathrm{N} 2-\mathrm{O} 3$ & 115.4 \\
$\mathrm{~N} 2-\mathrm{C} 1-\mathrm{H} 4$ & 107.1 \\
$\mathrm{~N} 2-\mathrm{C} 1-\mathrm{H} 5$ & 109.1 \\
$\mathrm{H} 4-\mathrm{C} 1-\mathrm{H} 5$ & 109.6
\end{tabular}


Table S778 Structural parameters of the CC2/TZVP-optimized excited-state equilibrium geometry of the $1^{3} A^{\prime \prime}\left({ }^{3} n \pi^{*}\right)$ state of nitrosomethane used for the statistical evaluations.

\begin{tabular}{ll}
$\begin{array}{l}\text { bond } \\
\text { or angle }\end{array}$ & $\begin{array}{l}\text { length } / \AA \\
\text { or angle } /{ }^{\circ}\end{array}$ \\
\hline $\mathrm{N} 2-\mathrm{O} 3$ & 1.261 \\
$\mathrm{C} 1-\mathrm{N} 2$ & 1.465 \\
$\mathrm{C} 1-\mathrm{H} 4$ & 1.085 \\
$\mathrm{C} 1-\mathrm{H} 5$ & 1.092 \\
$\mathrm{C} 1-\mathrm{N} 2-\mathrm{O} 3$ & 120.9 \\
$\mathrm{~N} 2-\mathrm{C} 1-\mathrm{H} 4$ & 107.6 \\
$\mathrm{~N} 2-\mathrm{C} 1-\mathrm{H} 5$ & 109.5 \\
$\mathrm{H} 4-\mathrm{C} 1-\mathrm{H} 5$ & 109.6
\end{tabular}


Table S779 Structural parameters of the CC2/TZVP-optimized excited-state equilibrium geometry of the $1^{1} A^{\prime \prime}\left({ }^{1} n \pi^{*}\right)$ state of propenoic acid anion used for the statistical evaluations.

\begin{tabular}{ll}
$\begin{array}{l}\text { bond } \\
\text { or angle }\end{array}$ & $\begin{array}{l}\text { length } / \AA \\
\text { or angle } /\end{array}$ \\
\hline $\mathrm{C} 2-\mathrm{O} 3$ & 1.315 \\
$\mathrm{C} 2-\mathrm{O} 4$ & 1.347 \\
$\mathrm{C} 1-\mathrm{C} 2$ & 1.390 \\
$\mathrm{C} 1-\mathrm{C} 5$ & 1.420 \\
$\mathrm{O} 4-\mathrm{C} 2-\mathrm{C} 1$ & 127.0 \\
$\mathrm{C} 2-\mathrm{C} 1-\mathrm{C} 5$ & 123.7 \\
$\mathrm{O} 3-\mathrm{C} 2-\mathrm{O} 4$ & 103.0
\end{tabular}


Table S780 Structural parameters of the CC2/TZVP-optimized excited-state equilibrium geometry of the $1^{1} A_{2}\left({ }^{1} n \pi^{*}\right)$ state of pyridine used for the statistical evaluations.

\begin{tabular}{ll}
$\begin{array}{l}\text { bond } \\
\text { or angle }\end{array}$ & $\begin{array}{l}\text { length } / \AA \\
\text { or angle / } /\end{array}$ \\
\hline $\mathrm{N} 1-\mathrm{C} 2$ & 1.316 \\
$\mathrm{C} 2-\mathrm{C} 4$ & 1.470 \\
$\mathrm{C} 4-\mathrm{C} 6$ & 1.392 \\
$\mathrm{C} 2-\mathrm{N} 1-\mathrm{C} 3$ & 135.4 \\
$\mathrm{~N} 1-\mathrm{C} 2-\mathrm{C} 4$ & 112.8 \\
$\mathrm{C} 2-\mathrm{C} 4-\mathrm{C} 6$ & 117.4 \\
$\mathrm{C} 4-\mathrm{C} 6-\mathrm{C} 5$ & 124.2
\end{tabular}


Table S781 Structural parameters of the CC2/TZVP-optimized excited-state equilibrium geometry of the $2{ }^{1} A^{\prime}\left({ }^{1} n \pi^{*}\right)$ state of pyridine used for the statistical evaluations.

\begin{tabular}{ll}
$\begin{array}{l}\text { bond } \\
\text { or angle }\end{array}$ & $\begin{array}{l}\text { length } / \AA \\
\text { or angle / } /\end{array}$ \\
\hline $\mathrm{N} 1-\mathrm{C} 2$ & 1.367 \\
$\mathrm{C} 2-\mathrm{C} 4$ & 1.377 \\
$\mathrm{C} 4-\mathrm{C} 6$ & 1.430 \\
$\mathrm{C} 2-\mathrm{N} 1-\mathrm{C} 3$ & 125.4 \\
$\mathrm{~N} 1-\mathrm{C} 2-\mathrm{C} 4$ & 113.5 \\
$\mathrm{C} 2-\mathrm{C} 4-\mathrm{C} 6$ & 119.0 \\
$\mathrm{C} 4-\mathrm{C} 6-\mathrm{C} 5$ & 118.5
\end{tabular}


Table S782 Structural parameters of the CC2/TZVP-optimized excited-state equilibrium geometry of the $1^{3} A_{1}\left({ }^{3} \pi \pi^{*}\right)$ state of pyridine used for the statistical evaluations.

\begin{tabular}{ll}
$\begin{array}{l}\text { bond } \\
\text { or angle }\end{array}$ & $\begin{array}{l}\text { length } / \AA \\
\text { or angle / }\end{array}$ \\
\hline $\mathrm{N} 1-\mathrm{C} 2$ & 1.337 \\
$\mathrm{C} 2-\mathrm{C} 4$ & 1.513 \\
$\mathrm{C} 4-\mathrm{C} 6$ & 1.391 \\
$\mathrm{C} 2-\mathrm{N} 1-\mathrm{C} 3$ & 117.6 \\
$\mathrm{~N} 1-\mathrm{C} 2-\mathrm{C} 4$ & 123.3 \\
$\mathrm{C} 2-\mathrm{C} 4-\mathrm{C} 6$ & 118.2 \\
$\mathrm{C} 4-\mathrm{C} 6-\mathrm{C} 5$ & 119.3
\end{tabular}


Table S783 Structural parameters of the CC2/TZVP-optimized excited-state equilibrium geometry of the $2^{3} A_{1}\left({ }^{3} \pi \pi^{*}\right)$ state of pyridine used for the statistical evaluations.

\begin{tabular}{ll}
$\begin{array}{l}\text { bond } \\
\text { or angle }\end{array}$ & $\begin{array}{l}\text { length } / \AA \\
\text { or angle / }\end{array}$ \\
\hline $\mathrm{N} 1-\mathrm{C} 2$ & 1.379 \\
$\mathrm{C} 2-\mathrm{C} 4$ & 1.419 \\
$\mathrm{C} 4-\mathrm{C} 6$ & 1.431 \\
$\mathrm{C} 2-\mathrm{N} 1-\mathrm{C} 3$ & 115.5 \\
$\mathrm{~N} 1-\mathrm{C} 2-\mathrm{C} 4$ & 124.5 \\
$\mathrm{C} 2-\mathrm{C} 4-\mathrm{C} 6$ & 119.0 \\
$\mathrm{C} 4-\mathrm{C} 6-\mathrm{C} 5$ & 117.4
\end{tabular}


Table S784 Structural parameters of the CC2/TZVP-optimized excited-state equilibrium geometry of the $2{ }^{1} A^{\prime}\left({ }^{1} \pi \pi^{*}\right)$ state of pyrrole used for the statistical evaluations.

\begin{tabular}{ll}
$\begin{array}{l}\text { bond } \\
\text { or angle }\end{array}$ & $\begin{array}{l}\text { length } / \AA \\
\text { or angle / }\end{array}$ \\
\hline N6-C7 & 1.418 \\
C7-C10 & 1.453 \\
C9-C10 & 1.465 \\
C7-N6-C8 & 113.6 \\
C10-C7-N6 & 103.4 \\
C7-C10-C9 & 108.2 \\
H1-N6 & 1.013
\end{tabular}


Table S785 Structural parameters of the CC2/TZVP-optimized excited-state equilibrium geometry of the $1{ }^{1} B_{2}\left({ }^{1} \pi \pi^{*}\right)$ state of pyrrole used for the statistical evaluations.

\begin{tabular}{ll}
$\begin{array}{l}\text { bond } \\
\text { or angle }\end{array}$ & $\begin{array}{l}\text { length } / \AA \\
\text { or angle / }\end{array}$ \\
\hline N6-C7 & 1.414 \\
C7-C10 & 1.461 \\
C9-C10 & 1.364 \\
C7-N6-C8 & 107.5 \\
C10-C7-N6 & 108.0 \\
C7-C10-C9 & 108.3 \\
H1-N6 & 1.005
\end{tabular}


Table S786 Structural parameters of the CC2/TZVP-optimized excited-state equilibrium geometry of the $2{ }^{1} A^{\prime}\left({ }^{1} \pi \pi^{*}\right)$ state of cis-PSB3 used for the statistical evaluations.

\begin{tabular}{ll}
$\begin{array}{l}\text { bond } \\
\text { or angle }\end{array}$ & $\begin{array}{l}\text { length } / \AA \\
\text { or angle } /\end{array}$ \\
\hline C1-C2 & 1.386 \\
C2-C3 & 1.435 \\
C3-C4 & 1.401 \\
C4-C5 & 1.447 \\
C5-N6 & 1.354 \\
C1-C2-C3 & 123.0 \\
C2-C3-C4 & 121.4 \\
C3-C4-C5 & 126.4 \\
C4-C5-N6 & 119.6 \\
N6-H13 & 1.012 \\
N6-H14 & 1.011
\end{tabular}


Table S787 Structural parameters of the CC2/TZVP-optimized excited-state equilibrium geometry of the $2{ }^{1} A^{\prime}\left({ }^{1} \pi \pi^{*}\right)$ state of 3Me-cis-PSB5 used for the statistical evaluations.

\begin{tabular}{ll} 
bond & $\begin{array}{c}\text { length } / \AA \\
\text { or angle }\end{array}$ \\
\hline $\mathrm{C} 10-\mathrm{C} 11$ & 1.367 \\
$\mathrm{C} 9-\mathrm{C} 10$ & 1.418 \\
$\mathrm{C} 8-\mathrm{C} 9$ & 1.389 \\
$\mathrm{C} 1-\mathrm{C} 8$ & 1.426 \\
$\mathrm{C} 1-\mathrm{C} 2$ & 1.388 \\
$\mathrm{C} 2-\mathrm{C} 3$ & 1.455 \\
$\mathrm{C} 4-\mathrm{C} 4$ & 1.403 \\
$\mathrm{C} 4-\mathrm{C} 5$ & 1.402 \\
$\mathrm{C} 5-\mathrm{N} 6$ & 1.347 \\
$\mathrm{C} 3-\mathrm{C} 7$ & 1.500 \\
$\mathrm{C} 11-\mathrm{C} 10-\mathrm{C} 9$ & 122.1 \\
$\mathrm{C} 10-\mathrm{C} 9-\mathrm{C} 8$ & 122.8 \\
$\mathrm{C} 9-\mathrm{C} 8-\mathrm{C} 1$ & 122.2 \\
$\mathrm{C} 8-\mathrm{C} 1-\mathrm{C} 2$ & 128.4 \\
$\mathrm{C} 1-\mathrm{C} 2-\mathrm{C} 3$ & 132.2 \\
$\mathrm{C} 2-\mathrm{C} 3-\mathrm{C} 4$ & 115.0 \\
$\mathrm{C} 3-\mathrm{C} 4-\mathrm{C} 5$ & 126.6 \\
$\mathrm{C} 4-\mathrm{C} 5-\mathrm{N} 6$ & 122.6 \\
$\mathrm{C} 2-\mathrm{C} 3-\mathrm{C} 7$ & 122.5 \\
$\mathrm{~N} 6-\mathrm{H} 16$ & 1.011 \\
$\mathrm{~N} 6-\mathrm{H} 17$ & 1.009
\end{tabular}


Table S788 Structural parameters of the CC2/TZVP-optimized excited-state equilibrium geometry of the $1{ }^{1} B_{u}\left({ }^{1} \pi \pi^{*}\right)$ state of stilbene used for the statistical evaluations.

\begin{tabular}{ll}
$\begin{array}{l}\text { bond } \\
\text { or angle }\end{array}$ & $\begin{array}{l}\text { length } / \AA \\
\text { or angle } /\end{array}$ \\
\hline C1-C8 & 1.419 \\
C1-C2 & 1.401 \\
C2-C3 & 1.435 \\
C3-C 4 & 1.379 \\
C4-C5 & 1.412 \\
C5-C6 & 1.402 \\
C6-C7 & 1.386 \\
C2-C7 & 1.442 \\
C $8-C 1-C 2$ & 124.6 \\
C1-C2-C3 & 119.7 \\
C1-C2-C7 & 123.0
\end{tabular}


Table S789 Structural parameters of the CC2/TZVP-optimized excited-state equilibrium geometry of the $1^{1} B\left({ }^{1} \pi \pi^{*}\right)$ state of stilbene used for the statistical evaluations.

\begin{tabular}{ll}
$\begin{array}{l}\text { bond } \\
\text { or angle }\end{array}$ & $\begin{array}{l}\text { length } / \AA \\
\text { or angle } /\end{array}$ \\
\hline C1-C8 & 1.405 \\
C1-C2 & 1.416 \\
C2-C3 & 1.419 \\
C3-C4 & 1.383 \\
C4-C5 & 1.416 \\
C5-C6 & 1.392 \\
C6-C7 & 1.397 \\
C2-C7 & 1.452 \\
C8-C1-C2 & 123.0 \\
C1-C2-C3 & 121.3 \\
C1-C2-C7 & 120.8
\end{tabular}


Table S790 Structural parameters of the CC2/TZVP-optimized excited-state equilibrium geometry of the $2{ }^{1} A^{\prime}\left({ }^{1} \pi \pi^{*}\right)$ state of styrene used for the statistical evaluations.

\begin{tabular}{ll}
$\begin{array}{l}\text { bond } \\
\text { or angle }\end{array}$ & $\begin{array}{l}\text { length } / \AA \\
\text { or angle } /{ }^{\circ}\end{array}$ \\
\hline C1-C2 & 1.361 \\
C1-C3 & 1.427 \\
C3-C4 & 1.446 \\
C3-C5 & 1.438 \\
C4-C6 & 1.427 \\
C5-C7 & 1.433 \\
C6-C8 & 1.417 \\
C7-C8 & 1.421
\end{tabular}


Table S791 Structural parameters of the CC2/TZVP-optimized excited-state equilibrium geometry of the $1^{3} A^{\prime}\left({ }^{3} \pi \pi^{*}\right)$ state of styrene used for the statistical evaluations.

\begin{tabular}{ll}
$\begin{array}{l}\text { bond } \\
\text { or angle }\end{array}$ & $\begin{array}{l}\text { length } / \AA \\
\text { or angle } /{ }^{\circ}\end{array}$ \\
\hline C1-C2 & 1.469 \\
C1-C3 & 1.377 \\
C3-C4 & 1.451 \\
C3-C5 & 1.453 \\
C4-C6 & 1.370 \\
C5-C7 & 1.379 \\
C6-C8 & 1.420 \\
C7-C8 & 1.407
\end{tabular}


Table S792 Structural parameters of the CC2/TZVP-optimized excited-state equilibrium geometry of the $1{ }^{1} B_{3 u}\left({ }^{1} n \pi^{*}\right)$ state of $s$-tetrazine used for the statistical evaluations.

\begin{tabular}{ll} 
bond & $\begin{array}{l}\text { length / } \AA \\
\text { or angle } \\
\text { or angle / }\end{array}$ \\
\hline N3-N4 & 1.336 \\
C2-N4 & 1.339 \\
C2-H7 & 1.080 \\
N3-C1-N5 & 122.3
\end{tabular}


Table S793 Structural parameters of the CC2/TZVP-optimized excited-state equilibrium geometry of the $2{ }^{1} A\left({ }^{1} n \pi^{*}\right)$ state of thymine used for the statistical evaluations.

\begin{tabular}{ll}
$\begin{array}{l}\text { bond } \\
\text { or angle }\end{array}$ & $\begin{array}{l}\text { length } / \AA \\
\text { or angle } /\end{array}$ \\
\hline N1-C6 & 1.376 \\
C5-N6 & 1.399 \\
N4-C5 & 1.386 \\
C3-C4 & 1.335 \\
C2-C3 & 1.438 \\
N1-C2 & 1.393 \\
C6-O9 & 1.230 \\
C4-O7 & 1.451 \\
C3-C8 & 1.500 \\
C2-H10 & 1.080 \\
N1-H11 & 1.012 \\
N5-H12 & 1.012
\end{tabular}


Table S794 Structural parameters of the CC2/TZVP-optimized excited-state equilibrium geometry of the $2{ }^{1} A^{\prime}\left({ }^{1} \pi \pi^{*}\right)$ state of uracil used for the statistical evaluations.

\begin{tabular}{ll}
$\begin{array}{l}\text { bond } \\
\text { or angle }\end{array}$ & $\begin{array}{c}\text { length } / \AA \\
\text { or angle } /\end{array}$ \\
\hline N1-C3 & 1.387 \\
N2-C3 & 1.394 \\
N2-C4 & 1.375 \\
C4-C5 & 1.353 \\
C5-C6 & 1.454 \\
N1-C6 & 1.415 \\
C3-O8 & 1.224 \\
C6-O7 & 1.229 \\
C4-H9 & 1.082 \\
C5-H12 & 1.079 \\
N2-H11 & 1.010 \\
N1-H10 & 1.015
\end{tabular}




\subsubsection{OM2/SCF-Optimized Ground-State Minima}


Table S795 Cartesian coordinates of the OM2/SCF-optimized ground-state equilibrium geometry of $9 \mathrm{H}$-adenine (ground-state energy: $-1777.68509 \mathrm{eV}$ ).

$\begin{array}{lrrr}15 & & & \\ & & & \\ \mathrm{~N} & -0.077180 & 0.096105 & -0.062322 \\ \mathrm{C} & 1.289889 & -0.031153 & -0.002430 \\ \mathrm{C} & 1.899016 & 1.270717 & 0.059393 \\ \mathrm{~N} & 0.847664 & 2.161761 & 0.033845 \\ \mathrm{C} & -0.322219 & 1.399798 & -0.039929 \\ \mathrm{~N} & 3.220770 & 1.520487 & 0.126938 \\ \mathrm{C} & 3.964181 & 0.411566 & 0.132617 \\ \mathrm{~N} & 3.534072 & -0.868078 & 0.079645 \\ \mathrm{C} & 2.214141 & -1.119102 & 0.012144 \\ \mathrm{~N} & 1.789679 & -2.410403 & -0.040881 \\ \mathrm{H} & 5.070388 & 0.547354 & 0.187249 \\ \mathrm{H} & 0.820519 & -2.631165 & -0.091416 \\ \mathrm{H} & 2.446238 & -3.158485 & -0.029893 \\ \mathrm{H} & 0.921935 & 3.166093 & 0.063257 \\ \mathrm{H} & -1.315116 & 1.852888 & -0.074102\end{array}$


Table S796 Cartesian coordinates of the OM2/SCF-optimized ground-state equilibrium geometry of acetaldehyde (ground-state energy: $-646.36576 \mathrm{eV}$ ).

$\begin{array}{lrrr}7 & & & \\ \mathrm{C} & -0.000682 & 0.003277 & 0.000176 \\ \mathrm{C} & 1.499532 & -0.002210 & -0.000117 \\ \mathrm{H} & -0.515402 & -1.011454 & 0.000327 \\ \mathrm{O} & -0.621012 & 1.055740 & 0.000265 \\ \mathrm{H} & 1.859018 & -0.525473 & 0.893299 \\ \mathrm{H} & 1.858699 & -0.525246 & -0.893791 \\ \mathrm{H} & 1.870489 & 1.026209 & -0.000066\end{array}$


Table S797 Cartesian coordinates of the OM2/SCF-optimized ground-state equilibrium geometry of acetone (ground-state energy: $-804.49598 \mathrm{eV}$ ).

$\begin{array}{lrrr}10 & & & \\ & & & \\ \mathrm{C} & 0.149596 & 0.004885 & -1.294236 \\ \mathrm{C} & -0.621606 & -0.039528 & 0.000850 \\ \mathrm{O} & -1.845954 & -0.109573 & 0.000563 \\ \mathrm{C} & 0.149000 & 0.004664 & 1.296299 \\ \mathrm{H} & -0.550864 & -0.037044 & -2.133100 \\ \mathrm{H} & 0.833698 & -0.849967 & -1.342897 \\ \mathrm{H} & 0.728288 & 0.934280 & -1.344144 \\ \mathrm{H} & -0.551780 & -0.038792 & 2.134822 \\ \mathrm{H} & 0.726481 & 0.934763 & 1.347152 \\ \mathrm{H} & 0.834196 & -0.849331 & 1.344578\end{array}$


Table S798 Cartesian coordinates of the OM2/SCF-optimized ground-state equilibrium geometry of acetophenone (ground-state energy: $-1471.70394 \mathrm{eV}$ ).

$\begin{array}{lrrr}17 & & & \\ \text { C } & 0.004604 & -0.004448 & 0.000311 \\ \mathrm{O} & 1.233414 & 0.013570 & 0.000673 \\ \mathrm{C} & -0.734803 & -1.316987 & -0.000052 \\ \mathrm{H} & -0.006543 & -2.133272 & 0.000095 \\ \mathrm{H} & -1.361652 & -1.386862 & -0.896114 \\ \mathrm{H} & -1.362242 & -1.387076 & 0.895581 \\ \mathrm{C} & -0.750104 & 1.281938 & 0.000170 \\ \mathrm{C} & -2.146528 & 1.334606 & 0.000052 \\ \mathrm{C} & 0.002823 & 2.461524 & 0.000156 \\ \mathrm{C} & -2.787328 & 2.569404 & -0.000072 \\ \mathrm{C} & -0.647103 & 3.691668 & 0.000019 \\ \mathrm{C} & -2.038969 & 3.745373 & -0.000091 \\ \mathrm{H} & -2.739998 & 0.413449 & 0.000069 \\ \mathrm{H} & 1.093857 & 2.399942 & 0.000255 \\ \mathrm{H} & -3.881504 & 2.618489 & -0.000156 \\ \mathrm{H} & -0.064099 & 4.618561 & 0.000003 \\ \mathrm{H} & -2.549401 & 4.715622 & -0.000192\end{array}$


Table S799 Cartesian coordinates of the OM2/SCF-optimized ground-state equilibrium geometry of acetylene (ground-state energy: $-282.94213 \mathrm{eV}$ ).

4

$\begin{array}{rrrr}\mathrm{C} & -0.606757 & 0.150681 & -0.010000 \\ \mathrm{C} & 0.608316 & 0.018127 & -0.010000 \\ \mathrm{H} & -1.641863 & 0.263600 & -0.010000 \\ \mathrm{H} & 1.643422 & -0.094794 & -0.010000\end{array}$


Table S800 Cartesian coordinates of the OM2/SCF-optimized ground-state equilibrium geometry of acrolein (ground-state energy: $-772.97312 \mathrm{eV}$ ).

8

$\begin{array}{lrrr}\text { C } & 0.000000 & 0.000000 & 0.000000 \\ \mathrm{C} & 1.334296 & 0.000000 & 0.000000 \\ \mathrm{C} & 2.089953 & 1.273538 & 0.000000 \\ \mathrm{O} & 3.312499 & 1.257427 & -0.000031 \\ \mathrm{H} & -0.578565 & -0.916925 & -0.000003 \\ \mathrm{H} & -0.571808 & 0.924604 & 0.000011 \\ \mathrm{H} & 1.930535 & -0.913564 & -0.000008 \\ \mathrm{H} & 1.481049 & 2.234747 & 0.000027\end{array}$


Table S801 Cartesian coordinates of the OM2/SCF-optimized groundstate equilibrium geometry of 2-amino-9-methylpurine (ground-state energy: $-1935.29327 \mathrm{eV})$.

$\begin{array}{lrrr}18 & & & \\ & & & \\ \mathrm{C} & -0.020535 & -0.062372 & 0.013576 \\ \mathrm{~N} & 1.354806 & 0.004762 & -0.026721 \\ \mathrm{~N} & -0.800530 & -1.169637 & 0.015005 \\ \mathrm{C} & -0.070368 & -2.291457 & -0.029579 \\ \mathrm{C} & 1.374809 & -2.355157 & -0.074604 \\ \mathrm{C} & 2.047002 & -1.115561 & -0.070125 \\ \mathrm{~N} & -0.503971 & -3.605757 & -0.042787 \\ \mathrm{C} & 0.661138 & -4.394184 & -0.093840 \\ \mathrm{~N} & 1.770499 & -3.675354 & -0.113021 \\ \mathrm{~N} & -0.662512 & 1.146117 & 0.057198 \\ \mathrm{C} & -1.865107 & -4.065408 & -0.010780 \\ \mathrm{H} & 3.149416 & -1.092479 & -0.103049 \\ \mathrm{H} & 0.617882 & -5.485571 & -0.114387 \\ \mathrm{H} & -1.654749 & 1.194452 & 0.088311 \\ \mathrm{H} & -0.144767 & 1.994687 & 0.058589 \\ \mathrm{H} & -2.551760 & -3.201187 & 0.027269 \\ \mathrm{H} & -2.040966 & -4.702744 & 0.886110 \\ \mathrm{H} & -2.095626 & -4.666659 & -0.920115\end{array}$


Table S802 Cartesian coordinates of the OM2/SCF-optimized ground-state equilibrium geometry of aniline (ground-state energy: $-1083.31437 \mathrm{eV}$ ).

$\begin{array}{lrrr}14 & & & \\ & & & \\ \mathrm{C} & -0.005977 & -0.019420 & -0.010001 \\ \mathrm{C} & 1.216997 & -0.721182 & -0.010001 \\ \mathrm{C} & 1.203086 & -2.109185 & -0.010001 \\ \mathrm{C} & -0.001633 & -2.810006 & -0.010001 \\ \mathrm{C} & -1.208524 & -2.112937 & -0.010001 \\ \mathrm{C} & -1.226758 & -0.724987 & -0.010001 \\ \mathrm{~N} & -0.008127 & 1.360237 & -0.010001 \\ \mathrm{H} & 0.837536 & 1.881141 & -0.010001 \\ \mathrm{H} & -0.855410 & 1.878499 & -0.010001 \\ \mathrm{H} & 2.160690 & -0.168472 & -0.010001 \\ \mathrm{H} & 2.153250 & -2.659730 & -0.010001 \\ \mathrm{H} & 0.000070 & -3.903597 & -0.010001 \\ \mathrm{H} & -2.156966 & -2.666439 & -0.010001 \\ \mathrm{H} & -2.172175 & -0.175232 & -0.010001\end{array}$


Table S803 Cartesian coordinates of the OM2/SCF-optimized ground-state equilibrium geometry of benzaldehyde (ground-state energy: $-1313.58895 \mathrm{eV}$ ).

$\begin{array}{lrrr}14 & & & \\ & & & \\ \mathrm{C} & -0.304692 & 2.018318 & -0.010001 \\ \mathrm{O} & -1.470097 & 2.390637 & -0.010001 \\ \mathrm{H} & 0.574274 & 2.742097 & -0.010001 \\ \mathrm{C} & 0.022643 & 0.572539 & -0.010001 \\ \mathrm{C} & 1.355003 & 0.148710 & -0.010001 \\ \mathrm{C} & -1.023967 & -0.355017 & -0.010001 \\ \mathrm{C} & 1.640233 & -1.212057 & -0.010001 \\ \mathrm{C} & -0.727026 & -1.714550 & -0.010001 \\ \mathrm{C} & 0.599519 & -2.140326 & -0.010001 \\ \mathrm{H} & 2.167131 & 0.886238 & -0.010001 \\ \mathrm{H} & -2.057368 & 0.000302 & -0.010001 \\ \mathrm{H} & 2.679683 & -1.555771 & -0.010001 \\ \mathrm{H} & -1.537192 & -2.450915 & -0.010001 \\ \mathrm{H} & 0.828477 & -3.212572 & -0.010001\end{array}$


Table S804 Cartesian coordinates of the OM2/SCF-optimized groundstate equilibrium geometry of $p$-benzoquinone (ground-state energy: $-1486.15480 \mathrm{eV})$.

$\begin{array}{lrrr}12 & & \\ \text { C } & 0.000000 & -0.002011 & 1.427302 \\ \text { C } & 0.000000 & -1.280605 & 0.670028 \\ \text { C } & 0.000000 & -1.280605 & -0.670028 \\ \text { C } & 0.000000 & -0.002011 & -1.427302 \\ \text { C } & 0.000000 & 1.276583 & -0.670028 \\ \text { C } & 0.000000 & 1.276584 & 0.670028 \\ \text { O } & 0.000000 & -0.002011 & 2.654380 \\ \text { O } & 0.000000 & -0.002007 & -2.654380 \\ \text { H } & 0.000000 & -2.194167 & 1.267991 \\ \text { H } & 0.000000 & -2.194167 & -1.267991 \\ \text { H } & 0.000000 & 2.190140 & -1.267998 \\ \text { H } & 0.000000 & 2.190139 & 1.267993\end{array}$


Table S805 Cartesian coordinates of the OM2/SCF-optimized ground-state equilibrium geometry of biphenylene (ground-state energy: $-1647.63341 \mathrm{eV}$ ).

$\begin{array}{lrrr}20 & & & \\ & & & \\ \mathrm{C} & -0.010000 & 0.720866 & 0.752390 \\ \mathrm{C} & -0.010000 & 1.456526 & 1.903835 \\ \mathrm{C} & -0.010000 & 0.692588 & 3.096395 \\ \mathrm{C} & -0.010000 & 0.720867 & -0.752390 \\ \mathrm{C} & -0.010000 & -0.720867 & -0.752390 \\ \mathrm{C} & -0.010000 & -0.720866 & 0.752390 \\ \mathrm{C} & -0.010000 & 1.456526 & -1.903835 \\ \mathrm{C} & -0.010000 & -1.456526 & -1.903835 \\ \mathrm{C} & -0.010000 & -1.456526 & 1.903835 \\ \mathrm{C} & -0.010000 & 0.692588 & -3.096395 \\ \mathrm{C} & -0.010000 & -0.692588 & -3.096394 \\ \mathrm{C} & -0.010000 & -0.692588 & 3.096394 \\ \mathrm{H} & -0.010000 & 2.546482 & 1.926746 \\ \mathrm{H} & -0.010000 & 2.546482 & -1.926746 \\ \mathrm{H} & -0.010000 & -2.546482 & -1.926746 \\ \mathrm{H} & -0.010000 & -2.546482 & 1.926746 \\ \mathrm{H} & -0.010000 & 1.227529 & 4.054251 \\ \mathrm{H} & -0.010000 & 1.227528 & -4.054251 \\ \mathrm{H} & -0.010000 & -1.227528 & -4.054251 \\ \mathrm{H} & -0.010000 & -1.227529 & 4.054250 \\ & & & \end{array}$


Table S806 Cartesian coordinates of the OM2/SCF-optimized ground-state equilibrium geometry of trans-butadiene (ground-state energy: $-599.12855 \mathrm{eV}$ ).

$\begin{array}{lrrr}10 & & & \\ & & & \\ \mathrm{C} & 0.015429 & 0.731442 & 0.000000 \\ \mathrm{C} & -0.015429 & -0.731442 & 0.000000 \\ \mathrm{C} & 1.112232 & -1.449704 & 0.000000 \\ \mathrm{C} & -1.112232 & 1.449704 & 0.000000 \\ \mathrm{H} & 1.103456 & -2.532834 & 0.000000 \\ \mathrm{H} & -1.103456 & 2.532833 & 0.000000 \\ \mathrm{H} & 2.089644 & -0.980388 & 0.000000 \\ \mathrm{H} & -2.089644 & 0.980388 & 0.000000 \\ \mathrm{H} & -1.004644 & -1.209790 & 0.000000 \\ \mathrm{H} & 1.004644 & 1.209790 & 0.000000\end{array}$


Table S807 Cartesian coordinates of the OM2/SCF-optimized ground-state equilibrium geometry of cytosine (ground-state energy: $-1555.44223 \mathrm{eV}$ ).

$\begin{array}{lrrr}13 & & & \\ & & & \\ \mathrm{~N} & 0.025130 & 0.005097 & 0.000169 \\ \mathrm{C} & 1.353321 & -0.001488 & -0.000037 \\ \mathrm{C} & 2.170970 & 1.193436 & -0.000190 \\ \mathrm{C} & 1.509683 & 2.396148 & -0.000116 \\ \mathrm{~N} & 0.156602 & 2.412131 & 0.000091 \\ \mathrm{C} & -0.638541 & 1.207284 & 0.000244 \\ \mathrm{~N} & 1.966886 & -1.219359 & -0.000107 \\ \mathrm{O} & -1.870027 & 1.376459 & 0.000432 \\ \mathrm{H} & 3.251128 & 1.127405 & -0.000355 \\ \mathrm{H} & 2.067989 & 3.350129 & -0.000224 \\ \mathrm{H} & 1.420131 & -2.052340 & -0.000004 \\ \mathrm{H} & 2.957006 & -1.315810 & -0.000259 \\ \mathrm{H} & -0.339317 & 3.297784 & 0.000146\end{array}$


Table S808 Cartesian coordinates of the OM2/SCF-optimized ground-state equilibrium geometry of diazomethane (ground-state energy: $-582.21189 \mathrm{eV}$ ).

$\begin{array}{lrrr}5 & & & \\ & & & \\ \mathrm{C} & 0.120962 & -0.013820 & -0.020000 \\ \mathrm{~N} & 1.426621 & -0.021229 & -0.020000 \\ \mathrm{~N} & 2.565187 & -0.027690 & -0.020000 \\ \mathrm{H} & -0.413590 & 0.919899 & -0.020000 \\ \mathrm{H} & -0.424156 & -0.941413 & -0.020000\end{array}$


Table S809 Cartesian coordinates of the OM2/SCF-optimized groundstate equilibrium geometry of dibenzo- $p$-dioxin (ground-state energy: $-2311.11660 \mathrm{eV})$.

$\begin{array}{lrrr}22 & & \\ \text { C } & -0.010000 & -3.549796 & 0.696824 \\ \text { C } & -0.010000 & -3.549796 & -0.696824 \\ \text { C } & -0.010000 & -2.355586 & -1.416175 \\ \text { C } & -0.010000 & -1.154885 & -0.703522 \\ \text { C } & -0.010000 & -1.154885 & 0.703522 \\ \text { C } & -0.010000 & -2.355586 & 1.416174 \\ \text { O } & -0.010000 & -0.000864 & -1.451312 \\ \text { C } & -0.010000 & 1.153157 & -0.703523 \\ \text { C } & -0.010000 & 1.153157 & 0.703523 \\ \text { O } & -0.010000 & -0.000864 & 1.451311 \\ \text { C } & -0.010000 & 2.353857 & -1.416176 \\ \text { C } & -0.010000 & 3.548065 & -0.696824 \\ \text { C } & -0.010000 & 3.548065 & 0.696824 \\ \text { C } & -0.010000 & 2.353857 & 1.416176 \\ \text { H } & -0.010000 & 4.498496 & 1.240001 \\ \text { H } & -0.010000 & -4.500222 & -1.240009 \\ \text { H } & -0.010000 & -2.336827 & -2.504915 \\ \text { H } & -0.010000 & -2.336827 & 2.504914 \\ \text { H } & -0.010000 & 2.335099 & -2.504916 \\ \text { H } & -0.010000 & 4.498496 & -1.240001 \\ \text { H } & -0.010000 & -4.500222 & 1.240009 \\ \text { H } & -0.010000 & 2.335099 & 2.504916\end{array}$


Table S810 Cartesian coordinates of the OM2/SCF-optimized ground-state equilibrium geometry of formaldehyde (ground-state energy: $-488.20983 \mathrm{eV}$ ).

4

$\begin{array}{rrrr}\mathrm{C} & -0.027039 & 0.051890 & 0.083062 \\ \mathrm{O} & 0.014921 & 1.066296 & 0.752713 \\ \mathrm{H} & 0.899137 & -0.496006 & -0.247332 \\ \mathrm{H} & -0.995102 & -0.412659 & -0.254872\end{array}$


Table S811 Cartesian coordinates of the OM2/SCF-optimized ground-state equilibrium geometry of trans-glyoxal (ground-state energy: $-946.53822 \mathrm{eV}$ ).

$\begin{array}{lrrr}6 & & & \\ \text { C } & 0.761660 & 0.000000 & 0.006900 \\ \mathrm{C} & -0.761660 & 0.000000 & -0.006900 \\ \mathrm{O} & 1.329552 & 0.000000 & 1.084199 \\ \mathrm{O} & -1.329552 & 0.000000 & -1.084199 \\ \mathrm{H} & 1.280050 & 0.000000 & -0.994366 \\ \mathrm{H} & -1.280050 & 0.000000 & 0.994366\end{array}$


Table S812 Cartesian coordinates of the OM2/SCF-optimized ground-state equilibrium geometry of $9 \mathrm{H}$-guanine (ground-state energy: $-2108.94672 \mathrm{eV}$ ).

$\begin{array}{rrrr}16 & & & \\ & & & \\ \mathrm{~N} & 0.164133 & 0.020923 & 0.016799 \\ \mathrm{C} & -0.115022 & -0.013212 & 1.391349 \\ \mathrm{~N} & -0.254981 & 1.205694 & 1.894654 \\ \mathrm{C} & -0.069877 & 2.077495 & 0.855534 \\ \mathrm{C} & -0.099317 & 3.523857 & 0.782253 \\ \mathrm{O} & -0.304085 & 4.370818 & 1.659051 \\ \mathrm{~N} & 0.156278 & 3.991288 & -0.541784 \\ \mathrm{H} & 0.151272 & 4.998280 & -0.668799 \\ \mathrm{C} & 0.403554 & 3.170062 & -1.631169 \\ \mathrm{~N} & 0.629418 & 3.789906 & -2.832195 \\ \mathrm{H} & 0.620488 & 4.778313 & -2.938193 \\ \mathrm{H} & 0.811240 & 3.240729 & -3.641594 \\ \mathrm{~N} & 0.436990 & 1.836088 & -1.594539 \\ \mathrm{C} & 0.197668 & 1.341187 & -0.346110 \\ \mathrm{H} & -0.200499 & -0.943841 & 1.950583 \\ \mathrm{H} & 0.313249 & -0.764924 & -0.596545\end{array}$


Table S813 Cartesian coordinates of the OM2/SCF-optimized ground-state equilibrium geometry of 1-hydroxy-2-acetonaphthone (ground-state energy: $-2343.21353 \mathrm{eV})$.

$\begin{array}{lrrr}24 & & & \\ & & & \\ \mathrm{C} & 0.107477 & 0.964221 & 0.001149 \\ \mathrm{C} & -1.162013 & 0.282204 & 0.000174 \\ \mathrm{C} & -1.156508 & -1.128924 & -0.001664 \\ \mathrm{C} & 0.083203 & -1.845168 & -0.002513 \\ \mathrm{C} & 1.274473 & -1.174374 & -0.001558 \\ \mathrm{C} & 1.312146 & 0.247723 & 0.000297 \\ \mathrm{C} & -2.379221 & 1.001181 & 0.001029 \\ \mathrm{C} & -3.569906 & 0.309987 & 0.000049 \\ \mathrm{C} & -3.577028 & -1.100532 & -0.001790 \\ \mathrm{C} & -2.398614 & -1.812272 & -0.002638 \\ \mathrm{C} & 2.554492 & 1.018261 & 0.001386 \\ \mathrm{C} & 3.901167 & 0.350148 & 0.000608 \\ \mathrm{O} & 0.044386 & 2.294415 & 0.002877 \\ \mathrm{O} & 2.486867 & 2.261125 & 0.003000 \\ \mathrm{H} & -4.522990 & 0.848412 & 0.000686 \\ \mathrm{H} & -4.539229 & -1.627371 & -0.002541 \\ \mathrm{H} & -2.404877 & -2.907866 & -0.004066 \\ \mathrm{H} & -2.347937 & 2.092907 & 0.002453 \\ \mathrm{H} & 0.053966 & -2.938774 & -0.003939 \\ \mathrm{H} & 2.222640 & -1.726438 & -0.002212 \\ \mathrm{H} & 0.987528 & 2.696829 & 0.003465 \\ \mathrm{H} & 4.678982 & 1.118961 & 0.001682 \\ \mathrm{H} & 4.000434 & -0.271914 & -0.895832 \\ \mathrm{H} & 4.000298 & -0.274281 & 0.895415\end{array}$


Table S814 Cartesian coordinates of the OM2/SCF-optimized ground-state equilibrium geometry of indole (ground-state energy: $-1337.80593 \mathrm{eV}$ ).

$\begin{array}{lrrr}16 & & & \\ & & & \\ \mathrm{C} & -0.489962 & -0.622481 & -0.010001 \\ \mathrm{C} & 0.001992 & 0.723586 & -0.010001 \\ \mathrm{~N} & -1.095443 & 1.574377 & -0.010001 \\ \mathrm{C} & -1.926848 & -0.536862 & -0.010001 \\ \mathrm{C} & -2.251432 & 0.809577 & -0.010001 \\ \mathrm{C} & 0.421598 & -1.685909 & -0.010001 \\ \mathrm{C} & 1.773863 & -1.390693 & -0.010001 \\ \mathrm{C} & 1.375112 & 1.015681 & -0.010001 \\ \mathrm{C} & 2.242033 & -0.062550 & -0.010001 \\ \mathrm{H} & -1.060400 & 2.581313 & -0.010001 \\ \mathrm{H} & -2.614970 & -1.365953 & -0.010001 \\ \mathrm{H} & -3.246070 & 1.249769 & -0.010001 \\ \mathrm{H} & 0.062531 & -2.720028 & -0.010001 \\ \mathrm{H} & 2.507645 & -2.205252 & -0.010001 \\ \mathrm{H} & 1.731405 & 2.048964 & -0.010001 \\ \mathrm{H} & 3.324529 & 0.116437 & -0.010001\end{array}$


Table S815 Cartesian coordinates of the OM2/SCF-optimized ground-state equilibrium geometry of maleimide (ground-state energy: $-1429.78790 \mathrm{eV}$ ).

$\begin{array}{lrrr}10 & & & \\ & & & \\ \mathrm{~N} & -0.000285 & 0.019581 & -0.010000 \\ \mathrm{C} & 1.148881 & 0.826897 & -0.010000 \\ \mathrm{C} & 0.670449 & 2.267582 & -0.010000 \\ \mathrm{C} & -0.669747 & 2.267973 & -0.010000 \\ \mathrm{C} & -1.148998 & 0.827559 & -0.010000 \\ \mathrm{O} & 2.304756 & 0.414542 & -0.010000 \\ \mathrm{O} & -2.305093 & 0.415823 & -0.010000 \\ \mathrm{H} & -0.000575 & -0.985621 & -0.010000 \\ \mathrm{H} & 1.367785 & 3.090495 & -0.010000 \\ \mathrm{H} & -1.366598 & 3.091297 & -0.010000\end{array}$


Table S816 Cartesian coordinates of the OM2/SCF-optimized ground-state equilibrium geometry of nitrosomethane (ground-state energy: $-715.10311 \mathrm{eV}$ ).

$\begin{array}{lrrr}6 & & & \\ \mathrm{C} & 0.038084 & 0.010712 & 0.004032 \\ \mathrm{~N} & 1.451905 & -0.033775 & -0.369026 \\ \mathrm{O} & 2.066827 & -0.830069 & 0.301888 \\ \mathrm{H} & -0.211240 & 1.036936 & 0.310053 \\ \mathrm{H} & -0.565493 & -0.239576 & -0.880279 \\ \mathrm{H} & -0.214376 & -0.682412 & 0.822466\end{array}$


Table S817 Cartesian coordinates of the OM2/SCF-optimized groundstate equilibrium geometry of propenoic acid anion (ground-state energy: $-1090.92406 \mathrm{eV})$.

$\begin{array}{lrrr}8 & & & \\ \text { C } & 0.011693 & -0.011672 & -0.010001 \\ \mathrm{C} & 1.561949 & -0.020849 & -0.010001 \\ \mathrm{O} & 2.083477 & 1.125325 & -0.010001 \\ \mathrm{O} & 2.099420 & -1.158871 & -0.010001 \\ \mathrm{C} & -0.701704 & -1.134876 & -0.010001 \\ \mathrm{H} & -0.483896 & 0.978686 & -0.010001 \\ \mathrm{H} & -1.794721 & -1.166692 & -0.010001 \\ \mathrm{H} & -0.194710 & -2.090679 & -0.010001\end{array}$


Table S818 Cartesian coordinates of the OM2/SCF-optimized ground-state equilibrium geometry of pyridine (ground-state energy: $-925.54092 \mathrm{eV}$ ).

$\begin{array}{lrrr}11 & & \\ \text { N } & -0.010000 & -0.002152 & 1.399879 \\ \mathrm{C} & -0.010000 & 1.142669 & 0.719697 \\ \mathrm{C} & -0.010000 & -1.148207 & 0.721779 \\ \mathrm{C} & -0.010000 & 1.193645 & -0.680505 \\ \mathrm{C} & -0.010000 & -1.201719 & -0.678327 \\ \mathrm{C} & -0.010000 & -0.004680 & -1.387209 \\ \mathrm{H} & -0.010000 & 2.087242 & 1.293259 \\ \mathrm{H} & -0.010000 & -2.091718 & 1.297071 \\ \mathrm{H} & -0.010000 & 2.151662 & -1.204526 \\ \mathrm{H} & -0.010000 & -2.160685 & -1.200611 \\ \mathrm{H} & -0.010000 & -0.005676 & -2.482693\end{array}$


Table S819 Cartesian coordinates of the OM2/SCF-optimized ground-state equilibrium geometry of pyrrole (ground-state energy: $-797.67339 \mathrm{eV}$ ).

$\begin{array}{lrrr}10 & & & \\ & & & \\ \mathrm{H} & -0.005832 & 1.732168 & -0.094350 \\ \mathrm{H} & 2.135922 & 0.326632 & 0.017072 \\ \mathrm{H} & -2.138867 & 0.314766 & 0.033062 \\ \mathrm{H} & -1.346355 & -2.261528 & 0.248334 \\ \mathrm{H} & 1.359333 & -2.254032 & 0.238201 \\ \mathrm{~N} & -0.002722 & 0.725328 & -0.009169 \\ \mathrm{C} & -1.125689 & -0.069085 & 0.062006 \\ \mathrm{C} & 1.125137 & -0.062845 & 0.053583 \\ \mathrm{C} & 0.713789 & -1.392042 & 0.167518 \\ \mathrm{C} & -0.706142 & -1.395979 & 0.172834\end{array}$


Table S820 Cartesian coordinates of the OM2/SCF-optimized ground-state equilibrium geometry of cis-PSB3 (ground-state energy: $-963.36632 \mathrm{eV}$ ).

$\begin{array}{lrrr}14 & & & \\ & & & \\ \mathrm{C} & 1.273962 & 0.003224 & -0.010001 \\ \mathrm{C} & -0.070456 & -0.024444 & -0.010001 \\ \mathrm{C} & -0.800283 & 1.221306 & -0.010001 \\ \mathrm{C} & -2.162874 & 1.341959 & -0.010001 \\ \mathrm{C} & -3.027248 & 0.205502 & -0.010001 \\ \mathrm{~N} & -4.330540 & 0.365803 & -0.010001 \\ \mathrm{H} & 1.858933 & -0.910900 & -0.010001 \\ \mathrm{H} & 1.836571 & 0.934325 & -0.010001 \\ \mathrm{H} & -0.608899 & -0.975539 & -0.010001 \\ \mathrm{H} & -0.199510 & 2.148766 & -0.010001 \\ \mathrm{H} & -2.617029 & 2.331865 & -0.010001 \\ \mathrm{H} & -2.597948 & -0.817606 & -0.010001 \\ \mathrm{H} & -4.975214 & -0.410613 & -0.010001 \\ \mathrm{H} & -4.760044 & 1.279920 & -0.010001\end{array}$


Table S821 Cartesian coordinates of the OM2/SCF-optimized ground-state equilibrium geometry of 3Me-cis-PSB5 (ground-state energy: -1690.85487 eV).

$\begin{array}{lrrr}25 & & & \\ & & & \\ \mathrm{C} & 0.061445 & 0.060353 & -0.571613 \\ \mathrm{C} & 1.425017 & 0.024005 & -0.532933 \\ \mathrm{C} & 2.249228 & -1.064211 & -0.085137 \\ \mathrm{C} & 3.556260 & -0.752201 & 0.289776 \\ \mathrm{C} & 4.485733 & -1.724407 & 0.701447 \\ \mathrm{~N} & 5.693605 & -1.369706 & 1.112700 \\ \mathrm{C} & 1.738596 & -2.463309 & -0.084306 \\ \mathrm{C} & -0.846160 & -0.896520 & 0.002087 \\ \mathrm{C} & -2.165342 & -0.838163 & -0.312480 \\ \mathrm{C} & -3.120375 & -1.767493 & 0.266439 \\ \mathrm{C} & -4.410682 & -1.725915 & -0.092952 \\ \mathrm{H} & -0.407241 & 0.946828 & -1.040365 \\ \mathrm{H} & 1.967519 & 0.910200 & -0.880873 \\ \mathrm{H} & 3.871446 & 0.291060 & 0.280124 \\ \mathrm{H} & 4.218342 & -2.800980 & 0.689739 \\ \mathrm{H} & 6.381971 & -2.039445 & 1.409102 \\ \mathrm{H} & 5.982878 & -0.406796 & 1.147945 \\ \mathrm{H} & 2.534451 & -3.182507 & -0.327528 \\ \mathrm{H} & 1.342216 & -2.697730 & 0.914065 \\ \mathrm{H} & 0.944042 & -2.575407 & -0.828138 \\ \mathrm{H} & -0.479466 & -1.627799 & 0.724280 \\ \mathrm{H} & -2.549128 & -0.098101 & -1.033781 \\ \mathrm{H} & -2.750048 & -2.488281 & 1.002235 \\ \mathrm{H} & -5.141054 & -2.405099 & 0.330439 \\ \mathrm{H} & -4.785283 & -1.012934 & -0.821336 \\ & & & \end{array}$


Table S822 Cartesian coordinates of the OM2/SCF-optimized ground-state equilibrium geometry of trans-stilbene (ground-state energy: $-1964.81216 \mathrm{eV}$ ).

$\begin{array}{lrrr}26 & & & \\ & & & \\ \mathrm{C} & 0.030115 & 0.685626 & -0.010005 \\ \mathrm{C} & -1.148961 & 1.557244 & -0.010005 \\ \mathrm{C} & -0.945102 & 2.946017 & -0.010005 \\ \mathrm{C} & -2.030457 & 3.813473 & -0.010005 \\ \mathrm{C} & -3.329272 & 3.308064 & -0.010005 \\ \mathrm{C} & -3.538053 & 1.931483 & -0.010005 \\ \mathrm{C} & -2.456667 & 1.055942 & -0.010005 \\ \mathrm{C} & -0.032532 & -0.659024 & -0.010005 \\ \mathrm{C} & 1.146540 & -1.530644 & -0.010005 \\ \mathrm{C} & 0.942677 & -2.919416 & -0.010005 \\ \mathrm{C} & 2.028032 & -3.786873 & -0.010005 \\ \mathrm{C} & 3.326849 & -3.281469 & -0.010005 \\ \mathrm{C} & 3.535631 & -1.904890 & -0.010005 \\ \mathrm{C} & 2.454247 & -1.029346 & -0.010005 \\ \mathrm{H} & -4.560183 & 1.534829 & -0.010005 \\ \mathrm{H} & -4.184236 & 3.993008 & -0.010005 \\ \mathrm{H} & -1.866358 & 4.897226 & -0.010005 \\ \mathrm{H} & 0.076631 & 3.346854 & -0.010005 \\ \mathrm{H} & -2.637125 & -0.025972 & -0.010005 \\ \mathrm{H} & 1.000476 & 1.206908 & -0.010005 \\ \mathrm{H} & -1.002892 & -1.180307 & -0.010005 \\ \mathrm{H} & -0.079056 & -3.320252 & -0.010005 \\ \mathrm{H} & 1.863932 & -4.870626 & -0.010005 \\ \mathrm{H} & 4.181808 & -3.966417 & -0.010005 \\ \mathrm{H} & 4.557760 & -1.508235 & -0.010005 \\ \mathrm{H} & 2.634710 & 0.052569 & -0.010005\end{array}$


Table S823 Cartesian coordinates of the OM2/SCF-optimized ground-state equilibrium geometry of styrene (ground-state energy: $-1139.66268 \mathrm{eV}$ ).

$\begin{array}{lrrr}16 & & & \\ \text { C } & 1.584336 & -0.064717 & -0.030001 \\ \mathrm{C} & 0.248276 & -0.090105 & -0.030001 \\ \mathrm{C} & 2.431543 & -1.266296 & -0.030001 \\ \mathrm{C} & 1.895697 & -2.559186 & -0.030001 \\ \mathrm{C} & 3.823756 & -1.095736 & -0.030001 \\ \mathrm{C} & 2.743587 & -3.662690 & -0.030001 \\ \mathrm{C} & 4.663589 & -2.203000 & -0.030001 \\ \mathrm{C} & 4.124957 & -3.488348 & -0.030001 \\ \mathrm{H} & 2.124100 & 0.896217 & -0.030001 \\ \mathrm{H} & -0.337583 & 0.821122 & -0.030001 \\ \mathrm{H} & -0.318825 & -1.013641 & -0.030001 \\ \mathrm{H} & 0.809602 & -2.708913 & -0.030001 \\ \mathrm{H} & 4.249701 & -0.084303 & -0.030001 \\ \mathrm{H} & 2.321391 & -4.674492 & -0.030001 \\ \mathrm{H} & 5.751155 & -2.066664 & -0.030001 \\ \mathrm{H} & 4.788179 & -4.360427 & -0.030001\end{array}$


Table S824 Cartesian coordinates of the OM2/SCF-optimized ground-state equilibrium geometry of $s$-tetrazine (ground-state energy: $-1136.06315 \mathrm{eV}$ ).

$\begin{array}{lrrr}\text { C } & -0.010000 & -0.013355 & 1.286758 \\ \mathrm{C} & -0.010000 & -0.013355 & -1.286758 \\ \mathrm{~N} & -0.010000 & 1.169162 & 0.626872 \\ \mathrm{~N} & -0.010000 & 1.169162 & -0.626872 \\ \mathrm{~N} & -0.010000 & -1.195874 & 0.626871 \\ \mathrm{~N} & -0.010000 & -1.195874 & -0.626871 \\ \mathrm{H} & -0.010000 & -0.013353 & -2.387879 \\ \mathrm{H} & -0.010000 & -0.013353 & 2.387879\end{array}$


Table S825 Cartesian coordinates of the OM2/SCF-optimized ground-state equilibrium geometry of thymine (ground-state energy: $-1816.79630 \mathrm{eV}$ ).

$\begin{array}{lrrr}15 & & & \\ & & & \\ \mathrm{~N} & 0.047832 & -0.009644 & -0.025084 \\ \mathrm{C} & 1.417914 & 0.040468 & 0.116820 \\ \mathrm{C} & 2.082944 & 1.228224 & 0.025758 \\ \mathrm{C} & 1.324441 & 2.467410 & -0.224449 \\ \mathrm{~N} & -0.064664 & 2.323564 & -0.364904 \\ \mathrm{C} & -0.746420 & 1.115570 & -0.274311 \\ \mathrm{O} & 1.827467 & 3.595743 & -0.309922 \\ \mathrm{C} & 3.560395 & 1.342965 & 0.149615 \\ \mathrm{O} & -1.981592 & 1.017594 & -0.401298 \\ \mathrm{H} & 1.954712 & -0.901835 & 0.306533 \\ \mathrm{H} & -0.436227 & -0.897740 & 0.049378 \\ \mathrm{H} & -0.620523 & 3.154459 & -0.541371 \\ \mathrm{H} & 3.829937 & 2.384837 & 0.354518 \\ \mathrm{H} & 4.034463 & 1.031540 & -0.796466 \\ \mathrm{H} & 3.931379 & 0.700235 & 0.964861\end{array}$


Table S826 Cartesian coordinates of the OM2/SCF-optimized ground-state equilibrium geometry of uracil (ground-state energy: $-1658.82481 \mathrm{eV}$ ).

$\begin{array}{lrrr}12 & & & \\ & & & \\ \mathrm{~N} & -0.684916 & 1.057934 & -0.010000 \\ \mathrm{~N} & -1.057734 & -1.274083 & -0.010000 \\ \mathrm{C} & -1.604172 & 0.019211 & -0.010000 \\ \mathrm{C} & 0.293960 & -1.526012 & -0.010000 \\ \mathrm{C} & 1.187241 & -0.500640 & -0.010000 \\ \mathrm{C} & 0.711143 & 0.885208 & -0.010000 \\ \mathrm{O} & 1.441770 & 1.884928 & -0.010000 \\ \mathrm{O} & -2.837400 & 0.189541 & -0.010000 \\ \mathrm{H} & 0.625875 & -2.577149 & -0.010000 \\ \mathrm{H} & -1.054067 & 2.003535 & -0.010000 \\ \mathrm{H} & -1.717950 & -2.044203 & -0.010000 \\ \mathrm{H} & 2.257990 & -0.652987 & -0.010000\end{array}$


4.1.10 DFT/B3LYP-Optimized Ground-State Minima 
Table S827 Cartesian coordinates of the DFT/TZVP-optimized ground-state equilibrium geometry of $9 \mathrm{H}$-adenine (ground-state energy: $-467.21546 E_{h}$ ).

$\begin{array}{lrrr}15 & & & \\ & & & \\ \mathrm{~N} & -1.8015385 & -0.2069325 & -0.0141318 \\ \mathrm{C} & -0.4185239 & -0.1892537 & 0.0009685 \\ \mathrm{C} & 0.0718378 & 1.1113976 & -0.1189330 \\ \mathrm{~N} & -1.0535108 & 1.8976189 & -0.2101817 \\ \mathrm{C} & -2.1377397 & 1.0486367 & -0.1407338 \\ \mathrm{~N} & 1.3538724 & 1.4808083 & -0.1346980 \\ \mathrm{C} & 2.1669691 & 0.4319237 & -0.0163934 \\ \mathrm{~N} & 1.8438421 & -0.8622819 & 0.1075262 \\ \mathrm{C} & 0.5478234 & -1.2055267 & 0.1171289 \\ \mathrm{~N} & 0.2159779 & -2.5108284 & 0.2250537 \\ \mathrm{H} & 3.2297595 & 0.6486992 & -0.0188202 \\ \mathrm{H} & -0.7481791 & -2.7798876 & 0.3159216 \\ \mathrm{H} & 0.9452287 & -3.1845544 & 0.3848483 \\ \mathrm{H} & -1.0657979 & 2.9003530 & -0.3083197 \\ \mathrm{H} & -3.1500211 & 1.4198278 & -0.1892354\end{array}$


Table S828 Cartesian coordinates of the DFT/TZVP-optimized ground-state equilibrium geometry of acetaldehyde (ground-state energy: $-153.80377 E_{h}$ ).

$\begin{array}{lrrr}7 & & & \\ \text { C } & -0.8417442 & 0.0106262 & -0.1393609 \\ \mathrm{C} & 0.6405457 & 0.0129067 & 0.1082381 \\ \mathrm{H} & -1.3058263 & -0.9967793 & -0.2126801 \\ \mathrm{O} & -1.5209414 & 0.9991989 & -0.2598934 \\ \mathrm{H} & 0.8613119 & -0.5447059 & 1.0239719 \\ \mathrm{H} & 1.1468655 & -0.5119652 & -0.7084396 \\ \mathrm{H} & 1.0197888 & 1.0307185 & 0.1881640\end{array}$


Table S829 Cartesian coordinates of the DFT/TZVP-optimized ground-state equilibrium geometry of acetone (ground-state energy: $-193.11306 E_{h}$ ).

$\begin{array}{lrrr}10 & & & \\ \text { C } & 0.1549059 & 0.0105844 & -1.2896941 \\ \mathrm{C} & -0.6410061 & -0.0382647 & 0.0000000 \\ \mathrm{O} & -1.8495176 & -0.1153348 & 0.0000000 \\ \mathrm{C} & 0.1549059 & 0.0105844 & 1.2896941 \\ \mathrm{H} & -0.5182157 & -0.0175285 & -2.1443548 \\ \mathrm{H} & 0.8463968 & -0.8356930 & -1.3401624 \\ \mathrm{H} & 0.7621749 & 0.9194368 & -1.3288390 \\ \mathrm{H} & -0.5182157 & -0.0175285 & 2.1443548 \\ \mathrm{H} & 0.7621749 & 0.9194368 & 1.3288390 \\ \mathrm{H} & 0.8463968 & -0.8356930 & 1.3401624\end{array}$


Table S830 Cartesian coordinates of the DFT/TZVP-optimized ground-state equilibrium geometry of acetophenone (ground-state energy: $-384.79234 E_{h}$ ).

$\begin{array}{lrrr}17 & & \\ & & & \\ \mathrm{C} & 1.3446888 & -1.2015122 & 0.0000000 \\ \mathrm{O} & 2.5463713 & -1.0140335 & 0.0000000 \\ \mathrm{C} & 0.7870552 & -2.6118454 & 0.0000000 \\ \mathrm{H} & 1.6172330 & -3.3148910 & 0.0000000 \\ \mathrm{H} & 0.1644510 & -2.7870686 & -0.8810012 \\ \mathrm{H} & 0.1644510 & -2.7870686 & 0.8810012 \\ \mathrm{C} & 0.3896411 & -0.0440933 & 0.0000000 \\ \mathrm{C} & -0.9989723 & -0.2143839 & 0.0000000 \\ \mathrm{C} & 0.9143402 & 1.2538337 & 0.0000000 \\ \mathrm{C} & -1.8450543 & 0.8895776 & 0.0000000 \\ \mathrm{C} & 0.0703075 & 2.3545601 & 0.0000000 \\ \mathrm{C} & -1.3120409 & 2.1746560 & 0.0000000 \\ \mathrm{H} & -1.4286628 & -1.2078563 & 0.0000000 \\ \mathrm{H} & 1.9901024 & 1.3739249 & 0.0000000 \\ \mathrm{H} & -2.9190013 & 0.7466605 & 0.0000000 \\ \mathrm{H} & 0.4863780 & 3.3549571 & 0.0000000 \\ \mathrm{H} & -1.9712880 & 3.0345829 & 0.0000000\end{array}$


Table S831 Cartesian coordinates of the DFT/TZVP-optimized ground-state equilibrium geometry of acetylene (ground-state energy: $-77.31120 E_{h}$ ).

4

$\begin{array}{lrrr}\mathrm{C} & 0.5991430 & 0.0000000 & 0.0002587 \\ \mathrm{C} & -0.5991430 & 0.0000000 & 0.0002587 \\ \mathrm{H} & 1.6622177 & 0.0000000 & -0.0002587 \\ \mathrm{H} & -1.6622177 & 0.0000000 & -0.0002587\end{array}$


Table S832 Cartesian coordinates of the DFT/TZVP-optimized ground-state equilibrium geometry of acrolein (ground-state energy: $-191.87653 E_{h}$ ).

$\begin{array}{lrrr}\mathrm{C} & -1.1219722 & -0.4866794 & 0.0000000 \\ \mathrm{C} & 0.2107874 & -0.4814076 & 0.0000000 \\ \mathrm{C} & 0.9704389 & 0.7803030 & 0.0000000 \\ \mathrm{O} & 2.1785890 & 0.8477231 & 0.0000000 \\ \mathrm{H} & -1.6947241 & -1.4055861 & 0.0000000 \\ \mathrm{H} & -1.6862757 & 0.4406603 & 0.0000000 \\ \mathrm{H} & 0.7943791 & -1.3960269 & 0.0000000 \\ \mathrm{H} & 0.3487776 & 1.7010136 & 0.0000000\end{array}$


Table S833 Cartesian coordinates of the DFT/TZVP-optimized groundstate equilibrium geometry of 2-amino-9-methylpurine (ground-state energy: $\left.-506.50588 E_{h}\right)$.

$\begin{array}{lrrr}18 & & \\ & & & \\ \mathrm{~N} & 1.8438912 & -1.6192803 & 0.0000000 \\ \mathrm{C} & 1.4378165 & -0.2937286 & 0.0000000 \\ \mathrm{C} & 0.0328223 & -0.2099839 & 0.0000000 \\ \mathrm{~N} & -0.4123515 & -1.5084030 & 0.0000000 \\ \mathrm{C} & 0.7308720 & -2.2953894 & 0.0000000 \\ \mathrm{C} & 2.1078991 & 0.9233849 & 0.0000000 \\ \mathrm{~N} & 1.4318492 & 2.0673389 & 0.0000000 \\ \mathrm{C} & 0.0781101 & 2.0102636 & 0.0000000 \\ \mathrm{~N} & -0.6841896 & 0.9041909 & 0.0000000 \\ \mathrm{~N} & -0.5662528 & 3.2077529 & 0.0000000 \\ \mathrm{C} & -1.7968923 & -1.9476596 & 0.0000000 \\ \mathrm{H} & 3.1916068 & 0.9854040 & 0.0000000 \\ \mathrm{H} & 0.6591523 & -3.3733712 & 0.0000000 \\ \mathrm{H} & -1.5691873 & 3.2357554 & 0.0000000 \\ \mathrm{H} & -0.0262033 & 4.0534901 & 0.0000000 \\ \mathrm{H} & -2.4270123 & -1.0604176 & 0.0000000 \\ \mathrm{H} & -2.0159652 & -2.5396736 & 0.8903551 \\ \mathrm{H} & -2.0159652 & -2.5396736 & -0.8903551\end{array}$


Table S834 Cartesian coordinates of the DFT/TZVP-optimized ground-state equilibrium geometry of aniline (ground-state energy: $-287.52121 E_{h}$ ).

$\begin{array}{lrrr}14 & & & \\ & & & \\ \text { C } & 0.0000000 & 0.0000000 & 0.9172655 \\ \text { C } & -1.2055657 & 0.0000000 & 0.1982195 \\ \text { C } & -1.1982902 & 0.0000000 & -1.1900206 \\ \text { C } & 0.0000000 & 0.0000000 & -1.8995676 \\ \text { C } & 1.1982902 & 0.0000000 & -1.1900206 \\ \text { C } & 1.2055657 & 0.0000000 & 0.1982195 \\ \text { N } & 0.0000000 & 0.0000000 & 2.2959617 \\ \text { H } & -0.8592538 & 0.0000000 & 2.8127242 \\ \text { H } & 0.8592538 & 0.0000000 & 2.8127242 \\ \text { H } & -2.1480632 & 0.0000000 & 0.7356401 \\ \text { H } & -2.1426650 & 0.0000000 & -1.7223759 \\ \text { H } & 0.0000000 & 0.0000000 & -2.9820342 \\ \text { H } & 2.1426650 & 0.0000000 & -1.7223759 \\ \text { H } & 2.1480632 & 0.0000000 & 0.7356401\end{array}$


Table S835 Cartesian coordinates of the DFT/TZVP-optimized ground-state equilibrium geometry of benzaldehyde (ground-state energy: $-345.48657 E_{h}$ ).

$\begin{array}{lrrr}14 & & & \\ & & & \\ \mathrm{C} & -0.5181562 & 2.2928330 & 0.0000000 \\ \mathrm{O} & -1.6346209 & 2.7601125 & 0.0000000 \\ \mathrm{H} & 0.3700151 & 2.9590077 & 0.0000000 \\ \mathrm{C} & -0.2022560 & 0.8474088 & 0.0000000 \\ \mathrm{C} & 1.1324737 & 0.4351110 & 0.0000000 \\ \mathrm{C} & -1.2254341 & -0.1074411 & 0.0000000 \\ \mathrm{C} & 1.4470132 & -0.9190865 & 0.0000000 \\ \mathrm{C} & -0.9105804 & -1.4576347 & 0.0000000 \\ \mathrm{C} & 0.4250062 & -1.8640046 & 0.0000000 \\ \mathrm{H} & 1.9220175 & 1.1793846 & 0.0000000 \\ \mathrm{H} & -2.2540721 & 0.2312539 & 0.0000000 \\ \mathrm{H} & 2.4821551 & -1.2377531 & 0.0000000 \\ \mathrm{H} & -1.7005610 & -2.1989481 & 0.0000000 \\ \mathrm{H} & 0.6670000 & -2.9202433 & 0.0000000\end{array}$


Table S836 Cartesian coordinates of the DFT/TZVP-optimized groundstate equilibrium geometry of $p$-benzoquinone (ground-state energy: $\left.-381.18842 E_{h}\right)$.

12

C $\quad 0.0000000$

1.5142801

0.0000000

C -1.1791166

0.6758869

$-1.1791166$

$-0.6758869$

0.0000000

C $\quad 0.0000000$

$-1.5142801$

C $\quad 1.1791166$

$-0.6758869$

0.0000000

1.1791166

$-0.6758869$

0.0000000

C

0.0000000

0.6758869

2.7672587

0.0000000

0.0000000

$-2.7672587$

0.0000000

$-2.1078802$

1.2331985

0.0000000

$-2.1078802$

$-1.2331985$

0.0000000

$\begin{array}{ll}\mathrm{H} & 2.1078802 \\ \mathrm{H} & 2.1078802\end{array}$

$-1.2331985$

0.0000000

1.2331985

0.0000000

0.0000000 
Table S837 Cartesian coordinates of the DFT/TZVP-optimized ground-state equilibrium geometry of biphenylene (ground-state energy: $-461.87983 E_{h}$ ).

$\begin{array}{lrrr}20 & & & \\ & & & \\ \mathrm{C} & 0.7101192 & 0.7540895 & 0.0000000 \\ \mathrm{C} & 1.4408235 & 1.9113649 & 0.0000000 \\ \mathrm{C} & 0.6928153 & 3.1149386 & 0.0000000 \\ \mathrm{C} & 0.7101192 & -0.7540895 & 0.0000000 \\ \mathrm{C} & -0.7101192 & -0.7540895 & 0.0000000 \\ \mathrm{C} & -0.7101192 & 0.7540895 & 0.0000000 \\ \mathrm{C} & 1.4408235 & -1.9113649 & 0.0000000 \\ \mathrm{C} & -1.4408235 & -1.9113649 & 0.0000000 \\ \mathrm{C} & -1.4408235 & 1.9113649 & 0.0000000 \\ \mathrm{C} & 0.6928153 & -3.1149386 & 0.0000000 \\ \mathrm{C} & -0.6928153 & -3.1149386 & 0.0000000 \\ \mathrm{C} & -0.6928153 & 3.1149386 & 0.0000000 \\ \mathrm{H} & 2.5236341 & 1.9320974 & 0.0000000 \\ \mathrm{H} & 2.5236341 & -1.9320974 & 0.0000000 \\ \mathrm{H} & -2.5236341 & -1.9320974 & 0.0000000 \\ \mathrm{H} & -2.5236341 & 1.9320974 & 0.0000000 \\ \mathrm{H} & 1.2198052 & 4.0617378 & 0.0000000 \\ \mathrm{H} & 1.2198052 & -4.0617378 & 0.0000000 \\ \mathrm{H} & -1.2198052 & -4.0617378 & 0.0000000 \\ \mathrm{H} & -1.2198052 & 4.0617378 & 0.0000000\end{array}$


Table S838 Cartesian coordinates of the DFT/TZVP-optimized groundstate equilibrium geometry of trans-butadiene (ground-state energy: $\left.-155.94279 E_{h}\right)$.

10

C $\quad-0.0518361$

C $\quad 0.0518361$

C $\quad 1.2053409$

C $\quad-1.2053409$

0.7253582

0.0000000

$-0.7253582 \quad 0.0000000$

$\mathrm{H} \quad 1.2315784$

$-1.3987917$

0.0000000

$-1.2315784$

1.3987917

0.0000000

$\mathrm{H}$

$-2.4810561$

0.0000000

$\mathrm{H} \quad 2.1613511$

2.4810561

0.0000000

$-2.1613511$

$-0.8858972$

0.0000000

$\mathrm{H} \quad-0.8862902-1.2753738 \quad 0.0000000$

0.8858972

0.0000000

$\mathrm{H} \quad 0.8862902 \quad 1.2753738 \quad 0.0000000$ 
Table S839 Cartesian coordinates of the DFT/TZVP-optimized ground-state equilibrium geometry of cytosine (ground-state energy: $-394.85947 E_{h}$ ).

$\begin{array}{lrrr}13 & & & \\ & & & \\ \mathrm{~N} & -1.0187568 & -0.8839661 & -0.1265482 \\ \mathrm{C} & 0.2944542 & -0.8900639 & -0.0591089 \\ \mathrm{C} & 1.0957361 & 0.2979707 & 0.0692284 \\ \mathrm{C} & 0.4214258 & 1.4711760 & 0.1164450 \\ \mathrm{~N} & -0.9290105 & 1.4795461 & 0.0437060 \\ \mathrm{C} & -1.7143312 & 0.2935717 & -0.0825431 \\ \mathrm{~N} & 0.9126142 & -2.0963720 & -0.1361769 \\ \mathrm{O} & -2.9229667 & 0.4047278 & -0.1381441 \\ \mathrm{H} & 2.1738031 & 0.2643503 & 0.1187923 \\ \mathrm{H} & 0.9131723 & 2.4310620 & 0.2114364 \\ \mathrm{H} & 0.3312917 & -2.9186934 & -0.1408498 \\ \mathrm{H} & 1.8950538 & -2.1951705 & 0.0463576 \\ \mathrm{H} & -1.4524861 & 2.3418612 & 0.0774052 \\ & & & \end{array}$


Table S840 Cartesian coordinates of the DFT/TZVP-optimized ground-state equilibrium geometry of diazomethane (ground-state energy: $-148.71393 E_{h}$ ).

5

$\begin{array}{lrrr}\mathrm{C} & 0.0000000 & 0.0000000 & -0.5411301 \\ \mathrm{~N} & 0.0000000 & 0.0000000 & 0.7510266 \\ \mathrm{~N} & 0.0000000 & 0.0000000 & 1.8850725 \\ \mathrm{H} & 0.9508998 & 0.0000000 & -1.0474845 \\ \mathrm{H} & -0.9508998 & 0.0000000 & -1.0474845\end{array}$


Table S841 Cartesian coordinates of the DFT/TZVP-optimized groundstate equilibrium geometry of dibenzo- $p$-dioxin (ground-state energy: $\left.-612.37165 E_{h}\right)$.

$\begin{array}{lrrr}22 & & & \\ & & & \\ \mathrm{C} & -3.5794873 & 0.6953452 & 0.0000000 \\ \mathrm{C} & -3.5794873 & -0.6953452 & 0.0000000 \\ \mathrm{C} & -2.3738826 & -1.3917553 & 0.0000000 \\ \mathrm{C} & -1.1740313 & -0.6973619 & 0.0000000 \\ \mathrm{C} & -1.1740313 & 0.6973619 & 0.0000000 \\ \mathrm{C} & -2.3738826 & 1.3917553 & 0.0000000 \\ \mathrm{O} & 0.0000000 & -1.4236640 & 0.0000000 \\ \mathrm{C} & 1.1740313 & -0.6973619 & 0.0000000 \\ \mathrm{C} & 1.1740313 & 0.6973619 & 0.0000000 \\ \mathrm{O} & 0.0000000 & 1.4236640 & 0.0000000 \\ \mathrm{C} & 2.3738826 & -1.3917553 & 0.0000000 \\ \mathrm{C} & 3.5794873 & -0.6953452 & 0.0000000 \\ \mathrm{C} & 3.5794873 & 0.6953452 & 0.0000000 \\ \mathrm{C} & 2.3738826 & 1.3917553 & 0.0000000 \\ \mathrm{H} & 4.5132112 & 1.2431718 & 0.0000000 \\ \mathrm{H} & -4.5132112 & -1.2431718 & 0.0000000 \\ \mathrm{H} & -2.3445318 & -2.4740051 & 0.0000000 \\ \mathrm{H} & -2.3445318 & 2.4740051 & 0.0000000 \\ \mathrm{H} & 2.3445318 & -2.4740051 & 0.0000000 \\ \mathrm{H} & 4.5132112 & -1.2431718 & 0.0000000 \\ \mathrm{H} & -4.5132112 & 1.2431718 & 0.0000000 \\ \mathrm{H} & 2.3445318 & 2.4740051 & 0.0000000\end{array}$


Table S842 Cartesian coordinates of the DFT/TZVP-optimized ground-state equilibrium geometry of formaldehyde (ground-state energy: $-114.49001 E_{h}$ ).

4

$\begin{array}{rrrr}\mathrm{C} & -0.0062763 & -0.0021242 & 0.0000000 \\ \mathrm{O} & 1.1254636 & 0.4003322 & 0.0000000 \\ \mathrm{H} & -0.5595937 & -0.1991040 & 0.9389762 \\ \mathrm{H} & -0.5595937 & -0.1991040 & -0.9389762\end{array}$


Table S843 Cartesian coordinates of the DFT/TZVP-optimized ground-state equilibrium geometry of trans-glyoxal (ground-state energy: $-227.79708 E_{h}$ ).

$\begin{array}{lrrr}\mathrm{C} & 0.7630763 & -0.0228837 & 0.0000000 \\ \mathrm{C} & -0.7630763 & 0.0228837 & 0.0000000 \\ \mathrm{O} & 1.3625513 & -1.0658085 & 0.0000000 \\ \mathrm{O} & -1.3625513 & 1.0658085 & 0.0000000 \\ \mathrm{H} & 1.2594799 & 0.9663370 & 0.0000000 \\ \mathrm{H} & -1.2594799 & -0.9663370 & 0.0000000\end{array}$


Table S844 Cartesian coordinates of the DFT/TZVP-optimized ground-state equilibrium geometry of $9 \mathrm{H}$-guanine (ground-state energy: $-542.45075 E_{h}$ ).

$\begin{array}{lrrr}16 & & & \\ & & & \\ \mathrm{~N} & -0.0151171 & -2.2277951 & 0.4149990 \\ \mathrm{C} & -0.2451266 & -2.2602161 & 1.7789668 \\ \mathrm{~N} & -0.3690160 & -1.0705400 & 2.2948708 \\ \mathrm{C} & -0.2156247 & -0.2066177 & 1.2306672 \\ \mathrm{C} & -0.2623150 & 1.2286400 & 1.1825494 \\ \mathrm{O} & -0.4458280 & 2.0529826 & 2.0525545 \\ \mathrm{~N} & -0.0524373 & 1.6695006 & -0.1709874 \\ \mathrm{H} & -0.1379264 & 2.6707541 & -0.2862214 \\ \mathrm{C} & 0.1652176 & 0.8716308 & -1.2619071 \\ \mathrm{~N} & 0.2886690 & 1.4979019 & -2.4770960 \\ \mathrm{H} & 0.6762334 & 2.4276898 & -2.5016925 \\ \mathrm{H} & 0.5933439 & 0.8900566 & -3.2215709 \\ \mathrm{~N} & 0.2111637 & -0.4328813 & -1.2000516 \\ \mathrm{C} & 0.0041724 & -0.9100903 & 0.0504241 \\ \mathrm{H} & -0.3078081 & -3.1906554 & 2.3214088 \\ \mathrm{H} & 0.1123991 & -3.0103603 & -0.2069135\end{array}$


Table S845 Cartesian coordinates of the DFT/TZVP-optimized groundstate equilibrium geometry of 1-hydroxy-2-acetonaphthone (ground-state energy: $-613.62068 E_{h}$ ).

$\begin{array}{lrrr}24 & & \\ & & & \\ \mathrm{C} & 0.1029397 & 1.0549888 & 0.0000000 \\ \mathrm{C} & -1.1454891 & 0.3553287 & 0.0000000 \\ \mathrm{C} & -1.1391523 & -1.0680540 & 0.0000000 \\ \mathrm{C} & 0.1039507 & -1.7636051 & 0.0000000 \\ \mathrm{C} & 1.2800310 & -1.0765127 & 0.0000000 \\ \mathrm{C} & 1.3182802 & 0.3490892 & 0.0000000 \\ \mathrm{C} & -2.3762369 & 1.0504145 & 0.0000000 \\ \mathrm{C} & -3.5648278 & 0.3601287 & 0.0000000 \\ \mathrm{C} & -3.5650069 & -1.0489989 & 0.0000000 \\ \mathrm{C} & -2.3799760 & -1.7460408 & 0.0000000 \\ \mathrm{C} & 2.5783380 & 1.0937721 & 0.0000000 \\ \mathrm{C} & 3.8973953 & 0.3556654 & 0.0000000 \\ \mathrm{O} & 0.0575088 & 2.3871344 & 0.0000000 \\ \mathrm{O} & 2.5893137 & 2.3339151 & 0.0000000 \\ \mathrm{H} & -4.5048502 & 0.8982600 & 0.0000000 \\ \mathrm{H} & -4.5069531 & -1.5847959 & 0.0000000 \\ \mathrm{H} & -2.3822635 & -2.8300652 & 0.0000000 \\ \mathrm{H} & -2.3622691 & 2.1318303 & 0.0000000 \\ \mathrm{H} & 0.0996262 & -2.8468581 & 0.0000000 \\ \mathrm{H} & 2.2128069 & -1.6244916 & 0.0000000 \\ \mathrm{H} & 1.0053123 & 2.7010077 & 0.0000000 \\ \mathrm{H} & 4.7040116 & 1.0855086 & 0.0000000 \\ \mathrm{H} & 3.9887552 & -0.2838105 & -0.8811587 \\ \mathrm{H} & 3.9887552 & -0.2838105 & 0.8811587\end{array}$


Table S846 Cartesian coordinates of the DFT/TZVP-optimized ground-state equilibrium geometry of indole (ground-state energy: $-363.70951 E_{h}$ ).

$\begin{array}{lrrr}16 & & & \\ & & & \\ \mathrm{C} & -0.5411383 & -0.5855747 & 0.0000000 \\ \mathrm{C} & -0.0476468 & 0.7459663 & 0.0000000 \\ \mathrm{~N} & -1.1423329 & 1.5848716 & 0.0000000 \\ \mathrm{C} & -1.9737767 & -0.4994745 & 0.0000000 \\ \mathrm{C} & -2.2975676 & 0.8273590 & 0.0000000 \\ \mathrm{C} & 0.3769313 & -1.6457751 & 0.0000000 \\ \mathrm{C} & 1.7322208 & -1.3631337 & 0.0000000 \\ \mathrm{C} & 1.3172558 & 1.0342955 & 0.0000000 \\ \mathrm{C} & 2.1980536 & -0.0356241 & 0.0000000 \\ \mathrm{H} & -1.1076793 & 2.5893040 & 0.0000000 \\ \mathrm{H} & -2.6728801 & -1.3200540 & 0.0000000 \\ \mathrm{H} & -3.2641200 & 1.3049951 & 0.0000000 \\ \mathrm{H} & 0.0288122 & -2.6721653 & 0.0000000 \\ \mathrm{H} & 2.4498796 & -2.1747122 & 0.0000000 \\ \mathrm{H} & 1.6792263 & 2.0560417 & 0.0000000 \\ \mathrm{H} & 3.2647622 & 0.1536805 & 0.0000000\end{array}$


Table S847 Cartesian coordinates of the DFT/TZVP-optimized ground-state equilibrium geometry of maleimide (ground-state energy: $-359.37512 E_{h}$ ).

$\begin{array}{lrrr}10 & & & \\ & & & \\ \mathrm{~N} & 0.0000000 & 0.0000000 & -1.1811889 \\ \mathrm{C} & -1.1550019 & 0.0000000 & -0.3978897 \\ \mathrm{C} & -0.6658357 & 0.0000000 & 1.0235261 \\ \mathrm{C} & 0.6658357 & 0.0000000 & 1.0235261 \\ \mathrm{C} & 1.1550019 & 0.0000000 & -0.3978897 \\ \mathrm{O} & -2.2920730 & 0.0000000 & -0.7985869 \\ \mathrm{O} & 2.2920730 & 0.0000000 & -0.7985869 \\ \mathrm{H} & 0.0000000 & 0.0000000 & -2.1897347 \\ \mathrm{H} & -1.3500965 & 0.0000000 & 1.8584124 \\ \mathrm{H} & 1.3500965 & 0.0000000 & 1.8584124\end{array}$


Table S848 Cartesian coordinates of the DFT/TZVP-optimized ground-state equilibrium geometry of nitrosomethane (ground-state energy: $-169.76879 E_{h}$ ).

$\begin{array}{lrrr}6 & & & \\ \mathrm{C} & -0.4136325 & 0.1639047 & 0.0000000 \\ \mathrm{~N} & 1.0814211 & 0.2583577 & 0.0000000 \\ \mathrm{O} & 1.6176469 & -0.8163644 & 0.0000000 \\ \mathrm{H} & -0.8268218 & 1.1698588 & 0.0000000 \\ \mathrm{H} & -0.7293068 & -0.3878784 & -0.8884913 \\ \mathrm{H} & -0.7293068 & -0.3878784 & 0.8884913\end{array}$


Table S849 Cartesian coordinates of the DFT/TZVP-optimized groundstate equilibrium geometry of propenoic acid anion (ground-state energy: $\left.-266.56810 E_{h}\right)$.

$\begin{array}{rrrr}\mathrm{C} & -0.2839773 & 0.4108519 & 0.0000000 \\ \mathrm{C} & 1.2568100 & 0.3618904 & 0.0000000 \\ \mathrm{O} & 1.8024857 & 1.4943357 & 0.0000000 \\ \mathrm{O} & 1.7847622 & -0.7740701 & 0.0000000 \\ \mathrm{C} & -1.0707540 & -0.6625499 & 0.0000000 \\ \mathrm{H} & -0.7136138 & 1.4115646 & 0.0000000 \\ \mathrm{H} & -2.1563206 & -0.5921696 & 0.0000000 \\ \mathrm{H} & -0.6193922 & -1.6498529 & 0.0000000\end{array}$


Table S850 Cartesian coordinates of the DFT/TZVP-optimized ground-state equilibrium geometry of pyridine (ground-state energy: $-248.21286 E_{h}$ ).

$\begin{array}{lrrr}11 & & & \\ & & & \\ \mathrm{~N} & 0.0000000 & 0.0000000 & 1.6031330 \\ \mathrm{C} & -1.1406161 & 0.0000000 & 0.9091617 \\ \mathrm{C} & 1.1406161 & 0.0000000 & 0.9091617 \\ \mathrm{C} & -1.1949873 & 0.0000000 & -0.4817100 \\ \mathrm{C} & 1.1949873 & 0.0000000 & -0.4817100 \\ \mathrm{C} & 0.0000000 & 0.0000000 & -1.1917023 \\ \mathrm{H} & -2.0547176 & 0.0000000 & 1.4947941 \\ \mathrm{H} & 2.0547176 & 0.0000000 & 1.4947941 \\ \mathrm{H} & -2.1511406 & 0.0000000 & -0.9903381 \\ \mathrm{H} & 2.1511406 & 0.0000000 & -0.9903381 \\ \mathrm{H} & 0.0000000 & 0.0000000 & -2.2752461\end{array}$


Table S851 Cartesian coordinates of the DFT/TZVP-optimized ground-state equilibrium geometry of pyrrole (ground-state energy: $-210.11117 E_{h}$ ).

$\begin{array}{lrrr}10 & & & \\ & & & \\ \mathrm{H} & 2.1432520 & -0.1336476 & 0.0000000 \\ \mathrm{H} & 0.7823982 & -0.0483823 & 2.1105645 \\ \mathrm{H} & 0.7823982 & -0.0483823 & -2.1105645 \\ \mathrm{H} & -1.8187204 & 0.1128883 & -1.3570539 \\ \mathrm{H} & -1.8187204 & 0.1128883 & 1.3570539 \\ \mathrm{~N} & 1.1400179 & -0.0701967 & 0.0000000 \\ \mathrm{C} & 0.3517742 & -0.0217478 & -1.1236010 \\ \mathrm{C} & 0.3517742 & -0.0217478 & 1.1236010 \\ \mathrm{C} & -0.9570870 & 0.0591640 & 0.7113639 \\ \mathrm{C} & -0.9570870 & 0.0591640 & -0.7113639\end{array}$


Table S852 Cartesian coordinates of the DFT/TZVP-optimized ground-state equilibrium geometry of cis-PSB3 (ground-state energy: $-249.96059 E_{h}$ ).

$\begin{array}{lrrr}14 & & & \\ \text { C } & 2.8936432 & -0.4502515 & 0.0000000 \\ \text { C } & 1.5365676 & -0.4372320 & 0.0000000 \\ \text { C } & 0.7315143 & 0.7299960 & 0.0000000 \\ \text { C } & -0.6746500 & 0.7926263 & 0.0000000 \\ \text { C } & -1.5592924 & -0.2569647 & 0.0000000 \\ \text { N } & -2.9245849 & -0.1210602 & 0.0000000 \\ \text { H } & 3.4508908 & -1.3777399 & 0.0000000 \\ \text { H } & 3.4673472 & 0.4700238 & 0.0000000 \\ \text { H } & 1.0300132 & -1.4000184 & 0.0000000 \\ \text { H } & 1.2567402 & 1.6801948 & 0.0000000 \\ \text { H } & -1.1078675 & 1.7910663 & 0.0000000 \\ \text { H } & -1.2169749 & -1.2841062 & 0.0000000 \\ \text { H } & -3.5262507 & -0.9225721 & 0.0000000 \\ \text { H } & -3.3570961 & 0.7860376 & 0.0000000\end{array}$


Table S853 Cartesian coordinates of the DFT/TZVP-optimized ground-state equilibrium geometry of 3Me-cis-PSB5 (ground-state energy: $-444.02575 E_{h}$ ).

$\begin{array}{lrrr}25 & & & \\ & & & \\ \mathrm{C} & -0.8410037 & 1.2635561 & 0.0000000 \\ \mathrm{C} & 0.5581443 & 1.2402261 & 0.0000000 \\ \mathrm{C} & 1.4857167 & 0.1950816 & 0.0000000 \\ \mathrm{C} & 2.8723295 & 0.5381114 & 0.0000000 \\ \mathrm{C} & 3.9423557 & -0.3045792 & 0.0000000 \\ \mathrm{~N} & 5.2521710 & 0.0980750 & 0.0000000 \\ \mathrm{C} & 1.0576411 & -1.2486265 & 0.0000000 \\ \mathrm{C} & -1.7909854 & 0.2209029 & 0.0000000 \\ \mathrm{C} & -3.1489959 & 0.4306193 & 0.0000000 \\ \mathrm{C} & -4.1236284 & -0.6165814 & 0.0000000 \\ \mathrm{C} & -5.4596568 & -0.4364732 & 0.0000000 \\ \mathrm{H} & -1.2713780 & 2.2624847 & 0.0000000 \\ \mathrm{H} & 1.0071524 & 2.2304816 & 0.0000000 \\ \mathrm{H} & 3.0920684 & 1.6042576 & 0.0000000 \\ \mathrm{H} & 3.8194684 & -1.3799384 & 0.0000000 \\ \mathrm{H} & 5.9995021 & -0.5696832 & 0.0000000 \\ \mathrm{H} & 5.4978585 & 1.0724747 & 0.0000000 \\ \mathrm{H} & 1.9017244 & -1.9357143 & 0.0000000 \\ \mathrm{H} & 0.4513227 & -1.4787062 & 0.8806437 \\ \mathrm{H} & 0.4513227 & -1.4787062 & -0.8806437 \\ \mathrm{H} & -1.4544668 & -0.8083875 & 0.0000000 \\ \mathrm{H} & -3.5189561 & 1.4539536 & 0.0000000 \\ \mathrm{H} & -3.7379257 & -1.6343991 & 0.0000000 \\ \mathrm{H} & -6.1444503 & -1.2744115 & 0.0000000 \\ \mathrm{H} & -5.8973308 & 0.5559821 & 0.0000000 \\ & & & \end{array}$


Table S854 Cartesian coordinates of the DFT/TZVP-optimized ground-state equilibrium geometry of trans-stilbene (ground-state energy: $-540.53261 E_{h}$ ).

$\begin{array}{lrrr}26 & & & \\ & & & \\ \mathrm{C} & -0.0398077 & 0.6702495 & 0.0000000 \\ \mathrm{C} & -1.2565476 & 1.4848287 & 0.0000000 \\ \mathrm{C} & -1.1318481 & 2.8829559 & 0.0000000 \\ \mathrm{C} & -2.2488945 & 3.7096011 & 0.0000000 \\ \mathrm{C} & -3.5250817 & 3.1572837 & 0.0000000 \\ \mathrm{C} & -3.6694289 & 1.7703526 & 0.0000000 \\ \mathrm{C} & -2.5542601 & 0.9460216 & 0.0000000 \\ \mathrm{C} & 0.0398077 & -0.6702495 & 0.0000000 \\ \mathrm{C} & 1.2565476 & -1.4848287 & 0.0000000 \\ \mathrm{C} & 1.1318481 & -2.8829559 & 0.0000000 \\ \mathrm{C} & 2.2488945 & -3.7096011 & 0.0000000 \\ \mathrm{C} & 3.5250817 & -3.1572837 & 0.0000000 \\ \mathrm{C} & 3.6694289 & -1.7703526 & 0.0000000 \\ \mathrm{C} & 2.5542601 & -0.9460216 & 0.0000000 \\ \mathrm{H} & -4.6598731 & 1.3302764 & 0.0000000 \\ \mathrm{H} & -4.3990441 & 3.7972441 & 0.0000000 \\ \mathrm{H} & -2.1215892 & 4.7857734 & 0.0000000 \\ \mathrm{H} & -0.1403806 & 3.3227676 & 0.0000000 \\ \mathrm{H} & -2.6959545 & -0.1275162 & 0.0000000 \\ \mathrm{H} & 0.8798515 & 1.2478208 & 0.0000000 \\ \mathrm{H} & -0.8798515 & -1.2478208 & 0.0000000 \\ \mathrm{H} & 0.1403806 & -3.3227676 & 0.0000000 \\ \mathrm{H} & 2.1215892 & -4.7857734 & 0.0000000 \\ \mathrm{H} & 4.3990441 & -3.7972441 & 0.0000000 \\ \mathrm{H} & 4.6598731 & -1.3302764 & 0.0000000 \\ \mathrm{H} & 2.6959545 & 0.1275162 & 0.0000000\end{array}$


Table S855 Cartesian coordinates of the DFT/TZVP-optimized ground-state equilibrium geometry of styrene (ground-state energy: $-309.54764 E_{h}$ ).

$\begin{array}{lrrr}16 & & & \\ & & & \\ \mathrm{C} & -0.9999353 & 1.6377073 & 0.0000000 \\ \mathrm{C} & -2.3331948 & 1.6815624 & 0.0000000 \\ \mathrm{C} & -0.1391103 & 0.4454696 & 0.0000000 \\ \mathrm{C} & -0.6411775 & -0.8647038 & 0.0000000 \\ \mathrm{C} & 1.2518848 & 0.6149107 & 0.0000000 \\ \mathrm{C} & 0.2155895 & -1.9557125 & 0.0000000 \\ \mathrm{C} & 2.1123202 & -0.4774444 & 0.0000000 \\ \mathrm{C} & 1.5973610 & -1.7688208 & 0.0000000 \\ \mathrm{H} & -0.4595924 & 2.5811245 & 0.0000000 \\ \mathrm{H} & -2.8561407 & 2.6294142 & 0.0000000 \\ \mathrm{H} & -2.9511086 & 0.7916955 & 0.0000000 \\ \mathrm{H} & -1.7109534 & -1.0336667 & 0.0000000 \\ \mathrm{H} & 1.6606920 & 1.6195784 & 0.0000000 \\ \mathrm{H} & -0.1940486 & -2.9590562 & 0.0000000 \\ \mathrm{H} & 3.1842770 & -0.3186690 & 0.0000000 \\ \mathrm{H} & 2.2631371 & -2.6233892 & 0.0000000\end{array}$


Table S856 Cartesian coordinates of the DFT/TZVP-optimized ground-state equilibrium geometry of $s$-tetrazine (ground-state energy: $-296.09627 E_{h}$ ).

8

$\begin{array}{lrrr}\mathrm{C} & 0.0000000 & 1.3603663 & 0.0000000 \\ \mathrm{C} & 0.0000000 & -1.3603663 & 0.0000000 \\ \mathrm{~N} & 1.1186954 & 0.6547551 & 0.0000000 \\ \mathrm{~N} & 1.1186954 & -0.6547551 & 0.0000000 \\ \mathrm{~N} & -1.1186954 & 0.6547551 & 0.0000000 \\ \mathrm{~N} & -1.1186954 & -0.6547551 & 0.0000000 \\ \mathrm{H} & 0.0000000 & -2.4386046 & 0.0000000 \\ \mathrm{H} & 0.0000000 & 2.4386046 & 0.0000000\end{array}$


Table S857 Cartesian coordinates of the DFT/TZVP-optimized ground-state equilibrium geometry of thymine (ground-state energy: $-454.05551 E_{h}$ ).

$\begin{array}{lrrr}15 & & & \\ & & & \\ \mathrm{~N} & -1.3252418 & -1.2179149 & -0.0004227 \\ \mathrm{C} & 0.0510028 & -1.1705668 & -0.0004600 \\ \mathrm{C} & 0.7477736 & -0.0166622 & -0.0002222 \\ \mathrm{C} & -0.0084675 & 1.2417408 & -0.0002593 \\ \mathrm{~N} & -1.4060772 & 1.0834543 & -0.0002166 \\ \mathrm{C} & -2.1345457 & -0.0917091 & 0.0002974 \\ \mathrm{O} & 0.4755453 & 2.3580623 & -0.0003761 \\ \mathrm{C} & 2.2512380 & 0.0451802 & 0.0002186 \\ \mathrm{O} & -3.3462175 & -0.1436960 & 0.0014459 \\ \mathrm{H} & 0.5387742 & -2.1376326 & -0.0004867 \\ \mathrm{H} & -1.8134147 & -2.1001012 & -0.0004703 \\ \mathrm{H} & -1.9496860 & 1.9370975 & -0.0001772 \\ \mathrm{H} & 2.5682513 & 1.0867575 & 0.0000214 \\ \mathrm{H} & 2.6758849 & -0.4373010 & -0.8832799 \\ \mathrm{H} & 2.6751801 & -0.4367089 & 0.8843876\end{array}$


Table S858 Cartesian coordinates of the DFT/TZVP-optimized ground-state equilibrium geometry of uracil (ground-state energy: $-414.75452 E_{h}$ ).

$\begin{array}{lrrr}12 & & & \\ & & & \\ \mathrm{~N} & -0.4820176 & 1.2241422 & 0.0000000 \\ \mathrm{~N} & -0.8457955 & -1.0598936 & 0.0000000 \\ \mathrm{C} & -1.4201177 & 0.2088053 & 0.0000000 \\ \mathrm{C} & 0.5096156 & -1.2804342 & 0.0000000 \\ \mathrm{C} & 1.3957506 & -0.2686484 & 0.0000000 \\ \mathrm{C} & 0.9249948 & 1.1100833 & 0.0000000 \\ \mathrm{O} & 1.6210005 & 2.1050469 & 0.0000000 \\ \mathrm{O} & -2.6186816 & 0.3861037 & 0.0000000 \\ \mathrm{H} & 0.8094439 & -2.3199945 & 0.0000000 \\ \mathrm{H} & -0.8545408 & 2.1652218 & 0.0000000 \\ \mathrm{H} & -1.4997349 & -1.8273928 & 0.0000000 \\ \mathrm{H} & 2.4600827 & -0.4430397 & 0.0000000\end{array}$




\subsubsection{OM2/SCF-OM2/MRCI Results on Significant Changes Be- tween Ground-State and Excited-State Minima}

Given are the differences in bond lengths (in $\AA$ ) and in bond angles (in ${ }^{\circ}$ ) (which were determined to change $\geq|0.03 \AA|$ or $\geq\left|5^{\circ}\right|$ at the (TD)DFT level) between the OM2/MRCI-optimized excited-state equilibrium geometry and the $\mathrm{OM}$ /SCF-optimized ground-state equilibrium geometry for each excited state of the 32-molecules benchmark set. ( 5 of the 15 excited states of dibenzo- $p$ dioxin were disregarded in this analysis, as the structural changes occurring in these states are smaller than the thresholds mentioned above.) 
Table S859 Structural differences between the $2{ }^{1} A\left({ }^{1} n \pi^{*}\right)$ state and the ground state of $9 \mathrm{H}$-adenine.

\begin{tabular}{ll} 
bond & length / $\AA$ \\
or angle & or angle / \\
\hline N1-C2 & -0.022 \\
C2-C9 & +0.018 \\
N4-C5 & +0.010 \\
N6-C7 & +0.051 \\
C9-N10 & -0.011
\end{tabular}


Table S860 Structural differences between the $2{ }^{1} A\left({ }^{1} n \pi^{*}\right)$ state and the ground state of acetaldehyde.

\begin{tabular}{ll}
$\begin{array}{l}\text { bond } \\
\text { or angle }\end{array}$ & $\begin{array}{l}\text { length } / \AA \\
\text { ongle / } / 0\end{array}$ \\
\hline C1-O4 4 +0.068 & -4.8
\end{tabular}


Table S861 Structural differences between the $1{ }^{3} A\left({ }^{3} n \pi^{*}\right)$ state and the ground state of acetaldehyde.

\begin{tabular}{ll} 
bond & length / $\AA$ \\
or angle & or angle / o \\
\hline $\mathrm{C} 1-\mathrm{O} 4$ & +0.048 \\
O4-C1-C2 & -5.7
\end{tabular}


Table S862 Structural differences between the $1{ }^{1} A^{\prime \prime}\left({ }^{1} n \pi^{*}\right)$ state and the ground state of acetone.

\begin{tabular}{ll}
$\begin{array}{l}\text { bond } \\
\text { or angle }\end{array}$ & $\begin{array}{l}\text { ongth / } \AA \\
\text { C2-03 } 3\end{array}$ \\
\hline 0.065
\end{tabular}


Table S863 Structural differences between the $1{ }^{3} A^{\prime \prime}\left({ }^{3} n \pi^{*}\right)$ state and the ground state of acetone.

\begin{tabular}{ll} 
bond & $\begin{array}{l}\text { length / } \AA \\
\text { or angle } \\
\text { or angle / o }\end{array}$ \\
\hline C2-03 & +0.040
\end{tabular}


Table S864 Structural differences between the $1{ }^{1} A^{\prime \prime}\left({ }^{1} n \pi^{*}\right)$ state and the ground state of acetophenone.

\begin{tabular}{|c|c|}
\hline $\begin{array}{l}\text { bond } \\
\text { or angle }\end{array}$ & $\begin{array}{l}\text { length / } \AA \\
\text { or angle / o }\end{array}$ \\
\hline $\mathrm{C} 7-\mathrm{C} 9$ & +0.020 \\
\hline $\mathrm{C} 7-\mathrm{C} 8$ & +0.029 \\
\hline $\mathrm{C} 1-\mathrm{C} 7$ & -0.073 \\
\hline $\mathrm{C} 1-\mathrm{O} 2$ & +0.060 \\
\hline
\end{tabular}


Table S865 Structural differences between the $2{ }^{1} A^{\prime}\left({ }^{1} \pi \pi^{*}\right)$ state and the ground state of acetophenone.

\begin{tabular}{|c|c|}
\hline $\begin{array}{l}\text { bond } \\
\text { or angle }\end{array}$ & $\begin{array}{l}\text { length / } \AA \\
\text { or angle / o }\end{array}$ \\
\hline C9-C11 & +0.032 \\
\hline $\mathrm{C} 8-\mathrm{C} 10$ & +0.033 \\
\hline $\mathrm{C} 1-\mathrm{C} 7$ & -0.022 \\
\hline $\mathrm{C} 1-\mathrm{O} 2$ & +0.012 \\
\hline
\end{tabular}


Table S866 Structural differences between the $1{ }^{3} A^{\prime \prime}\left({ }^{3} n \pi^{*}\right)$ state and the ground state of acetophenone.

\begin{tabular}{ll} 
bond & length / $\AA$ \\
or angle & or angle / o \\
\hline C1-C7 & -0.067 \\
C1-02 & +0.053
\end{tabular}


Table S867 Structural differences between the $1{ }^{3} A^{\prime}\left({ }^{3} \pi \pi^{*}\right)$ state and the ground state of acetophenone.

\begin{tabular}{ll} 
bond & $\begin{array}{l}\text { length / } \AA \\
\text { or angle }\end{array}$ \\
or angle / o \\
\hline C7-C9 & +0.067 \\
C11-C12 & +0.044 \\
C10-C12 & +0.045 \\
C8-C10 & -0.023 \\
C7-C8 & +0.065 \\
C1-C7 & -0.043 \\
C1-O2 & +0.023
\end{tabular}


Table S868 Structural differences between the $1{ }^{1} A_{2}\left({ }^{1} \pi \pi^{*}\right)$ state and the ground state of acetylene.

\begin{tabular}{ll} 
bond & length / $\AA$ \\
or angle & or angle / o \\
\hline $\mathrm{C} 1-\mathrm{C} 2$ & +0.104 \\
$\mathrm{C} 1-\mathrm{H} 4$ & +0.082 \\
$\mathrm{C} 1-\mathrm{C} 2-\mathrm{H} 3$ & -32.8
\end{tabular}


Table S869 Structural differences between the $1{ }^{1} B_{2}\left({ }^{1} \pi \pi^{*}\right)$ state and the ground state of acetylene.

\begin{tabular}{ll} 
bond & length / \\
or angle & or angle / o \\
\hline $\mathrm{C} 1-\mathrm{C} 2$ & +0.081 \\
$\mathrm{C} 1-\mathrm{H} 4$ & +0.097 \\
$\mathrm{C} 1-\mathrm{C} 2-\mathrm{H} 3$ & -40.5
\end{tabular}


Table S870 Structural differences between the $2{ }^{1} A_{2}\left({ }^{1} \pi \pi^{*}\right)$ state and the ground state of acetylene.

\begin{tabular}{ll} 
bond & length / $\AA$ \\
or angle & or angle / o \\
\hline $\mathrm{C} 1-\mathrm{C} 2$ & +0.100 \\
$\mathrm{C} 1-\mathrm{C} 2-\mathrm{H} 3$ & +0.0
\end{tabular}


Table S871 Structural differences between the $2{ }^{3} A_{2}\left({ }^{3} \pi \pi^{*}\right)$ state and the ground state of acetylene.

\begin{tabular}{ll} 
bond & length / $\AA$ \\
or angle & or angle / o \\
\hline $\mathrm{C} 1-\mathrm{C} 2$ & +0.103 \\
$\mathrm{C} 1-\mathrm{C} 2-\mathrm{H} 3$ & +0.0
\end{tabular}


Table S872 Structural differences between the $1{ }^{1} A_{u}\left({ }^{1} \pi \pi^{*}\right)$ state and the ground state of acetylene.

\begin{tabular}{ll} 
bond & $\begin{array}{l}\text { length / } \AA \\
\text { or angle } \\
\text { or angle / o }\end{array}$ \\
\hline $\mathrm{C} 1-\mathrm{C} 2$ & +0.139 \\
$\mathrm{C} 1-\mathrm{H} 4$ & +0.069 \\
$\mathrm{C} 1-\mathrm{C} 2-\mathrm{H} 3$ & -55.0
\end{tabular}


Table S873 Structural differences between the $1{ }^{1} B_{u}\left({ }^{1} \pi \pi^{*}\right)$ state and the ground state of acetylene.

\begin{tabular}{|c|c|}
\hline $\begin{array}{l}\text { bond } \\
\text { or angle }\end{array}$ & $\begin{array}{l}\text { length / } \AA \\
\text { or angle / o }\end{array}$ \\
\hline $\mathrm{C} 1-\mathrm{C} 2$ & +0.105 \\
\hline $\mathrm{C} 1-\mathrm{C} 2-\mathrm{H} 3$ & -36.8 \\
\hline
\end{tabular}


Table S874 Structural differences between the $2{ }^{1} A_{u}\left({ }^{1} \pi \pi^{*}\right)$ state and the ground state of acetylene.

$$
\begin{array}{ll}
\text { bond } & \begin{array}{l}
\text { length / } \AA \\
\text { or angle } \\
\text { or angle / o }
\end{array} \\
\hline \text { C1-C2 } & +0.096
\end{array}
$$


Table S875 Structural differences between the $1{ }^{3} B_{u}\left({ }^{3} \pi \pi^{*}\right)$ state and the ground state of acetylene.

\begin{tabular}{ll} 
bond & length / $\AA$ \\
or angle & or angle / o \\
\hline $\mathrm{C} 1-\mathrm{C} 2$ & +0.116 \\
$\mathrm{C} 1-\mathrm{C} 2-\mathrm{H} 3$ & -41.7
\end{tabular}


Table S876 Structural differences between the $1{ }^{3} A_{u}\left({ }^{3} \pi \pi^{*}\right)$ state and the ground state of acetylene.

\begin{tabular}{ll} 
bond & length / $\AA$ \\
or angle & or angle / o \\
\hline $\mathrm{C} 1-\mathrm{C} 2$ & +0.148 \\
$\mathrm{C} 1-\mathrm{H} 4$ & +0.067 \\
$\mathrm{C} 1-\mathrm{C} 2-\mathrm{H} 3$ & -56.3
\end{tabular}


Table S877 Structural differences between the $2{ }^{3} B_{u}\left({ }^{3} \pi \pi^{*}\right)$ state and the ground state of acetylene.

\begin{tabular}{ll} 
bond & $\begin{array}{l}\text { length / } \AA \\
\text { or angle }\end{array}$ \\
or angle / o \\
\hline C1-C2 & +0.119 \\
C1-C2-H3 & -29.5
\end{tabular}


Table S878 Structural differences between the $2{ }^{3} A_{u}\left({ }^{3} \pi \pi^{*}\right)$ state and the ground state of acetylene.

$\begin{array}{ll}\text { bond } & \begin{array}{l}\text { length / } \AA \\ \text { or angle }\end{array} \\ \text { or angle / o } \\ \text { C1-C2 } & +0.106 \\ \mathrm{C} 1-\mathrm{C} 2-\mathrm{H} 3 & -16.2\end{array}$


Table S879 Structural differences between the $1{ }^{1} A^{\prime \prime}\left({ }^{1} n \pi^{*}\right)$ state and the ground state of acrolein.

\begin{tabular}{|c|c|}
\hline $\begin{array}{l}\text { bond } \\
\text { or angle }\end{array}$ & $\begin{array}{l}\text { length / } \AA \\
\text { or angle / }\end{array}$ \\
\hline C3-04 & +0.072 \\
\hline $\mathrm{C} 2-\mathrm{C} 3$ & -0.084 \\
\hline $\mathrm{C} 1-\mathrm{C} 2$ & +0.051 \\
\hline $\mathrm{O} 4-\mathrm{C} 3-\mathrm{C} 2$ & +2.1 \\
\hline
\end{tabular}


Table S880 Structural differences between the $1{ }^{3} A^{\prime \prime}\left({ }^{3} n \pi^{*}\right)$ state and the ground state of acrolein.

\begin{tabular}{ll}
$\begin{array}{l}\text { bond } \\
\text { or angle }\end{array}$ & or angle / o \\
\hline C3-O4 & +0.064 \\
C2-C3 & -0.074 \\
C1-C2 & +0.042
\end{tabular}


Table S881 Structural differences between the $2{ }^{1} A\left({ }^{1} \pi \pi^{*}\right)$ state and the ground state of 2-amino-9-methylpurine.

\begin{tabular}{ll} 
bond & $\begin{array}{l}\text { length / } \AA \\
\text { or angle } \\
\text { or angle / o }\end{array}$ \\
\hline C1-N2 & -0.029 \\
C5-C6 & +0.033 \\
N2-C6 & +0.013 \\
C5-N9 & -0.042 \\
C8-N9 & +0.031 \\
N7-C8 & -0.010
\end{tabular}


Table S882 Structural differences between the $1{ }^{1} B_{2}\left({ }^{1} \pi \pi^{*}\right)$ state and the ground state of aniline.

\begin{tabular}{ll} 
bond & $\begin{array}{l}\text { length / } \AA \\
\text { or angle } \\
\text { or angle / o }\end{array}$ \\
\hline C2-C3 2 +0.028 & \\
C2-C1-C6 & +1.3
\end{tabular}


Table S883 Structural differences between the $1{ }^{1} A^{\prime \prime}\left({ }^{1} n \pi^{*}\right)$ state and the ground state of benzaldehyde.

\begin{tabular}{|c|c|}
\hline $\begin{array}{l}\text { bond } \\
\text { or angle }\end{array}$ & $\begin{array}{l}\text { length / } \AA \\
\text { or angle / }\end{array}$ \\
\hline $\mathrm{C} 4-\mathrm{C} 6$ & +0.021 \\
\hline $\mathrm{C} 4-\mathrm{C} 5$ & +0.032 \\
\hline $\mathrm{C} 1-\mathrm{C} 4$ & -0.082 \\
\hline $\mathrm{C} 1-\mathrm{O} 2$ & +0.065 \\
\hline
\end{tabular}


Table S884 Structural differences between the $2{ }^{1} A^{\prime}\left({ }^{1} \pi \pi^{*}\right)$ state and the ground state of benzaldehyde.

\begin{tabular}{ll} 
bond & length / $\AA$ \\
or angle & or angle / o \\
\hline C6-C8 & +0.028 \\
C5-C7 & +0.031 \\
C1-O2 & +0.013
\end{tabular}


Table S885 Structural differences between the $1{ }^{3} A^{\prime \prime}\left({ }^{3} n \pi^{*}\right)$ state and the ground state of benzaldehyde.

\begin{tabular}{|c|c|}
\hline $\begin{array}{l}\text { bond } \\
\text { or angle }\end{array}$ & $\begin{array}{l}\text { length / } \AA \\
\text { or angle / }\end{array}$ \\
\hline $\mathrm{C} 1-\mathrm{C} 4$ & -0.076 \\
\hline $\mathrm{C} 1-\mathrm{O} 2$ & +0.059 \\
\hline
\end{tabular}


Table S886 Structural differences between the $1{ }^{3} A^{\prime}\left({ }^{3} \pi \pi^{*}\right)$ state and the ground state of benzaldehyde.

\begin{tabular}{ll} 
bond & $\begin{array}{l}\text { length / } \AA \\
\text { or angle } \\
\text { or angle / o }\end{array}$ \\
\hline C4-C6 & +0.063 \\
C7-C9 & +0.038 \\
C5-C7 & -0.022 \\
C $4-$ C5 & +0.062 \\
C1-C4 & -0.050 \\
C1-O2 & +0.027
\end{tabular}


Table S887 Structural differences between the $1{ }^{1} A_{u}\left({ }^{1} n \pi^{*}\right)$ state and the ground state of $p$-benzoquinone.

\begin{tabular}{ll} 
bond & $\begin{array}{l}\text { length / } \AA \\
\text { or angle } \\
\text { or angle / o }\end{array}$ \\
\hline $\mathrm{C} 6-\mathrm{C} 1-\mathrm{C} 2$ & +3.3
\end{tabular}


Table S888 Structural differences between the $1{ }^{1} B_{1 g}\left({ }^{1} n \pi^{*}\right)$ state and the ground state of $p$-benzoquinone.

$$
\begin{array}{ll}
\text { bond } & \begin{array}{l}
\text { length / } \AA \\
\text { or angle } \\
\text { or angle / }
\end{array} \\
\hline \text { C6-C1-C2 } & -1.7
\end{array}
$$


Table S889 Structural differences between the $1{ }^{1} B_{1 u}\left({ }^{1} \pi \pi^{*}\right)$ state and the ground state of $p$-benzoquinone.

\begin{tabular}{|c|c|}
\hline $\begin{array}{l}\text { bond } \\
\text { or angle }\end{array}$ & $\begin{array}{l}\text { length / } \AA \\
\text { or angle / o }\end{array}$ \\
\hline $\mathrm{C} 2-\mathrm{C} 3$ & +0.041 \\
\hline $\mathrm{C} 6-\mathrm{C} 1-\mathrm{C} 2$ & +1.3 \\
\hline
\end{tabular}


Table S890 Structural differences between the $1{ }^{1} B_{3 g}\left({ }^{1} \pi \pi^{*}\right)$ state and the ground state of $p$-benzoquinone.

\begin{tabular}{ll} 
bond & $\begin{array}{l}\text { length / } \AA \\
\text { or angle } \\
\text { or angle / o }\end{array}$ \\
\hline C2-C3 & +0.068
\end{tabular}


Table S891 Structural differences between the $1{ }^{3} A_{u}\left({ }^{3} n \pi^{*}\right)$ state and the ground state of $p$-benzoquinone.

\begin{tabular}{ll} 
bond & $\begin{array}{l}\text { length / } \AA \\
\text { or angle } \\
\text { or angle / o }\end{array}$ \\
\hline C $6-\mathrm{C} 1-\mathrm{C} 2$ & +3.0
\end{tabular}


Table S892 Structural differences between the $1{ }^{3} B_{1 g}\left({ }^{3} n \pi^{*}\right)$ state and the ground state of $p$-benzoquinone.

$$
\begin{array}{ll}
\text { bond } & \text { length / } \\
\text { or angle } & \text { or angle / }
\end{array}
$$


Table S893 Structural differences between the $1{ }^{3} B_{3 g}\left({ }^{3} \pi \pi^{*}\right)$ state and the ground state of $p$-benzoquinone.

\begin{tabular}{ll} 
bond & $\begin{array}{l}\text { length / } \AA \\
\text { or angle } \\
\text { or angle / o }\end{array}$ \\
\hline C2-C3 & +0.074
\end{tabular}


Table S894 Structural differences between the $1{ }^{1} B_{3 g}\left({ }^{1} \pi \pi^{*}\right)$ state and the ground state of biphenylene.

\begin{tabular}{ll} 
bond & $\begin{array}{l}\text { length / } \AA \\
\text { or angle } \\
\text { or angle / o }\end{array}$ \\
\hline C1-C2 & +0.033 \\
C2-C3 & -0.029 \\
C3-C12 & +0.040 \\
C1-C6 & +0.054 \\
C1-C4 & -0.080
\end{tabular}


Table S895 Structural differences between the $1{ }^{1} B_{1 u}\left({ }^{1} \pi \pi^{*}\right)$ state and the ground state of biphenylene.

\begin{tabular}{ll}
$\begin{array}{l}\text { bond } \\
\text { or angle }\end{array}$ & $\begin{array}{l}\text { ongth } / \AA \\
\text { C1-C4 } 4\end{array}$ \\
\hline-0.040
\end{tabular}


Table S896 Structural differences between the $2{ }^{1} B_{1 u}\left({ }^{1} \pi \pi^{*}\right)$ state and the ground state of biphenylene.

\begin{tabular}{ll} 
bond & $\begin{array}{l}\text { length / } \AA \\
\text { or angle }\end{array}$ \\
\hline Or angle / 4 & -0.036
\end{tabular}


Table S897 Structural differences between the $1{ }^{1} B_{2 u}\left({ }^{1} \pi \pi^{*}\right)$ state and the ground state of biphenylene.

\begin{tabular}{ll} 
bond & length / $\AA$ \\
or angle & or angle / o \\
\hline C3-C12 & +0.059 \\
C1-C6 & +0.053
\end{tabular}


Table S898 Structural differences between the $1{ }^{1} B_{u}\left({ }^{1} \pi \pi^{*}\right)$ state and the ground state of trans-butadiene.

\begin{tabular}{|c|c|}
\hline $\begin{array}{l}\text { bond } \\
\text { or angle }\end{array}$ & $\begin{array}{l}\text { length / } \AA \\
\text { or angle / }\end{array}$ \\
\hline $\mathrm{C} 1-\mathrm{C} 2$ & +0.061 \\
\hline $\mathrm{C} 2-\mathrm{C} 3$ & -0.059 \\
\hline
\end{tabular}


Table S899 Structural differences between the $2{ }^{1} A_{g}\left({ }^{1} \pi \pi^{*}\right)$ state and the ground state of trans-butadiene.

$$
\begin{array}{ll}
\text { bond } & \begin{array}{l}
\text { length / } \AA \\
\text { or angle }
\end{array} \\
\hline \text { or angle / } 22 & +0.127
\end{array}
$$


Table S900 Structural differences between the $2{ }^{1} A\left({ }^{1} n \pi^{*}\right)$ state and the ground state of cytosine.

$\begin{array}{ll}\text { bond } & \begin{array}{l}\text { length / } \AA \\ \text { or angle }\end{array} \\ \text { or angle / }\end{array}$


Table S901 Structural differences between the $3{ }^{1} A\left({ }^{1} \pi \pi^{*}\right)$ state and the ground state of cytosine.

\begin{tabular}{ll} 
bond & $\begin{array}{l}\text { length / } \AA \\
\text { or angle }\end{array}$ \\
or angle / o \\
\hline N5-C6 & -0.019 \\
N1-C6 & -0.031 \\
N1-C2 & +0.066 \\
C2-C3 & -0.043 \\
C3-C4 & +0.053 \\
C4-N5 & +0.010 \\
C6-O8 & +0.028 \\
N1-C6-N5 & +5.3 \\
N1-C2-N7 & -2.4 \\
N5-C6-O8 & -2.9
\end{tabular}


Table S902 Structural differences between the $1{ }^{1} A_{2}\left({ }^{1} n \pi^{*}\right)$ state and the ground state of diazomethane.

\begin{tabular}{ll} 
bond & length / \\
or angle & or angle / o \\
\hline N2-N3 & +0.032 \\
H4-C1-H5 & +1.9
\end{tabular}


Table S903 Structural differences between the $1{ }^{1} B_{3 g}\left({ }^{1} \pi \pi^{*}\right)$ state and the ground state of dibenzo- $p$-dioxin.

\begin{tabular}{|c|c|}
\hline $\begin{array}{l}\text { bond } \\
\text { or angle }\end{array}$ & $\begin{array}{l}\text { length / } \AA \\
\text { or angle / }\end{array}$ \\
\hline $\mathrm{C} 4-\mathrm{C} 5$ & +0.035 \\
\hline $\mathrm{C} 1-\mathrm{C} 2$ & +0.031 \\
\hline
\end{tabular}


Table S904 Structural differences between the $1{ }^{1} B_{1 u}\left({ }^{1} \pi \pi^{*}\right)$ state and the ground state of dibenzo- $p$-dioxin.

\begin{tabular}{ll} 
bond & length / $\AA$ \\
or angle & or angle / o \\
\hline C4-C5 & +0.019 \\
C1-C2 & +0.025
\end{tabular}


Table S905 Structural differences between the $1{ }^{3} B_{3 g}\left({ }^{3} \pi \pi^{*}\right)$ state and the ground state of dibenzo- $p$-dioxin.

\begin{tabular}{ll} 
bond & length / $\AA$ \\
or angle & or angle / o \\
\hline C4-C5 & +0.038 \\
C1-C2 & +0.038
\end{tabular}


Table S906 Structural differences between the $1{ }^{3} B_{1 u}\left({ }^{3} \pi \pi^{*}\right)$ state and the ground state of dibenzo- $p$-dioxin.

\begin{tabular}{ll} 
bond & length / $\AA$ \\
or angle & or angle / o \\
\hline C4-C5 & +0.037 \\
C1-C2 & +0.038
\end{tabular}


Table S907 Structural differences between the $1{ }^{1} A_{u}\left({ }^{1} n \pi^{*}\right)$ state and the ground state of dibenzo- $p$-dioxin.

\begin{tabular}{ll} 
bond & length / \\
or angle & or angle / o \\
\hline C4-C7 & -0.022 \\
C4-C5 & +0.048 \\
C4-O7-C8 & +8.4
\end{tabular}


Table S908 Structural differences between the $1{ }^{1} B_{1 g}\left({ }^{1} n \pi^{*}\right)$ state and the ground state of dibenzo- $p$-dioxin.

$$
\begin{array}{ll}
\text { bond } & \begin{array}{l}
\text { length / } \AA \\
\text { or angle } \\
\text { or angle / }
\end{array} \\
\hline \text { C4-07-C8 }+8.7
\end{array}
$$


Table S909 Structural differences between the $1{ }^{1} B_{3 u}\left({ }^{1} n \pi^{*}\right)$ state and the ground state of dibenzo- $p$-dioxin.

$$
\begin{array}{ll}
\text { bond } & \begin{array}{l}
\text { length / } \AA \\
\text { or angle } \\
\text { or angle / o }
\end{array} \\
\hline \text { C4-07-C8 }+7.3
\end{array}
$$


Table S910 Structural differences between the $1{ }^{3} A_{u}\left({ }^{3} n \pi^{*}\right)$ state and the ground state of dibenzo- $p$-dioxin.

\begin{tabular}{ll} 
bond & length / $\AA$ \\
or angle & or angle / o \\
\hline C4-C5 & +0.049 \\
C4-07-C 8 & +8.4
\end{tabular}


Table S911 Structural differences between the $1{ }^{3} B_{3 u}\left({ }^{3} n \pi^{*}\right)$ state and the ground state of dibenzo- $p$-dioxin.

$$
\begin{aligned}
& \text { bond length / } \AA \\
& \text { or angle or angle / }{ }^{\circ}
\end{aligned}
$$


Table S912 Structural differences between the $1{ }^{3} B_{2 g}\left({ }^{3} n \pi^{*}\right)$ state and the ground state of dibenzo- $p$-dioxin.

\begin{tabular}{ll} 
bond & length / \\
or angle & or angle / o \\
\hline C4-C5 & +0.042 \\
C1-C2 & +0.019 \\
C4-O7-C8 & +7.0
\end{tabular}


Table S913 Structural differences between the $1{ }^{1} A^{\prime \prime}\left({ }^{1} n \pi^{*}\right)$ state and the ground state of betweenmaldehyde.

$$
\begin{array}{ll}
\text { bond } & \begin{array}{l}
\text { length / } \AA \\
\text { or angle } \\
\text { or angle / o }
\end{array} \\
\hline \mathrm{C}-\mathrm{O} & +0.063
\end{array}
$$


Table S914 Structural differences between the $1{ }^{3} A^{\prime \prime}\left({ }^{3} n \pi^{*}\right)$ state and the ground state of betweenmaldehyde.

$$
\begin{aligned}
& \text { bond length / } \AA \\
& \begin{array}{ll}
\text { or angle or angle / } \\
\hline \mathrm{C}-\mathrm{O} \\
+0.045
\end{array}
\end{aligned}
$$


Table S915 Structural differences between the $1{ }^{1} A_{u}\left({ }^{1} n \pi^{*}\right)$ state and the ground state of trans-glyoxal.

\begin{tabular}{ll} 
bond & $\begin{array}{l}\text { length / } \AA \\
\text { or angle }\end{array}$ \\
\hline or angle / 22 & -0.045
\end{tabular}


Table S916 Structural differences between the $1{ }^{3} A_{u}\left({ }^{3} n \pi^{*}\right)$ state and the ground state of trans-glyoxal.

\begin{tabular}{ll} 
bond & length / $\AA$ \\
or angle & or angle / o \\
\hline C1-C2 & -0.042 \\
C1-03 & +0.019
\end{tabular}


Table S917 Structural differences between the $2{ }^{1} A\left({ }^{1} \pi \pi^{*}\right)$ state and the ground state of $9 H$-guanine.

\begin{tabular}{|c|c|}
\hline $\begin{array}{l}\text { bond } \\
\text { or angle }\end{array}$ & $\begin{array}{l}\text { length / } \AA \\
\text { or angle / o }\end{array}$ \\
\hline $\mathrm{N} 7-\mathrm{C} 9$ & +0.008 \\
\hline C9-N13 & +0.118 \\
\hline $\mathrm{N} 13-\mathrm{C} 14$ & -0.054 \\
\hline C $4-C 14$ & +0.053 \\
\hline $\mathrm{C} 4-\mathrm{C} 5$ & +0.018 \\
\hline
\end{tabular}


Table S918 Structural differences between the $2{ }^{1} A^{\prime}\left({ }^{1} \pi \pi^{*}\right)$ state and the ground state of 1-hydroxy-2-acetonaphthone.

\begin{tabular}{ll} 
bond & length / $\AA$ \\
or angle & or angle / o \\
\hline C1-C6 6 & +0.062 \\
C4-C5 & +0.047 \\
C6-C11 & -0.001 \\
C1-013 & -0.066 \\
C11-014 & 0.066
\end{tabular}


Table S919 Structural differences between the $2{ }^{1} A^{\prime}\left({ }^{1} \pi \pi^{*}\right)$ state and the ground state of indole.

\begin{tabular}{ll} 
bond & length / $\AA$ \\
or angle & or angle / o \\
\hline N3-C5 & +0.001 \\
C4-C5 & +0.023 \\
C8-C9 & +0.028 \\
C2-N3 & -0.018
\end{tabular}


Table S920 Structural differences between the $3{ }^{1} A^{\prime}\left({ }^{1} \pi \pi^{*}\right)$ state and the ground state of indole.

\begin{tabular}{|c|c|}
\hline $\begin{array}{l}\text { bond } \\
\text { or angle }\end{array}$ & $\begin{array}{l}\text { length / } \AA \\
\text { or angle / }\end{array}$ \\
\hline $\mathrm{C} 6-\mathrm{C} 7$ & +0.025 \\
\hline $\mathrm{C} 1-\mathrm{C} 2$ & +0.018 \\
\hline
\end{tabular}


Table S921 Structural differences between the $1{ }^{1} B_{1}\left({ }^{1} n \pi^{*}\right)$ state and the ground state of maleimide.

\begin{tabular}{ll} 
bond & length / \\
or angle & or angle / \\
\hline $\mathrm{C} 2-\mathrm{C} 3$ & -0.055 \\
$\mathrm{C} 3-\mathrm{C} 4$ & +0.034 \\
$\mathrm{C} 2-\mathrm{O} 6$ & +0.021 \\
$\mathrm{~N} 1-\mathrm{C} 2-\mathrm{O} 6$ & -5.8 \\
$\mathrm{O} 6-\mathrm{C} 2-\mathrm{C} 3$ & +5.5
\end{tabular}


Table S922 Structural differences between the $1{ }^{1} A_{2}\left({ }^{1} n \pi^{*}\right)$ state and the ground state of maleimide.

\begin{tabular}{ll}
$\begin{array}{l}\text { bond } \\
\text { or angle }\end{array}$ & or angle / \\
\hline C2-C3 & -0.064 \\
C3-C4 & +0.053 \\
C2-06 & +0.044
\end{tabular}


Table S923 Structural differences between the $1{ }^{1} B_{2}\left({ }^{1} \pi \pi^{*}\right)$ state and the ground state of maleimide.

\begin{tabular}{ll} 
bond & length / $\AA$ \\
or angle & or angle / o \\
\hline $\mathrm{N} 1-\mathrm{C} 2$ & +0.060 \\
$\mathrm{C} 2-\mathrm{C} 3$ & -0.085 \\
$\mathrm{C} 3-\mathrm{C} 4$ & +0.067 \\
$\mathrm{~N} 1-\mathrm{C} 2-\mathrm{O} 6$ & -7.4 \\
O6-C2-C3 & +9.1
\end{tabular}


Table S924 Structural differences between the $2{ }^{1} B_{2}\left({ }^{1} \pi \pi^{*}\right)$ state and the ground state of maleimide.

\begin{tabular}{ll} 
bond & length / \\
or angle & or angle / o \\
\hline C2-C3 & -0.058 \\
C3-C4 & +0.074 \\
C2-06 & +0.041
\end{tabular}


Table S925 Structural differences between the $1{ }^{3} B_{1}\left({ }^{3} n \pi^{*}\right)$ state and the ground state of maleimide.

\begin{tabular}{ll} 
bond & $\begin{array}{l}\text { length / } \AA \\
\text { or angle } \\
\text { or angle / }\end{array}$ \\
\hline $\mathrm{C} 2-\mathrm{C} 3$ & -0.053 \\
$\mathrm{C} 3-\mathrm{C} 4$ & +0.032 \\
$\mathrm{C} 2-\mathrm{O} 6$ & +0.021 \\
$\mathrm{~N} 1-\mathrm{C} 2-\mathrm{O} 6$ & -5.0 \\
$\mathrm{O} 6-\mathrm{C} 2-\mathrm{C} 3$ & +4.7
\end{tabular}


Table S926 Structural differences between the $1{ }^{3} A_{2}\left({ }^{3} n \pi^{*}\right)$ state and the ground state of maleimide.

\begin{tabular}{ll}
$\begin{array}{l}\text { bond } \\
\text { or angle }\end{array}$ & or angle / o \\
\hline C2-C3 & -0.061 \\
C3-C4 & +0.051 \\
C2-06 & +0.042
\end{tabular}


Table S927 Structural differences between the $1{ }^{3} B_{2}\left({ }^{3} \pi \pi^{*}\right)$ state and the ground state of maleimide.

\begin{tabular}{ll} 
bond & $\begin{array}{l}\text { length / } \AA \\
\text { or angle } \\
\text { or angle / }\end{array}$ \\
\hline C2-C3 & -0.054 \\
C3-C4 & +0.152 \\
C2-O6 & +0.016 \\
$\mathrm{~N} 1-\mathrm{C} 2-\mathrm{O} 6$ & -0.7 \\
O6-C2-C3 & -0.4
\end{tabular}


Table S928 Structural differences between the $1{ }^{1} A^{\prime \prime}\left({ }^{1} n \pi^{*}\right)$ state and the ground state of nitrosomethane.

\begin{tabular}{|c|c|}
\hline $\begin{array}{l}\text { bond } \\
\text { or angle }\end{array}$ & $\begin{array}{l}\text { length / } \AA \\
\text { or angle / o }\end{array}$ \\
\hline $\mathrm{N} 2-\mathrm{O} 3$ & -0.009 \\
\hline $\mathrm{C} 1-\mathrm{N} 2-\mathrm{O} 3$ & +17.9 \\
\hline
\end{tabular}


Table S929 Structural differences between the $1{ }^{1} A^{\prime \prime}\left({ }^{1} n \pi^{*}\right)$ state and the ground state of propenoic acid anion.

\begin{tabular}{ll} 
bond & length / $\AA$ \\
or angle & or angle / o \\
\hline $\mathrm{C} 2-\mathrm{O} 4$ & +0.074 \\
$\mathrm{C} 1-\mathrm{C} 2$ & -0.139 \\
$\mathrm{C} 1-\mathrm{C} 5$ & +0.052 \\
$\mathrm{O} 4-\mathrm{C} 2-\mathrm{C} 1$ & +0.6 \\
$\mathrm{O} 3-\mathrm{C} 2-\mathrm{O} 4$ & -14.2
\end{tabular}


Table S930 Structural differences between the $1{ }^{1} A_{2}\left({ }^{1} n \pi^{*}\right)$ state and the ground state of pyridine.

\begin{tabular}{ll} 
bond & length / $\AA$ \\
or angle & or angle / \\
\hline $\mathrm{N} 1-\mathrm{C} 2$ & -0.022 \\
$\mathrm{C} 2-\mathrm{C} 4$ & +0.056 \\
$\mathrm{C} 2-\mathrm{N} 1-\mathrm{C} 3$ & +15.7 \\
$\mathrm{~N} 1-\mathrm{C} 2-\mathrm{C} 4$ & -9.5 \\
$\mathrm{C} 4-\mathrm{C} 6-\mathrm{C} 5$ & +4.1
\end{tabular}


Table S931 Structural differences between the $2{ }^{1} A^{\prime}\left({ }^{1} n \pi^{*}\right)$ state and the ground state of pyridine.

\begin{tabular}{ll} 
bond & $\begin{array}{l}\text { length / } \AA \\
\text { or angle } \\
\text { or angle / }\end{array}$ \\
\hline $\mathrm{C} 4-\mathrm{C} 6$ & +0.026 \\
$\mathrm{C} 2-\mathrm{N} 1-\mathrm{C} 3$ & +13.1 \\
$\mathrm{~N} 1-\mathrm{C} 2-\mathrm{C} 4$ & -9.0
\end{tabular}


Table S932 Structural differences between the $1{ }^{3} A_{1}\left({ }^{3} \pi \pi^{*}\right)$ state and the ground state of pyridine.

$$
\begin{array}{ll}
\begin{array}{l}
\text { bond } \\
\text { or angle }
\end{array} & \begin{array}{l}
\text { or angle / } / \\
\text { C2-C4 }
\end{array} \\
\hline & +0.087
\end{array}
$$


Table S933 Structural differences between the $2{ }^{3} A_{1}\left({ }^{3} \pi \pi^{*}\right)$ state and the ground state of pyridine.

\begin{tabular}{ll} 
bond & length / \\
or angle & or angle / o \\
\hline N1-C2 & +0.030 \\
C4-C6 & +0.040
\end{tabular}


Table S934 Structural differences between the $2{ }^{1} A^{\prime}\left({ }^{1} \pi \pi^{*}\right)$ state and the ground state of pyrrole.

\begin{tabular}{ll} 
bond & length / \\
or angle & or angle / o \\
\hline N6-C7 & -0.002 \\
C7-C10 & +0.078 \\
C9-C10 & +0.020
\end{tabular}


Table S935 Structural differences between the $1{ }^{1} B_{2}\left({ }^{1} \pi \pi^{*}\right)$ state and the ground state of pyrrole.

\begin{tabular}{|c|c|}
\hline $\begin{array}{l}\text { bond } \\
\text { or angle }\end{array}$ & $\begin{array}{l}\text { length / } \AA \\
\text { or angle / o }\end{array}$ \\
\hline $\mathrm{N} 6-\mathrm{C} 7$ & +0.014 \\
\hline $\mathrm{C} 7-\mathrm{C} 10$ & +0.059 \\
\hline $\mathrm{C} 9-\mathrm{C} 10$ & -0.040 \\
\hline
\end{tabular}


Table S936 Structural differences between the $2{ }^{1} A^{\prime}\left({ }^{1} \pi \pi^{*}\right)$ state and the ground state of cis-PSB3.

\begin{tabular}{ll} 
bond & length / $\AA$ \\
or angle & or angle / o \\
\hline C2-C3 & -0.029 \\
C3-C4 & +0.051 \\
C4-C5 & +0.006 \\
C5-N6 & +0.031 \\
C2-C3-C4 & -2.9
\end{tabular}


Table S937 Structural differences between the $2{ }^{1} A^{\prime}\left({ }^{1} \pi \pi^{*}\right)$ state and the ground state of 3Me-cis-PSB5.

\begin{tabular}{ll} 
bond & length / $\AA$ \\
or angle & or angle / o \\
\hline C1-C8 & -0.062 \\
C1-C2 & +0.088 \\
C2-C3 & -0.051 \\
C3-C4 & +0.057 \\
C4-C5 & -0.009 \\
C5-N6 & +0.022
\end{tabular}


Table S938 Structural differences between the $1{ }^{1} B_{u}\left({ }^{1} \pi \pi^{*}\right)$ state and the ground state of trans-stilbene.

\begin{tabular}{ll} 
bond & length / $\AA$ \\
or angle & or angle / o \\
\hline C1-C8 & +0.058 \\
C1-C2 & -0.055 \\
C2-C7 & +0.028
\end{tabular}


Table S939 Structural differences between the $1{ }^{1} B\left({ }^{1} \pi \pi^{*}\right)$ state and the ground state of stilbene.

\begin{tabular}{ll} 
bond & length / $\AA$ \\
or angle & or angle / o \\
\hline C1-C8 & +0.037 \\
C1-C2 & -0.030 \\
C2-C7 & +0.011
\end{tabular}


Table S940 Structural differences between the $2{ }^{1} A^{\prime}\left({ }^{1} \pi \pi^{*}\right)$ state and the ground state of styrene.

\begin{tabular}{ll} 
bond & $\begin{array}{l}\text { length / } \AA \\
\text { or angle } \\
\text { or angle / }\end{array}$ \\
\hline C1-C2 & +0.023 \\
C1-C3 & -0.037 \\
C3-C5 & +0.033 \\
C6-C8 & +0.018
\end{tabular}


Table S941 Structural differences between the $1{ }^{1} B_{3 u}\left({ }^{1} n \pi^{*}\right)$ state and the ground state of $s$-tetrazine.

\begin{tabular}{|c|c|}
\hline bond & length / $\AA$ \\
\hline or angle & or angle / o \\
\hline N3-C1-N5 & -6.2 \\
\hline
\end{tabular}


Table S942 Structural differences between the $2{ }^{1} A\left({ }^{1} n \pi^{*}\right)$ state and the ground state of thymine.

\begin{tabular}{ll} 
bond & length / $\AA$ \\
or angle & or angle / o \\
\hline C3-C4 & -0.071 \\
C2-C3 & +0.043 \\
C4-O7 & +0.090
\end{tabular}


Table S943 Structural differences between the $2{ }^{1} A^{\prime}\left({ }^{1} \pi \pi^{*}\right)$ state and the ground state of uracil.

\begin{tabular}{ll} 
bond & $\begin{array}{l}\text { length / } \\
\text { or angle }\end{array}$ \\
or angle / \\
\hline $\mathrm{N} 1-\mathrm{C} 3$ & -0.022 \\
$\mathrm{~N} 2-\mathrm{C} 3$ & +0.029 \\
$\mathrm{~N} 2-\mathrm{C} 4$ & -0.037 \\
$\mathrm{C} 4-\mathrm{C} 5$ & +0.117 \\
$\mathrm{C} 5-\mathrm{C} 6$ & -0.061 \\
$\mathrm{~N} 1-\mathrm{C} 6$ & +0.031 \\
$\mathrm{C} 3-\mathrm{O} 8$ & +0.006
\end{tabular}




\subsubsection{DFT-TDDFT Results on Significant Changes Between Ground- State and Excited-State Minima}

Given are the differences in bond lengths (in $\AA$ ) and in bond angles (in ${ }^{\circ}$ ) (which were determined to change $\geq|0.03 \AA|$ or $\geq\left|5^{\circ}\right|$ at the (TD)DFT level) between the TDDFT-optimized excited-state equilibrium geometry and the DFToptimized ground-state equilibrium geometry for each excited state of the 32molecules benchmark set. ( 5 of the 15 excited states of dibenzo- $p$-dioxin were disregarded in this analysis, as the structural changes occurring in these states are smaller than the thresholds mentioned above.) 
Table S944 Structural differences between the $2{ }^{1} A\left({ }^{1} n \pi^{*}\right)$ state and the ground state of $9 \mathrm{H}$-adenine.

\begin{tabular}{ll} 
bond & length / $\AA$ \\
or angle & or angle / \\
\hline N1-C2 & -0.047 \\
C2-C9 & +0.041 \\
N4-C5 & +0.033 \\
N6-C7 & +0.051 \\
C9-N10 & +0.031
\end{tabular}


Table S945 Structural differences between the $2{ }^{1} A\left({ }^{1} n \pi^{*}\right)$ state and the ground state of acetaldehyde.

\begin{tabular}{|c|c|}
\hline $\begin{array}{l}\text { bond } \\
\text { or angle }\end{array}$ & $\begin{array}{l}\text { length / } \AA \\
\text { or angle / }\end{array}$ \\
\hline $\mathrm{C} 1-04$ & +0.099 \\
\hline $\mathrm{O} 4-\mathrm{C} 1-\mathrm{C} 2$ & -7.5 \\
\hline
\end{tabular}


Table S946 Structural differences between the $1{ }^{3} A\left({ }^{3} n \pi^{*}\right)$ state and the ground state of acetaldehyde.

\begin{tabular}{|c|c|}
\hline $\begin{array}{l}\text { bond } \\
\text { or angle }\end{array}$ & $\begin{array}{l}\text { length / } \AA \\
\text { or angle / o }\end{array}$ \\
\hline $\mathrm{C} 1-\mathrm{O} 4$ & +0.100 \\
\hline $\mathrm{O} 4-\mathrm{C} 1-\mathrm{C} 2$ & -8.7 \\
\hline
\end{tabular}


Table S947 Structural differences between the $1{ }^{1} A^{\prime \prime}\left({ }^{1} n \pi^{*}\right)$ state and the ground state of acetone.

\begin{tabular}{ll} 
bond & $\begin{array}{l}\text { length / } \AA \\
\text { or angle } \\
\text { or angle / o }\end{array}$ \\
\hline C2-03 & +0.099
\end{tabular}


Table S948 Structural differences between the $1{ }^{3} A^{\prime \prime}\left({ }^{3} n \pi^{*}\right)$ state and the ground state of acetone.

$$
\begin{array}{ll}
\text { bond } & \begin{array}{l}
\text { length / } \AA \\
\text { or angle }
\end{array} \\
\hline \text { or angle / } 2-03 & +0.101
\end{array}
$$


Table S949 Structural differences between the $1{ }^{1} A^{\prime \prime}\left({ }^{1} n \pi^{*}\right)$ state and the ground state of acetophenone.

\begin{tabular}{|c|c|}
\hline $\begin{array}{l}\text { bond } \\
\text { or angle }\end{array}$ & $\begin{array}{l}\text { length / } \AA \\
\text { or angle / o }\end{array}$ \\
\hline $\mathrm{C} 7-\mathrm{C} 9$ & +0.033 \\
\hline $\mathrm{C} 7-\mathrm{C} 8$ & +0.032 \\
\hline $\mathrm{C} 1-\mathrm{C} 7$ & -0.103 \\
\hline $\mathrm{C} 1-\mathrm{O} 2$ & +0.081 \\
\hline
\end{tabular}


Table S950 Structural differences between the $2{ }^{1} A^{\prime}\left({ }^{1} \pi \pi^{*}\right)$ state and the ground state of acetophenone.

\begin{tabular}{|c|c|}
\hline $\begin{array}{l}\text { bond } \\
\text { or angle }\end{array}$ & $\begin{array}{l}\text { length / } \AA \\
\text { or angle / o }\end{array}$ \\
\hline C9-C11 & +0.041 \\
\hline $\mathrm{C} 8-\mathrm{C} 10$ & +0.046 \\
\hline $\mathrm{C} 1-\mathrm{C} 7$ & -0.039 \\
\hline $\mathrm{C} 1-\mathrm{O} 2$ & +0.040 \\
\hline
\end{tabular}


Table S951 Structural differences between the $1{ }^{3} A^{\prime \prime}\left({ }^{3} n \pi^{*}\right)$ state and the ground state of acetophenone.

\begin{tabular}{ll} 
bond & length / $\AA$ \\
or angle & or angle / o \\
\hline C1-C7 & -0.095 \\
C1-02 & +0.093
\end{tabular}


Table S952 Structural differences between the $1{ }^{3} A^{\prime}\left({ }^{3} \pi \pi^{*}\right)$ state and the ground state of acetophenone.

\begin{tabular}{|c|c|}
\hline bond & length / $\AA$ \\
\hline or angle & or angle / ${ }^{\circ}$ \\
\hline $\mathrm{C} 7-\mathrm{C} 9$ & +0.087 \\
\hline $\mathrm{C} 11-\mathrm{C} 12$ & +0.031 \\
\hline $\mathrm{C} 10-\mathrm{C} 12$ & +0.058 \\
\hline $\mathrm{C} 8-\mathrm{C} 10$ & -0.036 \\
\hline $\mathrm{C} 7-\mathrm{C} 8$ & +0.069 \\
\hline $\mathrm{C} 1-\mathrm{C} 7$ & -0.088 \\
\hline $\mathrm{C} 1-\mathrm{O} 2$ & +0.064 \\
\hline
\end{tabular}


Table S953 Structural differences between the $1{ }^{1} A_{2}\left({ }^{1} \pi \pi^{*}\right)$ state and the ground state of acetylene.

\begin{tabular}{ll} 
bond & length / \\
or angle & or angle / o \\
\hline $\mathrm{C} 1-\mathrm{C} 2$ & +0.141 \\
$\mathrm{C} 1-\mathrm{H} 4$ & +0.034 \\
$\mathrm{C} 1-\mathrm{C} 2-\mathrm{H} 3$ & -47.7
\end{tabular}


Table S954 Structural differences between the $1{ }^{1} B_{2}\left({ }^{1} \pi \pi^{*}\right)$ state and the ground state of acetylene.

\begin{tabular}{ll} 
bond & $\begin{array}{l}\text { length / } \AA \\
\text { or angle } \\
\text { or angle / o }\end{array}$ \\
\hline $\mathrm{C} 1-\mathrm{C} 2$ & +0.130 \\
$\mathrm{C} 1-\mathrm{H} 4$ & +0.030 \\
$\mathrm{C} 1-\mathrm{C} 2-\mathrm{H} 3$ & -36.3
\end{tabular}


Table S955 Structural differences between the $2{ }^{1} A_{2}\left({ }^{1} \pi \pi^{*}\right)$ state and the ground state of acetylene.

\begin{tabular}{ll} 
bond & length / $\AA$ \\
or angle & or angle / o \\
\hline $\mathrm{C} 1-\mathrm{C} 2$ & +0.125 \\
$\mathrm{C} 1-\mathrm{C} 2-\mathrm{H} 3$ & -5.7
\end{tabular}


Table S956 Structural differences between the $2{ }^{3} A_{2}\left({ }^{3} \pi \pi^{*}\right)$ state and the ground state of acetylene.

\begin{tabular}{ll} 
bond & length / $\AA$ \\
or angle & or angle / o \\
\hline $\mathrm{C} 1-\mathrm{C} 2$ & +0.132 \\
$\mathrm{C} 1-\mathrm{C} 2-\mathrm{H} 3$ & -14.0
\end{tabular}


Table S957 Structural differences between the $1{ }^{1} A_{u}\left({ }^{1} \pi \pi^{*}\right)$ state and the ground state of acetylene.

\begin{tabular}{ll} 
bond & length / $\AA$ \\
or angle & or angle / o \\
\hline $\mathrm{C} 1-\mathrm{C} 2$ & +0.168 \\
$\mathrm{C} 1-\mathrm{H} 4$ & +0.033 \\
$\mathrm{C} 1-\mathrm{C} 2-\mathrm{H} 3$ & -57.4
\end{tabular}


Table S958 Structural differences between the $1{ }^{1} B_{u}\left({ }^{1} \pi \pi^{*}\right)$ state and the ground state of acetylene.

\begin{tabular}{|c|c|}
\hline $\begin{array}{l}\text { bond } \\
\text { or angle }\end{array}$ & $\begin{array}{l}\text { length / } \AA \\
\text { or angle / }\end{array}$ \\
\hline $\mathrm{C} 1-\mathrm{C} 2$ & +0.132 \\
\hline $\mathrm{C} 1-\mathrm{C} 2-\mathrm{H} 3$ & -29.8 \\
\hline
\end{tabular}


Table S959 Structural differences between the $2{ }^{1} A_{u}\left({ }^{1} \pi \pi^{*}\right)$ state and the ground state of acetylene.

$$
\begin{array}{ll}
\text { bond } & \begin{array}{l}
\text { length / } \AA \\
\text { or angle }
\end{array} \\
\hline \text { or angle / } 22 & +0.125
\end{array}
$$


Table S960 Structural differences between the $1{ }^{3} B_{u}\left({ }^{3} \pi \pi^{*}\right)$ state and the ground state of acetylene.

\begin{tabular}{ll} 
bond & length / $\AA$ \\
or angle & or angle / o \\
\hline $\mathrm{C} 1-\mathrm{C} 2$ & +0.177 \\
$\mathrm{C} 1-\mathrm{C} 2-\mathrm{H} 3$ & -50.6
\end{tabular}


Table S961 Structural differences between the $1{ }^{3} A_{u}\left({ }^{3} \pi \pi^{*}\right)$ state and the ground state of acetylene.

\begin{tabular}{ll} 
bond & length / $\AA$ \\
or angle & or angle / o \\
\hline $\mathrm{C} 1-\mathrm{C} 2$ & +0.224 \\
$\mathrm{C} 1-\mathrm{H} 4$ & +0.033 \\
$\mathrm{C} 1-\mathrm{C} 2-\mathrm{H} 3$ & -63.1
\end{tabular}


Table S962 Structural differences between the $2{ }^{3} B_{u}\left({ }^{3} \pi \pi^{*}\right)$ state and the ground state of acetylene.

\begin{tabular}{|c|c|}
\hline $\begin{array}{l}\text { bond } \\
\text { or angle }\end{array}$ & $\begin{array}{l}\text { length / } \AA \\
\text { or angle / o }\end{array}$ \\
\hline $\mathrm{C} 1-\mathrm{C} 2$ & +0.161 \\
\hline $\mathrm{C} 1-\mathrm{C} 2-\mathrm{H} 3$ & -23.5 \\
\hline
\end{tabular}


Table S963 Structural differences between the $2{ }^{3} A_{u}\left({ }^{3} \pi \pi^{*}\right)$ state and the ground state of acetylene.

\begin{tabular}{|c|c|}
\hline $\begin{array}{l}\text { bond } \\
\text { or angle }\end{array}$ & $\begin{array}{l}\text { length / } \AA \\
\text { or angle / o }\end{array}$ \\
\hline $\mathrm{C} 1-\mathrm{C} 2$ & +0.134 \\
\hline $\mathrm{C} 1-\mathrm{C} 2-\mathrm{H} 3$ & -17.1 \\
\hline
\end{tabular}


Table S964 Structural differences between the $2{ }^{1} A\left({ }^{1} n \pi^{*}\right)$ state and the ground state of acrolein.

\begin{tabular}{|c|c|}
\hline $\begin{array}{l}\text { bond } \\
\text { or angle }\end{array}$ & $\begin{array}{l}\text { length / } \AA \\
\text { or angle / }\end{array}$ \\
\hline $\mathrm{C} 3-\mathrm{O} 4$ & +0.076 \\
\hline $\mathrm{C} 2-\mathrm{C} 3$ & -0.087 \\
\hline $\mathrm{C} 1-\mathrm{C} 2$ & +0.049 \\
\hline $\mathrm{O} 4-\mathrm{C} 3-\mathrm{C} 2$ & +5.7 \\
\hline
\end{tabular}


Table S965 Structural differences between the $1{ }^{3} A\left({ }^{3} n \pi^{*}\right)$ state and the ground state of acrolein.

\begin{tabular}{ll} 
bond & length / $\AA$ \\
or angle & or angle / o \\
\hline C3-O4 & +0.093 \\
C2-C3 & -0.081 \\
C1-C2 & +0.038
\end{tabular}


Table S966 Structural differences between the $2{ }^{1} A^{\prime}\left({ }^{1} \pi \pi^{*}\right)$ state and the ground state of 2-amino-9-methylpurine.

\begin{tabular}{ll} 
bond & length / $\AA$ \\
or angle & or angle / o \\
\hline C1-N2 & -0.030 \\
C5-C6 & +0.037 \\
N2-C6 & +0.050 \\
C5-N9 & -0.030 \\
C8-N9 & +0.045 \\
N7-C8 & -0.030
\end{tabular}


Table S967 Structural differences between the $1{ }^{1} B_{2}\left({ }^{1} \pi \pi^{*}\right)$ state and the ground state of aniline.

\begin{tabular}{ll} 
bond & $\begin{array}{l}\text { length / } \AA \\
\text { or angle } \\
\text { or angle / o }\end{array}$ \\
\hline $\mathrm{C} 2-\mathrm{C} 3$ & +0.040 \\
$\mathrm{C} 2-\mathrm{C} 1-\mathrm{C} 6$ & +5.3
\end{tabular}


Table S968 Structural differences between the $1{ }^{1} A^{\prime \prime}\left({ }^{1} n \pi^{*}\right)$ state and the ground state of benzaldehyde.

\begin{tabular}{|c|c|}
\hline $\begin{array}{l}\text { bond } \\
\text { or angle }\end{array}$ & $\begin{array}{l}\text { length / } \AA \\
\text { or angle / o }\end{array}$ \\
\hline $\mathrm{C} 4-\mathrm{C} 6$ & +0.031 \\
\hline $\mathrm{C} 4-\mathrm{C} 5$ & +0.033 \\
\hline $\mathrm{C} 1-\mathrm{C} 4$ & -0.086 \\
\hline $\mathrm{C} 1-\mathrm{O} 2$ & +0.081 \\
\hline
\end{tabular}


Table S969 Structural differences between the $2{ }^{1} A^{\prime}\left({ }^{1} \pi \pi^{*}\right)$ state and the ground state of benzaldehyde.

\begin{tabular}{ll} 
bond & $\begin{array}{l}\text { length / } \AA \\
\text { or angle } \\
\text { or angle / }\end{array}$ \\
\hline C6-C8 & +0.045 \\
C5-C7 & +0.045 \\
C1-02 & +0.042
\end{tabular}


Table S970 Structural differences between the $1{ }^{3} A^{\prime \prime}\left({ }^{3} n \pi^{*}\right)$ state and the ground state of benzaldehyde.

\begin{tabular}{|c|c|}
\hline $\begin{array}{l}\text { bond } \\
\text { or angle }\end{array}$ & $\begin{array}{l}\text { length / } \AA \\
\text { or angle / }\end{array}$ \\
\hline $\mathrm{C} 1-\mathrm{C} 4$ & -0.077 \\
\hline $\mathrm{C} 1-\mathrm{O} 2$ & +0.093 \\
\hline
\end{tabular}


Table S971 Structural differences between the $1{ }^{3} A^{\prime}\left({ }^{3} \pi \pi^{*}\right)$ state and the ground state of benzaldehyde.

\begin{tabular}{ll} 
bond & $\begin{array}{l}\text { length / } \AA \\
\text { or angle } \\
\text { or angle / o }\end{array}$ \\
\hline C4-C6 & +0.081 \\
C $7-$ C 9 & +0.055 \\
C5-C7 & -0.035 \\
C $4-$ C5 & +0.066 \\
C1-C4 & -0.085 \\
C1-O2 & +0.080
\end{tabular}


Table S972 Structural differences between the $1{ }^{1} A_{u}\left({ }^{1} n \pi^{*}\right)$ state and the ground state of $p$-benzoquinone.

\begin{tabular}{ll} 
bond & $\begin{array}{l}\text { length / } \AA \\
\text { or angle } \\
\text { or angle / o }\end{array}$ \\
\hline C $6-\mathrm{C} 1-\mathrm{C} 2$ & +9.5
\end{tabular}


Table S973 Structural differences between the $1{ }^{1} B_{1 g}\left({ }^{1} n \pi^{*}\right)$ state and the ground state of $p$-benzoquinone.

\begin{tabular}{ll} 
bond & $\begin{array}{l}\text { length / } \AA \\
\text { or angle } \\
\text { or angle / }\end{array}$ \\
\hline $\mathrm{C} 6-\mathrm{C} 1-\mathrm{C} 2$ & +6.3
\end{tabular}


Table S974 Structural differences between the $1{ }^{1} B_{1 u}\left({ }^{1} \pi \pi^{*}\right)$ state and the ground state of $p$-benzoquinone.

\begin{tabular}{|c|c|}
\hline $\begin{array}{l}\text { bond } \\
\text { or angle }\end{array}$ & $\begin{array}{l}\text { length / } \AA \\
\text { or angle / o }\end{array}$ \\
\hline $\mathrm{C} 2-\mathrm{C} 3$ & +0.043 \\
\hline $\mathrm{C} 6-\mathrm{C} 1-\mathrm{C} 2$ & +8.0 \\
\hline
\end{tabular}


Table S975 Structural differences between the $1{ }^{1} B_{3 g}\left({ }^{1} \pi \pi^{*}\right)$ state and the ground state of $p$-benzoquinone.

\begin{tabular}{ll} 
bond & $\begin{array}{l}\text { length / } \AA \\
\text { or angle } \\
\text { or angle / }\end{array}$ \\
\hline C2-C3 & +0.060
\end{tabular}


Table S976 Structural differences between the $1{ }^{3} A_{u}\left({ }^{3} n \pi^{*}\right)$ state and the ground state of $p$-benzoquinone.

\begin{tabular}{ll} 
bond & $\begin{array}{l}\text { length / } \AA \\
\text { or angle }\end{array}$ \\
\hline or $6-\mathrm{C} 1-\mathrm{C} 2$ & +9.8
\end{tabular}


Table S977 Structural differences between the $1{ }^{3} B_{1 g}\left({ }^{3} n \pi^{*}\right)$ state and the ground state of $p$-benzoquinone.

$$
\begin{array}{ll}
\text { bond } & \begin{array}{l}
\text { length / } \AA \\
\text { or angle } \\
\text { or angle / }
\end{array} \\
\hline \text { C6-C1-C2 } & +7.0
\end{array}
$$


Table S978 Structural differences between the $1{ }^{3} B_{3 g}\left({ }^{3} \pi \pi^{*}\right)$ state and the ground state of $p$-benzoquinone.

\begin{tabular}{ll} 
bond & $\begin{array}{l}\text { length / } \AA \\
\text { or angle } \\
\text { or angle / }\end{array}$ \\
\hline C2-C3 & +0.078
\end{tabular}


Table S979 Structural differences between the $1{ }^{1} B_{3 g}\left({ }^{1} \pi \pi^{*}\right)$ state and the ground state of biphenylene.

\begin{tabular}{ll} 
bond & $\begin{array}{l}\text { length / } \AA \\
\text { or angle } \\
\text { or angle / o }\end{array}$ \\
\hline C1-C2 & +0.030 \\
C2-C3 & -0.032 \\
C3-C12 & +0.048 \\
C1-C6 & +0.077 \\
C1-C4 & -0.092
\end{tabular}


Table S980 Structural differences between the $1{ }^{1} B_{1 u}\left({ }^{1} \pi \pi^{*}\right)$ state and the ground state of biphenylene.

$$
\begin{aligned}
& \text { bond length / } \AA \\
& \begin{array}{ll}
\text { or angle or angle / } \\
\hline \text { C1-C4 }
\end{array}
\end{aligned}
$$


Table S981 Structural differences between the $2{ }^{1} B_{1 u}\left({ }^{1} \pi \pi^{*}\right)$ state and the ground state of biphenylene.

\begin{tabular}{ll}
$\begin{array}{l}\text { bond } \\
\text { or angle }\end{array}$ & $\begin{array}{l}\text { ongth / } \AA \\
\text { C1-C4 } 4\end{array}$ \\
\hline-0.032
\end{tabular}


Table S982 Structural differences between the $1{ }^{1} B_{2 u}\left({ }^{1} \pi \pi^{*}\right)$ state and the ground state of biphenylene.

\begin{tabular}{ll} 
bond & $\begin{array}{l}\text { length / } \AA \\
\text { or angle }\end{array}$ \\
or angle / o \\
\hline C3-C12 & +0.068 \\
C1-C6 & +0.051
\end{tabular}


Table S983 Structural differences between the $1{ }^{1} B_{u}\left({ }^{1} \pi \pi^{*}\right)$ state and the ground state of trans-butadiene.

\begin{tabular}{ll} 
bond & length / $\AA$ \\
or angle & or angle / o \\
\hline C1-C2 & +0.087 \\
C2-C3 & -0.052
\end{tabular}


Table S984 Structural differences between the $2{ }^{1} A_{g}\left({ }^{1} \pi \pi^{*}\right)$ state and the ground state of trans-butadiene.

$$
\begin{array}{ll}
\text { bond } & \begin{array}{l}
\text { length / } \AA \\
\text { or angle } \\
\text { or angle / o }
\end{array} \\
\hline \text { C1-C2 } & +0.086
\end{array}
$$


Table S985 Structural differences between the $2{ }^{1} A\left({ }^{1} n \pi^{*}\right)$ state and the ground state of cytosine.

\begin{tabular}{ll} 
bond & $\begin{array}{l}\text { length / } \AA \\
\text { or angle }\end{array}$ \\
or angle / \\
\hline $\mathrm{N} 5-\mathrm{C} 6$ & -0.080 \\
$\mathrm{~N} 1-\mathrm{C} 6$ & -0.090 \\
$\mathrm{~N} 1-\mathrm{C} 2$ & +0.094 \\
$\mathrm{C} 2-\mathrm{C} 3$ & -0.070 \\
$\mathrm{C} 3-\mathrm{C} 4$ & +0.048 \\
$\mathrm{C} 4-\mathrm{N} 5$ & +0.069 \\
$\mathrm{C} 6-\mathrm{O} 8$ & +0.121 \\
$\mathrm{~N} 1-\mathrm{C} 6-\mathrm{N} 5$ & +10.7
\end{tabular}


Table S986 Structural differences between the $3{ }^{1} A\left({ }^{1} \pi \pi^{*}\right)$ state and the ground state of cytosine.

\begin{tabular}{ll} 
bond & $\begin{array}{l}\text { length / } \AA \\
\text { or angle } \\
\text { or angle / o }\end{array}$ \\
\hline $\mathrm{N} 5-\mathrm{C} 6$ & -0.067 \\
$\mathrm{~N} 1-\mathrm{C} 6$ & -0.082 \\
$\mathrm{~N} 1-\mathrm{C} 2$ & +0.110 \\
$\mathrm{C} 2-\mathrm{C} 3$ & -0.072 \\
$\mathrm{C} 3-\mathrm{C} 4$ & +0.058 \\
$\mathrm{C} 4-\mathrm{N} 5$ & +0.050 \\
$\mathrm{C} 6-\mathrm{O} 8$ & +0.116 \\
$\mathrm{~N} 1-\mathrm{C} 6-\mathrm{N} 5$ & +9.1 \\
$\mathrm{~N} 1-\mathrm{C} 2-\mathrm{N} 7$ & -5.4 \\
$\mathrm{~N} 5-\mathrm{C} 6-\mathrm{O} 8$ & -5.0
\end{tabular}


Table S987 Structural differences between the $1{ }^{1} A_{2}\left({ }^{1} n \pi^{*}\right)$ state and the ground state of diazomethane.

$$
\begin{array}{ll}
\begin{array}{l}
\text { bond } \\
\text { or angle }
\end{array} & \begin{array}{l}
\text { length } / \AA \\
\text { ongle / }
\end{array} \\
\hline \text { 2-N3 } & +0.061 \\
\text { H4-C1-H5 } & -5.3
\end{array}
$$


Table S988 Structural differences between the $1{ }^{1} B_{3 g}\left({ }^{1} \pi \pi^{*}\right)$ state and the ground state of dibenzo- $p$-dioxin.

\begin{tabular}{ll}
$\begin{array}{l}\text { bond } \\
\text { or angle }\end{array}$ & $\begin{array}{l}\text { length } / \AA \\
\text { ongle / } /\end{array}$ \\
\hline C4-C5 & +0.054 \\
C1-C2 & +0.045
\end{tabular}


Table S989 Structural differences between the $1{ }^{1} B_{1 u}\left({ }^{1} \pi \pi^{*}\right)$ state and the ground state of dibenzo- $p$-dioxin.

\begin{tabular}{ll} 
bond & length / $\AA$ \\
or angle & or angle / o \\
\hline C4-C5 & +0.042 \\
C1-C2 & +0.056
\end{tabular}


Table S990 Structural differences between the $1{ }^{3} B_{3 g}\left({ }^{3} \pi \pi^{*}\right)$ state and the ground state of dibenzo- $p$-dioxin.

\begin{tabular}{ll} 
bond & length / $\AA$ \\
or angle & or angle / o \\
\hline C4-C5 & +0.054 \\
C1-C2 & +0.052
\end{tabular}


Table S991 Structural differences between the $1{ }^{3} B_{1 u}\left({ }^{3} \pi \pi^{*}\right)$ state and the ground state of dibenzo- $p$-dioxin.

\begin{tabular}{ll} 
bond & length / $\AA$ \\
or angle & or angle / o \\
\hline C4-C5 & +0.036 \\
C1-C2 & +0.041
\end{tabular}


Table S992 Structural differences between the $1{ }^{1} A_{u}\left({ }^{1} n \pi^{*}\right)$ state and the ground state of dibenzo- $p$-dioxin.

\begin{tabular}{ll} 
bond & length / \\
or angle & or angle / o \\
\hline C4-C7 & -0.046 \\
C4-C5 & +0.073 \\
C4-O7-C8 & +8.8
\end{tabular}


Table S993 Structural differences between the $1{ }^{1} B_{1 g}\left({ }^{1} n \pi^{*}\right)$ state and the ground state of dibenzo- $p$-dioxin.

\begin{tabular}{ll} 
bond & $\begin{array}{l}\text { length / } \AA \\
\text { or angle } \\
\text { or angle / }\end{array}$ \\
\hline C4-07-C 8 & +8.5
\end{tabular}


Table S994 Structural differences between the $1{ }^{1} B_{3 u}\left({ }^{1} n \pi^{*}\right)$ state and the ground state of dibenzo- $p$-dioxin.

\begin{tabular}{|c|c|}
\hline bond & length / $\AA$ \\
\hline or angle & or angle / o \\
\hline $\mathrm{C} 4-07-\mathrm{C} 8$ & +5.1 \\
\hline
\end{tabular}


Table S995 Structural differences between the $1{ }^{3} A_{u}\left({ }^{3} n \pi^{*}\right)$ state and the ground state of dibenzo- $p$-dioxin.

\begin{tabular}{ll} 
bond & length / $\AA$ \\
or angle & or angle / o \\
\hline C4-C5 & +0.073 \\
C4-07-C 8 & +8.6
\end{tabular}


Table S996 Structural differences between the $1{ }^{3} B_{3 u}\left({ }^{3} n \pi^{*}\right)$ state and the ground state of dibenzo- $p$-dioxin.

\begin{tabular}{ll} 
bond & $\begin{array}{l}\text { length / } \AA \\
\text { or angle } \\
\text { or angle / o }\end{array}$ \\
\hline C4-07-C8 & +5.0
\end{tabular}


Table S997 Structural differences between the $1{ }^{3} B_{2 g}\left({ }^{3} n \pi^{*}\right)$ state and the ground state of dibenzo- $p$-dioxin.

\begin{tabular}{ll} 
bond & $\begin{array}{l}\text { length / } \AA \\
\text { or angle } \\
\text { or angle / }\end{array}$ \\
\hline C4-C5 & +0.053 \\
C1-C2 & +0.044 \\
C4-07-C 8 & +5.1
\end{tabular}


Table S998 Structural differences between the $1{ }^{1} A^{\prime \prime}\left({ }^{1} n \pi^{*}\right)$ state and the ground state of formaldehyde.

$$
\begin{array}{ll}
\text { bond } & \begin{array}{l}
\text { length / } \AA \\
\text { or angle } \\
\text { or angle / o }
\end{array} \\
\hline \text { C-O } & +0.095
\end{array}
$$


Table S999 Structural differences between the $1{ }^{3} A^{\prime \prime}\left({ }^{3} n \pi^{*}\right)$ state and the ground state of formaldehyde.

$$
\begin{array}{ll}
\text { bond } & \begin{array}{l}
\text { length / } \AA \\
\text { or angle } \\
\text { or angle / O }
\end{array} \\
\hline \text { C-O } & +0.097
\end{array}
$$


Table S1000 Structural differences between the $1{ }^{1} A_{u}\left({ }^{1} n \pi^{*}\right)$ state and the ground state of trans-glyoxal.

\begin{tabular}{ll} 
bond & $\begin{array}{l}\text { length / } \AA \\
\text { or angle }\end{array}$ \\
\hline or angle / 22 & -0.047
\end{tabular}


Table S1001 Structural differences between the $1{ }^{3} A_{u}\left({ }^{3} n \pi^{*}\right)$ state and the ground state of trans-glyoxal.

\begin{tabular}{ll} 
bond & $\begin{array}{l}\text { length / } \AA \\
\text { or angle }\end{array}$ \\
or angle / o \\
\hline C1-C2 & -0.056 \\
C1-03 & +0.032
\end{tabular}


Table S1002 Structural differences between the $2{ }^{1} A\left({ }^{1} \pi \pi^{*}\right)$ state and the ground state of $9 H$-guanine.

\begin{tabular}{ll} 
bond & length / \\
or angle & or angle / \\
\hline N7-C9 & +0.032 \\
C9-N13 & +0.168 \\
N13-C14 & -0.075 \\
C4-C14 & +0.087 \\
C4-C5 & +0.045
\end{tabular}


Table S1003 Structural differences between the $2{ }^{1} A^{\prime}\left({ }^{1} \pi \pi^{*}\right)$ state and the ground state of 1-hydroxy-2-acetonaphthone.

\begin{tabular}{ll} 
bond & $\begin{array}{l}\text { length / } \AA \\
\text { or angle }\end{array}$ or angle / o \\
\hline C1-C6 & +0.044 \\
C4-C5 & +0.050 \\
C6-C11 & -0.031 \\
C1-013 & -0.055 \\
C11-014 & 0.084
\end{tabular}


Table S1004 Structural differences between the $2{ }^{1} A^{\prime}\left({ }^{1} \pi \pi^{*}\right)$ state and the ground state of indole.

\begin{tabular}{ll} 
bond & length / $\AA$ \\
or angle & or angle / \\
\hline N3-C5 & -0.041 \\
C4-C5 & +0.062 \\
C $8-C 9$ & +0.057 \\
C2-N3 & +0.040
\end{tabular}


Table S1005 Structural differences between the $3{ }^{1} A^{\prime}\left({ }^{1} \pi \pi^{*}\right)$ state and the ground state of indole.

\begin{tabular}{ll} 
bond & length / $\AA$ \\
or angle & or angle / o \\
\hline $\mathrm{C} 6-\mathrm{C} 7$ & +0.046 \\
$\mathrm{C} 1-\mathrm{C} 2$ & +0.040
\end{tabular}


Table S1006 Structural differences between the $1{ }^{1} B_{1}\left({ }^{1} n \pi^{*}\right)$ state and the ground state of maleimide.

\begin{tabular}{ll} 
bond & length / \\
or angle & or angle / \\
\hline $\mathrm{C} 2-\mathrm{C} 3$ & -0.071 \\
$\mathrm{C} 3-\mathrm{C} 4$ & +0.052 \\
$\mathrm{C} 2-\mathrm{O} 6$ & +0.035 \\
$\mathrm{~N} 1-\mathrm{C} 2-\mathrm{O} 6$ & -7.2 \\
$\mathrm{O} 6-\mathrm{C} 2-\mathrm{C} 3$ & +5.8
\end{tabular}


Table S1007 Structural differences between the $1{ }^{1} A_{2}\left({ }^{1} n \pi^{*}\right)$ state and the ground state of maleimide.

\begin{tabular}{ll} 
bond & $\begin{array}{l}\text { length / } \AA \\
\text { or angle } \\
\text { or angle / }\end{array}$ \\
\hline C2-C3 3 -0.076 \\
C3-C4 & +0.067 \\
C2-06 & +0.058
\end{tabular}


Table S1008 Structural differences between the $1{ }^{1} B_{2}\left({ }^{1} \pi \pi^{*}\right)$ state and the ground state of maleimide.

\begin{tabular}{ll} 
bond & length / $\AA$ \\
or angle & or angle / \\
\hline $\mathrm{N} 1-\mathrm{C} 2$ & +0.123 \\
$\mathrm{C} 2-\mathrm{C} 3$ & -0.096 \\
$\mathrm{C} 3-\mathrm{C} 4$ & +0.090 \\
$\mathrm{~N} 1-\mathrm{C} 2-\mathrm{O} 6$ & -5.9 \\
O6-C2-C3 & +9.6
\end{tabular}


Table S1009 Structural differences between the $2{ }^{1} B_{2}\left({ }^{1} \pi \pi^{*}\right)$ state and the ground state of maleimide.

\begin{tabular}{ll} 
bond & $\begin{array}{l}\text { length / } \AA \\
\text { or angle } \\
\text { or angle / }\end{array}$ \\
\hline C2-C3 3 -0.048 \\
C3-C4 & +0.102 \\
C2-06 & +0.040
\end{tabular}


Table S1010 Structural differences between the $1{ }^{3} B_{1}\left({ }^{3} n \pi^{*}\right)$ state and the ground state of maleimide.

\begin{tabular}{ll} 
bond & $\begin{array}{l}\text { length / } \AA \\
\text { or angle } \\
\text { or angle / o }\end{array}$ \\
\hline $\mathrm{C} 2-\mathrm{C} 3$ & -0.073 \\
$\mathrm{C} 3-\mathrm{C} 4$ & +0.050 \\
$\mathrm{C} 2-06$ & +0.040 \\
$\mathrm{~N} 1-\mathrm{C} 2-\mathrm{O} 6$ & -6.7 \\
$\mathrm{O} 6-\mathrm{C} 2-\mathrm{C} 3$ & +5.3
\end{tabular}


Table S1011 Structural differences between the $1{ }^{3} A_{2}\left({ }^{3} n \pi^{*}\right)$ state and the ground state of maleimide.

\begin{tabular}{ll} 
bond & $\begin{array}{l}\text { length / } \AA \\
\text { or angle } \\
\text { or angle / }\end{array}$ \\
\hline C2-C3 3 -0.078 \\
C3-C4 & +0.064 \\
C2-06 & +0.061
\end{tabular}


Table S1012 Structural differences between the $1{ }^{3} B_{2}\left({ }^{3} \pi \pi^{*}\right)$ state and the ground state of maleimide.

\begin{tabular}{ll} 
bond & length / $\AA$ \\
or angle & or angle / o \\
\hline $\mathrm{C} 2-\mathrm{C} 3$ & -0.085 \\
$\mathrm{C} 3-\mathrm{C} 4$ & +0.075 \\
$\mathrm{C} 2-06$ & +0.045 \\
$\mathrm{~N} 1-\mathrm{C} 2-\mathrm{O} 6$ & -7.2 \\
$\mathrm{O} 6-\mathrm{C} 2-\mathrm{C} 3$ & +8.2
\end{tabular}


Table S1013 Structural differences between the $1{ }^{1} A^{\prime \prime}\left({ }^{1} n \pi^{*}\right)$ state and the ground state of nitrosomethane.

\begin{tabular}{|c|c|}
\hline $\begin{array}{l}\text { bond } \\
\text { or angle }\end{array}$ & $\begin{array}{l}\text { length / } \AA \\
\text { or angle / o }\end{array}$ \\
\hline $\mathrm{N} 2-\mathrm{O} 3$ & -0.033 \\
\hline $\mathrm{C} 1-\mathrm{N} 2-\mathrm{O} 3$ & +11.8 \\
\hline
\end{tabular}


Table S1014 Structural differences between the $1{ }^{1} A^{\prime \prime}\left({ }^{1} n \pi^{*}\right)$ state and the ground state of propenoic acid anion.

\begin{tabular}{ll} 
bond & $\begin{array}{l}\text { length / } \AA \\
\text { or angle } \\
\text { or angle / o }\end{array}$ \\
\hline $\mathrm{C} 2-\mathrm{O} 4$ & +0.033 \\
$\mathrm{C} 1-\mathrm{C} 2$ & -0.121 \\
$\mathrm{C} 1-\mathrm{C} 5$ & +0.081 \\
$\mathrm{O} 4-\mathrm{C} 2-\mathrm{C} 1$ & +12.4 \\
$\mathrm{O} 3-\mathrm{C} 2-\mathrm{O} 4$ & -22.2
\end{tabular}


Table S1015 Structural differences between the $1{ }^{1} A_{2}\left({ }^{1} n \pi^{*}\right)$ state and the ground state of pyridine.

\begin{tabular}{ll} 
bond & length / \\
or angle & or angle / \\
\hline $\mathrm{N} 1-\mathrm{C} 2$ & -0.035 \\
$\mathrm{C} 2-\mathrm{C} 4$ & +0.078 \\
$\mathrm{C} 2-\mathrm{N} 1-\mathrm{C} 3$ & +17.0 \\
$\mathrm{~N} 1-\mathrm{C} 2-\mathrm{C} 4$ & -9.9 \\
$\mathrm{C} 4-\mathrm{C} 6-\mathrm{C} 5$ & +5.3
\end{tabular}


Table S1016 Structural differences between the $2{ }^{1} A^{\prime}\left({ }^{1} n \pi^{*}\right)$ state and the ground state of pyridine.

\begin{tabular}{ll} 
bond & $\begin{array}{l}\text { length / } \AA \\
\text { or angle } \\
\text { or angle / o }\end{array}$ \\
\hline C4-C6 & +0.035 \\
C2-N1-C3 & +11.2 \\
N1-C2-C4 & -8.4
\end{tabular}


Table S1017 Structural differences between the $1{ }^{3} A_{1}\left({ }^{3} \pi \pi^{*}\right)$ state and the ground state of pyridine.

\begin{tabular}{ll}
$\begin{array}{l}\text { bond } \\
\text { or angle }\end{array}$ & $\begin{array}{l}\text { or angle / } \AA \\
\text { C2-C4 }\end{array}$ \\
\hline
\end{tabular}


Table S1018 Structural differences between the $2{ }^{3} A_{1}\left({ }^{3} \pi \pi^{*}\right)$ state and the ground state of pyridine.

\begin{tabular}{ll} 
bond & length / \\
or angle & or angle / o \\
\hline N1-C2 & +0.035 \\
C4-C6 & +0.039
\end{tabular}


Table S1019 Structural differences between the $2{ }^{1} A^{\prime}\left({ }^{1} \pi \pi^{*}\right)$ state and the ground state of pyrrole.

\begin{tabular}{ll} 
bond & $\begin{array}{l}\text { length / } \AA \\
\text { or angle } \\
\text { or angle / }\end{array}$ \\
\hline N6-C7 & +0.032 \\
C7-C10 & +0.049 \\
C9-C10 & +0.046
\end{tabular}


Table S1020 Structural differences between the $1{ }^{1} B_{2}\left({ }^{1} \pi \pi^{*}\right)$ state and the ground state of pyrrole.

\begin{tabular}{ll} 
bond & length / \\
or angle & or angle / o \\
\hline N6-C7 & +0.046 \\
C7-C10 & +0.077 \\
C9-C10 & -0.056
\end{tabular}


Table S1021 Structural differences between the $2{ }^{1} A^{\prime}\left({ }^{1} \pi \pi^{*}\right)$ state and the ground state of cis-PSB3.

\begin{tabular}{ll} 
bond & $\begin{array}{l}\text { length / } \AA \\
\text { or angle } \\
\text { or angle / }\end{array}$ \\
\hline C2-C3 & +0.068 \\
C3-C4 & -0.060 \\
C4-C5 & +0.100 \\
C5-N6 & -0.044 \\
C2-C3-C4 & -5.8
\end{tabular}


Table S1022 Structural differences between the $2{ }^{1} A^{\prime}\left({ }^{1} \pi \pi^{*}\right)$ state and the ground state of 3Me-cis-PSB5.

\begin{tabular}{ll} 
bond & $\begin{array}{l}\text { length / } \AA \\
\text { or angle } \\
\text { or angle / o }\end{array}$ \\
\hline C1-C8 & +0.049 \\
C1-C2 & -0.045 \\
C2-C3 & +0.086 \\
C3-C4 & -0.049 \\
C4-C5 & +0.046 \\
C5-N6 & -0.038
\end{tabular}


Table S1023 Structural differences between the $1{ }^{1} B_{u}\left({ }^{1} \pi \pi^{*}\right)$ state and the ground state of trans-stilbene.

\begin{tabular}{ll} 
bond & length / $\AA$ \\
or angle & or angle / o \\
\hline C1-C8 & +0.071 \\
C1-C2 & -0.053 \\
C2-C7 & +0.034
\end{tabular}


Table S1024 Structural differences between the $1{ }^{1} B\left({ }^{1} \pi \pi^{*}\right)$ state and the ground state of stilbene.

\begin{tabular}{ll} 
bond & length / $\AA$ \\
or angle & or angle / o \\
\hline C1-C8 & +0.070 \\
C1-C2 & -0.055 \\
C2-C7 & +0.045
\end{tabular}


Table S1025 Structural differences between the $2{ }^{1} A^{\prime}\left({ }^{1} \pi \pi^{*}\right)$ state and the ground state of styrene.

\begin{tabular}{ll} 
bond & $\begin{array}{l}\text { length / } \AA \\
\text { or angle } \\
\text { or angle / o }\end{array}$ \\
\hline C1-C2 & +0.064 \\
C1-C3 & -0.065 \\
C3-C5 & +0.070 \\
C6-C8 & +0.043
\end{tabular}


Table S1026 Structural differences between the $1{ }^{1} B_{3 u}\left({ }^{1} n \pi^{*}\right)$ state and the ground state of $s$-tetrazine.

\begin{tabular}{|c|c|}
\hline $\begin{array}{l}\text { bond } \\
\text { or angle }\end{array}$ & $\begin{array}{l}\text { length / } \AA \\
\text { or angle / o }\end{array}$ \\
\hline $\mathrm{N} 3-\mathrm{C} 1-\mathrm{N} 5$ & +5.4 \\
\hline
\end{tabular}


Table S1027 Structural differences between the $2{ }^{1} A\left({ }^{1} n \pi^{*}\right)$ state and the ground state of thymine.

\begin{tabular}{ll} 
bond & $\begin{array}{l}\text { length / } \AA \\
\text { or angle } \\
\text { or angle / o }\end{array}$ \\
\hline C3-C4 & -0.098 \\
C2-C3 & +0.056 \\
C4-07 & +0.113
\end{tabular}


Table S1028 Structural differences between the $2{ }^{1} A^{\prime}\left({ }^{1} \pi \pi^{*}\right)$ state and the ground state of uracil.

\begin{tabular}{ll} 
bond & $\begin{array}{l}\text { length / } \\
\text { or angle }\end{array}$ \\
or angle / \\
\hline $\mathrm{N} 1-\mathrm{C} 3$ & -0.068 \\
$\mathrm{~N} 2-\mathrm{C} 3$ & -0.067 \\
$\mathrm{~N} 2-\mathrm{C} 4$ & +0.074 \\
$\mathrm{C} 4-\mathrm{C} 5$ & +0.050 \\
$\mathrm{C} 5-\mathrm{C} 6$ & -0.058 \\
$\mathrm{~N} 1-\mathrm{C} 6$ & +0.127 \\
$\mathrm{C} 3-\mathrm{O} 8$ & +0.098
\end{tabular}


4.2 OM2-Optimized Minima for the Computation of ZPVEs

4.2.1 OM2/RHF-Optimized Ground-State Minima 
Table S1029 Cartesian coordinates of the OM2/SCF-optimized groundstate equilibrium geometry of 1,6-epoxy-10-annulene (ground-state energy: $-1723.54629 \mathrm{eV})$.

$\begin{array}{lrrr}\mathrm{O} & 0.00000 & 0.00000 & 0.00000 \\ \mathrm{C} & -0.88309 & 1.04917 & 0.00000 \\ \mathrm{C} & -0.88309 & -1.04917 & -0.00000 \\ \mathrm{C} & -1.30806 & 1.50084 & -1.25050 \\ \mathrm{C} & -1.30806 & -1.50084 & -1.25050 \\ \mathrm{C} & -1.26289 & 1.52294 & 1.29122 \\ \mathrm{C} & -1.26289 & -1.52294 & 1.29122 \\ \mathrm{C} & -1.22977 & 0.69695 & -2.39066 \\ \mathrm{C} & -1.22977 & -0.69695 & -2.39066 \\ \mathrm{C} & -1.27309 & 0.71174 & 2.39547 \\ \mathrm{C} & -1.27309 & -0.71174 & 2.39547 \\ \mathrm{H} & -1.71714 & 2.51170 & -1.33682 \\ \mathrm{H} & -1.71714 & -2.51170 & -1.33682 \\ \mathrm{H} & -1.53201 & 2.58051 & 1.38390 \\ \mathrm{H} & -1.53201 & -2.58051 & 1.38390 \\ \mathrm{H} & -1.33824 & 1.19614 & -3.36633 \\ \mathrm{H} & -1.33824 & -1.19614 & -3.36633 \\ \mathrm{H} & -1.44114 & 1.18475 & 3.37655 \\ \mathrm{H} & -1.44114 & -1.18475 & 3.37655\end{array}$


Table S1030 Cartesian coordinates of the OM2/SCF-optimized groundstate equilibrium geometry of 2-cyclopenten-1-one (ground-state energy: $-1058.64653 \mathrm{eV})$.

$\begin{array}{lrrr}\mathrm{C} & 0.00000 & 0.00000 & 0.00000 \\ \mathrm{C} & 1.52684 & 0.00000 & 0.00000 \\ \mathrm{C} & 1.90980 & 1.47416 & 0.00000 \\ \mathrm{C} & 0.66277 & 2.28176 & 0.00000 \\ \mathrm{C} & -0.39348 & 1.45309 & 0.00000 \\ \mathrm{O} & 3.05386 & 1.90935 & 0.00000 \\ \mathrm{H} & 0.66832 & 3.36149 & 0.00000 \\ \mathrm{H} & -1.44487 & 1.73972 & 0.00000 \\ \mathrm{H} & 1.94604 & -0.48322 & -0.90046 \\ \mathrm{H} & 1.94604 & -0.48322 & 0.90046 \\ \mathrm{H} & -0.41462 & -0.49628 & -0.90297 \\ \mathrm{H} & -0.41462 & -0.49628 & 0.90297\end{array}$


Table S1031 Cartesian coordinates of the OM2/SCF-optimized ground-state equilibrium geometry of 7-azaindole (ground-state energy: $-1408.29249 \mathrm{eV}$ ).

15

$\begin{array}{lrrr}\mathrm{C} & 0.00000 & 0.00000 & 0.00000 \\ \mathrm{~N} & 1.38400 & 0.00000 & 0.00000 \\ \mathrm{C} & 1.82481 & 1.31455 & 0.00000 \\ \mathrm{C} & 0.66465 & 2.16757 & 0.00000 \\ \mathrm{C} & -0.48043 & 1.30133 & 0.00000 \\ \mathrm{~N} & 3.10648 & 1.71801 & -0.00000 \\ \mathrm{C} & 3.26955 & 3.03300 & -0.00000 \\ \mathrm{C} & 2.20735 & 3.97160 & -0.00000 \\ \mathrm{C} & 0.88924 & 3.54837 & -0.00000 \\ \mathrm{H} & 1.98319 & -0.81071 & -0.00000 \\ \mathrm{H} & 4.30380 & 3.42468 & -0.00000 \\ \mathrm{H} & 2.44225 & 5.03860 & -0.00000 \\ \mathrm{H} & 0.05384 & 4.25534 & -0.00000 \\ \mathrm{H} & -1.51293 & 1.60741 & -0.00000 \\ \mathrm{H} & -0.58682 & -0.91579 & -0.00000\end{array}$


Table S1032 Cartesian coordinates of the OM2/SCF-optimized groundstate equilibrium geometry of $\beta$-dinaphthyleneoxide (ground-state energy: $-3060.76801 \mathrm{eV})$.

$\begin{array}{lrrr}\mathrm{C} & 0.00000 & 0.00000 & 0.00000 \\ \mathrm{C} & 1.37555 & 0.00000 & 0.00000 \\ \mathrm{C} & 2.09262 & 1.22195 & 0.00000 \\ \mathrm{C} & 1.37076 & 2.44055 & 0.10783 \\ \mathrm{C} & -0.04834 & 2.40959 & 0.07962 \\ \mathrm{C} & -0.71991 & 1.21277 & 0.00692 \\ \mathrm{C} & 3.51713 & 1.30479 & -0.02113 \\ \mathrm{C} & 4.09474 & 2.55566 & 0.28229 \\ \mathrm{C} & 3.39343 & 3.77380 & 0.42712 \\ \mathrm{C} & 2.03217 & 3.69604 & 0.29396 \\ \mathrm{O} & 5.46185 & 2.48833 & 0.39665 \\ \mathrm{C} & 5.77885 & 1.17917 & 0.12788 \\ \mathrm{C} & 4.64403 & 0.40501 & -0.19347 \\ \mathrm{C} & 4.87182 & -0.92732 & -0.65121 \\ \mathrm{C} & 6.19296 & -1.43725 & -0.54039 \\ \mathrm{C} & 7.28069 & -0.62431 & -0.08836 \\ \mathrm{C} & 7.10590 & 0.70100 & 0.21134 \\ \mathrm{C} & 6.45385 & -2.77592 & -0.93473 \\ \mathrm{C} & 5.45576 & -3.55354 & -1.47120 \\ \mathrm{C} & 4.16957 & -3.01042 & -1.67091 \\ \mathrm{C} & 3.88077 & -1.72530 & -1.27442 \\ \mathrm{H} & 7.91344 & 1.36741 & 0.50519 \\ \mathrm{H} & 8.27615 & -1.07951 & -0.01466 \\ \mathrm{H} & 2.89926 & -1.29378 & -1.48622 \\ \mathrm{H} & 7.46901 & -3.17594 & -0.81513 \\ \mathrm{H} & 5.65384 & -4.58799 & -1.77121 \\ \mathrm{H} & 3.40291 & -3.62310 & -2.15938 \\ \mathrm{H} & 3.93117 & 4.69825 & 0.62408 \\ \mathrm{H} & 1.41912 & 4.60348 & 0.35980 \\ \mathrm{H} & 1.92372 & -0.94396 & 0.05369 \\ \mathrm{H} & -0.60073 & 3.35685 & 0.12888 \\ \mathrm{H} & -1.81442 & 1.18716 & -0.02012 \\ \mathrm{H} & -0.55222 & -0.94698 & 0.00727\end{array}$


Table S1033 Cartesian coordinates of the OM2/SCF-optimized ground-state equilibrium geometry of acetaldehyde (ground-state energy: $-646.36576 \mathrm{eV}$ ).

7

$\begin{array}{lrrr}\mathrm{C} & 0.00000 & 0.00000 & 0.00000 \\ \mathrm{C} & 1.50022 & 0.00000 & 0.00000 \\ \mathrm{O} & 2.12440 & 1.05019 & 0.00000 \\ \mathrm{H} & -0.36723 & 1.02976 & 0.00000 \\ \mathrm{H} & -0.36123 & -0.52184 & 0.89354 \\ \mathrm{H} & -0.36123 & -0.52184 & -0.89354 \\ \mathrm{H} & 2.01123 & -1.01660 & 0.00000\end{array}$


Table S1034 Cartesian coordinates of the OM2/SCF-optimized ground-state equilibrium geometry of acetone (ground-state energy: $-804.50265 \mathrm{eV}$ ).

10

$\begin{array}{lrrr}\mathrm{C} & 0.00000 & 0.00000 & 0.00000 \\ \mathrm{C} & 1.50701 & 0.00000 & 0.00000 \\ \mathrm{O} & 2.13219 & 1.05570 & 0.00000 \\ \mathrm{C} & 2.23134 & -1.32153 & 0.00007 \\ \mathrm{H} & -0.35943 & 0.91960 & -0.47117 \\ \mathrm{H} & 3.21404 & -1.19289 & 0.46330 \\ \mathrm{H} & -0.33775 & -0.02516 & 1.04294 \\ \mathrm{H} & -0.39579 & -0.87211 & -0.53219 \\ \mathrm{H} & 1.66055 & -2.08511 & 0.54008 \\ \mathrm{H} & 2.36350 & -1.63460 & -1.04250\end{array}$


Table S1035 Cartesian coordinates of the OM2/SCF-optimized ground-state equilibrium geometry of acrolein (ground-state energy: $-772.97202 \mathrm{eV}$ ).

$\begin{array}{lrrr}8 & & & \\ & & & \\ \mathrm{C} & 0.00000 & 0.00000 & 0.00000 \\ \mathrm{C} & 1.33632 & 0.00000 & 0.00000 \\ \mathrm{C} & 2.04960 & 1.29576 & 0.00000 \\ \mathrm{O} & 1.42352 & 2.34799 & 0.00000 \\ \mathrm{H} & 3.18519 & 1.25627 & 0.00000 \\ \mathrm{H} & 1.92878 & -0.91755 & -0.00000 \\ \mathrm{H} & -0.55737 & 0.92963 & 0.00000 \\ \mathrm{H} & -0.57749 & -0.92019 & -0.00000\end{array}$


Table S1036 Cartesian coordinates of the OM2/SCF-optimized groundstate equilibrium geometry of aminobenzonitrile (ground-state energy: $-1408.50975 \mathrm{eV})$.

$\begin{array}{lrrr}\text { C } & 0.00000 & 0.00000 & 0.00000 \\ \text { C } & 2.76299 & 0.00000 & 0.00000 \\ \text { C } & 0.70018 & 0.00116 & -1.22582 \\ \mathrm{C} & 0.70018 & 0.00116 & 1.22582 \\ \mathrm{C} & 2.08619 & 0.00282 & 1.22600 \\ \mathrm{C} & 2.08619 & 0.00282 & -1.22600 \\ \mathrm{~N} & -1.38175 & 0.04224 & 0.00000 \\ \mathrm{C} & 4.19829 & -0.00360 & -0.00000 \\ \mathrm{~N} & 5.37062 & -0.00707 & -0.00000 \\ \mathrm{H} & 0.14420 & 0.01174 & -2.16602 \\ \mathrm{H} & 0.14420 & 0.01174 & 2.16602 \\ \mathrm{H} & 2.64558 & 0.00553 & 2.16578 \\ \mathrm{H} & 2.64558 & 0.00553 & -2.16578 \\ \mathrm{H} & -1.87258 & -0.19049 & -0.84037 \\ \mathrm{H} & -1.87258 & -0.19049 & 0.84037\end{array}$


Table S1037 Cartesian coordinates of the OM2/SCF-optimized ground-state equilibrium geometry of aniline (ground-state energy: $-1083.33795 \mathrm{eV}$ ).

$\begin{array}{lrrr}\text { C } & 0.00000 & 0.00000 & 0.00000 \\ \mathrm{C} & 2.79002 & 0.00000 & 0.00000 \\ \mathrm{C} & 0.70310 & 0.00081 & -1.21980 \\ \mathrm{C} & 0.70310 & 0.00081 & 1.21980 \\ \mathrm{C} & 2.09172 & 0.00285 & 1.20592 \\ \mathrm{C} & 2.09172 & 0.00285 & -1.20592 \\ \mathrm{~N} & -1.39083 & 0.04841 & 0.00000 \\ \mathrm{H} & 3.88399 & -0.00070 & -0.00000 \\ \mathrm{H} & 0.15157 & 0.01297 & -2.16444 \\ \mathrm{H} & 0.15157 & 0.01297 & 2.16444 \\ \mathrm{H} & 2.64303 & 0.00598 & 2.15502 \\ \mathrm{H} & 2.64303 & 0.00598 & -2.15502 \\ \mathrm{H} & -1.85988 & -0.25124 & -0.83618 \\ \mathrm{H} & -1.85988 & -0.25124 & 0.83618\end{array}$


Table S1038 Cartesian coordinates of the OM2/SCF-optimized ground-state equilibrium geometry of anisol (ground-state energy: $-1343.47005 \mathrm{eV}$ ).

$\begin{array}{rrrr}\text { 16 } & & & \\ \text { C } & 0.00000 & 0.00000 & 0.00000 \\ \text { C } & 1.39273 & 0.00000 & 0.00000 \\ \text { C } & 2.04997 & 1.23483 & 0.00000 \\ \text { C } & 1.35134 & 2.45427 & 0.00000 \\ \text { C } & -0.03587 & 2.41576 & 0.00000 \\ \text { C } & -0.71310 & 1.19547 & 0.00000 \\ \text { O } & 3.41287 & 1.39840 & 0.00000 \\ \text { C } & 4.20474 & 0.24855 & 0.00000 \\ \text { H } & 1.90508 & 3.39163 & 0.00000 \\ \text { H } & -0.60388 & 3.35418 & 0.00000 \\ \text { H } & -1.80693 & 1.17884 & 0.00000 \\ \text { H } & -0.53792 & -0.95647 & -0.00000 \\ \text { H } & 1.94563 & -0.93908 & -0.00000 \\ \text { H } & 4.03321 & -0.37417 & -0.91431 \\ \text { H } & 4.03321 & -0.37417 & 0.91431 \\ \text { H } & 5.24902 & 0.63000 & 0.00000\end{array}$


Table S1039 Cartesian coordinates of the OM2/SCF-optimized ground-state equilibrium geometry of anthracene (ground-state energy: $-1935.01727 \mathrm{eV}$ ).

\begin{tabular}{|c|c|c|c|}
\hline & 1.00000 & 2.41010 & 0.00000 \\
\hline & 1.00000 & -0.41010 & -0.00000 \\
\hline & 2.21439 & 1.71305 & 0.00000 \\
\hline & -0.21439 & 1.71305 & -0.00000 \\
\hline & 2.21439 & 0.28695 & 0.00000 \\
\hline & -0.21439 & 0.28695 & -0.00000 \\
\hline & 3.46809 & 2.40701 & 0.00000 \\
\hline & -1.46809 & 2.40701 & -0.00000 \\
\hline & 3.46809 & -0.40701 & 0.00000 \\
\hline & -1.46809 & -0.40701 & -0.00000 \\
\hline & 4.64323 & 1.71211 & 0.00000 \\
\hline & -2.64323 & 1.71211 & -0.00000 \\
\hline & 4.64323 & 0.28789 & 0.00000 \\
\hline & -2.64323 & 0.28789 & -0.00000 \\
\hline & 1.00000 & 3.50769 & 0.00000 \\
\hline & 1.00000 & -1.50769 & -0.00000 \\
\hline & 3.45899 & 3.50422 & 0.00000 \\
\hline & -1.45899 & 3.50422 & 0.00000 \\
\hline & 3.45899 & -1.50422 & 0.00000 \\
\hline & -1.45899 & -1.50422 & -0.00000 \\
\hline & 5.60586 & 2.23621 & 0.00000 \\
\hline & -3.60586 & 2.23621 & 0.00000 \\
\hline & 5.60586 & -0.23621 & 0.00000 \\
\hline & -3.60586 & -0.23621 & -0.00000 \\
\hline
\end{tabular}


Table S1040 Cartesian coordinates of the OM2/SCF-optimized ground-state equilibrium geometry of azulene (ground-state energy: $-1393.03828 \mathrm{eV}$ ).

$\begin{array}{lrrr}18 & & \\ \text { C } & 0.00000 & 1.54777 & 0.00000 \\ \text { C } & 0.00000 & 0.96398 & -1.26361 \\ \text { C } & 0.00000 & 0.96398 & 1.26361 \\ \text { C } & 0.00000 & -0.38304 & -1.60234 \\ \text { C } & 0.00000 & -0.38304 & 1.60234 \\ \text { C } & 0.00000 & -1.47347 & -0.74137 \\ \text { C } & 0.00000 & -1.47347 & 0.74137 \\ \text { C } & 0.00000 & -2.82860 & -1.14935 \\ \text { C } & 0.00000 & -2.82860 & 1.14935 \\ \text { C } & 0.00000 & -3.63210 & -0.00000 \\ \text { H } & 0.00000 & 2.65684 & 0.00000 \\ \text { H } & 0.00000 & 1.67176 & -2.11091 \\ \text { H } & 0.00000 & 1.67176 & 2.11091 \\ \text { H } & 0.00000 & -0.62029 & -2.68350 \\ \text { H } & 0.00000 & -0.62029 & 2.68350 \\ \text { H } & 0.00000 & -3.18077 & -2.17159 \\ \text { H } & 0.00000 & -3.18077 & 2.17159 \\ \text { H } & 0.00000 & -4.72015 & -0.00000\end{array}$


Table S1041 Cartesian coordinates of the OM2/SCF-optimized ground-state equilibrium geometry of benzaldehyde (ground-state energy: $-1313.58895 \mathrm{eV}$ ).

14

$\begin{array}{rrrr}\mathrm{C} & 0.00000 & 0.00000 & 0.00000 \\ \mathrm{C} & 1.39849 & 0.00000 & 0.00000 \\ \mathrm{C} & 2.07799 & 1.21440 & 0.00000 \\ \mathrm{C} & 1.36761 & 2.41289 & 0.00000 \\ \mathrm{C} & -0.02694 & 2.40844 & 0.00000 \\ \mathrm{C} & -0.71603 & 1.20088 & 0.00000 \\ \mathrm{C} & -0.71393 & -1.29913 & -0.00000 \\ \mathrm{O} & -0.08867 & -2.35072 & -0.00000 \\ \mathrm{H} & 1.93620 & -0.95133 & -0.00000 \\ \mathrm{H} & 3.17271 & 1.22814 & 0.00000 \\ \mathrm{H} & 1.90743 & 3.36721 & 0.00000 \\ \mathrm{H} & -0.57688 & 3.35510 & 0.00000 \\ \mathrm{H} & -1.81299 & 1.18757 & 0.00000 \\ \mathrm{H} & -1.85179 & -1.25785 & -0.00000\end{array}$


Table S1042 Cartesian coordinates of the OM2/SCF-optimized ground-state equilibrium geometry of benzene (ground-state energy: $-855.06716 \mathrm{eV}$ ).

$\begin{array}{lrrr}12 & & \\ \text { C } & 0.00000 & 1.39253 & 0.00000 \\ \text { C } & 0.00000 & 0.69626 & -1.20596 \\ \text { C } & 0.00000 & 0.69626 & 1.20596 \\ \text { C } & 0.00000 & -0.69626 & -1.20596 \\ \text { C } & 0.00000 & -0.69626 & 1.20596 \\ \text { C } & 0.00000 & -1.39253 & -0.00000 \\ \text { H } & 0.00000 & 2.48870 & 0.00000 \\ \text { H } & 0.00000 & 1.24435 & -2.15528 \\ \text { H } & 0.00000 & 1.24435 & 2.15528 \\ \text { H } & 0.00000 & -1.24435 & -2.15528 \\ \text { H } & 0.00000 & -1.24435 & 2.15528 \\ \text { H } & 0.00000 & -2.48870 & -0.00000\end{array}$


Table S1043 Cartesian coordinates of the OM2/SCF-optimized ground-state equilibrium geometry of benzonitrile (ground-state energy: $-1180.19567 \mathrm{eV}$ ).

$\begin{array}{lrrr}13 & & \\ \text { C } & 0.00000 & 1.40254 & 0.00000 \\ \text { C } & 0.00000 & 0.71133 & -1.21038 \\ \text { C } & 0.00000 & 0.71133 & 1.21038 \\ \text { C } & 0.00000 & -0.67997 & -1.22581 \\ \text { C } & 0.00000 & -0.67997 & 1.22581 \\ \text { C } & 0.00000 & -1.35300 & -0.00000 \\ \text { H } & 0.00000 & 2.49790 & 0.00000 \\ \text { H } & 0.00000 & 1.26433 & -2.15512 \\ \text { H } & 0.00000 & 1.26433 & 2.15512 \\ \text { H } & 0.00000 & -1.23537 & -2.16604 \\ \text { H } & 0.00000 & -1.23537 & 2.16604 \\ \text { C } & 0.00000 & -2.79211 & -0.00000 \\ \text { N } & 0.00000 & -3.96391 & -0.00000\end{array}$


Table S1044 Cartesian coordinates of the OM2/SCF-optimized ground-state equilibrium geometry of benzophenone ketyl radical (ground-state energy: $-2153.12295 \mathrm{eV})$.

$\begin{array}{lrrr}\mathrm{C} & 0.00000 & 0.00000 & 0.00000 \\ \mathrm{C} & 1.40080 & 0.00000 & 0.00000 \\ \mathrm{C} & 2.10328 & 1.21312 & 0.00000 \\ \mathrm{C} & 1.40331 & 2.41474 & 0.01809 \\ \mathrm{C} & 0.00961 & 2.41441 & 0.02813 \\ \mathrm{C} & -0.68862 & 1.20851 & 0.01855 \\ \mathrm{C} & 2.12557 & -1.27882 & -0.08122 \\ \mathrm{O} & 3.34128 & -1.26008 & -0.68313 \\ \mathrm{C} & 2.04678 & -2.31426 & 0.94242 \\ \mathrm{C} & 1.12073 & -2.22168 & 1.99409 \\ \mathrm{C} & 1.01639 & -3.26492 & 2.90675 \\ \mathrm{C} & 1.82306 & -4.39556 & 2.78184 \\ \mathrm{C} & 2.74767 & -4.48252 & 1.74150 \\ \mathrm{C} & 2.86339 & -3.45097 & 0.81765 \\ \mathrm{H} & 0.50208 & -1.32660 & 2.10607 \\ \mathrm{H} & 0.29940 & -3.19633 & 3.73342 \\ \mathrm{H} & 1.73338 & -5.21391 & 3.50525 \\ \mathrm{H} & 3.38524 & -5.36987 & 1.65253 \\ \mathrm{H} & 3.58888 & -3.50568 & 0.00288 \\ \mathrm{H} & 3.19806 & 1.21388 & 0.01353 \\ \mathrm{H} & 1.94910 & 3.36520 & 0.02948 \\ \mathrm{H} & -0.53720 & 3.36351 & 0.04259 \\ \mathrm{H} & -1.78460 & 1.21292 & 0.02042 \\ \mathrm{H} & -0.54521 & -0.94823 & -0.02928 \\ \mathrm{H} & 3.32509 & -0.59985 & -1.42725\end{array}$


Table S1045 Cartesian coordinates of the OM2/SCF-optimized ground-state equilibrium geometry of biphenyl (ground-state energy: $-1680.25705 \mathrm{eV}$ ).

$\begin{array}{lrrr}22 & & & \\ \mathrm{C} & 0.00000 & 0.00000 & 0.73902 \\ \mathrm{C} & 0.00000 & 0.00000 & -0.73902 \\ \mathrm{C} & 1.10349 & 0.50319 & 1.43623 \\ \mathrm{C} & 1.10349 & -0.50319 & -1.43623 \\ \mathrm{C} & -1.10349 & -0.50319 & 1.43623 \\ \mathrm{C} & -1.10349 & 0.50319 & -1.43623 \\ \mathrm{C} & 1.09730 & 0.50246 & 2.82752 \\ \mathrm{C} & 1.09730 & -0.50246 & -2.82752 \\ \mathrm{C} & -1.09730 & -0.50246 & 2.82752 \\ \mathrm{C} & -1.09730 & 0.50246 & -2.82752 \\ \mathrm{C} & 0.00000 & 0.00000 & 3.52347 \\ \mathrm{C} & 0.00000 & 0.00000 & -3.52347 \\ \mathrm{H} & 1.96049 & 0.90658 & 0.88709 \\ \mathrm{H} & 1.96049 & -0.90658 & -0.88709 \\ \mathrm{H} & -1.96049 & -0.90658 & 0.88709 \\ \mathrm{H} & -1.96049 & 0.90658 & -0.88709 \\ \mathrm{H} & 1.95768 & 0.89996 & 3.37857 \\ \mathrm{H} & 1.95768 & -0.89996 & -3.37857 \\ \mathrm{H} & -1.95768 & -0.89996 & 3.37857 \\ \mathrm{H} & -1.95768 & 0.89996 & -3.37857 \\ \mathrm{H} & 0.00000 & 0.00000 & 4.61912 \\ \mathrm{H} & 0.00000 & 0.00000 & -4.61912\end{array}$


Table S1046 Cartesian coordinates of the OM2/SCF-optimized ground-state equilibrium geometry of biphenylene (ground-state energy: $-1647.63341 \mathrm{eV}$ ).

$\begin{array}{lrrr}20 & & & \\ & & & \\ \mathrm{C} & 0.00000 & 0.00000 & 0.00000 \\ \mathrm{C} & 1.50478 & 0.00000 & 0.00000 \\ \mathrm{C} & 0.00000 & 1.44173 & 0.00000 \\ \mathrm{C} & 1.50478 & 1.44173 & 0.00000 \\ \mathrm{C} & -1.15145 & -0.73566 & 0.00000 \\ \mathrm{C} & 2.65622 & -0.73566 & -0.00000 \\ \mathrm{C} & -1.15145 & 2.17739 & -0.00000 \\ \mathrm{C} & 2.65622 & 2.17739 & 0.00000 \\ \mathrm{C} & -2.34400 & 0.02828 & 0.00000 \\ \mathrm{C} & 3.84878 & 0.02828 & -0.00000 \\ \mathrm{C} & -2.34400 & 1.41345 & -0.00000 \\ \mathrm{C} & 3.84878 & 1.41345 & 0.00000 \\ \mathrm{H} & -1.17436 & -1.82562 & 0.00000 \\ \mathrm{H} & 2.67914 & -1.82562 & -0.00000 \\ \mathrm{H} & -1.17436 & 3.26735 & -0.00000 \\ \mathrm{H} & 2.67914 & 3.26735 & 0.00000 \\ \mathrm{H} & -3.30186 & -0.50666 & 0.00000 \\ \mathrm{H} & 4.80664 & -0.50666 & -0.00000 \\ \mathrm{H} & -3.30186 & 1.94840 & -0.00000 \\ \mathrm{H} & 4.80664 & 1.94840 & 0.00000\end{array}$


Table S1047 Cartesian coordinates of the OM2/SCF-optimized ground-state equilibrium geometry of acetylene (ground-state energy: $-282.94213 \mathrm{eV}$ ).

$\begin{array}{lrrr}\text { C } & 0.00000 & 0.61114 & 0.00000 \\ \text { C } & 0.00000 & -0.61114 & -0.00000 \\ \text { H } & 0.00000 & 1.65239 & 0.00000 \\ \text { H } & 0.00000 & -1.65239 & -0.00000\end{array}$


Table S1048 Cartesian coordinates of the OM2/SCF-optimized ground-state equilibrium geometry of formaldehyde (ground-state energy: $-488.20983 \mathrm{eV}$ ).

$\begin{array}{llll}0 & 1.00000 & 1.00000 & 0.00000\end{array}$

C $-0.21623 \quad 1.00000 \quad 0.00000$

$\mathrm{H} \quad-0.82316 \quad 1.00000 \quad 0.94804$

$\mathrm{H} \quad-0.82316 \quad 1.00000 \quad-0.94804$ 
Table S1049 Cartesian coordinates of the OM2/SCF-optimized ground-state equilibrium geometry of cinnoline (ground-state energy: $-1535.81077 \mathrm{eV}$ ).

$\begin{array}{rrrr}16 & & & \\ & & & \\ \mathrm{C} & 0.00000 & 0.00000 & 0.00000 \\ \mathrm{C} & 1.42224 & 0.00000 & 0.00000 \\ \mathrm{C} & 2.12137 & 1.23077 & 0.00000 \\ \mathrm{C} & 1.40434 & 2.45377 & 0.00000 \\ \mathrm{C} & 0.03023 & 2.42593 & 0.00000 \\ \mathrm{C} & -0.67215 & 1.19801 & 0.00000 \\ \mathrm{~N} & 2.10515 & -1.21021 & -0.00000 \\ \mathrm{~N} & 3.33085 & -1.24801 & -0.00000 \\ \mathrm{C} & 4.10283 & -0.10160 & -0.00000 \\ \mathrm{C} & 3.54234 & 1.15244 & -0.00000 \\ \mathrm{H} & 1.95546 & 3.40010 & -0.00000 \\ \mathrm{H} & -0.54100 & 3.36151 & 0.00000 \\ \mathrm{H} & -1.76731 & 1.21694 & 0.00000 \\ \mathrm{H} & -0.52038 & -0.96029 & -0.00000 \\ \mathrm{H} & 4.14487 & 2.06478 & -0.00000 \\ \mathrm{H} & 5.19163 & -0.23912 & -0.00000\end{array}$


Table S1050 Cartesian coordinates of the OM2/SCF-optimized ground-state equilibrium geometry of cyanoacetylene (ground-state energy: $-607.63541 \mathrm{eV}$ ).

$\begin{array}{lrrr}\text { C } & 0.00000 & 0.61325 & 0.00000 \\ \text { C } & 0.00000 & -0.61325 & -0.00000 \\ \text { C } & 0.00000 & -1.99547 & -0.00000 \\ \text { N } & 0.00000 & -3.16914 & -0.00000 \\ \text { H } & 0.00000 & 1.65685 & 0.00000\end{array}$


Table S1051 Cartesian coordinates of the OM2/SCF-optimized groundstate equilibrium geometry of cyclohexadienyl radical (ground-state energy: $-868.93439 \mathrm{eV})$.

13

$\begin{array}{lrrr}\text { C } & 0.00000 & 0.00000 & 0.00000 \\ \mathrm{C} & 2.88414 & 0.00000 & 0.00000 \\ \mathrm{C} & 0.80960 & 1.23880 & 0.00000 \\ \mathrm{C} & 0.80960 & -1.23880 & -0.00000 \\ \mathrm{C} & 2.17960 & 1.22079 & 0.00000 \\ \mathrm{C} & 2.17960 & -1.22079 & -0.00000 \\ \mathrm{H} & -0.67265 & 0.00000 & -0.90416 \\ \mathrm{H} & -0.67265 & -0.00000 & 0.90416 \\ \mathrm{H} & 3.97907 & 0.00000 & 0.00000 \\ \mathrm{H} & 0.26137 & 2.18954 & -0.00000 \\ \mathrm{H} & 0.26137 & -2.18954 & -0.00000 \\ \mathrm{H} & 2.74619 & 2.16059 & 0.00000 \\ \mathrm{H} & 2.74619 & -2.16059 & -0.00000\end{array}$


Table S1052 Cartesian coordinates of the OM2/SCF-optimized ground-state equilibrium geometry of 2,3-diazabicyclo[2,2,1]hept-2-ene (ground-state energy: $-1183.58976 \mathrm{eV})$.

$\begin{array}{lrrr}\mathrm{N} & 0.00000 & 0.00000 & 0.00000 \\ \mathrm{C} & 1.50062 & 0.00000 & 0.00000 \\ \mathrm{C} & 1.77650 & 1.52091 & 0.00000 \\ \mathrm{C} & 0.75324 & 1.74317 & 1.13702 \\ \mathrm{~N} & -0.40455 & 0.94354 & 0.61547 \\ \mathrm{C} & 1.86753 & -0.36605 & 1.45884 \\ \mathrm{C} & 1.34833 & 0.84498 & 2.24875 \\ \mathrm{H} & 1.48911 & 2.01338 & -0.94391 \\ \mathrm{H} & 2.80803 & 1.78743 & 0.26943 \\ \mathrm{H} & 1.37255 & -1.30812 & 1.76159 \\ \mathrm{H} & 2.16634 & 1.37371 & 2.77743 \\ \mathrm{H} & 2.96414 & -0.48716 & 1.56368 \\ \mathrm{H} & 0.48894 & 2.77702 & 1.42956 \\ \mathrm{H} & 1.94928 & -0.62897 & -0.79207 \\ \mathrm{H} & 0.56970 & 0.56456 & 2.98309\end{array}$


Table S1053 Cartesian coordinates of the OM2/SCF-optimized ground-state equilibrium geometry of 4-(dimethylamino)-4'-cyanostilbene (ground-state energy: $-2833.37117 \mathrm{eV})$.

$\begin{array}{lrrr}\text { 35 } & & & \\ & & & \\ \mathrm{C} & 0.00000 & 0.00000 & 0.00000 \\ \mathrm{C} & 1.38844 & 0.00000 & 0.00000 \\ \mathrm{C} & 2.09946 & 1.21012 & 0.00000 \\ \mathrm{C} & 1.40938 & 2.43078 & -0.01609 \\ \mathrm{C} & 0.02019 & 2.45199 & -0.02033 \\ \mathrm{C} & -0.66475 & 1.23193 & -0.00902 \\ \mathrm{C} & 3.56490 & 1.17016 & 0.00727 \\ \mathrm{C} & 4.30491 & 2.12867 & 0.59541 \\ \mathrm{C} & 5.76841 & 2.10185 & 0.61337 \\ \mathrm{C} & 6.44937 & 2.78246 & 1.63294 \\ \mathrm{C} & 7.83424 & 2.77883 & 1.68691 \\ \mathrm{C} & 8.58053 & 2.09607 & 0.70192 \\ \mathrm{C} & 7.89316 & 1.42108 & -0.32860 \\ \mathrm{C} & 6.50622 & 1.42511 & -0.36562 \\ \mathrm{~N} & 9.98440 & 2.06376 & 0.76691 \\ \mathrm{C} & 10.66355 & 2.98861 & 1.63456 \\ \mathrm{C} & -2.10249 & 1.24359 & -0.01249 \\ \mathrm{~N} & -3.27446 & 1.25314 & -0.01533 \\ \mathrm{C} & 10.72472 & 1.56363 & -0.36029 \\ \mathrm{H} & 8.34210 & 3.30178 & 2.50079 \\ \mathrm{H} & 5.87795 & 3.31530 & 2.40485 \\ \mathrm{H} & 5.98822 & 0.90196 & -1.17996 \\ \mathrm{H} & 8.44701 & 0.88486 & -1.10304 \\ \mathrm{H} & 3.82986 & 2.97121 & 1.12089 \\ \mathrm{H} & 4.02866 & 0.30063 & -0.47435 \\ \mathrm{H} & 1.93390 & -0.94932 & 0.00986 \\ \mathrm{H} & 1.96615 & 3.37300 & -0.03696 \\ \mathrm{H} & -0.52988 & 3.39587 & -0.03521 \\ \mathrm{H} & -0.56568 & -0.93460 & 0.00698 \\ \mathrm{H} & 11.81760 & 1.58965 & -0.15537 \\ \mathrm{H} & 10.45438 & 0.50148 & -0.57514 \\ \mathrm{H} & 10.53743 & 2.16433 & -1.28974 \\ \mathrm{H} & 11.76545 & 2.85430 & 1.56795 \\ \mathrm{H} & 10.43322 & 4.05600 & 1.37584 \\ \mathrm{H} & 10.37447 & 2.82190 & 2.70071\end{array}$


Table S1054 Cartesian coordinates of the OM2/SCF-optimized groundstate equilibrium geometry of dimethylaminobenzonitrile (ground-state energy: $-1723.63758 \mathrm{eV})$.

$\begin{array}{lrrr}\text { C } & 0.00000 & 0.00000 & 0.00000 \\ \text { C } & 2.78187 & 0.00000 & 0.00000 \\ \text { C } & 0.71387 & 0.00187 & -1.22000 \\ \text { C } & 0.71387 & 0.00187 & 1.22000 \\ \mathrm{C} & 2.10022 & 0.00252 & 1.22186 \\ \mathrm{C} & 2.10022 & 0.00252 & -1.22186 \\ \mathrm{~N} & -1.39987 & 0.02510 & 0.00000 \\ \mathrm{C} & -2.11443 & -0.17725 & -1.23204 \\ \mathrm{C} & -2.11443 & -0.17725 & 1.23204 \\ \mathrm{C} & 4.21708 & -0.00358 & -0.00000 \\ \mathrm{~N} & 5.38945 & -0.00666 & -0.00000 \\ \mathrm{H} & 0.17982 & 0.00922 & -2.17181 \\ \mathrm{H} & 0.17982 & 0.00922 & 2.17181 \\ \mathrm{H} & 2.65606 & 0.00394 & 2.16413 \\ \mathrm{H} & 2.65606 & 0.00394 & -2.16413 \\ \mathrm{H} & -3.21204 & -0.13232 & -1.06255 \\ \mathrm{H} & -3.21204 & -0.13232 & 1.06255 \\ \mathrm{H} & -1.86105 & 0.61650 & -1.97682 \\ \mathrm{H} & -1.86105 & 0.61650 & 1.97682 \\ \mathrm{H} & -1.88112 & -1.17297 & -1.69225 \\ \mathrm{H} & -1.88112 & -1.17297 & 1.69225\end{array}$


Table S1055 Cartesian coordinates of the OM2/SCF-optimized ground-state equilibrium geometry of $N, N$-dimethyl-p-phenylenediamine (ground-state energy: $-1626.73548 \mathrm{eV})$.

$\begin{array}{lrrr}\text { C } & 0.00000 & 0.00000 & 0.00000 \\ \text { C } & 2.79067 & 0.00000 & 0.00000 \\ \text { C } & 0.70178 & -0.17149 & -1.20453 \\ \text { C } & 0.70274 & 0.16450 & 1.20519 \\ \mathrm{C} & 2.08928 & 0.16158 & 1.21073 \\ \mathrm{C} & 2.08887 & -0.16268 & -1.21020 \\ \mathrm{~N} & -1.42516 & 0.00707 & 0.04956 \\ \mathrm{C} & -2.07135 & 0.81020 & -0.95405 \\ \mathrm{C} & -2.00058 & -1.30450 & 0.21340 \\ \mathrm{~N} & 4.18227 & -0.04808 & 0.00604 \\ \mathrm{H} & 4.64920 & 0.37293 & 0.79010 \\ \mathrm{H} & 4.64868 & 0.13700 & -0.86466 \\ \mathrm{H} & 0.15214 & -0.30232 & -2.14331 \\ \mathrm{H} & 0.14098 & 0.29135 & 2.13613 \\ \mathrm{H} & 2.64548 & 0.27634 & 2.14599 \\ \mathrm{H} & 2.64494 & -0.29918 & -2.14294 \\ \mathrm{H} & -3.12965 & 1.00398 & -0.66315 \\ \mathrm{H} & -3.06738 & -1.22046 & 0.52398 \\ \mathrm{H} & -1.55786 & 1.78734 & -1.06382 \\ \mathrm{H} & -1.45596 & -1.87601 & 0.99308 \\ \mathrm{H} & -2.09049 & 0.31068 & -1.96443 \\ \mathrm{H} & -1.97089 & -1.90437 & -0.73875\end{array}$


Table S1056 Cartesian coordinates of the OM2/SCF-optimized ground-state equilibrium geometry of fluorene (ground-state energy: $-1807.92307 \mathrm{eV}$ ).

$\begin{array}{lrrr}23 & & & \\ \text { C } & 0.00000 & 0.00000 & 0.00000 \\ \text { C } & -0.94643 & 1.17322 & 0.00000 \\ \text { C } & -0.94643 & -1.17322 & -0.00000 \\ \text { C } & -2.29246 & 0.73477 & -0.00000 \\ \mathrm{C} & -2.29246 & -0.73477 & 0.00000 \\ \mathrm{C} & -0.64751 & 2.52739 & -0.00000 \\ \mathrm{C} & -0.64751 & -2.52739 & -0.00000 \\ \mathrm{C} & -3.34343 & 1.64182 & -0.00000 \\ \mathrm{C} & -3.34343 & -1.64182 & -0.00000 \\ \mathrm{C} & -1.70777 & 3.43771 & -0.00000 \\ \mathrm{C} & -1.70777 & -3.43771 & -0.00000 \\ \mathrm{C} & -3.03217 & 3.00281 & 0.00000 \\ \mathrm{C} & -3.03217 & -3.00281 & -0.00000 \\ \mathrm{H} & 0.63750 & 0.00000 & -0.90836 \\ \mathrm{H} & 0.63750 & 0.00000 & 0.90836 \\ \mathrm{H} & 0.38908 & 2.87775 & -0.00000 \\ \mathrm{H} & 0.38908 & -2.87775 & -0.00000 \\ \mathrm{H} & -4.38300 & 1.30154 & -0.00000 \\ \mathrm{H} & -4.38300 & -1.30154 & 0.00000 \\ \mathrm{H} & -1.49367 & 4.51316 & -0.00000 \\ \mathrm{H} & -1.49367 & -4.51316 & -0.00000 \\ \mathrm{H} & -3.84214 & 3.74166 & 0.00000 \\ \mathrm{H} & -3.84214 & -3.74166 & -0.00000\end{array}$


Table S1057 Cartesian coordinates of the OM2/SCF-optimized ground-state equilibrium geometry of glyoxal (ground-state energy: $-946.53822 \mathrm{eV}$ ).

$\begin{array}{lrrr}6 & & & \\ \text { C } & 0.23831 & -0.00000 & -0.00000 \\ \mathrm{C} & 1.76169 & 0.00000 & 0.00000 \\ \mathrm{O} & -0.33932 & 1.07211 & -0.00000 \\ \mathrm{O} & 2.33932 & -1.07211 & 0.00000 \\ \mathrm{H} & -0.27099 & -1.00592 & -0.00000 \\ \mathrm{H} & 2.27099 & 1.00592 & 0.00000\end{array}$


Table S1058 Cartesian coordinates of the OM2/SCF-optimized groundstate equilibrium geometry of hydrogen cyanide (ground-state energy: $-355.15537 \mathrm{eV})$.

3

$\begin{array}{llll}\mathrm{H} & 0.00000 & 0.00000 & 0.00000 \\ \mathrm{C} & 1.04586 & 0.00000 & 0.00000 \\ \mathrm{~N} & 2.21085 & 0.00000 & 0.00000\end{array}$


Table S1059 Cartesian coordinates of the OM2/SCF-optimized ground-state equilibrium geometry of formic acid (ground-state energy: $-820.00163 \mathrm{eV}$ ).

5

$\begin{array}{rrrr}\mathrm{C} & 0.00000 & 0.00000 & 0.00000 \\ \mathrm{O} & 1.35437 & 0.00000 & 0.00000 \\ \mathrm{O} & -0.58640 & 1.07608 & 0.00000 \\ \mathrm{H} & -0.42744 & -1.04553 & -0.00000 \\ \mathrm{H} & 1.69641 & 0.93253 & 0.00000\end{array}$


Table S1060 Cartesian coordinates of the OM2/SCF-optimized ground-state equilibrium geometry of hexatriene (ground-state energy: $-883.75673 \mathrm{eV}$ ).

$\begin{array}{lrrr}14 & & \\ & & & \\ \text { C } & 0.00000 & 0.67383 & 0.00000 \\ \text { C } & 0.00000 & -0.67383 & -0.00000 \\ \text { C } & 0.00000 & 1.42971 & 1.24699 \\ \text { C } & 0.00000 & -1.42971 & -1.24699 \\ \text { C } & 0.00000 & 2.76772 & 1.25167 \\ \text { C } & 0.00000 & -2.76772 & -1.25167 \\ \text { H } & 0.00000 & 1.24730 & -0.93696 \\ \text { H } & 0.00000 & -1.24730 & 0.93696 \\ \text { H } & -0.00000 & 0.85278 & 2.18257 \\ \text { H } & -0.00000 & -0.85278 & -2.18257 \\ \text { H } & 0.00000 & 3.33861 & 2.17204 \\ \text { H } & 0.00000 & -3.33861 & -2.17204 \\ \text { H } & 0.00000 & 3.34277 & 0.33268 \\ \text { H } & 0.00000 & -3.34277 & -0.33268\end{array}$


Table S1061 Cartesian coordinates of the OM2/SCF-optimized groundstate equilibrium geometry of trans-hydroquinone (ground-state energy: $-1517.19090 \mathrm{eV})$.

$\begin{array}{lrrr}\text { C } & 0.00000 & 0.00000 & 0.00000 \\ \mathrm{C} & 2.73110 & 0.00000 & 0.00000 \\ \mathrm{C} & 0.66879 & 1.23340 & 0.00000 \\ \mathrm{C} & 2.06231 & -1.23340 & 0.00000 \\ \mathrm{C} & 0.67374 & -1.22952 & -0.00000 \\ \mathrm{C} & 2.05735 & 1.22952 & -0.00000 \\ \mathrm{O} & -1.36309 & 0.08872 & 0.00000 \\ \mathrm{O} & 4.09419 & -0.08872 & 0.00000 \\ \mathrm{H} & 0.09438 & 2.15796 & -0.00000 \\ \mathrm{H} & 2.63672 & -2.15796 & 0.00000 \\ \mathrm{H} & 0.11409 & -2.16837 & -0.00000 \\ \mathrm{H} & 2.61701 & 2.16837 & -0.00000 \\ \mathrm{H} & -1.75144 & -0.82783 & 0.00000 \\ \mathrm{H} & 4.48254 & 0.82783 & -0.00000\end{array}$


Table S1062 Cartesian coordinates of the OM2/SCF-optimized ground-state equilibrium geometry of indole (ground-state energy: $-1337.80593 \mathrm{eV}$ ).

$\begin{array}{rrrr}16 & & & \\ & & & \\ \mathrm{C} & 0.00000 & 0.00000 & 0.00000 \\ \mathrm{~N} & 1.38608 & 0.00000 & 0.00000 \\ \mathrm{C} & 1.83190 & 1.31508 & 0.00000 \\ \mathrm{C} & 0.67889 & 2.16626 & 0.00000 \\ \mathrm{C} & -0.47222 & 1.30202 & 0.00000 \\ \mathrm{C} & 3.13824 & 1.82912 & -0.00000 \\ \mathrm{C} & 3.26632 & 3.20670 & -0.00000 \\ \mathrm{C} & 2.14304 & 4.05604 & -0.00000 \\ \mathrm{C} & 0.85236 & 3.55613 & -0.00000 \\ \mathrm{H} & 4.00553 & 1.16397 & -0.00000 \\ \mathrm{H} & 4.26788 & 3.65473 & -0.00000 \\ \mathrm{H} & 2.30556 & 5.14026 & -0.00000 \\ \mathrm{H} & -0.01769 & 4.22047 & -0.00000 \\ \mathrm{H} & 1.97091 & -0.82044 & -0.00000 \\ \mathrm{H} & -1.50358 & 1.61381 & -0.00000 \\ \mathrm{H} & -0.58665 & -0.91593 & -0.00000\end{array}$


Table S1063 Cartesian coordinates of the OM2/SCF-optimized groundstate equilibrium geometry of methyl-4-hydroxycinnamate (ground-state energy: $-2418.34284 \mathrm{eV})$.

$\begin{array}{lrrr}\text { C } & 0.00000 & 0.00000 & 0.00000 \\ \mathrm{C} & 1.46342 & 0.00000 & 0.00000 \\ \mathrm{C} & -0.66130 & 1.23822 & 0.00000 \\ \mathrm{C} & -0.73888 & -1.19333 & -0.00955 \\ \mathrm{C} & -2.04800 & 1.29517 & 0.00887 \\ \mathrm{C} & -2.12553 & -1.16444 & -0.00878 \\ \mathrm{C} & -2.76089 & 0.08756 & 0.00492 \\ \mathrm{C} & 2.19757 & -1.07429 & 0.33966 \\ \mathrm{C} & 3.67171 & -1.02003 & 0.32193 \\ \mathrm{O} & 4.45374 & -1.93133 & 0.59335 \\ \mathrm{O} & 4.14845 & 0.21074 & -0.04558 \\ \mathrm{C} & 5.54642 & 0.34581 & -0.08777 \\ \mathrm{O} & -4.12086 & 0.04220 & 0.00882 \\ \mathrm{H} & 1.95724 & 0.94001 & -0.28358 \\ \mathrm{H} & -0.07857 & 2.16801 & 0.00379 \\ \mathrm{H} & -0.22188 & -2.16026 & -0.02864 \\ \mathrm{H} & -2.57435 & 2.25133 & 0.01692 \\ \mathrm{H} & -2.72552 & -2.07131 & -0.01913 \\ \mathrm{H} & 1.78440 & -2.03382 & 0.64797 \\ \mathrm{H} & 5.81833 & 0.64292 & -1.12830 \\ \mathrm{H} & 5.82133 & 1.16148 & 0.62225 \\ \mathrm{H} & 6.06780 & -0.59326 & 0.18966 \\ \mathrm{H} & -4.48680 & 0.96822 & 0.01469\end{array}$


Table S1064 Cartesian coordinates of the OM2/SCF-optimized ground-state equilibrium geometry of naphthalene (ground-state energy: $-1395.14498 \mathrm{eV}$ ).

18

$\begin{array}{lrrr}\mathrm{C} & 1.00000 & 1.70707 & 0.00000 \\ \mathrm{C} & 1.00000 & 0.29293 & -0.00000 \\ \mathrm{C} & 2.23957 & 2.40435 & 0.00000 \\ \mathrm{C} & -0.23957 & 2.40435 & 0.00000 \\ \mathrm{C} & 2.23957 & -0.40435 & 0.00000 \\ \mathrm{C} & -0.23957 & -0.40435 & -0.00000 \\ \mathrm{C} & 3.42187 & 1.70755 & 0.00000 \\ \mathrm{C} & -1.42187 & 1.70755 & 0.00000 \\ \mathrm{C} & 3.42187 & 0.29245 & 0.00000 \\ \mathrm{C} & -1.42187 & 0.29245 & 0.00000 \\ \mathrm{H} & 2.23240 & 3.50136 & 0.00000 \\ \mathrm{H} & -0.23240 & 3.50136 & 0.00000 \\ \mathrm{H} & 2.23240 & -1.50136 & 0.00000 \\ \mathrm{H} & -0.23240 & -1.50136 & -0.00000 \\ \mathrm{H} & 4.38140 & 2.23734 & 0.00000 \\ \mathrm{H} & -2.38140 & 2.23734 & 0.00000 \\ \mathrm{H} & 4.38140 & -0.23734 & 0.00000 \\ \mathrm{H} & -2.38140 & -0.23734 & 0.00000\end{array}$


Table S1065 Cartesian coordinates of the OM2/SCF-optimized ground-state equilibrium geometry of octatetraene (ground-state energy: $-1168.39214 \mathrm{eV}$ ).

18

$\begin{array}{lrrr}\text { C } & 0.00000 & 0.00000 & 0.00000 \\ \text { C } & 1.33824 & 0.00000 & 0.00000 \\ \text { C } & 2.09834 & 1.24349 & 0.00000 \\ \text { C } & 3.44735 & 1.23829 & 0.00000 \\ \text { C } & 4.20811 & 2.47577 & -0.00000 \\ \text { C } & 5.55711 & 2.47057 & 0.00000 \\ \text { C } & 6.31722 & 3.71406 & -0.00000 \\ \mathrm{C} & 7.65545 & 3.71406 & 0.00000 \\ \text { H } & 4.01322 & 0.29625 & 0.00000 \\ \text { H } & 3.64224 & 3.41781 & -0.00000 \\ \text { H } & 1.52923 & 2.18294 & -0.00000 \\ \text { H } & 6.12622 & 1.53113 & 0.00000 \\ \text { H } & 1.91143 & -0.93800 & 0.00000 \\ \text { H } & 5.74402 & 4.65207 & -0.00000 \\ \text { H } & -0.57173 & 0.92103 & 0.00000 \\ \text { H } & 8.22718 & 2.79303 & 0.00000 \\ \text { H } & -0.57406 & -0.91836 & -0.00000 \\ \text { H } & 8.22952 & 4.63242 & 0.00000\end{array}$


Table S1066 Cartesian coordinates of the OM2/SCF-optimized groundstate equilibrium geometry of $o$-cyanobenzyl radical (ground-state energy: $-1321.71022 \mathrm{eV})$.

15

$\begin{array}{lrrr}\mathrm{C} & 0.00000 & 0.00000 & 0.00000 \\ \mathrm{C} & 1.42024 & 0.00000 & 0.00000 \\ \mathrm{C} & 2.12599 & 1.19207 & 0.00000 \\ \mathrm{C} & 1.45645 & 2.42268 & 0.00000 \\ \mathrm{C} & 0.06341 & 2.47028 & 0.00000 \\ \mathrm{C} & -0.64787 & 1.26925 & 0.00000 \\ \mathrm{C} & -2.08142 & 1.32172 & 0.00000 \\ \mathrm{~N} & -3.25310 & 1.37233 & 0.00000 \\ \mathrm{C} & -0.72794 & -1.19864 & -0.00000 \\ \mathrm{H} & 1.94906 & -0.95948 & -0.00000 \\ \mathrm{H} & 3.22133 & 1.17782 & 0.00000 \\ \mathrm{H} & 2.03117 & 3.35399 & 0.00000 \\ \mathrm{H} & -0.46375 & 3.42718 & 0.00000 \\ \mathrm{H} & -1.80753 & -1.19808 & -0.00000 \\ \mathrm{H} & -0.21677 & -2.15226 & -0.00000\end{array}$


Table S1067 Cartesian coordinates of the OM2/SCF-optimized ground-state equilibrium geometry of oxalylfluoride (ground-state energy: $-1849.20250 \mathrm{eV}$ ).

$\begin{array}{lrrr}6 & & & \\ C & 0.00000 & 0.00000 & 0.00000 \\ C & 1.52958 & 0.00000 & 0.00000 \\ O & -0.73756 & 0.96045 & 0.00000 \\ 0 & 2.26713 & -0.05719 & 0.95874 \\ \text { F } & -0.52890 & -1.24090 & 0.03063 \\ \text { F } & 2.05848 & 0.10446 & -1.23688\end{array}$


Table S1068 Cartesian coordinates of the OM2/SCF-optimized ground-state equilibrium geometry of benzoquinone (ground-state energy: $-1486.15480 \mathrm{eV}$ ).

12

$\begin{array}{lrrr}\text { C } & 0.00000 & 1.42730 & 0.00000 \\ \mathrm{C} & 0.00000 & -1.42730 & -0.00000 \\ \mathrm{C} & 1.27859 & 0.67003 & 0.00000 \\ \mathrm{C} & -1.27859 & 0.67003 & -0.00000 \\ \mathrm{C} & 1.27859 & -0.67003 & -0.00000 \\ \mathrm{C} & -1.27859 & -0.67003 & 0.00000 \\ \mathrm{O} & 0.00000 & 2.65438 & 0.00000 \\ \mathrm{O} & 0.00000 & -2.65438 & -0.00000 \\ \mathrm{H} & 2.19215 & 1.26799 & -0.00000 \\ \mathrm{H} & -2.19215 & 1.26799 & -0.00000 \\ \mathrm{H} & 2.19215 & -1.26799 & -0.00000 \\ \mathrm{H} & -2.19215 & -1.26799 & -0.00000\end{array}$


Table S1069 Cartesian coordinates of the OM2/SCF-optimized groundstate equilibrium geometry of diethynylbenzene (ground-state energy: $-1361.10914 \mathrm{eV})$.

$\begin{array}{lrrr}\text { C } & 0.00000 & 0.00000 & 1.37243 \\ \text { C } & 0.00000 & 0.00000 & -1.37243 \\ \text { C } & 0.00000 & 1.22652 & 0.69464 \\ \text { C } & -0.00000 & -1.22652 & 0.69464 \\ \text { C } & 0.00000 & -1.22652 & -0.69464 \\ \text { C } & 0.00000 & 1.22652 & -0.69464 \\ \text { C } & 0.00000 & -0.00000 & 2.80179 \\ \text { C } & 0.00000 & 0.00000 & -2.80179 \\ \text { C } & 0.00000 & -0.00000 & 4.02865 \\ \text { C } & 0.00000 & 0.00000 & -4.02865 \\ \text { H } & 0.00000 & -0.00000 & 5.06894 \\ \text { H } & 0.00000 & 0.00000 & -5.06894 \\ \text { H } & 0.00000 & 2.16359 & 1.25563 \\ \text { H } & -0.00000 & -2.16359 & 1.25563 \\ \text { H } & 0.00000 & -2.16359 & -1.25563 \\ \text { H } & 0.00000 & 2.16359 & -1.25563\end{array}$


Table S1070 Cartesian coordinates of the OM2/SCF-optimized groundstate equilibrium geometry of $p$-phenylenediamine (ground-state energy: $-1311.54560 \mathrm{eV})$.

$\begin{array}{lrrr}\text { C } & 0.00000 & 0.00000 & 0.00000 \\ \text { C } & 2.79292 & 0.00000 & 0.00000 \\ \text { C } & 0.70309 & -0.00106 & -1.21837 \\ \text { C } & 0.70309 & -0.00106 & 1.21837 \\ \text { C } & 2.08983 & 0.00106 & -1.21837 \\ \text { C } & 2.08983 & 0.00106 & 1.21837 \\ \text { N } & -1.39738 & 0.05788 & 0.00000 \\ \text { N } & 4.19029 & -0.05788 & 0.00000 \\ \text { H } & 0.14899 & 0.00850 & -2.16286 \\ \text { H } & 0.14899 & 0.00850 & 2.16286 \\ \text { H } & 2.64393 & -0.00850 & -2.16286 \\ \text { H } & 2.64393 & -0.00850 & 2.16286 \\ \text { H } & -1.84941 & -0.28600 & -0.83275 \\ \text { H } & -1.84941 & -0.28600 & 0.83275 \\ \text { H } & 4.64233 & 0.28600 & -0.83275 \\ \text { H } & 4.64233 & 0.28600 & 0.83275\end{array}$


Table S1071 Cartesian coordinates of the OM2/SCF-optimized ground-state equilibrium geometry of phenol (ground-state energy: $-1186.16110 \mathrm{eV}$ ).

$\begin{array}{lrrr}13 & & & \\ \text { C } & 0.00000 & 0.00000 & 0.00000 \\ \text { C } & 1.39125 & 0.00000 & 0.00000 \\ \text { C } & 2.05068 & 1.23542 & 0.00000 \\ \text { C } & 1.36216 & 2.45783 & 0.00000 \\ \text { C } & -0.02620 & 2.41893 & 0.00000 \\ \text { C } & -0.70657 & 1.20074 & 0.00000 \\ \text { O } & 3.41073 & 1.33839 & 0.00000 \\ \text { H } & 1.91968 & 3.39198 & 0.00000 \\ \text { H } & -0.59249 & 3.35831 & 0.00000 \\ \text { H } & -1.80032 & 1.18812 & 0.00000 \\ \text { H } & -0.54162 & -0.95371 & -0.00000 \\ \text { H } & 1.95696 & -0.93429 & -0.00000 \\ \text { H } & 3.81156 & 0.42708 & 0.00000\end{array}$


Table S1072 Cartesian coordinates of the OM2/SCF-optimized ground-state equilibrium geometry of porphine (ground-state energy: $-3608.24638 \mathrm{eV}$ ).

$\begin{array}{rrrr}\text { N } & 0.00000 & 0.00000 & 0.00000 \\ \mathrm{~N} & 4.12716 & 0.00000 & 0.00000 \\ \mathrm{C} & -0.80010 & 0.00000 & 1.11963 \\ \mathrm{C} & -0.80010 & -0.00000 & -1.11963 \\ \mathrm{C} & 4.92496 & 0.00000 & 1.13342 \\ \mathrm{C} & 4.92496 & 0.00000 & -1.13342 \\ \mathrm{C} & -2.16460 & 0.00000 & 0.69562 \\ \mathrm{C} & -2.16460 & -0.00000 & -0.69561 \\ \mathrm{C} & 6.33303 & -0.00000 & 0.67701 \\ \mathrm{C} & 6.33304 & 0.00000 & -0.67700 \\ \mathrm{C} & -0.35201 & 0.00000 & 2.47458 \\ \mathrm{C} & -0.35201 & -0.00000 & -2.47458 \\ \mathrm{C} & 4.51927 & 0.00000 & 2.44255 \\ \mathrm{C} & 4.51927 & 0.00000 & -2.44255 \\ \mathrm{C} & 0.96435 & 0.00000 & 2.84742 \\ \mathrm{C} & 0.96435 & -0.00000 & -2.84742 \\ \mathrm{C} & 3.13913 & 0.00000 & 2.81862 \\ \mathrm{C} & 3.13913 & 0.00000 & -2.81862 \\ \mathrm{~N} & 2.08532 & 0.00000 & 2.01299 \\ \mathrm{~N} & 2.08532 & -0.00000 & -2.01299 \\ \mathrm{C} & 1.39323 & 0.00000 & 4.26864 \\ \mathrm{C} & 1.39323 & -0.00000 & -4.26864 \\ \mathrm{C} & 2.74624 & 0.00000 & 4.25225 \\ \mathrm{C} & 2.74624 & 0.00000 & -4.25225 \\ \mathrm{H} & 1.01871 & 0.00000 & 0.00000 \\ \mathrm{H} & 3.11285 & -0.00000 & 0.00000 \\ \mathrm{H} & -3.02096 & 0.00000 & 1.35347 \\ \mathrm{H} & -3.02096 & -0.00000 & -1.35346 \\ \mathrm{H} & 7.17525 & -0.00000 & 1.35505 \\ \mathrm{H} & 7.17525 & 0.00000 & -1.35505 \\ \mathrm{H} & -1.13419 & 0.00000 & 3.24657 \\ \mathrm{H} & -1.13419 & -0.00000 & -3.24657 \\ \mathrm{H} & 5.26683 & 0.00000 & 3.23803 \\ \mathrm{H} & 5.26683 & 0.00000 & -3.23803 \\ \mathrm{H} & 0.71593 & 0.00000 & 5.11151 \\ \mathrm{H} & 0.71593 & -0.00000 & -5.11151 \\ \mathrm{H} & 3.44364 & 0.00000 & 5.07689 \\ \mathrm{H} & 3.44364 & 0.00000 & -5.07689\end{array}$


Table S1073 Cartesian coordinates of the OM2/SCF-optimized ground-state equilibrium geometry of propynal (ground-state energy: $-741.26682 \mathrm{eV}$ ).

$\begin{array}{lrrr}\text { H } & 0.00000 & 0.00000 & 0.00000 \\ \text { C } & 1.04396 & 0.00000 & 0.00000 \\ \text { C } & 2.27105 & 0.00000 & -0.00000 \\ \text { C } & 3.72083 & 0.00000 & 0.02386 \\ \text { O } & 4.30090 & 0.00000 & 1.09682 \\ \text { H } & 4.20961 & 0.00000 & -0.99536\end{array}$


Table S1074 Cartesian coordinates of the OM2/SCF-optimized ground-state equilibrium geometry of pyrene (ground-state energy: $-2190.54344 \mathrm{eV}$ ).

$\begin{array}{lrrr}\text { 26 } & & & \\ \text { C } & 0.00000 & 0.71507 & 0.00000 \\ \text { C } & 0.00000 & -0.71507 & -0.00000 \\ \text { C } & 0.00000 & 1.42222 & -1.22648 \\ \text { C } & 0.00000 & 1.42222 & 1.22648 \\ \text { C } & 0.00000 & -1.42222 & -1.22648 \\ \text { C } & 0.00000 & -1.42222 & 1.22648 \\ \text { C } & 0.00000 & 0.67884 & -2.46434 \\ \text { C } & 0.00000 & 0.67884 & 2.46434 \\ \text { C } & 0.00000 & -0.67884 & -2.46434 \\ \text { C } & 0.00000 & -0.67884 & 2.46434 \\ \text { C } & 0.00000 & 2.82353 & -1.21146 \\ \text { C } & 0.00000 & 2.82353 & 1.21146 \\ \text { C } & 0.00000 & -2.82353 & -1.21146 \\ \text { C } & 0.00000 & -2.82353 & 1.21146 \\ \text { C } & 0.00000 & 3.50944 & 0.00000 \\ \text { C } & 0.00000 & -3.50944 & -0.00000 \\ \text { H } & 0.00000 & 1.24615 & -3.40365 \\ \text { H } & 0.00000 & 1.24615 & 3.40365 \\ \text { H } & 0.00000 & -1.24615 & -3.40365 \\ \text { H } & 0.00000 & -1.24615 & 3.40365 \\ \text { H } & 0.00000 & 3.37841 & -2.15707 \\ \text { H } & 0.00000 & 3.37841 & 2.15707 \\ \text { H } & 0.00000 & -3.37841 & -2.15707 \\ \text { H } & 0.00000 & -3.37841 & 2.15707 \\ \text { H } & 0.00000 & 4.60585 & 0.00000 \\ \text { H } & 0.00000 & -4.60585 & -0.00000\end{array}$


Table S1075 Cartesian coordinates of the OM2/SCF-optimized ground-state equilibrium geometry of pyridine (ground-state energy: $-925.54092 \mathrm{eV}$ ).

11

$\begin{array}{llrr}\text { C } & 0.00000 & 1.40822 & 0.00000 \\ \text { C } & 0.00000 & 0.70042 & -1.19768 \\ \text { C } & 0.00000 & 0.70042 & 1.19768 \\ \text { C } & 0.00000 & -0.69973 & -1.14544 \\ \text { C } & 0.00000 & -0.69973 & 1.14544 \\ \text { N } & 0.00000 & -1.37887 & -0.00000 \\ \text { H } & 0.00000 & 2.50371 & 0.00000 \\ \text { H } & 0.00000 & 1.22358 & -2.15617 \\ \text { H } & 0.00000 & 1.22358 & 2.15617 \\ \text { H } & 0.00000 & -1.27416 & -2.08947 \\ \text { H } & 0.00000 & -1.27416 & 2.08947\end{array}$


Table S1076 Cartesian coordinates of the OM2/SCF-optimized groundstate equilibrium geometry of pyridone lactam (ground-state energy: $-1256.65368 \mathrm{eV})$.

$\begin{array}{rrrr}\mathrm{C} & 0.00000 & 0.00000 & 0.00000 \\ \mathrm{~N} & 1.36259 & 0.00000 & 0.00000 \\ \mathrm{C} & 2.13449 & 1.17864 & 0.00000 \\ \mathrm{C} & 1.39123 & 2.42896 & 0.00000 \\ \mathrm{C} & 0.03034 & 2.41666 & 0.00000 \\ \mathrm{C} & -0.69048 & 1.18826 & 0.00000 \\ \mathrm{O} & 3.37399 & 1.05964 & 0.00000 \\ \mathrm{H} & 1.97930 & 3.34261 & 0.00000 \\ \mathrm{H} & -0.54109 & 3.35369 & 0.00000 \\ \mathrm{H} & -1.77785 & 1.19756 & 0.00000 \\ \mathrm{H} & -0.53234 & -0.96616 & -0.00000 \\ \mathrm{H} & 1.86537 & -0.88426 & -0.00000\end{array}$


Table S1077 Cartesian coordinates of the OM2/SCF-optimized groundstate equilibrium geometry of pyridone lactim (ground-state energy: $-1256.71246 \mathrm{eV})$.

$\begin{array}{rrrr}\mathrm{C} & 0.00000 & 0.00000 & 0.00000 \\ \mathrm{~N} & 1.33357 & 0.00000 & 0.00000 \\ \mathrm{C} & 1.94026 & 1.19242 & 0.00000 \\ \mathrm{C} & 1.29493 & 2.44653 & 0.00000 \\ \mathrm{C} & -0.09136 & 2.40916 & 0.00000 \\ \mathrm{C} & -0.75394 & 1.17959 & 0.00000 \\ \mathrm{O} & 3.29983 & 1.21277 & 0.00000 \\ \mathrm{H} & 1.86877 & 3.36676 & 0.00000 \\ \mathrm{H} & -0.66519 & 3.34302 & 0.00000 \\ \mathrm{H} & -1.84298 & 1.13956 & 0.00000 \\ \mathrm{H} & -0.51865 & -0.97562 & -0.00000 \\ \mathrm{H} & 3.63349 & 0.27439 & 0.00000\end{array}$


Table S1078 Cartesian coordinates of the OM2/SCF-optimized ground-state equilibrium geometry of pyrimidine (ground-state energy: $-995.93937 \mathrm{eV}$ ).

$\begin{array}{lrrr}10 & & \\ \text { C } & 0.00000 & 1.29562 & 0.00000 \\ \text { N } & 0.00000 & 0.67561 & -1.18889 \\ \text { N } & 0.00000 & 0.67561 & 1.18889 \\ \text { C } & 0.00000 & -0.65480 & -1.19016 \\ \text { C } & 0.00000 & -0.65480 & 1.19016 \\ \text { C } & 0.00000 & -1.39080 & -0.00000 \\ \text { H } & 0.00000 & 2.40848 & 0.00000 \\ \text { H } & 0.00000 & -1.18017 & -2.16175 \\ \text { H } & 0.00000 & -1.18017 & 2.16175 \\ \text { H } & 0.00000 & -2.47784 & -0.00000\end{array}$


Table S1079 Cartesian coordinates of the OM2/SCF-optimized ground-state equilibrium geometry of quinoline (ground-state energy: $-1465.53555 \mathrm{eV}$ ).

17

$\begin{array}{lrrr}\mathrm{C} & 0.00000 & 0.00000 & 0.00000 \\ \mathrm{C} & 1.42802 & 0.00000 & 0.00000 \\ \mathrm{C} & 2.11880 & 1.24011 & 0.00000 \\ \mathrm{C} & 1.38604 & 2.45596 & 0.00000 \\ \mathrm{C} & 0.01353 & 2.42434 & 0.00000 \\ \mathrm{C} & -0.68069 & 1.19082 & 0.00000 \\ \mathrm{~N} & 2.08361 & -1.19842 & -0.00000 \\ \mathrm{C} & 3.39180 & -1.19500 & 0.00000 \\ \mathrm{C} & 4.17627 & -0.00269 & 0.00000 \\ \mathrm{C} & 3.54034 & 1.21082 & 0.00000 \\ \mathrm{H} & 1.93077 & 3.40760 & 0.00000 \\ \mathrm{H} & -0.56313 & 3.35645 & 0.00000 \\ \mathrm{H} & -1.77658 & 1.20260 & 0.00000 \\ \mathrm{H} & -0.51612 & -0.96321 & -0.00000 \\ \mathrm{H} & 4.10134 & 2.15325 & 0.00000 \\ \mathrm{H} & 3.92800 & -2.16354 & 0.00000 \\ \mathrm{H} & 5.26586 & -0.07441 & 0.00000\end{array}$


Table S1080 Cartesian coordinates of the OM2/SCF-optimized ground-state equilibrium geometry of quinoxaline (ground-state energy: $-1535.83453 \mathrm{eV}$ ).

$\begin{array}{lrrr}16 & & & \\ \text { C } & 0.00000 & 3.58170 & 0.70780 \\ \text { C } & 0.00000 & 3.58170 & -0.70780 \\ \text { C } & 0.00000 & 2.40599 & 1.41576 \\ \text { C } & 0.00000 & 2.40599 & -1.41576 \\ \text { C } & 0.00000 & 1.16669 & 0.71200 \\ \text { C } & 0.00000 & 1.16669 & -0.71200 \\ \text { N } & 0.00000 & -0.00728 & -1.41334 \\ \text { N } & 0.00000 & -0.00728 & 1.41334 \\ \text { C } & 0.00000 & -1.11390 & -0.72035 \\ \text { C } & 0.00000 & -1.11390 & 0.72035 \\ \text { H } & 0.00000 & 4.54257 & 1.23483 \\ \text { H } & 0.00000 & 4.54257 & -1.23483 \\ \text { H } & 0.00000 & 2.38335 & 2.50795 \\ \text { H } & 0.00000 & 2.38335 & -2.50795 \\ \text { H } & 0.00000 & -2.08456 & -1.24450 \\ \text { H } & 0.00000 & -2.08456 & 1.24450\end{array}$


Table S1081 Cartesian coordinates of the OM2/SCF-optimized ground-state equilibrium geometry of styrene (ground-state energy: $-1139.66268 \mathrm{eV}$ ).

$\begin{array}{rrrr}\text { 16 } & & & \\ \text { C } & 0.00000 & 0.00000 & 0.00000 \\ \text { C } & 1.39954 & 0.00000 & 0.00000 \\ \text { C } & 2.09431 & 1.20579 & 0.00000 \\ \text { C } & 1.40435 & 2.41515 & 0.00000 \\ \text { C } & 0.01072 & 2.42060 & 0.00000 \\ \text { C } & -0.69062 & 1.22082 & 0.00000 \\ \text { C } & -0.78564 & -1.24271 & -0.00000 \\ \text { C } & -0.25062 & -2.46724 & -0.00000 \\ \text { H } & 1.95370 & -0.94601 & -0.00000 \\ \text { H } & 3.19066 & 1.20317 & 0.00000 \\ \text { H } & 1.95605 & 3.36173 & 0.00000 \\ \text { H } & -0.53163 & 3.37309 & 0.00000 \\ \text { H } & -1.78806 & 1.22705 & 0.00000 \\ \text { H } & -1.88001 & -1.11201 & -0.00000 \\ \text { H } & 0.81967 & -2.63751 & -0.00000 \\ \text { H } & -0.86808 & -3.35735 & -0.00000\end{array}$


Table S1082 Cartesian coordinates of the OM2/SCF-optimized groundstate equilibrium geometry of syn-coumarin 153 (ground-state energy: $-4525.83696 \mathrm{eV})$.

$\begin{array}{lrrr}\mathrm{C} & 0.00000 & 0.00000 & 0.00000 \\ \mathrm{C} & 1.49481 & 0.00000 & 0.00000 \\ \mathrm{C} & 2.24848 & 1.19110 & 0.00000 \\ \mathrm{~N} & 1.59579 & 2.41536 & -0.03320 \\ \mathrm{C} & 0.16256 & 2.47230 & 0.15048 \\ \mathrm{C} & -0.49985 & 1.30846 & -0.58380 \\ \mathrm{C} & 3.67773 & 1.13701 & 0.01331 \\ \mathrm{C} & 4.47703 & 2.40554 & 0.04022 \\ \mathrm{C} & 3.67998 & 3.53116 & -0.59049 \\ \mathrm{C} & 2.33694 & 3.64412 & 0.12614 \\ \mathrm{C} & 2.20026 & -1.21757 & 0.01657 \\ \mathrm{C} & 3.60904 & -1.28681 & 0.02802 \\ \mathrm{C} & 4.33632 & -0.08003 & 0.02705 \\ \mathrm{C} & 4.20959 & -2.59386 & 0.03984 \\ \mathrm{C} & 3.45187 & -3.71673 & 0.03725 \\ \mathrm{C} & 1.99372 & -3.60265 & 0.02180 \\ \mathrm{O} & 1.43311 & -2.33693 & 0.01474 \\ \mathrm{O} & 1.15516 & -4.49818 & 0.01403 \\ \mathrm{C} & 5.71639 & -2.70497 & 0.05476 \\ \mathrm{~F} & 6.17142 & -3.98898 & 0.06513 \\ \mathrm{~F} & 6.31949 & -2.12550 & -1.02184 \\ \mathrm{~F} & 6.29875 & -2.11533 & 1.13719 \\ \mathrm{H} & 3.85641 & -4.72264 & 0.04543 \\ \mathrm{H} & 5.43072 & -0.10624 & 0.03761 \\ \mathrm{H} & 4.71880 & 2.65453 & 1.10314 \\ \mathrm{H} & 5.43697 & 2.26359 & -0.51088 \\ \mathrm{H} & 4.23448 & 4.49177 & -0.50169 \\ \mathrm{H} & 3.50549 & 3.31650 & -1.66756 \\ \mathrm{H} & 2.50638 & 3.87960 & 1.22515 \\ \mathrm{H} & 1.74977 & 4.50250 & -0.31336 \\ \mathrm{H} & -0.37675 & -0.85943 & -0.59531 \\ \mathrm{H} & -0.35923 & -0.11934 & 1.05167 \\ \mathrm{H} & -1.60483 & 1.37872 & -0.47528 \\ \mathrm{H} & -0.24259 & 1.36503 & -1.66485 \\ \mathrm{H} & -0.23400 & 3.44668 & -0.25903 \\ \mathrm{H} & -0.10687 & 2.43458 & 1.25325\end{array}$


Table S1083 Cartesian coordinates of the OM2/SCF-optimized ground-state equilibrium geometry of terylene (ground-state energy: $-4066.06048 \mathrm{eV}$ ).

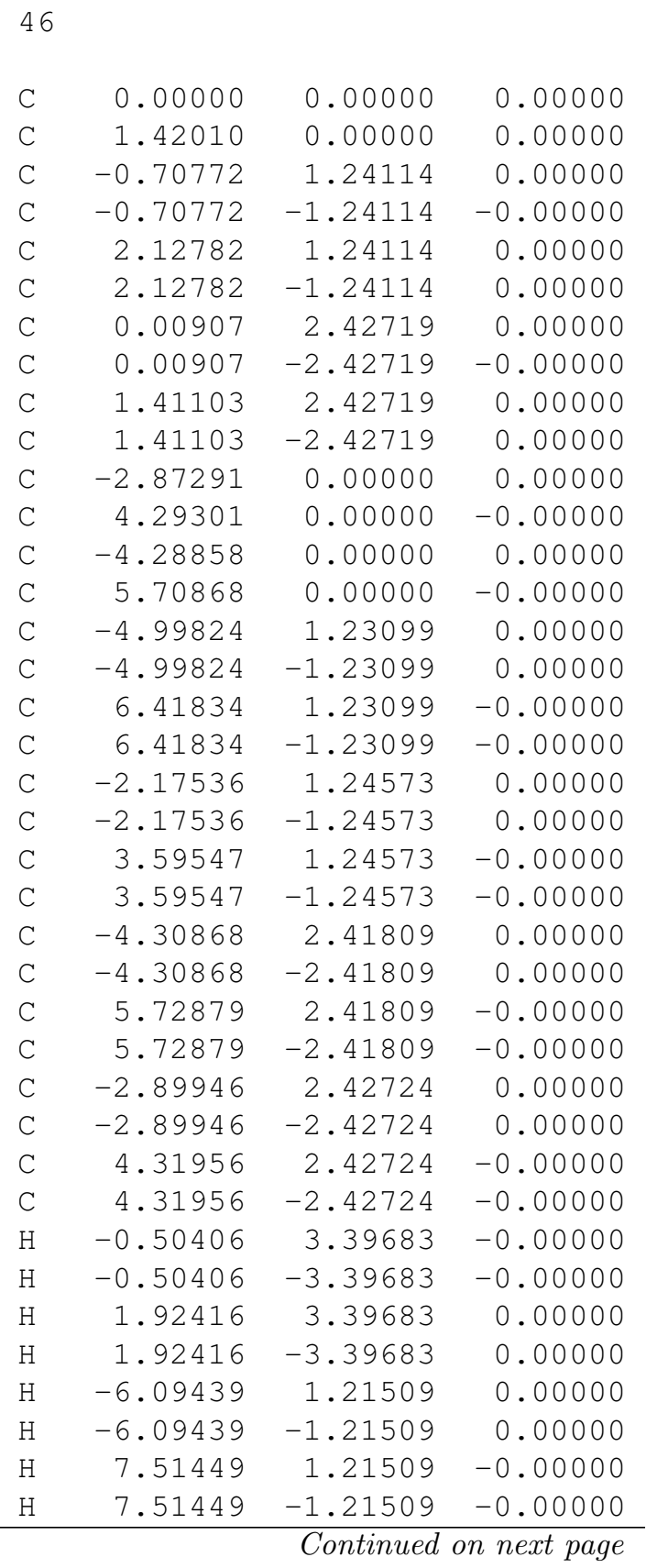

S1136 


\begin{tabular}{lrrr}
\multicolumn{5}{c}{ Continued from previous page } \\
\hline $\mathrm{H}$ & -4.84571 & 3.37356 & 0.00000 \\
$\mathrm{H}$ & -4.84571 & -3.37356 & 0.00000 \\
$\mathrm{H}$ & 6.26581 & 3.37356 & -0.00000 \\
$\mathrm{H}$ & 6.26581 & -3.37356 & -0.00000 \\
$\mathrm{H}$ & -2.39478 & 3.40131 & 0.00000 \\
$\mathrm{H}$ & -2.39478 & -3.40131 & -0.00000 \\
$\mathrm{H}$ & 3.81488 & 3.40131 & -0.00000 \\
$\mathrm{H}$ & 3.81488 & -3.40131 & 0.00000
\end{tabular}


Table S1084 Cartesian coordinates of the OM2/SCF-optimized ground-state equilibrium geometry of $s$-tetrazine (ground-state energy: $-1136.06315 \mathrm{eV}$ ).

$\begin{array}{lrrr}8 & & \\ & & & \\ \mathrm{C} & 0.00000 & 0.00000 & 1.28676 \\ \mathrm{C} & 0.00000 & 0.00000 & -1.28676 \\ \mathrm{~N} & 0.00000 & 1.18252 & 0.62687 \\ \mathrm{~N} & -0.00000 & -1.18252 & 0.62687 \\ \mathrm{~N} & 0.00000 & 1.18252 & -0.62687 \\ \mathrm{~N} & 0.00000 & -1.18252 & -0.62687 \\ \mathrm{H} & 0.00000 & 0.00000 & 2.38788 \\ \mathrm{H} & 0.00000 & 0.00000 & -2.38788\end{array}$


Table S1085 Cartesian coordinates of the OM2/SCF-optimized ground-state equilibrium geometry of toluene (ground-state energy: $-1013.02515 \mathrm{eV}$ ).

$\begin{array}{lrrr}15 & & & \\ \text { C } & 0.00000 & 0.00000 & 0.00000 \\ \text { C } & 0.00000 & 2.79084 & 0.00000 \\ \text { C } & -0.00042 & 0.70236 & 1.20972 \\ \text { C } & -0.00042 & 0.70236 & -1.20972 \\ \text { C } & -0.00035 & 2.09370 & -1.20587 \\ \text { C } & -0.00035 & 2.09370 & 1.20587 \\ \mathrm{C} & 0.02623 & -1.49506 & -0.00000 \\ \text { H } & -0.00122 & 3.88637 & 0.00000 \\ \text { H } & -0.00157 & 0.15480 & 2.15957 \\ \text { H } & -0.00157 & 0.15480 & -2.15957 \\ \text { H } & -0.00125 & 2.64355 & -2.15465 \\ \text { H } & -0.00125 & 2.64355 & 2.15465 \\ \text { H } & 1.07459 & -1.83576 & -0.00000 \\ \text { H } & -0.47722 & -1.89064 & 0.89575 \\ \text { H } & -0.47722 & -1.89064 & -0.89575\end{array}$


Table S1086 Cartesian coordinates of the OM2/SCF-optimized ground-state equilibrium geometry of stilbene (ground-state energy: $-1964.83029 \mathrm{eV}$ ).

$\begin{array}{lrrr}\text { C6 } & & & \\ \text { C } & 0.00000 & 0.00000 & 0.00000 \\ \text { C } & 1.34501 & 0.00000 & 0.00000 \\ \text { C } & -0.78282 & 1.24129 & 0.00000 \\ \text { C } & 2.12783 & -1.24128 & 0.00525 \\ \text { C } & -0.30560 & 2.39837 & -0.62773 \\ \text { C } & 1.65061 & -2.40101 & -0.61757 \\ \text { C } & -2.03357 & 1.25671 & 0.63227 \\ \mathrm{C} & 3.37858 & -1.25402 & 0.63758 \\ \text { C } & -1.07065 & 3.56042 & -0.60938 \\ \mathrm{C} & 2.41566 & -3.56297 & -0.59431 \\ \mathrm{C} & -2.78784 & 2.42425 & 0.64901 \\ \mathrm{C} & 4.13284 & -2.42148 & 0.65927 \\ \mathrm{C} & -2.30805 & 3.57676 & 0.02946 \\ \mathrm{C} & 3.65306 & -3.57660 & 0.04460 \\ \mathrm{H} & -0.57293 & -0.93669 & 0.02706 \\ \mathrm{H} & 1.91794 & 0.93680 & 0.02309 \\ \mathrm{H} & 0.66218 & 2.38642 & -1.14149 \\ \mathrm{H} & 0.68282 & -2.39123 & -1.13138 \\ \mathrm{H} & -2.40882 & 0.35047 & 1.12167 \\ \mathrm{H} & 3.75382 & -0.34572 & 1.12314 \\ \mathrm{H} & -0.69906 & 4.46651 & -1.10229 \\ \mathrm{H} & 2.04407 & -4.47114 & -1.08338 \\ \mathrm{H} & -3.76297 & 2.43940 & 1.14959 \\ \mathrm{H} & 5.10797 & -2.43451 & 1.15991 \\ \mathrm{H} & -2.90627 & 4.49454 & 0.04197 \\ \mathrm{H} & 4.25127 & -4.49433 & 0.06099\end{array}$


Table S1087 Cartesian coordinates of the OM2/SCF-optimized ground-state equilibrium geometry of vinyl radical (ground-state energy: $-297.43236 \mathrm{eV}$ ).

$\begin{array}{lrrr}\mathrm{C} & 0.00000 & 0.00000 & 0.00000 \\ \mathrm{C} & 1.30496 & 0.00000 & 0.00000 \\ \mathrm{H} & -0.59366 & 0.90975 & 0.00000 \\ \mathrm{H} & -0.55117 & -0.92982 & 0.00000 \\ \mathrm{H} & 2.15956 & 0.64672 & 0.00000\end{array}$


4.2.2 OM2/ROHF-Optimized Excited-State Minima 
Table S1088 Cartesian coordinates of the OM2/ROHF-optimized excited-state equilibrium geometry of the $2{ }^{1} A\left({ }^{1} n \pi^{*}\right)$ state of 2-cyclopenten-1-one (excitedstate energy: $-1055.29227 \mathrm{eV})$.

$\begin{array}{lrrr}\mathrm{C} & 0.00000 & 0.00000 & 0.00000 \\ \mathrm{C} & 1.53180 & 0.00000 & 0.00000 \\ \mathrm{C} & 1.88315 & 1.49895 & 0.00000 \\ \mathrm{C} & 0.70570 & 2.26917 & 0.00011 \\ \mathrm{C} & -0.39404 & 1.43930 & 0.00013 \\ \mathrm{O} & 3.11503 & 1.79839 & -0.00005 \\ \mathrm{H} & 0.72966 & 3.35053 & 0.00014 \\ \mathrm{H} & -1.42730 & 1.75785 & 0.00021 \\ \mathrm{H} & 1.97212 & -0.45420 & -0.91100 \\ \mathrm{H} & 1.97210 & -0.45416 & 0.91103 \\ \mathrm{H} & -0.39925 & -0.51404 & -0.90346 \\ \mathrm{H} & -0.39926 & -0.51422 & 0.90335\end{array}$


Table S1089 Cartesian coordinates of the OM2/ROHF-optimized excited-state equilibrium geometry of the $2{ }^{1} A\left({ }^{1} \pi \pi^{*}\right)$ state of $\beta$-dinaphthyleneoxide (excitedstate energy: $-3056.40545 \mathrm{eV})$.

33

$\begin{array}{lrrr}\mathrm{C} & 0.00000 & 0.00000 & 0.00000 \\ \mathrm{C} & 1.39039 & 0.00000 & 0.00000 \\ \mathrm{C} & 2.09024 & 1.21357 & 0.00000 \\ \mathrm{C} & 1.38041 & 2.44776 & 0.11455 \\ \mathrm{C} & -0.03873 & 2.41390 & 0.07718 \\ \mathrm{C} & -0.70994 & 1.20944 & 0.00794 \\ \mathrm{C} & 3.52329 & 1.29543 & -0.04223 \\ \mathrm{C} & 4.12971 & 2.56084 & 0.30476 \\ \mathrm{C} & 3.43474 & 3.75713 & 0.45759 \\ \mathrm{C} & 2.05272 & 3.67901 & 0.32444 \\ \mathrm{O} & 5.50331 & 2.47743 & 0.37498 \\ \mathrm{C} & 5.78888 & 1.17851 & 0.01452 \\ \mathrm{C} & 4.60097 & 0.43936 & -0.34876 \\ \mathrm{C} & 4.78000 & -0.87970 & -0.88738 \\ \mathrm{C} & 6.08391 & -1.45021 & -0.76673 \\ \mathrm{C} & 7.18384 & -0.70160 & -0.27508 \\ \mathrm{C} & 7.06768 & 0.63443 & 0.09282 \\ \mathrm{C} & 6.27898 & -2.78383 & -1.21388 \\ \mathrm{C} & 5.25004 & -3.47866 & -1.81764 \\ \mathrm{C} & 4.00423 & -2.86789 & -2.02189 \\ \mathrm{C} & 3.77023 & -1.57603 & -1.56417 \\ \mathrm{H} & 7.91532 & 1.23310 & 0.41367 \\ \mathrm{H} & 8.16681 & -1.18668 & -0.22192 \\ \mathrm{H} & 2.81188 & -1.09059 & -1.76467 \\ \mathrm{H} & 7.26093 & -3.25346 & -1.07617 \\ \mathrm{H} & 5.40676 & -4.50854 & -2.15805 \\ \mathrm{H} & 3.21268 & -3.41422 & -2.54589 \\ \mathrm{H} & 3.95396 & 4.68545 & 0.67780 \\ \mathrm{H} & 1.45408 & 4.59344 & 0.42343 \\ \mathrm{H} & 1.93670 & -0.94574 & 0.03737 \\ \mathrm{H} & -0.59500 & 3.35908 & 0.10801 \\ \mathrm{H} & -1.80527 & 1.19073 & -0.02318 \\ \mathrm{H} & -0.54690 & -0.94893 & -0.00583\end{array}$


Table S1090 Cartesian coordinates of the OM2/ROHF-optimized excited-state equilibrium geometry of the $2{ }^{1} A\left({ }^{1} n \pi^{*}\right)$ state of acetaldehyde (excited-state energy: $-642.98870 \mathrm{eV}$ ).

7

$\begin{array}{rrrr}\mathrm{C} & 0.00000 & 0.00000 & 0.00000 \\ \mathrm{C} & 1.49182 & 0.00000 & 0.00000 \\ \mathrm{O} & 2.01653 & 1.17142 & 0.00000 \\ \mathrm{H} & -0.36821 & 0.26749 & 1.00148 \\ \mathrm{H} & -0.35075 & -1.00245 & -0.27449 \\ \mathrm{H} & -0.35984 & 0.73550 & -0.72946 \\ \mathrm{H} & 2.02984 & -0.81884 & 0.48568\end{array}$


Table S1091 Cartesian coordinates of the OM2/ROHF-optimized excited-state equilibrium geometry of the $2{ }^{1} A\left({ }^{1} n \pi^{*}\right)$ state of acetone (excited-state energy: $-800.99372 \mathrm{eV})$.

10

$\begin{array}{lrrr}\mathrm{C} & 0.00000 & 0.00000 & 0.00000 \\ \mathrm{C} & 1.49616 & 0.00000 & 0.00000 \\ \mathrm{O} & 2.02970 & 1.17700 & 0.00000 \\ \mathrm{C} & 2.23274 & -1.01213 & 0.81947 \\ \mathrm{H} & -0.35830 & 0.67206 & -0.78952 \\ \mathrm{H} & 3.29620 & -0.98458 & 0.55178 \\ \mathrm{H} & -0.37444 & 0.35060 & 0.97432 \\ \mathrm{H} & -0.35346 & -1.02186 & -0.18994 \\ \mathrm{H} & 2.12060 & -0.78044 & 1.89007 \\ \mathrm{H} & 1.81951 & -2.00690 & 0.60759\end{array}$


Table S1092 Cartesian coordinates of the OM2/ROHF-optimized excited-state equilibrium geometry of the $1{ }^{1} A^{\prime \prime}\left({ }^{1} n \pi^{*}\right)$ state of acrolein (excited-state energy: $-769.73661 \mathrm{eV})$.

8

$\begin{array}{lrrr}\text { C } & 0.00000 & 0.00000 & 0.00000 \\ \text { C } & 1.36224 & 0.00000 & 0.00000 \\ \text { C } & 2.07398 & 1.21284 & 0.00000 \\ \text { O } & 1.46873 & 2.35234 & 0.00000 \\ \text { H } & 3.17245 & 1.27645 & 0.00000 \\ \text { H } & 1.93870 & -0.92845 & -0.00000 \\ \text { H } & -0.55935 & 0.92340 & 0.00000 \\ \text { H } & -0.55840 & -0.92269 & -0.00000\end{array}$


Table S1093 Cartesian coordinates of the OM2/ROHF-optimized excited-state equilibrium geometry of the $1^{3} A^{\prime \prime}\left({ }^{3} n \pi^{*}\right)$ state of acrolein (excited-state energy: $-769.94576 \mathrm{eV})$.

8

$\begin{array}{rrrr}\text { C } & 0.00000 & 0.00000 & 0.00000 \\ \text { C } & 1.35695 & 0.00000 & 0.00000 \\ \text { C } & 2.07497 & 1.21822 & 0.00000 \\ \text { O } & 1.47386 & 2.35386 & 0.00000 \\ \text { H } & 3.17023 & 1.26893 & 0.00000 \\ \text { H } & 1.93973 & -0.92524 & -0.00000 \\ \text { H } & -0.56304 & 0.92036 & 0.00000 \\ \text { H } & -0.55647 & -0.92352 & -0.00000\end{array}$


Table S1094 Cartesian coordinates of the OM2/ROHF-optimized excited-state equilibrium geometry of the $2{ }^{1} A\left({ }^{1} \pi \pi^{*}\right)$ state of aniline (excited-state energy: $-1079.85996 \mathrm{eV})$.

$\begin{array}{lrrr}\text { C } & 0.00000 & 0.00000 & 0.00000 \\ \text { C } & 2.65684 & 0.00000 & 0.00000 \\ \text { C } & 0.65663 & 0.50350 & -1.23150 \\ \text { C } & 0.65663 & 0.50350 & 1.23150 \\ \text { C } & 1.99896 & 0.24788 & 1.23805 \\ \text { C } & 1.99896 & 0.24788 & -1.23805 \\ \text { N } & -0.07301 & -1.38252 & 0.00000 \\ \text { H } & 3.73478 & -0.19634 & -0.00000 \\ \text { H } & 0.09056 & 0.94244 & -2.04824 \\ \text { H } & 0.09056 & 0.94244 & 2.04824 \\ \text { H } & 2.60925 & 0.37511 & 2.13830 \\ \text { H } & 2.60925 & 0.37511 & -2.13830 \\ \text { H } & 0.07300 & -1.90934 & -0.84064 \\ \text { H } & 0.07300 & -1.90934 & 0.84064\end{array}$


Table S1095 Cartesian coordinates of the OM2/ROHF-optimized excited-state equilibrium geometry of the $2{ }^{1} A\left({ }^{1} \pi \pi^{*}\right)$ state of anisol (excited-state energy: $-1340.83951 \mathrm{eV})$.

$\begin{array}{lrrr}\text { C } & 0.00000 & 0.00000 & 0.00000 \\ \text { C } & 2.80815 & 0.00000 & 0.00000 \\ \text { C } & 0.74914 & 1.25005 & 0.00000 \\ \text { C } & 0.74864 & -1.25068 & -0.00000 \\ \text { C } & 2.12026 & 1.22651 & 0.00000 \\ \text { C } & 2.11992 & -1.22593 & 0.00000 \\ \text { O } & -1.24503 & 0.00052 & 0.00000 \\ \text { C } & -2.49384 & -2.82933 & 0.00000 \\ \text { H } & 3.90588 & 0.00015 & -0.00000 \\ \text { H } & 0.18256 & 2.18368 & -0.00000 \\ \text { H } & 0.18078 & -2.18241 & -0.00000 \\ \text { H } & 2.69587 & 2.15802 & 0.00000 \\ \text { H } & 2.69525 & -2.15756 & 0.00000 \\ \text { H } & -2.29586 & -1.78588 & 0.00000 \\ \text { H } & -2.65008 & -3.34733 & -0.92010 \\ \text { H } & -2.65008 & -3.34733 & 0.92010\end{array}$


Table S1096 Cartesian coordinates of the OM2/ROHF-optimized excited-state equilibrium geometry of the $1{ }^{1} B_{2 u}\left({ }^{1} \pi \pi^{*}\right)$ state of anthracene (excited-state energy: $-1930.90435 \mathrm{eV}$ ).

$\begin{array}{lrrr}\text { C } & 1.00000 & 2.40302 & 0.00000 \\ \mathrm{C} & 1.00000 & -0.40302 & -0.00000 \\ \mathrm{C} & 2.23369 & 1.71722 & 0.00000 \\ \mathrm{C} & -0.23369 & 1.71722 & -0.00000 \\ \mathrm{C} & 2.23369 & 0.28278 & 0.00000 \\ \mathrm{C} & -0.23369 & 0.28278 & -0.00000 \\ \mathrm{C} & 3.46069 & 2.40309 & 0.00000 \\ \mathrm{C} & -1.46069 & 2.40309 & -0.00000 \\ \mathrm{C} & 3.46069 & -0.40309 & 0.00000 \\ \mathrm{C} & -1.46069 & -0.40309 & -0.00000 \\ \mathrm{C} & 4.66723 & 1.69590 & 0.00000 \\ \mathrm{C} & -2.66723 & 1.69590 & -0.00000 \\ \mathrm{C} & 4.66723 & 0.30410 & 0.00000 \\ \mathrm{C} & -2.66723 & 0.30410 & -0.00000 \\ \mathrm{H} & 1.00000 & 3.50199 & 0.00000 \\ \mathrm{H} & 1.00000 & -1.50199 & -0.00000 \\ \mathrm{H} & 3.46915 & 3.49981 & 0.00000 \\ \mathrm{H} & -1.46915 & 3.49981 & -0.00000 \\ \mathrm{H} & 3.46915 & -1.49981 & 0.00000 \\ \mathrm{H} & -1.46915 & -1.49981 & -0.00000 \\ \mathrm{H} & 5.61644 & 2.24319 & 0.00000 \\ \mathrm{H} & -3.61644 & 2.24319 & 0.00000 \\ \mathrm{H} & 5.61644 & -0.24319 & 0.00000 \\ \mathrm{H} & -3.61644 & -0.24319 & -0.00000\end{array}$


Table S1097 Cartesian coordinates of the OM2/ROHF-optimized excited-state equilibrium geometry of the $1{ }^{1} B_{1}\left({ }^{1} \pi \pi^{*}\right)$ state of azulene (excited-state energy: $-1391.18933 \mathrm{eV})$.

$\begin{array}{lrrr}\text { C } & 0.00000 & 1.55930 & 0.00000 \\ \mathrm{C} & 0.00000 & 0.96192 & -1.27504 \\ \mathrm{C} & 0.00000 & 0.96192 & 1.27504 \\ \mathrm{C} & 0.00000 & -0.36085 & -1.61641 \\ \mathrm{C} & 0.00000 & -0.36085 & 1.61641 \\ \mathrm{C} & 0.00000 & -1.45074 & -0.68923 \\ \mathrm{C} & 0.00000 & -1.45074 & 0.68923 \\ \mathrm{C} & 0.00000 & -2.86312 & -1.12587 \\ \mathrm{C} & 0.00000 & -2.86312 & 1.12587 \\ \mathrm{C} & 0.00000 & -3.68685 & -0.00000 \\ \mathrm{H} & 0.00000 & 2.66207 & 0.00000 \\ \mathrm{H} & 0.00000 & 1.68174 & -2.11861 \\ \mathrm{H} & 0.00000 & 1.68174 & 2.11861 \\ \mathrm{H} & 0.00000 & -0.63723 & -2.68048 \\ \mathrm{H} & 0.00000 & -0.63723 & 2.68048 \\ \mathrm{H} & 0.00000 & -3.18242 & -2.16566 \\ \mathrm{H} & 0.00000 & -3.18242 & 2.16566 \\ \mathrm{H} & 0.00000 & -4.76663 & -0.00000\end{array}$


Table S1098 Cartesian coordinates of the OM2/ROHF-optimized excited-state equilibrium geometry of the $2{ }^{2} A\left({ }^{2} \pi \pi^{*}\right)$ state of benzophenone ketyl radical (excited-state energy: $-2152.79742 \mathrm{eV}$ ).

$\begin{array}{rrrr}\mathrm{C} & 0.00000 & 0.00000 & 0.00000 \\ \mathrm{C} & 1.39898 & 0.00000 & 0.00000 \\ \mathrm{C} & 2.10344 & 1.21032 & 0.00000 \\ \mathrm{C} & 1.40440 & 2.41338 & 0.01259 \\ \mathrm{C} & 0.01151 & 2.41385 & 0.02151 \\ \mathrm{C} & -0.68803 & 1.20980 & 0.01536 \\ \mathrm{C} & 2.12444 & -1.28167 & -0.07232 \\ \mathrm{O} & 3.32264 & -1.26827 & -0.70531 \\ \mathrm{C} & 2.08463 & -2.28490 & 0.98707 \\ \mathrm{C} & 1.18195 & -2.16455 & 2.05242 \\ \mathrm{C} & 1.10639 & -3.17891 & 3.00119 \\ \mathrm{C} & 1.91970 & -4.30445 & 2.89477 \\ \mathrm{C} & 2.81996 & -4.41881 & 1.83826 \\ \mathrm{C} & 2.90609 & -3.41614 & 0.87864 \\ \mathrm{H} & 0.55599 & -1.27266 & 2.14727 \\ \mathrm{H} & 0.40527 & -3.08961 & 3.83945 \\ \mathrm{H} & 1.85364 & -5.09944 & 3.64649 \\ \mathrm{H} & 3.46279 & -5.30367 & 1.76256 \\ \mathrm{H} & 3.61301 & -3.49270 & 0.04954 \\ \mathrm{H} & 3.19798 & 1.20927 & 0.01365 \\ \mathrm{H} & 1.95134 & 3.36315 & 0.01930 \\ \mathrm{H} & -0.53435 & 3.36372 & 0.03287 \\ \mathrm{H} & -1.78396 & 1.21496 & 0.01820 \\ \mathrm{H} & -0.54523 & -0.94817 & -0.02360 \\ \mathrm{H} & 3.27796 & -0.63489 & -1.47181\end{array}$


Table S1099 Cartesian coordinates of the OM2/ROHF-optimized excited-state equilibrium geometry of the $2{ }^{1} A\left({ }^{1} \pi \pi^{*}\right)$ state of acetylene (excited-state energy: $-278.22029 \mathrm{eV})$.

$\begin{array}{lrrr}\mathrm{C} & 0.00000 & 0.00000 & 0.00000 \\ \mathrm{C} & 1.35426 & 0.00000 & 0.00000 \\ \mathrm{H} & -0.64074 & 0.89761 & 0.00000 \\ \mathrm{H} & 1.99501 & -0.89761 & 0.00000\end{array}$


Table S1100 Cartesian coordinates of the OM2/ROHF-optimized excited-state equilibrium geometry of the $2{ }^{1} A\left({ }^{1} n \pi^{*}\right)$ state of formaldehyde (excited-state energy: $-485.03594 \mathrm{eV}$ ).

$\begin{array}{lrrr}0 & 1.00000 & 1.00000 & 0.00000\end{array}$

C $\quad-0.26524 \quad 0.86076-0.04755$

$\mathrm{H} \quad-0.83209 \quad 0.86851 \quad 0.87473$

$\mathrm{H} \quad-0.77203 \quad 0.96260 \quad-0.99875$ 
Table S1101 Cartesian coordinates of the OM2/ROHF-optimized excited-state equilibrium geometry of the $1^{3} A\left({ }^{3} n \pi^{*}\right)$ state of formaldehyde (excited-state energy: $-485.41560 \mathrm{eV}$ ).

4

$\begin{array}{lrrr}\mathrm{O} & 1.00000 & 1.00000 & 0.00000 \\ \mathrm{C} & -0.26159 & 1.09399 & 0.00611 \\ \mathrm{H} & -0.72862 & 1.44021 & 0.94401 \\ \mathrm{H} & -0.73474 & 1.47875 & -0.91354\end{array}$


Table S1102 Cartesian coordinates of the OM2/ROHF-optimized excited-state equilibrium geometry of the $1^{1} A^{\prime \prime}\left({ }^{1} n \pi^{*}\right)$ state of cinnoline (excited-state energy: $-1532.59892 \mathrm{eV})$.

$\begin{array}{lrrr}\text { C } & 0.00000 & 0.00000 & 0.00000 \\ \text { C } & 1.39225 & 0.00000 & 0.00000 \\ \text { C } & 2.19072 & 1.19266 & 0.00000 \\ \text { C } & 1.48862 & 2.40860 & 0.00000 \\ \text { C } & 0.09585 & 2.42148 & 0.00000 \\ \text { C } & -0.64107 & 1.23838 & 0.00000 \\ \text { N } & 2.17262 & -1.18637 & -0.00000 \\ \text { N } & 3.37663 & -1.21277 & -0.00000 \\ \mathrm{C} & 4.23350 & -0.17032 & -0.00000 \\ \mathrm{C} & 3.61238 & 1.09662 & -0.00000 \\ \text { H } & 2.05145 & 3.34555 & -0.00000 \\ \text { H } & -0.42924 & 3.38332 & 0.00000 \\ \text { H } & -1.73481 & 1.27558 & 0.00000 \\ \text { H } & -0.54809 & -0.93955 & -0.00000 \\ \text { H } & 4.22603 & 1.98878 & -0.00000 \\ \text { H } & 5.31331 & -0.32644 & -0.00000\end{array}$


Table S1103 Cartesian coordinates of the OM2/ROHF-optimized excitedstate equilibrium geometry of the $2^{2} A\left({ }^{2} \pi \pi^{*}\right)$ state of cyclohexadienyl radical (excited-state energy: $-868.65688 \mathrm{eV}$ ).

13

$\begin{array}{lrrr}\mathrm{C} & 0.00000 & 0.00000 & 0.00000 \\ \mathrm{C} & 1.47845 & 0.00000 & 0.00000 \\ \mathrm{C} & 2.21137 & 1.15165 & 0.00000 \\ \mathrm{C} & 1.57595 & 2.40813 & -0.00004 \\ \mathrm{C} & 0.17017 & 2.48747 & -0.00011 \\ \mathrm{C} & -0.59180 & 1.35483 & -0.00015 \\ \mathrm{H} & 2.17541 & 3.32414 & -0.00001 \\ \mathrm{H} & 3.30826 & 1.11240 & 0.00004 \\ \mathrm{H} & -0.30488 & 3.47693 & -0.00020 \\ \mathrm{H} & 1.97254 & -0.98017 & 0.00003 \\ \mathrm{H} & -1.68780 & 1.41526 & -0.00033 \\ \mathrm{H} & -0.36866 & -0.56323 & 0.90382 \\ \mathrm{H} & -0.36868 & -0.56348 & -0.90365\end{array}$


Table S1104 Cartesian coordinates of the OM2/ROHF-optimized excited-state equilibrium geometry of the $2{ }^{1} A\left({ }^{1} n \pi^{*}\right)$ state of 2,3-diazabicyclo[2,2,1]hept-2ene (excited-state energy: $-1180.02764 \mathrm{eV})$.

15

$\begin{array}{lrrr}\mathrm{N} & 0.00000 & 0.00000 & 0.00000 \\ \mathrm{C} & 1.54258 & 0.00000 & 0.00000 \\ \mathrm{C} & 1.74241 & 1.53510 & 0.00000 \\ \mathrm{C} & 0.78776 & 1.74060 & 1.20122 \\ \mathrm{~N} & -0.40621 & 0.93671 & 0.64644 \\ \mathrm{C} & 1.88942 & -0.39028 & 1.44653 \\ \mathrm{C} & 1.37306 & 0.80046 & 2.26827 \\ \mathrm{H} & 1.37443 & 2.01103 & -0.92086 \\ \mathrm{H} & 2.78060 & 1.84492 & 0.20343 \\ \mathrm{H} & 1.38961 & -1.33200 & 1.72983 \\ \mathrm{H} & 2.19244 & 1.31804 & 2.80627 \\ \mathrm{H} & 2.98683 & -0.51385 & 1.54208 \\ \mathrm{H} & 0.49229 & 2.76626 & 1.50695 \\ \mathrm{H} & 1.95834 & -0.61440 & -0.82608 \\ \mathrm{H} & 0.59734 & 0.49500 & 2.99067\end{array}$


Table S1105 Cartesian coordinates of the OM2/ROHF-optimized excitedstate equilibrium geometry of the $2{ }^{1} A\left({ }^{1} \pi \pi^{*}\right)$ state of 4 -(dimethylamino)- $4^{\prime}$ cyanostilbene (excited-state energy: $-2829.02728 \mathrm{eV}$ ).

$\begin{array}{lrrr}\text { 35 } & & & \\ & & & \\ \mathrm{C} & 0.00000 & 0.00000 & 0.00000 \\ \mathrm{C} & 1.37866 & 0.00000 & 0.00000 \\ \mathrm{C} & 2.10990 & 1.22033 & 0.00000 \\ \mathrm{C} & 1.39704 & 2.44843 & 0.00032 \\ \mathrm{C} & 0.01408 & 2.45992 & 0.00060 \\ \mathrm{C} & -0.67485 & 1.23687 & 0.00027 \\ \mathrm{C} & 3.53398 & 1.16176 & -0.00026 \\ \mathrm{C} & 4.34994 & 2.28563 & -0.00208 \\ \mathrm{C} & 5.77253 & 2.19755 & 0.00027 \\ \mathrm{C} & 6.53639 & 3.39983 & -0.00314 \\ \mathrm{C} & 7.91088 & 3.37397 & 0.00031 \\ \mathrm{C} & 8.60385 & 2.12866 & 0.00567 \\ \mathrm{C} & 7.85297 & 0.92620 & 0.00744 \\ \mathrm{C} & 6.47338 & 0.96124 & 0.00543 \\ \mathrm{~N} & 9.99021 & 2.09675 & 0.01016 \\ \mathrm{C} & 10.75263 & 3.31752 & 0.00798 \\ \mathrm{C} & -2.10407 & 1.24575 & 0.00023 \\ \mathrm{~N} & -3.27832 & 1.25382 & -0.00015 \\ \mathrm{C} & 10.69279 & 0.84066 & 0.01723 \\ \mathrm{H} & 8.47403 & 4.31087 & -0.00118 \\ \mathrm{H} & 6.00813 & 4.36090 & -0.00767 \\ \mathrm{H} & 5.91507 & 0.01875 & 0.00769 \\ \mathrm{H} & 8.36688 & -0.03876 & 0.01091 \\ \mathrm{H} & 3.90850 & 3.28757 & -0.00515 \\ \mathrm{H} & 3.98620 & 0.15428 & 0.00064 \\ \mathrm{H} & 1.92804 & -0.95075 & -0.00011 \\ \mathrm{H} & 1.94551 & 3.39790 & 0.00056 \\ \mathrm{H} & -0.54284 & 3.40152 & 0.00095 \\ \mathrm{H} & -0.57032 & -0.93293 & -0.00014 \\ \mathrm{H} & 11.79145 & 1.00082 & 0.01956 \\ \mathrm{H} & 10.43663 & 0.23857 & 0.92392 \\ \mathrm{H} & 10.44168 & 0.23127 & -0.88598 \\ \mathrm{H} & 11.84219 & 3.10386 & 0.01223 \\ \mathrm{H} & 10.53231 & 3.93154 & -0.89996 \\ \mathrm{H} & 10.52661 & 3.93798 & 0.91023\end{array}$


Table S1106 Cartesian coordinates of the OM2/ROHF-optimized excited-state equilibrium geometry of the $1^{1} A_{u}\left({ }^{1} n \pi^{*}\right)$ state of glyoxal (excited-state energy: $-943.91037 \mathrm{eV})$.

6

$\begin{array}{llll}\text { C } & 0.24949 & -0.00000 & -0.00000\end{array}$

$\begin{array}{lrrr}\text { C } & 1.75051 & 0.00000 & 0.00000\end{array}$

$\begin{array}{lrrr}0 & -0.31293 & 1.09054 & -0.00000\end{array}$

o $\quad 2.31293-1.09054 \quad 0.00000$

$\mathrm{H} \quad-0.18119-1.02447-0.00000$

H $\quad 2.18119 \quad 1.02447 \quad 0.00000$ 
Table S1107 Cartesian coordinates of the OM2/ROHF-optimized excited-state equilibrium geometry of the $2{ }^{1} A\left({ }^{1} n \pi^{*}\right)$ state of formic acid (excited-state energy: $-816.11420 \mathrm{eV}$ ).

5

$\begin{array}{rrrr}\mathrm{C} & 0.00000 & 0.00000 & 0.00000 \\ \mathrm{O} & 1.34723 & 0.00000 & 0.00000 \\ \mathrm{O} & -0.24883 & 1.31234 & 0.00000 \\ \mathrm{H} & -0.48662 & -0.70504 & 0.70608 \\ \mathrm{H} & 1.67388 & 0.65832 & -0.67451\end{array}$


Table S1108 Cartesian coordinates of the OM2/ROHF-optimized excited-state equilibrium geometry of the $1{ }^{1} B_{2 u}\left({ }^{1} \pi \pi^{*}\right)$ state of naphthalene (excited-state energy: $-1390.22292 \mathrm{eV}$ ).

$\begin{array}{lrrr}\text { C } & 0.00000 & 0.00000 & 0.00000 \\ \text { C } & 1.46750 & 0.00000 & 0.00000 \\ \text { C } & -0.64379 & 1.24270 & 0.00000 \\ \text { C } & 2.11129 & 1.18831 & 0.36365 \\ \text { C } & -0.64379 & -1.24271 & 0.00001 \\ \mathrm{C} & 2.11130 & -1.18831 & -0.36363 \\ \mathrm{C} & 0.04724 & 2.43744 & 0.28211 \\ \mathrm{C} & 1.42025 & 2.41331 & 0.44346 \\ \mathrm{C} & 0.04725 & -2.43744 & -0.28213 \\ \mathrm{C} & 1.42026 & -2.41331 & -0.44347 \\ \mathrm{H} & -1.67367 & 1.29737 & -0.37537 \\ \mathrm{H} & 3.14115 & 1.13074 & 0.73863 \\ \mathrm{H} & -1.67366 & -1.29739 & 0.37541 \\ \mathrm{H} & 3.14118 & -1.13074 & -0.73857 \\ \mathrm{H} & -0.50529 & 3.38064 & 0.33908 \\ \mathrm{H} & 1.97277 & 3.33191 & 0.66497 \\ \mathrm{H} & -0.50527 & -3.38065 & -0.33913 \\ \mathrm{H} & 1.97280 & -3.33190 & -0.66498\end{array}$


Table S1109 Cartesian coordinates of the OM2/ROHF-optimized excited-state equilibrium geometry of the $1^{1} B_{u}\left({ }^{1} \pi \pi^{*}\right)$ state of octatetraene (excited-state energy: $-1163.46652 \mathrm{eV}$ ).

$\begin{array}{lrrr}18 & & \\ \text { C } & 0.00000 & 0.70525 & 0.00000 \\ \text { C } & 0.00000 & -0.70525 & -0.00000 \\ \text { C } & 0.00000 & 1.41910 & 1.19215 \\ \text { C } & 0.00000 & -1.41910 & -1.19215 \\ \text { C } & 0.00000 & 2.84949 & 1.19022 \\ \text { C } & 0.00000 & -2.84949 & -1.19022 \\ \text { C } & 0.00000 & 3.56580 & 2.33629 \\ \text { C } & 0.00000 & -3.56580 & -2.33629 \\ \text { H } & 0.00000 & 1.24072 & -0.95887 \\ \text { H } & 0.00000 & -1.24072 & 0.95887 \\ \text { H } & 0.00000 & 0.89191 & 2.15492 \\ \text { H } & 0.00000 & -0.89191 & -2.15492 \\ \text { H } & 0.00000 & 3.35828 & 0.21504 \\ \text { H } & 0.00000 & -3.35828 & -0.21504 \\ \text { H } & 0.00000 & 3.08465 & 3.30721 \\ \text { H } & 0.00000 & -3.08465 & -3.30721 \\ \text { H } & 0.00000 & 4.64815 & 2.33255 \\ \text { H } & 0.00000 & -4.64815 & -2.33255\end{array}$


Table S1110 Cartesian coordinates of the OM2/ROHF-optimized excited-state equilibrium geometry of the $1{ }^{1} A_{u}\left({ }^{1} n \pi^{*}\right)$ state of oxalylfluoride (excited-state energy: $-1845.42605 \mathrm{eV}$ ).

$\begin{array}{lrrr}6 & & & \\ \text { C } & 0.25348 & -0.00000 & -0.00000 \\ \text { C } & 1.74652 & 0.00000 & 0.00000 \\ \text { O } & -0.36312 & 1.07084 & -0.00000 \\ 0 & 2.36312 & -1.07084 & 0.00000 \\ \text { F } & -0.26404 & -1.22494 & -0.00000 \\ \text { F } & 2.26404 & 1.22494 & 0.00000\end{array}$


Table S1111 Cartesian coordinates of the OM2/ROHF-optimized excited-state equilibrium geometry of the $1{ }^{1} B_{2 g}\left({ }^{1} n \pi^{*}\right)$ state of $p$-benzoquinone (excitedstate energy: $-1483.30507 \mathrm{eV})$.

12

$\begin{array}{lrrr}\text { C } & 0.00000 & 1.45779 & 0.00000 \\ \mathrm{C} & 0.00000 & -1.45779 & -0.00000 \\ \mathrm{C} & 1.23927 & 0.67160 & 0.00000 \\ \mathrm{C} & -1.23927 & 0.67160 & -0.00000 \\ \mathrm{C} & 1.23927 & -0.67160 & -0.00000 \\ \mathrm{C} & -1.23927 & -0.67160 & 0.00000 \\ \mathrm{O} & 0.00000 & 2.69411 & 0.00000 \\ \mathrm{O} & 0.00000 & -2.69411 & -0.00000 \\ \mathrm{H} & 2.13776 & 1.28882 & -0.00000 \\ \mathrm{H} & -2.13776 & 1.28882 & -0.00000 \\ \mathrm{H} & 2.13776 & -1.28882 & -0.00000 \\ \mathrm{H} & -2.13776 & -1.28882 & -0.00000\end{array}$


Table S1112 Cartesian coordinates of the OM2/ROHF-optimized excited-state equilibrium geometry of the $2{ }^{1} A\left({ }^{1} \pi \pi^{*}\right)$ state of $p$-phenylenediamine (excitedstate energy: $-1307.05045 \mathrm{eV})$.

$\begin{array}{lrrr}16 & & & \\ \text { C } & 0.00000 & 0.00000 & 0.00000 \\ \mathrm{C} & 1.43376 & 0.00000 & 0.00000 \\ \mathrm{C} & 2.10912 & 1.21325 & 0.00000 \\ \mathrm{C} & 1.35371 & 2.43186 & -0.00000 \\ \mathrm{C} & -0.08005 & 2.43187 & 0.00000 \\ \mathrm{C} & -0.75541 & 1.21862 & 0.00001 \\ \mathrm{~N} & 2.01622 & 3.62202 & -0.00002 \\ \mathrm{~N} & -0.66252 & -1.19015 & -0.00001 \\ \mathrm{H} & 3.16965 & 1.25514 & 0.31251 \\ \mathrm{H} & -0.60320 & 3.35532 & -0.31252 \\ \mathrm{H} & 1.95691 & -0.92346 & 0.31251 \\ \mathrm{H} & -1.81593 & 1.17673 & -0.31253 \\ \mathrm{H} & 1.54572 & 4.48361 & 0.18108 \\ \mathrm{H} & 2.99643 & 3.67602 & -0.18109 \\ \mathrm{H} & -0.19199 & -2.05173 & -0.18109 \\ \mathrm{H} & -1.64272 & -1.24417 & 0.18108\end{array}$


Table S1113 Cartesian coordinates of the OM2/ROHF-optimized excited-state equilibrium geometry of the $2{ }^{1} A\left({ }^{1} n \pi^{*}\right)$ state of propynal (excited-state energy: $-737.85551 \mathrm{eV})$.

$\begin{array}{lrrr}\mathrm{H} & 0.00000 & 0.00000 & 0.00000 \\ \mathrm{C} & 1.04008 & 0.00000 & 0.00000 \\ \mathrm{C} & 2.27020 & 0.00000 & -0.05751 \\ \mathrm{C} & 3.65934 & -0.05845 & -0.04531 \\ \mathrm{O} & 4.21080 & 0.69702 & 0.87316 \\ \mathrm{H} & 4.26323 & -0.42987 & -0.87213\end{array}$


Table S1114 Cartesian coordinates of the OM2/ROHF-optimized excited-state equilibrium geometry of the $2{ }^{1} A\left({ }^{1} n \pi^{*}\right)$ state of pyrimidine (excited-state energy: $-991.98766 \mathrm{eV}$ ).

10

$\begin{array}{lrrr}\mathrm{C} & 0.00000 & 0.00000 & 0.00000 \\ \mathrm{C} & 1.42547 & 0.00000 & 0.00000 \\ \mathrm{C} & 2.00902 & 1.30056 & 0.00000 \\ \mathrm{~N} & 1.26479 & 2.35840 & 0.00835 \\ \mathrm{C} & -0.11016 & 2.37214 & -0.06483 \\ \mathrm{~N} & -0.66047 & 1.11206 & 0.00836 \\ \mathrm{H} & 2.01179 & -0.90568 & -0.00882 \\ \mathrm{H} & 3.10549 & 1.37205 & -0.00725 \\ \mathrm{H} & -0.69030 & 3.26833 & 0.16896 \\ \mathrm{H} & -0.51410 & -0.97111 & -0.00724\end{array}$


Table S1115 Cartesian coordinates of the OM2/ROHF-optimized excited-state equilibrium geometry of the $2{ }^{1} A\left({ }^{1} n \pi^{*}\right)$ state of quinoxaline (excited-state energy: $-1532.58846 \mathrm{eV}$ ).

$\begin{array}{rrrr}\text { 16 } & & & \\ \text { C } & 0.00000 & 0.00000 & 0.00000 \\ \text { N } & 1.32195 & 0.00000 & 0.00000 \\ \text { C } & 2.10832 & 1.08927 & 0.00000 \\ \text { C } & 1.41199 & 2.39683 & -0.00001 \\ \text { N } & 0.06927 & 2.35222 & -0.00000 \\ \text { C } & -0.66851 & 1.25530 & -0.00000 \\ \text { C } & 2.18100 & 3.56721 & -0.00001 \\ \text { C } & 3.57270 & 3.49603 & -0.00002 \\ \text { C } & 4.22632 & 2.26867 & -0.00001 \\ \text { C } & 3.50866 & 1.07417 & 0.00000 \\ \text { H } & 5.32067 & 2.23227 & 0.00001 \\ \text { H } & 4.15326 & 4.42440 & -0.00002 \\ \text { H } & 4.00709 & 0.10505 & 0.00001 \\ \text { H } & 1.65504 & 4.52167 & -0.00002 \\ \text { H } & -1.76111 & 1.28939 & -0.00000 \\ \text { H } & -0.58149 & -0.92563 & -0.00000\end{array}$


Table S1116 Cartesian coordinates of the OM2/ROHF-optimized excited-state equilibrium geometry of the $1^{3} A^{\prime}\left({ }^{3} \pi \pi^{*}\right)$ state of quinoxaline (excited-state energy: $-1532.82059 \mathrm{eV}$ ).

$\begin{array}{lrrr}\text { C } & 0.00000 & 0.00000 & 0.00000 \\ \text { C } & 1.40099 & 0.00000 & 0.00000 \\ \text { C } & 2.11110 & 1.29200 & 0.00000 \\ \text { C } & 1.36017 & 2.47475 & 0.00000 \\ \text { C } & -0.03032 & 2.42221 & 0.00000 \\ \text { C } & -0.70095 & 1.20204 & 0.00000 \\ \text { N } & 2.17036 & -1.10575 & -0.00000 \\ \mathrm{C} & 3.49331 & -1.11962 & -0.00000 \\ \mathrm{C} & 4.17779 & 0.12575 & -0.00000 \\ \mathrm{~N} & 3.45698 & 1.23517 & -0.00000 \\ \mathrm{H} & 1.89887 & 3.42253 & -0.00000 \\ \mathrm{H} & -0.59998 & 3.35726 & 0.00000 \\ \mathrm{H} & -1.79567 & 1.18177 & 0.00000 \\ \mathrm{H} & -0.51139 & -0.96279 & -0.00000 \\ \mathrm{H} & 4.06394 & -2.05119 & -0.00000 \\ \mathrm{H} & 5.27009 & 0.14333 & -0.00000\end{array}$


Table S1117 Cartesian coordinates of the OM2/ROHF-optimized excited-state equilibrium geometry of the $2{ }^{1} A\left({ }^{1} \pi \pi^{*}\right)$ state of syn-coumarin 153 (excitedstate energy: $-4522.87986 \mathrm{eV})$.

$\begin{array}{lrrr}\text { 36 } & & & \\ \text { C } & 0.00000 & 0.00000 & 0.00000 \\ \text { C } & 1.49060 & 0.00000 & 0.00000 \\ \text { C } & 2.24210 & 1.17453 & 0.00000 \\ \text { N } & 1.60296 & 2.42031 & -0.05052 \\ \text { C } & 0.18530 & 2.46779 & 0.22838 \\ \text { C } & -0.51527 & 1.33170 & -0.51471 \\ \text { C } & 3.66672 & 1.10452 & 0.00688 \\ \text { C } & 4.49249 & 2.34951 & 0.05316 \\ \text { C } & 3.70754 & 3.53129 & -0.48436 \\ \text { C } & 2.36686 & 3.60807 & 0.24225 \\ \text { C } & 2.16330 & -1.28623 & 0.04509 \\ \text { C } & 3.62444 & -1.31210 & 0.02660 \\ \text { C } & 4.33850 & -0.14319 & -0.00589 \\ \text { C } & 4.26791 & -2.64372 & -0.05989 \\ \text { C } & 3.64174 & -3.68559 & -0.61101 \\ \text { C } & 2.26473 & -3.47138 & -1.20647 \\ \text { O } & 1.52772 & -2.36833 & 0.09377 \\ \text { O } & 2.05998 & -2.94018 & -2.27005 \\ \text { C } & 5.63464 & -2.78585 & 0.55943 \\ \text { F } & 6.05562 & -4.07765 & 0.67770 \\ \text { F } & 6.62645 & -2.16422 & -0.14973 \\ \text { F } & 5.73713 & -2.26193 & 1.81192 \\ \text { H } & 4.06511 & -4.68566 & -0.69816 \\ \text { H } & 5.43142 & -0.14674 & -0.04161 \\ \text { H } & 4.77922 & 2.53024 & 1.11761 \\ \text { H } & 5.42662 & 2.20915 & -0.53871 \\ \text { H } & 4.28029 & 4.47150 & -0.32475 \\ \text { H } & 3.52610 & 3.40188 & -1.57312 \\ \text { H } & 2.53998 & 3.73072 & 1.36027 \\ \text { H } & 1.80415 & 4.51914 & -0.11058 \\ \text { H } & -0.36894 & -0.83424 & -0.63370 \\ \text { H } & -0.35144 & -0.17751 & 1.04580 \\ \text { H } & -1.61449 & 1.40113 & -0.35726 \\ \text { H } & -0.30273 & 1.42377 & -1.60216 \\ \text { H } & -0.23534 & 3.45530 & -0.11799 \\ \text { H } & -0.02054 & 2.38498 & 1.34364\end{array}$


Table S1118 Cartesian coordinates of the OM2/ROHF-optimized excited-state equilibrium geometry of the $2{ }^{1} A^{\prime}\left({ }^{1} \pi \pi^{*}\right)$ state of terylene (excited-state energy: $-4062.68849 \mathrm{eV})$.

\begin{tabular}{|c|c|c|c|}
\hline C & 0.00000 & 0.00000 & 0.00000 \\
\hline $\mathrm{C}$ & 1.39849 & 0.00000 & 0.00000 \\
\hline C & 2.09095 & 1.24699 & 0.00000 \\
\hline C & 1.34794 & 2.45648 & 0.00000 \\
\hline C & -0.06992 & 2.41475 & 0.00000 \\
\hline C & -0.72272 & 1.20080 & 0.00000 \\
\hline C & 3.51628 & 1.30099 & 0.00000 \\
\hline C & 4.26836 & 0.06112 & 0.00000 \\
\hline C & 3.58503 & -1.18511 & 0.00000 \\
\hline C & 2.16450 & -1.23131 & 0.00000 \\
\hline C & 5.68056 & 0.07198 & 0.00000 \\
\hline C & 6.40258 & -1.10333 & 0.00000 \\
\hline C & 5.75453 & -2.35811 & 0.00000 \\
\hline C & 4.33400 & -2.40431 & 0.00000 \\
\hline C & 1.51646 & -2.48610 & 0.00000 \\
\hline$C$ & 2.23846 & -3.66141 & 0.00000 \\
\hline$C$ & 3.65067 & -3.65054 & 0.00000 \\
\hline C & 4.40275 & -4.89041 & 0.00000 \\
\hline C & 5.82808 & -4.83640 & 0.00000 \\
\hline C & 6.52054 & -3.58942 & 0.00000 \\
\hline C & 6.57108 & -6.04590 & 0.00000 \\
\hline C & 7.98894 & -6.00417 & 0.00000 \\
\hline C & 8.64175 & -4.79021 & 0.00000 \\
\hline C & 7.91904 & -3.58941 & 0.00000 \\
\hline C & 5.89295 & -7.29178 & 0.00000 \\
\hline C & 4.51502 & -7.32534 & 0.00000 \\
\hline C & 3.77060 & -6.13787 & 0.00000 \\
\hline C & 2.02609 & 3.70235 & 0.00000 \\
\hline C & 3.40403 & 3.73591 & 0.00000 \\
\hline C & 4.14845 & 2.54844 & 0.00000 \\
\hline $\mathrm{H}$ & 7.49832 & -1.05005 & 0.00000 \\
\hline $\mathrm{H}$ & 1.69563 & -4.61472 & 0.00000 \\
\hline $\mathrm{H}$ & 6.22340 & 1.02529 & 0.00000 \\
\hline $\mathrm{H}$ & 0.42072 & -2.53938 & 0.00000 \\
\hline $\mathrm{H}$ & 9.73746 & -4.75397 & 0.00000 \\
\hline $\mathrm{H}$ & 3.98738 & -8.28633 & 0.00000 \\
\hline $\mathrm{H}$ & 3.93166 & 4.69690 & 0.00000 \\
\hline
\end{tabular}

S1173 


\begin{tabular}{lrrr}
\multicolumn{4}{c}{ Continued from previous page } \\
\hline $\mathrm{H}$ & -1.81843 & 1.16454 & 0.00000 \\
$\mathrm{H}$ & 8.48118 & -2.64754 & 0.00000 \\
$\mathrm{H}$ & 2.67634 & -6.21355 & 0.00000 \\
$\mathrm{H}$ & 5.24271 & 2.62412 & 0.00000 \\
$\mathrm{H}$ & -0.56214 & -0.94187 & 0.00000 \\
$\mathrm{H}$ & 8.55255 & -6.94442 & 0.00000 \\
$\mathrm{H}$ & 6.47690 & -8.21954 & 0.00000 \\
$\mathrm{H}$ & 1.44215 & 4.63011 & 0.00000 \\
$\mathrm{H}$ & -0.63352 & 3.35501 & 0.00000
\end{tabular}


Table S1119 Cartesian coordinates of the OM2/ROHF-optimized excited-state equilibrium geometry of the $2{ }^{1} A\left({ }^{1} \pi \pi^{*}\right)$ state of trans-stilbene (excited-state energy: $-1960.77267 \mathrm{eV})$.

\begin{tabular}{|c|c|c|c|}
\hline & 0.00000 & 0.00000 & 0.00000 \\
\hline & 1.36963 & 0.00000 & 0.00000 \\
\hline & -0.75745 & 1.24514 & 0.00000 \\
\hline & 2.12708 & 0.05922 & -1.24373 \\
\hline & -0.07482 & 2.46771 & -0.07910 \\
\hline & 1.44445 & 0.19638 & -2.46115 \\
\hline & -2.16139 & 1.22076 & 0.05278 \\
\hline & 3.53103 & 0.00534 & -1.22189 \\
\hline & -0.80344 & 3.65726 & -0.10392 \\
\hline & 2.17308 & 0.27775 & -3.64818 \\
\hline & -2.86845 & 2.41382 & 0.02762 \\
\hline & 4.23808 & 0.08722 & -2.41240 \\
\hline & -2.19013 & 3.63262 & -0.05205 \\
\hline & 3.55977 & 0.22477 & -3.62603 \\
\hline & -0.59687 & -0.93216 & 0.01111 \\
\hline & 1.96650 & -0.05543 & 0.93058 \\
\hline & 1.01800 & 2.47806 & -0.12521 \\
\hline & 0.35163 & 0.24293 & -2.46930 \\
\hline & -2.69000 & 0.26255 & 0.11765 \\
\hline & 4.05963 & -0.10503 & -0.26784 \\
\hline & -0.27407 & 4.61533 & -0.16681 \\
\hline & 1.64371 & 0.38614 & -4.60217 \\
\hline & -3.96405 & 2.40334 & 0.06972 \\
\hline & 5.33369 & 0.04467 & -2.40394 \\
\hline & -2.75815 & 4.56953 & -0.07277 \\
\hline & 12778 & 0.29003 & -4.56 \\
\hline
\end{tabular}




\subsubsection{OM2/MRCI-Optimized Ground- and Excited-State Minima}

The OM2/MRCI-optimized geometries used for the computation of ZPVEs and $\triangle \mathrm{ZPVE}$ are presented below as part of the data of the SKF benchmark set. 


\subsection{Send-Kühn-Furche Benchmark Set on 0-0 Transition Energies}

4.3.1 OM2/MRCI-Optimized Ground- and Excited-State Minima 
Table S1120 Employed active space, reference configurations, and Cartesian coordinates of the OM2/MRCISD-optimized ground-state equilibrium geometry of 1,6-epoxy-10-annulene (ground-state energy: $-1724.48597 \mathrm{eV}$ ).

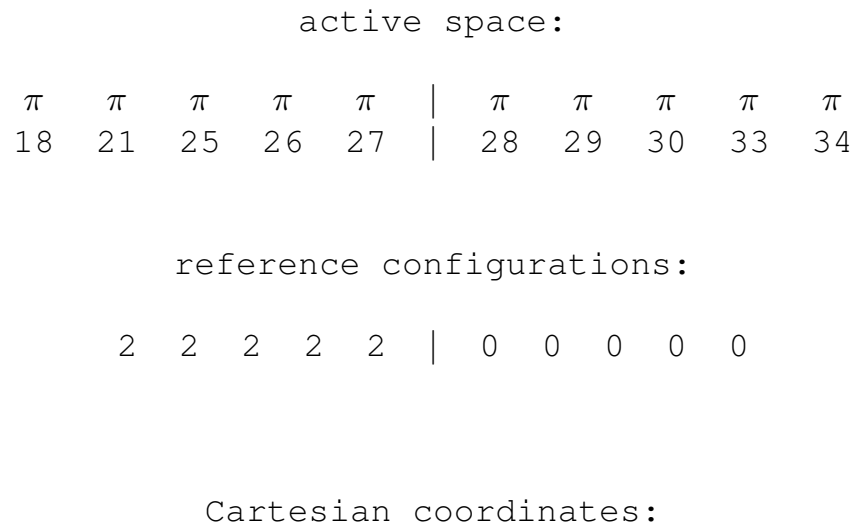


Table S1121 Employed active space, reference configurations, and Cartesian coordinates of the OM2/MRCISD-optimized excited-state equilibrium geometry of the $1^{1} B_{1}\left({ }^{1} \pi \pi^{*}\right)$ state of 1,6-epoxy-10-annulene (excited-state energy: $-1721.26634 \mathrm{eV})$.

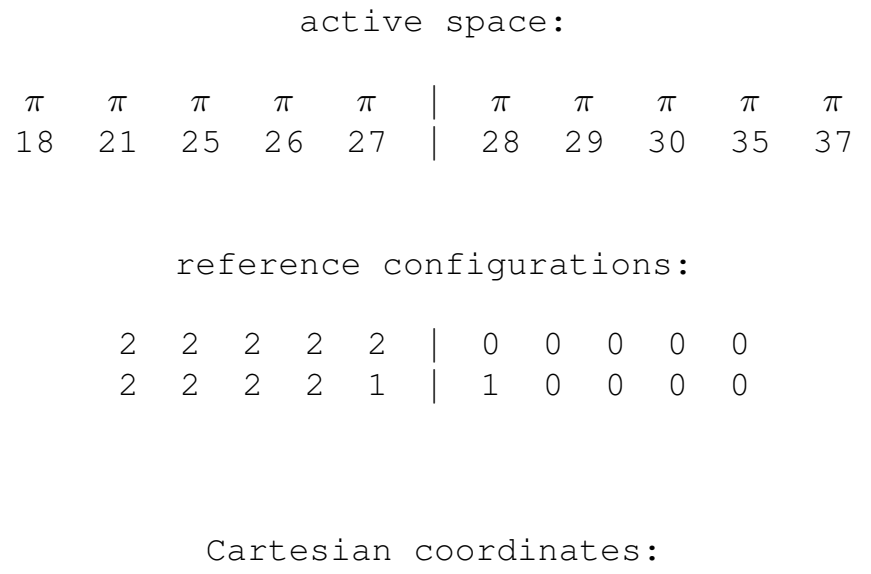

19

$\begin{array}{llll}0 & 1.00000 & 0.00000 & 0.86268\end{array}$

C $\quad 1.00000 \quad 1.08565 \quad 0.04692$

$\begin{array}{llll}\text { C } & 1.00000 & -1.08565 & 0.04692\end{array}$

$\begin{array}{llll}\text { C } & 2.30889 & 1.60087 & -0.21528\end{array}$

$\begin{array}{llll}\text { C } & -0.30889 & 1.60087 & -0.21528\end{array}$

C $\quad 2.30889-1.60087-0.21528$

C $\quad-0.30889-1.60087-0.21528$

C $\quad 3.36113 \quad 0.72373 \quad-0.35791$

$\begin{array}{llll}\text { C } & -1.36113 & 0.72373 & -0.35791\end{array}$

$\begin{array}{llll}\text { C } & 3.36113 & -0.72373 & -0.35791\end{array}$

$\begin{array}{llll}\text { C } & -1.36113 & -0.72373 & -0.35791\end{array}$

$\mathrm{H} \quad 2.46261 \quad 2.67556 \quad-0.31164$

$\mathrm{H} \quad-0.46261 \quad 2.67556 \quad-0.31164$

$\mathrm{H} \quad \begin{array}{llll}2.46261 & -2.67556 & -0.31164\end{array}$

$\mathrm{H} \quad-0.46261 \quad-2.67556 \quad-0.31164$

$\begin{array}{llll}\mathrm{H} & 4.35392 & 1.17133 & -0.52710\end{array}$

$\mathrm{H} \quad-2.35392 \quad 1.17133 \quad-0.52710$

$\mathrm{H} \quad 4.35392 \quad-1.17133 \quad-0.52710$

$\mathrm{H} \quad-2.35392 \quad-1.17133 \quad-0.52710$ 
Table S1122 Employed active space, reference configurations, and Cartesian coordinates of the OM2/MRCISD-optimized ground-state equilibrium geometry of 2-cyclopenten-1-one (ground-state energy: -1059.04759 eV).

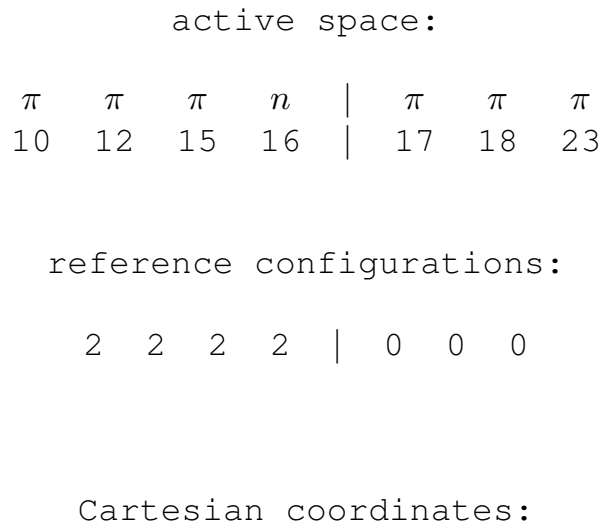


Table S1123 Employed active space, reference configurations, and Cartesian coordinates of the OM2/MRCISD-optimized excited-state equilibrium geometry of the $2^{1} A\left({ }^{1} n \pi^{*}\right)$ state of 2-cyclopenten-1-one (excited-state energy: $-1055.55491 \mathrm{eV})$.

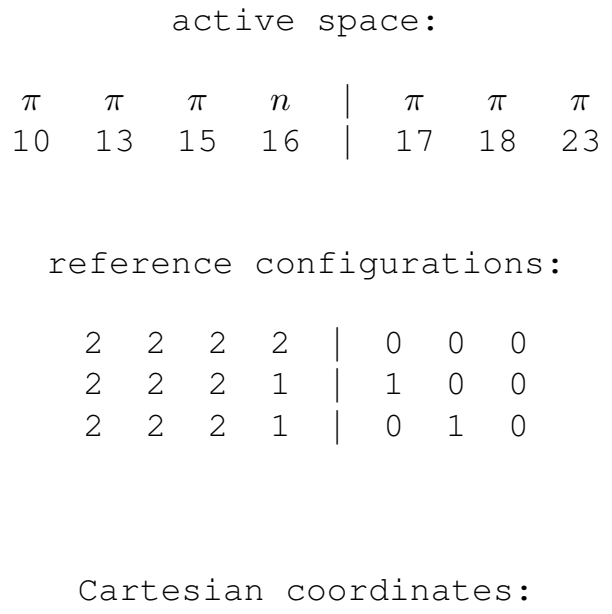


Table S1124 Employed active space, reference configurations, and Cartesian coordinates of the OM2/MRCISD-optimized ground-state equilibrium geometry of 7-azaindole (ground-state energy: $-1409.32842 \mathrm{eV}$ ).

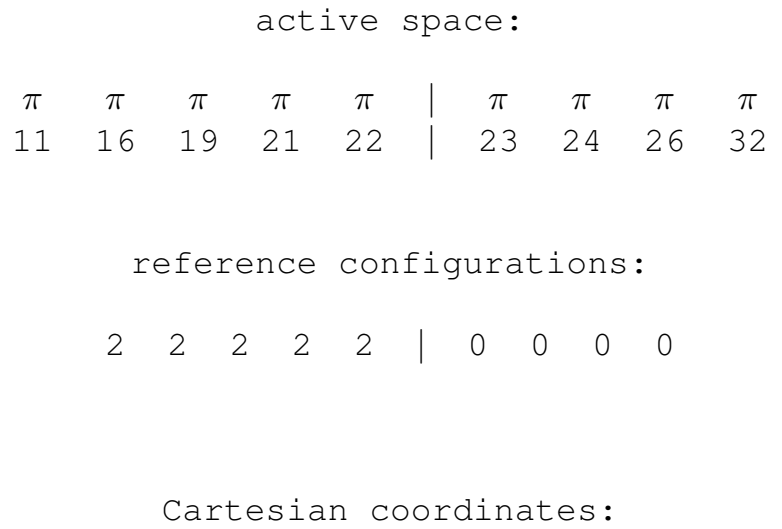


Table S1125 Employed active space, reference configurations, and Cartesian coordinates of the OM2/MRCISD-optimized excited-state equilibrium geometry of the $2{ }^{1} A\left({ }^{1} \pi \pi^{*}\right)$ state of 7 -azaindole (excited-state energy: $-1405.35672 \mathrm{eV}$ ).

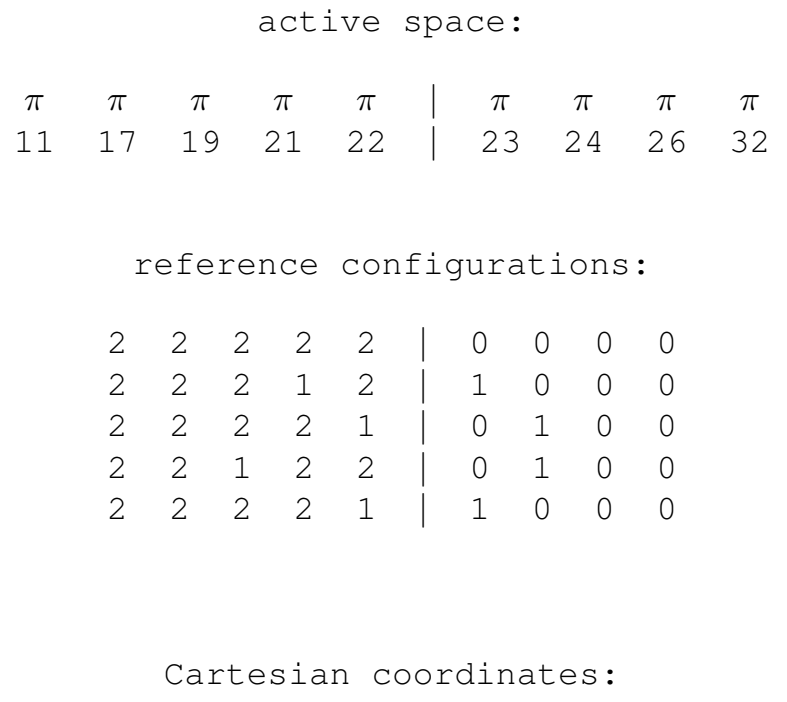

15

$\begin{array}{lrrr}\mathrm{C} & 0.00000 & 0.00000 & 0.00000 \\ \mathrm{~N} & 1.39045 & 0.00000 & 0.00000 \\ \mathrm{C} & 1.85590 & 1.29443 & 0.00000 \\ \mathrm{C} & 0.66450 & 2.18463 & -0.00000 \\ \mathrm{C} & -0.47255 & 1.33042 & 0.00001 \\ \mathrm{~N} & 3.14851 & 1.67211 & -0.00000 \\ \mathrm{C} & 3.31639 & 3.01470 & 0.00000 \\ \mathrm{C} & 2.25153 & 3.97132 & -0.00000 \\ \mathrm{C} & 0.89170 & 3.56939 & 0.00000 \\ \mathrm{H} & 1.97997 & -0.82137 & 0.00000 \\ \mathrm{H} & 4.35098 & 3.39569 & 0.00001 \\ \mathrm{H} & 2.49755 & 5.03704 & 0.00001 \\ \mathrm{H} & 0.07603 & 4.29182 & 0.00001 \\ \mathrm{H} & -1.50870 & 1.63062 & 0.00001 \\ \mathrm{H} & -0.59305 & -0.90750 & -0.00000\end{array}$


Table S1126 Employed active space, reference configurations, and Cartesian coordinates of the OM2/MRCISD-optimized ground-state equilibrium geometry of $\beta$-dinaphthyleneoxide (ground-state energy: $-3061.84239 \mathrm{eV}$ ).

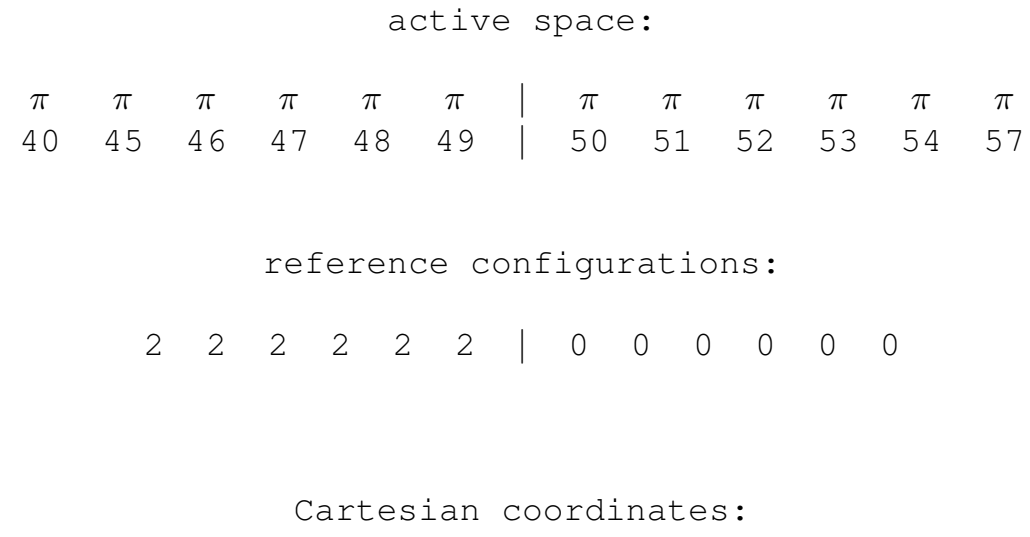

\begin{tabular}{lrrr}
33 & & & \\
C & 0.00000 & 0.00000 & 0.00000 \\
C & 1.37885 & 0.00000 & 0.00000 \\
C & 2.10903 & 1.21456 & 0.00000 \\
C & 1.38012 & 2.43754 & 0.08450 \\
C & -0.04058 & 2.40819 & 0.05235 \\
C & -0.71726 & 1.20965 & -0.00294 \\
C & 3.53671 & 1.29367 & -0.00603 \\
C & 4.09674 & 2.57376 & 0.22561 \\
C & 3.39491 & 3.79065 & 0.32417 \\
C & 2.02872 & 3.70240 & 0.22726 \\
O & 5.46269 & 2.53713 & 0.35405 \\
C & 5.80129 & 1.21971 & 0.17121 \\
C & 4.67873 & 0.40216 & -0.10700 \\
C & 4.95412 & -0.95524 & -0.46222 \\
C & 6.30384 & -1.40426 & -0.35857 \\
C & 7.37007 & -0.53130 & 0.01832 \\
C & 7.14188 & 0.79917 & 0.26624 \\
C & 6.61568 & -2.75730 & -0.66244 \\
C & 5.63885 & -3.62015 & -1.10821 \\
C & 4.32455 & -3.15481 & -1.29209 \\
C & 3.98791 & -1.85552 & -0.97629 \\
H & 7.92744 & 1.51001 & 0.51174 \\
H & 8.38851 & -0.93601 & 0.06729 \\
H & 2.97647 & -1.49923 & -1.18624 \\
\hline & & Continued & on next page
\end{tabular}

S1184 


\begin{tabular}{lrrr}
\multicolumn{4}{c}{ Continued from previous page } \\
\hline H & 7.65065 & -3.10186 & -0.54246 \\
H & 5.87717 & -4.66472 & -1.33417 \\
H & 3.56533 & -3.83877 & -1.68868 \\
H & 3.92918 & 4.72520 & 0.47862 \\
H & 1.40992 & 4.60535 & 0.29940 \\
H & 1.91343 & -0.95078 & 0.06542 \\
H & -0.59047 & 3.35749 & 0.07939 \\
H & -1.81150 & 1.18717 & -0.03634 \\
H & -0.54997 & -0.94817 & -0.00015
\end{tabular}


Table S1127 Employed active space, reference configurations, and Cartesian coordinates of the OM2/MRCISD-optimized excited-state equilibrium geometry of the $2{ }^{1} A\left({ }^{1} \pi \pi^{*}\right)$ state of $\beta$-dinaphthyleneoxide (excited-state energy: $-3057.91154 \mathrm{eV})$.

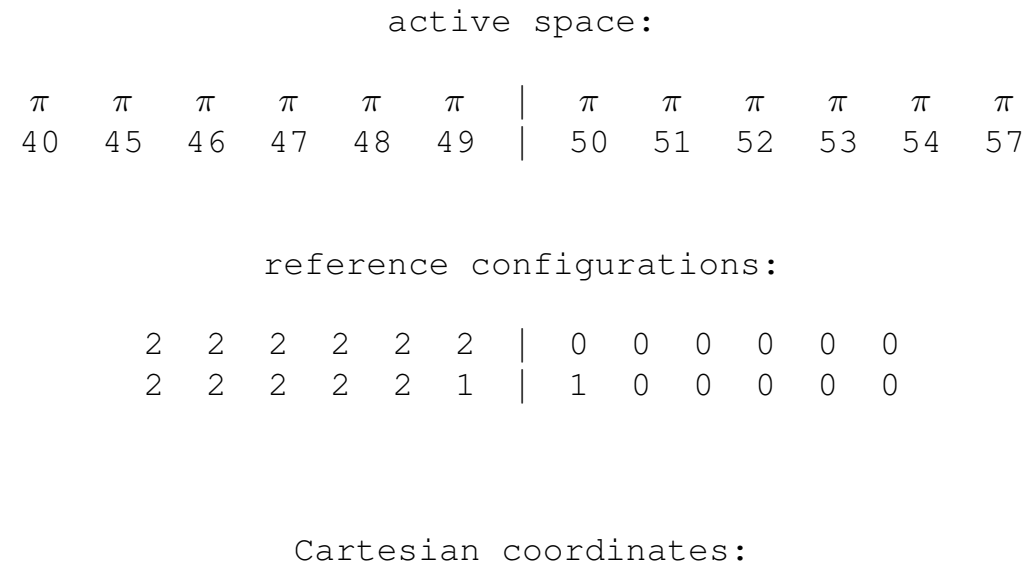

\begin{tabular}{lrrr}
33 & & & \\
C & 0.00000 & 0.00000 & 0.00000 \\
C & 1.39415 & 0.00000 & 0.00000 \\
C & 2.10396 & 1.20733 & 0.00000 \\
C & 1.38989 & 2.44476 & 0.10666 \\
C & -0.02881 & 2.41282 & 0.06852 \\
C & -0.70666 & 1.20527 & 0.00286 \\
C & 3.54434 & 1.28249 & -0.02502 \\
C & 4.13095 & 2.57531 & 0.28090 \\
C & 3.43771 & 3.76998 & 0.40972 \\
C & 2.05135 & 3.68535 & 0.29316 \\
O & 5.49987 & 2.50648 & 0.39040 \\
C & 5.81025 & 1.19689 & 0.10881 \\
C & 4.63222 & 0.42126 & -0.23723 \\
C & 4.86226 & -0.92227 & -0.70942 \\
C & 6.19438 & -1.43525 & -0.58773 \\
C & 7.27328 & -0.63589 & -0.13123 \\
C & 7.10472 & 0.70694 & 0.20214 \\
C & 6.44177 & -2.77624 & -0.98232 \\
C & 5.43265 & -3.54610 & -1.53996 \\
C & 4.16262 & -3.00119 & -1.74524 \\
C & 3.87915 & -1.69930 & -1.33488 \\
H & 7.92997 & 1.34418 & 0.50344 \\
H & 8.27742 & -1.07542 & -0.09096 \\
\hline & & $C 094144 d$ &
\end{tabular}

Continued on next page

S1186 


\begin{tabular}{lrrr}
\multicolumn{5}{c}{ Continued from previous page } \\
\hline $\mathrm{H}$ & 2.89908 & -1.27138 & -1.55535 \\
$\mathrm{H}$ & 7.44498 & -3.19719 & -0.83990 \\
$\mathrm{H}$ & 5.63215 & -4.58209 & -1.83486 \\
$\mathrm{H}$ & 3.38284 & -3.60259 & -2.22414 \\
$\mathrm{H}$ & 3.95367 & 4.70483 & 0.60394 \\
$\mathrm{H}$ & 1.44858 & 4.59536 & 0.40119 \\
$\mathrm{H}$ & 1.92814 & -0.95080 & 0.05566 \\
$\mathrm{H}$ & -0.58396 & 3.35895 & 0.09163 \\
$\mathrm{H}$ & -1.80146 & 1.19183 & -0.03302 \\
$\mathrm{H}$ & -0.54401 & -0.95028 & -0.01019
\end{tabular}


Table S1128 Employed active space, reference configurations, and Cartesian coordinates of the OM2/MRCISD-optimized ground-state equilibrium geometry of acetaldehyde (ground-state energy: $-646.46387 \mathrm{eV}$ ).

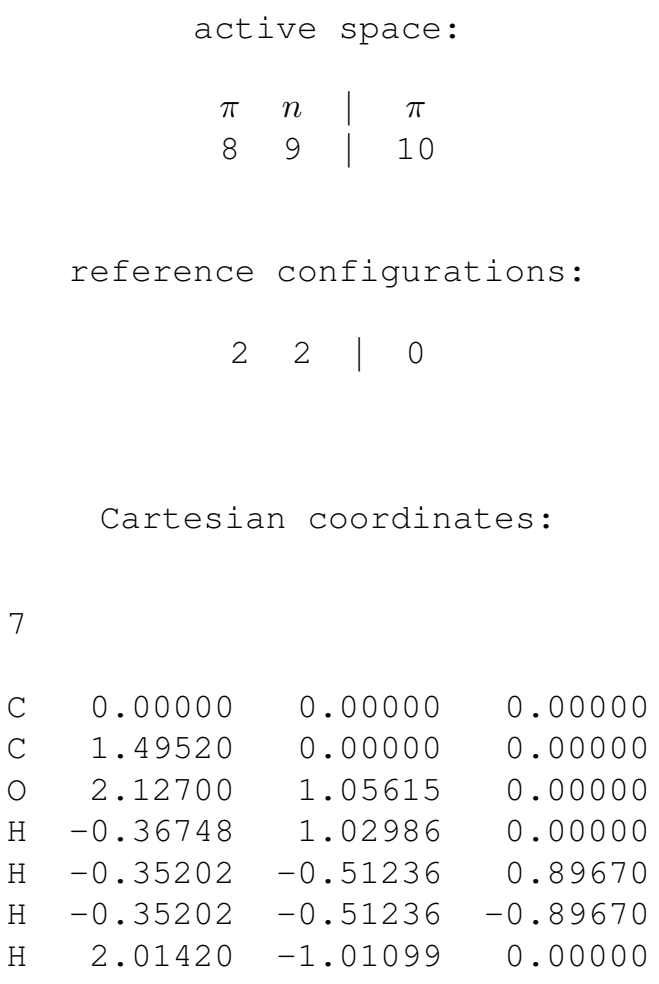


Table S1129 Employed active space, reference configurations, and Cartesian coordinates of the OM2/MRCISD-optimized excited-state equilibrium geometry of the $2{ }^{1} A\left({ }^{1} n \pi^{*}\right)$ state of acetaldehyde (excited-state energy: $\left.-642.81015 \mathrm{eV}\right)$.

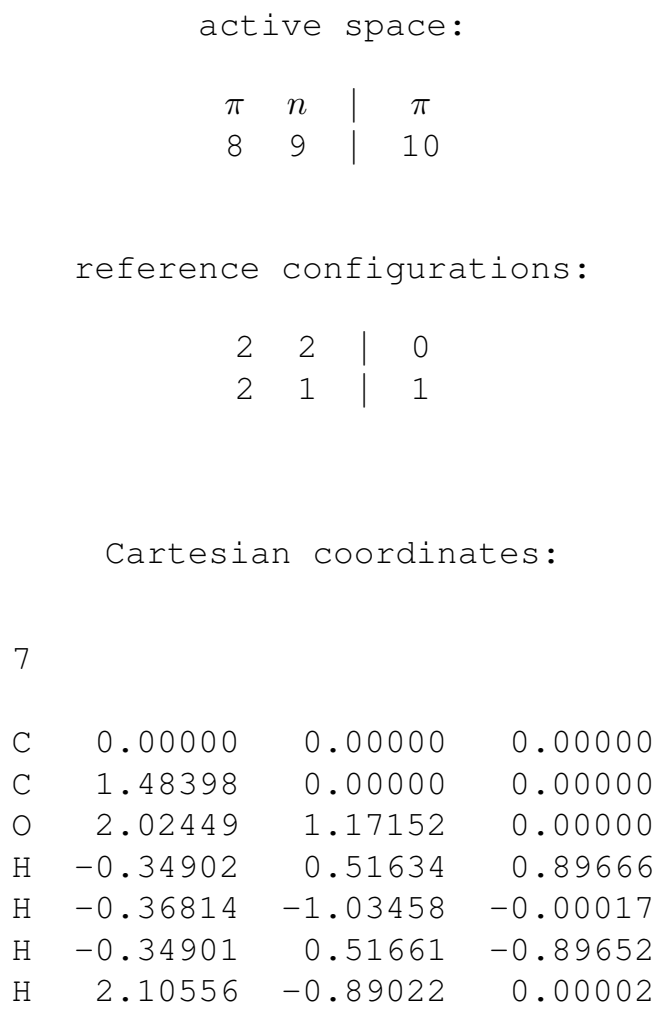


Table S1130 Employed active space, reference configurations, and Cartesian coordinates of the OM2/MRCISD-optimized ground-state equilibrium geometry of acetone (ground-state energy: $-804.60569 \mathrm{eV}$ ).

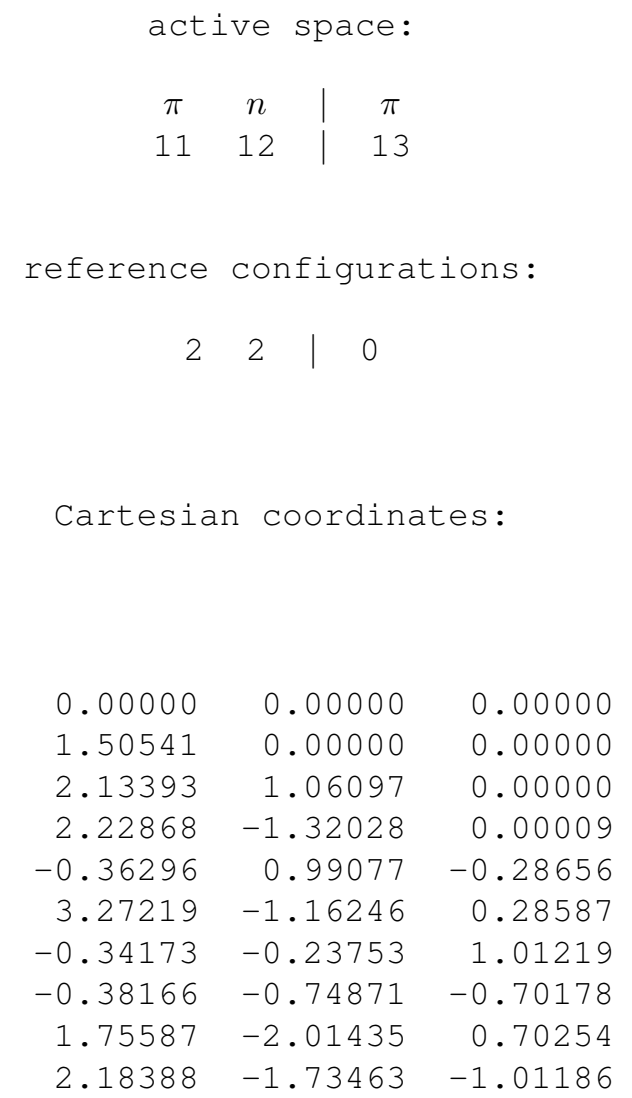


Table S1131 Employed active space, reference configurations, and Cartesian coordinates of the OM2/MRCISD-optimized excited-state equilibrium geometry of the $2{ }^{1} A\left({ }^{1} n \pi^{*}\right)$ state of acetone (excited-state energy: $-800.79104 \mathrm{eV}$ ).

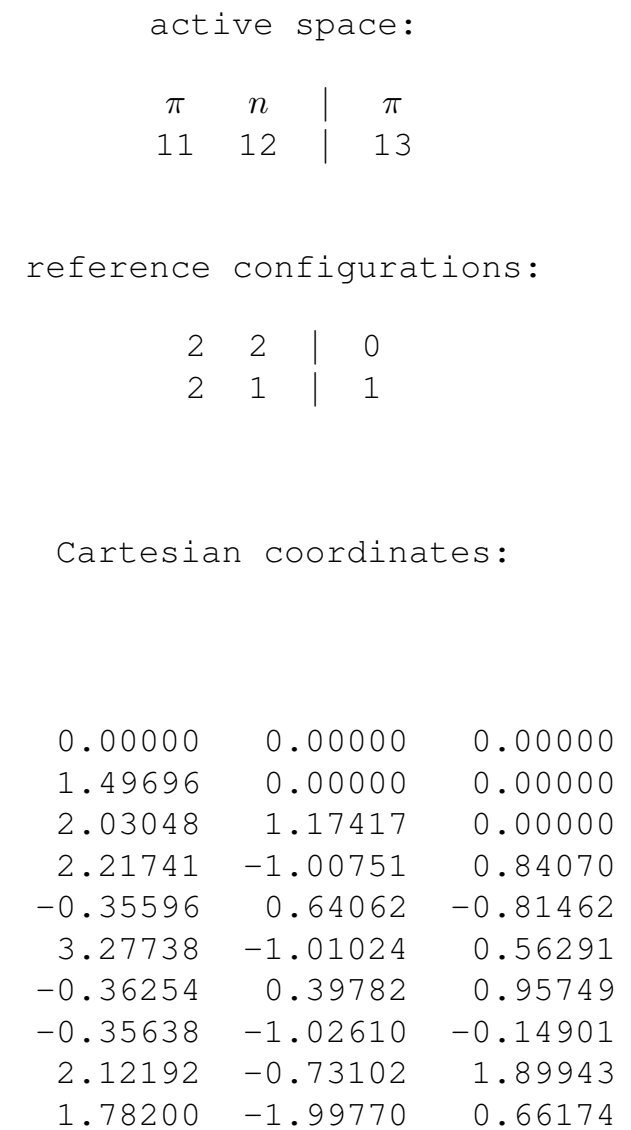


Table S1132 Employed active space, reference configurations, and Cartesian coordinates of the OM2/MRCISD-optimized ground-state equilibrium geometry of acrolein (ground-state energy: $-773.08219 \mathrm{eV}$ ).

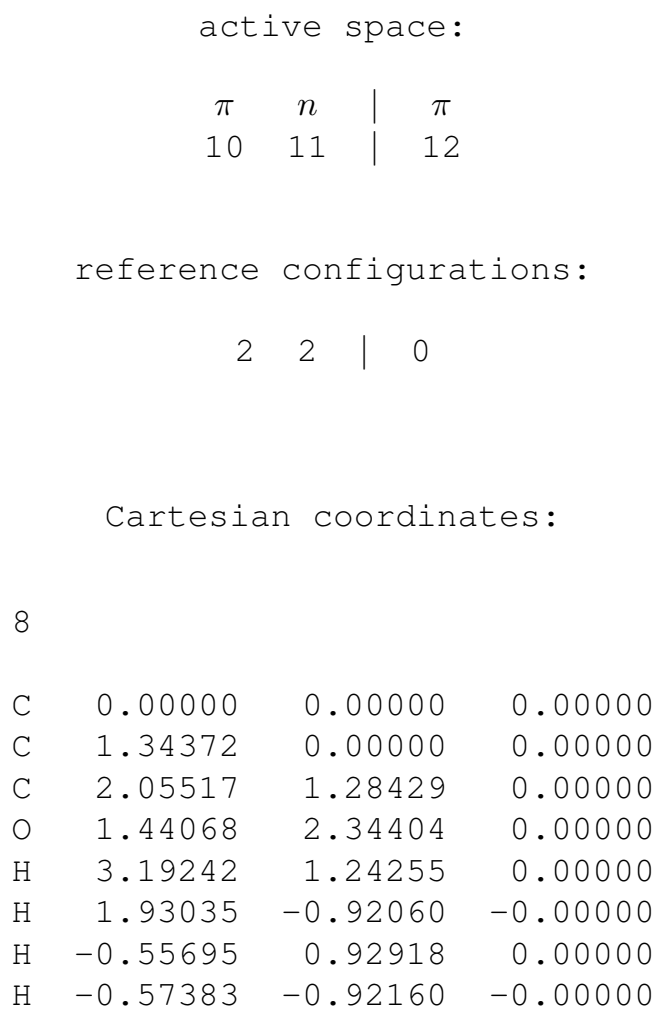


Table S1133 Employed active space, reference configurations, and Cartesian coordinates of the OM2/MRCISD-optimized excited-state equilibrium geometry of the $1{ }^{1} A^{\prime \prime}\left({ }^{1} n \pi^{*}\right)$ state of acrolein (excited-state energy: $-769.40695 \mathrm{eV}$ ).

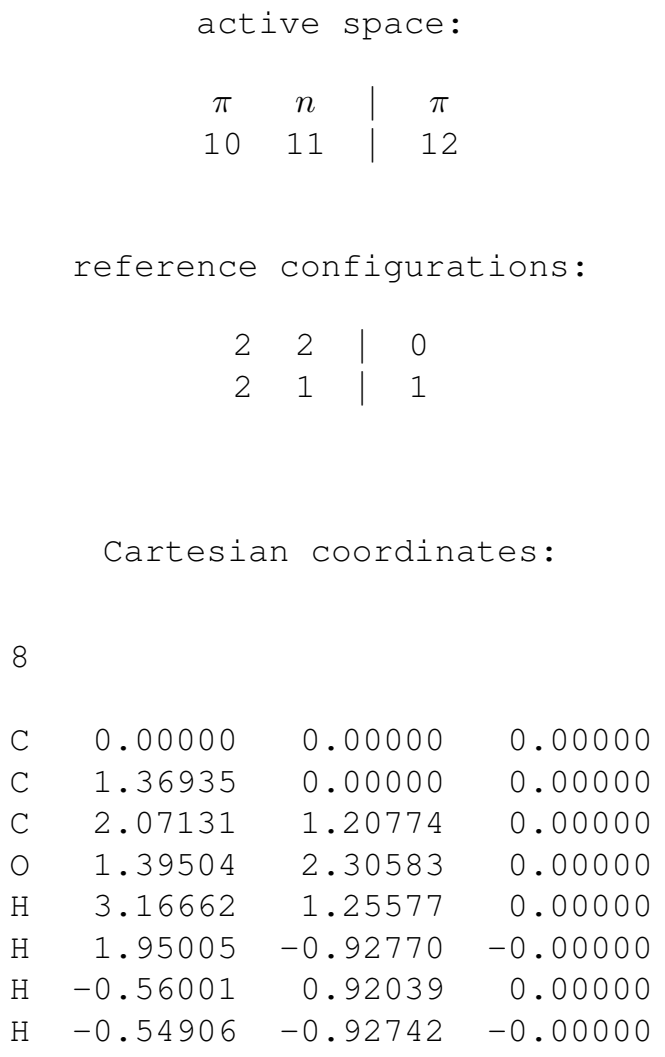


Table S1134 Employed active space, reference configurations, and Cartesian coordinates of the OM2/MRCISD-optimized excited-state equilibrium geometry of the $1^{3} A^{\prime \prime}\left({ }^{3} n \pi^{*}\right)$ state of acrolein (excited-state energy: $-769.55722 \mathrm{eV}$ ).

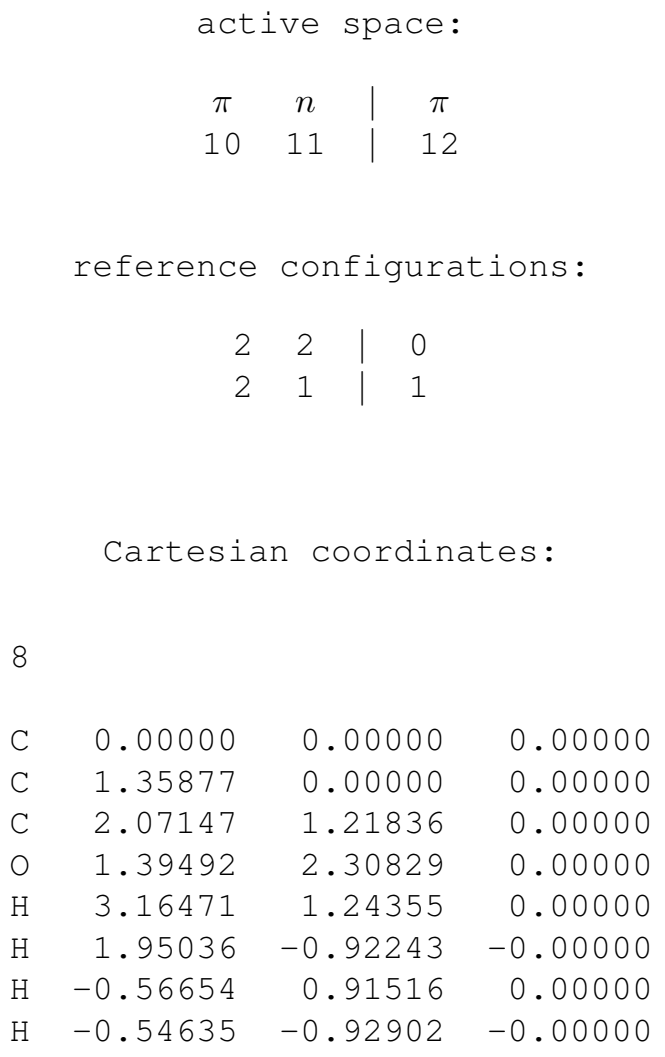


Table S1135 Employed active space, reference configurations, and Cartesian coordinates of the OM2/MRCISD-optimized ground-state equilibrium geometry of aminobenzonitrile (ground-state energy: $-1409.43613 \mathrm{eV}$ ).

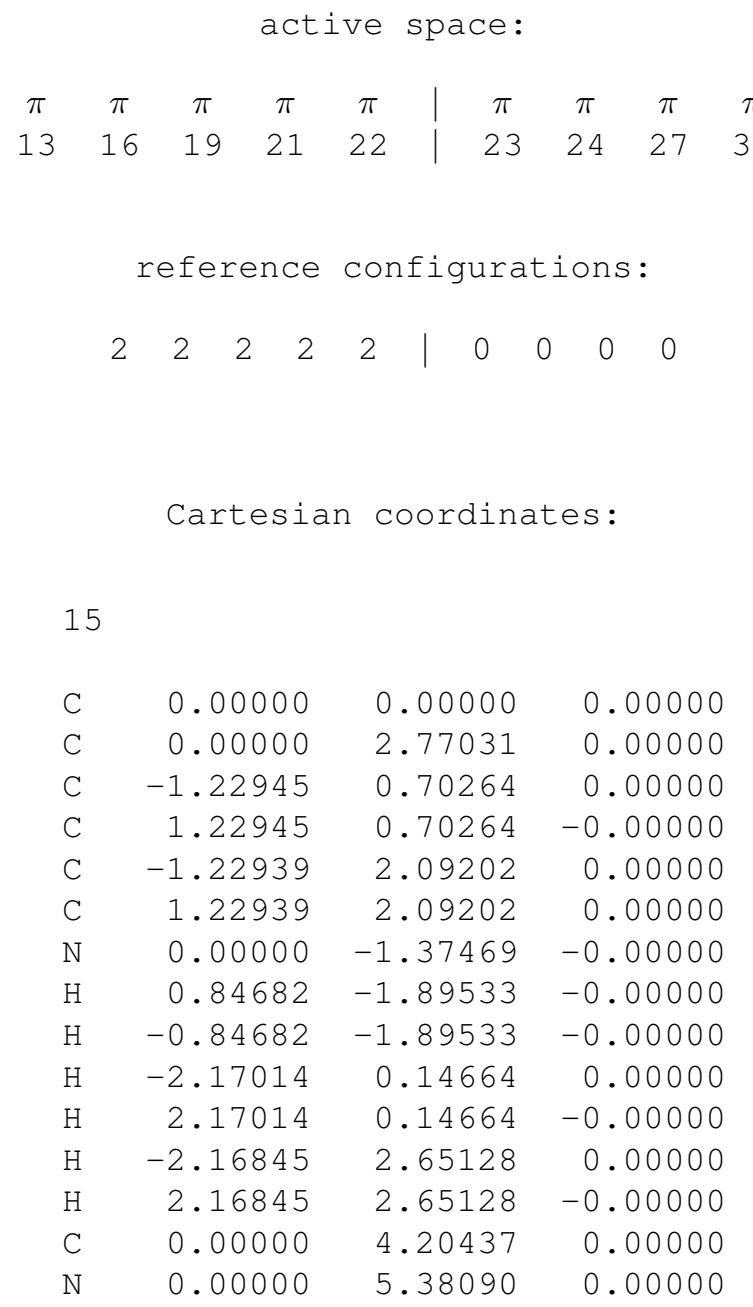


Table S1136 Employed active space, reference configurations, and Cartesian coordinates of the OM2/MRCISD-optimized excited-state equilibrium geometry of the $1{ }^{1} B_{u}\left({ }^{1} \pi \pi^{*}\right)$ state of aminobenzonitrile (excited-state energy: $-1405.54415 \mathrm{eV})$.

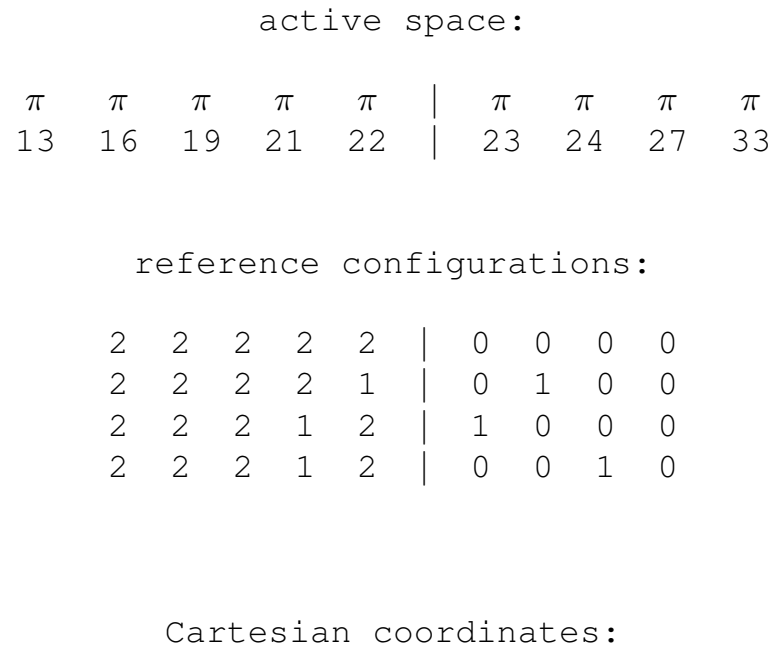


Table S1137 Employed active space, reference configurations, and Cartesian coordinates of the OM2/MRCISD-optimized ground-state equilibrium geometry of aniline (ground-state energy: $-1084.03224 \mathrm{eV}$ ).

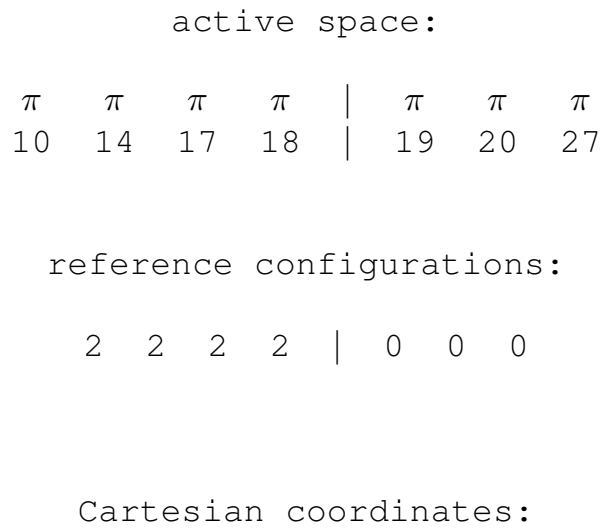


Table S1138 Employed active space, reference configurations, and Cartesian coordinates of the OM2/MRCISD-optimized excited-state equilibrium geometry of the $2{ }^{1} A\left({ }^{1} \pi \pi^{*}\right)$ state of aniline (excited-state energy: $-1080.06598 \mathrm{eV}$ ).

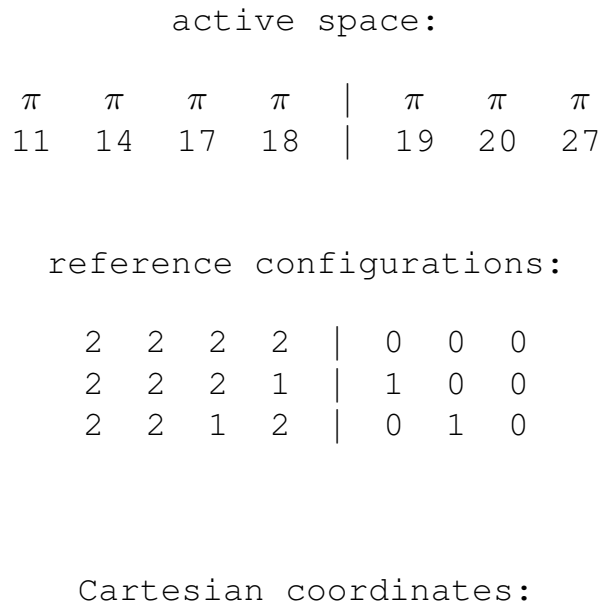


Table S1139 Employed active space, reference configurations, and Cartesian coordinates of the OM2/MRCISD-optimized ground-state equilibrium geometry of anisol (ground-state energy: $-1344.21574 \mathrm{eV}$ ).

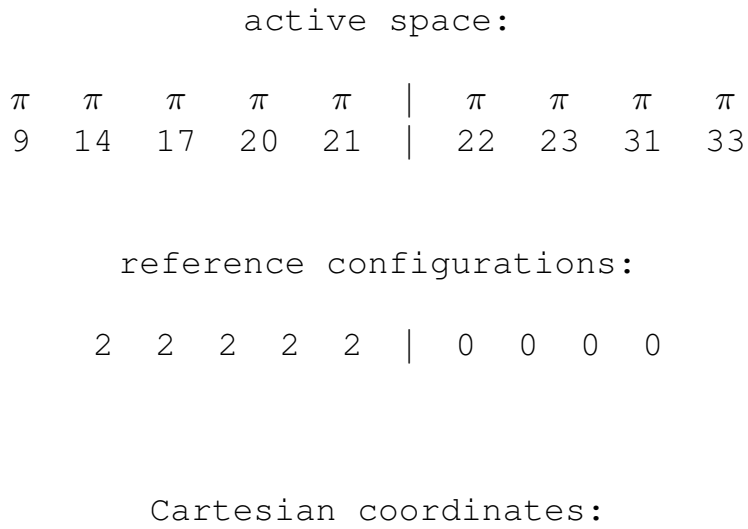

$\begin{array}{llll}\mathrm{H} & 5.25382 & 0.63166 & 0.00000\end{array}$ 
Table S1140 Employed active space, reference configurations, and Cartesian coordinates of the OM2/MRCISD-optimized excited-state equilibrium geometry of the $2{ }^{1} A\left({ }^{1} \pi \pi^{*}\right)$ state of anisol (excited-state energy: $-1340.06931 \mathrm{eV}$ ).

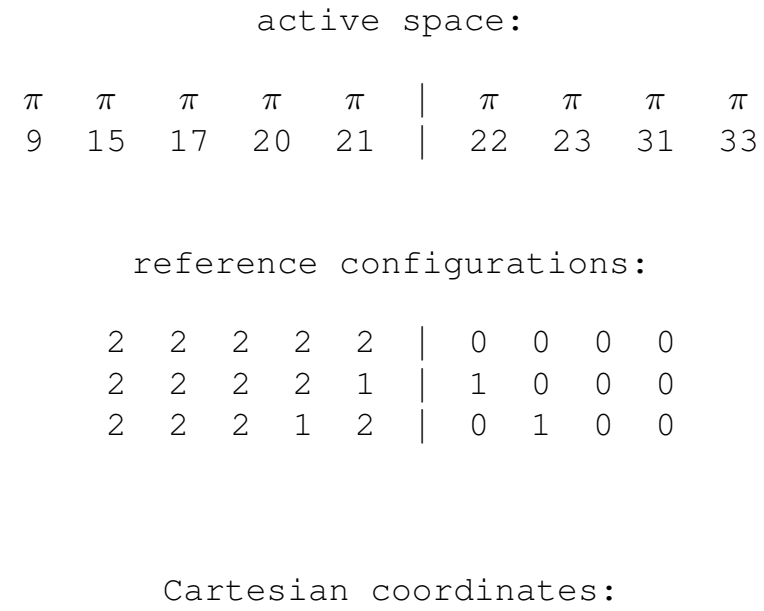

$\begin{array}{llll}\mathrm{H} & 5.26836 & 0.70661 & 0.00000\end{array}$ 
Table S1141 Employed active space, reference configurations, and Cartesian coordinates of the OM2/MRCISD-optimized ground-state equilibrium geometry of anthracene (ground-state energy: $-1936.68785 \mathrm{eV}$ ).

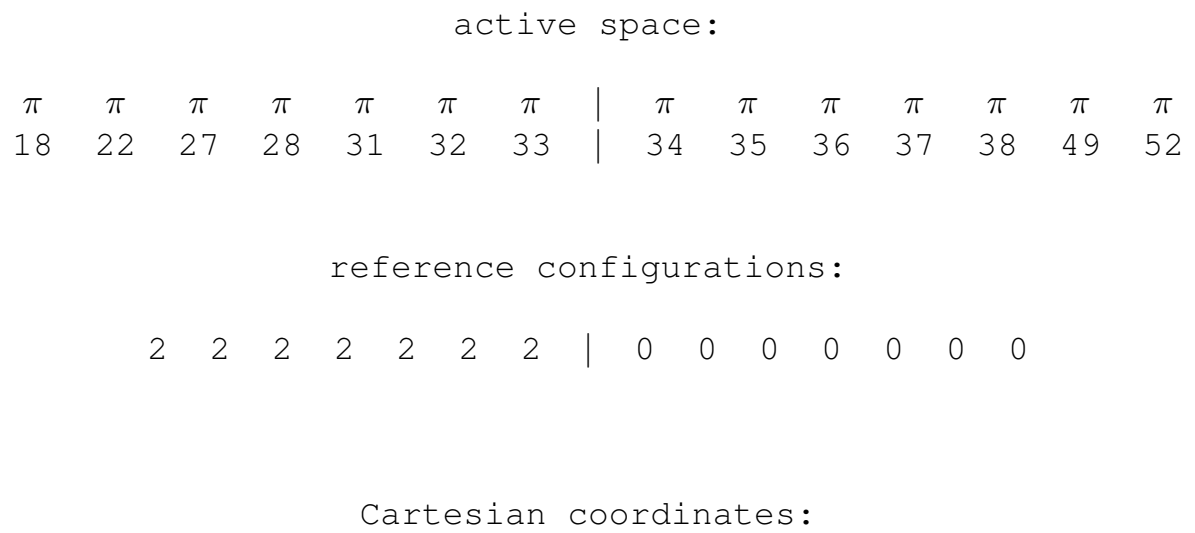


Table S1142 Employed active space, reference configurations, and Cartesian coordinates of the OM2/MRCISD-optimized excited-state equilibrium geometry of the $1^{1} B_{2 u}\left({ }^{1} \pi \pi^{*}\right)$ state of anthracene (excited-state energy: $-1932.81478 \mathrm{eV})$.

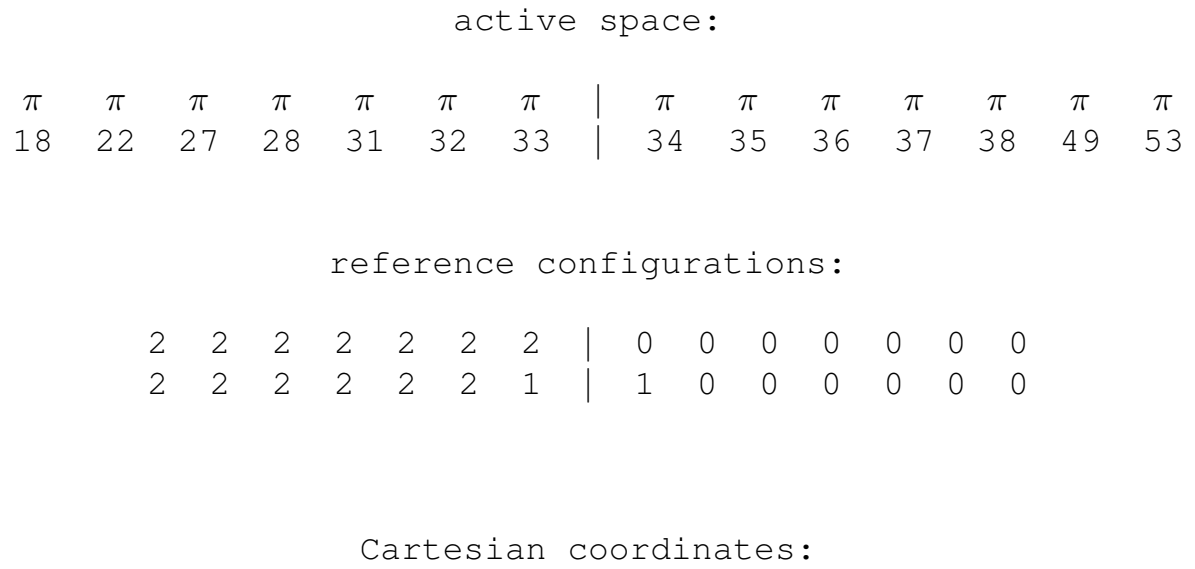

Continued on next page 
Continued from previous page

$\begin{array}{llll}\mathrm{H} & -3.61923 & -0.24388 & -0.00000\end{array}$ 
Table S1143 Employed active space, reference configurations, and Cartesian coordinates of the OM2/MRCISD-optimized ground-state equilibrium geometry of azulene (ground-state energy: $-1394.36421 \mathrm{eV}$ ).

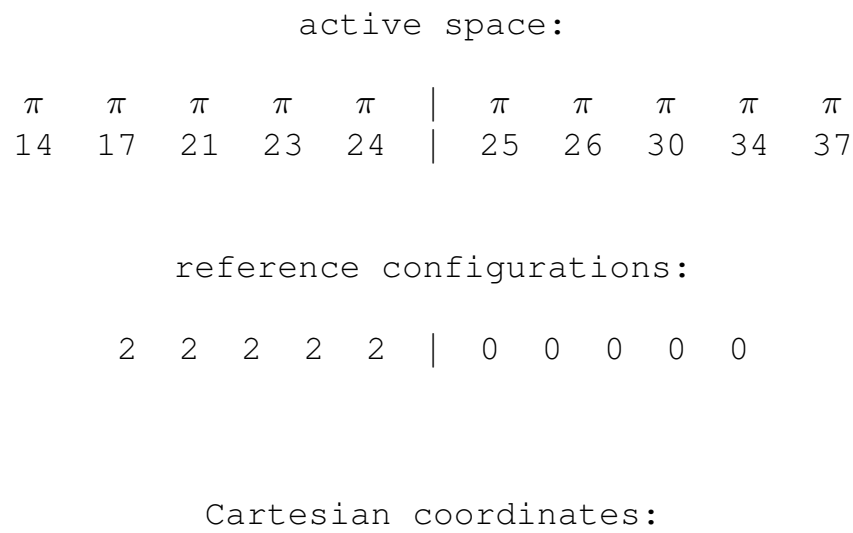


Table S1144 Employed active space, reference configurations, and Cartesian coordinates of the OM2/MRCISD-optimized excited-state equilibrium geometry of the $1{ }^{1} B_{1}\left({ }^{1} \pi \pi^{*}\right)$ state of azulene (excited-state energy: $\left.-1392.81136 \mathrm{eV}\right)$.

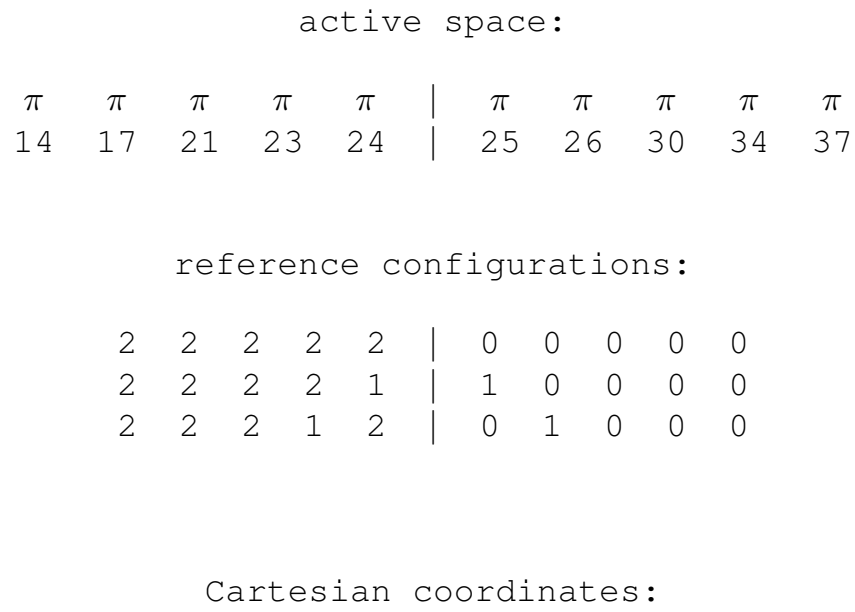


Table S1145 Employed active space, reference configurations, and Cartesian coordinates of the OM2/MRCISD-optimized ground-state equilibrium geometry of benzaldehyde (ground-state energy: $-1314.52437 \mathrm{eV}$ ).

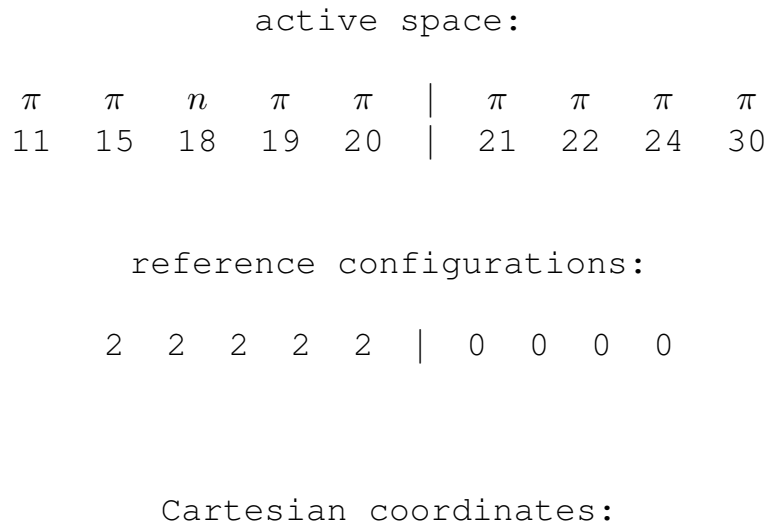


Table S1146 Employed active space, reference configurations, and Cartesian coordinates of the OM2/MRCISD-optimized excited-state equilibrium geometry of the $2^{1} A^{\prime}\left({ }^{1} \pi \pi^{*}\right)$ state of benzaldehyde (excited-state energy: $-1310.34073 \mathrm{eV})$.

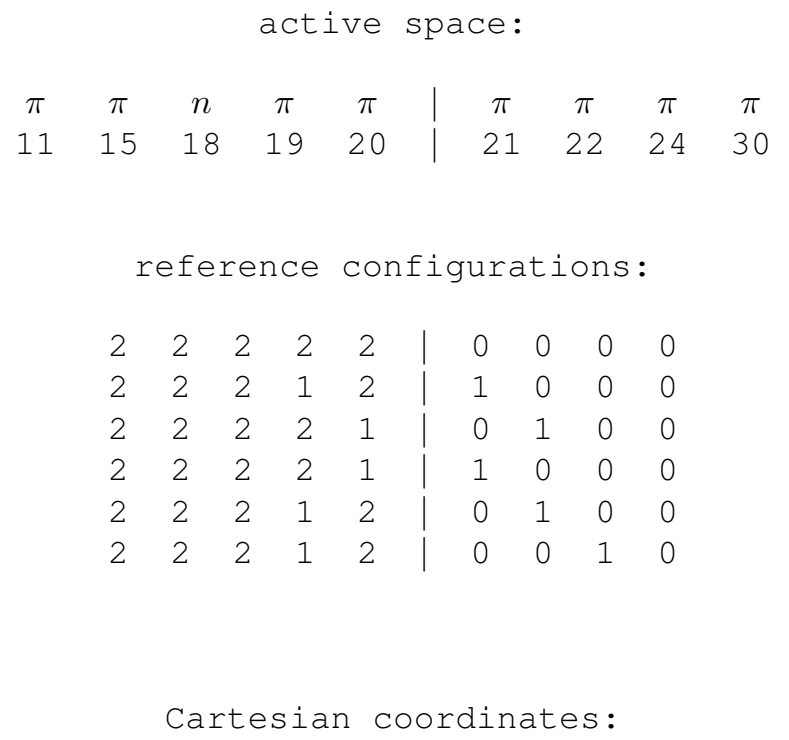

14

$\begin{array}{lrrr}\mathrm{C} & 0.00000 & 0.00000 & 0.00000 \\ \mathrm{C} & 1.42891 & 0.00000 & 0.00000 \\ \mathrm{C} & 2.13256 & 1.23265 & 0.00000 \\ \mathrm{C} & 1.42840 & 2.45971 & 0.00000 \\ \mathrm{C} & 0.01472 & 2.46249 & 0.00000 \\ \mathrm{C} & -0.70607 & 1.23857 & 0.00000 \\ \mathrm{C} & -0.70604 & -1.27456 & -0.00000 \\ \mathrm{O} & -0.06677 & -2.33242 & -0.00000 \\ \mathrm{H} & 1.95967 & -0.95329 & -0.00000 \\ \mathrm{H} & 3.22675 & 1.23139 & 0.00000 \\ \mathrm{H} & 1.97602 & 3.40495 & 0.00000 \\ \mathrm{H} & -0.53017 & 3.41188 & 0.00000 \\ \mathrm{H} & -1.80055 & 1.24449 & 0.00000 \\ \mathrm{H} & -1.83870 & -1.24685 & -0.00000\end{array}$


Table S1147 Employed active space, reference configurations, and Cartesian coordinates of the OM2/MRCISD-optimized excited-state equilibrium geometry of the $1^{1} A^{\prime \prime}\left({ }^{1} n \pi^{*}\right)$ state of benzaldehyde (excited-state energy: $-1310.77409 \mathrm{eV})$.

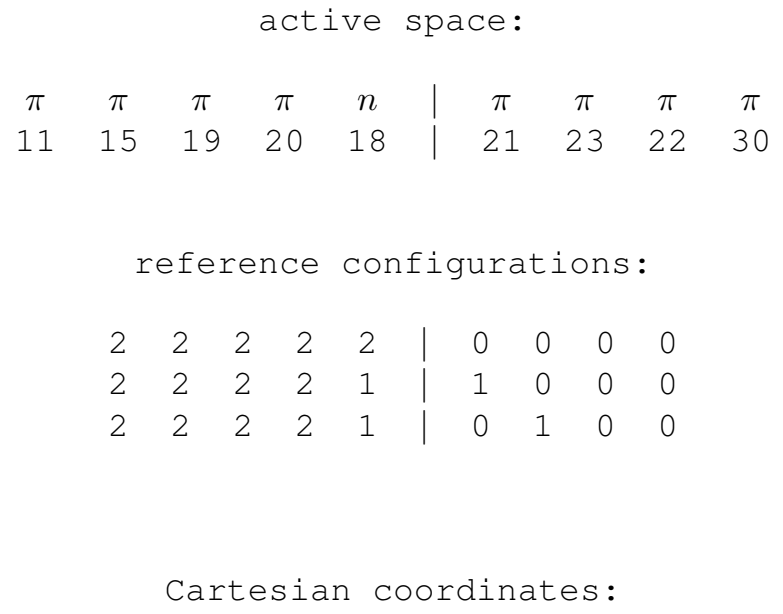


Table S1148 Employed active space, reference configurations, and Cartesian coordinates of the OM2/MRCISD-optimized excited-state equilibrium geometry of the $1^{3} A^{\prime \prime}\left({ }^{3} n \pi^{*}\right)$ state of benzaldehyde (excited-state energy: $-1310.98889 \mathrm{eV})$.

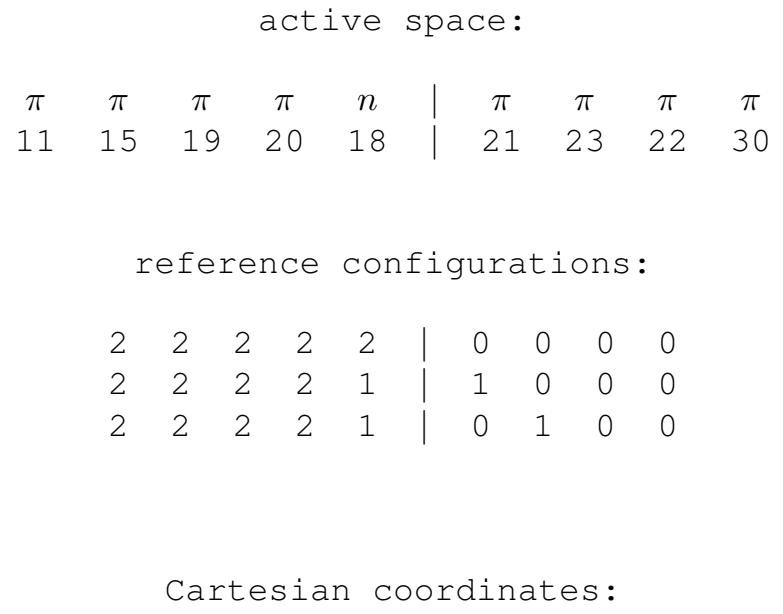


Table S1149 Employed active space, reference configurations, and Cartesian coordinates of the OM2/MRCISD-optimized ground-state equilibrium geometry of benzene (ground-state energy: $-855.78570 \mathrm{eV}$ ).

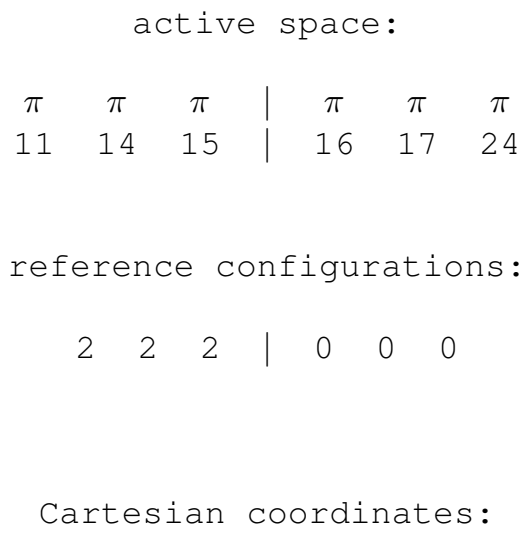

12

$\begin{array}{lrrr}\text { C } & 0.00000 & 1.39632 & 0.00000 \\ \text { C } & 0.00000 & 0.69816 & -1.20925 \\ \text { C } & 0.00000 & 0.69816 & 1.20925 \\ \text { C } & 0.00000 & -0.69816 & -1.20925 \\ \text { C } & 0.00000 & -0.69816 & 1.20925 \\ \text { C } & 0.00000 & -1.39632 & -0.00000 \\ \text { H } & 0.00000 & 2.49219 & 0.00000 \\ \text { H } & 0.00000 & 1.24610 & -2.15830 \\ \text { H } & 0.00000 & 1.24610 & 2.15830 \\ \text { H } & 0.00000 & -1.24610 & -2.15830 \\ \text { H } & 0.00000 & -1.24610 & 2.15830 \\ \text { H } & 0.00000 & -2.49219 & -0.00000\end{array}$


Table S1150 Employed active space, reference configurations, and Cartesian coordinates of the OM2/MRCISD-optimized excited-state equilibrium geometry of the $1{ }^{1} B_{1 u}\left({ }^{1} \pi \pi^{*}\right)$ state of benzene (excited-state energy: $\left.-851.41609 \mathrm{eV}\right)$.

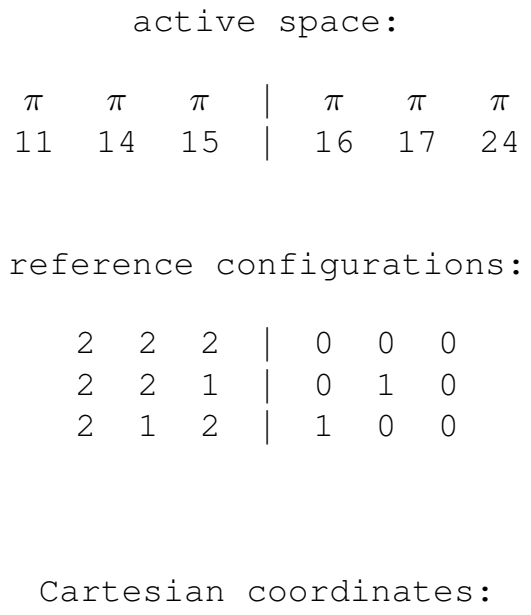

12

C $\quad 0.00000$

$1.41940 \quad 0.00000$

C 0.00000

$0.70970-1.22924$

C 0.00000

$0.70970 \quad 1.22924$

C $\quad 0.00000-0.70970-1.22924$

C $\quad 0.00000 \quad-0.70970 \quad 1.22924$

$\begin{array}{llll}\text { C } & 0.00000 & -1.41940 & -0.00000\end{array}$

$\begin{array}{llll}\mathrm{H} & 0.00000 & 2.51351 & 0.00000\end{array}$

$\mathrm{H} \quad 0.00000 \quad 1.25675 \quad-2.17676$

$\begin{array}{llll}\mathrm{H} & 0.00000 & 1.25675 & 2.17676\end{array}$

$\mathrm{H} \quad 0.00000 \quad-1.25675 \quad-2.17676$

$\mathrm{H} \quad 0.00000 \quad-1.25675 \quad 2.17676$

$\mathrm{H} \quad 0.00000 \quad-2.51351-0.00000$ 
Table S1151 Employed active space, reference configurations, and Cartesian coordinates of the OM2/MRCISD-optimized ground-state equilibrium geometry of benzonitrile (ground-state energy: $-1181.13549 \mathrm{eV}$ ).

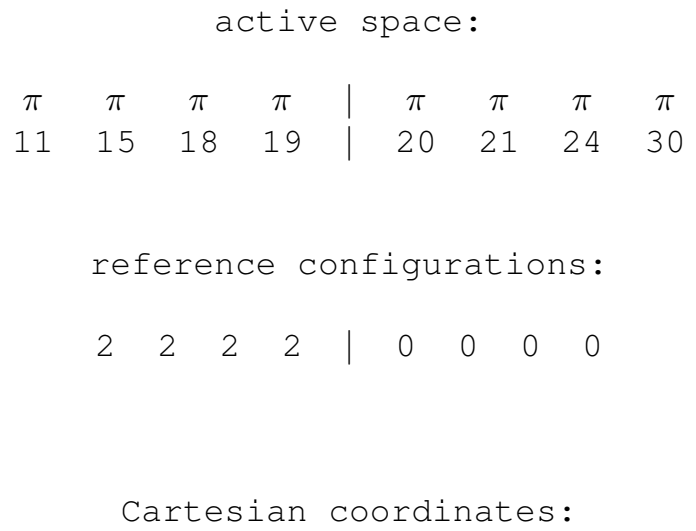


Table S1152 Employed active space, reference configurations, and Cartesian coordinates of the OM2/MRCISD-optimized excited-state equilibrium geometry of the $1{ }^{1} B_{1}\left({ }^{1} \pi \pi^{*}\right)$ state of benzonitrile (excited-state energy: $-1176.89542 \mathrm{eV})$.

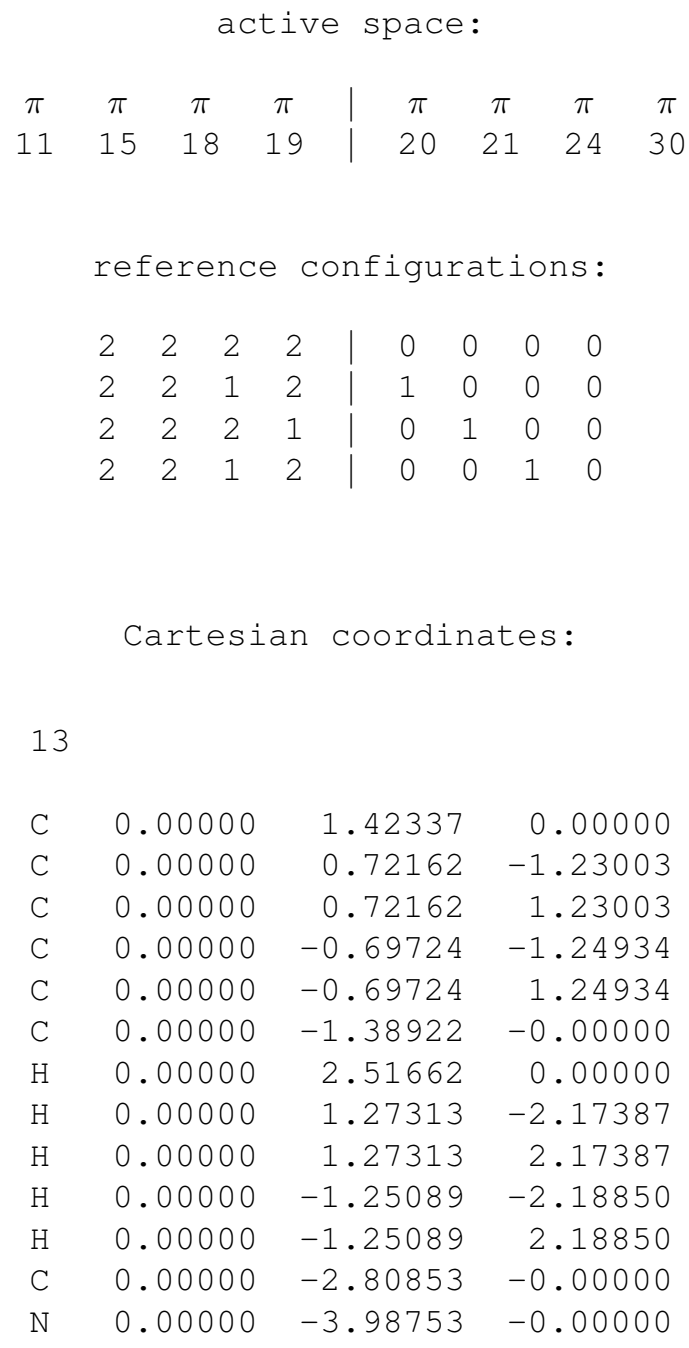


Table S1153 Employed active space, reference configurations, and Cartesian coordinates of the OM2/MRCISD-optimized ground-state equilibrium geometry of benzophenone ketyl radical (ground-state energy: -2153.79469 eV).

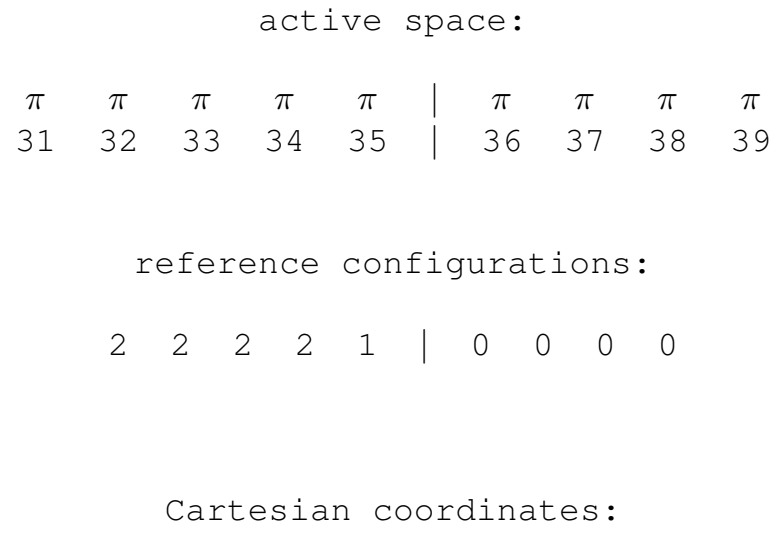

25

$\begin{array}{llll}C & 0.00000 & 0.00000 & 0.00000\end{array}$

C $\quad 1.40120 \quad 0.00000 \quad 0.00000$

$\begin{array}{llll}\text { C } & 2.10899 & 1.21021 & 0.00000\end{array}$

C $\quad 1.41357 \quad 2.41539 \quad 0.04131$

$\begin{array}{lrrr}\text { C } & 0.01746 & 2.41788 & 0.05284\end{array}$

$\begin{array}{lrrr}\text { C } & -0.68580 & 1.21161 & 0.04066\end{array}$

C $\quad 2.11782-1.28654-0.08054$

$\begin{array}{llll}0 & 3.24057 & -1.28522 & -0.83504\end{array}$

$\begin{array}{llll}\text { C } & 2.20694 & -2.21046 & 1.04240\end{array}$

$\begin{array}{llll}\text { C } & 1.32091 & -2.09121 & 2.12613\end{array}$

$\begin{array}{llll}\text { C } & 1.33699 & -3.05358 & 3.13033\end{array}$

$\begin{array}{llll}\text { C } & 2.22157 & -4.13224 & 3.06109\end{array}$

$\begin{array}{llll}\text { C } & 3.10160 & -4.24713 & 1.98241\end{array}$

$\begin{array}{llll}\text { C } & 3.09770 & -3.29460 & 0.96939\end{array}$

$\mathrm{H} \quad 0.64032 \quad-1.23703 \quad 2.18870$

$\mathrm{H} \quad 0.66345 \quad-2.95657 \quad 3.98888$

$\mathrm{H} \quad \begin{array}{llll}2.22688 & -4.88673 & 3.85557\end{array}$

$\mathrm{H} \quad 3.79983 \quad-5.08964 \quad 1.93836$

$\begin{array}{llll}\mathrm{H} & 3.78174 & -3.37272 & 0.12128\end{array}$

$\begin{array}{llll}\mathrm{H} & 3.20329 & 1.20546 & 0.00224\end{array}$

$\begin{array}{llll}\mathrm{H} & 1.96118 & 3.36070 & 0.07219\end{array}$

$\begin{array}{llll}\mathrm{H} & -0.52661 & 3.36856 & 0.06068\end{array}$

$\begin{array}{lrrr}\mathrm{H} & -1.78016 & 1.21866 & 0.06107\end{array}$

$\begin{array}{llll}\mathrm{H} & -0.54341 & -0.94866 & -0.02449\end{array}$

$\begin{array}{llll}\mathrm{H} & 3.13363 & -0.62726 & -1.57317\end{array}$ 
Table S1154 Employed active space, reference configurations, and Cartesian coordinates of the OM2/MRCISD-optimized excited-state equilibrium geometry of the $2{ }^{2} A\left({ }^{2} \pi \pi^{*}\right)$ state of benzophenone ketyl radical (excited-state energy: $-2151.07486 \mathrm{eV})$.

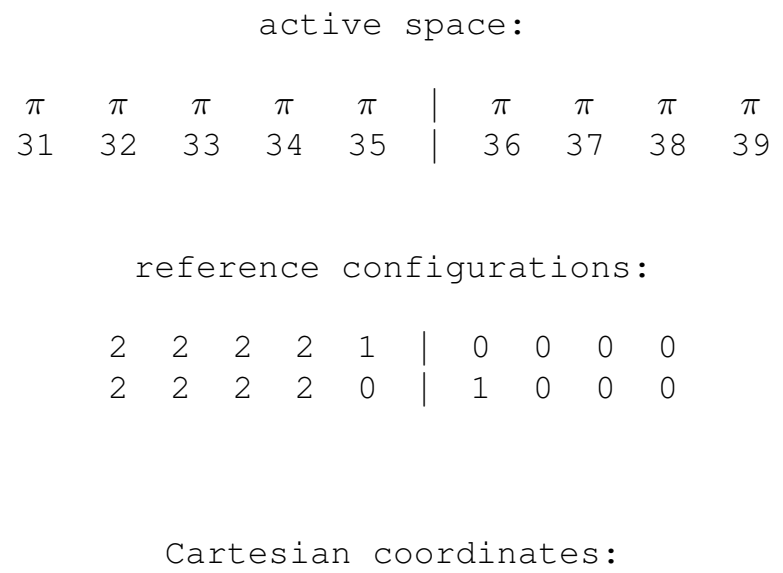


Continued from previous page

\begin{tabular}{rrrr}
\hline $\mathrm{H}$ & -0.50763 & -0.95969 & -0.14983 \\
$\mathrm{H}$ & 3.78411 & -0.60253 & 0.18712
\end{tabular}


Table S1155 Employed active space, reference configurations, and Cartesian coordinates of the OM2/MRCISD-optimized ground-state equilibrium geometry of biphenyl (ground-state energy: $-1681.53106 \mathrm{eV}$ ).

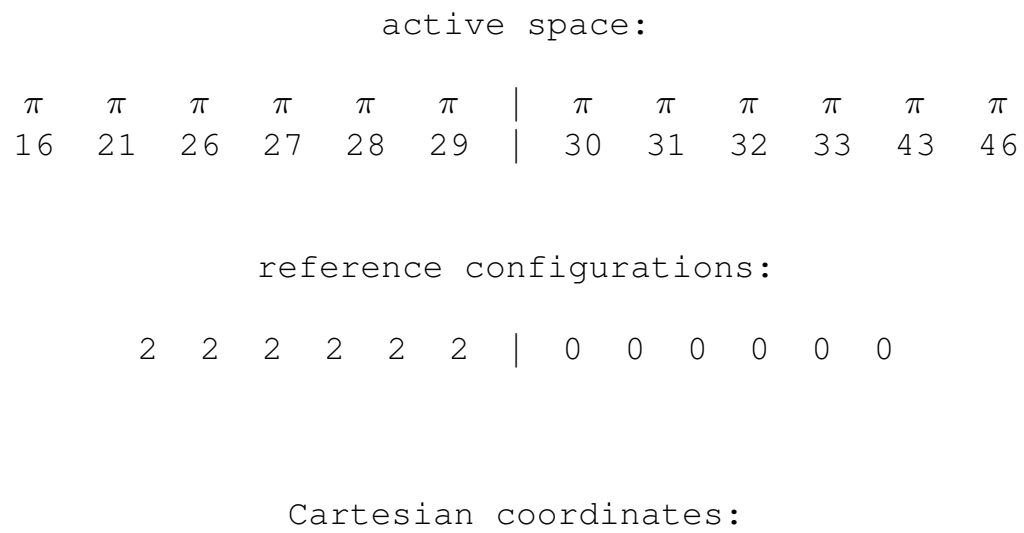


Table S1156 Employed active space, reference configurations, and Cartesian coordinates of the OM2/MRCISD-optimized excited-state equilibrium geometry of the $1{ }^{1} B_{1 u}\left({ }^{1} \pi \pi^{*}\right)$ state of biphenyl (excited-state energy: $\left.-1676.70470 \mathrm{eV}\right)$.

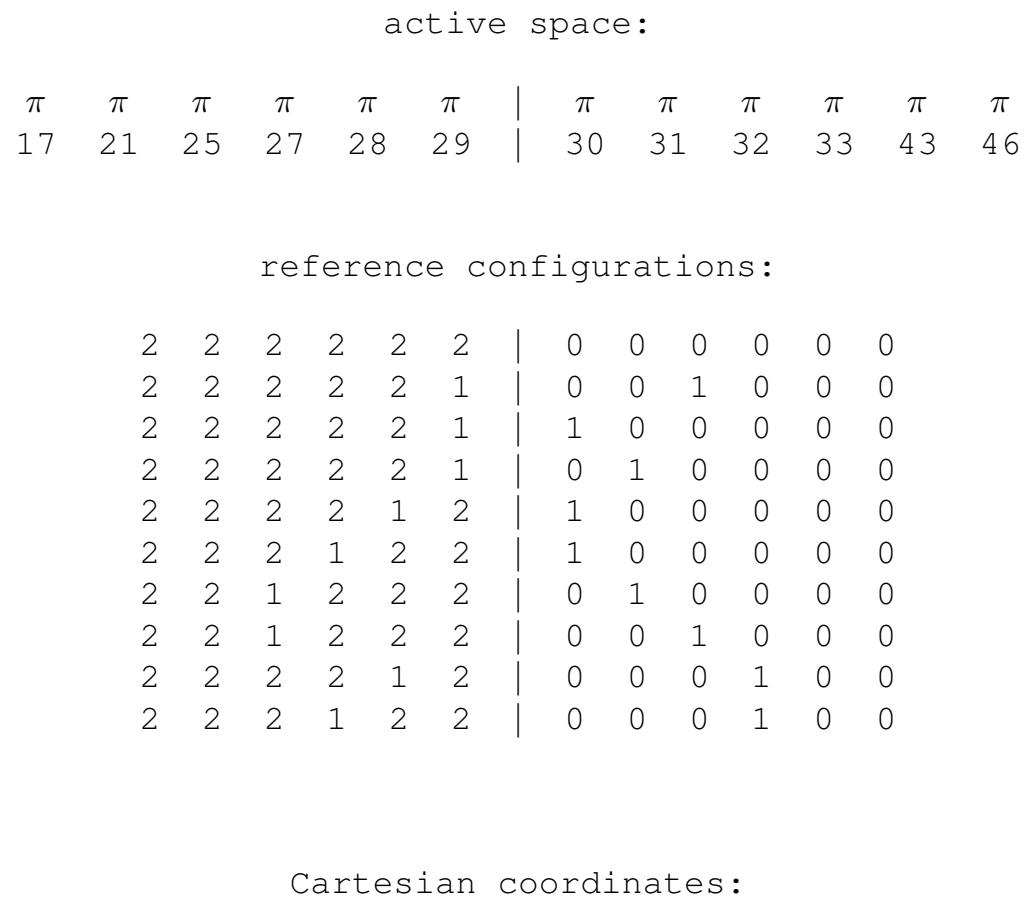

\begin{tabular}{lrrr}
22 & & & \\
C & 0.00000 & 0.00000 & 0.70941 \\
C & 0.00000 & 0.00000 & -0.70941 \\
C & 1.23126 & 0.00000 & 1.45729 \\
C & 1.23126 & 0.00000 & -1.45729 \\
C & -1.23126 & 0.00000 & 1.45729 \\
C & -1.23126 & -0.00000 & -1.45729 \\
C & 1.21577 & 0.00000 & 2.83778 \\
C & 1.21577 & 0.00000 & -2.83778 \\
C & -1.21577 & 0.00000 & 2.83778 \\
C & -1.21577 & 0.00000 & -2.83778 \\
C & 0.00000 & -0.00000 & 3.54468 \\
C & 0.00000 & 0.00000 & -3.54468 \\
H & 2.19255 & 0.00000 & 0.93035 \\
H & 2.19255 & 0.00000 & -0.93035 \\
H & -2.19255 & 0.00000 & 0.93035 \\
H & -2.19255 & -0.00000 & -0.93035 \\
\hline
\end{tabular}

Continued on next page 


\begin{tabular}{lrrr}
\multicolumn{4}{c}{ Continued from previous page } \\
\hline $\mathrm{H}$ & 2.15893 & 0.00000 & 3.39751 \\
$\mathrm{H}$ & 2.15893 & 0.00000 & -3.39751 \\
$\mathrm{H}$ & -2.15893 & 0.00000 & 3.39751 \\
$\mathrm{H}$ & -2.15893 & -0.00000 & -3.39751 \\
$\mathrm{H}$ & 0.00000 & -0.00000 & 4.63903 \\
$\mathrm{H}$ & 0.00000 & 0.00000 & -4.63903
\end{tabular}


Table S1157 Employed active space, reference configurations, and Cartesian coordinates of the OM2/MRCISD-optimized ground-state equilibrium geometry of biphenylene (ground-state energy: $-1649.05835 \mathrm{eV}$ ).

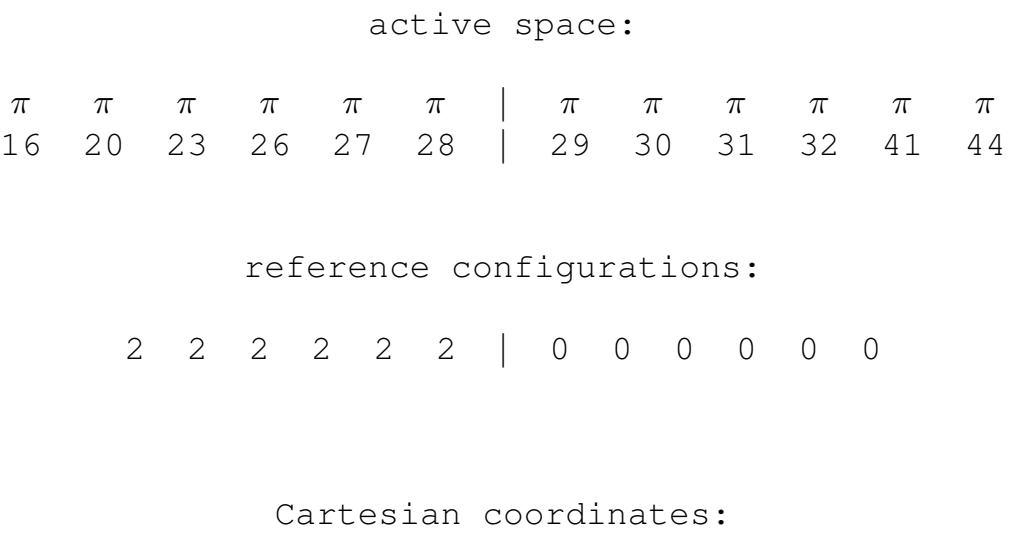


Table S1158 Employed active space, reference configurations, and Cartesian coordinates of the OM2/MRCISD-optimized excited-state equilibrium geometry of the $1{ }^{1} B_{3 u}\left({ }^{1} \pi \pi^{*}\right)$ state of biphenylene (excited-state energy: $-1645.36751 \mathrm{eV})$.

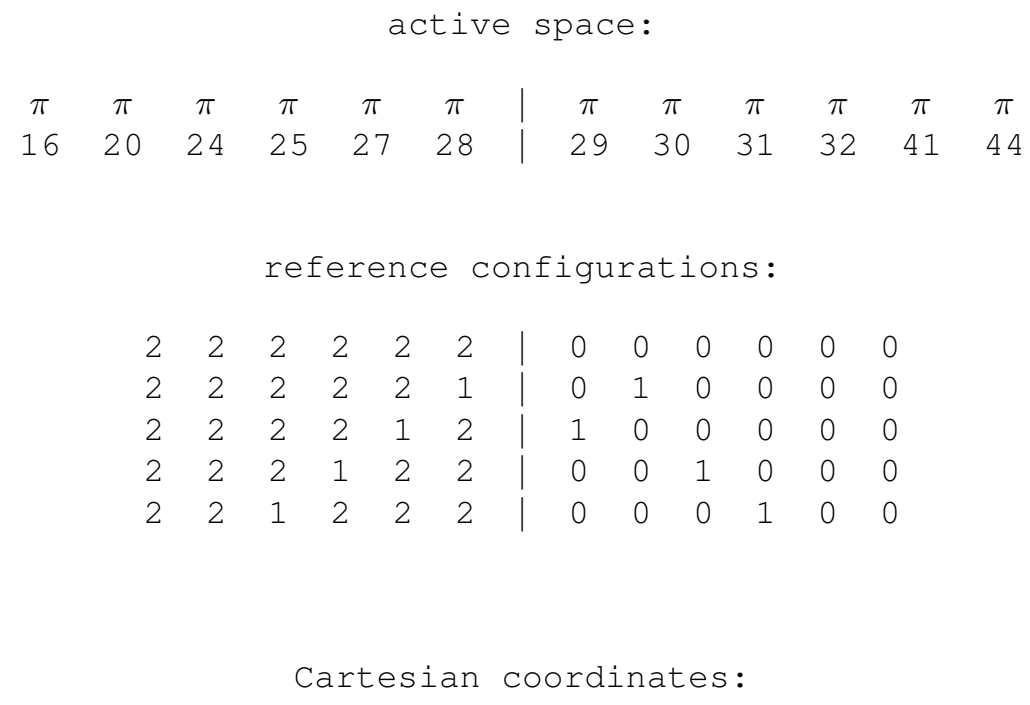

20

$\begin{array}{lrrr}\mathrm{C} & 0.00000 & 0.00000 & 0.00000 \\ \mathrm{C} & 1.46504 & 0.00000 & 0.00000 \\ \mathrm{C} & 0.00000 & 1.45755 & 0.00000 \\ \mathrm{C} & 1.46504 & 1.45755 & 0.00000 \\ \mathrm{C} & -1.17722 & -0.74794 & 0.00000 \\ \mathrm{C} & 2.64225 & -0.74794 & -0.00000 \\ \mathrm{C} & -1.17722 & 2.20549 & -0.00000 \\ \mathrm{C} & 2.64225 & 2.20549 & 0.00000 \\ \mathrm{C} & -2.36111 & 0.02639 & 0.00000 \\ \mathrm{C} & 3.82615 & 0.02639 & -0.00000 \\ \mathrm{C} & -2.36111 & 1.43116 & -0.00000 \\ \mathrm{C} & 3.82615 & 1.43116 & 0.00000 \\ \mathrm{H} & -1.19588 & -1.83585 & 0.00000 \\ \mathrm{H} & 2.66092 & -1.83585 & -0.00000 \\ \mathrm{H} & -1.19588 & 3.29340 & -0.00000 \\ \mathrm{H} & 2.66092 & 3.29340 & 0.00000 \\ \mathrm{H} & -3.32565 & -0.49728 & 0.00000 \\ \mathrm{H} & 4.79069 & -0.49728 & -0.00000 \\ \mathrm{H} & -3.32565 & 1.95482 & -0.00000 \\ \mathrm{H} & 4.79069 & 1.95482 & 0.00000\end{array}$


Table S1159 Employed active space, reference configurations, and Cartesian coordinates of the OM2/MRCISD-optimized ground-state equilibrium geometry of acetylene (ground-state energy: $-283.51212 \mathrm{eV}$ ).

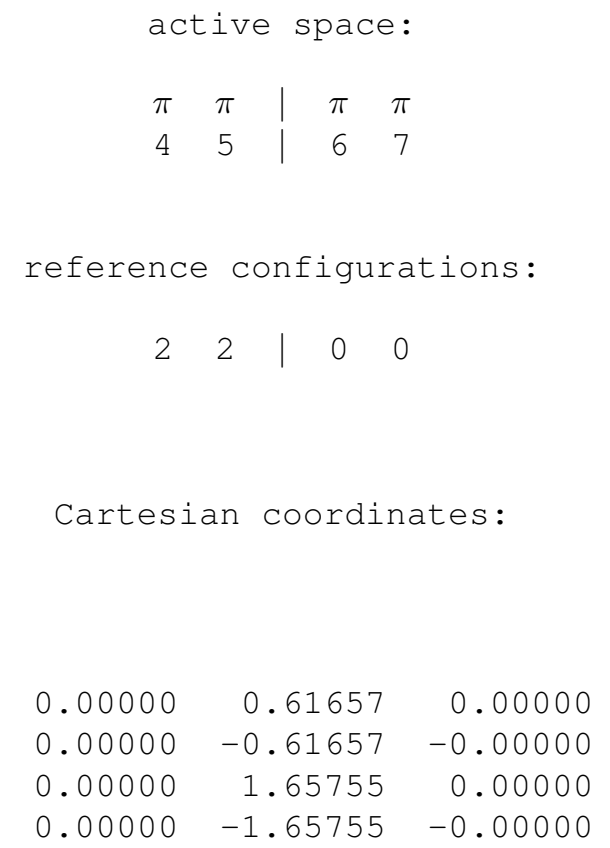


Table S1160 Employed active space, reference configurations, and Cartesian coordinates of the OM2/MRCISD-optimized excited-state equilibrium geometry of the $2{ }^{1} A\left({ }^{1} \pi \pi^{*}\right)$ state of acetylene (excited-state energy: $-278.29425 \mathrm{eV}$ ).

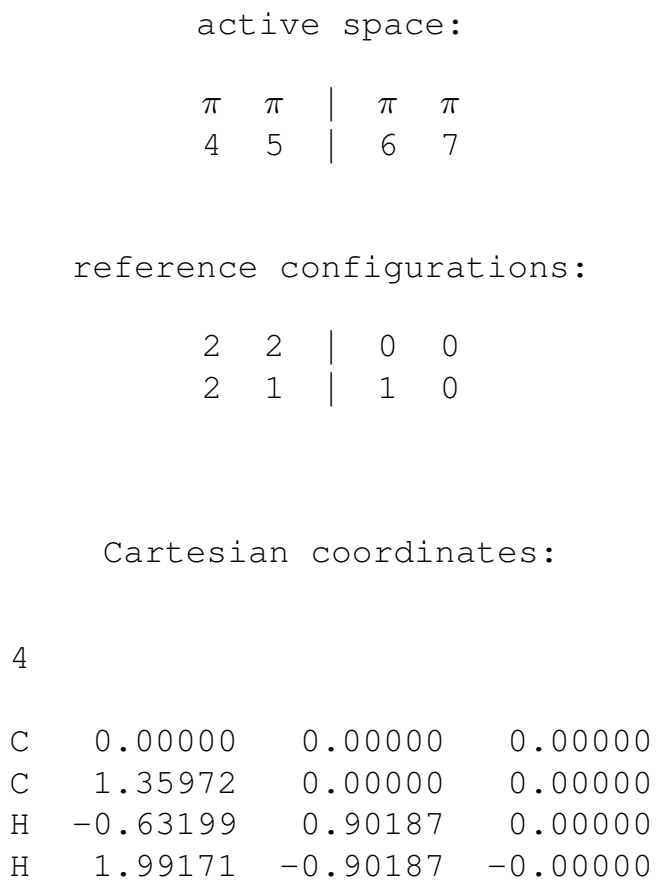


Table S1161 Employed active space, reference configurations, and Cartesian coordinates of the OM2/MRCISD-optimized ground-state equilibrium geometry of formaldehyde (ground-state energy: $-488.47484 \mathrm{eV}$ ).

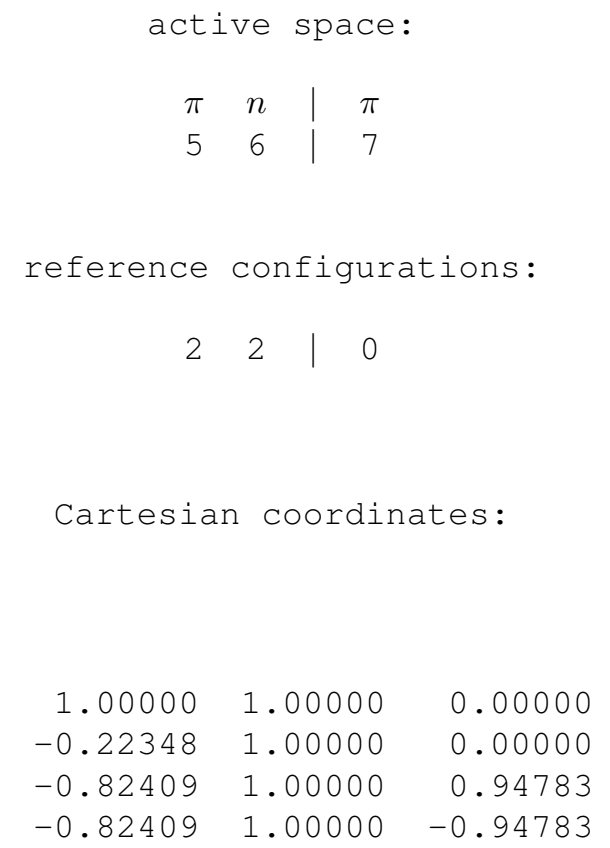


Table S1162 Employed active space, reference configurations, and Cartesian coordinates of the OM2/MRCISD-optimized excited-state equilibrium geometry of the $2{ }^{1} A\left({ }^{1} n \pi^{*}\right)$ state of formaldehyde (excited-state energy: $\left.-485.05556 \mathrm{eV}\right)$.

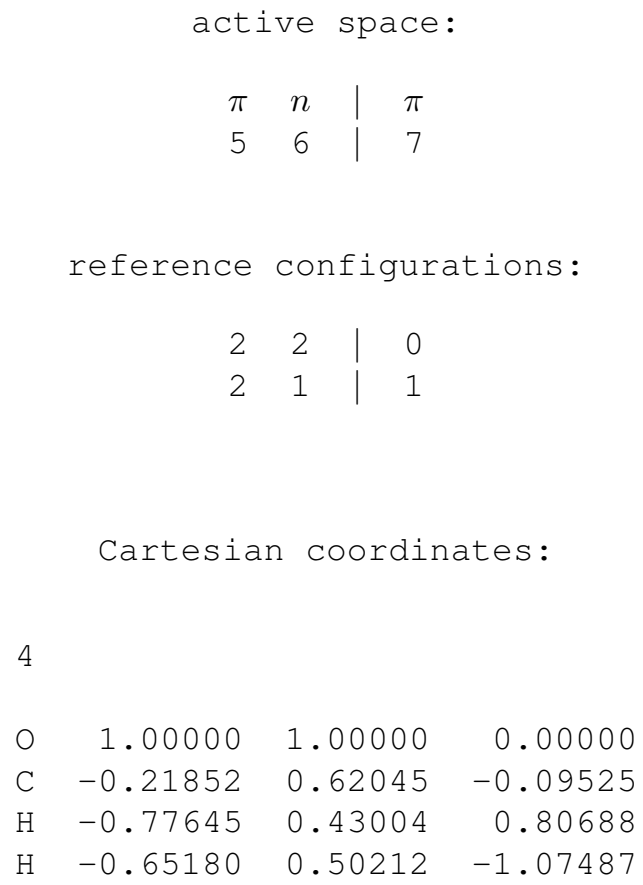


Table S1163 Employed active space, reference configurations, and Cartesian coordinates of the OM2/MRCISD-optimized excited-state equilibrium geometry of the $1^{3} A\left({ }^{3} n \pi^{*}\right)$ state of formaldehyde (excited-state energy: $\left.-485.35944 \mathrm{eV}\right)$.

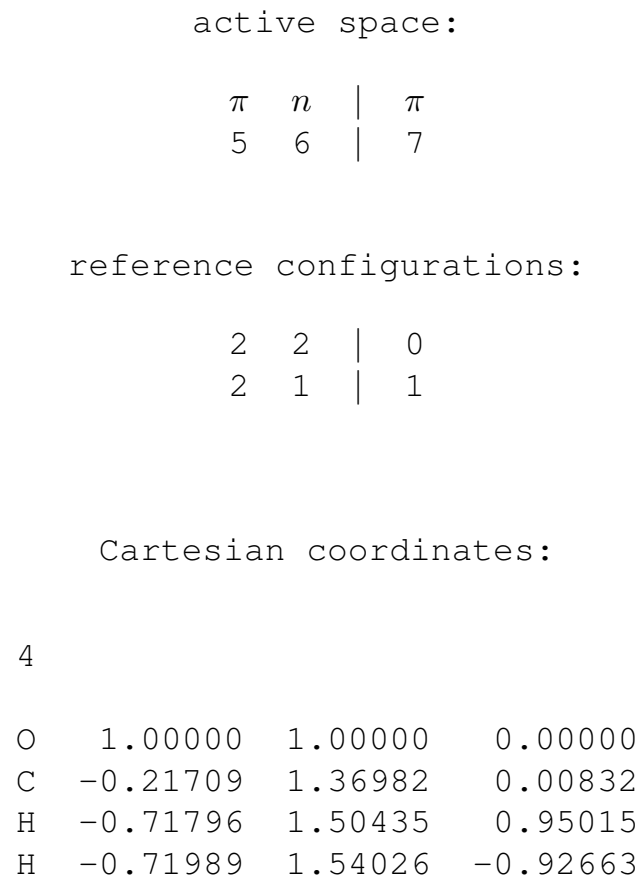


Table S1164 Employed active space, reference configurations, and Cartesian coordinates of the OM2/MRCISD-optimized ground-state equilibrium geometry of cinnoline (ground-state energy: $-1537.06020 \mathrm{eV}$ ).

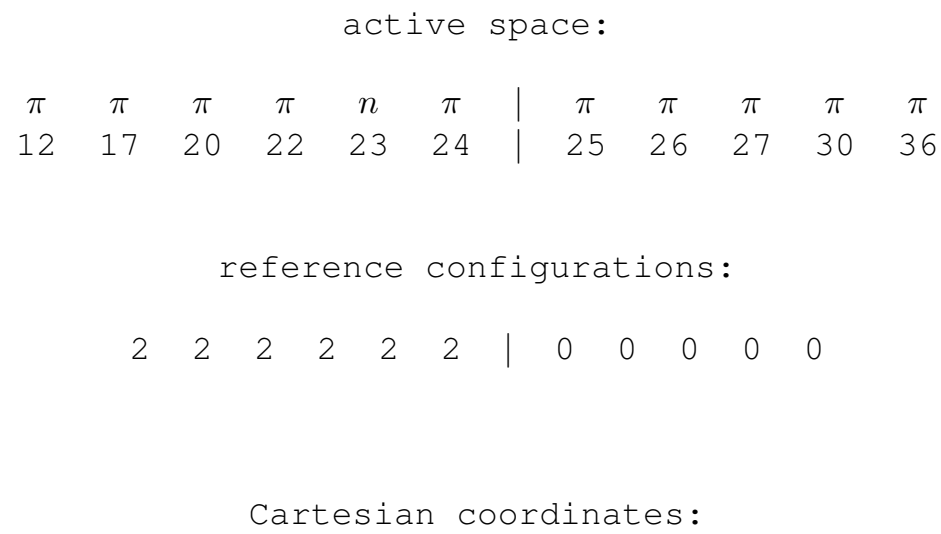


Table S1165 Employed active space, reference configurations, and Cartesian coordinates of the OM2/MRCISD-optimized excited-state equilibrium geometry of the $1^{1} A^{\prime \prime}\left({ }^{1} n \pi^{*}\right)$ state of cinnoline (excited-state energy: $-1533.70655 \mathrm{eV}$ ).

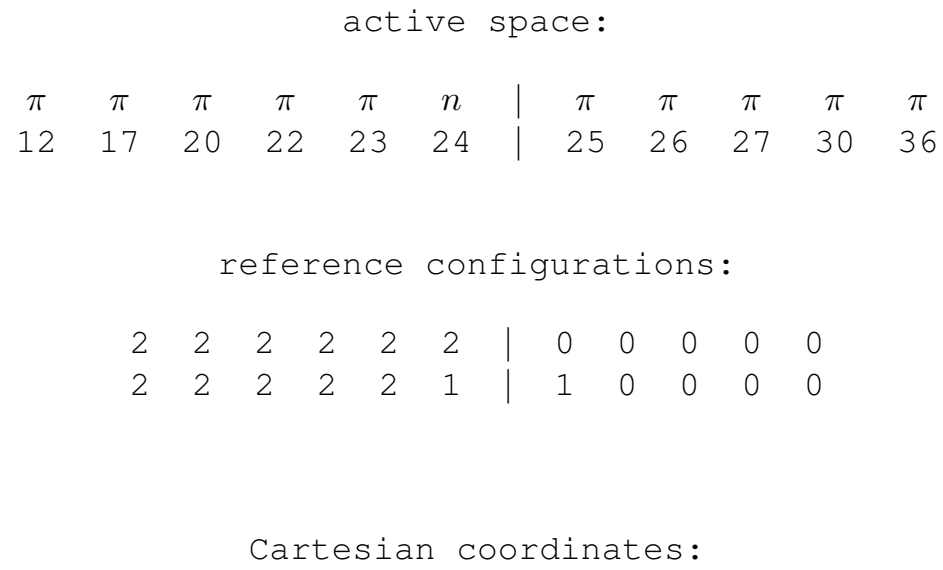


Table S1166 Employed active space, reference configurations, and Cartesian coordinates of the OM2/MRCISD-optimized ground-state equilibrium geometry of cyanoacetylene (ground-state energy: $-608.00605 \mathrm{eV}$ ).

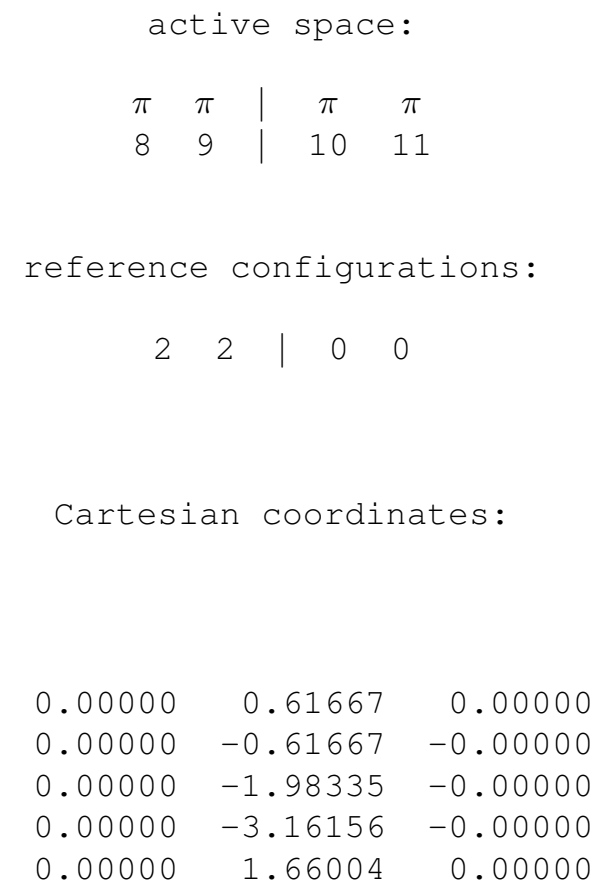


Table S1167 Employed active space, reference configurations, and Cartesian coordinates of the OM2/MRCISD-optimized excited-state equilibrium geometry of the $2^{1} A^{\prime}\left({ }^{1} \pi \pi^{*}\right)$ state of cyanoacetylene (excited-state energy: $-602.45268 \mathrm{eV})$.

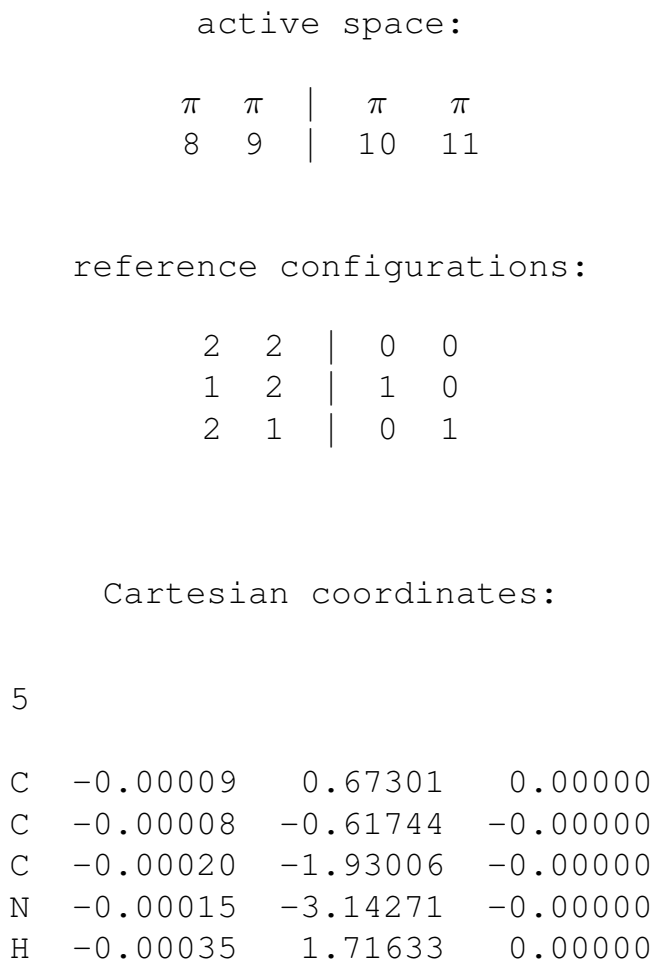


Table S1168 Employed active space, reference configurations, and Cartesian coordinates of the OM2/MRCISD-optimized ground-state equilibrium geometry of cyclohexadienyl radical (ground-state energy: $-869.43673 \mathrm{eV}$ ).

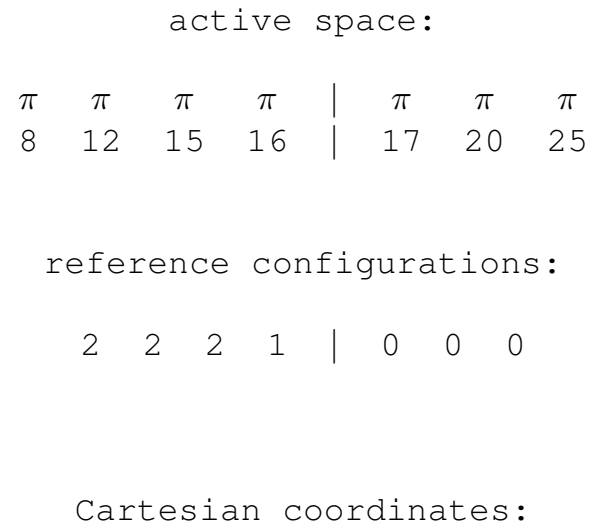


Table S1169 Employed active space, reference configurations, and Cartesian coordinates of the OM2/MRCISD-optimized excited-state equilibrium geometry of the $2^{2} A\left({ }^{2} \pi \pi^{*}\right)$ state of cyclohexadienyl radical (excited-state energy: $-867.28805 \mathrm{eV})$.

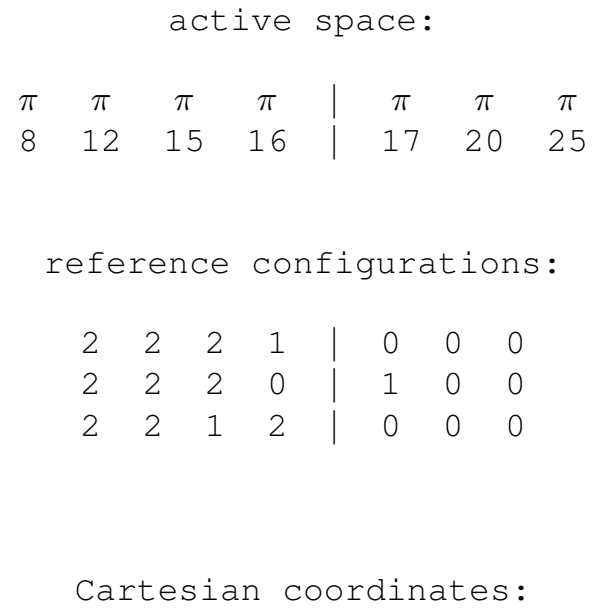


Table S1170 Employed active space, reference configurations, and Cartesian coordinates of the OM2/MRCISD-optimized ground-state equilibrium geometry of 2,3-diazabicyclo[2,2,1]hept-2-ene (ground-state energy: -1183.71045 eV).

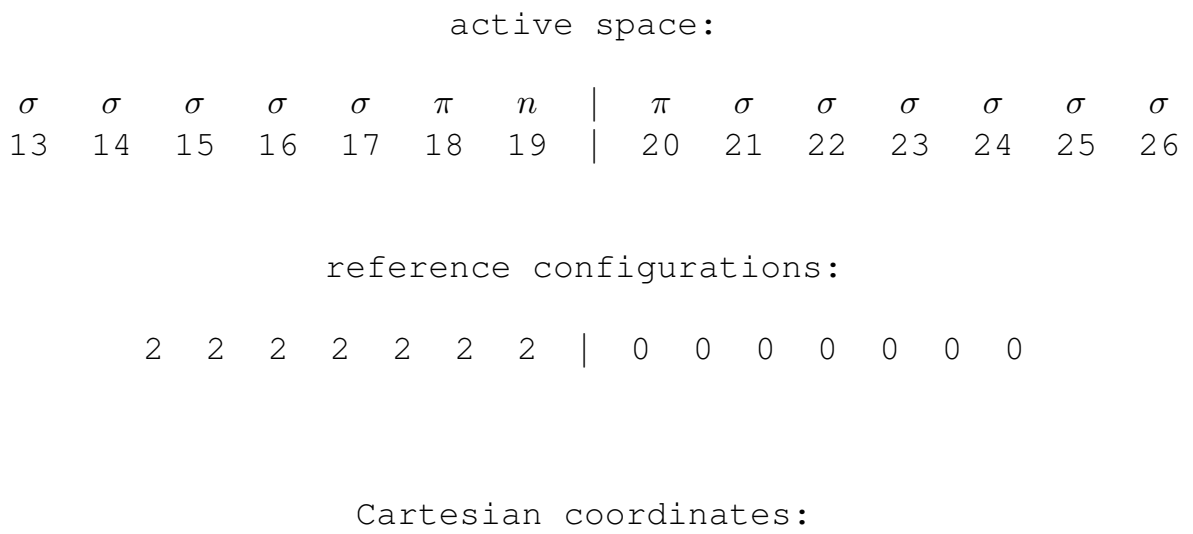


Table S1171 Employed active space, reference configurations, and Cartesian coordinates of the OM2/MRCISD-optimized excited-state equilibrium geometry of the $2{ }^{1} A\left({ }^{1} n \pi^{*}\right)$ state of 2,3-diazabicyclo[2,2,1]hept-2-ene (excited-state energy: $-1180.10100 \mathrm{eV})$.

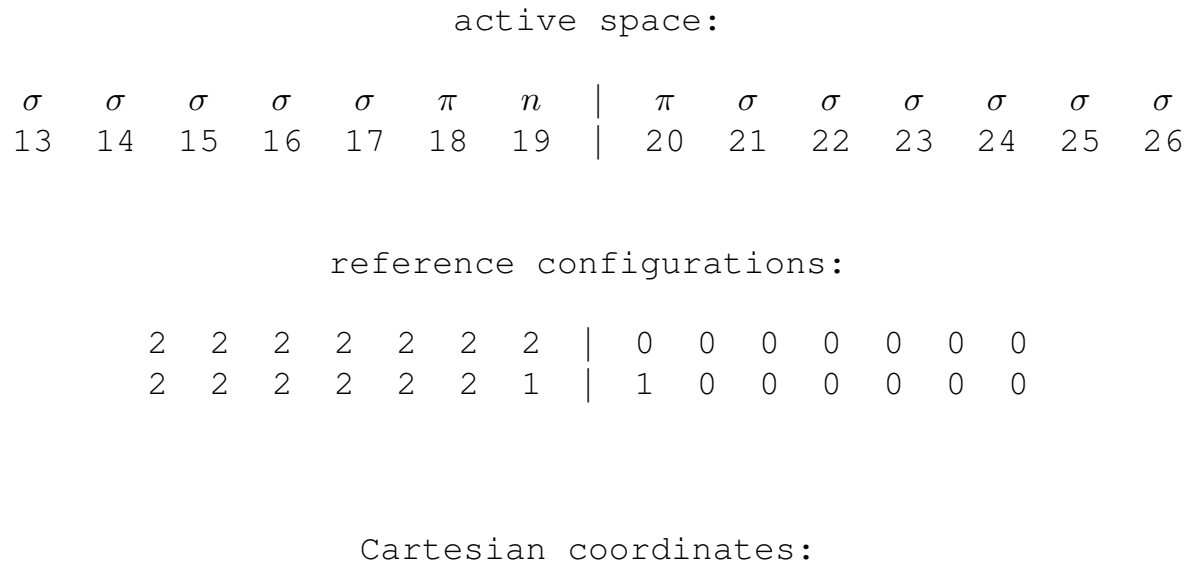


Table S1172 Employed active space, reference configurations, and Cartesian coordinates of the OM2/MRCISD-optimized ground-state equilibrium geometry of 4-(dimethylamino)-4'-cyanostilbene (ground-state energy: $-2834.40712 \mathrm{eV}$ ).

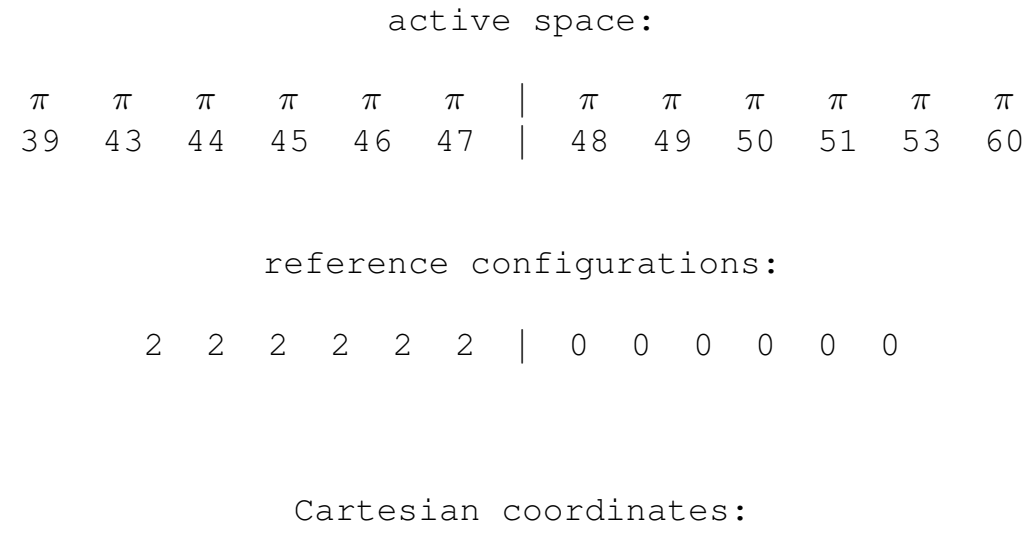

\begin{tabular}{rrrr}
35 & & & \\
& & & \\
$\mathrm{C}$ & 0.00000 & 0.00000 & 0.00000 \\
$\mathrm{C}$ & 1.38934 & 0.00000 & 0.00000 \\
$\mathrm{C}$ & 2.10902 & 1.20927 & 0.00000 \\
$\mathrm{C}$ & 1.41529 & 2.43059 & 0.00001 \\
$\mathrm{C}$ & 0.02488 & 2.45281 & 0.00001 \\
$\mathrm{C}$ & -0.66454 & 1.23362 & 0.00000 \\
$\mathrm{C}$ & 3.57122 & 1.13690 & -0.00001 \\
$\mathrm{C}$ & 4.37917 & 2.22565 & 0.00004 \\
$\mathrm{C}$ & 5.83924 & 2.15491 & 0.00002 \\
$\mathrm{C}$ & 6.56551 & 3.35827 & 0.00007 \\
$\mathrm{C}$ & 7.95233 & 3.35678 & 0.00006 \\
$\mathrm{C}$ & 8.65847 & 2.13263 & 0.00001 \\
$\mathrm{C}$ & 7.92995 & 0.92229 & -0.00004 \\
$\mathrm{C}$ & 6.54204 & 0.94053 & -0.00003 \\
$\mathrm{~N}$ & 10.05906 & 2.11980 & -0.00000 \\
$\mathrm{C}$ & 10.79480 & 3.35248 & 0.00001 \\
$\mathrm{C}$ & -2.10192 & 1.24850 & -0.00001 \\
$\mathrm{~N}$ & -3.27285 & 1.26058 & -0.00002 \\
$\mathrm{C}$ & 10.77226 & 0.87400 & 0.00004 \\
$\mathrm{H}$ & 8.49296 & 4.30615 & 0.00009 \\
$\mathrm{H}$ & 6.02707 & 4.31622 & 0.00012 \\
$\mathrm{H}$ & 5.99660 & -0.01267 & -0.00009 \\
$\mathrm{H}$ & 8.45406 & -0.03655 & -0.00008 \\
$\mathrm{H}$ & 3.95412 & 3.24283 & 0.00007 \\
\hline & & Continued & on next page
\end{tabular}




\begin{tabular}{lrrr}
\multicolumn{4}{c}{ Continued from previous page } \\
\hline $\mathrm{H}$ & 3.99562 & 0.12232 & -0.00004 \\
$\mathrm{H}$ & 1.93107 & -0.95296 & 0.00000 \\
$\mathrm{H}$ & 1.96544 & 3.37795 & 0.00002 \\
$\mathrm{H}$ & -0.52319 & 3.39774 & 0.00001 \\
$\mathrm{H}$ & -0.56619 & -0.93387 & 0.00000 \\
$\mathrm{H}$ & 11.87106 & 1.04597 & 0.00010 \\
$\mathrm{H}$ & 10.52760 & 0.26283 & 0.90631 \\
$\mathrm{H}$ & 10.52770 & 0.26282 & -0.90626 \\
$\mathrm{H}$ & 11.89029 & 3.16054 & -0.00009 \\
$\mathrm{H}$ & 10.56127 & 3.96800 & -0.90622 \\
$\mathrm{H}$ & 10.56141 & 3.96792 & 0.90633
\end{tabular}


Table S1173 Employed active space, reference configurations, and Cartesian coordinates of the OM2/MRCISD-optimized excited-state equilibrium geometry of the $2{ }^{1} A\left({ }^{1} \pi \pi^{*}\right)$ state of 4 -(dimethylamino)-4'-cyanostilbene (excited-state energy: $-2830.62911 \mathrm{eV})$.

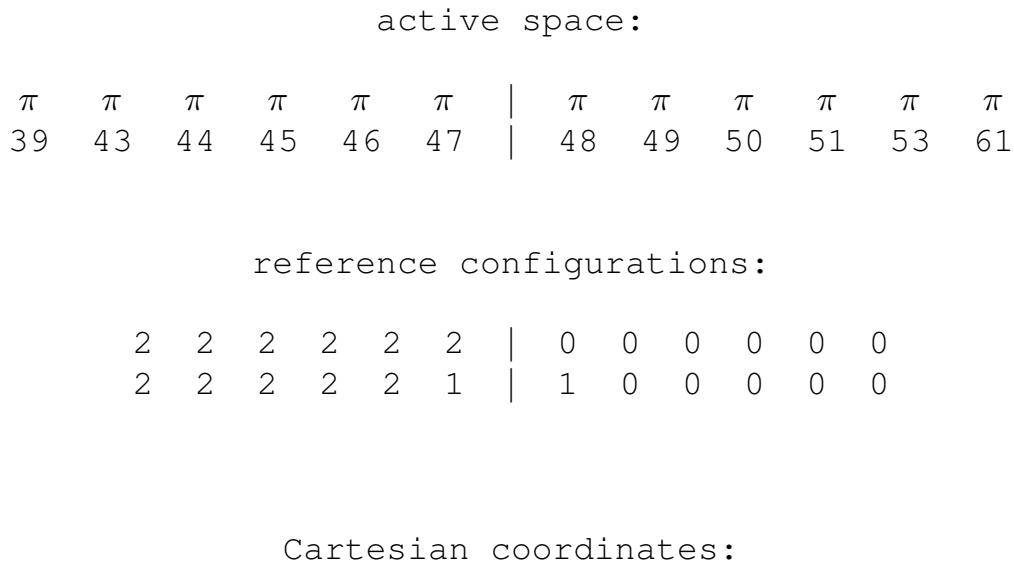




\begin{tabular}{lrrr}
\multicolumn{5}{c}{ Continued from previous page } \\
\hline $\mathrm{H}$ & 3.90260 & 3.28994 & 0.00010 \\
$\mathrm{H}$ & 3.98319 & 0.15894 & 0.00003 \\
$\mathrm{H}$ & 1.92834 & -0.94980 & -0.00003 \\
$\mathrm{H}$ & 1.94087 & 3.40492 & -0.00000 \\
$\mathrm{H}$ & -0.54673 & 3.40302 & -0.00002 \\
$\mathrm{H}$ & -0.56977 & -0.93386 & 0.00001 \\
$\mathrm{H}$ & 11.80150 & 1.08703 & 0.00076 \\
$\mathrm{H}$ & 10.45952 & 0.30824 & 0.90548 \\
$\mathrm{H}$ & 10.45965 & 0.30816 & -0.90408 \\
$\mathrm{H}$ & 11.83095 & 3.18444 & 0.00073 \\
$\mathrm{H}$ & 10.51190 & 4.00097 & -0.90412 \\
$\mathrm{H}$ & 10.51183 & 4.00084 & 0.90560
\end{tabular}


Table S1174 Employed active space, reference configurations, and Cartesian coordinates of the OM2/MRCISD-optimized ground-state equilibrium geometry of dimethylaminobenzonitrile (ground-state energy: $-1724.63377 \mathrm{eV}$ ).

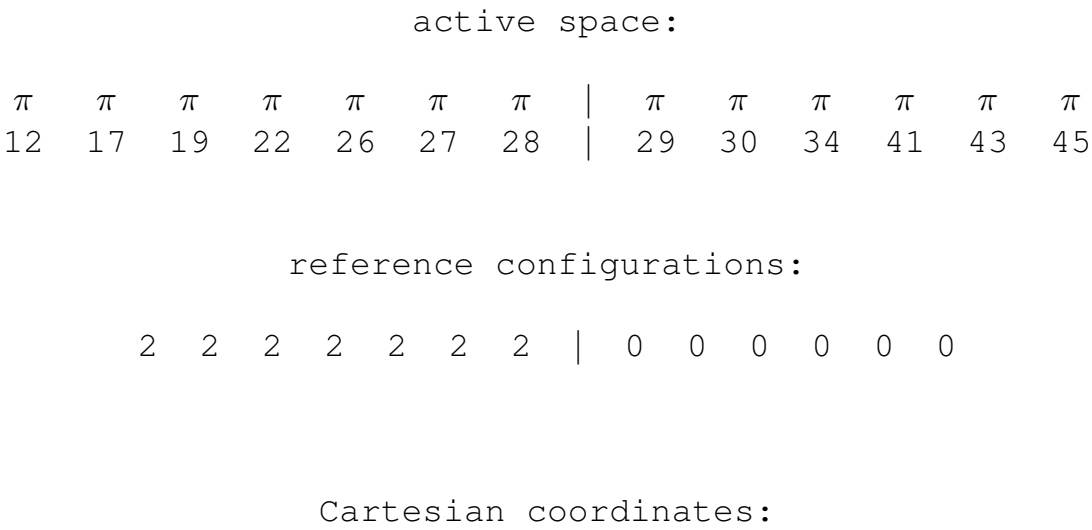


Table S1175 Employed active space, reference configurations, and Cartesian coordinates of the OM2/MRCISD-optimized excited-state equilibrium geometry of the $1^{1} B\left({ }^{1} \pi \pi^{*}\right)$ state of dimethylaminobenzonitrile (excited-state energy: $-1720.71695 \mathrm{eV})$.

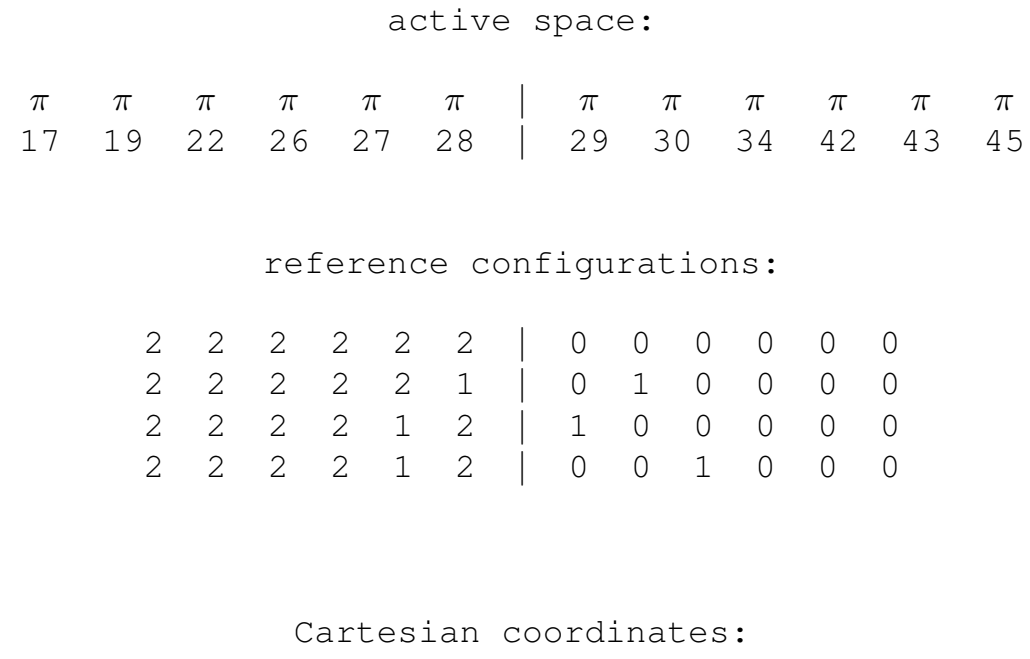

21

$\begin{array}{rrrr}\text { C } & 0.00000 & 0.00000 & 0.00000 \\ \mathrm{C} & 0.00000 & 2.80003 & 0.00000 \\ \mathrm{C} & 0.00000 & 0.71186 & 1.24542 \\ \mathrm{C} & 0.00000 & 0.71186 & -1.24542 \\ \mathrm{C} & 0.00000 & 2.12738 & -1.24718 \\ \mathrm{C} & -0.00000 & 2.12738 & 1.24718 \\ \mathrm{C} & 0.00000 & 4.22981 & 0.00000 \\ \mathrm{~N} & 0.00000 & 5.40732 & 0.00000 \\ \mathrm{~N} & 0.00000 & -1.38023 & -0.00000 \\ \mathrm{C} & -0.00002 & -2.11914 & 1.23686 \\ \mathrm{C} & 0.00002 & -2.11914 & -1.23686 \\ \mathrm{H} & 0.00001 & 0.17928 & 2.19643 \\ \mathrm{H} & -0.00001 & 0.17928 & -2.19643 \\ \mathrm{H} & 0.00001 & 2.68360 & -2.18318 \\ \mathrm{H} & -0.00001 & 2.68360 & 2.18318 \\ \mathrm{H} & -0.00001 & -3.21337 & 1.04635 \\ \mathrm{H} & 0.00001 & -3.21337 & -1.04635 \\ \mathrm{H} & 0.90401 & -1.88096 & 1.84632 \\ \mathrm{H} & -0.90401 & -1.88096 & -1.84632 \\ \mathrm{H} & -0.90408 & -1.88096 & 1.84628 \\ \mathrm{H} & 0.90408 & -1.88096 & -1.84628\end{array}$


Table S1176 Employed active space, reference configurations, and Cartesian coordinates of the OM2/MRCISD-optimized ground-state equilibrium geometry of $N, N$-dimethyl-p-phenylenediamine (ground-state energy: $-1627.40320 \mathrm{eV}$ ).

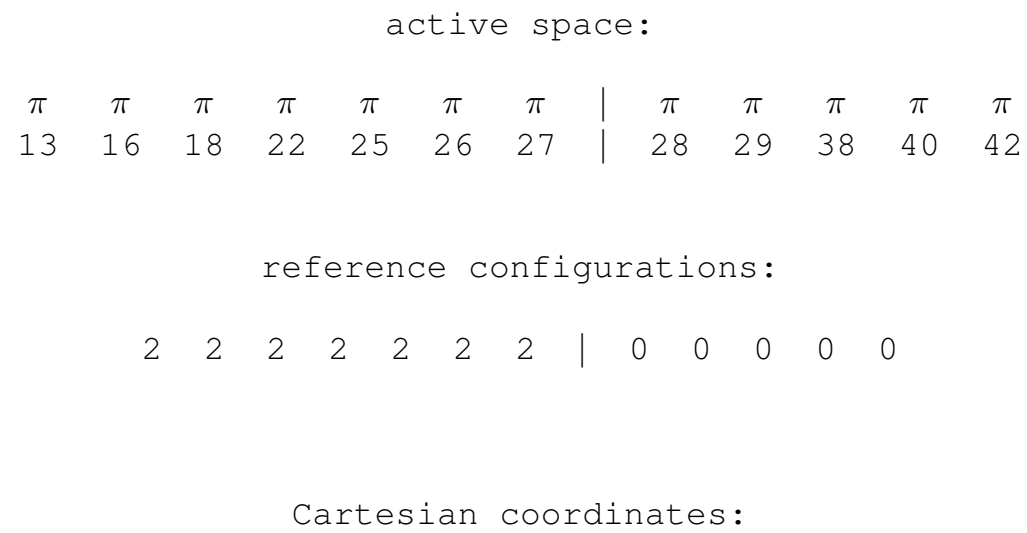


Table S1177 Employed active space, reference configurations, and Cartesian coordinates of the OM2/MRCISD-optimized excited-state equilibrium geometry of the $2{ }^{1} A_{1}\left({ }^{1} \pi \pi^{*}\right)$ state of $N, N$-dimethyl-p-phenylenediamine (excited-state energy: $-1622.75792 \mathrm{eV})$.

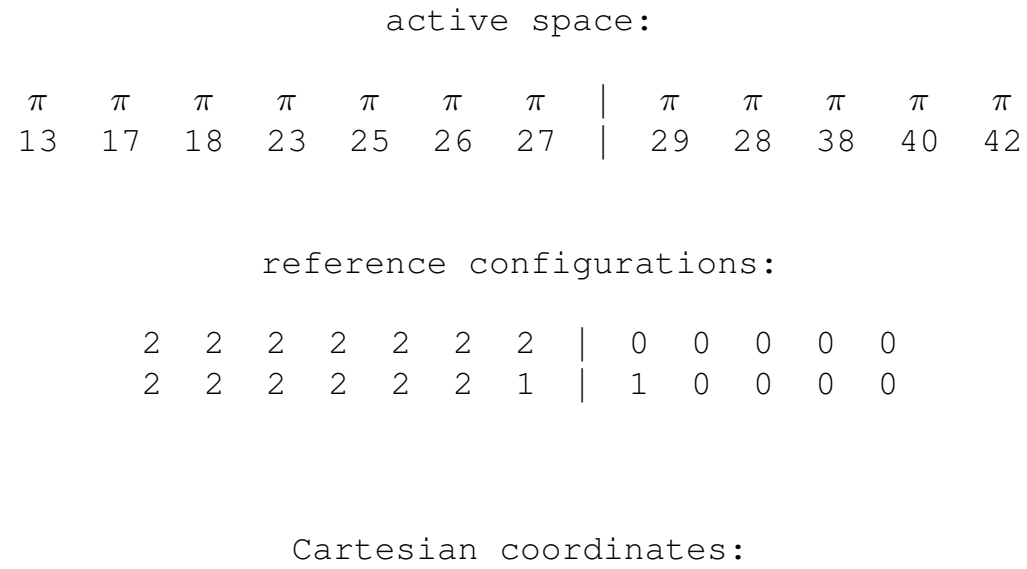


Table S1178 Employed active space, reference configurations, and Cartesian coordinates of the OM2/MRCISD-optimized ground-state equilibrium geometry of fluorene (ground-state energy: $-1809.16225 \mathrm{eV}$ ).

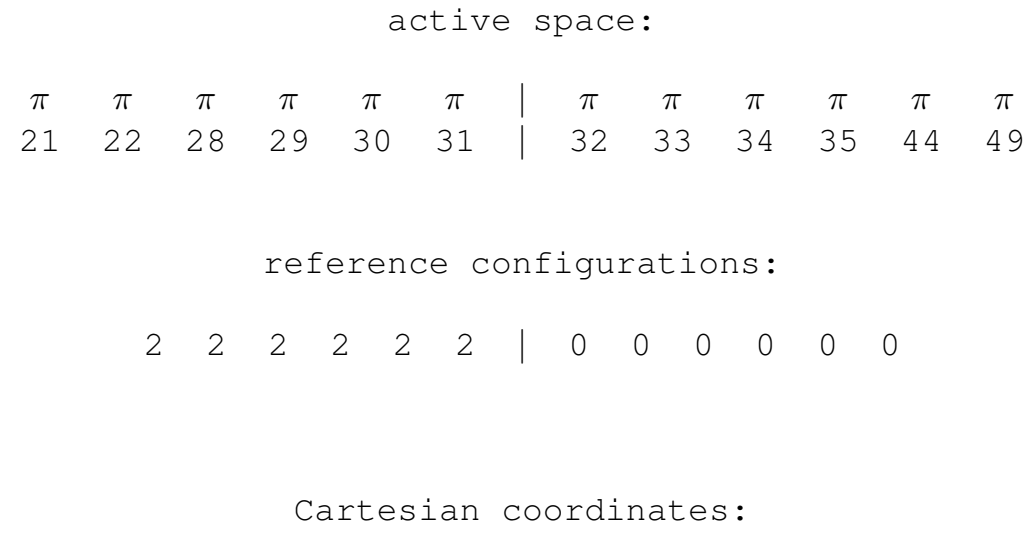

23

$\begin{array}{lrrr}\text { C } & 0.00000 & 0.00000 & 0.00000\end{array}$

$\begin{array}{lrrr}\text { C } & -0.94745 & 1.17254 & 0.00000\end{array}$

C $\quad-0.94745-1.17254-0.00000$

$\begin{array}{llll}\text { C } & -2.29742 & 0.73447 & -0.00000\end{array}$

$\begin{array}{llll}\text { C } & -2.29742 & -0.73447 & 0.00000\end{array}$

C $\quad-0.64661 \quad 2.53039-0.00000$

C $\quad-0.64661-2.53039-0.00000$

C $\quad-3.35058 \quad 1.64488-0.00000$

$\begin{array}{llll}\text { C } & -3.35058 & -1.64488 & -0.00000\end{array}$

C $\quad-1.70866 \quad 3.44365-0.00000$

$\begin{array}{llll}\text { C } & -1.70866 & -3.44365 & -0.00000\end{array}$

$\begin{array}{llll}\text { C } & -3.03722 & 3.00877 & 0.00000\end{array}$

$\begin{array}{llll}\text { C } & -3.03722 & -3.00877 & -0.00000\end{array}$

$\begin{array}{llll}\mathrm{H} & 0.62124 & 0.00000 & -0.91073\end{array}$

$\begin{array}{llll}\mathrm{H} & 0.62124 & 0.00000 & 0.91073\end{array}$

$\begin{array}{llll}\mathrm{H} & 0.39002 & 2.87982 & -0.00000\end{array}$

$\mathrm{H} \quad 0.39002 \quad-2.87982 \quad-0.00000$

$\mathrm{H} \quad-4.39013 \quad 1.30540 \quad-0.00000$

$\mathrm{H} \quad-4.39013 \quad-1.30540 \quad 0.00000$

$\mathrm{H} \quad-1.49401 \quad 4.51870 \quad-0.00000$

$\mathrm{H} \quad-1.49401-4.51870 \quad-0.00000$

$\begin{array}{lrrr}\mathrm{H} & -3.84615 & 3.74836 & 0.00000\end{array}$

$\mathrm{H} \quad-3.84615 \quad-3.74836-0.00000$ 
Table S1179 Employed active space, reference configurations, and Cartesian coordinates of the OM2/MRCISD-optimized excited-state equilibrium geometry of the $2{ }^{1} A^{\prime}\left({ }^{1} \pi \pi^{*}\right)$ state of fluorene (excited-state energy: $-1804.92031 \mathrm{eV}$ ).

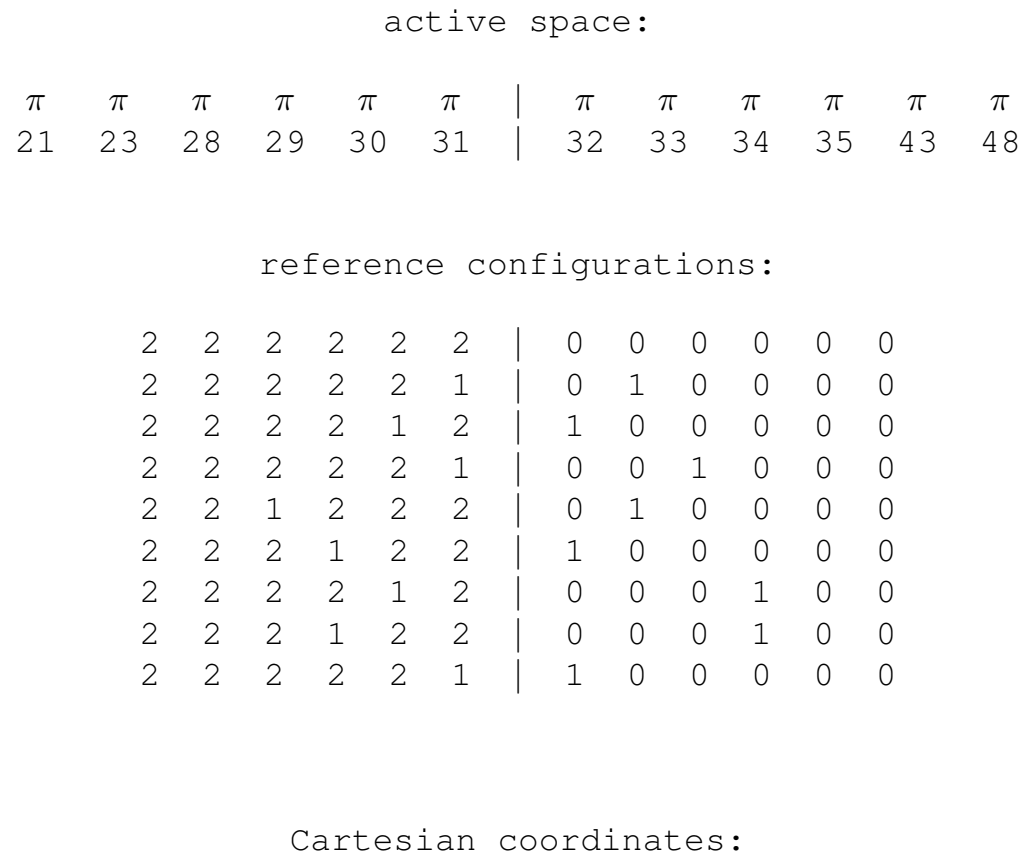

\begin{tabular}{lrrr}
23 & & & \\
C & 0.00000 & 0.00000 & 0.00000 \\
C & 1.39400 & 0.00000 & 0.00000 \\
C & 2.05544 & 1.23330 & 0.00000 \\
C & 1.31759 & 2.45239 & 0.00000 \\
C & -0.06855 & 2.43637 & 0.00000 \\
C & -0.72266 & 1.19797 & 0.00000 \\
C & 3.46170 & 1.54663 & -0.00000 \\
C & 3.59207 & 2.98991 & -0.00000 \\
C & 2.23593 & 3.64055 & -0.00000 \\
C & 4.60761 & 0.70879 & -0.00000 \\
C & 5.88067 & 1.34200 & -0.00000 \\
C & 6.00596 & 2.75503 & -0.00000 \\
C & 4.87279 & 3.60385 & -0.00000 \\
H & 2.10353 & 4.24194 & 0.91155 \\
H & 2.10353 & 4.24194 & -0.91155 \\
H & 4.97808 & 4.68968 & -0.00000 \\
H & -0.64390 & 3.36723 & -0.00000 \\
\hline & & Continued on next page
\end{tabular}

S1244 


\begin{tabular}{lrrr}
\multicolumn{4}{c}{ Continued from previous page } \\
\hline $\mathrm{H}$ & 4.51521 & -0.37831 & -0.00000 \\
$\mathrm{H}$ & 1.95975 & -0.93587 & -0.00000 \\
$\mathrm{H}$ & 7.00825 & 3.19944 & -0.00000 \\
$\mathrm{H}$ & -1.81795 & 1.16638 & 0.00000 \\
$\mathrm{H}$ & 6.78519 & 0.72434 & -0.00000 \\
$\mathrm{H}$ & -0.54170 & -0.95296 & -0.00000
\end{tabular}


Table S1180 Employed active space, reference configurations, and Cartesian coordinates of the OM2/MRCISD-optimized ground-state equilibrium geometry of glyoxal (ground-state energy: $-947.06697 \mathrm{eV}$ ).

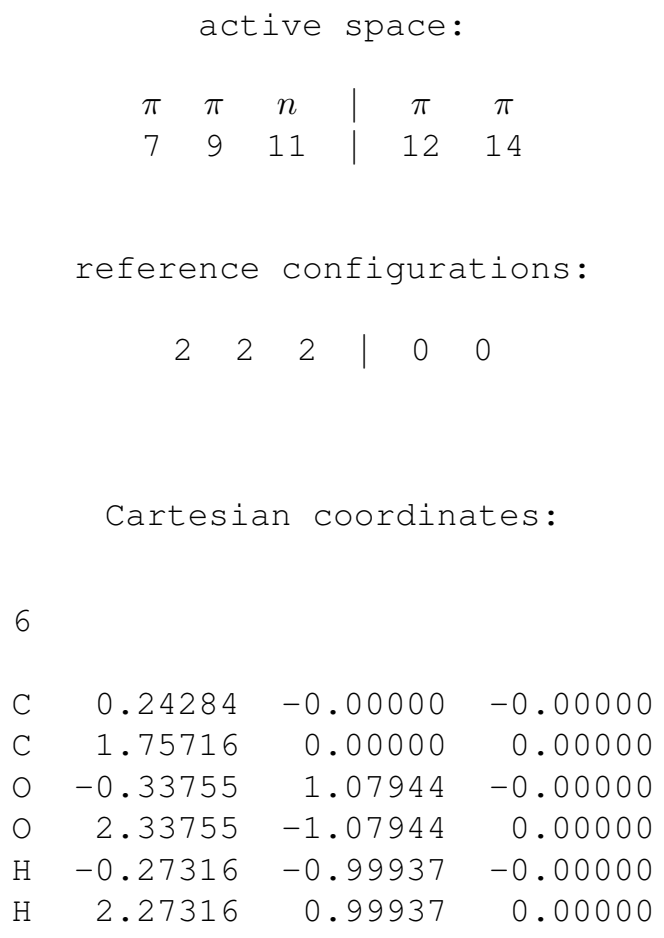


Table S1181 Employed active space, reference configurations, and Cartesian coordinates of the OM2/MRCISD-optimized excited-state equilibrium geometry of the $1{ }^{1} A_{u}\left({ }^{1} n \pi^{*}\right)$ state of glyoxal (excited-state energy: $-944.06855 \mathrm{eV}$ ).

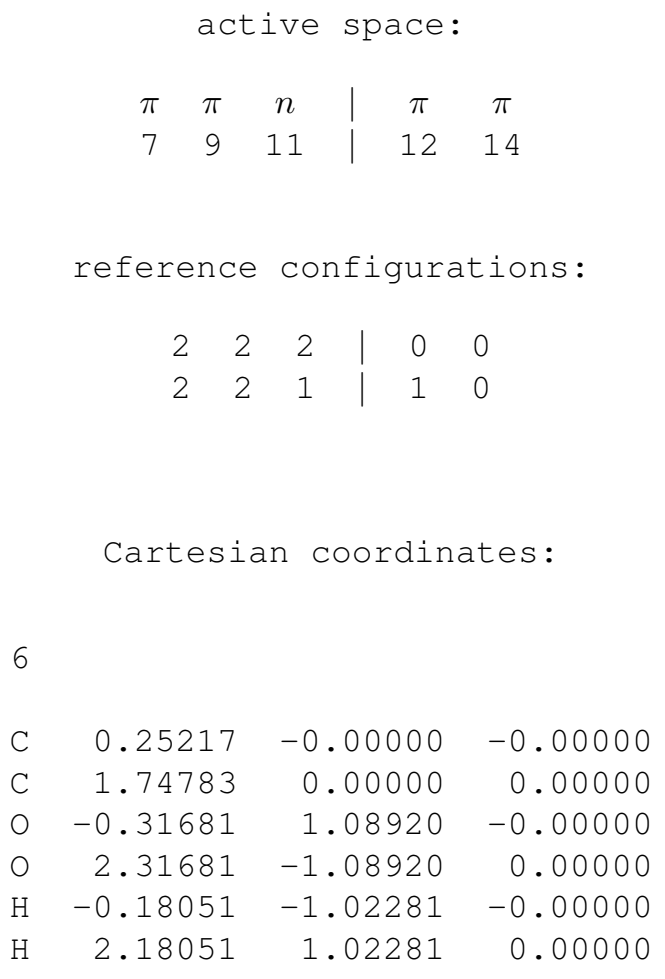


Table S1182 Employed active space, reference configurations, and Cartesian coordinates of the OM2/MRCISD-optimized ground-state equilibrium geometry of hydrogen cyanide (ground-state energy: $-355.82905 \mathrm{eV}$ ).

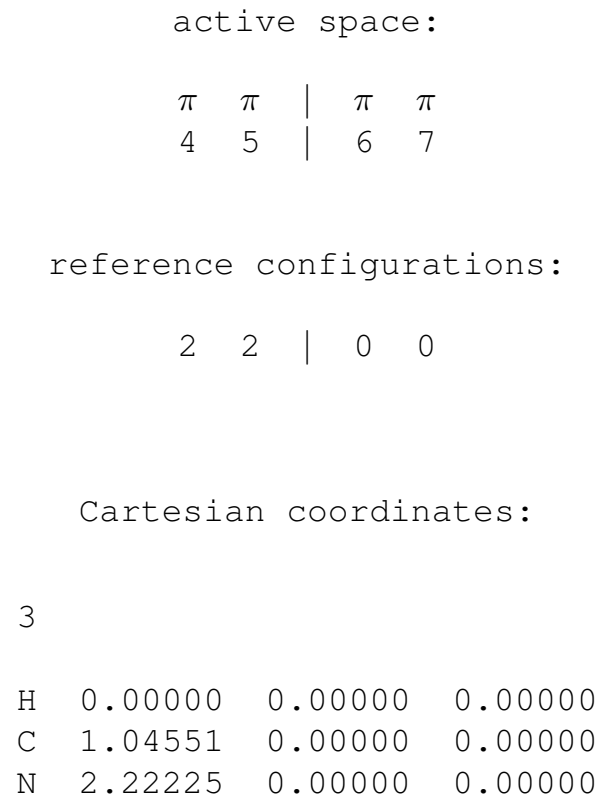


Table S1183 Employed active space, reference configurations, and Cartesian coordinates of the OM2/MRCISD-optimized excited-state equilibrium geometry of the $1^{1} A^{\prime \prime}\left({ }^{1} \pi \pi^{*}\right)$ state of hydrogen cyanide (excited-state energy: $-349.04573 \mathrm{eV})$.

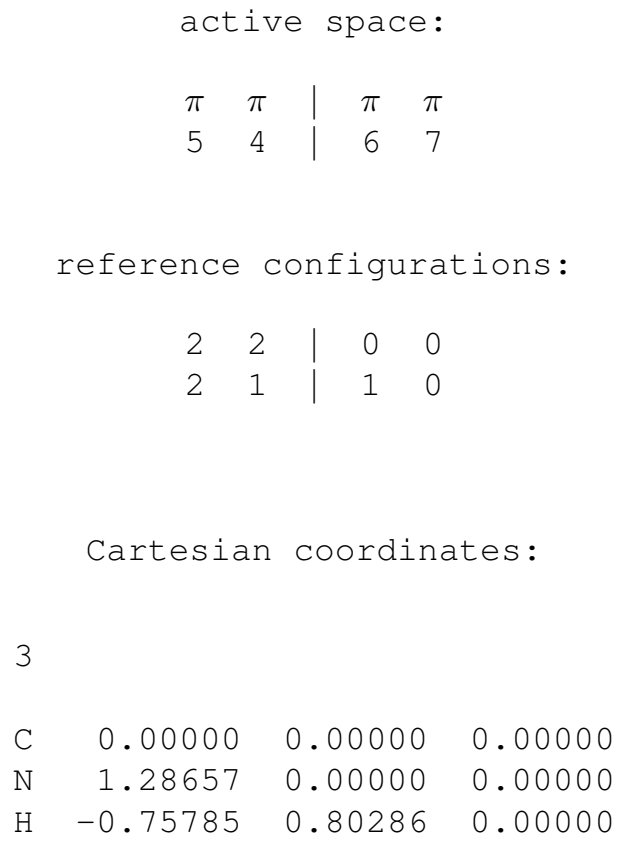


Table S1184 Employed active space, reference configurations, and Cartesian coordinates of the OM2/MRCISD-optimized ground-state equilibrium geometry of formic acid (ground-state energy: $-820.12451 \mathrm{eV}$ ).

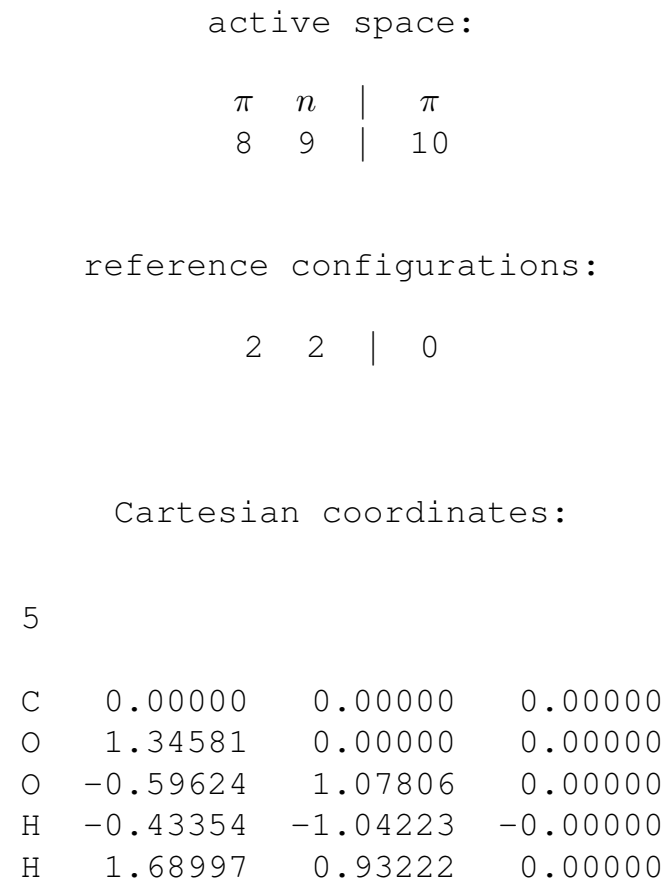


Table S1185 Employed active space, reference configurations, and Cartesian coordinates of the OM2/MRCISD-optimized excited-state equilibrium geometry of the $2{ }^{1} A\left({ }^{1} n \pi^{*}\right)$ state of formic acid (excited-state energy: $-815.76005 \mathrm{eV}$ ).

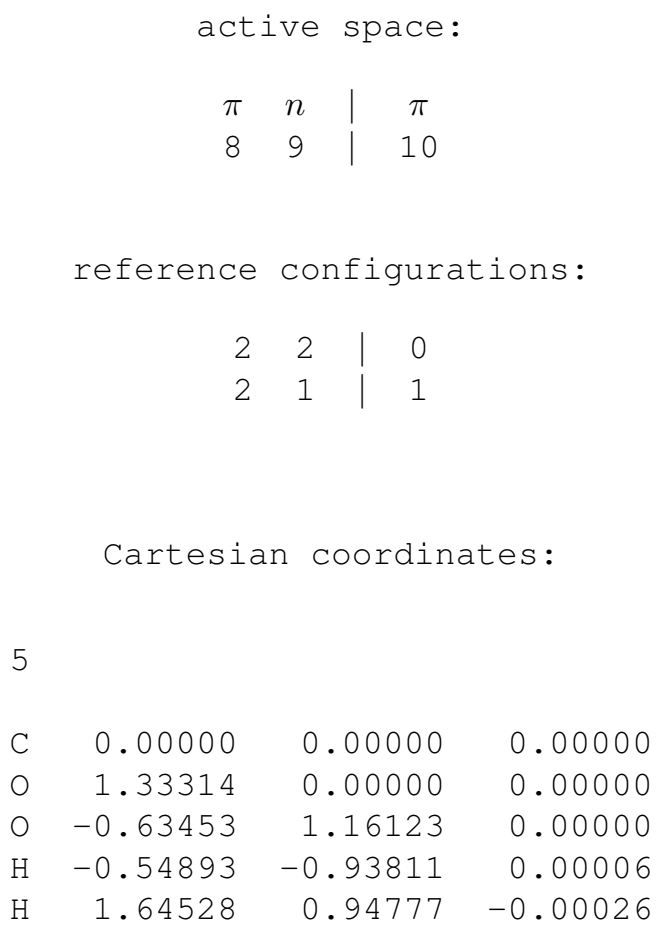


Table S1186 Employed active space, reference configurations, and Cartesian coordinates of the OM2/MRCISD-optimized ground-state equilibrium geometry of hexatriene (ground-state energy: $-884.59550 \mathrm{eV}$ ).

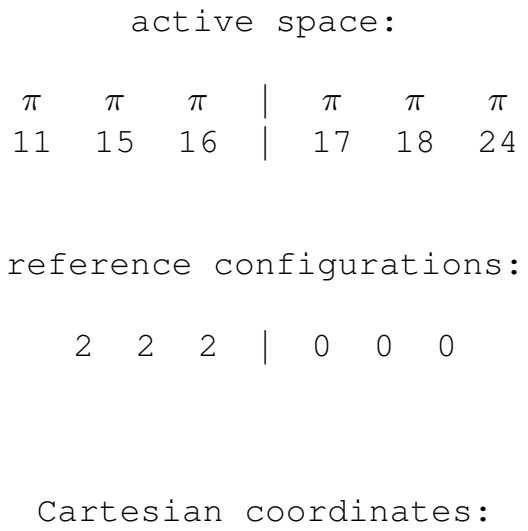


Table S1187 Employed active space, reference configurations, and Cartesian coordinates of the OM2/MRCISD-optimized excited-state equilibrium geometry of the $1{ }^{1} B_{u}\left({ }^{1} \pi \pi^{*}\right)$ state of hexatriene (excited-state energy: $-879.53064 \mathrm{eV}$ ).

\begin{tabular}{ccc|ccc}
$\pi$ & $\pi$ & $\pi$ & $\pi$ & $\pi$ & $\pi$ \\
11 & 15 & 16 & 17 & 18 & 24 \\
reference & configurations: \\
2 & 2 & 2 & 0 & 0 & 0 \\
2 & 2 & 1 & 1 & 0 & 0
\end{tabular}

Cartesian coordinates:

14

$\begin{array}{llll}C & 0.00000 & 0.70515 & 0.00000\end{array}$

C $\quad 0.00000-0.70515-0.00000$

C $\quad 0.00000 \quad 1.42720 \quad 1.21002$

C $\quad 0.00000-1.42720-1.21002$

$\begin{array}{llll}\text { C } & 0.00000 & 2.80381 & 1.23783\end{array}$

C $\quad 0.00000-2.80381-1.23783$

$\mathrm{H} \quad 0.00000 \quad 1.24764-0.95408$

$\mathrm{H} \quad 0.00000 \quad-1.24764 \quad 0.95408$

$\begin{array}{lrrr}\mathrm{H} & 0.00000 & 0.86594 & 2.15564\end{array}$

$\begin{array}{llll}\mathrm{H} & 0.00000 & -0.86594 & -2.15564\end{array}$

$\begin{array}{llll}\mathrm{H} & 0.00000 & 3.34825 & 2.17176\end{array}$

$\mathrm{H} \quad 0.00000 \quad-3.34825 \quad-2.17176$

$\begin{array}{llll}\mathrm{H} & 0.00000 & 3.38405 & 0.32419\end{array}$

H $\quad 0.00000 \quad-3.38405 \quad-0.32419$ 
Table S1188 Employed active space, reference configurations, and Cartesian coordinates of the OM2/MRCISD-optimized ground-state equilibrium geometry of trans-hydroquinone (ground-state energy: $-1517.92634 \mathrm{eV}$ ).

\begin{tabular}{lcccc|ccc}
\multicolumn{10}{c}{ active space: } \\
$\pi$ & $\pi$ & $\pi$ & $\pi$ & $\pi$ & $\pi$ & $\pi$ & $\pi$ \\
12 & 13 & 18 & 20 & 21 & 22 & 23 & 30
\end{tabular}

reference configurations:

\begin{tabular}{lllll|lll}
2 & 2 & 2 & 2 & 2 & 0 & 0 & 0
\end{tabular}

Cartesian coordinates:

14

$\begin{array}{lrrr}\mathrm{C} & 0.00000 & 0.00000 & 0.00000 \\ \mathrm{C} & 2.73753 & 0.00000 & 0.00000 \\ \mathrm{C} & 0.67023 & 1.23731 & 0.00000 \\ \mathrm{C} & 2.06729 & -1.23731 & 0.00000 \\ \mathrm{C} & 0.67508 & -1.23333 & -0.00000 \\ \mathrm{C} & 2.06245 & 1.23333 & -0.00000 \\ \mathrm{O} & -1.36164 & 0.08917 & 0.00000 \\ \mathrm{O} & 4.09917 & -0.08917 & 0.00000 \\ \mathrm{H} & 0.09543 & 2.16138 & -0.00000 \\ \mathrm{H} & 2.64210 & -2.16138 & 0.00000 \\ \mathrm{H} & 0.11498 & -2.17174 & -0.00000 \\ \mathrm{H} & 2.62254 & 2.17174 & -0.00000 \\ \mathrm{H} & -1.75054 & -0.82727 & 0.00000 \\ \mathrm{H} & 4.48806 & 0.82727 & -0.00000\end{array}$


Table S1189 Employed active space, reference configurations, and Cartesian coordinates of the OM2/MRCISD-optimized excited-state equilibrium geometry of the $1^{1} B_{u}\left({ }^{1} \pi \pi^{*}\right)$ state of trans-hydroquinone (excited-state energy: $-1514.07610 \mathrm{eV})$.

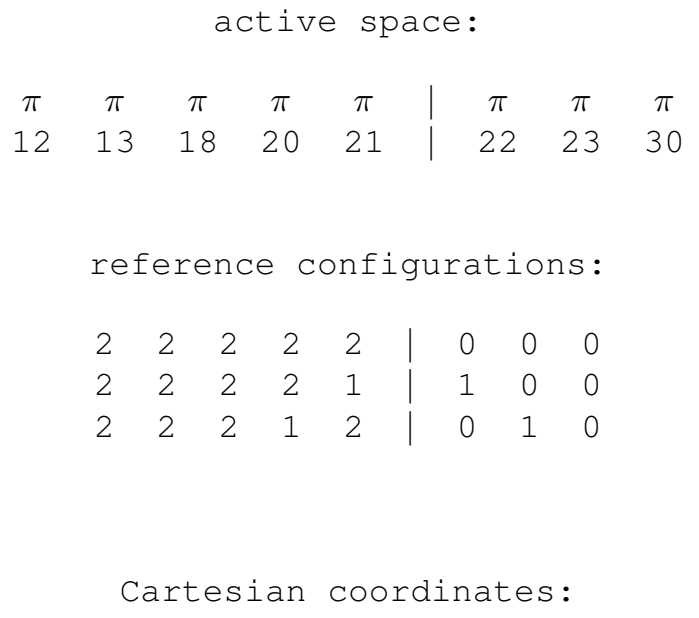

14

$\begin{array}{lrrr}\mathrm{C} & 0.00000 & 0.00000 & 0.00000 \\ \mathrm{C} & 2.73733 & 0.00000 & 0.00000 \\ \mathrm{C} & 0.66153 & 1.26564 & 0.00000 \\ \mathrm{C} & 2.07581 & -1.26564 & 0.00000 \\ \mathrm{C} & 0.66429 & -1.26260 & -0.00000 \\ \mathrm{C} & 2.07304 & 1.26260 & -0.00000 \\ \mathrm{O} & -1.34540 & 0.08828 & 0.00000 \\ \mathrm{O} & 4.08273 & -0.08828 & 0.00000 \\ \mathrm{H} & 0.08021 & 2.18195 & -0.00000 \\ \mathrm{H} & 2.65713 & -2.18195 & 0.00000 \\ \mathrm{H} & 0.09825 & -2.19310 & -0.00000 \\ \mathrm{H} & 2.63908 & 2.19310 & -0.00000 \\ \mathrm{H} & -1.74704 & -0.82432 & 0.00000 \\ \mathrm{H} & 4.48437 & 0.82432 & -0.00000\end{array}$


Table S1190 Employed active space, reference configurations, and Cartesian coordinates of the OM2/MRCISD-optimized ground-state equilibrium geometry of indole (ground-state energy: $-1338.81436 \mathrm{eV}$ ).

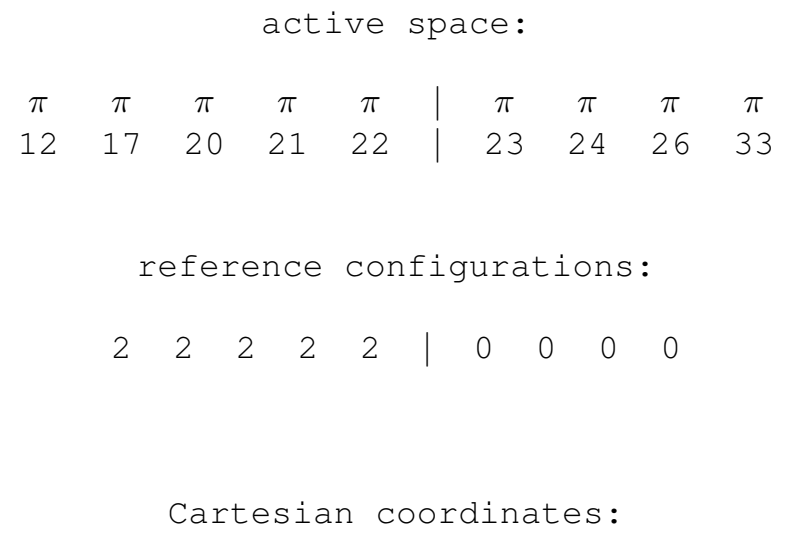

$\begin{array}{lrrr}16 & & & \\ \mathrm{C} & 0.00000 & 0.00000 & 0.00000 \\ \mathrm{~N} & 1.38603 & 0.00000 & 0.00000 \\ \mathrm{C} & 1.83381 & 1.31363 & 0.00000 \\ \mathrm{C} & 0.67975 & 2.17054 & 0.00000 \\ \mathrm{C} & -0.47256 & 1.31014 & 0.00000 \\ \mathrm{C} & 3.14390 & 1.82488 & -0.00000 \\ \mathrm{C} & 3.27614 & 3.20691 & -0.00000 \\ \mathrm{C} & 2.15326 & 4.06050 & -0.00000 \\ \mathrm{C} & 0.85671 & 3.56273 & -0.00000 \\ \mathrm{H} & 4.00989 & 1.15819 & -0.00000 \\ \mathrm{H} & 4.27888 & 3.65129 & -0.00000 \\ \mathrm{H} & 2.31798 & 5.14428 & -0.00000 \\ \mathrm{H} & -0.01080 & 4.22964 & -0.00000 \\ \mathrm{H} & 1.97033 & -0.82145 & -0.00000 \\ \mathrm{H} & -1.50451 & 1.62057 & -0.00000 \\ \mathrm{H} & -0.58880 & -0.91338 & -0.00000\end{array}$


Table S1191 Employed active space, reference configurations, and Cartesian coordinates of the OM2/MRCISD-optimized excited-state equilibrium geometry of the $2{ }^{1} A^{\prime}\left({ }^{1} \pi \pi^{*}\right)$ state of indole (excited-state energy: $-1334.86531 \mathrm{eV}$ ).

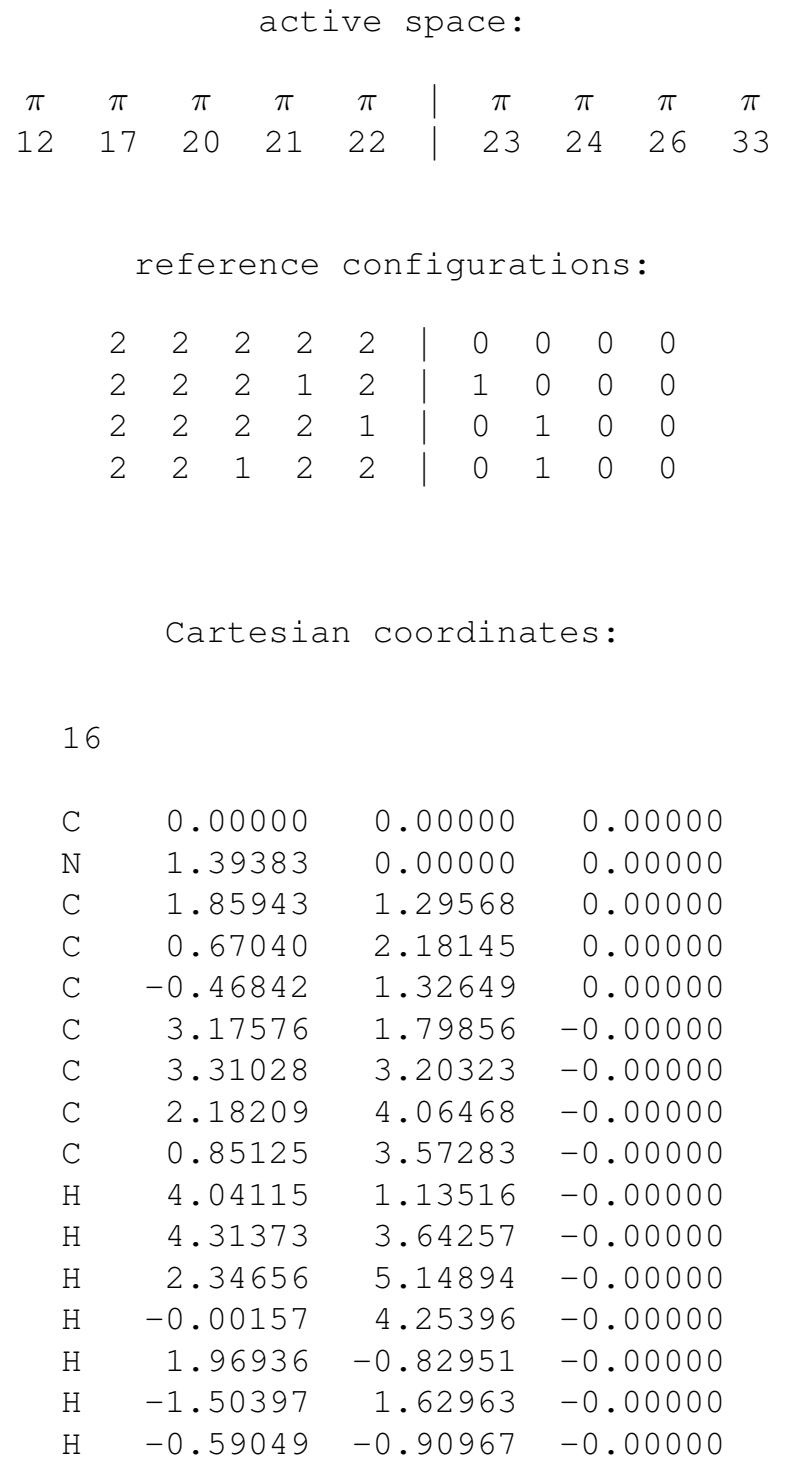


Table S1192 Employed active space, reference configurations, and Cartesian coordinates of the OM2/MRCISD-optimized ground-state equilibrium geometry of methyl-4-hydroxycinnamate (ground-state energy: $-2419.44858 \mathrm{eV}$ ).

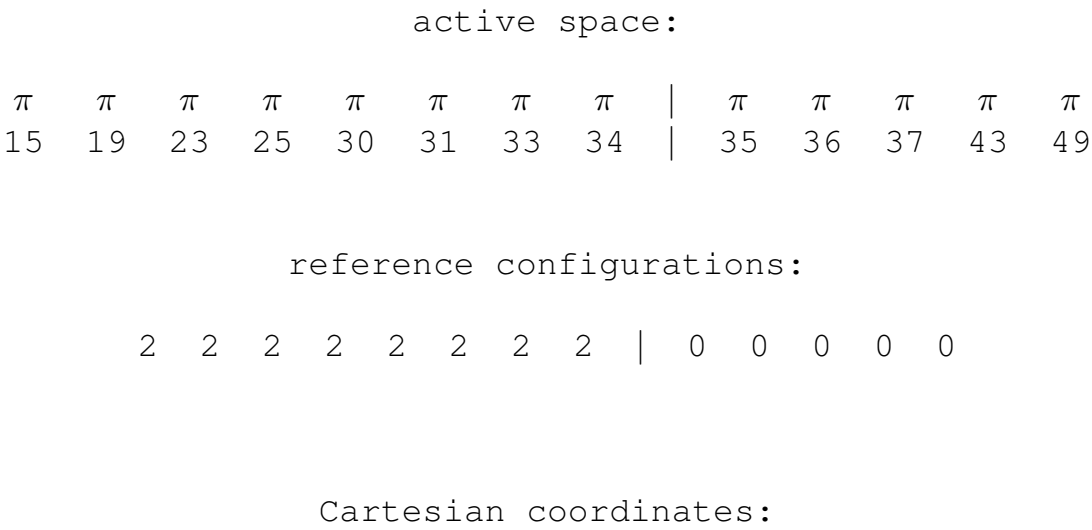


Table S1193 Employed active space, reference configurations, and Cartesian coordinates of the OM2/MRCISD-optimized excited-state equilibrium geometry of the $2{ }^{1} A\left({ }^{1} \pi \pi^{*}\right)$ state of methyl-4-hydroxycinnamate (excited-state energy: $-2415.51056 \mathrm{eV})$.

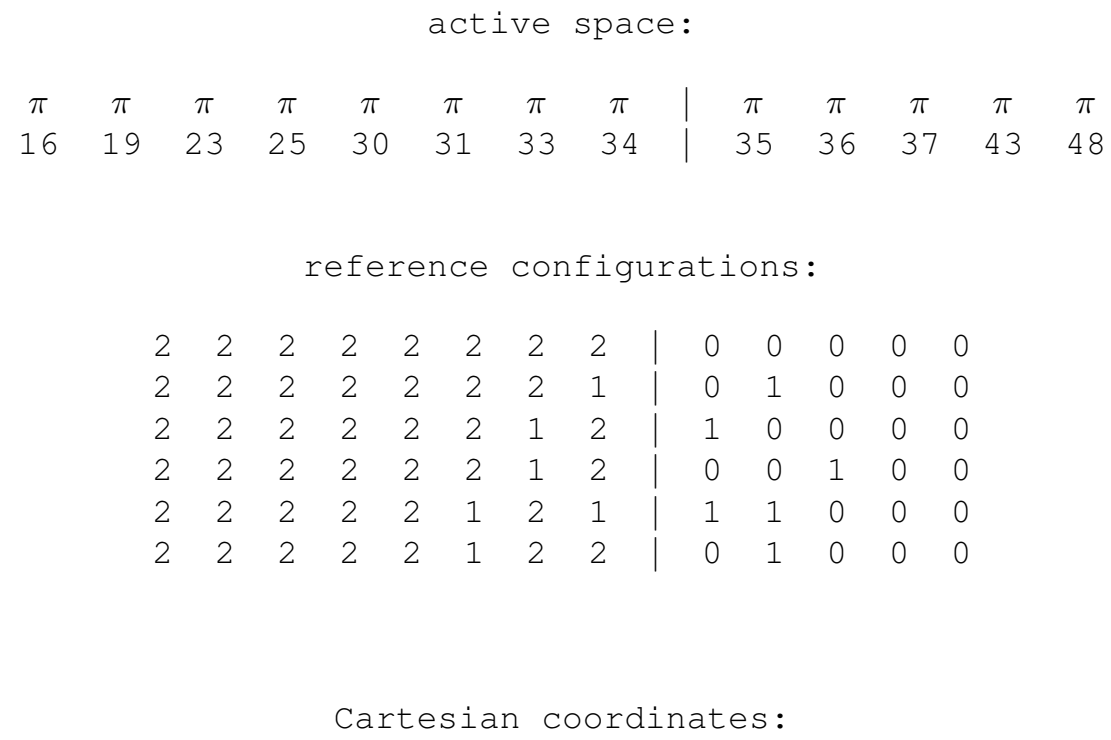

\begin{tabular}{lrrr}
23 & & \\
C & 0.00000 & 0.00000 & 0.00000 \\
$\mathrm{C}$ & 1.41579 & 0.00000 & 0.00000 \\
$\mathrm{C}$ & 2.13480 & 1.23685 & 0.00000 \\
$\mathrm{C}$ & 1.42987 & 2.48608 & 0.00002 \\
$\mathrm{C}$ & 0.01643 & 2.50840 & 0.00002 \\
$\mathrm{C}$ & -0.66501 & 1.25883 & 0.00001 \\
$\mathrm{C}$ & 3.56905 & 1.28795 & -0.00000 \\
$\mathrm{C}$ & 4.36082 & 0.17560 & -0.00002 \\
$\mathrm{C}$ & 5.82863 & 0.29682 & -0.00001 \\
$\mathrm{O}$ & 6.65056 & -0.62203 & -0.00001 \\
$\mathrm{O}$ & -2.01184 & 1.17875 & 0.00002 \\
$\mathrm{O}$ & 6.25789 & 1.60025 & 0.00001 \\
$\mathrm{C}$ & 7.65345 & 1.76436 & 0.00003 \\
$\mathrm{H}$ & 3.98494 & -0.84798 & -0.00003 \\
$\mathrm{H}$ & 4.04151 & 2.27890 & 0.00001 \\
$\mathrm{H}$ & 1.98926 & 3.42623 & 0.00002 \\
$\mathrm{H}$ & -0.53590 & 3.44800 & 0.00002 \\
$\mathrm{H}$ & -0.57956 & -0.91866 & 0.00000 \\
$\mathrm{H}$ & 1.94906 & -0.95352 & -0.00000 \\
\hline & & Continued & on next page
\end{tabular}

S1259 


\begin{tabular}{lrrr}
\multicolumn{5}{c}{ Continued from previous page } \\
\hline $\mathrm{H}$ & -2.40745 & 2.09400 & 0.00002 \\
$\mathrm{H}$ & 7.81272 & 2.86569 & 0.00036 \\
$\mathrm{H}$ & 8.10388 & 1.31204 & 0.90909 \\
$\mathrm{H}$ & 8.10381 & 1.31263 & -0.90936
\end{tabular}


Table S1194 Employed active space, reference configurations, and Cartesian coordinates of the OM2/MRCISD-optimized ground-state equilibrium geometry of naphthalene (ground-state energy: $-1396.33929 \mathrm{eV}$ ).

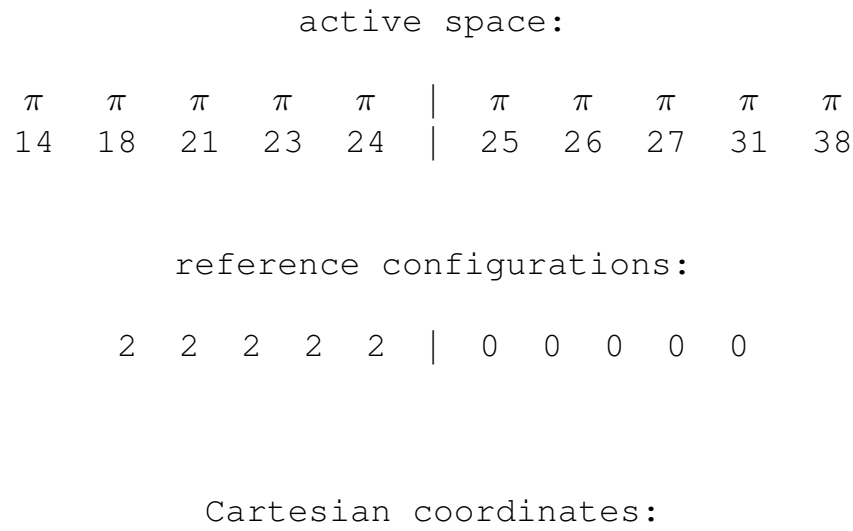


Table S1195 Employed active space, reference configurations, and Cartesian coordinates of the OM2/MRCISD-optimized excited-state equilibrium geometry of the $1{ }^{1} B_{2 u}\left({ }^{1} \pi \pi^{*}\right)$ state of naphthalene (excited-state energy: $-1391.69406 \mathrm{eV})$.

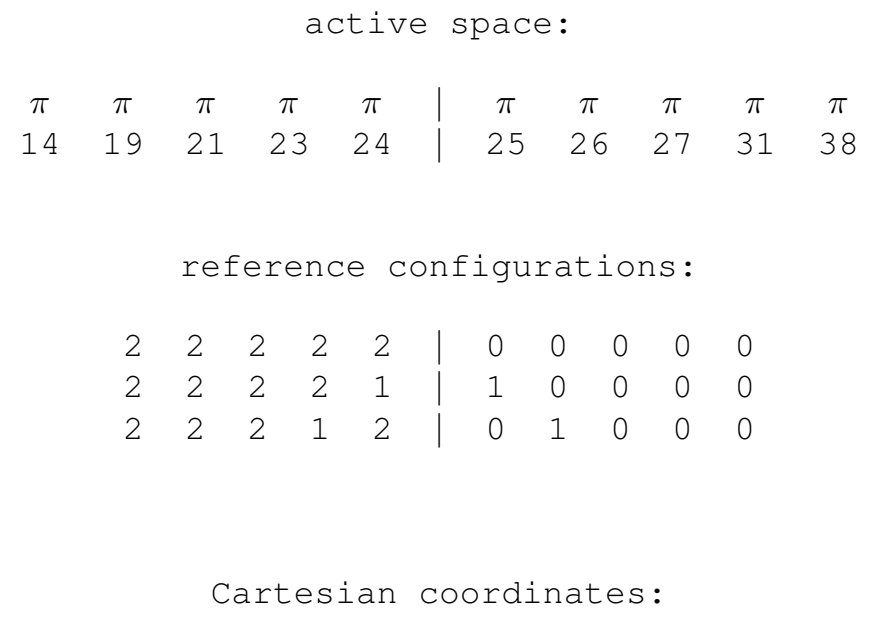

18

C $\quad 1.00000$

1.721160 .00000

C 1.00000

$0.27884-0.00000$

C $\quad 2.23207$

2. 40686

0.00000

$-0.23207$

2.40686

0.00000

2.23207
-0.23207

$-0.40686$

0.00000

3. 45178

$-0.40686$

$-0.00000$

$-1.45178$

1.69338

0.00000

1.69338

0.00000

3. 45178

0.30662

0.00000

$-1.45178$

0.30662

$-0.00000$

2. 24478

3. 50312

0.00000

$-0.24478$

3. 50312

0.00000

2. 24478

$-1.50312$

0.00000

$-0.24478$

$-1.50312$

$-0.00000$

4.39669

2. 24727

0.00000

$-2.39669$

2. 24727

0.00000

4.39669

$-0.24727$

0.00000

$\mathrm{H} \quad-2.39669$

$-0.24727-0.00000$ 
Table S1196 Employed active space, reference configurations, and Cartesian coordinates of the OM2/MRCISD-optimized ground-state equilibrium geometry of octatetraene (ground-state energy: $-1169.49316 \mathrm{eV}$ ).

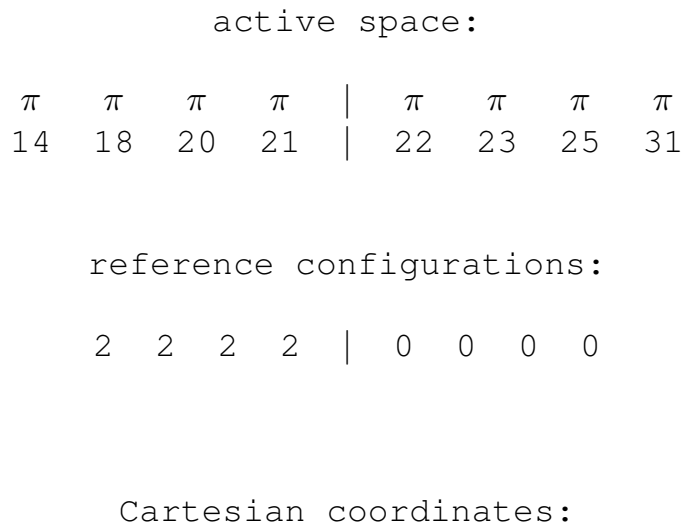


Table S1197 Employed active space, reference configurations, and Cartesian coordinates of the OM2/MRCISD-optimized excited-state equilibrium geometry of the $1{ }^{1} B_{u}\left({ }^{1} \pi \pi^{*}\right)$ state of octatetraene (excited-state energy: $-1164.98727 \mathrm{eV})$.

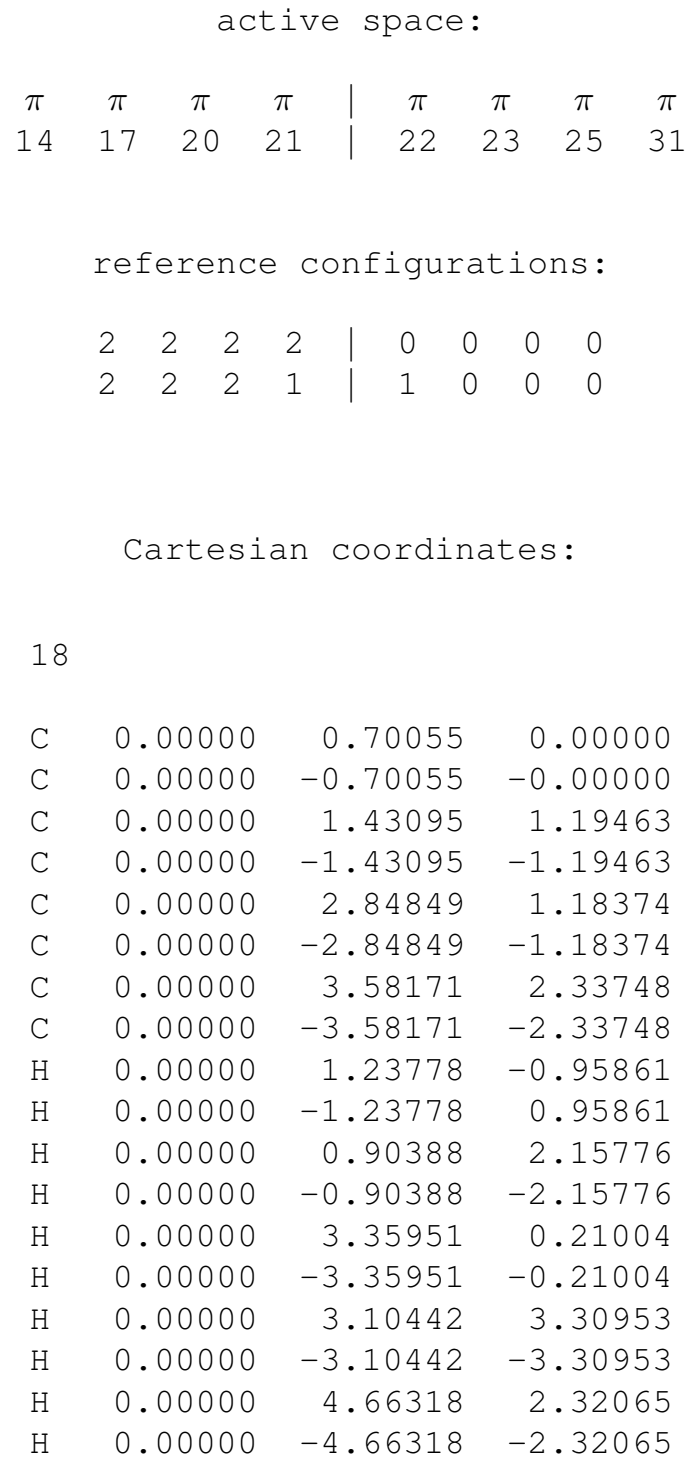


Table S1198 Employed active space, reference configurations, and Cartesian coordinates of the OM2/MRCISD-optimized ground-state equilibrium geometry of o-cyanobenzyl radical (ground-state energy: $-1322.57076 \mathrm{eV}$ ).

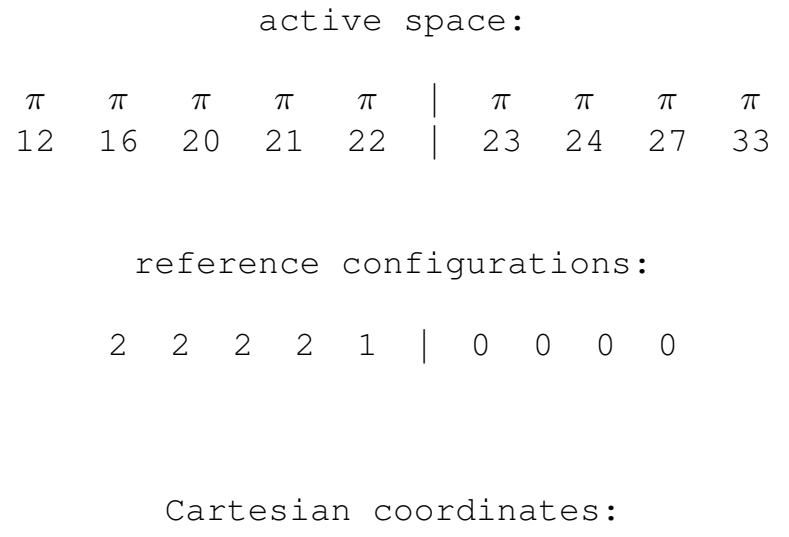

$\begin{array}{lrrr}15 & & & \\ & & & \\ \mathrm{C} & 0.00000 & 0.00000 & 0.00000 \\ \mathrm{C} & 1.42353 & 0.00000 & 0.00000 \\ \mathrm{C} & 2.13094 & 1.19310 & 0.00000 \\ \mathrm{C} & 1.46113 & 2.42663 & 0.00000 \\ \mathrm{C} & 0.06583 & 2.47507 & 0.00000 \\ \mathrm{C} & -0.64750 & 1.27268 & 0.00000 \\ \mathrm{C} & -2.07999 & 1.32541 & 0.00000 \\ \mathrm{~N} & -3.25549 & 1.37468 & 0.00000 \\ \mathrm{C} & -0.72738 & -1.19544 & -0.00000 \\ \mathrm{H} & 1.95140 & -0.95980 & -0.00000 \\ \mathrm{H} & 3.22605 & 1.17835 & 0.00000 \\ \mathrm{H} & 2.03640 & 3.35732 & 0.00000 \\ \mathrm{H} & -0.46071 & 3.43206 & 0.00000 \\ \mathrm{H} & -1.80662 & -1.19459 & -0.00000 \\ \mathrm{H} & -0.21769 & -2.14941 & -0.00000\end{array}$


Table S1199 Employed active space, reference configurations, and Cartesian coordinates of the OM2/MRCISD-optimized excited-state equilibrium geometry of the $2^{2} A^{\prime \prime}\left({ }^{2} \pi \pi^{*}\right)$ state of $o$-cyanobenzyl radical (excited-state energy: $-1320.20534 \mathrm{eV})$.

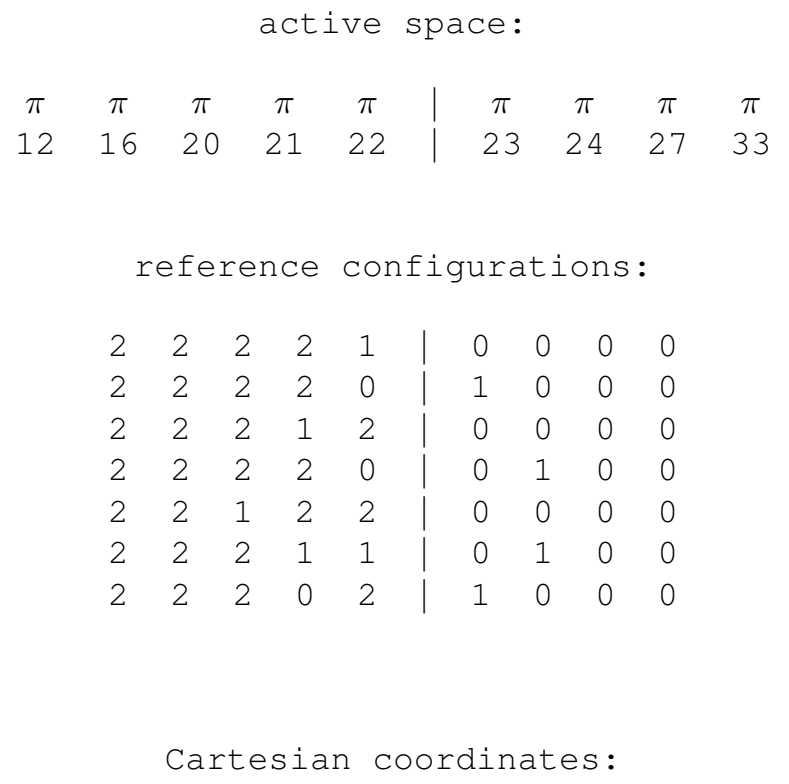

15

$\begin{array}{lrrr}\mathrm{C} & 0.00000 & 0.00000 & 0.00000 \\ \mathrm{C} & 1.41720 & 0.00000 & 0.00000 \\ \mathrm{C} & 2.14026 & 1.20313 & 0.00000 \\ \mathrm{C} & 1.47252 & 2.46057 & 0.00000 \\ \mathrm{C} & 0.09558 & 2.53947 & 0.00000 \\ \mathrm{C} & -0.66783 & 1.32848 & 0.00000 \\ \mathrm{C} & -2.06608 & 1.38975 & 0.00000 \\ \mathrm{~N} & -3.25072 & 1.44263 & 0.00000 \\ \mathrm{C} & -0.74628 & -1.15866 & -0.00000 \\ \mathrm{H} & 1.94914 & -0.95753 & -0.00000 \\ \mathrm{H} & 3.23370 & 1.18106 & 0.00000 \\ \mathrm{H} & 2.07471 & 3.37575 & 0.00000 \\ \mathrm{H} & -0.42504 & 3.49882 & 0.00000 \\ \mathrm{H} & -1.82601 & -1.13629 & -0.00000 \\ \mathrm{H} & -0.26473 & -2.12851 & -0.00000\end{array}$


Table S1200 Employed active space, reference configurations, and Cartesian coordinates of the OM2/MRCISD-optimized ground-state equilibrium geometry of oxalylfluoride (ground-state energy: $-1849.57602 \mathrm{eV}$ ).

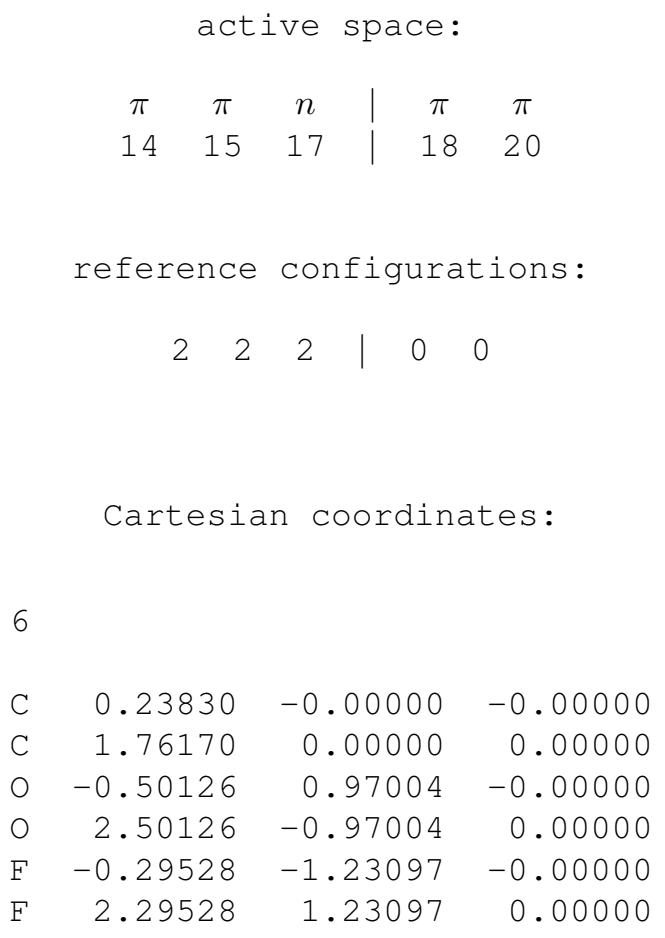


Table S1201 Employed active space, reference configurations, and Cartesian coordinates of the OM2/MRCISD-optimized excited-state equilibrium geometry of the $1{ }^{1} A_{u}\left({ }^{1} n \pi^{*}\right)$ state of oxalylfluoride (excited-state energy: $-1845.51972 \mathrm{eV})$.

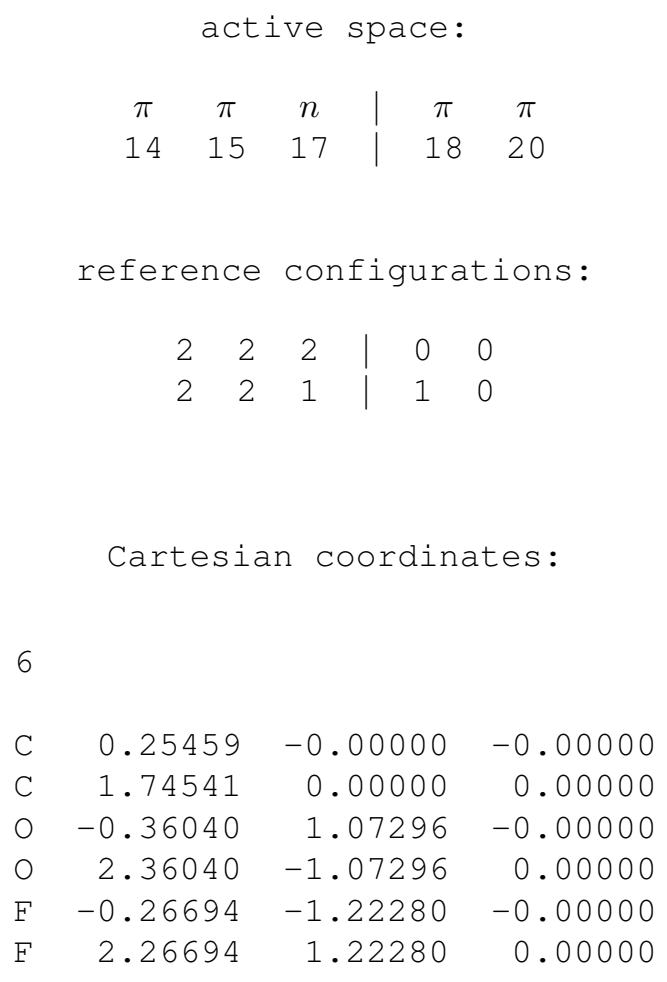


Table S1202 Employed active space, reference configurations, and Cartesian coordinates of the OM2/MRCISD-optimized ground-state equilibrium geometry of $p$-benzoquinone (ground-state energy: $-1487.20623 \mathrm{eV}$ ).

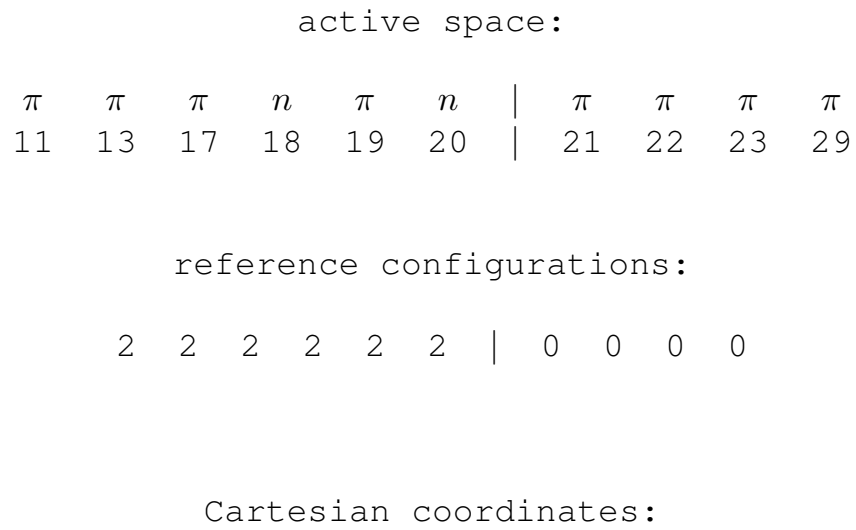


Table S1203 Employed active space, reference configurations, and Cartesian coordinates of the OM2/MRCISD-optimized excited-state equilibrium geometry of the $1{ }^{1} B_{1 g}\left({ }^{1} \pi \pi^{*}\right)$ state of $p$-benzoquinone (excited-state energy: $-1482.96859 \mathrm{eV})$.

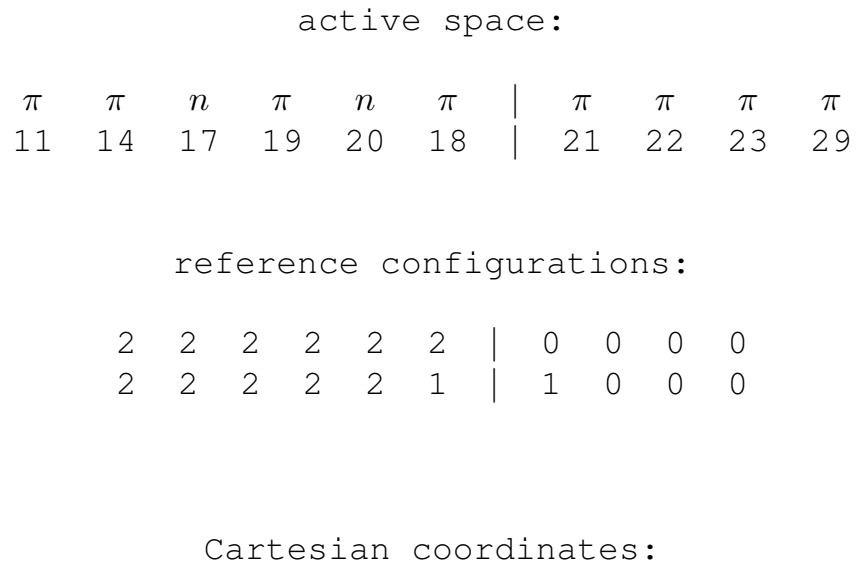


Table S1204 Employed active space, reference configurations, and Cartesian coordinates of the OM2/MRCISD-optimized excited-state equilibrium geometry of the $1{ }^{1} B_{2 g}\left({ }^{1} n \pi^{*}\right)$ state of $p$-benzoquinone (excited-state energy: $-1484.54231 \mathrm{eV})$.

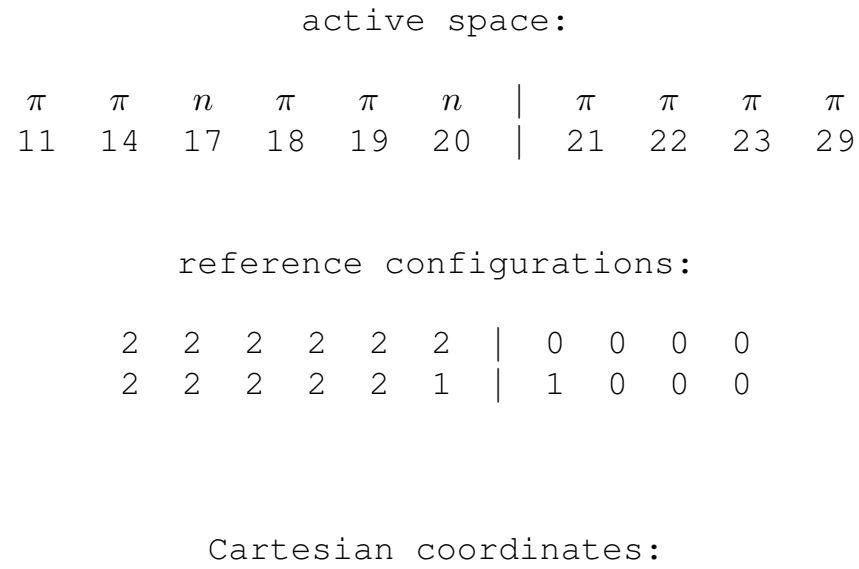


Table S1205 Employed active space, reference configurations, and Cartesian coordinates of the OM2/MRCISD-optimized ground-state equilibrium geometry of diethynylbenzene (ground-state energy: $-1361.90301 \mathrm{eV}$ ).

\begin{tabular}{lccc|cccc} 
active space: \\
$\pi$ & $\pi$ & $\pi$ & $\pi$ & $\pi$ & $\pi$ & $\pi$ & $\pi$ \\
16 & 19 & 22 & 23 & 24 & 25 & 27 & 33
\end{tabular}

reference configurations:

\begin{tabular}{llll|llll}
2 & 2 & 2 & 2 & 0 & 0 & 0 & 0
\end{tabular}

Cartesian coordinates:

$\begin{array}{lrrr}\text { 16 } & & & \\ & & & \\ \text { C } & 0.00000 & 0.00000 & 1.37454 \\ \text { C } & 0.00000 & 0.00000 & -1.37454 \\ \text { C } & 0.00000 & 1.22747 & 0.69513 \\ \text { C } & -0.00000 & -1.22747 & 0.69513 \\ \text { C } & 0.00000 & -1.22747 & -0.69513 \\ \text { C } & 0.00000 & 1.22747 & -0.69513 \\ \text { C } & 0.00000 & -0.00000 & 2.80228 \\ \text { C } & 0.00000 & 0.00000 & -2.80228 \\ \text { C } & 0.00000 & -0.00000 & 4.03297 \\ \text { C } & 0.00000 & 0.00000 & -4.03297 \\ \text { H } & 0.00000 & -0.00000 & 5.07316 \\ \text { H } & 0.00000 & 0.00000 & -5.07316 \\ \text { H } & 0.00000 & 2.16450 & 1.25613 \\ \text { H } & -0.00000 & -2.16450 & 1.25613 \\ \text { H } & 0.00000 & -2.16450 & -1.25613 \\ \text { H } & 0.00000 & 2.16450 & -1.25613\end{array}$


Table S1206 Employed active space, reference configurations, and Cartesian coordinates of the OM2/MRCISD-optimized excited-state equilibrium geometry of the $1{ }^{1} B_{2 u}\left({ }^{1} \pi \pi^{*}\right)$ state of diethynylbenzene (excited-state energy: $-1357.18261 \mathrm{eV})$.

\begin{tabular}{lccc|cccc} 
active space: \\
$\pi$ & $\pi$ & $\pi$ & $\pi$ & $\pi$ & $\pi$ & $\pi$ & $\pi$ \\
16 & 19 & 22 & 23 & 24 & 25 & 27 & 33
\end{tabular}

reference configurations:
2

Cartesian coordinates:

16

$\begin{array}{lrrr}\mathrm{C} & 0.00000 & 0.00000 & 1.39610 \\ \mathrm{C} & 0.00000 & 0.00000 & -1.39610 \\ \mathrm{C} & 0.00000 & 1.24686 & 0.70775 \\ \mathrm{C} & -0.00000 & -1.24686 & 0.70775 \\ \mathrm{C} & 0.00000 & -1.24686 & -0.70775 \\ \mathrm{C} & 0.00000 & 1.24686 & -0.70775 \\ \mathrm{C} & 0.00000 & -0.00000 & 2.79680 \\ \mathrm{C} & 0.00000 & 0.00000 & -2.79680 \\ \mathrm{C} & 0.00000 & -0.00000 & 4.03024 \\ \mathrm{C} & 0.00000 & 0.00000 & -4.03024 \\ \mathrm{H} & 0.00000 & -0.00000 & 5.07089 \\ \mathrm{H} & 0.00000 & 0.00000 & -5.07089 \\ \mathrm{H} & 0.00000 & 2.18296 & 1.26678 \\ \mathrm{H} & -0.00000 & -2.18296 & 1.26678 \\ \mathrm{H} & 0.00000 & -2.18296 & -1.26678 \\ \mathrm{H} & 0.00000 & 2.18296 & -1.26678\end{array}$


Table S1207 Employed active space, reference configurations, and Cartesian coordinates of the OM2/MRCISD-optimized ground-state equilibrium geometry of $p$-phenylenediamine (ground-state energy: $-1312.18689 \mathrm{eV}$ ).

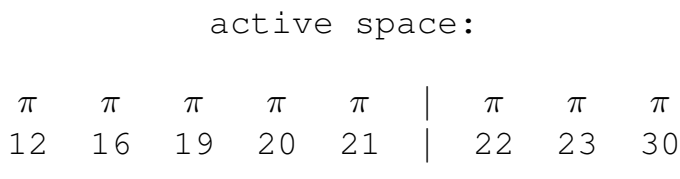

reference configurations:

$\begin{array}{lllllllll}2 & 2 & 2 & 2 & 2 & 0 & 0 & 0\end{array}$

Cartesian coordinates:

16

$\begin{array}{llll}C & 0.00000 & 0.00000 & 1.39992\end{array}$

C $\quad 0.00000 \quad 0.00000-1.39992$

$\begin{array}{lrrr}\text { C } & 0.00000 & 1.22391 & 0.69504\end{array}$

$\begin{array}{lrrr}C & -0.00000 & -1.22391 & 0.69504\end{array}$

C $\quad 0.00000-1.22391-0.69504$

$\begin{array}{llll}\text { C } & 0.00000 & 1.22391 & -0.69504\end{array}$

$\begin{array}{llll}\mathrm{N} & 0.00000 & -0.00000 & 2.78369\end{array}$

$\begin{array}{llll}\mathrm{N} & 0.00000 & 0.00000 & -2.78369\end{array}$

$\begin{array}{lrrr}\mathrm{H} & 0.00000 & 2.16817 & 1.24965\end{array}$

$\mathrm{H} \quad-0.00000 \quad-2.16817 \quad 1.24965$

$\begin{array}{llll}\mathrm{H} & 0.00000 & -2.16817 & -1.24965\end{array}$

$\begin{array}{llll}\mathrm{H} & 0.00000 & 2.16817 & -1.24965\end{array}$

$\begin{array}{llll}\mathrm{H} & 0.00000 & 0.84647 & 3.30206\end{array}$

$\begin{array}{llll}\mathrm{H} & 0.00000 & -0.84647 & 3.30206\end{array}$

$\begin{array}{llll}\mathrm{H} & 0.00000 & 0.84647 & -3.30206\end{array}$

$\mathrm{H} \quad 0.00000 \quad-0.84647 \quad-3.30206$ 
Table S1208 Employed active space, reference configurations, and Cartesian coordinates of the OM2/MRCISD-optimized excited-state equilibrium geometry of the $2^{1} A\left({ }^{1} \pi \pi^{*}\right)$ state of $p$-phenylenediamine (excited-state energy: $-1308.65421 \mathrm{eV})$.

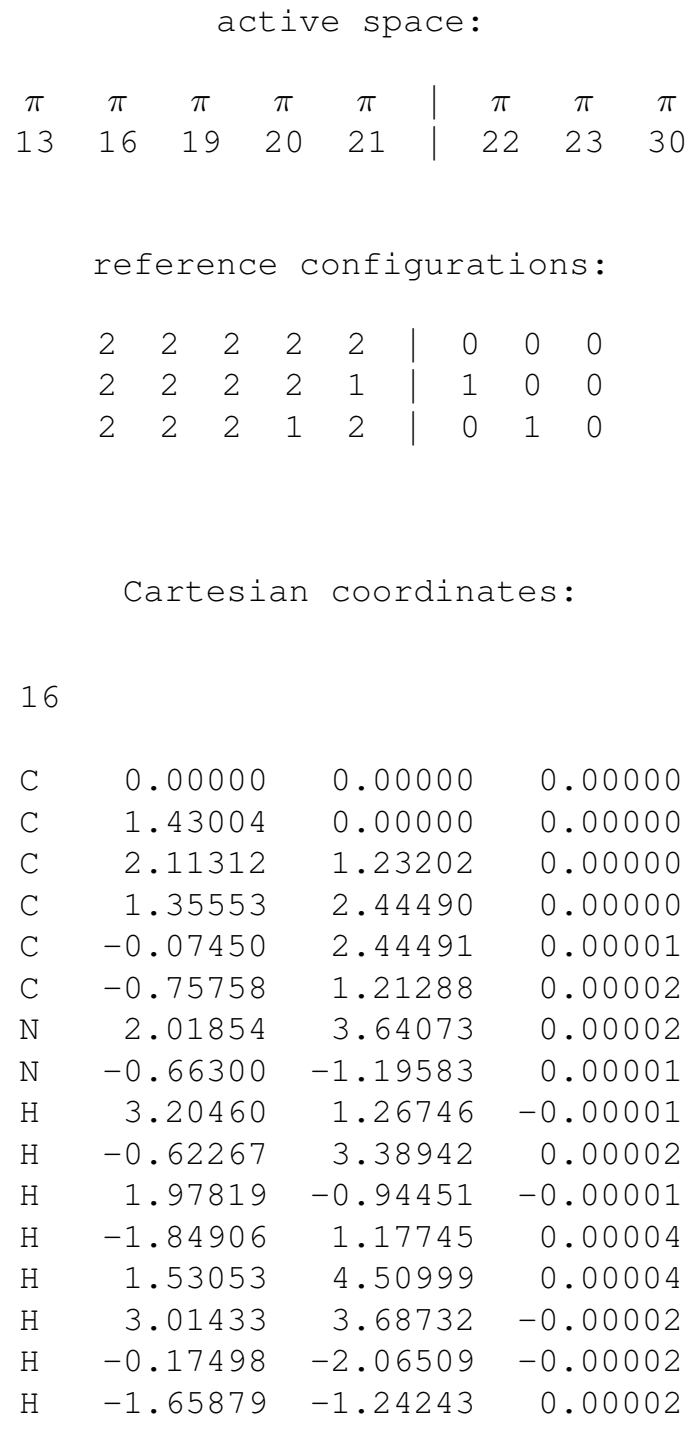


Table S1209 Employed active space, reference configurations, and Cartesian coordinates of the OM2/MRCISD-optimized ground-state equilibrium geometry of phenol (ground-state energy: $-1186.87973 \mathrm{eV}$ ).

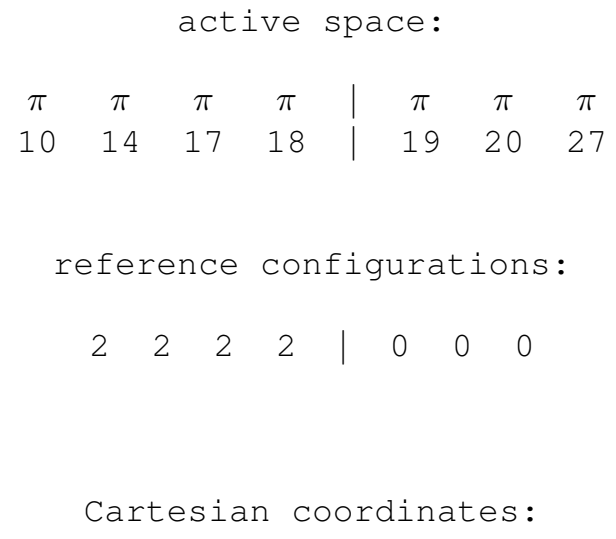

$\begin{array}{lrrr}13 & & & \\ \text { C } & 0.00000 & 0.00000 & 0.00000 \\ \mathrm{C} & 1.39501 & 0.00000 & 0.00000 \\ \mathrm{C} & 2.05698 & 1.23810 & 0.00000 \\ \mathrm{C} & 1.36704 & 2.46395 & 0.00000 \\ \mathrm{C} & -0.02527 & 2.42632 & 0.00000 \\ \mathrm{C} & -0.70722 & 1.20486 & 0.00000 \\ \mathrm{O} & 3.41639 & 1.34151 & 0.00000 \\ \mathrm{H} & 1.92541 & 3.39792 & 0.00000 \\ \mathrm{H} & -0.59077 & 3.36525 & 0.00000 \\ \mathrm{H} & -1.80122 & 1.19262 & 0.00000 \\ \mathrm{H} & -0.54184 & -0.95265 & -0.00000 \\ \mathrm{H} & 1.96068 & -0.93472 & -0.00000 \\ \mathrm{H} & 3.81691 & 0.42996 & 0.00000\end{array}$


Table S1210 Employed active space, reference configurations, and Cartesian coordinates of the OM2/MRCISD-optimized excited-state equilibrium geometry of the $2{ }^{1} A\left({ }^{1} \pi \pi^{*}\right)$ state of phenol (excited-state energy: $-1182.73589 \mathrm{eV}$ ).

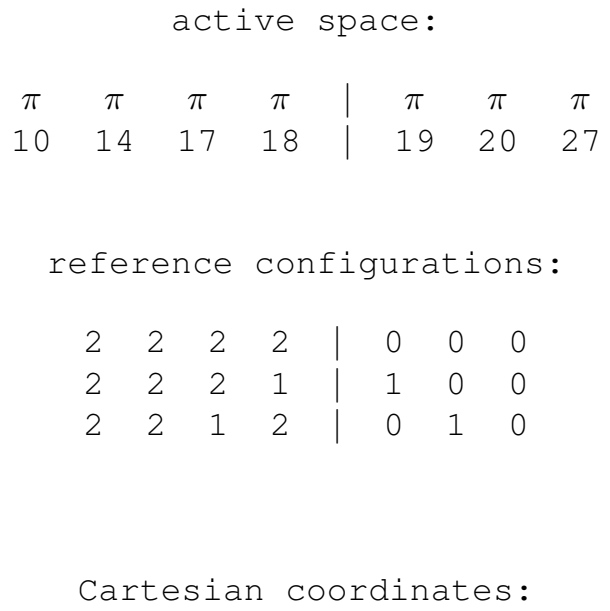


Table S1211 Employed active space, reference configurations, and Cartesian coordinates of the OM2/MRCISD-optimized ground-state equilibrium geometry of porphyrin (ground-state energy: $-3609.13544 \mathrm{eV}$ ).

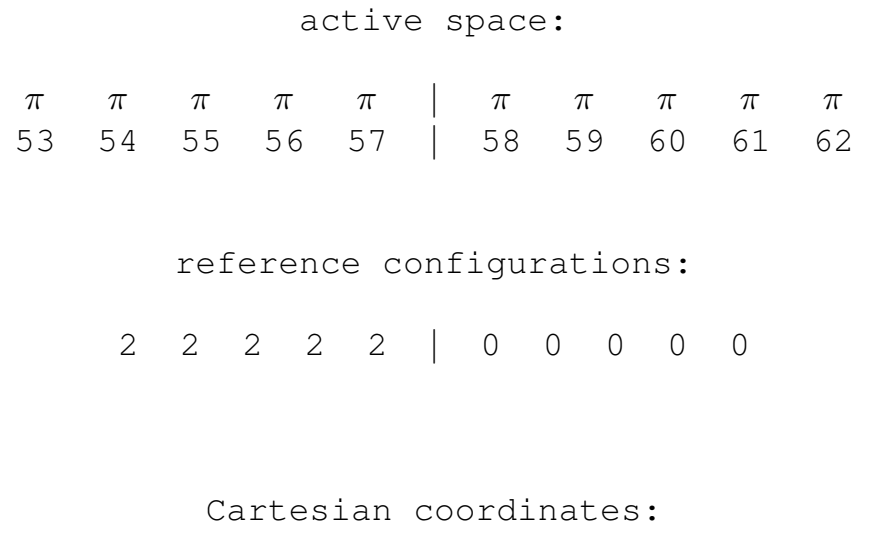

\begin{tabular}{lrrr}
38 & & & \\
& & & \\
$\mathrm{~N}$ & 2.05769 & 0.00000 & 0.00000 \\
$\mathrm{~N}$ & 0.00000 & 2.01774 & 0.00000 \\
$\mathrm{~N}$ & -2.05769 & 0.00000 & 0.00000 \\
$\mathrm{~N}$ & 0.00000 & -2.01774 & -0.00000 \\
$\mathrm{C}$ & 2.85290 & 1.12408 & 0.00000 \\
$\mathrm{C}$ & 2.85290 & -1.12408 & -0.00000 \\
$\mathrm{C}$ & -2.85290 & 1.12408 & 0.00000 \\
$\mathrm{C}$ & -2.85290 & -1.12408 & 0.00000 \\
$\mathrm{C}$ & 1.08573 & 2.83659 & 0.00000 \\
$\mathrm{C}$ & -1.08573 & 2.83659 & 0.00000 \\
$\mathrm{C}$ & 1.08573 & -2.83659 & -0.00000 \\
$\mathrm{C}$ & -1.08573 & -2.83659 & -0.00000 \\
$\mathrm{C}$ & 4.23862 & 0.68748 & 0.00000 \\
$\mathrm{C}$ & 4.23862 & -0.68748 & -0.00000 \\
$\mathrm{C}$ & -4.23862 & 0.68748 & 0.00000 \\
$\mathrm{C}$ & -4.23862 & -0.68748 & 0.00000 \\
$\mathrm{C}$ & 0.67679 & 4.26468 & -0.00000 \\
$\mathrm{C}$ & -0.67679 & 4.26468 & 0.00000 \\
$\mathrm{C}$ & 0.67679 & -4.26468 & -0.00000 \\
$\mathrm{C}$ & -0.67679 & -4.26468 & -0.00000 \\
$\mathrm{C}$ & 2.43169 & 2.45840 & 0.00000 \\
$\mathrm{C}$ & 2.43169 & -2.45840 & -0.00000 \\
$\mathrm{C}$ & -2.43169 & 2.45840 & 0.00000 \\
$\mathrm{C}$ & -2.43169 & -2.45840 & -0.00000 \\
\hline & & Continued & on next page
\end{tabular}




\begin{tabular}{lrrr}
\multicolumn{5}{c}{ Continued from previous page } \\
\hline $\mathrm{H}$ & 5.08937 & 1.35479 & 0.00000 \\
$\mathrm{H}$ & 5.08937 & -1.35479 & -0.00000 \\
$\mathrm{H}$ & -5.08937 & 1.35479 & 0.00000 \\
$\mathrm{H}$ & -5.08937 & -1.35479 & 0.00000 \\
$\mathrm{H}$ & 1.36425 & 5.09810 & -0.00000 \\
$\mathrm{H}$ & -1.36425 & 5.09810 & 0.00000 \\
$\mathrm{H}$ & 1.36425 & -5.09810 & -0.00000 \\
$\mathrm{H}$ & -1.36425 & -5.09810 & -0.00000 \\
$\mathrm{H}$ & 3.20005 & 3.23978 & 0.00000 \\
$\mathrm{H}$ & 3.20005 & -3.23978 & -0.00000 \\
$\mathrm{H}$ & -3.20005 & 3.23978 & 0.00000 \\
$\mathrm{H}$ & -3.20005 & -3.23978 & 0.00000 \\
$\mathrm{H}$ & 1.04217 & 0.00000 & -0.00000 \\
$\mathrm{H}$ & -1.04217 & 0.00000 & 0.00000
\end{tabular}


Table S1212 Employed active space, reference configurations, and Cartesian coordinates of the OM2/MRCISD-optimized excited-state equilibrium geometry of the $1{ }^{1} B_{3 u}\left({ }^{1} \pi \pi^{*}\right)$ state of porphyrin (excited-state energy: $-3607.17218 \mathrm{eV}$ ).

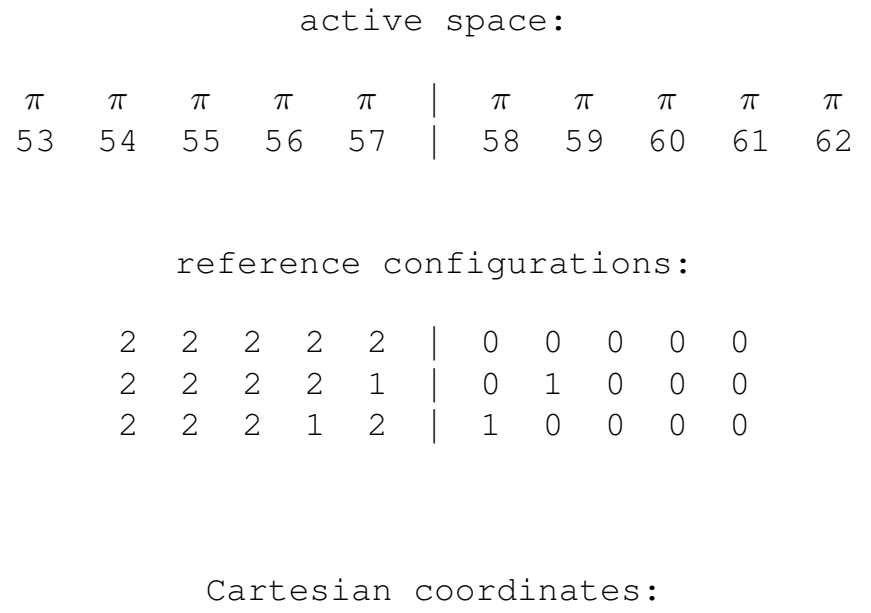

\begin{tabular}{lrrr}
38 & & & \\
& & & \\
$\mathrm{~N}$ & 2.05574 & 0.00000 & 0.00000 \\
$\mathrm{~N}$ & 0.00000 & 2.02813 & 0.00000 \\
$\mathrm{~N}$ & -2.05574 & 0.00000 & 0.00000 \\
$\mathrm{~N}$ & 0.00000 & -2.02813 & -0.00000 \\
$\mathrm{C}$ & 2.85405 & 1.12450 & 0.00000 \\
$\mathrm{C}$ & 2.85405 & -1.12450 & -0.00000 \\
$\mathrm{C}$ & -2.85405 & 1.12450 & 0.00000 \\
$\mathrm{C}$ & -2.85405 & -1.12450 & 0.00000 \\
$\mathrm{C}$ & 1.08674 & 2.85091 & 0.00000 \\
$\mathrm{C}$ & -1.08674 & 2.85091 & 0.00000 \\
$\mathrm{C}$ & 1.08674 & -2.85091 & -0.00000 \\
$\mathrm{C}$ & -1.08674 & -2.85091 & -0.00000 \\
$\mathrm{C}$ & 4.23800 & 0.68963 & 0.00000 \\
$\mathrm{C}$ & 4.23800 & -0.68963 & -0.00000 \\
$\mathrm{C}$ & -4.23800 & 0.68963 & 0.00000 \\
$\mathrm{C}$ & -4.23800 & -0.68963 & 0.00000 \\
$\mathrm{C}$ & 0.67791 & 4.27241 & -0.00000 \\
$\mathrm{C}$ & -0.67791 & 4.27241 & 0.00000 \\
$\mathrm{C}$ & 0.67791 & -4.27241 & -0.00000 \\
$\mathrm{C}$ & -0.67791 & -4.27241 & -0.00000 \\
$\mathrm{C}$ & 2.43708 & 2.46258 & 0.00000 \\
$\mathrm{C}$ & 2.43708 & -2.46258 & -0.00000 \\
$\mathrm{C}$ & -2.43708 & 2.46258 & 0.00000 \\
\hline & & Continued & on next page
\end{tabular}

S1280 


\begin{tabular}{lrrr}
\multicolumn{5}{c}{ Continued from previous page } \\
\hline C & -2.43708 & -2.46258 & -0.00000 \\
H & 5.08999 & 1.35544 & 0.00000 \\
H & 5.08999 & -1.35544 & -0.00000 \\
H & -5.08999 & 1.35544 & 0.00000 \\
H & -5.08999 & -1.35544 & 0.00000 \\
H & 1.36289 & 5.10707 & -0.00000 \\
H & -1.36289 & 5.10707 & 0.00000 \\
H & 1.36289 & -5.10707 & -0.00000 \\
H & -1.36289 & -5.10707 & -0.00000 \\
H & 3.20705 & 3.24192 & 0.00000 \\
H & 3.20705 & -3.24192 & -0.00000 \\
H & -3.20705 & 3.24192 & 0.00000 \\
H & -3.20705 & -3.24192 & 0.00000 \\
H & 1.04184 & 0.00000 & -0.00000 \\
H & -1.04184 & 0.00000 & 0.00000
\end{tabular}


Table S1213 Employed active space, reference configurations, and Cartesian coordinates of the OM2/MRCISD-optimized ground-state equilibrium geometry of propynal (ground-state energy: $-741.71221 \mathrm{eV}$ ).

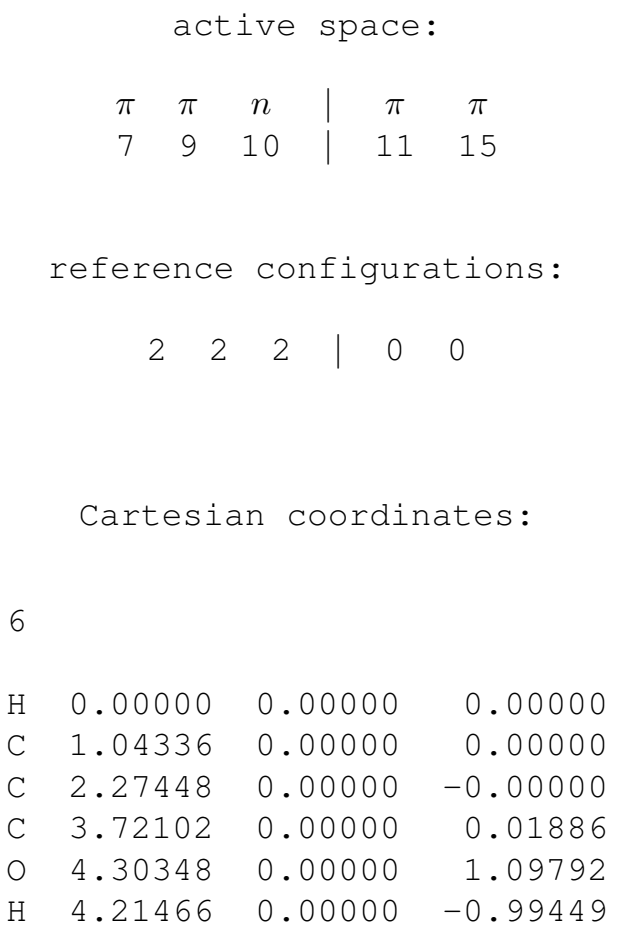


Table S1214 Employed active space, reference configurations, and Cartesian coordinates of the OM2/MRCISD-optimized excited-state equilibrium geometry of the $2{ }^{1} A\left({ }^{1} n \pi^{*}\right)$ state of propynal (excited-state energy: $-738.22028 \mathrm{eV}$ ).

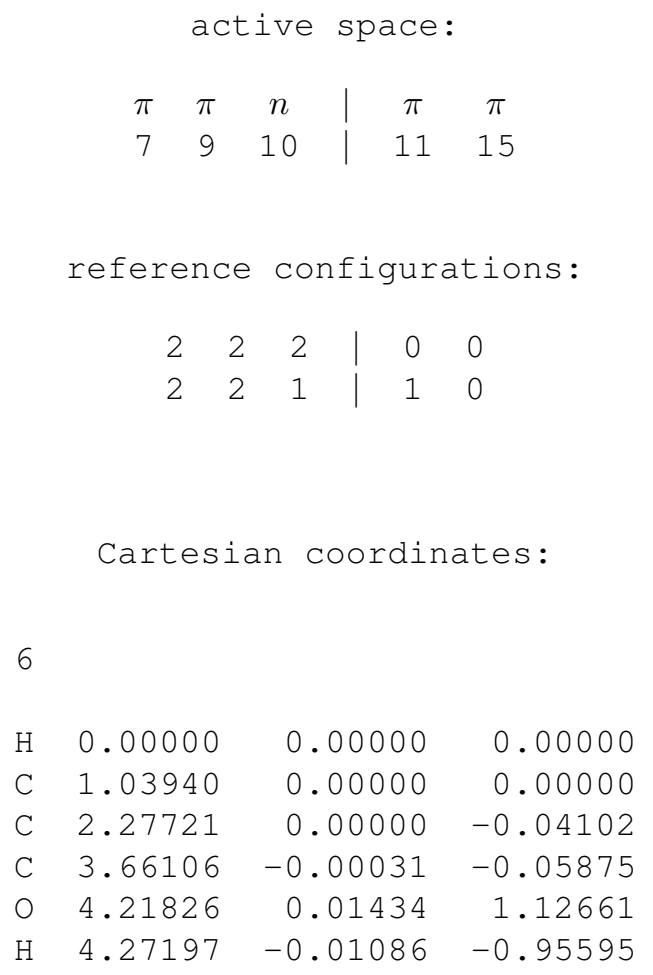


Table S1215 Employed active space, reference configurations, and Cartesian coordinates of the OM2/MRCISD-optimized ground-state equilibrium geometry of pyrene (ground-state energy: $-2192.40525 \mathrm{eV}$ ).

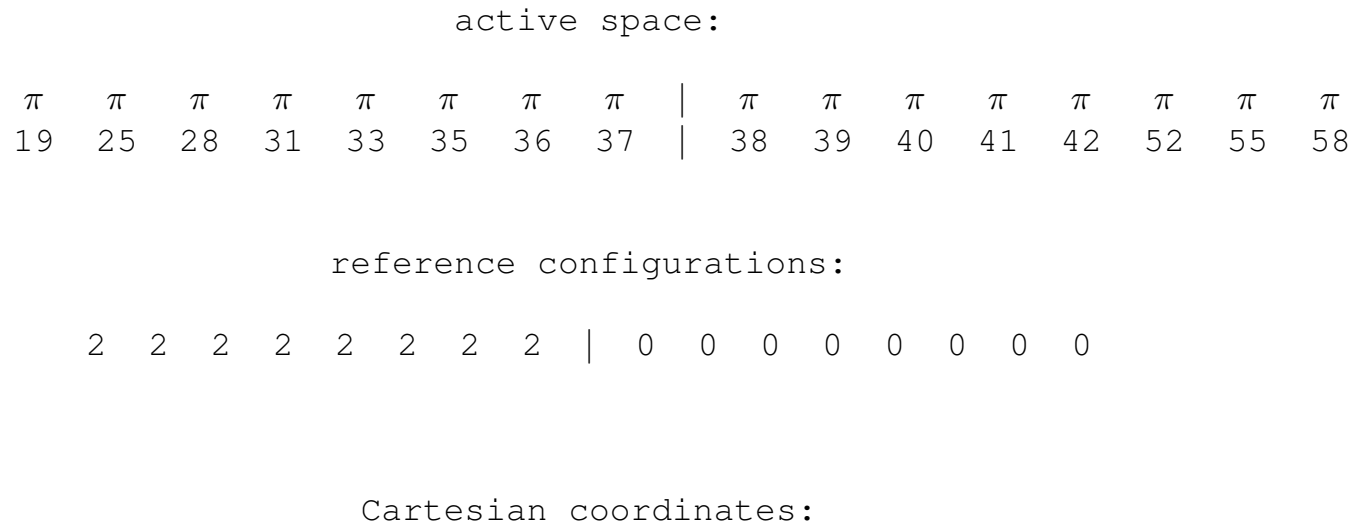


Continued from previous page

$\begin{array}{rrrr}\mathrm{H} & 0.00000 & 4.61212 & 0.00000 \\ \mathrm{H} & 0.00000 & -4.61212 & -0.00000\end{array}$

S1285 
Table S1216 Employed active space, reference configurations, and Cartesian coordinates of the OM2/MRCISD-optimized excited-state equilibrium geometry of the $2{ }^{1} A\left({ }^{1} \pi \pi^{*}\right)$ state of pyrene (excited-state energy: $-2189.04742 \mathrm{eV}$ ).

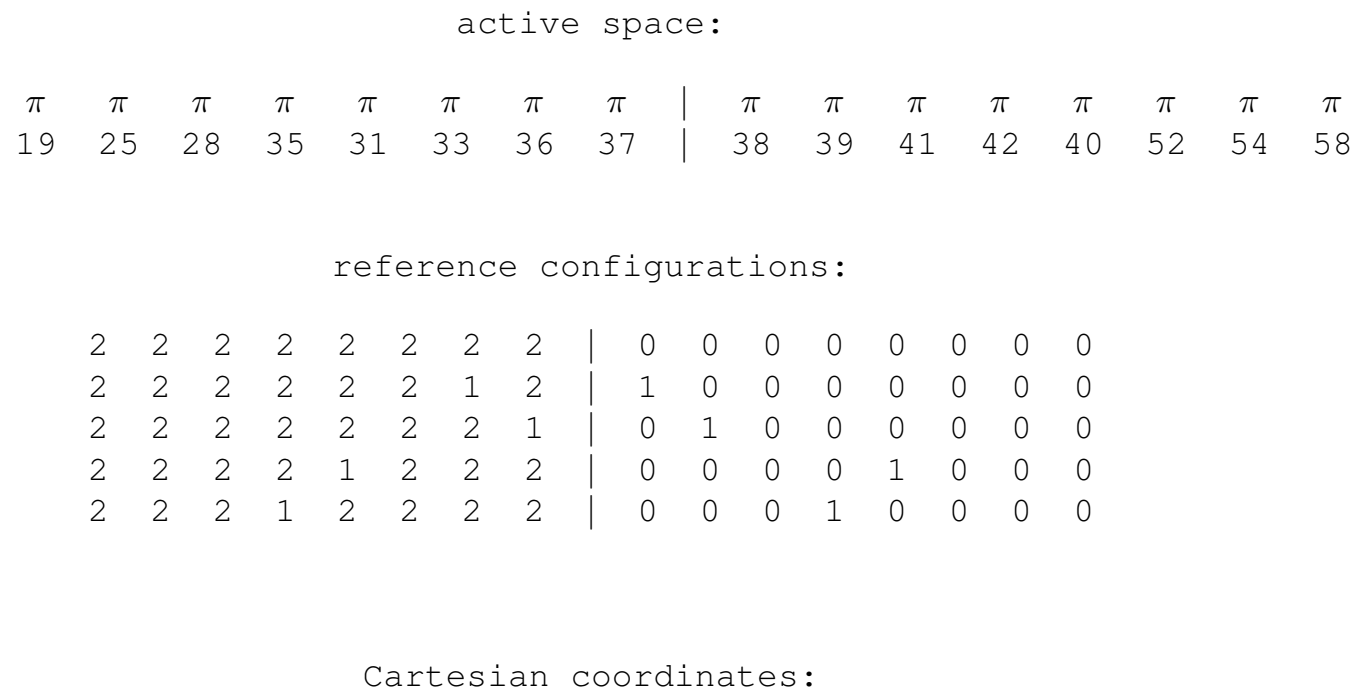

\begin{tabular}{lrrr}
26 & & & \\
& & & \\
$\mathrm{C}$ & 0.00000 & 0.00000 & 0.00000 \\
$\mathrm{C}$ & 1.40345 & 0.00000 & 0.00000 \\
$\mathrm{C}$ & 2.11716 & 1.21535 & 0.00000 \\
$\mathrm{C}$ & 1.38866 & 2.46059 & -0.00002 \\
$\mathrm{C}$ & -0.05389 & 2.44061 & -0.00002 \\
$\mathrm{C}$ & -0.72540 & 1.20144 & -0.00002 \\
$\mathrm{C}$ & 3.54160 & 1.25256 & 0.00002 \\
$\mathrm{C}$ & 4.22043 & 2.45540 & 0.00003 \\
$\mathrm{C}$ & 3.51602 & 3.69403 & -0.00000 \\
$\mathrm{C}$ & 2.07348 & 3.67404 & -0.00000 \\
$\mathrm{C}$ & -0.75830 & 3.67924 & -0.00006 \\
$\mathrm{C}$ & -0.07946 & 4.88208 & -0.00003 \\
$\mathrm{C}$ & 1.34497 & 4.91928 & -0.00001 \\
$\mathrm{C}$ & 2.05869 & 6.13463 & 0.00001 \\
$\mathrm{C}$ & 3.46213 & 6.13464 & -0.00001 \\
$\mathrm{C}$ & 4.18754 & 4.93320 & -0.00002 \\
$\mathrm{H}$ & 5.31708 & 2.47293 & 0.00006 \\
$\mathrm{H}$ & -0.63129 & 5.82994 & -0.00004 \\
$\mathrm{H}$ & 4.09342 & 0.30469 & 0.00004 \\
$\mathrm{H}$ & -1.85495 & 3.66171 & -0.00009 \\
$\mathrm{H}$ & 5.28324 & 4.95705 & -0.00002 \\
\hline & & Continued & on next page
\end{tabular}

S1286 


\begin{tabular}{lrrr}
\multicolumn{4}{c}{ Continued from previous page } \\
\hline $\mathrm{H}$ & 1.51276 & 7.08495 & 0.00002 \\
$\mathrm{H}$ & 1.94938 & -0.95032 & 0.00002 \\
$\mathrm{H}$ & -1.82111 & 1.17759 & -0.00003 \\
$\mathrm{H}$ & 4.00068 & 7.08890 & -0.00002 \\
$\mathrm{H}$ & -0.53855 & -0.95426 & 0.00000
\end{tabular}


Table S1217 Employed active space, reference configurations, and Cartesian coordinates of the OM2/MRCISD-optimized ground-state equilibrium geometry of pyridine (ground-state energy: $-926.29033 \mathrm{eV}$ ).

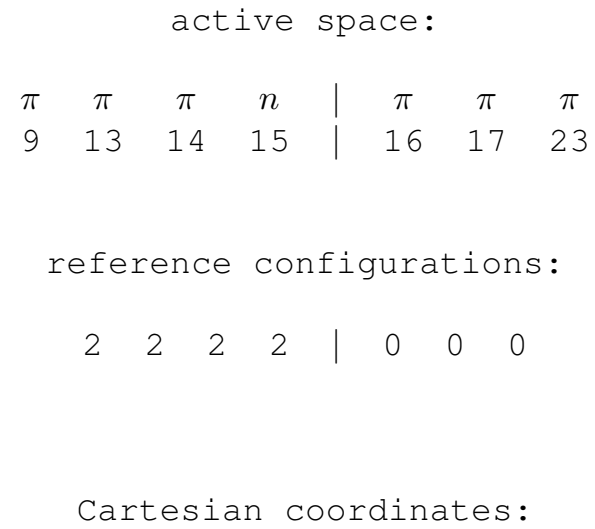

$\begin{array}{lrrr}\text { C } & 0.00000 & 1.41275 & 0.00000 \\ \text { C } & 0.00000 & 0.70226 & -1.20044 \\ \text { C } & 0.00000 & 0.70226 & 1.20044 \\ \text { C } & 0.00000 & -0.70153 & -1.14864 \\ \text { C } & 0.00000 & -0.70153 & 1.14864 \\ \text { N } & 0.00000 & -1.38378 & -0.00000 \\ \text { H } & 0.00000 & 2.50755 & 0.00000 \\ \text { H } & 0.00000 & 1.22509 & -2.15909 \\ \text { H } & 0.00000 & 1.22509 & 2.15909 \\ \text { H } & 0.00000 & -1.27549 & -2.09196 \\ \text { H } & 0.00000 & -1.27549 & 2.09196\end{array}$


Table S1218 Employed active space, reference configurations, and Cartesian coordinates of the OM2/MRCISD-optimized excited-state equilibrium geometry of the $2{ }^{1} A\left({ }^{1} n \pi^{*}\right)$ state of pyridine (excited-state energy: $-921.97652 \mathrm{eV}$ ).

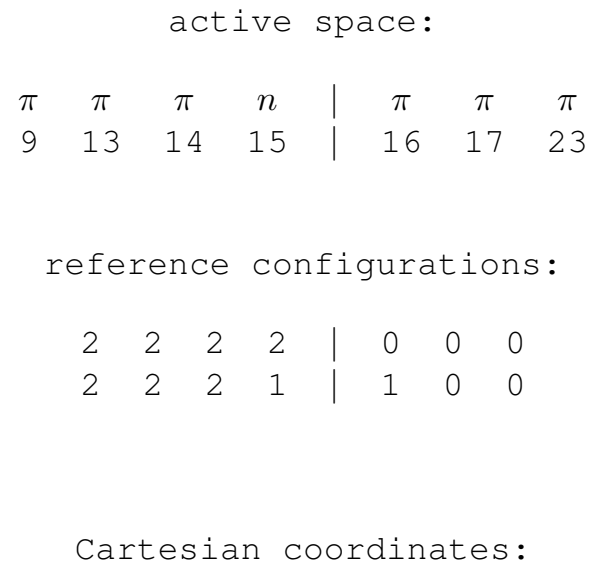


Table S1219 Employed active space, reference configurations, and Cartesian coordinates of the OM2/MRCISD-optimized ground-state equilibrium geometry of pyridone lactam (ground-state energy: $-1257.38046 \mathrm{eV}$ ).

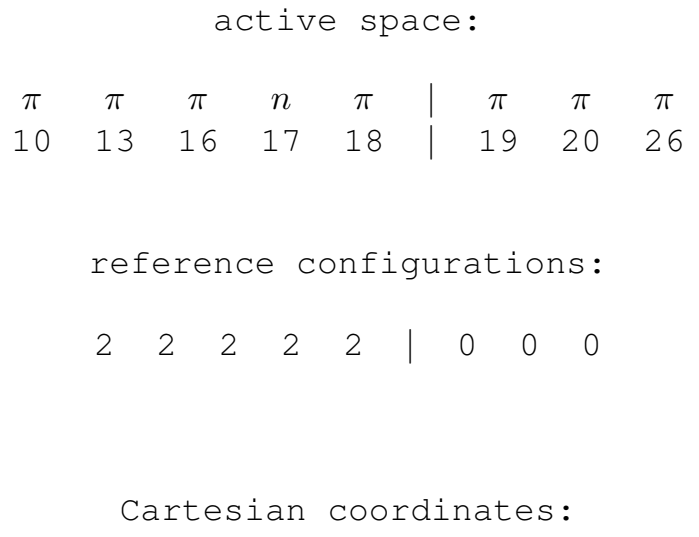

12

$\begin{array}{lrrr}\mathrm{C} & 0.00000 & 0.00000 & 0.00000 \\ \mathrm{~N} & 1.36561 & 0.00000 & 0.00000 \\ \mathrm{C} & 2.13438 & 1.17692 & 0.00000 \\ \mathrm{C} & 1.39498 & 2.43292 & 0.00000 \\ \mathrm{C} & 0.02816 & 2.42414 & 0.00000 \\ \mathrm{C} & -0.69187 & 1.19441 & 0.00000 \\ \mathrm{O} & 3.37667 & 1.06603 & 0.00000 \\ \mathrm{H} & 1.98527 & 3.34631 & 0.00000 \\ \mathrm{H} & -0.54005 & 3.36141 & 0.00000 \\ \mathrm{H} & -1.78015 & 1.20158 & 0.00000 \\ \mathrm{H} & -0.53207 & -0.96408 & -0.00000 \\ \mathrm{H} & 1.86761 & -0.88510 & -0.00000\end{array}$


Table S1220 Employed active space, reference configurations, and Cartesian coordinates of the OM2/MRCISD-optimized excited-state equilibrium geometry of the $3{ }^{1} A\left({ }^{1} n \pi^{*}\right)$ state of pyridone lactam (excited-state energy: $-1253.35196 \mathrm{eV})$.

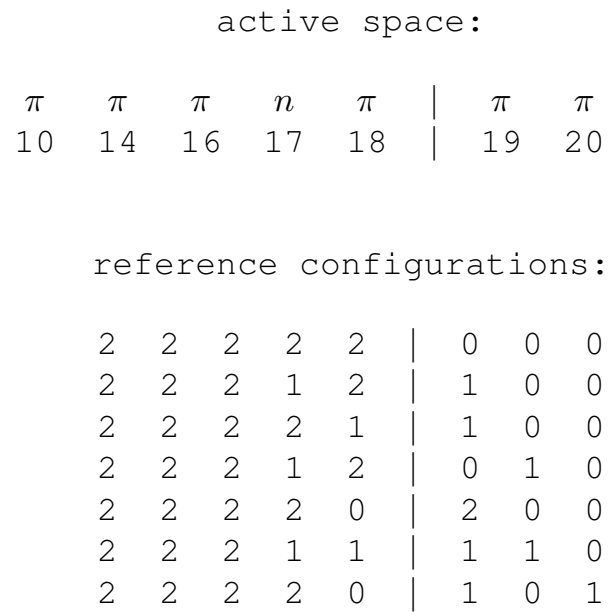

Cartesian coordinates:

12

$\begin{array}{rrrr}\mathrm{C} & 0.00000 & 0.00000 & 0.00000 \\ \mathrm{~N} & 1.39404 & 0.00000 & 0.00000 \\ \mathrm{C} & 2.06068 & 1.20816 & 0.00000 \\ \mathrm{C} & 1.41884 & 2.44043 & 0.00002 \\ \mathrm{C} & 0.00189 & 2.43713 & 0.00004 \\ \mathrm{C} & -0.68070 & 1.21026 & 0.00001 \\ \mathrm{O} & 3.37703 & 1.13119 & -0.00002 \\ \mathrm{H} & 1.99776 & 3.35815 & 0.00001 \\ \mathrm{H} & -0.53468 & 3.38010 & 0.00005 \\ \mathrm{H} & -1.77570 & 1.20022 & 0.00001 \\ \mathrm{H} & -0.51023 & -0.96501 & -0.00002 \\ \mathrm{H} & 1.91930 & -0.86381 & -0.00003\end{array}$


Table S1221 Employed active space, reference configurations, and Cartesian coordinates of the OM2/MRCISD-optimized ground-state equilibrium geometry of pyridone lactim (ground-state energy: $-1257.45030 \mathrm{eV}$ ).

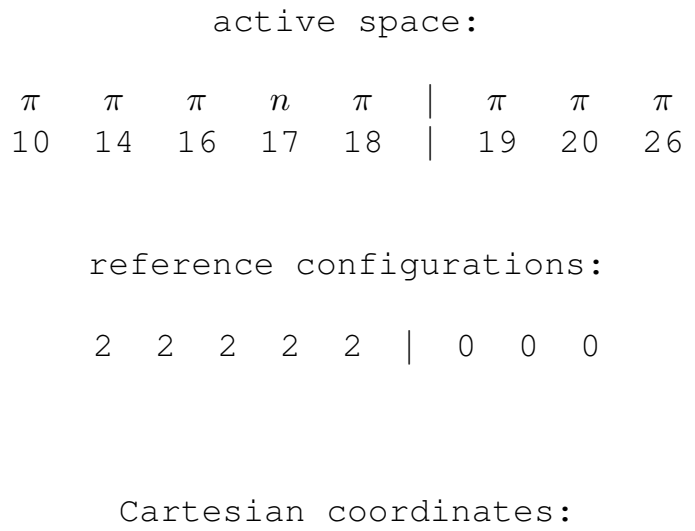


Table S1222 Employed active space, reference configurations, and Cartesian coordinates of the OM2/MRCISD-optimized excited-state equilibrium geometry of the $1{ }^{1} A^{\prime \prime}\left({ }^{1} n \pi^{*}\right)$ state of pyridone lactim (excited-state energy: $-1252.76544 \mathrm{eV})$.

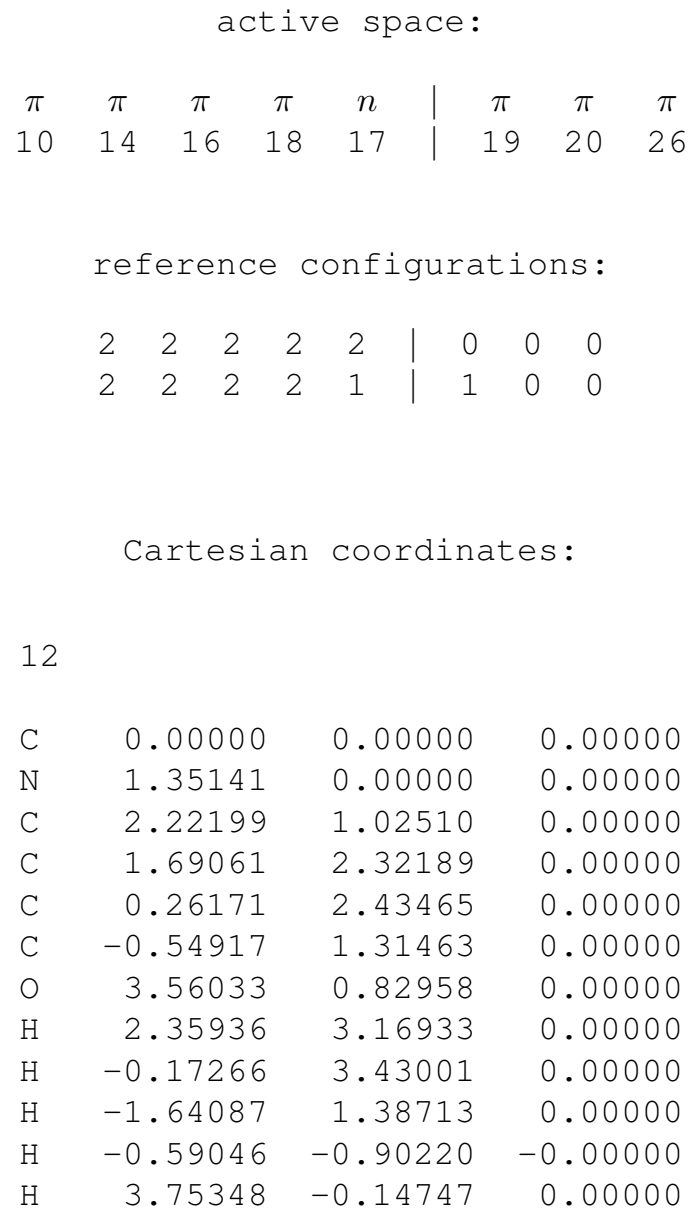


Table S1223 Employed active space, reference configurations, and Cartesian coordinates of the OM2/MRCISD-optimized ground-state equilibrium geometry of pyrimidine (ground-state energy: $-996.71297 \mathrm{eV}$ ).

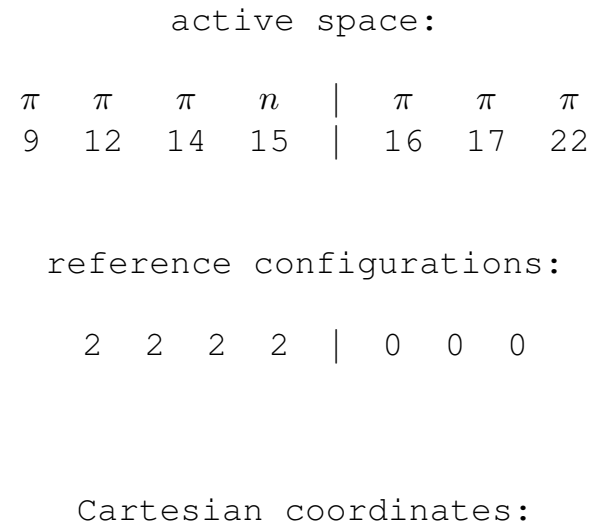

$\mathrm{H} \quad 0.00000 \quad-2.48094-0.00000$ 
Table S1224 Employed active space, reference configurations, and Cartesian coordinates of the OM2/MRCISD-optimized excited-state equilibrium geometry of the $2{ }^{1} A\left({ }^{1} n \pi^{*}\right)$ state of pyrimidine (excited-state energy: $-992.67685 \mathrm{eV}$ ).

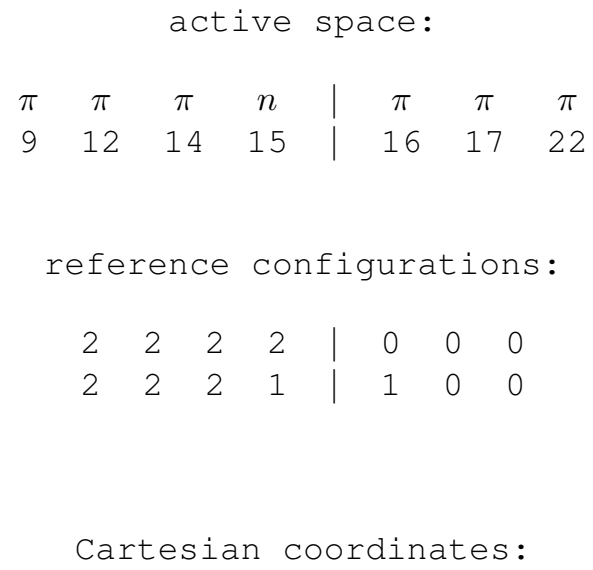


Table S1225 Employed active space, reference configurations, and Cartesian coordinates of the OM2/MRCISD-optimized excited-state equilibrium geometry of the $4^{1} A\left({ }^{1} \pi \pi^{*}\right)$ state of pyrimidine (excited-state energy: $-992.00390 \mathrm{eV}$ ).

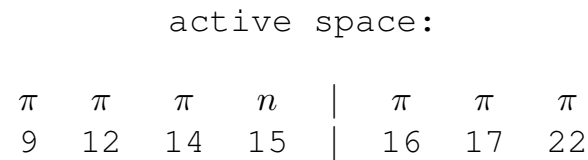

reference configurations:

$\begin{array}{llll:lll}2 & 2 & 2 & 2 & 0 & 0 & 0 \\ 2 & 2 & 1 & 2 & 0 & 1 & 0 \\ 2 & 2 & 2 & 1 & 0 & 1 & 0 \\ 2 & 2 & 2 & 1 & 1 & 0 & 0 \\ 2 & 1 & 2 & 2 & 1 & 0 & 0\end{array}$

Cartesian coordinates:

10

$\begin{array}{llll}C & 0.00000 & 0.00000 & 0.00000\end{array}$

C $\quad 1.42965 \quad 0.00000 \quad 0.00000$

$\mathrm{N} \quad 2.13635 \quad 1.16296 \quad 0.00000$

C $\quad 1.41587 \quad 2.32605-0.00000$

$\mathrm{N} \quad 0.05180 \quad 2.43183 \quad-0.00001$

C $\quad-0.65665 \quad 1.26993-0.00001$

$\mathrm{H} \quad 1.99318 \quad 3.27448 \quad-0.00000$

$\mathrm{H} \quad 1.98156-0.94959 \quad 0.00001$

$\mathrm{H} \quad-1.75365 \quad 1.32402 \quad-0.00001$

$\begin{array}{llll}\mathrm{H} & -0.56576 & -0.92945 & 0.00001\end{array}$ 
Table S1226 Employed active space, reference configurations, and Cartesian coordinates of the OM2/MRCISD-optimized ground-state equilibrium geometry of quinoline (ground-state energy: $-1466.75784 \mathrm{eV}$ ).

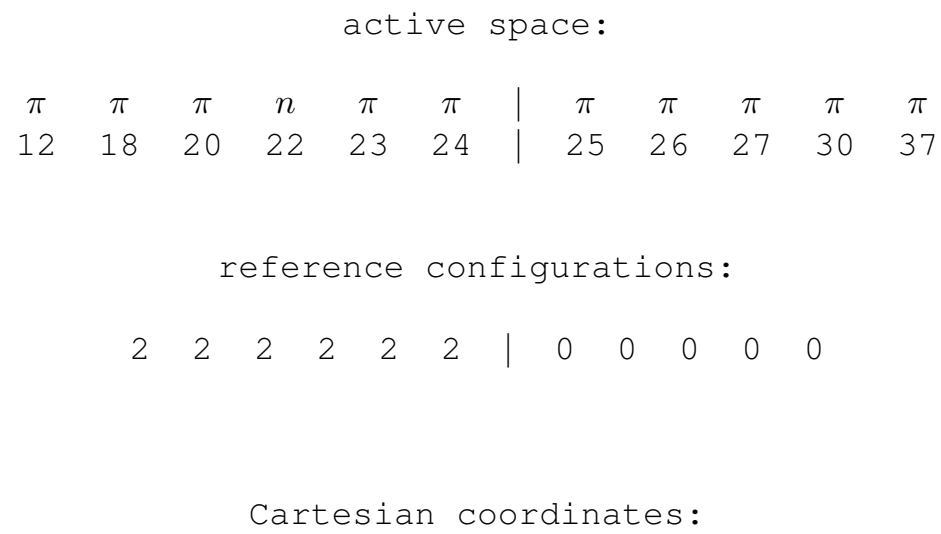


Table S1227 Employed active space, reference configurations, and Cartesian coordinates of the OM2/MRCISD-optimized excited-state equilibrium geometry of the $1{ }^{1} A^{\prime \prime}\left({ }^{1} n \pi^{*}\right)$ state of quinoline (excited-state energy: $\left.-1462.80859 \mathrm{eV}\right)$.

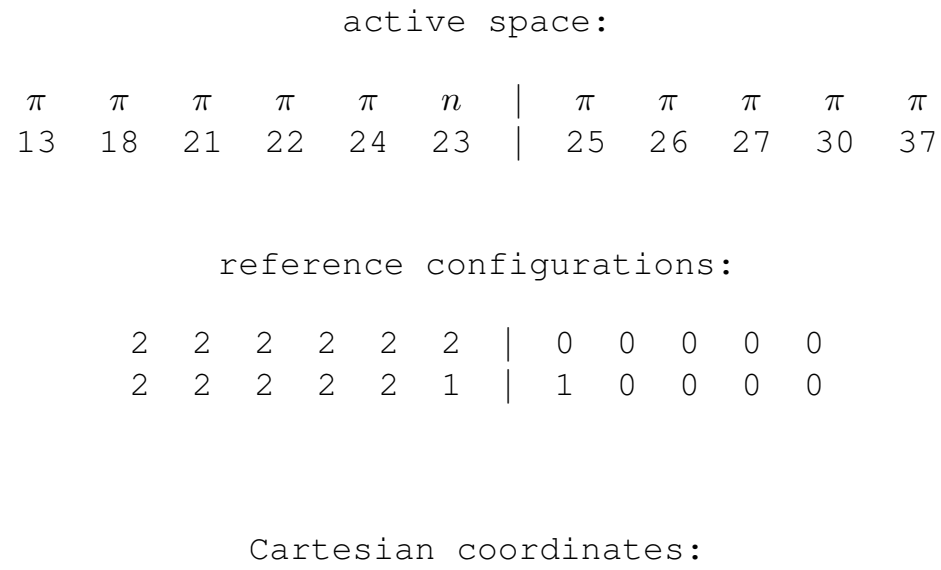


Table S1228 Employed active space, reference configurations, and Cartesian coordinates of the OM2/MRCISD-optimized excited-state equilibrium geometry of the $1^{3} A^{\prime}\left({ }^{3} \pi \pi^{*}\right)$ state of quinoline (excited-state energy: $-1464.00472 \mathrm{eV}$ ).

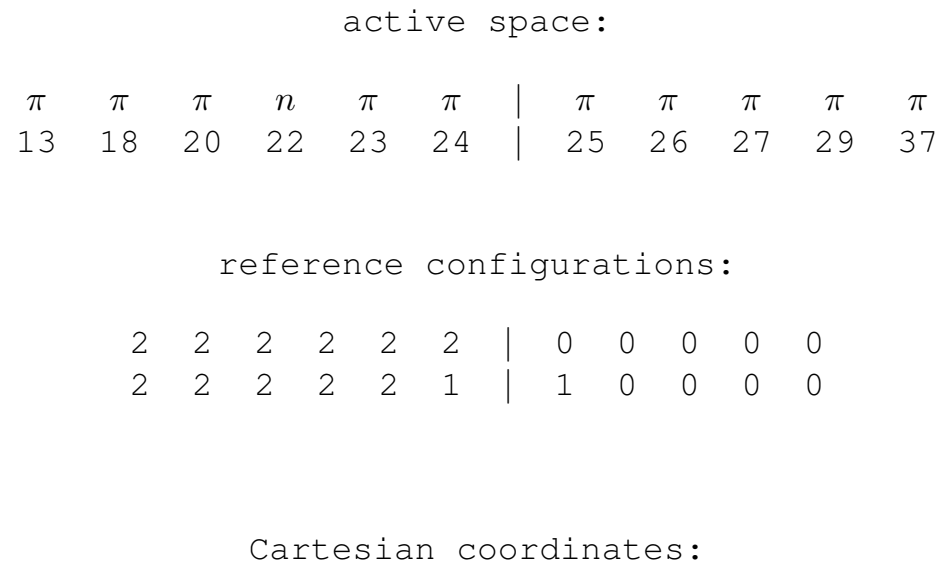


Table S1229 Employed active space, reference configurations, and Cartesian coordinates of the OM2/MRCISD-optimized ground-state equilibrium geometry of quinoxaline (ground-state energy: $-1537.09091 \mathrm{eV}$ ).

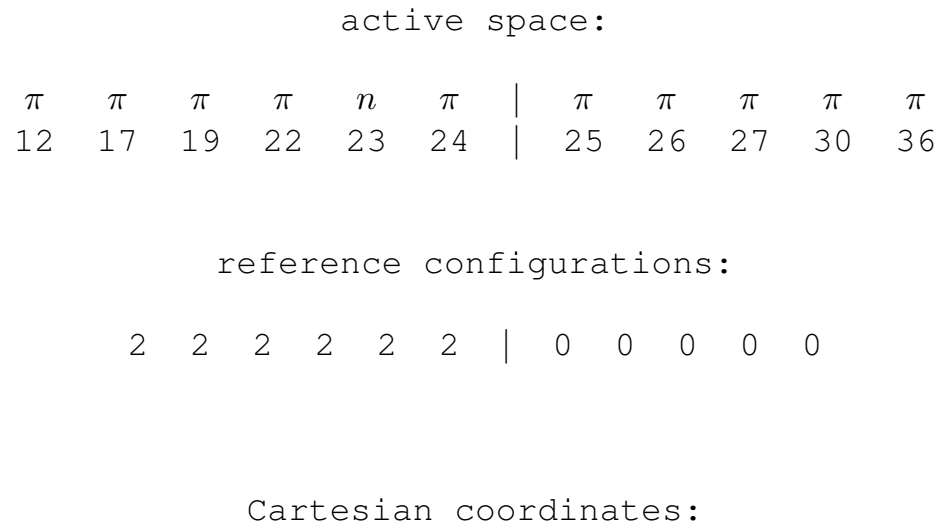


Table S1230 Employed active space, reference configurations, and Cartesian coordinates of the OM2/MRCISD-optimized excited-state equilibrium geometry of the $2{ }^{1} A_{1}\left({ }^{1} \pi \pi^{*}\right)$ state of quinoxaline (excited-state energy: $-1533.30157 \mathrm{eV}$ ).

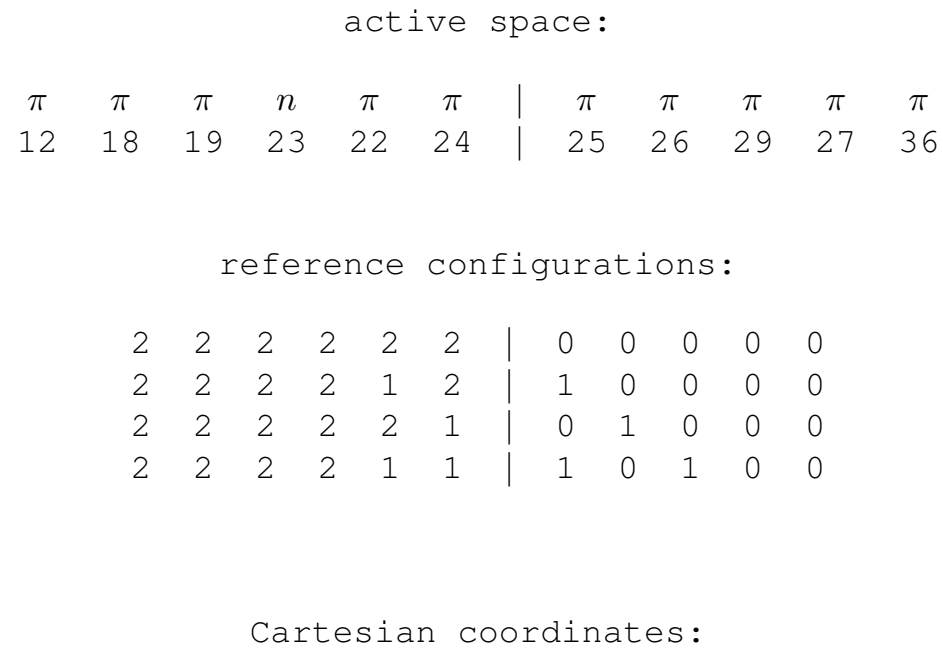


Table S1231 Employed active space, reference configurations, and Cartesian coordinates of the OM2/MRCISD-optimized excited-state equilibrium geometry of the $2{ }^{1} A\left({ }^{1} n \pi^{*}\right)$ state of quinoxaline (excited-state energy: $-1533.70350 \mathrm{eV}$ ).

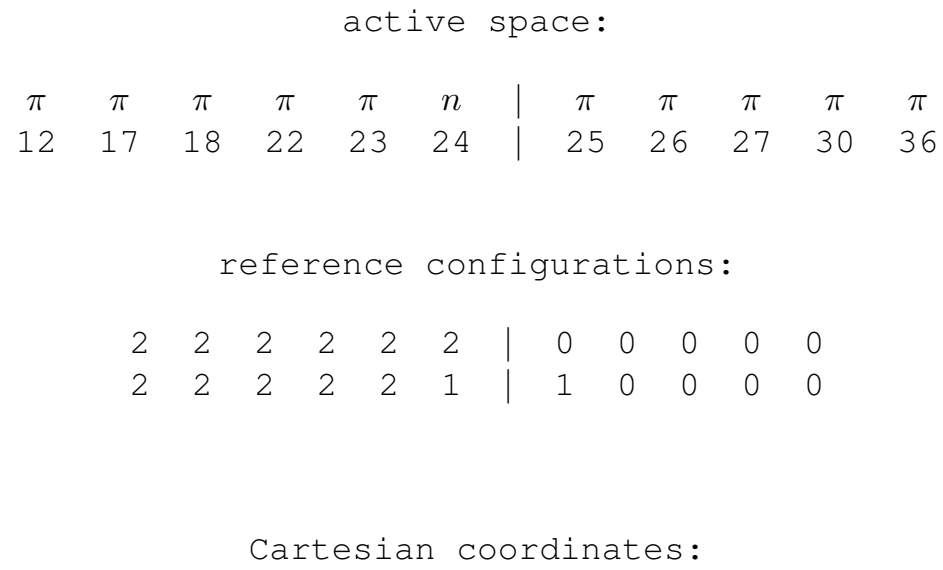


Table S1232 Employed active space, reference configurations, and Cartesian coordinates of the OM2/MRCISD-optimized excited-state equilibrium geometry of the $1^{3} A^{\prime}\left({ }^{3} \pi \pi^{*}\right)$ state of quinoxaline (excited-state energy: $-1534.28132 \mathrm{eV}$ ).

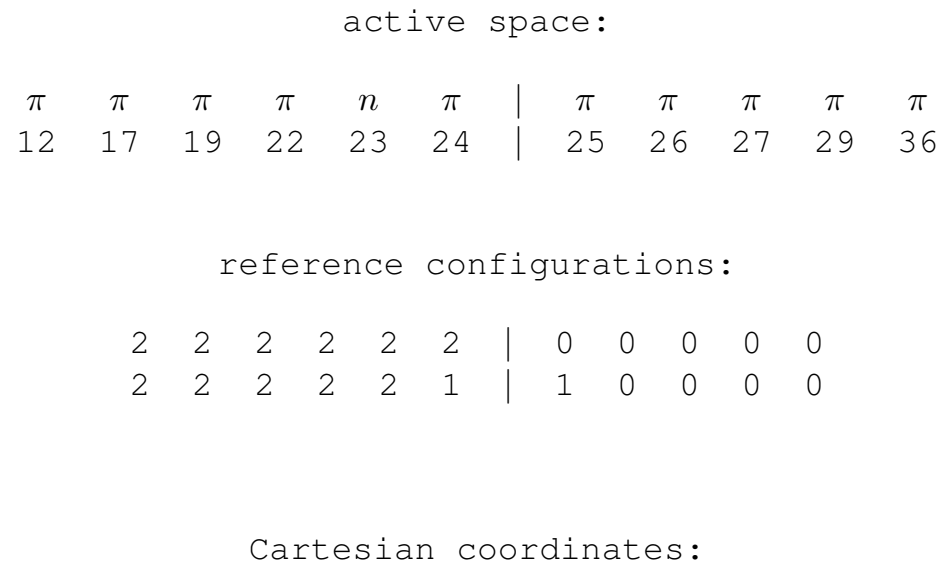


Table S1233 Employed active space, reference configurations, and Cartesian coordinates of the OM2/MRCISD-optimized ground-state equilibrium geometry of styrene (ground-state energy: $-1140.63898 \mathrm{eV}$ ).

\begin{tabular}{cccc|cccc}
\multicolumn{10}{c}{ active space: } \\
$\pi$ & $\pi$ & $\pi$ & $\pi$ & $\pi$ & $\pi$ & $\pi$ & $\pi$ \\
13 & 16 & 19 & 20 & 21 & 22 & 23 & 32
\end{tabular}

reference configurations:

\begin{tabular}{llll|llll}
2 & 2 & 2 & 2 & 0 & 0 & 0 & 0
\end{tabular}

Cartesian coordinates:

$\begin{array}{lrrr}16 & & & \\ & & & \\ \text { C } & 0.00000 & 0.00000 & 0.00000 \\ \text { C } & 1.40345 & 0.00000 & 0.00000 \\ \text { C } & 2.10017 & 1.20860 & 0.00000 \\ \text { C } & 1.40862 & 2.42142 & 0.00000 \\ \text { C } & 0.01154 & 2.42719 & 0.00000 \\ \text { C } & -0.69197 & 1.22439 & 0.00000 \\ \text { C } & -0.78486 & -1.24062 & -0.00000 \\ \text { C } & -0.24562 & -2.47291 & -0.00000 \\ \text { H } & 1.95731 & -0.94593 & -0.00000 \\ \text { H } & 3.19620 & 1.20587 & 0.00000 \\ \text { H } & 1.96053 & 3.36760 & 0.00000 \\ \text { H } & -0.53052 & 3.37949 & 0.00000 \\ \text { H } & -1.78916 & 1.23087 & 0.00000 \\ \text { H } & -1.87910 & -1.11405 & -0.00000 \\ \text { H } & 0.82486 & -2.64010 & -0.00000 \\ \text { H } & -0.86480 & -3.36151 & -0.00000\end{array}$


Table S1234 Employed active space, reference configurations, and Cartesian coordinates of the OM2/MRCISD-optimized excited-state equilibrium geometry of the $2{ }^{1} A\left({ }^{1} \pi \pi^{*}\right)$ state of styrene (excited-state energy: $-1136.48867 \mathrm{eV}$ ).

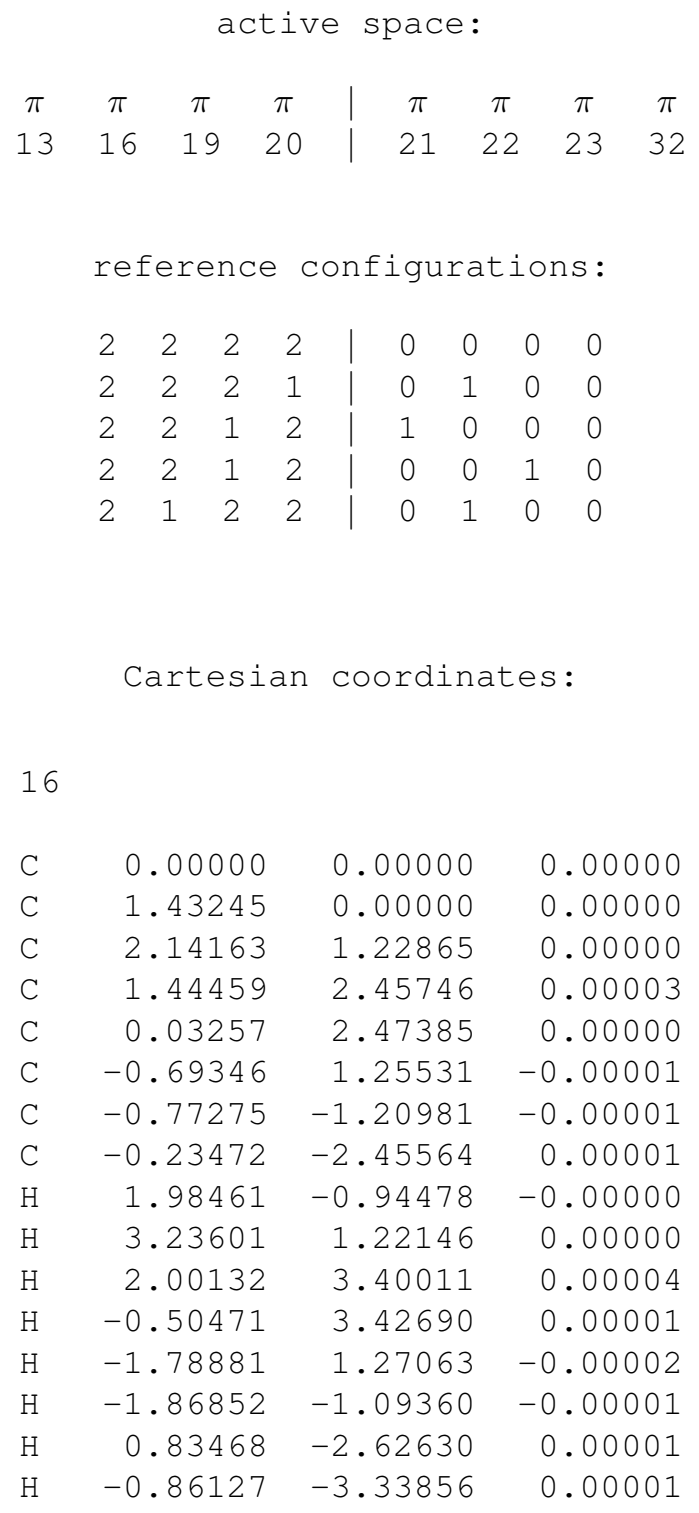


Table S1235 Employed active space, reference configurations, and Cartesian coordinates of the OM2/MRCISD-optimized ground-state equilibrium geometry of syn-coumarin 153 (ground-state energy: $-4525.95749 \mathrm{eV}$ ).

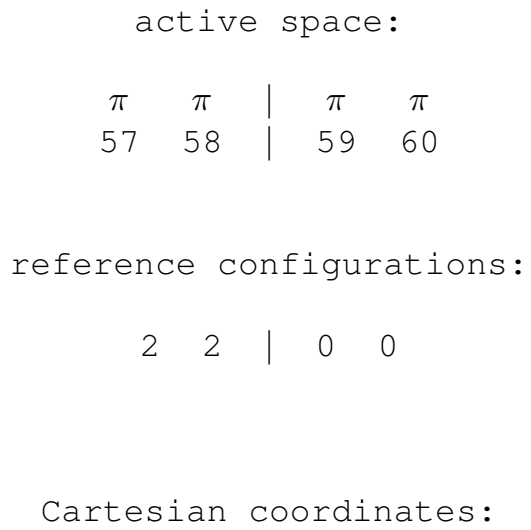




\begin{tabular}{lrrr}
\multicolumn{5}{c}{ Continued from previous page } \\
\hline $\mathrm{H}$ & 4.72024 & 2.65780 & 1.09956 \\
$\mathrm{H}$ & 5.43451 & 2.26833 & -0.51664 \\
$\mathrm{H}$ & 4.23114 & 4.49635 & -0.50268 \\
$\mathrm{H}$ & 3.50238 & 3.32128 & -1.66874 \\
$\mathrm{H}$ & 2.50369 & 3.88143 & 1.22479 \\
$\mathrm{H}$ & 1.74631 & 4.50294 & -0.31408 \\
$\mathrm{H}$ & -0.37712 & -0.86054 & -0.59356 \\
$\mathrm{H}$ & -0.35955 & -0.11656 & 1.05198 \\
$\mathrm{H}$ & -1.60468 & 1.37720 & -0.48055 \\
$\mathrm{H}$ & -0.24007 & 1.36283 & -1.66730 \\
$\mathrm{H}$ & -0.23451 & 3.44576 & -0.26428 \\
$\mathrm{H}$ & -0.11016 & 2.43593 & 1.24989
\end{tabular}


Table S1236 Employed active space, reference configurations, and Cartesian coordinates of the OM2/MRCISD-optimized excited-state equilibrium geometry of the $2{ }^{1} A\left({ }^{1} \pi \pi^{*}\right)$ state of syn-coumarin 153 (excited-state energy: $-4522.27538 \mathrm{eV})$.

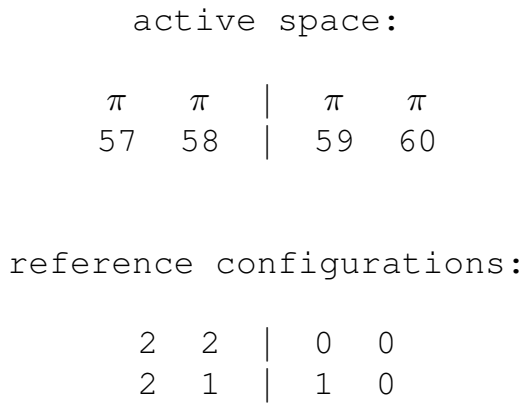




\begin{tabular}{lrrr}
\multicolumn{5}{c}{ Continued from previous page } \\
\hline $\mathrm{H}$ & 5.43866 & -0.03361 & -0.01423 \\
$\mathrm{H}$ & 4.69821 & 2.75743 & 1.02084 \\
$\mathrm{H}$ & 5.39042 & 2.34721 & -0.59771 \\
$\mathrm{H}$ & 4.13827 & 4.54750 & -0.62346 \\
$\mathrm{H}$ & 3.40650 & 3.32711 & -1.73678 \\
$\mathrm{H}$ & 2.47918 & 3.95243 & 1.16154 \\
$\mathrm{H}$ & 1.65574 & 4.49729 & -0.37008 \\
$\mathrm{H}$ & -0.37241 & -0.88526 & -0.55190 \\
$\mathrm{H}$ & -0.35943 & -0.07075 & 1.05681 \\
$\mathrm{H}$ & -1.60400 & 1.34735 & -0.57083 \\
$\mathrm{H}$ & -0.19961 & 1.32475 & -1.70673 \\
$\mathrm{H}$ & -0.25413 & 3.43328 & -0.33437 \\
$\mathrm{H}$ & -0.19436 & 2.43950 & 1.19262
\end{tabular}


Table S1237 Employed active space, reference configurations, and Cartesian coordinates of the OM2/MRCISD-optimized ground-state equilibrium geometry of terylene (ground-state energy: $-4067.44869 \mathrm{eV}$ ).

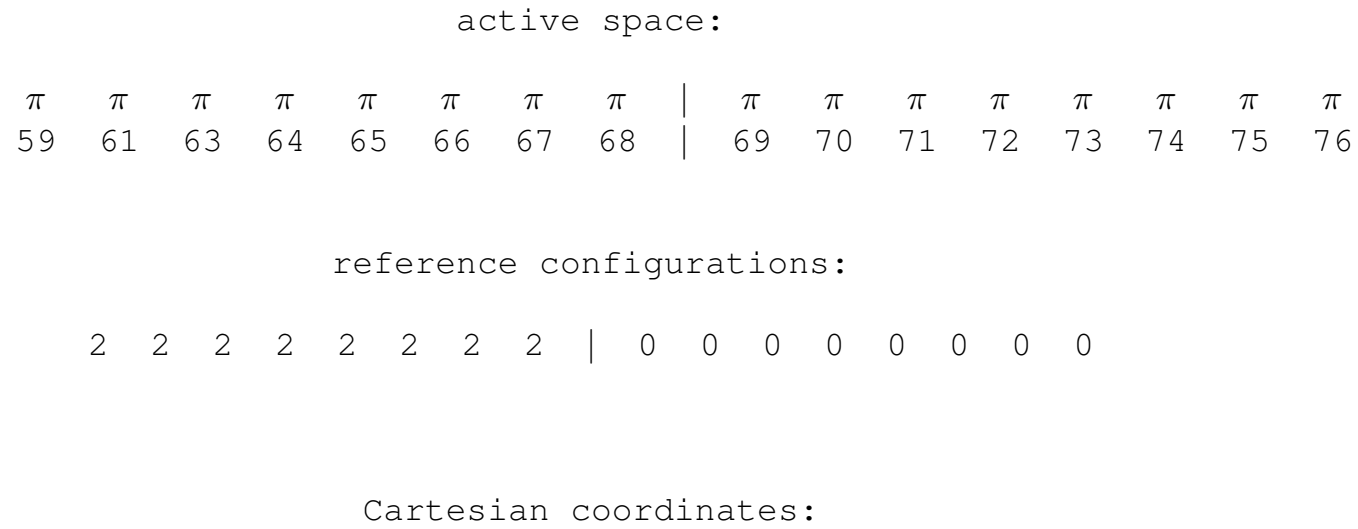

Continued on next page 


\begin{tabular}{lrrr}
\multicolumn{4}{c}{ Continued from previous page } \\
\hline C & 5.73029 & 2.41927 & -0.00000 \\
$\mathrm{C}$ & 5.73029 & -2.41927 & -0.00000 \\
$\mathrm{C}$ & -2.89846 & 2.42924 & 0.00000 \\
$\mathrm{C}$ & -2.89846 & -2.42924 & 0.00000 \\
$\mathrm{C}$ & 4.32494 & 2.42924 & -0.00000 \\
$\mathrm{C}$ & 4.32494 & -2.42924 & -0.00000 \\
$\mathrm{H}$ & -0.50108 & 3.39723 & -0.00000 \\
$\mathrm{H}$ & -0.50108 & -3.39723 & -0.00000 \\
$\mathrm{H}$ & 1.92756 & 3.39723 & 0.00000 \\
$\mathrm{H}$ & 1.92756 & -3.39723 & 0.00000 \\
$\mathrm{H}$ & -6.09276 & 1.21756 & 0.00000 \\
$\mathrm{H}$ & -6.09276 & -1.21756 & 0.00000 \\
$\mathrm{H}$ & 7.51924 & 1.21756 & -0.00000 \\
$\mathrm{H}$ & 7.51924 & -1.21756 & -0.00000 \\
$\mathrm{H}$ & -4.84271 & 3.37386 & 0.00000 \\
$\mathrm{H}$ & -4.84271 & -3.37386 & 0.00000 \\
$\mathrm{H}$ & 6.26919 & 3.37386 & -0.00000 \\
$\mathrm{H}$ & 6.26919 & -3.37386 & -0.00000 \\
$\mathrm{H}$ & -2.39189 & 3.40250 & 0.00000 \\
$\mathrm{H}$ & -2.39189 & -3.40250 & -0.00000 \\
$\mathrm{H}$ & 3.81837 & 3.40250 & -0.00000 \\
$\mathrm{H}$ & 3.81837 & -3.40250 & 0.00000
\end{tabular}


Table S1238 Employed active space, reference configurations, and Cartesian coordinates of the OM2/MRCISD-optimized excited-state equilibrium geometry of the $2{ }^{1} A^{\prime}\left({ }^{1} \pi \pi^{*}\right)$ state of terylene (excited-state energy: $\left.-4064.61406 \mathrm{eV}\right)$.

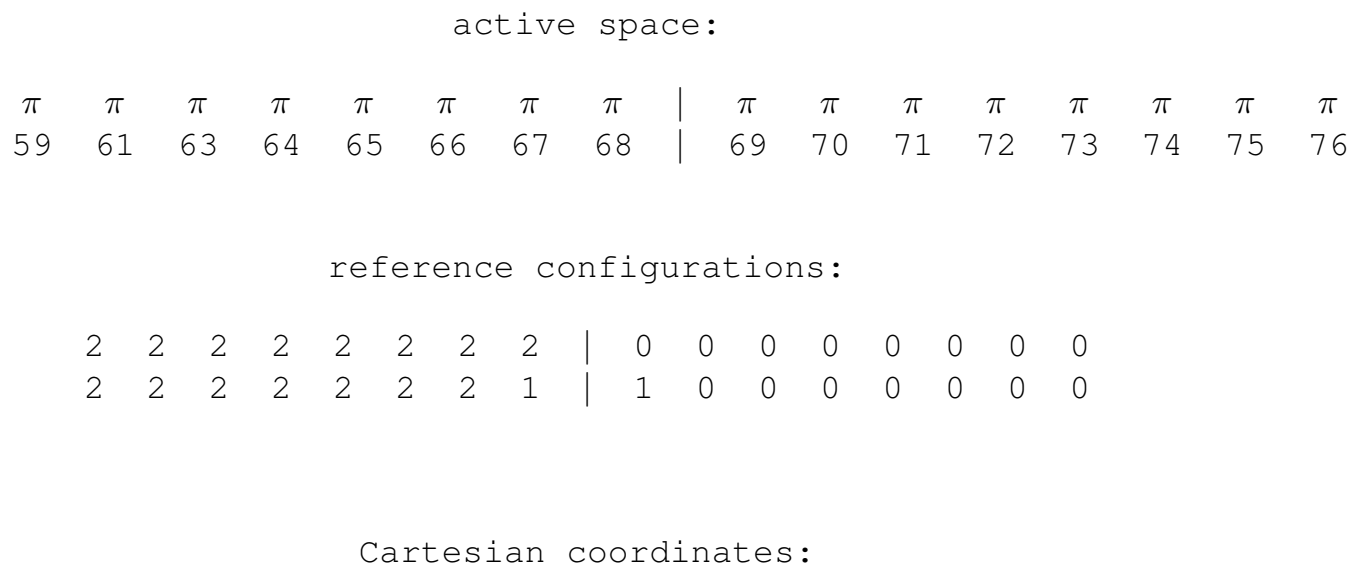




\begin{tabular}{lrrl}
\multicolumn{4}{c}{ Continued from previous page } \\
\hline $\mathrm{C}$ & 5.91242 & -7.28012 & 0.00000 \\
$\mathrm{C}$ & 4.52974 & -7.31514 & 0.00000 \\
$\mathrm{C}$ & 3.78471 & -6.13528 & 0.00000 \\
$\mathrm{C}$ & 2.02308 & 3.70537 & 0.00000 \\
$\mathrm{C}$ & 3.40576 & 3.74038 & 0.00000 \\
$\mathrm{C}$ & 4.15078 & 2.56052 & 0.00000 \\
$\mathrm{H}$ & 7.50530 & -1.03524 & 0.00000 \\
$\mathrm{H}$ & 1.70822 & -4.61132 & 0.00000 \\
$\mathrm{H}$ & 6.22727 & 1.03656 & 0.00000 \\
$\mathrm{H}$ & 0.43019 & -2.53952 & 0.00000 \\
$\mathrm{H}$ & 9.75159 & -4.73592 & 0.00000 \\
$\mathrm{H}$ & 4.00752 & -8.27932 & 0.00000 \\
$\mathrm{H}$ & 3.92799 & 4.70456 & 0.00000 \\
$\mathrm{H}$ & -1.81609 & 1.16117 & 0.00000 \\
$\mathrm{H}$ & 8.49302 & -2.63007 & 0.00000 \\
$\mathrm{H}$ & 2.69029 & -6.20964 & 0.00000 \\
$\mathrm{H}$ & 5.24520 & 2.63488 & 0.00000 \\
$\mathrm{H}$ & -0.55753 & -0.94468 & 0.00000 \\
$\mathrm{H}$ & 8.57242 & -6.92715 & 0.00000 \\
$\mathrm{H}$ & 6.49487 & -8.20874 & 0.00000 \\
$\mathrm{H}$ & 1.44063 & 4.63399 & 0.00000 \\
$\mathrm{H}$ & -0.63692 & 3.35240 & 0.00000
\end{tabular}

S1313 
Table S1239 Employed active space, reference configurations, and Cartesian coordinates of the OM2/MRCISD-optimized ground-state equilibrium geometry of $s$-tetrazine (ground-state energy: $-1136.91045 \mathrm{eV}$ ).

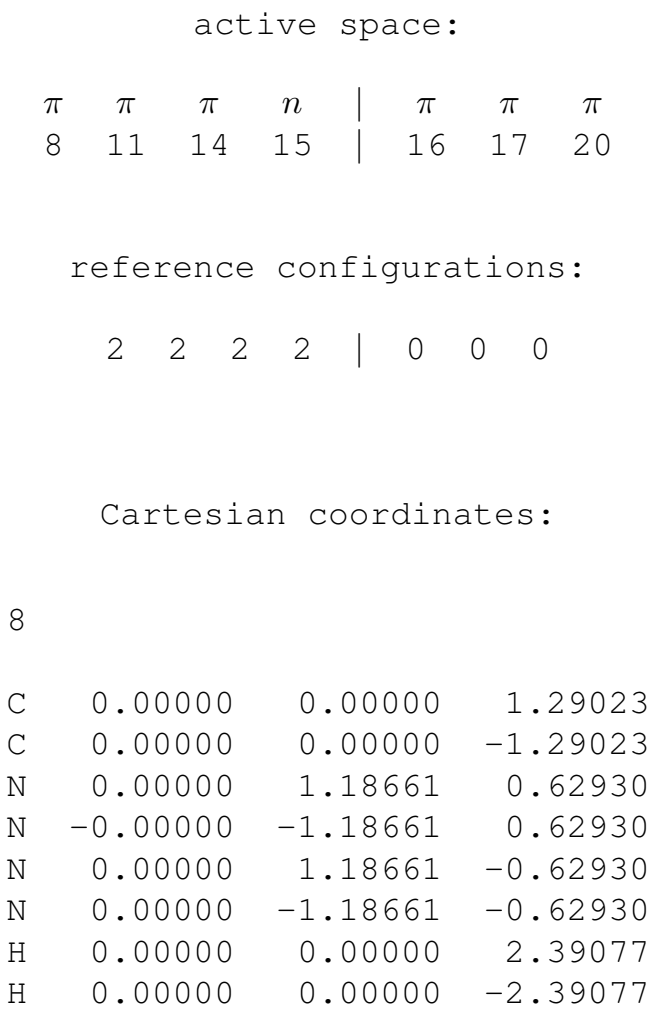


Table S1240 Employed active space, reference configurations, and Cartesian coordinates of the OM2/MRCISD-optimized excited-state equilibrium geometry of the $1^{1} B_{3 u}\left({ }^{1} n \pi^{*}\right)$ state of $s$-tetrazine (excited-state energy: $-1134.07136 \mathrm{eV})$.

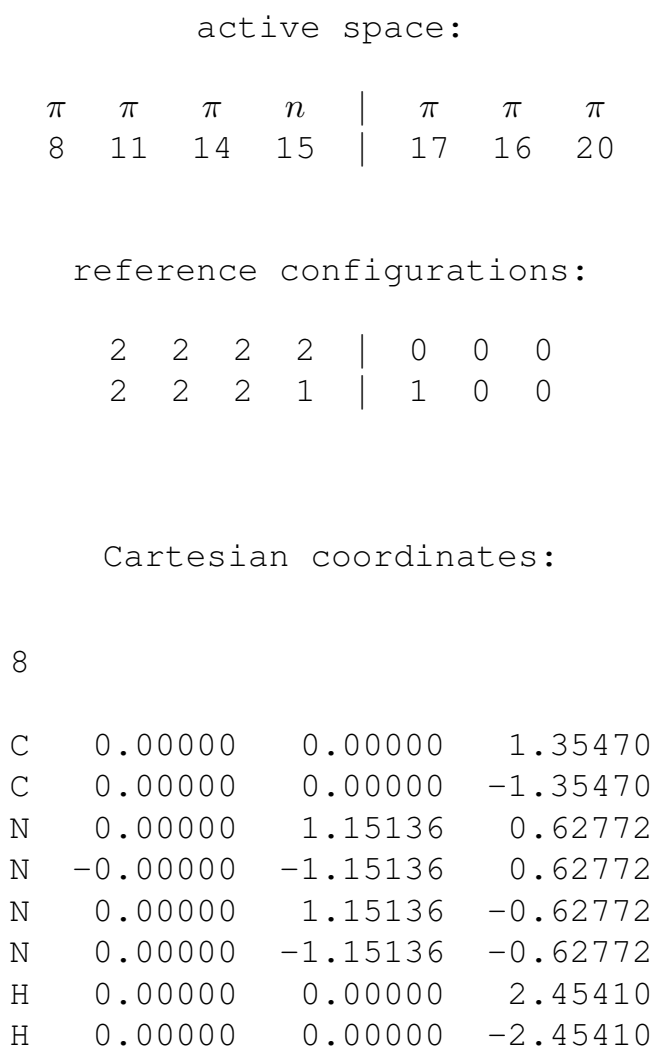


Table S1241 Employed active space, reference configurations, and Cartesian coordinates of the OM2/MRCISD-optimized ground-state equilibrium geometry of toluene (ground-state energy: $-1013.76275 \mathrm{eV}$ ).

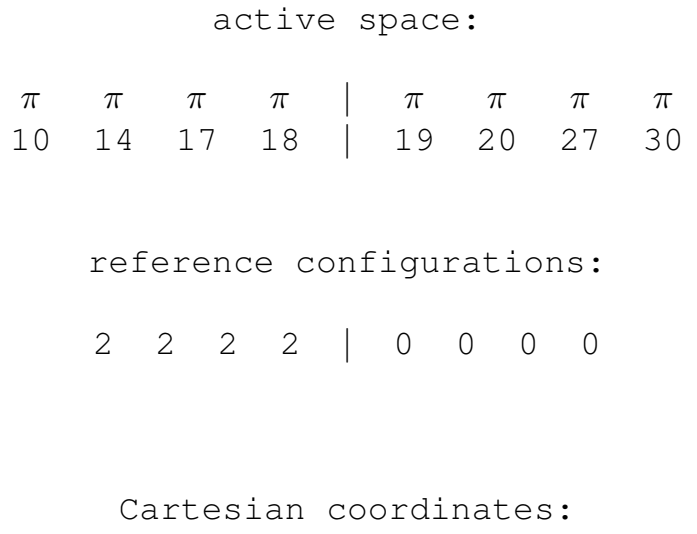

15

$\begin{array}{lrrr}\text { C } & 0.00000 & 0.00000 & 0.00000 \\ \text { C } & 1.49412 & 0.00000 & 0.00000 \\ \text { C } & -0.71261 & 1.20743 & 0.00000 \\ \text { C } & -0.69606 & -1.21853 & -0.00000 \\ \text { C } & -2.10807 & 1.19407 & -0.00000 \\ \mathrm{C} & -2.09080 & -1.22447 & -0.00000 \\ \mathrm{C} & -2.79821 & -0.01981 & -0.00000 \\ \mathrm{H} & 1.88661 & 1.02885 & 0.00000 \\ \mathrm{H} & 1.86782 & -0.52538 & 0.89563 \\ \mathrm{H} & 1.86782 & -0.52538 & -0.89563 \\ \mathrm{H} & -0.17159 & 2.16137 & 0.00000 \\ \mathrm{H} & -0.14182 & -2.16385 & -0.00000 \\ \mathrm{H} & -2.66421 & 2.13862 & -0.00000 \\ \mathrm{H} & -2.63407 & -2.17654 & -0.00000 \\ \mathrm{H} & -3.89357 & -0.02773 & -0.00000\end{array}$


Table S1242 Employed active space, reference configurations, and Cartesian coordinates of the OM2/MRCISD-optimized excited-state equilibrium geometry of the $2{ }^{1} A^{\prime}\left({ }^{1} \pi \pi^{*}\right)$ state of toluene (excited-state energy: $-1009.47783 \mathrm{eV}$ ).

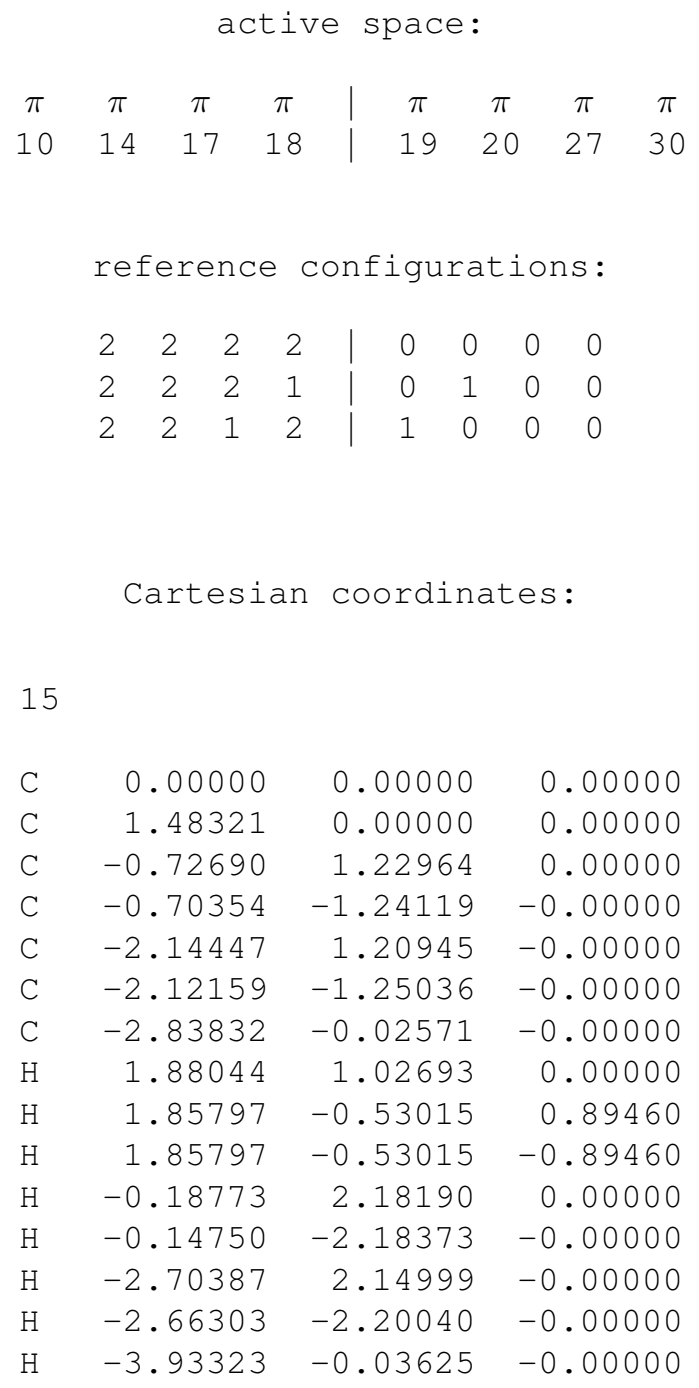


Table S1243 Employed active space, reference configurations, and Cartesian coordinates of the OM2/MRCISD-optimized ground-state equilibrium geometry of trans-stilbene (ground-state energy: $-1966.42529 \mathrm{eV}$ ).

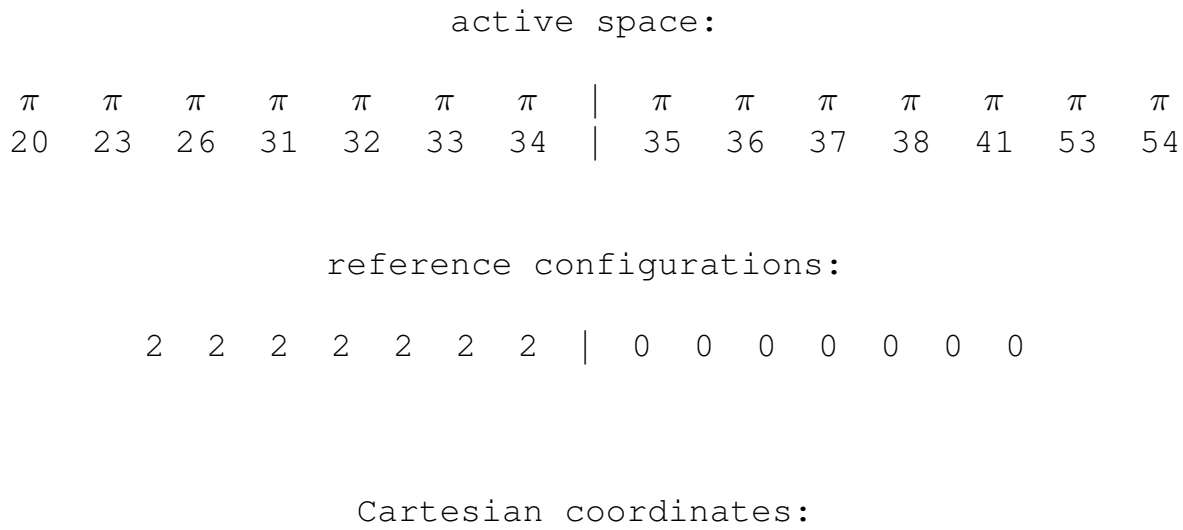


Continued from previous page

\begin{tabular}{rrrr}
\hline $\mathrm{H}$ & 0.00000 & 3.33959 & 0.07766 \\
$\mathrm{H}$ & 0.00000 & -3.33959 & -0.07766
\end{tabular}


Table S1244 Employed active space, reference configurations, and Cartesian coordinates of the OM2/MRCISD-optimized excited-state equilibrium geometry of the $2{ }^{1} A\left({ }^{1} \pi \pi^{*}\right)$ state of trans-stilbene (excited-state energy: $-1962.05577 \mathrm{eV})$.

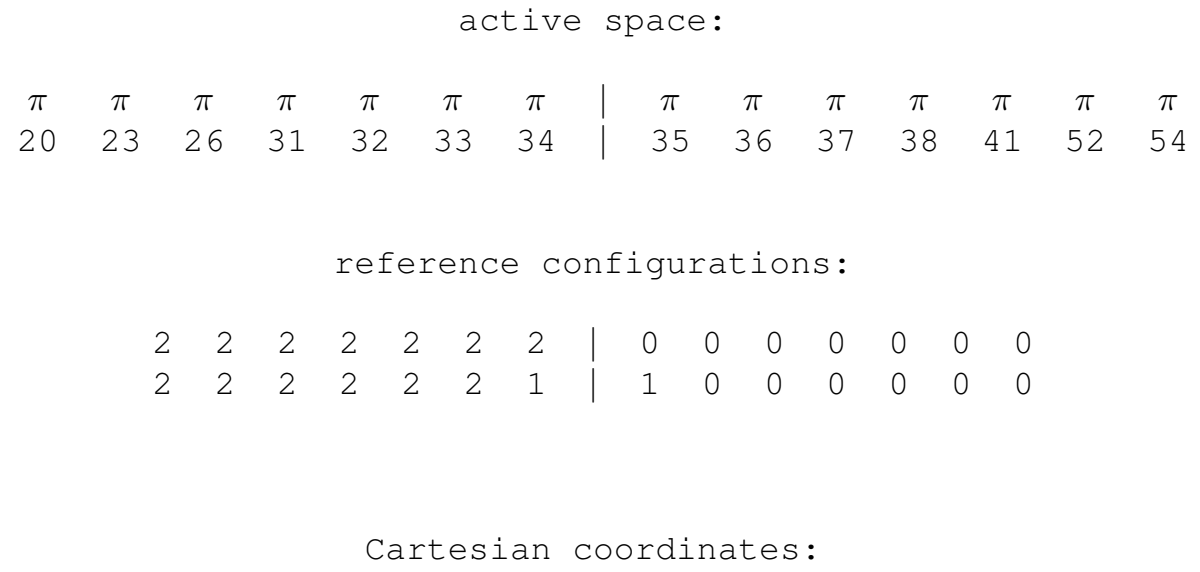

\begin{tabular}{lrrr}
26 & & & \\
& & & \\
$\mathrm{C}$ & 0.00000 & 0.00000 & 0.00000 \\
$\mathrm{C}$ & 1.43209 & 0.00000 & 0.00000 \\
$\mathrm{C}$ & 2.11020 & 1.25859 & 0.00000 \\
$\mathrm{C}$ & 1.38337 & 2.43879 & -0.00002 \\
$\mathrm{C}$ & -0.01650 & 2.41846 & 0.00002 \\
$\mathrm{C}$ & -0.70082 & 1.19023 & 0.00000 \\
$\mathrm{C}$ & 2.11287 & -1.23692 & 0.00003 \\
$\mathrm{C}$ & 3.51297 & -1.34431 & 0.00005 \\
$\mathrm{C}$ & 4.19376 & -2.58122 & -0.00006 \\
$\mathrm{C}$ & 3.51567 & -3.83982 & -0.00029 \\
$\mathrm{C}$ & 4.24253 & -5.02002 & -0.00040 \\
$\mathrm{C}$ & 5.64241 & -4.99965 & -0.00015 \\
$\mathrm{C}$ & 6.32673 & -3.77142 & -0.00002 \\
$\mathrm{C}$ & 5.62586 & -2.58119 & -0.00000 \\
$\mathrm{H}$ & 4.12416 & -0.42963 & 0.00016 \\
$\mathrm{H}$ & 1.50163 & -2.15160 & -0.00002 \\
$\mathrm{H}$ & 2.42028 & -3.87126 & -0.00043 \\
$\mathrm{H}$ & 3.20559 & 1.29002 & -0.00002 \\
$\mathrm{H}$ & 3.71878 & -5.98365 & -0.00058 \\
$\mathrm{H}$ & 1.90714 & 3.40241 & -0.00004 \\
$\mathrm{H}$ & 6.20584 & -5.93853 & -0.00021 \\
$\mathrm{H}$ & -0.57992 & 3.35734 & 0.00004 \\
$\mathrm{H}$ & 7.42284 & -3.76729 & 0.00006 \\
\hline & & Continued & on next page
\end{tabular}

S1320 


\begin{tabular}{lrrr}
\multicolumn{5}{c}{ Continued from previous page } \\
\hline $\mathrm{H}$ & -1.79693 & 1.18612 & 0.00001 \\
$\mathrm{H}$ & 6.15702 & -1.62101 & 0.00010 \\
$\mathrm{H}$ & -0.53117 & -0.96017 & -0.00004
\end{tabular}


Table S1245 Employed active space, reference configurations, and Cartesian coordinates of the OM2/MRCISD-optimized ground-state equilibrium geometry of vinyl radical (ground-state energy: $-297.56521 \mathrm{eV}$ ).

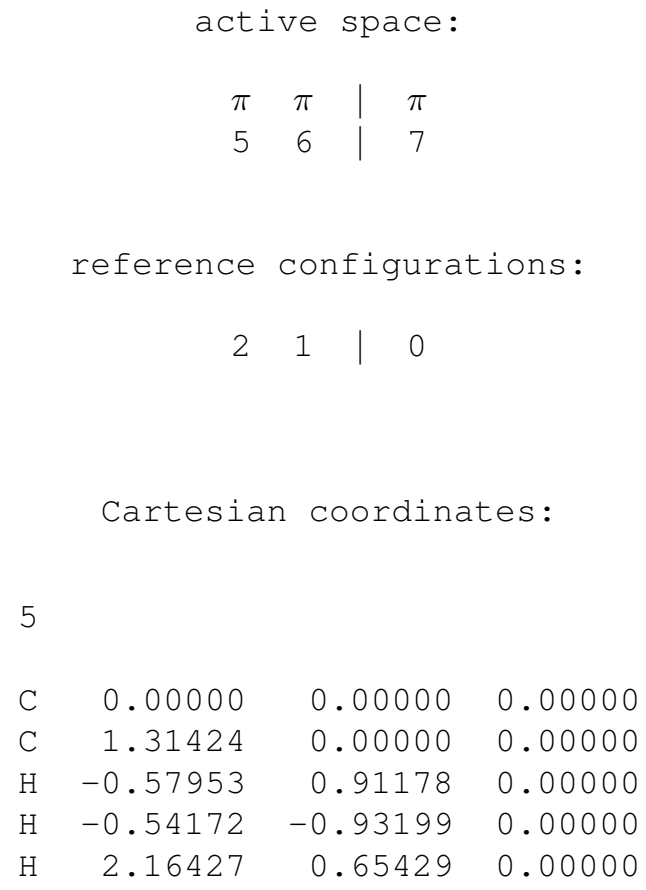


Table S1246 Employed active space, reference configurations, and Cartesian coordinates of the OM2/MRCISD-optimized excited-state equilibrium geometry of the $2^{2} A\left({ }^{2} \pi \sigma^{*}\right)$ state of vinyl radical (excited-state energy: $-295.18717 \mathrm{eV}$ ).

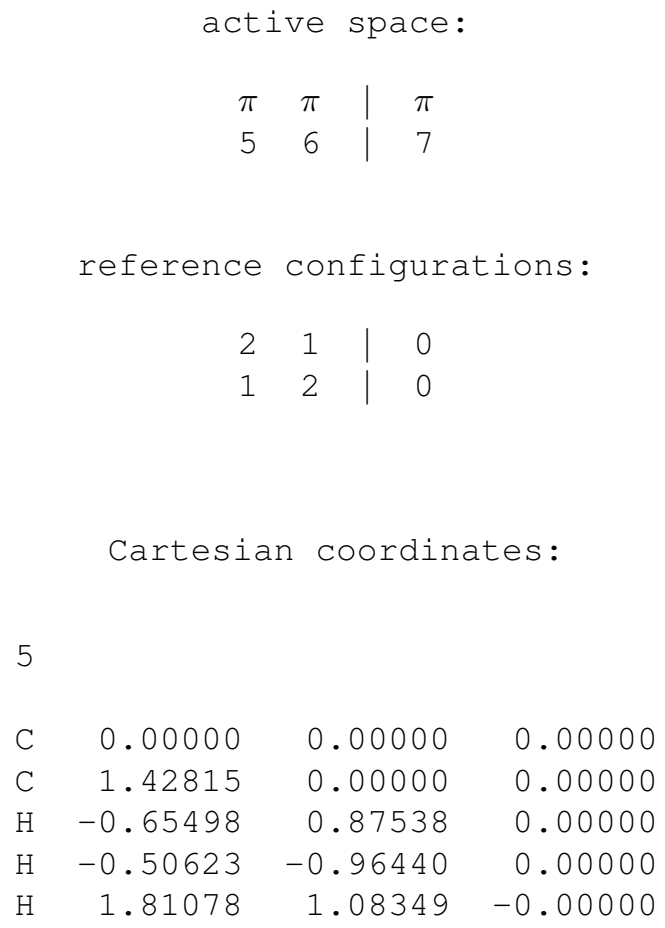

$\mathrm{H} \quad 1.81078 \quad 1.08349 \quad-0.00000$ 
4.3.2 OM3/MRCI-Optimized Ground- and Excited-State Minima 
Table S1247 Employed active space, reference configurations, and Cartesian coordinates of the OM3/MRCISD-optimized ground-state equilibrium geometry of 1,6-epoxy-10-annulene (ground-state energy: -1705.96631 eV).

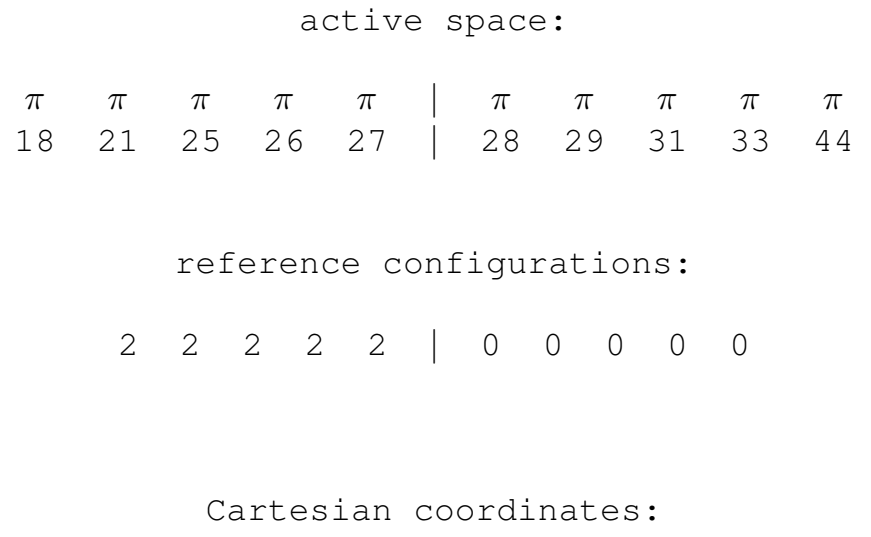

19

$\begin{array}{lrrr}\mathrm{O} & 1.00000 & 0.00000 & 0.90084 \\ \mathrm{C} & 1.00000 & 1.06635 & 0.00037 \\ \mathrm{C} & 1.00000 & -1.06635 & 0.00037 \\ \mathrm{C} & 2.28262 & 1.52368 & -0.38756 \\ \mathrm{C} & -0.28262 & 1.52368 & -0.38756 \\ \mathrm{C} & 2.28262 & -1.52368 & -0.38756 \\ \mathrm{C} & -0.28262 & -1.52368 & -0.38756 \\ \mathrm{C} & 3.41335 & 0.70769 & -0.32784 \\ \mathrm{C} & -1.41335 & 0.70769 & -0.32784 \\ \mathrm{C} & 3.41335 & -0.70769 & -0.32784 \\ \mathrm{C} & -1.41335 & -0.70769 & -0.32784 \\ \mathrm{H} & 2.38530 & 2.56064 & -0.72062 \\ \mathrm{H} & -0.38530 & 2.56064 & -0.72062 \\ \mathrm{H} & 2.38530 & -2.56064 & -0.72062 \\ \mathrm{H} & -0.38530 & -2.56064 & -0.72062 \\ \mathrm{H} & 4.39548 & 1.19602 & -0.46349 \\ \mathrm{H} & -2.39548 & 1.19602 & -0.46349 \\ \mathrm{H} & 4.39548 & -1.19602 & -0.46349 \\ \mathrm{H} & -2.39548 & -1.19602 & -0.46349\end{array}$


Table S1248 Employed active space, reference configurations, and Cartesian coordinates of the OM3/MRCISD-optimized excited-state equilibrium geometry of the $1^{1} B_{1}\left({ }^{1} \pi \pi^{*}\right)$ state of 1,6-epoxy-10-annulene (excited-state energy: $-1702.72399 \mathrm{eV})$.

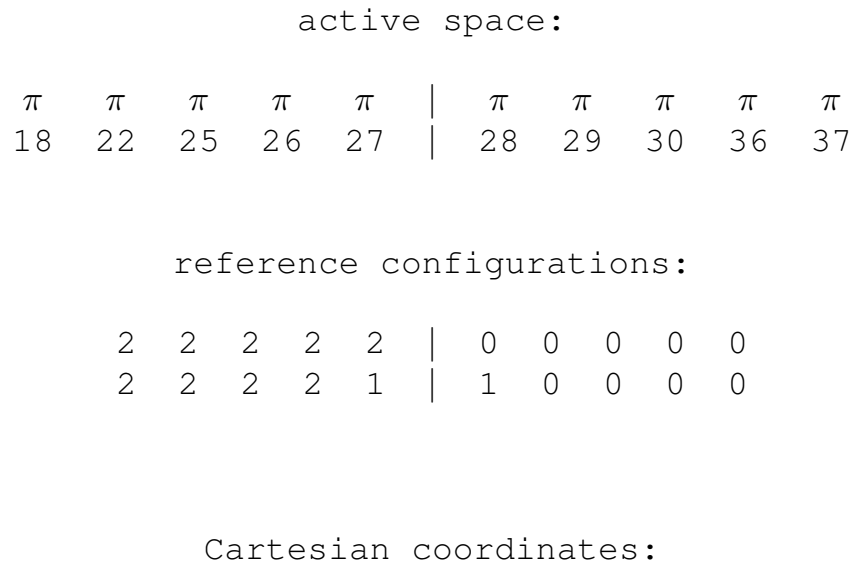


Table S1249 Employed active space, reference configurations, and Cartesian coordinates of the OM3/MRCISD-optimized ground-state equilibrium geometry of 2-cyclopenten-1-one (ground-state energy: -1053.50373 eV).

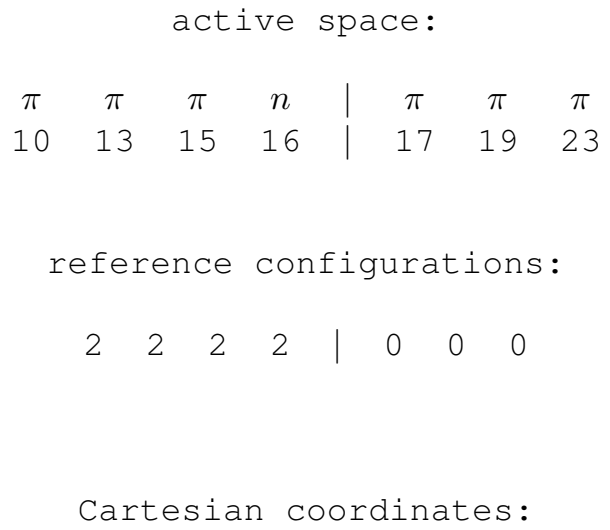


Table S1250 Employed active space, reference configurations, and Cartesian coordinates of the OM3/MRCISD-optimized excited-state equilibrium geometry of the $2{ }^{1} A\left({ }^{1} n \pi^{*}\right)$ state of 2-cyclopenten-1-one (excited-state energy: $-1049.98694 \mathrm{eV})$.

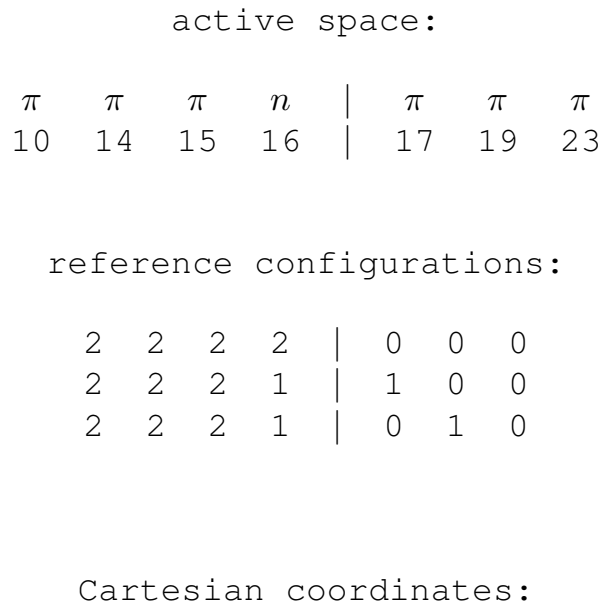


Table S1251 Employed active space, reference configurations, and Cartesian coordinates of the OM3/MRCISD-optimized ground-state equilibrium geometry of 7-azaindole (ground-state energy: $-1396.17799 \mathrm{eV}$ ).

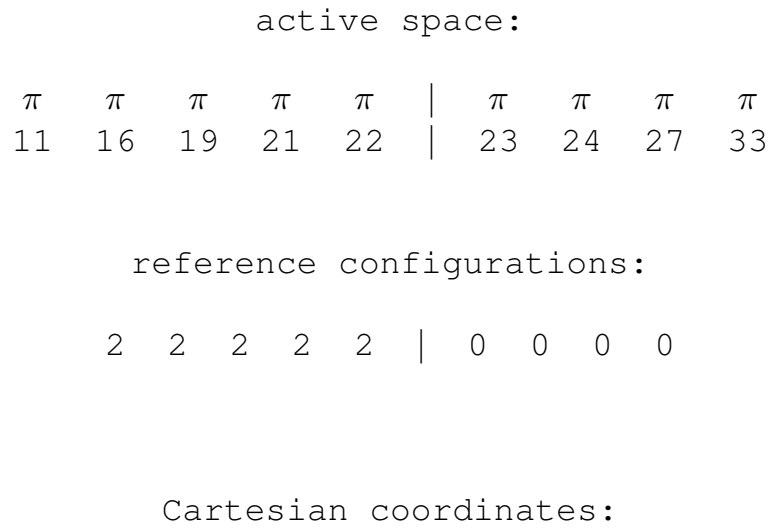


Table S1252 Employed active space, reference configurations, and Cartesian coordinates of the OM3/MRCISD-optimized excited-state equilibrium geometry of the $2{ }^{1} A\left({ }^{1} \pi \pi^{*}\right)$ state of 7 -azaindole (excited-state energy: $-1392.17798 \mathrm{eV}$ ).

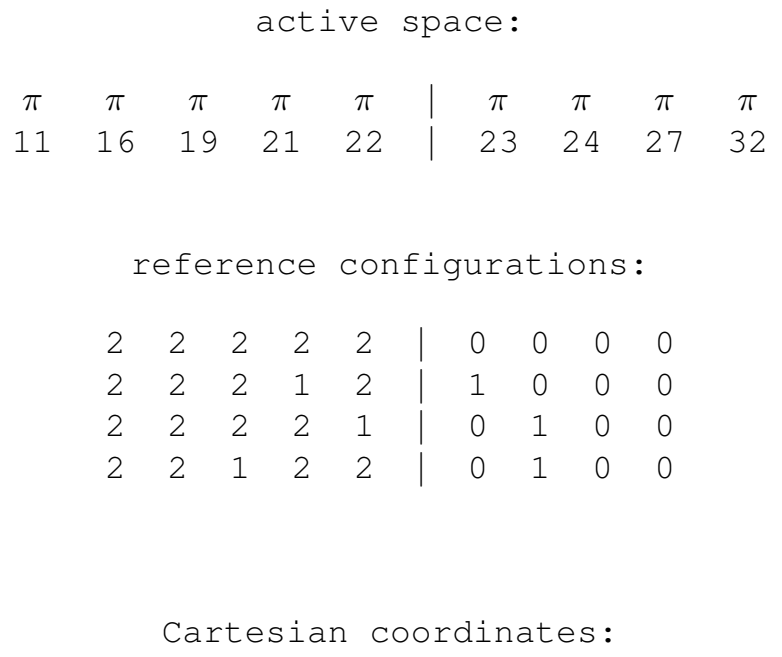


Table S1253 Employed active space, reference configurations, and Cartesian coordinates of the OM3/MRCISD-optimized ground-state equilibrium geometry of $\beta$-dinaphthyleneoxide (ground-state energy: $-3017.86254 \mathrm{eV}$ ).

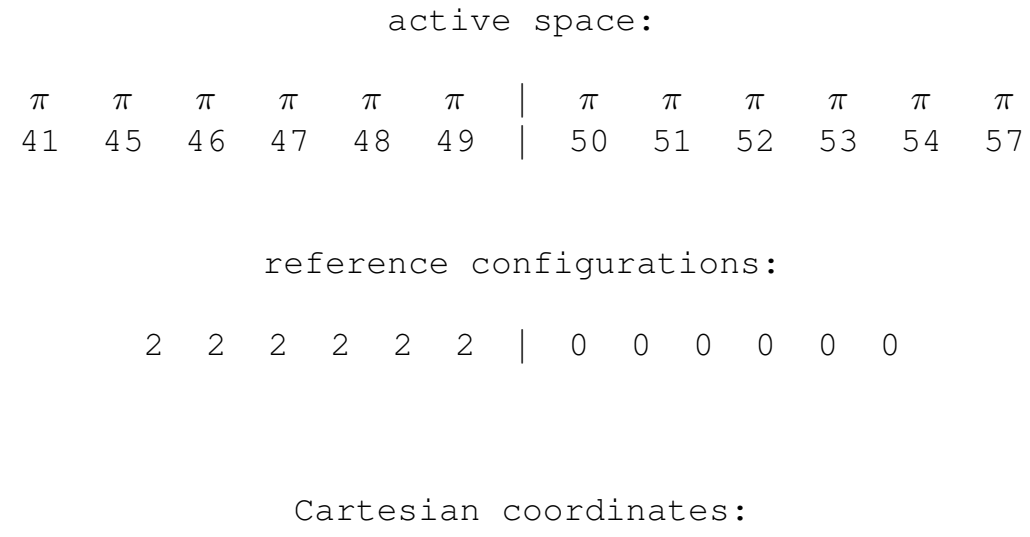

\begin{tabular}{lrrr}
33 & & & \\
C & 0.00000 & 0.00000 & 0.00000 \\
C & 1.38307 & 0.00000 & 0.00000 \\
C & 2.13346 & 1.20866 & 0.00000 \\
C & 1.39698 & 2.44124 & 0.04942 \\
C & -0.02980 & 2.41222 & 0.02546 \\
C & -0.71784 & 1.21468 & -0.01266 \\
C & 3.57053 & 1.28559 & -0.00194 \\
C & 4.13055 & 2.58156 & 0.15256 \\
C & 3.42152 & 3.80040 & 0.21404 \\
C & 2.05091 & 3.71321 & 0.14439 \\
O & 5.51976 & 2.56690 & 0.25674 \\
C & 5.85545 & 1.22023 & 0.13525 \\
C & 4.72766 & 0.38222 & -0.07177 \\
C & 5.00463 & -1.00761 & -0.32222 \\
C & 6.37073 & -1.44414 & -0.23675 \\
C & 7.44305 & -0.53353 & 0.03801 \\
C & 7.20369 & 0.80957 & 0.21068 \\
C & 6.68680 & -2.81943 & -0.45071 \\
C & 5.70318 & -3.73545 & -0.77064 \\
C & 4.36879 & -3.30030 & -0.91537 \\
C & 4.03174 & -1.97777 & -0.69133 \\
H & 7.99112 & 1.54678 & 0.39737 \\
H & 8.47344 & -0.92635 & 0.06273 \\
H & 2.98871 & -1.66178 & -0.85177 \\
\hline & & Continued & on next page
\end{tabular}




\begin{tabular}{lrrr}
\multicolumn{5}{c}{ Continued from previous page } \\
\hline H & 7.74139 & -3.13489 & -0.37077 \\
H & 5.95043 & -4.79810 & -0.92338 \\
H & 3.59050 & -4.02890 & -1.19780 \\
H & 3.96480 & 4.74568 & 0.31237 \\
H & 1.42819 & 4.62146 & 0.20699 \\
H & 1.91342 & -0.96420 & 0.05061 \\
H & -0.57551 & 3.37109 & 0.05453 \\
H & -1.81897 & 1.19773 & -0.04283 \\
H & -0.55279 & -0.95434 & -0.00267
\end{tabular}


Table S1254 Employed active space, reference configurations, and Cartesian coordinates of the OM3/MRCISD-optimized excited-state equilibrium geometry of the $2{ }^{1} A\left({ }^{1} \pi \pi^{*}\right)$ state of $\beta$-dinaphthyleneoxide (excited-state energy: $-3013.90339 \mathrm{eV})$.

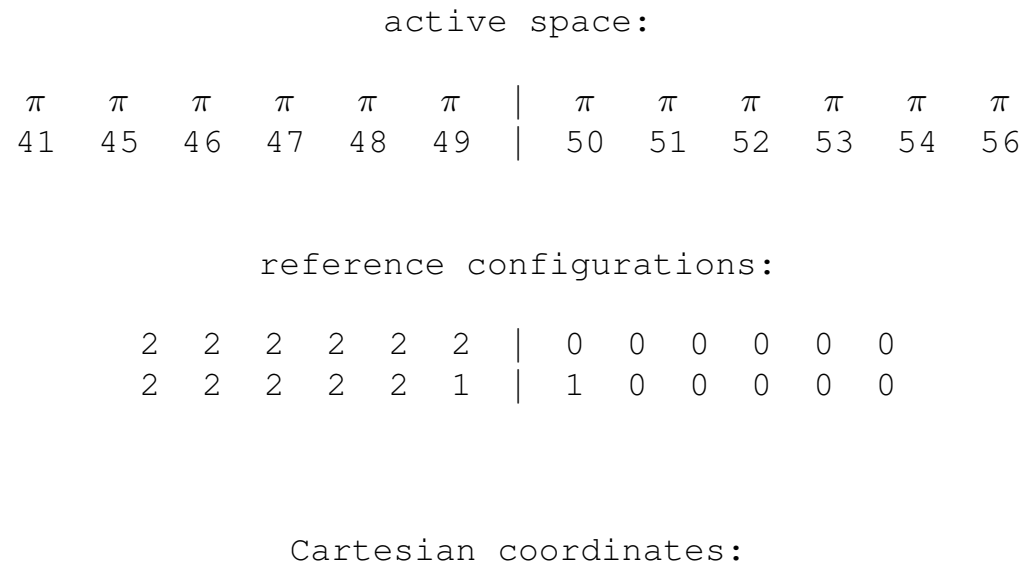

\begin{tabular}{lrrr}
33 & & & \\
C & 0.00000 & 0.00000 & 0.00000 \\
C & 1.39909 & 0.00000 & 0.00000 \\
C & 2.12641 & 1.20289 & 0.00000 \\
C & 1.40656 & 2.45041 & 0.09638 \\
C & -0.01822 & 2.41737 & 0.07239 \\
C & -0.70617 & 1.21077 & 0.00212 \\
C & 3.57532 & 1.27605 & -0.03236 \\
C & 4.16750 & 2.58018 & 0.23925 \\
C & 3.46761 & 3.77480 & 0.35663 \\
C & 2.07698 & 3.69377 & 0.26339 \\
O & 5.56112 & 2.52105 & 0.32922 \\
C & 5.85765 & 1.18246 & 0.05721 \\
C & 4.66552 & 0.40564 & -0.25995 \\
C & 4.88029 & -0.96050 & -0.69955 \\
C & 6.22134 & -1.48355 & -0.59097 \\
C & 7.31324 & -0.67161 & -0.17645 \\
C & 7.15055 & 0.67943 & 0.13549 \\
C & 6.45439 & -2.84038 & -0.96027 \\
C & 5.42639 & -3.63497 & -1.45619 \\
C & 4.14784 & -3.09174 & -1.64290 \\
C & 3.88207 & -1.77002 & -1.26892 \\
H & 7.98530 & 1.31840 & 0.43300 \\
H & 8.32274 & -1.11592 & -0.15573 \\
\hline & & $C 094144 d$ &
\end{tabular}

Continued on next page

S1333 


\begin{tabular}{lrrr}
\multicolumn{5}{c}{ Continued from previous page } \\
\hline $\mathrm{H}$ & 2.89601 & -1.34370 & -1.49813 \\
$\mathrm{H}$ & 7.47796 & -3.24415 & -0.87071 \\
$\mathrm{H}$ & 5.61810 & -4.68750 & -1.71930 \\
$\mathrm{H}$ & 3.33969 & -3.71588 & -2.05737 \\
$\mathrm{H}$ & 3.99220 & 4.72117 & 0.50766 \\
$\mathrm{H}$ & 1.47102 & 4.60871 & 0.37583 \\
$\mathrm{H}$ & 1.92788 & -0.95960 & 0.07835 \\
$\mathrm{H}$ & -0.57016 & 3.37119 & 0.13842 \\
$\mathrm{H}$ & -1.80724 & 1.20277 & -0.03486 \\
$\mathrm{H}$ & -0.54690 & -0.95619 & -0.03263
\end{tabular}


Table S1255 Employed active space, reference configurations, and Cartesian coordinates of the OM3/MRCISD-optimized ground-state equilibrium geometry of acetaldehyde (ground-state energy: $-648.69790 \mathrm{eV}$ ).

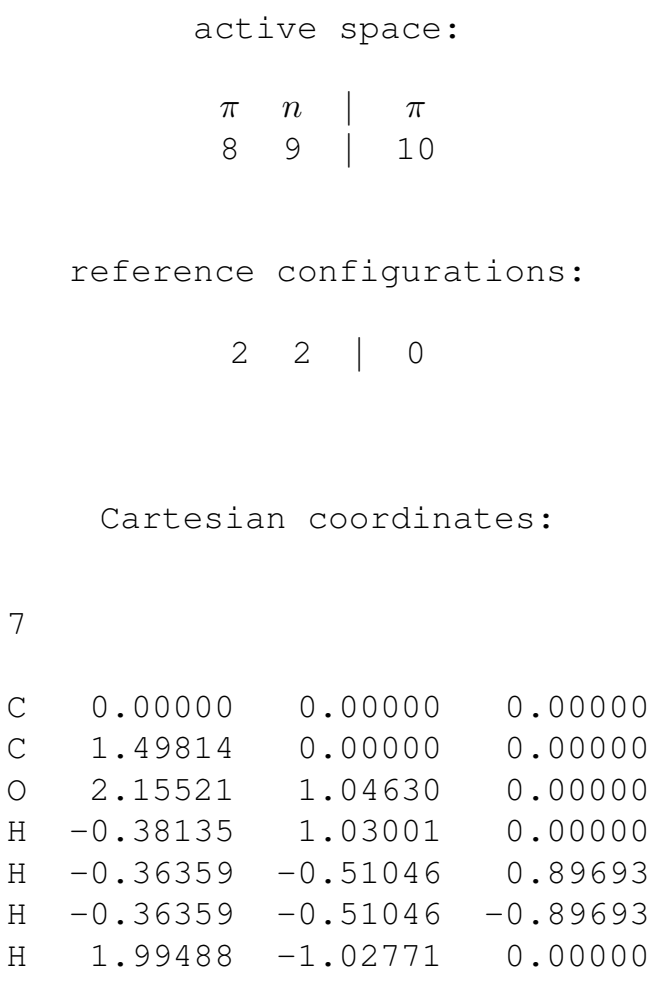


Table S1256 Employed active space, reference configurations, and Cartesian coordinates of the OM3/MRCISD-optimized excited-state equilibrium geometry of the $2{ }^{1} A\left({ }^{1} n \pi^{*}\right)$ state of acetaldehyde (excited-state energy: $\left.-644.99578 \mathrm{eV}\right)$.

\begin{tabular}{|c|c|c|c|}
\hline \multirow[b]{2}{*}{ reference } & $\begin{array}{l}n \\
9\end{array}$ & \begin{tabular}{l|c}
2 & $\pi$ \\
$y$ & 10
\end{tabular} & $\pi$ \\
\hline & \multicolumn{3}{|c|}{ configurations: } \\
\hline & & 0 & \\
\hline & & \begin{tabular}{l|l}
1 & 1
\end{tabular} & \\
\hline \multicolumn{4}{|c|}{ Cartesian coordinates: } \\
\hline & & & \\
\hline 0.00000 & & 0.00000 & 0.00000 \\
\hline 1.48559 & & 0.00000 & 0.00000 \\
\hline 2.05386 & & 1.17347 & 0.00000 \\
\hline-0.35736 & & 0.51709 & 0.89739 \\
\hline-0.38293 & & 1.03436 & -0.00035 \\
\hline-0.35738 & & 0.51768 & -0.89705 \\
\hline 2.08983 & & 0.90856 & 0.00001 \\
\hline
\end{tabular}


Table S1257 Employed active space, reference configurations, and Cartesian coordinates of the OM3/MRCISD-optimized ground-state equilibrium geometry of acetone (ground-state energy: $-803.97246 \mathrm{eV}$ ).

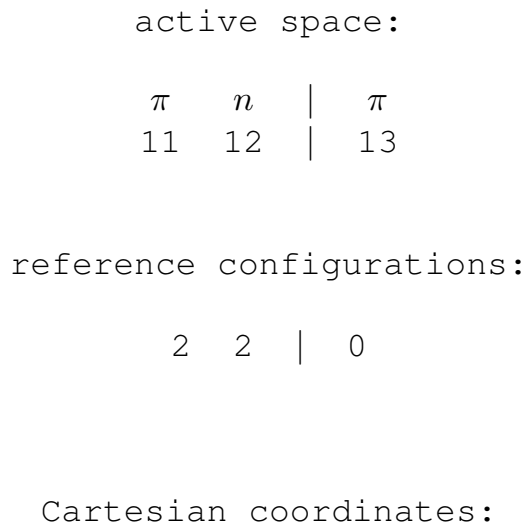

H $\quad 2.13131-1.78061-1.00167$ 
Table S1258 Employed active space, reference configurations, and Cartesian coordinates of the OM3/MRCISD-optimized excited-state equilibrium geometry of the $2{ }^{1} A\left({ }^{1} n \pi^{*}\right)$ state of acetone (excited-state energy: $-800.12765 \mathrm{eV}$ ).

\begin{tabular}{|c|c|c|}
\hline \multirow[b]{2}{*}{ reference } & \begin{tabular}{c|c}
$n$ & $\pi$ \\
12 &
\end{tabular} & | $\pi$ \\
\hline & \multicolumn{2}{|c|}{ configurations: } \\
\hline 2 & 0 & 0 \\
\hline 2 & 11 & 1 \\
\hline \multicolumn{3}{|c|}{ Cartesian coordinates: } \\
\hline 0.00000 & 0.00000 & 0.00000 \\
\hline 1.49755 & 0.00000 & 0.00000 \\
\hline 2.04335 & 1.18995 & 0.00000 \\
\hline 2.24165 & -1.02821 & 0.79483 \\
\hline-0.36926 & 0.64458 & -0.81197 \\
\hline 3.29865 & -1.03783 & 0.48857 \\
\hline-0.37586 & 0.38957 & 0.95911 \\
\hline-0.36784 & -1.02465 & -0.16200 \\
\hline 2.18684 & -0.78589 & 1.86778 \\
\hline 1.80688 & -2.02216 & 0.60910 \\
\hline
\end{tabular}


Table S1259 Employed active space, reference configurations, and Cartesian coordinates of the OM3/MRCISD-optimized ground-state equilibrium geometry of acrolein (ground-state energy: $-772.80638 \mathrm{eV}$ ).

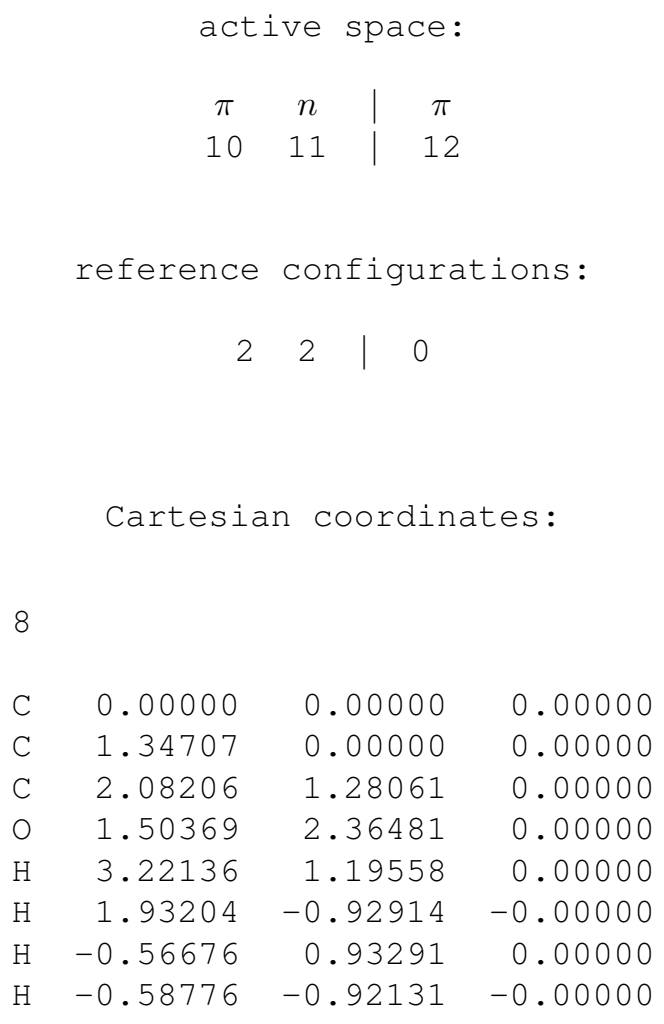


Table S1260 Employed active space, reference configurations, and Cartesian coordinates of the OM3/MRCISD-optimized excited-state equilibrium geometry of the $1{ }^{1} A^{\prime \prime}\left({ }^{1} n \pi^{*}\right)$ state of acrolein (excited-state energy: $-769.10480 \mathrm{eV}$ ).

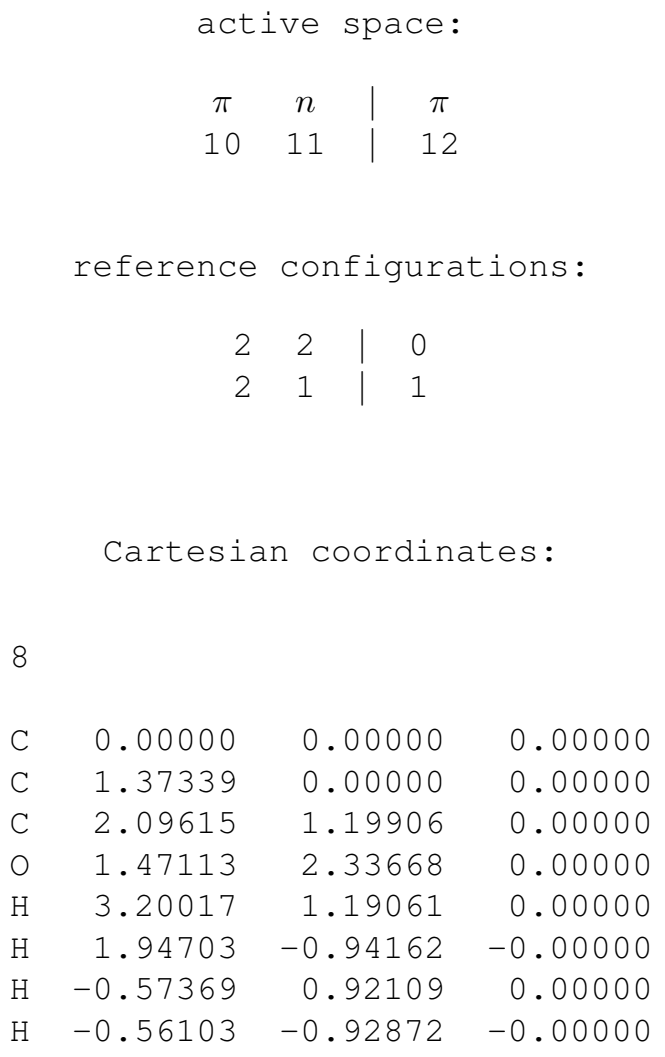


Table S1261 Employed active space, reference configurations, and Cartesian coordinates of the OM3/MRCISD-optimized excited-state equilibrium geometry of the $1^{3} A^{\prime \prime}\left({ }^{3} n \pi^{*}\right)$ state of acrolein (excited-state energy: $-769.26038 \mathrm{eV}$ ).

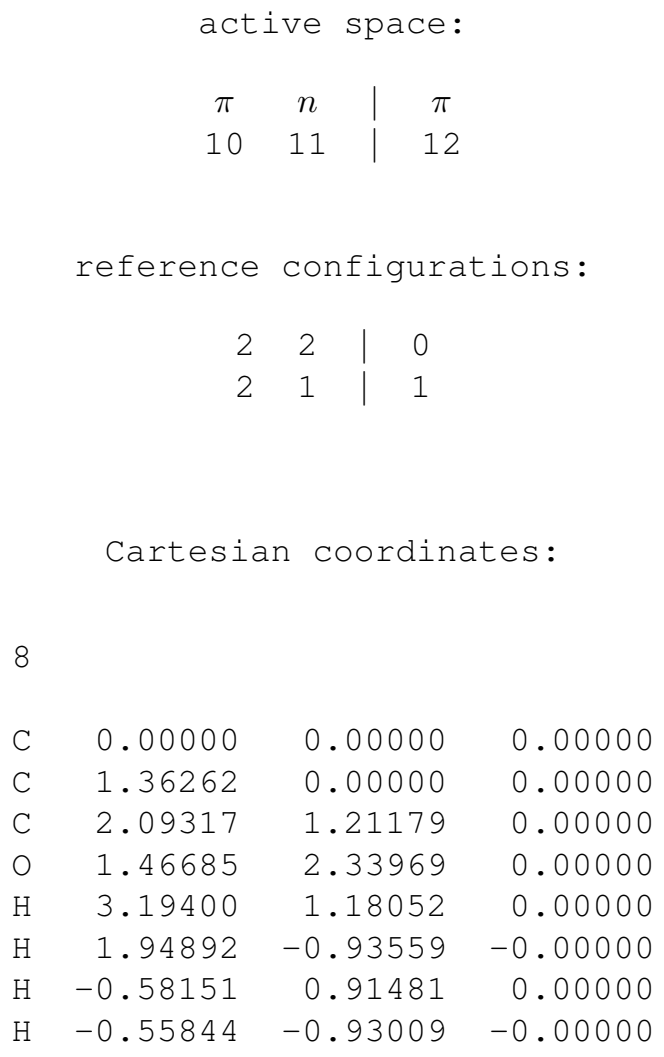


Table S1262 Employed active space, reference configurations, and Cartesian coordinates of the OM3/MRCISD-optimized ground-state equilibrium geometry of aminobenzonitrile (ground-state energy: $-1396.37250 \mathrm{eV}$ ).

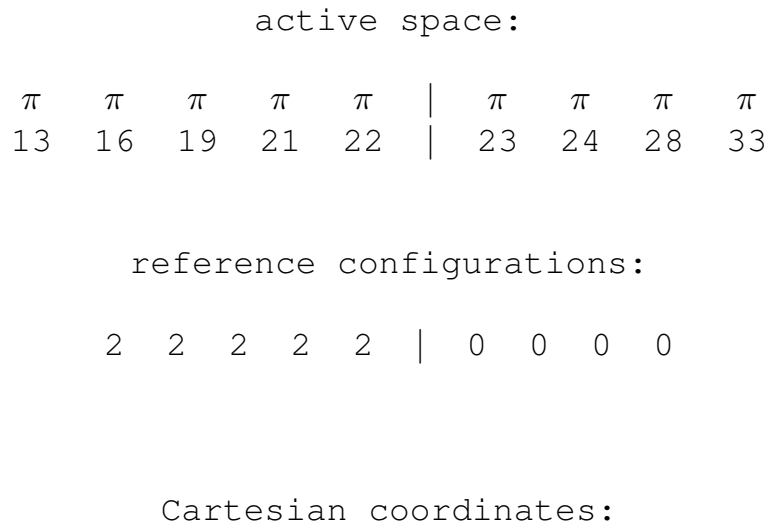


Table S1263 Employed active space, reference configurations, and Cartesian coordinates of the OM3/MRCISD-optimized excited-state equilibrium geometry of the $1{ }^{1} B_{u}\left({ }^{1} \pi \pi^{*}\right)$ state of aminobenzonitrile (excited-state energy: $-1392.56122 \mathrm{eV})$.

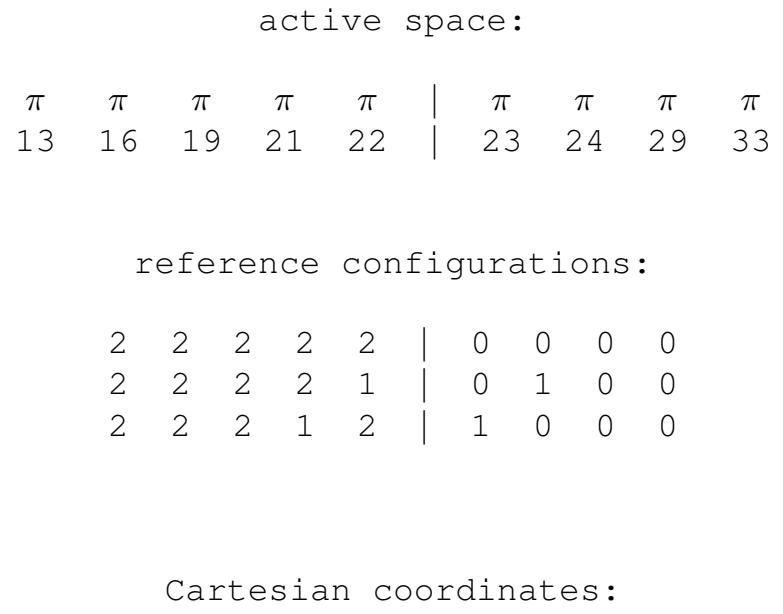


Table S1264 Employed active space, reference configurations, and Cartesian coordinates of the OM3/MRCISD-optimized ground-state equilibrium geometry of aniline (ground-state energy: $-1070.59300 \mathrm{eV}$ ).

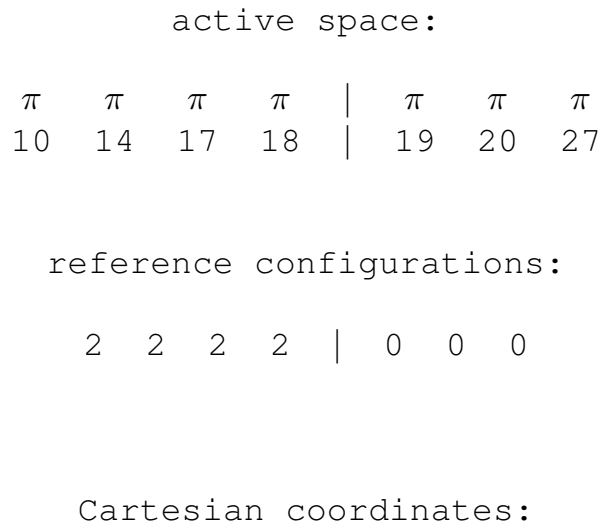


Table S1265 Employed active space, reference configurations, and Cartesian coordinates of the OM3/MRCISD-optimized excited-state equilibrium geometry of the $2{ }^{1} A\left({ }^{1} \pi \pi^{*}\right)$ state of aniline (excited-state energy: $-1066.73378 \mathrm{eV}$ ).

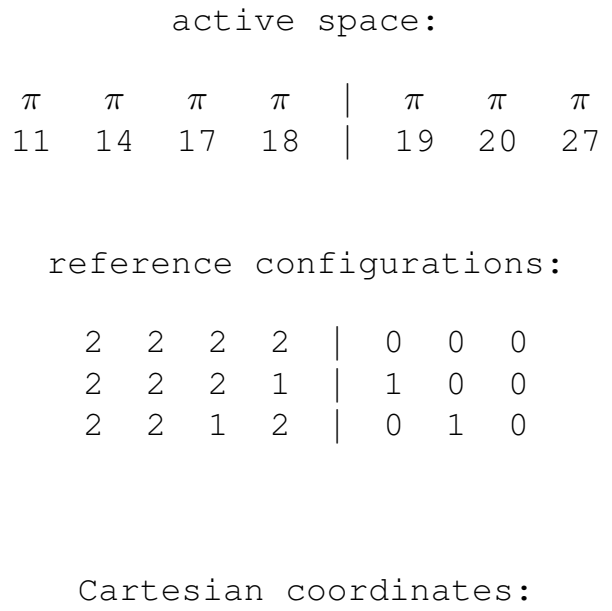


Table S1266 Employed active space, reference configurations, and Cartesian coordinates of the OM3/MRCISD-optimized ground-state equilibrium geometry of anisol (ground-state energy: $-1333.18177 \mathrm{eV}$ ).

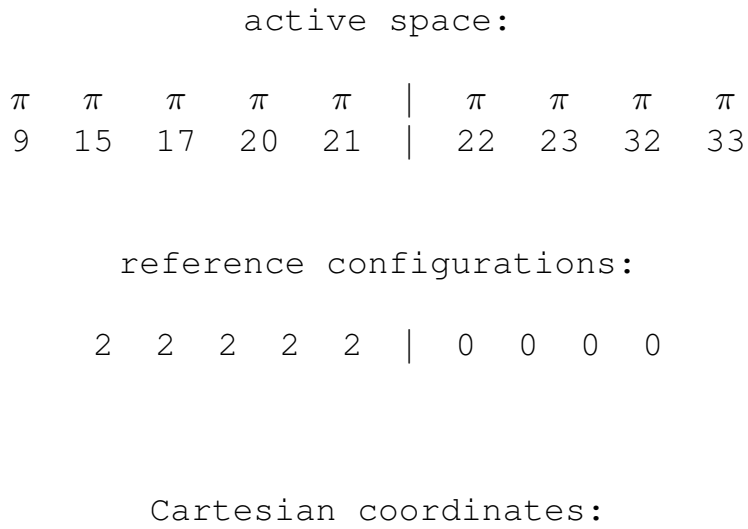


Table S1267 Employed active space, reference configurations, and Cartesian coordinates of the OM3/MRCISD-optimized excited-state equilibrium geometry of the $2{ }^{1} A\left({ }^{1} \pi \pi^{*}\right)$ state of anisol (excited-state energy: $\left.-1329.01763 \mathrm{eV}\right)$.

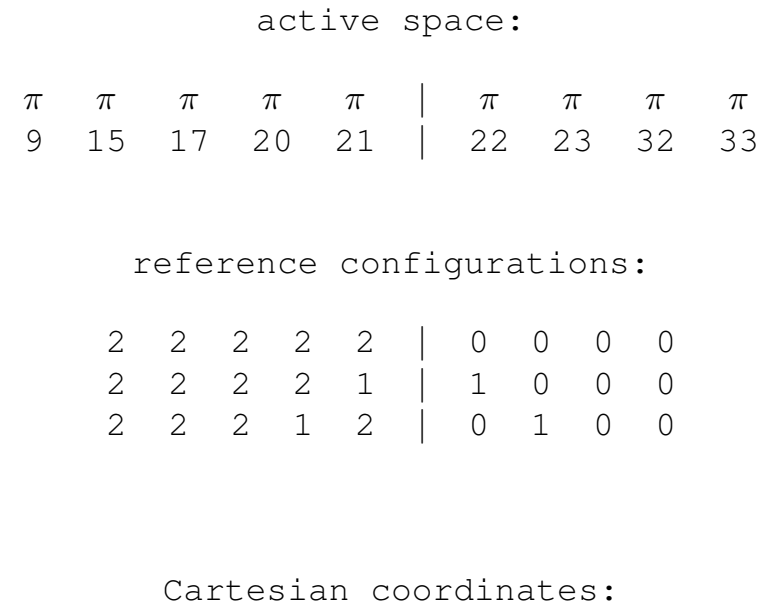

$\mathrm{H} \quad 5.31614 \quad 0.64320 \quad-0.00006$ 
Table S1268 Employed active space, reference configurations, and Cartesian coordinates of the OM3/MRCISD-optimized ground-state equilibrium geometry of anthracene (ground-state energy: $-1900.15664 \mathrm{eV}$ ).

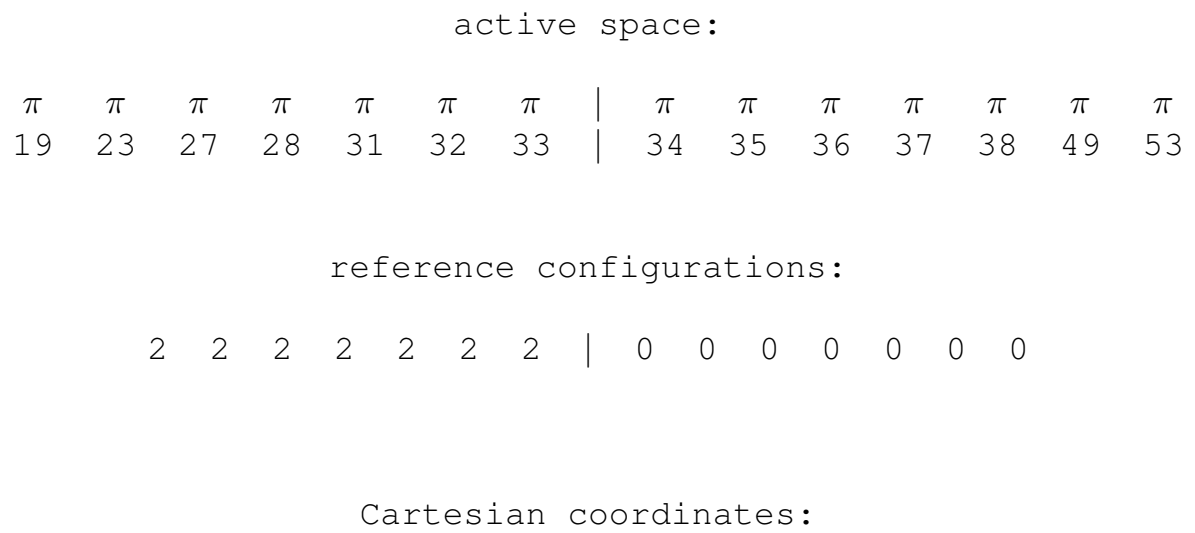

$\begin{array}{rr}2.41453 & 0.00000 \\ -0.41453 & -0.00000 \\ 1.71820 & 0.00000 \\ 1.71820 & -0.00000 \\ 0.28180 & 0.00000 \\ 0.28180 & 0.00000 \\ 2.41297 & 0.00000 \\ 2.41297 & -0.00000 \\ -0.41297 & 0.00000 \\ -0.41297 & 0.00000 \\ 1.71439 & 0.00000 \\ 1.71439 & -0.00000 \\ 0.28561 & 0.00000 \\ 0.28561 & 0.00000 \\ 3.51759 & 0.00000 \\ -1.51759 & -0.00000 \\ 3.51590 & 0.00000 \\ 3.51590 & -0.00000 \\ -1.51590 & 0.00000 \\ -1.51590 & 0.00000 \\ 2.24504 & 0.00000 \\ 2.24504 & 0.00000 \\ -0.24504 & 0.00000 \\ -0.24504 & 0.00000\end{array}$


Table S1269 Employed active space, reference configurations, and Cartesian coordinates of the OM3/MRCISD-optimized excited-state equilibrium geometry of the $1^{1} B_{2 u}\left({ }^{1} \pi \pi^{*}\right)$ state of anthracene (excited-state energy: $-1896.27907 \mathrm{eV})$.

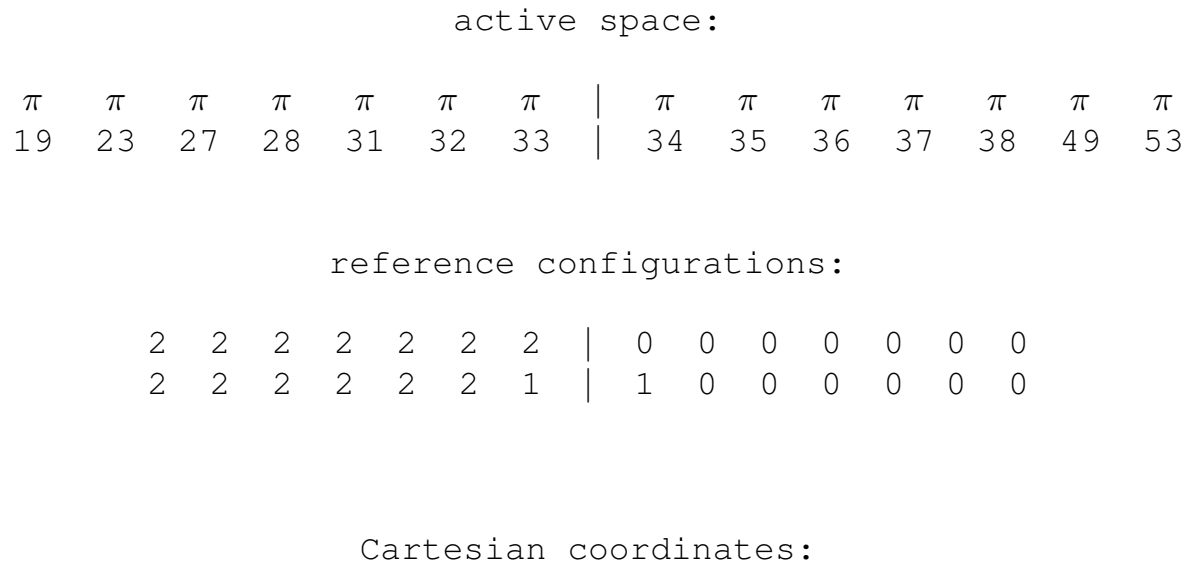


Continued from previous page

$\begin{array}{llll}\mathrm{H} & -3.64622 & -0.25064 & -0.00000\end{array}$

S1350 
Table S1270 Employed active space, reference configurations, and Cartesian coordinates of the OM3/MRCISD-optimized ground-state equilibrium geometry of azulene (ground-state energy: $-1368.20770 \mathrm{eV}$ ).

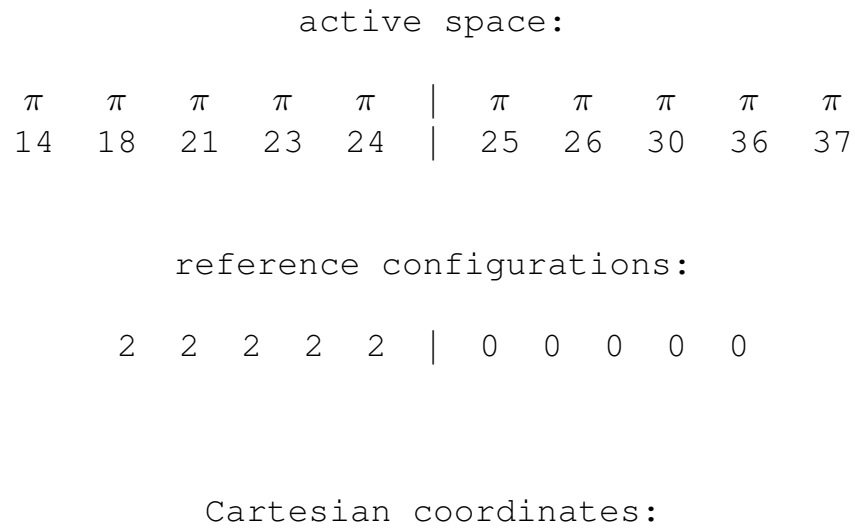

$\mathrm{H} \quad 0.00000 \quad-4.74023 \quad-0.00000$ 
Table S1271 Employed active space, reference configurations, and Cartesian coordinates of the OM3/MRCISD-optimized excited-state equilibrium geometry of the $1{ }^{1} B_{1}\left({ }^{1} \pi \pi^{*}\right)$ state of azulene (excited-state energy: $\left.-1366.67663 \mathrm{eV}\right)$.

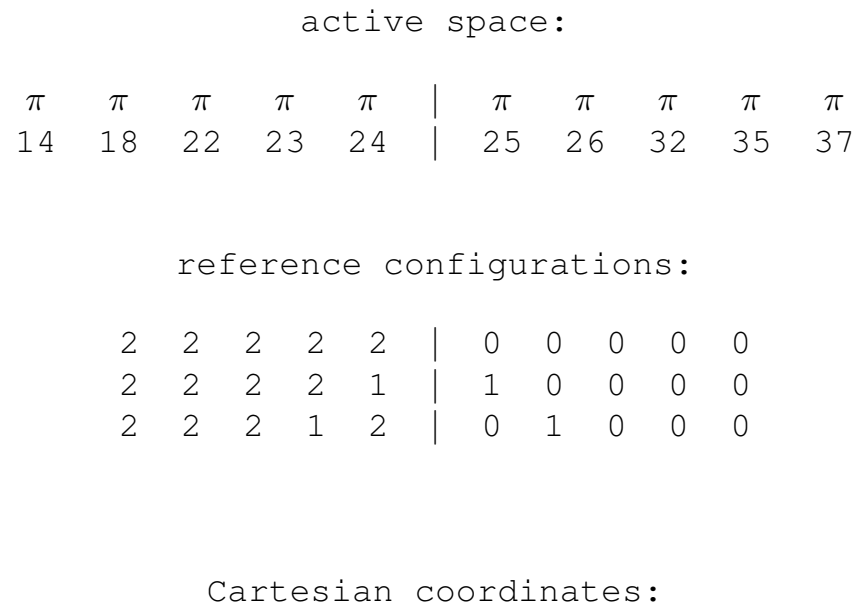

18

C $\quad 0.00000$

1.587490 .00000

C 0.00000

$0.98506-1.27815$

C $\quad 0.00000$

$0.98506 \quad 1.27815$

C $\quad 0.00000$

$-0.36023-1.62080$

$\begin{array}{llll}\text { C } & 0.00000 & -0.36023 & 1.62080\end{array}$

$\begin{array}{llll}\text { C } & 0.00000 & -1.45499 & -0.70741\end{array}$

$\begin{array}{llll}\text { C } & 0.00000 & -1.45499 & 0.70741\end{array}$

$\begin{array}{llll}\text { C } & 0.00000 & -2.85918 & -1.13597\end{array}$

$\begin{array}{llll}\text { C } & 0.00000 & -2.85918 & 1.13597\end{array}$

C $\quad 0.00000 \quad-3.69286-0.00000$

H $\quad 0.00000 \quad 2.69510 \quad 0.00000$

$\mathrm{H} \quad 0.00000 \quad 1.70118 \quad-2.13085$

H $\quad 0.00000 \quad 1.70118 \quad 2.13085$

$\mathrm{H} \quad 0.00000 \quad-0.62622 \quad-2.69373$

$\mathrm{H} \quad 0.00000 \quad-0.62622 \quad 2.69373$

$\mathrm{H} \quad 0.00000 \quad-3.18768-2.18076$

$\mathrm{H} \quad 0.00000 \quad-3.18768 \quad 2.18076$

$\mathrm{H} \quad 0.00000 \quad-4.77951-0.00000$ 
Table S1272 Employed active space, reference configurations, and Cartesian coordinates of the OM3/MRCISD-optimized ground-state equilibrium geometry of benzaldehyde (ground-state energy: $-1304.00598 \mathrm{eV}$ ).

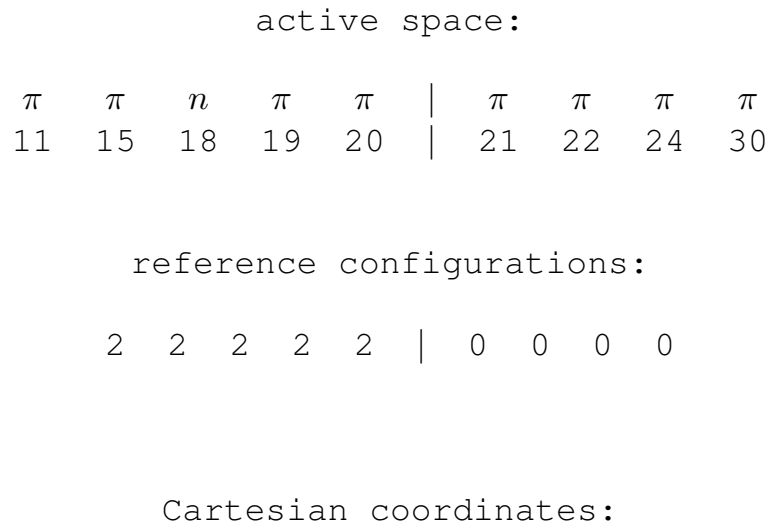


Table S1273 Employed active space, reference configurations, and Cartesian coordinates of the OM3/MRCISD-optimized excited-state equilibrium geometry of the $2^{1} A^{\prime}\left({ }^{1} \pi \pi^{*}\right)$ state of benzaldehyde (excited-state energy: $-1299.81519 \mathrm{eV})$.

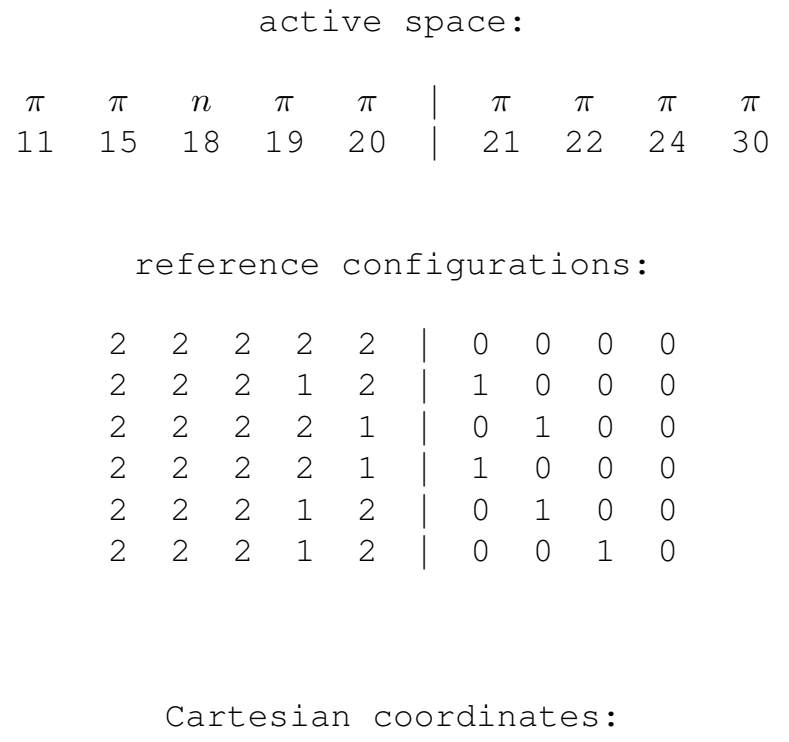

14

$\begin{array}{lrrr}\mathrm{C} & 0.00000 & 0.00000 & 0.00000 \\ \mathrm{C} & 1.43500 & 0.00000 & 0.00000 \\ \mathrm{C} & 2.15063 & 1.23301 & 0.00000 \\ \mathrm{C} & 1.45139 & 2.46927 & 0.00000 \\ \mathrm{C} & 0.03137 & 2.47725 & 0.00000 \\ \mathrm{C} & -0.69637 & 1.25044 & 0.00000 \\ \mathrm{C} & -0.72547 & -1.27661 & -0.00000 \\ \mathrm{O} & -0.12700 & -2.36171 & -0.00000 \\ \mathrm{H} & 1.96861 & -0.96045 & -0.00000 \\ \mathrm{H} & 3.25123 & 1.22358 & 0.00000 \\ \mathrm{H} & 2.00682 & 3.41727 & 0.00000 \\ \mathrm{H} & -0.51389 & 3.43385 & 0.00000 \\ \mathrm{H} & -1.79741 & 1.26084 & 0.00000 \\ \mathrm{H} & -1.86091 & -1.20169 & -0.00000\end{array}$


Table S1274 Employed active space, reference configurations, and Cartesian coordinates of the OM3/MRCISD-optimized excited-state equilibrium geometry of the $1^{1} A^{\prime \prime}\left({ }^{1} n \pi^{*}\right)$ state of benzaldehyde (excited-state energy: $-1300.30699 \mathrm{eV})$.

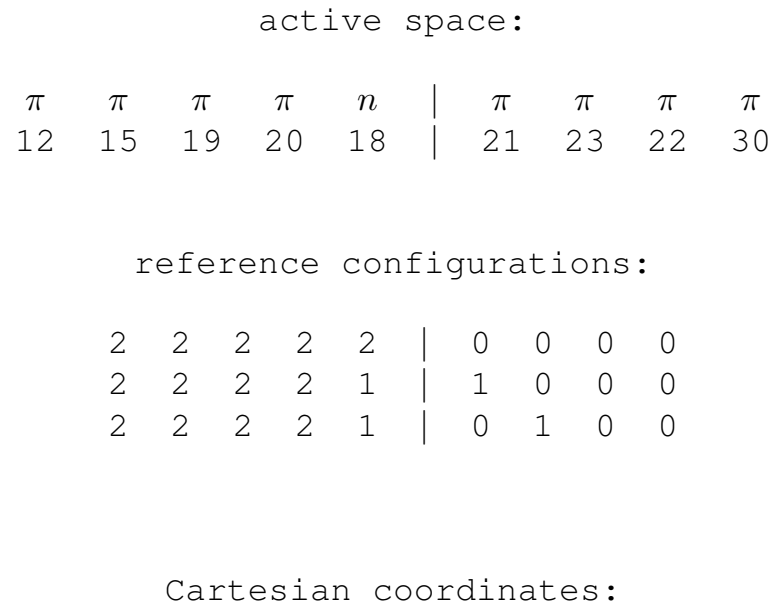


Table S1275 Employed active space, reference configurations, and Cartesian coordinates of the OM3/MRCISD-optimized excited-state equilibrium geometry of the $1^{3} A^{\prime \prime}\left({ }^{3} n \pi^{*}\right)$ state of benzaldehyde (excited-state energy: $-1300.53484 \mathrm{eV})$.

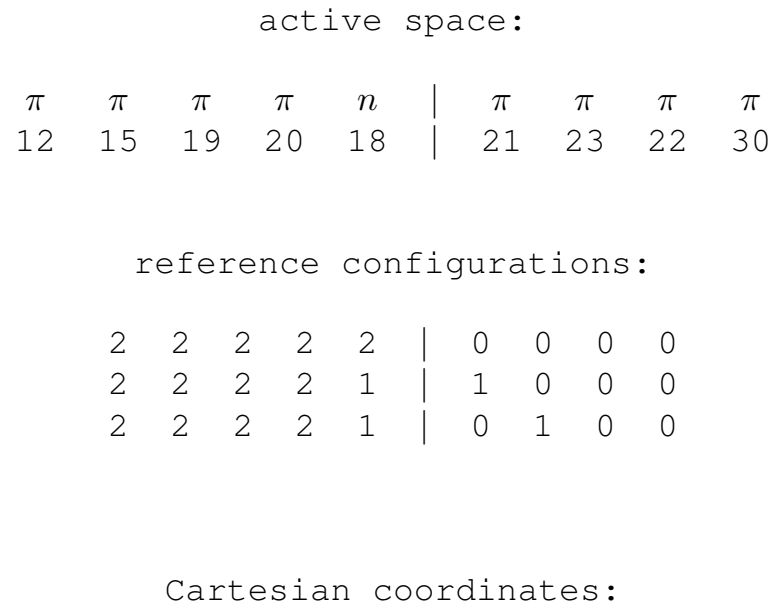


Table S1276 Employed active space, reference configurations, and Cartesian coordinates of the OM3/MRCISD-optimized ground-state equilibrium geometry of benzene (ground-state energy: $-839.85573 \mathrm{eV}$ ).

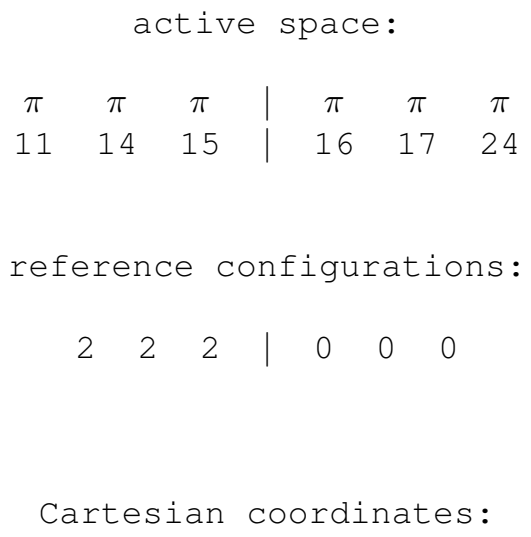

12

$\begin{array}{llrr}\text { C } & 0.00000 & 1.40197 & 0.00000 \\ \text { C } & 0.00000 & 0.70099 & -1.21414 \\ \text { C } & 0.00000 & 0.70099 & 1.21414 \\ \text { C } & 0.00000 & -0.70099 & -1.21414 \\ \text { C } & 0.00000 & -0.70099 & 1.21414 \\ \text { C } & 0.00000 & -1.40197 & -0.00000 \\ \text { H } & 0.00000 & 2.50417 & 0.00000 \\ \text { H } & 0.00000 & 1.25209 & -2.16868 \\ \text { H } & 0.00000 & 1.25209 & 2.16868 \\ \text { H } & 0.00000 & -1.25209 & -2.16868 \\ \text { H } & 0.00000 & -1.25209 & 2.16868 \\ \text { H } & 0.00000 & -2.50417 & -0.00000\end{array}$


Table S1277 Employed active space, reference configurations, and Cartesian coordinates of the OM3/MRCISD-optimized excited-state equilibrium geometry of the $1{ }^{1} B_{1 u}\left({ }^{1} \pi \pi^{*}\right)$ state of benzene (excited-state energy: $\left.-835.50269 \mathrm{eV}\right)$.

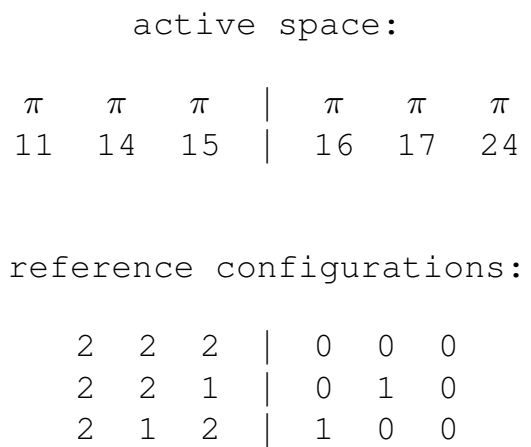

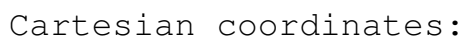

H $\quad 0.00000 \quad-2.52584 \quad-0.00000$ 
Table S1278 Employed active space, reference configurations, and Cartesian coordinates of the OM3/MRCISD-optimized ground-state equilibrium geometry of benzonitrile (ground-state energy: $-1165.57263 \mathrm{eV}$ ).

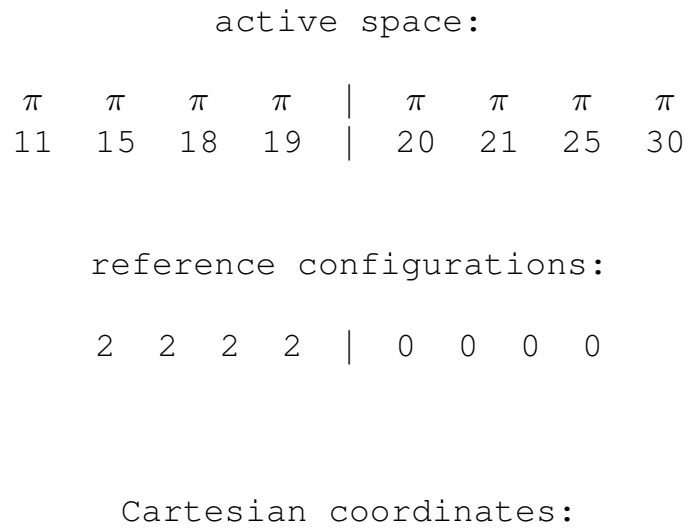


Table S1279 Employed active space, reference configurations, and Cartesian coordinates of the OM3/MRCISD-optimized excited-state equilibrium geometry of the $1{ }^{1} B_{1}\left({ }^{1} \pi \pi^{*}\right)$ state of benzonitrile (excited-state energy: $-1161.33723 \mathrm{eV})$.

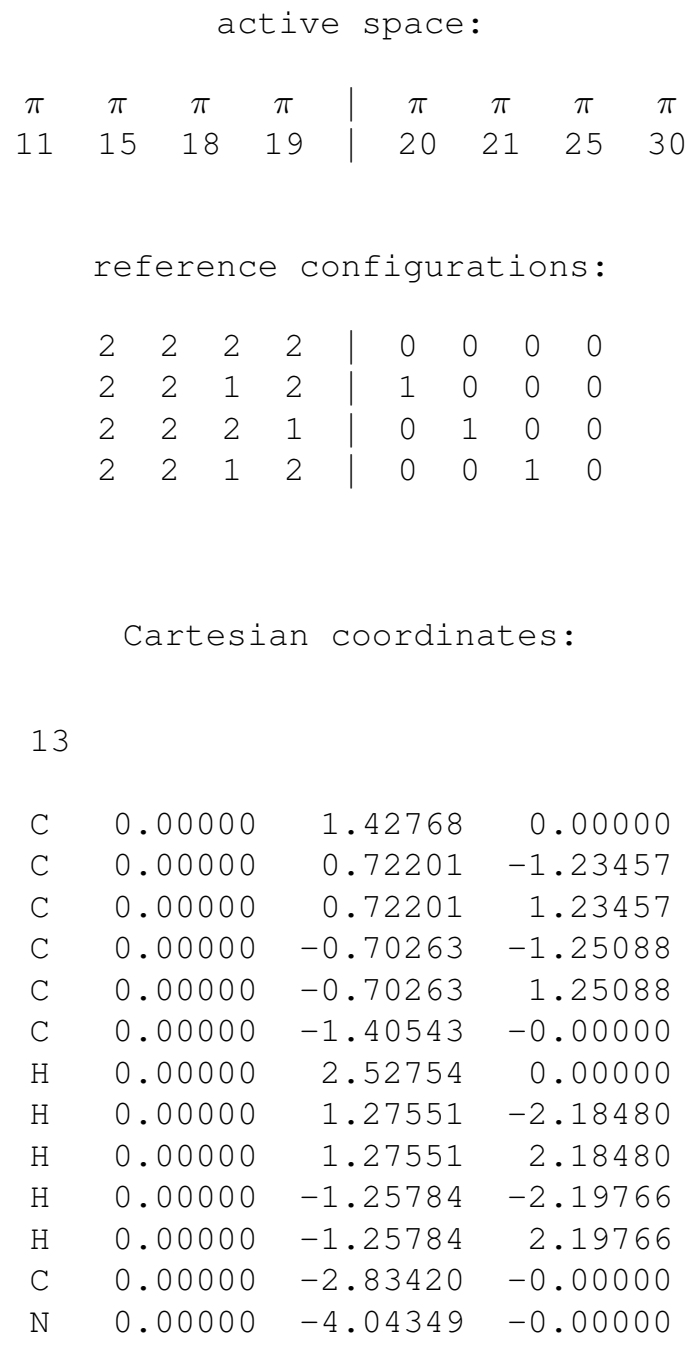


Table S1280 Employed active space, reference configurations, and Cartesian coordinates of the OM3/MRCISD-optimized ground-state equilibrium geometry of biphenyl (ground-state energy: $-1649.98001 \mathrm{eV}$ ).

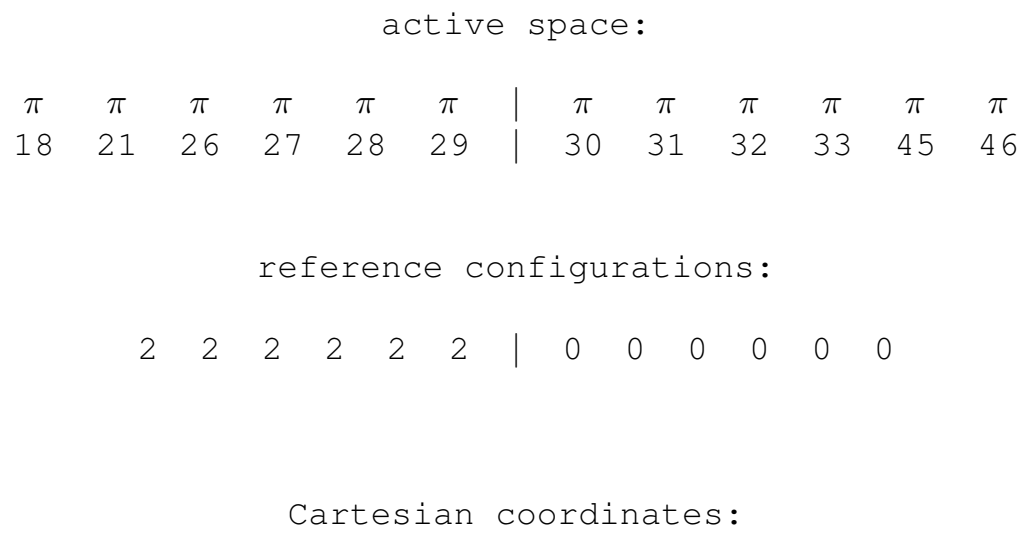


Table S1281 Employed active space, reference configurations, and Cartesian coordinates of the OM3/MRCISD-optimized excited-state equilibrium geometry of the $1{ }^{1} B_{1 u}\left({ }^{1} \pi \pi^{*}\right)$ state of biphenyl (excited-state energy: $-1645.12856 \mathrm{eV}$ ).

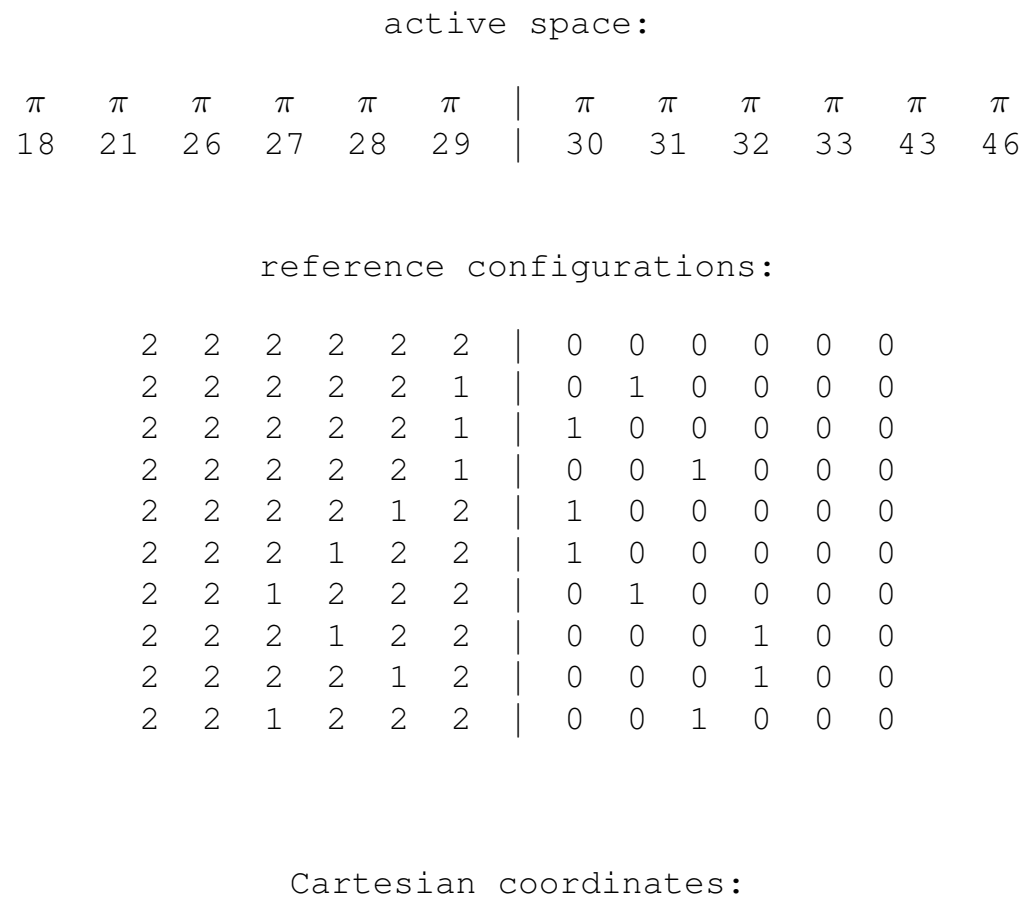

\begin{tabular}{lrrr}
22 & & \\
& & & \\
C & 0.00000 & 0.00000 & 0.71260 \\
C & 0.00000 & 0.00000 & -0.71260 \\
C & 1.23443 & 0.00000 & 1.46813 \\
C & 1.23443 & 0.00000 & -1.46813 \\
C & -1.23443 & 0.00000 & 1.46813 \\
C & -1.23443 & -0.00000 & -1.46813 \\
C & 1.22071 & 0.00000 & 2.85351 \\
C & 1.22071 & 0.00000 & -2.85351 \\
C & -1.22071 & 0.00000 & 2.85351 \\
C & -1.22071 & 0.00000 & -2.85351 \\
C & 0.00000 & -0.00000 & 3.56340 \\
C & 0.00000 & 0.00000 & -3.56340 \\
H & 2.19938 & 0.00000 & 0.93478 \\
H & 2.19938 & 0.00000 & -0.93478 \\
H & -2.19938 & 0.00000 & 0.93478 \\
H & -2.19938 & -0.00000 & -0.93478 \\
\hline & & Continued on next page
\end{tabular}

S1362 


\begin{tabular}{lrrr}
\multicolumn{4}{c}{ Continued from previous page } \\
\hline $\mathrm{H}$ & 2.16944 & 0.00000 & 3.41574 \\
$\mathrm{H}$ & 2.16944 & 0.00000 & -3.41574 \\
$\mathrm{H}$ & -2.16944 & 0.00000 & 3.41574 \\
$\mathrm{H}$ & -2.16944 & -0.00000 & -3.41574 \\
$\mathrm{H}$ & 0.00000 & -0.00000 & 4.66439 \\
$\mathrm{H}$ & 0.00000 & 0.00000 & -4.66439
\end{tabular}


Table S1282 Employed active space, reference configurations, and Cartesian coordinates of the OM3/MRCISD-optimized ground-state equilibrium geometry of biphenylene (ground-state energy: $-1617.95326 \mathrm{eV}$ ).

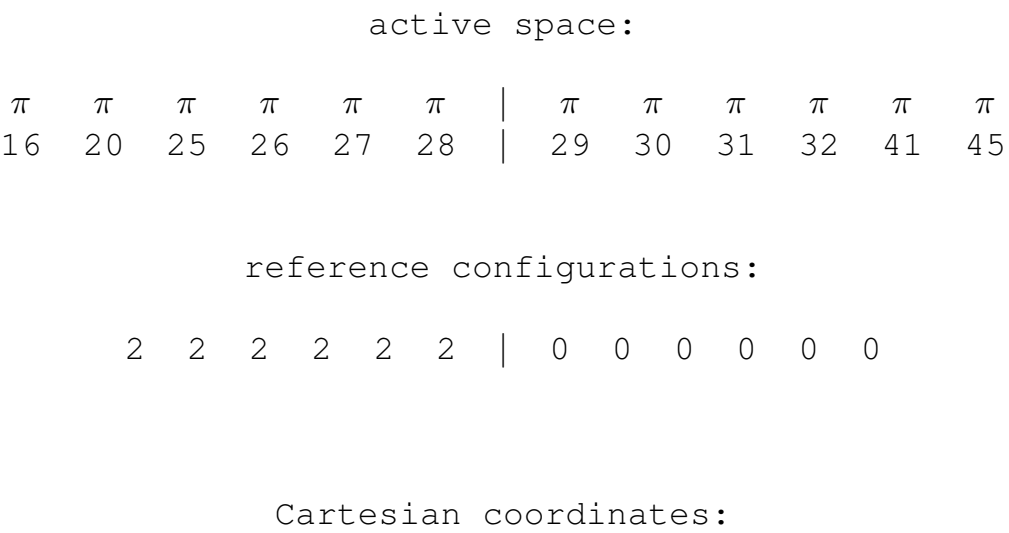


Table S1283 Employed active space, reference configurations, and Cartesian coordinates of the OM3/MRCISD-optimized excited-state equilibrium geometry of the $1{ }^{1} B_{3 u}\left({ }^{1} \pi \pi^{*}\right)$ state of biphenylene (excited-state energy: $-1614.29451 \mathrm{eV})$.

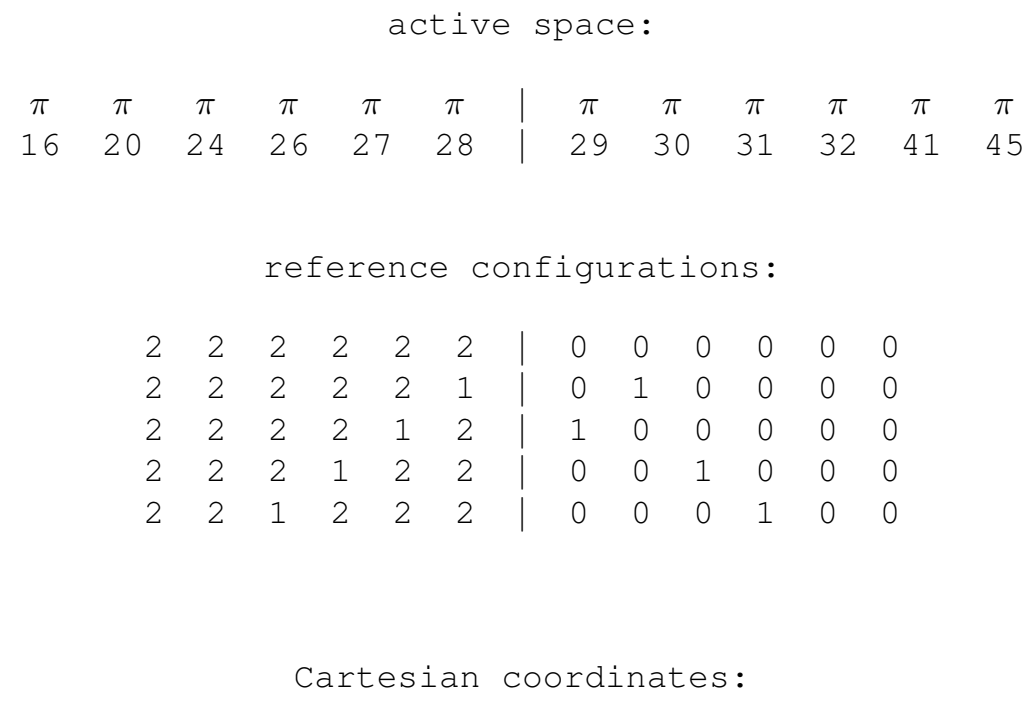

20

$\begin{array}{lrrr}\text { C } & 0.00000 & 0.00000 & 0.00000 \\ \mathrm{C} & 1.46917 & 0.00000 & 0.00000 \\ \mathrm{C} & 0.00000 & 1.46306 & 0.00000 \\ \mathrm{C} & 1.46917 & 1.46306 & 0.00000 \\ \mathrm{C} & -1.18456 & -0.74870 & 0.00000 \\ \mathrm{C} & 2.65373 & -0.74870 & -0.00000 \\ \mathrm{C} & -1.18456 & 2.21176 & -0.00000 \\ \mathrm{C} & 2.65373 & 2.21176 & 0.00000 \\ \mathrm{C} & -2.37359 & 0.02636 & 0.00000 \\ \mathrm{C} & 3.84276 & 0.02636 & -0.00000 \\ \mathrm{C} & -2.37359 & 1.43670 & -0.00000 \\ \mathrm{C} & 3.84276 & 1.43670 & 0.00000 \\ \mathrm{H} & -1.20000 & -1.84343 & 0.00000 \\ \mathrm{H} & 2.66917 & -1.84343 & -0.00000 \\ \mathrm{H} & -1.20000 & 3.30650 & -0.00000 \\ \mathrm{H} & 2.66917 & 3.30650 & 0.00000 \\ \mathrm{H} & -3.34325 & -0.50066 & 0.00000 \\ \mathrm{H} & 4.81243 & -0.50066 & -0.00000 \\ \mathrm{H} & -3.34325 & 1.96372 & -0.00000 \\ \mathrm{H} & 4.81243 & 1.96372 & 0.00000\end{array}$


Table S1284 Employed active space, reference configurations, and Cartesian coordinates of the OM3/MRCISD-optimized ground-state equilibrium geometry of acetylene (ground-state energy: $-277.82502 \mathrm{eV}$ ).

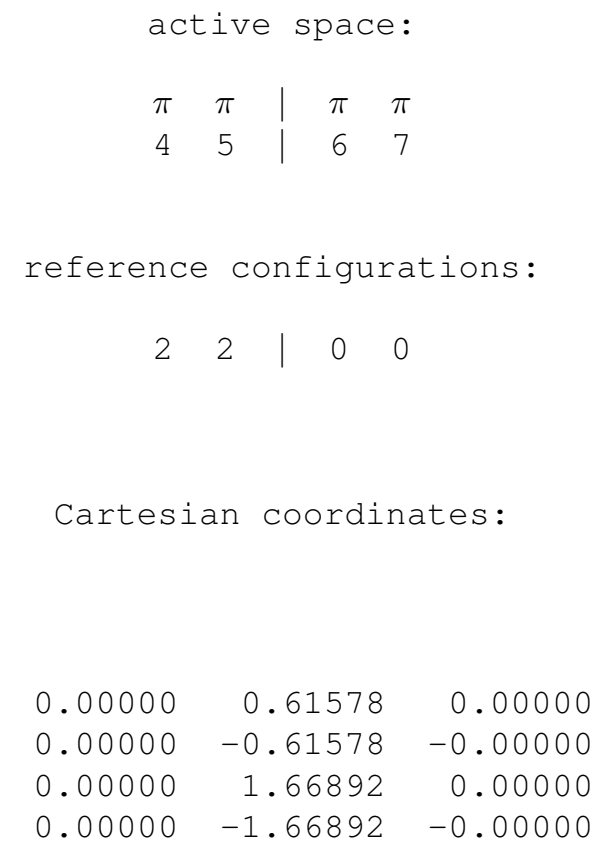


Table S1285 Employed active space, reference configurations, and Cartesian coordinates of the OM3/MRCISD-optimized excited-state equilibrium geometry of the $2{ }^{1} A\left({ }^{1} \pi \pi^{*}\right)$ state of acetylene (excited-state energy: $-272.88702 \mathrm{eV}$ ).

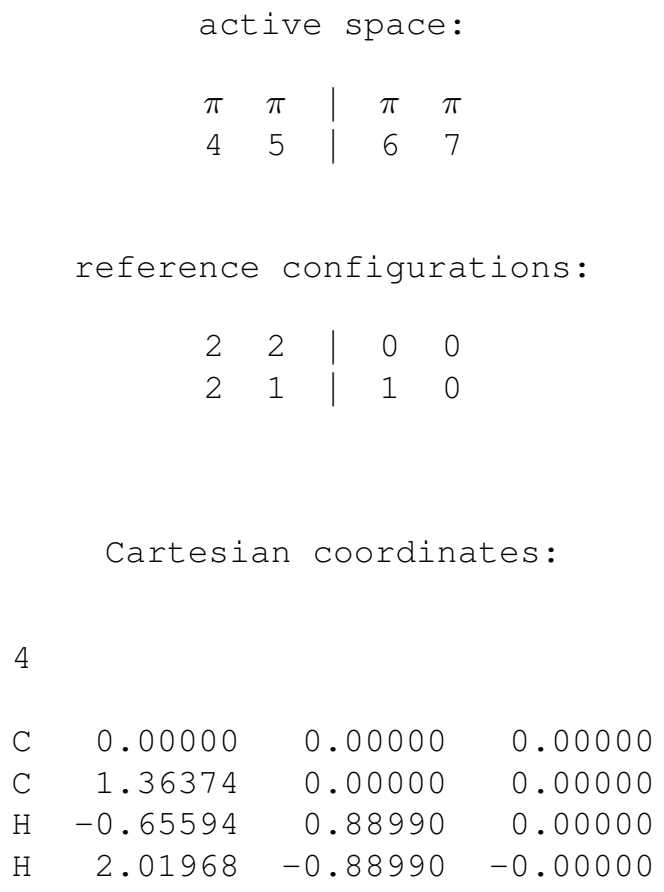


Table S1286 Employed active space, reference configurations, and Cartesian coordinates of the OM3/MRCISD-optimized ground-state equilibrium geometry of formaldehyde (ground-state energy: $-493.54110 \mathrm{eV}$ ).

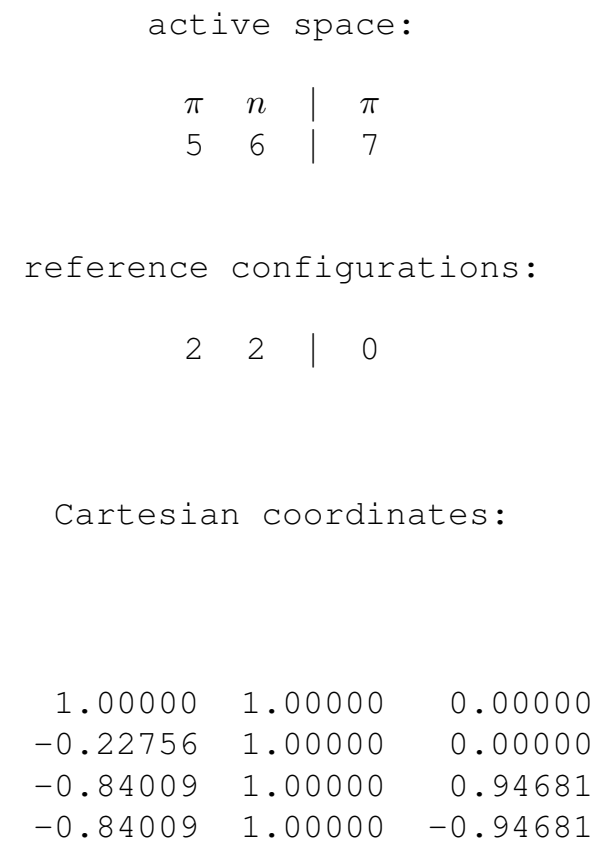


Table S1287 Employed active space, reference configurations, and Cartesian coordinates of the OM3/MRCISD-optimized excited-state equilibrium geometry of the $2{ }^{1} A\left({ }^{1} n \pi^{*}\right)$ state of formaldehyde (excited-state energy: $-490.07723 \mathrm{eV}$ ).

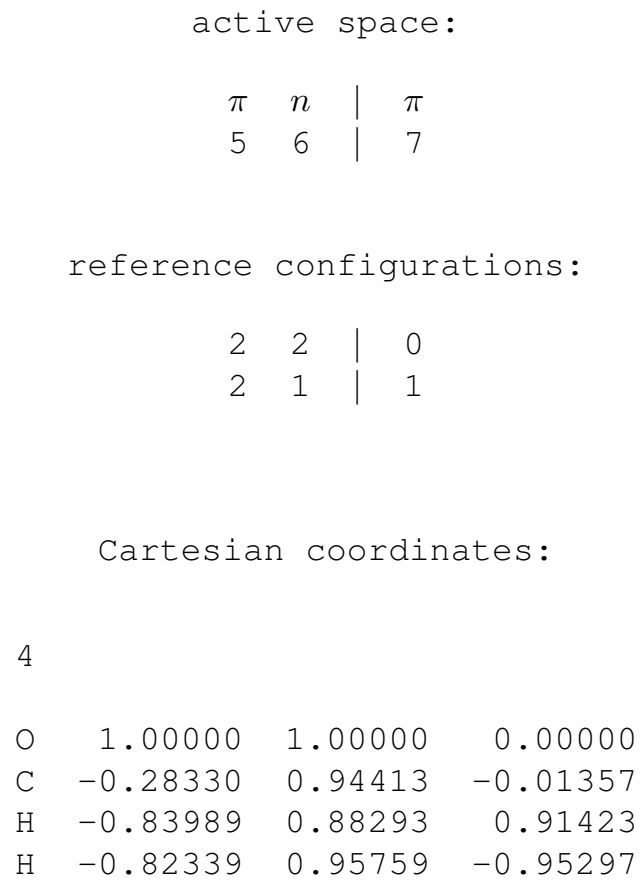

$\mathrm{H} \quad-0.82339 \quad 0.95759 \quad-0.95297$ 
Table S1288 Employed active space, reference configurations, and Cartesian coordinates of the OM3/MRCISD-optimized excited-state equilibrium geometry of the $1^{3} A\left({ }^{3} n \pi^{*}\right)$ state of formaldehyde (excited-state energy: $\left.-490.40596 \mathrm{eV}\right)$.

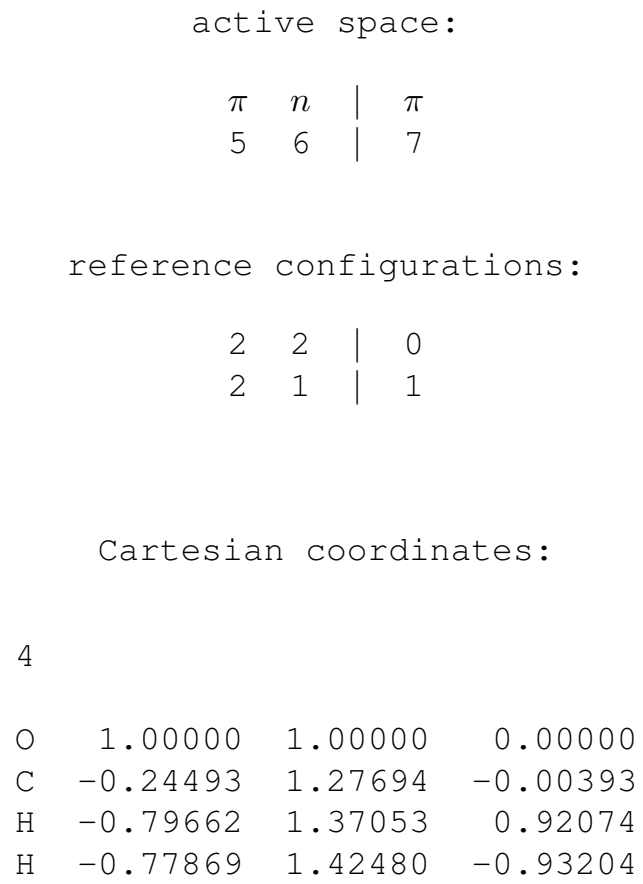

$\mathrm{H} \quad-0.77869 \quad 1.42480 \quad-0.93204$ 
Table S1289 Employed active space, reference configurations, and Cartesian coordinates of the OM3/MRCISD-optimized ground-state equilibrium geometry of cinnoline (ground-state energy: $-1521.15466 \mathrm{eV}$ ).

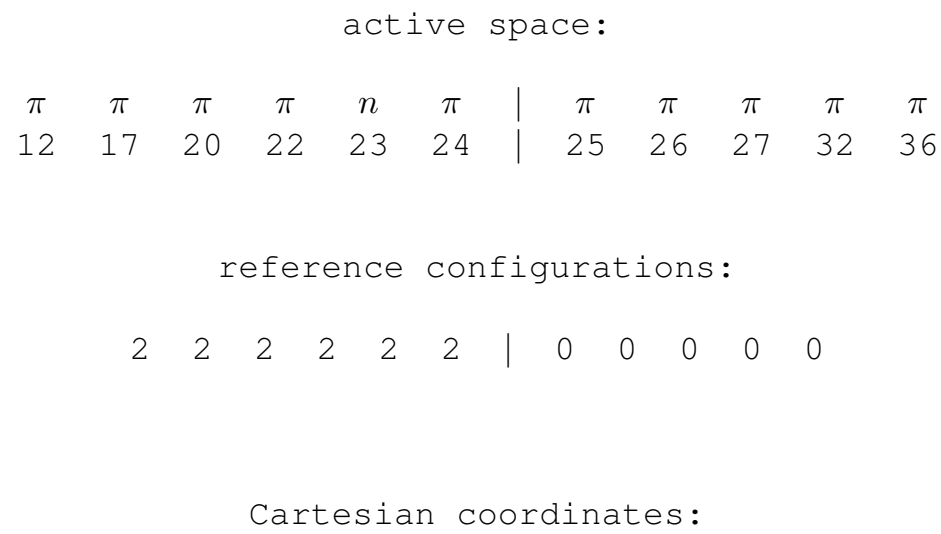


Table S1290 Employed active space, reference configurations, and Cartesian coordinates of the OM3/MRCISD-optimized excited-state equilibrium geometry of the $1^{1} A^{\prime \prime}\left({ }^{1} n \pi^{*}\right)$ state of cinnoline (excited-state energy: $-1517.97036 \mathrm{eV}$ ).

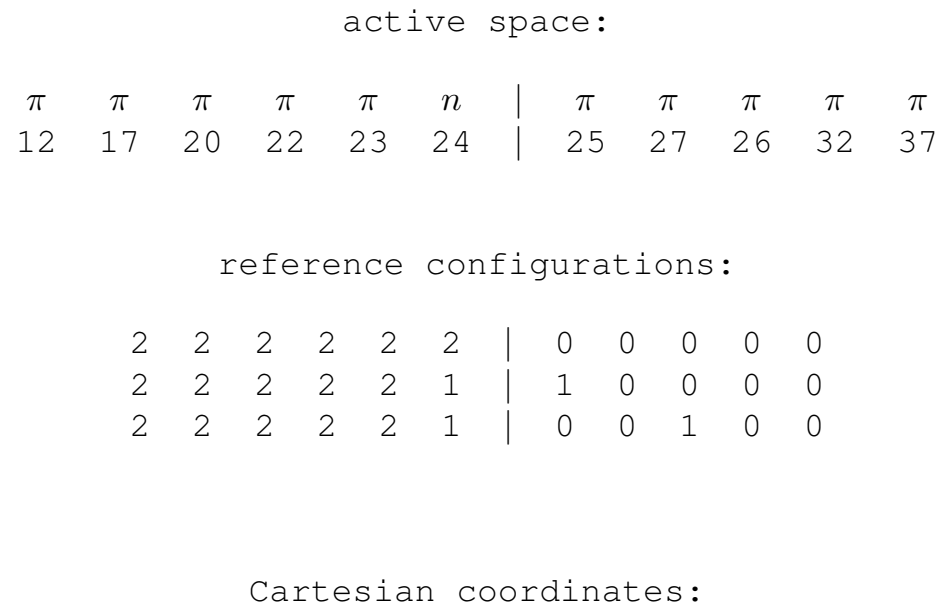


Table S1291 Employed active space, reference configurations, and Cartesian coordinates of the OM3/MRCISD-optimized ground-state equilibrium geometry of cyanoacetylene (ground-state energy: $-603.18431 \mathrm{eV}$ ).

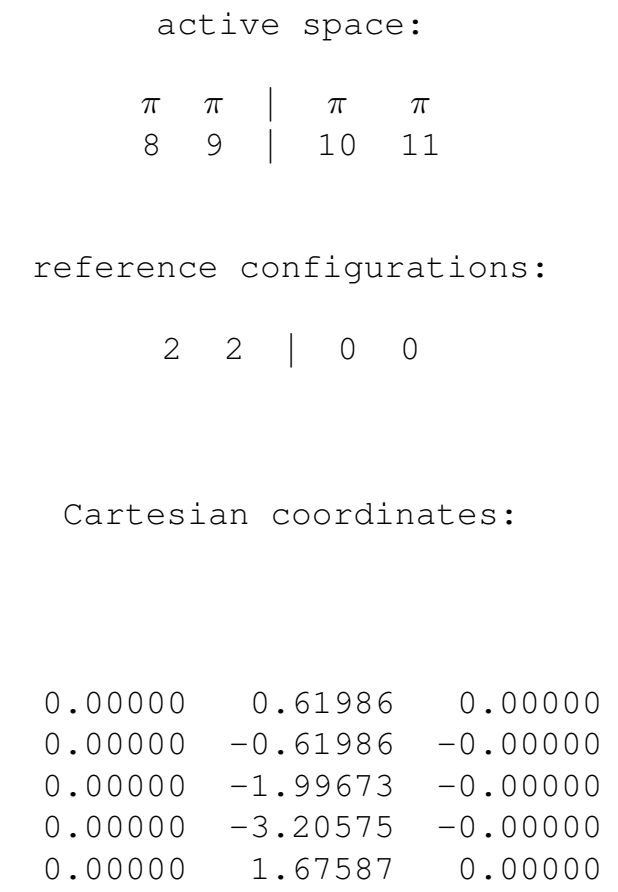


Table S1292 Employed active space, reference configurations, and Cartesian coordinates of the OM3/MRCISD-optimized excited-state equilibrium geometry of the $2^{1} A^{\prime}\left({ }^{1} \pi \pi^{*}\right)$ state of cyanoacetylene (excited-state energy: $-597.58518 \mathrm{eV})$.

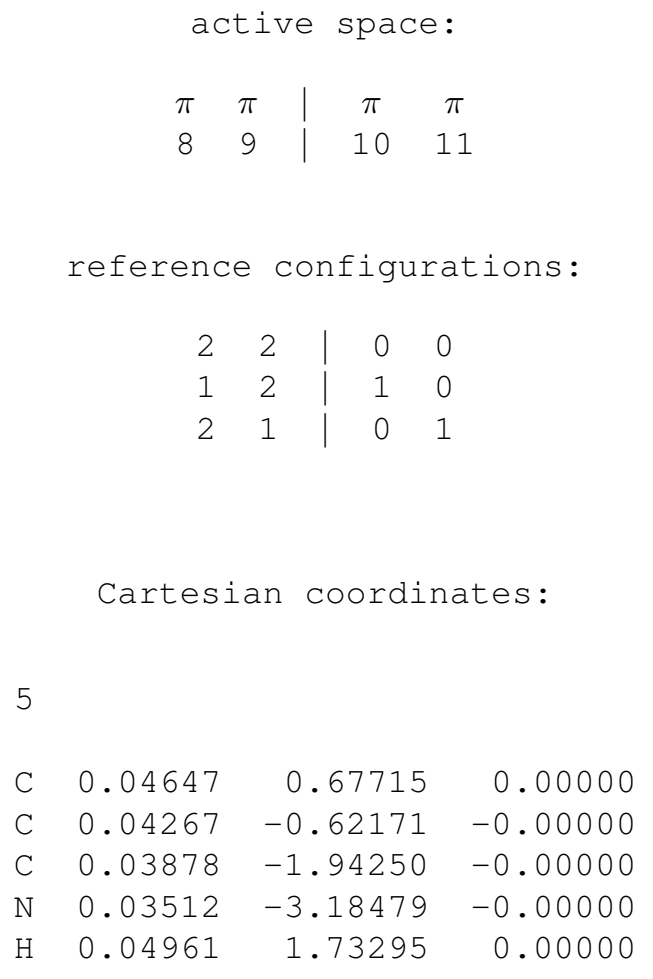


Table S1293 Employed active space, reference configurations, and Cartesian coordinates of the OM3/MRCISD-optimized ground-state equilibrium geometry of cyclohexadienyl radical (ground-state energy: $-853.37141 \mathrm{eV}$ ).

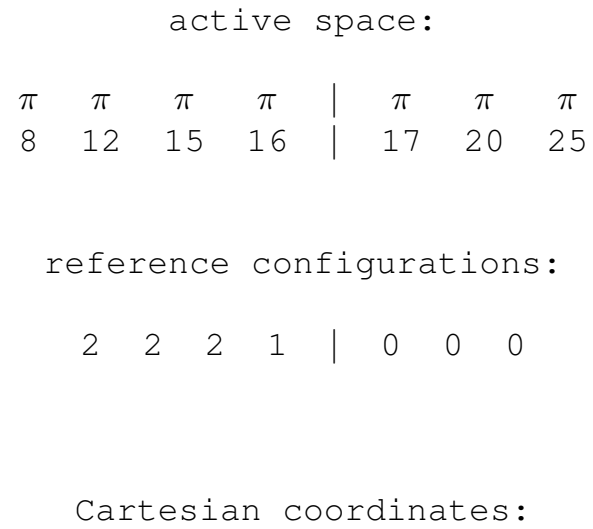


Table S1294 Employed active space, reference configurations, and Cartesian coordinates of the OM3/MRCISD-optimized excited-state equilibrium geometry of the $2^{2} A\left({ }^{2} \pi \pi^{*}\right)$ state of cyclohexadienyl radical (excited-state energy: $-851.24652 \mathrm{eV})$.

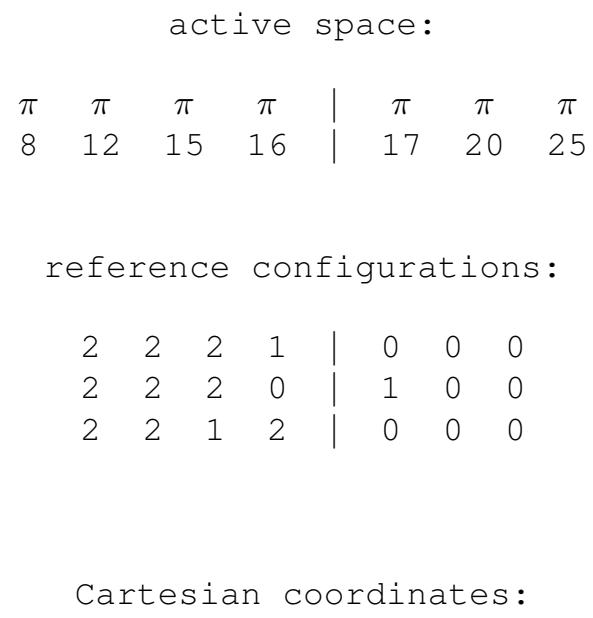


Table S1295 Employed active space, reference configurations, and Cartesian coordinates of the OM3/MRCISD-optimized ground-state equilibrium geometry of 4-(dimethylamino)-4'-cyanostilbene (ground-state energy: $-2794.30938 \mathrm{eV}$ ).

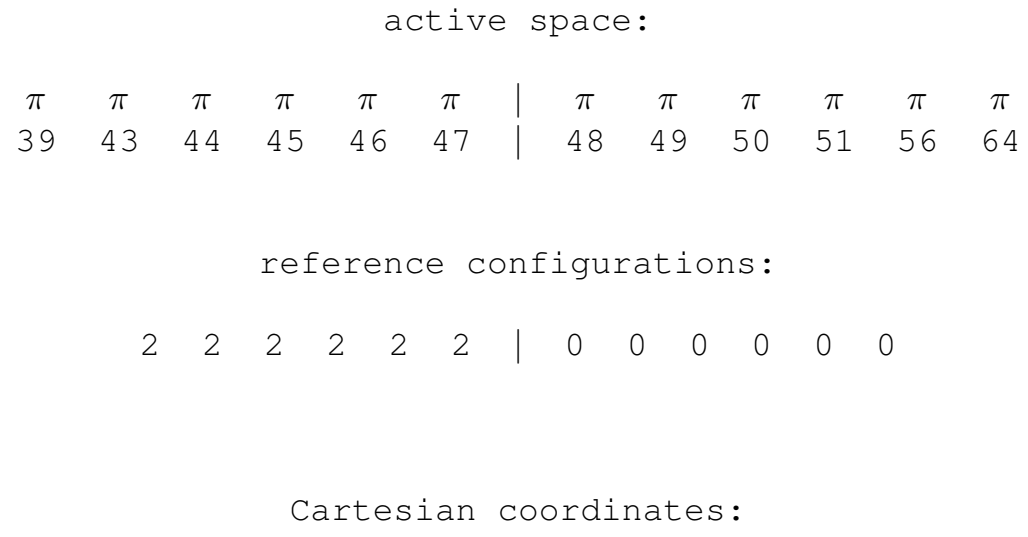

\begin{tabular}{rrrr}
35 & & & \\
& & & \\
$\mathrm{C}$ & 0.00000 & 0.00000 & 0.00000 \\
$\mathrm{C}$ & 1.39433 & 0.00000 & 0.00000 \\
$\mathrm{C}$ & 2.12215 & 1.21153 & 0.00000 \\
$\mathrm{C}$ & 1.41788 & 2.43393 & 0.00000 \\
$\mathrm{C}$ & 0.02248 & 2.45432 & 0.00001 \\
$\mathrm{C}$ & -0.67731 & 1.23380 & 0.00001 \\
$\mathrm{C}$ & 3.59115 & 1.14569 & -0.00002 \\
$\mathrm{C}$ & 4.41642 & 2.22682 & -0.00001 \\
$\mathrm{C}$ & 5.88294 & 2.16377 & -0.00005 \\
$\mathrm{C}$ & 6.61725 & 3.36976 & -0.00001 \\
$\mathrm{C}$ & 8.00835 & 3.37126 & -0.00004 \\
$\mathrm{C}$ & 8.72682 & 2.14553 & -0.00010 \\
$\mathrm{C}$ & 7.98988 & 0.93141 & -0.00014 \\
$\mathrm{C}$ & 6.59775 & 0.94889 & -0.00011 \\
$\mathrm{~N}$ & 10.15205 & 2.13490 & -0.00014 \\
$\mathrm{C}$ & 10.90260 & 3.38427 & -0.00011 \\
$\mathrm{C}$ & -2.12361 & 1.24721 & 0.00005 \\
$\mathrm{~N}$ & -3.32462 & 1.25834 & 0.00009 \\
$\mathrm{C}$ & 10.88391 & 0.87452 & -0.00019 \\
$\mathrm{H}$ & 8.54763 & 4.32775 & -0.00001 \\
$\mathrm{H}$ & 6.07517 & 4.33267 & 0.00003 \\
$\mathrm{H}$ & 6.05109 & -0.01073 & -0.00014 \\
$\mathrm{H}$ & 8.51567 & -0.03281 & -0.00020 \\
$\mathrm{H}$ & 3.99141 & 3.25053 & 0.00002 \\
\hline & & Continued & on next page
\end{tabular}




\begin{tabular}{lrrr}
\multicolumn{4}{c}{ Continued from previous page } \\
\hline $\mathrm{H}$ & 4.01472 & 0.12439 & -0.00004 \\
$\mathrm{H}$ & 1.93927 & -0.95816 & -0.00001 \\
$\mathrm{H}$ & 1.97008 & 3.38731 & -0.00000 \\
$\mathrm{H}$ & -0.52720 & 3.40667 & 0.00001 \\
$\mathrm{H}$ & -0.56617 & -0.94235 & 0.00000 \\
$\mathrm{H}$ & 11.98686 & 1.05668 & -0.00024 \\
$\mathrm{H}$ & 10.64412 & 0.25738 & 0.91004 \\
$\mathrm{H}$ & 10.64404 & 0.25741 & -0.91043 \\
$\mathrm{H}$ & 12.00271 & 3.18569 & -0.00018 \\
$\mathrm{H}$ & 10.67196 & 4.00492 & -0.91030 \\
$\mathrm{H}$ & 10.67206 & 4.00482 & 0.91018
\end{tabular}


Table S1296 Employed active space, reference configurations, and Cartesian coordinates of the OM3/MRCISD-optimized excited-state equilibrium geometry of the $2{ }^{1} A\left({ }^{1} \pi \pi^{*}\right)$ state of 4 -(dimethylamino)-4'-cyanostilbene (excited-state energy: $-2790.61998 \mathrm{eV})$.

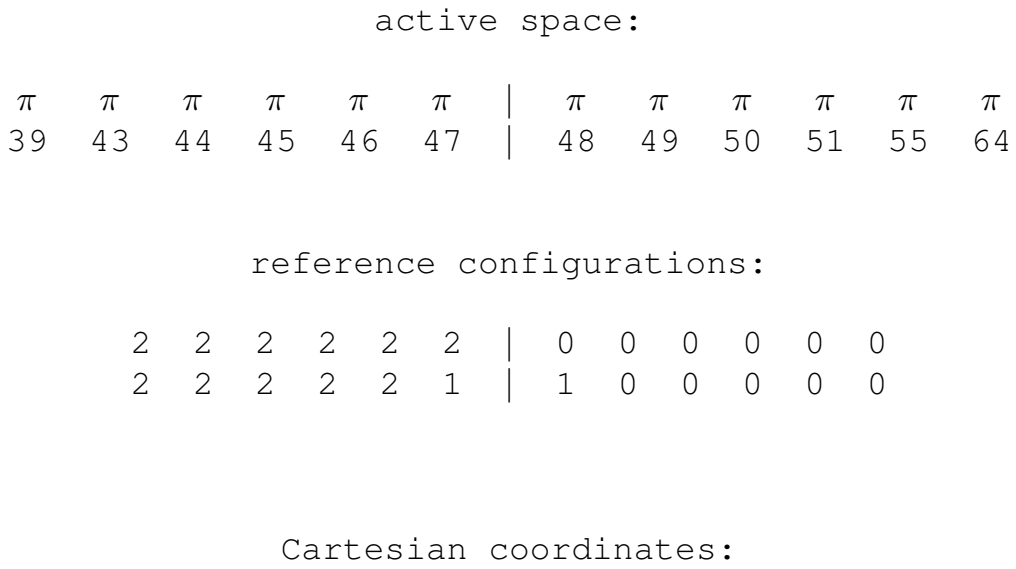




\begin{tabular}{lrrr}
\multicolumn{5}{c}{ Continued from previous page } \\
\hline $\mathrm{H}$ & 3.94403 & 3.29490 & 0.00003 \\
$\mathrm{H}$ & 4.00334 & 0.16038 & -0.00001 \\
$\mathrm{H}$ & 1.93640 & -0.95517 & -0.00002 \\
$\mathrm{H}$ & 1.94627 & 3.41185 & 0.00006 \\
$\mathrm{H}$ & -0.54992 & 3.41048 & 0.00008 \\
$\mathrm{H}$ & -0.56920 & -0.94258 & 0.00000 \\
$\mathrm{H}$ & 11.91609 & 1.07840 & -0.00005 \\
$\mathrm{H}$ & 10.57254 & 0.28645 & 0.90814 \\
$\mathrm{H}$ & 10.57251 & 0.28647 & -0.90819 \\
$\mathrm{H}$ & 11.94718 & 3.18797 & -0.00001 \\
$\mathrm{H}$ & 10.62802 & 4.01948 & -0.90821 \\
$\mathrm{H}$ & 10.62806 & 4.01940 & 0.90833
\end{tabular}


Table S1297 Employed active space, reference configurations, and Cartesian coordinates of the OM3/MRCISD-optimized ground-state equilibrium geometry of dimethylaminobenzonitrile (ground-state energy: -1705.52029 eV).

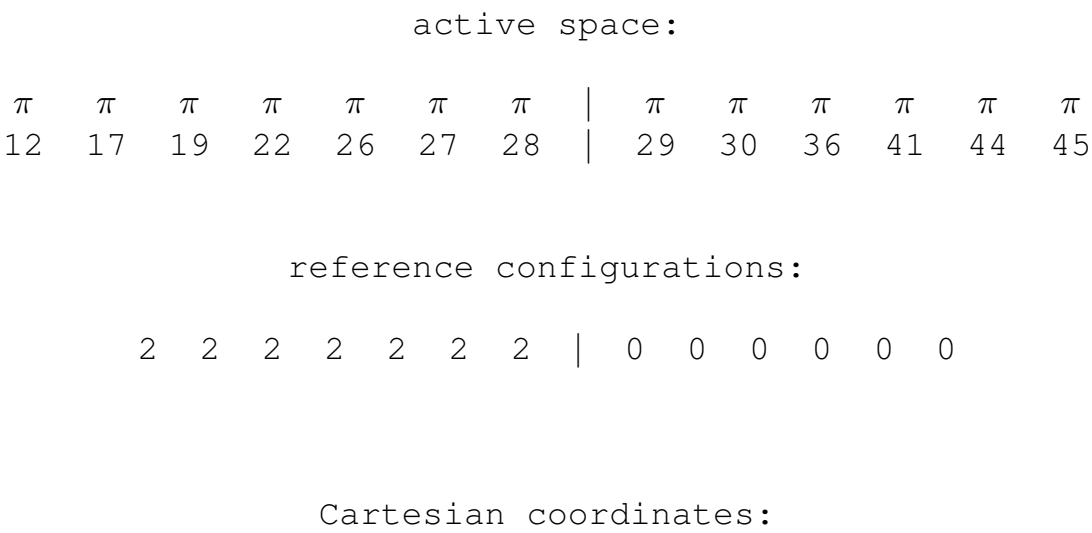


Table S1298 Employed active space, reference configurations, and Cartesian coordinates of the OM3/MRCISD-optimized excited-state equilibrium geometry of the $1^{1} B\left({ }^{1} \pi \pi^{*}\right)$ state of dimethylaminobenzonitrile (excited-state energy: $-1701.72331 \mathrm{eV})$.

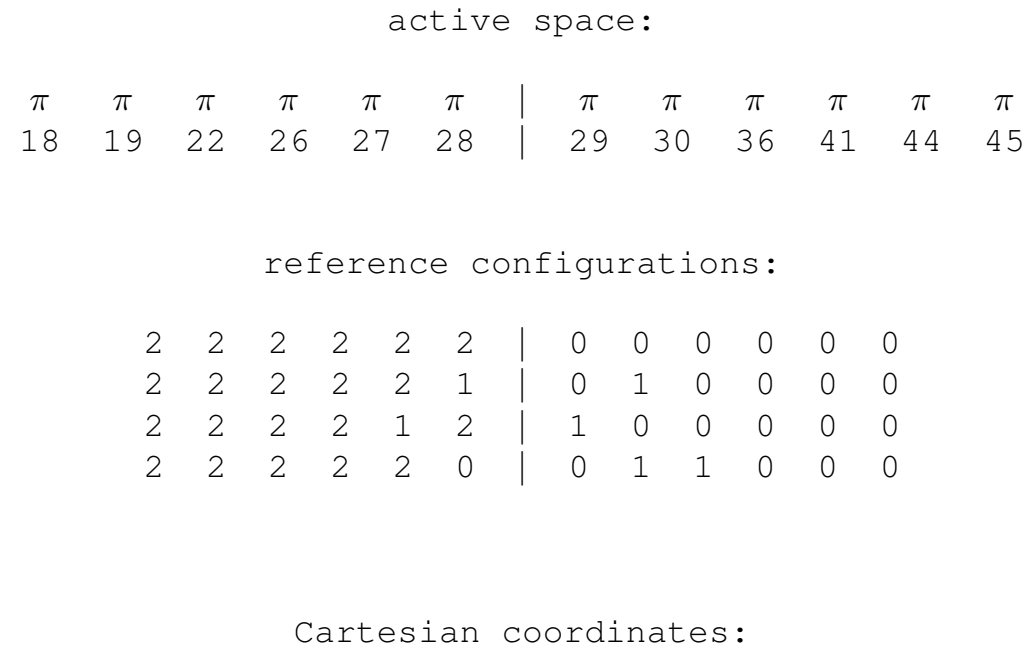

21

$\begin{array}{lrrr}\mathrm{C} & 0.00000 & 0.00000 & 0.00000 \\ \mathrm{C} & 0.00000 & 2.81639 & 0.00000 \\ \mathrm{C} & 0.00000 & 0.71584 & 1.24911 \\ \mathrm{C} & 0.00000 & 0.71584 & -1.24911 \\ \mathrm{C} & -0.00001 & 2.13565 & -1.24802 \\ \mathrm{C} & 0.00001 & 2.13565 & 1.24802 \\ \mathrm{C} & 0.00000 & 4.25682 & 0.00000 \\ \mathrm{~N} & 0.00000 & 5.46440 & 0.00000 \\ \mathrm{~N} & 0.00000 & -1.40496 & -0.00000 \\ \mathrm{C} & -0.00002 & -2.16475 & 1.25216 \\ \mathrm{C} & 0.00002 & -2.16475 & -1.25216 \\ \mathrm{H} & -0.00000 & 0.18303 & 2.20618 \\ \mathrm{H} & 0.00000 & 0.18303 & -2.20618 \\ \mathrm{H} & -0.00002 & 2.69273 & -2.19137 \\ \mathrm{H} & 0.00002 & 2.69273 & 2.19137 \\ \mathrm{H} & -0.00001 & -3.26258 & 1.05194 \\ \mathrm{H} & 0.00001 & -3.26258 & -1.05194 \\ \mathrm{H} & 0.90699 & -1.92875 & 1.86650 \\ \mathrm{H} & -0.90699 & -1.92875 & -1.86650 \\ \mathrm{H} & -0.90703 & -1.92876 & 1.86648 \\ \mathrm{H} & 0.90703 & -1.92876 & -1.86648\end{array}$


Table S1299 Employed active space, reference configurations, and Cartesian coordinates of the OM3/MRCISD-optimized ground-state equilibrium geometry of $N, N$-dimethyl-p-phenylenediamine (ground-state energy: $-1610.38612 \mathrm{eV}$ ).

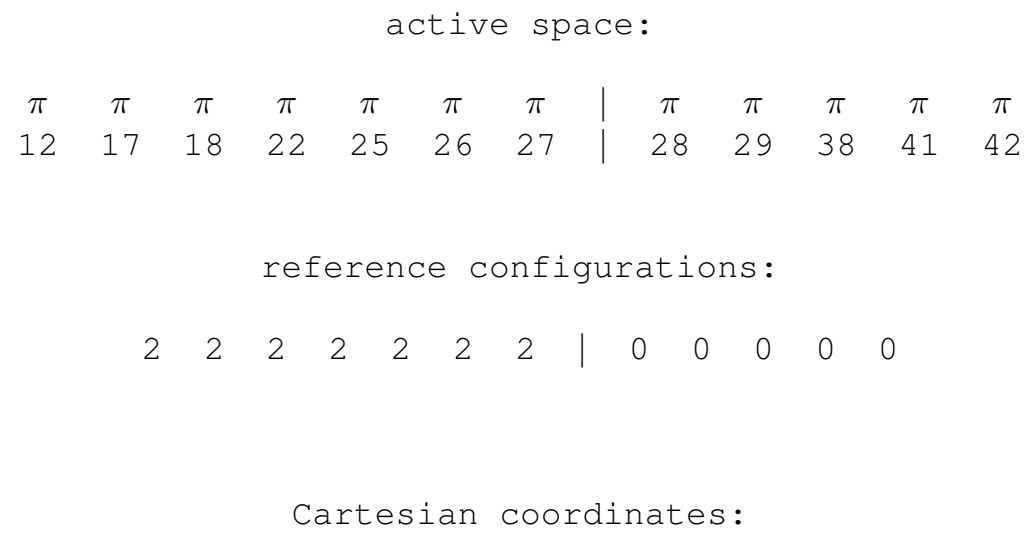


Table S1300 Employed active space, reference configurations, and Cartesian coordinates of the OM3/MRCISD-optimized excited-state equilibrium geometry of the $2{ }^{1} A_{1}\left({ }^{1} \pi \pi^{*}\right)$ state of $N, N$-dimethyl-p-phenylenediamine (excited-state energy: $-1605.93101 \mathrm{eV})$.

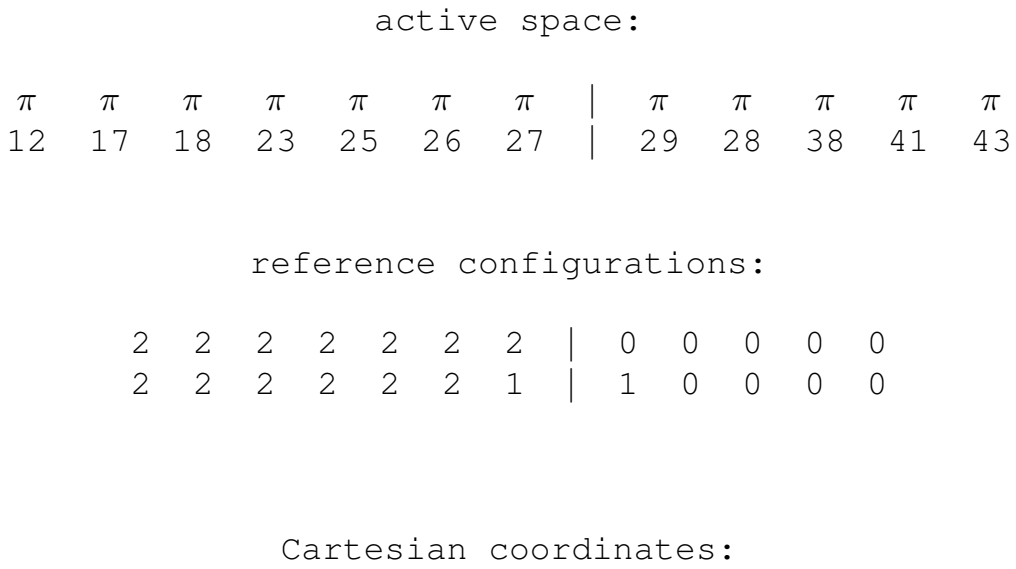


Table S1301 Employed active space, reference configurations, and Cartesian coordinates of the OM3/MRCISD-optimized ground-state equilibrium geometry of fluorene (ground-state energy: $-1775.14165 \mathrm{eV}$ ).

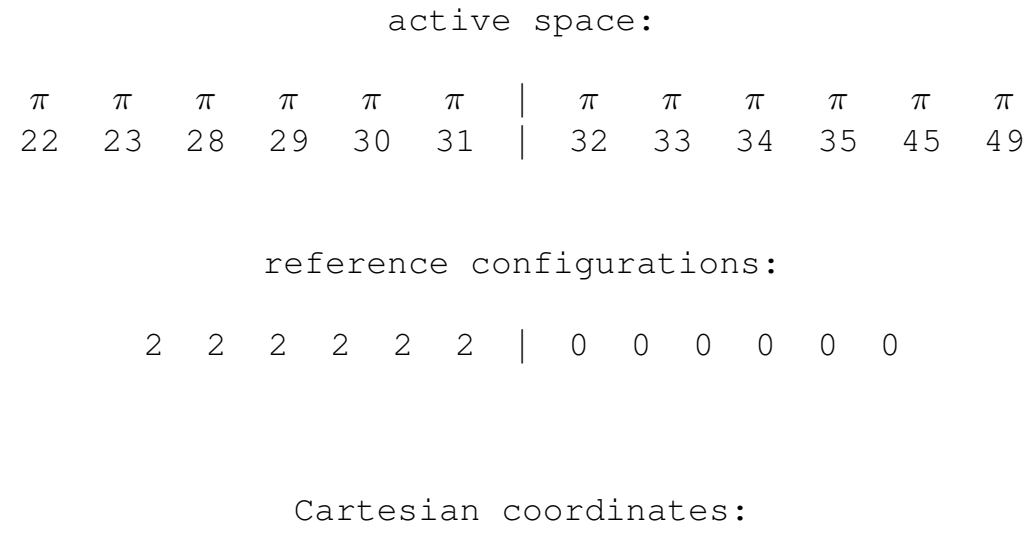

23

$\begin{array}{lrrr}C & 0.00000 & 0.00000 & 0.00000\end{array}$

$\begin{array}{lrrr}\text { C } & -0.94615 & 1.18037 & 0.00000\end{array}$

C $\quad-0.94615-1.18037-0.00000$

$\begin{array}{llll}\text { C } & -2.30255 & 0.73880 & -0.00000\end{array}$

$\begin{array}{llll}\text { C } & -2.30255 & -0.73880 & 0.00000\end{array}$

C $\quad-0.64608 \quad 2.54463 \quad-0.00000$

C $\quad-0.64608-2.54463-0.00000$

C $\quad-3.35686 \quad 1.65763-0.00000$

$\begin{array}{llll}\text { C } & -3.35686 & -1.65763 & -0.00000\end{array}$

C $\quad-1.71024 \quad 3.46329-0.00000$

$\begin{array}{llll}\text { C } & -1.71024 & -3.46329 & -0.00000\end{array}$

$\begin{array}{llll}\text { C } & -3.04397 & 3.02697 & 0.00000\end{array}$

$\begin{array}{llll}\text { C } & -3.04397 & -3.02697 & -0.00000\end{array}$

$\begin{array}{llll}\mathrm{H} & 0.63248 & 0.00000 & -0.90969\end{array}$

$\begin{array}{llll}\mathrm{H} & 0.63248 & 0.00000 & 0.90969\end{array}$

$\begin{array}{llll}\mathrm{H} & 0.39743 & 2.89311 & -0.00000\end{array}$

$\mathrm{H} \quad 0.39743 \quad-2.89311-0.00000$

$\mathrm{H} \quad-4.40234 \quad 1.31565 \quad-0.00000$

$\begin{array}{llll}\mathrm{H} & -4.40234 & -1.31565 & 0.00000\end{array}$

$\begin{array}{llll}\mathrm{H} & -1.49289 & 4.54407 & -0.00000\end{array}$

$\mathrm{H} \quad-1.49289-4.54407 \quad-0.00000$

$\begin{array}{lrrr}\mathrm{H} & -3.85790 & 3.77027 & 0.00000\end{array}$

$\mathrm{H} \quad-3.85790 \quad-3.77027 \quad-0.00000$ 
Table S1302 Employed active space, reference configurations, and Cartesian coordinates of the OM3/MRCISD-optimized excited-state equilibrium geometry of the $2{ }^{1} A^{\prime}\left({ }^{1} \pi \pi^{*}\right)$ state of fluorene (excited-state energy: $-1770.92124 \mathrm{eV}$ ).

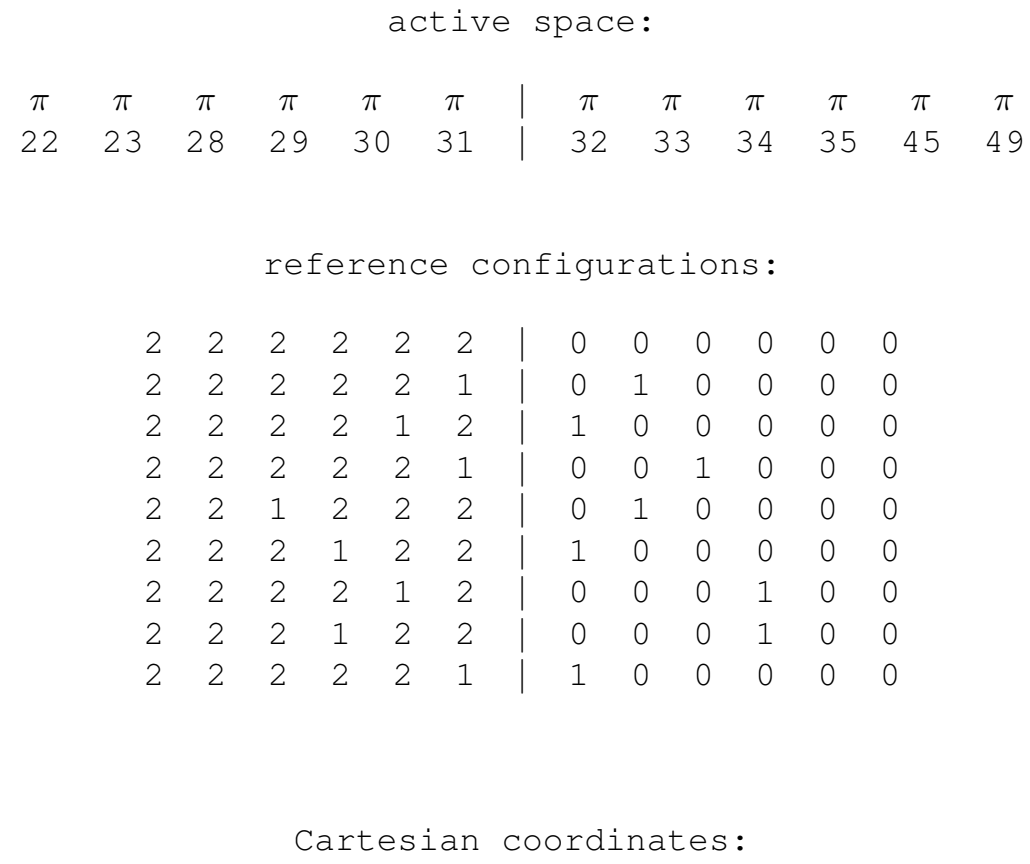

\begin{tabular}{lrrr}
23 & & & \\
C & 0.00000 & 0.00000 & 0.00000 \\
C & 1.39820 & 0.00000 & 0.00000 \\
C & 2.07065 & 1.23403 & 0.00000 \\
C & 1.33088 & 2.45913 & 0.00000 \\
C & -0.06056 & 2.44262 & 0.00000 \\
C & -0.72266 & 1.20365 & 0.00000 \\
C & 3.48410 & 1.54306 & -0.00000 \\
C & 3.62165 & 2.99490 & -0.00000 \\
C & 2.25774 & 3.64468 & -0.00000 \\
C & 4.63622 & 0.70230 & -0.00000 \\
C & 5.91679 & 1.33291 & -0.00000 \\
C & 6.04711 & 2.75139 & -0.00000 \\
C & 4.91191 & 3.60663 & -0.00000 \\
H & 2.12798 & 4.26288 & 0.90999 \\
H & 2.12798 & 4.26288 & -0.90999 \\
H & 5.01774 & 4.69839 & -0.00000 \\
H & -0.63408 & 3.38186 & -0.00000 \\
\hline & & Continued on next page
\end{tabular}

S1386 


\begin{tabular}{lrrr}
\multicolumn{4}{c}{ Continued from previous page } \\
\hline $\mathrm{H}$ & 4.54072 & -0.39128 & -0.00000 \\
$\mathrm{H}$ & 1.96603 & -0.94217 & -0.00000 \\
$\mathrm{H}$ & 7.05654 & 3.19541 & -0.00000 \\
$\mathrm{H}$ & -1.82413 & 1.17578 & 0.00000 \\
$\mathrm{H}$ & 6.82391 & 0.70827 & -0.00000 \\
$\mathrm{H}$ & -0.54577 & -0.95768 & -0.00000
\end{tabular}


Table S1303 Employed active space, reference configurations, and Cartesian coordinates of the OM3/MRCISD-optimized ground-state equilibrium geometry of glyoxal (ground-state energy: $-957.55599 \mathrm{eV}$ ).

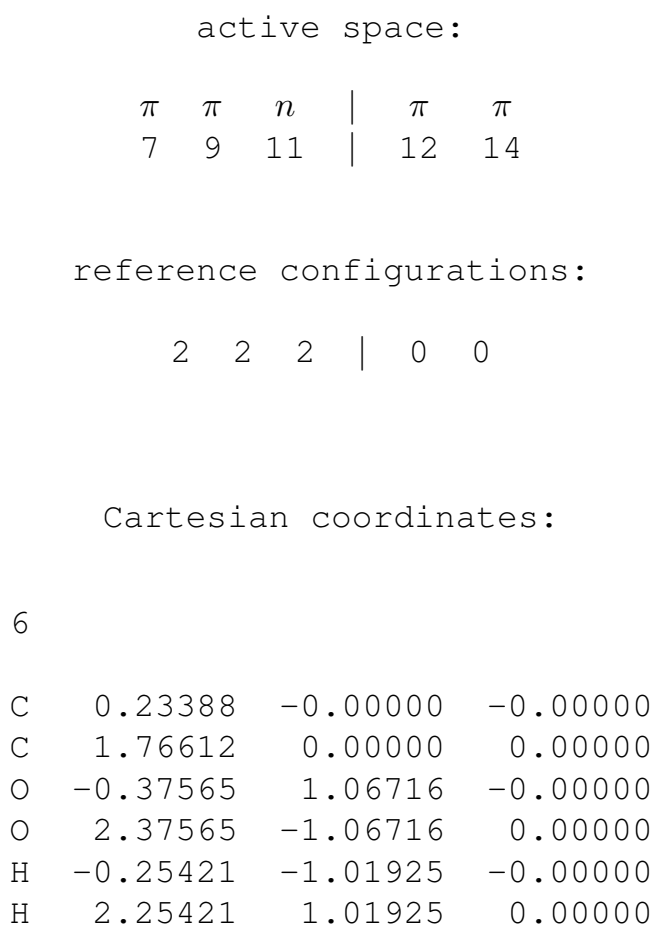


Table S1304 Employed active space, reference configurations, and Cartesian coordinates of the OM3/MRCISD-optimized excited-state equilibrium geometry of the $1{ }^{1} A_{u}\left({ }^{1} n \pi^{*}\right)$ state of glyoxal (excited-state energy: $-954.55475 \mathrm{eV}$ ).

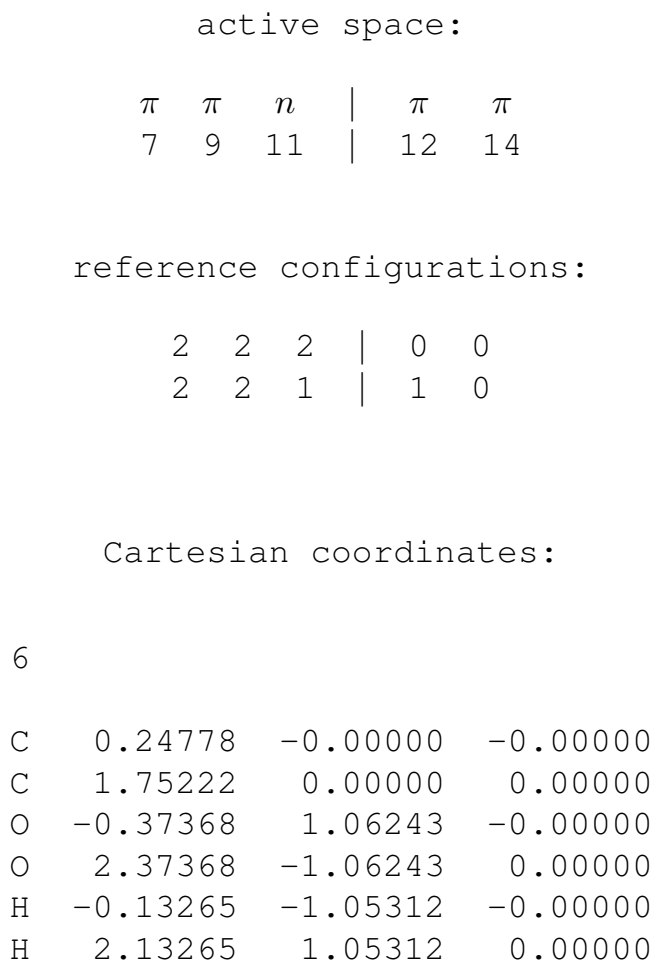


Table S1305 Employed active space, reference configurations, and Cartesian coordinates of the OM3/MRCISD-optimized ground-state equilibrium geometry of hydrogen cyanide (ground-state energy: $-355.35555 \mathrm{eV}$ ).

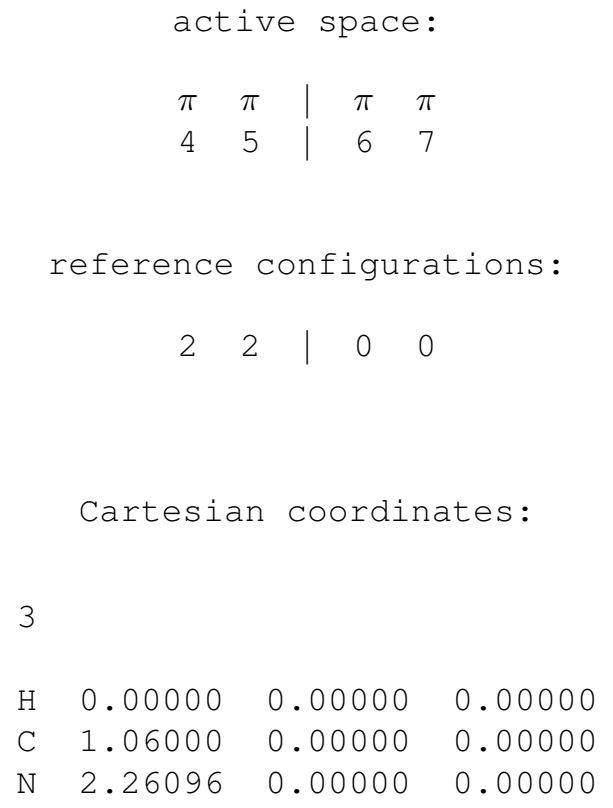


Table S1306 Employed active space, reference configurations, and Cartesian coordinates of the OM3/MRCISD-optimized excited-state equilibrium geometry of the $1^{1} A^{\prime \prime}\left({ }^{1} \pi \pi^{*}\right)$ state of hydrogen cyanide (excited-state energy: $-349.04582 \mathrm{eV})$.

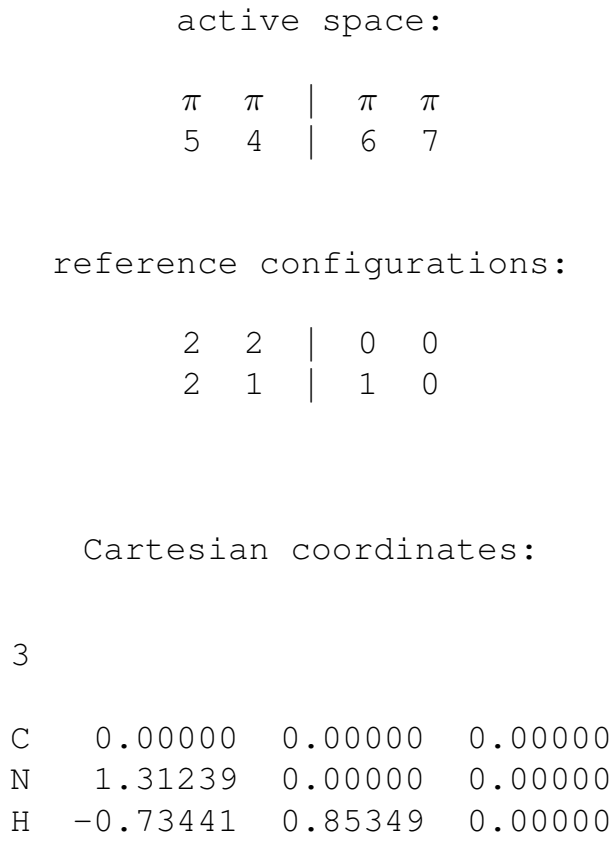


Table S1307 Employed active space, reference configurations, and Cartesian coordinates of the OM3/MRCISD-optimized ground-state equilibrium geometry of formic acid (ground-state energy: $-833.00973 \mathrm{eV}$ ).

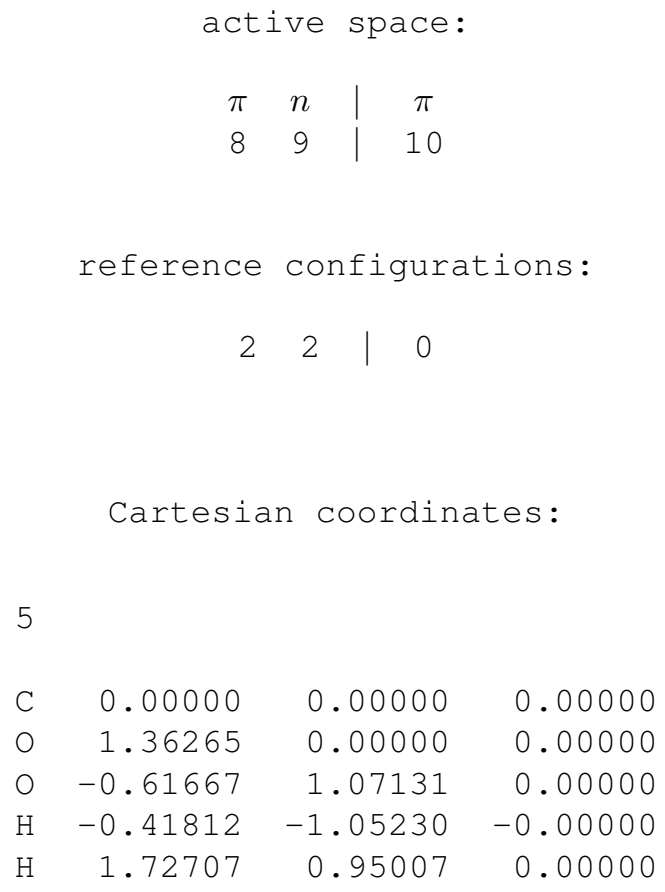

$\begin{array}{llll}\mathrm{H} & 1.72707 & 0.95007 & 0.00000\end{array}$ 
Table S1308 Employed active space, reference configurations, and Cartesian coordinates of the OM3/MRCISD-optimized excited-state equilibrium geometry of the $2{ }^{1} A\left({ }^{1} n \pi^{*}\right)$ state of formic acid (excited-state energy: $-828.61619 \mathrm{eV}$ ).

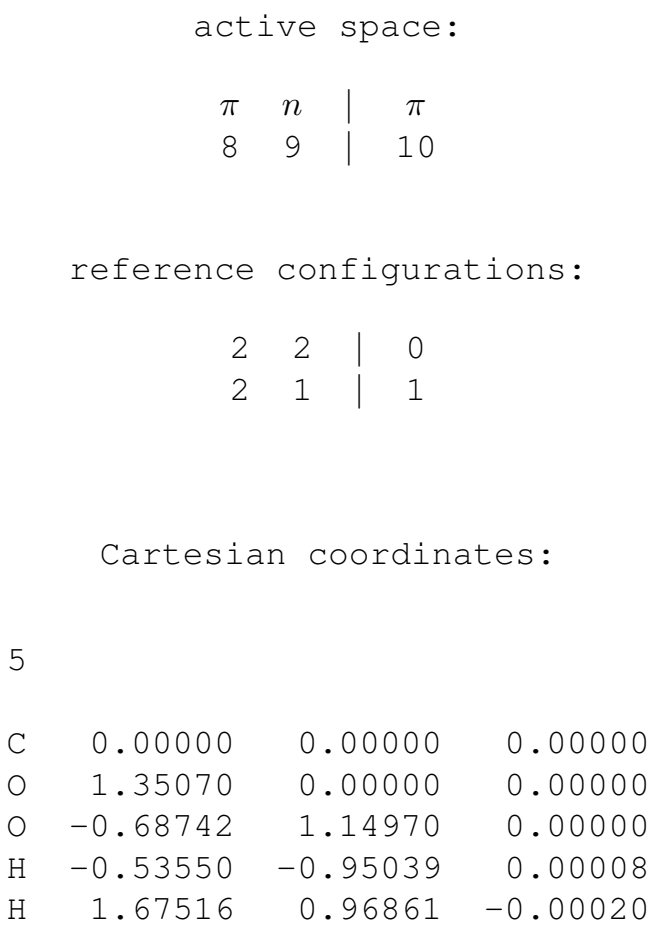


Table S1309 Employed active space, reference configurations, and Cartesian coordinates of the OM3/MRCISD-optimized ground-state equilibrium geometry of hexatriene (ground-state energy: $-868.23660 \mathrm{eV}$ ).

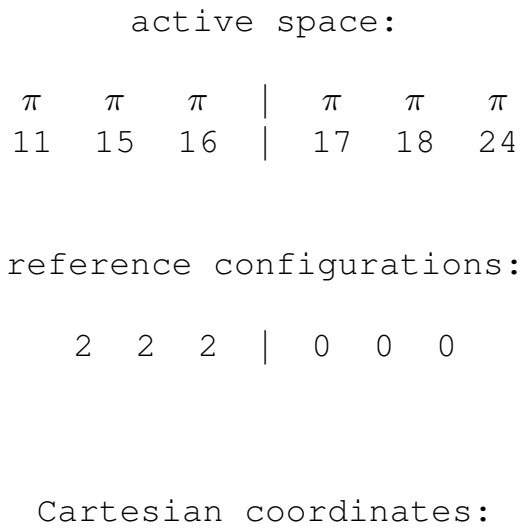


Table S1310 Employed active space, reference configurations, and Cartesian coordinates of the OM3/MRCISD-optimized excited-state equilibrium geometry of the $1{ }^{1} B_{u}\left({ }^{1} \pi \pi^{*}\right)$ state of hexatriene (excited-state energy: $-863.14827 \mathrm{eV}$ ).

\begin{tabular}{ccc|ccc}
$\pi$ & $\pi$ & $\pi$ & $\pi$ & $\pi$ & $\pi$ \\
11 & 15 & 16 & 17 & 18 & 24 \\
reference & configurations: \\
2 & 2 & 2 & 0 & 0 & 0 \\
2 & 2 & 1 & 1 & 0 & 0
\end{tabular}

Cartesian coordinates:

14

$\begin{array}{lrrr}C & 0.00000 & 0.70766 & 0.00000\end{array}$

C $\quad 0.00000-0.70766-0.00000$

C $\quad 0.00000 \quad 1.45774 \quad 1.19864$

C $\quad 0.00000-1.45774-1.19864$

$\begin{array}{llll}\text { C } & 0.00000 & 2.83837 & 1.21958\end{array}$

C $\quad 0.00000-2.83837-1.21958$

$\mathrm{H} \quad 0.00000 \quad 1.24223-0.96652$

$\mathrm{H} \quad 0.00000 \quad-1.24223 \quad 0.96652$

$\begin{array}{lrrr}\mathrm{H} & 0.00000 & 0.90327 & 2.15599\end{array}$

$\mathrm{H} \quad 0.00000 \quad-0.90327 \quad-2.15599$

$\begin{array}{llll}\mathrm{H} & 0.00000 & 3.40057 & 2.15143\end{array}$

$\mathrm{H} \quad 0.00000 \quad-3.40057 \quad-2.15143$

$\begin{array}{llll}\mathrm{H} & 0.00000 & 3.42075 & 0.29873\end{array}$

$\mathrm{H} \quad 0.00000 \quad-3.42075 \quad-0.29873$ 
Table S1311 Employed active space, reference configurations, and Cartesian coordinates of the OM3/MRCISD-optimized ground-state equilibrium geometry of trans-hydroquinone (ground-state energy: $-1517.83018 \mathrm{eV}$ ).

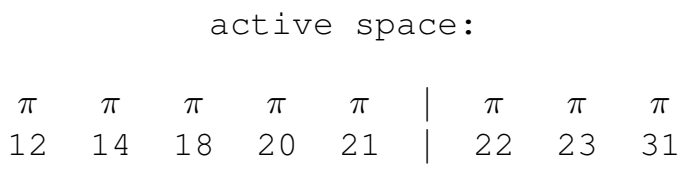

reference configurations:

\begin{tabular}{lllll|llll}
2 & 2 & 2 & 2 & 2 & 0 & 0 & 0
\end{tabular}

Cartesian coordinates:

14

$\begin{array}{lrrr}\mathrm{C} & 0.00000 & 0.00000 & 0.00000 \\ \mathrm{C} & 2.76784 & 0.00000 & 0.00000 \\ \mathrm{C} & 0.68311 & 1.23518 & 0.00000 \\ \mathrm{C} & 2.08473 & -1.23518 & 0.00000 \\ \mathrm{C} & 0.68779 & -1.23203 & -0.00000 \\ \mathrm{C} & 2.08005 & 1.23203 & -0.00000 \\ \mathrm{O} & -1.38107 & 0.08593 & 0.00000 \\ \mathrm{O} & 4.14891 & -0.08593 & 0.00000 \\ \mathrm{H} & 0.10846 & 2.16794 & -0.00000 \\ \mathrm{H} & 2.65938 & -2.16794 & 0.00000 \\ \mathrm{H} & 0.12657 & -2.17698 & -0.00000 \\ \mathrm{H} & 2.64127 & 2.17698 & -0.00000 \\ \mathrm{H} & -1.77727 & -0.85110 & 0.00000 \\ \mathrm{H} & 4.54511 & 0.85110 & -0.00000\end{array}$


Table S1312 Employed active space, reference configurations, and Cartesian coordinates of the OM3/MRCISD-optimized excited-state equilibrium geometry of the $1^{1} B_{u}\left({ }^{1} \pi \pi^{*}\right)$ state of trans-hydroquinone (excited-state energy: $-1513.92435 \mathrm{eV})$.

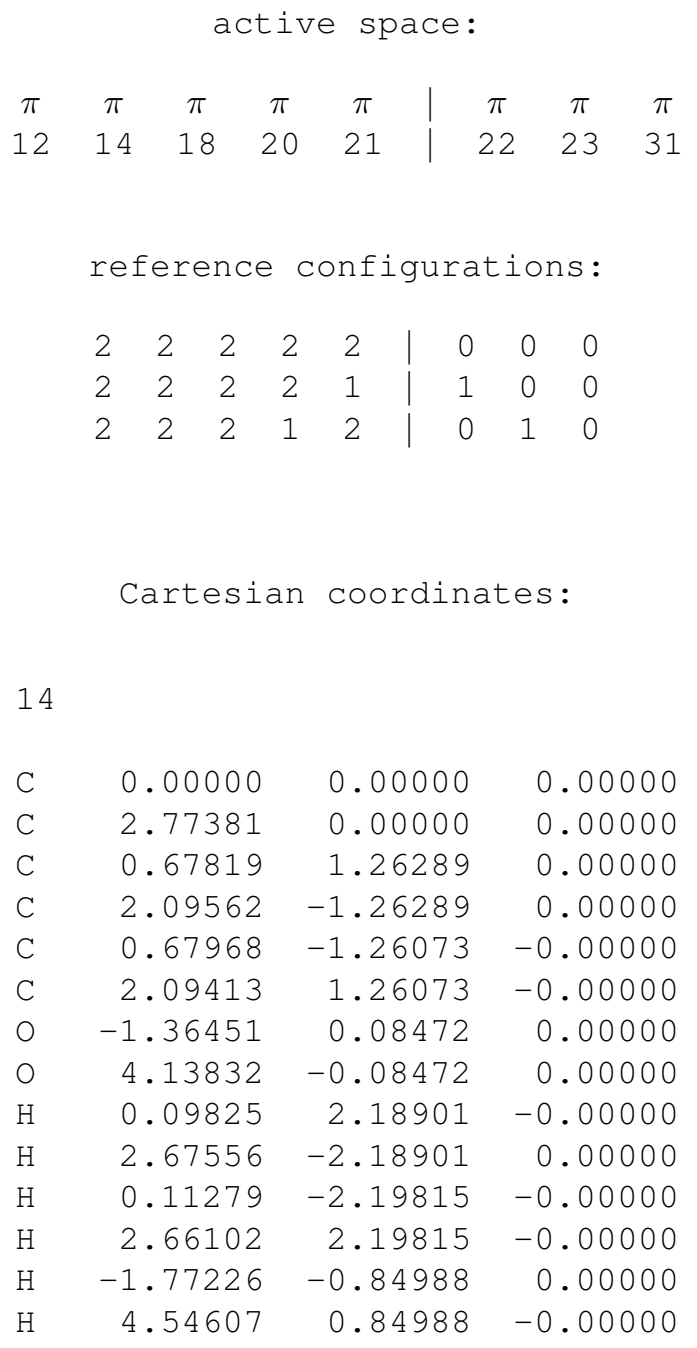


Table S1313 Employed active space, reference configurations, and Cartesian coordinates of the OM3/MRCISD-optimized ground-state equilibrium geometry of indole (ground-state energy: $-1320.38627 \mathrm{eV}$ ).

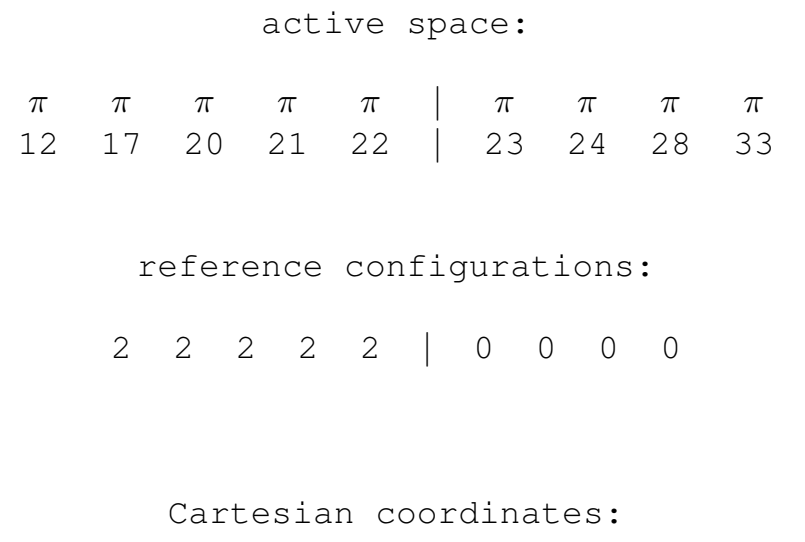

$\begin{array}{lrrr}16 & & & \\ & & & \\ \mathrm{C} & 0.00000 & 0.00000 & 0.00000 \\ \mathrm{~N} & 1.41329 & 0.00000 & 0.00000 \\ \mathrm{C} & 1.85001 & 1.34645 & 0.00000 \\ \mathrm{C} & 0.67293 & 2.18840 & 0.00000 \\ \mathrm{C} & -0.47330 & 1.31677 & 0.00000 \\ \mathrm{C} & 3.15757 & 1.88598 & -0.00000 \\ \mathrm{C} & 3.26948 & 3.27356 & -0.00000 \\ \mathrm{C} & 2.12770 & 4.11290 & -0.00000 \\ \mathrm{C} & 0.83655 & 3.59018 & -0.00000 \\ \mathrm{H} & 4.03792 & 1.22884 & -0.00000 \\ \mathrm{H} & 4.27055 & 3.73663 & -0.00000 \\ \mathrm{H} & 2.27504 & 5.20509 & -0.00000 \\ \mathrm{H} & -0.04906 & 4.24422 & -0.00000 \\ \mathrm{H} & 2.02386 & -0.84262 & -0.00000 \\ \mathrm{H} & -1.51324 & 1.62298 & -0.00000 \\ \mathrm{H} & -0.58528 & -0.92673 & -0.00000\end{array}$


Table S1314 Employed active space, reference configurations, and Cartesian coordinates of the OM3/MRCISD-optimized excited-state equilibrium geometry of the $2{ }^{1} A^{\prime}\left({ }^{1} \pi \pi^{*}\right)$ state of indole (excited-state energy: $-1316.49074 \mathrm{eV}$ ).

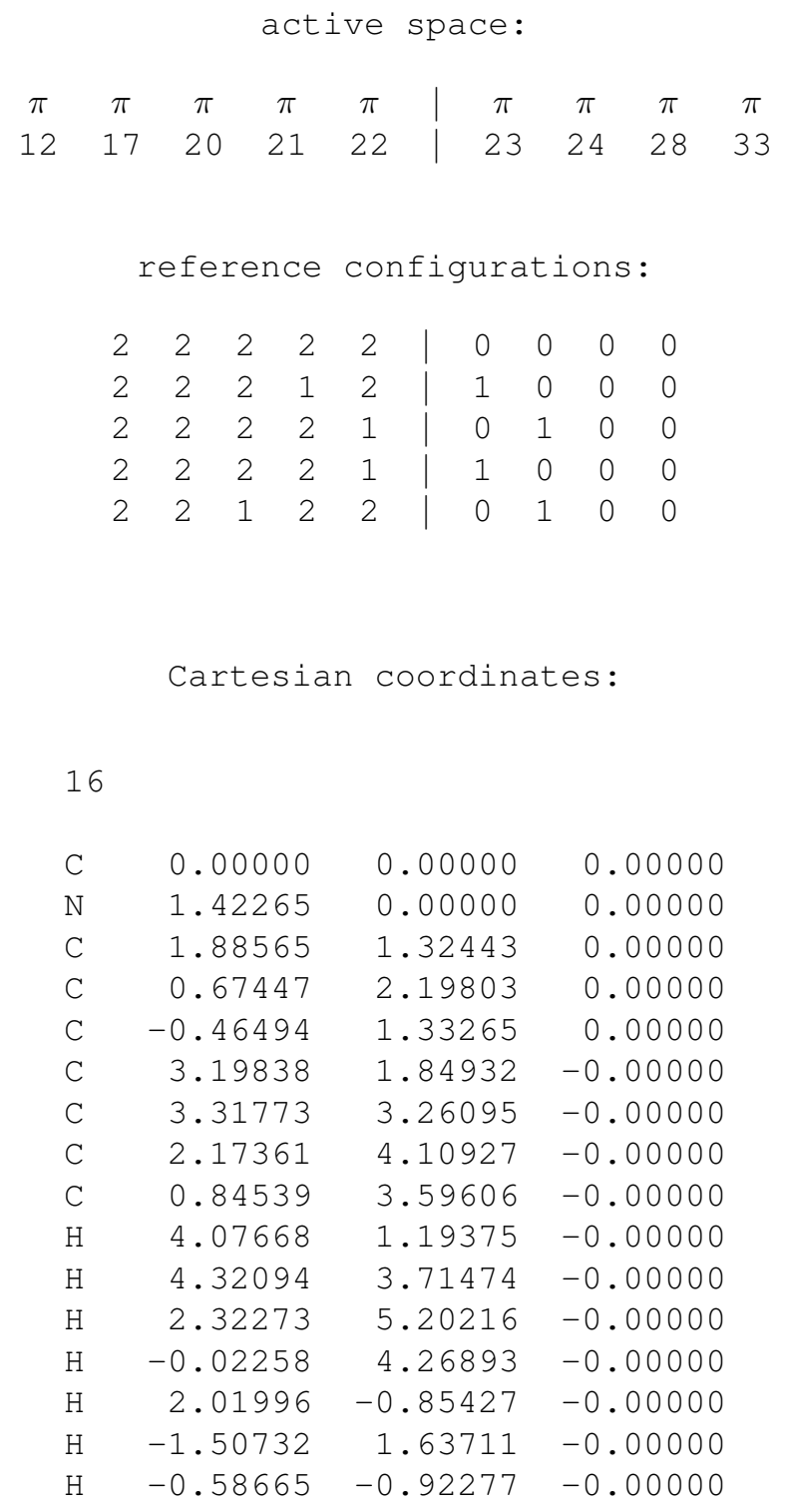


Table S1315 Employed active space, reference configurations, and Cartesian coordinates of the OM3/MRCISD-optimized ground-state equilibrium geometry of methyl-4-hydroxycinnamate (ground-state energy: -2416.32919 eV).

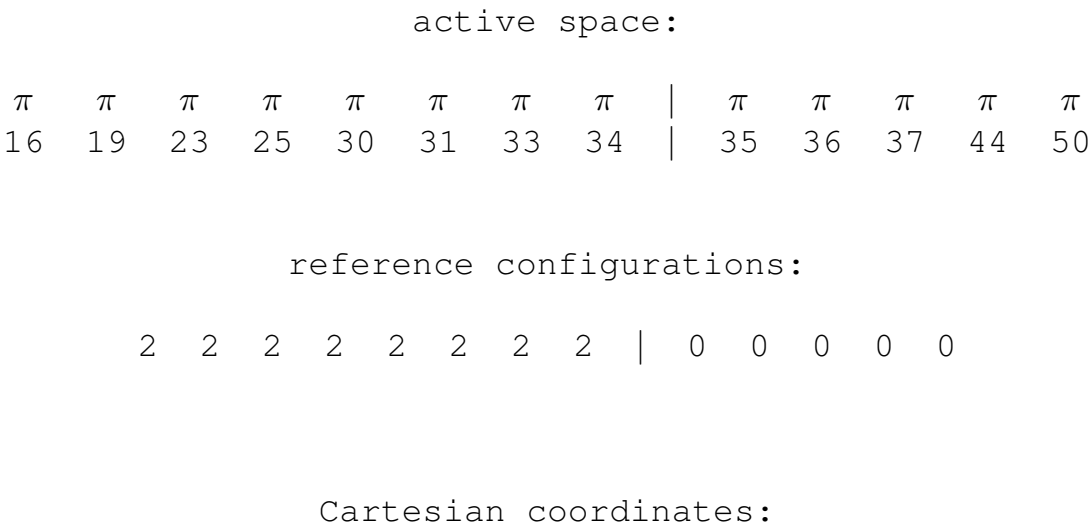


Table S1316 Employed active space, reference configurations, and Cartesian coordinates of the OM3/MRCISD-optimized excited-state equilibrium geometry of the $2{ }^{1} A\left({ }^{1} \pi \pi^{*}\right)$ state of methyl-4-hydroxycinnamate (excited-state energy: $-2412.38088 \mathrm{eV})$.

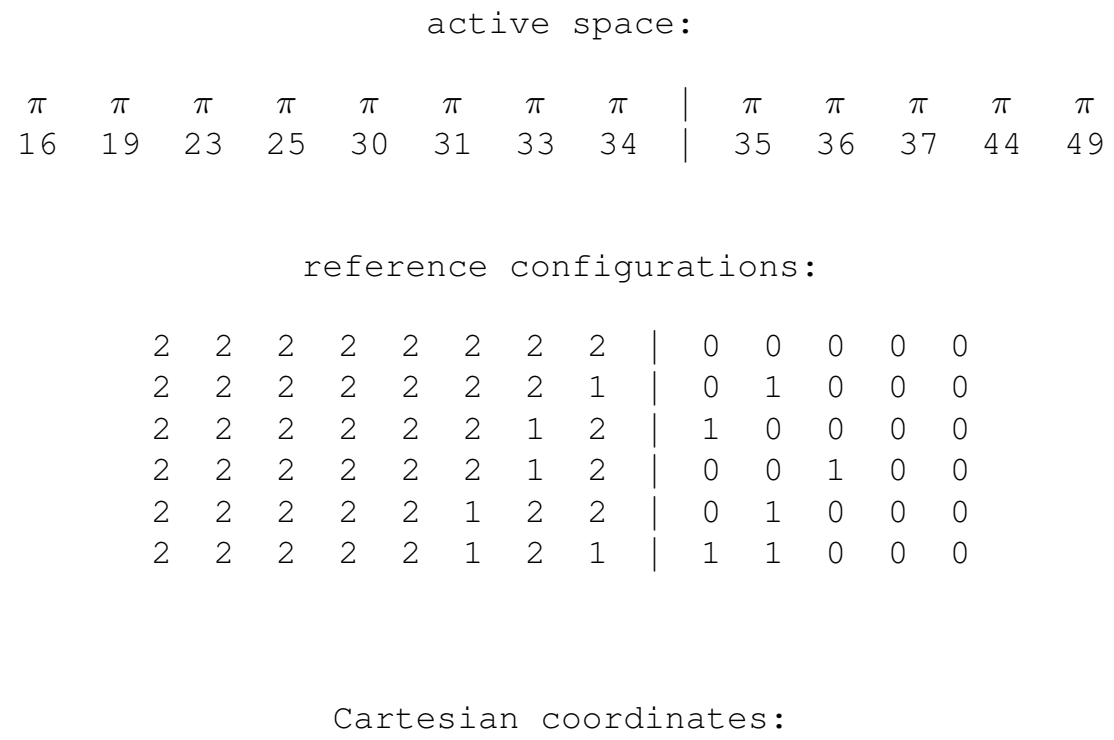

\begin{tabular}{lrrr}
23 & & & \\
$\mathrm{C}$ & 0.00000 & 0.00000 & 0.00000 \\
$\mathrm{C}$ & 1.42136 & 0.00000 & 0.00000 \\
$\mathrm{C}$ & 2.14944 & 1.24081 & 0.00000 \\
$\mathrm{C}$ & 1.42783 & 2.49016 & 0.00005 \\
$\mathrm{C}$ & 0.00919 & 2.50495 & 0.00014 \\
$\mathrm{C}$ & -0.68230 & 1.25485 & 0.00005 \\
$\mathrm{C}$ & 3.58927 & 1.29355 & 0.00004 \\
$\mathrm{C}$ & 4.40275 & 0.18979 & 0.00015 \\
$\mathrm{C}$ & 5.87403 & 0.28889 & 0.00019 \\
$\mathrm{O}$ & 6.69535 & -0.63606 & -0.00002 \\
$\mathrm{O}$ & -2.04918 & 1.17568 & 0.00009 \\
$\mathrm{O}$ & 6.32176 & 1.61931 & 0.00048 \\
$\mathrm{C}$ & 7.74191 & 1.75693 & 0.00059 \\
$\mathrm{H}$ & 4.02426 & -0.83972 & 0.00015 \\
$\mathrm{H}$ & 4.05255 & 2.29677 & 0.00004 \\
$\mathrm{H}$ & 1.98463 & 3.43925 & 0.00001 \\
$\mathrm{H}$ & -0.54858 & 3.44889 & 0.00019 \\
$\mathrm{H}$ & -0.57764 & -0.92879 & -0.00002 \\
$\mathrm{H}$ & 1.95753 & -0.95929 & -0.00004 \\
\hline & & \multicolumn{3}{c}{ Continued } \\
& & &
\end{tabular}

S1401 


\begin{tabular}{lrrr}
\multicolumn{5}{c}{ Continued from previous page } \\
\hline H & -2.45131 & 2.11242 & 0.00010 \\
H & 7.93496 & 2.85707 & 0.00214 \\
H & 8.19305 & 1.30818 & 0.90971 \\
H & 8.19282 & 1.31084 & -0.91004
\end{tabular}


Table S1317 Employed active space, reference configurations, and Cartesian coordinates of the OM3/MRCISD-optimized ground-state equilibrium geometry of naphthalene (ground-state energy: $-1370.10774 \mathrm{eV}$ ).

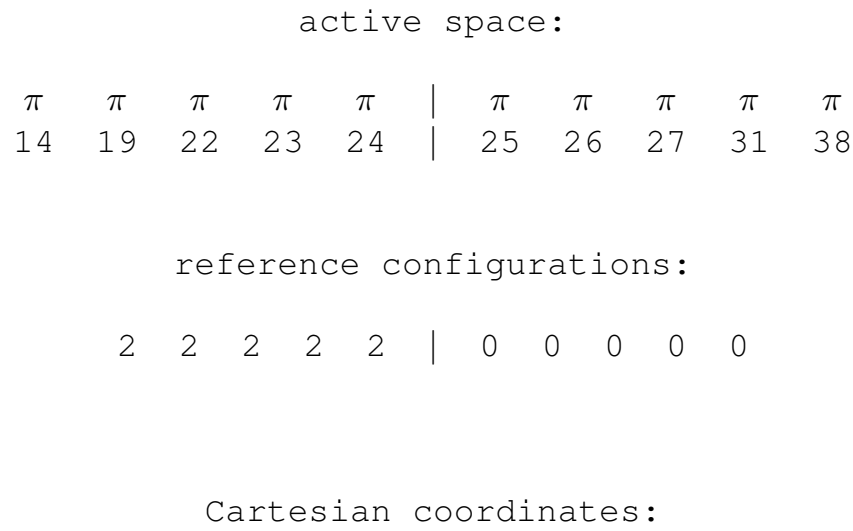


Table S1318 Employed active space, reference configurations, and Cartesian coordinates of the OM3/MRCISD-optimized excited-state equilibrium geometry of the $1{ }^{1} B_{2 u}\left({ }^{1} \pi \pi^{*}\right)$ state of naphthalene (excited-state energy: $-1365.44599 \mathrm{eV})$.

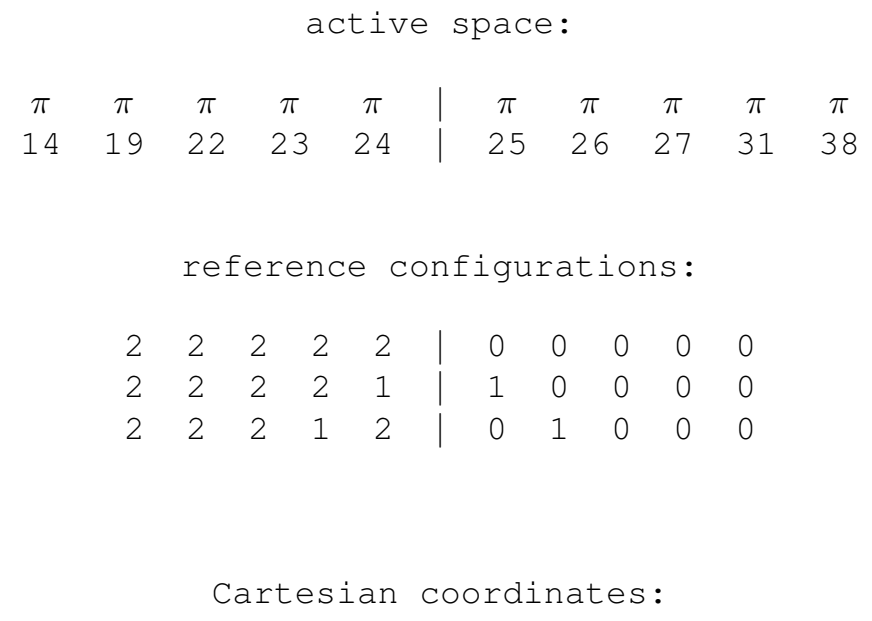

18

C $\quad 1.00000$

$1.72557 \quad 0.00000$

C $\quad 1.00000$

$0.27443-0.00000$

$\begin{array}{lrrr}\text { C } & 2.23992 & 2.40952 & 0.00000\end{array}$

$\begin{array}{lrrr}\text { C } & -0.23992 & 2.40952 & 0.00000\end{array}$

$\begin{array}{llll}\text { C } & 2.23992 & -0.40952 & 0.00000\end{array}$

$\begin{array}{llll}\text { C } & -0.23992 & -0.40952 & 0.00000\end{array}$

$\begin{array}{llll}\text { C } & 3.46610 & 1.69558 & 0.00000\end{array}$

C $\quad-1.46610 \quad 1.69558 \quad 0.00000$

$\begin{array}{llll}\text { C } & 3.46610 & 0.30442 & 0.00000\end{array}$

$\begin{array}{llll}\text { C } & -1.46610 & 0.30442 & 0.00000\end{array}$

$\begin{array}{llll}\mathrm{H} & 2.25129 & 3.51196 & 0.00000\end{array}$

$\mathrm{H} \quad-0.25129 \quad 3.51196 \quad 0.00000$

$\mathrm{H} \quad 2.25129 \quad-1.51196 \quad 0.00000$

$\mathrm{H} \quad-0.25129-1.51196 \quad 0.00000$

$\begin{array}{llll}\mathrm{H} & 4.41528 & 2.25457 & 0.00000\end{array}$

$\mathrm{H} \quad-2.41528 \quad 2.25457 \quad 0.00000$

$\mathrm{H} \quad 4.41528 \quad-0.25457 \quad 0.00000$

$\mathrm{H} \quad-2.41528 \quad-0.25457 \quad 0.00000$ 
Table S1319 Employed active space, reference configurations, and Cartesian coordinates of the OM3/MRCISD-optimized ground-state equilibrium geometry of octatetraene (ground-state energy: $-1147.81666 \mathrm{eV}$ ).

\begin{tabular}{lccc|cccc} 
active space: \\
$\pi$ & $\pi$ & $\pi$ & $\pi$ & $\pi$ & $\pi$ & $\pi$ & $\pi$ \\
14 & 18 & 20 & 21 & 22 & 23 & 27 & 32
\end{tabular}

reference configurations:

\begin{tabular}{llll|llll}
2 & 2 & 2 & 2 & 0 & 0 & 0 & 0
\end{tabular}

Cartesian coordinates:

$\begin{array}{lrrr}18 & & & \\ & & & \\ \mathrm{C} & 0.00000 & 0.00000 & 0.00000 \\ \mathrm{C} & 1.35198 & 0.00000 & 0.00000 \\ \mathrm{C} & 2.13286 & 1.23133 & 0.00000 \\ \mathrm{C} & 3.49802 & 1.23051 & 0.00000 \\ \mathrm{C} & 4.27888 & 2.45454 & -0.00000 \\ \mathrm{C} & 5.64404 & 2.45372 & 0.00000 \\ \mathrm{C} & 6.42492 & 3.68505 & -0.00000 \\ \mathrm{C} & 7.77690 & 3.68505 & 0.00000 \\ \mathrm{H} & 4.05506 & 0.27603 & 0.00000 \\ \mathrm{H} & 3.72184 & 3.40902 & -0.00000 \\ \mathrm{H} & 1.57267 & 2.18352 & -0.00000 \\ \mathrm{H} & 6.20424 & 1.50154 & 0.00000 \\ \mathrm{H} & 1.91888 & -0.94962 & 0.00000 \\ \mathrm{H} & 5.85802 & 4.63467 & -0.00000 \\ \mathrm{H} & -0.57534 & 0.92650 & 0.00000 \\ \mathrm{H} & 8.35224 & 2.75856 & 0.00000 \\ \mathrm{H} & -0.58446 & -0.91952 & -0.00000 \\ \mathrm{H} & 8.36136 & 4.60458 & 0.00000\end{array}$


Table S1320 Employed active space, reference configurations, and Cartesian coordinates of the OM3/MRCISD-optimized excited-state equilibrium geometry of the $1^{1} B_{u}\left({ }^{1} \pi \pi^{*}\right)$ state of octatetraene (excited-state energy: $-1143.29424 \mathrm{eV})$.

\begin{tabular}{|c|c|c|c|c|c|c|c|c|}
\hline$\pi$ & $\pi$ & $\pi$ & $\pi$ & $\pi$ & & $\pi$ & $\pi$ & $\pi$ \\
\hline \multirow[t]{5}{*}{14} & 18 & 20 & 21 & 22 & & 3 & 27 & 32 \\
\hline & \multicolumn{3}{|c|}{ reference } & \multicolumn{5}{|c|}{ configurations: } \\
\hline & 2 & 22 & 2 & 0 & 0 & 0 & 0 & \\
\hline & 2 & 22 & 1 & 1 & 0 & 0 & 0 & \\
\hline & \multicolumn{8}{|c|}{ Cartesian coordinates: } \\
\hline \multicolumn{9}{|c|}{18} \\
\hline $\mathrm{C}$ & 0 . & 00000 & & 0.7027 & & & .000 & 00 \\
\hline $\mathrm{C}$ & 0 . & 00000 & & -0.7027 & & & .000 & 00 \\
\hline $\mathrm{C}$ & 0 . & 00000 & & 1.4596 & & & .183 & 81 \\
\hline $\mathrm{C}$ & 0 . & 00000 & & -1.4596 & & & .183 & \\
\hline $\mathrm{C}$ & 0 . & 00000 & & 2.8824 & & & .173 & 78 \\
\hline $\mathrm{C}$ & 0 . & 00000 & & -2.8824 & & & .173 & \\
\hline $\mathrm{C}$ & 0 . & 00000 & & 3.6366 & & & .318 & \\
\hline C & 0 . & 00000 & & -3.6366 & & & .318 & 70 \\
\hline $\mathrm{H}$ & 0 . & 00000 & & 1.2338 & & & .969 & \\
\hline $\mathrm{H}$ & 0 . & 00000 & & -1.2338 & & & .969 & 37 \\
\hline $\mathrm{H}$ & 0 . & 00000 & & 0.9378 & & & .157 & \\
\hline $\mathrm{H}$ & 0 . & 00000 & & -0.9378 & & & .157 & \\
\hline $\mathrm{H}$ & 0 . & 00000 & & 3.3864 & & & .188 & 70 \\
\hline $\mathrm{H}$ & 0 . & 00000 & & -3.3864 & & & $.18 \varepsilon$ & \\
\hline $\mathrm{H}$ & 0 . & 00000 & & 3.1752 & & & .306 & \\
\hline $\mathrm{H}$ & 0 . & 00000 & & -3.1752 & & & .306 & \\
\hline $\mathrm{H}$ & 0 . & 00000 & & 4.7251 & & & .295 & 00 \\
\hline $\mathrm{H}$ & 0 . & 00000 & & -4.7251 & & & .295 & 00 \\
\hline
\end{tabular}


Table S1321 Employed active space, reference configurations, and Cartesian coordinates of the OM3/MRCISD-optimized ground-state equilibrium geometry of o-cyanobenzyl radical (ground-state energy: $-1304.30569 \mathrm{eV}$ ).

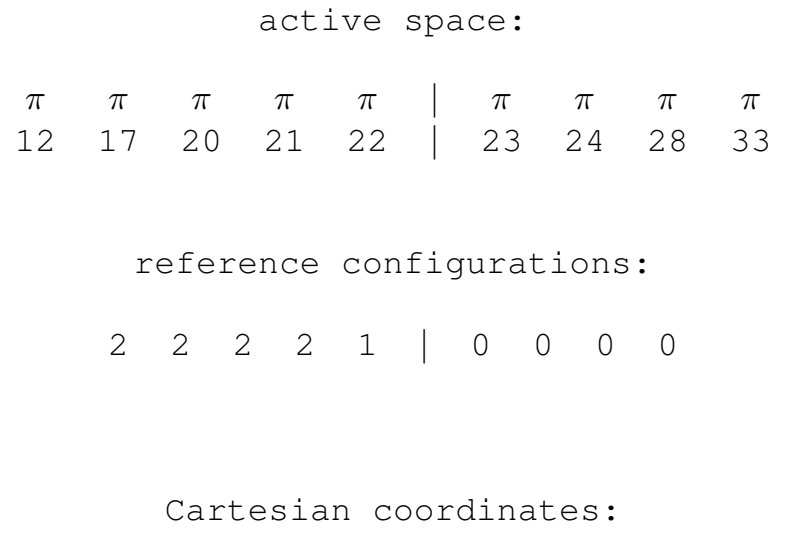

$\begin{array}{lrrr}15 & & & \\ & & & \\ \mathrm{C} & 0.00000 & 0.00000 & 0.00000 \\ \mathrm{C} & 1.43087 & 0.00000 & 0.00000 \\ \mathrm{C} & 2.14851 & 1.19290 & 0.00000 \\ \mathrm{C} & 1.48035 & 2.43326 & 0.00000 \\ \mathrm{C} & 0.07977 & 2.48250 & 0.00000 \\ \mathrm{C} & -0.64860 & 1.28215 & 0.00000 \\ \mathrm{C} & -2.08980 & 1.34761 & 0.00000 \\ \mathrm{~N} & -3.29525 & 1.40784 & 0.00000 \\ \mathrm{C} & -0.72562 & -1.20191 & -0.00000 \\ \mathrm{H} & 1.95736 & -0.96797 & -0.00000 \\ \mathrm{H} & 3.24993 & 1.17238 & 0.00000 \\ \mathrm{H} & 2.06095 & 3.36849 & 0.00000 \\ \mathrm{H} & -0.44573 & 3.44826 & 0.00000 \\ \mathrm{H} & -1.81240 & -1.21665 & -0.00000 \\ \mathrm{H} & -0.21512 & -2.16337 & -0.00000\end{array}$


Table S1322 Employed active space, reference configurations, and Cartesian coordinates of the OM3/MRCISD-optimized excited-state equilibrium geometry of the $2^{2} A^{\prime \prime}\left({ }^{2} \pi \pi^{*}\right)$ state of $o$-cyanobenzyl radical (excited-state energy: $-1301.93545 \mathrm{eV})$.

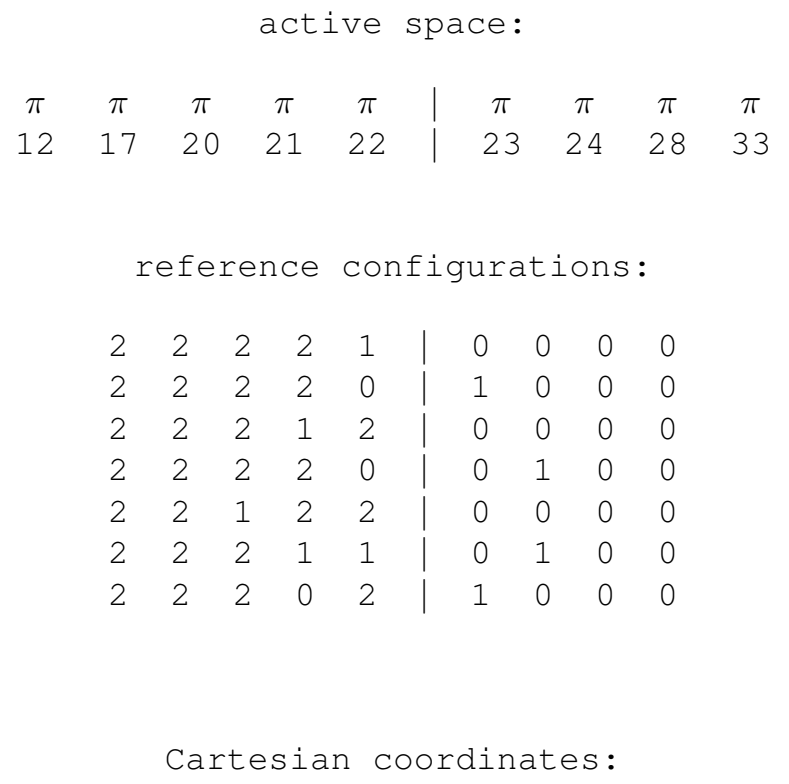

15

$\begin{array}{lrrr}\mathrm{C} & 0.00000 & 0.00000 & 0.00000 \\ \mathrm{C} & 1.42420 & 0.00000 & 0.00000 \\ \mathrm{C} & 2.15828 & 1.20342 & 0.00000 \\ \mathrm{C} & 1.49234 & 2.46830 & 0.00000 \\ \mathrm{C} & 0.11046 & 2.54875 & 0.00000 \\ \mathrm{C} & -0.66869 & 1.34039 & 0.00000 \\ \mathrm{C} & -2.07651 & 1.41463 & 0.00000 \\ \mathrm{~N} & -3.29025 & 1.47966 & 0.00000 \\ \mathrm{C} & -0.74512 & -1.16414 & -0.00000 \\ \mathrm{H} & 1.95506 & -0.96548 & -0.00000 \\ \mathrm{H} & 3.25801 & 1.17516 & 0.00000 \\ \mathrm{H} & 2.10055 & 3.38740 & 0.00000 \\ \mathrm{H} & -0.40852 & 3.51718 & 0.00000 \\ \mathrm{H} & -1.83277 & -1.15704 & -0.00000 \\ \mathrm{H} & -0.26367 & -2.14171 & -0.00000\end{array}$


Table S1323 Employed active space, reference configurations, and Cartesian coordinates of the OM3/MRCISD-optimized ground-state equilibrium geometry of oxalylfluoride (ground-state energy: $-1862.92511 \mathrm{eV}$ ).

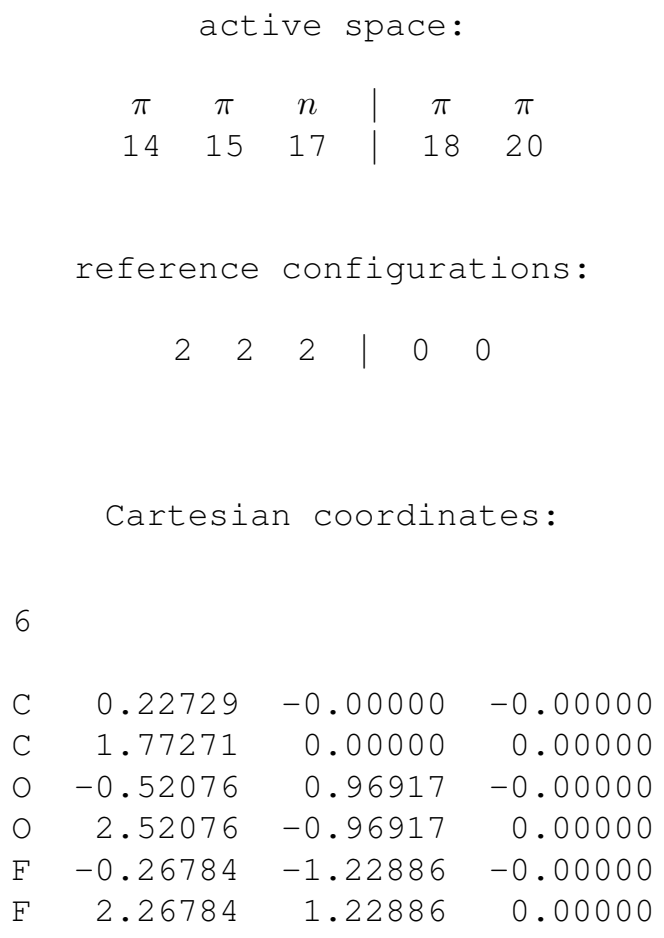


Table S1324 Employed active space, reference configurations, and Cartesian coordinates of the OM3/MRCISD-optimized excited-state equilibrium geometry of the $1{ }^{1} A_{u}\left({ }^{1} n \pi^{*}\right)$ state of oxalylfluoride (excited-state energy: $-1858.70420 \mathrm{eV})$.

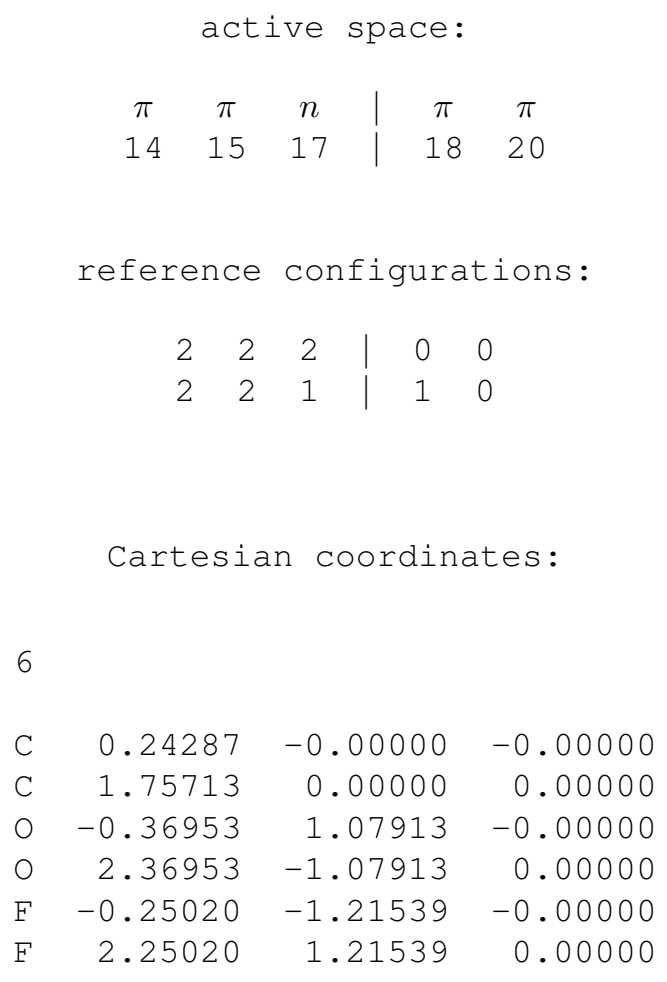


Table S1325 Employed active space, reference configurations, and Cartesian coordinates of the OM3/MRCISD-optimized ground-state equilibrium geometry of $p$-benzoquinone (ground-state energy: $-1487.35376 \mathrm{eV}$ ).

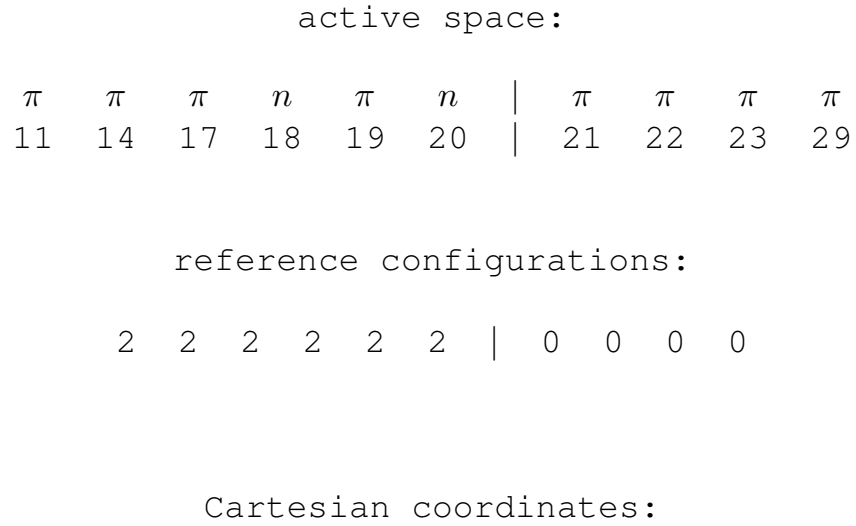


Table S1326 Employed active space, reference configurations, and Cartesian coordinates of the OM3/MRCISD-optimized excited-state equilibrium geometry of the $1{ }^{1} B_{1 g}\left({ }^{1} \pi \pi^{*}\right)$ state of $p$-benzoquinone (excited-state energy: $-1483.09168 \mathrm{eV})$.

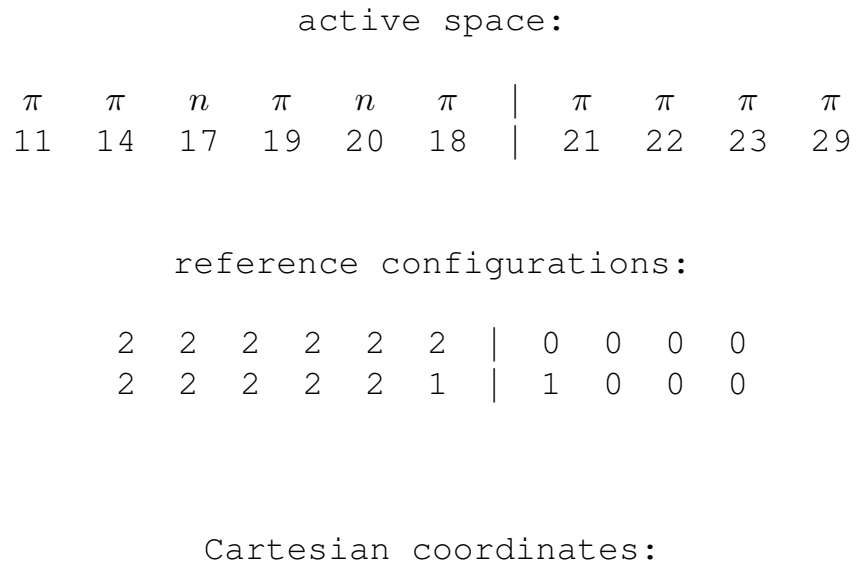


Table S1327 Employed active space, reference configurations, and Cartesian coordinates of the OM3/MRCISD-optimized excited-state equilibrium geometry of the $1{ }^{1} B_{2 g}\left({ }^{1} n \pi^{*}\right)$ state of $p$-benzoquinone (excited-state energy: $-1484.74681 \mathrm{eV})$.

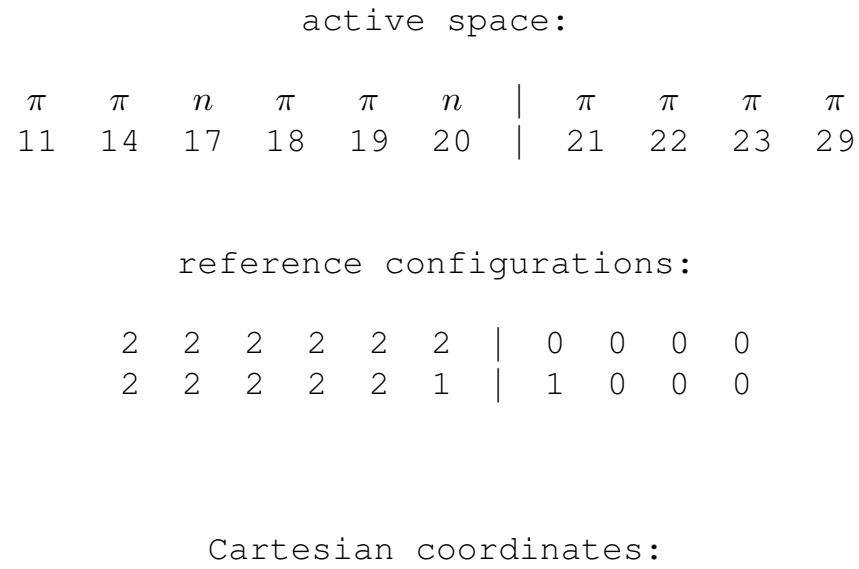


Table S1328 Employed active space, reference configurations, and Cartesian coordinates of the OM3/MRCISD-optimized ground-state equilibrium geometry of diethynylbenzene (ground-state energy: $-1336.04986 \mathrm{eV}$ ).

\begin{tabular}{lccc|cccc}
\multicolumn{10}{c}{ active space: } \\
\begin{tabular}{cccc|cccc}
$\pi$ & $\pi$ & $\pi$ & $\pi$ & $\pi$ & $\pi$ & $\pi$ & $\pi$ \\
16 & 19 & 22 & 23 & 24 & 25 & 27 & 34
\end{tabular}
\end{tabular}

reference configurations:

\begin{tabular}{llll|llll}
2 & 2 & 2 & 2 & 0 & 0 & 0 & 0
\end{tabular}

Cartesian coordinates:

$\begin{array}{lrrr}\text { 16 } & & \\ & & & \\ \text { C } & 0.00000 & 0.00000 & 1.38916 \\ \text { C } & 0.00000 & 0.00000 & -1.38916 \\ \text { C } & 0.00000 & 1.22816 & 0.69755 \\ \text { C } & -0.00000 & -1.22816 & 0.69755 \\ \text { C } & 0.00000 & -1.22816 & -0.69755 \\ \text { C } & 0.00000 & 1.22816 & -0.69755 \\ \text { C } & 0.00000 & -0.00000 & 2.82431 \\ \text { C } & 0.00000 & 0.00000 & -2.82431 \\ \text { C } & 0.00000 & -0.00000 & 4.06044 \\ \text { C } & 0.00000 & 0.00000 & -4.06044 \\ \text { H } & 0.00000 & -0.00000 & 5.11231 \\ \text { H } & 0.00000 & 0.00000 & -5.11231 \\ \text { H } & 0.00000 & 2.17309 & 1.25956 \\ \text { H } & -0.00000 & -2.17309 & 1.25956 \\ \text { H } & 0.00000 & -2.17309 & -1.25956 \\ \text { H } & 0.00000 & 2.17309 & -1.25956\end{array}$


Table S1329 Employed active space, reference configurations, and Cartesian coordinates of the OM3/MRCISD-optimized excited-state equilibrium geometry of the $1{ }^{1} B_{2 u}\left({ }^{1} \pi \pi^{*}\right)$ state of diethynylbenzene (excited-state energy: $-1331.33936 \mathrm{eV})$.

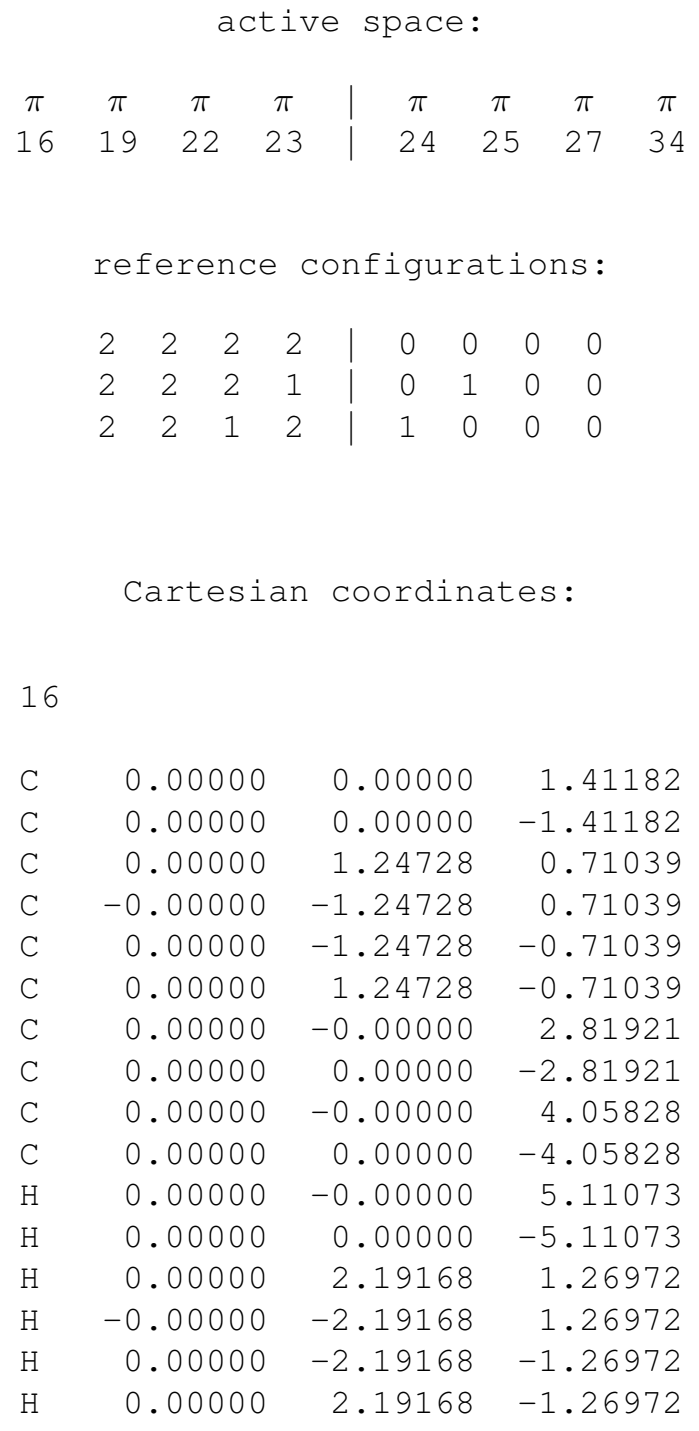


Table S1330 Employed active space, reference configurations, and Cartesian coordinates of the OM3/MRCISD-optimized ground-state equilibrium geometry of $p$-phenylenediamine (ground-state energy: $-1301.20962 \mathrm{eV}$ ).

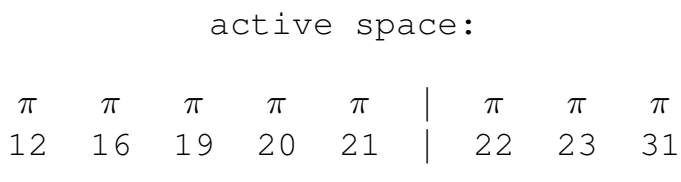

reference configurations:

\begin{tabular}{lllll|llll}
2 & 2 & 2 & 2 & 2 & 0 & 0 & 0
\end{tabular}

Cartesian coordinates:

16

$\begin{array}{llll}C & 0.00000 & 0.00000 & 1.41352\end{array}$

C $\quad 0.00000 \quad 0.00000-1.41352$

$\begin{array}{lrrr}\text { C } & 0.00000 & 1.22677 & 0.69717\end{array}$

$\begin{array}{lrrr}C & -0.00000 & -1.22677 & 0.69717\end{array}$

C $\quad 0.00000-1.22677-0.69717$

$\begin{array}{llll}\text { C } & 0.00000 & 1.22677 & -0.69717\end{array}$

$\begin{array}{llll}\mathrm{N} & 0.00000 & -0.00000 & 2.82100\end{array}$

$\begin{array}{llll}\mathrm{N} & 0.00000 & 0.00000 & -2.82100\end{array}$

$\begin{array}{lrrr}\mathrm{H} & 0.00000 & 2.17721 & 1.25171\end{array}$

$\mathrm{H} \quad-0.00000 \quad-2.17721 \quad 1.25171$

$\mathrm{H} \quad 0.00000 \quad-2.17721 \quad-1.25171$

$\begin{array}{llll}\mathrm{H} & 0.00000 & 2.17721 & -1.25171\end{array}$

$\begin{array}{llll}\mathrm{H} & 0.00000 & 0.86709 & 3.37065\end{array}$

$\begin{array}{llll}\mathrm{H} & 0.00000 & -0.86709 & 3.37065\end{array}$

$\begin{array}{llll}\mathrm{H} & 0.00000 & 0.86709 & -3.37065\end{array}$

H $\quad 0.00000 \quad-0.86709 \quad-3.37065$ 
Table S1331 Employed active space, reference configurations, and Cartesian coordinates of the OM3/MRCISD-optimized excited-state equilibrium geometry of the $2{ }^{1} A\left({ }^{1} \pi \pi^{*}\right)$ state of $p$-phenylenediamine (excited-state energy: $-1297.85839 \mathrm{eV})$.

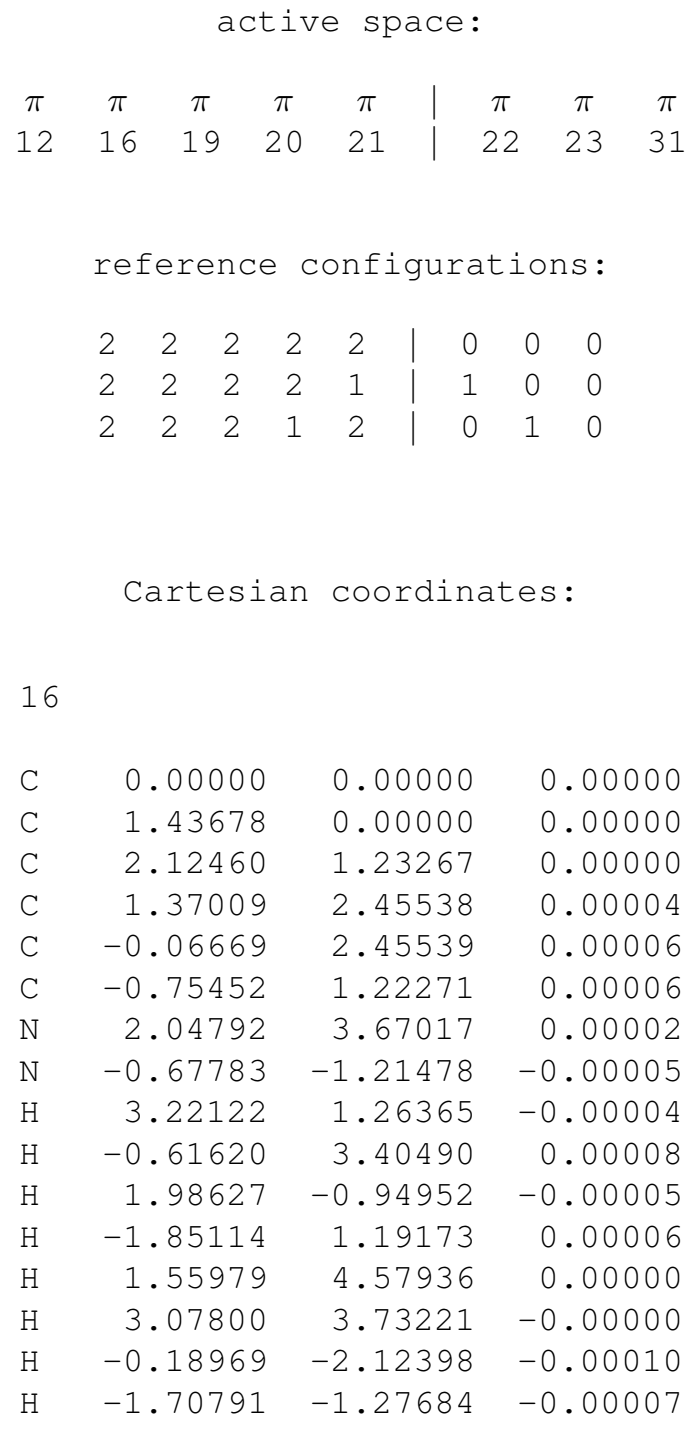


Table S1332 Employed active space, reference configurations, and Cartesian coordinates of the OM3/MRCISD-optimized ground-state equilibrium geometry of phenol (ground-state energy: $-1178.60624 \mathrm{eV}$ ).

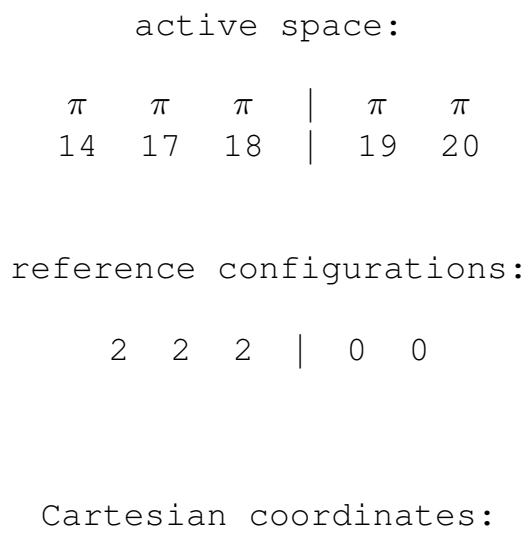

$\begin{array}{llll}\mathrm{H} & 3.85835 & 0.39578 & 0.00000\end{array}$ 
Table S1333 Employed active space, reference configurations, and Cartesian coordinates of the OM3/MRCISD-optimized excited-state equilibrium geometry of the $2{ }^{1} A^{\prime}\left({ }^{1} \pi \pi^{*}\right)$ state of phenol (excited-state energy: $-1173.73514 \mathrm{eV}$ ).

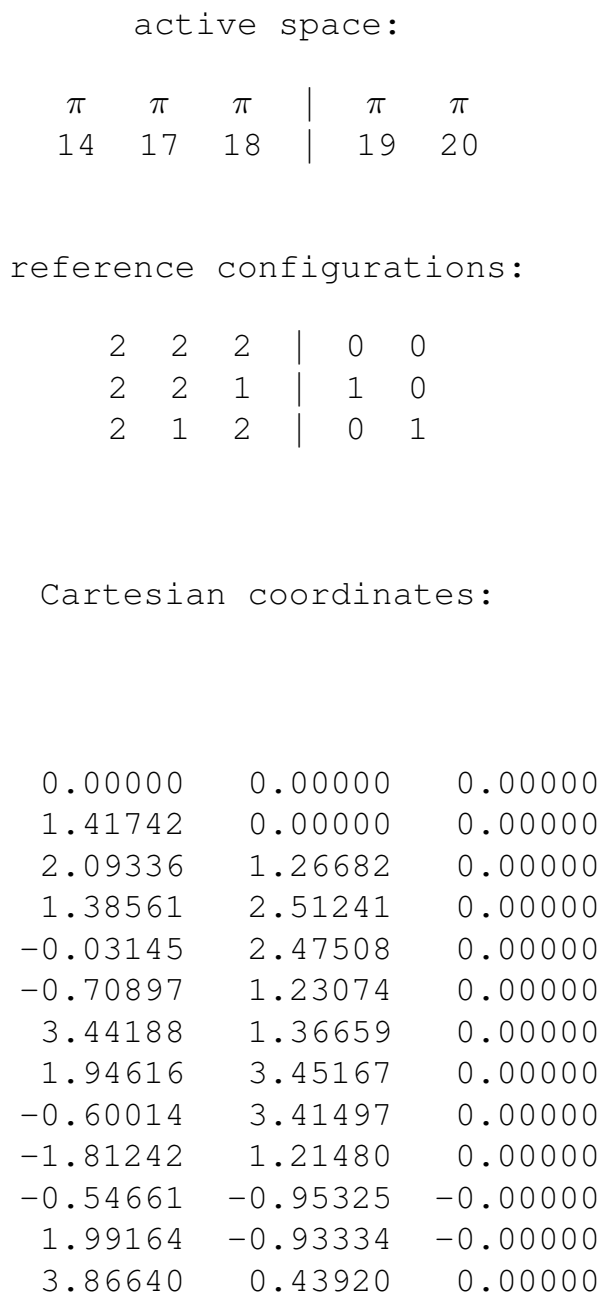


Table S1334 Employed active space, reference configurations, and Cartesian coordinates of the OM3/MRCISD-optimized ground-state equilibrium geometry of porphyrin (ground-state energy: $-3567.52040 \mathrm{eV}$ ).

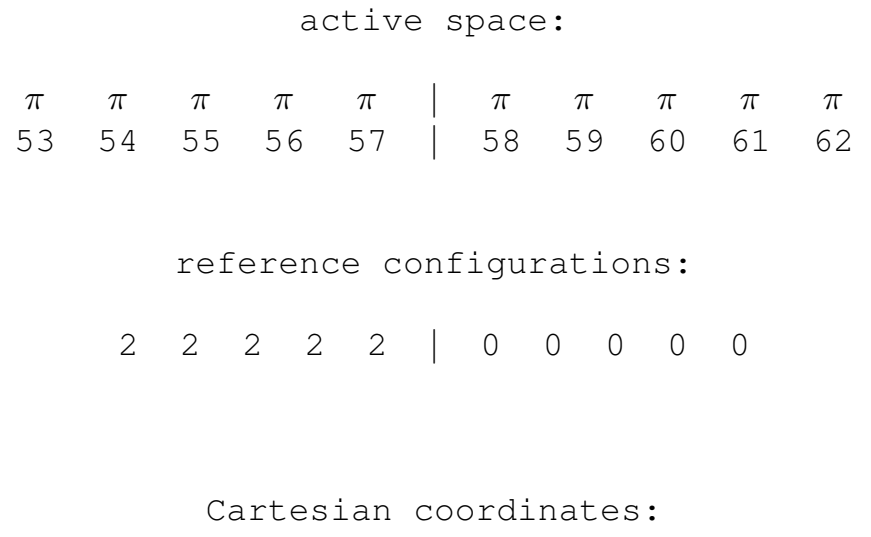

\begin{tabular}{lrrr}
38 & & & \\
& & & \\
$\mathrm{~N}$ & 2.07017 & 0.00000 & 0.00000 \\
$\mathrm{~N}$ & 0.00000 & 2.03630 & 0.00000 \\
$\mathrm{~N}$ & -2.07017 & 0.00000 & 0.00000 \\
$\mathrm{~N}$ & 0.00000 & -2.03630 & -0.00000 \\
$\mathrm{C}$ & 2.88723 & 1.14264 & 0.00000 \\
$\mathrm{C}$ & 2.88723 & -1.14264 & -0.00000 \\
$\mathrm{C}$ & -2.88723 & 1.14264 & 0.00000 \\
$\mathrm{C}$ & -2.88723 & -1.14264 & 0.00000 \\
$\mathrm{C}$ & 1.10388 & 2.87192 & 0.00000 \\
$\mathrm{C}$ & -1.10388 & 2.87192 & 0.00000 \\
$\mathrm{C}$ & 1.10388 & -2.87192 & -0.00000 \\
$\mathrm{C}$ & -1.10388 & -2.87192 & -0.00000 \\
$\mathrm{C}$ & 4.28108 & 0.68323 & 0.00000 \\
$\mathrm{C}$ & 4.28108 & -0.68323 & -0.00000 \\
$\mathrm{C}$ & -4.28108 & 0.68323 & 0.00000 \\
$\mathrm{C}$ & -4.28108 & -0.68323 & 0.00000 \\
$\mathrm{C}$ & 0.67823 & 4.30695 & -0.00000 \\
$\mathrm{C}$ & -0.67823 & 4.30695 & 0.00000 \\
$\mathrm{C}$ & 0.67823 & -4.30695 & -0.00000 \\
$\mathrm{C}$ & -0.67823 & -4.30695 & -0.00000 \\
$\mathrm{C}$ & 2.45731 & 2.47879 & 0.00000 \\
$\mathrm{C}$ & 2.45731 & -2.47879 & -0.00000 \\
$\mathrm{C}$ & -2.45731 & 2.47879 & 0.00000 \\
$\mathrm{C}$ & -2.45731 & -2.47879 & -0.00000 \\
\hline & & Continued & on $n$ next page
\end{tabular}

S1420 


\begin{tabular}{lrrr}
\multicolumn{5}{c}{ Continued from previous page } \\
\hline $\mathrm{H}$ & 5.13477 & 1.35723 & 0.00000 \\
$\mathrm{H}$ & 5.13477 & -1.35723 & -0.00000 \\
$\mathrm{H}$ & -5.13477 & 1.35723 & 0.00000 \\
$\mathrm{H}$ & -5.13477 & -1.35723 & 0.00000 \\
$\mathrm{H}$ & 1.36643 & 5.14898 & -0.00000 \\
$\mathrm{H}$ & -1.36643 & 5.14898 & 0.00000 \\
$\mathrm{H}$ & 1.36643 & -5.14898 & -0.00000 \\
$\mathrm{H}$ & -1.36643 & -5.14898 & -0.00000 \\
$\mathrm{H}$ & 3.22943 & 3.26391 & 0.00000 \\
$\mathrm{H}$ & 3.22943 & -3.26391 & -0.00000 \\
$\mathrm{H}$ & -3.22943 & 3.26391 & 0.00000 \\
$\mathrm{H}$ & -3.22943 & -3.26391 & 0.00000 \\
$\mathrm{H}$ & 1.02511 & 0.00000 & -0.00000 \\
$\mathrm{H}$ & -1.02511 & 0.00000 & 0.00000
\end{tabular}


Table S1335 Employed active space, reference configurations, and Cartesian coordinates of the OM3/MRCISD-optimized excited-state equilibrium geometry of the $1{ }^{1} B_{3 u}\left({ }^{1} \pi \pi^{*}\right)$ state of porphyrin (excited-state energy: $\left.-3565.49464 \mathrm{eV}\right)$.

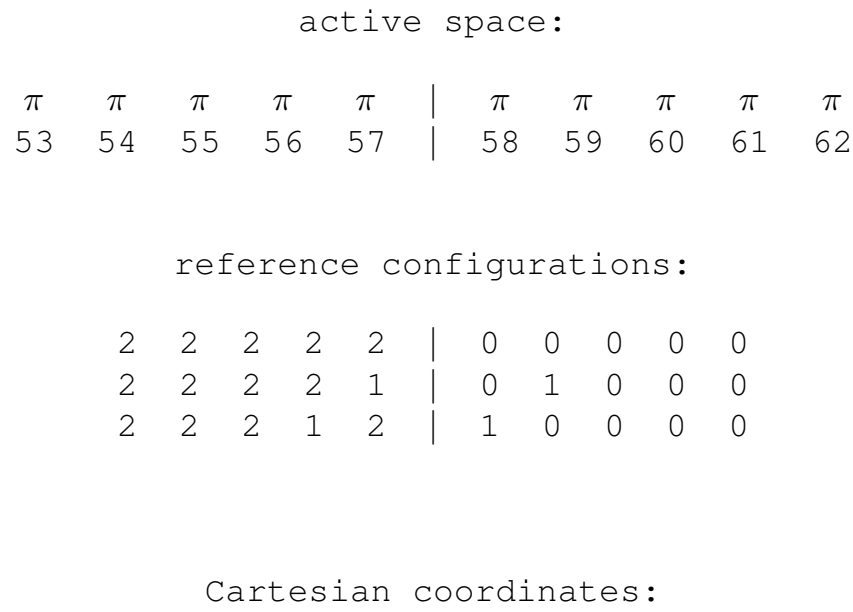




\begin{tabular}{lrrr}
\multicolumn{5}{c}{ Continued from previous page } \\
\hline C & -2.46474 & -2.48248 & -0.00000 \\
H & 5.13513 & 1.35804 & 0.00000 \\
H & 5.13513 & -1.35804 & -0.00000 \\
H & -5.13513 & 1.35804 & 0.00000 \\
H & -5.13513 & -1.35804 & 0.00000 \\
H & 1.36413 & 5.16121 & -0.00000 \\
$\mathrm{H}$ & -1.36413 & 5.16121 & 0.00000 \\
$\mathrm{H}$ & 1.36413 & -5.16121 & -0.00000 \\
$\mathrm{H}$ & -1.36413 & -5.16121 & -0.00000 \\
$\mathrm{H}$ & 3.23985 & 3.26447 & 0.00000 \\
$\mathrm{H}$ & 3.23985 & -3.26447 & -0.00000 \\
$\mathrm{H}$ & -3.23985 & 3.26447 & 0.00000 \\
$\mathrm{H}$ & -3.23985 & -3.26447 & 0.00000 \\
$\mathrm{H}$ & 1.02477 & 0.00000 & -0.00000 \\
$\mathrm{H}$ & -1.02477 & 0.00000 & 0.00000
\end{tabular}


Table S1336 Employed active space, reference configurations, and Cartesian coordinates of the OM3/MRCISD-optimized ground-state equilibrium geometry of propynal (ground-state energy: $-741.85993 \mathrm{eV}$ ).

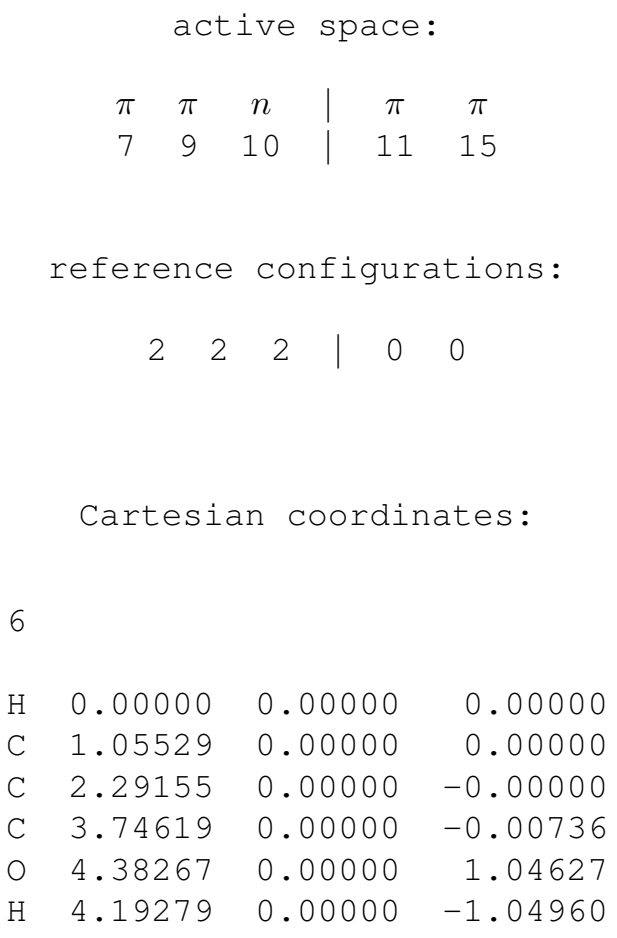


Table S1337 Employed active space, reference configurations, and Cartesian coordinates of the OM3/MRCISD-optimized excited-state equilibrium geometry of the $2{ }^{1} A\left({ }^{1} n \pi^{*}\right)$ state of propynal (excited-state energy: $-738.36288 \mathrm{eV}$ ).

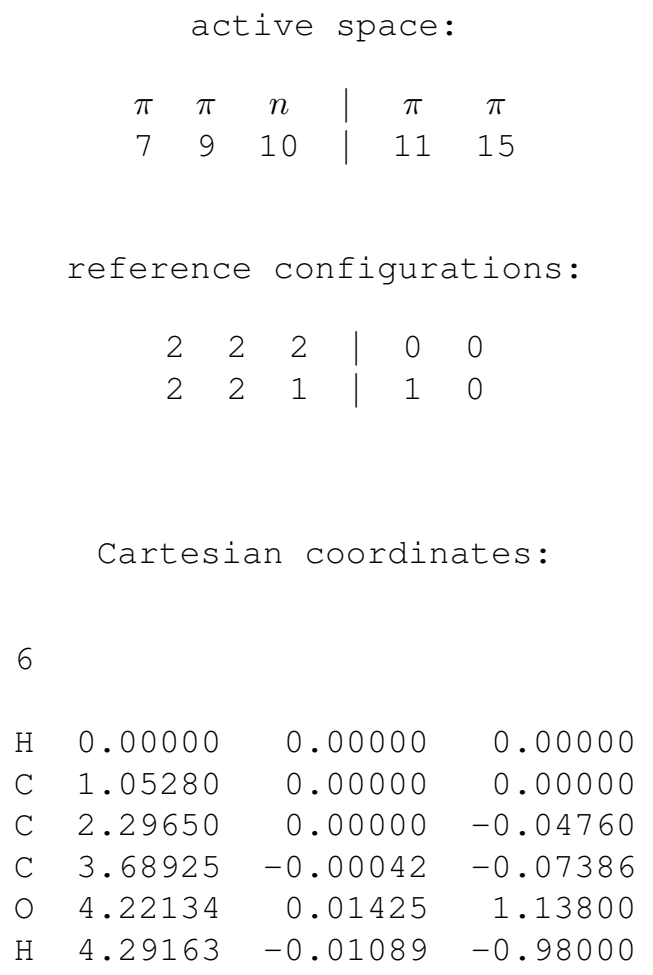


Table S1338 Employed active space, reference configurations, and Cartesian coordinates of the OM3/MRCISD-optimized ground-state equilibrium geometry of pyrene (ground-state energy: $-2150.59558 \mathrm{eV}$ ).

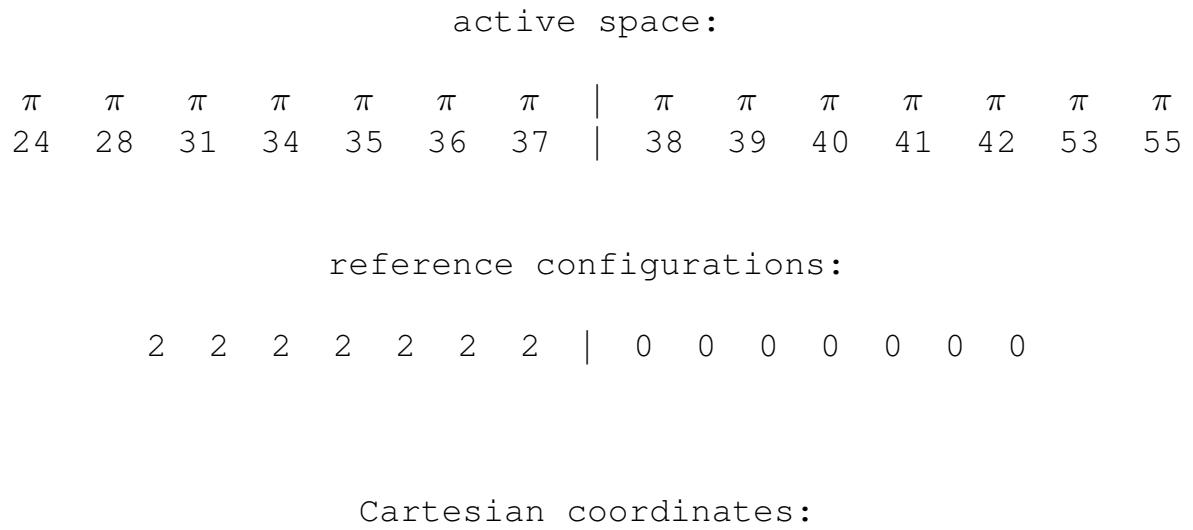


Continued from previous page

\begin{tabular}{rrrr}
\hline $\mathrm{H}$ & 0.00000 & 4.63666 & 0.00000 \\
$\mathrm{H}$ & 0.00000 & -4.63666 & -0.00000
\end{tabular}

S1427 
Table S1339 Employed active space, reference configurations, and Cartesian coordinates of the OM3/MRCISD-optimized excited-state equilibrium geometry of the $1{ }^{1} B_{2 u}\left({ }^{1} \pi \pi^{*}\right)$ state of pyrene (excited-state energy: $\left.-2147.11724 \mathrm{eV}\right)$.

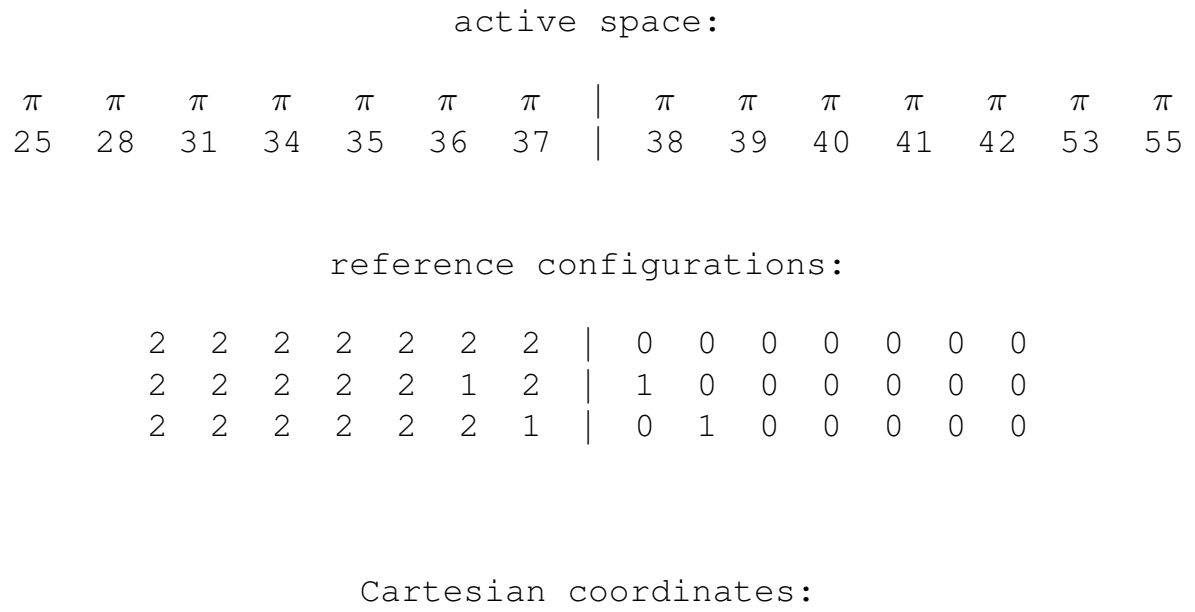

Continued on next page 


\begin{tabular}{lrrr}
\multicolumn{5}{c}{ Continued from previous page } \\
\hline $\mathrm{H}$ & 4.10311 & -2.17420 & 0.00000 \\
$\mathrm{H}$ & -3.94328 & 0.00000 & 0.00000 \\
$\mathrm{H}$ & 5.34008 & 0.00000 & -0.00000
\end{tabular}


Table S1340 Employed active space, reference configurations, and Cartesian coordinates of the OM3/MRCISD-optimized ground-state equilibrium geometry of pyridine (ground-state energy: $-915.63390 \mathrm{eV}$ ).

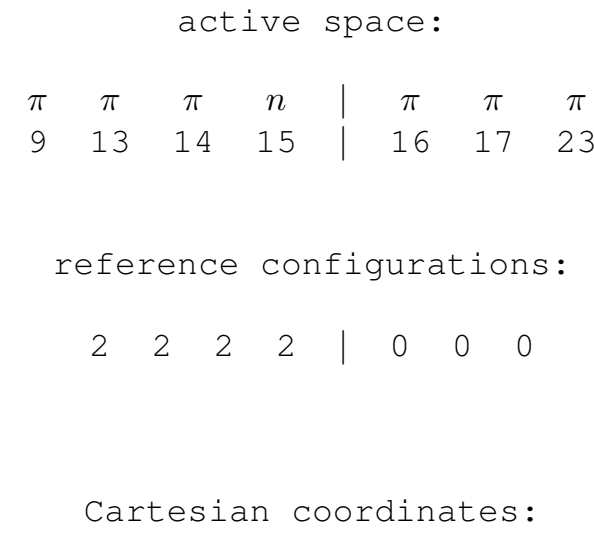

11

$\begin{array}{lrrr}\text { C } & 0.00000 & 1.42406 & 0.00000 \\ \text { C } & 0.00000 & 0.71614 & -1.20849 \\ \text { C } & 0.00000 & 0.71614 & 1.20849 \\ \text { C } & 0.00000 & -0.69430 & -1.16988 \\ \text { C } & 0.00000 & -0.69430 & 1.16988 \\ \text { N } & 0.00000 & -1.38987 & -0.00000 \\ \text { H } & 0.00000 & 2.52491 & 0.00000 \\ \text { H } & 0.00000 & 1.24853 & -2.16929 \\ \text { H } & 0.00000 & 1.24853 & 2.16929 \\ \text { H } & 0.00000 & -1.28175 & -2.11346 \\ \text { H } & 0.00000 & -1.28175 & 2.11346\end{array}$


Table S1341 Employed active space, reference configurations, and Cartesian coordinates of the OM3/MRCISD-optimized excited-state equilibrium geometry of the $2{ }^{1} A\left({ }^{1} n \pi^{*}\right)$ state of pyridine (excited-state energy: $-911.55433 \mathrm{eV}$ ).

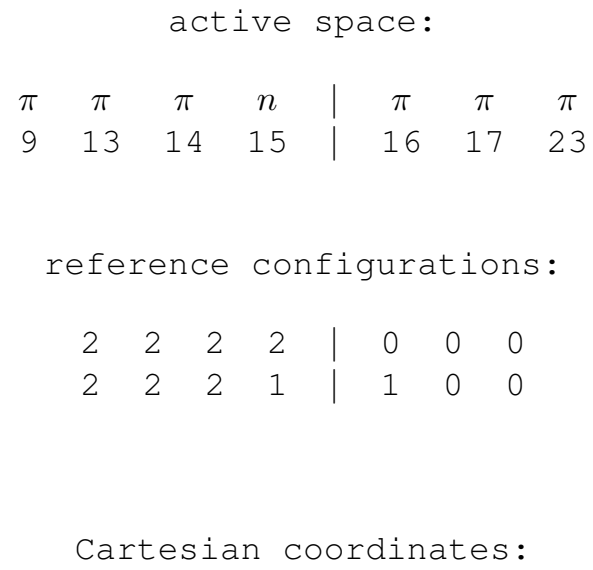


Table S1342 Employed active space, reference configurations, and Cartesian coordinates of the OM3/MRCISD-optimized ground-state equilibrium geometry of pyridone lactam (ground-state energy: $-1254.73170 \mathrm{eV}$ ).

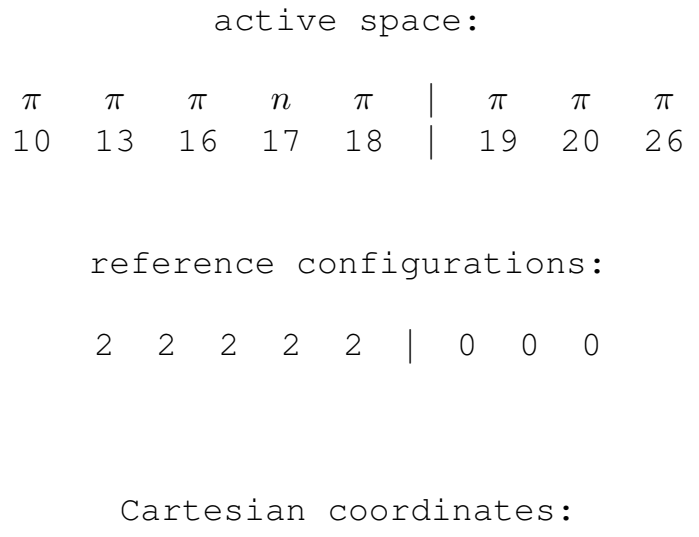

12

$\begin{array}{rrrr}\mathrm{C} & 0.00000 & 0.00000 & 0.00000 \\ \mathrm{~N} & 1.38848 & 0.00000 & 0.00000 \\ \mathrm{C} & 2.18461 & 1.19885 & 0.00000 \\ \mathrm{C} & 1.41157 & 2.43919 & 0.00000 \\ \mathrm{C} & 0.03784 & 2.43222 & 0.00000 \\ \mathrm{C} & -0.68870 & 1.20509 & 0.00000 \\ \mathrm{O} & 3.43005 & 1.08541 & 0.00000 \\ \mathrm{H} & 1.99723 & 3.36385 & 0.00000 \\ \mathrm{H} & -0.52743 & 3.37912 & 0.00000 \\ \mathrm{H} & -1.78271 & 1.21384 & 0.00000 \\ \mathrm{H} & -0.53090 & -0.97446 & -0.00000 \\ \mathrm{H} & 1.90661 & -0.91260 & -0.00000\end{array}$


Table S1343 Employed active space, reference configurations, and Cartesian coordinates of the OM3/MRCISD-optimized excited-state equilibrium geometry of the $3{ }^{1} A\left({ }^{1} n \pi^{*}\right)$ state of pyridone lactam (excited-state energy: $-1250.57097 \mathrm{eV})$.

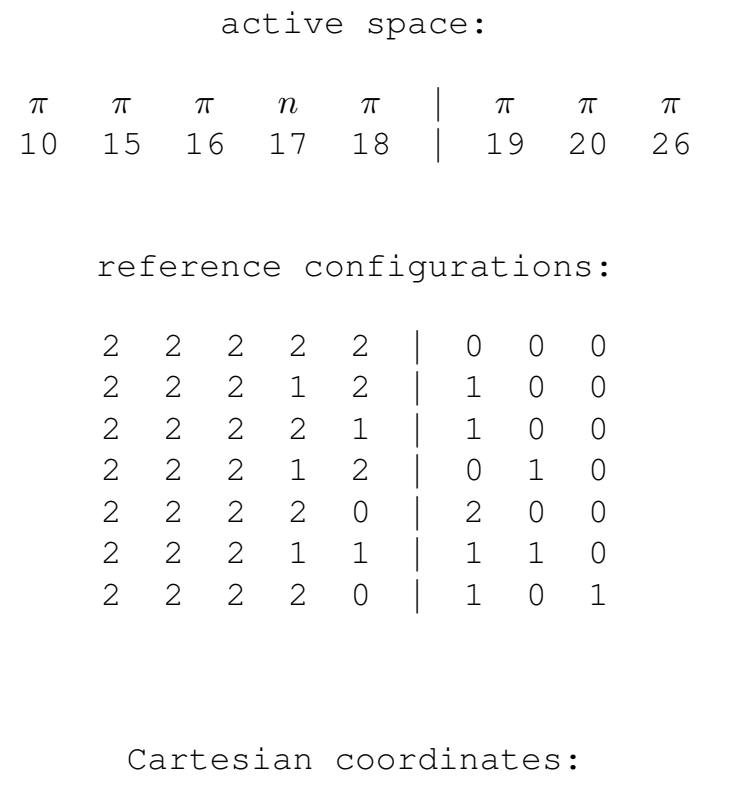

12

$\begin{array}{rrrr}\mathrm{C} & 0.00000 & 0.00000 & 0.00000 \\ \mathrm{~N} & 1.42013 & 0.00000 & 0.00000 \\ \mathrm{C} & 2.10212 & 1.23032 & 0.00000 \\ \mathrm{C} & 1.43306 & 2.45403 & 0.00016 \\ \mathrm{C} & 0.01111 & 2.44784 & 0.00033 \\ \mathrm{C} & -0.67599 & 1.21882 & 0.00017 \\ \mathrm{O} & 3.43532 & 1.16409 & -0.00014 \\ \mathrm{H} & 2.00655 & 3.38316 & 0.00017 \\ \mathrm{H} & -0.52864 & 3.39681 & 0.00049 \\ \mathrm{H} & -1.77700 & 1.20927 & 0.00018 \\ \mathrm{H} & -0.50119 & -0.97781 & -0.00016 \\ \mathrm{H} & 1.96592 & -0.88874 & -0.00010\end{array}$


Table S1344 Employed active space, reference configurations, and Cartesian coordinates of the OM3/MRCISD-optimized ground-state equilibrium geometry of pyridone lactim (ground-state energy: $-1254.72461 \mathrm{eV}$ ).

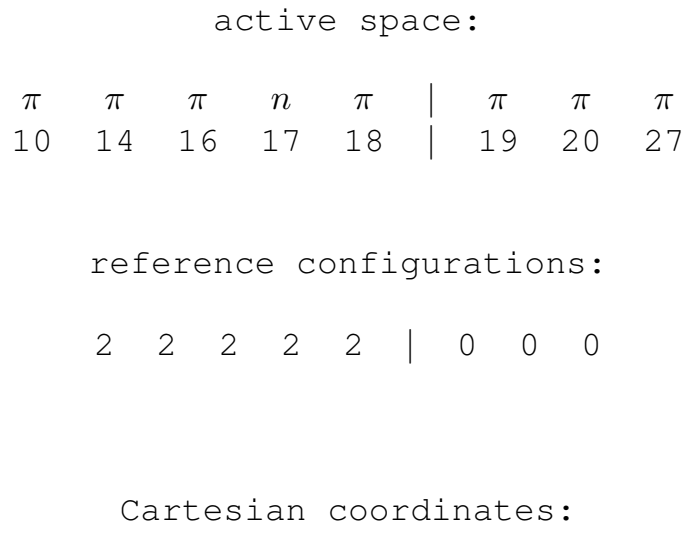

12

$\begin{array}{lrrr}\mathrm{C} & 0.00000 & 0.00000 & 0.00000 \\ \mathrm{~N} & 1.35965 & 0.00000 & 0.00000 \\ \mathrm{C} & 1.99007 & 1.21128 & 0.00000 \\ \mathrm{C} & 1.32412 & 2.46263 & 0.00000 \\ \mathrm{C} & -0.07332 & 2.42880 & 0.00000 \\ \mathrm{C} & -0.74725 & 1.19752 & 0.00000 \\ \mathrm{O} & 3.37174 & 1.22887 & 0.00000 \\ \mathrm{H} & 1.89509 & 3.39417 & 0.00000 \\ \mathrm{H} & -0.64440 & 3.37015 & 0.00000 \\ \mathrm{H} & -1.84342 & 1.16362 & 0.00000 \\ \mathrm{H} & -0.51143 & -0.98639 & -0.00000 \\ \mathrm{H} & 3.71525 & 0.27096 & 0.00000\end{array}$


Table S1345 Employed active space, reference configurations, and Cartesian coordinates of the OM3/MRCISD-optimized excited-state equilibrium geometry of the $1^{1} A^{\prime \prime}\left({ }^{1} n \pi^{*}\right)$ state of pyridone lactim (excited-state energy: $-1250.16711 \mathrm{eV})$.

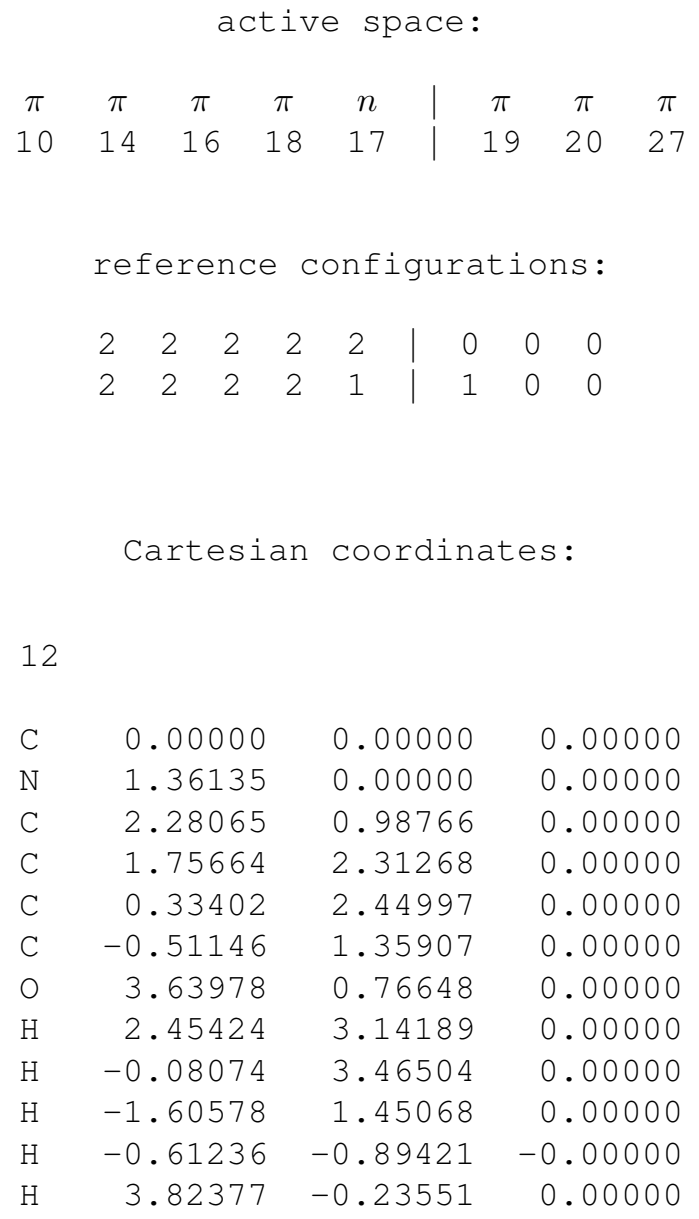


Table S1346 Employed active space, reference configurations, and Cartesian coordinates of the OM3/MRCISD-optimized ground-state equilibrium geometry of pyrimidine (ground-state energy: $-991.33594 \mathrm{eV}$ ).

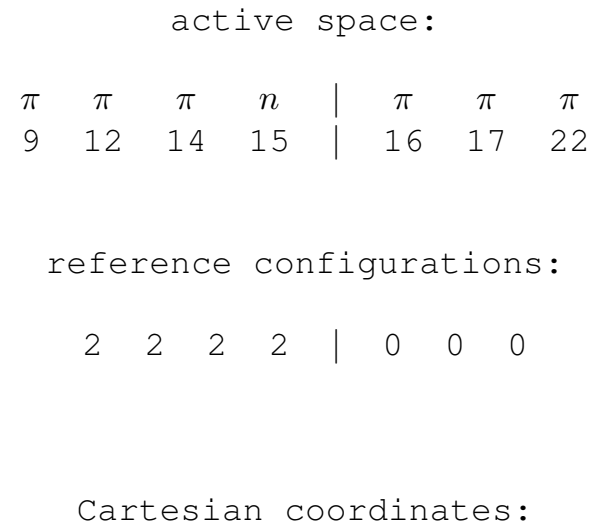

$\mathrm{H} \quad 0.00000 \quad-1.20772 \quad 2.18562$

H $\quad 0.00000 \quad-2.51261-0.00000$ 
Table S1347 Employed active space, reference configurations, and Cartesian coordinates of the OM3/MRCISD-optimized excited-state equilibrium geometry of the $2{ }^{1} A\left({ }^{1} n \pi^{*}\right)$ state of pyrimidine (excited-state energy: $-987.50977 \mathrm{eV}$ ).

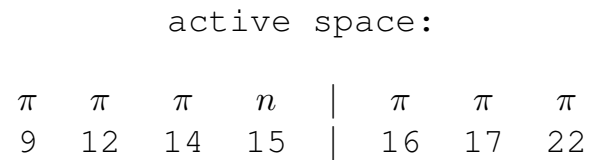

reference configurations:

\begin{tabular}{llll|lll}
2 & 2 & 2 & 2 & 0 & 0 & 0 \\
2 & 2 & 2 & 1 & 1 & 0 & 0
\end{tabular}

Cartesian coordinates:

10

$\begin{array}{llll}\text { C } & 0.00000 & 0.00000 & 0.00000\end{array}$

C $\quad 1.43742 \quad 0.00000 \quad 0.00000$

C $\quad 2.03168 \quad 1.30882 \quad 0.00000$

$\mathrm{N} \quad \begin{array}{llll}\mathrm{N} & 1.25831 & 2.38639 & -0.00005\end{array}$

C $\quad-0.13109 \quad 2.43482-0.00001$

$\mathrm{N} \quad-0.66139 \quad 1.14971-0.00003$

$\begin{array}{llll}\mathrm{H} & 2.02452 & -0.91137 & 0.00005\end{array}$

$\mathrm{H} \quad 3.13056 \quad 1.41072 \quad 0.00000$

$\begin{array}{llll}\mathrm{H} & -0.72291 & 3.35352 & -0.00006\end{array}$

$\begin{array}{llll}\mathrm{H} & -0.54711 & -0.95843 & 0.00001\end{array}$ 
Table S1348 Employed active space, reference configurations, and Cartesian coordinates of the OM3/MRCISD-optimized excited-state equilibrium geometry of the $4^{1} A\left({ }^{1} \pi \pi^{*}\right)$ state of pyrimidine (excited-state energy: $-986.43990 \mathrm{eV}$ ).

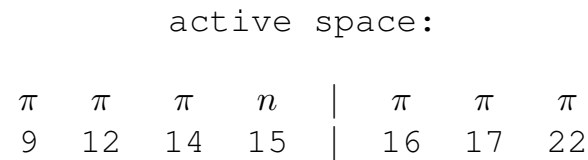

reference configurations:

\begin{tabular}{|c|c|c|c|c|c|}
\hline & 2 & 2 & 2 & 0 & 0 \\
\hline 2 & 2 & 2 & 1 & 1 & 0 \\
\hline & 2 & 2 & 1 & 0 & 1 \\
\hline & 2 & 1 & 2 & 0 & 1 \\
\hline & 1 & 2 & 2 & 1 & 0 \\
\hline
\end{tabular}

Cartesian coordinates:

10

$\begin{array}{llll}C & 0.00000 & 0.00000 & 0.00000\end{array}$

$\begin{array}{llll}\text { C } & 1.43817 & 0.00000 & 0.00000\end{array}$

$\mathrm{N} \quad 2.15905 \quad 1.18475 \quad 0.00000$

C $\quad 1.42744 \quad 2.36911-0.00001$

$\begin{array}{lrrr}\mathrm{N} & 0.03847 & 2.46245 & -0.00002\end{array}$

C $\quad-0.67208 \quad 1.27148 \quad-0.00002$

$\begin{array}{llll}\mathrm{H} & 2.00364 & 3.32541 & -0.00001\end{array}$

$\begin{array}{lrrr}\mathrm{H} & 2.00611 & -0.94866 & 0.00001\end{array}$

$\mathrm{H} \quad-1.77619 \quad 1.33026 \quad-0.00002$

$\mathrm{H} \quad-0.56498 \quad-0.93769 \quad-0.00000$ 
Table S1349 Employed active space, reference configurations, and Cartesian coordinates of the OM3/MRCISD-optimized ground-state equilibrium geometry of quinoline (ground-state energy: $-1445.82390 \mathrm{eV}$ ).

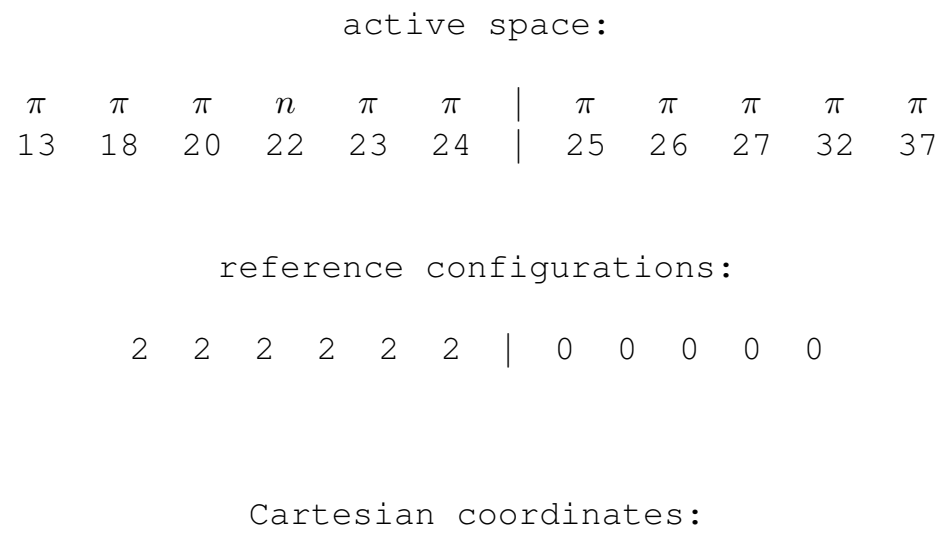


Table S1350 Employed active space, reference configurations, and Cartesian coordinates of the OM3/MRCISD-optimized excited-state equilibrium geometry of the $1{ }^{1} A^{\prime \prime}\left({ }^{1} n \pi^{*}\right)$ state of quinoline (excited-state energy: $\left.-1442.02482 \mathrm{eV}\right)$.

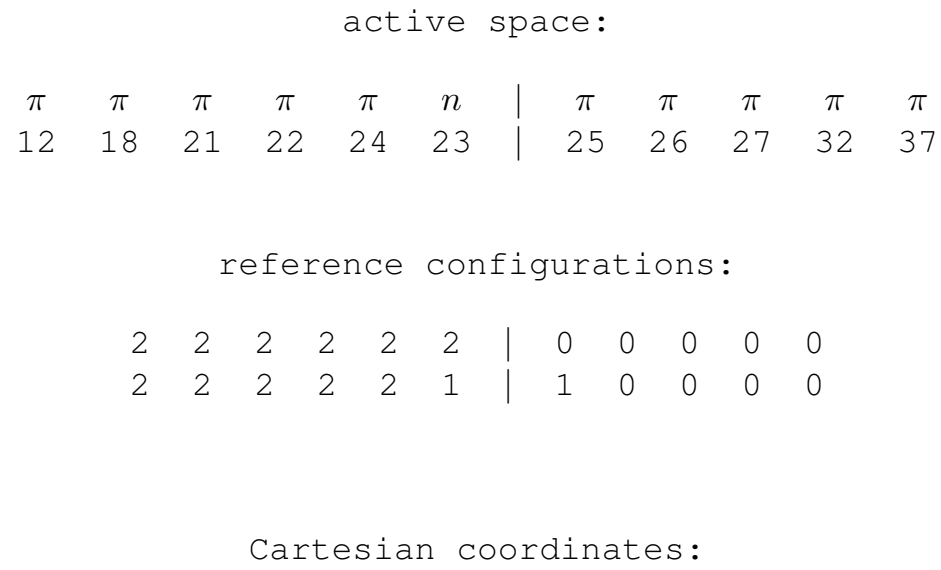


Table S1351 Employed active space, reference configurations, and Cartesian coordinates of the OM3/MRCISD-optimized excited-state equilibrium geometry of the $1{ }^{3} A^{\prime}\left({ }^{3} \pi \pi^{*}\right)$ state of quinoline (excited-state energy: $-1443.05994 \mathrm{eV}$ ).

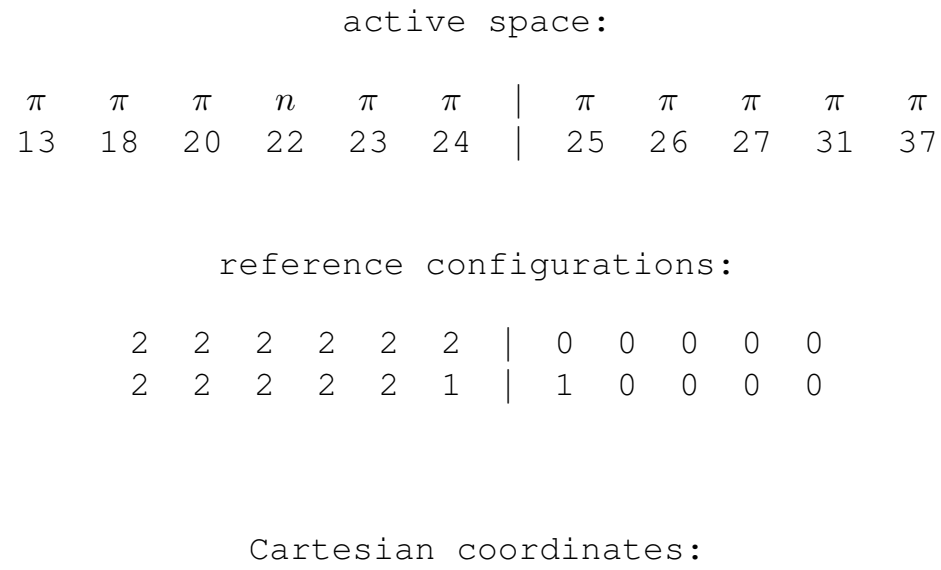


Table S1352 Employed active space, reference configurations, and Cartesian coordinates of the OM3/MRCISD-optimized ground-state equilibrium geometry of quinoxaline (ground-state energy: $-1521.47189 \mathrm{eV}$ ).

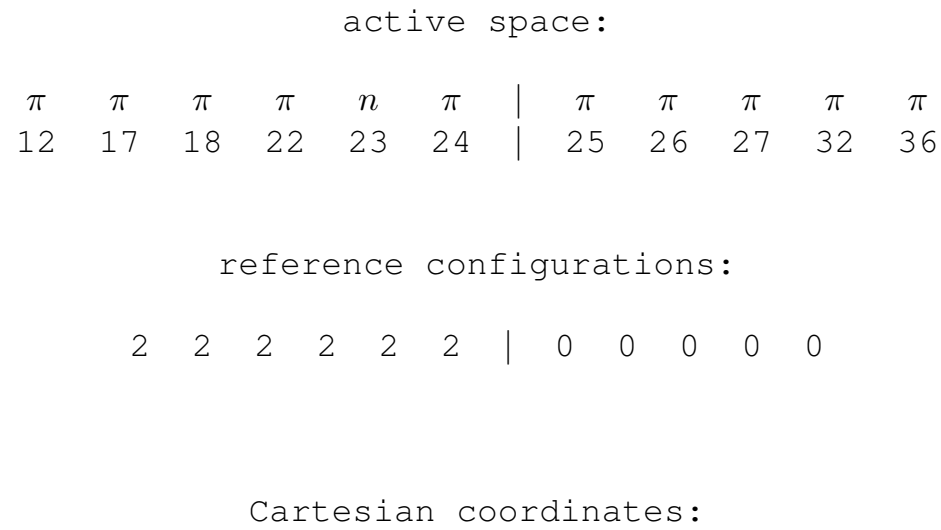


Table S1353 Employed active space, reference configurations, and Cartesian coordinates of the OM3/MRCISD-optimized excited-state equilibrium geometry of the $2{ }^{1} A_{1}\left({ }^{1} \pi \pi^{*}\right)$ state of quinoxaline (excited-state energy: $-1517.59732 \mathrm{eV}$ ).

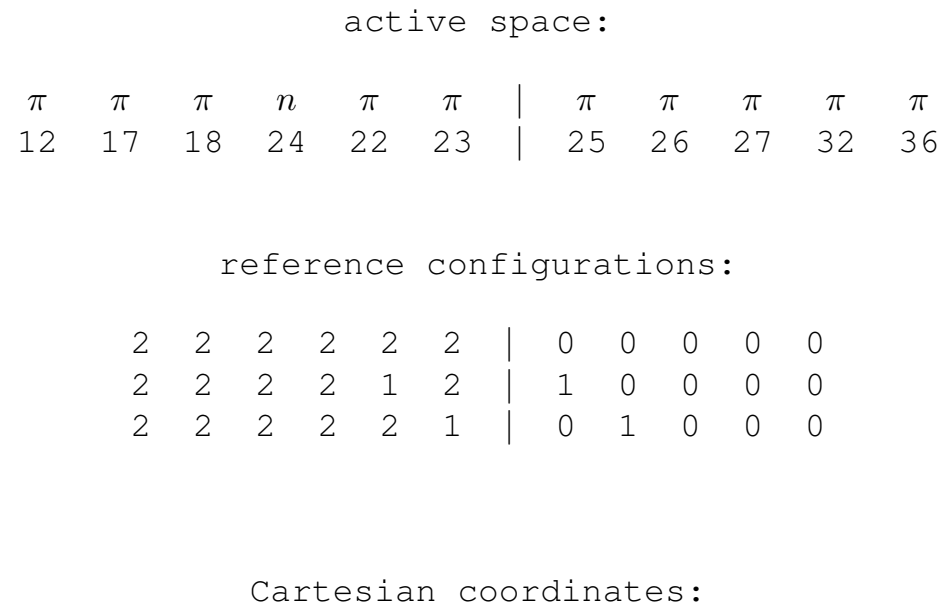


Table S1354 Employed active space, reference configurations, and Cartesian coordinates of the OM3/MRCISD-optimized excited-state equilibrium geometry of the $2{ }^{1} A\left({ }^{1} n \pi^{*}\right)$ state of quinoxaline (excited-state energy: $-1518.06720 \mathrm{eV}$ ).

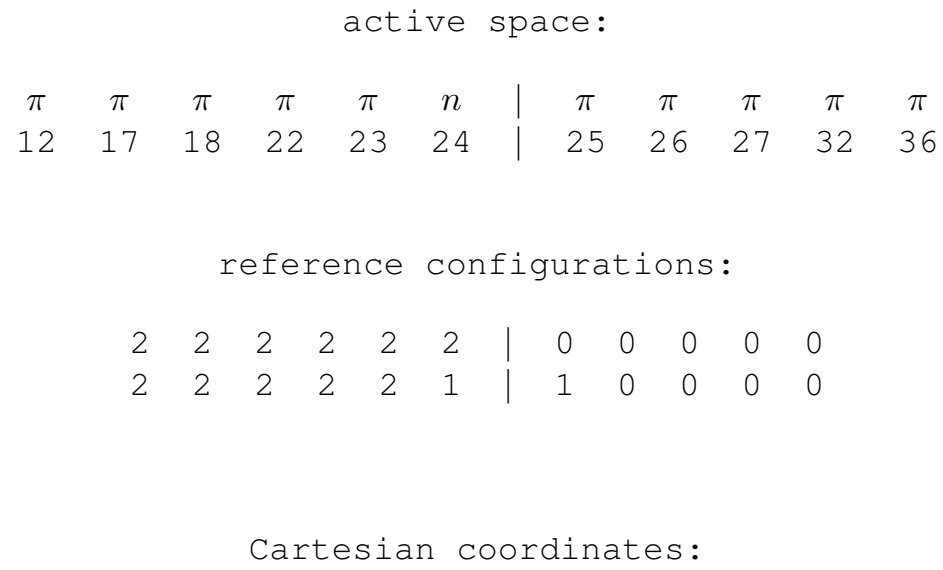


Table S1355 Employed active space, reference configurations, and Cartesian coordinates of the OM3/MRCISD-optimized excited-state equilibrium geometry of the $1^{3} A^{\prime}\left({ }^{3} \pi \pi^{*}\right)$ state of quinoxaline (excited-state energy: $-1518.61840 \mathrm{eV}$ ).

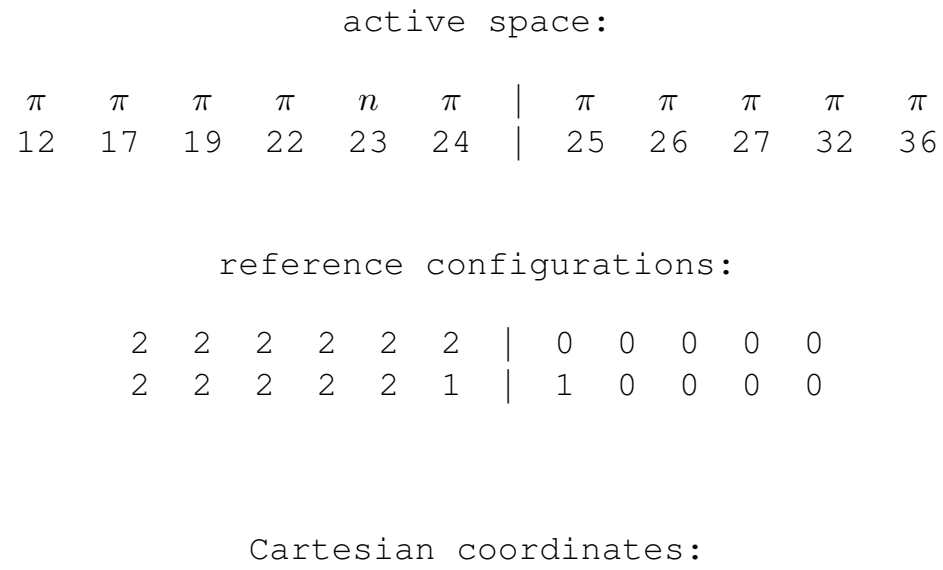

$\mathrm{H} \quad 5.29253 \quad 0.14698-0.00000$ 
Table S1356 Employed active space, reference configurations, and Cartesian coordinates of the OM3/MRCISD-optimized ground-state equilibrium geometry of styrene (ground-state energy: $-1119.34644 \mathrm{eV}$ ).

\begin{tabular}{lccc|cccc}
\multicolumn{10}{c}{ active space: } \\
$\pi$ & $\pi$ & $\pi$ & $\pi$ & $\pi$ & $\pi$ & $\pi$ & $\pi$ \\
13 & 16 & 19 & 20 & 21 & 22 & 24 & 32
\end{tabular}

reference configurations:

\begin{tabular}{llll|llll}
2 & 2 & 2 & 2 & 0 & 0 & 0 & 0
\end{tabular}

Cartesian coordinates:

$\begin{array}{lrrr}16 & & & \\ & & & \\ \mathrm{C} & 0.00000 & 0.00000 & 0.00000 \\ \mathrm{C} & 1.40983 & 0.00000 & 0.00000 \\ \mathrm{C} & 2.11690 & 1.20882 & 0.00000 \\ \mathrm{C} & 1.42895 & 2.43001 & 0.00000 \\ \mathrm{C} & 0.02642 & 2.44135 & 0.00000 \\ \mathrm{C} & -0.68383 & 1.23627 & 0.00000 \\ \mathrm{C} & -0.79100 & -1.24586 & -0.00000 \\ \mathrm{C} & -0.27092 & -2.49060 & -0.00000 \\ \mathrm{H} & 1.96214 & -0.95405 & -0.00000 \\ \mathrm{H} & 3.21915 & 1.19949 & 0.00000 \\ \mathrm{H} & 1.98841 & 3.37917 & 0.00000 \\ \mathrm{H} & -0.51560 & 3.40088 & 0.00000 \\ \mathrm{H} & -1.78702 & 1.24744 & 0.00000 \\ \mathrm{H} & -1.89039 & -1.10591 & -0.00000 \\ \mathrm{H} & 0.80275 & -2.67962 & -0.00000 \\ \mathrm{H} & -0.89954 & -3.38075 & -0.00000\end{array}$


Table S1357 Employed active space, reference configurations, and Cartesian coordinates of the OM3/MRCISD-optimized excited-state equilibrium geometry of the $2{ }^{1} A\left({ }^{1} \pi \pi^{*}\right)$ state of styrene (excited-state energy: $-1115.21207 \mathrm{eV}$ ).

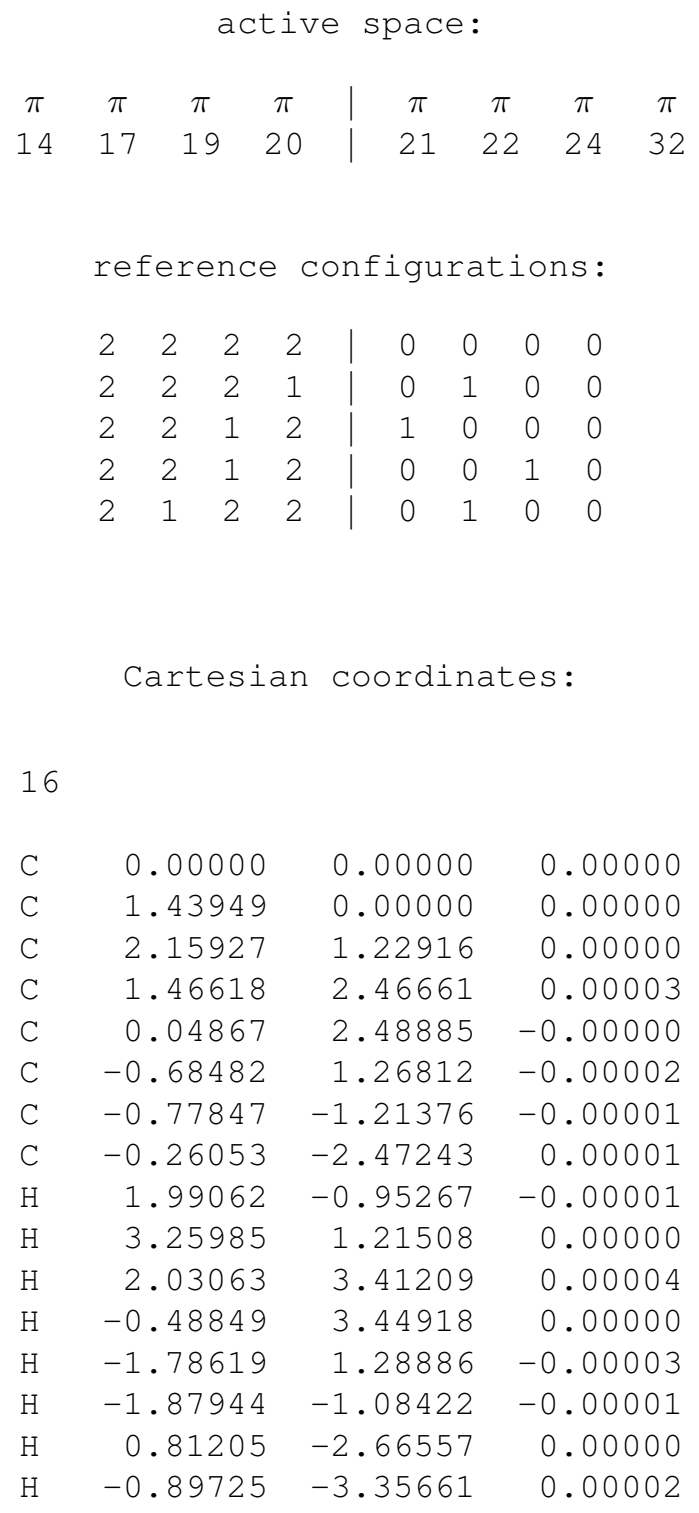


Table S1358 Employed active space, reference configurations, and Cartesian coordinates of the OM3/MRCISD-optimized ground-state equilibrium geometry of syn-coumarin 153 (ground-state energy: -4505.17949 eV).

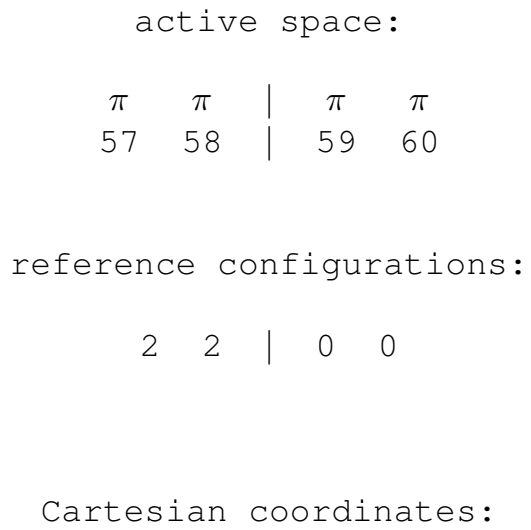




\begin{tabular}{lrrr}
\multicolumn{5}{c}{ Continued from previous page } \\
\hline H & 4.72456 & 2.72125 & 1.04743 \\
H & 5.42826 & 2.32046 & -0.56564 \\
H & 4.21196 & 4.55257 & -0.58470 \\
H & 3.48258 & 3.36306 & -1.72865 \\
H & 2.46970 & 4.03130 & 1.15117 \\
H & 1.68480 & 4.53073 & -0.42052 \\
H & -0.38120 & -0.87800 & -0.57486 \\
H & -0.35862 & -0.11648 & 1.05778 \\
H & -1.64292 & 1.34514 & -0.50478 \\
H & -0.28144 & 1.34050 & -1.68868 \\
H & -0.26829 & 3.46676 & -0.35518 \\
H & -0.21897 & 2.51805 & 1.20476
\end{tabular}


Table S1359 Employed active space, reference configurations, and Cartesian coordinates of the OM3/MRCISD-optimized excited-state equilibrium geometry of the $2{ }^{1} A\left({ }^{1} \pi \pi^{*}\right)$ state of syn-coumarin 153 (excited-state energy: $-4501.68862 \mathrm{eV})$.

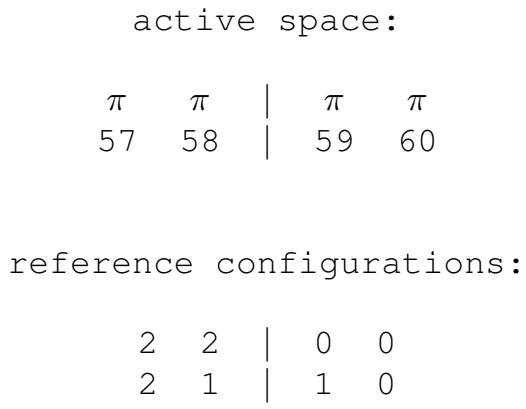




\begin{tabular}{lrrr}
\multicolumn{5}{c}{ Continued from previous page } \\
\hline $\mathrm{H}$ & 5.44923 & -0.01213 & -0.03634 \\
$\mathrm{H}$ & 4.74292 & 2.76780 & 1.00006 \\
$\mathrm{H}$ & 5.39500 & 2.37666 & -0.63419 \\
$\mathrm{H}$ & 4.15339 & 4.60310 & -0.59455 \\
$\mathrm{H}$ & 3.42093 & 3.41799 & -1.73786 \\
$\mathrm{H}$ & 2.45724 & 4.01416 & 1.17009 \\
$\mathrm{H}$ & 1.63339 & 4.53975 & -0.37036 \\
$\mathrm{H}$ & -0.37598 & -0.88747 & -0.55704 \\
$\mathrm{H}$ & -0.35517 & -0.10572 & 1.06059 \\
$\mathrm{H}$ & -1.64854 & 1.33525 & -0.52016 \\
$\mathrm{H}$ & -0.28048 & 1.34003 & -1.69292 \\
$\mathrm{H}$ & -0.28479 & 3.46219 & -0.33112 \\
$\mathrm{H}$ & -0.22884 & 2.48539 & 1.20962
\end{tabular}


Table S1360 Employed active space, reference configurations, and Cartesian coordinates of the OM3/MRCISD-optimized ground-state equilibrium geometry of terylene (ground-state energy: $-3989.95339 \mathrm{eV}$ ).

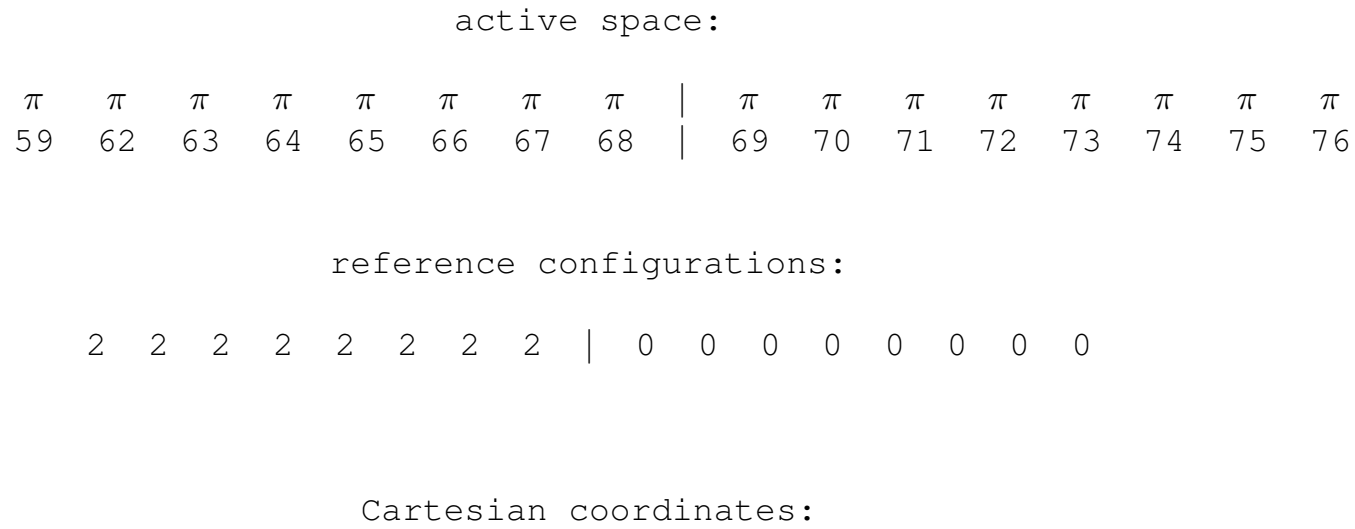




\begin{tabular}{lrrr}
\multicolumn{4}{c}{ Continued from previous page } \\
\hline C & 5.76113 & 2.43205 & -0.00000 \\
$\mathrm{C}$ & 5.76113 & -2.43205 & -0.00000 \\
$\mathrm{C}$ & -2.91673 & 2.43829 & 0.00000 \\
$\mathrm{C}$ & -2.91673 & -2.43829 & 0.00000 \\
$\mathrm{C}$ & 4.35094 & 2.43829 & -0.00000 \\
$\mathrm{C}$ & 4.35094 & -2.43829 & -0.00000 \\
$\mathrm{H}$ & -0.50492 & 3.40956 & -0.00000 \\
$\mathrm{H}$ & -0.50492 & -3.40956 & -0.00000 \\
$\mathrm{H}$ & 1.93913 & 3.40956 & 0.00000 \\
$\mathrm{H}$ & 1.93913 & -3.40956 & 0.00000 \\
$\mathrm{H}$ & -6.12362 & 1.22410 & 0.00000 \\
$\mathrm{H}$ & -6.12362 & -1.22410 & 0.00000 \\
$\mathrm{H}$ & 7.55783 & 1.22410 & -0.00000 \\
$\mathrm{H}$ & 7.55783 & -1.22410 & -0.00000 \\
$\mathrm{H}$ & -4.86802 & 3.39252 & 0.00000 \\
$\mathrm{H}$ & -4.86802 & -3.39252 & 0.00000 \\
$\mathrm{H}$ & 6.30223 & 3.39252 & -0.00000 \\
$\mathrm{H}$ & 6.30223 & -3.39252 & -0.00000 \\
$\mathrm{H}$ & -2.40256 & 3.41460 & 0.00000 \\
$\mathrm{H}$ & -2.40256 & -3.41460 & -0.00000 \\
$\mathrm{H}$ & 3.83677 & 3.41460 & -0.00000 \\
$\mathrm{H}$ & 3.83677 & -3.41460 & 0.00000
\end{tabular}

S1453 
Table S1361 Employed active space, reference configurations, and Cartesian coordinates of the OM3/MRCISD-optimized excited-state equilibrium geometry of the $2{ }^{1} A^{\prime}\left({ }^{1} \pi \pi^{*}\right)$ state of terylene (excited-state energy: $\left.-3987.11986 \mathrm{eV}\right)$.

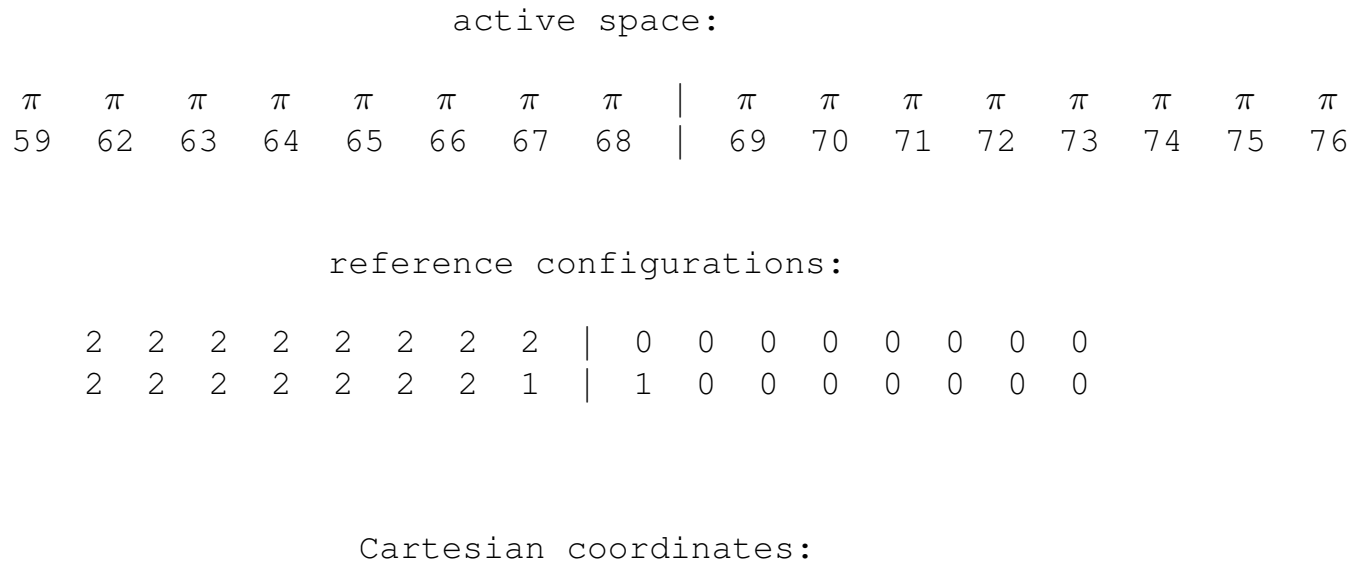




\begin{tabular}{lrrl}
\multicolumn{4}{c}{ Continued from previous page } \\
\hline $\mathrm{C}$ & 5.95336 & -7.31001 & 0.00000 \\
$\mathrm{C}$ & 4.56588 & -7.34879 & 0.00000 \\
$\mathrm{C}$ & 3.81918 & -6.16443 & 0.00000 \\
$\mathrm{C}$ & 2.02739 & 3.72387 & 0.00000 \\
$\mathrm{C}$ & 3.41487 & 3.76264 & 0.00000 \\
$\mathrm{C}$ & 4.16156 & 2.57828 & 0.00000 \\
$\mathrm{H}$ & 7.54116 & -1.03428 & 0.00000 \\
$\mathrm{H}$ & 1.72993 & -4.63462 & 0.00000 \\
$\mathrm{H}$ & 6.25079 & 1.04847 & 0.00000 \\
$\mathrm{H}$ & 0.43957 & -2.55187 & 0.00000 \\
$\mathrm{H}$ & 9.81076 & -4.74484 & 0.00000 \\
$\mathrm{H}$ & 4.04186 & -8.31896 & 0.00000 \\
$\mathrm{H}$ & 3.93889 & 4.73281 & 0.00000 \\
$\mathrm{H}$ & -1.83002 & 1.15871 & 0.00000 \\
$\mathrm{H}$ & 8.53483 & -2.63215 & 0.00000 \\
$\mathrm{H}$ & 2.71826 & -6.23581 & 0.00000 \\
$\mathrm{H}$ & 5.26249 & 2.64965 & 0.00000 \\
$\mathrm{H}$ & -0.55410 & -0.95399 & 0.00000 \\
$\mathrm{H}$ & 8.62859 & -6.94931 & 0.00000 \\
$\mathrm{H}$ & 6.54212 & -8.24199 & 0.00000 \\
$\mathrm{H}$ & 1.43864 & 4.65584 & 0.00000 \\
$\mathrm{H}$ & -0.64785 & 3.36317 & 0.00000
\end{tabular}

S1455 
Table S1362 Employed active space, reference configurations, and Cartesian coordinates of the OM3/MRCISD-optimized ground-state equilibrium geometry of $s$-tetrazine (ground-state energy: $-1141.75409 \mathrm{eV}$ ).

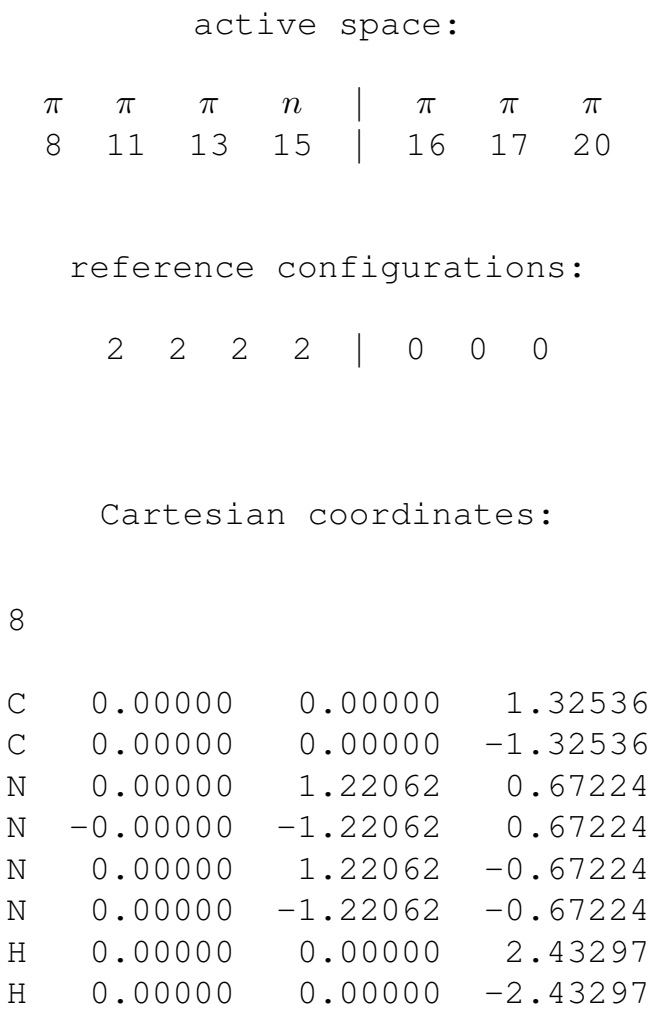


Table S1363 Employed active space, reference configurations, and Cartesian coordinates of the OM3/MRCISD-optimized excited-state equilibrium geometry of the $1^{1} B_{3 u}\left({ }^{1} n \pi^{*}\right)$ state of $s$-tetrazine (excited-state energy: $-1138.83474 \mathrm{eV})$.

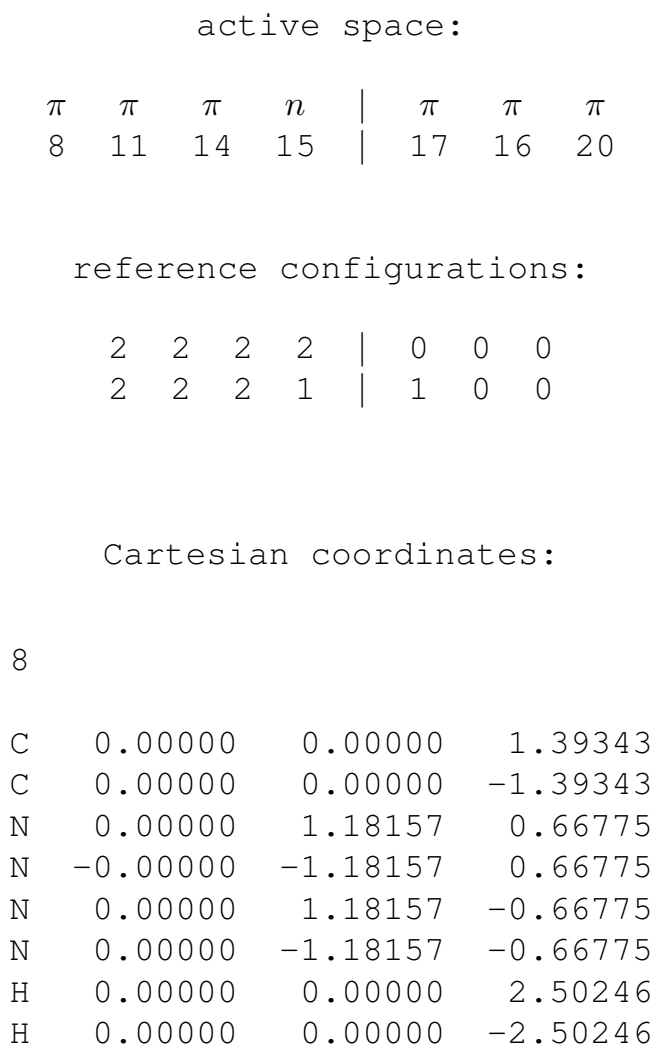


Table S1364 Employed active space, reference configurations, and Cartesian coordinates of the OM3/MRCISD-optimized ground-state equilibrium geometry of toluene (ground-state energy: $-994.96718 \mathrm{eV}$ ).

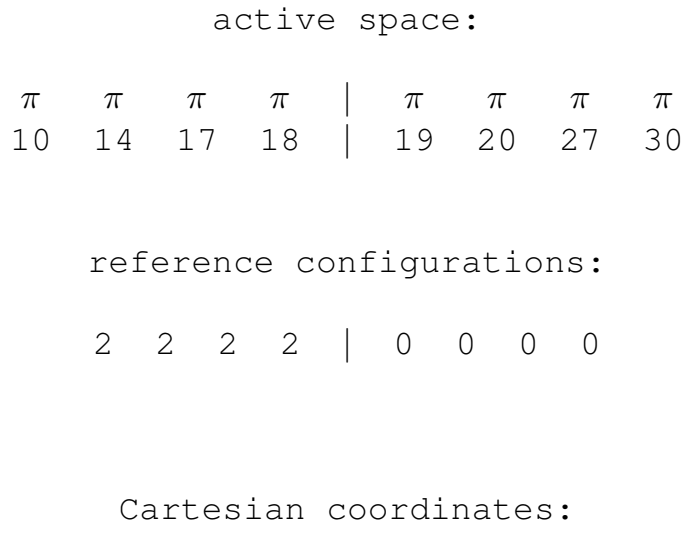

15

C $\quad 0.00000$

C $\quad 1.49839$

0.00000

0.00000

$-0.72086$

0.00000

0.00000

C $\quad-0.70600$

1.21058

0.00000

C $\quad-2.12160$

$-1.22061-0.00000$

C $\quad-2.10616$

$1.19991-0.00000$

C $\quad-2.81625$

$-1.22712-0.00000$

$\mathrm{H} \quad 1.90232$

$-0.01777$

$-0.00000$

1.88275

1.02923

0.00000

1.88275

$-0.52698$

0.89621

$-0.17678$

$-0.52698$

$-0.89621$

$\begin{array}{ll}2.16966 & 0.00000\end{array}$

$-0.15022$

$-2.17221$

$-0.00000$

$-2.67895$

$2.15099-0.00000$

$\mathrm{H} \quad-2.65193$

$-2.18495-0.00000$

$\mathrm{H} \quad-3.91791$

$-0.02485$

$-0.00000$ 
Table S1365 Employed active space, reference configurations, and Cartesian coordinates of the OM3/MRCISD-optimized excited-state equilibrium geometry of the $2{ }^{1} A^{\prime}\left({ }^{1} \pi \pi^{*}\right)$ state of toluene (excited-state energy: $-990.70281 \mathrm{eV}$ ).

\begin{tabular}{|c|c|c|c|c|c|c|c|c|}
\hline \multirow{2}{*}{$\begin{array}{c}\pi \\
10\end{array}$} & $\pi$ & $\pi$ & $\pi$ & $\pi$ & \multicolumn{2}{|c|}{$\pi$} & $\pi$ & $\pi$ \\
\hline & 14 & 7 & 18 & 19 & 2 & & 27 & 30 \\
\hline & $r e f$ & ence & & onfigu & cat & ior & s: & \\
\hline & 2 & 2 & 2 & 0 & 0 & 0 & 0 & \\
\hline & 2 & 2 & 1 & 0 & 1 & 0 & 0 & \\
\hline & 2 & 1 & 2 & 1 & 0 & 0 & 0 & \\
\hline & & cesi & an & coord & nat & es & & \\
\hline 15 & & & & & & & & \\
\hline C & & 0000 & & 0.000 & & & $.00 C$ & 000 \\
\hline$C$ & & 8649 & & 0.000 & & & .000 & 000 \\
\hline C & -0 . & 3562 & & 1.2328 & & & .00 & 000 \\
\hline C & -0 . & 1419 & & -1.2439 & & -0 & .00 & 000 \\
\hline C & -2 . & 5879 & & 1.215 & & & .000 & 000 \\
\hline C & -2 . & 3776 & & -1.2532 & & -0 & .00 & 000 \\
\hline C & -2 . & 5726 & & 0.0238 & & -0 & .000 & 000 \\
\hline $\mathrm{H}$ & & 9589 & & 1.0269 & & & .000 & \\
\hline $\mathrm{H}$ & & 7171 & & 0.5323 & & & .894 & \\
\hline $\mathrm{H}$ & & 7171 & & 0.5323 & & -0 & .894 & 472 \\
\hline $\mathrm{H}$ & -0 . & 9374 & & 2.1906 & & & $.00 C$ & 000 \\
\hline $\mathrm{H}$ & -0 . & 5681 & & 2.1929 & & -0 & .000 & 000 \\
\hline $\mathrm{H}$ & -2 . & 1942 & & 2.1624 & & -0 & $.00 C$ & 000 \\
\hline H & -2 . & 8183 & & 2.2090 & & -0 & .000 & \\
\hline$H$ & -3. & & & 0.0335 & & & & 000 \\
\hline
\end{tabular}


Table S1366 Employed active space, reference configurations, and Cartesian coordinates of the OM3/MRCISD-optimized ground-state equilibrium geometry of trans-stilbene (ground-state energy: $-1929.56253 \mathrm{eV}$ ).

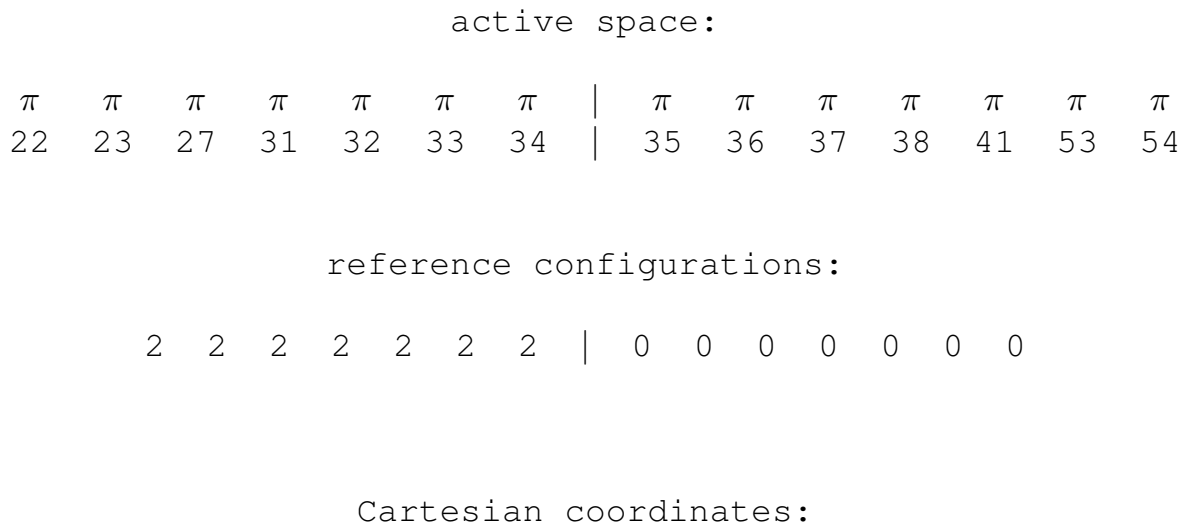


Continued from previous page

\begin{tabular}{rrrr}
\hline $\mathrm{H}$ & 0.00000 & 3.35978 & 0.04052 \\
$\mathrm{H}$ & 0.00000 & -3.35978 & -0.04052
\end{tabular}

S1461 
Table S1367 Employed active space, reference configurations, and Cartesian coordinates of the OM3/MRCISD-optimized excited-state equilibrium geometry of the $2{ }^{1} A\left({ }^{1} \pi \pi^{*}\right)$ state of trans-stilbene (excited-state energy: $-1925.17599 \mathrm{eV})$.

\begin{tabular}{|c|c|c|c|c|c|c|c|c|c|c|c|c|c|c|c|}
\hline$\pi$ & $\pi$ & $\pi$ & $\pi$ & $\pi$ & $\pi$ & $\pi$ & $\pi$ & & $\pi$ & $\pi$ & $\pi$ & & $\pi$ & $\pi$ & $\pi$ \\
\hline \multirow[t]{4}{*}{22} & 23 & 27 & 31 & 32 & 33 & 34 & 35 & & 36 & 37 & 38 & & 41 & 52 & 54 \\
\hline & \multicolumn{14}{|c|}{ reference c } & \\
\hline & & 2 & 2 & 2 & 2 & 2 & 0 & 0 & 0 & 0 & 0 & 0 & 0 & & \\
\hline & & 2 & 2 & 2 & 2 & 1 & 1 & 0 & 0 & 0 & 0 & 0 & 0 & & \\
\hline
\end{tabular}

\begin{tabular}{lrrr}
26 & & & \\
& & & \\
$\mathrm{C}$ & 0.00000 & 0.00000 & 0.00000 \\
$\mathrm{C}$ & 1.43883 & 0.00000 & 0.00000 \\
$\mathrm{C}$ & 2.11054 & 1.27015 & 0.00000 \\
$\mathrm{C}$ & 1.37703 & 2.45181 & 0.00004 \\
$\mathrm{C}$ & -0.02835 & 2.42665 & 0.00005 \\
$\mathrm{C}$ & -0.71009 & 1.19091 & 0.00006 \\
$\mathrm{C}$ & 2.13264 & -1.23583 & -0.00014 \\
$\mathrm{C}$ & 3.53588 & -1.35653 & -0.00026 \\
$\mathrm{C}$ & 4.22891 & -2.59272 & -0.00047 \\
$\mathrm{C}$ & 3.55731 & -3.86247 & -0.00035 \\
$\mathrm{C}$ & 4.28912 & -5.04576 & -0.00045 \\
$\mathrm{C}$ & 5.69450 & -5.02110 & -0.00072 \\
$\mathrm{C}$ & 6.37643 & -3.78510 & -0.00087 \\
$\mathrm{C}$ & 5.66788 & -2.59351 & -0.00072 \\
$\mathrm{H}$ & 4.14931 & -0.43592 & -0.00012 \\
$\mathrm{H}$ & 1.51942 & -2.15647 & -0.00020 \\
$\mathrm{H}$ & 2.45574 & -3.89658 & 0.00002 \\
$\mathrm{H}$ & 3.21198 & 1.30478 & -0.00009 \\
$\mathrm{H}$ & 3.76466 & -6.01595 & -0.00015 \\
$\mathrm{H}$ & 1.90033 & 3.42275 & -0.00006 \\
$\mathrm{H}$ & 6.26466 & -5.96355 & -0.00069 \\
$\mathrm{H}$ & -0.59848 & 3.36918 & 0.00003 \\
$\mathrm{H}$ & 7.47870 & -3.77610 & -0.00102 \\
\hline & & Continued & on next page
\end{tabular}

S1462 


\begin{tabular}{lrrr}
\multicolumn{5}{c}{ Continued from previous page } \\
\hline $\mathrm{H}$ & -1.81211 & 1.18133 & -0.00006 \\
$\mathrm{H}$ & 6.19777 & -1.62580 & -0.00066 \\
$\mathrm{H}$ & -0.52899 & -0.96825 & -0.00015
\end{tabular}

S1463 
Table S1368 Employed active space, reference configurations, and Cartesian coordinates of the OM3/MRCISD-optimized ground-state equilibrium geometry of vinyl radical (ground-state energy: $-292.11589 \mathrm{eV}$ ).

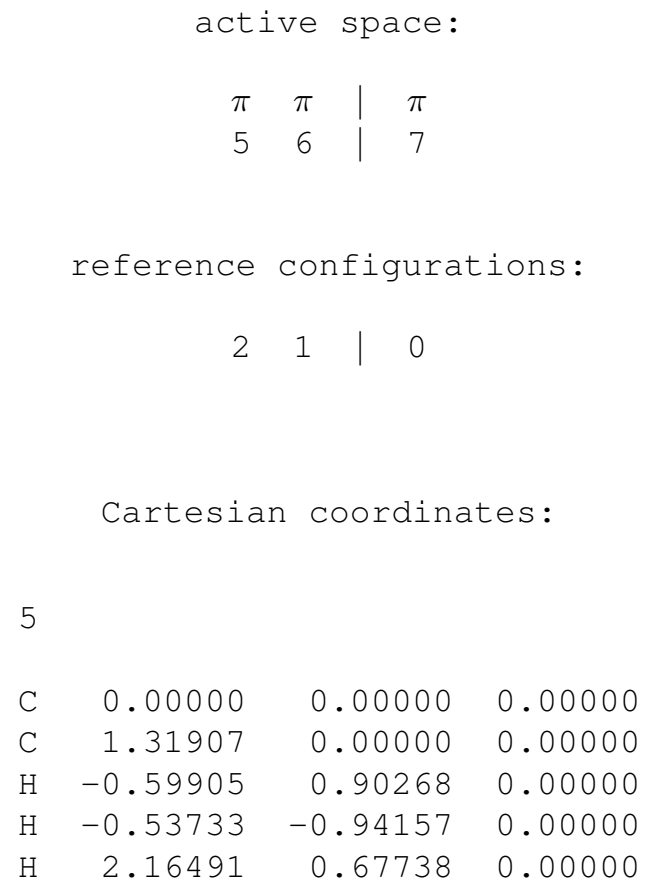


Table S1369 Employed active space, reference configurations, and Cartesian coordinates of the OM3/MRCISD-optimized excited-state equilibrium geometry of the $2^{2} A\left({ }^{2} \pi \sigma^{*}\right)$ state of vinyl radical (excited-state energy: $-289.62115 \mathrm{eV}$ ).

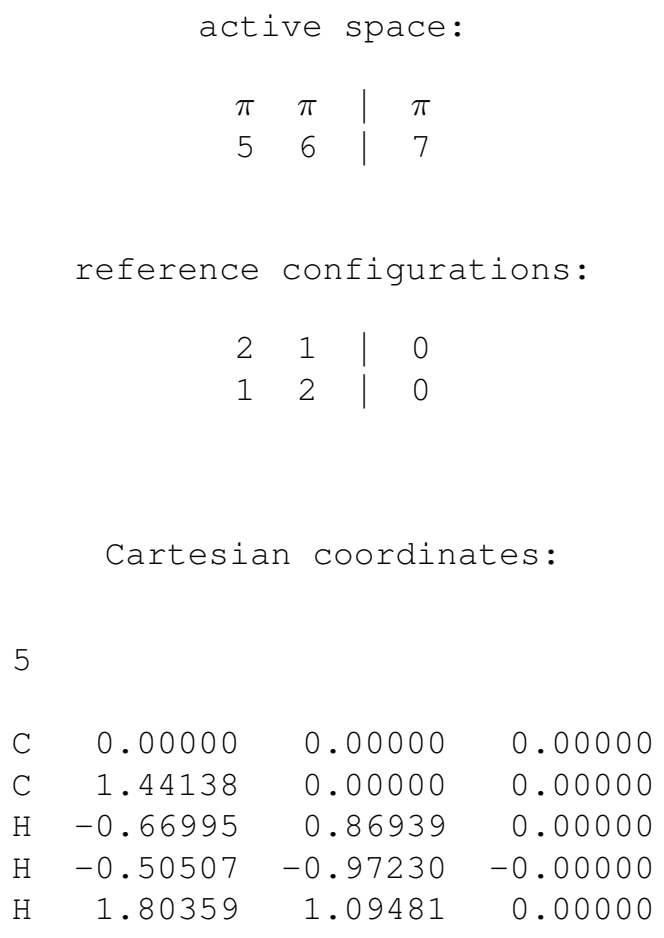


4.4 Winter-Graf-Leutwyler-Hättig Benchmark Set on 00 Transition Energies 

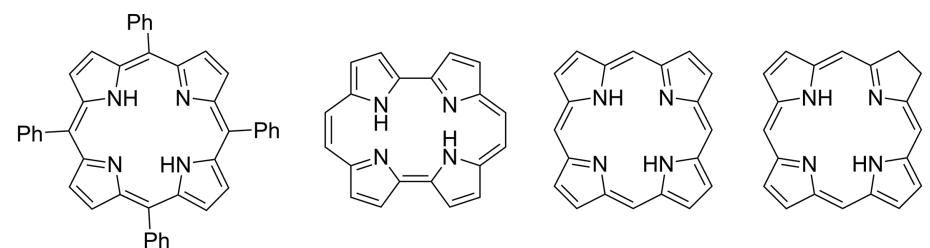

Now

tetraphenylporphine

porphycene

porphine

chlorin

tetracene
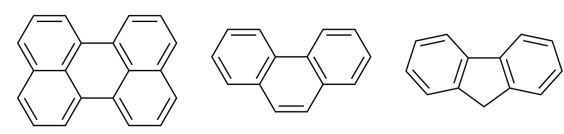

phenanthrene

fluorene
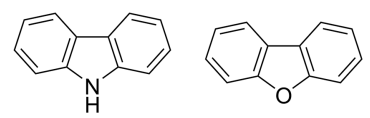

dibenzofuran

carbazole

pyrrolo[3,2-h]quinoline<smiles>NCCc1c[nH]c2ccccc12</smiles><smiles>C/C=C/C=C/C=C/C=C/C</smiles><smiles>COc1ccc(O)c(C(=O)O)c1</smiles><smiles>O=C(O)c1ccccc1O</smiles><smiles>O=C(O)CCc1ccccc1</smiles>

tryptamine

2,4,6,8-decatetraene

5-methoxysalicylic acid

salicylic acid

3-phenyl-1-propionic acid
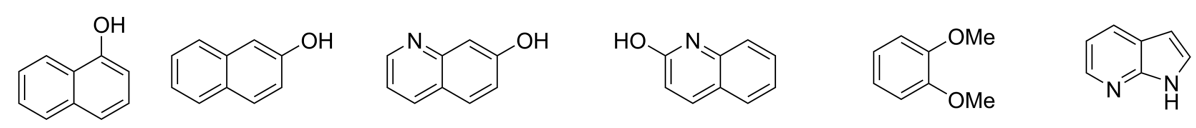

1-naphthol

2-naphthol

7-hydroxyquinoline

2-hydroxyquinoline

o-dimethoxybenzene

7-azaindole
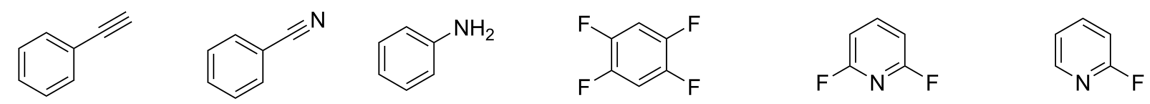

phenylacetylene

benzonitrile

aniline

tetrafluorobenzene

2,6-difluoropyridine

2-fluoropyridine
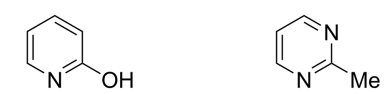

2-hydroxypyridine

2-methylpyrimidine

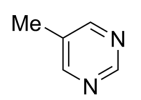

5-methylpyrimidine<smiles>c1cnccn1</smiles><smiles>O=c1cccc[nH]1</smiles><smiles>Oc1ccccc1O</smiles><smiles>Oc1cccc(O)c1</smiles>

resorcinol

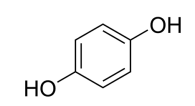

hydroquinone

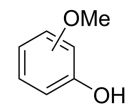

o/m/p-methoxyphenol pyrazine

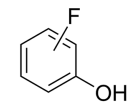

o/m/p-fluorophenol catechol<smiles>Cc1ccccc1O</smiles>

olm/p-creso

Figure S14 Structural formulas of the molecules in the WGLH benchmark set on $0-0$ transition energies. 
4.4.1 OM2/MRCI-Optimized Ground- and Excited-State Minima 
Table S1370 Employed active space, reference configurations, and Cartesian coordinates of the OM2/MRCISD-optimized ground-state equilibrium geometry of tetraphenylporphine (ground-state energy: $-6909.60542 \mathrm{eV}$ ).

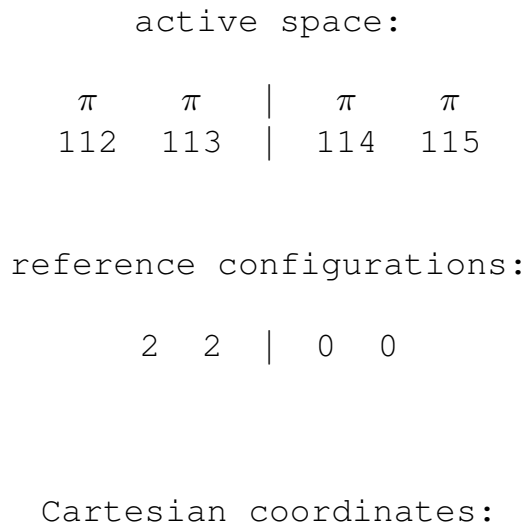

\begin{tabular}{rrrr}
$\mathrm{N}$ & 2.06631 & 0.00000 & 0.00000 \\
$\mathrm{~N}$ & -2.06631 & 0.00000 & 0.00000 \\
$\mathrm{~N}$ & 0.00000 & -2.02923 & -0.01558 \\
$\mathrm{~N}$ & 0.00000 & 2.02923 & -0.01558 \\
$\mathrm{C}$ & 2.86524 & -1.12550 & -0.02363 \\
$\mathrm{C}$ & 2.86524 & 1.12550 & -0.02363 \\
$\mathrm{C}$ & -2.86524 & 1.12550 & -0.02363 \\
$\mathrm{C}$ & -2.86524 & -1.12550 & -0.02363 \\
$\mathrm{C}$ & -1.08500 & -2.84874 & 0.00875 \\
$\mathrm{C}$ & 1.08500 & -2.84874 & 0.00875 \\
$\mathrm{C}$ & 1.08500 & 2.84874 & 0.00875 \\
$\mathrm{C}$ & -1.08500 & 2.84874 & 0.00875 \\
$\mathrm{C}$ & 2.43438 & 2.46184 & -0.00833 \\
$\mathrm{C}$ & 2.43438 & -2.46184 & -0.00833 \\
$\mathrm{C}$ & -2.43438 & -2.46184 & -0.00833 \\
$\mathrm{C}$ & -2.43438 & 2.46184 & -0.00833 \\
$\mathrm{C}$ & 4.24857 & -0.68550 & -0.07787 \\
$\mathrm{C}$ & 4.24857 & 0.68550 & -0.07787 \\
$\mathrm{C}$ & -4.24857 & 0.68550 & -0.07787 \\
$\mathrm{C}$ & -4.24857 & -0.68550 & -0.07787 \\
$\mathrm{C}$ & -0.67772 & -4.27045 & 0.07230 \\
$\mathrm{C}$ & 0.67772 & -4.27045 & 0.07230 \\
$\mathrm{C}$ & 0.67772 & 4.27045 & 0.07230 \\
$\mathrm{C}$ & -0.67772 & 4.27045 & 0.07230 \\
\hline & & Continued & on next page
\end{tabular}




\begin{tabular}{|c|c|c|c|}
\hline \multicolumn{4}{|c|}{ Continued from previous page } \\
\hline C & 3.47902 & 3.50771 & -0.01449 \\
\hline$C$ & 3.47902 & -3.50771 & -0.01449 \\
\hline$C$ & -3.47902 & -3.50771 & -0.01449 \\
\hline$C$ & -3.47902 & 3.50771 & -0.01449 \\
\hline$C$ & 4.33757 & 3.64134 & 1.08182 \\
\hline C & 4.33757 & -3.64134 & 1.08182 \\
\hline C & -4.33757 & -3.64134 & 1.08182 \\
\hline C & -4.33757 & 3.64134 & 1.08182 \\
\hline C & 3.60216 & 4.35748 & -1.11891 \\
\hline$C$ & 3.60216 & -4.35748 & -1.1 \\
\hline C & -3.60216 & -4.35748 & -1.11891 \\
\hline C & -3.60216 & 4.35748 & -1.1 \\
\hline C & 5.31807 & 4.62884 & \\
\hline C & 5.31807 & -4.62884 & 1 . \\
\hline C & -5.31807 & $-4 \cdot 6$ & 1.0 \\
\hline C & -5.31807 & 4.6 & 1 . \\
\hline C & 4.589 & 5.3 & -1 \\
\hline C & 4.5 & -5.3 & -1 \\
\hline C & -4.58900 & -5.3 & -1 \\
\hline C & -4.58900 & 5.3 & -1 \\
\hline C & 5.445 & 5.2 & -0 . \\
\hline C & 5.445 & -5.4 & -0 . \\
\hline C & -5.445 & -5.4 & -0 . \\
\hline C & -5.44559 & 5.4 & -0 . \\
\hline H & 5.09296 & -1.3 & -0 . \\
\hline $\mathrm{H}$ & 5.09296 & 1.3 & -0 \\
\hline $\mathrm{H}$ & -5.09296 & 1.3 & -0 \\
\hline $\mathrm{H}$ & -5.09296 & -1.3 & -0 \\
\hline $\mathrm{H}$ & -1.36510 & -5.1 & 88 \\
\hline $\mathrm{H}$ & 1.36510 & -5.1 & 0 \\
\hline $\mathrm{H}$ & 1.36510 & 5.1 & 0.1 \\
\hline $\mathrm{H}$ & -1.36510 & 5.1 & 0.1 \\
\hline $\mathrm{H}$ & 4.22743 & 2.97936 & 1.9 \\
\hline $\mathrm{H}$ & 4.22743 & -2.97936 & 1.9 \\
\hline $\mathrm{H}$ & -4.22743 & -2.97936 & 1.9457 \\
\hline H & -4.22743 & 2.97936 & 1.94571 \\
\hline $\mathrm{H}$ & 2.92835 & 4.24288 & -1.97267 \\
\hline H & 2.92835 & -4.24288 & -1.97267 \\
\hline $\mathrm{H}$ & -2.92835 & -4.24288 & -1.97267 \\
\hline $\mathrm{H}$ & -2.92835 & 4.24288 & -1.97267 \\
\hline $\mathrm{H}$ & 5.99109 & 4.74253 & 1.92548 \\
\hline $\mathrm{H}$ & 5.99109 & -4.74253 & 1.92548 \\
\hline $\mathrm{H}$ & -5.99109 & -4.74253 & 1.92548 \\
\hline $\mathrm{H}$ & -5.99109 & 4.74253 & 1.92548 \\
\hline
\end{tabular}

S1470 


\begin{tabular}{lrrr}
\multicolumn{4}{c}{ Continued from previous page } \\
\hline $\mathrm{H}$ & 4.69318 & 6.00629 & -1.98426 \\
$\mathrm{H}$ & 4.69318 & -6.00629 & -1.98426 \\
$\mathrm{H}$ & -4.69318 & -6.00629 & -1.98426 \\
$\mathrm{H}$ & -4.69318 & 6.00629 & -1.98426 \\
$\mathrm{H}$ & 6.21995 & 6.25024 & -0.03766 \\
$\mathrm{H}$ & 6.21995 & -6.25024 & -0.03766 \\
$\mathrm{H}$ & -6.21995 & -6.25024 & -0.03766 \\
$\mathrm{H}$ & -6.21995 & 6.25024 & -0.03766 \\
$\mathrm{H}$ & 1.04938 & 0.00000 & 0.01831 \\
$\mathrm{H}$ & -1.04938 & 0.00000 & 0.01831
\end{tabular}


Table S1371 Employed active space, reference configurations, and Cartesian coordinates of the OM2/MRCISD-optimized excited-state equilibrium geometry of the $1{ }^{1} B_{2}\left({ }^{1} \pi \pi^{*}\right)$ state of tetraphenylporphine (excited-state energy: $-6907.15803 \mathrm{eV})$.

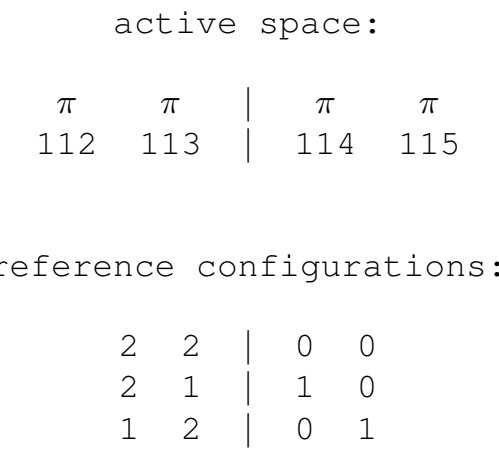

Cartesian coordinates:

\begin{tabular}{lrrr}
78 & & & \\
$\mathrm{~N}$ & 2.06653 & 0.00000 & 0.00000 \\
$\mathrm{~N}$ & -2.06653 & 0.00000 & 0.00000 \\
$\mathrm{~N}$ & 0.00000 & -2.03700 & -0.02854 \\
$\mathrm{~N}$ & 0.00000 & 2.03700 & -0.02854 \\
$\mathrm{C}$ & 2.86760 & -1.12657 & -0.03915 \\
$\mathrm{C}$ & 2.86760 & 1.12657 & -0.03915 \\
$\mathrm{C}$ & -2.86760 & 1.12657 & -0.03915 \\
$\mathrm{C}$ & -2.86760 & -1.12657 & -0.03915 \\
$\mathrm{C}$ & -1.08582 & -2.85823 & 0.01142 \\
$\mathrm{C}$ & 1.08582 & -2.85823 & 0.01142 \\
$\mathrm{C}$ & 1.08582 & 2.85823 & 0.01142 \\
$\mathrm{C}$ & -1.08582 & 2.85823 & 0.01142 \\
$\mathrm{C}$ & 2.43920 & 2.46477 & -0.01732 \\
$\mathrm{C}$ & 2.43920 & -2.46477 & -0.01732 \\
$\mathrm{C}$ & -2.43920 & -2.46477 & -0.01732 \\
$\mathrm{C}$ & -2.43920 & 2.46477 & -0.01732 \\
$\mathrm{C}$ & 4.24977 & -0.68570 & -0.12339 \\
$\mathrm{C}$ & 4.24977 & 0.68570 & -0.12339 \\
$\mathrm{C}$ & -4.24977 & 0.68570 & -0.12339 \\
$\mathrm{C}$ & -4.24977 & -0.68570 & -0.12339 \\
$\mathrm{C}$ & -0.67899 & -4.27427 & 0.10699 \\
$\mathrm{C}$ & 0.67899 & -4.27427 & 0.10699 \\
\hline & & Continued \\
& & &
\end{tabular}

S1472 


\begin{tabular}{|c|c|c|c|}
\hline \multicolumn{4}{|c|}{ ontinued from previous page } \\
\hline C & 0.67899 & 4.27427 & 0.10699 \\
\hline $\mathcal{C}$ & -0.67899 & 4.27427 & 0.10699 \\
\hline U & 3.48249 & 3.50814 & -0.02812 \\
\hline U & 3.48249 & -3.50814 & -0.02812 \\
\hline C & -3.48249 & -3.50814 & -0.02812 \\
\hline U & -3.48249 & 3.50814 & -0.02812 \\
\hline- & 4.39468 & 3.59464 & 1.02984 \\
\hline C & 4.39468 & -3.59464 & 1.02984 \\
\hline$c$ & -4.39468 & -3.59464 & 1.02984 \\
\hline C & -4.39468 & 3.59464 & 1.02984 \\
\hline$C$ & 3.55386 & 4.40643 & -1.09909 \\
\hline$C$ & 3.55386 & -4.40643 & -1.09909 \\
\hline$C$ & -3.55386 & -4.40643 & -1.09909 \\
\hline$C$ & -3.55386 & 4.40643 & -1.09909 \\
\hline$C$ & 5.37490 & 4.58213 & 1.01127 \\
\hline C & 5.37490 & -4.58213 & 1.01127 \\
\hline C & -5.37490 & -4.58213 & 1.01127 \\
\hline C & -5.37490 & 4.58213 & 1.01127 \\
\hline C & 4.54196 & 5.38610 & -1.10715 \\
\hline C & 4.54196 & -5.38610 & -1.10715 \\
\hline C & -4.54196 & -5.38610 & -1.10715 \\
\hline C & -4.54196 & 5.38610 & -1.10715 \\
\hline$C$ & 5.45094 & 5.47528 & -0 . \\
\hline C & 5.45094 & -5.47528 & -0 . \\
\hline C & -5.45094 & -5.47528 & -0 \\
\hline C & -5.45094 & 5.47 & -0 \\
\hline $\mathrm{H}$ & 5.0 & $-1 \cdot 35$ & -0 \\
\hline $\mathrm{H}$ & 5.0 & 1.3 & -0 . \\
\hline $\mathrm{H}$ & -5.0 & 1.3 & -0 . \\
\hline $\mathrm{H}$ & -5.0 & $-1 \cdot 3$ & -0.1 \\
\hline $\mathrm{H}$ & -1.36369 & -5.10438 & 0.17977 \\
\hline $\mathrm{H}$ & 1.3 & -5.1 & 0.17977 \\
\hline $\mathrm{H}$ & 1.36369 & 5.10438 & 0.17977 \\
\hline $\mathrm{H}$ & -1.36369 & 5.10438 & 0.17977 \\
\hline $\mathrm{H}$ & 4.32396 & 2.89783 & 1.87028 \\
\hline $\mathrm{H}$ & 4.32396 & -2.89783 & 1.87028 \\
\hline $\mathrm{H}$ & -4.32396 & -2.89783 & 1.87028 \\
\hline $\mathrm{H}$ & -4.32396 & 2.89783 & 1.87028 \\
\hline H & 2.84026 & 4.32835 & -1.92418 \\
\hline $\mathrm{H}$ & 2.84026 & -4.32835 & -1.92418 \\
\hline $\mathrm{H}$ & -2.84026 & -4.32835 & -1.92418 \\
\hline $\mathrm{H}$ & -2.84026 & 4.32835 & -1.92418 \\
\hline $\mathrm{H}$ & 6.08835 & 4.65908 & 1.83966 \\
\hline $\mathrm{H}$ & 6.08835 & -4.65908 & 1.83966 \\
\hline
\end{tabular}

\section{S1473}




\begin{tabular}{lrrr}
\multicolumn{5}{c}{ Continued from previous page } \\
\hline H & -6.08835 & -4.65908 & 1.83966 \\
H & -6.08835 & 4.65908 & 1.83966 \\
H & 4.60584 & 6.09007 & -1.94465 \\
H & 4.60584 & -6.09007 & -1.94465 \\
$\mathrm{H}$ & -4.60584 & -6.09007 & -1.94465 \\
$\mathrm{H}$ & -4.60584 & 6.09007 & -1.94465 \\
$\mathrm{H}$ & 6.22573 & 6.24981 & -0.06569 \\
$\mathrm{H}$ & 6.22573 & -6.24981 & -0.06569 \\
$\mathrm{H}$ & -6.22573 & -6.24981 & -0.06569 \\
$\mathrm{H}$ & -6.22573 & 6.24981 & -0.06569 \\
$\mathrm{H}$ & 1.05073 & 0.00000 & 0.02551 \\
$\mathrm{H}$ & -1.05073 & 0.00000 & 0.02551
\end{tabular}


Table S1372 Employed active space, reference configurations, and Cartesian coordinates of the OM2/MRCISD-optimized ground-state equilibrium geometry of porphycene (ground-state energy: $-3609.08194 \mathrm{eV}$ ).

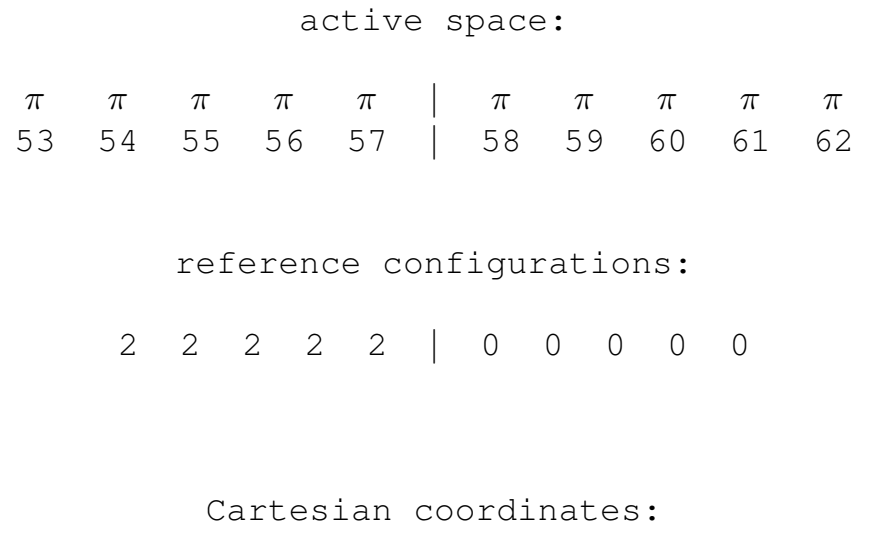

\begin{tabular}{lrrr}
38 & & & \\
& & & \\
$\mathrm{~N}$ & 0.00000 & 0.00000 & 0.00000 \\
$\mathrm{~N}$ & 3.86265 & 0.00000 & 0.00000 \\
$\mathrm{~N}$ & 1.72818 & 1.88351 & 0.00000 \\
$\mathrm{~N}$ & 2.13447 & -1.88351 & 0.00000 \\
$\mathrm{C}$ & -1.19354 & 0.67020 & 0.00000 \\
$\mathrm{C}$ & 5.05619 & -0.67020 & 0.00000 \\
$\mathrm{C}$ & -0.22672 & -1.35471 & 0.00000 \\
$\mathrm{C}$ & 4.08937 & 1.35471 & -0.00000 \\
$\mathrm{C}$ & -2.25131 & -0.32949 & 0.00000 \\
$\mathrm{C}$ & 6.11396 & 0.32949 & 0.00000 \\
$\mathrm{C}$ & -1.65500 & -1.56959 & 0.00000 \\
$\mathrm{C}$ & 5.51765 & 1.56959 & 0.00000 \\
$\mathrm{C}$ & -1.39316 & 2.06909 & 0.00000 \\
$\mathrm{C}$ & 5.25581 & -2.06909 & 0.00000 \\
$\mathrm{C}$ & 0.95675 & 2.99952 & -0.00000 \\
$\mathrm{C}$ & 2.90590 & -2.99952 & 0.00000 \\
$\mathrm{C}$ & 3.02410 & 2.28612 & -0.00000 \\
$\mathrm{C}$ & 0.83855 & -2.28612 & 0.00000 \\
$\mathrm{C}$ & 1.82233 & 4.19698 & -0.00000 \\
$\mathrm{C}$ & 2.04032 & -4.19698 & 0.00000 \\
$\mathrm{C}$ & 3.11253 & 3.74965 & -0.00000 \\
$\mathrm{C}$ & 0.75012 & -3.74965 & 0.00000 \\
$\mathrm{C}$ & -0.45288 & 3.08217 & -0.00000 \\
$\mathrm{C}$ & 4.31553 & -3.08217 & 0.00000 \\
\hline & & Continued & on next page
\end{tabular}




\begin{tabular}{lrrr}
\multicolumn{4}{c}{ Continued from previous page } \\
\hline $\mathrm{H}$ & 0.93080 & 0.49494 & -0.00000 \\
$\mathrm{H}$ & 2.93185 & -0.49494 & -0.00000 \\
$\mathrm{H}$ & -3.30840 & -0.10260 & 0.00000 \\
$\mathrm{H}$ & 7.17105 & 0.10260 & 0.00000 \\
$\mathrm{H}$ & -2.13098 & -2.53861 & 0.00000 \\
$\mathrm{H}$ & 5.99363 & 2.53861 & -0.00000 \\
$\mathrm{H}$ & -2.45299 & 2.37328 & 0.00000 \\
$\mathrm{H}$ & 6.31564 & -2.37328 & 0.00000 \\
$\mathrm{H}$ & 1.46290 & 5.21660 & -0.00000 \\
$\mathrm{H}$ & 2.39975 & -5.21660 & 0.00000 \\
$\mathrm{H}$ & 4.03040 & 4.31684 & -0.00000 \\
$\mathrm{H}$ & -0.16775 & -4.31684 & 0.00000 \\
$\mathrm{H}$ & -0.84833 & 4.11333 & -0.00000 \\
$\mathrm{H}$ & 4.71098 & -4.11333 & 0.00000
\end{tabular}


Table S1373 Employed active space, reference configurations, and Cartesian coordinates of the OM2/MRCISD-optimized excited-state equilibrium geometry of the $1{ }^{1} B_{u}\left({ }^{1} \pi \pi^{*}\right)$ state of porphycene (excited-state energy: $-3607.10727 \mathrm{eV}$ ).

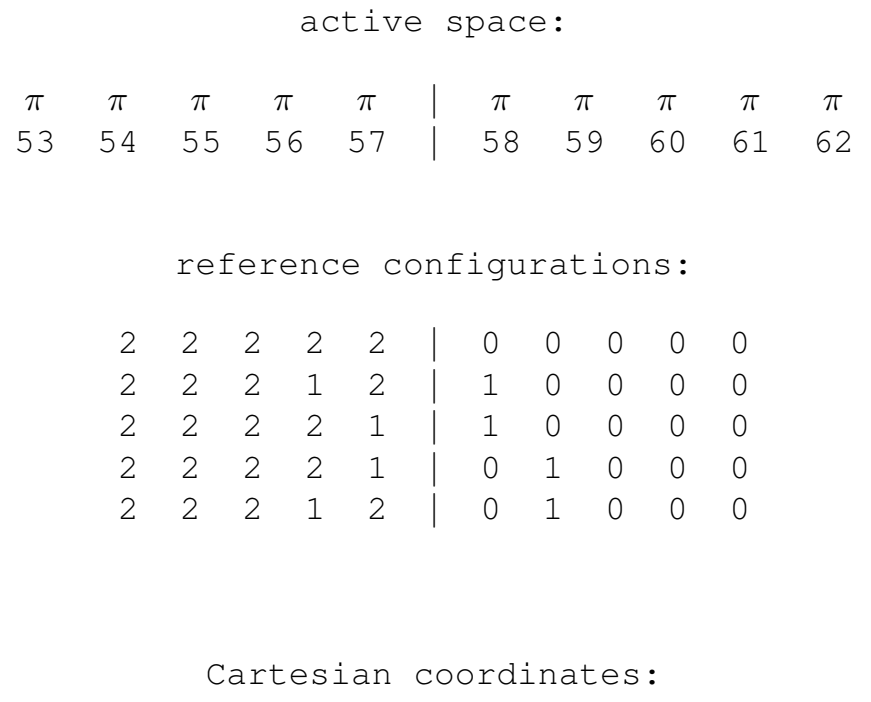

\begin{tabular}{lrrr}
38 & & & \\
& & & \\
$\mathrm{~N}$ & 0.00000 & 0.00000 & 0.00000 \\
$\mathrm{~N}$ & 3.86694 & 0.00000 & 0.00000 \\
$\mathrm{~N}$ & 1.75158 & 1.89652 & 0.00000 \\
$\mathrm{~N}$ & 2.11536 & -1.89652 & 0.00000 \\
$\mathrm{C}$ & -1.18664 & 0.68567 & 0.00000 \\
$\mathrm{C}$ & 5.05358 & -0.68567 & 0.00000 \\
$\mathrm{C}$ & -0.24545 & -1.35538 & 0.00000 \\
$\mathrm{C}$ & 4.11239 & 1.35538 & -0.00000 \\
$\mathrm{C}$ & -2.25687 & -0.30541 & 0.00000 \\
$\mathrm{C}$ & 6.12381 & 0.30541 & 0.00000 \\
$\mathrm{C}$ & -1.67496 & -1.55307 & 0.00000 \\
$\mathrm{C}$ & 5.54190 & 1.55307 & 0.00000 \\
$\mathrm{C}$ & -1.37787 & 2.08560 & 0.00000 \\
$\mathrm{C}$ & 5.24481 & -2.08560 & 0.00000 \\
$\mathrm{C}$ & 0.98547 & 3.01607 & -0.00000 \\
$\mathrm{C}$ & 2.88147 & -3.01607 & 0.00000 \\
$\mathrm{C}$ & 3.05556 & 2.30368 & -0.00000 \\
$\mathrm{C}$ & 0.81138 & -2.30368 & 0.00000 \\
$\mathrm{C}$ & 1.84923 & 4.20555 & -0.00000 \\
$\mathrm{C}$ & 2.01771 & -4.20555 & 0.00000 \\
$\mathrm{C}$ & 3.14348 & 3.75642 & -0.00000 \\
\hline & & Continued & on next page
\end{tabular}

S1477 


\begin{tabular}{lrrr}
\multicolumn{4}{c}{ Continued from previous page } \\
\hline $\mathrm{C}$ & 0.72347 & -3.75642 & 0.00000 \\
$\mathrm{C}$ & -0.43294 & 3.09568 & -0.00000 \\
$\mathrm{C}$ & 4.29988 & -3.09568 & 0.00000 \\
$\mathrm{H}$ & 0.93116 & 0.46982 & -0.00000 \\
$\mathrm{H}$ & 2.93578 & -0.46982 & -0.00000 \\
$\mathrm{H}$ & -3.31089 & -0.06560 & 0.00000 \\
$\mathrm{H}$ & 7.17783 & 0.06560 & 0.00000 \\
$\mathrm{H}$ & -2.16169 & -2.51630 & 0.00000 \\
$\mathrm{H}$ & 6.02863 & 2.51630 & -0.00000 \\
$\mathrm{H}$ & -2.43504 & 2.39536 & 0.00000 \\
$\mathrm{H}$ & 6.30198 & -2.39536 & 0.00000 \\
$\mathrm{H}$ & 1.49442 & 5.22634 & -0.00000 \\
$\mathrm{H}$ & 2.37252 & -5.22634 & 0.00000 \\
$\mathrm{H}$ & 4.05846 & 4.32663 & -0.00000 \\
$\mathrm{H}$ & -0.19152 & -4.32663 & 0.00000 \\
$\mathrm{H}$ & -0.82445 & 4.12877 & -0.00000 \\
$\mathrm{H}$ & 4.69139 & -4.12877 & 0.00000
\end{tabular}


Table S1374 Employed active space, reference configurations, and Cartesian coordinates of the OM2/MRCISD-optimized ground-state equilibrium geometry of porphine (ground-state energy: $-3609.13544 \mathrm{eV}$ ).

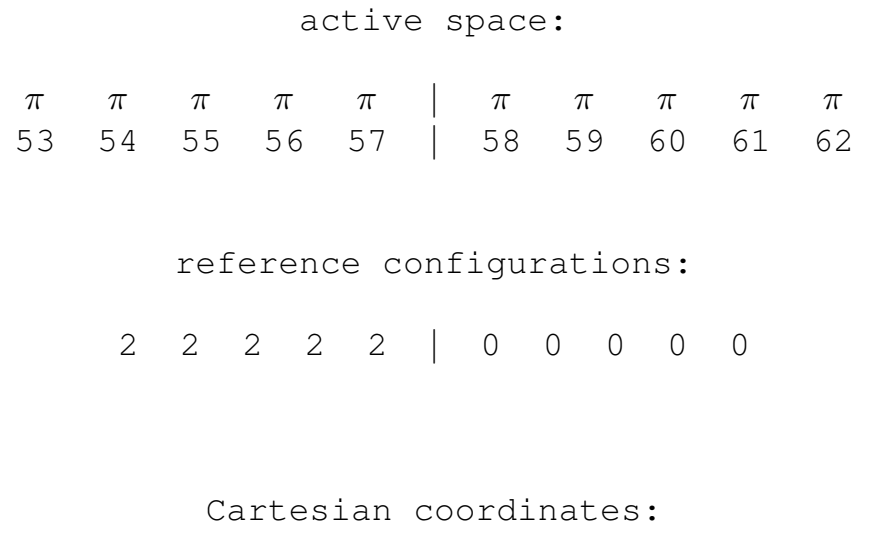

38

\begin{tabular}{lrrr}
$\mathrm{N}$ & 2.05769 & 0.00000 & 0.00000 \\
$\mathrm{~N}$ & 0.00000 & 2.01774 & 0.00000 \\
$\mathrm{~N}$ & -2.05769 & 0.00000 & 0.00000 \\
$\mathrm{~N}$ & 0.00000 & -2.01774 & -0.00000 \\
$\mathrm{C}$ & 2.85290 & 1.12408 & 0.00000 \\
$\mathrm{C}$ & 2.85290 & -1.12408 & -0.00000 \\
$\mathrm{C}$ & -2.85290 & 1.12408 & 0.00000 \\
$\mathrm{C}$ & -2.85290 & -1.12408 & 0.00000 \\
$\mathrm{C}$ & 1.08573 & 2.83659 & 0.00000 \\
$\mathrm{C}$ & -1.08573 & 2.83659 & 0.00000 \\
$\mathrm{C}$ & 1.08573 & -2.83659 & -0.00000 \\
$\mathrm{C}$ & -1.08573 & -2.83659 & -0.00000 \\
$\mathrm{C}$ & 4.23862 & 0.68748 & 0.00000 \\
$\mathrm{C}$ & 4.23862 & -0.68748 & -0.00000 \\
$\mathrm{C}$ & -4.23862 & 0.68748 & 0.00000 \\
$\mathrm{C}$ & -4.23862 & -0.68748 & 0.00000 \\
$\mathrm{C}$ & 0.67679 & 4.26468 & -0.00000 \\
$\mathrm{C}$ & -0.67679 & 4.26468 & 0.00000 \\
$\mathrm{C}$ & 0.67679 & -4.26468 & -0.00000 \\
$\mathrm{C}$ & -0.67679 & -4.26468 & -0.00000 \\
$\mathrm{C}$ & 2.43169 & 2.45840 & 0.00000 \\
$\mathrm{C}$ & 2.43169 & -2.45840 & -0.00000 \\
$\mathrm{C}$ & -2.43169 & 2.45840 & 0.00000 \\
$\mathrm{C}$ & -2.43169 & -2.45840 & -0.00000 \\
\hline & & Continued & on next page
\end{tabular}




\begin{tabular}{lrrr}
\multicolumn{5}{c}{ Continued from previous page } \\
\hline $\mathrm{H}$ & 5.08937 & 1.35479 & 0.00000 \\
$\mathrm{H}$ & 5.08937 & -1.35479 & -0.00000 \\
$\mathrm{H}$ & -5.08937 & 1.35479 & 0.00000 \\
$\mathrm{H}$ & -5.08937 & -1.35479 & 0.00000 \\
$\mathrm{H}$ & 1.36425 & 5.09810 & -0.00000 \\
$\mathrm{H}$ & -1.36425 & 5.09810 & 0.00000 \\
$\mathrm{H}$ & 1.36425 & -5.09810 & -0.00000 \\
$\mathrm{H}$ & -1.36425 & -5.09810 & -0.00000 \\
$\mathrm{H}$ & 3.20005 & 3.23978 & 0.00000 \\
$\mathrm{H}$ & 3.20005 & -3.23978 & -0.00000 \\
$\mathrm{H}$ & -3.20005 & 3.23978 & 0.00000 \\
$\mathrm{H}$ & -3.20005 & -3.23978 & 0.00000 \\
$\mathrm{H}$ & 1.04217 & 0.00000 & -0.00000 \\
$\mathrm{H}$ & -1.04217 & 0.00000 & 0.00000
\end{tabular}

S1480 
Table S1375 Employed active space, reference configurations, and Cartesian coordinates of the OM2/MRCISD-optimized excited-state equilibrium geometry of the $1{ }^{1} B_{3 u}\left({ }^{1} \pi \pi^{*}\right)$ state of porphine (excited-state energy: $-3607.17218 \mathrm{eV}$ ).

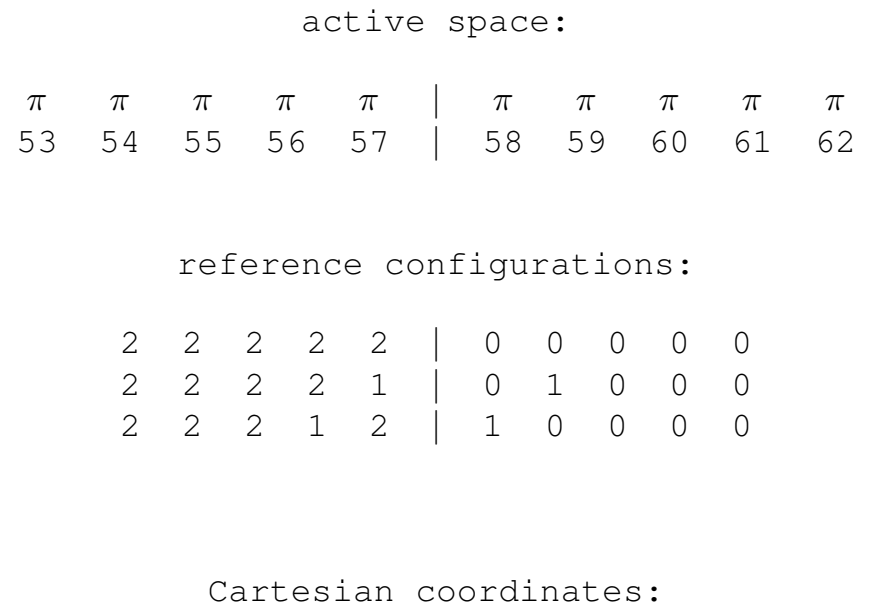

\begin{tabular}{lrrr}
38 & & & \\
& & & \\
$\mathrm{~N}$ & 2.05574 & 0.00000 & 0.00000 \\
$\mathrm{~N}$ & 0.00000 & 2.02813 & 0.00000 \\
$\mathrm{~N}$ & -2.05574 & 0.00000 & 0.00000 \\
$\mathrm{~N}$ & 0.00000 & -2.02813 & -0.00000 \\
$\mathrm{C}$ & 2.85405 & 1.12450 & 0.00000 \\
$\mathrm{C}$ & 2.85405 & -1.12450 & -0.00000 \\
$\mathrm{C}$ & -2.85405 & 1.12450 & 0.00000 \\
$\mathrm{C}$ & -2.85405 & -1.12450 & 0.00000 \\
$\mathrm{C}$ & 1.08674 & 2.85091 & 0.00000 \\
$\mathrm{C}$ & -1.08674 & 2.85091 & 0.00000 \\
$\mathrm{C}$ & 1.08674 & -2.85091 & -0.00000 \\
$\mathrm{C}$ & -1.08674 & -2.85091 & -0.00000 \\
$\mathrm{C}$ & 4.23800 & 0.68963 & 0.00000 \\
$\mathrm{C}$ & 4.23800 & -0.68963 & -0.00000 \\
$\mathrm{C}$ & -4.23800 & 0.68963 & 0.00000 \\
$\mathrm{C}$ & -4.23800 & -0.68963 & 0.00000 \\
$\mathrm{C}$ & 0.67791 & 4.27241 & -0.00000 \\
$\mathrm{C}$ & -0.67791 & 4.27241 & 0.00000 \\
$\mathrm{C}$ & 0.67791 & -4.27241 & -0.00000 \\
$\mathrm{C}$ & -0.67791 & -4.27241 & -0.00000 \\
$\mathrm{C}$ & 2.43708 & 2.46258 & 0.00000 \\
$\mathrm{C}$ & 2.43708 & -2.46258 & -0.00000 \\
$\mathrm{C}$ & -2.43708 & 2.46258 & 0.00000 \\
\hline & & Continued & on next page
\end{tabular}

S1481 


\begin{tabular}{lrrr}
\multicolumn{5}{c}{ Continued from previous page } \\
\hline C & -2.43708 & -2.46258 & -0.00000 \\
H & 5.08999 & 1.35544 & 0.00000 \\
H & 5.08999 & -1.35544 & -0.00000 \\
H & -5.08999 & 1.35544 & 0.00000 \\
H & -5.08999 & -1.35544 & 0.00000 \\
H & 1.36289 & 5.10707 & -0.00000 \\
H & -1.36289 & 5.10707 & 0.00000 \\
H & 1.36289 & -5.10707 & -0.00000 \\
H & -1.36289 & -5.10707 & -0.00000 \\
H & 3.20705 & 3.24192 & 0.00000 \\
H & 3.20705 & -3.24192 & -0.00000 \\
H & -3.20705 & 3.24192 & 0.00000 \\
H & -3.20705 & -3.24192 & 0.00000 \\
H & 1.04184 & 0.00000 & -0.00000 \\
H & -1.04184 & 0.00000 & 0.00000
\end{tabular}


Table S1376 Employed active space, reference configurations, and Cartesian coordinates of the OM2/MRCISD-optimized ground-state equilibrium geometry of chlorin (ground-state energy: $-3640.23699 \mathrm{eV}$ ).

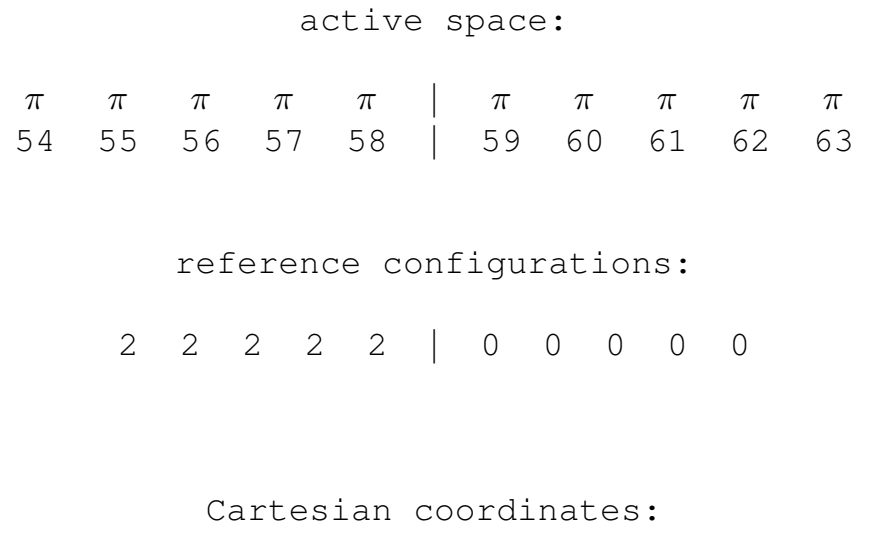

\begin{tabular}{lrrr}
40 & & & \\
& & & \\
$\mathrm{~N}$ & 0.00000 & 0.00000 & 0.00000 \\
$\mathrm{~N}$ & 0.00000 & 4.10015 & 0.00000 \\
$\mathrm{~N}$ & 2.05153 & 2.08441 & 0.00000 \\
$\mathrm{~N}$ & -2.05153 & 2.08441 & 0.00000 \\
$\mathrm{C}$ & 1.09365 & -0.78881 & 0.00000 \\
$\mathrm{C}$ & -1.09365 & -0.78881 & 0.00000 \\
$\mathrm{C}$ & 0.75922 & -2.28186 & 0.00000 \\
$\mathrm{C}$ & -0.75922 & -2.28186 & -0.00000 \\
$\mathrm{C}$ & 1.08587 & 4.92075 & 0.00000 \\
$\mathrm{C}$ & -1.08587 & 4.92075 & -0.00000 \\
$\mathrm{C}$ & 0.67851 & 6.34195 & -0.00000 \\
$\mathrm{C}$ & -0.67851 & 6.34195 & 0.00000 \\
$\mathrm{C}$ & 2.84570 & 0.95566 & -0.00000 \\
$\mathrm{C}$ & -2.84570 & 0.95566 & 0.00000 \\
$\mathrm{C}$ & 2.85261 & 3.20820 & -0.00000 \\
$\mathrm{C}$ & -2.85261 & 3.20820 & 0.00000 \\
$\mathrm{C}$ & 4.22930 & 1.38669 & -0.00000 \\
$\mathrm{C}$ & -4.22930 & 1.38669 & 0.00000 \\
$\mathrm{C}$ & 4.23717 & 2.75866 & -0.00000 \\
$\mathrm{C}$ & -4.23717 & 2.75866 & 0.00000 \\
$\mathrm{C}$ & 2.42615 & -0.38271 & 0.00000 \\
$\mathrm{C}$ & -2.42615 & -0.38271 & 0.00000 \\
$\mathrm{C}$ & 2.43321 & 4.53916 & 0.00000 \\
$\mathrm{C}$ & -2.43321 & 4.53916 & -0.00000 \\
\hline & & Continued & on next page
\end{tabular}

S1483 


\begin{tabular}{lrrr}
\multicolumn{4}{c}{ Continued from previous page } \\
\hline $\mathrm{H}$ & 1.03608 & 2.09367 & 0.00000 \\
$\mathrm{H}$ & -1.03608 & 2.09367 & 0.00000 \\
$\mathrm{H}$ & 1.36316 & 7.17854 & -0.00000 \\
$\mathrm{H}$ & -1.36316 & 7.17854 & -0.00000 \\
$\mathrm{H}$ & 5.07553 & 0.71531 & -0.00000 \\
$\mathrm{H}$ & -5.07553 & 0.71531 & 0.00000 \\
$\mathrm{H}$ & 5.09132 & 3.42239 & -0.00000 \\
$\mathrm{H}$ & -5.09132 & 3.42239 & 0.00000 \\
$\mathrm{H}$ & 3.20715 & -1.14933 & 0.00000 \\
$\mathrm{H}$ & -3.20715 & -1.14933 & 0.00000 \\
$\mathrm{H}$ & 3.20234 & 5.32079 & -0.00000 \\
$\mathrm{H}$ & -3.20234 & 5.32079 & -0.00000 \\
$\mathrm{H}$ & 1.18188 & -2.76194 & 0.90416 \\
$\mathrm{H}$ & 1.18188 & -2.76194 & -0.90416 \\
$\mathrm{H}$ & -1.18188 & -2.76194 & -0.90416 \\
$\mathrm{H}$ & -1.18188 & -2.76194 & 0.90416
\end{tabular}


Table S1377 Employed active space, reference configurations, and Cartesian coordinates of the OM2/MRCISD-optimized excited-state equilibrium geometry of the $1{ }^{1} B_{2}\left({ }^{1} \pi \pi^{*}\right)$ state of chlorin (excited-state energy: $-3638.24096 \mathrm{eV}$ ).

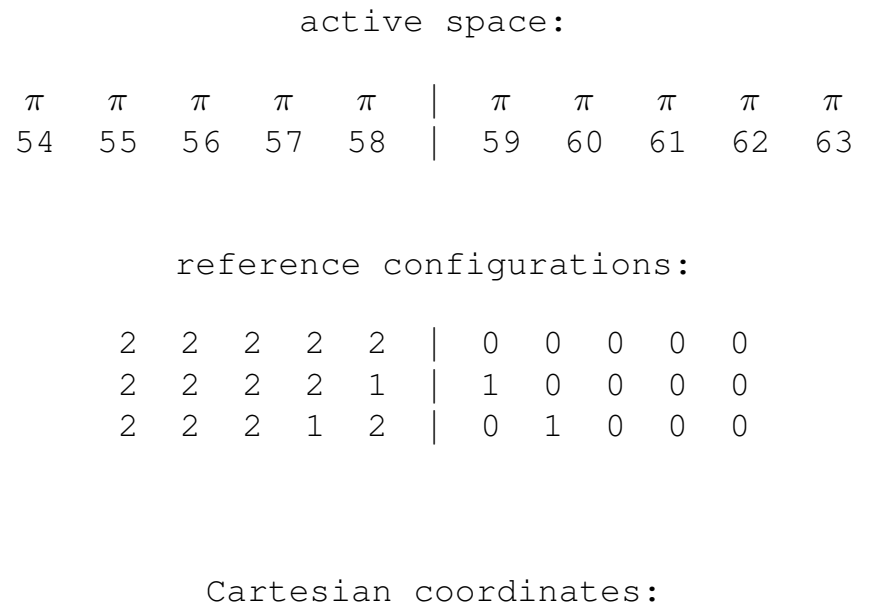

\begin{tabular}{lrrr}
40 & & & \\
& & & \\
$\mathrm{~N}$ & 0.00000 & 0.00000 & 0.00000 \\
$\mathrm{~N}$ & 0.00000 & 4.10489 & 0.00000 \\
$\mathrm{~N}$ & 2.05201 & 2.08541 & 0.00000 \\
$\mathrm{~N}$ & -2.05201 & 2.08541 & 0.00000 \\
$\mathrm{C}$ & 1.09371 & -0.79324 & 0.00000 \\
$\mathrm{C}$ & -1.09371 & -0.79324 & 0.00000 \\
$\mathrm{C}$ & 0.75964 & -2.28358 & 0.00000 \\
$\mathrm{C}$ & -0.75964 & -2.28358 & -0.00000 \\
$\mathrm{C}$ & 1.08667 & 4.92800 & 0.00000 \\
$\mathrm{C}$ & -1.08667 & 4.92800 & -0.00000 \\
$\mathrm{C}$ & 0.67787 & 6.35147 & -0.00000 \\
$\mathrm{C}$ & -0.67787 & 6.35147 & 0.00000 \\
$\mathrm{C}$ & 2.84983 & 0.95543 & -0.00000 \\
$\mathrm{C}$ & -2.84983 & 0.95543 & 0.00000 \\
$\mathrm{C}$ & 2.85501 & 3.20852 & -0.00000 \\
$\mathrm{C}$ & -2.85501 & 3.20852 & 0.00000 \\
$\mathrm{C}$ & 4.23153 & 1.38743 & -0.00000 \\
$\mathrm{C}$ & -4.23153 & 1.38743 & 0.00000 \\
$\mathrm{C}$ & 4.23748 & 2.76378 & -0.00000 \\
$\mathrm{C}$ & -4.23748 & 2.76378 & 0.00000 \\
$\mathrm{C}$ & 2.43150 & -0.38423 & 0.00000 \\
$\mathrm{C}$ & -2.43150 & -0.38423 & 0.00000 \\
$\mathrm{C}$ & 2.43789 & 4.54443 & 0.00000 \\
\hline & & Continued & on next page
\end{tabular}

S1485 


\begin{tabular}{lrrr}
\multicolumn{4}{c}{ Continued from previous page } \\
\hline $\mathrm{C}$ & -2.43789 & 4.54443 & -0.00000 \\
$\mathrm{H}$ & 1.03795 & 2.09263 & 0.00000 \\
$\mathrm{H}$ & -1.03795 & 2.09263 & 0.00000 \\
$\mathrm{H}$ & 1.36366 & 7.18620 & -0.00000 \\
$\mathrm{H}$ & -1.36366 & 7.18620 & -0.00000 \\
$\mathrm{H}$ & 5.08043 & 0.71881 & -0.00000 \\
$\mathrm{H}$ & -5.08043 & 0.71881 & 0.00000 \\
$\mathrm{H}$ & 5.09149 & 3.42686 & -0.00000 \\
$\mathrm{H}$ & -5.09149 & 3.42686 & 0.00000 \\
$\mathrm{H}$ & 3.21182 & -1.15203 & 0.00000 \\
$\mathrm{H}$ & -3.21182 & -1.15203 & 0.00000 \\
$\mathrm{H}$ & 3.20649 & 5.32432 & -0.00000 \\
$\mathrm{H}$ & -3.20649 & 5.32432 & -0.00000 \\
$\mathrm{H}$ & 1.18169 & -2.76384 & 0.90416 \\
$\mathrm{H}$ & 1.18169 & -2.76384 & -0.90416 \\
$\mathrm{H}$ & -1.18169 & -2.76384 & -0.90416 \\
$\mathrm{H}$ & -1.18169 & -2.76384 & 0.90416
\end{tabular}


Table S1378 Employed active space, reference configurations, and Cartesian coordinates of the OM2/MRCISD-optimized ground-state equilibrium geometry of tetracene (ground-state energy: $-2476.39417 \mathrm{eV}$ ).

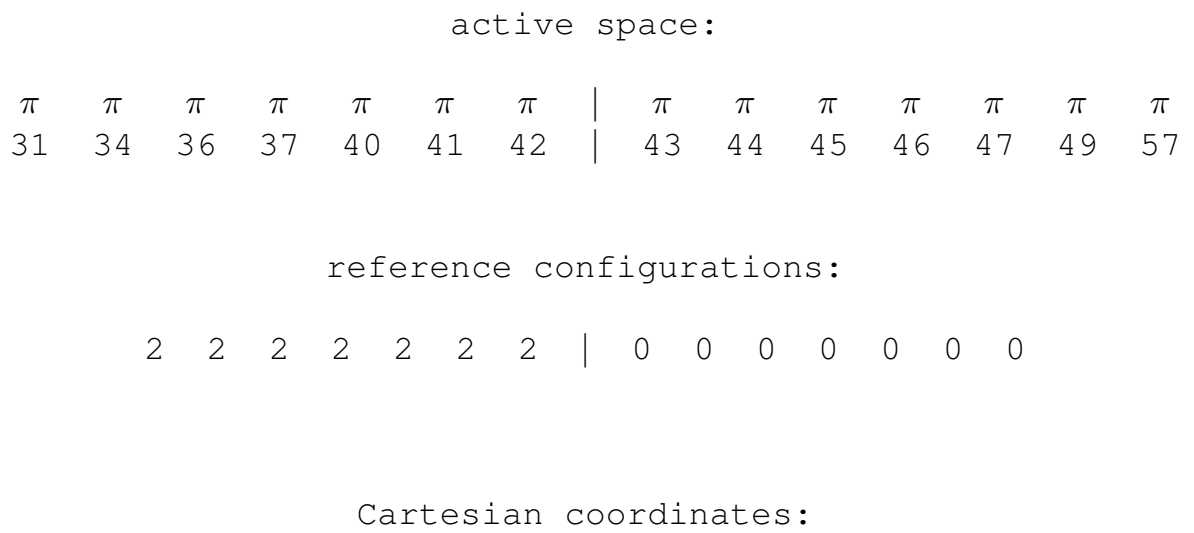




\begin{tabular}{lrrr}
\multicolumn{5}{c}{ Continued from previous page } \\
\hline $\mathrm{H}$ & 4.68578 & -1.50588 & 0.00000 \\
$\mathrm{H}$ & -2.68578 & -1.50588 & 0.00000 \\
$\mathrm{H}$ & 6.83340 & 2.23754 & 0.00000 \\
$\mathrm{H}$ & -4.83340 & 2.23754 & 0.00000 \\
$\mathrm{H}$ & 6.83340 & -0.23754 & 0.00000 \\
$\mathrm{H}$ & -4.83340 & -0.23754 & 0.00000
\end{tabular}

S1488 
Table S1379 Employed active space, reference configurations, and Cartesian coordinates of the OM2/MRCISD-optimized excited-state equilibrium geometry of the $1{ }^{1} B_{2 u}\left({ }^{1} \pi \pi^{*}\right)$ state of tetracene (excited-state energy: $-2473.12828 \mathrm{eV}$ ).

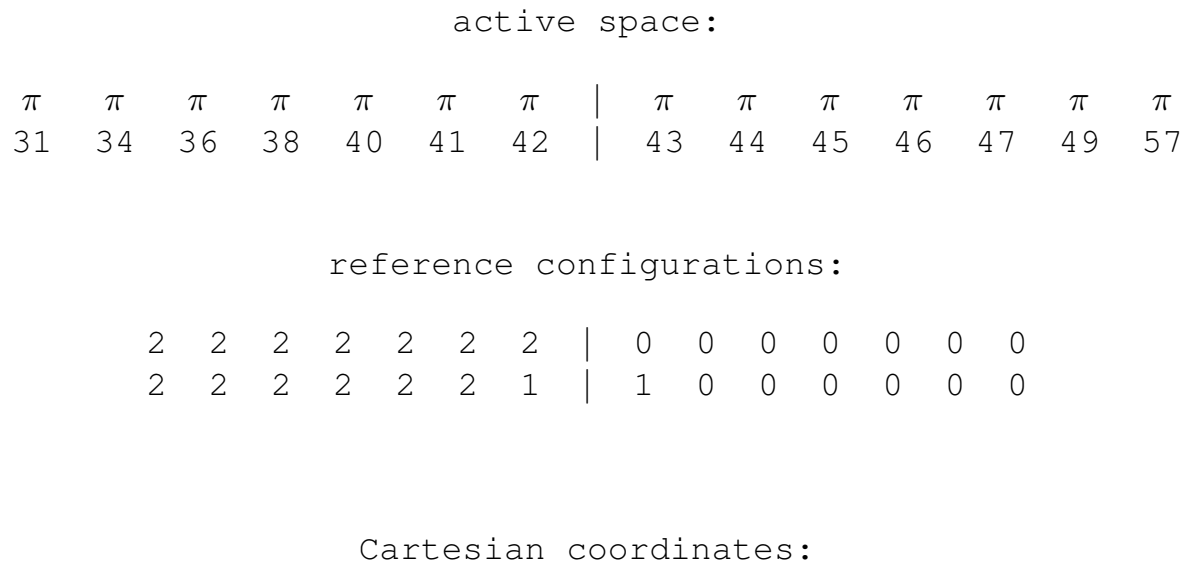




\begin{tabular}{lrrr}
\multicolumn{4}{c}{ Continued from previous page } \\
\hline $\mathrm{H}$ & 4.69257 & -1.50208 & 0.00000 \\
$\mathrm{H}$ & -2.69257 & -1.50208 & 0.00000 \\
$\mathrm{H}$ & 6.83955 & 2.24083 & 0.00000 \\
$\mathrm{H}$ & -4.83955 & 2.24083 & 0.00000 \\
$\mathrm{H}$ & 6.83955 & -0.24083 & 0.00000 \\
$\mathrm{H}$ & -4.83955 & -0.24083 & -0.00000
\end{tabular}


Table S1380 Employed active space, reference configurations, and Cartesian coordinates of the OM2/MRCISD-optimized ground-state equilibrium geometry of perylene (ground-state energy: $-2731.44021 \mathrm{eV}$ ).

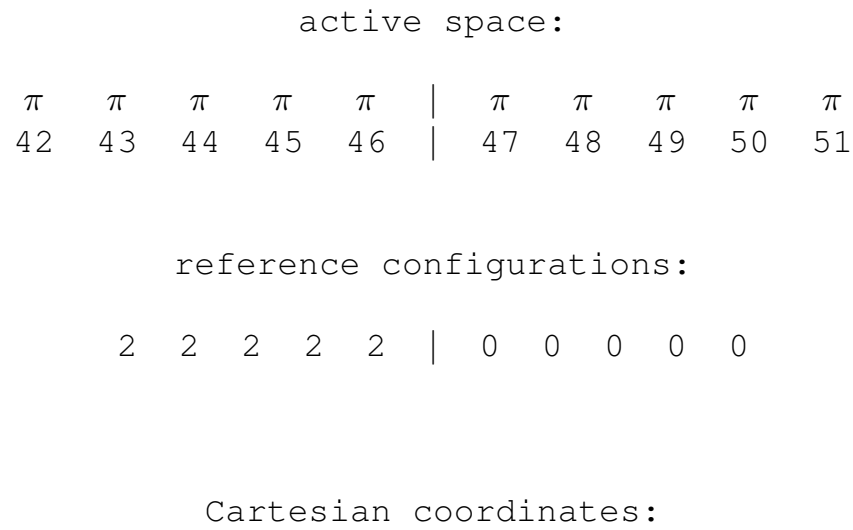




\begin{tabular}{lrrr}
\multicolumn{4}{c}{ Continued from previous page } \\
\hline $\mathrm{H}$ & 0.95405 & 3.40329 & -0.00000 \\
$\mathrm{H}$ & 0.95405 & -3.40329 & 0.00000 \\
$\mathrm{H}$ & -0.95405 & 3.40329 & 0.00000 \\
$\mathrm{H}$ & -0.95405 & -3.40329 & 0.00000 \\
$\mathrm{H}$ & 3.40737 & 3.37314 & -0.00000 \\
$\mathrm{H}$ & 3.40737 & -3.37314 & 0.00000 \\
$\mathrm{H}$ & -3.40737 & 3.37314 & 0.00000 \\
$\mathrm{H}$ & -3.40737 & -3.37314 & 0.00000
\end{tabular}


Table S1381 Employed active space, reference configurations, and Cartesian coordinates of the OM2/MRCISD-optimized excited-state equilibrium geometry of the $1{ }^{1} B_{3 u}\left({ }^{1} \pi \pi^{*}\right)$ state of perylene (excited-state energy: $-2727.99994 \mathrm{eV}$ ).

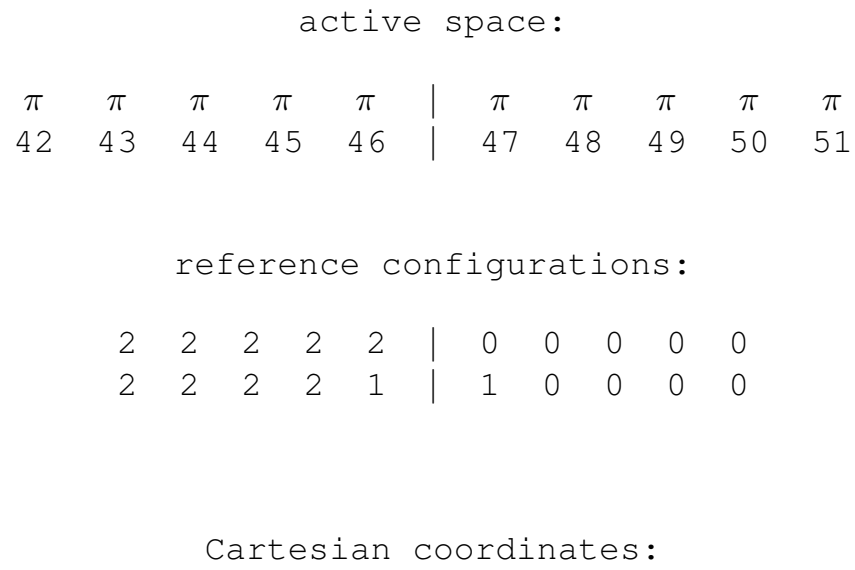

Continued on next page

S1493 


\begin{tabular}{lrrr}
\multicolumn{5}{c}{ Continued from previous page } \\
\hline H & 0.94428 & 3.41491 & -0.00000 \\
H & 0.94428 & -3.41491 & 0.00000 \\
H & -0.94428 & 3.41491 & 0.00000 \\
H & -0.94428 & -3.41491 & 0.00000 \\
H & 3.40402 & 3.37630 & -0.00000 \\
H & 3.40402 & -3.37630 & 0.00000 \\
H & -3.40402 & 3.37630 & 0.00000 \\
H & -3.40402 & -3.37630 & 0.00000
\end{tabular}


Table S1382 Employed active space, reference configurations, and Cartesian coordinates of the OM2/MRCISD-optimized ground-state equilibrium geometry of phenanthrene (ground-state energy: $-1936.72317 \mathrm{eV}$ ).

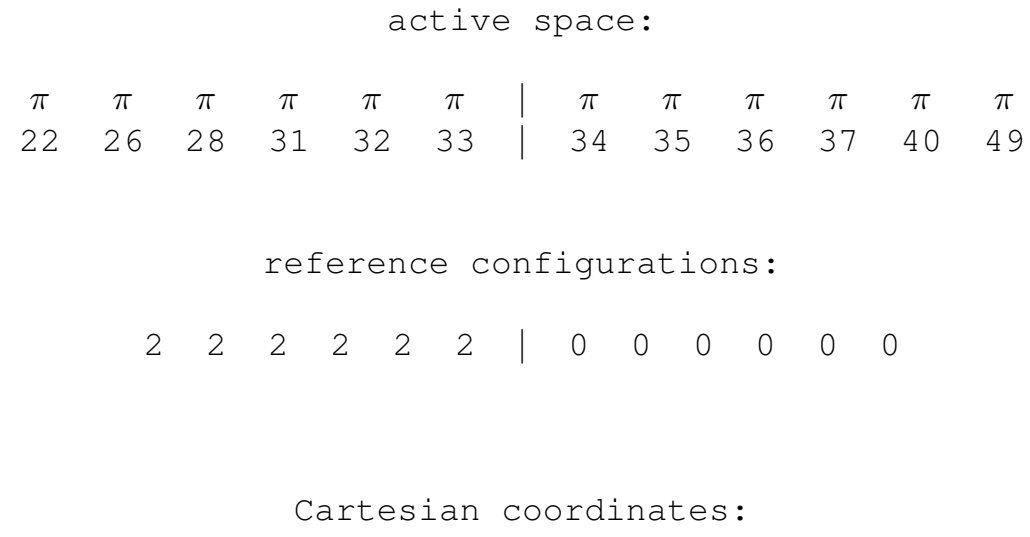

24

$\begin{array}{lrrr}\text { C } & 1.21830 & 0.68224 & 0.00000\end{array}$

C $\quad 1.21830 \quad-0.68224-0.00000$

C $\quad-0.01889 \quad 1.41183-0.00000$

C $\quad-0.01889-1.41183-0.00000$

C $\quad-1.25467 \quad 0.72424 \quad-0.00000$

C $\quad-1.25467-0.72424-0.00000$

$\mathrm{H} \quad 2.15767 \quad 1.24883 \quad 0.00000$

$\mathrm{H} \quad 2.15767-1.24883-0.00000$

$\begin{array}{llll}\text { C } & 0.00472 & 2.82660 & -0.00000\end{array}$

$\begin{array}{llll}\text { C } & 0.00472 & -2.82660 & -0.00000\end{array}$

$\begin{array}{llll}\text { C } & -1.17807 & 3.54281 & -0.00000\end{array}$

$\begin{array}{llll}\text { C } & -1.17807 & -3.54281 & -0.00000\end{array}$

$\begin{array}{llll}\text { C } & -2.40978 & 2.86365 & -0.00000\end{array}$

$\begin{array}{llll}\text { C } & -2.40978 & -2.86365 & -0.00000\end{array}$

$\begin{array}{llll}\text { C } & -2.44949 & 1.48007 & -0.00000\end{array}$

$\begin{array}{llll}\text { C } & -2.44949 & -1.48007 & -0.00000\end{array}$

$\mathrm{H} \quad 0.97045 \quad 3.34689-0.00000$

$\mathrm{H} \quad 0.97045 \quad-3.34689-0.00000$

$\mathrm{H} \quad-1.16498 \quad 4.63805 \quad-0.00000$

$\mathrm{H} \quad-1.16498 \quad-4.63805 \quad-0.00000$

$\mathrm{H} \quad-3.34140 \quad 3.44075-0.00000$

$\mathrm{H} \quad-3.34140 \quad-3.44075-0.00000$

$\begin{array}{llll}\mathrm{H} & -3.42147 & 0.97132 & -0.00000\end{array}$

$\mathrm{H} \quad-3.42147 \quad-0.97132 \quad-0.00000$ 
Table S1383 Employed active space, reference configurations, and Cartesian coordinates of the OM2/MRCISD-optimized excited-state equilibrium geometry of the $2{ }^{1} A_{1}\left({ }^{1} \pi \pi^{*}\right)$ state of phenanthrene (excited-state energy: $-1932.90369 \mathrm{eV})$.

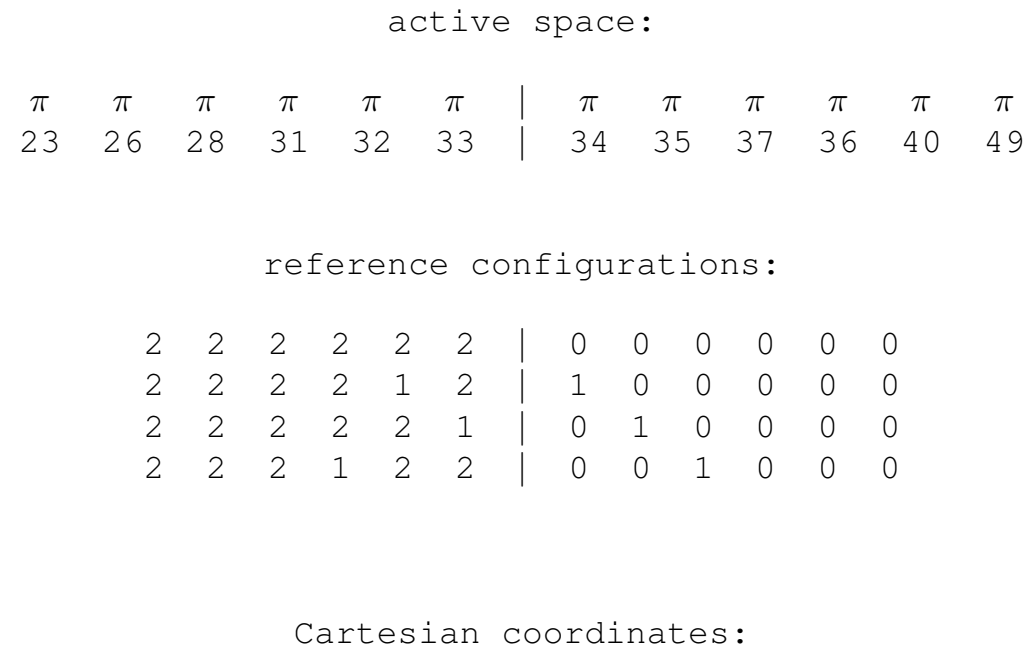

\begin{tabular}{lrrr}
24 & & & \\
& & & \\
$\mathrm{C}$ & 1.20269 & 0.69649 & 0.00000 \\
$\mathrm{C}$ & 1.20269 & -0.69649 & -0.00000 \\
$\mathrm{C}$ & -0.01078 & 1.41813 & -0.00000 \\
$\mathrm{C}$ & -0.01078 & -1.41813 & -0.00000 \\
$\mathrm{C}$ & -1.28103 & 0.70868 & -0.00000 \\
$\mathrm{C}$ & -1.28103 & -0.70868 & -0.00000 \\
$\mathrm{H}$ & 2.15262 & 1.24462 & -0.00000 \\
$\mathrm{H}$ & 2.15262 & -1.24462 & -0.00000 \\
$\mathrm{C}$ & 0.00061 & 2.82915 & -0.00000 \\
$\mathrm{C}$ & 0.00061 & -2.82915 & -0.00000 \\
$\mathrm{C}$ & -1.19550 & 3.54905 & -0.00000 \\
$\mathrm{C}$ & -1.19550 & -3.54905 & -0.00000 \\
$\mathrm{C}$ & -2.42857 & 2.86821 & -0.00000 \\
$\mathrm{C}$ & -2.42857 & -2.86821 & -0.00000 \\
$\mathrm{C}$ & -2.47187 & 1.47593 & -0.00000 \\
$\mathrm{C}$ & -2.47187 & -1.47593 & -0.00000 \\
$\mathrm{H}$ & 0.96035 & 3.36014 & -0.00000 \\
$\mathrm{H}$ & 0.96035 & -3.36014 & -0.00000 \\
$\mathrm{H}$ & -1.18003 & 4.64381 & -0.00000 \\
$\mathrm{H}$ & -1.18003 & -4.64381 & -0.00000 \\
$\mathrm{H}$ & -3.36080 & 3.44374 & -0.00000 \\
\hline & & Continued on next page
\end{tabular}

S1496 


\begin{tabular}{lrrr}
\multicolumn{5}{c}{ Continued from previous page } \\
\hline $\mathrm{H}$ & -3.36080 & -3.44374 & -0.00000 \\
$\mathrm{H}$ & -3.44780 & 0.97462 & -0.00000 \\
$\mathrm{H}$ & -3.44780 & -0.97462 & -0.00000
\end{tabular}


Table S1384 Employed active space, reference configurations, and Cartesian coordinates of the OM2/MRCISD-optimized ground-state equilibrium geometry of fluorene (ground-state energy: $-1809.16225 \mathrm{eV}$ ).

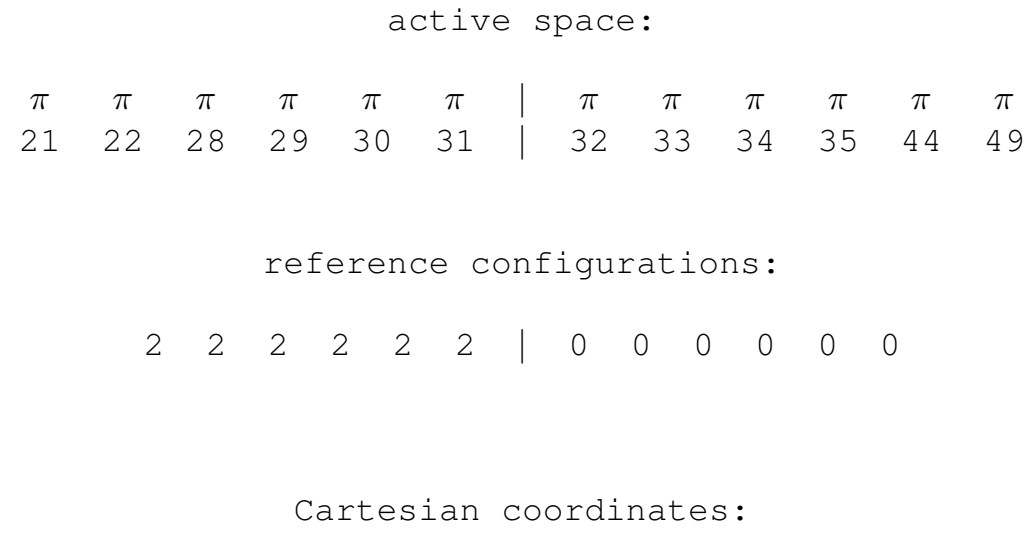

23

$\begin{array}{lrrr}C & 0.00000 & 0.00000 & 0.00000\end{array}$

$\begin{array}{lrrr}\text { C } & -0.94745 & 1.17254 & 0.00000\end{array}$

C $\quad-0.94745-1.17254-0.00000$

$\begin{array}{llll}\text { C } & -2.29742 & 0.73447 & -0.00000\end{array}$

$\begin{array}{llll}\text { C } & -2.29742 & -0.73447 & 0.00000\end{array}$

C $\quad-0.64661 \quad 2.53039-0.00000$

C $\quad-0.64661-2.53039-0.00000$

C $\quad-3.35058 \quad 1.64488-0.00000$

$\begin{array}{llll}\text { C } & -3.35058 & -1.64488 & -0.00000\end{array}$

C $\quad-1.70866 \quad 3.44365-0.00000$

$\begin{array}{llll}\text { C } & -1.70866 & -3.44365 & -0.00000\end{array}$

$\begin{array}{llll}\text { C } & -3.03722 & 3.00877 & 0.00000\end{array}$

$\begin{array}{llll}\text { C } & -3.03722 & -3.00877 & -0.00000\end{array}$

$\begin{array}{llll}\mathrm{H} & 0.62124 & 0.00000 & -0.91073\end{array}$

$\begin{array}{llll}\mathrm{H} & 0.62124 & 0.00000 & 0.91073\end{array}$

$\mathrm{H} \quad 0.39002 \quad 2.87983 \quad-0.00000$

$\mathrm{H} \quad 0.39002 \quad-2.87983 \quad-0.00000$

$\mathrm{H} \quad-4.39013 \quad 1.30540 \quad-0.00000$

$\mathrm{H} \quad-4.39013 \quad-1.30540 \quad 0.00000$

$\mathrm{H} \quad-1.49401 \quad 4.51870 \quad-0.00000$

$\mathrm{H} \quad-1.49401 \quad-4.51870 \quad-0.00000$

$\begin{array}{lrrr}\mathrm{H} & -3.84615 & 3.74837 & 0.00000\end{array}$

$\mathrm{H} \quad-3.84615 \quad-3.74837 \quad-0.00000$ 
Table S1385 Employed active space, reference configurations, and Cartesian coordinates of the OM2/MRCISD-optimized excited-state equilibrium geometry of the $1{ }^{1} B_{2}\left({ }^{1} \pi \pi^{*}\right)$ state of fluorene (excited-state energy: $\left.-1804.81462 \mathrm{eV}\right)$.

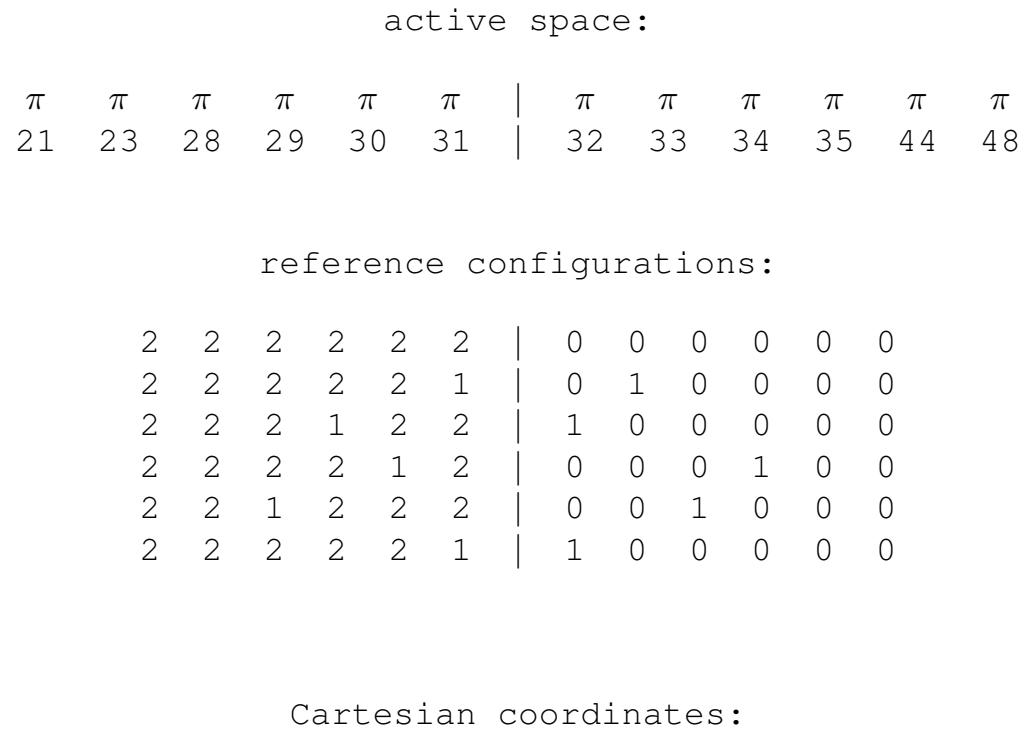

\begin{tabular}{lrrr}
23 & & & \\
C & 0.00000 & 0.00000 & 0.00000 \\
C & -0.95083 & 1.16465 & 0.00000 \\
C & -0.95083 & -1.16465 & -0.00000 \\
C & -2.31667 & 0.72008 & -0.00000 \\
C & -2.31667 & -0.72008 & 0.00000 \\
C & -0.64453 & 2.53343 & -0.00000 \\
C & -0.64453 & -2.53343 & -0.00000 \\
C & -3.38024 & 1.64623 & -0.00000 \\
C & -3.38024 & -1.64623 & -0.00000 \\
C & -1.71442 & 3.44899 & -0.00000 \\
C & -1.71442 & -3.44899 & -0.00000 \\
C & -3.05471 & 3.01487 & 0.00000 \\
C & -3.05471 & -3.01487 & -0.00000 \\
H & 0.61476 & 0.00000 & -0.91151 \\
H & 0.61476 & 0.00000 & 0.91151 \\
H & 0.39064 & 2.88080 & -0.00000 \\
H & 0.39064 & -2.88080 & -0.00000 \\
H & -4.41814 & 1.30891 & -0.00000 \\
H & -4.41814 & -1.30891 & 0.00000 \\
H & -1.50052 & 4.52439 & -0.00000 \\
\hline & & Continued on next page
\end{tabular}

S1499 


\begin{tabular}{lrrr}
\multicolumn{5}{c}{ Continued from previous page } \\
\hline $\mathrm{H}$ & -1.50052 & -4.52439 & -0.00000 \\
$\mathrm{H}$ & -3.85860 & 3.75952 & 0.00000 \\
$\mathrm{H}$ & -3.85860 & -3.75952 & -0.00000
\end{tabular}


Table S1386 Employed active space, reference configurations, and Cartesian coordinates of the OM2/MRCISD-optimized ground-state equilibrium geometry of carbazole (ground-state energy: $-1879.44028 \mathrm{eV}$ ).

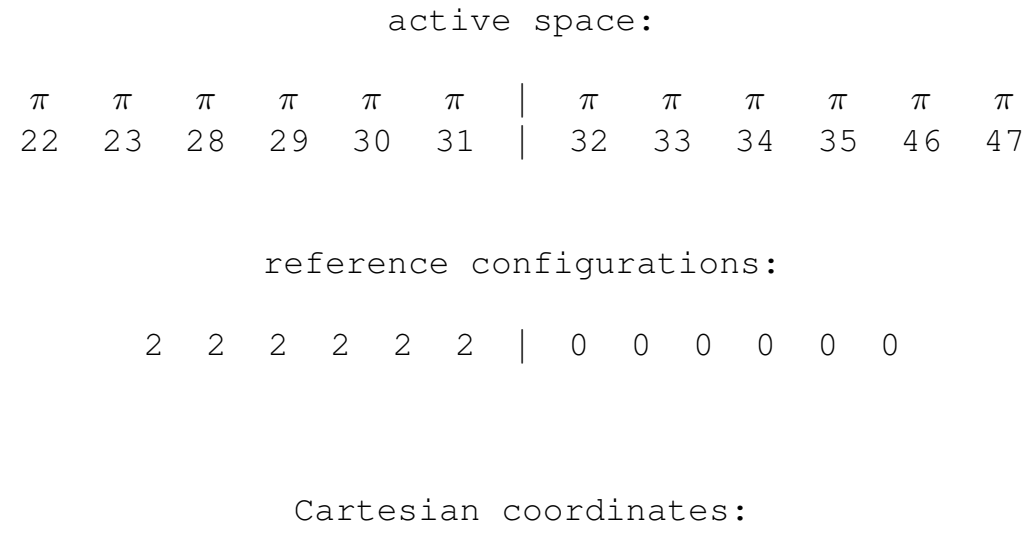

22

N $\quad 0.00000$

$\mathrm{H} \quad 1.00585$

0.00000

0.00000

$\begin{array}{ll}\text { C } & -0.81228 \\ \text { C } & -0.81228\end{array}$

0.00000

0.00000

1.12881

0.00000

C -2.19090

$-1.12881-0.00000$

C $\quad-2.19090$

$0.72651-0.00000$

C $\quad-0.44462$

$-0.72651$

0.00000

C $\quad-0.44462$

$2.48240-0.00000$

C -3.19860

$-2.48240-0.00000$

C -3.19860

$1.69194-0.00000$

C -1.47405

$-1.69194-0.00000$

C $\quad-1.47405$

$3.41968-0.00000$

C $\quad-2.82517$

$-3.41968$

$-0.00000$

$3.03480 \quad 0.00000$

$-2.82517$

$-3.03480$

$-0.00000$

0.60594

2.78076

$-0.00000$

0.60594

$-2.78076$

$-0.00000$

$-4.25179$

1.39607

$-0.00000$

$-4.25179$

$-1.39607$

0.00000

$\mathrm{H}-1.22504$

4.48797

$-0.00000$

$-1.22504$

$-4.48797$

$-0.00000$

$-3.59826$

3. 81078

0.00000

$\mathrm{H}-3.59826-3.81078-0.00000$ 
Table S1387 Employed active space, reference configurations, and Cartesian coordinates of the OM2/MRCISD-optimized excited-state equilibrium geometry of the $2{ }^{1} A_{1}\left({ }^{1} \pi \pi^{*}\right)$ state of carbazole (excited-state energy: $-1875.69642 \mathrm{eV}$ ).

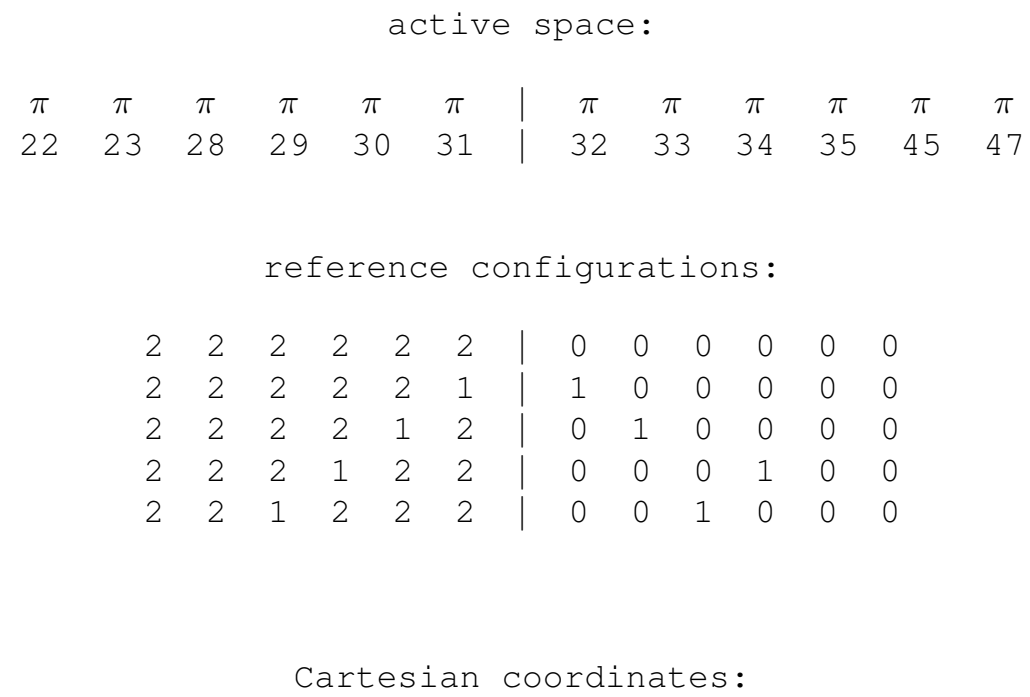

\begin{tabular}{lrrr}
22 & & & \\
& & & \\
$\mathrm{~N}$ & 0.00000 & 0.00000 & 0.00000 \\
$\mathrm{H}$ & 1.01131 & 0.00000 & 0.00000 \\
$\mathrm{C}$ & -0.79250 & 1.13229 & 0.00000 \\
$\mathrm{C}$ & -0.79250 & -1.13229 & -0.00000 \\
$\mathrm{C}$ & -2.19576 & 0.71513 & -0.00000 \\
$\mathrm{C}$ & -2.19576 & -0.71513 & 0.00000 \\
$\mathrm{C}$ & -0.41738 & 2.48131 & -0.00000 \\
$\mathrm{C}$ & -0.41738 & -2.48131 & -0.00000 \\
$\mathrm{C}$ & -3.19934 & 1.69271 & -0.00000 \\
$\mathrm{C}$ & -3.19934 & -1.69271 & -0.00000 \\
$\mathrm{C}$ & -1.45616 & 3.43182 & -0.00000 \\
$\mathrm{C}$ & -1.45616 & -3.43182 & -0.00000 \\
$\mathrm{C}$ & -2.80722 & 3.04397 & 0.00000 \\
$\mathrm{C}$ & -2.80722 & -3.04397 & -0.00000 \\
$\mathrm{H}$ & 0.63258 & 2.78150 & -0.00000 \\
$\mathrm{H}$ & 0.63258 & -2.78150 & -0.00000 \\
$\mathrm{H}$ & -4.25449 & 1.41032 & -0.00000 \\
$\mathrm{H}$ & -4.25449 & -1.41032 & 0.00000 \\
$\mathrm{H}$ & -1.20222 & 4.49656 & -0.00000 \\
$\mathrm{H}$ & -1.20222 & -4.49656 & -0.00000 \\
$\mathrm{H}$ & -3.58161 & 3.82127 & 0.00000 \\
\hline & & Continued & on next page
\end{tabular}

S1502 
Continued from previous page

$\begin{array}{llll}\mathrm{H} & -3.58161 & -3.82127 & -0.00000\end{array}$

S1503 
Table S1388 Employed active space, reference configurations, and Cartesian coordinates of the OM2/MRCISD-optimized ground-state equilibrium geometry of dibenzofuran (ground-state energy: $-1982.05409 \mathrm{eV}$ ).

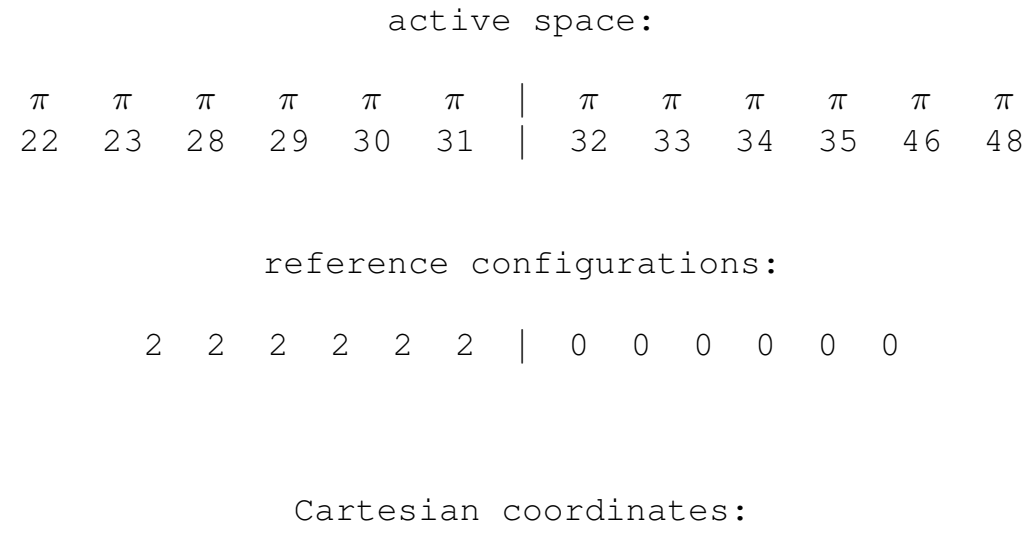

21

$\begin{array}{lrrr}0 & 0.00000 & 0.00000 & 0.00000\end{array}$

$\begin{array}{lrrr}\text { C } & -0.83382 & 1.09516 & 0.00000\end{array}$

C $\quad-0.83382-1.09516-0.00000$

C $\quad-2.21171 \quad 0.72665-0.00000$

$\begin{array}{llll}\text { C } & -2.21171 & -0.72665 & 0.00000\end{array}$

C $\quad-0.39344 \quad 2.41963-0.00000$

C $\quad-0.39344-2.41963-0.00000$

C $\quad-3.18728 \quad 1.72183 \quad-0.00000$

$\begin{array}{llll}\text { C } & -3.18728 & -1.72183 & -0.00000\end{array}$

$\begin{array}{llll}\text { C } & -1.39737 & 3.39012 & -0.00000\end{array}$

$\begin{array}{llll}\text { C } & -1.39737 & -3.39012 & -0.00000\end{array}$

$\begin{array}{llll}\text { C } & -2.75916 & 3.05169 & 0.00000\end{array}$

$\begin{array}{llll}\text { C } & -2.75916 & -3.05169 & -0.00000\end{array}$

$\mathrm{H} \quad 0.66544 \quad 2.66636 \quad-0.00000$

$\mathrm{H} \quad 0.66544 \quad-2.66636 \quad-0.00000$

$\mathrm{H} \quad-4.24962 \quad 1.46512 \quad-0.00000$

$\mathrm{H} \quad-4.24962 \quad-1.46512 \quad 0.00000$

$\mathrm{H} \quad-1.11140 \quad 4.44834 \quad-0.00000$

$\mathrm{H} \quad-1.11140 \quad-4.44834 \quad-0.00000$

$\begin{array}{lrrr}\mathrm{H} & -3.50478 & 3.85370 & 0.00000\end{array}$

$\mathrm{H} \quad-3.50478 \quad-3.85370 \quad-0.00000$ 
Table S1389 Employed active space, reference configurations, and Cartesian coordinates of the OM2/MRCISD-optimized excited-state equilibrium geometry of the $2{ }^{1} A_{1}\left({ }^{1} \pi \pi^{*}\right)$ state of dibenzofuran (excited-state energy: $-1978.09641 \mathrm{eV})$.

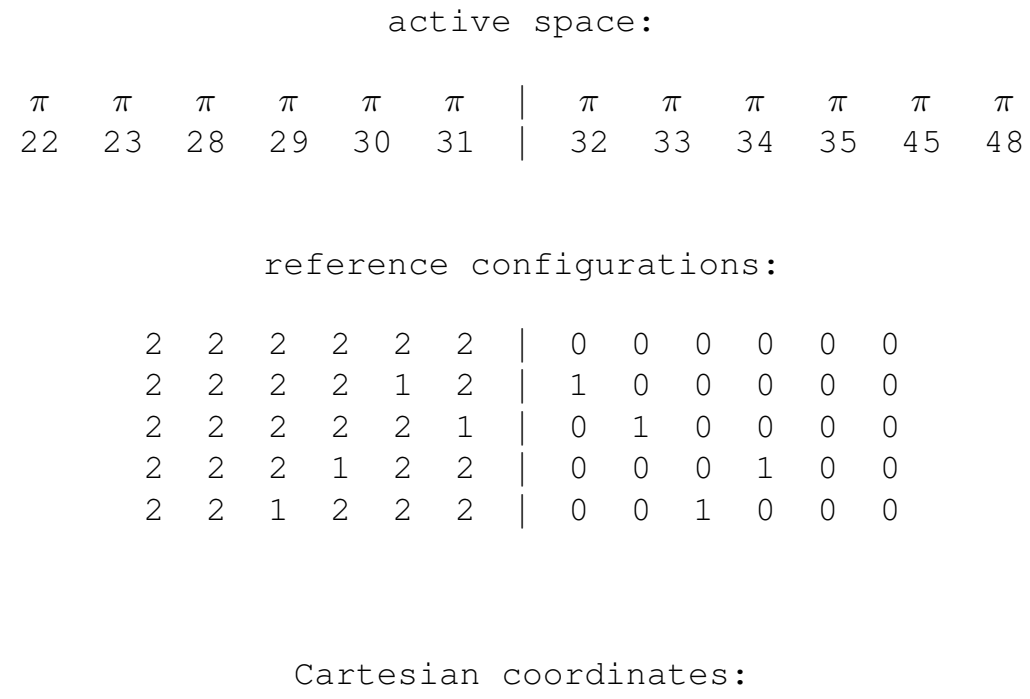

21

\begin{tabular}{lrrr}
$\mathrm{O}$ & 0.00000 & 0.00000 & 0.00000 \\
$\mathrm{C}$ & -0.81619 & 1.09532 & 0.00000 \\
$\mathrm{C}$ & -0.81619 & -1.09532 & -0.00000 \\
$\mathrm{C}$ & -2.21828 & 0.71264 & -0.00000 \\
$\mathrm{C}$ & -2.21828 & -0.71264 & 0.00000 \\
$\mathrm{C}$ & -0.36763 & 2.42111 & -0.00000 \\
$\mathrm{C}$ & -0.36763 & -2.42111 & -0.00000 \\
$\mathrm{C}$ & -3.19127 & 1.72314 & -0.00000 \\
$\mathrm{C}$ & -3.19127 & -1.72314 & -0.00000 \\
$\mathrm{C}$ & -1.37960 & 3.39983 & -0.00000 \\
$\mathrm{C}$ & -1.37960 & -3.39983 & -0.00000 \\
$\mathrm{C}$ & -2.74627 & 3.05942 & 0.00000 \\
$\mathrm{C}$ & -2.74627 & -3.05942 & -0.00000 \\
$\mathrm{H}$ & 0.69074 & 2.66751 & -0.00000 \\
$\mathrm{H}$ & 0.69074 & -2.66751 & -0.00000 \\
$\mathrm{H}$ & -4.25522 & 1.47779 & -0.00000 \\
$\mathrm{H}$ & -4.25522 & -1.47779 & 0.00000 \\
$\mathrm{H}$ & -1.09224 & 4.45615 & -0.00000 \\
$\mathrm{H}$ & -1.09224 & -4.45615 & -0.00000 \\
$\mathrm{H}$ & -3.49055 & 3.86398 & 0.00000 \\
\hline & & Continued on next page
\end{tabular}

S1505 
Continued from previous page

$\mathrm{H} \quad-3.49055 \quad-3.86398 \quad-0.00000$

S1506 
Table S1390 Employed active space, reference configurations, and Cartesian coordinates of the OM2/MRCISD-optimized ground-state equilibrium geometry of pyrrolo[3,2- $h]$ quinoline (ground-state energy: $-1949.55083 \mathrm{eV}$ ).

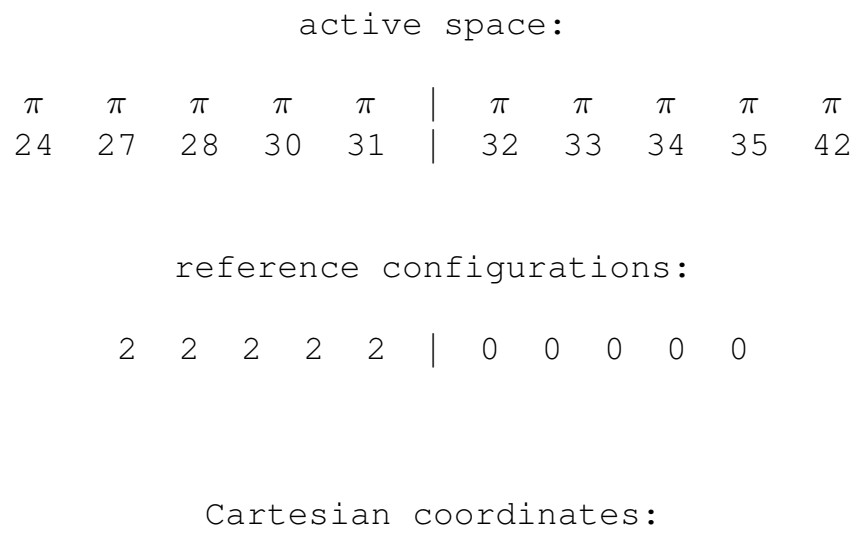

$\mathrm{H} \quad-1.49338 \quad 1.57917 \quad 0.00000$ 
Table S1391 Employed active space, reference configurations, and Cartesian coordinates of the OM2/MRCISD-optimized excited-state equilibrium geometry of the $2{ }^{1} A^{\prime}\left({ }^{1} \pi \pi^{*}\right)$ state of pyrrolo[3,2- $\left.h\right]$ quinoline (excited-state energy: $-1945.71939 \mathrm{eV})$.

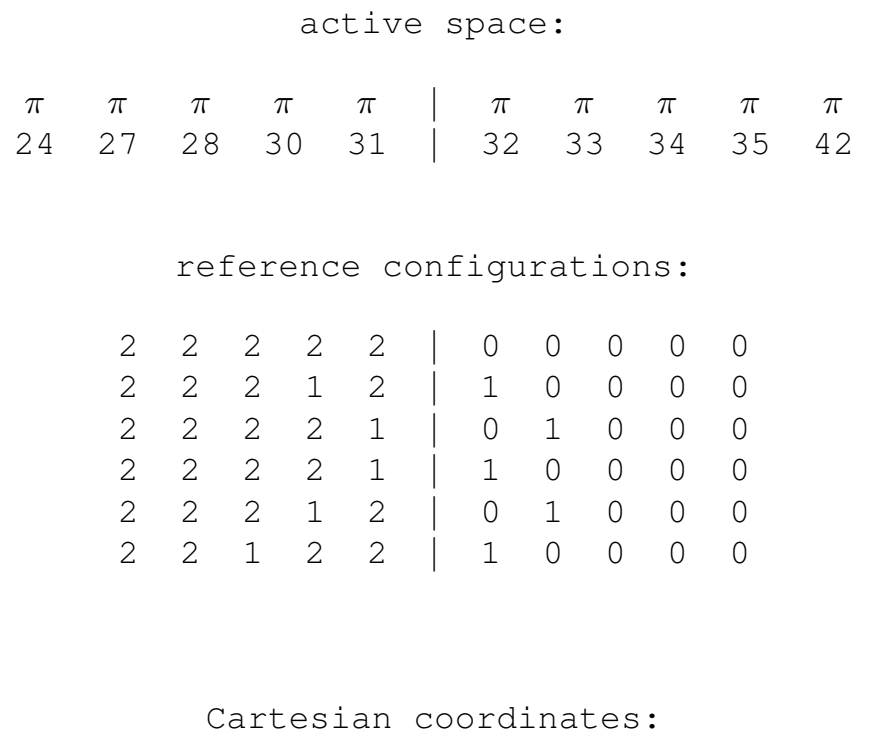

21

\begin{tabular}{lrrr}
$\mathrm{N}$ & 0.00000 & 0.00000 & 0.00000 \\
$\mathrm{C}$ & 1.37841 & 0.00000 & 0.00000 \\
$\mathrm{C}$ & 1.82727 & 1.40220 & 0.00000 \\
$\mathrm{C}$ & 0.64911 & 2.19014 & 0.00000 \\
$\mathrm{C}$ & -0.45449 & 1.29702 & 0.00000 \\
$\mathrm{C}$ & 3.20180 & 1.69638 & 0.00000 \\
$\mathrm{C}$ & 4.11278 & 0.62298 & 0.00000 \\
$\mathrm{C}$ & 3.69014 & -0.72584 & 0.00000 \\
$\mathrm{C}$ & 2.27490 & -1.08992 & 0.00000 \\
$\mathrm{C}$ & 4.60673 & -1.80124 & 0.00000 \\
$\mathrm{C}$ & 4.11870 & -3.10589 & 0.00000 \\
$\mathrm{C}$ & 2.71874 & -3.33583 & 0.00000 \\
$\mathrm{~N}$ & 1.81912 & -2.35522 & 0.00000 \\
$\mathrm{H}$ & 2.35566 & -4.37835 & 0.00000 \\
$\mathrm{H}$ & 4.80722 & -3.95515 & 0.00000 \\
$\mathrm{H}$ & 5.68418 & -1.60508 & 0.00000 \\
$\mathrm{H}$ & 3.54850 & 2.73133 & 0.00000 \\
$\mathrm{H}$ & 5.18798 & 0.83615 & 0.00000 \\
$\mathrm{H}$ & -0.56938 & -0.83497 & 0.00000 \\
\hline & & Continued on next page
\end{tabular}

S1508 
Continued from previous page

$\begin{array}{llll}\mathrm{H} & 0.58301 & 3.26633 & 0.00000\end{array}$

$\mathrm{H} \quad-1.50685 \quad 1.56658 \quad 0.00000$

S1509 
Table S1392 Employed active space, reference configurations, and Cartesian coordinates of the OM2/MRCISD-optimized ground-state equilibrium geometry of tryptamine (anti, $\mathrm{Ph}$ ) (ground-state energy: $-1881.89535 \mathrm{eV}$ ).

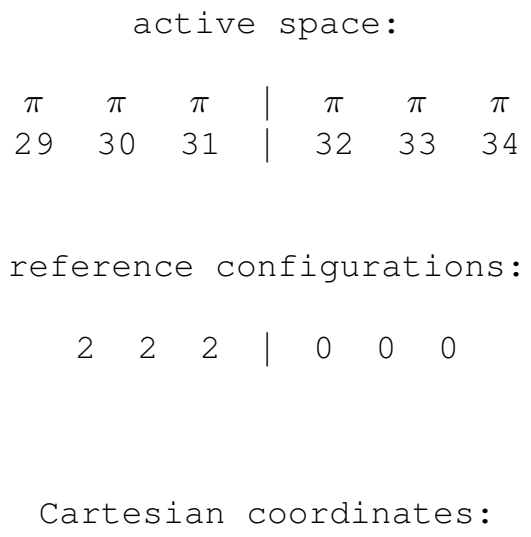

24

$\begin{array}{llll}\text { C } & 0.00000 & 0.00000 & 0.00000\end{array}$

$\begin{array}{llll}\text { C } & 1.39884 & 0.00000 & 0.00000\end{array}$

$\begin{array}{llll}\text { C } & 2.10511 & 1.25426 & 0.00000\end{array}$

C $\quad 1.43087 \quad 2.48506 \quad-0.00521$

C $\quad 0.04335 \quad 2.44068-0.00952$

C $\quad-0.65872 \quad 1.22352-0.00554$

$\begin{array}{llll}\text { C } & 2.40377 & -1.03814 & 0.00722\end{array}$

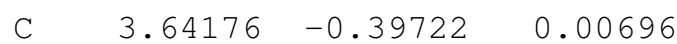

$\begin{array}{lrrr}\mathrm{N} & 3.46325 & 0.97786 & 0.00561\end{array}$

$\begin{array}{llll}\text { C } & 2.15314 & -2.49883 & -0.01739\end{array}$

$\begin{array}{llll}\text { C } & 1.73380 & -2.92433 & -1.43103\end{array}$

$\begin{array}{llll}\mathrm{N} & 1.33281 & -4.29838 & -1.39620\end{array}$

$\mathrm{H} \quad \begin{array}{llll}\mathrm{H} & 1.98022 & 3.43001 & -0.00703\end{array}$

$\begin{array}{llll}\mathrm{H} & -0.52308 & 3.38003 & -0.01784\end{array}$

$\mathrm{H} \quad-1.75452 \quad 1.24735 \quad-0.00641$

$\begin{array}{llll}\mathrm{H} & -0.55296 & -0.94387 & 0.00846\end{array}$

$\begin{array}{llll}\mathrm{H} & 4.20150 & 1.66324 & 0.00492\end{array}$

$\begin{array}{llll}\mathrm{H} & 4.62345 & -0.86321 & 0.00665\end{array}$

$\begin{array}{llll}\mathrm{H} & 1.33561 & -2.75232 & 0.69613\end{array}$

$\begin{array}{llll}\mathrm{H} & 3.07480 & -3.05482 & 0.28720\end{array}$

H $\quad 2.60080 \quad-2.72752 \quad-2.14381$

$\mathrm{H} \quad \begin{array}{llll}\mathrm{H} & 0.87462 & -2.28463 & -1.76724\end{array}$

$\mathrm{H} \quad \begin{array}{llll}2.08815 & -4.91753 & -1.12784\end{array}$

$\mathrm{H} \quad 0.95346 \quad-4.62066 \quad-2.27815$ 
Table S1393 Employed active space, reference configurations, and Cartesian coordinates of the OM2/MRCISD-optimized excited-state equilibrium geometry of the $2{ }^{1} A\left({ }^{1} \pi \pi^{*}\right)$ state of tryptamine (anti, $\mathrm{Ph}$ ) (excited-state energy: $-1877.41797 \mathrm{eV})$.

active space:
\begin{tabular}{ccc|ccc}
$\pi$ & $\pi$ & $\pi$ & $\pi$ & $\pi$ & $\pi$ \\
28 & 30 & 31 & 32 & 33 & 34 \\
reference configurations: \\
2 & 2 & 2 & 0 & 0 & 0 \\
2 & 1 & 2 & 1 & 0 & 0 \\
2 & 2 & 1 & 0 & 1 & 0 \\
2 & 2 & 1 & 1 & 0 & 0
\end{tabular}

Cartesian coordinates:

\begin{tabular}{lrrr}
24 & & & \\
& & & \\
$\mathrm{C}$ & 0.00000 & 0.00000 & 0.00000 \\
$\mathrm{C}$ & 1.39402 & 0.00000 & 0.00000 \\
$\mathrm{C}$ & 2.11123 & 1.30393 & 0.00000 \\
$\mathrm{C}$ & 1.42489 & 2.53510 & -0.01313 \\
$\mathrm{C}$ & 0.03154 & 2.47399 & -0.02531 \\
$\mathrm{C}$ & -0.65989 & 1.23848 & -0.01201 \\
$\mathrm{C}$ & 2.40022 & -1.01055 & 0.02837 \\
$\mathrm{C}$ & 3.67049 & -0.34333 & 0.03612 \\
$\mathrm{~N}$ & 3.45585 & 1.02564 & 0.02373 \\
$\mathrm{C}$ & 2.20173 & -2.47870 & -0.03620 \\
$\mathrm{C}$ & 1.94116 & -2.87052 & -1.48701 \\
$\mathrm{~N}$ & 1.72062 & -4.28236 & -1.56648 \\
$\mathrm{H}$ & 1.96697 & 3.48361 & -0.02360 \\
$\mathrm{H}$ & -0.54749 & 3.40260 & -0.05145 \\
$\mathrm{H}$ & -1.75783 & 1.25497 & -0.01315 \\
$\mathrm{H}$ & -0.56189 & -0.93735 & 0.02284 \\
$\mathrm{H}$ & 4.19009 & 1.72026 & 0.03841 \\
$\mathrm{H}$ & 4.65194 & -0.79998 & 0.05086 \\
$\mathrm{H}$ & 1.33944 & -2.77231 & 0.60070 \\
$\mathrm{H}$ & 3.11198 & -3.00230 & 0.34814 \\
$\mathrm{H}$ & 2.81934 & -2.53126 & -2.12802 \\
\hline & & Continued & on next page
\end{tabular}

S1511 


\begin{tabular}{llll}
\multicolumn{5}{c}{ Continued from previous page } \\
\hline $\mathrm{H}$ & 1.03591 & -2.32346 & -1.86123 \\
$\mathrm{H}$ & 2.39556 & -4.83388 & -1.05291 \\
$\mathrm{H}$ & 1.65802 & -4.62715 & -2.51387
\end{tabular}


Table S1394 Employed active space, reference configurations, and Cartesian coordinates of the OM2/MRCISD-optimized ground-state equilibrium geometry of tryptamine (anti, py) (ground-state energy: $-1882.10156 \mathrm{eV}$ ).

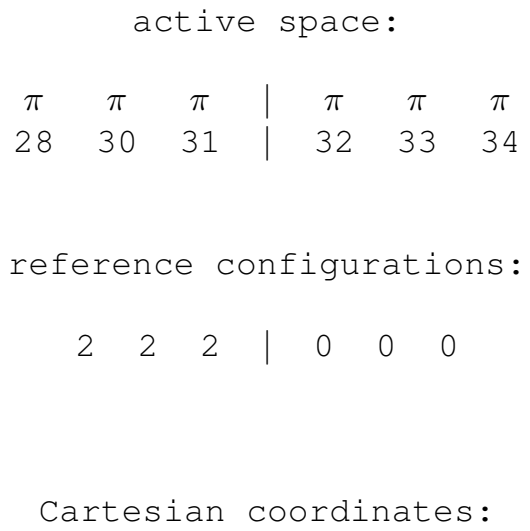


Table S1395 Employed active space, reference configurations, and Cartesian coordinates of the OM2/MRCISD-optimized excited-state equilibrium geometry of the $2{ }^{1} A\left({ }^{1} \pi \pi^{*}\right)$ state of tryptamine (anti, py) (excited-state energy: $-1877.42046 \mathrm{eV})$.

active space:
\begin{tabular}{ccc|ccc}
$\pi$ & $\pi$ & $\pi$ & $\pi$ & $\pi$ & $\pi$ \\
28 & 30 & 31 & 32 & 33 & 34 \\
reference configurations: \\
2 & 2 & 2 & 0 & 0 & 0 \\
2 & 1 & 2 & 1 & 0 & 0 \\
2 & 2 & 1 & 0 & 1 & 0 \\
2 & 2 & 1 & 1 & 0 & 0
\end{tabular}

Cartesian coordinates:

\begin{tabular}{lrrr}
24 & & & \\
& & & \\
$\mathrm{C}$ & 0.00000 & 0.00000 & 0.00000 \\
$\mathrm{C}$ & 1.39495 & 0.00000 & 0.00000 \\
$\mathrm{C}$ & 2.10982 & 1.30455 & 0.00000 \\
$\mathrm{C}$ & 1.42230 & 2.53483 & -0.01386 \\
$\mathrm{C}$ & 0.02854 & 2.47304 & -0.02848 \\
$\mathrm{C}$ & -0.66165 & 1.23737 & -0.01259 \\
$\mathrm{C}$ & 2.40257 & -1.00943 & 0.03992 \\
$\mathrm{C}$ & 3.67207 & -0.33941 & 0.05322 \\
$\mathrm{~N}$ & 3.45523 & 1.02847 & 0.03220 \\
$\mathrm{C}$ & 2.21260 & -2.47828 & -0.02231 \\
$\mathrm{C}$ & 1.96890 & -2.87946 & -1.47450 \\
$\mathrm{~N}$ & 1.79749 & -4.29922 & -1.55106 \\
$\mathrm{H}$ & 1.96348 & 3.48380 & -0.02698 \\
$\mathrm{H}$ & -0.55082 & 3.40121 & -0.05852 \\
$\mathrm{H}$ & -1.75954 & 1.25252 & -0.01454 \\
$\mathrm{H}$ & -0.56179 & -0.93812 & 0.02319 \\
$\mathrm{H}$ & 4.18825 & 1.72461 & 0.04108 \\
$\mathrm{H}$ & 4.65312 & -0.79628 & 0.07061 \\
$\mathrm{H}$ & 1.34397 & -2.76508 & 0.61486 \\
$\mathrm{H}$ & 3.11553 & -3.00254 & 0.36544 \\
$\mathrm{H}$ & 2.85087 & -2.57136 & -2.09884 \\
\hline & \multicolumn{3}{c}{ Continued on next page }
\end{tabular}

$\mathrm{S} 1514$ 
Continued from previous page

$\begin{array}{llll}\mathrm{H} & 1.06884 & -2.30857 & -1.86979 \\ \mathrm{H} & 1.15308 & -4.66943 & -0.86394 \\ \mathrm{H} & 1.52042 & -4.61735 & -2.46954\end{array}$

S1515 
Table S1396 Employed active space, reference configurations, and Cartesian coordinates of the OM2/MRCISD-optimized ground-state equilibrium geometry of tryptamine (anti, up) (ground-state energy: $-1881.93159 \mathrm{eV}$ ).

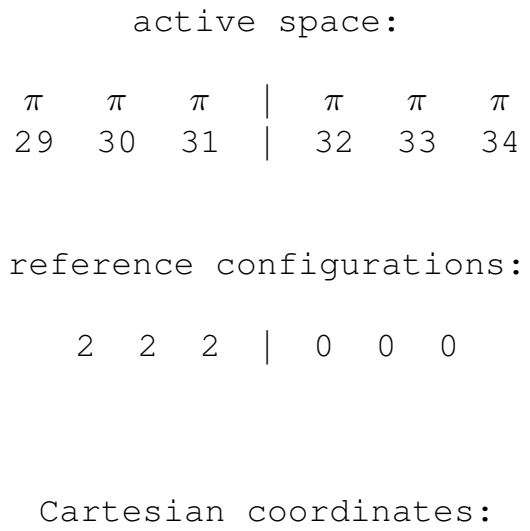


Table S1397 Employed active space, reference configurations, and Cartesian coordinates of the OM2/MRCISD-optimized excited-state equilibrium geometry of the $2{ }^{1} A\left({ }^{1} \pi \pi^{*}\right)$ state of tryptamine (anti, up) (excited-state energy: $-1877.24769 \mathrm{eV})$.

active space:
\begin{tabular}{ccc|ccc}
$\pi$ & $\pi$ & $\pi$ & $\pi$ & $\pi$ & $\pi$ \\
29 & 30 & 31 & 32 & 33 & 34 \\
reference configurations: \\
2 & 2 & 2 & 0 & 0 & 0 \\
2 & 1 & 2 & 1 & 0 & 0 \\
2 & 2 & 1 & 0 & 1 & 0 \\
2 & 2 & 1 & 1 & 0 & 0
\end{tabular}

Cartesian coordinates:

\begin{tabular}{lrrr}
24 & & & \\
& & & \\
$\mathrm{C}$ & 0.00000 & 0.00000 & 0.00000 \\
$\mathrm{C}$ & 1.39535 & 0.00000 & 0.00000 \\
$\mathrm{C}$ & 2.11014 & 1.30142 & 0.00000 \\
$\mathrm{C}$ & 1.42617 & 2.53290 & -0.00742 \\
$\mathrm{C}$ & 0.03143 & 2.47586 & -0.01242 \\
$\mathrm{C}$ & -0.66167 & 1.24195 & -0.00511 \\
$\mathrm{C}$ & 2.39739 & -1.01227 & 0.02916 \\
$\mathrm{C}$ & 3.66664 & -0.34857 & 0.01870 \\
$\mathrm{~N}$ & 3.45474 & 1.02275 & 0.01157 \\
$\mathrm{C}$ & 2.19623 & -2.47072 & -0.05218 \\
$\mathrm{C}$ & 2.05354 & -2.88073 & -1.53449 \\
$\mathrm{~N}$ & 1.84693 & -4.28802 & -1.67691 \\
$\mathrm{H}$ & 1.96968 & 3.48027 & -0.01847 \\
$\mathrm{H}$ & -0.54478 & 3.40556 & -0.03066 \\
$\mathrm{H}$ & -1.75901 & 1.25729 & -0.00509 \\
$\mathrm{H}$ & -0.56307 & -0.93711 & 0.01903 \\
$\mathrm{H}$ & 4.19026 & 1.71652 & 0.01812 \\
$\mathrm{H}$ & 4.64745 & -0.80605 & 0.01795 \\
$\mathrm{H}$ & 1.27736 & -2.75582 & 0.50980 \\
$\mathrm{H}$ & 3.06156 & -3.00378 & 0.40700 \\
$\mathrm{H}$ & 2.98483 & -2.57284 & -2.08270 \\
\hline & & Continued & on next page
\end{tabular}




\begin{tabular}{lrrr}
\multicolumn{5}{c}{ Continued from previous page } \\
\hline $\mathrm{H}$ & 1.19253 & -2.31802 & -1.98262 \\
$\mathrm{H}$ & 0.98985 & -4.60553 & -1.23796 \\
$\mathrm{H}$ & 2.61529 & -4.83697 & -1.30743
\end{tabular}

S1518 
Table S1398 Employed active space, reference configurations, and Cartesian coordinates of the OM2/MRCISD-optimized ground-state equilibrium geometry of tryptamine (Ph, out) (ground-state energy: $-1882.10464 \mathrm{eV}$ ).

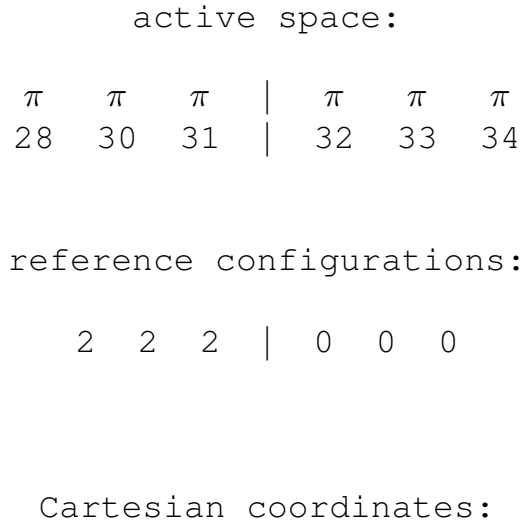


Table S1399 Employed active space, reference configurations, and Cartesian coordinates of the OM2/MRCISD-optimized excited-state equilibrium geometry of the $2{ }^{1} A\left({ }^{1} \pi \pi^{*}\right)$ state of tryptamine (Ph, out) (excited-state energy: $-1877.41850 \mathrm{eV})$.

active space:
\begin{tabular}{ccc|ccc}
$\pi$ & $\pi$ & $\pi$ & $\pi$ & $\pi$ & $\pi$ \\
28 & 30 & 31 & 32 & 33 & 34 \\
reference configurations: \\
2 & 2 & 2 & 0 & 0 & 0 \\
2 & 1 & 2 & 1 & 0 & 0 \\
2 & 2 & 1 & 0 & 1 & 0 \\
2 & 2 & 1 & 1 & 0 & 0
\end{tabular}

Cartesian coordinates:

\begin{tabular}{lrrr}
24 & & & \\
& & & \\
$\mathrm{C}$ & 0.00000 & 0.00000 & 0.00000 \\
$\mathrm{~N}$ & 1.38547 & 0.00000 & 0.00000 \\
$\mathrm{C}$ & 1.86961 & 1.28608 & 0.00000 \\
$\mathrm{C}$ & 0.69462 & 2.19644 & 0.00539 \\
$\mathrm{C}$ & -0.46281 & 1.35981 & 0.00620 \\
$\mathrm{C}$ & 3.19254 & 1.77221 & 0.02481 \\
$\mathrm{C}$ & 3.34967 & 3.15906 & 0.03148 \\
$\mathrm{C}$ & 2.23660 & 4.03353 & 0.04915 \\
$\mathrm{C}$ & 0.91015 & 3.57426 & 0.03375 \\
$\mathrm{C}$ & -1.88452 & 1.77663 & -0.04527 \\
$\mathrm{C}$ & -2.33911 & 1.84256 & -1.50581 \\
$\mathrm{~N}$ & -1.69320 & 2.92861 & -2.17013 \\
$\mathrm{H}$ & 4.04530 & 1.08922 & 0.02215 \\
$\mathrm{H}$ & 4.35726 & 3.58616 & 0.02401 \\
$\mathrm{H}$ & 2.42288 & 5.11538 & 0.06996 \\
$\mathrm{H}$ & 0.07071 & 4.27361 & 0.05261 \\
$\mathrm{H}$ & 1.95782 & -0.83312 & 0.01228 \\
$\mathrm{H}$ & -0.60315 & -0.89879 & -0.01688 \\
$\mathrm{H}$ & -2.00017 & 2.77591 & 0.42817 \\
$\mathrm{H}$ & -2.50671 & 1.04381 & 0.51487 \\
$\mathrm{H}$ & -3.44859 & 1.99240 & -1.52662 \\
\hline & & Continued & on next page
\end{tabular}

S1520 


\begin{tabular}{llrl}
\multicolumn{5}{c}{ Continued from previous page } \\
\hline $\mathrm{H}$ & -2.13718 & 0.83771 & -1.99839 \\
$\mathrm{H}$ & -0.70414 & 2.99533 & -1.96828 \\
$\mathrm{H}$ & -1.83153 & 2.92733 & -3.17175
\end{tabular}


Table S1400 Employed active space, reference configurations, and Cartesian coordinates of the OM2/MRCISD-optimized ground-state equilibrium geometry of tryptamine (Ph, up) (ground-state energy: $-1881.91969 \mathrm{eV})$.

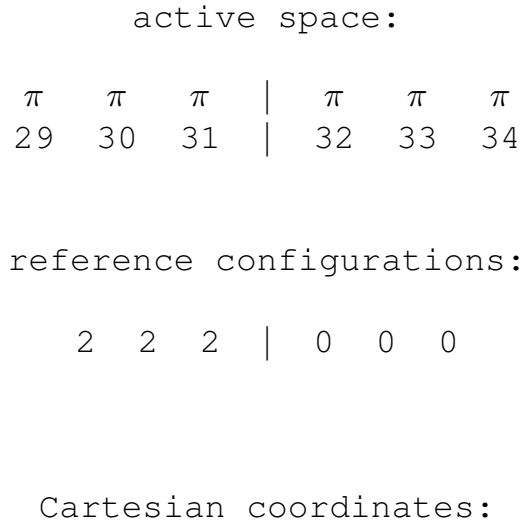


Table S1401 Employed active space, reference configurations, and Cartesian coordinates of the OM2/MRCISD-optimized excited-state equilibrium geometry of the $2{ }^{1} A\left({ }^{1} \pi \pi^{*}\right)$ state of tryptamine (Ph, up) (excited-state energy: $-1877.23069 \mathrm{eV})$.

active space:
\begin{tabular}{ccc|ccc}
$\pi$ & $\pi$ & $\pi$ & $\pi$ & $\pi$ & $\pi$ \\
29 & 30 & 31 & 32 & 33 & 34 \\
reference configurations: \\
2 & 2 & 2 & 0 & 0 & 0 \\
2 & 1 & 2 & 1 & 0 & 0 \\
2 & 2 & 1 & 0 & 1 & 0 \\
2 & 2 & 1 & 1 & 0 & 0
\end{tabular}

Cartesian coordinates:

\begin{tabular}{lrrr}
24 & & & \\
& & & \\
$\mathrm{C}$ & 0.00000 & 0.00000 & 0.00000 \\
$\mathrm{C}$ & 1.39470 & 0.00000 & 0.00000 \\
$\mathrm{C}$ & 2.10826 & 1.30100 & 0.00000 \\
$\mathrm{C}$ & 1.42570 & 2.53017 & -0.02298 \\
$\mathrm{C}$ & 0.02987 & 2.47455 & -0.04148 \\
$\mathrm{C}$ & -0.66264 & 1.24096 & -0.02627 \\
$\mathrm{C}$ & 2.39678 & -1.01233 & 0.04047 \\
$\mathrm{C}$ & 3.66735 & -0.34765 & 0.05214 \\
$\mathrm{~N}$ & 3.45371 & 1.02223 & 0.03102 \\
$\mathrm{C}$ & 2.20317 & -2.47381 & -0.00084 \\
$\mathrm{C}$ & 2.25521 & -2.95300 & -1.47384 \\
$\mathrm{~N}$ & 1.18692 & -2.42827 & -2.25842 \\
$\mathrm{H}$ & 1.96897 & 3.47794 & -0.04031 \\
$\mathrm{H}$ & -0.54522 & 3.40409 & -0.07421 \\
$\mathrm{H}$ & -1.76019 & 1.25534 & -0.03482 \\
$\mathrm{H}$ & -0.56363 & -0.93743 & 0.03673 \\
$\mathrm{H}$ & 4.18778 & 1.71693 & 0.06690 \\
$\mathrm{H}$ & 4.64867 & -0.80411 & 0.05789 \\
$\mathrm{H}$ & 1.21493 & -2.73523 & 0.44267 \\
$\mathrm{H}$ & 2.99765 & -2.97807 & 0.59037 \\
$\mathrm{H}$ & 2.21413 & -4.07219 & -1.46823 \\
\hline & \multicolumn{3}{c}{ Continued on next page }
\end{tabular}

S1523 


\begin{tabular}{lrrr}
\multicolumn{5}{c}{ Continued from previous page } \\
\hline $\mathrm{H}$ & 3.24189 & -2.64723 & -1.91290 \\
$\mathrm{H}$ & 1.23075 & -1.41992 & -2.35046 \\
$\mathrm{H}$ & 0.27253 & -2.67803 & -1.89774
\end{tabular}


Table S1402 Employed active space, reference configurations, and Cartesian coordinates of the OM2/MRCISD-optimized ground-state equilibrium geometry of tryptamine (py, out) (ground-state energy: $-1882.10304 \mathrm{eV}$ ).

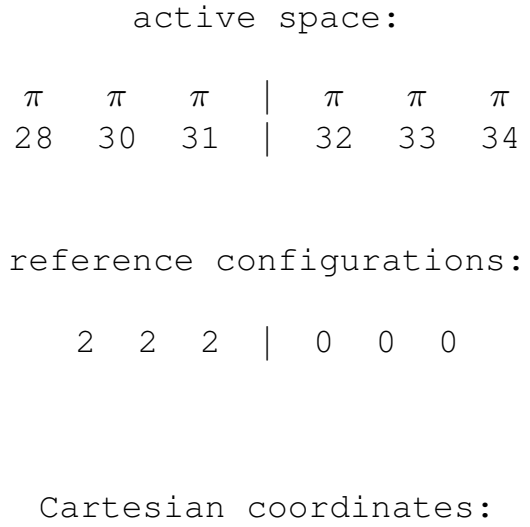


Table S1403 Employed active space, reference configurations, and Cartesian coordinates of the OM2/MRCISD-optimized excited-state equilibrium geometry of the $2^{1} A\left({ }^{1} \pi \pi^{*}\right)$ state of tryptamine (py, out) (excited-state energy: $-1877.41131 \mathrm{eV})$.

active space:
\begin{tabular}{ccc|ccc}
$\pi$ & $\pi$ & $\pi$ & $\pi$ & $\pi$ & $\pi$ \\
28 & 30 & 31 & 32 & 33 & 34 \\
reference configurations: \\
2 & 2 & 2 & 0 & 0 & 0 \\
2 & 1 & 2 & 1 & 0 & 0 \\
2 & 2 & 1 & 0 & 1 & 0 \\
2 & 2 & 1 & 1 & 0 & 0
\end{tabular}

Cartesian coordinates:

\begin{tabular}{lrrr}
24 & & & \\
& & & \\
$\mathrm{C}$ & 0.00000 & 0.00000 & 0.00000 \\
$\mathrm{~N}$ & 1.38523 & 0.00000 & 0.00000 \\
$\mathrm{C}$ & 1.86861 & 1.28670 & 0.00000 \\
$\mathrm{C}$ & 0.69364 & 2.19722 & 0.00271 \\
$\mathrm{C}$ & -0.46351 & 1.35997 & -0.00273 \\
$\mathrm{C}$ & 3.19229 & 1.77009 & 0.03145 \\
$\mathrm{C}$ & 3.35240 & 3.15662 & 0.05331 \\
$\mathrm{C}$ & 2.24093 & 4.03214 & 0.08000 \\
$\mathrm{C}$ & 0.91457 & 3.57444 & 0.05441 \\
$\mathrm{C}$ & -1.88557 & 1.77854 & -0.03439 \\
$\mathrm{C}$ & -2.20459 & 2.41094 & -1.39140 \\
$\mathrm{~N}$ & -2.07470 & 1.43047 & -2.42349 \\
$\mathrm{H}$ & 4.04378 & 1.08563 & 0.02303 \\
$\mathrm{H}$ & 4.36067 & 3.58216 & 0.04512 \\
$\mathrm{H}$ & 2.42871 & 5.11348 & 0.11617 \\
$\mathrm{H}$ & 0.07738 & 4.27728 & 0.09098 \\
$\mathrm{H}$ & 1.95803 & -0.83282 & 0.00898 \\
$\mathrm{H}$ & -0.60626 & -0.89623 & -0.01962 \\
$\mathrm{H}$ & -2.06554 & 2.50732 & 0.78370 \\
$\mathrm{H}$ & -2.54497 & 0.89437 & 0.12161 \\
$\mathrm{H}$ & -1.52767 & 3.30717 & -1.54549 \\
\hline & & Continued & on next page
\end{tabular}

S1526 


\begin{tabular}{llll}
\multicolumn{5}{c}{ Continued from previous page } \\
\hline $\mathrm{H}$ & -3.25441 & 2.80008 & -1.36751 \\
$\mathrm{H}$ & -2.10372 & 1.82621 & -3.35458 \\
$\mathrm{H}$ & -1.23681 & 0.86941 & -2.32884
\end{tabular}


Table S1404 Employed active space, reference configurations, and Cartesian coordinates of the OM2/MRCISD-optimized ground-state equilibrium geometry of tryptamine (py, up) (ground-state energy: $-1881.91896 \mathrm{eV}$ ).

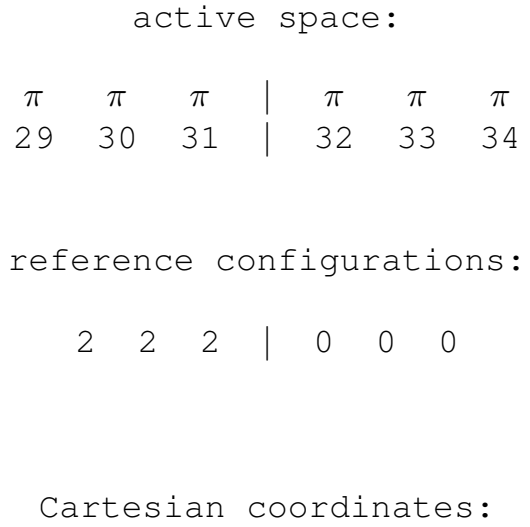


Table S1405 Employed active space, reference configurations, and Cartesian coordinates of the OM2/MRCISD-optimized excited-state equilibrium geometry of the $2^{1} A\left({ }^{1} \pi \pi^{*}\right)$ state of tryptamine (py, up) (excited-state energy: $-1877.22272 \mathrm{eV})$.

active space:
\begin{tabular}{ccc|ccc}
$\pi$ & $\pi$ & $\pi$ & $\pi$ & $\pi$ & $\pi$ \\
29 & 30 & 31 & 32 & 33 & 34 \\
reference configurations: \\
2 & 2 & 2 & 0 & 0 & 0 \\
2 & 1 & 2 & 1 & 0 & 0 \\
2 & 2 & 1 & 0 & 1 & 0 \\
2 & 2 & 1 & 1 & 0 & 0
\end{tabular}

Cartesian coordinates:

\begin{tabular}{lrrr}
24 & & & \\
& & & \\
$\mathrm{C}$ & 0.00000 & 0.00000 & 0.00000 \\
$\mathrm{C}$ & 1.39516 & 0.00000 & 0.00000 \\
$\mathrm{C}$ & 2.10878 & 1.30170 & 0.00000 \\
$\mathrm{C}$ & 1.42536 & 2.53229 & -0.02006 \\
$\mathrm{C}$ & 0.03020 & 2.47580 & -0.03343 \\
$\mathrm{C}$ & -0.66216 & 1.24178 & -0.01874 \\
$\mathrm{C}$ & 2.39811 & -1.01204 & 0.02682 \\
$\mathrm{C}$ & 3.66756 & -0.34708 & 0.02287 \\
$\mathrm{~N}$ & 3.45367 & 1.02434 & 0.01356 \\
$\mathrm{C}$ & 2.19051 & -2.47224 & -0.00406 \\
$\mathrm{C}$ & 1.88472 & -2.91529 & -1.45605 \\
$\mathrm{~N}$ & 2.98788 & -2.69460 & -2.33494 \\
$\mathrm{H}$ & 1.96887 & 3.47968 & -0.04024 \\
$\mathrm{H}$ & -0.54566 & 3.40508 & -0.06731 \\
$\mathrm{H}$ & -1.75977 & 1.25654 & -0.02380 \\
$\mathrm{H}$ & -0.56391 & -0.93659 & 0.03005 \\
$\mathrm{H}$ & 4.18827 & 1.71896 & 0.03021 \\
$\mathrm{H}$ & 4.64952 & -0.80205 & 0.02405 \\
$\mathrm{H}$ & 1.34272 & -2.73990 & 0.66148 \\
$\mathrm{H}$ & 3.10465 & -2.99610 & 0.36277 \\
$\mathrm{H}$ & 0.98526 & -2.35551 & -1.82031 \\
\hline & & Continued \\
& &
\end{tabular}

S1529 


\begin{tabular}{llll}
\multicolumn{5}{c}{ Continued from previous page } \\
\hline $\mathrm{H}$ & 1.62634 & -4.00467 & -1.43312 \\
$\mathrm{H}$ & 3.83332 & -3.16360 & -2.02650 \\
$\mathrm{H}$ & 3.19343 & -1.70855 & -2.45033
\end{tabular}

S1530 
Table S1406 Employed active space, reference configurations, and Cartesian coordinates of the OM2/MRCISD-optimized ground-state equilibrium geometry of 2,4,6,8-decatetraene (ground-state energy: $-1485.22049 \mathrm{eV}$ ).

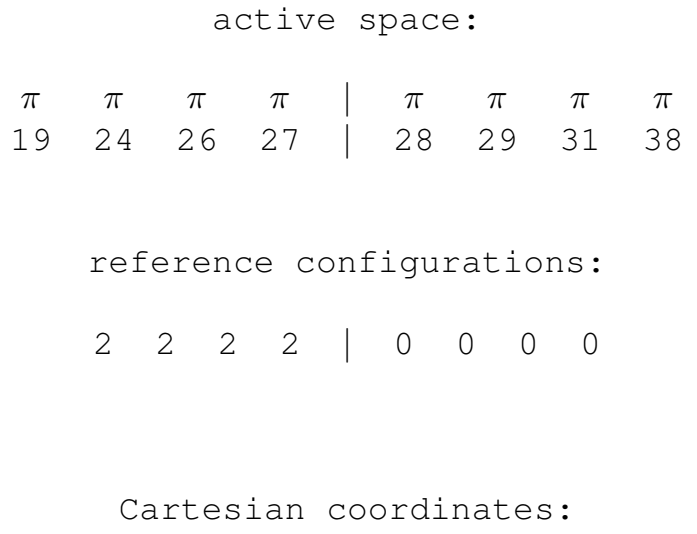

24

C 0.00000

C $\quad 1.44582$

$\begin{array}{lr}\text { C } & -0.70887 \\ \text { C } & 2.15470\end{array}$

0.00000

0.00000

0.00000

0.00000

1.16339

0.00000

C $\quad-2.15912$

$-1.16339$

0.00000

$1.16211-0.00000$

C $\quad 3.60494$

$-1.16211$

$-0.00000$

$-2.86427$

2. 32032

$-0.00000$

C $\quad 4.31009$

$-2.32032$

0.00000

$-4.35056$

$2.34473-0.00000$

5.79639

$-2.34473-0.00000$

$-0.51277$

$-0.97150-0.00000$

1.95859

0.97150

$-0.00000$

$-0.19780$

2.13587

0.00000

1.64363

$-2.13587$

0.00000

$-2.66900$

0.18928

$-0.00000$

4.11482

$-0.18928$

$-0.00000$

$-2.35638$

3.29354

0.00000

3. 80220

$-3.29354$

0.00000

$-4.76081$

1. 32212

$-0.00000$

6.20663

$-1.32212$

$-0.00000$

$-4.70074$

2.86622

0.89830

$-4.70074$

2.86622

$-0.89830$

6.14656

$-2.86622$

0.89830

$\mathrm{H} \quad 6.14656$

$-2.86622$

$-0.89830$ 
Table S1407 Employed active space, reference configurations, and Cartesian coordinates of the OM2/MRCISD-optimized excited-state equilibrium geometry of the $1{ }^{1} B_{u}\left({ }^{1} \pi \pi^{*}\right)$ state of $2,4,6,8$-decatetraene (excited-state energy: $-1480.97506 \mathrm{eV})$.

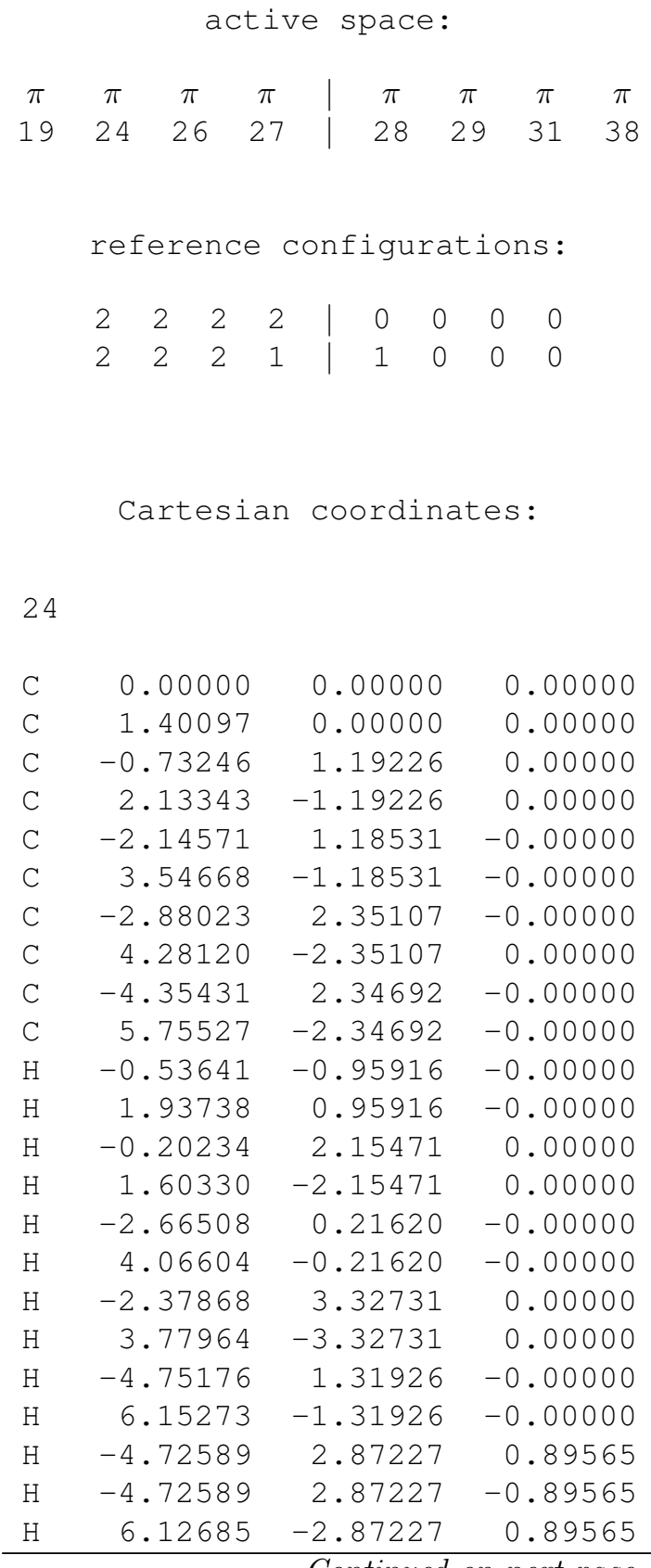

Continued on next page

S1532 
Continued from previous page

$\begin{array}{llll}\mathrm{H} & 6.12685 & -2.87227 & -0.89565\end{array}$

S1533 
Table S1408 Employed active space, reference configurations, and Cartesian coordinates of the OM2/MRCISD-optimized ground-state equilibrium geometry of 5-methoxysalicylic acid (ground-state energy: $-2465.65380 \mathrm{eV}$ ).

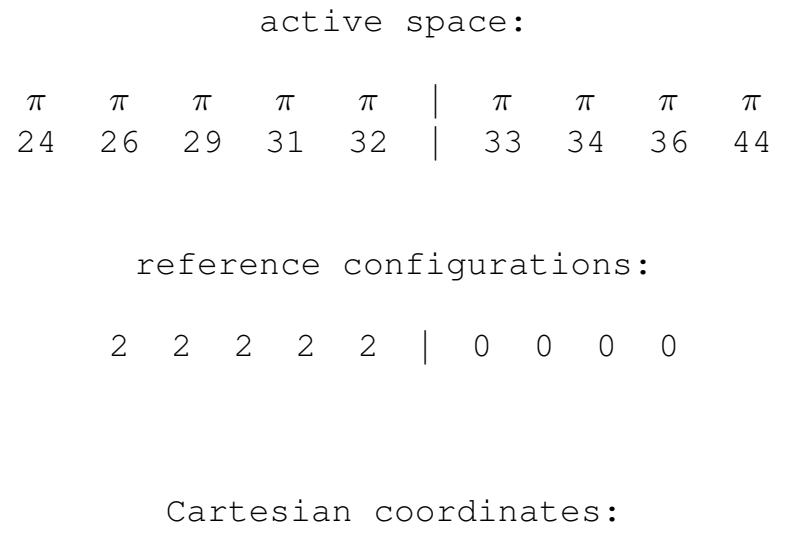

$\begin{array}{lrrr}20 & & & \\ & & & \\ \mathrm{C} & 0.00000 & 0.00000 & 0.00000 \\ \mathrm{C} & 1.41905 & 0.00000 & 0.00000 \\ \mathrm{C} & 2.09497 & 1.20979 & 0.00000 \\ \mathrm{C} & 1.35562 & 2.42082 & 0.00000 \\ \mathrm{C} & -0.04129 & 2.45717 & 0.00000 \\ \mathrm{C} & -0.71143 & 1.22169 & 0.00000 \\ \mathrm{O} & 2.17160 & 3.53148 & 0.00000 \\ \mathrm{C} & 1.54622 & 4.77881 & 0.00000 \\ \mathrm{C} & -2.17737 & 1.13235 & 0.00000 \\ \mathrm{O} & -2.82583 & 0.07064 & 0.00000 \\ \mathrm{O} & -0.58720 & -1.20492 & -0.00000 \\ \mathrm{O} & -2.82863 & 2.31373 & 0.00000 \\ \mathrm{H} & 2.38944 & 5.50676 & 0.00000 \\ \mathrm{H} & 0.94210 & 4.92784 & 0.91482 \\ \mathrm{H} & 0.94210 & 4.92784 & -0.91482 \\ \mathrm{H} & -0.61334 & 3.38088 & 0.00000 \\ \mathrm{H} & 1.94416 & -0.95285 & -0.00000 \\ \mathrm{H} & 3.18393 & 1.25774 & 0.00000 \\ \mathrm{H} & -1.59176 & -1.11216 & -0.00000 \\ \mathrm{H} & -3.80987 & 2.16085 & 0.00000\end{array}$


Table S1409 Employed active space, reference configurations, and Cartesian coordinates of the OM2/MRCISD-optimized excited-state equilibrium geometry of the $2{ }^{1} A^{\prime}\left({ }^{1} \pi \pi^{*}\right)$ state of 5-methoxysalicylic acid (excited-state energy: $-2462.14844 \mathrm{eV})$.

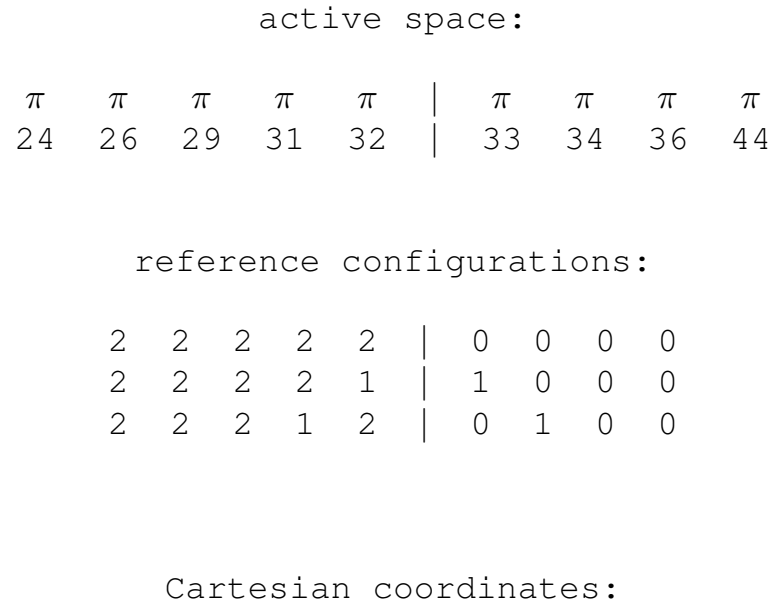


Table S1410 Employed active space, reference configurations, and Cartesian coordinates of the OM2/MRCISD-optimized ground-state equilibrium geometry of salicylic acid (ground-state energy: $-1977.42844 \mathrm{eV}$ ).

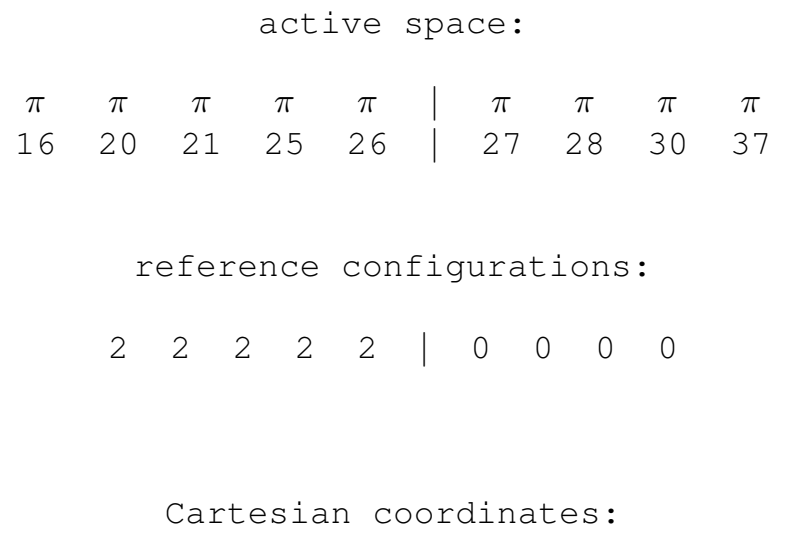

$\begin{array}{lrrr}16 & & & \\ & & & \\ \text { C } & 0.00000 & 0.00000 & 0.00000 \\ \text { C } & 1.38934 & 0.00000 & 0.00000 \\ \text { C } & 2.06509 & 1.24107 & 0.00000 \\ \text { C } & 1.32801 & 2.44809 & 0.00000 \\ \text { C } & -0.07391 & 2.42615 & 0.00000 \\ \text { C } & -0.73066 & 1.19938 & 0.00000 \\ \text { O } & 3.40675 & 1.18989 & 0.00000 \\ \mathrm{C} & 2.10600 & 3.69435 & 0.00000 \\ \text { O } & 3.34879 & 3.74791 & 0.00000 \\ \mathrm{O} & 1.38204 & 4.82873 & 0.00000 \\ \mathrm{H} & -0.62730 & 3.36759 & 0.00000 \\ \mathrm{H} & 1.97059 & -0.91937 & -0.00000 \\ \mathrm{H} & -1.82294 & 1.16647 & 0.00000 \\ \mathrm{H} & -0.53831 & -0.95537 & -0.00000 \\ \mathrm{H} & 3.80096 & 2.12165 & 0.00000 \\ \mathrm{H} & 1.98422 & 5.61881 & 0.00000\end{array}$


Table S1411 Employed active space, reference configurations, and Cartesian coordinates of the OM2/MRCISD-optimized excited-state equilibrium geometry of the $2^{1} A^{\prime}\left({ }^{1} \pi \pi^{*}\right)$ state of salicylic acid (excited-state energy: $-1973.84564 \mathrm{eV})$.

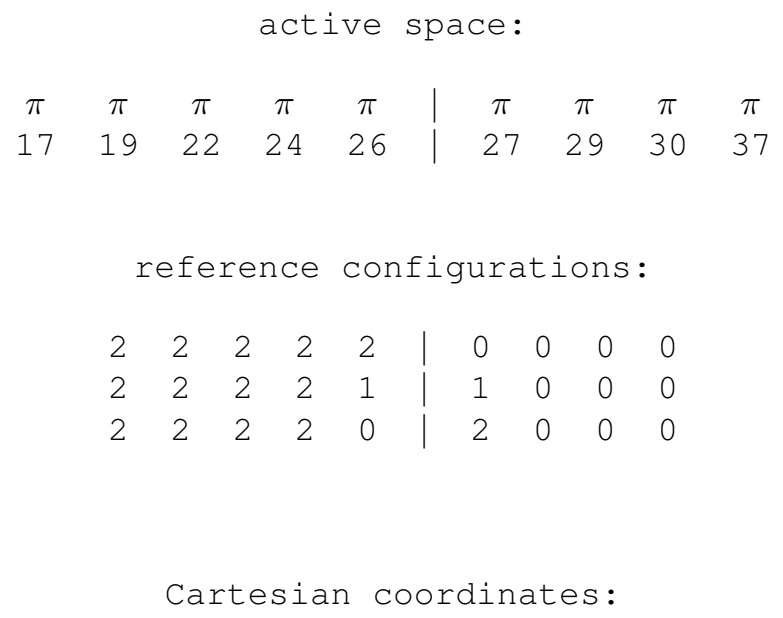


Table S1412 Employed active space, reference configurations, and Cartesian coordinates of the OM2/MRCISD-optimized ground-state equilibrium geometry of gauche-3-phenyl-1-propionic acid (ground-state energy: -1961.36747 eV).

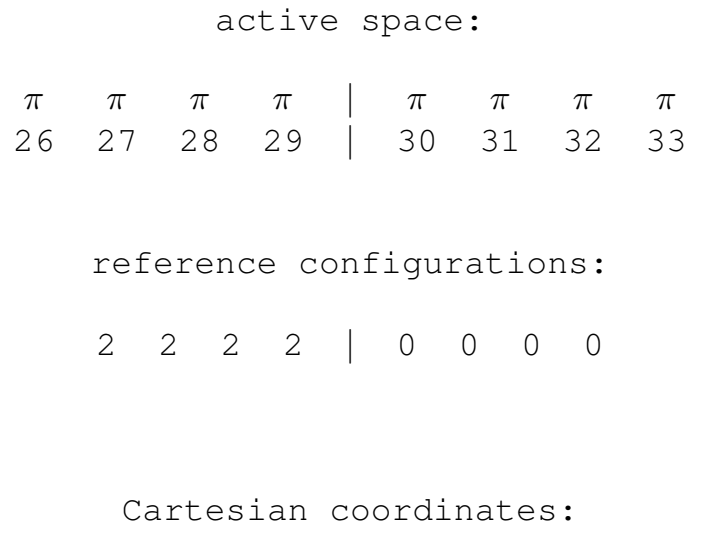

21

$\begin{array}{lrrr}\text { C } & 0.00000 & 0.00000 & 0.00000 \\ \text { C } & 2.78953 & 0.00000 & 0.00000 \\ \text { C } & 0.69997 & 1.21260 & 0.00000 \\ \text { C } & 0.69658 & -1.21521 & -0.00283 \\ \text { C } & 2.09335 & 1.20912 & 0.00192 \\ \mathrm{C} & 2.09002 & -1.20762 & -0.00040 \\ \mathrm{C} & -1.50598 & 0.00302 & 0.01820 \\ \mathrm{C} & -1.98439 & -0.05860 & 1.45895 \\ \mathrm{C} & -1.67929 & -1.43088 & 2.01180 \\ \mathrm{O} & -1.51380 & -1.40991 & 3.36026 \\ \mathrm{O} & -1.59609 & -2.50518 & 1.41814 \\ \mathrm{H} & 3.88510 & -0.00059 & -0.00465 \\ \mathrm{H} & 0.15346 & 2.16377 & -0.00447 \\ \mathrm{H} & 0.14757 & -2.15926 & 0.00216 \\ \mathrm{H} & 2.64323 & 2.15738 & 0.00204 \\ \mathrm{H} & 2.63967 & -2.15582 & -0.00032 \\ \mathrm{H} & -1.88837 & 0.93862 & -0.45169 \\ \mathrm{H} & -1.89590 & -0.86472 & -0.55508 \\ \mathrm{H} & -1.49045 & 0.70948 & 2.08374 \\ \mathrm{H} & -3.08663 & 0.07050 & 1.51621 \\ \mathrm{H} & -1.30637 & -2.31747 & 3.70212\end{array}$


Table S1413 Employed active space, reference configurations, and Cartesian coordinates of the OM2/MRCISD-optimized excited-state equilibrium geometry of the $2{ }^{1} A\left({ }^{1} \pi \pi^{*}\right)$ state of gauche-3-phenyl-1-propionic acid (excited-state energy: $-1955.91522 \mathrm{eV})$.

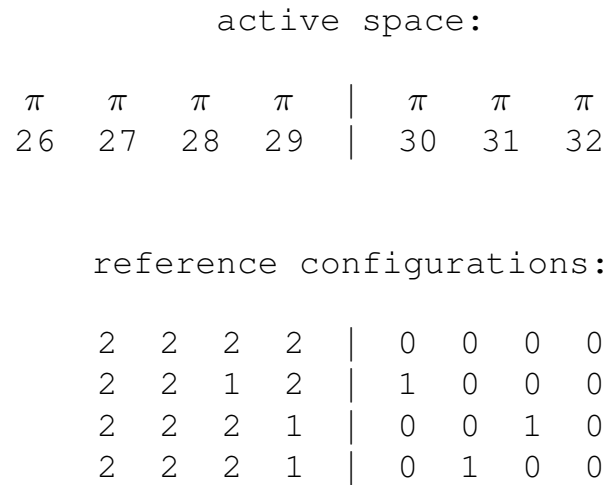

Cartesian coordinates:

21

$\begin{array}{lrrr}\mathrm{C} & 0.00000 & 0.00000 & 0.00000 \\ \mathrm{C} & 2.80994 & 0.00000 & 0.00000 \\ \mathrm{C} & 0.71012 & 1.23914 & 0.00000 \\ \mathrm{C} & 0.71239 & -1.20217 & -0.28117 \\ \mathrm{C} & 2.11109 & 1.20333 & 0.17034 \\ \mathrm{C} & 2.11660 & -1.20645 & -0.16527 \\ \mathrm{C} & -1.46079 & -0.03882 & 0.29198 \\ \mathrm{C} & -1.67179 & -0.49289 & 1.73282 \\ \mathrm{C} & -1.34414 & -1.96312 & 1.82806 \\ \mathrm{O} & -0.98584 & -2.32315 & 3.08864 \\ \mathrm{O} & -1.38795 & -2.82704 & 0.95335 \\ \mathrm{H} & 3.90571 & 0.00176 & 0.00484 \\ \mathrm{H} & 0.17228 & 2.18739 & -0.06787 \\ \mathrm{H} & 0.16798 & -2.10762 & -0.55170 \\ \mathrm{H} & 2.65059 & 2.12221 & 0.42390 \\ \mathrm{H} & 2.66645 & -2.14839 & -0.25095 \\ \mathrm{H} & -1.90387 & 0.97697 & 0.16809 \\ \mathrm{H} & -1.96756 & -0.74084 & -0.40566 \\ \mathrm{H} & -1.03247 & 0.07736 & 2.43206 \\ \mathrm{H} & -2.73636 & -0.37855 & 2.02654 \\ \mathrm{H} & -0.76768 & -3.28951 & 3.12770\end{array}$


Table S1414 Employed active space, reference configurations, and Cartesian coordinates of the OM2/MRCISD-optimized ground-state equilibrium geometry of anti-3-phenyl-1-propionic acid (ground-state energy: -1961.39288 eV).

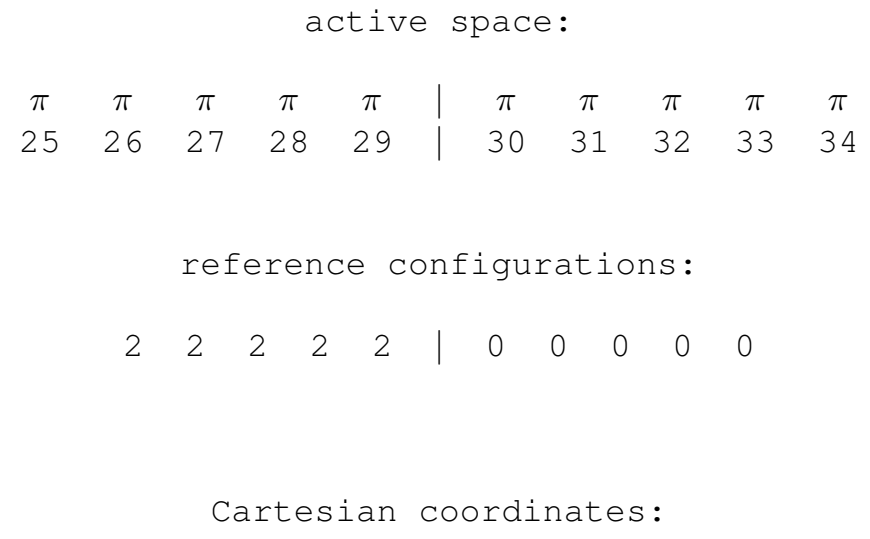

21

$\begin{array}{llll}C & 0.00000 & 0.00000 & 0.00000\end{array}$

$\begin{array}{lrrr}\text { C } & 2.79221 & 0.00000 & 0.00000\end{array}$

$\begin{array}{llll}\text { C } & 0.70188 & -0.00169 & -1.21235\end{array}$

C $\quad 0.70195 \quad-0.00042 \quad 1.21224$

C $\quad 2.09563 \quad-0.00091 \quad 1.20949$

C $\quad 2.09552-0.00204-1.20940$

$\begin{array}{llll}\text { C } & -1.50464 & 0.03897 & -0.00221\end{array}$

$\begin{array}{lrrr}\text { C } & -1.92478 & 1.49825 & 0.05240\end{array}$

$\begin{array}{llll}\text { C } & -3.42836 & 1.55381 & -0.09309\end{array}$

$\begin{array}{llll}0 & -3.99697 & 2.44131 & 0.76896\end{array}$

$\begin{array}{llll}0 & -4.14918 & 0.92305 & -0.86275\end{array}$

$\begin{array}{lrrr}\mathrm{H} & 3.88612 & 0.00373 & 0.00001\end{array}$

$\begin{array}{llll}\mathrm{H} & 0.15459 & -0.01101 & -2.16044\end{array}$

$\begin{array}{llll}\mathrm{H} & 0.15530 & -0.00796 & 2.16100\end{array}$

$\mathrm{H} \quad 2.64610 \quad-0.00526 \quad 2.15843$

$\mathrm{H} \quad \begin{array}{llll}\mathrm{H} & 2.64593 & -0.00788 & -2.15834\end{array}$

$\mathrm{H} \quad-1.90530 \quad-0.44163 \quad-0.92020$

$\mathrm{H} \quad-1.90317 \quad-0.50819 \quad 0.88165$

$\begin{array}{llll}\mathrm{H} & -1.61459 & 1.97793 & 1.00029\end{array}$

$\begin{array}{llll}\mathrm{H} & -1.49715 & 2.06372 & -0.80221\end{array}$

$\begin{array}{llll}\mathrm{H} & -4.98112 & 2.46423 & 0.64995\end{array}$ 
Table S1415 Employed active space, reference configurations, and Cartesian coordinates of the OM2/MRCISD-optimized excited-state equilibrium geometry of the $1^{1} A^{\prime \prime}\left({ }^{1} \pi \pi^{*}\right)$ state of anti-3-phenyl-1-propionic acid (excited-state energy: $-1956.47234 \mathrm{eV})$.

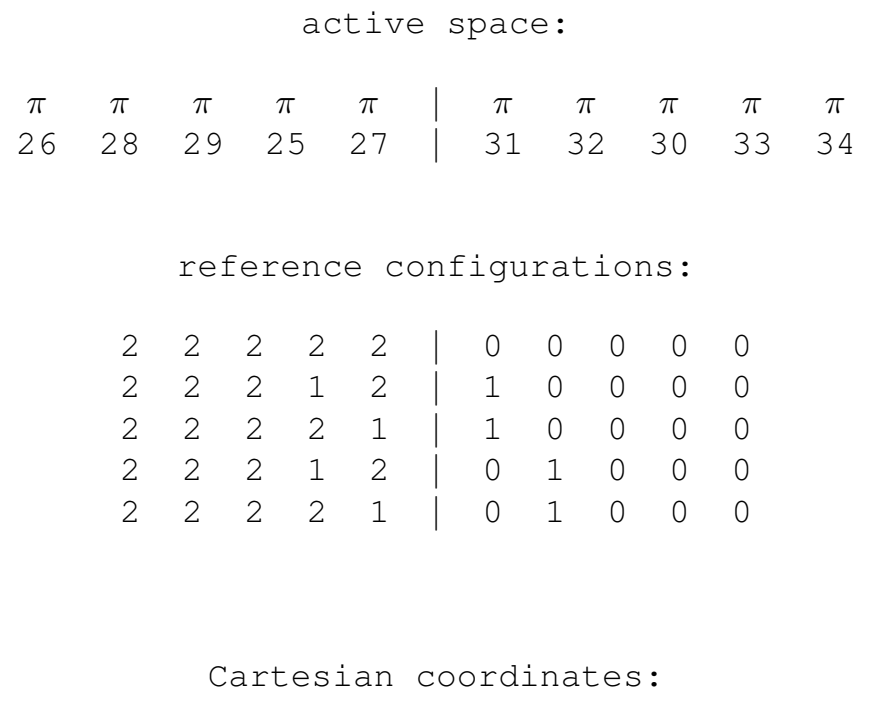

21

\begin{tabular}{lrrr}
$\mathrm{C}$ & 0.00000 & 0.00000 & 0.00000 \\
$\mathrm{C}$ & 2.80155 & 0.00000 & 0.00000 \\
$\mathrm{C}$ & 0.70147 & -0.00019 & -1.21175 \\
$\mathrm{C}$ & 0.70147 & -0.00019 & 1.21175 \\
$\mathrm{C}$ & 2.09459 & 0.00174 & 1.20430 \\
$\mathrm{C}$ & 2.09459 & 0.00174 & -1.20430 \\
$\mathrm{C}$ & -1.50470 & 0.03492 & 0.00000 \\
$\mathrm{C}$ & -1.92352 & 1.49967 & 0.00000 \\
$\mathrm{C}$ & -3.43308 & 1.56115 & 0.00000 \\
$\mathrm{O}$ & -3.96633 & 2.81879 & 0.00000 \\
$\mathrm{O}$ & -4.16223 & 0.49868 & -0.00000 \\
$\mathrm{H}$ & 3.89185 & -0.01558 & -0.00000 \\
$\mathrm{H}$ & 0.15449 & -0.00535 & -2.15980 \\
$\mathrm{H}$ & 0.15449 & -0.00535 & 2.15980 \\
$\mathrm{H}$ & 2.64292 & -0.00099 & 2.15585 \\
$\mathrm{H}$ & 2.64292 & -0.00099 & -2.15585 \\
$\mathrm{H}$ & -1.90518 & -0.47847 & -0.90101 \\
$\mathrm{H}$ & -1.90518 & -0.47847 & 0.90101 \\
$\mathrm{H}$ & -1.57841 & 2.02361 & 0.90791 \\
$\mathrm{H}$ & -1.57841 & 2.02361 & -0.90791 \\
\hline & & Continued on next page
\end{tabular}

S1541 
Continued from previous page

$\begin{array}{llll}\mathrm{H} & -4.95883 & 2.71160 & 0.00000\end{array}$ 
Table S1416 Employed active space, reference configurations, and Cartesian coordinates of the OM2/MRCISD-optimized ground-state equilibrium geometry of cis-1-naphthol (ground-state energy: $-1727.32028 \mathrm{eV}$ ).

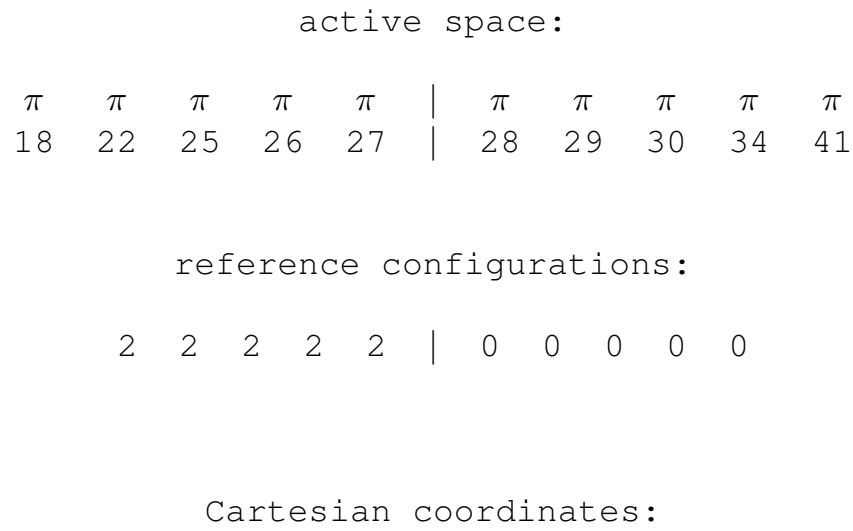


Table S1417 Employed active space, reference configurations, and Cartesian coordinates of the OM2/MRCISD-optimized excited-state equilibrium geometry of the $2{ }^{1} A\left({ }^{1} \pi \pi^{*}\right)$ state of cis-1-naphthol (excited-state energy: $-1723.59995 \mathrm{eV})$.

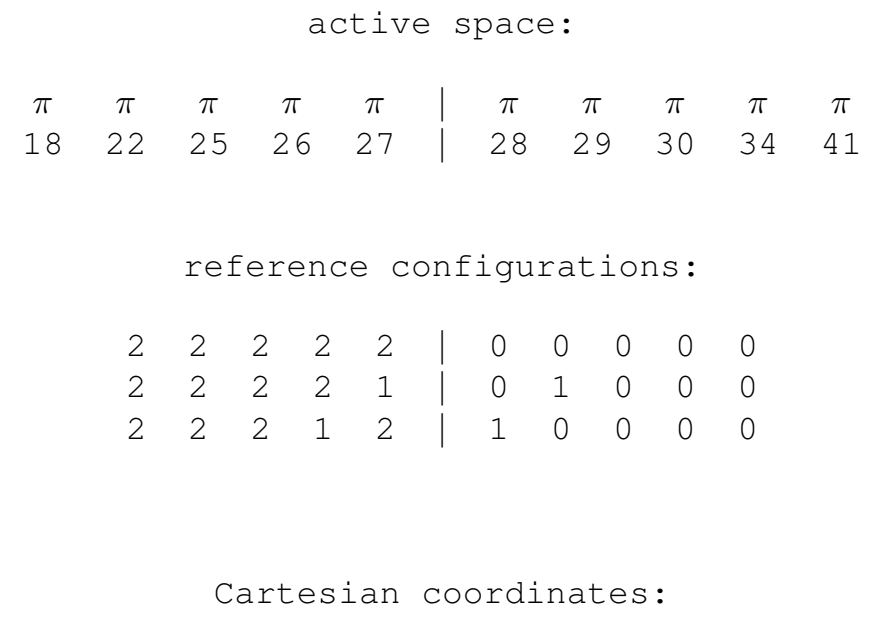

19

$\begin{array}{llll}\text { C } & 0.00000 & 0.00000 & 0.00000\end{array}$

$\begin{array}{llll}\text { C } & 1.41297 & 0.00000 & 0.00000\end{array}$

$\begin{array}{lllr}\text { C } & 2.11728 & 1.29622 & 0.00000\end{array}$

$\begin{array}{llll}\text { C } & 1.36831 & 2.48744 & -0.00001\end{array}$

$\begin{array}{llll}\text { C } & -0.03033 & 2.44558 & 0.00000\end{array}$

$\begin{array}{llll}\text { C } & -0.71285 & 1.20266 & 0.00000\end{array}$

$\begin{array}{llll}\text { C } & 2.15984 & -1.19935 & 0.00002\end{array}$

$\begin{array}{llll}\text { C } & 3.56512 & -1.18086 & 0.00001\end{array}$

$\begin{array}{llll}\text { C } & 4.27361 & 0.03811 & -0.00001\end{array}$

$\begin{array}{llll}\text { C } & 3.54330 & 1.25521 & -0.00000\end{array}$

$\begin{array}{lrrr}0 & 4.31631 & 2.35077 & -0.00000\end{array}$

$\mathrm{H} \quad-0.53744 \quad-0.95618 \quad-0.00000$

$\mathrm{H} \quad-1.80720 \quad 1.19339 \quad 0.00000$

$\mathrm{H} \quad-0.60615 \quad 3.37589 \quad 0.00001$

$\mathrm{H} \quad 1.87514 \quad 3.46476 \quad-0.00001$

$\mathrm{H} \quad 1.63024 \quad-2.15918 \quad 0.00003$

$\begin{array}{llll}\mathrm{H} & 4.12251 & -2.12263 & 0.00001\end{array}$

$\begin{array}{llll}\mathrm{H} & 5.36092 & 0.06988 & -0.00001\end{array}$

$\mathrm{H} \quad 3.75388 \quad 3.17250 \quad-0.00001$ 
Table S1418 Employed active space, reference configurations, and Cartesian coordinates of the OM2/MRCISD-optimized ground-state equilibrium geometry of trans-1-naphthol (ground-state energy: $-1727.37483 \mathrm{eV}$ ).

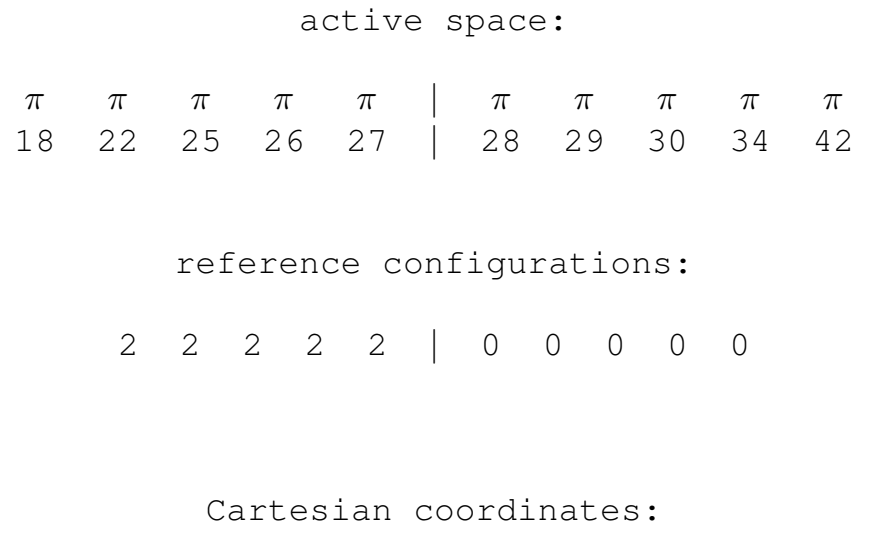

19

$\begin{array}{llll}C & 0.00000 & 0.00000 & 0.00000\end{array}$

$\begin{array}{llll}\text { C } & 1.43588 & 0.00000 & 0.00000\end{array}$

C $\quad 2.08947 \quad 1.25752 \quad 0.00000$

C $\quad 1.32443 \quad 2.46055 \quad 0.00000$

C $\quad-0.05464 \quad 2.41133 \quad 0.00000$

C $\quad-0.73936 \quad 1.17546 \quad 0.00000$

C $\quad 2.18341-1.20572-0.00000$

C $\quad 3.56231-1.14458-0.00000$

$\begin{array}{llll}\text { C } & 4.22760 & 0.10390 & -0.00000\end{array}$

C $\quad 3.51258 \quad 1.28394 \quad-0.00000$

$\begin{array}{llll}0 & -0.56139 & -1.23156 & -0.00000\end{array}$

$\begin{array}{llll}\mathrm{H} & 4.02672 & 2.25197 & -0.00000\end{array}$

$\begin{array}{llll}\mathrm{H} & 5.32364 & 0.12219 & -0.00000\end{array}$

$\mathrm{H} \quad 4.15678 \quad-2.06445 \quad-0.00000$

$\mathrm{H} \quad \begin{array}{llll}\mathrm{H} & 1.65080 & -2.15851 & -0.00000\end{array}$

$\mathrm{H} \quad \begin{array}{llll}\mathrm{H} & 1.84850 & 3.42162 & -0.00000\end{array}$

$\begin{array}{llll}\mathrm{H} & -0.64157 & 3.33723 & 0.00000\end{array}$

$\mathrm{H} \quad-1.83076 \quad 1.15246 \quad 0.00000$

$\mathrm{H} \quad-1.55270 \quad-1.14564 \quad-0.00000$ 
Table S1419 Employed active space, reference configurations, and Cartesian coordinates of the OM2/MRCISD-optimized excited-state equilibrium geometry of the $2{ }^{1} A^{\prime}\left({ }^{1} \pi \pi^{*}\right)$ state of trans-1-naphthol (excited-state energy: $-1723.71315 \mathrm{eV})$.

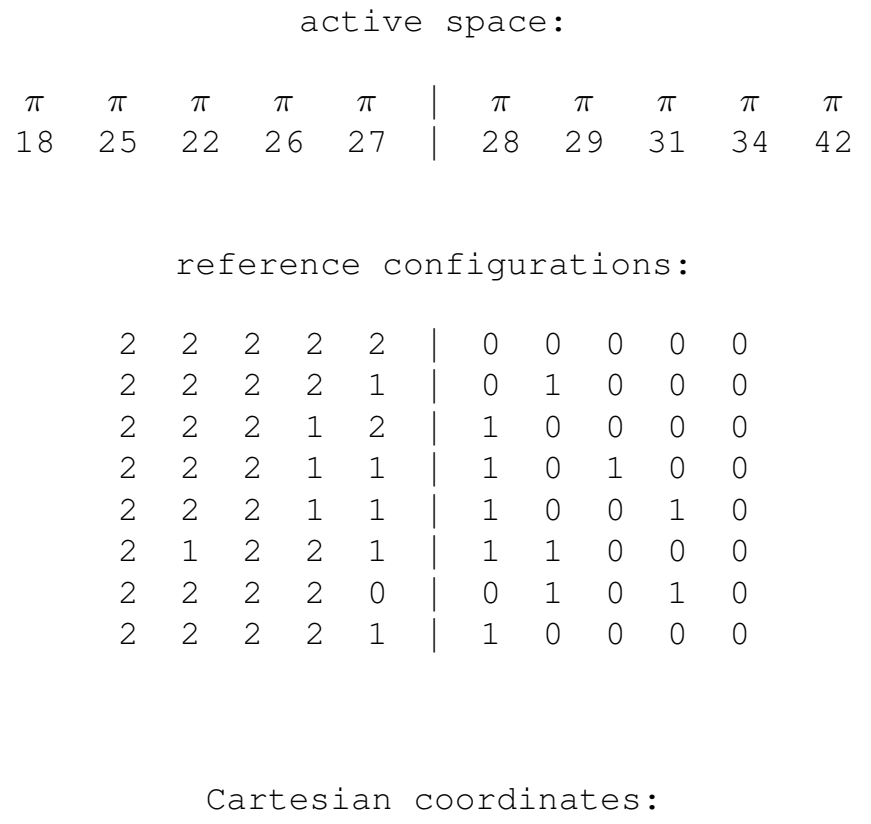

\begin{tabular}{lrrr}
19 & & & \\
& & & \\
$\mathrm{C}$ & 0.00000 & 0.00000 & 0.00000 \\
$\mathrm{C}$ & 1.42618 & 0.00000 & 0.00000 \\
$\mathrm{C}$ & 2.09493 & 1.31349 & 0.00000 \\
$\mathrm{C}$ & 1.31714 & 2.49220 & 0.00000 \\
$\mathrm{C}$ & -0.08915 & 2.43450 & 0.00000 \\
$\mathrm{C}$ & -0.76280 & 1.19537 & 0.00000 \\
$\mathrm{C}$ & 2.18930 & -1.18296 & -0.00000 \\
$\mathrm{C}$ & 3.58806 & -1.10517 & -0.00000 \\
$\mathrm{C}$ & 4.24488 & 0.15175 & -0.00000 \\
$\mathrm{C}$ & 3.50798 & 1.34319 & -0.00000 \\
$\mathrm{O}$ & -0.57384 & -1.21412 & -0.00000 \\
$\mathrm{H}$ & 4.02702 & 2.30955 & -0.00000 \\
$\mathrm{H}$ & 5.33899 & 0.18588 & -0.00000 \\
$\mathrm{H}$ & 4.18612 & -2.02203 & -0.00000 \\
$\mathrm{H}$ & 1.68062 & -2.14940 & -0.00000 \\
$\mathrm{H}$ & 1.81733 & 3.46739 & -0.00000 \\
$\mathrm{H}$ & -0.67195 & 3.36129 & 0.00000 \\
\hline & & \multicolumn{3}{c}{ Continued on next page }
\end{tabular}

S1546 
Continued from previous page

\begin{tabular}{rrrr}
\hline $\mathrm{H}$ & -1.85389 & 1.15298 & 0.00000 \\
$\mathrm{H}$ & -1.56470 & -1.11898 & -0.00000
\end{tabular}


Table S1420 Employed active space, reference configurations, and Cartesian coordinates of the OM2/MRCISD-optimized ground-state equilibrium geometry of cis-2-naphthol (ground-state energy: $-1727.35886 \mathrm{eV}$ ).

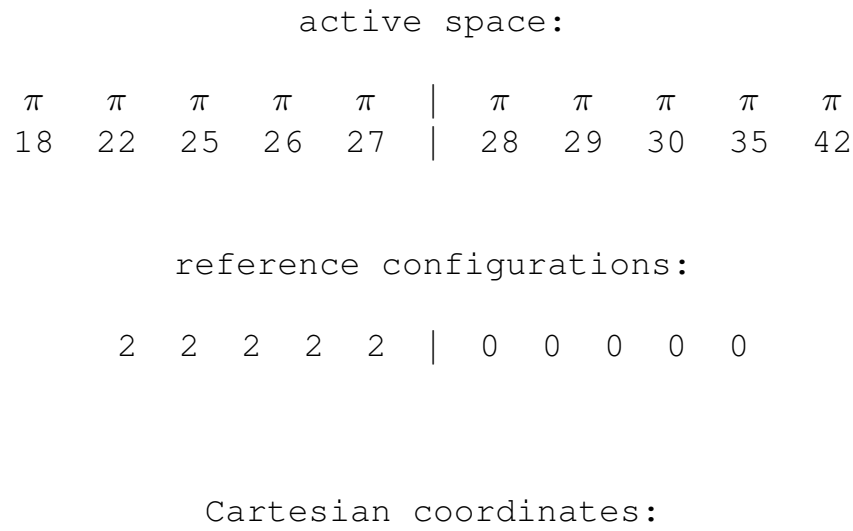

$\mathrm{H} \quad-2.42495 \quad 0.43821 \quad 0.00000$ 
Table S1421 Employed active space, reference configurations, and Cartesian coordinates of the OM2/MRCISD-optimized excited-state equilibrium geometry of the $2^{1} A^{\prime}\left({ }^{1} \pi \pi^{*}\right)$ state of cis-2-naphthol (excited-state energy: $-1723.71222 \mathrm{eV})$.

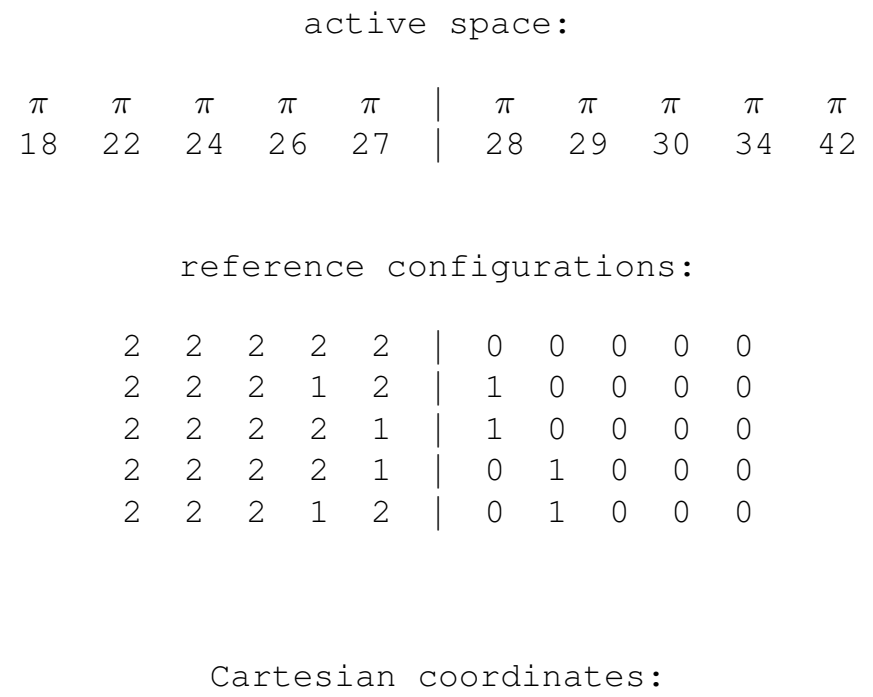

19

$\begin{array}{lrrr}\mathrm{C} & 0.00000 & 0.00000 & 0.00000 \\ \mathrm{C} & 1.40757 & 0.00000 & 0.00000 \\ \mathrm{C} & 2.12113 & 1.29322 & 0.00000 \\ \mathrm{C} & 1.39063 & 2.50386 & 0.00000 \\ \mathrm{C} & -0.00919 & 2.50395 & 0.00000 \\ \mathrm{C} & -0.68095 & 1.24221 & 0.00000 \\ \mathrm{C} & 2.15304 & -1.20000 & -0.00000 \\ \mathrm{C} & 3.55336 & -1.16472 & -0.00000 \\ \mathrm{C} & 4.23744 & 0.07187 & -0.00000 \\ \mathrm{C} & 3.53502 & 1.28280 & -0.00000 \\ \mathrm{O} & -2.01762 & 1.33103 & 0.00000 \\ \mathrm{H} & -0.56132 & -0.93772 & -0.00000 \\ \mathrm{H} & 4.08054 & 2.23302 & -0.00000 \\ \mathrm{H} & 5.33359 & 0.07882 & -0.00000 \\ \mathrm{H} & 4.12449 & -2.09787 & -0.00000 \\ \mathrm{H} & 1.62792 & -2.16157 & -0.00000 \\ \mathrm{H} & 1.93055 & 3.45729 & -0.00000 \\ \mathrm{H} & -0.59606 & 3.41830 & 0.00000 \\ \mathrm{H} & -2.42171 & 0.42040 & 0.00000\end{array}$


Table S1422 Employed active space, reference configurations, and Cartesian coordinates of the OM2/MRCISD-optimized ground-state equilibrium geometry of trans-2-naphthol (ground-state energy: $-1727.33445 \mathrm{eV}$ ).

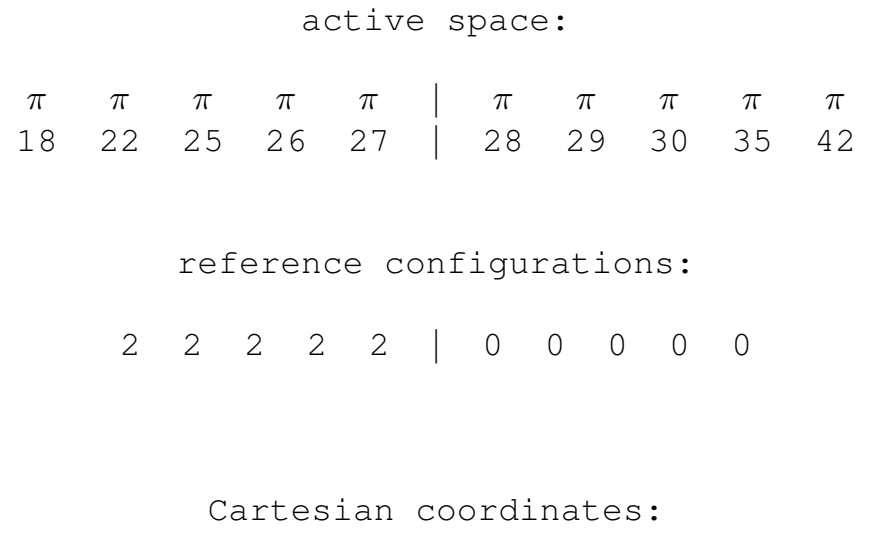

19

$\begin{array}{llll}C & 0.00000 & 0.00000 & 0.00000\end{array}$

C $\quad 1.41965 \quad 0.00000 \quad 0.00000$

$\begin{array}{llll}\mathrm{C} & 2.13125 & 1.23006 & 0.00000\end{array}$

C $\quad 1.41509 \quad 2.46203 \quad 0.00000$

C $\quad 0.03774 \quad 2.46842 \quad 0.00000$

$\begin{array}{lrrr}\text { C } & -0.65746 & 1.22496 & 0.00000\end{array}$

C $\quad 2.14929-1.22440-0.00000$

C $\quad 3.52735-1.20945-0.00000$

$\begin{array}{llll}\text { C } & 4.23525 & 0.01683 & -0.00000\end{array}$

$\begin{array}{llll}\text { C } & 3.55469 & 1.21562 & -0.00000\end{array}$

$\begin{array}{llll}0 & -2.01183 & 1.17206 & 0.00000\end{array}$

$\begin{array}{llll}\mathrm{H} & -0.56682 & -0.92877 & -0.00000\end{array}$

$\mathrm{H} \quad 4.09734 \quad 2.16895 \quad-0.00000$

$\mathrm{H} \quad \begin{array}{llll}5.33036 & -0.00068 & -0.00000\end{array}$

$\mathrm{H} \quad 4.09233 \quad-2.14848 \quad-0.00000$

$\mathrm{H} \quad \begin{array}{llll}\mathrm{H} & 1.59734 & -2.17102 & -0.00000\end{array}$

$\mathrm{H} \quad \begin{array}{llll}1.97628 & 3.40448 & -0.00000\end{array}$

$\mathrm{H} \quad-0.52933 \quad 3.40380 \quad 0.00000$

$\mathrm{H} \quad-2.38064 \quad 2.09649 \quad 0.00000$ 
Table S1423 Employed active space, reference configurations, and Cartesian coordinates of the OM2/MRCISD-optimized excited-state equilibrium geometry of the $2{ }^{1} A^{\prime}\left({ }^{1} \pi \pi^{*}\right)$ state of trans-2-naphthol (excited-state energy: $-1723.70993 \mathrm{eV})$.

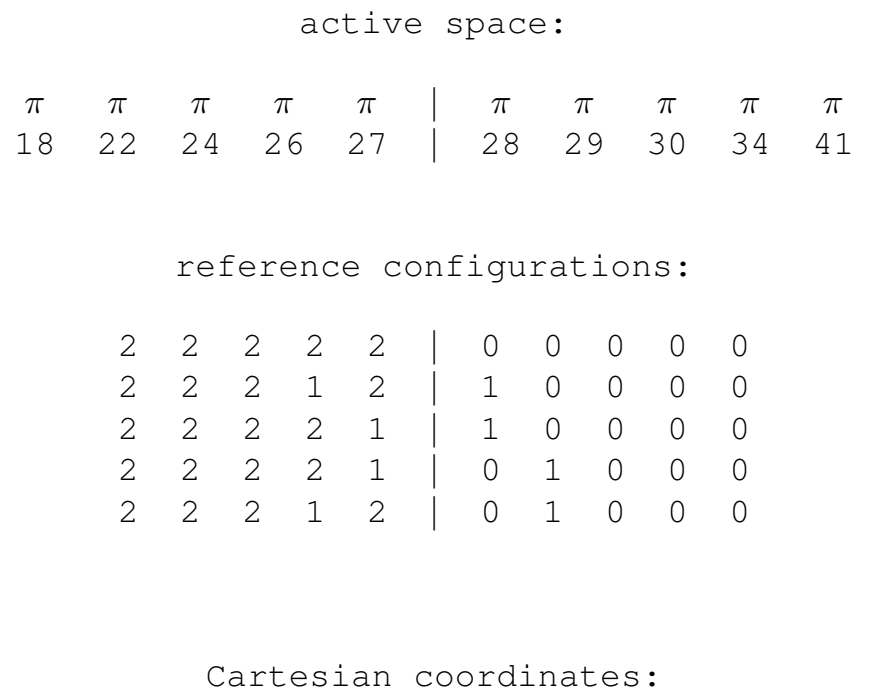

19

$\begin{array}{lrrr}\text { C } & 0.00000 & 0.00000 & 0.00000 \\ \mathrm{C} & 1.40771 & 0.00000 & 0.00000 \\ \mathrm{C} & 2.12620 & 1.28926 & 0.00000 \\ \mathrm{C} & 1.40206 & 2.50475 & 0.00000 \\ \mathrm{C} & 0.00202 & 2.50534 & 0.00000 \\ \mathrm{C} & -0.67571 & 1.24699 & 0.00000 \\ \mathrm{C} & 2.14908 & -1.20159 & -0.00000 \\ \mathrm{C} & 3.54958 & -1.17187 & -0.00000 \\ \mathrm{C} & 4.23757 & 0.06204 & -0.00000 \\ \mathrm{C} & 3.53958 & 1.27527 & -0.00000 \\ \mathrm{O} & -2.01227 & 1.16353 & 0.00000 \\ \mathrm{H} & -0.57794 & -0.92238 & -0.00000 \\ \mathrm{H} & 4.08877 & 2.22376 & -0.00000 \\ \mathrm{H} & 5.33395 & 0.06566 & -0.00000 \\ \mathrm{H} & 4.11736 & -2.10679 & -0.00000 \\ \mathrm{H} & 1.62022 & -2.16089 & -0.00000 \\ \mathrm{H} & 1.94598 & 3.45608 & -0.00000 \\ \mathrm{H} & -0.56788 & 3.43543 & 0.00000 \\ \mathrm{H} & -2.41137 & 2.07622 & 0.00000\end{array}$


Table S1424 Employed active space, reference configurations, and Cartesian coordinates of the OM2/MRCISD-optimized ground-state equilibrium geometry of cis-7-hydroxyquinoline (ground-state energy: $-1797.86253 \mathrm{eV}$ ).

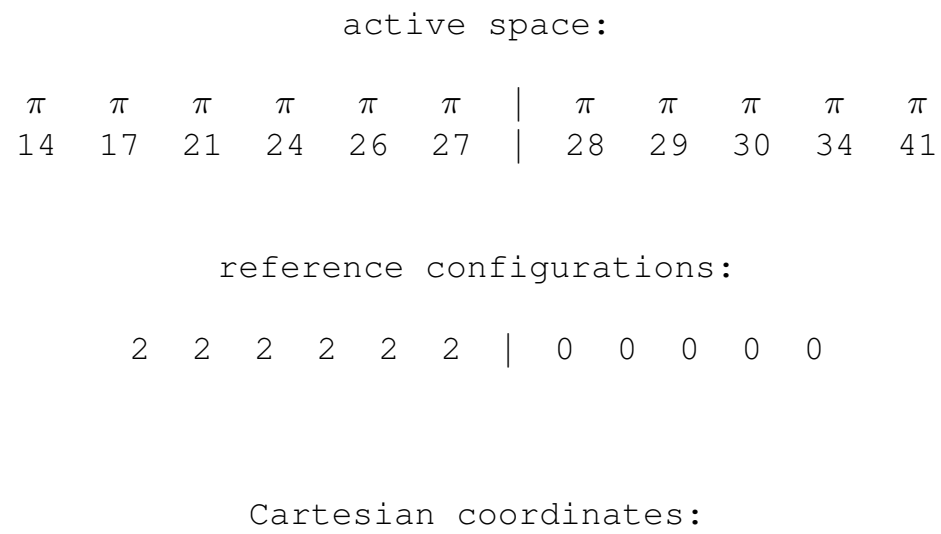


Table S1425 Employed active space, reference configurations, and Cartesian coordinates of the OM2/MRCISD-optimized excited-state equilibrium geometry of the $2{ }^{1} A^{\prime}\left({ }^{1} \pi \pi^{*}\right)$ state of cis-7-hydroxyquinoline (excited-state energy: $-1794.32659 \mathrm{eV})$.

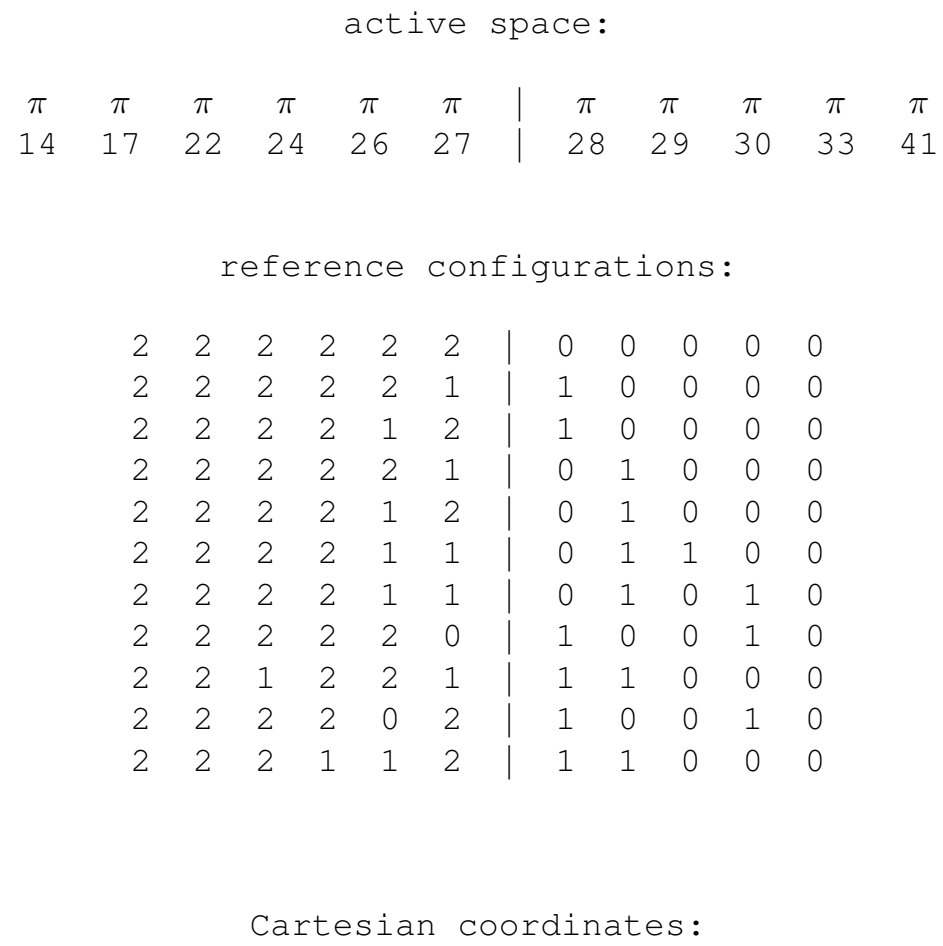

\begin{tabular}{lrrr}
18 & & \\
& & & \\
$\mathrm{C}$ & 0.00000 & 0.00000 & 0.00000 \\
$\mathrm{C}$ & 1.41044 & 0.00000 & 0.00000 \\
$\mathrm{C}$ & 2.16464 & 1.27470 & 0.00000 \\
$\mathrm{C}$ & 1.43938 & 2.48687 & 0.00000 \\
$\mathrm{C}$ & 0.02238 & 2.42310 & 0.00000 \\
$\mathrm{C}$ & -0.71926 & 1.20532 & 0.00000 \\
$\mathrm{C}$ & 2.16607 & -1.19694 & -0.00000 \\
$\mathrm{C}$ & 3.55875 & -1.11501 & -0.00000 \\
$\mathrm{C}$ & 4.19095 & 0.16377 & -0.00000 \\
$\mathrm{~N}$ & 3.51689 & 1.31779 & -0.00000 \\
$\mathrm{O}$ & -0.74096 & 3.53538 & 0.00000 \\
$\mathrm{H}$ & 5.29311 & 0.20553 & -0.00000 \\
$\mathrm{H}$ & -0.54091 & -0.95199 & -0.00000 \\
$\mathrm{H}$ & -1.80491 & 1.23914 & 0.00000 \\
\hline & \multicolumn{3}{c}{ Continued on next page }
\end{tabular}

$\mathrm{S} 1553$ 


\begin{tabular}{lrrr}
\multicolumn{4}{c}{ Continued from previous page } \\
\hline $\mathrm{H}$ & 1.96771 & 3.43975 & -0.00000 \\
$\mathrm{H}$ & 1.65917 & -2.16666 & -0.00000 \\
$\mathrm{H}$ & 4.17003 & -2.02101 & -0.00000 \\
$\mathrm{H}$ & -0.16281 & 4.34781 & 0.00000
\end{tabular}


Table S1426 Employed active space, reference configurations, and Cartesian coordinates of the OM2/MRCISD-optimized ground-state equilibrium geometry of trans-7-hydroxyquinoline (ground-state energy: -1797.82115 eV).

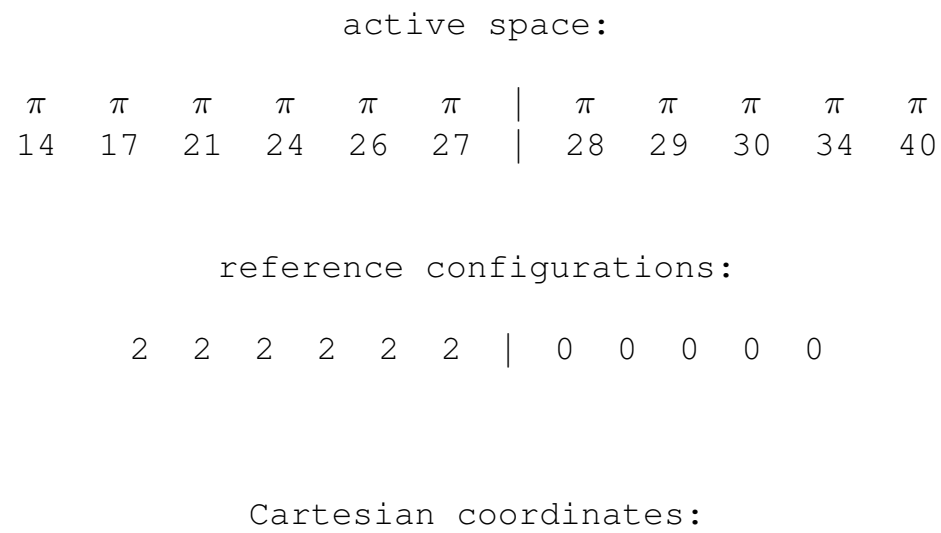


Table S1427 Employed active space, reference configurations, and Cartesian coordinates of the OM2/MRCISD-optimized excited-state equilibrium geometry of the $2{ }^{1} A\left({ }^{1} \pi \pi^{*}\right)$ state of trans-7-hydroxyquinoline (excited-state energy: $-1794.24477 \mathrm{eV})$.

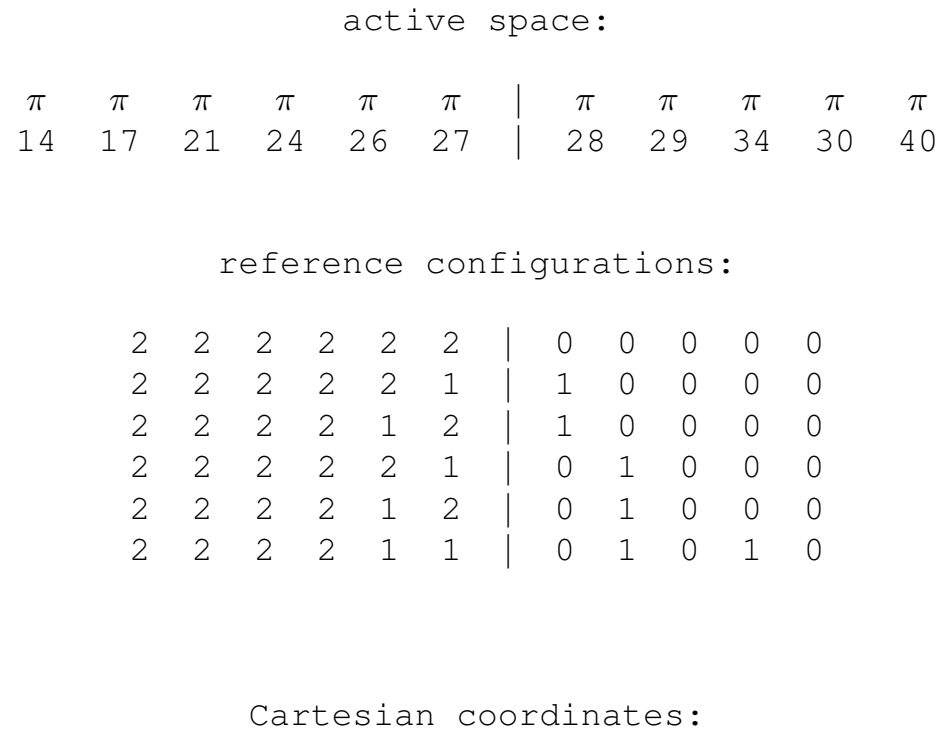

$\begin{array}{lrrr}\mathrm{C} & 0.00000 & 0.00000 & 0.00000 \\ \mathrm{C} & 1.41121 & 0.00000 & 0.00000 \\ \mathrm{C} & 2.16739 & 1.27210 & 0.00000 \\ \mathrm{C} & 1.44813 & 2.48853 & 0.00002 \\ \mathrm{C} & 0.02969 & 2.42423 & 0.00003 \\ \mathrm{C} & -0.71267 & 1.20798 & 0.00002 \\ \mathrm{C} & 2.16171 & -1.19942 & -0.00002 \\ \mathrm{C} & 3.55371 & -1.12206 & 0.00002 \\ \mathrm{C} & 4.18935 & 0.15381 & -0.00002 \\ \mathrm{~N} & 3.51759 & 1.30916 & -0.00004 \\ \mathrm{O} & -0.58075 & 3.62699 & 0.00006 \\ \mathrm{H} & 5.29133 & 0.19313 & -0.00002 \\ \mathrm{H} & -0.54211 & -0.95159 & -0.00002 \\ \mathrm{H} & -1.80284 & 1.22568 & 0.00002 \\ \mathrm{H} & 1.95975 & 3.44643 & 0.00003 \\ \mathrm{H} & 1.65148 & -2.16788 & -0.00002 \\ \mathrm{H} & 4.16187 & -2.03042 & 0.00005 \\ \mathrm{H} & -1.57075 & 3.50881 & 0.00007\end{array}$


Table S1428 Employed active space, reference configurations, and Cartesian coordinates of the OM2/MRCISD-optimized ground-state equilibrium geometry of 2-hydroxyquinoline (enol) (ground-state energy: $-1797.58484 \mathrm{eV}$ ).

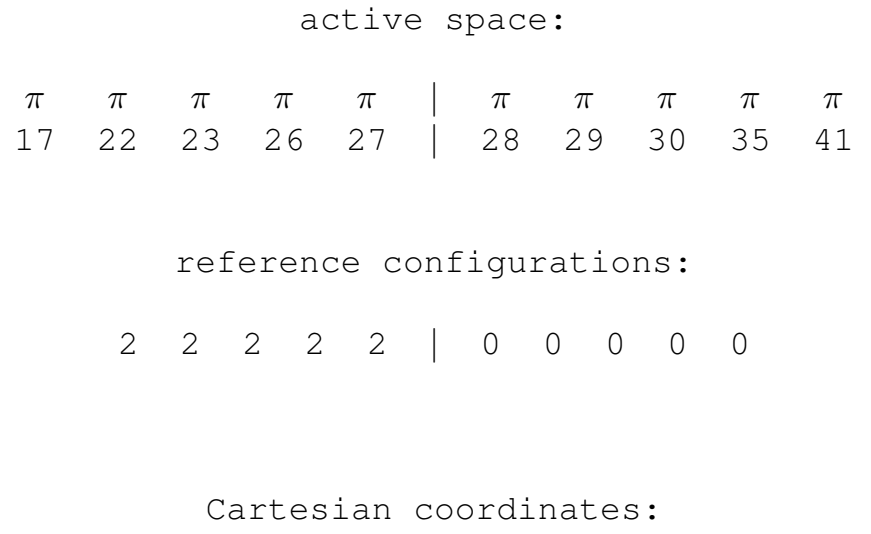

18

$\begin{array}{lrrr}\mathrm{N} & 0.00000 & 0.00000 & 0.00000 \\ \mathrm{C} & 1.36763 & 0.00000 & 0.00000 \\ \mathrm{C} & 2.13422 & 1.20191 & 0.00000 \\ \mathrm{C} & 1.44327 & 2.44947 & 0.00000 \\ \mathrm{C} & 0.06970 & 2.45512 & 0.00000 \\ \mathrm{C} & -0.59541 & 1.18081 & 0.00000 \\ \mathrm{C} & 2.04733 & -1.25725 & -0.00000 \\ \mathrm{C} & 3.42515 & -1.28978 & -0.00000 \\ \mathrm{C} & 4.18127 & -0.09352 & -0.00000 \\ \mathrm{C} & 3.55255 & 1.13458 & -0.00000 \\ \mathrm{O} & -1.94848 & 1.10957 & 0.00000 \\ \mathrm{H} & 4.13260 & 2.06499 & -0.00000 \\ \mathrm{H} & 5.27482 & -0.15466 & -0.00000 \\ \mathrm{H} & 3.95330 & -2.24979 & -0.00000 \\ \mathrm{H} & 1.44562 & -2.16810 & -0.00000 \\ \mathrm{H} & 2.01533 & 3.38469 & -0.00000 \\ \mathrm{H} & -0.50835 & 3.37983 & 0.00000 \\ \mathrm{H} & -2.32594 & 2.03186 & 0.00000\end{array}$


Table S1429 Employed active space, reference configurations, and Cartesian coordinates of the OM2/MRCISD-optimized excited-state equilibrium geometry of the $2{ }^{1} A^{\prime}\left({ }^{1} \pi \pi^{*}\right)$ state of 2-hydroxyquinoline (enol) (excited-state energy: $-1794.13068 \mathrm{eV})$.

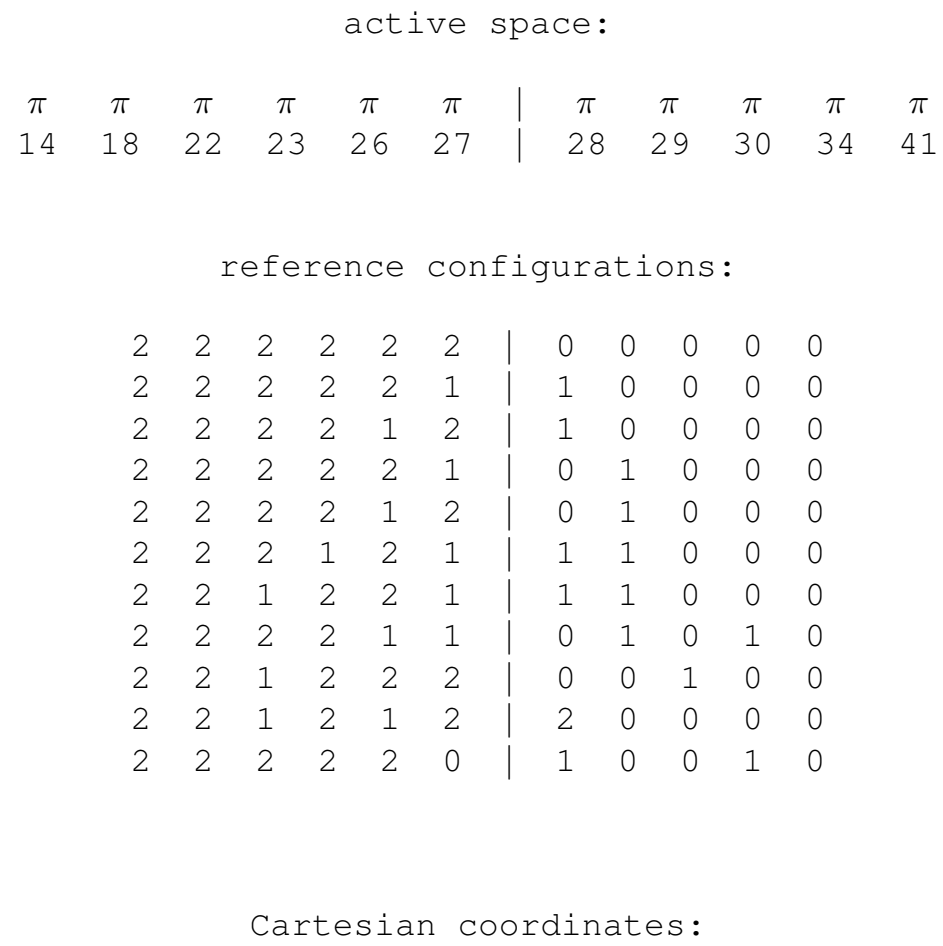

\begin{tabular}{lrrr}
18 & & \\
& & & \\
$\mathrm{~N}$ & 0.00000 & 0.00000 & 0.00000 \\
$\mathrm{C}$ & 1.34956 & 0.00000 & 0.00000 \\
$\mathrm{C}$ & 2.13644 & 1.25869 & 0.00000 \\
$\mathrm{C}$ & 1.44635 & 2.49003 & 0.00000 \\
$\mathrm{C}$ & 0.04571 & 2.48752 & 0.00000 \\
$\mathrm{C}$ & -0.60106 & 1.20785 & 0.00000 \\
$\mathrm{C}$ & 2.04174 & -1.24077 & -0.00000 \\
$\mathrm{C}$ & 3.44177 & -1.26676 & -0.00000 \\
$\mathrm{C}$ & 4.18623 & -0.06528 & -0.00000 \\
$\mathrm{C}$ & 3.54432 & 1.18300 & -0.00000 \\
$\mathrm{O}$ & -1.95093 & 1.10533 & 0.00000 \\
$\mathrm{H}$ & 4.13653 & 2.10465 & -0.00000 \\
$\mathrm{H}$ & 5.28123 & -0.11103 & -0.00000 \\
$\mathrm{H}$ & 3.96810 & -2.22572 & -0.00000 \\
\hline & \multicolumn{3}{c}{ Continued on next page }
\end{tabular}

$\mathrm{S} 1558$ 


\begin{tabular}{lrrr}
\multicolumn{4}{c}{ Continued from previous page } \\
\hline $\mathrm{H}$ & 1.45792 & -2.16326 & -0.00000 \\
$\mathrm{H}$ & 1.99881 & 3.43406 & -0.00000 \\
$\mathrm{H}$ & -0.53299 & 3.40920 & 0.00000 \\
$\mathrm{H}$ & -2.35136 & 2.01930 & 0.00000
\end{tabular}

S1559 
Table S1430 Employed active space, reference configurations, and Cartesian coordinates of the OM2/MRCISD-optimized ground-state equilibrium geometry of 2-hydroxyquinoline (keto) (ground-state energy: -1798.15028 eV).

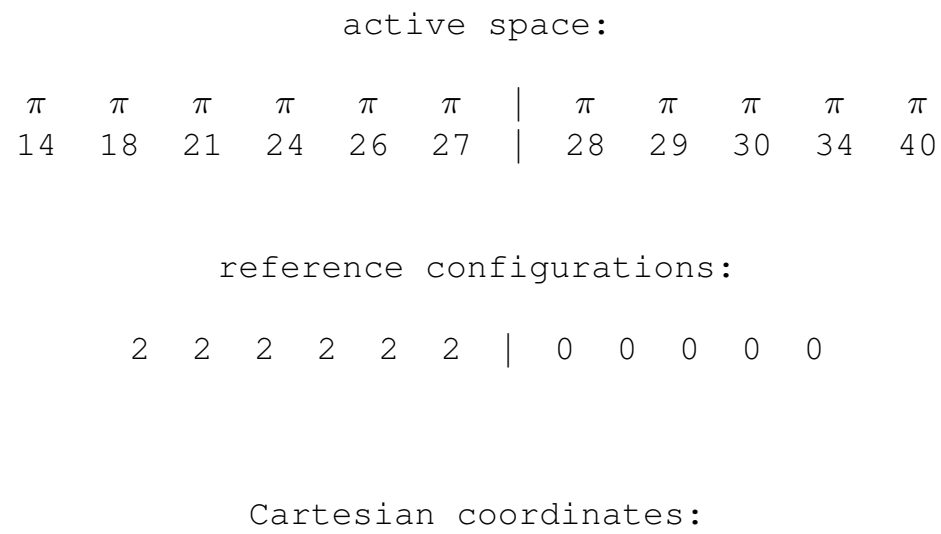


Table S1431 Employed active space, reference configurations, and Cartesian coordinates of the OM2/MRCISD-optimized excited-state equilibrium geometry of the $2{ }^{1} A^{\prime}\left({ }^{1} \pi \pi^{*}\right)$ state of 2-hydroxyquinoline (keto) (excited-state energy: $-1794.59704 \mathrm{eV})$.

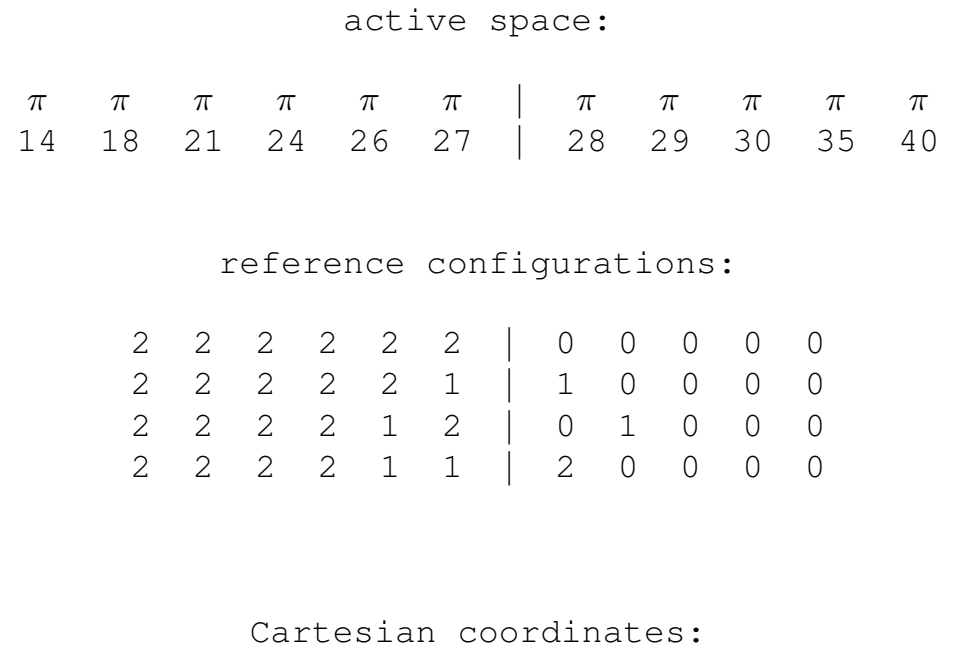


Table S1432 Employed active space, reference configurations, and Cartesian coordinates of the OM2/MRCISD-optimized ground-state equilibrium geometry of trans-o-dimethoxybenzene (ground-state energy: -1832.40174 eV).

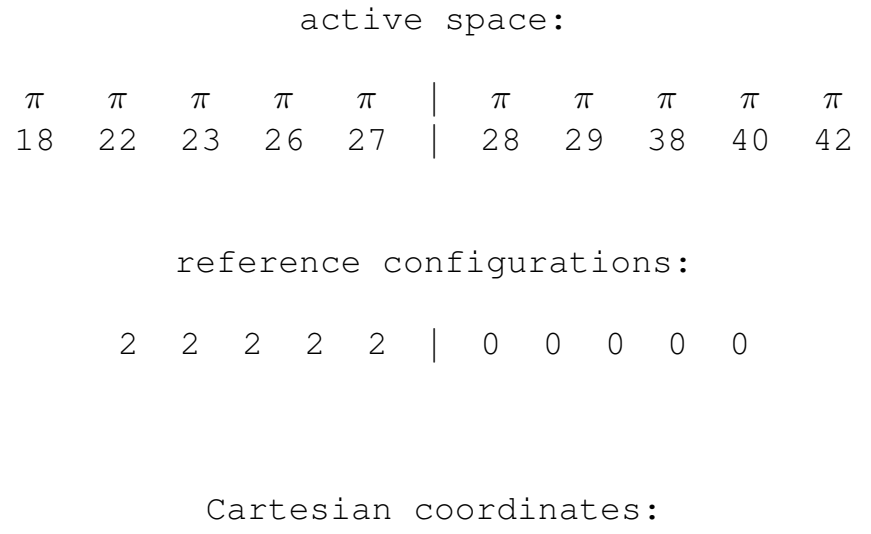

20

$\begin{array}{llll}C & 0.00000 & 0.00000 & 0.00000\end{array}$

$\begin{array}{llll}\text { C } & 1.42699 & 0.00000 & 0.00000\end{array}$

C $\quad-0.70013 \quad 1.21013 \quad 0.00000$

C $\quad 2.12713 \quad 1.21013 \quad 0.00000$

$\begin{array}{llll}\text { C } & 0.01681 & 2.41313 & 0.00000\end{array}$

C $\quad 1.41018 \quad 2.41313 \quad 0.00000$

$\begin{array}{llll}0 & -0.55771 & -1.24663 & -0.00000\end{array}$

$\begin{array}{llll}0 & 1.98471 & -1.24663 & 0.00000\end{array}$

$\begin{array}{llll}\text { C } & -1.95062 & -1.32137 & -0.00000\end{array}$

$\begin{array}{llll}\text { C } & 3.37761 & -1.32137 & 0.00000\end{array}$

$\mathrm{H} \quad-1.79071 \quad 1.22425-0.00000$

$\mathrm{H} \quad 3.21771 \quad 1.22425 \quad 0.00000$

$\mathrm{H} \quad-0.52942 \quad 3.36199 \quad-0.00000$

$\mathrm{H} \quad 1.95641 \quad 3.36199 \quad 0.00000$

$\mathrm{H} \quad-2.16637-2.41125 \quad-0.00000$

$\mathrm{H} \quad 3.59337 \quad-2.41125 \quad 0.00000$

$\mathrm{H} \quad-2.39658 \quad-0.84967 \quad 0.91370$

$\mathrm{H} \quad-2.39658 \quad-0.84967 \quad-0.91370$

$\mathrm{H} \quad 3.82358 \quad-0.84967 \quad-0.91370$

$\begin{array}{llll}\mathrm{H} & 3.82358 & -0.84967 & 0.91370\end{array}$ 
Table S1433 Employed active space, reference configurations, and Cartesian coordinates of the OM2/MRCISD-optimized excited-state equilibrium geometry of the $2{ }^{1} A^{\prime}\left({ }^{1} \pi \pi^{*}\right)$ state of trans-o-dimethoxybenzene (excited-state energy: $-1828.44910 \mathrm{eV})$.

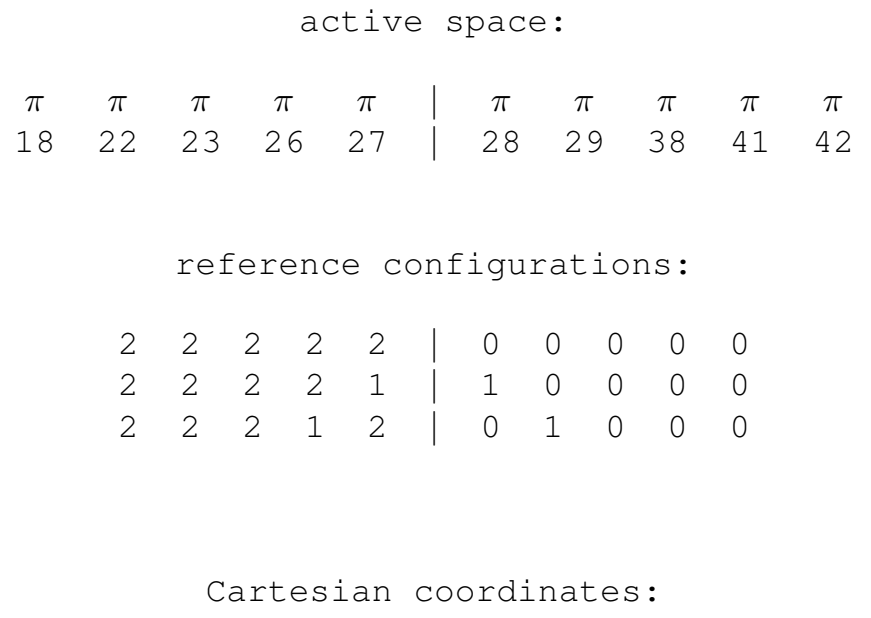

20

$\begin{array}{llll}\text { C } & 0.00000 & 0.00000 & 0.00000\end{array}$

$\begin{array}{llll}\text { C } & 1.40861 & 0.00000 & 0.00000\end{array}$

$\begin{array}{llll}\mathrm{C} & 2.07774 & 1.26839 & 0.00000\end{array}$

C $\quad 1.31999 \quad 2.49483 \quad 0.00000$

$\begin{array}{llll}\text { C } & -0.11375 & 2.46397 & 0.00000\end{array}$

$\begin{array}{lrrr}\text { C } & -0.74404 & 1.20424 & 0.00000\end{array}$

$\begin{array}{llll}0 & 2.08093 & 3.60992 & 0.00000\end{array}$

C $\quad 1.42594 \quad 4.84586 \quad 0.00000$

$\begin{array}{llll}\mathrm{O} & 3.41546 & 1.44996 & -0.00000\end{array}$

$\begin{array}{llll}\mathrm{C} & 4.22770 & 0.31118 & -0.00000\end{array}$

$\begin{array}{lrrr}\mathrm{H} & -0.69231 & 3.38375 & 0.00000\end{array}$

$\mathrm{H} \quad 1.97230 \quad-0.92898-0.00000$

$\mathrm{H} \quad-1.83890 \quad 1.15583 \quad 0.00000$

$\mathrm{H} \quad-0.53319-0.95748 \quad-0.00000$

$\begin{array}{llll}\mathrm{H} & 2.25034 & 5.59071 & 0.00000\end{array}$

$\mathrm{H} \quad 5.26270 \quad 0.71516 \quad-0.00000$

$\begin{array}{llll}\mathrm{H} & 0.79643 & 4.98064 & 0.91392\end{array}$

$\mathrm{H} \quad 4.06656 \quad-0.31211 \quad 0.91392$

$\mathrm{H} \quad 0.79643 \quad 4.98064 \quad-0.91392$

$\mathrm{H} \quad 4.06656 \quad-0.31211 \quad-0.91392$ 
Table S1434 Employed active space, reference configurations, and Cartesian coordinates of the OM2/MRCISD-optimized ground-state equilibrium geometry of 7-azaindole (ground-state energy: $-1409.32842 \mathrm{eV}$ ).

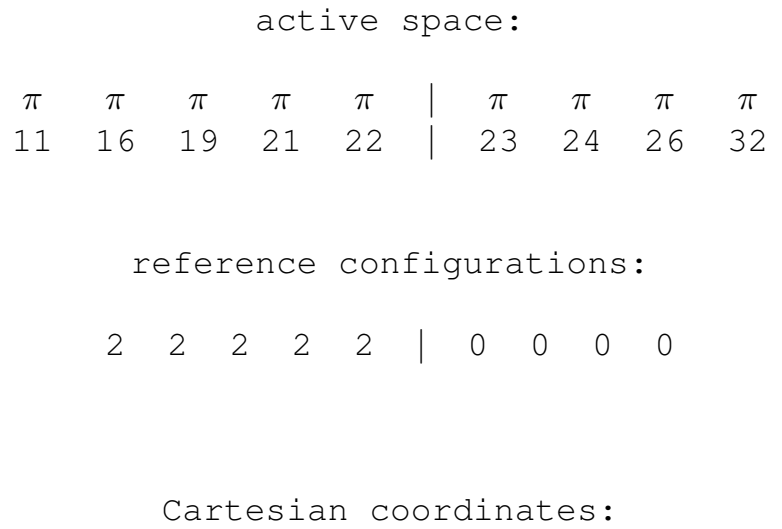


Table S1435 Employed active space, reference configurations, and Cartesian coordinates of the OM2/MRCISD-optimized excited-state equilibrium geometry of the $2{ }^{1} A^{\prime}\left({ }^{1} \pi \pi^{*}\right)$ state of 7 -azaindole (excited-state energy: $-1405.35672 \mathrm{eV}$ ).

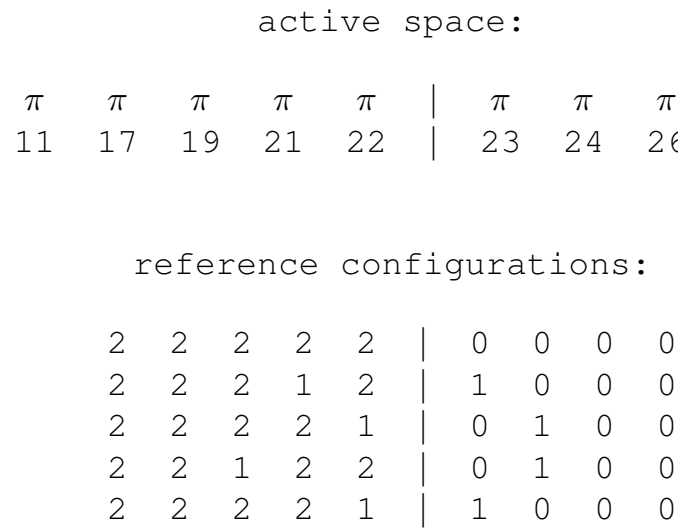

Cartesian coordinates:

15

$\begin{array}{lrrr}\mathrm{C} & 0.00000 & 0.00000 & 0.00000 \\ \mathrm{~N} & 1.39045 & 0.00000 & 0.00000 \\ \mathrm{C} & 1.85592 & 1.29442 & 0.00000 \\ \mathrm{C} & 0.66452 & 2.18463 & 0.00000 \\ \mathrm{C} & -0.47254 & 1.33042 & 0.00000 \\ \mathrm{~N} & 3.14853 & 1.67209 & -0.00000 \\ \mathrm{C} & 3.31642 & 3.01469 & -0.00000 \\ \mathrm{C} & 2.25156 & 3.97131 & -0.00000 \\ \mathrm{C} & 0.89173 & 3.56939 & -0.00000 \\ \mathrm{H} & 1.97995 & -0.82138 & -0.00000 \\ \mathrm{H} & 4.35101 & 3.39567 & -0.00000 \\ \mathrm{H} & 2.49758 & 5.03702 & -0.00000 \\ \mathrm{H} & 0.07605 & 4.29182 & -0.00000 \\ \mathrm{H} & -1.50869 & 1.63064 & -0.00000 \\ \mathrm{H} & -0.59304 & -0.90750 & -0.00000\end{array}$


Table S1436 Employed active space, reference configurations, and Cartesian coordinates of the OM2/MRCISD-optimized ground-state equilibrium geometry of phenylacetylene (ground-state energy: $-1109.15388 \mathrm{eV}$ ).

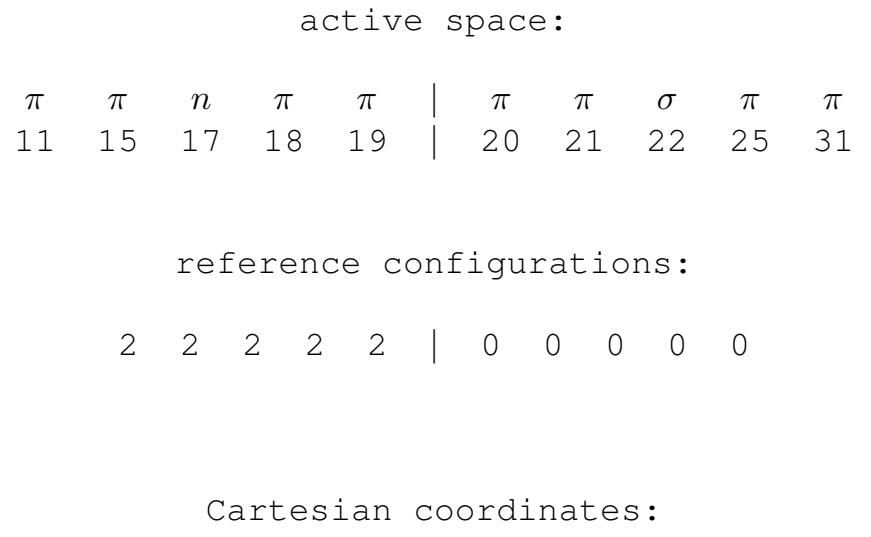

14

C $\quad 0.00000 \quad 0.00000 \quad-1.33826$

C $\quad 1.21638 \quad 0.00000-0.65205$

C $\quad-1.21638 \quad 0.00000-0.65205$

C $\quad 1.22994 \quad 0.00000 \quad 0.74486$

C $\quad-1.22994 \quad 0.00000 \quad 0.74486$

C $\quad 0.00000 \quad 0.00000 \quad 1.41562$

$\mathrm{H} \quad 0.00000 \quad 0.00000 \quad-2.43483$

$\mathrm{H} \quad 2.16177 \quad 0.00000 \quad-1.19993$

$\mathrm{H} \quad-2.16177 \quad 0.00000 \quad-1.19993$

$\mathrm{H} \quad 2.17196 \quad 0.00000 \quad 1.29408$

$\begin{array}{llll}\mathrm{H} & -2.17196 & 0.00000 & 1.29408\end{array}$

$\begin{array}{llll}\text { C } & 0.00000 & 0.00000 & 2.84624\end{array}$

$\begin{array}{llll}\text { C } & 0.00000 & 0.00000 & 4.08683\end{array}$

$\begin{array}{llll}\mathrm{H} & 0.00000 & 0.00000 & 5.12751\end{array}$ 
Table S1437 Employed active space, reference configurations, and Cartesian coordinates of the OM2/MRCISD-optimized excited-state equilibrium geometry of the $1{ }^{1} B_{2}\left({ }^{1} \pi \pi^{*}\right)$ state of phenylacetylene (excited-state energy: $-1104.94662 \mathrm{eV})$.

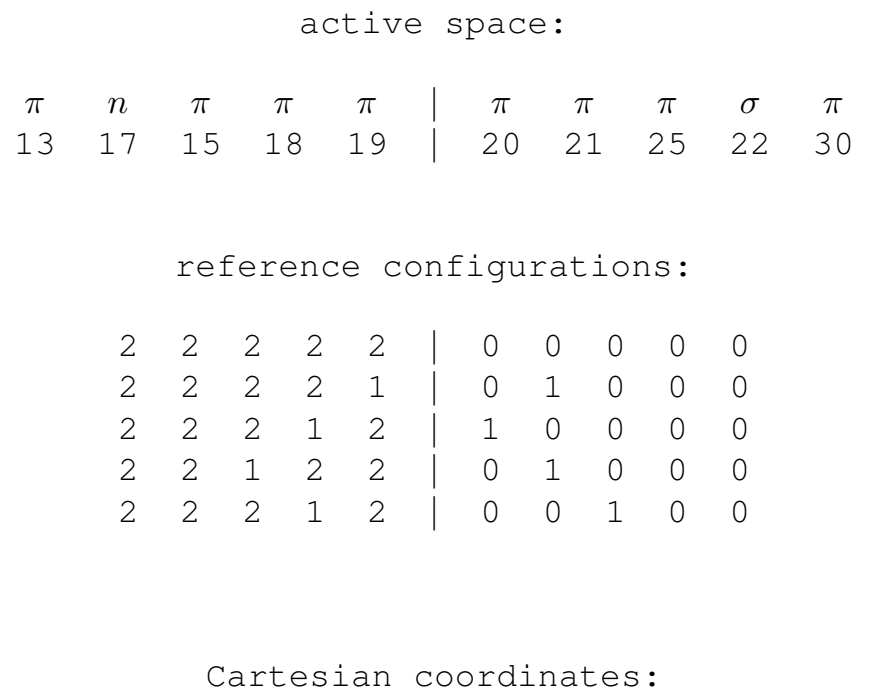

14

$\begin{array}{lrrr}\mathrm{C} & 0.00000 & 0.00000 & -1.35741 \\ \mathrm{C} & 1.23309 & 0.00000 & -0.66475 \\ \mathrm{C} & -1.23309 & 0.00000 & -0.66475 \\ \mathrm{C} & 1.25109 & 0.00000 & 0.75499 \\ \mathrm{C} & -1.25109 & 0.00000 & 0.75499 \\ \mathrm{C} & 0.00000 & 0.00000 & 1.44349 \\ \mathrm{H} & 0.00000 & 0.00000 & -2.45320 \\ \mathrm{H} & 2.17632 & 0.00000 & -1.21357 \\ \mathrm{H} & -2.17632 & 0.00000 & -1.21357 \\ \mathrm{H} & 2.19158 & 0.00000 & 1.30394 \\ \mathrm{H} & -2.19158 & 0.00000 & 1.30394 \\ \mathrm{C} & 0.00000 & 0.00000 & 2.85118 \\ \mathrm{C} & 0.00000 & 0.00000 & 4.09435 \\ \mathrm{H} & 0.00000 & 0.00000 & 5.13495\end{array}$


Table S1438 Employed active space, reference configurations, and Cartesian coordinates of the OM2/MRCISD-optimized ground-state equilibrium geometry of benzonitrile (ground-state energy: $-1181.13549 \mathrm{eV}$ ).

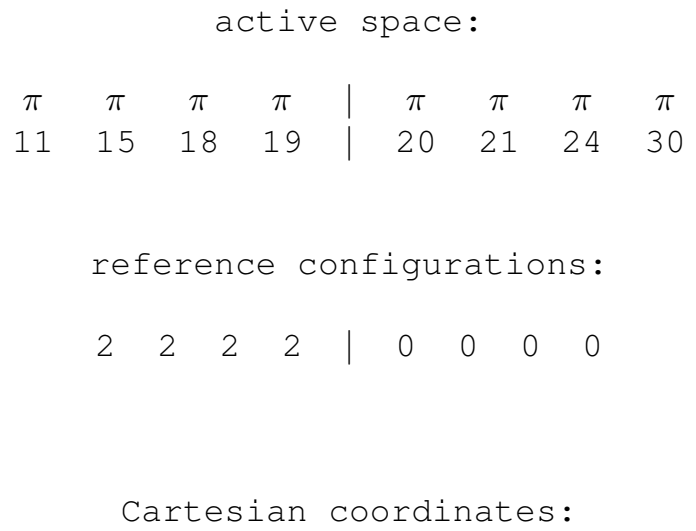


Table S1439 Employed active space, reference configurations, and Cartesian coordinates of the OM2/MRCISD-optimized excited-state equilibrium geometry of the $1{ }^{1} B_{1}\left({ }^{1} \pi \pi^{*}\right)$ state of benzonitrile (excited-state energy: $-1176.89542 \mathrm{eV})$.

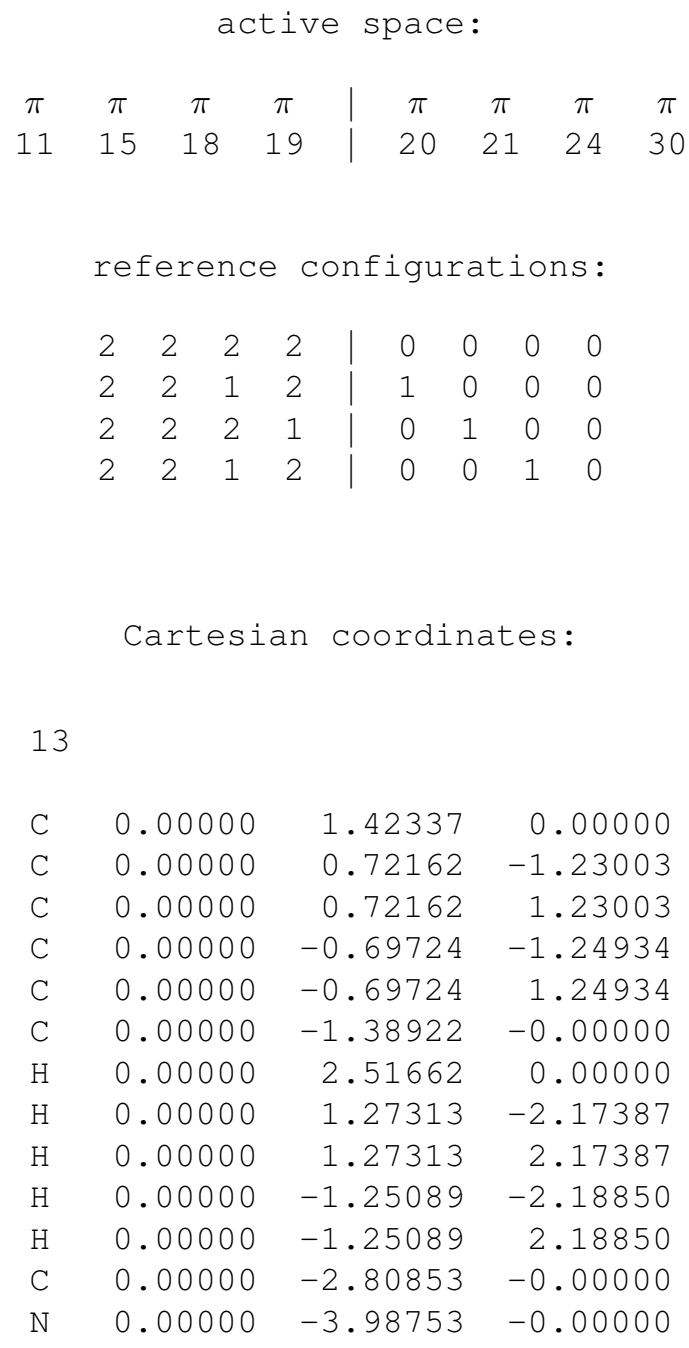


Table S1440 Employed active space, reference configurations, and Cartesian coordinates of the OM2/MRCISD-optimized ground-state equilibrium geometry of aniline (ground-state energy: $-1084.03224 \mathrm{eV}$ ).

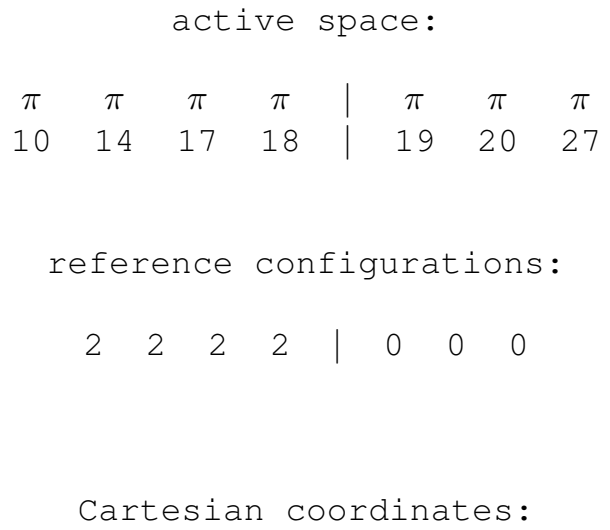


Table S1441 Employed active space, reference configurations, and Cartesian coordinates of the OM2/MRCISD-optimized excited-state equilibrium geometry of the $1{ }^{1} B_{2}\left({ }^{1} \pi \pi^{*}\right)$ state of aniline (excited-state energy: $-1080.06598 \mathrm{eV}$ ).

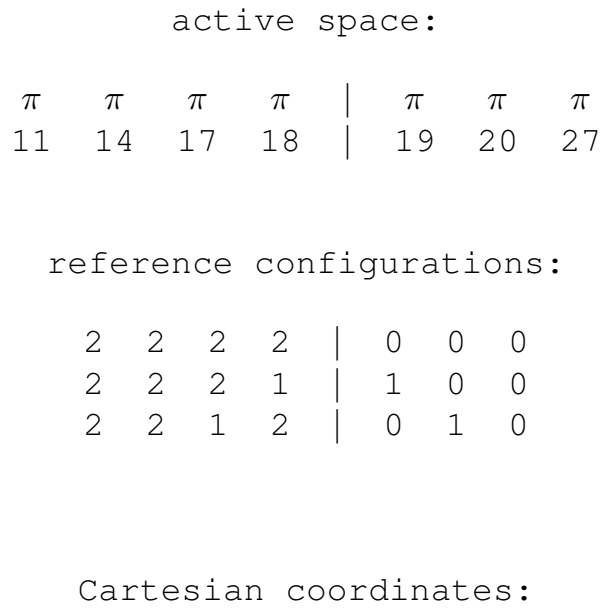


Table S1442 Employed active space, reference configurations, and Cartesian coordinates of the OM2/MRCISD-optimized ground-state equilibrium geometry of tetrafluorobenzene (ground-state energy: $-2659.86078 \mathrm{eV}$ ).

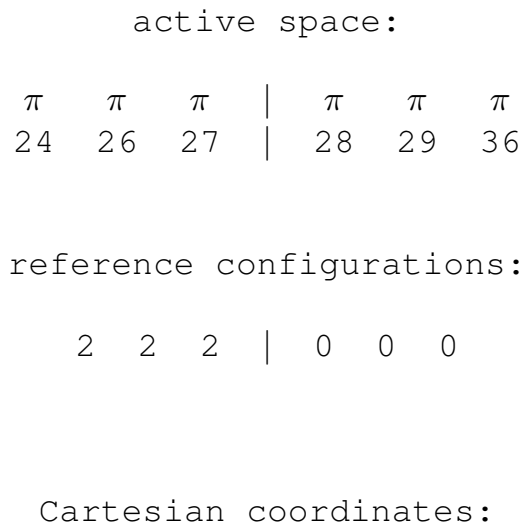


Table S1443 Employed active space, reference configurations, and Cartesian coordinates of the OM2/MRCISD-optimized excited-state equilibrium geometry of the $1{ }^{1} B_{1}\left({ }^{1} \pi \pi^{*}\right)$ state of tetrafluorobenzene (excited-state energy: $-2655.78918 \mathrm{eV})$.

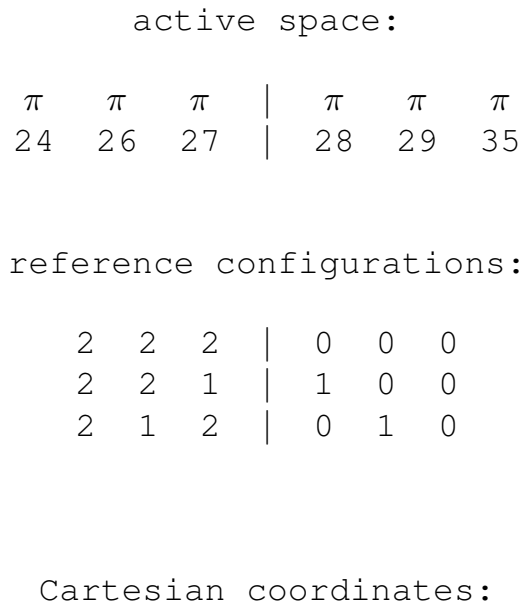


Table S1444 Employed active space, reference configurations, and Cartesian coordinates of the OM2/MRCISD-optimized ground-state equilibrium geometry of 2,6-difluoropyridine (ground-state energy: $-1828.34245 \mathrm{eV}$ ).

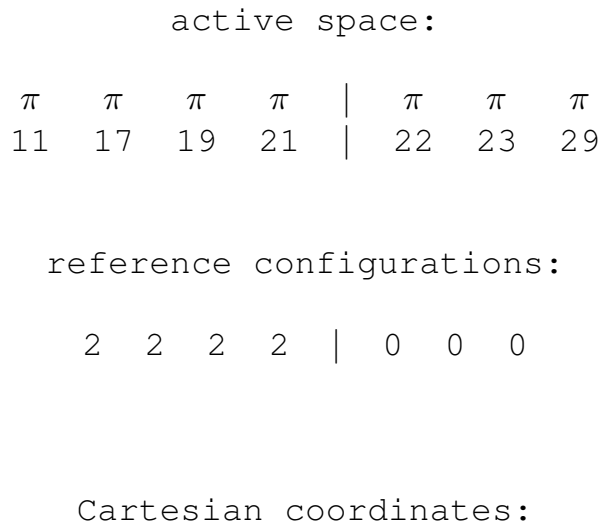

$\mathrm{H} \quad 0.00000-1.23985-2.17243$

$\mathrm{H} \quad 0.00000 \quad-1.23985 \quad 2.17243$ 
Table S1445 Employed active space, reference configurations, and Cartesian coordinates of the OM2/MRCISD-optimized excited-state equilibrium geometry of the $1^{1} B_{1}\left({ }^{1} \pi \pi^{*}\right)$ state of 2,6-difluoropyridine (excited-state energy: $-1823.94627 \mathrm{eV})$.

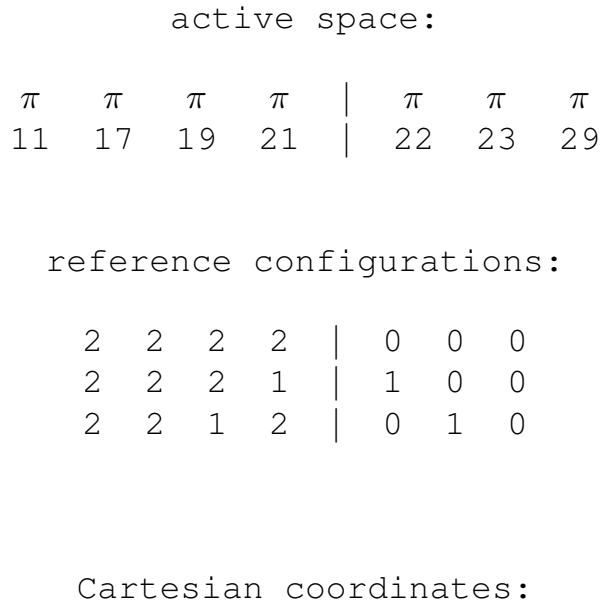

$\begin{array}{lrrr}\mathrm{N} & 0.00000 & 1.42358 & 0.00000 \\ \mathrm{C} & 0.00000 & -1.49930 & -0.00000 \\ \mathrm{C} & 0.00000 & 0.66186 & -1.12870 \\ \mathrm{C} & 0.00000 & 0.66186 & 1.12870 \\ \mathrm{C} & 0.00000 & -0.76753 & -1.21891 \\ \mathrm{C} & 0.00000 & -0.76753 & 1.21891 \\ \mathrm{H} & 0.00000 & -2.58620 & -0.00000 \\ \mathrm{~F} & 0.00000 & 1.35647 & -2.27418 \\ \mathrm{~F} & 0.00000 & 1.35647 & 2.27418 \\ \mathrm{H} & 0.00000 & -1.25433 & -2.18947 \\ \mathrm{H} & 0.00000 & -1.25433 & 2.18947\end{array}$


Table S1446 Employed active space, reference configurations, and Cartesian coordinates of the OM2/MRCISD-optimized ground-state equilibrium geometry of 2-fluoropyridine (ground-state energy: $-1377.35660 \mathrm{eV}$ ).

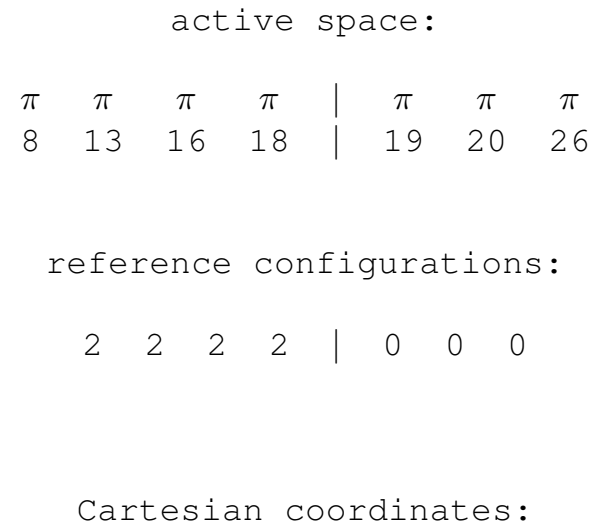


Table S1447 Employed active space, reference configurations, and Cartesian coordinates of the OM2/MRCISD-optimized excited-state equilibrium geometry of the $2{ }^{1} A^{\prime}\left({ }^{1} \pi \pi^{*}\right)$ state of 2 -fluoropyridine (excited-state energy: $-1372.92493 \mathrm{eV})$.

active space:
\begin{tabular}{cccc|ccc}
$\pi$ & $\pi$ & $\pi$ & $\pi$ & $\pi$ & $\pi$ & $\pi$ \\
8 & 13 & 16 & 18 & 19 & 20 & 26 \\
\\
reference configurations: \\
2 & 2 & 2 & 2 & 0 & 0 & 0 \\
2 & 2 & 2 & 1 & 1 & 0 & 0 \\
2 & 2 & 1 & 2 & 0 & 1 & 0 \\
2 & 2 & 2 & 1 & 0 & 1 & 0 \\
2 & 2 & 1 & 2 & 1 & 0 & 0
\end{tabular}

Cartesian coordinates:

11

$\begin{array}{lrrr}\mathrm{C} & 0.00000 & 0.00000 & 0.00000 \\ \mathrm{C} & 1.42800 & 0.00000 & 0.00000 \\ \mathrm{~N} & 2.26792 & 1.06955 & 0.00000 \\ \mathrm{C} & 1.64828 & 2.27679 & 0.00000 \\ \mathrm{C} & 0.22483 & 2.42566 & 0.00000 \\ \mathrm{C} & -0.61699 & 1.27994 & 0.00000 \\ \mathrm{~F} & 2.05078 & -1.19235 & -0.00000 \\ \mathrm{H} & 2.27891 & 3.17863 & 0.00000 \\ \mathrm{H} & -0.21424 & 3.42574 & 0.00000 \\ \mathrm{H} & -1.70121 & 1.37731 & 0.00000 \\ \mathrm{H} & -0.56218 & -0.92763 & -0.00000\end{array}$


Table S1448 Employed active space, reference configurations, and Cartesian coordinates of the OM2/MRCISD-optimized ground-state equilibrium geometry of 2-hydroxypyridine (ground-state energy: $-1257.44772 \mathrm{eV}$ ).

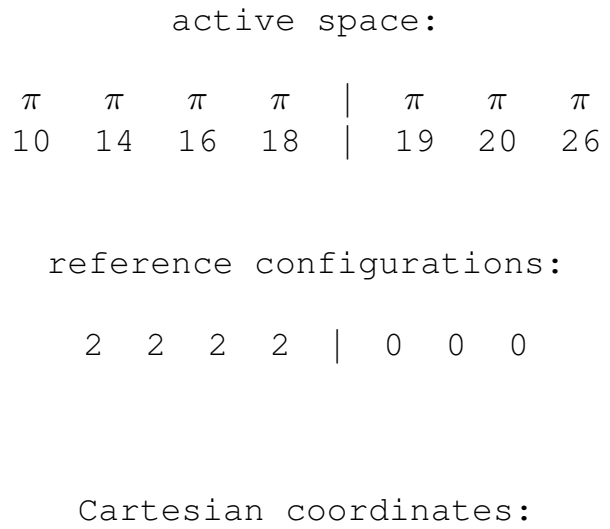

$\begin{array}{llll}\mathrm{H} & 3.67572 & 2.23151 & 0.00000\end{array}$ 
Table S1449 Employed active space, reference configurations, and Cartesian coordinates of the OM2/MRCISD-optimized excited-state equilibrium geometry of the $2{ }^{1} A^{\prime}\left({ }^{1} \pi \pi^{*}\right)$ state of 2-hydroxypyridine (excited-state energy: $-1253.19179 \mathrm{eV})$.

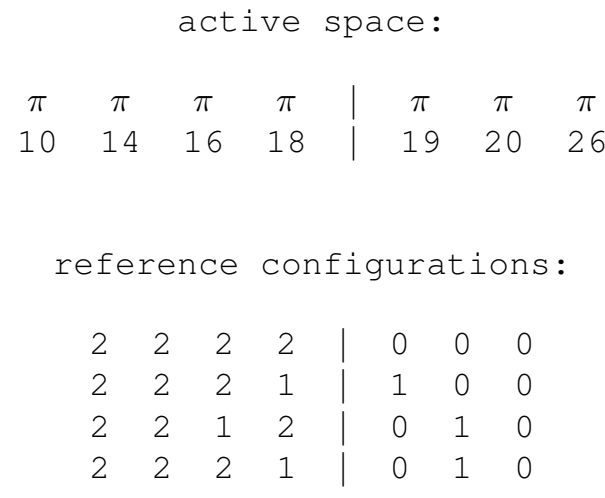

Cartesian coordinates:

12

$\begin{array}{lrrr}\mathrm{C} & 0.00000 & 0.00000 & 0.00000 \\ \mathrm{C} & 1.42153 & 0.00000 & 0.00000 \\ \mathrm{C} & 2.04352 & 1.28673 & 0.00000 \\ \mathrm{~N} & 1.42807 & 2.51365 & 0.00000 \\ \mathrm{C} & 0.07598 & 2.47057 & 0.00000 \\ \mathrm{C} & -0.66644 & 1.24982 & 0.00000 \\ \mathrm{O} & 3.38668 & 1.28748 & 0.00000 \\ \mathrm{H} & -0.47111 & 3.42616 & 0.00000 \\ \mathrm{H} & -1.75963 & 1.28594 & 0.00000 \\ \mathrm{H} & -0.55453 & -0.93617 & -0.00000 \\ \mathrm{H} & 2.01700 & -0.90647 & -0.00000 \\ \mathrm{H} & 3.71447 & 2.23047 & 0.00000\end{array}$


Table S1450 Employed active space, reference configurations, and Cartesian coordinates of the OM2/MRCISD-optimized ground-state equilibrium geometry of 2-methylpyrimidine (ground-state energy: $-1154.51426 \mathrm{eV}$ ).

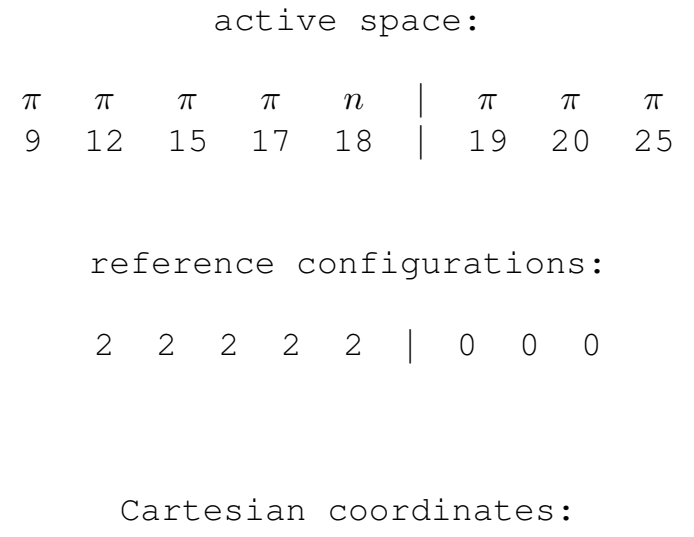

13

$\begin{array}{lrrr}\mathrm{C} & 0.00000 & 0.00000 & 0.00000 \\ \mathrm{C} & 1.40314 & 0.00000 & 0.00000 \\ \mathrm{C} & 2.03369 & 1.25403 & 0.00000 \\ \mathrm{~N} & 1.33919 & 2.39269 & 0.00000 \\ \mathrm{C} & -0.00960 & 2.29099 & 0.00000 \\ \mathrm{~N} & -0.70554 & 1.13252 & 0.00000 \\ \mathrm{C} & -0.79475 & 3.58208 & 0.00000 \\ \mathrm{H} & 3.13633 & 1.31178 & 0.00000 \\ \mathrm{H} & -0.54730 & -0.95890 & -0.00000 \\ \mathrm{H} & 1.97373 & -0.92523 & -0.00000 \\ \mathrm{H} & -1.86784 & 3.36023 & 0.00000 \\ \mathrm{H} & -0.52657 & 4.14423 & 0.89675 \\ \mathrm{H} & -0.52657 & 4.14423 & -0.89675\end{array}$


Table S1451 Employed active space, reference configurations, and Cartesian coordinates of the OM2/MRCISD-optimized excited-state equilibrium geometry of the $1^{1} A^{\prime \prime}\left({ }^{1} n \pi^{*}\right)$ state of 2-methylpyrimidine (excited-state energy: $-1150.37011 \mathrm{eV})$.

\begin{tabular}{lcccc|ccc} 
active space : \\
$\pi$ & $\pi$ & $\pi$ & $\pi$ & $n$ & $\pi$ & $\pi$ & $\pi$ \\
9 & 12 & 15 & 17 & 18 & 19 & 20 & 25
\end{tabular}

reference configurations:

\begin{tabular}{lllll|lll}
2 & 2 & 2 & 2 & 2 & 0 & 0 & 0 \\
2 & 2 & 2 & 2 & 1 & 1 & 0 & 0
\end{tabular}

Cartesian coordinates:

13

$\begin{array}{lrrr}\mathrm{C} & 0.00000 & 0.00000 & 0.00000 \\ \mathrm{C} & 1.45609 & 0.00000 & 0.00000 \\ \mathrm{C} & 2.05398 & 1.25995 & 0.00000 \\ \mathrm{~N} & 1.37388 & 2.39375 & 0.00000 \\ \mathrm{C} & -0.03477 & 2.37256 & 0.00000 \\ \mathrm{~N} & -0.60109 & 1.16915 & 0.00000 \\ \mathrm{C} & -0.78727 & 3.66714 & 0.00000 \\ \mathrm{H} & 3.15328 & 1.30584 & 0.00000 \\ \mathrm{H} & -0.53574 & -0.94300 & -0.00000 \\ \mathrm{H} & 2.00385 & -0.93484 & -0.00000 \\ \mathrm{H} & -1.86598 & 3.45702 & 0.00000 \\ \mathrm{H} & -0.51625 & 4.21430 & 0.89875 \\ \mathrm{H} & -0.51625 & 4.21430 & -0.89875\end{array}$


Table S1452 Employed active space, reference configurations, and Cartesian coordinates of the OM2/MRCISD-optimized ground-state equilibrium geometry of 5-methylpyrimidine (ground-state energy: $-1154.62809 \mathrm{eV}$ ).

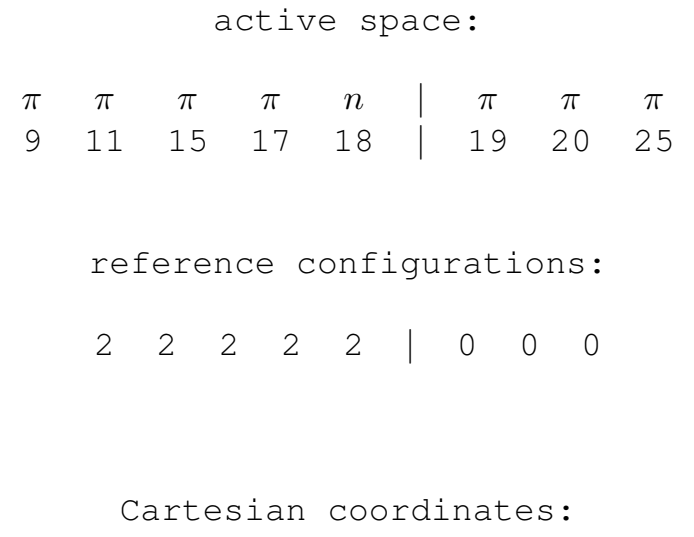

13

$\begin{array}{llll}C & 0.00000 & 0.00000 & 0.00000\end{array}$

$\begin{array}{llll}\mathrm{N} & 1.34581 & 0.00000 & 0.00000\end{array}$

C $\quad 1.96505 \quad 1.18136 \quad 0.00000$

$\begin{array}{llll}\text { C } & 1.24557 & 2.39322 & 0.00000\end{array}$

C $\quad-0.16115 \quad 2.28752 \quad 0.00000$

$\begin{array}{llll}\mathrm{N} & -0.77270 & 1.10296 & 0.00000\end{array}$

$\begin{array}{lrrr}\text { C } & 1.92781 & 3.71864 & 0.00000\end{array}$

$\mathrm{H} \quad-0.51329-0.98540-0.00000$

$\begin{array}{llll}\mathrm{H} & -0.78031 & 3.20131 & 0.00000\end{array}$

$\begin{array}{llll}\mathrm{H} & 3.06949 & 1.19987 & 0.00000\end{array}$

$\begin{array}{llll}\mathrm{H} & 3.02125 & 3.58417 & 0.00000\end{array}$

$\mathrm{H} \quad 1.64168 \quad 4.27160 \quad-0.89731$

$\begin{array}{llll}\mathrm{H} & 1.64168 & 4.27160 & 0.89731\end{array}$ 
Table S1453 Employed active space, reference configurations, and Cartesian coordinates of the OM2/MRCISD-optimized excited-state equilibrium geometry of the $1^{1} A^{\prime \prime}\left({ }^{1} n \pi^{*}\right)$ state of 5-methylpyrimidine (excited-state energy: $-1150.53591 \mathrm{eV})$.

\begin{tabular}{lcccc|ccc} 
active space : \\
$\pi$ & $\pi$ & $\pi$ & $\pi$ & $n$ & $\pi$ & $\pi$ & $\pi$ \\
9 & 12 & 15 & 17 & 18 & 19 & 20 & 25
\end{tabular}

reference configurations:
\begin{tabular}{lllll|lll}
2 & 2 & 2 & 2 & 2 & 0 & 0 & 0 \\
2 & 2 & 2 & 2 & 1 & 1 & 0 & 0
\end{tabular}

Cartesian coordinates:

13

$\begin{array}{lrrr}\mathrm{C} & 0.00000 & 0.00000 & 0.00000 \\ \mathrm{~N} & 1.39392 & 0.00000 & 0.00000 \\ \mathrm{C} & 2.10183 & 1.09784 & 0.00000 \\ \mathrm{C} & 1.50734 & 2.38685 & 0.00000 \\ \mathrm{C} & 0.04564 & 2.37205 & 0.00000 \\ \mathrm{~N} & -0.56097 & 1.22289 & 0.00000 \\ \mathrm{C} & 2.25704 & 3.66421 & 0.00000 \\ \mathrm{H} & -0.56257 & -0.93067 & -0.00000 \\ \mathrm{H} & -0.48185 & 3.32605 & 0.00000 \\ \mathrm{H} & 3.20116 & 1.04793 & 0.00000 \\ \mathrm{H} & 3.34093 & 3.45732 & 0.00000 \\ \mathrm{H} & 2.01511 & 4.23170 & -0.89921 \\ \mathrm{H} & 2.01511 & 4.23170 & 0.89921\end{array}$


Table S1454 Employed active space, reference configurations, and Cartesian coordinates of the OM2/MRCISD-optimized ground-state equilibrium geometry of pyrazine (ground-state energy: $-996.70231 \mathrm{eV}$ ).

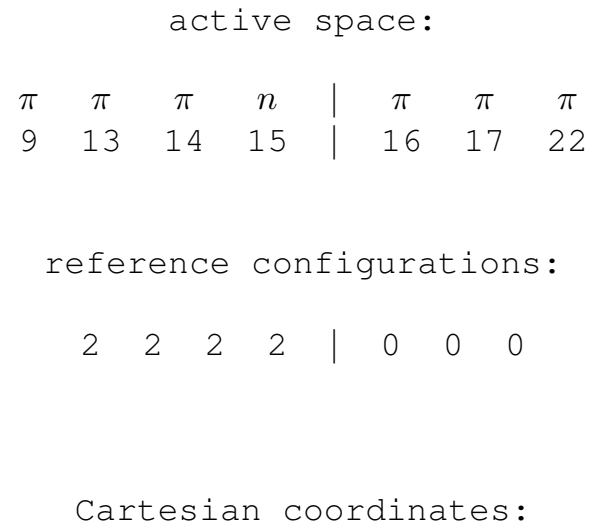


Table S1455 Employed active space, reference configurations, and Cartesian coordinates of the OM2/MRCISD-optimized excited-state equilibrium geometry of the $1{ }^{1} B_{u}\left({ }^{1} n \pi^{*}\right)$ state of pyrazine (excited-state energy: $-992.72997 \mathrm{eV}$ ).

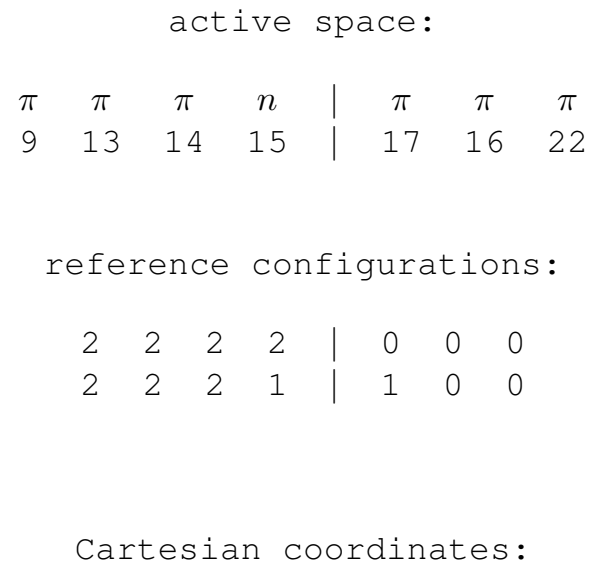


Table S1456 Employed active space, reference configurations, and Cartesian coordinates of the OM2/MRCISD-optimized ground-state equilibrium geometry of 2-pyridone (ground-state energy: $-1257.38046 \mathrm{eV}$ ).

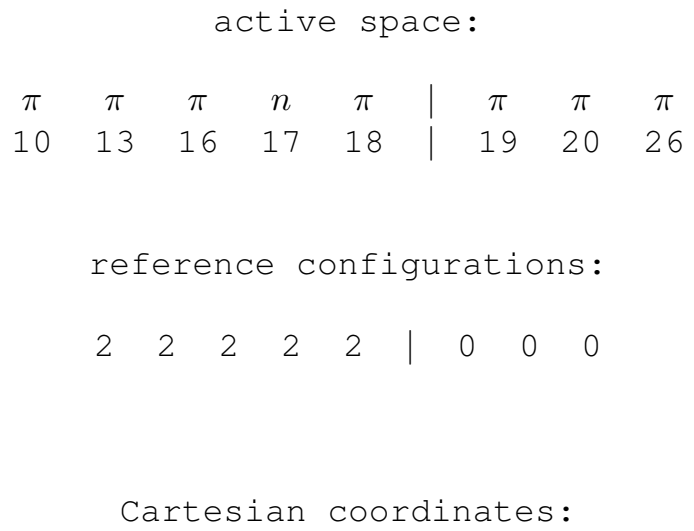


Table S1457 Employed active space, reference configurations, and Cartesian coordinates of the OM2/MRCISD-optimized excited-state equilibrium geometry of the $2{ }^{1} A^{\prime}\left({ }^{1} \pi \pi^{*}\right)$ state of 2-pyridone (excited-state energy: $\left.-1253.80109 \mathrm{eV}\right)$.

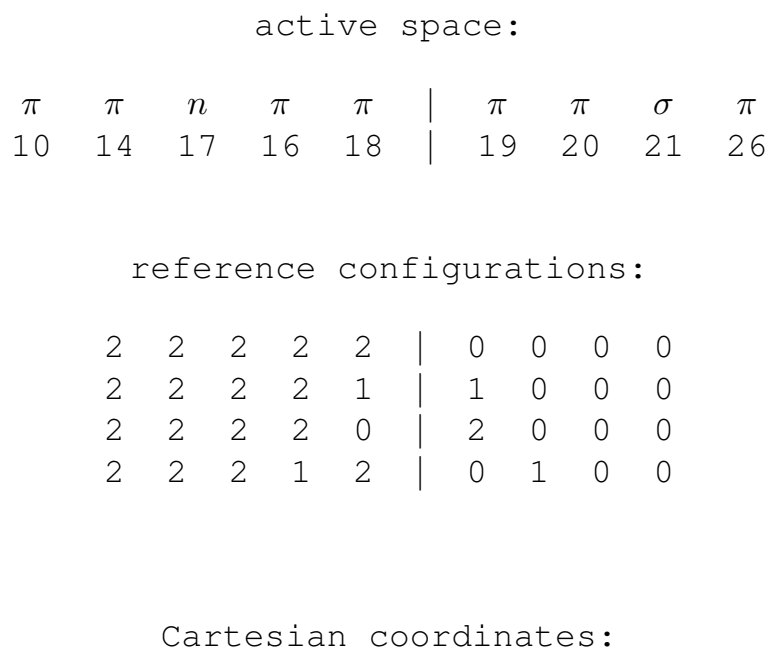


Table S1458 Employed active space, reference configurations, and Cartesian coordinates of the OM2/MRCISD-optimized ground-state equilibrium geometry of catechol (ground-state energy: $-1517.94387 \mathrm{eV}$ ).

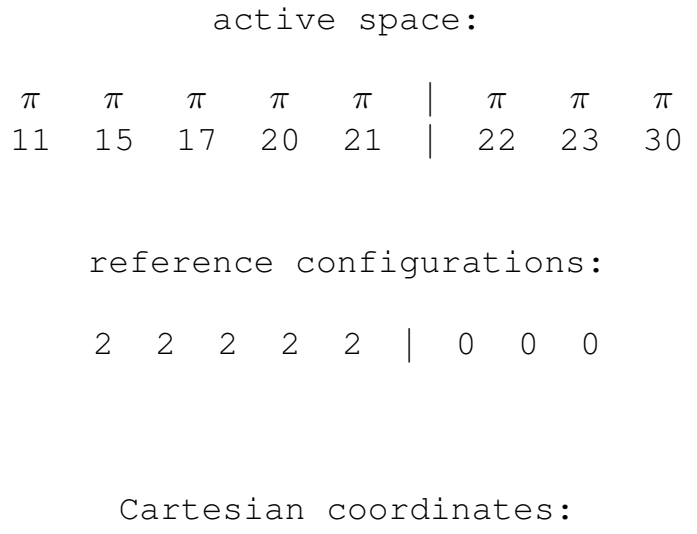

14

$\begin{array}{llll}\text { C } & 0.00000 & 0.00000 & 0.00000\end{array}$

$\begin{array}{llll}\text { C } & 1.39873 & 0.00000 & 0.00000\end{array}$

C $\quad 2.06293 \quad 1.23001 \quad 0.00000$

C $\quad 1.34165 \quad 2.45113 \quad 0.00000$

C $\quad-0.05964 \quad 2.43470 \quad 0.00000$

C $\quad-0.71533 \quad 1.20045 \quad 0.00000$

$0 \quad 3.42023 \quad 1.37914 \quad 0.00000$

$\begin{array}{llll}0 & 1.96073 & 3.65718 & 0.00000\end{array}$

$\begin{array}{llll}\mathrm{H} & -0.60491 & 3.37689 & 0.00000\end{array}$

$\mathrm{H} \quad-1.80978 \quad 1.17662 \quad 0.00000$

$\mathrm{H} \quad-0.53591 \quad-0.95398 \quad-0.00000$

$\begin{array}{llll}\mathrm{H} & 1.96309 & -0.93549 & -0.00000\end{array}$

$\begin{array}{llll}\mathrm{H} & 3.84899 & 0.48327 & 0.00000\end{array}$

$\begin{array}{llll}\mathrm{H} & 2.94494 & 3.50501 & 0.00000\end{array}$ 
Table S1459 Employed active space, reference configurations, and Cartesian coordinates of the OM2/MRCISD-optimized excited-state equilibrium geometry of the $2{ }^{1} A\left({ }^{1} \pi \pi^{*}\right)$ state of catechol (excited-state energy: $-1514.00256 \mathrm{eV}$ ).

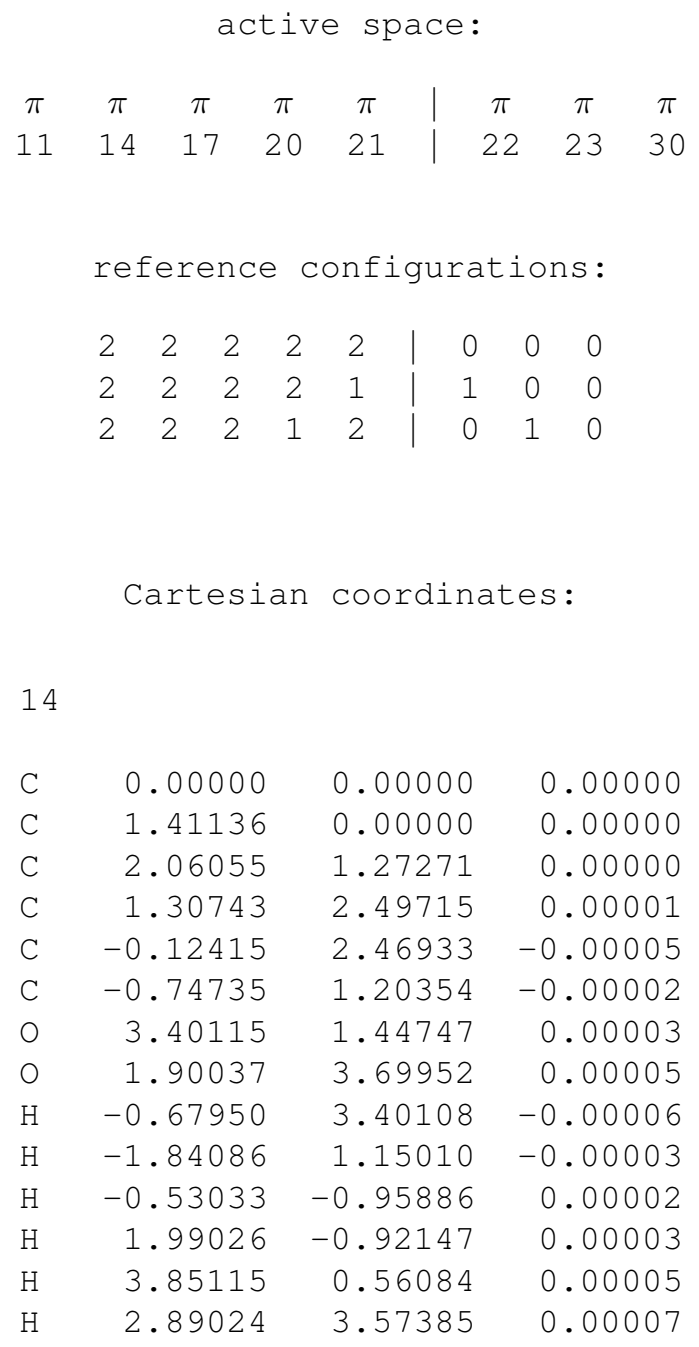


Table S1460 Employed active space, reference configurations, and Cartesian coordinates of the OM2/MRCISD-optimized ground-state equilibrium geometry of resorcinol (isomer 3) (ground-state energy: $-1517.97094 \mathrm{eV}$ ).

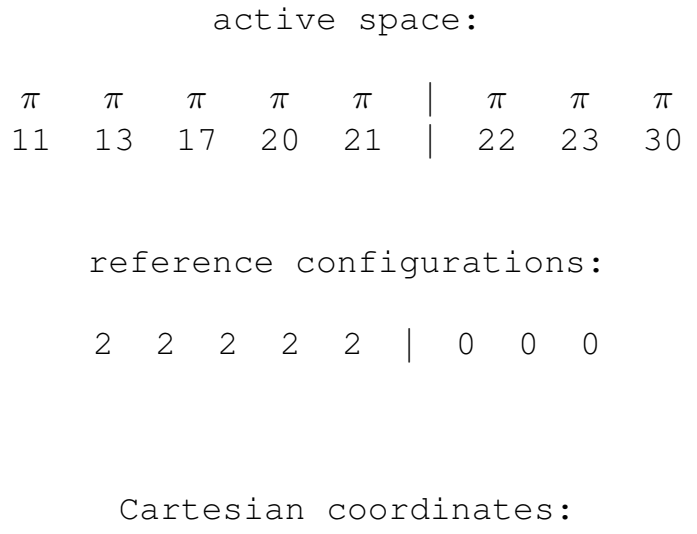

14

$\begin{array}{llll}\text { C } & 1.42986 & 0.00000 & 0.00000\end{array}$

C $\quad 0.68976 \quad 1.19235 \quad 0.00000$

C $\quad 0.68976-1.19235-0.00000$

C $\quad-0.71762 \quad 1.21672 \quad-0.00000$

C $\quad-0.71762-1.21672-0.00000$

C $\quad-1.40000 \quad 0.00000-0.00000$

$\mathrm{H} \quad 2.51862-0.00000 \quad-0.00000$

$\mathrm{H} \quad-2.49604 \quad 0.00000 \quad-0.00000$

$\begin{array}{llll}\mathrm{H} & -1.23584 & 2.17187 & -0.00000\end{array}$

$\mathrm{H} \quad-1.23584-2.17187 \quad-0.00000$

$\begin{array}{llll}0 & 1.28718 & 2.41609 & 0.00000\end{array}$

$\begin{array}{llll}0 & 1.28718 & -2.41609 & -0.00000\end{array}$

$\mathrm{H} \quad 2.27645 \quad 2.30485 \quad-0.00000$

$\mathrm{H} \quad 2.27645 \quad-2.30485 \quad-0.00000$ 
Table S1461 Employed active space, reference configurations, and Cartesian coordinates of the OM2/MRCISD-optimized excited-state equilibrium geometry of the $1{ }^{1} B_{2}\left({ }^{1} \pi \pi^{*}\right)$ state of resorcinol (isomer 3) (excited-state energy: $-1513.96359 \mathrm{eV})$.

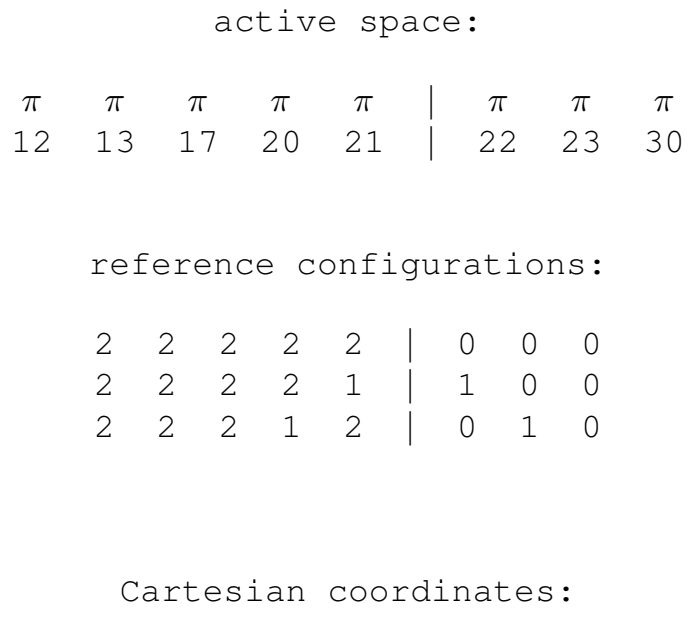

14

$\begin{array}{lrrr}\mathrm{C} & 1.50163 & 0.00000 & 0.00000 \\ \mathrm{C} & 0.73387 & 1.20105 & 0.00000 \\ \mathrm{C} & 0.73387 & -1.20105 & -0.00000 \\ \mathrm{C} & -0.69410 & 1.22714 & -0.00000 \\ \mathrm{C} & -0.69410 & -1.22714 & -0.00000 \\ \mathrm{C} & -1.40000 & 0.00000 & -0.00000 \\ \mathrm{H} & 2.58719 & -0.00000 & -0.00000 \\ \mathrm{H} & -2.49023 & 0.00000 & -0.00000 \\ \mathrm{H} & -1.20637 & 2.18756 & -0.00000 \\ \mathrm{H} & -1.20637 & -2.18756 & -0.00000 \\ \mathrm{O} & 1.31300 & 2.42186 & -0.00000 \\ \mathrm{O} & 1.31300 & -2.42186 & -0.00000 \\ \mathrm{H} & 2.30461 & 2.32394 & -0.00000 \\ \mathrm{H} & 2.30461 & -2.32394 & -0.00000\end{array}$


Table S1462 Employed active space, reference configurations, and Cartesian coordinates of the OM2/MRCISD-optimized ground-state equilibrium geometry of resorcinol (isomer 1) (ground-state energy: -1517.98159 eV).

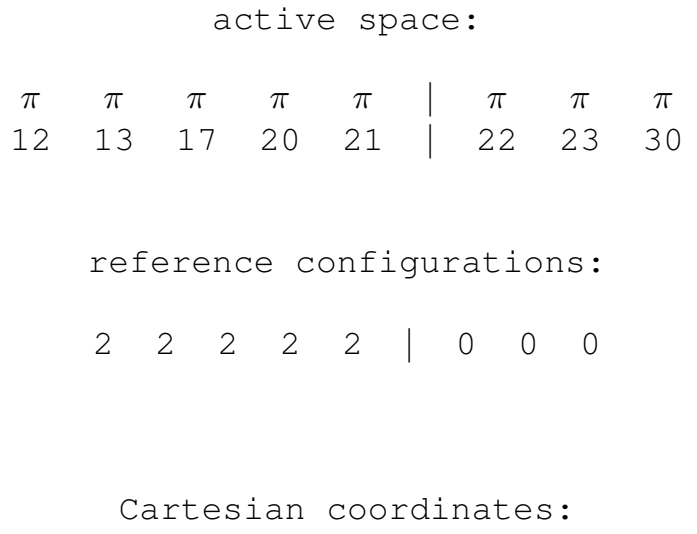

14

$\begin{array}{lrrr}\text { C } & 0.00000 & 0.00000 & 0.00000 \\ \text { C } & 1.40144 & 0.00000 & 0.00000 \\ \text { C } & 2.02015 & 1.26119 & 0.00000 \\ \text { C } & 1.29482 & 2.46506 & 0.00000 \\ \mathrm{C} & -0.10088 & 2.40416 & 0.00000 \\ \mathrm{C} & -0.76861 & 1.18111 & 0.00000 \\ \mathrm{O} & 3.38186 & 1.22679 & 0.00000 \\ \mathrm{O} & -0.72311 & -1.15364 & -0.00000 \\ \mathrm{H} & 1.81506 & 3.42392 & 0.00000 \\ \mathrm{H} & -0.68013 & 3.33454 & 0.00000 \\ \mathrm{H} & -1.85325 & 1.11407 & 0.00000 \\ \mathrm{H} & 1.99425 & -0.90836 & -0.00000 \\ \mathrm{H} & 3.73532 & 2.15753 & 0.00000 \\ \mathrm{H} & -0.10456 & -1.93363 & -0.00000\end{array}$


Table S1463 Employed active space, reference configurations, and Cartesian coordinates of the OM2/MRCISD-optimized excited-state equilibrium geometry of the $2{ }^{1} A\left({ }^{1} \pi \pi^{*}\right)$ state of resorcinol (isomer 1) (excited-state energy: $-1513.95665 \mathrm{eV})$.

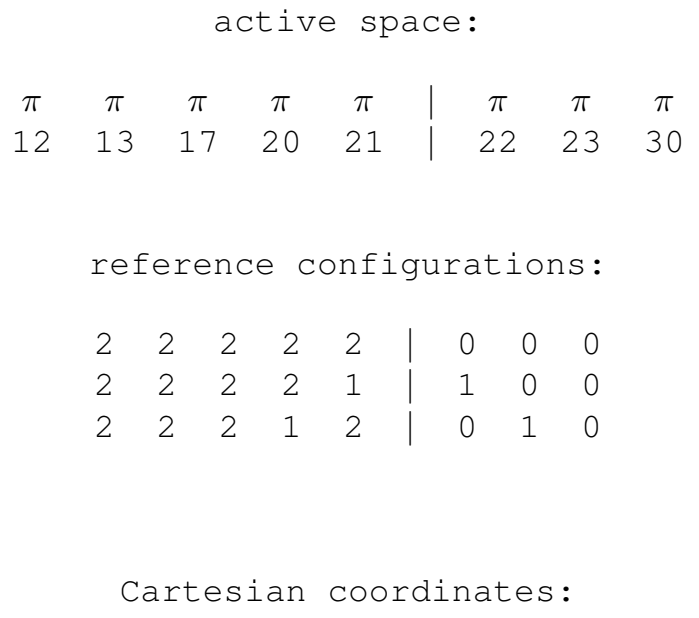

14

$\begin{array}{lrrr}\mathrm{C} & 0.00000 & 0.00000 & 0.00000 \\ \mathrm{C} & 1.42616 & 0.00000 & 0.00000 \\ \mathrm{C} & 2.02491 & 1.29437 & 0.00000 \\ \mathrm{C} & 1.27448 & 2.50902 & 0.00006 \\ \mathrm{C} & -0.13844 & 2.44510 & 0.00008 \\ \mathrm{C} & -0.79199 & 1.18747 & 0.00006 \\ \mathrm{O} & 3.37599 & 1.28396 & -0.00003 \\ \mathrm{O} & -0.71434 & -1.14719 & -0.00002 \\ \mathrm{H} & 1.79282 & 3.47118 & 0.00009 \\ \mathrm{H} & -0.72681 & 3.36343 & 0.00013 \\ \mathrm{H} & -1.87706 & 1.10436 & 0.00008 \\ \mathrm{H} & 2.02734 & -0.89989 & -0.00003 \\ \mathrm{H} & 3.71950 & 2.22001 & 0.00000 \\ \mathrm{H} & -0.09385 & -1.92691 & -0.00005\end{array}$


Table S1464 Employed active space, reference configurations, and Cartesian coordinates of the OM2/MRCISD-optimized ground-state equilibrium geometry of resorcinol (isomer 2) (ground-state energy: $-1517.96685 \mathrm{eV}$ ).

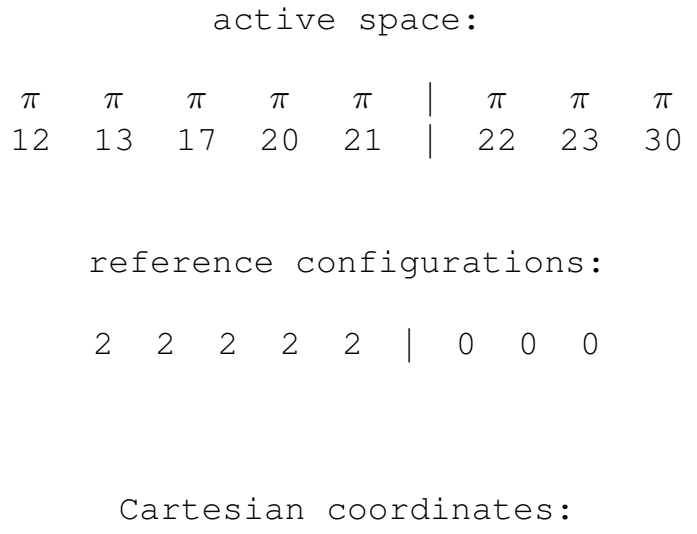

14

$\begin{array}{llll}\text { C } & 1.44100 & 0.00000 & 0.00000\end{array}$

$\begin{array}{llll}\text { C } & 0.69617 & 1.18918 & 0.00000\end{array}$

$\begin{array}{llll}\text { C } & 0.69617 & -1.18918 & -0.00000\end{array}$

C $\quad-0.71079 \quad 1.21323-0.00000$

C $\quad-0.71079-1.21323-0.00000$

C $\quad-1.40000 \quad 0.00000-0.00000$

$\mathrm{H} \quad 2.52252 \quad-0.00000 \quad-0.00000$

$\mathrm{H} \quad-2.49586 \quad 0.00000-0.00000$

$\mathrm{H} \quad-1.24796 \quad 2.16285-0.00000$

$\mathrm{H} \quad-1.24796 \quad-2.16285-0.00000$

$\begin{array}{lrrr}0 & 1.44172 & 2.32889 & 0.00000\end{array}$

$\begin{array}{llll}0 & 1.44172 & -2.32889 & -0.00000\end{array}$

$\begin{array}{llll}\mathrm{H} & 0.83683 & 3.11982 & -0.00000\end{array}$

$\mathrm{H} \quad \begin{array}{llll}\mathrm{H} & 0.83683 & -3.11982 & -0.00000\end{array}$ 
Table S1465 Employed active space, reference configurations, and Cartesian coordinates of the OM2/MRCISD-optimized excited-state equilibrium geometry of the $2^{1} A^{\prime}\left({ }^{1} \pi \pi^{*}\right)$ state of resorcinol (isomer 2) (excited-state energy: $-1513.93036 \mathrm{eV})$.

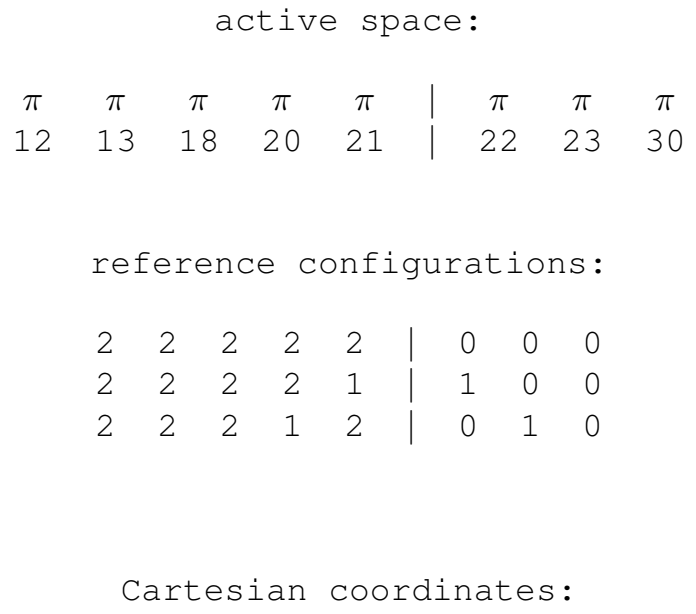

$\mathrm{H} \quad-1.57260 \quad 3.52935 \quad 0.00000$ 
Table S1466 Employed active space, reference configurations, and Cartesian coordinates of the OM2/MRCISD-optimized ground-state equilibrium geometry of cis-hydroquinone (ground-state energy: $-1517.92299 \mathrm{eV}$ ).

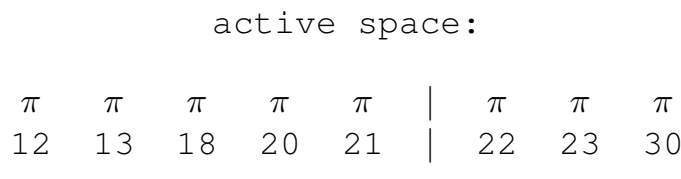

reference configurations:

$\begin{array}{lllllllll}2 & 2 & 2 & 2 & 2 & 0 & 0 & 0\end{array}$

Cartesian coordinates:

14

$\begin{array}{lrrr}\text { C } & 1.23303 & 0.69750 & 0.00000\end{array}$

C $\quad 1.23303-0.69750-0.00000$

C $\quad 0.00000 \quad 1.36863 \quad 0.00000$

C $\quad 0.00000-1.36863-0.00000$

$\begin{array}{llll}\text { C } & -1.23787 & 0.69477 & -0.00000\end{array}$

$\begin{array}{llll}\text { C } & -1.23787 & -0.69477 & -0.00000\end{array}$

$\begin{array}{llll}\mathrm{H} & 2.17185 & 1.25636 & 0.00000\end{array}$

$\mathrm{H} \quad 2.17185-1.25636-0.00000$

$\mathrm{H} \quad-2.16144 \quad 1.27083 \quad-0.00000$

$\mathrm{H} \quad-2.16144-1.27083-0.00000$

$\begin{array}{llll}0 & -0.09128 & 2.72997 & -0.00000\end{array}$

$\begin{array}{llll}0 & -0.09128 & -2.72997 & -0.00000\end{array}$

$\begin{array}{llll}\mathrm{H} & 0.82416 & 3.12137 & -0.00000\end{array}$

$\mathrm{H} \quad 0.82416 \quad-3.12137 \quad-0.00000$ 
Table S1467 Employed active space, reference configurations, and Cartesian coordinates of the OM2/MRCISD-optimized excited-state equilibrium geometry of the $2{ }^{1} A_{1}\left({ }^{1} \pi \pi^{*}\right)$ state of cis-hydroquinone (excited-state energy: $-1514.06821 \mathrm{eV})$.

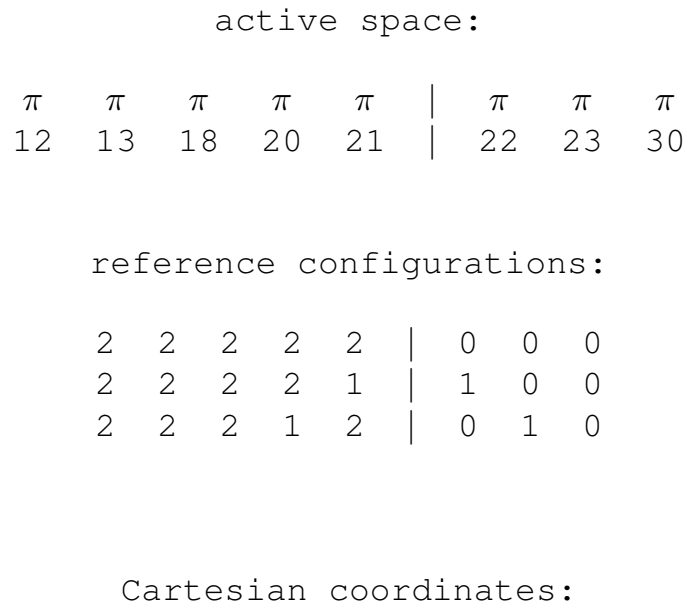


Table S1468 Employed active space, reference configurations, and Cartesian coordinates of the OM2/MRCISD-optimized ground-state equilibrium geometry of trans-hydroquinone (ground-state energy: $-1517.92634 \mathrm{eV}$ ).

\begin{tabular}{lcccc|ccc}
\multicolumn{10}{c}{ active space: } \\
$\pi$ & $\pi$ & $\pi$ & $\pi$ & $\pi$ & $\pi$ & $\pi$ & $\pi$ \\
12 & 13 & 18 & 20 & 21 & 22 & 23 & 30
\end{tabular}

reference configurations:

\begin{tabular}{lllll|lll}
2 & 2 & 2 & 2 & 2 & 0 & 0 & 0
\end{tabular}

Cartesian coordinates:

14

$\begin{array}{lrrr}\mathrm{C} & 0.00000 & 0.00000 & 0.00000 \\ \mathrm{C} & 2.73753 & 0.00000 & 0.00000 \\ \mathrm{C} & 0.67023 & 1.23731 & 0.00000 \\ \mathrm{C} & 2.06729 & -1.23731 & 0.00000 \\ \mathrm{C} & 0.67508 & -1.23333 & -0.00000 \\ \mathrm{C} & 2.06245 & 1.23333 & -0.00000 \\ \mathrm{O} & -1.36164 & 0.08917 & 0.00000 \\ \mathrm{O} & 4.09917 & -0.08917 & 0.00000 \\ \mathrm{H} & 0.09543 & 2.16138 & -0.00000 \\ \mathrm{H} & 2.64210 & -2.16138 & 0.00000 \\ \mathrm{H} & 0.11498 & -2.17174 & -0.00000 \\ \mathrm{H} & 2.62254 & 2.17174 & -0.00000 \\ \mathrm{H} & -1.75054 & -0.82727 & 0.00000 \\ \mathrm{H} & 4.48806 & 0.82727 & -0.00000\end{array}$


Table S1469 Employed active space, reference configurations, and Cartesian coordinates of the OM2/MRCISD-optimized excited-state equilibrium geometry of the $1^{1} B_{u}\left({ }^{1} \pi \pi^{*}\right)$ state of trans-hydroquinone (excited-state energy: $-1514.07610 \mathrm{eV})$.

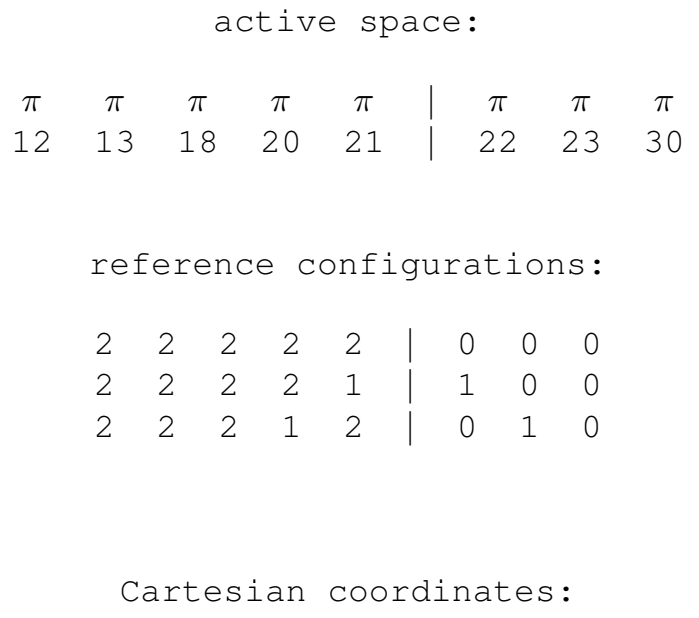

14

$\begin{array}{lrrr}\mathrm{C} & 0.00000 & 0.00000 & 0.00000 \\ \mathrm{C} & 2.73733 & 0.00000 & 0.00000 \\ \mathrm{C} & 0.66153 & 1.26564 & 0.00000 \\ \mathrm{C} & 2.07581 & -1.26564 & 0.00000 \\ \mathrm{C} & 0.66429 & -1.26260 & -0.00000 \\ \mathrm{C} & 2.07304 & 1.26260 & -0.00000 \\ \mathrm{O} & -1.34540 & 0.08828 & 0.00000 \\ \mathrm{O} & 4.08273 & -0.08828 & 0.00000 \\ \mathrm{H} & 0.08021 & 2.18195 & -0.00000 \\ \mathrm{H} & 2.65713 & -2.18195 & 0.00000 \\ \mathrm{H} & 0.09825 & -2.19310 & -0.00000 \\ \mathrm{H} & 2.63908 & 2.19310 & -0.00000 \\ \mathrm{H} & -1.74704 & -0.82432 & 0.00000 \\ \mathrm{H} & 4.48437 & 0.82432 & -0.00000\end{array}$


Table S1470 Employed active space, reference configurations, and Cartesian coordinates of the OM2/MRCISD-optimized ground-state equilibrium geometry of o-methoxyphenol (ground-state energy: $-1675.28899 \mathrm{eV}$ ).

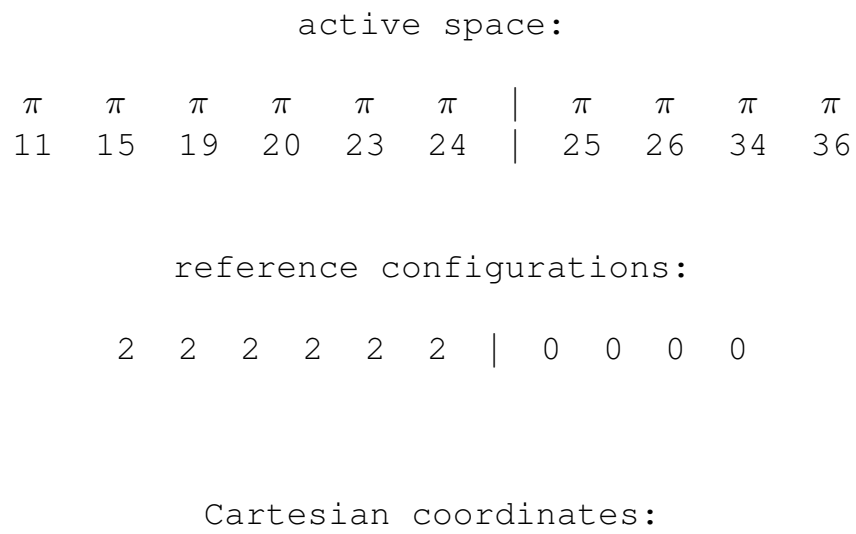


Table S1471 Employed active space, reference configurations, and Cartesian coordinates of the OM2/MRCISD-optimized excited-state equilibrium geometry of the $2{ }^{1} A\left({ }^{1} \pi \pi^{*}\right)$ state of $o$-methoxyphenol (excited-state energy: $-1671.34122 \mathrm{eV})$.

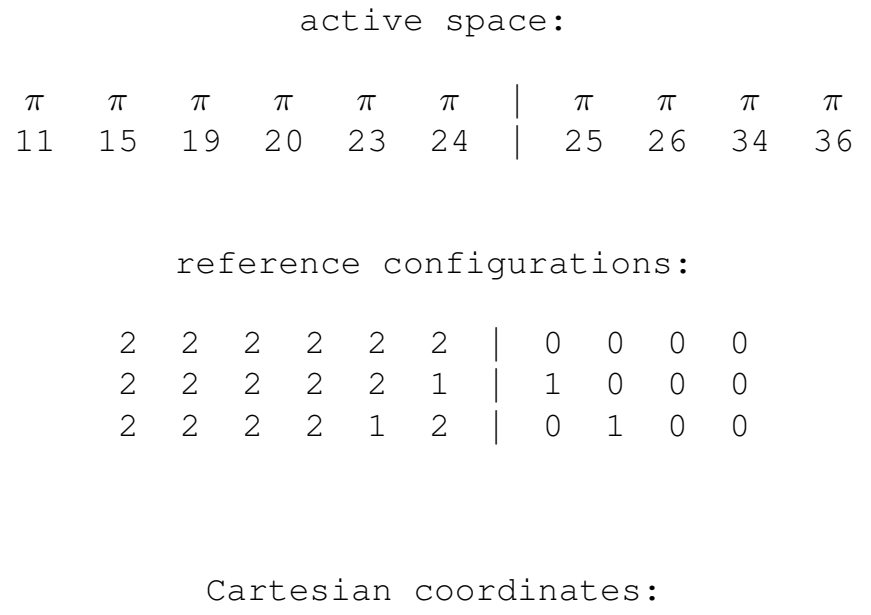

17

$\begin{array}{llll}\text { C } & 0.00000 & 0.00000 & 0.00000\end{array}$

$\begin{array}{llll}\text { C } & 1.41599 & 0.00000 & 0.00000\end{array}$

C $2.16753 \quad 1.19545 \quad 0.00000$

$\begin{array}{llll}\text { C } & 1.43607 & 2.42231 & 0.00001\end{array}$

$\begin{array}{llll}\text { C } & -0.00276 & 2.42579 & 0.00004\end{array}$

$\begin{array}{llll}\text { C } & -0.74198 & 1.19881 & 0.00002\end{array}$

$\begin{array}{llll}0 & 1.95546 & 3.67741 & 0.00002\end{array}$

$\begin{array}{llll}\text { C } & 3.35022 & 3.79968 & 0.00001\end{array}$

$\begin{array}{llll}0 & -0.71338 & 3.56361 & 0.00007\end{array}$

$\mathrm{H} \quad-1.82642 \quad 1.22432 \quad 0.00003$

$\mathrm{H} \quad-0.53314 \quad-0.95645 \quad-0.00001$

$\mathrm{H} \quad 1.94926 \quad-0.95786 \quad-0.00001$

$\mathrm{H} \quad 3.25380 \quad 1.18329-0.00001$

$\mathrm{H} \quad-0.08440 \quad 4.33827 \quad 0.00006$

H $\quad 3.53529 \quad 4.89562 \quad 0.00005$

$\begin{array}{llll}\mathrm{H} & 3.80242 & 3.33703 & 0.91299\end{array}$

$\mathrm{H} \quad 3.80240 \quad 3.33710 \quad-0.91300$ 
Table S1472 Employed active space, reference configurations, and Cartesian coordinates of the OM2/MRCISD-optimized ground-state equilibrium geometry of $m$-methoxyphenol (isomer 2) (ground-state energy: $-1675.31235 \mathrm{eV}$ ).

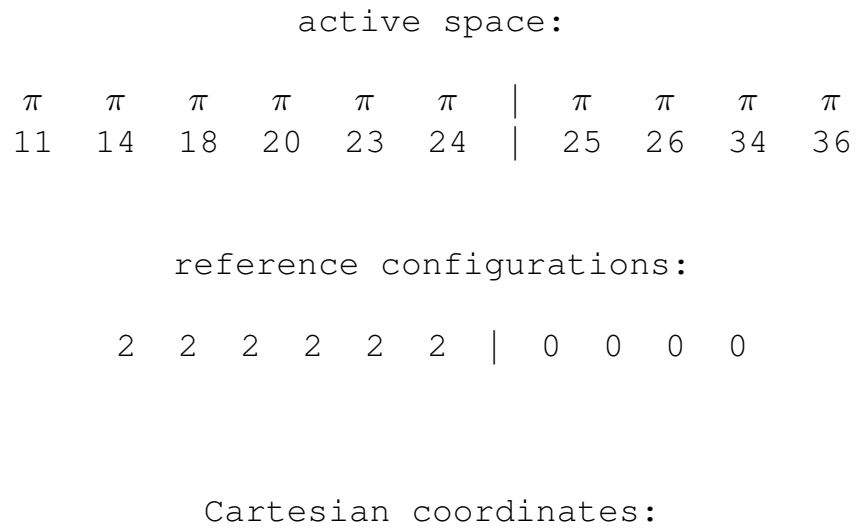


Table S1473 Employed active space, reference configurations, and Cartesian coordinates of the OM2/MRCISD-optimized excited-state equilibrium geometry of the $2{ }^{1} A\left({ }^{1} \pi \pi^{*}\right)$ state of $m$-methoxyphenol (isomer 2) (excited-state energy: $-1671.30011 \mathrm{eV})$.

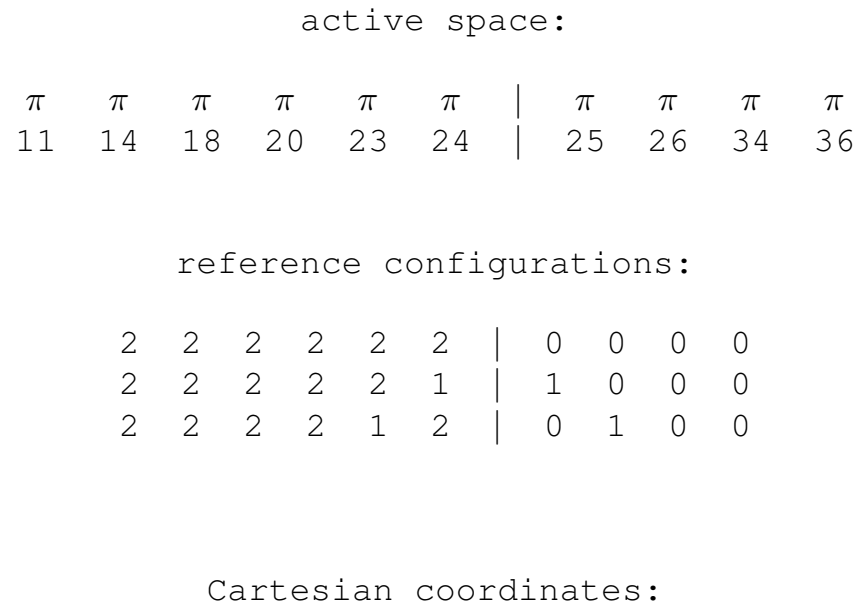


Table S1474 Employed active space, reference configurations, and Cartesian coordinates of the OM2/MRCISD-optimized ground-state equilibrium geometry of $m$-methoxyphenol (isomer 4) (ground-state energy: $-1675.32336 \mathrm{eV}$ ).

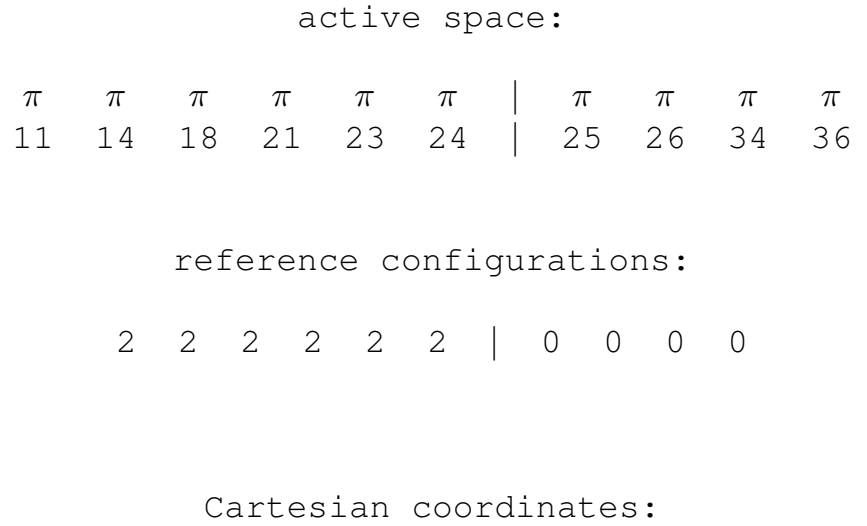


Table S1475 Employed active space, reference configurations, and Cartesian coordinates of the OM2/MRCISD-optimized excited-state equilibrium geometry of the $2{ }^{1} A\left({ }^{1} \pi \pi^{*}\right)$ state of $m$-methoxyphenol (isomer 4) (excited-state energy: $-1671.29262 \mathrm{eV})$.

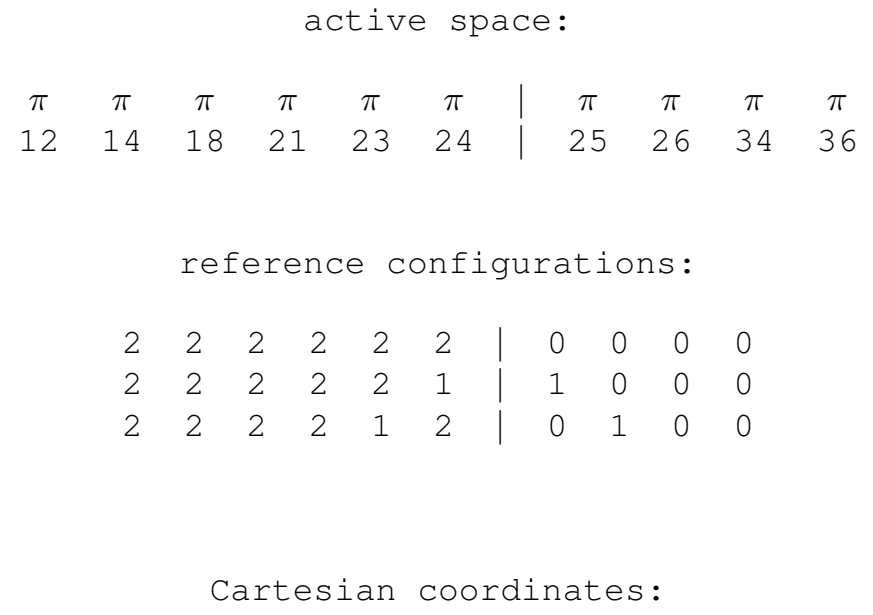

17

$\begin{array}{llll}\text { C } & 0.00000 & 0.00000 & 0.00000\end{array}$

C $\quad 1.41274 \quad 0.00000 \quad 0.00000$

$\begin{array}{llll}\mathrm{C} & 2.11975 & 1.22961 & 0.00000\end{array}$

$\begin{array}{llll}\text { C } & 1.38759 & 2.45622 & 0.00002\end{array}$

$\begin{array}{llll}\text { C } & -0.03658 & 2.51733 & 0.00002\end{array}$

$\begin{array}{lrrr}\text { C } & -0.69018 & 1.24890 & 0.00000\end{array}$

$\begin{array}{llll}0 & 2.20089 & 3.54390 & 0.00001\end{array}$

$\begin{array}{llll}\text { C } & 1.60184 & 4.80804 & 0.00003\end{array}$

O $\quad-2.04052 \quad 1.32209-0.00000$

$\mathrm{H} \quad-0.60914 \quad 3.43361 \quad 0.00003$

$\mathrm{H} \quad-0.56318 \quad-0.93689-0.00001$

$\mathrm{H} \quad \begin{array}{llll}1.96121 & -0.94284 & -0.00000\end{array}$

$\mathrm{H} \quad 3.20816 \quad 1.25963-0.00000$

$\begin{array}{llll}\mathrm{H} & 2.45475 & 5.52057 & 0.00002\end{array}$

$\mathrm{H} \quad 0.97398 \quad 4.96854 \quad-0.91271$

H $\quad 0.97402 \quad 4.96852 \quad 0.91280$

$\begin{array}{llll}\mathrm{H} & -2.42623 & 0.40274 & -0.00001\end{array}$ 
Table S1476 Employed active space, reference configurations, and Cartesian coordinates of the OM2/MRCISD-optimized ground-state equilibrium geometry of trans-p-methoxyphenol (ground-state energy: $-1675.26525 \mathrm{eV}$ ).

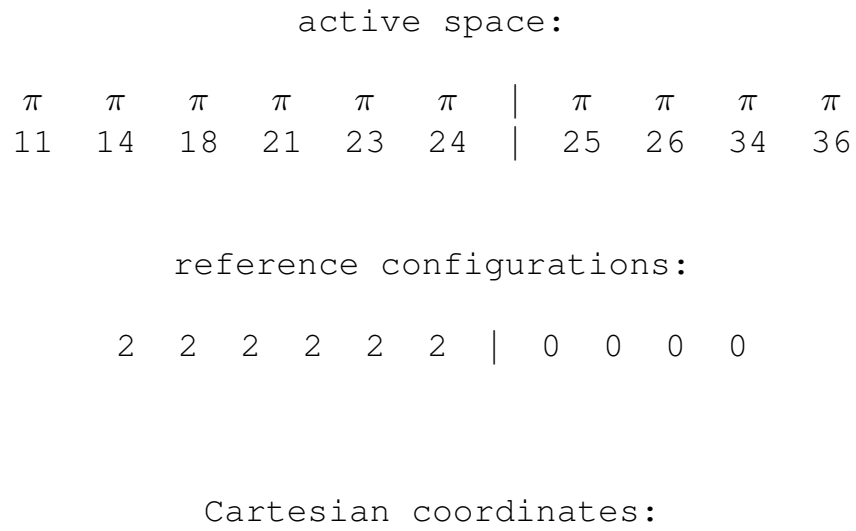


Table S1477 Employed active space, reference configurations, and Cartesian coordinates of the OM2/MRCISD-optimized excited-state equilibrium geometry of the $2{ }^{1} A\left({ }^{1} \pi \pi^{*}\right)$ state of trans-p-methoxyphenol (excited-state energy: $-1671.40770 \mathrm{eV})$.

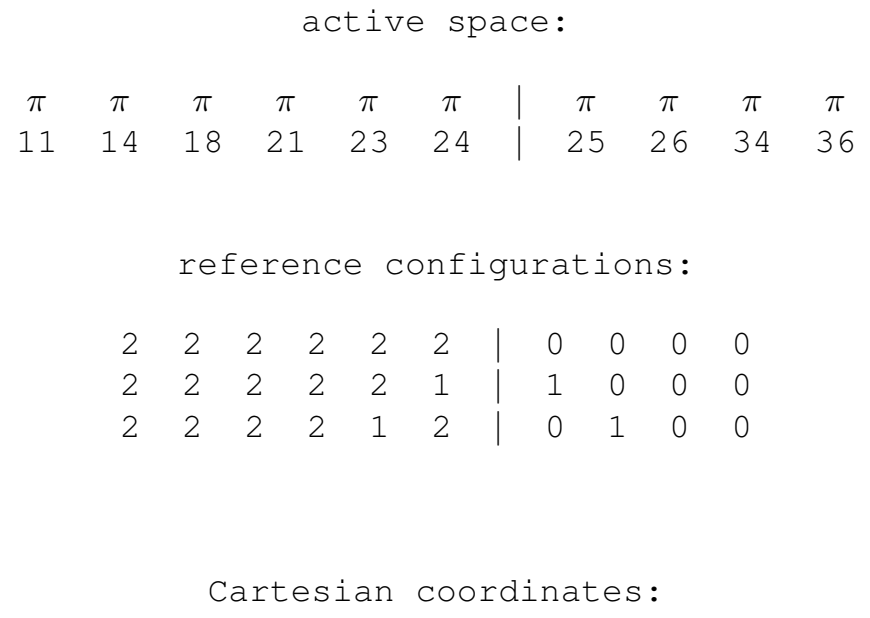

17

$\begin{array}{lrrr}\mathrm{C} & 0.00000 & 0.00000 & 0.00000 \\ \mathrm{C} & 1.43000 & 0.00000 & 0.00000 \\ \mathrm{C} & 2.09232 & 1.24650 & 0.00000 \\ \mathrm{C} & 1.28799 & 2.42364 & 0.00003 \\ \mathrm{C} & -0.13899 & 2.42642 & 0.00005 \\ \mathrm{C} & -0.80156 & 1.17954 & 0.00003 \\ \mathrm{O} & 1.83853 & 3.65526 & 0.00004 \\ \mathrm{O} & -0.49949 & -1.25984 & -0.00004 \\ \mathrm{C} & -1.88866 & -1.42990 & -0.00005 \\ \mathrm{H} & -1.88726 & 1.13277 & 0.00002 \\ \mathrm{H} & 1.97090 & -0.94166 & -0.00003 \\ \mathrm{H} & 3.17968 & 1.30912 & -0.00001 \\ \mathrm{H} & 2.83291 & 3.58268 & 0.00001 \\ \mathrm{H} & -0.67817 & 3.36888 & 0.00006 \\ \mathrm{H} & -2.03336 & -2.53154 & -0.00005 \\ \mathrm{H} & -2.35953 & -0.98577 & 0.91281 \\ \mathrm{H} & -2.35950 & -0.98579 & -0.91294\end{array}$


Table S1478 Employed active space, reference configurations, and Cartesian coordinates of the OM2/MRCISD-optimized ground-state equilibrium geometry of cis-p-methoxyphenol (ground-state energy: -1675.26303 eV).

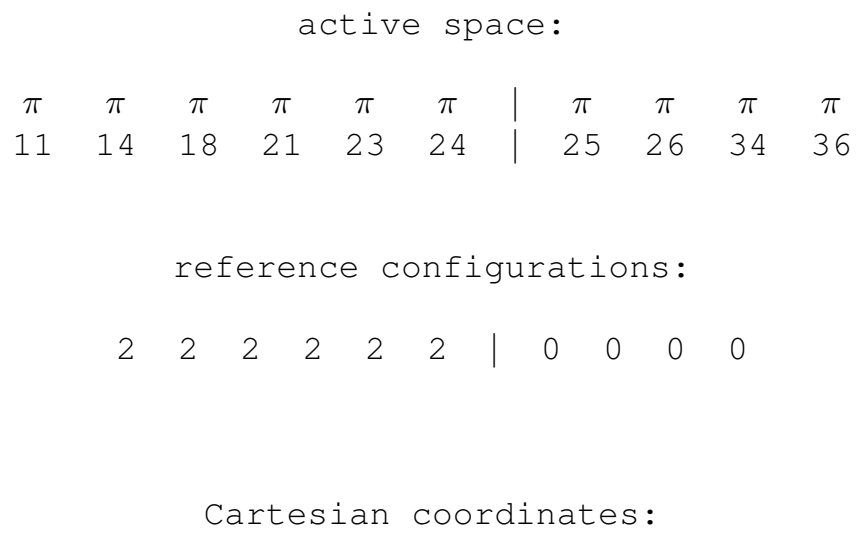


Table S1479 Employed active space, reference configurations, and Cartesian coordinates of the OM2/MRCISD-optimized excited-state equilibrium geometry of the $2^{1} A\left({ }^{1} \pi \pi^{*}\right)$ state of cis-p-methoxyphenol (excited-state energy: $-1671.39844 \mathrm{eV})$.

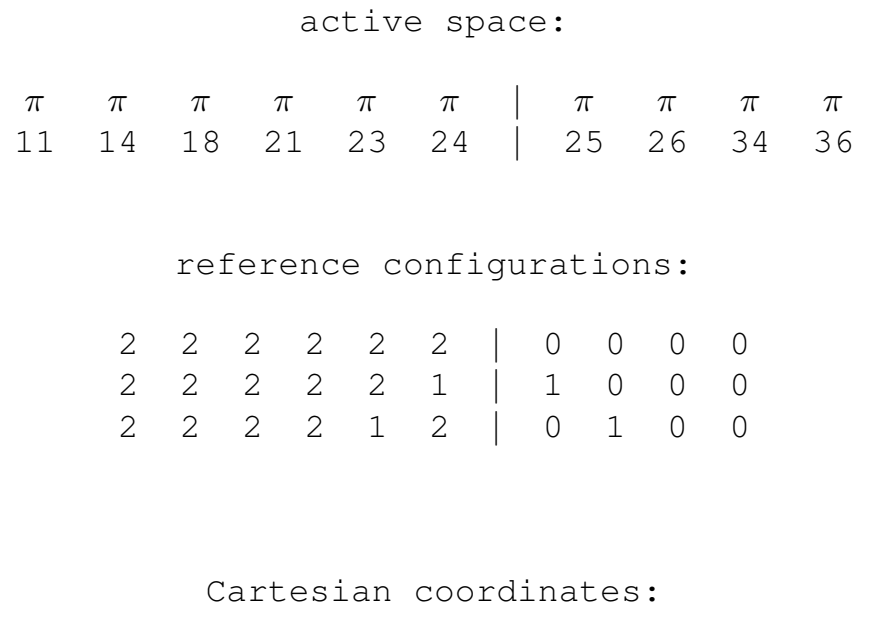

17

$\begin{array}{llll}\text { C } & 0.00000 & 0.00000 & 0.00000\end{array}$

$\begin{array}{llll}\text { C } & 1.42837 & 0.00000 & 0.00000\end{array}$

$\begin{array}{lllr}\mathrm{C} & 2.23051 & 1.18130 & 0.00000\end{array}$

$\begin{array}{llll}\text { C } & 1.57581 & 2.43316 & -0.00001\end{array}$

$\begin{array}{llll}\text { C } & 0.15076 & 2.42982 & -0.00001\end{array}$

$\begin{array}{llll}\text { C } & -0.65357 & 1.25023 & -0.00001\end{array}$

$\begin{array}{llll}0 & 2.18403 & -1.12506 & 0.00000\end{array}$

$\begin{array}{llll}\text { C } & 1.54117 & -2.36791 & 0.00000\end{array}$

$\begin{array}{llll}0 & -0.40804 & 3.65764 & -0.00001\end{array}$

$\mathrm{H} \quad-0.57172 \quad-0.92448 \quad 0.00000$

$\begin{array}{lrrr}\mathrm{H} & 3.31317 & 1.09988 & 0.00000\end{array}$

$\mathrm{H} \quad 2.11861 \quad 3.37246 \quad-0.00001$

$\mathrm{H} \quad-1.74184 \quad 1.31751 \quad-0.00001$

$\mathrm{H} \quad 2.36938 \quad-3.10848 \quad-0.00001$

$\begin{array}{llll}\mathrm{H} & 0.90888 & -2.50689 & 0.91301\end{array}$

$\mathrm{H} \quad 0.90887-2.50688-0.91300$

$\mathrm{H} \quad-1.40221 \quad 3.58009 \quad-0.00001$ 
Table S1480 Employed active space, reference configurations, and Cartesian coordinates of the OM2/MRCISD-optimized ground-state equilibrium geometry of cis-o-fluorophenol (ground-state energy: $-1637.93047 \mathrm{eV}$ ).

\begin{tabular}{lcccc|ccc} 
active space : \\
$\pi$ & $\pi$ & $\pi$ & $\pi$ & $\pi$ & $\pi$ & $\pi$ & $\pi$ \\
10 & 13 & 17 & 20 & 21 & 22 & 23 & 30
\end{tabular}

reference configurations:

\begin{tabular}{lllll|lll}
2 & 2 & 2 & 2 & 2 & 0 & 0 & 0
\end{tabular}

Cartesian coordinates:

$\begin{array}{lrrr}13 & & & \\ \text { C } & 0.00000 & 0.00000 & 0.00000 \\ \text { C } & 1.39729 & 0.00000 & 0.00000 \\ \text { C } & 2.04763 & 1.23395 & 0.00000 \\ \text { C } & 1.34233 & 2.45522 & 0.00000 \\ \text { C } & -0.06254 & 2.43781 & 0.00000 \\ \text { C } & -0.71430 & 1.20354 & 0.00000 \\ \text { F } & 3.40727 & 1.27659 & 0.00000 \\ \text { O } & 1.94452 & 3.67127 & 0.00000 \\ \text { H } & -0.60810 & 3.37949 & 0.00000 \\ \text { H } & -1.80914 & 1.17915 & 0.00000 \\ \text { H } & -0.53792 & -0.95215 & -0.00000 \\ \text { H } & 1.97655 & -0.92182 & -0.00000 \\ \text { H } & 2.93180 & 3.54433 & 0.00000\end{array}$


Table S1481 Employed active space, reference configurations, and Cartesian coordinates of the OM2/MRCISD-optimized excited-state equilibrium geometry of the $2{ }^{1} A\left({ }^{1} \pi \pi^{*}\right)$ state of cis-o-fluorophenol (excited-state energy: $-1633.85974 \mathrm{eV})$.

\begin{tabular}{lcccc|ccc} 
active space \\
$\pi$ & $\pi$ & $\pi$ & $\pi$ & $\pi$ & $\pi$ & $\pi$ & $\pi$ \\
9 & 13 & 17 & 20 & 21 & 22 & 23 & 30
\end{tabular}

reference configurations:

\begin{tabular}{lllll|llll}
2 & 2 & 2 & 2 & 2 & 0 & 0 & 0
\end{tabular}

\begin{tabular}{lllll|lll}
2 & 2 & 2 & 2 & 1 & 1 & 0 & 0
\end{tabular}

\begin{tabular}{lllll|lll}
2 & 2 & 2 & 1 & 2 & 0 & 1 & 0
\end{tabular}

Cartesian coordinates:

13

$\begin{array}{llll}\text { C } & 0.00000 & 0.00000 & 0.00000\end{array}$

$\begin{array}{llll}\text { C } & 1.41469 & 0.00000 & 0.00000\end{array}$

$\begin{array}{lllr}\text { C } & 2.05616 & 1.26632 & 0.00000\end{array}$

$\begin{array}{llll}\text { C } & 1.31641 & 2.49602 & -0.00000\end{array}$

$\begin{array}{lrrr}\text { C } & -0.11359 & 2.47210 & 0.00001\end{array}$

$\begin{array}{lrrr}\text { C } & -0.74677 & 1.20511 & 0.00001\end{array}$

F $\quad 3.40702 \quad 1.34000-0.00001$

$\begin{array}{llll}0 & 1.89866 & 3.70443 & -0.00001\end{array}$

$\mathrm{H} \quad-0.66828 \quad 3.40495 \quad 0.00001$

$\mathrm{H} \quad-1.83904 \quad 1.15870 \quad 0.00001$

$\mathrm{H} \quad-0.53002-0.95871 \quad-0.00001$

$\mathrm{H} \quad 2.00163 \quad-0.91260 \quad-0.00002$

$\mathrm{H} \quad 2.89034 \quad 3.59883 \quad-0.00001$ 
Table S1482 Employed active space, reference configurations, and Cartesian coordinates of the OM2/MRCISD-optimized ground-state equilibrium geometry of trans-o-fluorophenol (ground-state energy: $-1637.85794 \mathrm{eV}$ ).

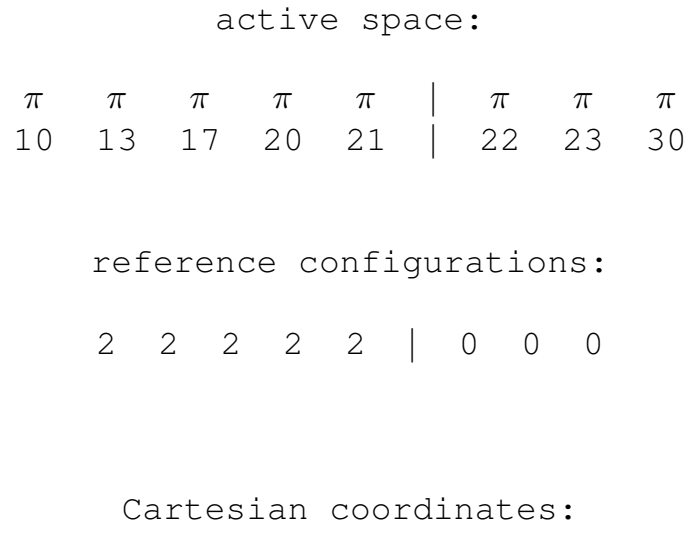

13

$\begin{array}{lrrr}\text { C } & 0.00000 & 0.00000 & 0.00000 \\ \text { C } & 1.39542 & 0.00000 & 0.00000 \\ \text { C } & 2.13088 & 1.20679 & 0.00000 \\ \text { C } & 1.44745 & 2.43174 & 0.00000 \\ \text { C } & 0.05015 & 2.42816 & 0.00000 \\ \text { C } & -0.66712 & 1.22856 & 0.00000 \\ \text { F } & 2.05523 & -1.17758 & -0.00000 \\ \text { O } & 3.48312 & 1.09180 & 0.00000 \\ \text { H } & 2.00552 & 3.37103 & 0.00000 \\ \text { H } & -0.48787 & 3.38168 & 0.00000 \\ \text { H } & -1.76052 & 1.24860 & 0.00000 \\ \text { H } & -0.53957 & -0.94606 & -0.00000 \\ \text { H } & 3.88851 & 2.00270 & 0.00000\end{array}$


Table S1483 Employed active space, reference configurations, and Cartesian coordinates of the OM2/MRCISD-optimized excited-state equilibrium geometry of the $2{ }^{1} A\left({ }^{1} \pi \pi^{*}\right)$ state of trans-o-fluorophenol (excited-state energy: $-1633.80064 \mathrm{eV})$.

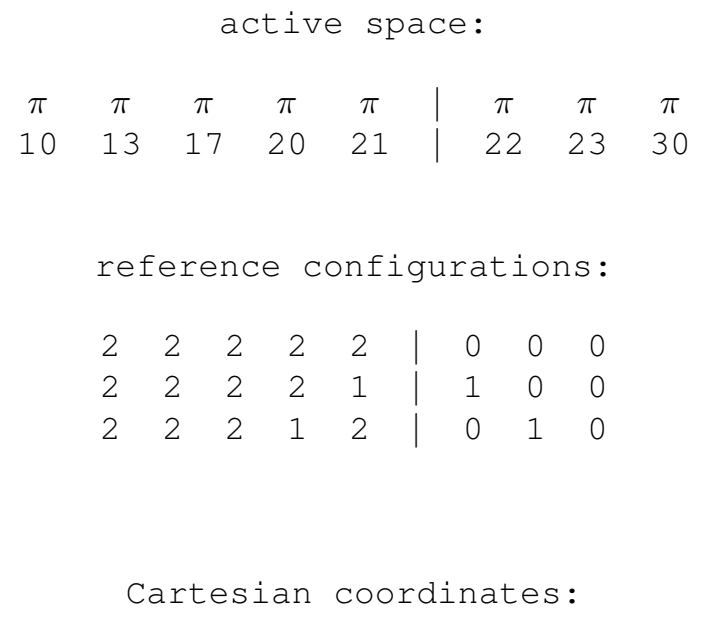

13

$\begin{array}{lrrr}\text { C } & 0.00000 & 0.00000 & 0.00000 \\ \mathrm{C} & 1.42389 & 0.00000 & 0.00000 \\ \mathrm{C} & 2.16983 & 1.22568 & 0.00000 \\ \mathrm{C} & 1.48945 & 2.48413 & -0.00005 \\ \mathrm{C} & 0.07516 & 2.47083 & -0.00011 \\ \mathrm{C} & -0.65157 & 1.25245 & -0.00004 \\ \mathrm{~F} & 2.08472 & -1.16798 & 0.00003 \\ \mathrm{O} & 3.50609 & 1.10317 & 0.00004 \\ \mathrm{H} & 2.05236 & 3.41658 & -0.00007 \\ \mathrm{H} & -0.46755 & 3.42029 & -0.00015 \\ \mathrm{H} & -1.74646 & 1.28470 & -0.00005 \\ \mathrm{H} & -0.54138 & -0.94050 & 0.00003 \\ \mathrm{H} & 3.92513 & 2.00957 & 0.00006\end{array}$


Table S1484 Employed active space, reference configurations, and Cartesian coordinates of the OM2/MRCISD-optimized ground-state equilibrium geometry of cis-m-fluorophenol (ground-state energy: $-1638.02543 \mathrm{eV}$ ).

\begin{tabular}{lcccc|ccc} 
active space : \\
$\pi$ & $\pi$ & $\pi$ & $\pi$ & $\pi$ & $\pi$ & $\pi$ & $\pi$ \\
10 & 13 & 17 & 20 & 21 & 22 & 23 & 30
\end{tabular}

reference configurations:

\begin{tabular}{lllll|lll}
2 & 2 & 2 & 2 & 2 & 0 & 0 & 0
\end{tabular}

Cartesian coordinates:

13

$\begin{array}{lrrr}\text { C } & 0.00000 & 0.00000 & 0.00000 \\ \text { C } & 1.39900 & 0.00000 & 0.00000 \\ \text { C } & 2.18665 & 1.15474 & 0.00000 \\ \text { C } & 1.48092 & 2.36775 & 0.00000 \\ \text { C } & 0.07290 & 2.43004 & 0.00000 \\ \text { C } & -0.64971 & 1.23779 & 0.00000 \\ \text { F } & 2.03872 & -1.19732 & -0.00000 \\ \text { O } & 2.10892 & 3.57492 & 0.00000 \\ \text { H } & -0.41647 & 3.40106 & 0.00000 \\ \text { H } & -1.74454 & 1.27132 & 0.00000 \\ \text { H } & -0.54653 & -0.93911 & -0.00000 \\ \text { H } & 3.26984 & 1.10008 & 0.00000 \\ \text { H } & 3.09494 & 3.43832 & 0.00000\end{array}$


Table S1485 Employed active space, reference configurations, and Cartesian coordinates of the OM2/MRCISD-optimized excited-state equilibrium geometry of the $2{ }^{1} A\left({ }^{1} \pi \pi^{*}\right)$ state of cis- $m$-fluorophenol (excited-state energy: $-1633.92027 \mathrm{eV})$.

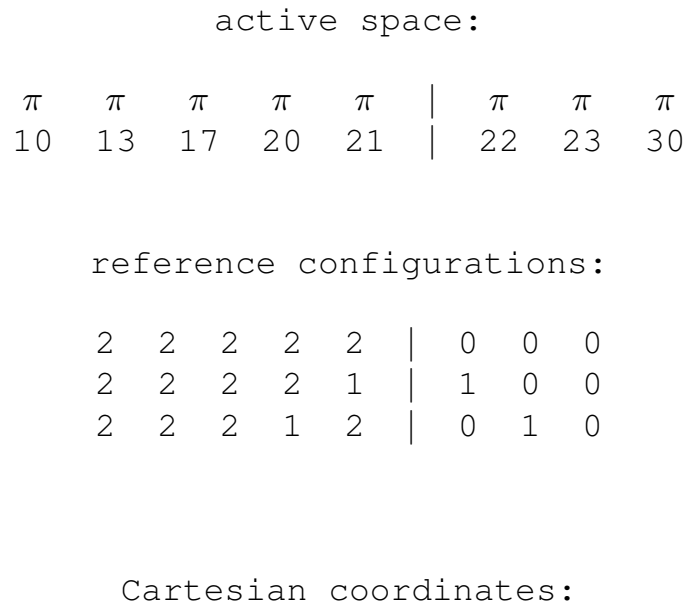

$\begin{array}{llll}\mathrm{H} & 3.08905 & 3.47068 & 0.00001\end{array}$ 
Table S1486 Employed active space, reference configurations, and Cartesian coordinates of the OM2/MRCISD-optimized ground-state equilibrium geometry of trans- $m$-fluorophenol (ground-state energy: $-1638.01816 \mathrm{eV}$ ).

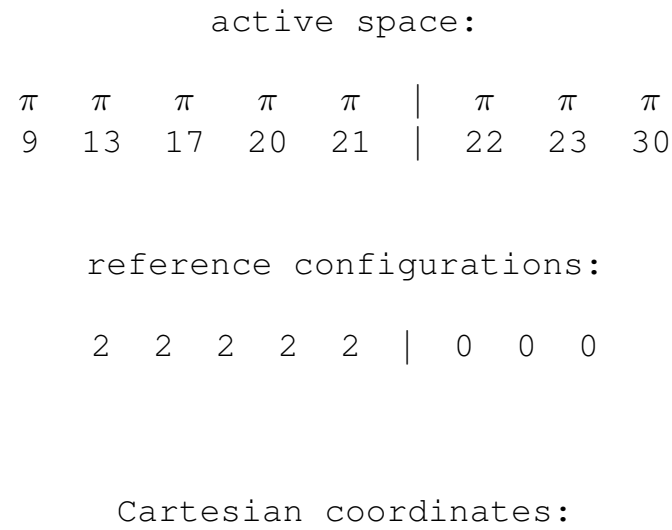


Table S1487 Employed active space, reference configurations, and Cartesian coordinates of the OM2/MRCISD-optimized excited-state equilibrium geometry of the $2^{1} A^{\prime}\left({ }^{1} \pi \pi^{*}\right)$ state of trans- $m$-fluorophenol (excited-state energy: $-1633.90471 \mathrm{eV})$.

\begin{tabular}{lcccc|ccc} 
active space \\
$\pi$ & $\pi$ & $\pi$ & $\pi$ & $\pi$ & $\pi$ & $\pi$ & $\pi$ \\
9 & 13 & 17 & 20 & 21 & 22 & 23 & 30
\end{tabular}

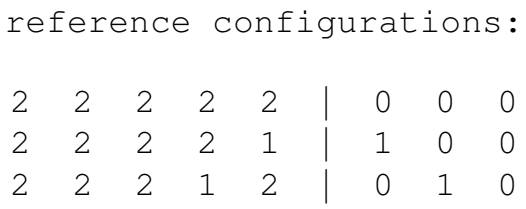

Cartesian coordinates:

13

$\begin{array}{lrrr}\text { C } & 0.00000 & 0.00000 & 0.00000 \\ \mathrm{C} & 1.42020 & 0.00000 & 0.00000 \\ \mathrm{C} & 2.00688 & 1.30119 & 0.00000 \\ \mathrm{C} & 1.24554 & 2.51305 & 0.00000 \\ \mathrm{C} & -0.17011 & 2.43278 & 0.00000 \\ \mathrm{C} & -0.80722 & 1.16881 & 0.00000 \\ \mathrm{O} & 3.35405 & 1.30397 & 0.00000 \\ \mathrm{~F} & -0.62905 & -1.19675 & -0.00000 \\ \mathrm{H} & 1.75243 & 3.47950 & 0.00000 \\ \mathrm{H} & -0.76677 & 3.34605 & 0.00000 \\ \mathrm{H} & -1.89218 & 1.07606 & 0.00000 \\ \mathrm{H} & 2.01270 & -0.90238 & -0.00000 \\ \mathrm{H} & 3.69272 & 2.24215 & 0.00000\end{array}$


Table S1488 Employed active space, reference configurations, and Cartesian coordinates of the OM2/MRCISD-optimized ground-state equilibrium geometry of $p$-fluorophenol (ground-state energy: $-1637.99822 \mathrm{eV}$ ).

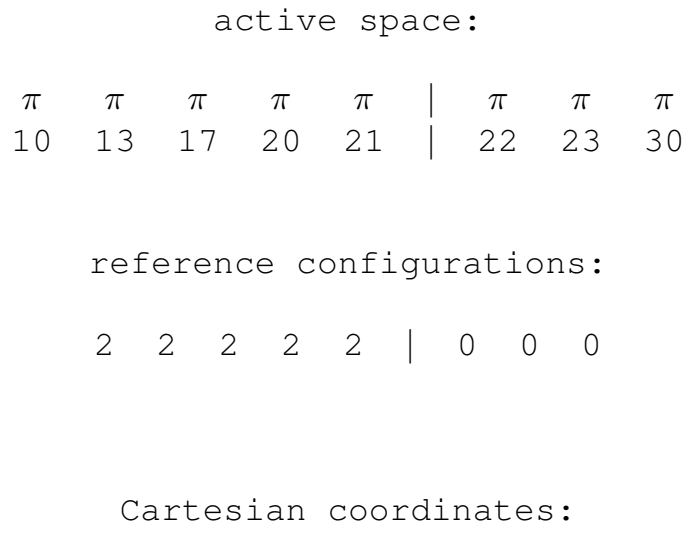

13

$\begin{array}{rrrr}\text { C } & 0.00000 & 0.00000 & 0.00000 \\ \text { C } & 1.39173 & 0.00000 & 0.00000 \\ \text { C } & 2.05903 & 1.24128 & 0.00000 \\ \text { C } & 1.38713 & 2.47629 & 0.00000 \\ \text { C } & -0.00720 & 2.47622 & 0.00000 \\ \text { C } & -0.65742 & 1.23737 & 0.00000 \\ \text { O } & 3.41896 & 1.15296 & 0.00000 \\ \text { F } & -2.01630 & 1.23516 & 0.00000 \\ \text { H } & 1.94677 & 3.41413 & 0.00000 \\ \text { H } & -0.58281 & 3.39984 & 0.00000 \\ \text { H } & -0.57407 & -0.92480 & -0.00000 \\ \text { H } & 1.97029 & -0.92130 & -0.00000 \\ \text { H } & 3.81080 & 2.06823 & 0.00000\end{array}$


Table S1489 Employed active space, reference configurations, and Cartesian coordinates of the OM2/MRCISD-optimized excited-state equilibrium geometry of the $2^{1} A^{\prime}\left({ }^{1} \pi \pi^{*}\right)$ state of $p$-fluorophenol (excited-state energy: $-1633.98380 \mathrm{eV})$.

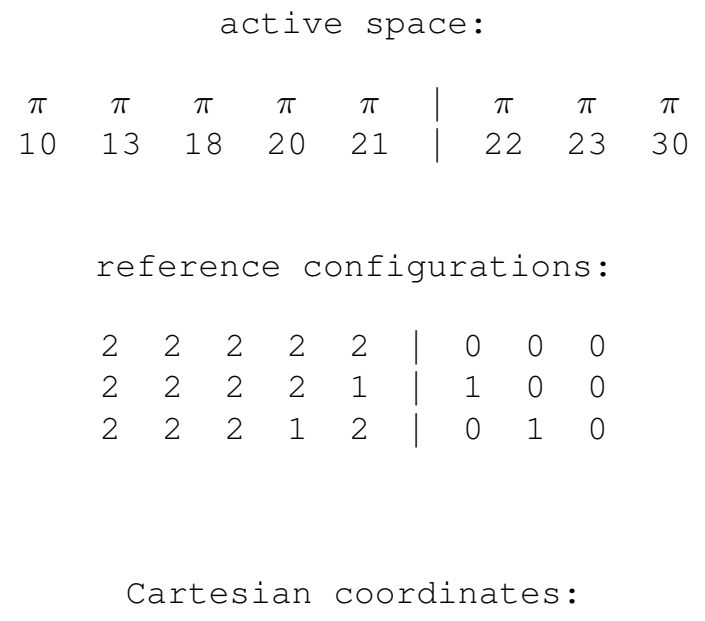

13

$\begin{array}{lrrr}\mathrm{C} & 0.00000 & 0.00000 & 0.00000 \\ \mathrm{C} & 1.41438 & 0.00000 & 0.00000 \\ \mathrm{C} & 2.07456 & 1.26907 & 0.00000 \\ \mathrm{C} & 1.40575 & 2.53435 & 0.00000 \\ \mathrm{C} & -0.00685 & 2.52945 & 0.00000 \\ \mathrm{C} & -0.64925 & 1.26166 & 0.00000 \\ \mathrm{O} & 3.41786 & 1.18545 & 0.00000 \\ \mathrm{~F} & -2.00060 & 1.26166 & 0.00000 \\ \mathrm{H} & 1.97055 & 3.46578 & 0.00000 \\ \mathrm{H} & -0.59043 & 3.44427 & 0.00000 \\ \mathrm{H} & -0.57680 & -0.91868 & -0.00000 \\ \mathrm{H} & 1.99799 & -0.91489 & -0.00000 \\ \mathrm{H} & 3.81933 & 2.09851 & 0.00000\end{array}$


Table S1490 Employed active space, reference configurations, and Cartesian coordinates of the OM2/MRCISD-optimized ground-state equilibrium geometry of cis-o-cresol (ground-state energy: $-1344.72299 \mathrm{eV}$ ).

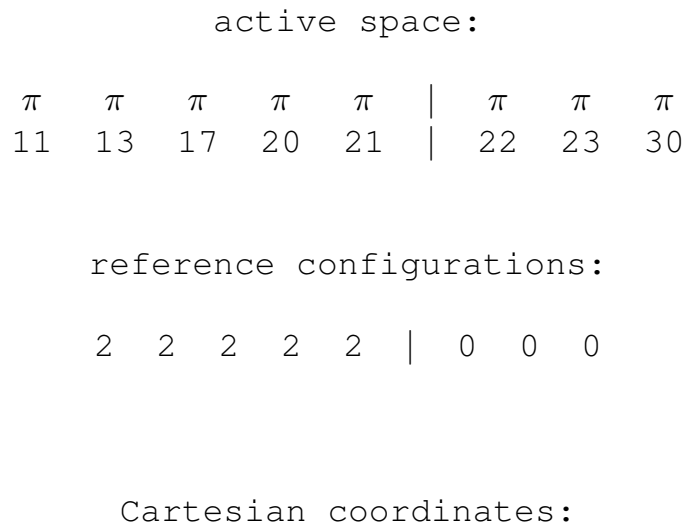

$\begin{array}{rr}0.00000 & 0.00000 \\ 0.00000 & 0.00000 \\ 1.20361 & 0.00000 \\ 2.42719 & 0.00000 \\ 2.46828 & 0.00000 \\ 1.23978 & 0.00000 \\ 3.76796 & 0.00000 \\ -0.95350 & -0.00000 \\ 1.18716 & 0.00000 \\ 3.36597 & 0.00000 \\ 1.15282 & 0.00000 \\ 3.85126 & 0.90086 \\ 4.60146 & 0.00000 \\ 3.85126 & -0.90086 \\ 2.06507 & 0.00000 \\ -0.92048 & -0.00000\end{array}$


Table S1491 Employed active space, reference configurations, and Cartesian coordinates of the OM2/MRCISD-optimized excited-state equilibrium geometry of the $2{ }^{1} A\left({ }^{1} \pi \pi^{*}\right)$ state of cis-o-cresol (excited-state energy: $-1340.51910 \mathrm{eV}$ ).

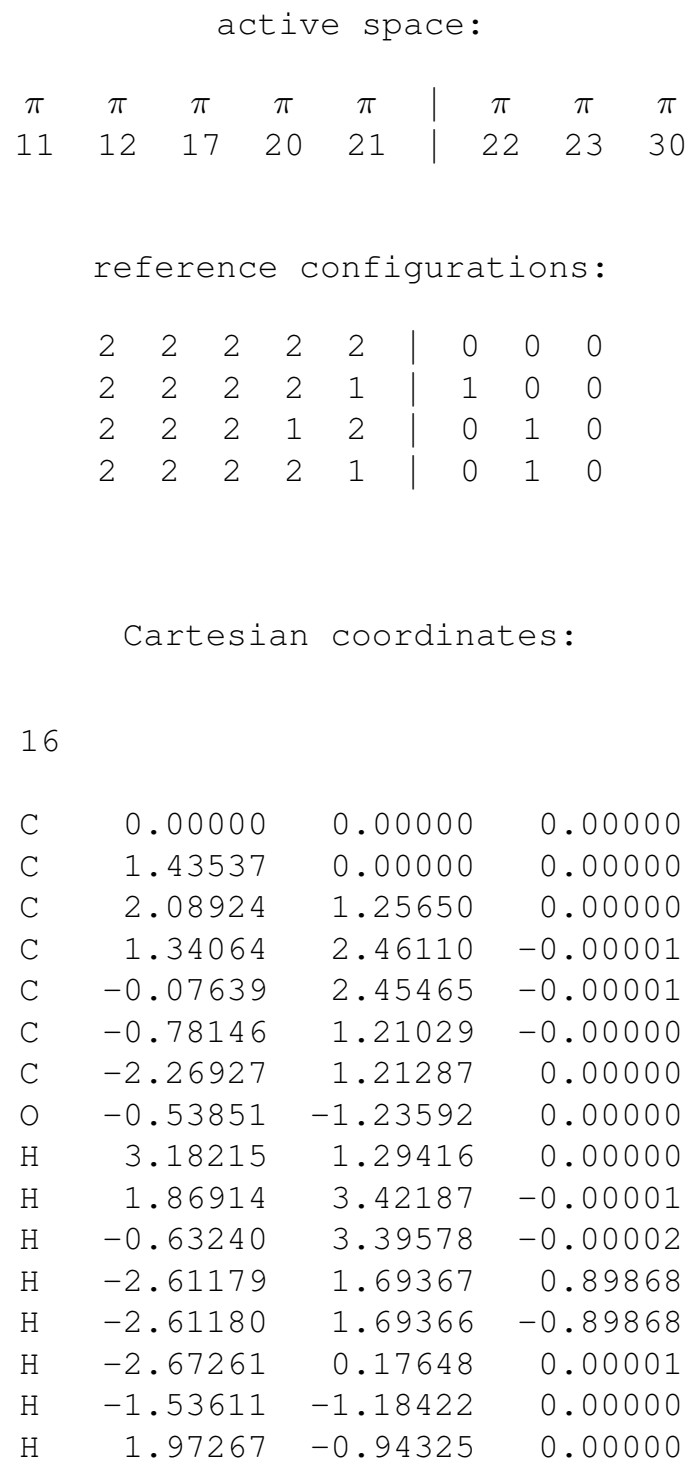


Table S1492 Employed active space, reference configurations, and Cartesian coordinates of the OM2/MRCISD-optimized ground-state equilibrium geometry of trans-o-cresol (ground-state energy: $-1344.76486 \mathrm{eV}$ ).

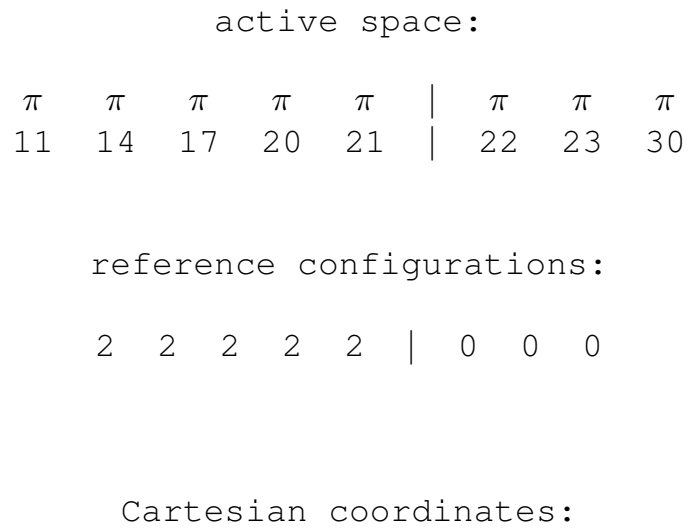

$\mathrm{H} \quad 3.94426 \quad 1.78260 \quad 0.89439$ 
Table S1493 Employed active space, reference configurations, and Cartesian coordinates of the OM2/MRCISD-optimized excited-state equilibrium geometry of the $2{ }^{1} A\left({ }^{1} \pi \pi^{*}\right)$ state of trans-o-cresol (excited-state energy: $-1340.61346 \mathrm{eV})$.

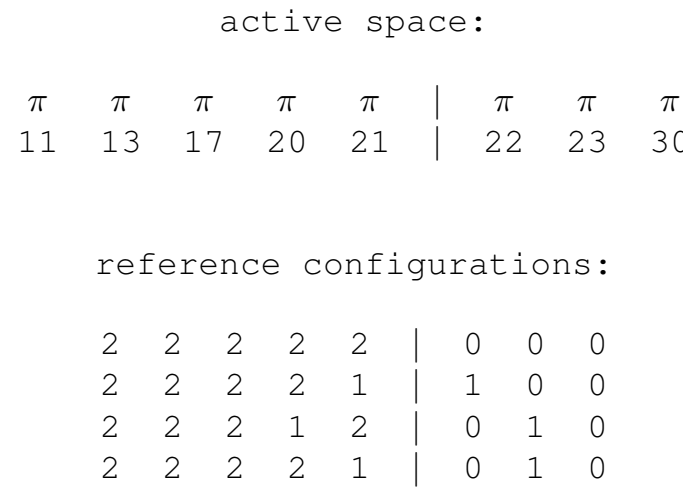

Cartesian coordinates:

16

$\begin{array}{lrrr}\mathrm{C} & 0.00000 & 0.00000 & 0.00000 \\ \mathrm{C} & 1.41435 & 0.00000 & 0.00000 \\ \mathrm{C} & 2.12146 & 1.24109 & 0.00000 \\ \mathrm{C} & 1.34263 & 2.44899 & 0.00005 \\ \mathrm{C} & -0.09020 & 2.45911 & 0.00005 \\ \mathrm{C} & -0.74679 & 1.20674 & 0.00003 \\ \mathrm{C} & 3.60506 & 1.32582 & -0.00003 \\ \mathrm{O} & 2.06597 & 3.58856 & 0.00005 \\ \mathrm{H} & -0.64237 & 3.39881 & 0.00004 \\ \mathrm{H} & -1.84027 & 1.16969 & 0.00004 \\ \mathrm{H} & -0.53623 & -0.95654 & -0.00003 \\ \mathrm{H} & 1.97201 & -0.93927 & -0.00002 \\ \mathrm{H} & 1.45124 & 4.37334 & 0.00008 \\ \mathrm{H} & 4.04136 & 0.31173 & -0.00015 \\ \mathrm{H} & 3.92544 & 1.84830 & -0.89507 \\ \mathrm{H} & 3.92548 & 1.84812 & 0.89508\end{array}$


Table S1494 Employed active space, reference configurations, and Cartesian coordinates of the OM2/MRCISD-optimized ground-state equilibrium geometry of cis- $m$-cresol (ground-state energy: $-1344.75668 \mathrm{eV}$ ).

\begin{tabular}{ccccc|ccc}
\multicolumn{10}{c}{ active space: } \\
\begin{tabular}{ccccc|ccc}
$\pi$ & $\pi$ & $\pi$ & $\pi$ & $\pi$ & $\pi$ & $\pi$ & $\pi$ \\
11 & 12 & 17 & 20 & 21 & 22 & 23 & 30
\end{tabular}
\end{tabular}

reference configurations:

\begin{tabular}{lllll|lll}
2 & 2 & 2 & 2 & 2 & 0 & 0 & 0
\end{tabular}

Cartesian coordinates:

$\begin{array}{lrrr}16 & & & \\ & & & \\ \text { C } & 0.00000 & 0.00000 & 0.00000 \\ \text { C } & 1.39332 & 0.00000 & 0.00000 \\ \text { C } & 2.11230 & 1.20000 & 0.00000 \\ \text { C } & 1.43610 & 2.42959 & 0.00000 \\ \text { C } & 0.03417 & 2.46682 & 0.00000 \\ \text { C } & -0.65649 & 1.24525 & 0.00000 \\ \text { C } & 2.20199 & 3.71656 & 0.00000 \\ \text { O } & -2.01924 & 1.17775 & 0.00000 \\ \text { H } & -0.58361 & -0.91791 & -0.00000 \\ \text { H } & 1.93188 & -0.95487 & -0.00000 \\ \text { H } & 3.20710 & 1.18111 & 0.00000 \\ \text { H } & -0.50755 & 3.41555 & 0.00000 \\ \text { H } & -2.39398 & 2.10018 & 0.00000 \\ \text { H } & 1.95322 & 4.28650 & 0.89775 \\ \text { H } & 3.28597 & 3.51671 & 0.00000 \\ \text { H } & 1.95322 & 4.28650 & -0.89775\end{array}$


Table S1495 Employed active space, reference configurations, and Cartesian coordinates of the OM2/MRCISD-optimized excited-state equilibrium geometry of the $2{ }^{1} A\left({ }^{1} \pi \pi^{*}\right)$ state of cis-m-cresol (excited-state energy: $\left.-1340.54907 \mathrm{eV}\right)$.

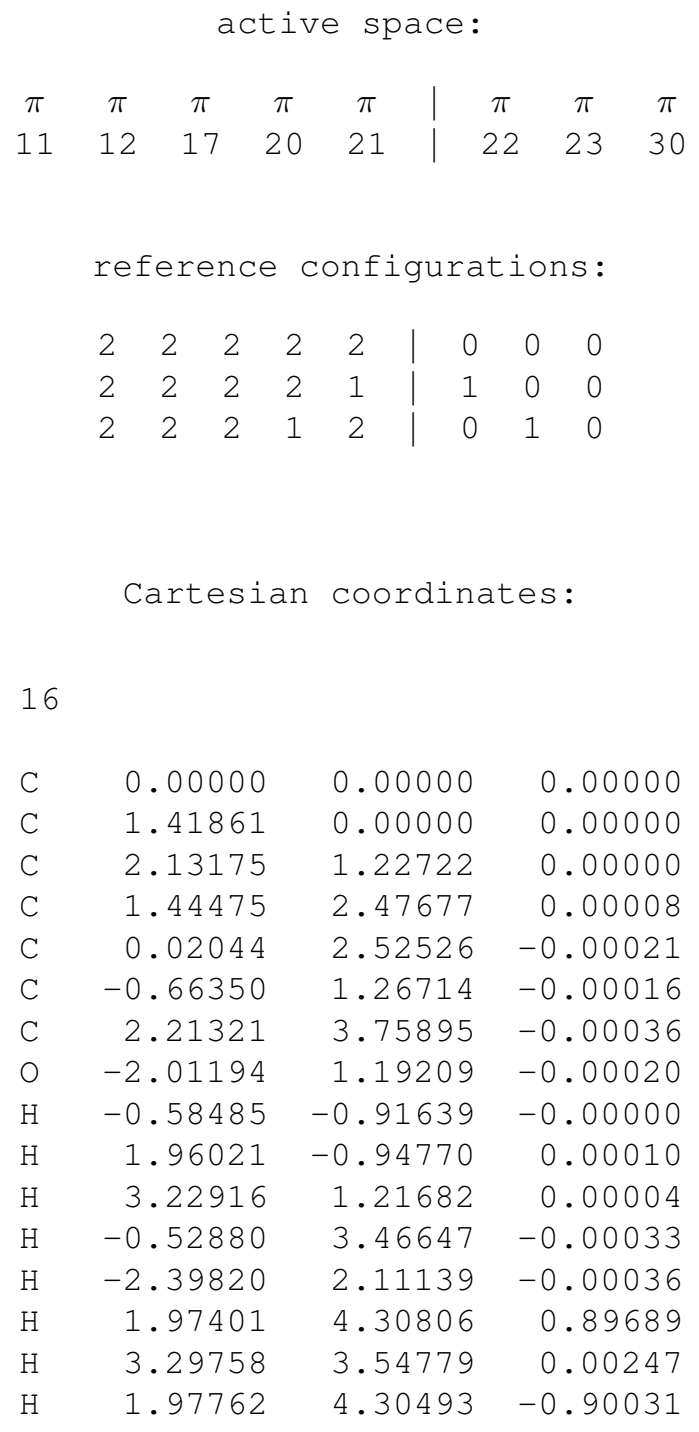


Table S1496 Employed active space, reference configurations, and Cartesian coordinates of the OM2/MRCISD-optimized ground-state equilibrium geometry of trans-m-cresol (ground-state energy: $-1344.76023 \mathrm{eV}$ ).

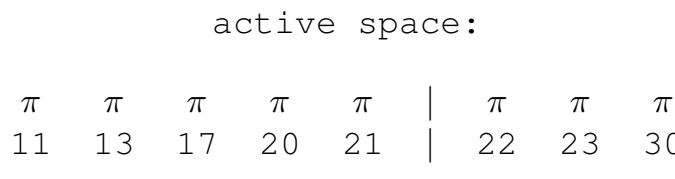

reference configurations:

$\begin{array}{lllllllll}2 & 2 & 2 & 2 & 2 & 0 & 0 & 0\end{array}$

Cartesian coordinates:

$\begin{array}{lrrr}16 & & & \\ & & & \\ \mathrm{C} & 0.00000 & 0.00000 & 0.00000 \\ \mathrm{C} & 1.39522 & 0.00000 & 0.00000 \\ \mathrm{C} & 2.10661 & 1.20241 & 0.00000 \\ \mathrm{C} & 1.42303 & 2.42984 & 0.00000 \\ \mathrm{C} & 0.02308 & 2.46650 & 0.00000 \\ \mathrm{C} & -0.66221 & 1.23939 & 0.00000 \\ \mathrm{C} & 2.18548 & 3.71915 & 0.00000 \\ \mathrm{O} & -2.02308 & 1.33931 & 0.00000 \\ \mathrm{H} & -0.56612 & -0.93411 & -0.00000 \\ \mathrm{H} & 1.93580 & -0.95365 & -0.00000 \\ \mathrm{H} & 3.20149 & 1.18863 & 0.00000 \\ \mathrm{H} & -0.53613 & 3.39970 & 0.00000 \\ \mathrm{H} & -2.41904 & 0.42584 & 0.00000 \\ \mathrm{H} & 1.93348 & 4.28856 & 0.89741 \\ \mathrm{H} & 3.27037 & 3.52371 & 0.00000 \\ \mathrm{H} & 1.93348 & 4.28856 & -0.89741\end{array}$


Table S1497 Employed active space, reference configurations, and Cartesian coordinates of the OM2/MRCISD-optimized excited-state equilibrium geometry of the $2^{1} A^{\prime}\left({ }^{1} \pi \pi^{*}\right)$ state of trans-m-cresol (excited-state energy: $-1340.55320 \mathrm{eV})$.

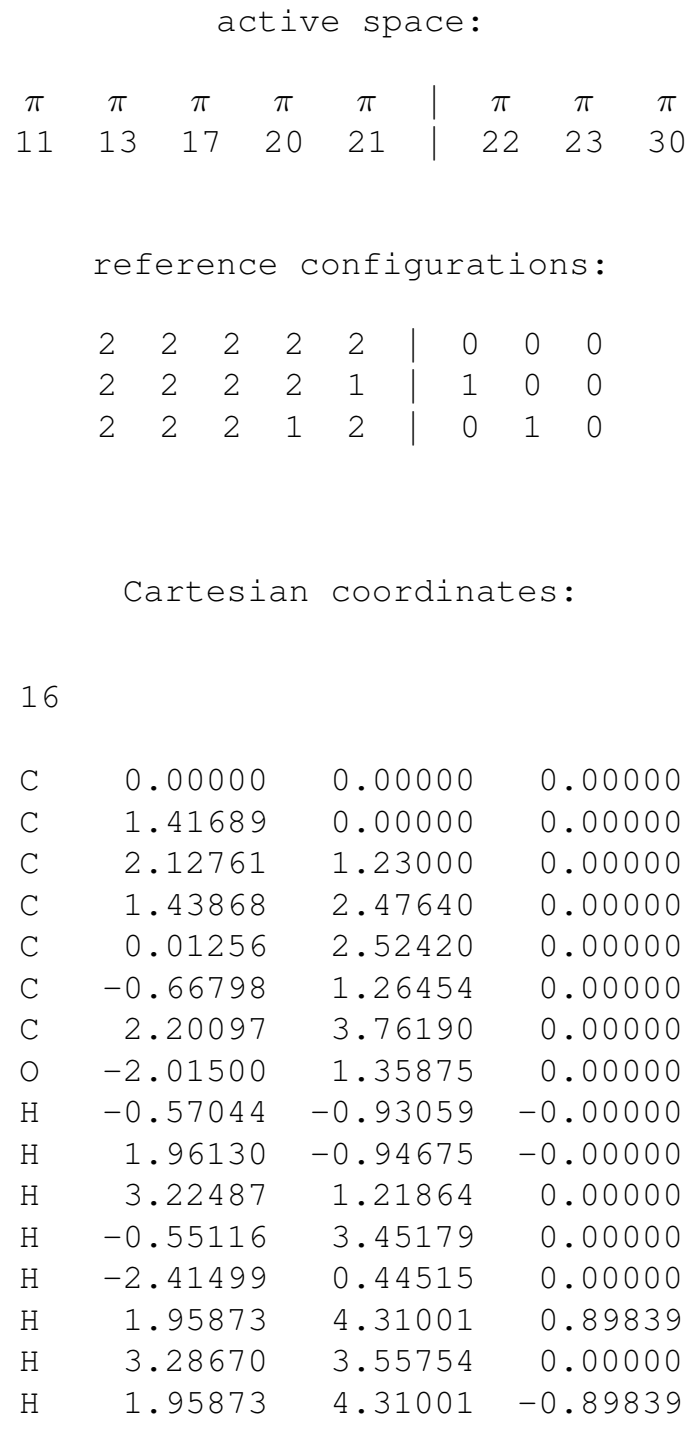


Table S1498 Employed active space, reference configurations, and Cartesian coordinates of the OM2/MRCISD-optimized ground-state equilibrium geometry of $p$-cresol (ground-state energy: $-1344.74914 \mathrm{eV}$ ).

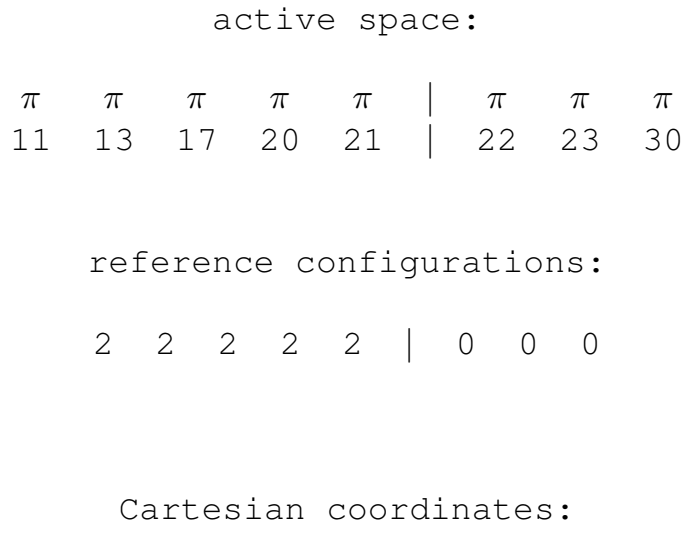

16

C $\quad 0.00000$

0.00000

0.00000

C $\quad 1.40658$

0.00000

0.00000

C 2.11485

1.19829

0.00000

C $\quad 1.38068$

2.39935

0.00000

$-0.02314$

2. 42672

0.00000

C $\quad-0.70799$

1. 21130

0.00000

2.13518

3. 53622

0.00000

$-0.73563$

$-1.30344$

$-0.00000$

$\mathrm{H} \quad-0.56109$

3. 37788

0.00000

$-1.80485$

1.20457

0.00000

1.95085

$-0.95150$

$-0.00000$

3.20261

1. 23196

0.00000

1.53313

4.32922

0.00000

$-0.47298$

$-1.86966$

0.89780

$-0.47298$

$-1.86966$

$-0.89780$

$\mathrm{H} \quad-1.82485$

$-1.13086-0.00000$ 
Table S1499 Employed active space, reference configurations, and Cartesian coordinates of the OM2/MRCISD-optimized excited-state equilibrium geometry of the $2{ }^{1} A\left({ }^{1} \pi \pi^{*}\right)$ state of $p$-cresol (excited-state energy: $-1340.60278 \mathrm{eV}$ ).

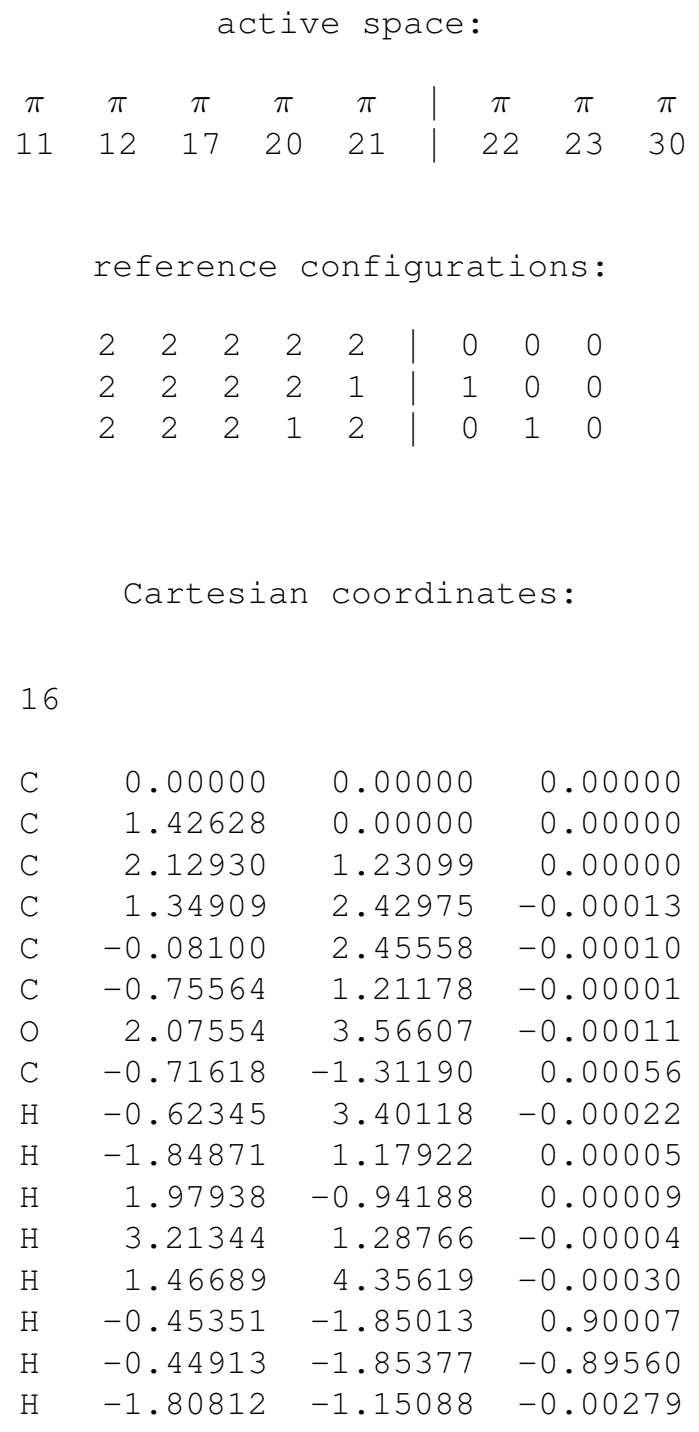


4.4.2 OM3/MRCI-Optimized Ground- and Excited-State Minima 
Table S1500 Employed active space, reference configurations, and Cartesian coordinates of the OM3/MRCISD-optimized ground-state equilibrium geometry of tetraphenylporphine (ground-state energy: $-6805.30218 \mathrm{eV}$ ).

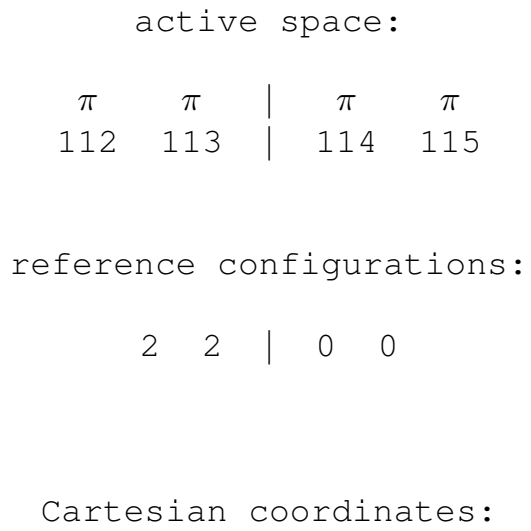

\begin{tabular}{rrrr}
$\mathrm{N}$ & 2.07523 & 0.00000 & 0.00000 \\
$\mathrm{~N}$ & -2.07523 & 0.00000 & 0.00000 \\
$\mathrm{~N}$ & 0.00000 & -2.04324 & -0.01139 \\
$\mathrm{~N}$ & 0.00000 & 2.04324 & -0.01139 \\
$\mathrm{C}$ & 2.89409 & -1.14625 & -0.01249 \\
$\mathrm{C}$ & 2.89409 & 1.14625 & -0.01249 \\
$\mathrm{C}$ & -2.89409 & 1.14625 & -0.01249 \\
$\mathrm{C}$ & -2.89409 & -1.14625 & -0.01249 \\
$\mathrm{C}$ & -1.10373 & -2.87993 & 0.00139 \\
$\mathrm{C}$ & 1.10373 & -2.87993 & 0.00139 \\
$\mathrm{C}$ & 1.10373 & 2.87993 & 0.00139 \\
$\mathrm{C}$ & -1.10373 & 2.87993 & 0.00139 \\
$\mathrm{C}$ & 2.46032 & 2.48980 & -0.00640 \\
$\mathrm{C}$ & 2.46032 & -2.48980 & -0.00640 \\
$\mathrm{C}$ & -2.46032 & -2.48980 & -0.00640 \\
$\mathrm{C}$ & -2.46032 & 2.48980 & -0.00640 \\
$\mathrm{C}$ & 4.28089 & -0.68640 & -0.04141 \\
$\mathrm{C}$ & 4.28089 & 0.68640 & -0.04141 \\
$\mathrm{C}$ & -4.28089 & 0.68640 & -0.04141 \\
$\mathrm{C}$ & -4.28089 & -0.68640 & -0.04141 \\
$\mathrm{C}$ & -0.67869 & -4.30898 & 0.03579 \\
$\mathrm{C}$ & 0.67869 & -4.30898 & 0.03579 \\
$\mathrm{C}$ & 0.67869 & 4.30898 & 0.03579 \\
$\mathrm{C}$ & -0.67869 & 4.30898 & 0.03579 \\
\hline & & $C 0 n t i n u e d$ & on \\
& & $-10 x t$ page
\end{tabular}




\begin{tabular}{|c|c|c|c|}
\hline \multicolumn{4}{|c|}{ Continued from previous page } \\
\hline $\bar{C}$ & 3.51089 & 3.54253 & -0.01040 \\
\hline$C$ & 3.51089 & -3.54253 & -0.01040 \\
\hline$C$ & -3.51089 & -3.54253 & -0.01040 \\
\hline C & -3.51089 & 3.54253 & -0.01040 \\
\hline$C$ & 4.24662 & 3.80804 & 5644 \\
\hline C & 4.24662 & -3.80804 & \\
\hline C & -4.24662 & -3.80804 & 644 \\
\hline C & -4.24662 & 3.80804 & 644 \\
\hline C & 3.76789 & 4.27367 & -1.18203 \\
\hline$C$ & 3.76789 & -4.27367 & -1.18203 \\
\hline C & -3.76789 & -4.27367 & -1.18203 \\
\hline C & -3.76789 & 4.27367 & -1.1 \\
\hline C & 5.23118 & 4.79875 & 1.1 \\
\hline C & 5.23118 & -4.79875 & 39 \\
\hline C & -5.23118 & -4.79875 & 39 \\
\hline C & -5.23118 & 4.79875 & 39 \\
\hline C & 4.75577 & 5.2 & -1 \\
\hline C & 4.75577 & -5.2 & -1 . \\
\hline C & -4.75577 & -5.2 & -1 \\
\hline C & -4.75577 & 5.2 & -1 . \\
\hline C & 5.48732 & 5.5 & -0 . \\
\hline C & 5.487 & -5.5 & -0 . \\
\hline C & -5.48732 & -5.5 & -0 . \\
\hline C & -5.48732 & 5.5 & -0 . \\
\hline H & 5.1 & $-1 \cdot 3$ & -0 . \\
\hline $\mathrm{H}$ & 5.1 & 1.3 & -0 . \\
\hline $\mathrm{H}$ & -5.13658 & 1.35608 & -0 . \\
\hline $\mathrm{H}$ & -5.13658 & -1.35608 & -0. \\
\hline $\mathrm{H}$ & -1.36390 & -5.15234 & 430 \\
\hline $\mathrm{H}$ & 1.36390 & -5.1 & 0. \\
\hline $\mathrm{H}$ & 1.36390 & 234 & 0.0 \\
\hline $\mathrm{H}$ & -1.36390 & 5.1 & 0.0 \\
\hline $\mathrm{H}$ & 4.03858 & 3.23773 & 2 . \\
\hline $\mathrm{H}$ & 4.03858 & -3.23773 & 452 \\
\hline $\mathrm{H}$ & -4.03858 & -3.23773 & 2.07452 \\
\hline $\mathrm{H}$ & -4.03858 & 3.23773 & 7452 \\
\hline $\mathrm{H}$ & 3.18917 & 4.06308 & -2 . \\
\hline $\mathrm{H}$ & 3.18917 & -4.06308 & -2.09410 \\
\hline $\mathrm{H}$ & -3.18917 & -4.06308 & -2.09410 \\
\hline $\mathrm{H}$ & -3.18917 & 4.06308 & -2.09410 \\
\hline $\mathrm{H}$ & 5.80658 & 5.01022 & 2.06346 \\
\hline $\mathrm{H}$ & 5.80658 & -5.01022 & 2.06346 \\
\hline $\mathrm{H}$ & -5.80658 & -5.01022 & 2.06346 \\
\hline $\mathrm{H}$ & -5.80658 & 5.01022 & 2.06346 \\
\hline
\end{tabular}

\section{S1632}




\begin{tabular}{lrrr}
\multicolumn{5}{c}{ Continued from previous page } \\
\hline H & 4.95913 & 5.83440 & -2.10183 \\
H & 4.95913 & -5.83440 & -2.10183 \\
H & -4.95913 & -5.83440 & -2.10183 \\
H & -4.95913 & 5.83440 & -2.10183 \\
H & 6.26522 & 6.30486 & -0.02404 \\
H & 6.26522 & -6.30486 & -0.02404 \\
H & -6.26522 & -6.30486 & -0.02404 \\
H & -6.26522 & 6.30486 & -0.02404 \\
H & 1.02862 & 0.00000 & 0.01026 \\
H & -1.02862 & 0.00000 & 0.01026
\end{tabular}


Table S1501 Employed active space, reference configurations, and Cartesian coordinates of the OM3/MRCISD-optimized excited-state equilibrium geometry of the $1{ }^{1} B_{2}\left({ }^{1} \pi \pi^{*}\right)$ state of tetraphenylporphine (excited-state energy: $-6802.84183 \mathrm{eV})$.

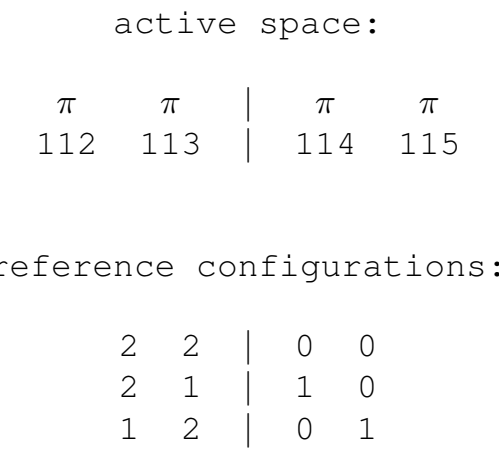

Cartesian coordinates:

\begin{tabular}{lrrr}
78 & & & \\
& & & \\
$\mathrm{~N}$ & 2.07503 & 0.00000 & 0.00000 \\
$\mathrm{~N}$ & -2.07503 & 0.00000 & 0.00000 \\
$\mathrm{~N}$ & 0.00000 & -2.05313 & -0.02536 \\
$\mathrm{~N}$ & 0.00000 & 2.05313 & -0.02536 \\
$\mathrm{C}$ & 2.89571 & -1.14736 & -0.02764 \\
$\mathrm{C}$ & 2.89571 & 1.14736 & -0.02764 \\
$\mathrm{C}$ & -2.89571 & 1.14736 & -0.02764 \\
$\mathrm{C}$ & -2.89571 & -1.14736 & -0.02764 \\
$\mathrm{C}$ & -1.10470 & -2.89172 & 0.00269 \\
$\mathrm{C}$ & 1.10470 & -2.89172 & 0.00269 \\
$\mathrm{C}$ & 1.10470 & 2.89172 & 0.00269 \\
$\mathrm{C}$ & -1.10470 & 2.89172 & 0.00269 \\
$\mathrm{C}$ & 2.46501 & 2.49301 & -0.01587 \\
$\mathrm{C}$ & 2.46501 & -2.49301 & -0.01587 \\
$\mathrm{C}$ & -2.46501 & -2.49301 & -0.01587 \\
$\mathrm{C}$ & -2.46501 & 2.49301 & -0.01587 \\
$\mathrm{C}$ & 4.28195 & -0.68650 & -0.08710 \\
$\mathrm{C}$ & 4.28195 & 0.68650 & -0.08710 \\
$\mathrm{C}$ & -4.28195 & 0.68650 & -0.08710 \\
$\mathrm{C}$ & -4.28195 & -0.68650 & -0.08710 \\
$\mathrm{C}$ & -0.68007 & -4.31495 & 0.06998 \\
$\mathrm{C}$ & 0.68007 & -4.31495 & 0.06998 \\
\hline & & Continued & on next page
\end{tabular}

S1634 


\begin{tabular}{|c|c|c|c|}
\hline \multicolumn{4}{|c|}{ atinued from previous page } \\
\hline $\mathrm{C}$ & 0.68007 & 4.31495 & 0.06998 \\
\hline & -0.68007 & 4.31495 & 0.06998 \\
\hline & 3.51515 & 3.54233 & -0.02499 \\
\hline & 3.51515 & -3.54233 & -0.02499 \\
\hline & -3.51515 & -3.54233 & -0.02499 \\
\hline & -3.51515 & 3.54233 & -0.02499 \\
\hline & 4.34331 & 3.72291 & 1.09611 \\
\hline & 4.34331 & -3.72291 & 1.09611 \\
\hline & -4.34331 & -3.72291 & \\
\hline & -4.343 & 3.72291 & \\
\hline & 3.682 & 4.35935 & -1 \\
\hline & 3.6 & $-4 \cdot 3$ & -1 \\
\hline & -3.6 & -4 . & -1 \\
\hline & -3 & & -1 \\
\hline & & & \\
\hline & 5 . & -4 & \\
\hline & -5 & -4 & \\
\hline & -5 & 4.7 & \\
\hline & 4.6 & & -1 \\
\hline & & -5 & -1 \\
\hline & -4.6 & -5.34452 & -1 \\
\hline & -4.6 & 5.3 & -1 \\
\hline & 5. & & -0 \\
\hline & 5. & -5 . & -0 \\
\hline & -5.4 & -5 . & -0 \\
\hline & -5.4 & & -0 \\
\hline & 5 . & -1 & -0 \\
\hline & 5 . & & -0 \\
\hline & -5 & & -0 \\
\hline & -5 & -1 & -0 \\
\hline & -1 & -5 & \\
\hline & & -5 . & \\
\hline & & & \\
\hline & $-1 \cdot 3$ & & \\
\hline[ & & & \\
\hline $\mathrm{H}$ & & -3.0 & \\
\hline I & -4.20456 & -3.0 & \\
\hline $\mathrm{H}$ & -4.204 & & \\
\hline $\mathrm{H}$ & 3.0 & 4.2 & -2 \\
\hline H & & -4.214 & -2.033 \\
\hline $\mathrm{H}$ & -3.03409 & -4.21497 & -2.03370 \\
\hline 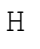 & -3.034 & 4.21497 & -2.03370 \\
\hline . & 5.9 & 4.8 & 1.96323 \\
\hline 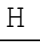 & 5.97409 & -4.85819 & 1.96323 \\
\hline
\end{tabular}

Continued on next page

\section{S1635}




\begin{tabular}{lrrr}
\multicolumn{5}{c}{ Continued from previous page } \\
\hline H & -5.97409 & -4.85819 & 1.96323 \\
H & -5.97409 & 4.85819 & 1.96323 \\
H & 4.80579 & 5.98328 & -2.05109 \\
H & 4.80579 & -5.98328 & -2.05109 \\
H & -4.80579 & -5.98328 & -2.05109 \\
H & -4.80579 & 5.98328 & -2.05109 \\
H & 6.27375 & 6.30230 & -0.05403 \\
H & 6.27375 & -6.30230 & -0.05403 \\
H & -6.27375 & -6.30230 & -0.05403 \\
H & -6.27375 & 6.30230 & -0.05403 \\
H & 1.02965 & 0.00000 & 0.02032 \\
H & -1.02965 & 0.00000 & 0.02032
\end{tabular}


Table S1502 Employed active space, reference configurations, and Cartesian coordinates of the OM3/MRCISD-optimized ground-state equilibrium geometry of porphycene (ground-state energy: $-3568.04390 \mathrm{eV}$ ).

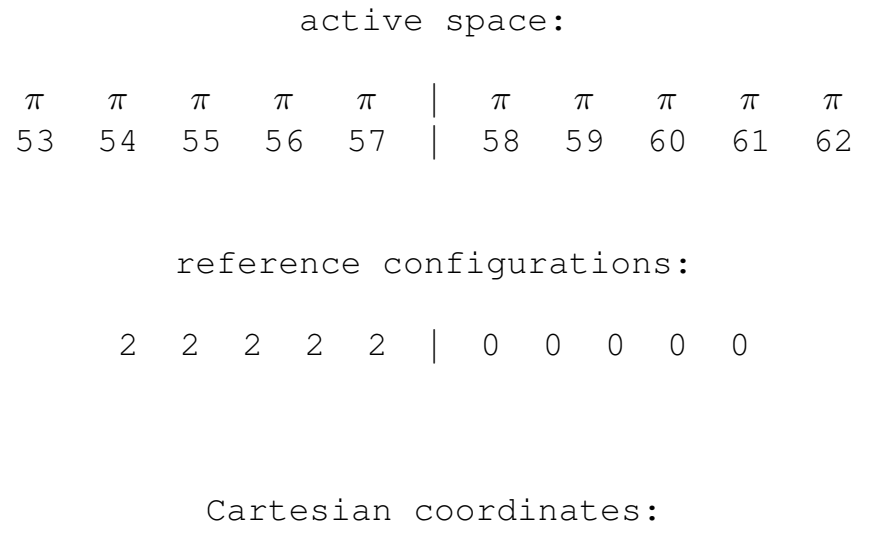

\begin{tabular}{lrrr}
38 & & & \\
& & & \\
$\mathrm{~N}$ & 0.00000 & 0.00000 & 0.00000 \\
$\mathrm{~N}$ & 3.82665 & 0.00000 & 0.00000 \\
$\mathrm{~N}$ & 1.55283 & 1.87864 & 0.00000 \\
$\mathrm{~N}$ & 2.27306 & -1.87955 & 0.00000 \\
$\mathrm{C}$ & -1.26222 & 0.57983 & 0.00000 \\
$\mathrm{C}$ & 5.09053 & -0.57478 & 0.00000 \\
$\mathrm{C}$ & -0.15492 & -1.37563 & 0.00000 \\
$\mathrm{C}$ & 3.97992 & 1.38239 & -0.00000 \\
$\mathrm{C}$ & -2.26866 & -0.51550 & 0.00000 \\
$\mathrm{C}$ & 6.09051 & 0.50495 & 0.00000 \\
$\mathrm{C}$ & -1.58855 & -1.69718 & 0.00000 \\
$\mathrm{C}$ & 5.40778 & 1.70133 & 0.00000 \\
$\mathrm{C}$ & -1.55509 & 1.96381 & 0.00000 \\
$\mathrm{C}$ & 5.38108 & -1.97151 & 0.00000 \\
$\mathrm{C}$ & 0.74578 & 3.00915 & -0.00000 \\
$\mathrm{C}$ & 3.07547 & -3.01265 & 0.00000 \\
$\mathrm{C}$ & 2.87471 & 2.28973 & -0.00000 \\
$\mathrm{C}$ & 0.94424 & -2.29029 & 0.00000 \\
$\mathrm{C}$ & 1.63208 & 4.20379 & -0.00000 \\
$\mathrm{C}$ & 2.20328 & -4.19799 & 0.00000 \\
$\mathrm{C}$ & 2.92066 & 3.75826 & -0.00000 \\
$\mathrm{C}$ & 0.89977 & -3.75266 & 0.00000 \\
$\mathrm{C}$ & -0.66859 & 3.03632 & -0.00000 \\
$\mathrm{C}$ & 4.50193 & -3.03513 & 0.00000 \\
\hline & & Continued & on \\
& & &
\end{tabular}




\begin{tabular}{lrrr}
\multicolumn{4}{c}{ Continued from previous page } \\
\hline $\mathrm{H}$ & 0.99264 & 0.76057 & -0.00000 \\
$\mathrm{H}$ & 2.83338 & -0.76083 & -0.00000 \\
$\mathrm{H}$ & -3.34474 & -0.35309 & 0.00000 \\
$\mathrm{H}$ & 7.16710 & 0.34852 & 0.00000 \\
$\mathrm{H}$ & -1.98872 & -2.70687 & 0.00000 \\
$\mathrm{H}$ & 5.81763 & 2.70767 & -0.00000 \\
$\mathrm{H}$ & -2.63543 & 2.20579 & 0.00000 \\
$\mathrm{H}$ & 6.46312 & -2.20706 & 0.00000 \\
$\mathrm{H}$ & 1.27003 & 5.23006 & -0.00000 \\
$\mathrm{H}$ & 2.55957 & -5.22589 & 0.00000 \\
$\mathrm{H}$ & 3.83698 & 4.34132 & -0.00000 \\
$\mathrm{H}$ & -0.01144 & -4.34461 & 0.00000 \\
$\mathrm{H}$ & -1.10956 & 4.05181 & -0.00000 \\
$\mathrm{H}$ & 4.93691 & -4.05350 & 0.00000
\end{tabular}


Table S1503 Employed active space, reference configurations, and Cartesian coordinates of the OM3/MRCISD-optimized excited-state equilibrium geometry of the $1^{1} B_{u}\left({ }^{1} \pi \pi^{*}\right)$ state of porphycene (excited-state energy: $\left.-3566.01947 \mathrm{eV}\right)$.

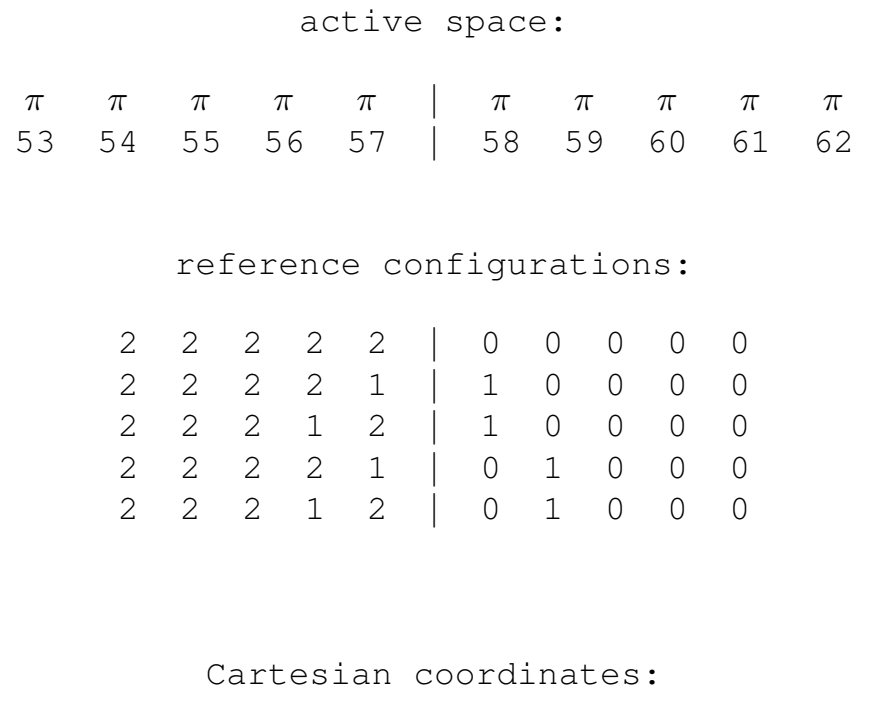

\begin{tabular}{lrrr}
38 & & & \\
& & & \\
$\mathrm{~N}$ & 0.00000 & 0.00000 & 0.00000 \\
$\mathrm{~N}$ & 3.84316 & 0.00000 & 0.00000 \\
$\mathrm{~N}$ & 1.56314 & 1.88003 & 0.00000 \\
$\mathrm{~N}$ & 2.28002 & -1.88003 & 0.00000 \\
$\mathrm{C}$ & -1.25433 & 0.57330 & 0.00000 \\
$\mathrm{C}$ & 5.09749 & -0.57330 & 0.00000 \\
$\mathrm{C}$ & -0.15283 & -1.39595 & 0.00000 \\
$\mathrm{C}$ & 3.99599 & 1.39595 & -0.00000 \\
$\mathrm{C}$ & -2.25601 & -0.51701 & 0.00000 \\
$\mathrm{C}$ & 6.09917 & 0.51701 & 0.00000 \\
$\mathrm{C}$ & -1.57320 & -1.70851 & 0.00000 \\
$\mathrm{C}$ & 5.41636 & 1.70851 & 0.00000 \\
$\mathrm{C}$ & -1.55238 & 1.97133 & 0.00000 \\
$\mathrm{C}$ & 5.39555 & -1.97133 & 0.00000 \\
$\mathrm{C}$ & 0.76071 & 3.00886 & -0.00000 \\
$\mathrm{C}$ & 3.08246 & -3.00886 & 0.00000 \\
$\mathrm{C}$ & 2.89336 & 2.30522 & -0.00000 \\
$\mathrm{C}$ & 0.94980 & -2.30522 & 0.00000 \\
$\mathrm{C}$ & 1.62451 & 4.20070 & -0.00000 \\
$\mathrm{C}$ & 2.21865 & -4.20070 & 0.00000 \\
$\mathrm{C}$ & 2.93330 & 3.76393 & -0.00000 \\
\hline & & Continued & on next page
\end{tabular}

S1639 


\begin{tabular}{lrrr}
\multicolumn{4}{c}{ Continued from previous page } \\
\hline $\mathrm{C}$ & 0.90986 & -3.76393 & 0.00000 \\
$\mathrm{C}$ & -0.66941 & 3.03252 & -0.00000 \\
$\mathrm{C}$ & 4.51257 & -3.03252 & 0.00000 \\
$\mathrm{H}$ & 0.96309 & 0.70762 & -0.00000 \\
$\mathrm{H}$ & 2.88008 & -0.70762 & -0.00000 \\
$\mathrm{H}$ & -3.33269 & -0.35823 & 0.00000 \\
$\mathrm{H}$ & 7.17585 & 0.35823 & 0.00000 \\
$\mathrm{H}$ & -1.98219 & -2.71432 & 0.00000 \\
$\mathrm{H}$ & 5.82535 & 2.71432 & -0.00000 \\
$\mathrm{H}$ & -2.63313 & 2.20438 & 0.00000 \\
$\mathrm{H}$ & 6.47629 & -2.20438 & 0.00000 \\
$\mathrm{H}$ & 1.26225 & 5.22632 & -0.00000 \\
$\mathrm{H}$ & 2.58091 & -5.22632 & 0.00000 \\
$\mathrm{H}$ & 3.83950 & 4.36185 & -0.00000 \\
$\mathrm{H}$ & 0.00366 & -4.36185 & 0.00000 \\
$\mathrm{H}$ & -1.10180 & 4.05183 & -0.00000 \\
$\mathrm{H}$ & 4.94496 & -4.05183 & 0.00000
\end{tabular}


Table S1504 Employed active space, reference configurations, and Cartesian coordinates of the OM3/MRCISD-optimized ground-state equilibrium geometry of porphine (ground-state energy: $-3567.52040 \mathrm{eV}$ ).

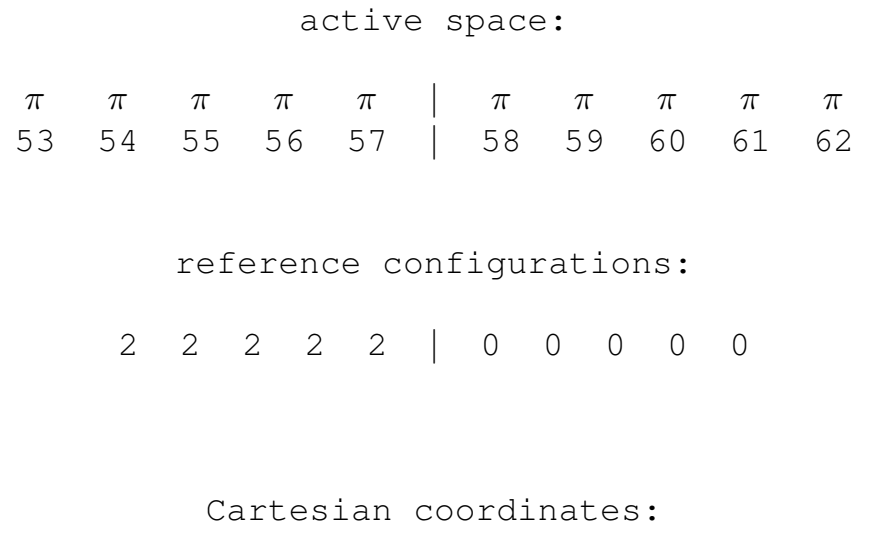

\begin{tabular}{lrrr}
38 & & & \\
& & & \\
$\mathrm{~N}$ & 2.07017 & 0.00000 & 0.00000 \\
$\mathrm{~N}$ & 0.00000 & 2.03630 & 0.00000 \\
$\mathrm{~N}$ & -2.07017 & 0.00000 & 0.00000 \\
$\mathrm{~N}$ & 0.00000 & -2.03630 & -0.00000 \\
$\mathrm{C}$ & 2.88723 & 1.14264 & 0.00000 \\
$\mathrm{C}$ & 2.88723 & -1.14264 & -0.00000 \\
$\mathrm{C}$ & -2.88723 & 1.14264 & 0.00000 \\
$\mathrm{C}$ & -2.88723 & -1.14264 & 0.00000 \\
$\mathrm{C}$ & 1.10388 & 2.87192 & 0.00000 \\
$\mathrm{C}$ & -1.10388 & 2.87192 & 0.00000 \\
$\mathrm{C}$ & 1.10388 & -2.87192 & -0.00000 \\
$\mathrm{C}$ & -1.10388 & -2.87192 & -0.00000 \\
$\mathrm{C}$ & 4.28108 & 0.68323 & 0.00000 \\
$\mathrm{C}$ & 4.28108 & -0.68323 & -0.00000 \\
$\mathrm{C}$ & -4.28108 & 0.68323 & 0.00000 \\
$\mathrm{C}$ & -4.28108 & -0.68323 & 0.00000 \\
$\mathrm{C}$ & 0.67823 & 4.30695 & -0.00000 \\
$\mathrm{C}$ & -0.67823 & 4.30695 & 0.00000 \\
$\mathrm{C}$ & 0.67823 & -4.30695 & -0.00000 \\
$\mathrm{C}$ & -0.67823 & -4.30695 & -0.00000 \\
$\mathrm{C}$ & 2.45731 & 2.47879 & 0.00000 \\
$\mathrm{C}$ & 2.45731 & -2.47879 & -0.00000 \\
$\mathrm{C}$ & -2.45731 & 2.47879 & 0.00000 \\
$\mathrm{C}$ & -2.45731 & -2.47879 & -0.00000 \\
\hline & & Continued & on $n$ next page
\end{tabular}




\begin{tabular}{lrrr}
\multicolumn{5}{c}{ Continued from previous page } \\
\hline $\mathrm{H}$ & 5.13477 & 1.35723 & 0.00000 \\
$\mathrm{H}$ & 5.13477 & -1.35723 & -0.00000 \\
$\mathrm{H}$ & -5.13477 & 1.35723 & 0.00000 \\
$\mathrm{H}$ & -5.13477 & -1.35723 & 0.00000 \\
$\mathrm{H}$ & 1.36643 & 5.14898 & -0.00000 \\
$\mathrm{H}$ & -1.36643 & 5.14898 & 0.00000 \\
$\mathrm{H}$ & 1.36643 & -5.14898 & -0.00000 \\
$\mathrm{H}$ & -1.36643 & -5.14898 & -0.00000 \\
$\mathrm{H}$ & 3.22943 & 3.26391 & 0.00000 \\
$\mathrm{H}$ & 3.22943 & -3.26391 & -0.00000 \\
$\mathrm{H}$ & -3.22943 & 3.26391 & 0.00000 \\
$\mathrm{H}$ & -3.22943 & -3.26391 & 0.00000 \\
$\mathrm{H}$ & 1.02511 & 0.00000 & -0.00000 \\
$\mathrm{H}$ & -1.02511 & 0.00000 & 0.00000
\end{tabular}


Table S1505 Employed active space, reference configurations, and Cartesian coordinates of the OM3/MRCISD-optimized excited-state equilibrium geometry of the $1{ }^{1} B_{3 u}\left({ }^{1} \pi \pi^{*}\right)$ state of porphine (excited-state energy: $-3565.49464 \mathrm{eV}$ ).

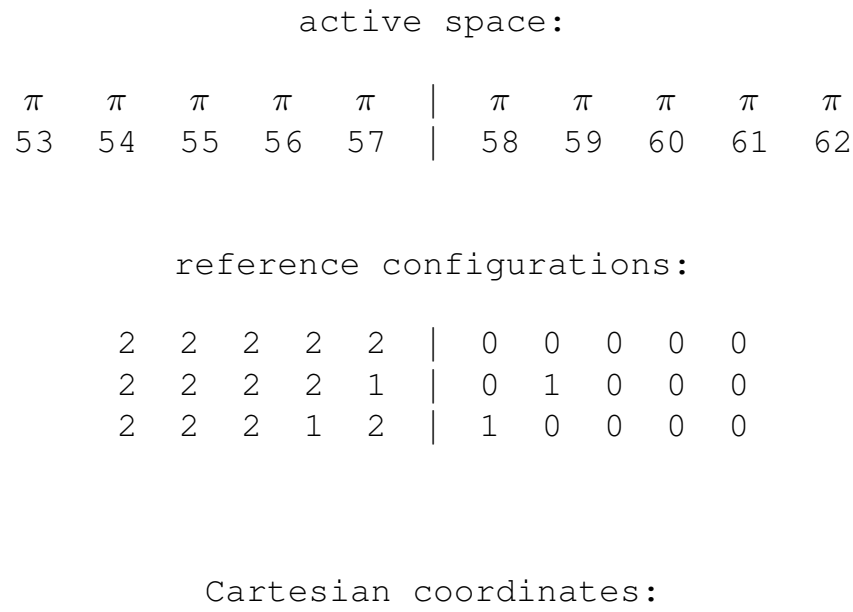




\begin{tabular}{lrrr}
\multicolumn{5}{c}{ Continued from previous page } \\
\hline $\mathrm{C}$ & -2.46474 & -2.48248 & -0.00000 \\
$\mathrm{H}$ & 5.13513 & 1.35804 & 0.00000 \\
$\mathrm{H}$ & 5.13513 & -1.35804 & -0.00000 \\
$\mathrm{H}$ & -5.13513 & 1.35804 & 0.00000 \\
$\mathrm{H}$ & -5.13513 & -1.35804 & 0.00000 \\
$\mathrm{H}$ & 1.36413 & 5.16121 & -0.00000 \\
$\mathrm{H}$ & -1.36413 & 5.16121 & 0.00000 \\
$\mathrm{H}$ & 1.36413 & -5.16121 & -0.00000 \\
$\mathrm{H}$ & -1.36413 & -5.16121 & -0.00000 \\
$\mathrm{H}$ & 3.23985 & 3.26447 & 0.00000 \\
$\mathrm{H}$ & 3.23985 & -3.26447 & -0.00000 \\
$\mathrm{H}$ & -3.23985 & 3.26447 & 0.00000 \\
$\mathrm{H}$ & -3.23985 & -3.26447 & 0.00000 \\
$\mathrm{H}$ & 1.02477 & 0.00000 & -0.00000 \\
$\mathrm{H}$ & -1.02477 & 0.00000 & 0.00000
\end{tabular}


Table S1506 Employed active space, reference configurations, and Cartesian coordinates of the OM3/MRCISD-optimized ground-state equilibrium geometry of tetracene (ground-state energy: $-2429.54076 \mathrm{eV}$ ).

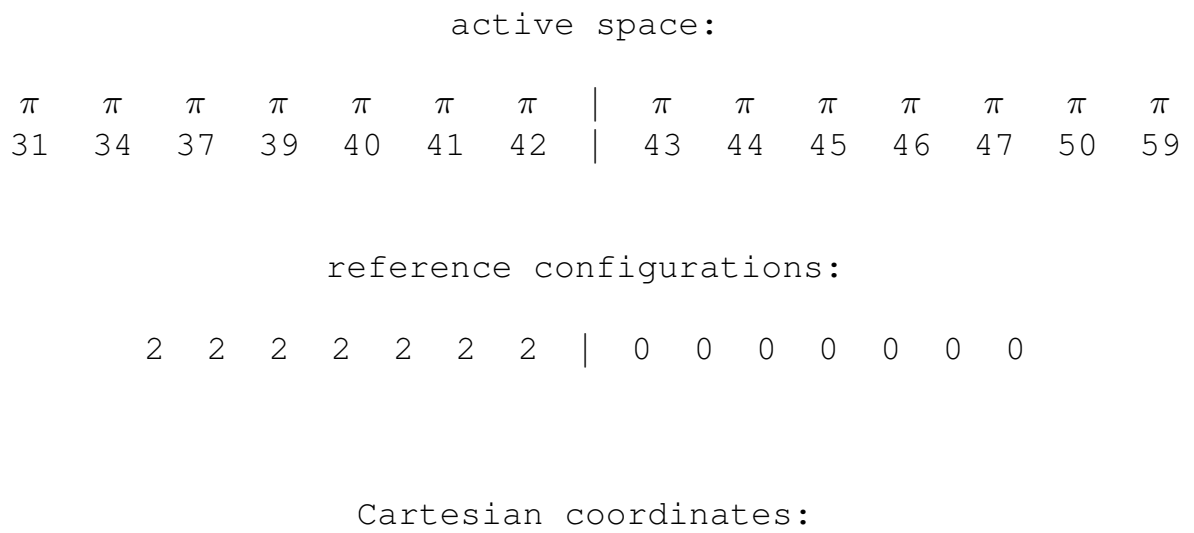




\begin{tabular}{lrrr}
\multicolumn{4}{c}{ Continued from previous page } \\
\hline $\mathrm{H}$ & 4.70852 & -1.51548 & 0.00000 \\
$\mathrm{H}$ & -2.70852 & -1.51548 & 0.00000 \\
$\mathrm{H}$ & 6.86834 & 2.24435 & 0.00000 \\
$\mathrm{H}$ & -4.86834 & 2.24435 & 0.00000 \\
$\mathrm{H}$ & 6.86834 & -0.24435 & 0.00000 \\
$\mathrm{H}$ & -4.86834 & -0.24435 & -0.00000
\end{tabular}


Table S1507 Employed active space, reference configurations, and Cartesian coordinates of the OM3/MRCISD-optimized excited-state equilibrium geometry of the $1{ }^{1} B_{2 u}\left({ }^{1} \pi \pi^{*}\right)$ state of tetracene (excited-state energy: $\left.-2426.27569 \mathrm{eV}\right)$.

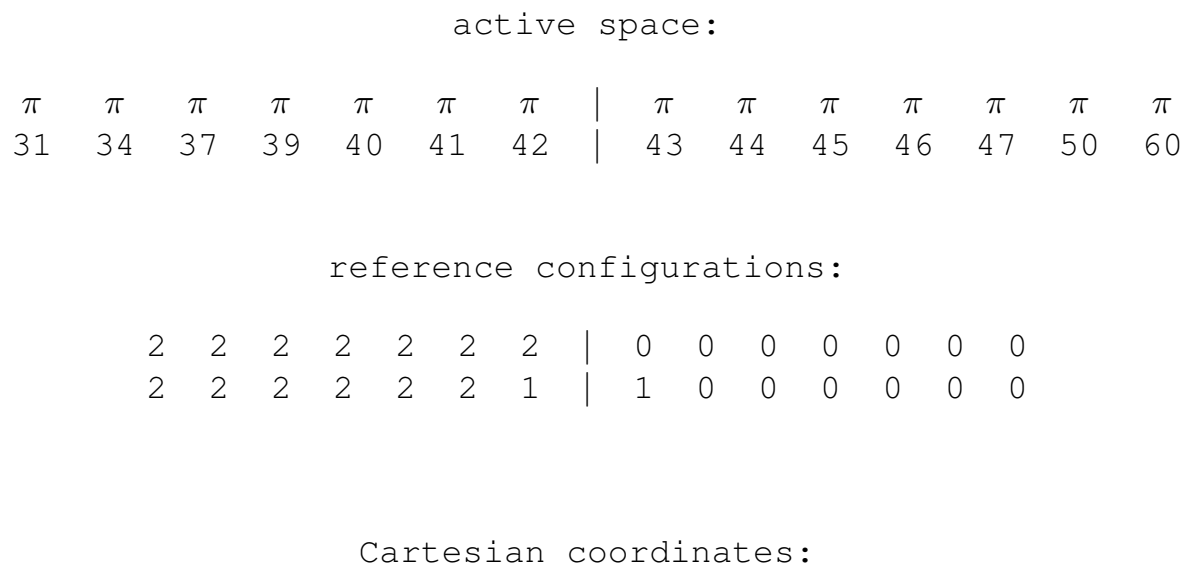

Continued on next page 


\begin{tabular}{lrrr}
\multicolumn{4}{c}{ Continued from previous page } \\
\hline $\mathrm{H}$ & 4.71555 & -1.51148 & 0.00000 \\
$\mathrm{H}$ & -2.71555 & -1.51148 & 0.00000 \\
$\mathrm{H}$ & 6.87459 & 2.24773 & 0.00000 \\
$\mathrm{H}$ & -4.87459 & 2.24773 & 0.00000 \\
$\mathrm{H}$ & 6.87459 & -0.24773 & 0.00000 \\
$\mathrm{H}$ & -4.87459 & -0.24773 & -0.00000
\end{tabular}


Table S1508 Employed active space, reference configurations, and Cartesian coordinates of the OM3/MRCISD-optimized ground-state equilibrium geometry of perylene (ground-state energy: $-2679.56291 \mathrm{eV}$ ).

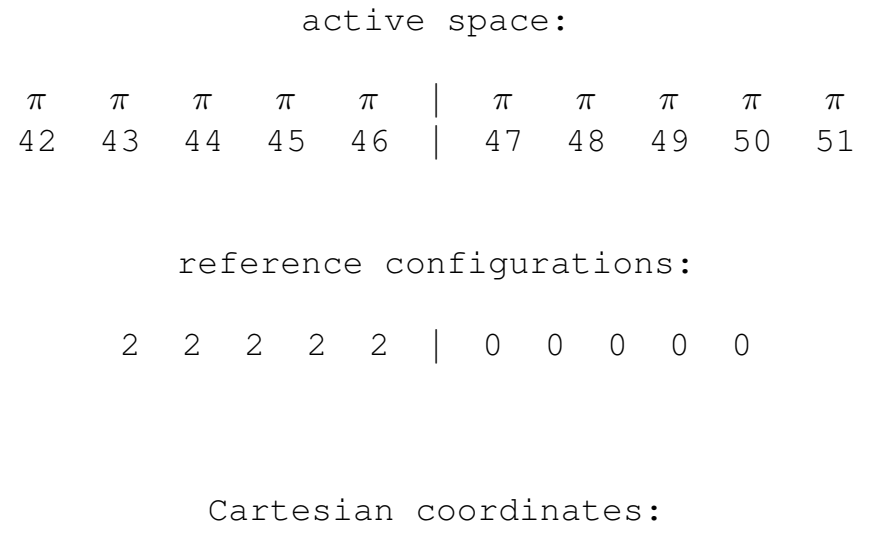

\begin{tabular}{lrrr}
32 & & & \\
& & & \\
C & 1.43501 & 0.00000 & 0.00000 \\
C & -1.43501 & 0.00000 & 0.00000 \\
C & 2.86628 & 0.00000 & -0.00000 \\
C & -2.86628 & 0.00000 & 0.00000 \\
C & 0.73688 & 1.25343 & -0.00000 \\
C & 0.73688 & -1.25343 & 0.00000 \\
C & -0.73688 & 1.25343 & 0.00000 \\
C & -0.73688 & -1.25343 & 0.00000 \\
C & 3.57345 & 1.23732 & -0.00000 \\
C & 3.57345 & -1.23732 & -0.00000 \\
C & -3.57345 & 1.23732 & 0.00000 \\
C & -3.57345 & -1.23732 & 0.00000 \\
C & 1.47107 & 2.43949 & -0.00000 \\
C & 1.47107 & -2.43949 & 0.00000 \\
C & -1.47107 & 2.43949 & 0.00000 \\
C & -1.47107 & -2.43949 & 0.00000 \\
C & 2.88179 & 2.43196 & -0.00000 \\
C & 2.88179 & -2.43196 & 0.00000 \\
C & -2.88179 & 2.43196 & 0.00000 \\
C & -2.88179 & -2.43196 & 0.00000 \\
H & 4.67586 & 1.22122 & -0.00000 \\
H & 4.67586 & -1.22122 & -0.00000 \\
H & -4.67586 & 1.22122 & 0.00000 \\
H & -4.67586 & -1.22122 & 0.00000 \\
\hline & & Continued & on next page
\end{tabular}




\begin{tabular}{lrrr}
\multicolumn{5}{c}{ Continued from previous page } \\
\hline H & 0.95678 & 3.41559 & -0.00000 \\
H & 0.95678 & -3.41559 & 0.00000 \\
H & -0.95678 & 3.41559 & 0.00000 \\
H & -0.95678 & -3.41559 & 0.00000 \\
H & 3.42417 & 3.39167 & -0.00000 \\
H & 3.42417 & -3.39167 & 0.00000 \\
H & -3.42417 & 3.39167 & 0.00000 \\
H & -3.42417 & -3.39167 & 0.00000
\end{tabular}


Table S1509 Employed active space, reference configurations, and Cartesian coordinates of the OM3/MRCISD-optimized excited-state equilibrium geometry of the $1{ }^{1} B_{3 u}\left({ }^{1} \pi \pi^{*}\right)$ state of perylene (excited-state energy: $-2676.12384 \mathrm{eV}$ ).

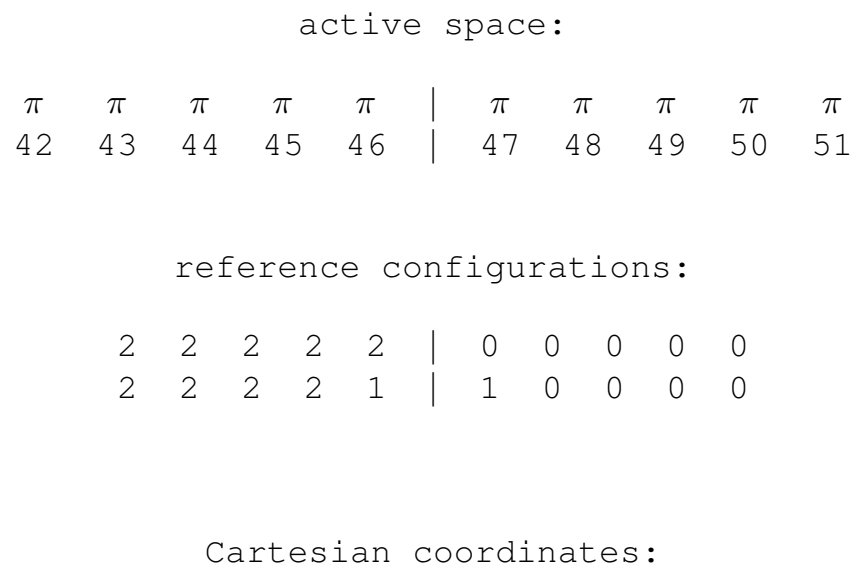

Continued on next page 


\begin{tabular}{lrrr}
\multicolumn{5}{c}{ Continued from previous page } \\
\hline H & 0.94621 & 3.42752 & -0.00000 \\
H & 0.94621 & -3.42752 & 0.00000 \\
H & -0.94621 & 3.42752 & 0.00000 \\
H & -0.94621 & -3.42752 & 0.00000 \\
H & 3.42038 & 3.39476 & -0.00000 \\
H & 3.42038 & -3.39476 & 0.00000 \\
H & -3.42038 & 3.39476 & 0.00000 \\
H & -3.42038 & -3.39476 & 0.00000
\end{tabular}


Table S1510 Employed active space, reference configurations, and Cartesian coordinates of the OM3/MRCISD-optimized ground-state equilibrium geometry of phenanthrene (ground-state energy: $-1900.16587 \mathrm{eV}$ ).

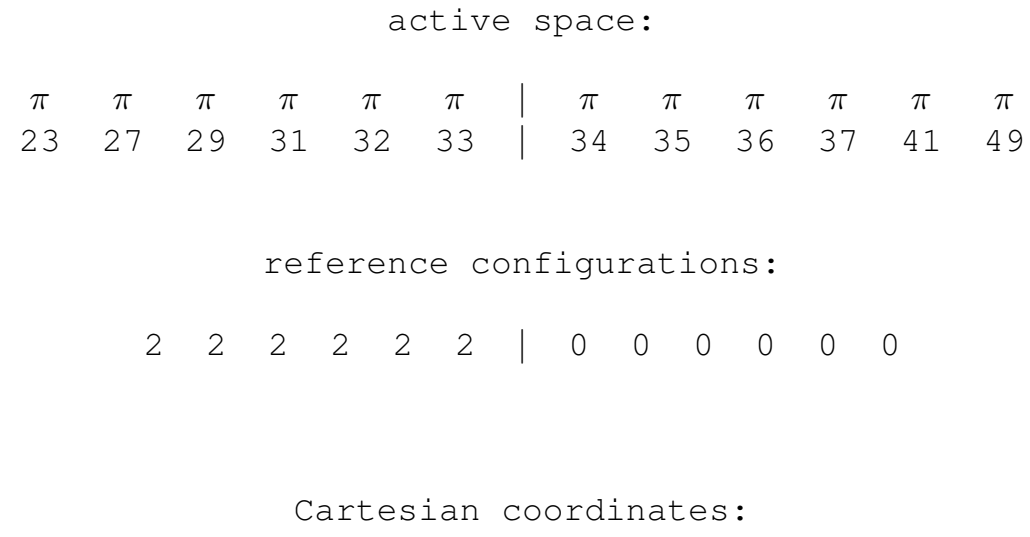

24

$\begin{array}{lrrr}\text { C } & 1.21884 & 0.68449 & 0.00000\end{array}$

C $\quad 1.21884-0.68449-0.00000$

C $\quad-0.02139 \quad 1.42161-0.00000$

C $\quad-0.02139-1.42161-0.00000$

C $\quad-1.26311 \quad 0.72859-0.00000$

C $\quad-1.26311-0.72859-0.00000$

$\mathrm{H} \quad 2.16291 \quad 1.25480 \quad 0.00000$

$\mathrm{H} \quad 2.16291-1.25480-0.00000$

$\begin{array}{lrrr}\text { C } & -0.00429 & 2.84283 & -0.00000\end{array}$

C $\quad-0.00429-2.84283-0.00000$

$\begin{array}{llll}\text { C } & -1.19094 & 3.56255 & -0.00000\end{array}$

$\begin{array}{llll}\text { C } & -1.19094 & -3.56255 & -0.00000\end{array}$

$\begin{array}{llll}\text { C } & -2.42642 & 2.87912 & -0.00000\end{array}$

$\begin{array}{llll}\text { C } & -2.42642 & -2.87912 & -0.00000\end{array}$

C $\quad-2.46140 \quad 1.49035 \quad-0.00000$

$\begin{array}{llll}\text { C } & -2.46140 & -1.49035 & -0.00000\end{array}$

$\mathrm{H} \quad \begin{array}{llll}0.96716 & 3.36524 & -0.00000\end{array}$

$\mathrm{H} \quad 0.96716 \quad-3.36524 \quad-0.00000$

$\mathrm{H} \quad-1.17760 \quad 4.66409 \quad-0.00000$

$\mathrm{H} \quad-1.17760 \quad-4.66409-0.00000$

$\begin{array}{llll}\mathrm{H} & -3.36497 & 3.45694 & -0.00000\end{array}$

$\mathrm{H} \quad-3.36497 \quad-3.45694 \quad-0.00000$

$\mathrm{H} \quad-3.43696 \quad 0.97525 \quad-0.00000$

$\mathrm{H} \quad-3.43696 \quad-0.97525 \quad-0.00000$ 
Table S1511 Employed active space, reference configurations, and Cartesian coordinates of the OM3/MRCISD-optimized excited-state equilibrium geometry of the $2{ }^{1} A_{1}\left({ }^{1} \pi \pi^{*}\right)$ state of phenanthrene (excited-state energy: $-1896.36195 \mathrm{eV})$.

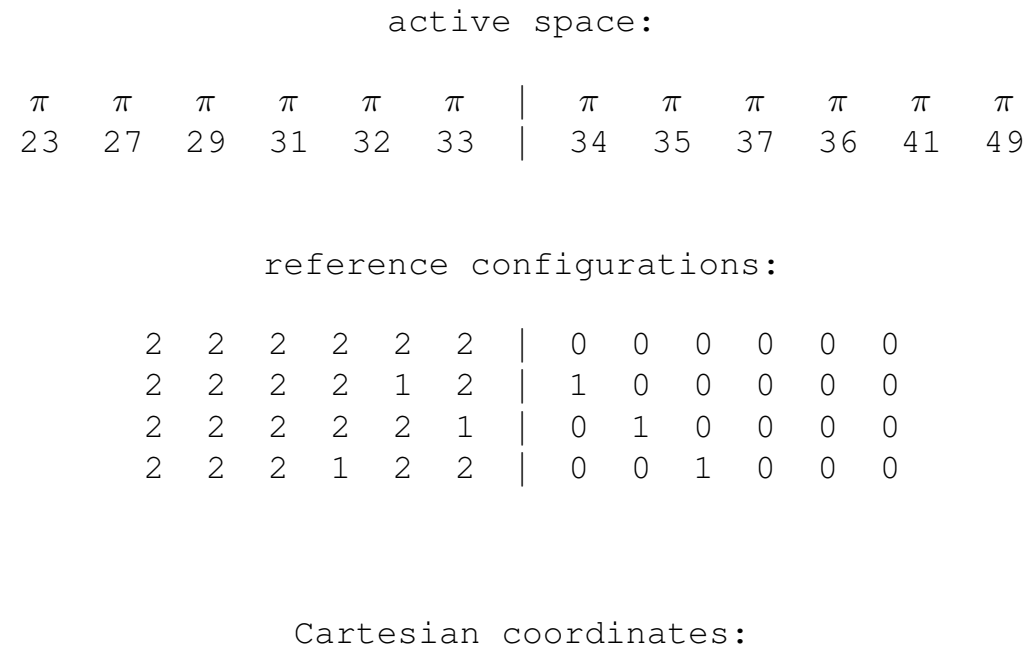

\begin{tabular}{lrrr}
24 & & & \\
& & & \\
$\mathrm{C}$ & 1.20264 & 0.69895 & 0.00000 \\
$\mathrm{C}$ & 1.20264 & -0.69895 & -0.00000 \\
$\mathrm{C}$ & -0.01288 & 1.42806 & -0.00000 \\
$\mathrm{C}$ & -0.01288 & -1.42806 & -0.00000 \\
$\mathrm{C}$ & -1.29034 & 0.71233 & -0.00000 \\
$\mathrm{C}$ & -1.29034 & -0.71233 & -0.00000 \\
$\mathrm{H}$ & 2.15771 & 1.25019 & 0.00000 \\
$\mathrm{H}$ & 2.15771 & -1.25019 & -0.00000 \\
$\mathrm{C}$ & -0.00814 & 2.84526 & -0.00000 \\
$\mathrm{C}$ & -0.00814 & -2.84526 & -0.00000 \\
$\mathrm{C}$ & -1.20812 & 3.56901 & -0.00000 \\
$\mathrm{C}$ & -1.20812 & -3.56901 & -0.00000 \\
$\mathrm{C}$ & -2.44494 & 2.88401 & -0.00000 \\
$\mathrm{C}$ & -2.44494 & -2.88401 & -0.00000 \\
$\mathrm{C}$ & -2.48399 & 1.48642 & -0.00000 \\
$\mathrm{C}$ & -2.48399 & -1.48642 & -0.00000 \\
$\mathrm{H}$ & 0.95728 & 3.37848 & -0.00000 \\
$\mathrm{H}$ & 0.95728 & -3.37848 & -0.00000 \\
$\mathrm{H}$ & -1.19212 & 4.67007 & -0.00000 \\
$\mathrm{H}$ & -1.19212 & -4.67007 & -0.00000 \\
$\mathrm{H}$ & -3.38400 & 3.46044 & -0.00000 \\
\hline & & Continued on next page
\end{tabular}

S1654 


\begin{tabular}{lrrr}
\multicolumn{5}{c}{ Continued from previous page } \\
\hline $\mathrm{H}$ & -3.38400 & -3.46044 & -0.00000 \\
$\mathrm{H}$ & -3.46389 & 0.97951 & -0.00000 \\
$\mathrm{H}$ & -3.46389 & -0.97951 & -0.00000
\end{tabular}


Table S1512 Employed active space, reference configurations, and Cartesian coordinates of the OM3/MRCISD-optimized ground-state equilibrium geometry of fluorene (ground-state energy: $-1775.14165 \mathrm{eV}$ ).

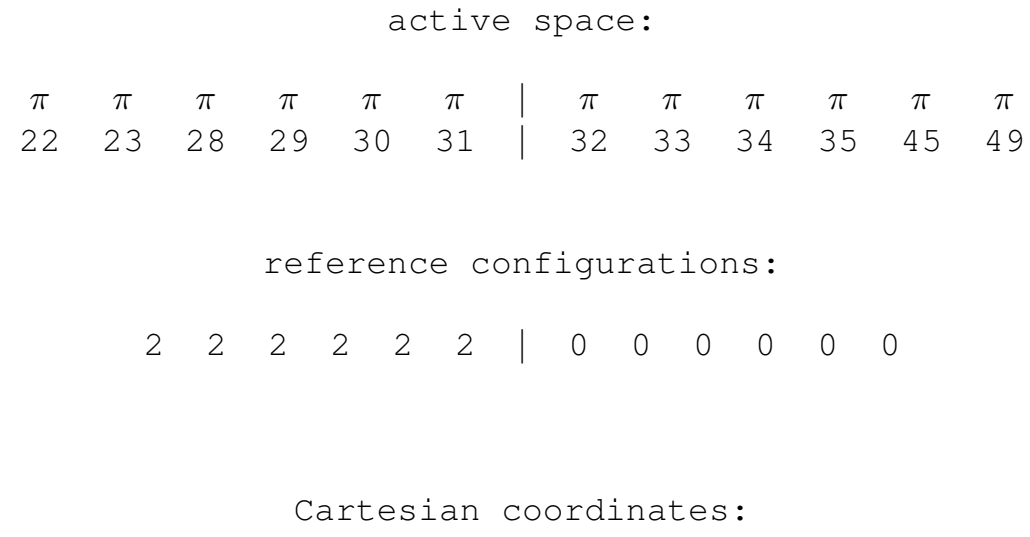

23

$\begin{array}{lrrr}C & 0.00000 & 0.00000 & 0.00000\end{array}$

$\begin{array}{lrrr}\text { C } & -0.94615 & 1.18037 & 0.00000\end{array}$

C $\quad-0.94615-1.18037-0.00000$

$\begin{array}{llll}\text { C } & -2.30255 & 0.73880 & -0.00000\end{array}$

$\begin{array}{llll}\text { C } & -2.30255 & -0.73880 & 0.00000\end{array}$

C $\quad-0.64608 \quad 2.54463 \quad-0.00000$

C $\quad-0.64608-2.54463-0.00000$

C $\quad-3.35686 \quad 1.65763-0.00000$

$\begin{array}{llll}\text { C } & -3.35686 & -1.65763 & -0.00000\end{array}$

C $\quad-1.71024 \quad 3.46329-0.00000$

$\begin{array}{llll}\text { C } & -1.71024 & -3.46329 & -0.00000\end{array}$

$\begin{array}{llll}\text { C } & -3.04397 & 3.02697 & 0.00000\end{array}$

$\begin{array}{llll}\text { C } & -3.04397 & -3.02697 & -0.00000\end{array}$

$\begin{array}{llll}\mathrm{H} & 0.63248 & 0.00000 & -0.90969\end{array}$

$\begin{array}{llll}\mathrm{H} & 0.63248 & 0.00000 & 0.90969\end{array}$

$\begin{array}{llll}\mathrm{H} & 0.39743 & 2.89311 & -0.00000\end{array}$

$\mathrm{H} \quad 0.39743 \quad-2.89311-0.00000$

$\mathrm{H} \quad-4.40234 \quad 1.31565 \quad-0.00000$

$\begin{array}{llll}\mathrm{H} & -4.40234 & -1.31565 & 0.00000\end{array}$

$\begin{array}{llll}\mathrm{H} & -1.49289 & 4.54407 & -0.00000\end{array}$

$\mathrm{H} \quad-1.49289-4.54407 \quad-0.00000$

$\begin{array}{lrrr}\mathrm{H} & -3.85790 & 3.77027 & 0.00000\end{array}$

$\mathrm{H} \quad-3.85790 \quad-3.77027 \quad-0.00000$ 
Table S1513 Employed active space, reference configurations, and Cartesian coordinates of the OM3/MRCISD-optimized excited-state equilibrium geometry of the $1{ }^{1} B_{2}\left({ }^{1} \pi \pi^{*}\right)$ state of fluorene (excited-state energy: $\left.-1770.80415 \mathrm{eV}\right)$.

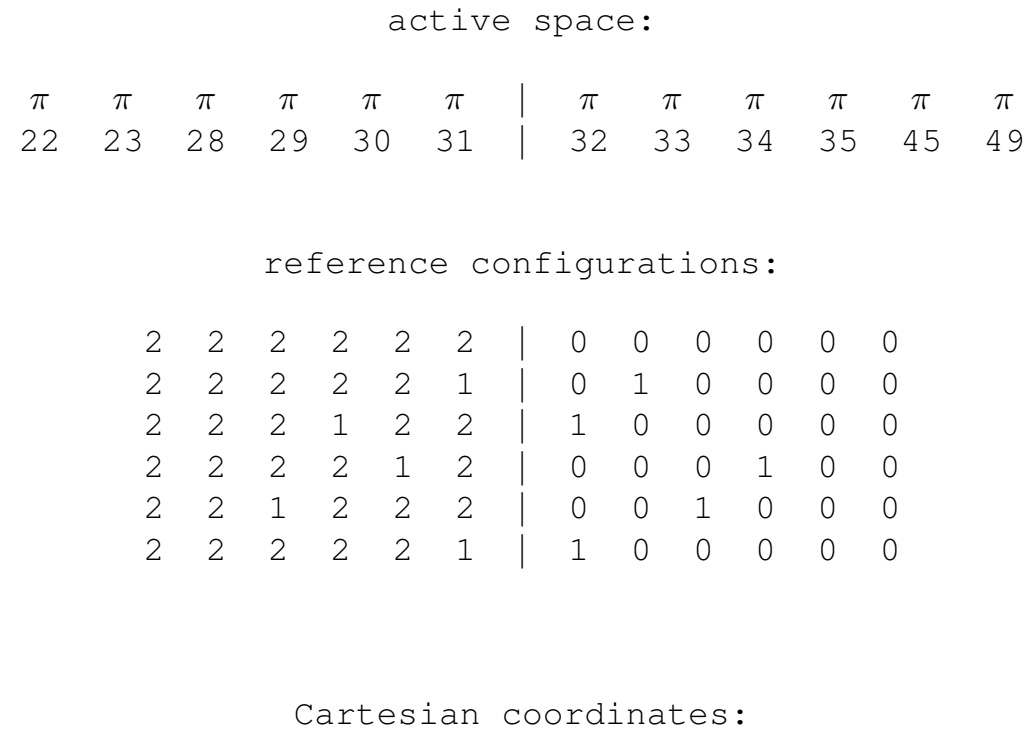

\begin{tabular}{lrrr}
23 & & & \\
C & 0.00000 & 0.00000 & 0.00000 \\
C & -0.94966 & 1.17225 & 0.00000 \\
C & -0.94966 & -1.17225 & -0.00000 \\
C & -2.32251 & 0.72367 & -0.00000 \\
C & -2.32251 & -0.72367 & 0.00000 \\
C & -0.64381 & 2.54742 & -0.00000 \\
C & -0.64381 & -2.54742 & -0.00000 \\
C & -3.38718 & 1.65882 & -0.00000 \\
C & -3.38718 & -1.65882 & -0.00000 \\
C & -1.71595 & 3.46824 & -0.00000 \\
C & -1.71595 & -3.46824 & -0.00000 \\
C & -3.06178 & 3.03283 & 0.00000 \\
C & -3.06178 & -3.03283 & -0.00000 \\
H & 0.62435 & 0.00000 & -0.91144 \\
H & 0.62435 & 0.00000 & 0.91144 \\
H & 0.39813 & 2.89403 & -0.00000 \\
H & 0.39813 & -2.89403 & -0.00000 \\
H & -4.43113 & 1.31928 & -0.00000 \\
H & -4.43113 & -1.31928 & 0.00000 \\
H & -1.49937 & 4.54943 & -0.00000 \\
\hline & & Continued on next page
\end{tabular}

$\mathrm{S} 1657$ 


\begin{tabular}{lrrr}
\multicolumn{5}{c}{ Continued from previous page } \\
\hline $\mathrm{H}$ & -1.49937 & -4.54943 & -0.00000 \\
$\mathrm{H}$ & -3.87042 & 3.78139 & 0.00000 \\
$\mathrm{H}$ & -3.87042 & -3.78139 & -0.00000
\end{tabular}

S1658 
Table S1514 Employed active space, reference configurations, and Cartesian coordinates of the OM3/MRCISD-optimized ground-state equilibrium geometry of carbazole (ground-state energy: $-1850.73918 \mathrm{eV}$ ).

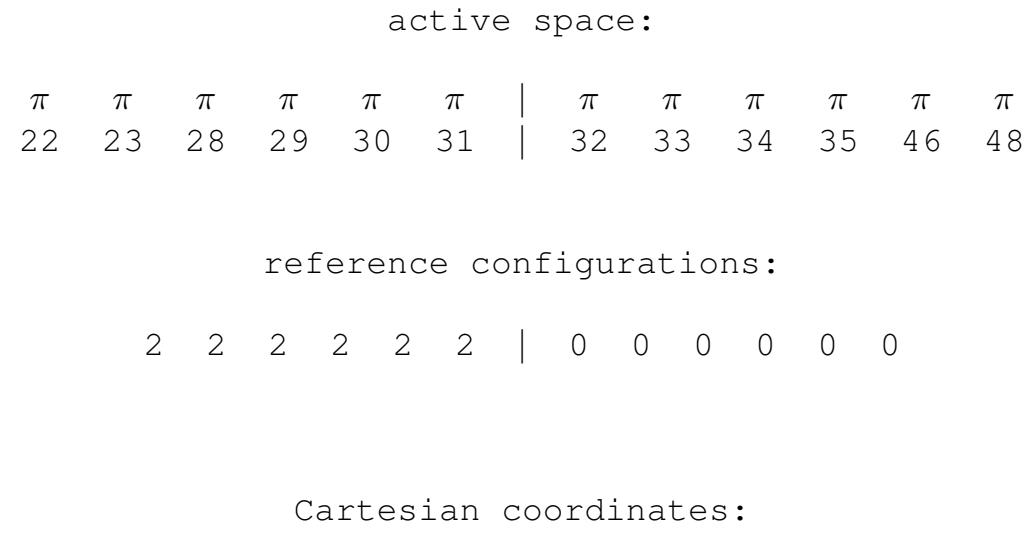

22

$\begin{array}{lrrr}\mathrm{N} & 0.00000 & 0.00000 & 0.00000 \\ \mathrm{H} & 1.03762 & 0.00000 & 0.00000 \\ \mathrm{C} & -0.83304 & 1.14619 & 0.00000 \\ \mathrm{C} & -0.83304 & -1.14619 & -0.00000 \\ \mathrm{C} & -2.21669 & 0.72868 & -0.00000 \\ \mathrm{C} & -2.21669 & -0.72868 & 0.00000 \\ \mathrm{C} & -0.47786 & 2.51144 & -0.00000 \\ \mathrm{C} & -0.47786 & -2.51144 & -0.00000 \\ \mathrm{C} & -3.23128 & 1.69718 & -0.00000 \\ \mathrm{C} & -3.23128 & -1.69718 & -0.00000 \\ \mathrm{C} & -1.51603 & 3.44477 & -0.00000 \\ \mathrm{C} & -1.51603 & -3.44477 & -0.00000 \\ \mathrm{C} & -2.87022 & 3.04794 & 0.00000 \\ \mathrm{C} & -2.87022 & -3.04794 & -0.00000 \\ \mathrm{H} & 0.57616 & 2.81809 & -0.00000 \\ \mathrm{H} & 0.57616 & -2.81809 & -0.00000 \\ \mathrm{H} & -4.28807 & 1.38918 & -0.00000 \\ \mathrm{H} & -4.28807 & -1.38918 & 0.00000 \\ \mathrm{H} & -1.27336 & 4.52097 & -0.00000 \\ \mathrm{H} & -1.27336 & -4.52097 & -0.00000 \\ \mathrm{H} & -3.65401 & 3.82153 & 0.00000 \\ \mathrm{H} & -3.65401 & -3.82153 & -0.00000\end{array}$


Table S1515 Employed active space, reference configurations, and Cartesian coordinates of the OM3/MRCISD-optimized excited-state equilibrium geometry of the $2{ }^{1} A_{1}\left({ }^{1} \pi \pi^{*}\right)$ state of carbazole (excited-state energy: $-1847.09957 \mathrm{eV}$ ).

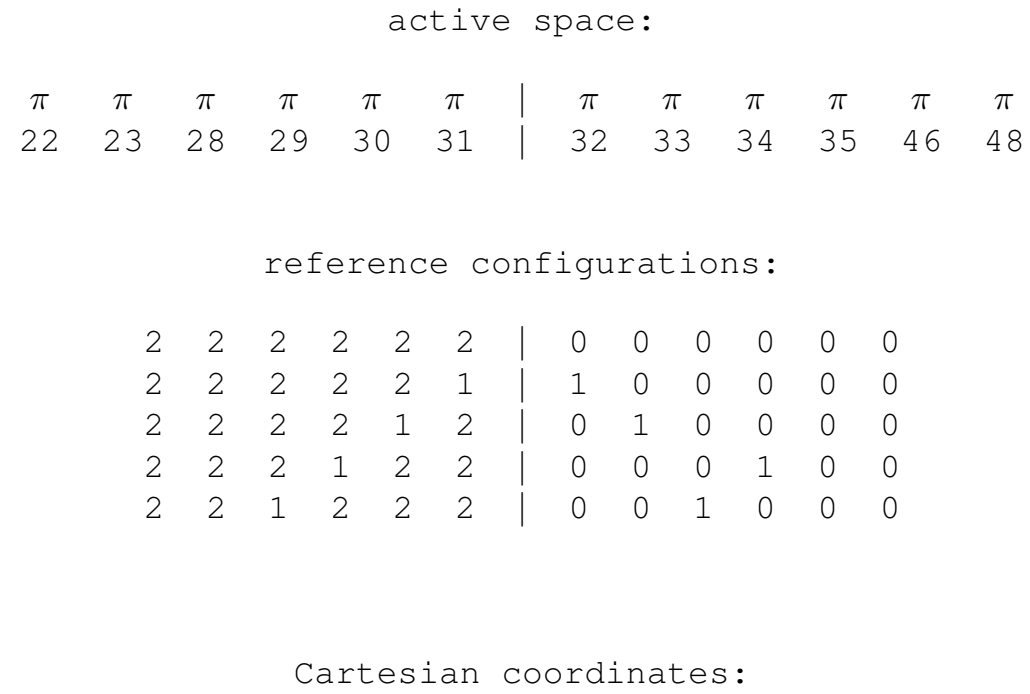

\begin{tabular}{lrrr}
22 & & & \\
& & & \\
$\mathrm{~N}$ & 0.00000 & 0.00000 & 0.00000 \\
$\mathrm{H}$ & 1.04426 & 0.00000 & 0.00000 \\
$\mathrm{C}$ & -0.81065 & 1.15302 & 0.00000 \\
$\mathrm{C}$ & -0.81065 & -1.15302 & -0.00000 \\
$\mathrm{C}$ & -2.21768 & 0.71855 & -0.00000 \\
$\mathrm{C}$ & -2.21768 & -0.71855 & 0.00000 \\
$\mathrm{C}$ & -0.44495 & 2.51014 & -0.00000 \\
$\mathrm{C}$ & -0.44495 & -2.51014 & -0.00000 \\
$\mathrm{C}$ & -3.22684 & 1.69821 & -0.00000 \\
$\mathrm{C}$ & -3.22684 & -1.69821 & -0.00000 \\
$\mathrm{C}$ & -1.49318 & 3.45854 & -0.00000 \\
$\mathrm{C}$ & -1.49318 & -3.45854 & -0.00000 \\
$\mathrm{C}$ & -2.84515 & 3.05857 & 0.00000 \\
$\mathrm{C}$ & -2.84515 & -3.05857 & -0.00000 \\
$\mathrm{H}$ & 0.60830 & 2.81842 & -0.00000 \\
$\mathrm{H}$ & 0.60830 & -2.81842 & -0.00000 \\
$\mathrm{H}$ & -4.28604 & 1.40592 & -0.00000 \\
$\mathrm{H}$ & -4.28604 & -1.40592 & 0.00000 \\
$\mathrm{H}$ & -1.24417 & 4.53056 & -0.00000 \\
$\mathrm{H}$ & -1.24417 & -4.53056 & -0.00000 \\
$\mathrm{H}$ & -3.63113 & 3.83307 & 0.00000 \\
\hline & & Continued & on next page
\end{tabular}

S1660 
Continued from previous page

$\mathrm{H} \quad-3.63113 \quad-3.83307 \quad-0.00000$

S1661 
Table S1516 Employed active space, reference configurations, and Cartesian coordinates of the OM3/MRCISD-optimized ground-state equilibrium geometry of dibenzofuran (ground-state energy: $-1958.73940 \mathrm{eV}$ ).

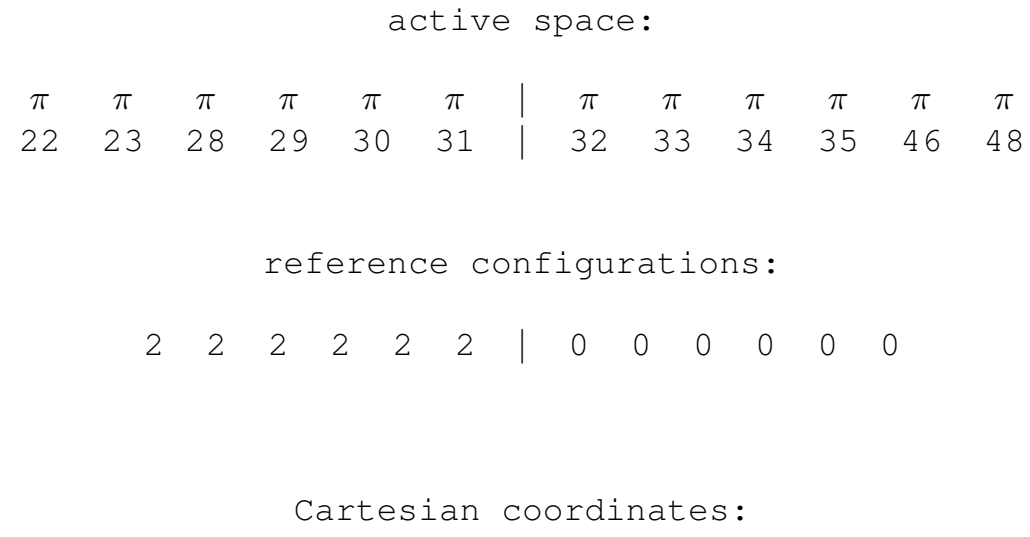

21

$\begin{array}{lrrr}0 & 0.00000 & 0.00000 & 0.00000\end{array}$

$\begin{array}{lrrr}\text { C } & -0.85572 & 1.10532 & 0.00000\end{array}$

C $\quad-0.85572-1.10532-0.00000$

$\begin{array}{llll}\text { C } & -2.23647 & 0.73138 & -0.00000\end{array}$

$\begin{array}{llll}\text { C } & -2.23647 & -0.73138 & 0.00000\end{array}$

C $\quad-0.42714 \quad 2.43787-0.00000$

C $\quad-0.42714-2.43787 \quad-0.00000$

C $\quad-3.22061 \quad 1.72712-0.00000$

$\begin{array}{llll}\text { C } & -3.22061 & -1.72712 & -0.00000\end{array}$

$\begin{array}{llll}\text { C } & -1.43580 & 3.41119 & -0.00000\end{array}$

$\begin{array}{llll}\text { C } & -1.43580 & -3.41119 & -0.00000\end{array}$

$\begin{array}{lrrr}\text { C } & -2.80100 & 3.06507 & 0.00000\end{array}$

$\begin{array}{llll}\text { C } & -2.80100 & -3.06507 & -0.00000\end{array}$

$\mathrm{H} \quad 0.63772 \quad 2.69083 \quad-0.00000$

$\mathrm{H} \quad 0.63772 \quad-2.69083 \quad-0.00000$

$\mathrm{H} \quad-4.28697 \quad 1.45964 \quad-0.00000$

$\mathrm{H} \quad-4.28697 \quad-1.45964 \quad 0.00000$

$\mathrm{H} \quad-1.15134 \quad 4.47618 \quad-0.00000$

$\mathrm{H} \quad-1.15134 \quad-4.47618 \quad-0.00000$

$\begin{array}{lrrr}\mathrm{H} & -3.55604 & 3.86688 & 0.00000\end{array}$

$\mathrm{H} \quad-3.55604 \quad-3.86688 \quad-0.00000$ 
Table S1517 Employed active space, reference configurations, and Cartesian coordinates of the OM3/MRCISD-optimized excited-state equilibrium geometry of the $2{ }^{1} A_{1}\left({ }^{1} \pi \pi^{*}\right)$ state of dibenzofuran (excited-state energy: $-1954.74634 \mathrm{eV})$.

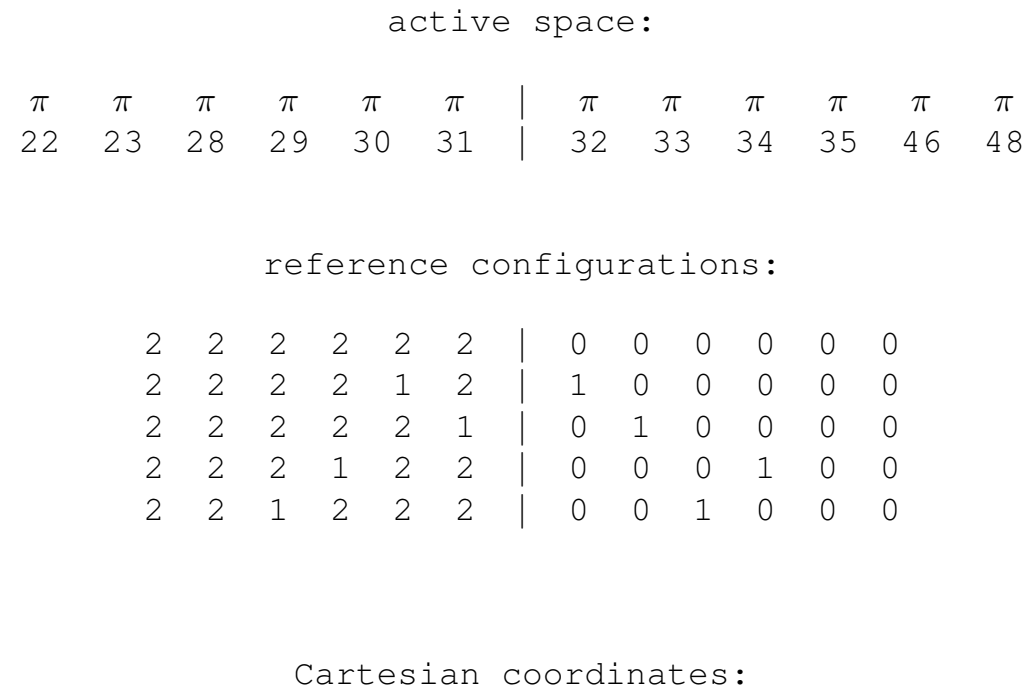

\begin{tabular}{lrrr}
21 & & & \\
& & & \\
$\mathrm{O}$ & 0.00000 & 0.00000 & 0.00000 \\
$\mathrm{C}$ & -0.83939 & 1.10444 & 0.00000 \\
$\mathrm{C}$ & -0.83939 & -1.10444 & -0.00000 \\
$\mathrm{C}$ & -2.24483 & 0.71674 & -0.00000 \\
$\mathrm{C}$ & -2.24483 & -0.71674 & 0.00000 \\
$\mathrm{C}$ & -0.40428 & 2.43952 & -0.00000 \\
$\mathrm{C}$ & -0.40428 & -2.43952 & -0.00000 \\
$\mathrm{C}$ & -3.22724 & 1.72765 & -0.00000 \\
$\mathrm{C}$ & -3.22724 & -1.72765 & -0.00000 \\
$\mathrm{C}$ & -1.42112 & 3.42035 & -0.00000 \\
$\mathrm{C}$ & -1.42112 & -3.42035 & -0.00000 \\
$\mathrm{C}$ & -2.79185 & 3.07270 & 0.00000 \\
$\mathrm{C}$ & -2.79185 & -3.07270 & -0.00000 \\
$\mathrm{H}$ & 0.65983 & 2.69298 & -0.00000 \\
$\mathrm{H}$ & 0.65983 & -2.69298 & -0.00000 \\
$\mathrm{H}$ & -4.29529 & 1.47081 & -0.00000 \\
$\mathrm{H}$ & -4.29529 & -1.47081 & 0.00000 \\
$\mathrm{H}$ & -1.13554 & 4.48375 & -0.00000 \\
$\mathrm{H}$ & -1.13554 & -4.48375 & -0.00000 \\
$\mathrm{H}$ & -3.54539 & 3.87686 & 0.00000 \\
\hline & & Continued & on next page
\end{tabular}

S1663 
Continued from previous page

$\mathrm{H} \quad-3.54539-3.87686 \quad-0.00000$ 
Table S1518 Employed active space, reference configurations, and Cartesian coordinates of the OM3/MRCISD-optimized ground-state equilibrium geometry of pyrrolo[3,2- $h]$ quinoline (ground-state energy: $-1926.11960 \mathrm{eV}$ ).

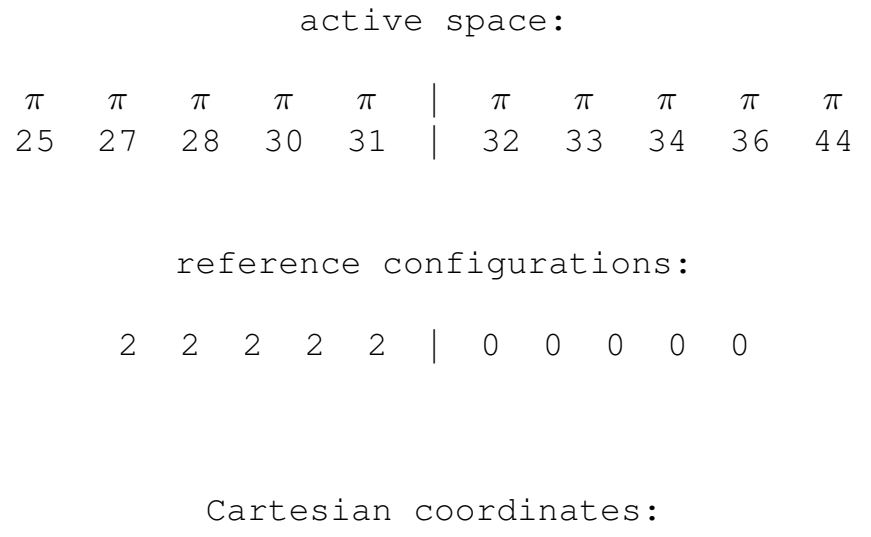

21

$\begin{array}{lrrr}\mathrm{N} & 0.00000 & 0.00000 & 0.00000 \\ \mathrm{C} & 1.40493 & 0.00000 & 0.00000 \\ \mathrm{C} & 1.85587 & 1.36436 & 0.00000 \\ \mathrm{C} & 0.68331 & 2.19217 & 0.00000 \\ \mathrm{C} & -0.43258 & 1.34105 & 0.00000 \\ \mathrm{C} & 3.25445 & 1.64574 & 0.00000 \\ \mathrm{C} & 4.15519 & 0.60080 & 0.00000 \\ \mathrm{C} & 3.72319 & -0.77156 & 0.00000 \\ \mathrm{C} & 2.33005 & -1.10804 & 0.00000 \\ \mathrm{C} & 4.64335 & -1.85308 & 0.00000 \\ \mathrm{C} & 4.17871 & -3.15915 & 0.00000 \\ \mathrm{C} & 2.77642 & -3.40072 & 0.00000 \\ \mathrm{~N} & 1.87287 & -2.40460 & 0.00000 \\ \mathrm{H} & 2.39786 & -4.44718 & 0.00000 \\ \mathrm{H} & 4.87729 & -4.00675 & 0.00000 \\ \mathrm{H} & 5.72462 & -1.64009 & 0.00000 \\ \mathrm{H} & 3.58816 & 2.69542 & 0.00000 \\ \mathrm{H} & 5.23946 & 0.79606 & 0.00000 \\ \mathrm{H} & -0.59988 & -0.85171 & 0.00000 \\ \mathrm{H} & 0.65872 & 3.27639 & 0.00000 \\ \mathrm{H} & -1.49182 & 1.61902 & 0.00000\end{array}$


Table S1519 Employed active space, reference configurations, and Cartesian coordinates of the OM3/MRCISD-optimized excited-state equilibrium geometry of the $2{ }^{1} A^{\prime}\left({ }^{1} \pi \pi^{*}\right)$ state of pyrrolo[3,2- $\left.h\right]$ quinoline (excited-state energy: $-1922.29509 \mathrm{eV})$.

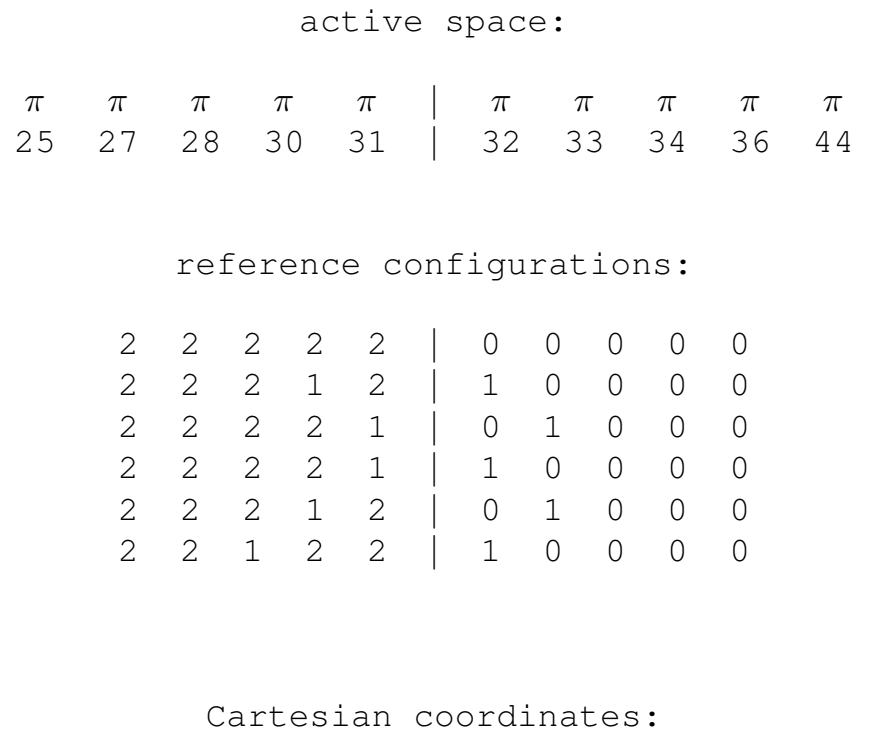

21

\begin{tabular}{lrrr}
$\mathrm{N}$ & 0.00000 & 0.00000 & 0.00000 \\
$\mathrm{C}$ & 1.40232 & 0.00000 & 0.00000 \\
$\mathrm{C}$ & 1.84636 & 1.41712 & 0.00000 \\
$\mathrm{C}$ & 0.66552 & 2.21151 & 0.00000 \\
$\mathrm{C}$ & -0.45234 & 1.33054 & 0.00000 \\
$\mathrm{C}$ & 3.22542 & 1.71348 & 0.00000 \\
$\mathrm{C}$ & 4.15011 & 0.64243 & 0.00000 \\
$\mathrm{C}$ & 3.74197 & -0.71691 & 0.00000 \\
$\mathrm{C}$ & 2.31863 & -1.08823 & 0.00000 \\
$\mathrm{C}$ & 4.66669 & -1.79416 & 0.00000 \\
$\mathrm{C}$ & 4.20335 & -3.11222 & 0.00000 \\
$\mathrm{C}$ & 2.80117 & -3.37105 & 0.00000 \\
$\mathrm{~N}$ & 1.87971 & -2.38198 & 0.00000 \\
$\mathrm{H}$ & 2.43447 & -4.42004 & 0.00000 \\
$\mathrm{H}$ & 4.91143 & -3.95287 & 0.00000 \\
$\mathrm{H}$ & 5.74723 & -1.58109 & 0.00000 \\
$\mathrm{H}$ & 3.56734 & 2.75680 & 0.00000 \\
$\mathrm{H}$ & 5.22915 & 0.86670 & 0.00000 \\
$\mathrm{H}$ & -0.59149 & -0.85819 & 0.00000 \\
\hline & & Continued on next page
\end{tabular}

S1666 
Continued from previous page

\begin{tabular}{rrrr}
\hline $\mathrm{H}$ & 0.60841 & 3.29500 & 0.00000 \\
$\mathrm{H}$ & -1.51403 & 1.60058 & 0.00000
\end{tabular}


Table S1520 Employed active space, reference configurations, and Cartesian coordinates of the OM3/MRCISD-optimized ground-state equilibrium geometry of tryptamine (anti, $\mathrm{Ph}$ ) (ground-state energy: $-1860.38871 \mathrm{eV}$ ).

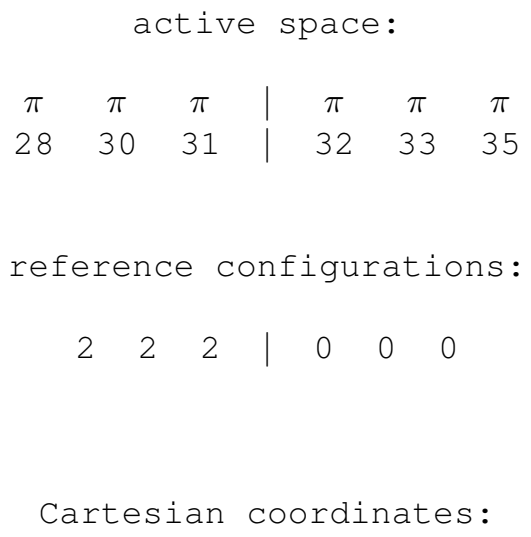

24

$\begin{array}{lrrr}\mathrm{C} & 0.00000 & 0.00000 & 0.00000 \\ \mathrm{C} & 1.40625 & 0.00000 & 0.00000 \\ \mathrm{C} & 2.10440 & 1.26458 & 0.00000 \\ \mathrm{C} & 1.41946 & 2.49916 & 0.00067 \\ \mathrm{C} & 0.02776 & 2.44927 & -0.00420 \\ \mathrm{C} & -0.66935 & 1.22118 & -0.00642 \\ \mathrm{C} & 2.41424 & -1.04663 & 0.00398 \\ \mathrm{C} & 3.66757 & -0.40767 & -0.00549 \\ \mathrm{~N} & 3.49594 & 0.99248 & 0.00564 \\ \mathrm{C} & 2.17479 & -2.51179 & -0.10491 \\ \mathrm{C} & 1.96294 & -2.91542 & -1.57077 \\ \mathrm{~N} & 1.71902 & -4.34706 & -1.68901 \\ \mathrm{H} & 1.96936 & 3.44987 & 0.01599 \\ \mathrm{H} & -0.54777 & 3.39052 & -0.01027 \\ \mathrm{H} & -1.77219 & 1.23812 & -0.00663 \\ \mathrm{H} & -0.55148 & -0.95276 & 0.01891 \\ \mathrm{H} & 4.25601 & 1.70166 & 0.00370 \\ \mathrm{H} & 4.65845 & -0.87594 & -0.01228 \\ \mathrm{H} & 1.27752 & -2.79504 & 0.50105 \\ \mathrm{H} & 3.04513 & -3.07109 & 0.33001 \\ \mathrm{H} & 2.85663 & -2.56313 & -2.18604 \\ \mathrm{H} & 1.07179 & -2.36686 & -1.99011 \\ \mathrm{H} & 2.41774 & -4.93490 & -1.17242 \\ \mathrm{H} & 1.69413 & -4.69740 & -2.67785\end{array}$


Table S1521 Employed active space, reference configurations, and Cartesian coordinates of the OM3/MRCISD-optimized excited-state equilibrium geometry of the $2{ }^{1} A\left({ }^{1} \pi \pi^{*}\right)$ state of tryptamine (anti, $\mathrm{Ph}$ ) (excited-state energy: $-1855.77118 \mathrm{eV})$.

active space:
\begin{tabular}{ccc|ccc}
$\pi$ & $\pi$ & $\pi$ & $\pi$ & $\pi$ & $\pi$ \\
28 & 30 & 31 & 32 & 33 & 35 \\
reference configurations: \\
2 & 2 & 2 & 0 & 0 & 0 \\
2 & 1 & 2 & 1 & 0 & 0 \\
2 & 2 & 1 & 0 & 1 & 0 \\
2 & 2 & 1 & 1 & 0 & 0
\end{tabular}

Cartesian coordinates:

\begin{tabular}{lrrr}
24 & & & \\
& & & \\
$\mathrm{C}$ & 0.00000 & 0.00000 & 0.00000 \\
$\mathrm{C}$ & 1.40853 & 0.00000 & 0.00000 \\
$\mathrm{C}$ & 2.09983 & 1.28402 & 0.00000 \\
$\mathrm{C}$ & 1.43746 & 2.51225 & -0.00246 \\
$\mathrm{C}$ & 0.01531 & 2.46249 & 0.01036 \\
$\mathrm{C}$ & -0.66379 & 1.24283 & 0.00976 \\
$\mathrm{C}$ & 2.41222 & -1.03068 & 0.00966 \\
$\mathrm{C}$ & 3.72547 & -0.36235 & 0.04824 \\
$\mathrm{~N}$ & 3.49900 & 1.00463 & 0.01739 \\
$\mathrm{C}$ & 2.24201 & -2.48919 & -0.15563 \\
$\mathrm{C}$ & 2.80394 & -2.96150 & -1.50698 \\
$\mathrm{~N}$ & 2.71101 & -4.40793 & -1.61806 \\
$\mathrm{H}$ & 1.97806 & 3.46576 & -0.02193 \\
$\mathrm{H}$ & -0.54439 & 3.40793 & 0.02894 \\
$\mathrm{H}$ & -1.77047 & 1.24711 & 0.01841 \\
$\mathrm{H}$ & -0.55704 & -0.94605 & 0.00619 \\
$\mathrm{H}$ & 4.24476 & 1.74023 & 0.02975 \\
$\mathrm{H}$ & 4.71225 & -0.82908 & 0.05936 \\
$\mathrm{H}$ & 1.16058 & -2.76054 & -0.08981 \\
$\mathrm{H}$ & 2.76326 & -3.01449 & 0.68699 \\
$\mathrm{H}$ & 3.87123 & -2.56931 & -1.62249 \\
\hline & & Continued & on next page
\end{tabular}

S1669 


\begin{tabular}{lrrr}
\multicolumn{5}{c}{ Continued from previous page } \\
\hline $\mathrm{H}$ & 2.20789 & -2.49529 & -2.34348 \\
$\mathrm{H}$ & 3.13525 & -4.92410 & -0.80967 \\
$\mathrm{H}$ & 3.14005 & -4.79490 & -2.49299
\end{tabular}


Table S1522 Employed active space, reference configurations, and Cartesian coordinates of the OM3/MRCISD-optimized ground-state equilibrium geometry of tryptamine (anti, py) (ground-state energy: $-1860.39427 \mathrm{eV}$ ).

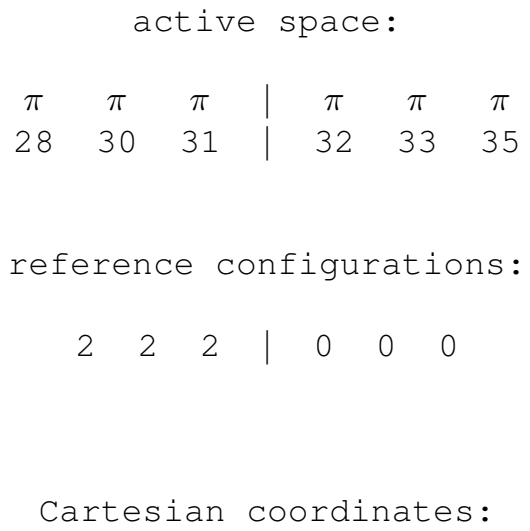


Table S1523 Employed active space, reference configurations, and Cartesian coordinates of the OM3/MRCISD-optimized excited-state equilibrium geometry of the $2{ }^{1} A\left({ }^{1} \pi \pi^{*}\right)$ state of tryptamine (anti, py) (excited-state energy: $-1855.78541 \mathrm{eV})$.

active space:
\begin{tabular}{ccc|ccc}
$\pi$ & $\pi$ & $\pi$ & $\pi$ & $\pi$ & $\pi$ \\
28 & 30 & 31 & 32 & 33 & 35 \\
reference configurations: \\
2 & 2 & 2 & 0 & 0 & 0 \\
2 & 1 & 2 & 1 & 0 & 0 \\
2 & 2 & 1 & 0 & 1 & 0 \\
2 & 2 & 1 & 1 & 0 & 0
\end{tabular}

Cartesian coordinates:

\begin{tabular}{lrrr}
24 & & & \\
& & & \\
$\mathrm{C}$ & 0.00000 & 0.00000 & 0.00000 \\
$\mathrm{C}$ & 1.40891 & 0.00000 & 0.00000 \\
$\mathrm{C}$ & 2.09955 & 1.28239 & 0.00000 \\
$\mathrm{C}$ & 1.43964 & 2.51092 & -0.00527 \\
$\mathrm{C}$ & 0.01662 & 2.46077 & 0.00359 \\
$\mathrm{C}$ & -0.66309 & 1.24268 & 0.00664 \\
$\mathrm{C}$ & 2.41189 & -1.02910 & 0.01307 \\
$\mathrm{C}$ & 3.72850 & -0.36009 & 0.04808 \\
$\mathrm{~N}$ & 3.50215 & 1.00355 & 0.01612 \\
$\mathrm{C}$ & 2.24829 & -2.48599 & -0.17110 \\
$\mathrm{C}$ & 2.66020 & -2.89482 & -1.59465 \\
$\mathrm{~N}$ & 2.61727 & -4.34141 & -1.74317 \\
$\mathrm{H}$ & 1.97994 & 3.46392 & -0.02876 \\
$\mathrm{H}$ & -0.54223 & 3.40723 & 0.01666 \\
$\mathrm{H}$ & -1.76989 & 1.24759 & 0.01434 \\
$\mathrm{H}$ & -0.55716 & -0.94596 & 0.01211 \\
$\mathrm{H}$ & 4.24630 & 1.74092 & 0.02736 \\
$\mathrm{H}$ & 4.71339 & -0.83158 & 0.05833 \\
$\mathrm{H}$ & 1.18299 & -2.77236 & 0.01840 \\
$\mathrm{H}$ & 2.87433 & -3.03625 & 0.57627 \\
$\mathrm{H}$ & 3.71697 & -2.55034 & -1.79560 \\
\hline & & Continued \\
& &
\end{tabular}

S1672 


\begin{tabular}{llll}
\multicolumn{5}{c}{ Continued from previous page } \\
\hline $\mathrm{H}$ & 2.00267 & -2.34309 & -2.34281 \\
$\mathrm{H}$ & 1.72886 & -4.77287 & -1.39111 \\
$\mathrm{H}$ & 2.74917 & -4.66806 & -2.73082
\end{tabular}


Table S1524 Employed active space, reference configurations, and Cartesian coordinates of the OM3/MRCISD-optimized ground-state equilibrium geometry of tryptamine (anti, up) (ground-state energy: $-1860.27034 \mathrm{eV}$ ).

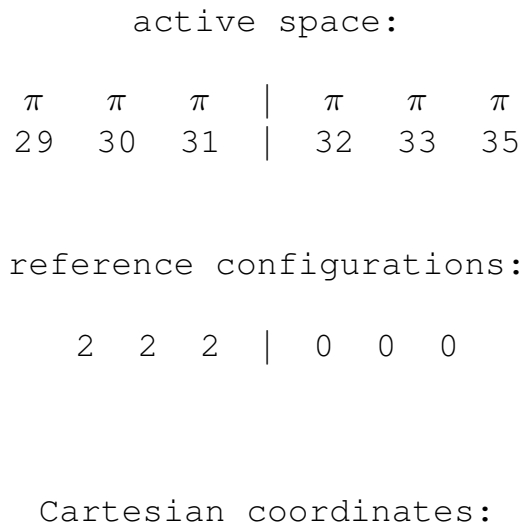


Table S1525 Employed active space, reference configurations, and Cartesian coordinates of the OM3/MRCISD-optimized excited-state equilibrium geometry of the $2{ }^{1} A\left({ }^{1} \pi \pi^{*}\right)$ state of tryptamine (anti, up) (excited-state energy: $-1855.64453 \mathrm{eV})$.

active space:
\begin{tabular}{ccc|ccc}
$\pi$ & $\pi$ & $\pi$ & $\pi$ & $\pi$ & $\pi$ \\
29 & 30 & 31 & 32 & 33 & 35 \\
reference configurations: \\
2 & 2 & 2 & 0 & 0 & 0 \\
2 & 1 & 2 & 1 & 0 & 0 \\
2 & 2 & 1 & 0 & 1 & 0 \\
2 & 2 & 1 & 1 & 0 & 0
\end{tabular}

Cartesian coordinates:

\begin{tabular}{lrrr}
24 & & & \\
& & & \\
$\mathrm{C}$ & 0.00000 & 0.00000 & 0.00000 \\
$\mathrm{C}$ & 1.40965 & 0.00000 & 0.00000 \\
$\mathrm{C}$ & 2.10263 & 1.27882 & 0.00000 \\
$\mathrm{C}$ & 1.44184 & 2.50879 & -0.00063 \\
$\mathrm{C}$ & 0.01663 & 2.45821 & 0.00294 \\
$\mathrm{C}$ & -0.66554 & 1.24356 & 0.00329 \\
$\mathrm{C}$ & 2.40857 & -1.03130 & 0.00934 \\
$\mathrm{C}$ & 3.72260 & -0.36626 & 0.00816 \\
$\mathrm{~N}$ & 3.50627 & 0.99981 & 0.00168 \\
$\mathrm{C}$ & 2.22927 & -2.48093 & -0.12767 \\
$\mathrm{C}$ & 2.32955 & -2.91872 & -1.62213 \\
$\mathrm{~N}$ & 2.16249 & -4.34602 & -1.83667 \\
$\mathrm{H}$ & 1.98107 & 3.46202 & -0.00120 \\
$\mathrm{H}$ & -0.54002 & 3.40687 & 0.00065 \\
$\mathrm{H}$ & -1.77131 & 1.24794 & 0.00597 \\
$\mathrm{H}$ & -0.55734 & -0.94573 & 0.01146 \\
$\mathrm{H}$ & 4.25345 & 1.73389 & 0.00085 \\
$\mathrm{H}$ & 4.70581 & -0.84216 & 0.00195 \\
$\mathrm{H}$ & 1.23235 & -2.77927 & 0.27919 \\
$\mathrm{H}$ & 3.00578 & -3.02544 & 0.46756 \\
$\mathrm{H}$ & 3.33760 & -2.60525 & -2.02507 \\
\hline & \multicolumn{3}{c}{ Continued on next page }
\end{tabular}

S1675 


\begin{tabular}{llrl}
\multicolumn{5}{c}{ Continued from previous page } \\
\hline $\mathrm{H}$ & 1.54765 & -2.36119 & -2.21262 \\
$\mathrm{H}$ & 1.21721 & -4.68753 & -1.52795 \\
$\mathrm{H}$ & 2.86442 & -4.91659 & -1.29977
\end{tabular}


Table S1526 Employed active space, reference configurations, and Cartesian coordinates of the OM3/MRCISD-optimized ground-state equilibrium geometry of tryptamine (Ph, out) (ground-state energy: $-1860.35934 \mathrm{eV}$ ).

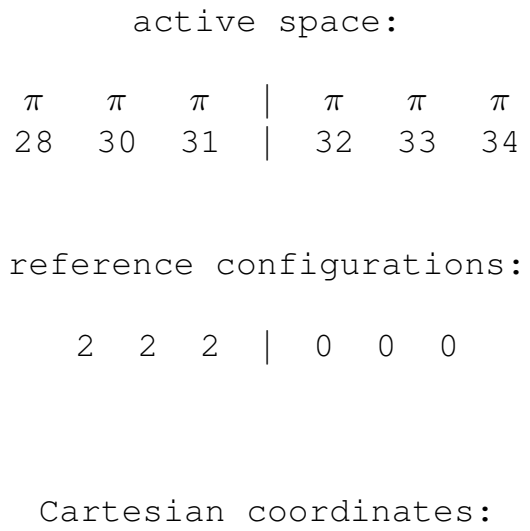


Table S1527 Employed active space, reference configurations, and Cartesian coordinates of the OM3/MRCISD-optimized excited-state equilibrium geometry of the $2{ }^{1} A\left({ }^{1} \pi \pi^{*}\right)$ state of tryptamine (Ph, out) (excited-state energy: $-1855.73466 \mathrm{eV})$.

active space:
\begin{tabular}{ccc|ccc}
$\pi$ & $\pi$ & $\pi$ & $\pi$ & $\pi$ & $\pi$ \\
28 & 30 & 31 & 32 & 33 & 35 \\
reference configurations: \\
2 & 2 & 2 & 0 & 0 & 0 \\
2 & 1 & 2 & 1 & 0 & 0 \\
2 & 2 & 1 & 0 & 1 & 0 \\
2 & 2 & 1 & 1 & 0 & 0
\end{tabular}

Cartesian coordinates:

\begin{tabular}{lrrr}
24 & & & \\
& & & \\
$\mathrm{C}$ & 0.00000 & 0.00000 & 0.00000 \\
$\mathrm{~N}$ & 1.38105 & 0.00000 & 0.00000 \\
$\mathrm{C}$ & 1.88367 & 1.34202 & 0.00000 \\
$\mathrm{C}$ & 0.73521 & 2.23120 & -0.00569 \\
$\mathrm{C}$ & -0.44697 & 1.41091 & -0.02574 \\
$\mathrm{C}$ & 3.20617 & 1.78954 & -0.00356 \\
$\mathrm{C}$ & 3.38786 & 3.20408 & -0.00733 \\
$\mathrm{C}$ & 2.29865 & 4.07385 & -0.00668 \\
$\mathrm{C}$ & 0.96278 & 3.62326 & -0.00673 \\
$\mathrm{C}$ & -1.84805 & 1.81640 & -0.24135 \\
$\mathrm{C}$ & -2.19557 & 1.78197 & -1.75130 \\
$\mathrm{~N}$ & -1.66041 & 2.94581 & -2.43446 \\
$\mathrm{H}$ & 4.05726 & 1.10089 & -0.00868 \\
$\mathrm{H}$ & 4.41385 & 3.59988 & -0.00807 \\
$\mathrm{H}$ & 2.48407 & 5.16479 & -0.00442 \\
$\mathrm{H}$ & 0.12202 & 4.32650 & 0.02605 \\
$\mathrm{H}$ & 1.98763 & -0.85341 & 0.03536 \\
$\mathrm{H}$ & -0.62588 & -0.89531 & 0.00324 \\
$\mathrm{H}$ & -2.00851 & 2.85078 & 0.14659 \\
$\mathrm{H}$ & -2.52840 & 1.13489 & 0.32332 \\
$\mathrm{H}$ & -3.31407 & 1.78084 & -1.87377 \\
\hline & & Continued & on next page
\end{tabular}

$\mathrm{S} 1678$ 


\begin{tabular}{llrl}
\multicolumn{5}{c}{ Continued from previous page } \\
\hline $\mathrm{H}$ & -1.84706 & 0.78799 & -2.18486 \\
$\mathrm{H}$ & -0.70183 & 3.21639 & -2.11557 \\
$\mathrm{H}$ & -1.62929 & 2.85538 & -3.47752
\end{tabular}


Table S1528 Employed active space, reference configurations, and Cartesian coordinates of the OM3/MRCISD-optimized ground-state equilibrium geometry of tryptamine (py, out) (ground-state energy: $-1860.36178 \mathrm{eV}$ ).

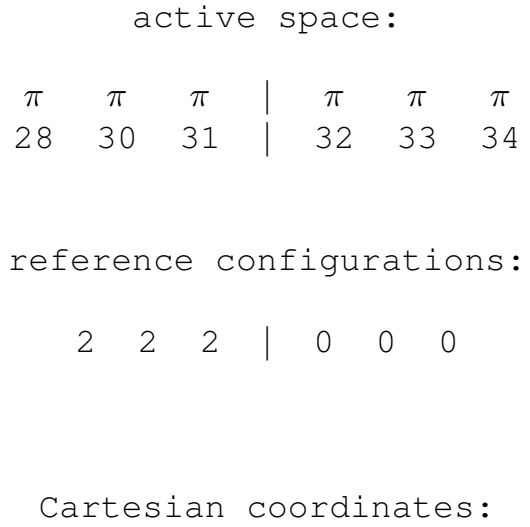


Table S1529 Employed active space, reference configurations, and Cartesian coordinates of the OM3/MRCISD-optimized excited-state equilibrium geometry of the $2^{1} A\left({ }^{1} \pi \pi^{*}\right)$ state of tryptamine (py, out) (excited-state energy: $-1855.71889 \mathrm{eV})$.

active space:
\begin{tabular}{ccc|ccc}
$\pi$ & $\pi$ & $\pi$ & $\pi$ & $\pi$ & $\pi$ \\
28 & 30 & 31 & 32 & 33 & 35 \\
reference configurations: \\
2 & 2 & 2 & 0 & 0 & 0 \\
2 & 1 & 2 & 1 & 0 & 0 \\
2 & 2 & 1 & 0 & 1 & 0 \\
2 & 2 & 1 & 1 & 0 & 0
\end{tabular}

Cartesian coordinates:

\begin{tabular}{lrrr}
24 & & & \\
& & & \\
$\mathrm{C}$ & 0.00000 & 0.00000 & 0.00000 \\
$\mathrm{~N}$ & 1.38212 & 0.00000 & 0.00000 \\
$\mathrm{C}$ & 1.88474 & 1.34196 & 0.00000 \\
$\mathrm{C}$ & 0.73633 & 2.23063 & -0.00128 \\
$\mathrm{C}$ & -0.44622 & 1.40852 & -0.01947 \\
$\mathrm{C}$ & 3.20741 & 1.78800 & -0.00459 \\
$\mathrm{C}$ & 3.39104 & 3.20311 & -0.00778 \\
$\mathrm{C}$ & 2.30244 & 4.07311 & 0.00627 \\
$\mathrm{C}$ & 0.96673 & 3.62287 & 0.01247 \\
$\mathrm{C}$ & -1.85007 & 1.83000 & -0.18050 \\
$\mathrm{C}$ & -2.12783 & 2.27916 & -1.63676 \\
$\mathrm{~N}$ & -2.14825 & 1.13166 & -2.53441 \\
$\mathrm{H}$ & 4.05782 & 1.09865 & -0.00564 \\
$\mathrm{H}$ & 4.41719 & 3.59793 & -0.02606 \\
$\mathrm{H}$ & 2.48852 & 5.16433 & 0.01184 \\
$\mathrm{H}$ & 0.12756 & 4.32867 & 0.06446 \\
$\mathrm{H}$ & 1.98845 & -0.85395 & 0.02246 \\
$\mathrm{H}$ & -0.62838 & -0.89274 & 0.00536 \\
$\mathrm{H}$ & -2.05955 & 2.67156 & 0.51925 \\
$\mathrm{H}$ & -2.54262 & 0.99107 & 0.08106 \\
$\mathrm{H}$ & -1.37421 & 3.07391 & -1.92362 \\
\hline & & Continued & on next page
\end{tabular}

S1681 


\begin{tabular}{llrl}
\multicolumn{5}{c}{ Continued from previous page } \\
\hline $\mathrm{H}$ & -3.12954 & 2.78093 & -1.68470 \\
$\mathrm{H}$ & -2.16989 & 1.39119 & -3.55064 \\
$\mathrm{H}$ & -1.32292 & 0.50027 & -2.39917
\end{tabular}


Table S1530 Employed active space, reference configurations, and Cartesian coordinates of the OM3/MRCISD-optimized ground-state equilibrium geometry of 2,4,6,8-decatetraene (ground-state energy: $-1457.86896 \mathrm{eV}$ ).

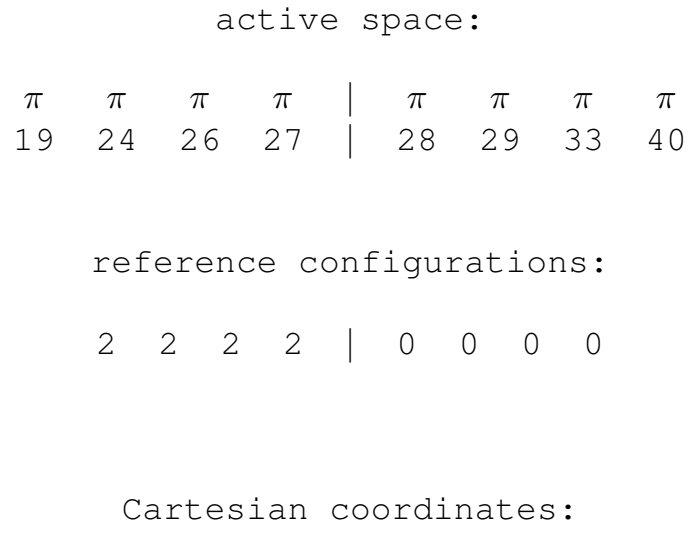

24

$\begin{array}{lrrr}\text { C } & 0.00000 & 0.00000 & 0.00000 \\ \mathrm{C} & 1.45163 & 0.00000 & 0.00000 \\ \mathrm{C} & -0.73411 & 1.15370 & 0.00000 \\ \mathrm{C} & 2.18573 & -1.15370 & 0.00000 \\ \mathrm{C} & -2.19016 & 1.15179 & -0.00000 \\ \mathrm{C} & 3.64179 & -1.15179 & -0.00000 \\ \mathrm{C} & -2.91998 & 2.30075 & -0.00000 \\ \mathrm{C} & 4.37161 & -2.30075 & 0.00000 \\ \mathrm{C} & -4.41042 & 2.32154 & -0.00000 \\ \mathrm{C} & 5.86205 & -2.32154 & -0.00000 \\ \mathrm{H} & -0.50629 & -0.98216 & -0.00000 \\ \mathrm{H} & 1.95792 & 0.98216 & -0.00000 \\ \mathrm{H} & -0.22990 & 2.13678 & 0.00000 \\ \mathrm{H} & 1.68153 & -2.13678 & 0.00000 \\ \mathrm{H} & -2.69236 & 0.16783 & -0.00000 \\ \mathrm{H} & 4.14398 & -0.16783 & -0.00000 \\ \mathrm{H} & -2.41735 & 3.28420 & 0.00000 \\ \mathrm{H} & 3.86898 & -3.28420 & 0.00000 \\ \mathrm{H} & -4.82454 & 1.29516 & -0.00000 \\ \mathrm{H} & 6.27617 & -1.29516 & -0.00000 \\ \mathrm{H} & -4.77316 & 2.83886 & 0.90032 \\ \mathrm{H} & -4.77316 & 2.83886 & -0.90032 \\ \mathrm{H} & 6.22478 & -2.83886 & 0.90032 \\ \mathrm{H} & 6.22478 & -2.83886 & -0.90032\end{array}$


Table S1531 Employed active space, reference configurations, and Cartesian coordinates of the OM3/MRCISD-optimized excited-state equilibrium geometry of the $1{ }^{1} B_{u}\left({ }^{1} \pi \pi^{*}\right)$ state of $2,4,6,8$-decatetraene (excited-state energy: $-1453.62570 \mathrm{eV})$.

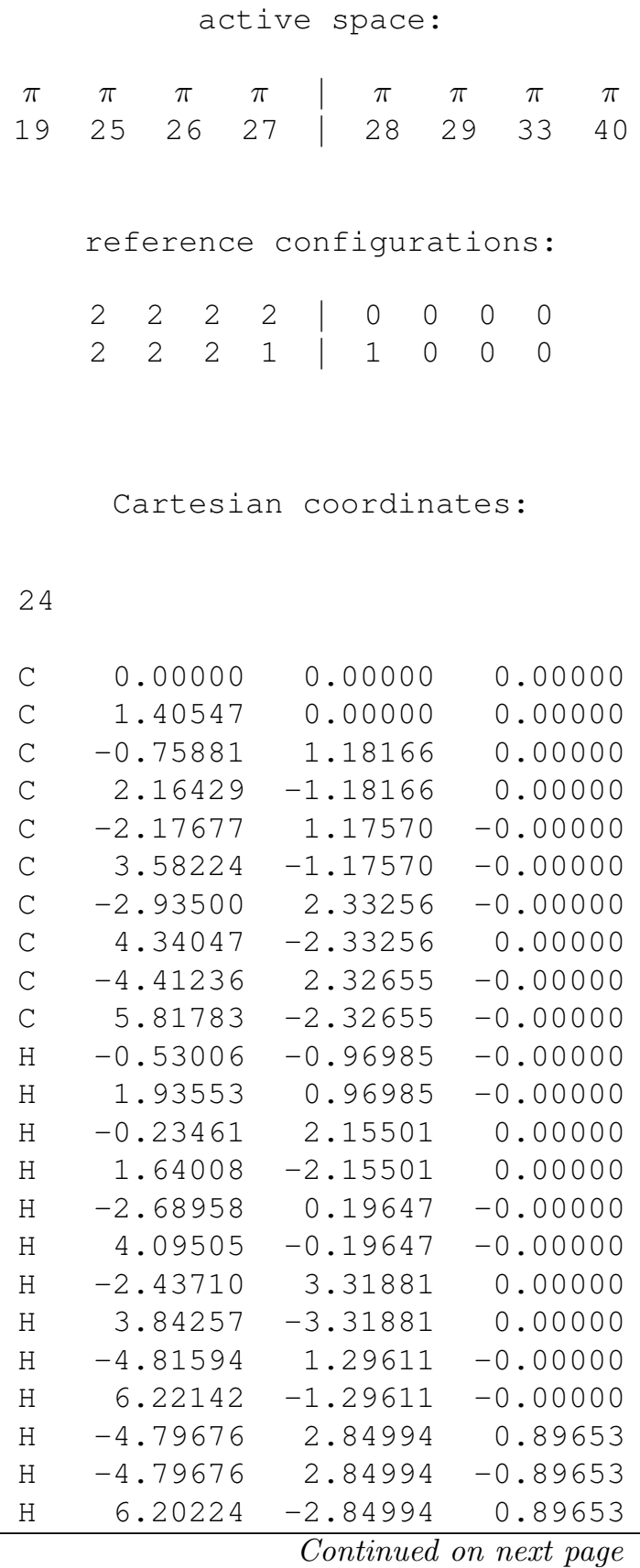

S1684 
Continued from previous page

\begin{tabular}{llll}
\hline $\mathrm{H}$ & 6.20224 & -2.84994 & -0.89653
\end{tabular}

S1685 
Table S1532 Employed active space, reference configurations, and Cartesian coordinates of the OM3/MRCISD-optimized ground-state equilibrium geometry of 5-methoxysalicylic acid (ground-state energy: $-2475.63617 \mathrm{eV}$ ).

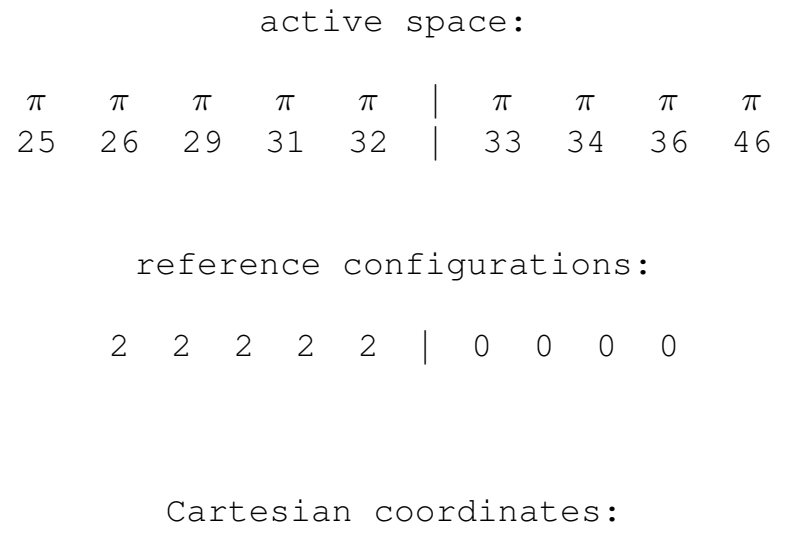

$\begin{array}{lrrr}20 & & & \\ & & & \\ \mathrm{C} & 0.00000 & 0.00000 & 0.00000 \\ \mathrm{C} & 1.42413 & 0.00000 & 0.00000 \\ \mathrm{C} & 2.11406 & 1.20966 & 0.00000 \\ \mathrm{C} & 1.38449 & 2.43037 & 0.00000 \\ \mathrm{C} & -0.01632 & 2.46569 & 0.00000 \\ \mathrm{C} & -0.70761 & 1.23423 & 0.00000 \\ \mathrm{O} & 2.22915 & 3.55955 & 0.00000 \\ \mathrm{C} & 1.57686 & 4.81728 & 0.00000 \\ \mathrm{C} & -2.18210 & 1.16873 & 0.00000 \\ \mathrm{O} & -2.85677 & 0.11611 & 0.00000 \\ \mathrm{O} & -0.59768 & -1.22242 & -0.00000 \\ \mathrm{O} & -2.81694 & 2.38243 & 0.00000 \\ \mathrm{H} & 2.41182 & 5.56485 & 0.00000 \\ \mathrm{H} & 0.98958 & 4.97589 & 0.91725 \\ \mathrm{H} & 0.98958 & 4.97589 & -0.91725 \\ \mathrm{H} & -0.58985 & 3.39705 & 0.00000 \\ \mathrm{H} & 1.94929 & -0.96054 & -0.00000 \\ \mathrm{H} & 3.21046 & 1.24549 & 0.00000 \\ \mathrm{H} & -1.62301 & -1.12308 & -0.00000 \\ \mathrm{H} & -3.82532 & 2.25491 & 0.00000\end{array}$


Table S1533 Employed active space, reference configurations, and Cartesian coordinates of the OM3/MRCISD-optimized excited-state equilibrium geometry of the $2{ }^{1} A^{\prime}\left({ }^{1} \pi \pi^{*}\right)$ state of 5-methoxysalicylic acid (excited-state energy: $-2472.21106 \mathrm{eV})$.

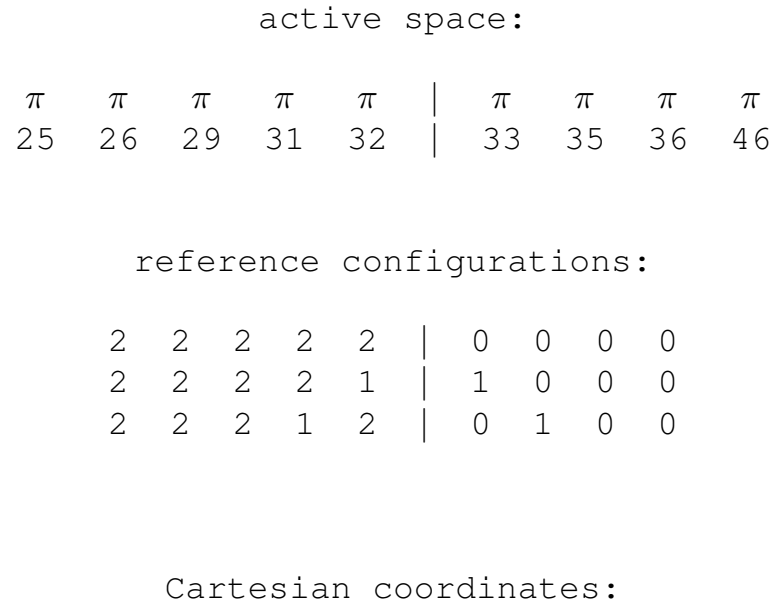


Table S1534 Employed active space, reference configurations, and Cartesian coordinates of the OM3/MRCISD-optimized ground-state equilibrium geometry of salicylic acid (ground-state energy: $-1982.55657 \mathrm{eV}$ ).

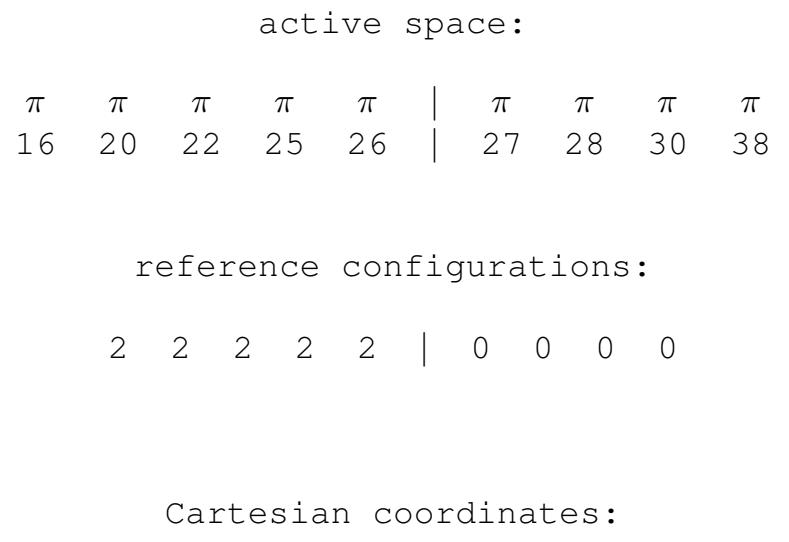

$\begin{array}{lrrr}16 & & & \\ & & & \\ \text { C } & 0.00000 & 0.00000 & 0.00000 \\ \text { C } & 1.39482 & 0.00000 & 0.00000 \\ \text { C } & 2.08611 & 1.23690 & 0.00000 \\ \text { C } & 1.35236 & 2.45460 & 0.00000 \\ \text { C } & -0.05556 & 2.43268 & 0.00000 \\ \text { C } & -0.72615 & 1.20743 & 0.00000 \\ \text { O } & 3.44721 & 1.17102 & 0.00000 \\ \mathrm{C} & 2.13487 & 3.70699 & 0.00000 \\ \text { O } & 3.38413 & 3.76084 & 0.00000 \\ \mathrm{O} & 1.39574 & 4.85337 & 0.00000 \\ \mathrm{H} & -0.60804 & 3.38325 & 0.00000 \\ \mathrm{H} & 1.97521 & -0.92846 & -0.00000 \\ \mathrm{H} & -1.82518 & 1.18332 & 0.00000 \\ \mathrm{H} & -0.54352 & -0.95980 & -0.00000 \\ \mathrm{H} & 3.85612 & 2.12125 & 0.00000 \\ \mathrm{H} & 2.00055 & 5.67140 & 0.00000\end{array}$


Table S1535 Employed active space, reference configurations, and Cartesian coordinates of the OM3/MRCISD-optimized excited-state equilibrium geometry of the $2^{1} A^{\prime}\left({ }^{1} \pi \pi^{*}\right)$ state of salicylic acid (excited-state energy: $-1979.05582 \mathrm{eV})$.

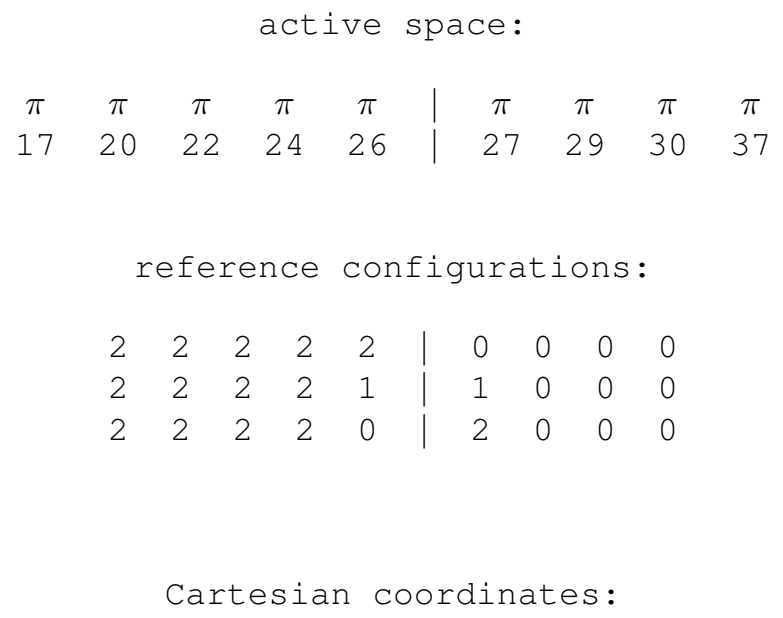


Table S1536 Employed active space, reference configurations, and Cartesian coordinates of the OM3/MRCISD-optimized ground-state equilibrium geometry of gauche-3-phenyl-1-propionic acid (ground-state energy: $-1952.93978 \mathrm{eV}$ ).

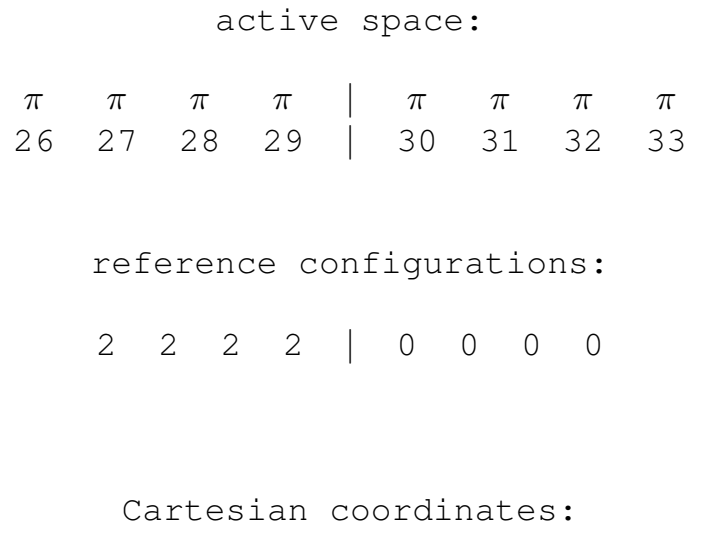

21

$\begin{array}{lrrr}\text { C } & 0.00000 & 0.00000 & 0.00000 \\ \text { C } & 2.80841 & 0.00000 & 0.00000 \\ \text { C } & 0.70911 & 1.21525 & 0.00000 \\ \text { C } & 0.70770 & -1.21547 & -0.00354 \\ \text { C } & 2.10743 & 1.21277 & 0.00245 \\ \text { C } & 2.10612 & -1.21268 & -0.00094 \\ \text { C } & -1.51288 & 0.00456 & 0.01951 \\ \mathrm{C} & -2.02670 & 0.07049 & 1.45436 \\ \mathrm{C} & -1.76979 & -1.24373 & 2.16309 \\ \mathrm{O} & -1.57091 & -1.05436 & 3.51613 \\ \mathrm{O} & -1.74946 & -2.38639 & 1.69959 \\ \mathrm{H} & 3.91005 & -0.00085 & -0.00647 \\ \mathrm{H} & 0.16010 & 2.17176 & -0.00321 \\ \mathrm{H} & 0.15774 & -2.16836 & -0.00241 \\ \mathrm{H} & 2.65898 & 2.16737 & 0.00260 \\ \mathrm{H} & 2.65767 & -2.16679 & -0.00075 \\ \mathrm{H} & -1.89176 & 0.88827 & -0.55161 \\ \mathrm{H} & -1.90276 & -0.91062 & -0.48885 \\ \mathrm{H} & -1.54403 & 0.89881 & 2.01695 \\ \mathrm{H} & -3.13014 & 0.23316 & 1.47620 \\ \mathrm{H} & -1.39065 & -1.93516 & 3.98673\end{array}$


Table S1537 Employed active space, reference configurations, and Cartesian coordinates of the OM3/MRCISD-optimized excited-state equilibrium geometry of the $2{ }^{1} A\left({ }^{1} \pi \pi^{*}\right)$ state of gauche-3-phenyl-1-propionic acid (excited-state energy: $-1947.47913 \mathrm{eV})$.

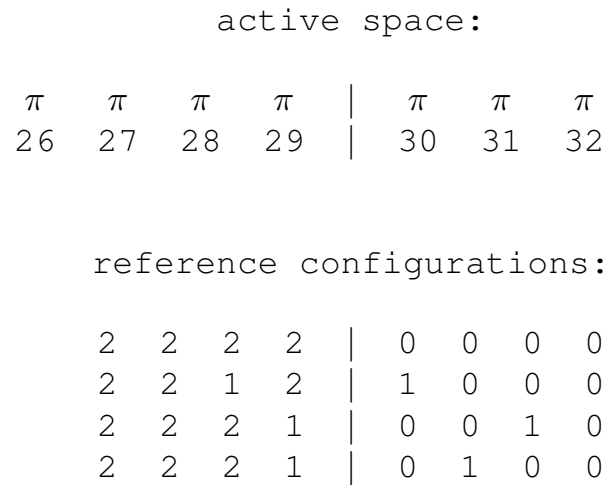

Cartesian coordinates:

21

$\begin{array}{lrrr}\mathrm{C} & 0.00000 & 0.00000 & 0.00000 \\ \mathrm{C} & 2.83006 & 0.00000 & 0.00000 \\ \mathrm{C} & 0.72010 & 1.24316 & 0.00000 \\ \mathrm{C} & 0.72522 & -1.18985 & -0.33493 \\ \mathrm{C} & 2.12745 & 1.20854 & 0.15462 \\ \mathrm{C} & 2.13210 & -1.20688 & -0.18890 \\ \mathrm{C} & -1.44913 & -0.05109 & 0.35937 \\ \mathrm{C} & -1.62802 & -0.47083 & 1.82197 \\ \mathrm{C} & -1.31139 & -1.94253 & 1.98456 \\ \mathrm{O} & -0.85720 & -2.22054 & 3.25929 \\ \mathrm{O} & -1.43010 & -2.86073 & 1.17012 \\ \mathrm{H} & 3.93104 & -0.00391 & 0.03492 \\ \mathrm{H} & 0.17704 & 2.19661 & -0.06167 \\ \mathrm{H} & 0.18336 & -2.08368 & -0.67342 \\ \mathrm{H} & 2.67425 & 2.13983 & 0.37429 \\ \mathrm{H} & 2.68286 & -2.15585 & -0.27278 \\ \mathrm{H} & -1.91579 & 0.95530 & 0.21021 \\ \mathrm{H} & -1.97852 & -0.77357 & -0.31104 \\ \mathrm{H} & -0.97633 & 0.13168 & 2.49015 \\ \mathrm{H} & -2.68403 & -0.32818 & 2.14648 \\ \mathrm{H} & -0.63321 & -3.20446 & 3.36050\end{array}$


Table S1538 Employed active space, reference configurations, and Cartesian coordinates of the OM3/MRCISD-optimized ground-state equilibrium geometry of anti-3-phenyl-1-propionic acid (ground-state energy: $-1952.99728 \mathrm{eV}$ ).

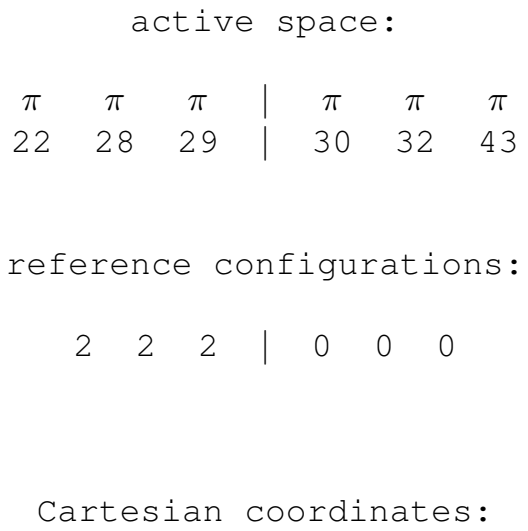


Table S1539 Employed active space, reference configurations, and Cartesian coordinates of the OM3/MRCISD-optimized excited-state equilibrium geometry of the $1^{1} A^{\prime \prime}\left({ }^{1} \pi \pi^{*}\right)$ state of anti-3-phenyl-1-propionic acid (excited-state energy: $-1948.37456 \mathrm{eV})$.

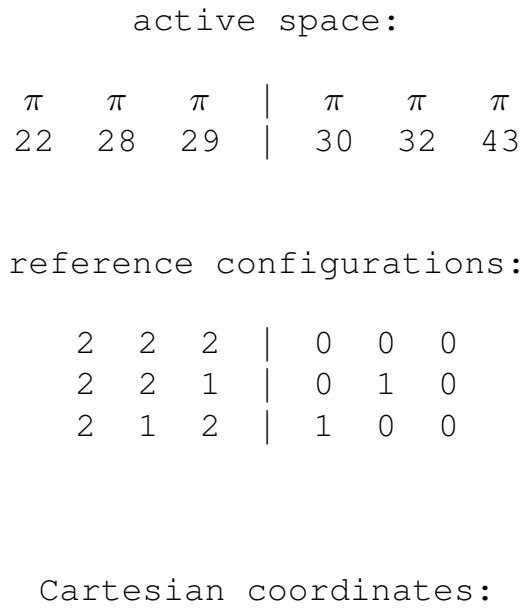


Table S1540 Employed active space, reference configurations, and Cartesian coordinates of the OM3/MRCISD-optimized ground-state equilibrium geometry of cis-1-naphthol (ground-state energy: $-1708.97211 \mathrm{eV}$ ).

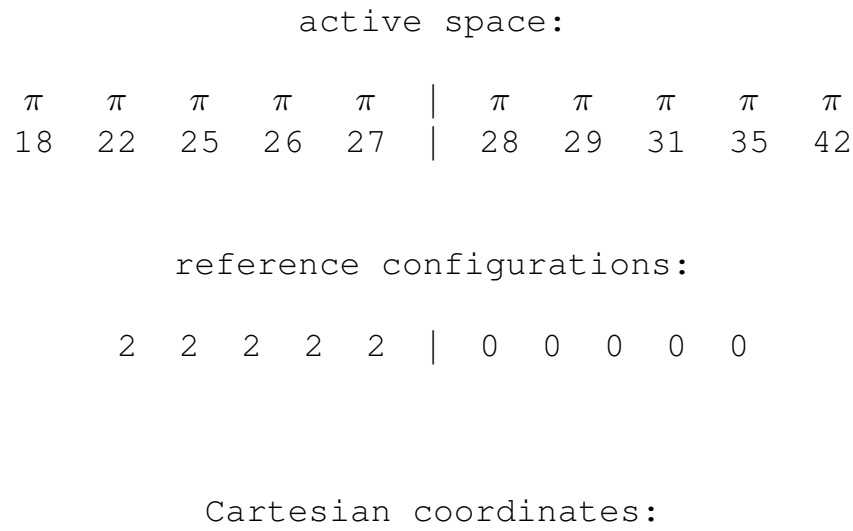

$\begin{array}{llll}\mathrm{H} & 3.74232 & 3.18567 & -0.00000\end{array}$ 
Table S1541 Employed active space, reference configurations, and Cartesian coordinates of the OM3/MRCISD-optimized excited-state equilibrium geometry of the $2{ }^{1} A\left({ }^{1} \pi \pi^{*}\right)$ state of cis-1-naphthol (excited-state energy: $-1705.25795 \mathrm{eV})$.

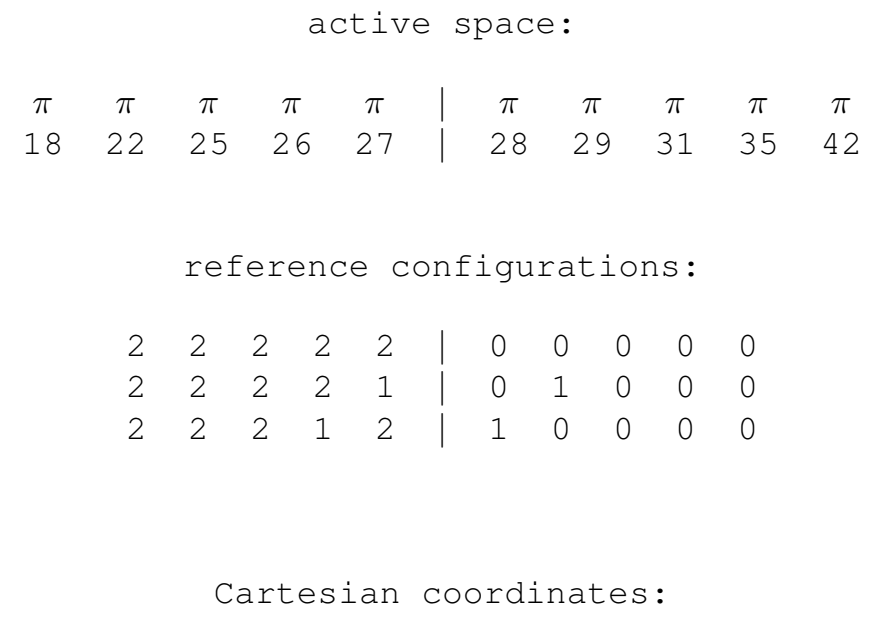

19

$\begin{array}{llll}\mathrm{C} & 0.00000 & 0.00000 & 0.00000\end{array}$

$\begin{array}{llll}\text { C } & 1.41930 & 0.00000 & 0.00000\end{array}$

$\begin{array}{llll}\text { C } & 2.12503 & 1.30717 & 0.00000\end{array}$

$\begin{array}{llll}\text { C } & 1.36189 & 2.49689 & 0.00001\end{array}$

$\begin{array}{llll}\text { C } & -0.04259 & 2.45309 & 0.00003\end{array}$

$\begin{array}{lrrr}\text { C } & -0.72331 & 1.20334 & 0.00001\end{array}$

$\begin{array}{llll}\text { C } & 2.17765 & -1.19953 & -0.00001\end{array}$

$\begin{array}{llll}\text { C } & 3.58774 & -1.17367 & -0.00002\end{array}$

$\begin{array}{llll}\text { C } & 4.28725 & 0.05624 & -0.00002\end{array}$

$\begin{array}{llll}\text { C } & 3.55883 & 1.27971 & -0.00003\end{array}$

$\begin{array}{llll}0 & 4.33438 & 2.39291 & -0.00005\end{array}$

$\mathrm{H} \quad-0.53502 \quad-0.96450 \quad-0.00001$

$\mathrm{H} \quad-1.82386 \quad 1.18796 \quad 0.00001$

$\begin{array}{llll}\mathrm{H} & -0.62357 & 3.38767 & 0.00004\end{array}$

$\begin{array}{llll}\mathrm{H} & 1.86737 & 3.48168 & 0.00002\end{array}$

$\mathrm{H} \quad 1.64819-2.16631-0.00000$

$\mathrm{H} \quad 4.15531-2.11677-0.00002$

$\mathrm{H} \quad 5.38182 \quad 0.09221-0.00003$

$\begin{array}{llll}\mathrm{H} & 3.75630 & 3.23128 & -0.00004\end{array}$ 
Table S1542 Employed active space, reference configurations, and Cartesian coordinates of the OM3/MRCISD-optimized ground-state equilibrium geometry of trans-1-naphthol (ground-state energy: $-1709.02879 \mathrm{eV}$ ).

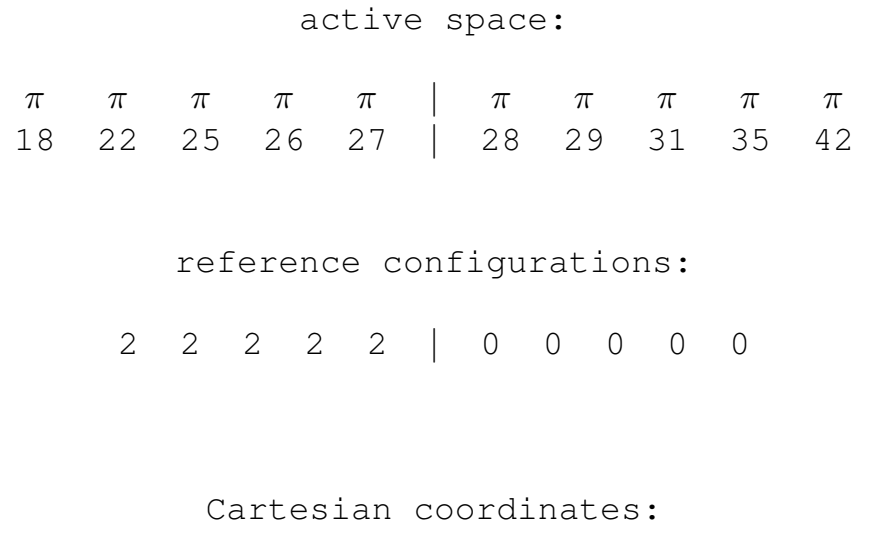

19

$\begin{array}{llll}C & 0.00000 & 0.00000 & 0.00000\end{array}$

$\begin{array}{llll}\text { C } & 1.44271 & 0.00000 & 0.00000\end{array}$

C $\quad 2.10744 \quad 1.26189 \quad 0.00000$

C $\quad 1.34093 \quad 2.47144 \quad 0.00000$

C $\quad-0.04326 \quad 2.42778 \quad 0.00000$

C $\quad-0.72921 \quad 1.18754 \quad 0.00000$

C $\quad 2.19895-1.20932-0.00000$

C $\quad 3.58310-1.15466-0.00000$

$\begin{array}{llll}\text { C } & 4.25511 & 0.09646 & -0.00000\end{array}$

C $\quad 3.53757 \quad 1.28063-0.00000$

$\begin{array}{llll}0 & -0.58459 & -1.24016 & -0.00000\end{array}$

$\begin{array}{llll}\mathrm{H} & 4.05452 & 2.25412 & -0.00000\end{array}$

$\begin{array}{llll}\mathrm{H} & 5.35738 & 0.11332 & -0.00000\end{array}$

$\mathrm{H} \quad \begin{array}{llll}\mathrm{H} & .17684 & -2.08251 & -0.00000\end{array}$

$\mathrm{H} \quad \begin{array}{llll}\mathrm{H} & 1.66298 & -2.16864 & -0.00000\end{array}$

$\mathrm{H} \quad \begin{array}{llll}\mathrm{H} & 1.87285 & 3.43528 & -0.00000\end{array}$

$\begin{array}{llll}\mathrm{H} & -0.63200 & 3.35978 & 0.00000\end{array}$

$\begin{array}{lrrr}\mathrm{H} & -1.82689 & 1.16584 & 0.00000\end{array}$

$\mathrm{H} \quad-1.59690 \quad-1.14151 \quad-0.00000$ 
Table S1543 Employed active space, reference configurations, and Cartesian coordinates of the OM3/MRCISD-optimized excited-state equilibrium geometry of the $2{ }^{1} A^{\prime}\left({ }^{1} \pi \pi^{*}\right)$ state of trans-1-naphthol (excited-state energy: $-1705.38229 \mathrm{eV})$.

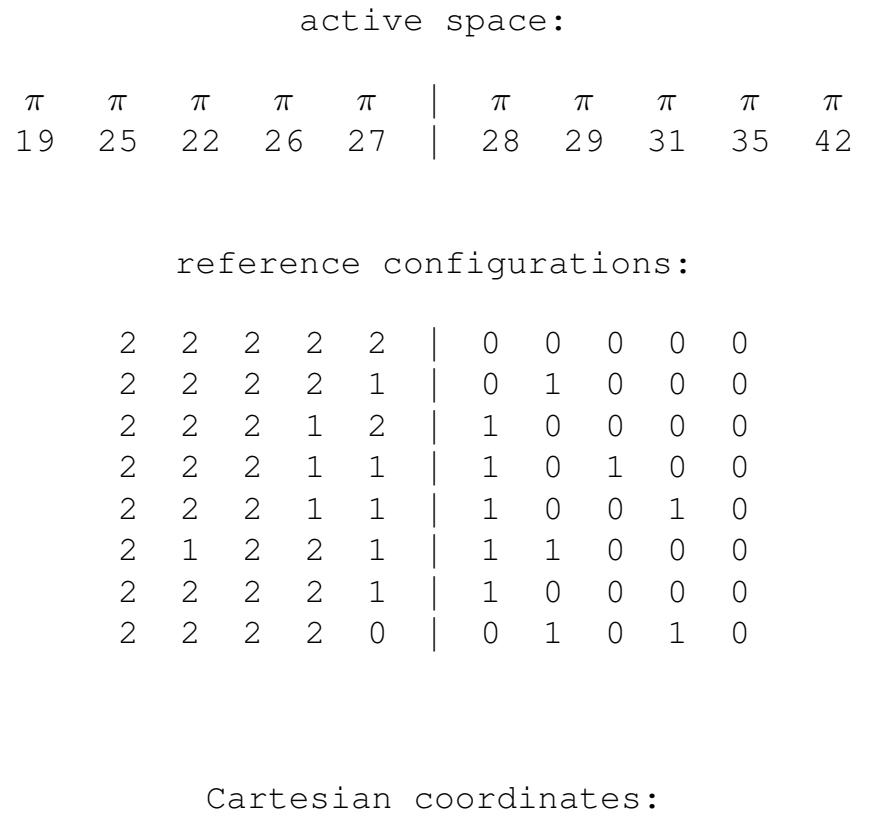

\begin{tabular}{lrrr}
19 & & & \\
& & & \\
$\mathrm{C}$ & 0.00000 & 0.00000 & 0.00000 \\
$\mathrm{C}$ & 1.43281 & 0.00000 & 0.00000 \\
$\mathrm{C}$ & 2.11530 & 1.31890 & 0.00000 \\
$\mathrm{C}$ & 1.33632 & 2.50434 & 0.00000 \\
$\mathrm{C}$ & -0.07530 & 2.45196 & 0.00000 \\
$\mathrm{C}$ & -0.75128 & 1.20885 & 0.00000 \\
$\mathrm{C}$ & 2.20345 & -1.18621 & -0.00000 \\
$\mathrm{C}$ & 3.60881 & -1.11663 & -0.00000 \\
$\mathrm{C}$ & 4.27348 & 0.14165 & -0.00000 \\
$\mathrm{C}$ & 3.53487 & 1.33872 & -0.00000 \\
$\mathrm{O}$ & -0.59380 & -1.22263 & -0.00000 \\
$\mathrm{H}$ & 4.05841 & 2.30958 & -0.00000 \\
$\mathrm{H}$ & 5.37389 & 0.17359 & -0.00000 \\
$\mathrm{H}$ & 4.20463 & -2.04247 & -0.00000 \\
$\mathrm{H}$ & 1.69101 & -2.15892 & -0.00000 \\
$\mathrm{H}$ & 1.84433 & 3.48231 & -0.00000 \\
$\mathrm{H}$ & -0.65985 & 3.38507 & 0.00000 \\
\hline & & Continued on next page
\end{tabular}


Continued from previous page

$\begin{array}{rrrr}\mathrm{H} & -1.84870 & 1.16845 & 0.00000 \\ \mathrm{H} & -1.60598 & -1.11684 & -0.00000\end{array}$

S1698 
Table S1544 Employed active space, reference configurations, and Cartesian coordinates of the OM3/MRCISD-optimized ground-state equilibrium geometry of cis-2-naphthol (ground-state energy: $-1709.02452 \mathrm{eV}$ ).

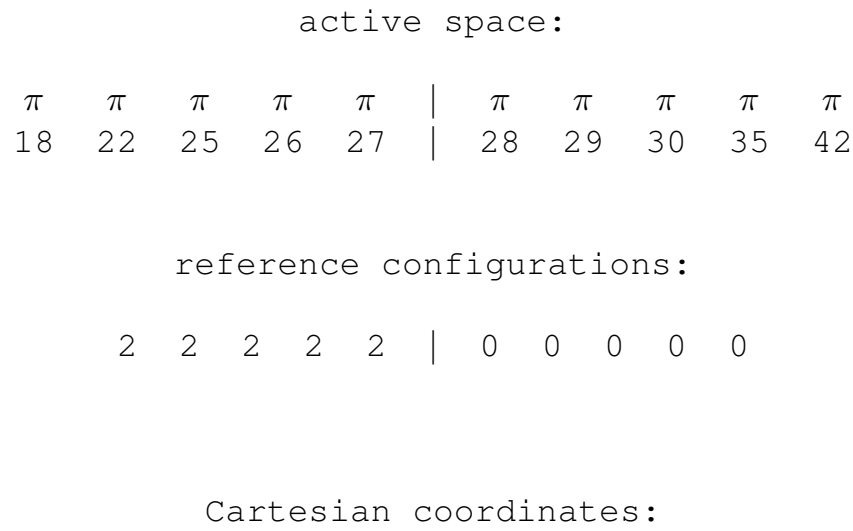


Table S1545 Employed active space, reference configurations, and Cartesian coordinates of the OM3/MRCISD-optimized excited-state equilibrium geometry of the $2^{1} A^{\prime}\left({ }^{1} \pi \pi^{*}\right)$ state of cis-2-naphthol (excited-state energy: $-1705.37421 \mathrm{eV})$.

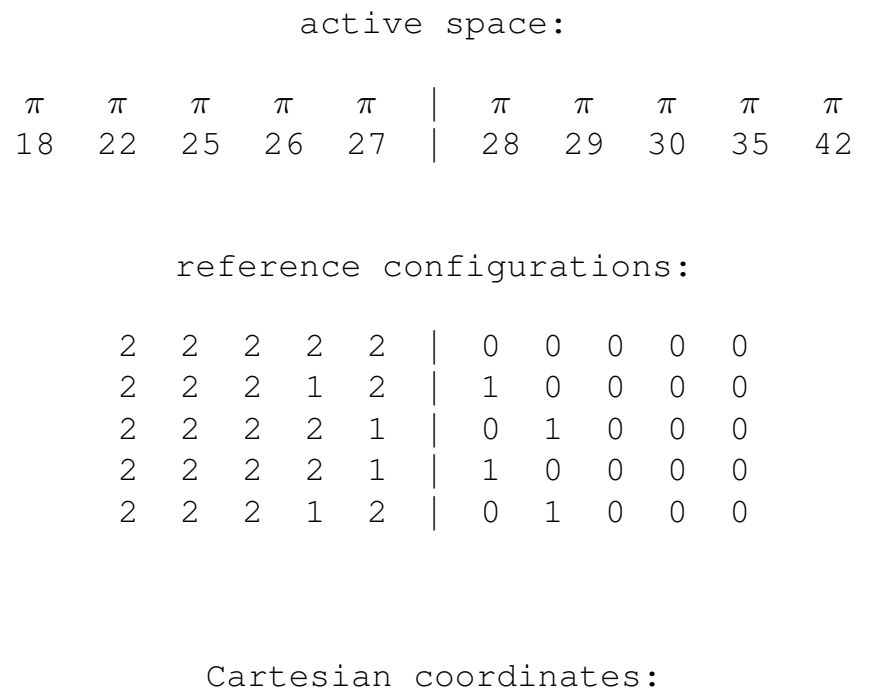

19

$\begin{array}{lrrr}\mathrm{C} & 0.00000 & 0.00000 & 0.00000 \\ \mathrm{C} & 1.41324 & 0.00000 & 0.00000 \\ \mathrm{C} & 2.12631 & 1.30679 & 0.00000 \\ \mathrm{C} & 1.37644 & 2.51304 & 0.00000 \\ \mathrm{C} & -0.02799 & 2.49903 & 0.00000 \\ \mathrm{C} & -0.70481 & 1.23457 & 0.00000 \\ \mathrm{C} & 2.17313 & -1.19861 & -0.00000 \\ \mathrm{C} & 3.57901 & -1.15753 & -0.00000 \\ \mathrm{C} & 4.25983 & 0.08768 & -0.00000 \\ \mathrm{C} & 3.54601 & 1.29883 & -0.00000 \\ \mathrm{O} & -2.05867 & 1.30882 & 0.00000 \\ \mathrm{H} & -0.55729 & -0.94716 & -0.00000 \\ \mathrm{H} & 4.08845 & 2.25778 & -0.00000 \\ \mathrm{H} & 5.36207 & 0.10139 & -0.00000 \\ \mathrm{H} & 4.15734 & -2.09373 & -0.00000 \\ \mathrm{H} & 1.64971 & -2.16806 & -0.00000 \\ \mathrm{H} & 1.91051 & 3.47680 & -0.00000 \\ \mathrm{H} & -0.62215 & 3.41776 & 0.00000 \\ \mathrm{H} & -2.46204 & 0.37347 & 0.00000\end{array}$


Table S1546 Employed active space, reference configurations, and Cartesian coordinates of the OM3/MRCISD-optimized ground-state equilibrium geometry of trans-2-naphthol (ground-state energy: $-1709.00465 \mathrm{eV}$ ).

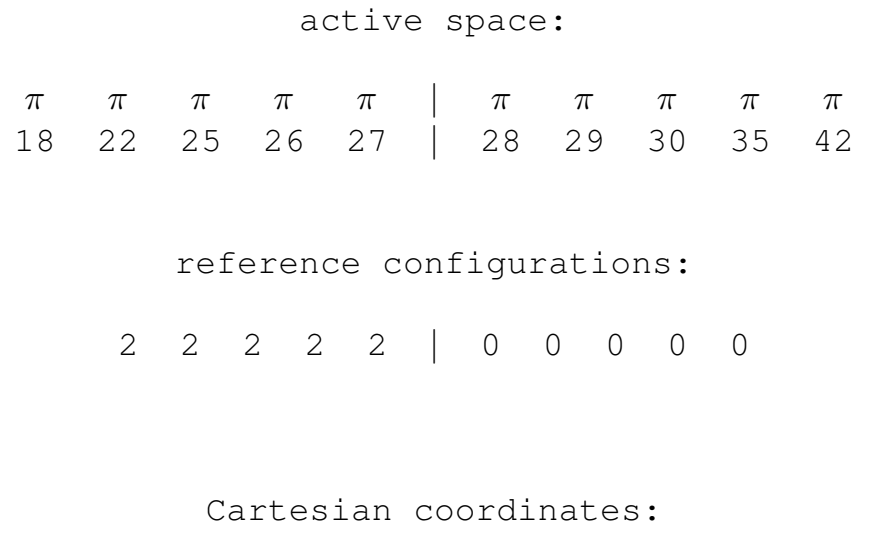

19

$\begin{array}{llll}C & 0.00000 & 0.00000 & 0.00000\end{array}$

$\begin{array}{llll}\text { C } & 1.42616 & 0.00000 & 0.00000\end{array}$

C $\quad 2.13495 \quad 1.24156 \quad 0.00000$

C $\quad 1.40077 \quad 2.47054 \quad 0.00000$

$\begin{array}{lrrr}\text { C } & 0.01873 & 2.46508 & 0.00000\end{array}$

$\begin{array}{lrrr}\text { C } & -0.67948 & 1.21727 & 0.00000\end{array}$

C $\quad 2.17007-1.22384-0.00000$

$\begin{array}{llll}\text { C } & 3.55327 & -1.20301 & -0.00000\end{array}$

$\begin{array}{llll}\text { C } & 4.25688 & 0.03226 & -0.00000\end{array}$

$\begin{array}{llll}\text { C } & 3.56524 & 1.23067 & -0.00000\end{array}$

$\begin{array}{lrrr}0 & -2.05040 & 1.15339 & 0.00000\end{array}$

$\mathrm{H} \quad-0.56080 \quad-0.94063 \quad-0.00000$

$\begin{array}{llll}\mathrm{H} & 4.10383 & 2.19319 & -0.00000\end{array}$

$\begin{array}{llll}\mathrm{H} & 5.35843 & 0.02198 & -0.00000\end{array}$

$\mathrm{H} \quad 4.12593 \quad-2.14471 \quad-0.00000$

$\mathrm{H} \quad \begin{array}{llll}\mathrm{H} & 1.61896 & -2.17809 & -0.00000\end{array}$

$\mathrm{H} \quad \begin{array}{llll}\mathrm{H} & 1.95717 & 3.42276 & -0.00000\end{array}$

$\mathrm{H} \quad-0.55785 \quad 3.40186 \quad 0.00000$

$\mathrm{H} \quad-2.43556 \quad 2.09474 \quad 0.00000$ 
Table S1547 Employed active space, reference configurations, and Cartesian coordinates of the OM3/MRCISD-optimized excited-state equilibrium geometry of the $2{ }^{1} A^{\prime}\left({ }^{1} \pi \pi^{*}\right)$ state of trans-2-naphthol (excited-state energy: $-1705.37392 \mathrm{eV})$.

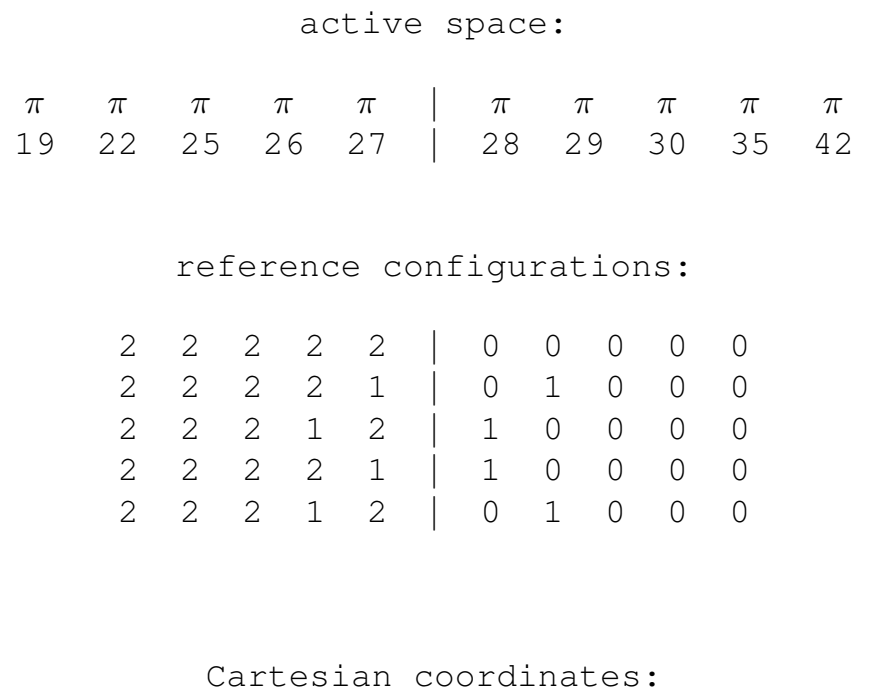

19

$\begin{array}{lrrr}\mathrm{C} & 0.00000 & 0.00000 & 0.00000 \\ \mathrm{C} & 1.41378 & 0.00000 & 0.00000 \\ \mathrm{C} & 2.13060 & 1.30325 & 0.00000 \\ \mathrm{C} & 1.38627 & 2.51407 & 0.00000 \\ \mathrm{C} & -0.01783 & 2.50071 & 0.00000 \\ \mathrm{C} & -0.69997 & 1.23829 & 0.00000 \\ \mathrm{C} & 2.17070 & -1.19955 & -0.00000 \\ \mathrm{C} & 3.57675 & -1.16304 & -0.00000 \\ \mathrm{C} & 4.26026 & 0.08008 & -0.00000 \\ \mathrm{C} & 3.54983 & 1.29314 & -0.00000 \\ \mathrm{O} & -2.05228 & 1.14626 & 0.00000 \\ \mathrm{H} & -0.57128 & -0.93495 & -0.00000 \\ \mathrm{H} & 4.09536 & 2.25075 & -0.00000 \\ \mathrm{H} & 5.36278 & 0.09151 & -0.00000 \\ \mathrm{H} & 4.15254 & -2.10050 & -0.00000 \\ \mathrm{H} & 1.64404 & -2.16710 & -0.00000 \\ \mathrm{H} & 1.92377 & 3.47610 & -0.00000 \\ \mathrm{H} & -0.59795 & 3.43207 & 0.00000 \\ \mathrm{H} & -2.46642 & 2.07680 & 0.00000\end{array}$


Table S1548 Employed active space, reference configurations, and Cartesian coordinates of the OM3/MRCISD-optimized ground-state equilibrium geometry of cis-7-hydroxyquinoline (ground-state energy: $-1784.83720 \mathrm{eV}$ ).

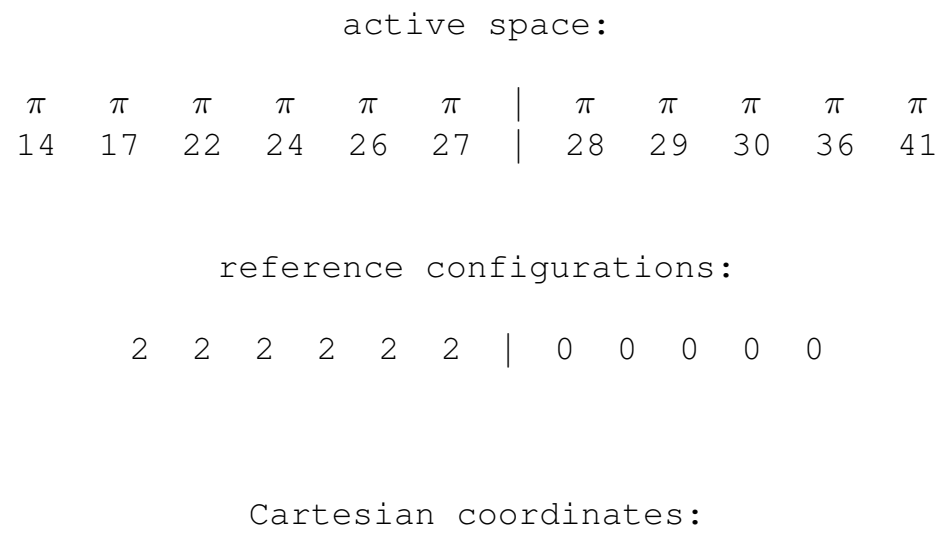


Table S1549 Employed active space, reference configurations, and Cartesian coordinates of the OM3/MRCISD-optimized excited-state equilibrium geometry of the $2{ }^{1} A^{\prime}\left({ }^{1} \pi \pi^{*}\right)$ state of cis-7-hydroxyquinoline (excited-state energy: $-1781.26523 \mathrm{eV})$.

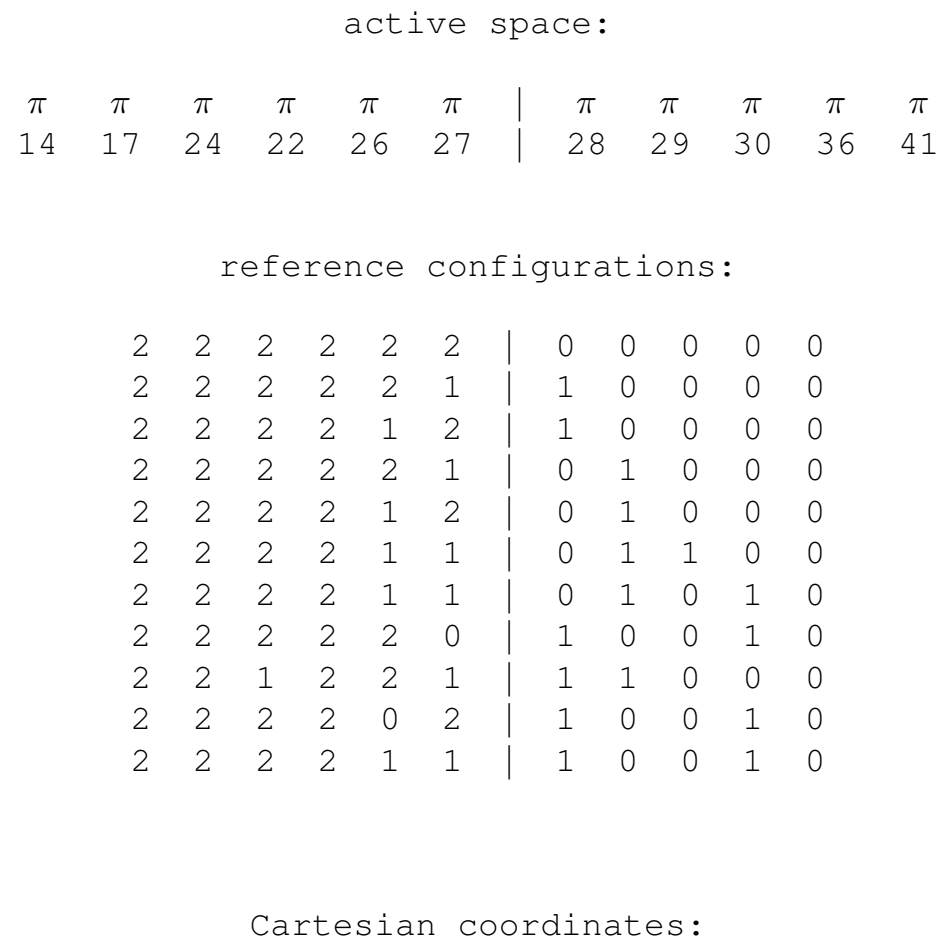

\begin{tabular}{lrrr}
18 & & \\
& & & \\
$\mathrm{C}$ & 0.00000 & 0.00000 & 0.00000 \\
$\mathrm{C}$ & 1.41564 & 0.00000 & 0.00000 \\
$\mathrm{C}$ & 2.16066 & 1.29388 & 0.00000 \\
$\mathrm{C}$ & 1.41908 & 2.50458 & 0.00000 \\
$\mathrm{C}$ & -0.00405 & 2.44245 & 0.00000 \\
$\mathrm{C}$ & -0.72623 & 1.20896 & 0.00000 \\
$\mathrm{C}$ & 2.18952 & -1.19218 & -0.00000 \\
$\mathrm{C}$ & 3.58792 & -1.11400 & -0.00000 \\
$\mathrm{C}$ & 4.22867 & 0.16852 & -0.00000 \\
$\mathrm{~N}$ & 3.53551 & 1.33762 & -0.00000 \\
$\mathrm{O}$ & -0.78271 & 3.56869 & 0.00000 \\
$\mathrm{H}$ & 5.33744 & 0.22834 & -0.00000 \\
$\mathrm{H}$ & -0.54191 & -0.95877 & -0.00000 \\
$\mathrm{H}$ & -1.81974 & 1.23031 & 0.00000 \\
\hline & \multicolumn{3}{c}{ Continued on next page }
\end{tabular}




\begin{tabular}{lrrr}
\multicolumn{4}{c}{ Continued from previous page } \\
\hline $\mathrm{H}$ & 1.94272 & 3.46716 & -0.00000 \\
$\mathrm{H}$ & 1.68269 & -2.16885 & -0.00000 \\
$\mathrm{H}$ & 4.19965 & -2.02707 & -0.00000 \\
$\mathrm{H}$ & -0.19423 & 4.40125 & 0.00000
\end{tabular}


Table S1550 Employed active space, reference configurations, and Cartesian coordinates of the OM3/MRCISD-optimized ground-state equilibrium geometry of trans-7-hydroxyquinoline (ground-state energy: $-1784.79831 \mathrm{eV}$ ).

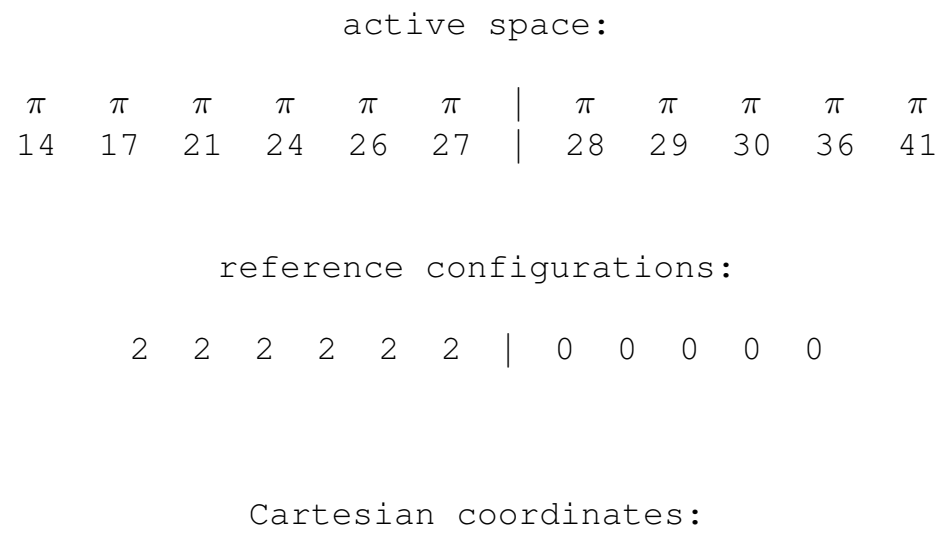


Table S1551 Employed active space, reference configurations, and Cartesian coordinates of the OM3/MRCISD-optimized excited-state equilibrium geometry of the $2{ }^{1} A^{\prime}\left({ }^{1} \pi \pi^{*}\right)$ state of trans-7-hydroxyquinoline (excited-state energy: $-1781.18781 \mathrm{eV})$.

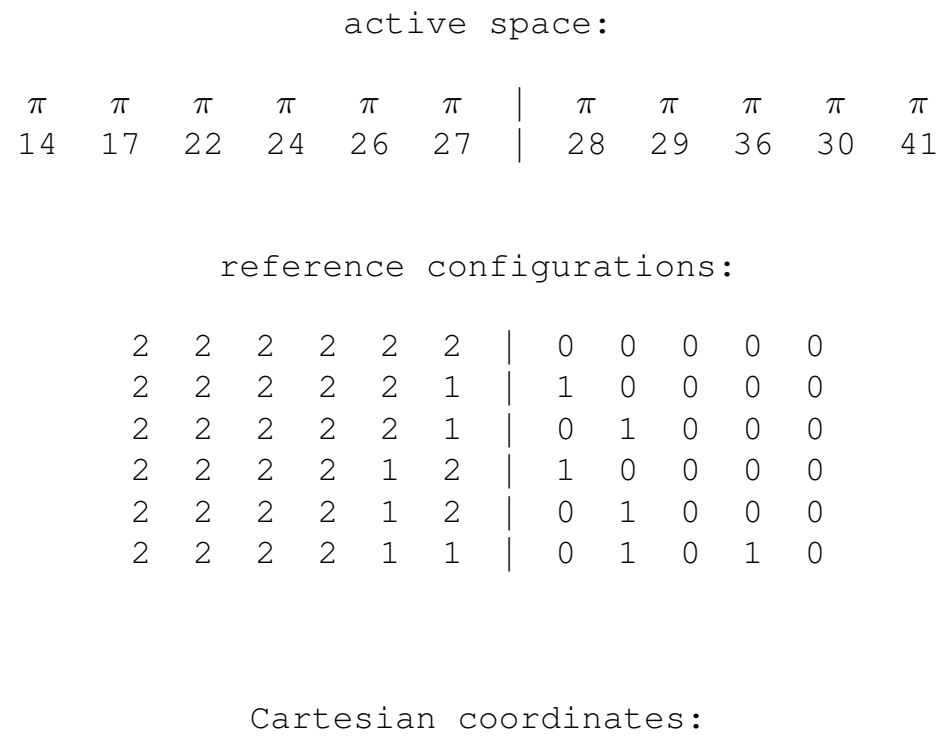

18

$\begin{array}{lrrr}\mathrm{C} & 0.00000 & 0.00000 & 0.00000 \\ \mathrm{C} & 1.41722 & 0.00000 & 0.00000 \\ \mathrm{C} & 2.16385 & 1.29178 & 0.00000 \\ \mathrm{C} & 1.42636 & 2.50611 & 0.00000 \\ \mathrm{C} & 0.00368 & 2.44301 & 0.00000 \\ \mathrm{C} & -0.71965 & 1.20997 & 0.00000 \\ \mathrm{C} & 2.18563 & -1.19525 & -0.00000 \\ \mathrm{C} & 3.58328 & -1.12147 & -0.00000 \\ \mathrm{C} & 4.22761 & 0.15806 & -0.00000 \\ \mathrm{~N} & 3.53772 & 1.32854 & -0.00000 \\ \mathrm{O} & -0.63065 & 3.65619 & 0.00000 \\ \mathrm{H} & 5.33646 & 0.21448 & -0.00000 \\ \mathrm{H} & -0.54263 & -0.95857 & -0.00000 \\ \mathrm{H} & -1.81651 & 1.21838 & 0.00000 \\ \mathrm{H} & 1.93573 & 3.47343 & -0.00000 \\ \mathrm{H} & 1.67543 & -2.17056 & -0.00000 \\ \mathrm{H} & 4.19187 & -2.03695 & -0.00000 \\ \mathrm{H} & -1.64130 & 3.52252 & 0.00000\end{array}$


Table S1552 Employed active space, reference configurations, and Cartesian coordinates of the OM3/MRCISD-optimized ground-state equilibrium geometry of 2-hydroxyquinoline (enol) (ground-state energy: $-1784.55023 \mathrm{eV}$ ).

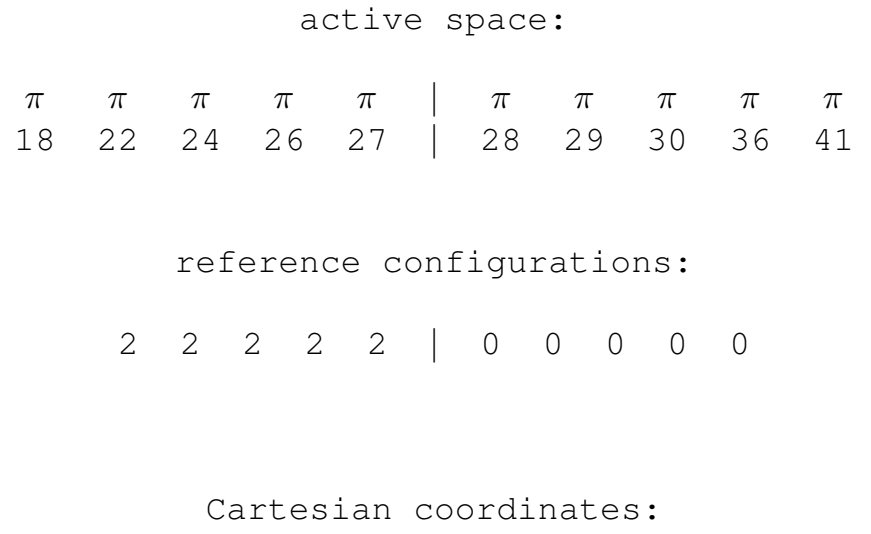

18

$\begin{array}{lrrr}\mathrm{N} & 0.00000 & 0.00000 & 0.00000 \\ \mathrm{C} & 1.38820 & 0.00000 & 0.00000 \\ \mathrm{C} & 2.14490 & 1.21988 & 0.00000 \\ \mathrm{C} & 1.43298 & 2.46097 & 0.00000 \\ \mathrm{C} & 0.05351 & 2.46218 & 0.00000 \\ \mathrm{C} & -0.62677 & 1.19045 & 0.00000 \\ \mathrm{C} & 2.08356 & -1.25755 & -0.00000 \\ \mathrm{C} & 3.46587 & -1.27825 & -0.00000 \\ \mathrm{C} & 4.21333 & -0.06838 & -0.00000 \\ \mathrm{C} & 3.57091 & 1.15785 & -0.00000 \\ \mathrm{O} & -2.00175 & 1.11904 & 0.00000 \\ \mathrm{H} & 4.14567 & 2.09874 & -0.00000 \\ \mathrm{H} & 5.31378 & -0.11971 & -0.00000 \\ \mathrm{H} & 4.00677 & -2.23842 & -0.00000 \\ \mathrm{H} & 1.48668 & -2.17942 & -0.00000 \\ \mathrm{H} & 2.00094 & 3.40532 & -0.00000 \\ \mathrm{H} & -0.52354 & 3.39507 & 0.00000 \\ \mathrm{H} & -2.38913 & 2.05995 & 0.00000\end{array}$


Table S1553 Employed active space, reference configurations, and Cartesian coordinates of the OM3/MRCISD-optimized excited-state equilibrium geometry of the $2{ }^{1} A^{\prime}\left({ }^{1} \pi \pi^{*}\right)$ state of 2-hydroxyquinoline (enol) (excited-state energy: $-1781.09955 \mathrm{eV})$.

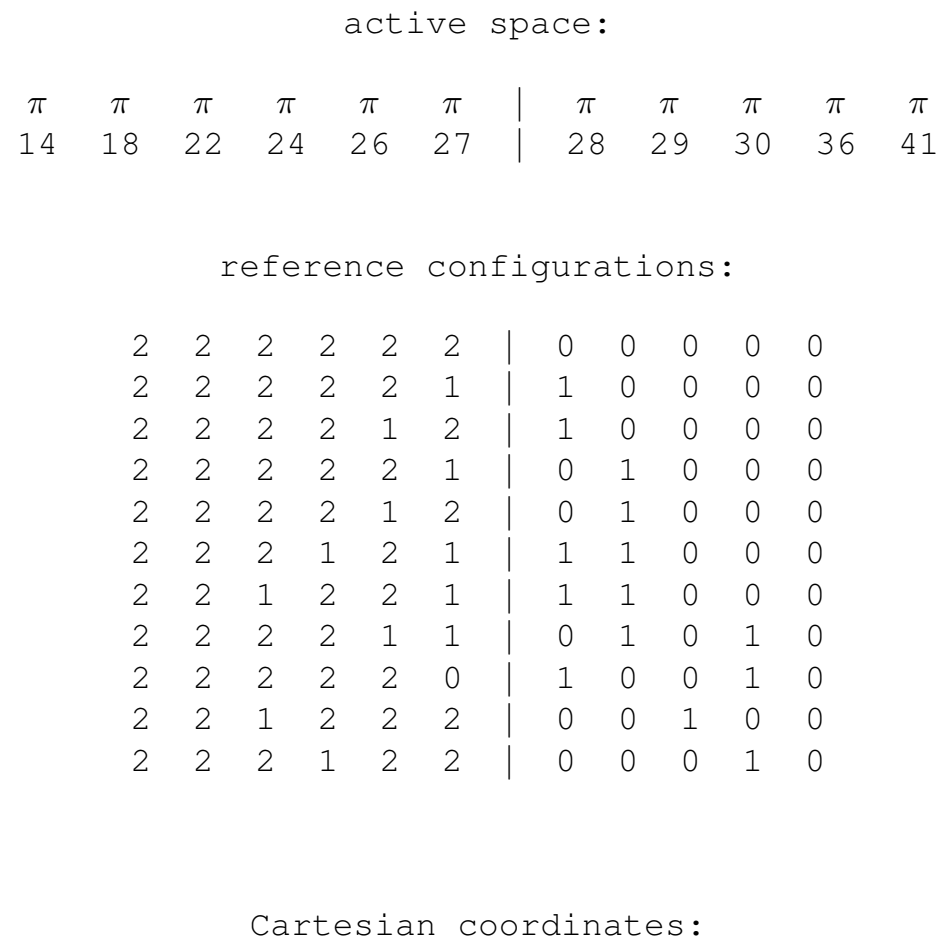

\begin{tabular}{lrrr}
18 & & \\
& & & \\
$\mathrm{~N}$ & 0.00000 & 0.00000 & 0.00000 \\
$\mathrm{C}$ & 1.37099 & 0.00000 & 0.00000 \\
$\mathrm{C}$ & 2.14386 & 1.28069 & 0.00000 \\
$\mathrm{C}$ & 1.42530 & 2.50410 & 0.00000 \\
$\mathrm{C}$ & 0.02212 & 2.49457 & 0.00000 \\
$\mathrm{C}$ & -0.63665 & 1.21128 & 0.00000 \\
$\mathrm{C}$ & 2.08121 & -1.23756 & -0.00000 \\
$\mathrm{C}$ & 3.48883 & -1.24652 & -0.00000 \\
$\mathrm{C}$ & 4.21892 & -0.03084 & -0.00000 \\
$\mathrm{C}$ & 3.55747 & 1.21619 & -0.00000 \\
$\mathrm{O}$ & -2.00947 & 1.10942 & 0.00000 \\
$\mathrm{H}$ & 4.14308 & 2.14941 & -0.00000 \\
$\mathrm{H}$ & 5.32086 & -0.06079 & -0.00000 \\
$\mathrm{H}$ & 4.02972 & -2.20453 & -0.00000 \\
\hline & \multicolumn{3}{c}{ Continued on next page }
\end{tabular}

S1709 


\begin{tabular}{lrrr}
\multicolumn{5}{c}{ Continued from previous page } \\
\hline $\mathrm{H}$ & 1.50669 & -2.17344 & -0.00000 \\
$\mathrm{H}$ & 1.97230 & 3.45876 & -0.00000 \\
$\mathrm{H}$ & -0.55979 & 3.42177 & 0.00000 \\
$\mathrm{H}$ & -2.41953 & 2.04236 & 0.00000
\end{tabular}


Table S1554 Employed active space, reference configurations, and Cartesian coordinates of the OM3/MRCISD-optimized ground-state equilibrium geometry of 2-hydroxyquinoline (keto) (ground-state energy: -1785.19367 eV).

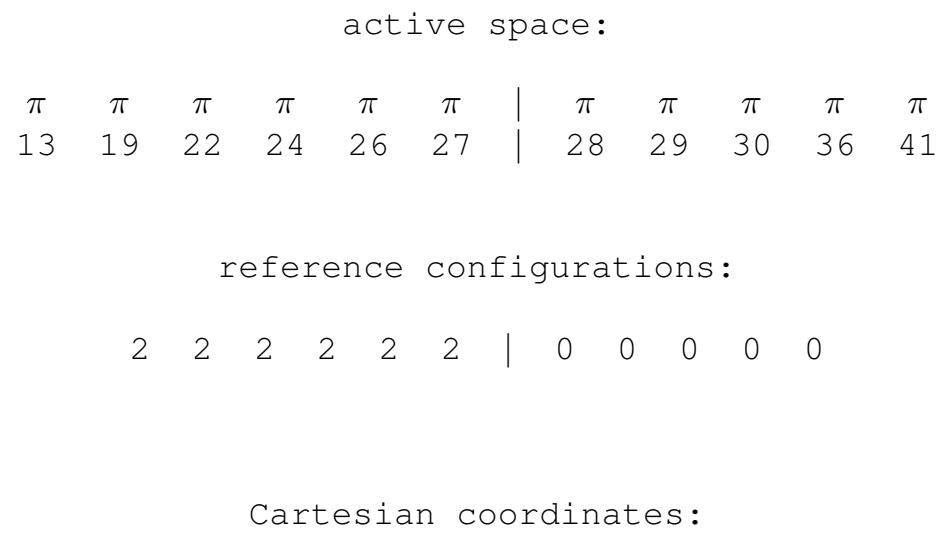


Table S1555 Employed active space, reference configurations, and Cartesian coordinates of the OM3/MRCISD-optimized excited-state equilibrium geometry of the $2{ }^{1} A^{\prime}\left({ }^{1} \pi \pi^{*}\right)$ state of 2-hydroxyquinoline (keto) (excited-state energy: $-1781.68532 \mathrm{eV})$.

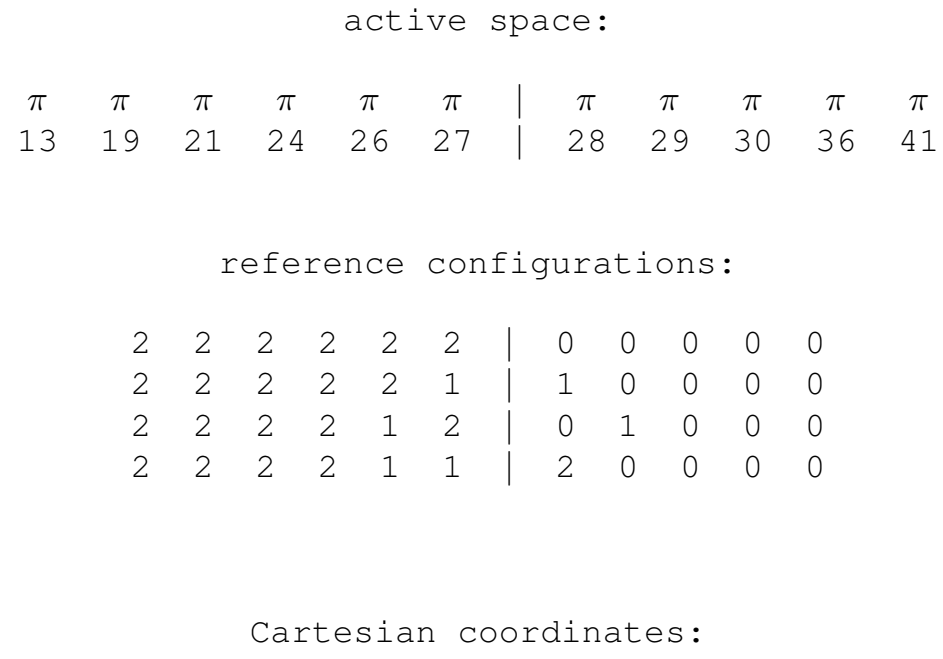

18

$\begin{array}{llll}C & 0.00000 & 0.00000 & 0.00000\end{array}$

C $\quad 1.42250 \quad 0.00000 \quad 0.00000$

C $\quad 2.09762 \quad 1.31511 \quad 0.00000$

C $\quad 1.33820 \quad 2.52502 \quad 0.00000$

C $\quad-0.06540 \quad 2.45912 \quad 0.00000$

C $\quad-0.72227 \quad 1.20942 \quad 0.00000$

C $\quad 2.19945-1.18087-0.00000$

$\begin{array}{llll}\text { C } & 3.59939 & -1.13972 & -0.00000\end{array}$

$\begin{array}{llll}\mathrm{C} & 4.30802 & 0.10994 & -0.00000\end{array}$

$\begin{array}{llll}\mathrm{N} & 3.47280 & 1.32237 & -0.00000\end{array}$

$\begin{array}{lrrr}0 & 5.54230 & 0.32977 & -0.00000\end{array}$

$\mathrm{H} \quad-0.53096 \quad-0.96190 \quad-0.00000$

$\mathrm{H} \quad-1.82503 \quad 1.17946 \quad 0.00000$

$\mathrm{H} \quad-0.65314 \quad 3.38847 \quad 0.00000$

$\mathrm{H} \quad 1.85684 \quad 3.49252-0.00000$

$\mathrm{H} \quad 1.68512-2.15395-0.00000$

$\mathrm{H} \quad 4.20591-2.04509-0.00000$

$\mathrm{H} \quad 3.98601 \quad 2.24150 \quad-0.00000$ 
Table S1556 Employed active space, reference configurations, and Cartesian coordinates of the OM3/MRCISD-optimized ground-state equilibrium geometry of trans-o-dimethoxybenzene (ground-state energy: $-1826.25078 \mathrm{eV}$ ).

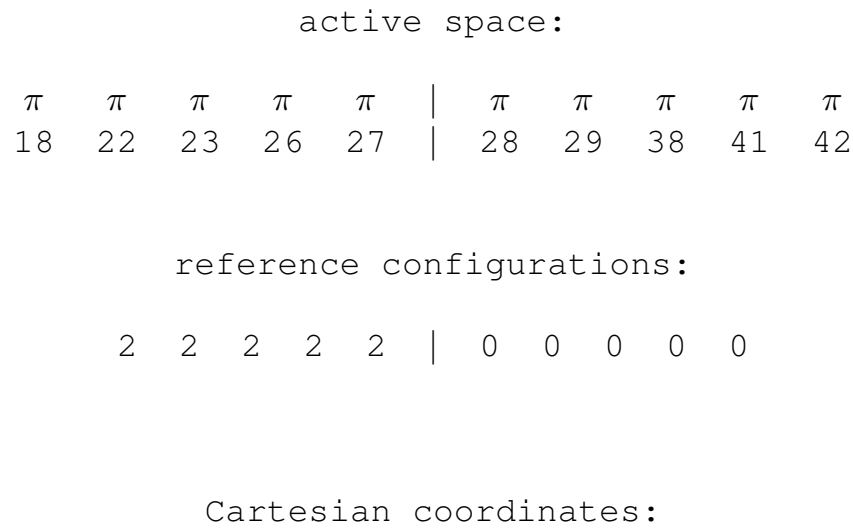


Table S1557 Employed active space, reference configurations, and Cartesian coordinates of the OM3/MRCISD-optimized excited-state equilibrium geometry of the $2{ }^{1} A^{\prime}\left({ }^{1} \pi \pi^{*}\right)$ state of trans-o-dimethoxybenzene (excited-state energy: $-1822.26582 \mathrm{eV})$.

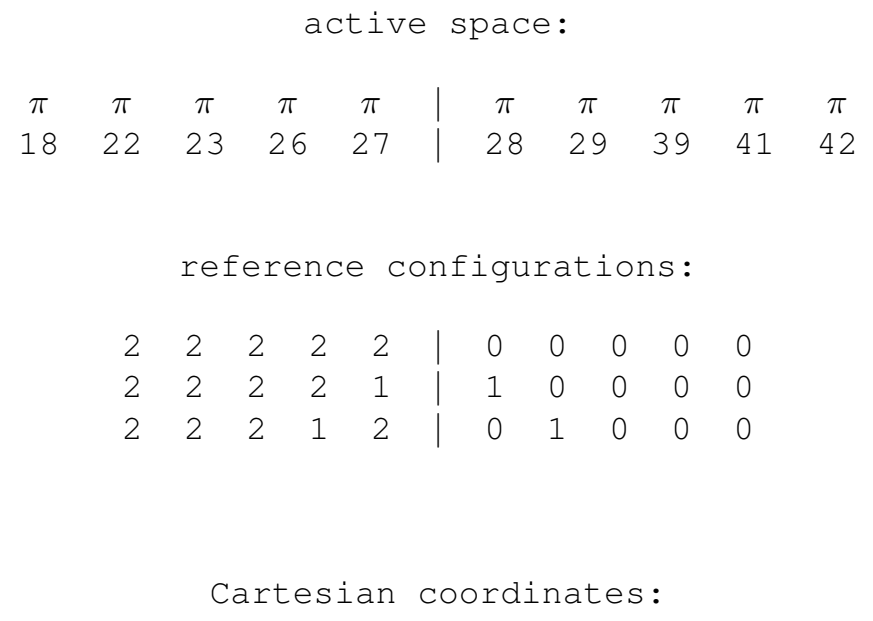

20

$\begin{array}{llll}\mathrm{C} & 0.00000 & 0.00000 & 0.00000\end{array}$

C $\quad 1.41415 \quad 0.00000 \quad 0.00000$

C $2.10154 \quad 1.26275 \quad 0.00000$

$\begin{array}{llll}\text { C } & 1.34768 & 2.50109 & 0.00000\end{array}$

$\begin{array}{llll}\text { C } & -0.08971 & 2.47034 & 0.00000\end{array}$

$\begin{array}{llll}\text { C } & -0.73911 & 1.21412 & 0.00000\end{array}$

$\begin{array}{llll}0 & 2.12745 & 3.63146 & 0.00000\end{array}$

$\begin{array}{llll}\text { C } & 1.43954 & 4.87959 & 0.00000\end{array}$

$\begin{array}{llll}\text { O } & 3.46377 & 1.43634 & -0.00000\end{array}$

$\begin{array}{llll}\mathrm{C} & 4.25661 & 0.25209 & -0.00000\end{array}$

$\mathrm{H} \quad-0.66542 \quad 3.39965 \quad 0.00000$

$\mathrm{H} \quad 1.97531-0.93817 \quad-0.00000$

$\mathrm{H} \quad-1.84028 \quad 1.17732 \quad 0.00000$

$\mathrm{H} \quad-0.53836-0.96129-0.00000$

$\begin{array}{llll}\mathrm{H} & 2.24957 & 5.64546 & 0.00000\end{array}$

H $\quad 5.30892 \quad 0.61995-0.00000$

$\mathrm{H} \quad 0.81121 \quad 5.01900 \quad 0.91509$

$\mathrm{H} \quad 4.09190 \quad-0.37009 \quad 0.91509$

$\mathrm{H} \quad 0.81121 \quad 5.01900 \quad-0.91509$

$\mathrm{H} \quad 4.09190 \quad-0.37009 \quad-0.91509$ 
Table S1558 Employed active space, reference configurations, and Cartesian coordinates of the OM3/MRCISD-optimized ground-state equilibrium geometry of 7-azaindole (ground-state energy: $-1396.17799 \mathrm{eV}$ ).

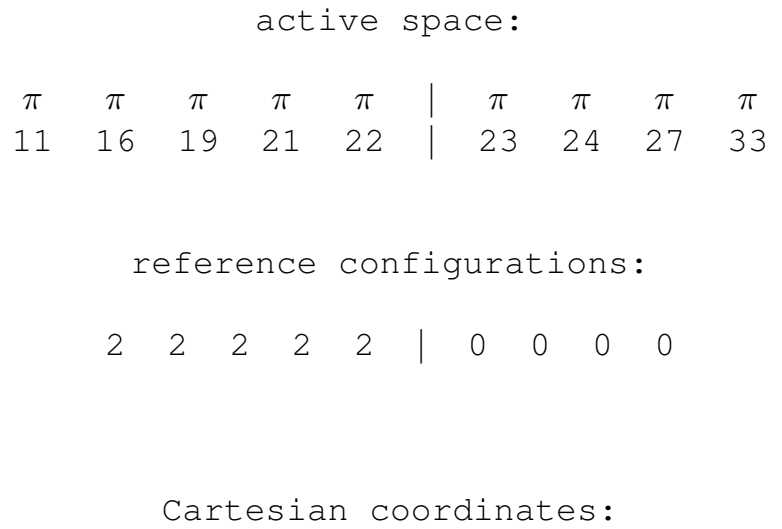


Table S1559 Employed active space, reference configurations, and Cartesian coordinates of the OM3/MRCISD-optimized excited-state equilibrium geometry of the $2{ }^{1} A^{\prime}\left({ }^{1} \pi \pi^{*}\right)$ state of 7 -azaindole (excited-state energy: $\left.-1392.17798 \mathrm{eV}\right)$.

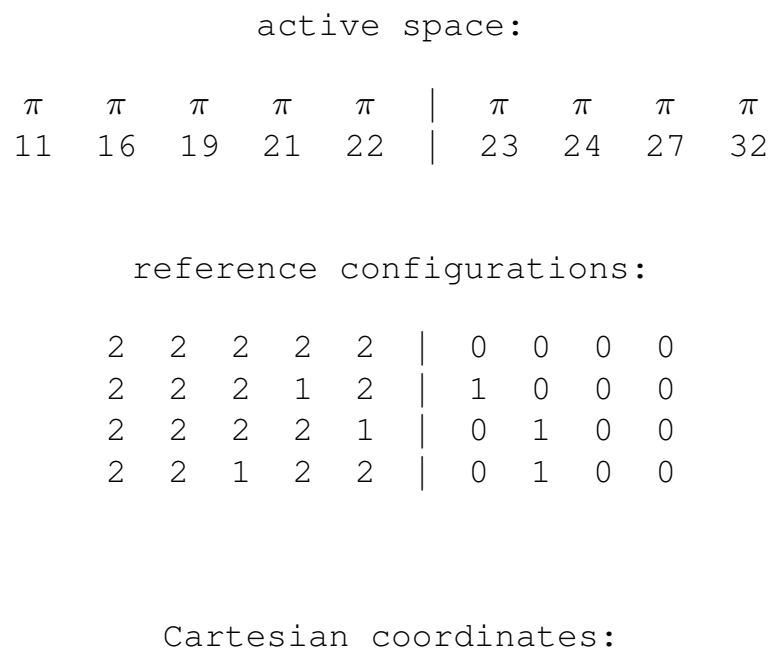


Table S1560 Employed active space, reference configurations, and Cartesian coordinates of the OM3/MRCISD-optimized ground-state equilibrium geometry of phenylacetylene (ground-state energy: $-1088.29402 \mathrm{eV}$ ).

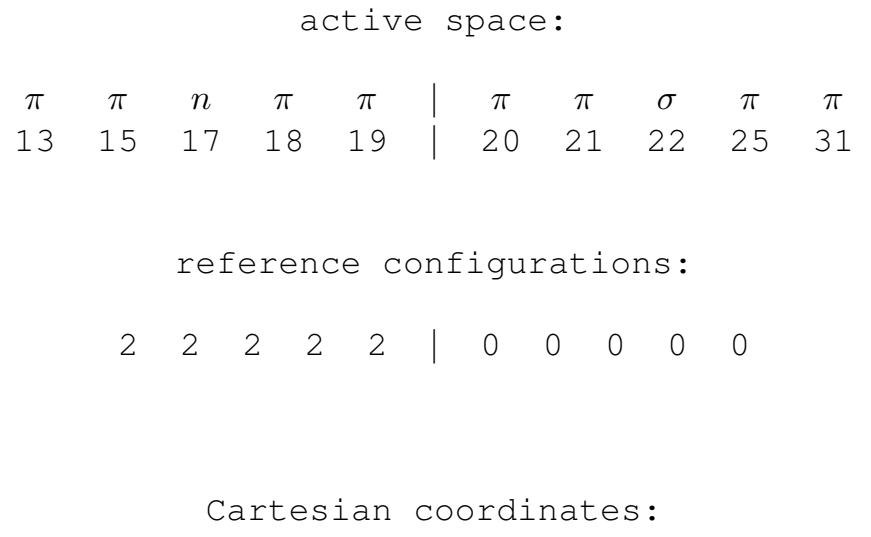

14

$\begin{array}{llll}\text { C } & 0.00000 & 0.00000 & -1.34691\end{array}$

C $\quad 1.22030 \quad 0.00000 \quad-0.65632$

C $\quad-1.22030 \quad 0.00000 \quad-0.65632$

$\begin{array}{llll}\text { C } & 1.23057 & 0.00000 & 0.74521\end{array}$

C $\quad-1.23057 \quad 0.00000 \quad 0.74521$

C $\quad 0.00000 \quad 0.00000 \quad 1.42870$

$\mathrm{H} \quad 0.00000 \quad 0.00000-2.44965$

$\mathrm{H} \quad 2.17186 \quad 0.00000 \quad-1.20638$

$\mathrm{H} \quad-2.17186 \quad 0.00000 \quad-1.20638$

$\mathrm{H} \quad 2.17968 \quad 0.00000 \quad 1.29588$

$\begin{array}{llll}\mathrm{H} & -2.17968 & 0.00000 & 1.29588\end{array}$

$\begin{array}{llll}\text { C } & 0.00000 & 0.00000 & 2.86666\end{array}$

$\begin{array}{llll}\text { C } & 0.00000 & 0.00000 & 4.11302\end{array}$

$\begin{array}{llll}\mathrm{H} & 0.00000 & 0.00000 & 5.16499\end{array}$ 
Table S1561 Employed active space, reference configurations, and Cartesian coordinates of the OM3/MRCISD-optimized excited-state equilibrium geometry of the $1{ }^{1} B_{2}\left({ }^{1} \pi \pi^{*}\right)$ state of phenylacetylene (excited-state energy: $-1084.09887 \mathrm{eV})$.

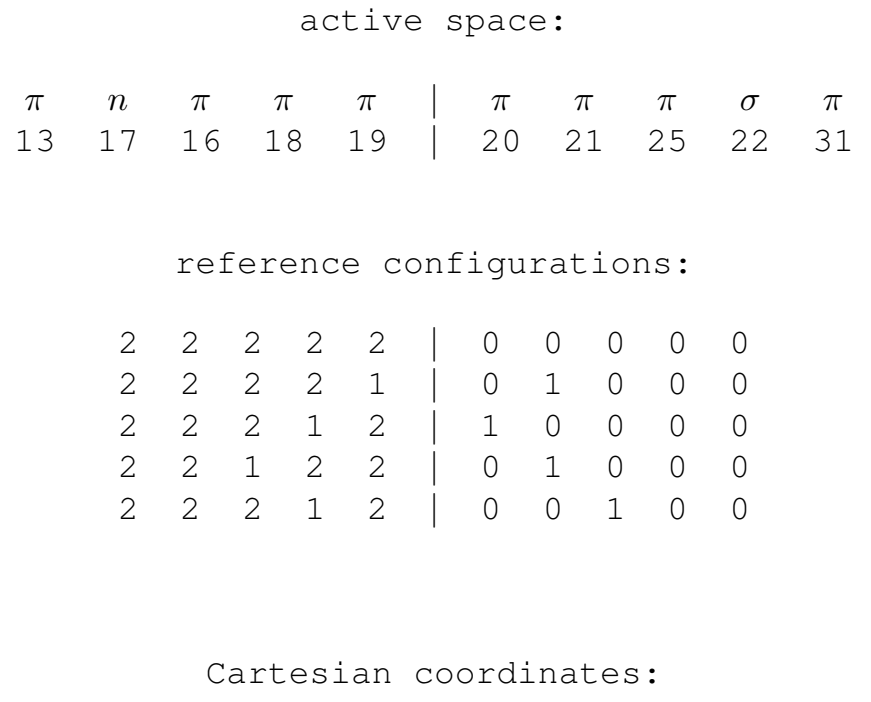

14

$\begin{array}{lrrr}\mathrm{C} & 0.00000 & 0.00000 & -1.36648 \\ \mathrm{C} & 1.23702 & 0.00000 & -0.66929 \\ \mathrm{C} & -1.23702 & 0.00000 & -0.66929 \\ \mathrm{C} & 1.25194 & 0.00000 & 0.75551 \\ \mathrm{C} & -1.25194 & 0.00000 & 0.75551 \\ \mathrm{C} & 0.00000 & 0.00000 & 1.45725 \\ \mathrm{H} & 0.00000 & 0.00000 & -2.46844 \\ \mathrm{H} & 2.18663 & 0.00000 & -1.22003 \\ \mathrm{H} & -2.18663 & 0.00000 & -1.22003 \\ \mathrm{H} & 2.19996 & 0.00000 & 1.30540 \\ \mathrm{H} & -2.19996 & 0.00000 & 1.30540 \\ \mathrm{C} & 0.00000 & 0.00000 & 2.87181 \\ \mathrm{C} & 0.00000 & 0.00000 & 4.12086 \\ \mathrm{H} & 0.00000 & 0.00000 & 5.17301\end{array}$


Table S1562 Employed active space, reference configurations, and Cartesian coordinates of the OM3/MRCISD-optimized ground-state equilibrium geometry of benzonitrile (ground-state energy: $-1165.57263 \mathrm{eV}$ ).

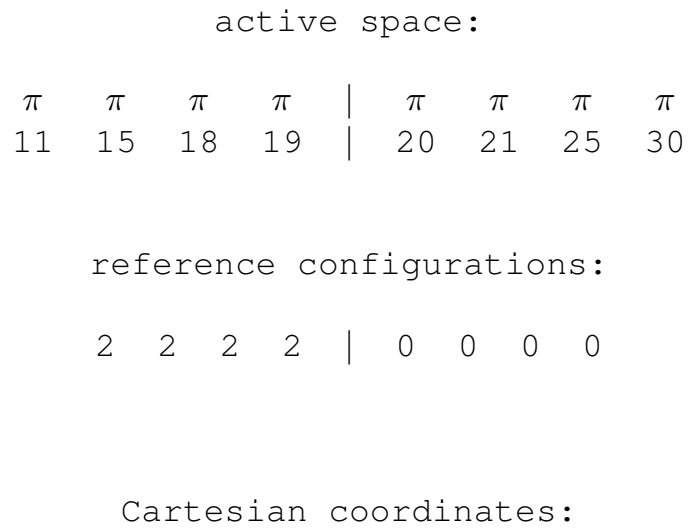


Table S1563 Employed active space, reference configurations, and Cartesian coordinates of the OM3/MRCISD-optimized excited-state equilibrium geometry of the $1^{1} B_{1}\left({ }^{1} \pi \pi^{*}\right)$ state of benzonitrile (excited-state energy: $-1161.33723 \mathrm{eV})$.

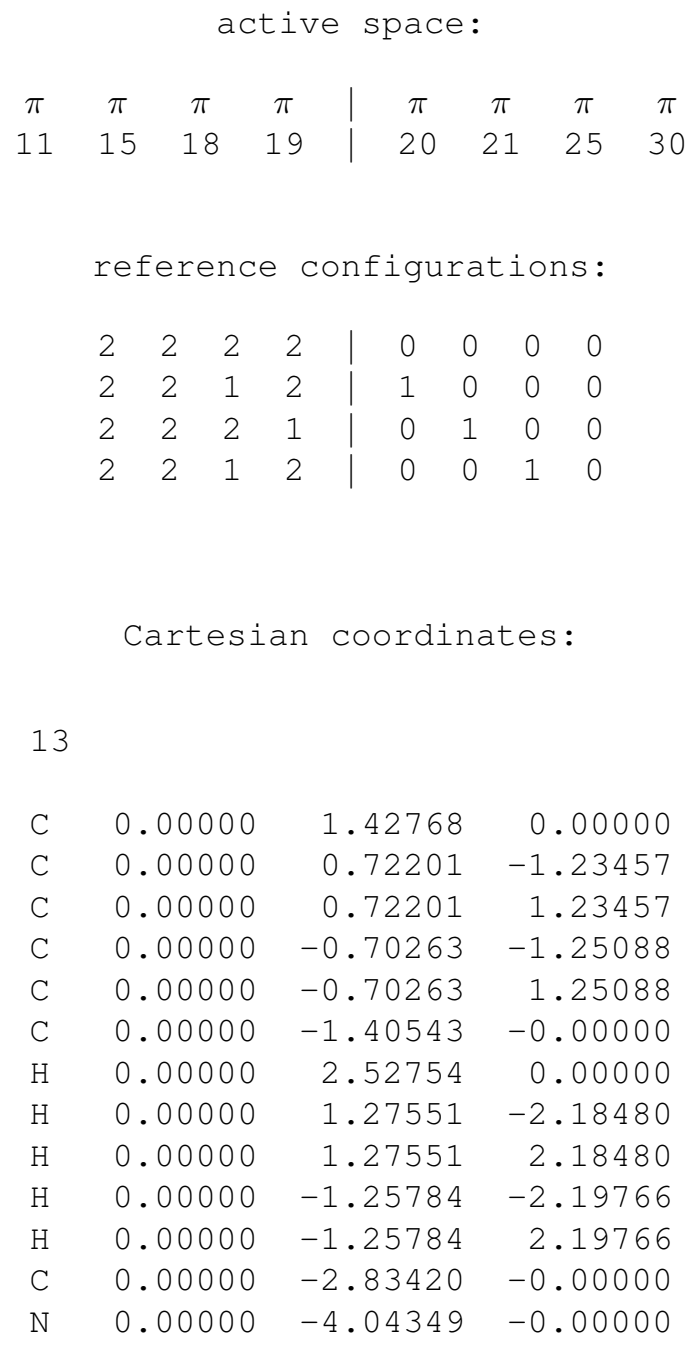


Table S1564 Employed active space, reference configurations, and Cartesian coordinates of the OM3/MRCISD-optimized ground-state equilibrium geometry of aniline (ground-state energy: $-1070.59300 \mathrm{eV}$ ).

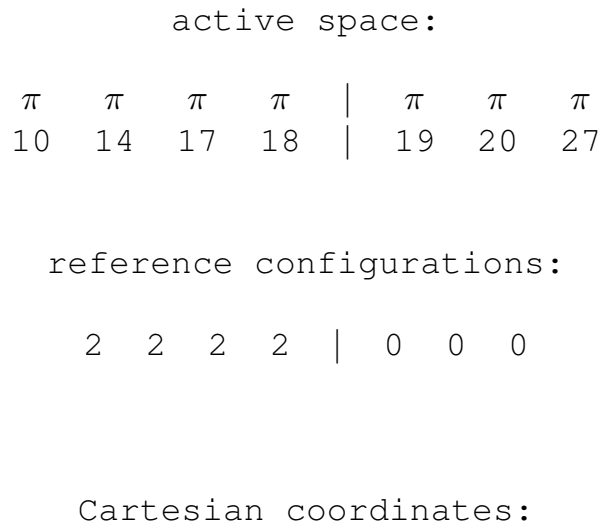


Table S1565 Employed active space, reference configurations, and Cartesian coordinates of the OM3/MRCISD-optimized excited-state equilibrium geometry of the $1{ }^{1} B_{2}\left({ }^{1} \pi \pi^{*}\right)$ state of aniline (excited-state energy: $-1066.73378 \mathrm{eV}$ ).

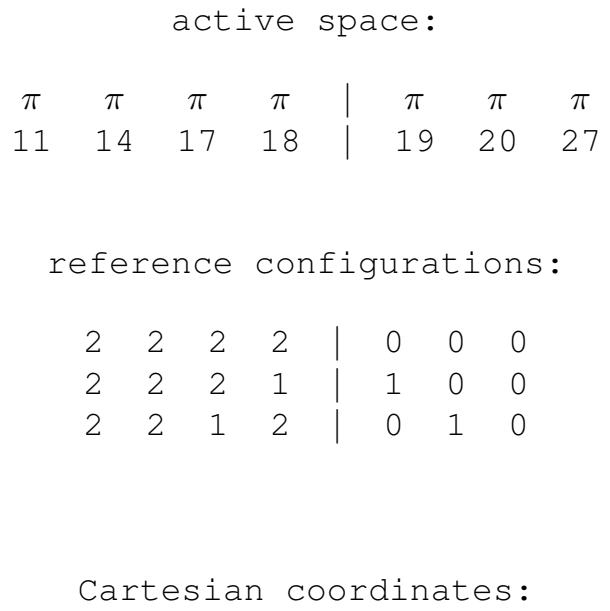


Table S1566 Employed active space, reference configurations, and Cartesian coordinates of the OM3/MRCISD-optimized ground-state equilibrium geometry of tetrafluorobenzene (ground-state energy: $-2649.59602 \mathrm{eV}$ ).

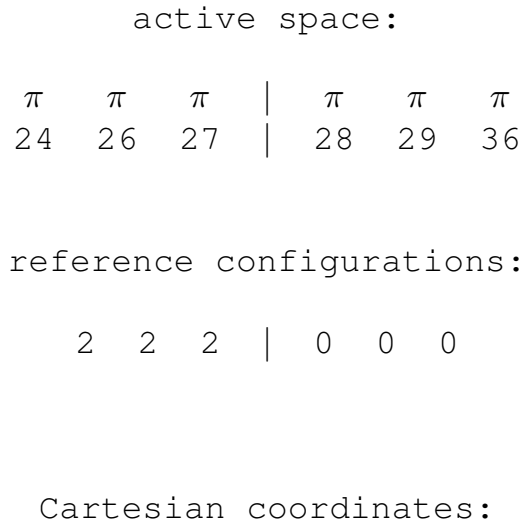


Table S1567 Employed active space, reference configurations, and Cartesian coordinates of the OM3/MRCISD-optimized excited-state equilibrium geometry of the $1{ }^{1} B_{1}\left({ }^{1} \pi \pi^{*}\right)$ state of tetrafluorobenzene (excited-state energy: $-2645.56637 \mathrm{eV})$.

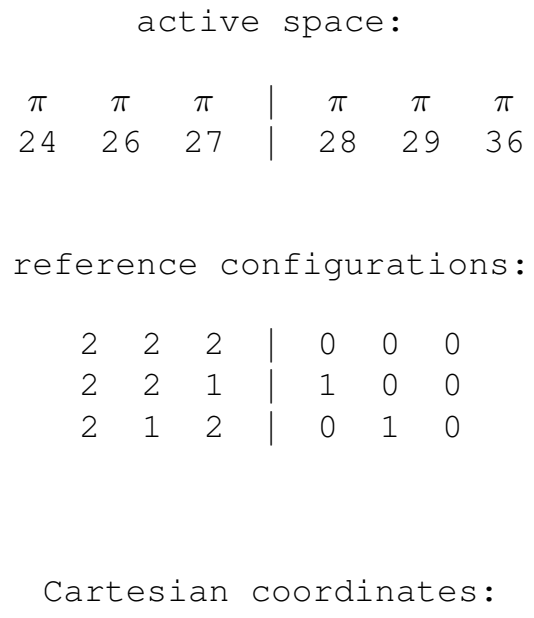

12

$\begin{array}{lrrr}\text { C } & -0.03619 & 1.46775 & 0.00000\end{array}$

C $\quad-0.03619-1.46775-0.00000$

C $\quad-0.03619 \quad 0.72262-1.21583$

C $\quad-0.03619 \quad 0.72262 \quad 1.21583$

$\begin{array}{llll}\text { C } & -0.03619 & -0.72262 & -1.21583\end{array}$

$\begin{array}{llll}\text { C } & -0.03619 & -0.72262 & 1.21583\end{array}$

$\mathrm{H} \quad-0.03619 \quad 2.55314 \quad 0.00000$

$\mathrm{H} \quad-0.03619-2.55314-0.00000$

F $\quad-0.03620 \quad 1.36827 \quad-2.36675$

F $\quad-0.03620 \quad 1.36827 \quad 2.36675$

$\mathrm{F} \quad-0.03620 \quad-1.36827 \quad-2.36675$

$\mathrm{F} \quad-0.03620 \quad-1.36827 \quad 2.36675$ 
Table S1568 Employed active space, reference configurations, and Cartesian coordinates of the OM3/MRCISD-optimized ground-state equilibrium geometry of 2,6-difluoropyridine (ground-state energy: $-1820.62177 \mathrm{eV}$ ).

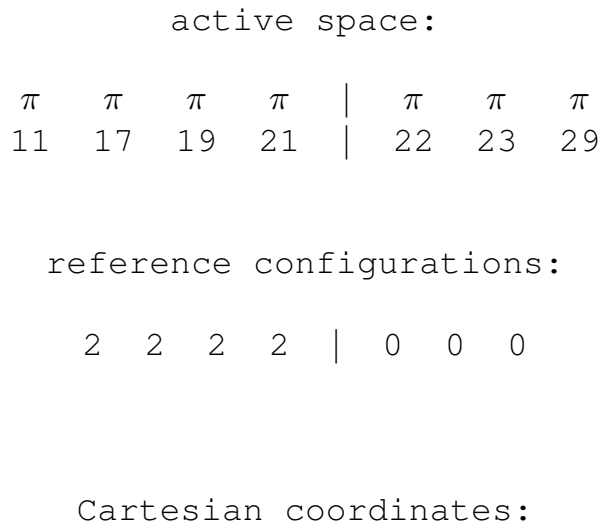

$\mathrm{H} \quad 0.00000 \quad-1.26238-2.17999$

H $\quad 0.00000 \quad-1.26238 \quad 2.17999$ 
Table S1569 Employed active space, reference configurations, and Cartesian coordinates of the OM3/MRCISD-optimized excited-state equilibrium geometry of the $1^{1} B_{1}\left({ }^{1} \pi \pi^{*}\right)$ state of 2,6-difluoropyridine (excited-state energy: $-1816.13704 \mathrm{eV})$.

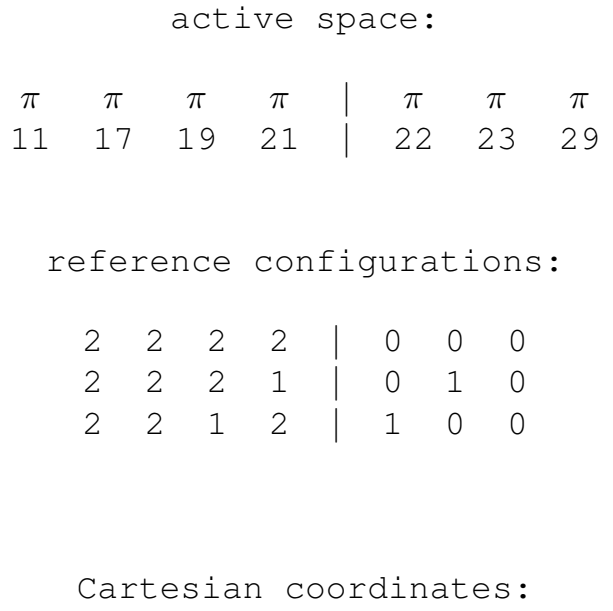

$\begin{array}{lrrr}\mathrm{N} & 0.00000 & 1.42030 & 0.00000 \\ \mathrm{C} & 0.00000 & -1.49539 & -0.00000 \\ \mathrm{C} & 0.00000 & 0.67098 & -1.15835 \\ \mathrm{C} & 0.00000 & 0.67098 & 1.15835 \\ \mathrm{C} & 0.00000 & -0.76769 & -1.23121 \\ \mathrm{C} & 0.00000 & -0.76769 & 1.23121 \\ \mathrm{H} & 0.00000 & -2.58916 & -0.00000 \\ \mathrm{~F} & 0.00000 & 1.36316 & -2.28521 \\ \mathrm{~F} & 0.00000 & 1.36316 & 2.28521 \\ \mathrm{H} & 0.00000 & -1.27133 & -2.20042 \\ \mathrm{H} & 0.00000 & -1.27133 & 2.20042\end{array}$


Table S1570 Employed active space, reference configurations, and Cartesian coordinates of the OM3/MRCISD-optimized ground-state equilibrium geometry of 2-fluoropyridine (ground-state energy: $-1368.16885 \mathrm{eV}$ ).

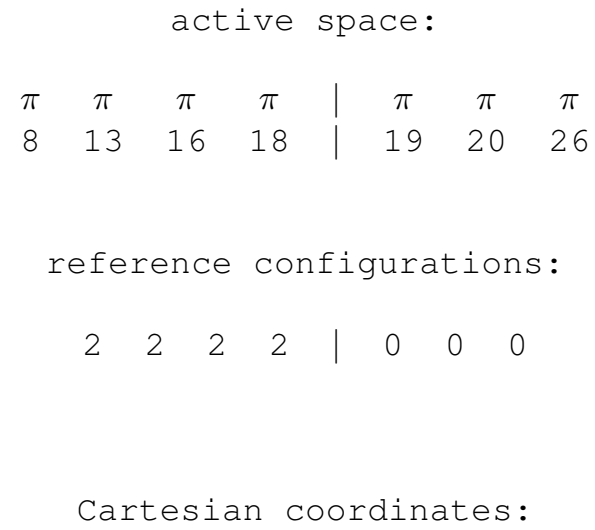


Table S1571 Employed active space, reference configurations, and Cartesian coordinates of the OM3/MRCISD-optimized excited-state equilibrium geometry of the $2{ }^{1} A^{\prime}\left({ }^{1} \pi \pi^{*}\right)$ state of 2 -fluoropyridine (excited-state energy: $-1363.65758 \mathrm{eV})$.

active space:
\begin{tabular}{cccc|ccc}
$\pi$ & $\pi$ & $\pi$ & $\pi$ & $\pi$ & $\pi$ & $\pi$ \\
8 & 13 & 16 & 18 & 19 & 20 & 26 \\
\\
reference configurations: \\
2 & 2 & 2 & 2 & 0 & 0 & 0 \\
2 & 2 & 2 & 1 & 0 & 1 & 0 \\
2 & 2 & 1 & 2 & 1 & 0 & 0 \\
2 & 2 & 2 & 1 & 1 & 0 & 0 \\
2 & 2 & 1 & 2 & 0 & 1 & 0
\end{tabular}

Cartesian coordinates:

11

$\begin{array}{lrrr}\mathrm{C} & 0.00000 & 0.00000 & 0.00000 \\ \mathrm{C} & 1.43667 & 0.00000 & 0.00000 \\ \mathrm{~N} & 2.26015 & 1.10717 & 0.00000 \\ \mathrm{C} & 1.62660 & 2.32979 & 0.00000 \\ \mathrm{C} & 0.19416 & 2.45251 & 0.00000 \\ \mathrm{C} & -0.62981 & 1.28277 & 0.00000 \\ \mathrm{~F} & 2.06697 & -1.16947 & -0.00000 \\ \mathrm{H} & 2.26482 & 3.23538 & 0.00000 \\ \mathrm{H} & -0.26809 & 3.44879 & 0.00000 \\ \mathrm{H} & -1.72199 & 1.36555 & 0.00000 \\ \mathrm{H} & -0.56673 & -0.93241 & -0.00000\end{array}$


Table S1572 Employed active space, reference configurations, and Cartesian coordinates of the OM3/MRCISD-optimized ground-state equilibrium geometry of 2-hydroxypyridine (ground-state energy: $-1254.72143 \mathrm{eV}$ ).

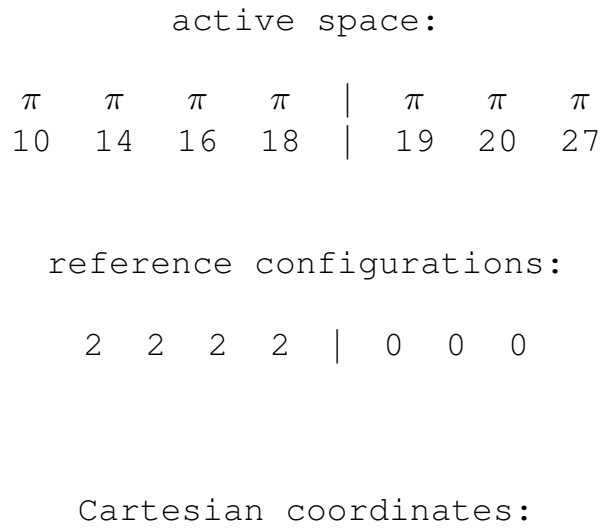

$\begin{array}{llll}\mathrm{H} & 3.73501 & 2.24909 & 0.00000\end{array}$ 
Table S1573 Employed active space, reference configurations, and Cartesian coordinates of the OM3/MRCISD-optimized excited-state equilibrium geometry of the $2{ }^{1} A^{\prime}\left({ }^{1} \pi \pi^{*}\right)$ state of 2-hydroxypyridine (excited-state energy: $-1250.35066 \mathrm{eV})$.

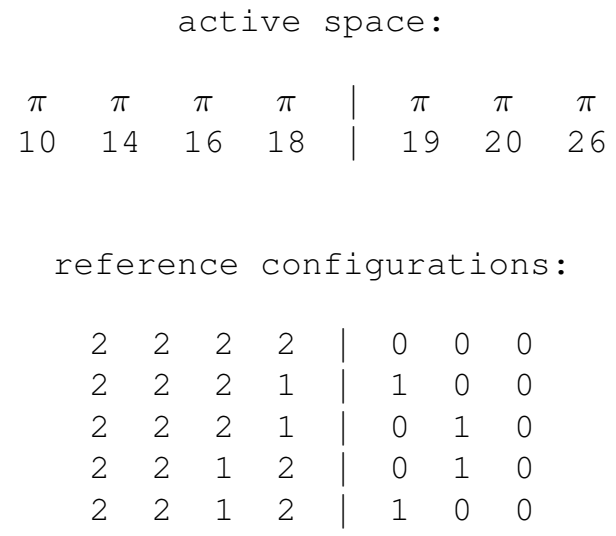

Cartesian coordinates:

12

$\begin{array}{lrrr}\mathrm{C} & 0.00000 & 0.00000 & 0.00000 \\ \mathrm{C} & 1.42863 & 0.00000 & 0.00000 \\ \mathrm{C} & 2.06640 & 1.28785 & 0.00000 \\ \mathrm{~N} & 1.42407 & 2.52238 & 0.00000 \\ \mathrm{C} & 0.05039 & 2.48401 & 0.00000 \\ \mathrm{C} & -0.68430 & 1.25075 & 0.00000 \\ \mathrm{O} & 3.43125 & 1.29818 & 0.00000 \\ \mathrm{H} & -0.48789 & 3.45267 & 0.00000 \\ \mathrm{H} & -1.78376 & 1.27533 & 0.00000 \\ \mathrm{H} & -0.55396 & -0.94435 & -0.00000 \\ \mathrm{H} & 2.02133 & -0.91657 & -0.00000 \\ \mathrm{H} & 3.76436 & 2.26309 & 0.00000\end{array}$


Table S1574 Employed active space, reference configurations, and Cartesian coordinates of the OM3/MRCISD-optimized ground-state equilibrium geometry of 2-methylpyrimidine (ground-state energy: $-1146.30664 \mathrm{eV}$ ).

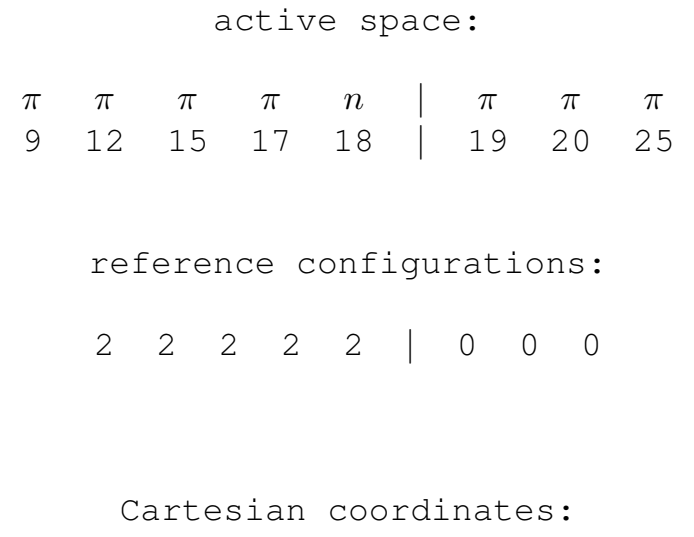

13

$\begin{array}{lrrr}\mathrm{C} & 0.00000 & 0.00000 & 0.00000 \\ \mathrm{C} & 1.40954 & 0.00000 & 0.00000 \\ \mathrm{C} & 2.05962 & 1.25123 & 0.00000 \\ \mathrm{~N} & 1.36570 & 2.41941 & 0.00000 \\ \mathrm{C} & -0.01132 & 2.33888 & 0.00000 \\ \mathrm{~N} & -0.71697 & 1.15486 & 0.00000 \\ \mathrm{C} & -0.79011 & 3.63498 & 0.00000 \\ \mathrm{H} & 3.16923 & 1.31201 & 0.00000 \\ \mathrm{H} & -0.56579 & -0.95637 & -0.00000 \\ \mathrm{H} & 1.97754 & -0.93482 & -0.00000 \\ \mathrm{H} & -1.87167 & 3.42389 & 0.00000 \\ \mathrm{H} & -0.53342 & 4.20549 & 0.89762 \\ \mathrm{H} & -0.53342 & 4.20549 & -0.89762\end{array}$


Table S1575 Employed active space, reference configurations, and Cartesian coordinates of the OM3/MRCISD-optimized excited-state equilibrium geometry of the $1^{1} A^{\prime \prime}\left({ }^{1} n \pi^{*}\right)$ state of 2-methylpyrimidine (excited-state energy: $-1142.31731 \mathrm{eV})$.

\begin{tabular}{lcccc|ccc} 
active space : \\
$\pi$ & $\pi$ & $\pi$ & $\pi$ & $n$ & $\pi$ & $\pi$ & $\pi$ \\
9 & 12 & 15 & 17 & 18 & 19 & 20 & 26
\end{tabular}

reference configurations:
\begin{tabular}{lllll|lll}
2 & 2 & 2 & 2 & 2 & 0 & 0 & 0 \\
2 & 2 & 2 & 2 & 1 & 1 & 0 & 0
\end{tabular}

Cartesian coordinates:

13

$\begin{array}{lrrr}\mathrm{C} & 0.00000 & 0.00000 & 0.00000 \\ \mathrm{C} & 1.44412 & 0.00000 & 0.00000 \\ \mathrm{C} & 2.04177 & 1.30352 & 0.00000 \\ \mathrm{~N} & 1.29034 & 2.39927 & 0.00000 \\ \mathrm{C} & -0.11705 & 2.42846 & 0.00000 \\ \mathrm{~N} & -0.66601 & 1.14971 & 0.00000 \\ \mathrm{C} & -0.90250 & 3.69566 & 0.00000 \\ \mathrm{H} & 3.14320 & 1.39081 & 0.00000 \\ \mathrm{H} & -0.54165 & -0.96007 & -0.00000 \\ \mathrm{H} & 2.02530 & -0.91536 & -0.00000 \\ \mathrm{H} & -1.98035 & 3.44470 & -0.00000 \\ \mathrm{H} & -0.67568 & 4.26104 & 0.90331 \\ \mathrm{H} & -0.67568 & 4.26104 & -0.90331\end{array}$


Table S1576 Employed active space, reference configurations, and Cartesian coordinates of the OM3/MRCISD-optimized ground-state equilibrium geometry of 5-methylpyrimidine (ground-state energy: $-1146.34075 \mathrm{eV}$ ).

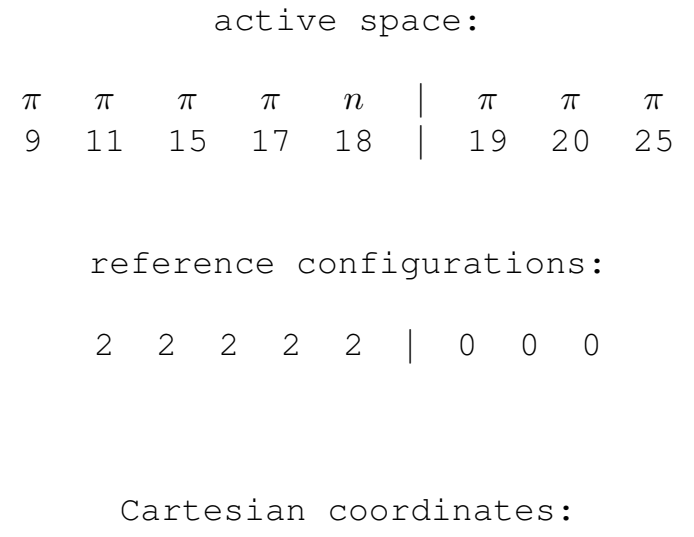

13

$\begin{array}{llll}C & 0.00000 & 0.00000 & 0.00000\end{array}$

$\begin{array}{llll}\mathrm{N} & 1.37276 & 0.00000 & 0.00000\end{array}$

C $\quad 2.00483 \quad 1.20263 \quad 0.00000$

C $\quad 1.28713 \quad 2.42450 \quad 0.00000$

$\begin{array}{llll}\mathrm{C} & -0.12812 & 2.33467 & 0.00000\end{array}$

$\mathrm{N} \quad-0.76966 \quad 1.13750 \quad 0.00000$

$\begin{array}{lrrr}\mathrm{C} & 1.98212 & 3.74705 & 0.00000\end{array}$

$\mathrm{H} \quad-0.52375-0.98613-0.00000$

$\mathrm{H} \quad-0.75132 \quad 3.25454 \quad 0.00000$

$\begin{array}{llll}\mathrm{H} & 3.11654 & 1.20381 & 0.00000\end{array}$

$\begin{array}{llll}\mathrm{H} & 3.08089 & 3.60779 & 0.00000\end{array}$

$\mathrm{H} \quad 1.71223 \quad 4.30952 \quad-0.89962$

$\begin{array}{llll}\mathrm{H} & 1.71223 & 4.30952 & 0.89962\end{array}$ 
Table S1577 Employed active space, reference configurations, and Cartesian coordinates of the OM3/MRCISD-optimized excited-state equilibrium geometry of the $1^{1} A^{\prime \prime}\left({ }^{1} n \pi^{*}\right)$ state of 5-methylpyrimidine (excited-state energy: $-1142.41274 \mathrm{eV})$.

\begin{tabular}{lcccc|ccc} 
active space : \\
$\pi$ & $\pi$ & $\pi$ & $\pi$ & $n$ & $\pi$ & $\pi$ & $\pi$ \\
9 & 11 & 15 & 17 & 18 & 19 & 20 & 25
\end{tabular}

reference configurations:
\begin{tabular}{lllll|lll}
2 & 2 & 2 & 2 & 2 & 0 & 0 & 0 \\
2 & 2 & 2 & 2 & 1 & 1 & 0 & 0
\end{tabular}

Cartesian coordinates:

13

$\begin{array}{lrrr}\mathrm{C} & 0.00000 & 0.00000 & 0.00000 \\ \mathrm{~N} & 1.39688 & 0.00000 & 0.00000 \\ \mathrm{C} & 2.19740 & 1.05786 & 0.00000 \\ \mathrm{C} & 1.64587 & 2.39444 & 0.00000 \\ \mathrm{C} & 0.19295 & 2.43062 & 0.00000 \\ \mathrm{~N} & -0.49581 & 1.29830 & 0.00000 \\ \mathrm{C} & 2.46876 & 3.62711 & 0.00000 \\ \mathrm{H} & -0.61593 & -0.90177 & -0.00000 \\ \mathrm{H} & -0.32250 & 3.40704 & 0.00000 \\ \mathrm{H} & 3.29573 & 0.92890 & 0.00000 \\ \mathrm{H} & 3.54586 & 3.35403 & 0.00000 \\ \mathrm{H} & 2.28012 & 4.21011 & -0.90382 \\ \mathrm{H} & 2.28012 & 4.21011 & 0.90382\end{array}$


Table S1578 Employed active space, reference configurations, and Cartesian coordinates of the OM3/MRCISD-optimized ground-state equilibrium geometry of pyrazine (ground-state energy: $-991.33625 \mathrm{eV}$ ).

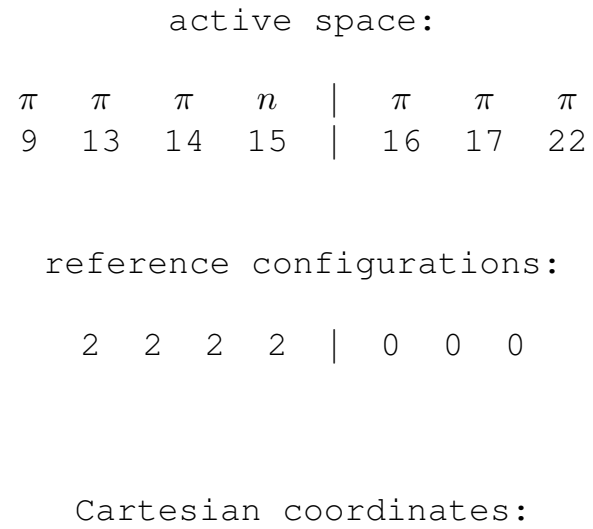


Table S1579 Employed active space, reference configurations, and Cartesian coordinates of the OM3/MRCISD-optimized excited-state equilibrium geometry of the $1{ }^{1} B_{u}\left({ }^{1} n \pi^{*}\right)$ state of pyrazine (excited-state energy: $\left.-987.20078 \mathrm{eV}\right)$.

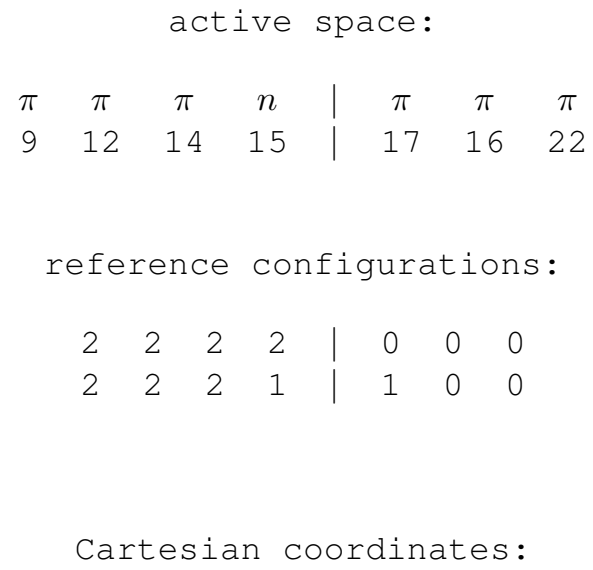


Table S1580 Employed active space, reference configurations, and Cartesian coordinates of the OM3/MRCISD-optimized ground-state equilibrium geometry of 2-pyridone (ground-state energy: $-1254.73170 \mathrm{eV}$ ).

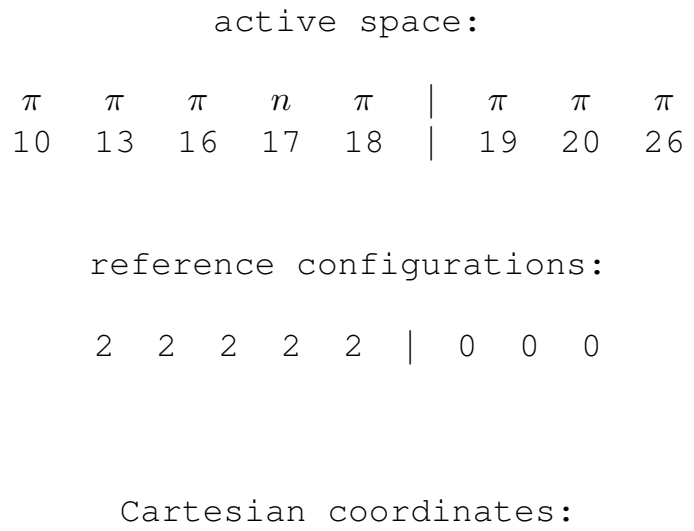


Table S1581 Employed active space, reference configurations, and Cartesian coordinates of the OM3/MRCISD-optimized excited-state equilibrium geometry of the $2{ }^{1} A^{\prime}\left({ }^{1} \pi \pi^{*}\right)$ state of 2-pyridone (excited-state energy: $\left.-1251.07935 \mathrm{eV}\right)$.

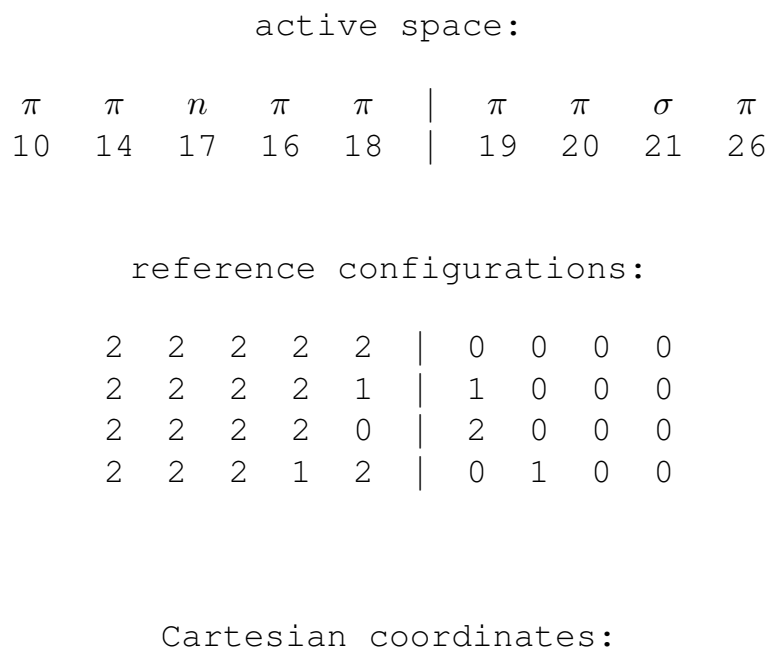


Table S1582 Employed active space, reference configurations, and Cartesian coordinates of the OM3/MRCISD-optimized ground-state equilibrium geometry of catechol (ground-state energy: $-1517.84083 \mathrm{eV}$ ).

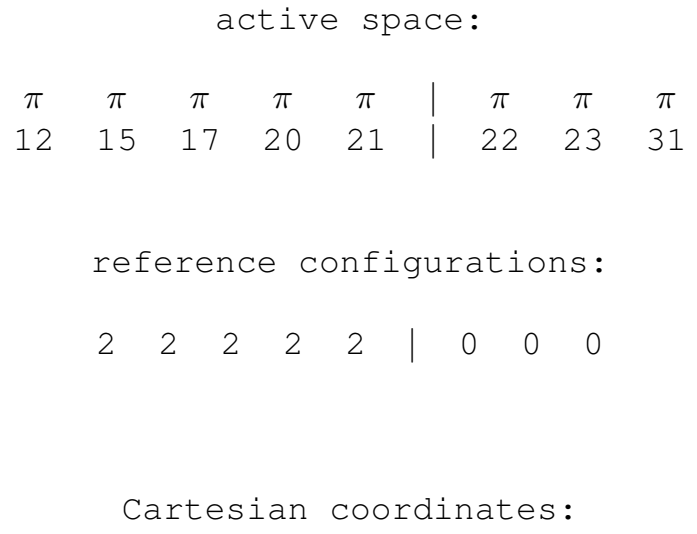

14

$\begin{array}{llll}C & 0.00000 & 0.00000 & 0.00000\end{array}$

C $\quad 1.40266 \quad 0.00000 \quad 0.00000$

C $\quad 2.08424 \quad 1.22674 \quad 0.00000$

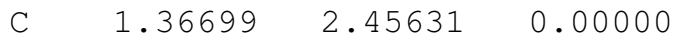

C $\quad-0.03972 \quad 2.43951 \quad 0.00000$

C $\quad-0.71165 \quad 1.20908 \quad 0.00000$

$0 \quad 3.46370 \quad 1.35572 \quad 0.00000$

$\begin{array}{llll}0 & 1.98784 & 3.68470 & 0.00000\end{array}$

$\begin{array}{llll}\mathrm{H} & -0.58156 & 3.39200 & 0.00000\end{array}$

$\mathrm{H} \quad-1.81255 \quad 1.19409 \quad 0.00000$

$\begin{array}{llll}\mathrm{H} & -0.54137 & -0.95812 & -0.00000\end{array}$

$\mathrm{H} \quad \begin{array}{llll}\mathrm{H} & 1.96888 & -0.94176 & -0.00000\end{array}$

$\begin{array}{llll}\mathrm{H} & 3.89157 & 0.43504 & 0.00000\end{array}$

H $\quad 2.99692 \quad 3.54596 \quad 0.00000$ 
Table S1583 Employed active space, reference configurations, and Cartesian coordinates of the OM3/MRCISD-optimized excited-state equilibrium geometry of the $2{ }^{1} A\left({ }^{1} \pi \pi^{*}\right)$ state of catechol (excited-state energy: $-1513.86675 \mathrm{eV}$ ).

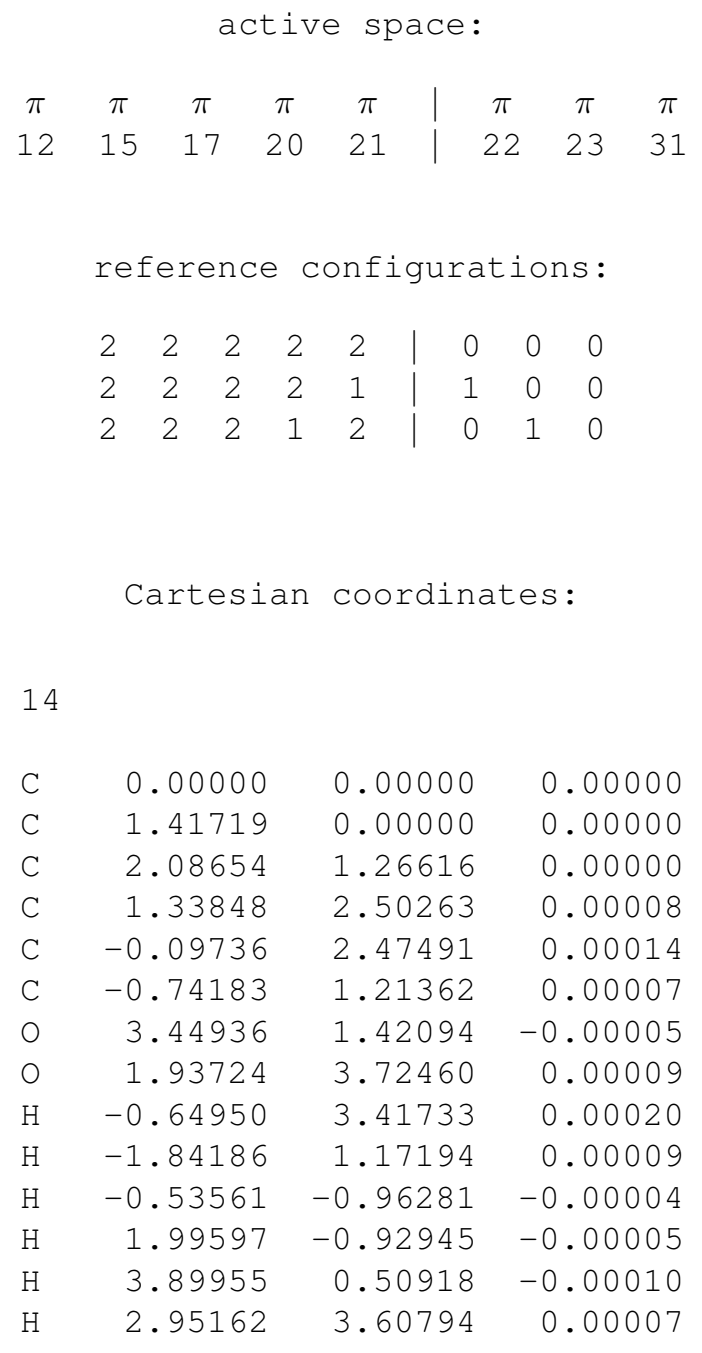


Table S1584 Employed active space, reference configurations, and Cartesian coordinates of the OM3/MRCISD-optimized ground-state equilibrium geometry of resorcinol (isomer 3) (ground-state energy: $-1517.85140 \mathrm{eV}$ ).

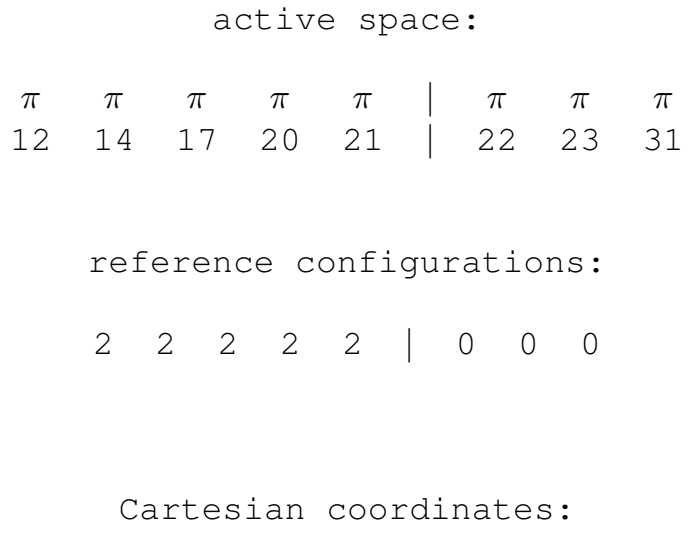

14

$\begin{array}{llll}\text { C } & 1.43013 & 0.00000 & 0.00000\end{array}$

C $\quad 0.69962 \quad 1.20358 \quad 0.00000$

C $\quad 0.69962-1.20358-0.00000$

C $\quad-0.71182 \quad 1.21959-0.00000$

C $\quad-0.71182-1.21959-0.00000$

C $\quad-1.40000 \quad 0.00000-0.00000$

$\mathrm{H} \quad 2.52495 \quad-0.00000 \quad-0.00000$

$\mathrm{H} \quad-2.50231 \quad 0.00000 \quad-0.00000$

$\mathrm{H} \quad-1.23511 \quad 2.18046 \quad-0.00000$

$\mathrm{H} \quad-1.23511-2.18046-0.00000$

$\begin{array}{llll}0 & 1.31238 & 2.44179 & -0.00000\end{array}$

$\begin{array}{llll}0 & 1.31238 & -2.44179 & -0.00000\end{array}$

$\begin{array}{llll}\mathrm{H} & 2.32307 & 2.32428 & -0.00000\end{array}$

$\mathrm{H} \quad 2.32307 \quad-2.32428 \quad-0.00000$ 
Table S1585 Employed active space, reference configurations, and Cartesian coordinates of the OM3/MRCISD-optimized excited-state equilibrium geometry of the $1{ }^{1} B_{2}\left({ }^{1} \pi \pi^{*}\right)$ state of resorcinol (isomer 3) (excited-state energy: $-1513.82542 \mathrm{eV})$.

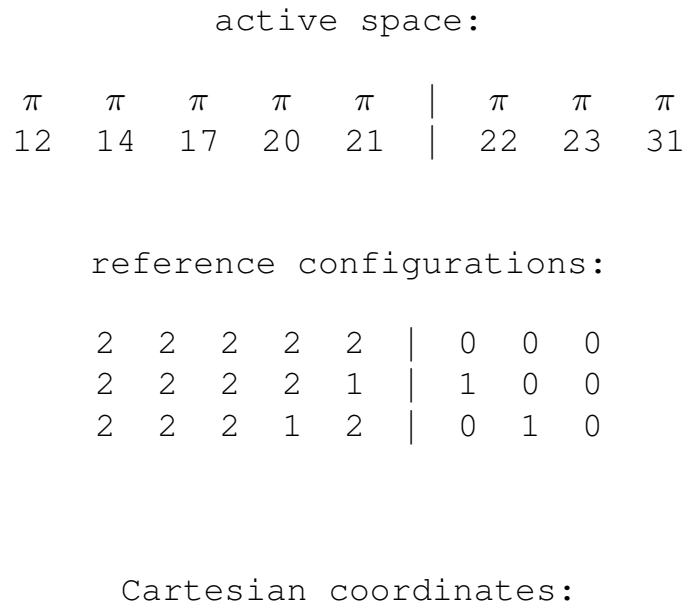


Table S1586 Employed active space, reference configurations, and Cartesian coordinates of the OM3/MRCISD-optimized ground-state equilibrium geometry of resorcinol (isomer 1) (ground-state energy: $-1517.87102 \mathrm{eV}$ ).

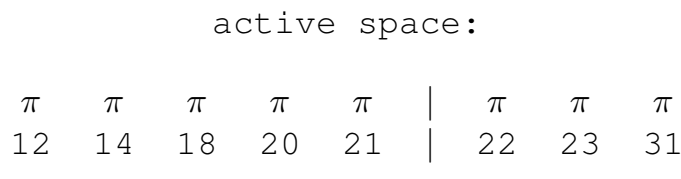

reference configurations:

$\begin{array}{lllllllll}2 & 2 & 2 & 2 & 2 & 0 & 0 & 0\end{array}$

Cartesian coordinates:

14

$\begin{array}{rrrr}\mathrm{C} & 0.00000 & 0.00000 & 0.00000 \\ \mathrm{C} & 1.40674 & 0.00000 & 0.00000 \\ \mathrm{C} & 2.05349 & 1.25083 & 0.00000 \\ \mathrm{C} & 1.33155 & 2.46277 & 0.00000 \\ \mathrm{C} & -0.06925 & 2.41945 & 0.00000 \\ \mathrm{C} & -0.75025 & 1.19714 & 0.00000 \\ \mathrm{O} & 3.43422 & 1.20190 & 0.00000 \\ \mathrm{O} & -0.73905 & -1.16669 & -0.00000 \\ \mathrm{H} & 1.86632 & 3.42090 & 0.00000 \\ \mathrm{H} & -0.64183 & 3.36126 & 0.00000 \\ \mathrm{H} & -1.84311 & 1.14430 & 0.00000 \\ \mathrm{H} & 1.99138 & -0.92210 & -0.00000 \\ \mathrm{H} & 3.80628 & 2.14902 & 0.00000 \\ \mathrm{H} & -0.11189 & -1.96803 & -0.00000\end{array}$


Table S1587 Employed active space, reference configurations, and Cartesian coordinates of the OM3/MRCISD-optimized excited-state equilibrium geometry of the $2{ }^{1} A\left({ }^{1} \pi \pi^{*}\right)$ state of resorcinol (isomer 1) (excited-state energy: $-1513.82674 \mathrm{eV})$.

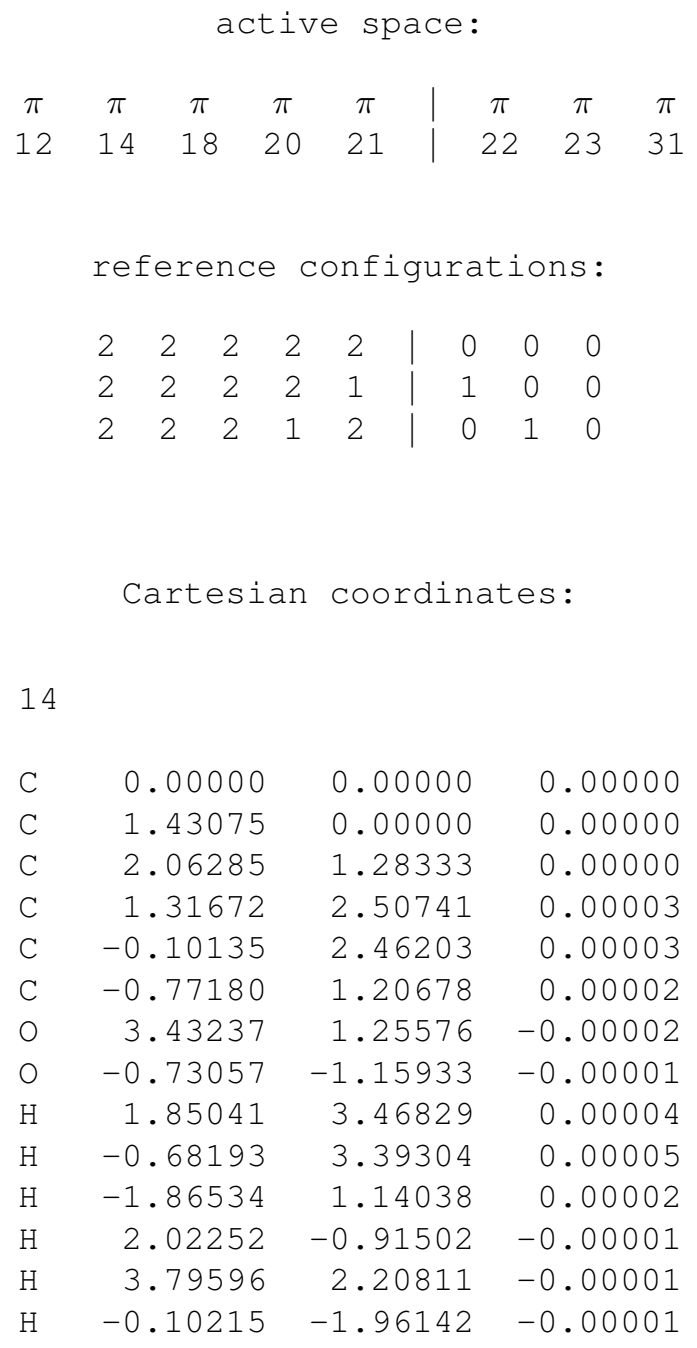


Table S1588 Employed active space, reference configurations, and Cartesian coordinates of the OM3/MRCISD-optimized ground-state equilibrium geometry of resorcinol (isomer 2) (ground-state energy: $-1517.85575 \mathrm{eV}$ ).

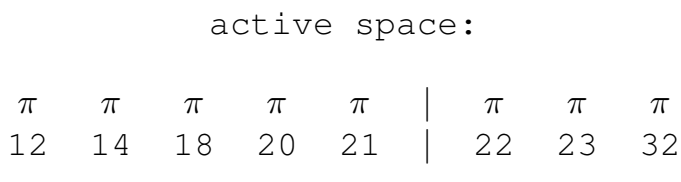

reference configurations:

$\begin{array}{lllllllll}2 & 2 & 2 & 2 & 2 & 0 & 0 & 0\end{array}$

Cartesian coordinates:

14

$\begin{array}{llll}\text { C } & 1.43949 & 0.00000 & 0.00000\end{array}$

$\begin{array}{llll}C & 0.70553 & 1.20087 & 0.00000\end{array}$

C $\quad 0.70553-1.20087-0.00000$

C $\quad-0.70642 \quad 1.21633-0.00000$

C $\quad-0.70642-1.21633-0.00000$

$\begin{array}{lrrr}\text { C } & -1.40000 & 0.00000 & -0.00000\end{array}$

$\mathrm{H} \quad 2.52915 \quad-0.00000 \quad-0.00000$

$\begin{array}{llll}\mathrm{H} & -2.50205 & 0.00000 & -0.00000\end{array}$

$\mathrm{H} \quad-1.24657 \quad 2.17153 \quad-0.00000$

$\mathrm{H} \quad-1.24657-2.17153 \quad-0.00000$

$\begin{array}{lrrr}0 & 1.46050 & 2.35758 & 0.00000\end{array}$

$\begin{array}{llll}0 & 1.46050 & -2.35758 & -0.00000\end{array}$

$\begin{array}{llll}\mathrm{H} & 0.84237 & 3.16608 & -0.00000\end{array}$

$\begin{array}{llll}\mathrm{H} & 0.84237 & -3.16608 & -0.00000\end{array}$ 
Table S1589 Employed active space, reference configurations, and Cartesian coordinates of the OM3/MRCISD-optimized excited-state equilibrium geometry of the $2^{1} A^{\prime}\left({ }^{1} \pi \pi^{*}\right)$ state of resorcinol (isomer 2) (excited-state energy: $-1513.79875 \mathrm{eV})$.

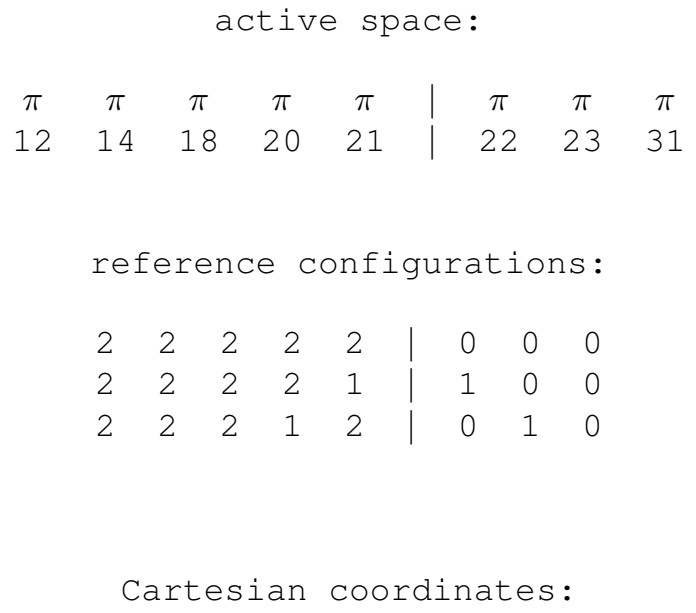

$\mathrm{H} \quad-1.61070 \quad 3.55695 \quad 0.00000$ 
Table S1590 Employed active space, reference configurations, and Cartesian coordinates of the OM3/MRCISD-optimized ground-state equilibrium geometry of cis-hydroquinone (ground-state energy: $-1517.82390 \mathrm{eV}$ ).

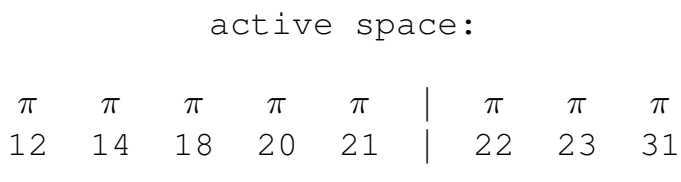

reference configurations:

$\begin{array}{lllllllll}2 & 2 & 2 & 2 & 2 & 0 & 0 & 0\end{array}$

Cartesian coordinates:

14

$\begin{array}{lrrr}\text { C } & 1.23193 & 0.69945 & 0.00000\end{array}$

C $\quad 1.23193-0.69945-0.00000$

$\begin{array}{llll}\text { C } & 0.00000 & 1.38382 & 0.00000\end{array}$

C $\quad 0.00000-1.38382-0.00000$

C $\quad-1.23547 \quad 0.69756-0.00000$

$\begin{array}{llll}\text { C } & -1.23547 & -0.69756 & -0.00000\end{array}$

$\begin{array}{llll}\mathrm{H} & 2.17724 & 1.25946 & 0.00000\end{array}$

$\mathrm{H} \quad 2.17724 \quad-1.25946 \quad-0.00000$

$\mathrm{H} \quad-2.16796 \quad 1.27316 \quad-0.00000$

$\mathrm{H} \quad-2.16796-1.27316-0.00000$

$\begin{array}{llll}0 & -0.08668 & 2.76473 & -0.00000\end{array}$

$\begin{array}{llll}0 & -0.08668 & -2.76473 & -0.00000\end{array}$

$\begin{array}{llll}\mathrm{H} & 0.84970 & 3.16257 & -0.00000\end{array}$

$\begin{array}{llll}\mathrm{H} & 0.84970 & -3.16257 & -0.00000\end{array}$ 
Table S1591 Employed active space, reference configurations, and Cartesian coordinates of the OM3/MRCISD-optimized excited-state equilibrium geometry of the $2{ }^{1} A_{1}\left({ }^{1} \pi \pi^{*}\right)$ state of cis-hydroquinone (excited-state energy: $-1513.91366 \mathrm{eV})$.

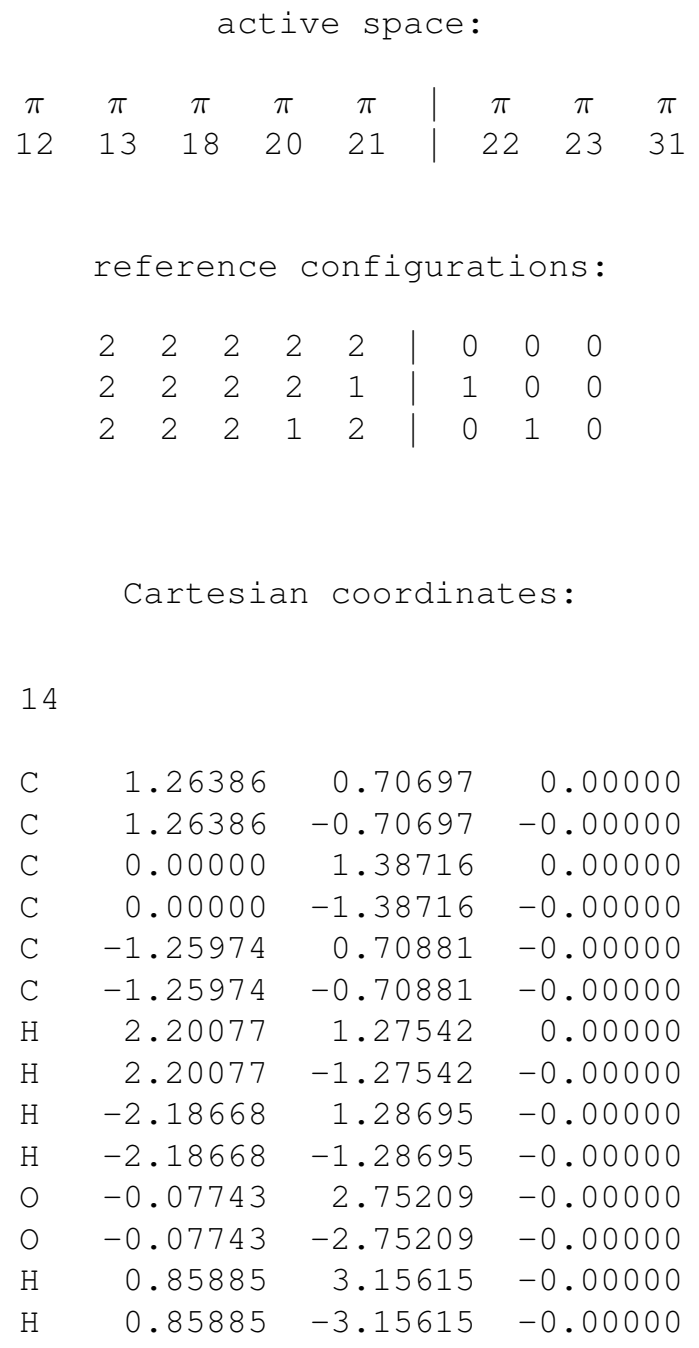


Table S1592 Employed active space, reference configurations, and Cartesian coordinates of the OM3/MRCISD-optimized ground-state equilibrium geometry of trans-hydroquinone (ground-state energy: $-1517.83018 \mathrm{eV}$ ).

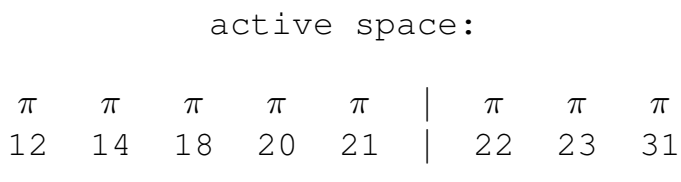

reference configurations:

\begin{tabular}{lllll|llll}
2 & 2 & 2 & 2 & 2 & 0 & 0 & 0
\end{tabular}

Cartesian coordinates:

14

$\begin{array}{lrrr}\mathrm{C} & 0.00000 & 0.00000 & 0.00000 \\ \mathrm{C} & 2.76784 & 0.00000 & 0.00000 \\ \mathrm{C} & 0.68311 & 1.23518 & 0.00000 \\ \mathrm{C} & 2.08473 & -1.23518 & 0.00000 \\ \mathrm{C} & 0.68779 & -1.23203 & -0.00000 \\ \mathrm{C} & 2.08005 & 1.23203 & -0.00000 \\ \mathrm{O} & -1.38107 & 0.08593 & 0.00000 \\ \mathrm{O} & 4.14891 & -0.08593 & 0.00000 \\ \mathrm{H} & 0.10846 & 2.16794 & -0.00000 \\ \mathrm{H} & 2.65938 & -2.16794 & 0.00000 \\ \mathrm{H} & 0.12657 & -2.17698 & -0.00000 \\ \mathrm{H} & 2.64127 & 2.17698 & -0.00000 \\ \mathrm{H} & -1.77727 & -0.85110 & 0.00000 \\ \mathrm{H} & 4.54511 & 0.85110 & -0.00000\end{array}$


Table S1593 Employed active space, reference configurations, and Cartesian coordinates of the OM3/MRCISD-optimized excited-state equilibrium geometry of the $1^{1} B_{u}\left({ }^{1} \pi \pi^{*}\right)$ state of trans-hydroquinone (excited-state energy: $-1513.92435 \mathrm{eV})$.

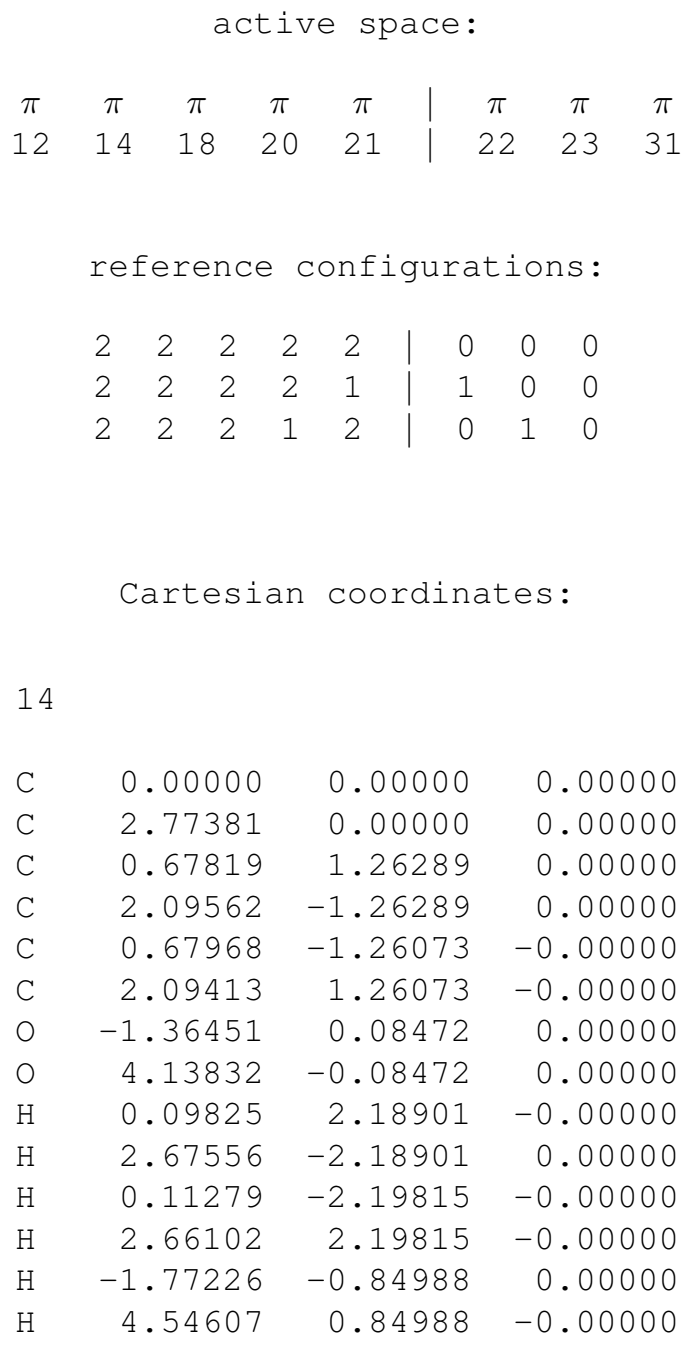


Table S1594 Employed active space, reference configurations, and Cartesian coordinates of the OM3/MRCISD-optimized ground-state equilibrium geometry of o-methoxyphenol (ground-state energy: $-1672.16181 \mathrm{eV}$ ).

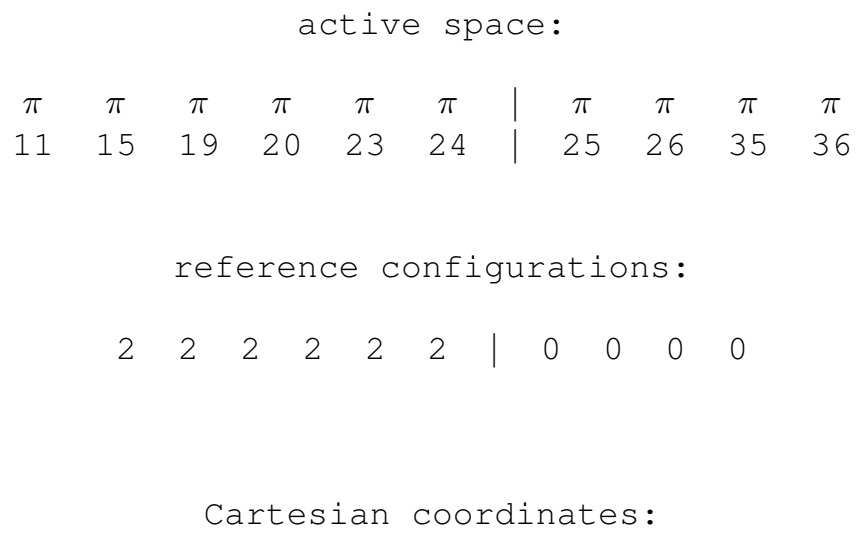


Table S1595 Employed active space, reference configurations, and Cartesian coordinates of the OM3/MRCISD-optimized excited-state equilibrium geometry of the $2{ }^{1} A\left({ }^{1} \pi \pi^{*}\right)$ state of $o$-methoxyphenol (excited-state energy: $-1668.17811 \mathrm{eV})$.

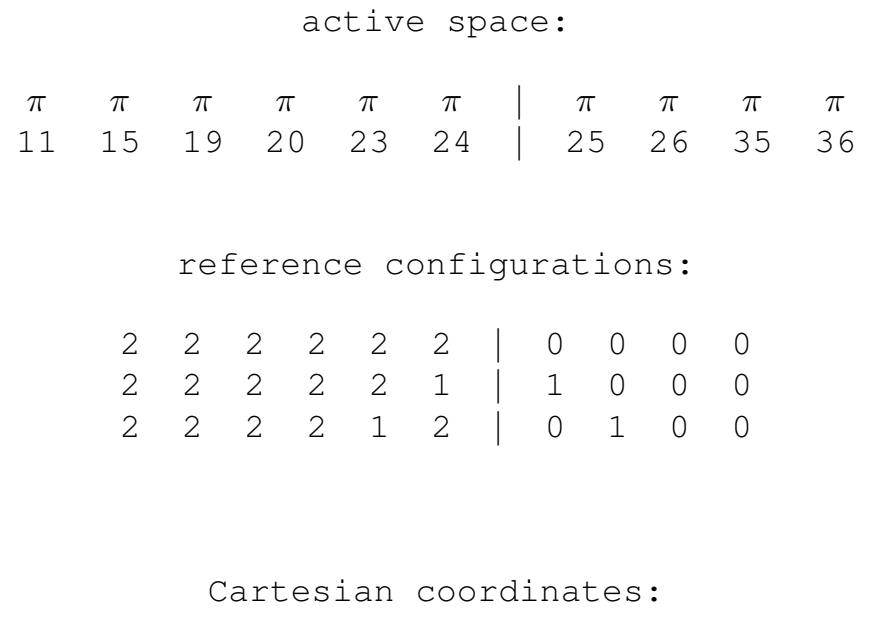

17

$\begin{array}{llll}\text { C } & 0.00000 & 0.00000 & 0.00000\end{array}$

$\begin{array}{llll}\text { C } & 1.42130 & 0.00000 & 0.00000\end{array}$

$\begin{array}{llll}\text { C } & 2.16805 & 1.20618 & 0.00000\end{array}$

$\begin{array}{llll}\text { C } & 1.44543 & 2.44122 & 0.00002\end{array}$

$\begin{array}{llll}\text { C } & -0.00128 & 2.44599 & 0.00004\end{array}$

$\begin{array}{llll}\text { C } & -0.73430 & 1.20972 & 0.00004\end{array}$

$\begin{array}{llll}0 & 1.97676 & 3.71660 & 0.00002\end{array}$

$\begin{array}{llll}\text { C } & 3.39999 & 3.80991 & -0.00002\end{array}$

$\begin{array}{llll}0 & -0.73732 & 3.59155 & 0.00005\end{array}$

$\mathrm{H} \quad-1.82624 \quad 1.23713 \quad 0.00004$

$\mathrm{H} \quad-0.54042 \quad-0.95921 \quad-0.00002$

$\mathrm{H} \quad 1.96237 \quad-0.96022 \quad-0.00004$

$\begin{array}{llll}\mathrm{H} & 3.26107 & 1.19338 & -0.00004\end{array}$

$\begin{array}{lrrr}\mathrm{H} & -0.11418 & 4.39993 & 0.00005\end{array}$

$\mathrm{H} \quad 3.61630 \quad 4.90378 \quad-0.00004$

$\mathrm{H} \quad 3.85302 \quad 3.34659 \quad 0.91385$

H $\quad 3.85295 \quad 3.34657 \quad-0.91390$ 
Table S1596 Employed active space, reference configurations, and Cartesian coordinates of the OM3/MRCISD-optimized ground-state equilibrium geometry of $m$-methoxyphenol (isomer 2) (ground-state energy: $-1672.17835 \mathrm{eV}$ ).

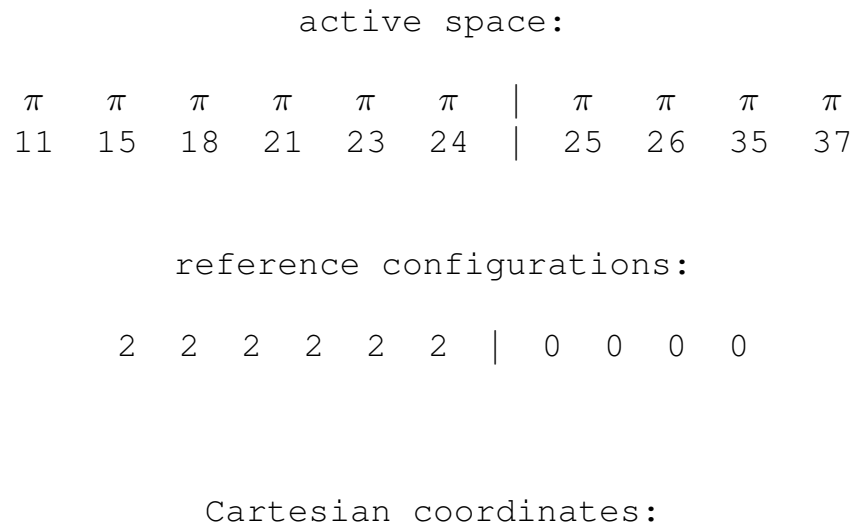


Table S1597 Employed active space, reference configurations, and Cartesian coordinates of the OM3/MRCISD-optimized excited-state equilibrium geometry of the $2{ }^{1} A\left({ }^{1} \pi \pi^{*}\right)$ state of $m$-methoxyphenol (isomer 2) (excited-state energy: $-1668.14449 \mathrm{eV})$.

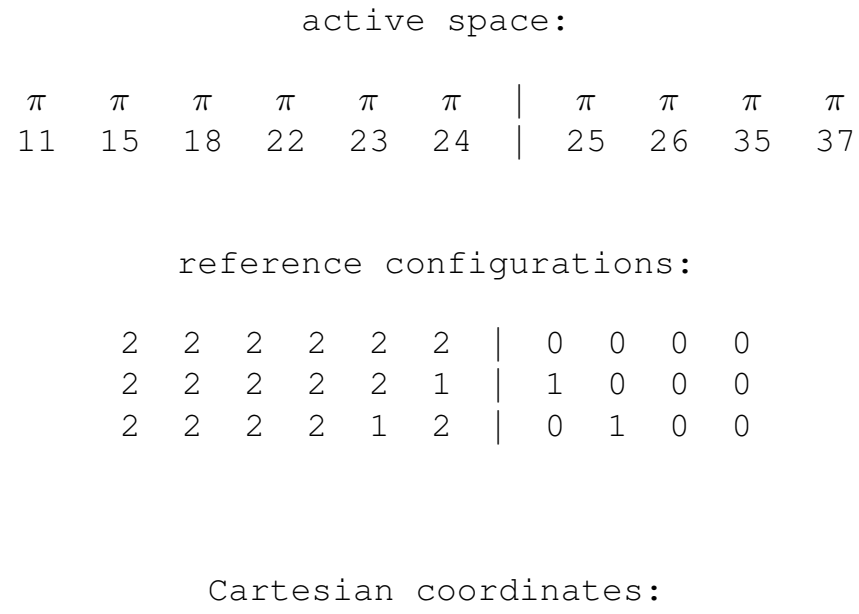


Table S1598 Employed active space, reference configurations, and Cartesian coordinates of the OM3/MRCISD-optimized ground-state equilibrium geometry of $m$-methoxyphenol (isomer 4) (ground-state energy: $-1672.19588 \mathrm{eV}$ ).

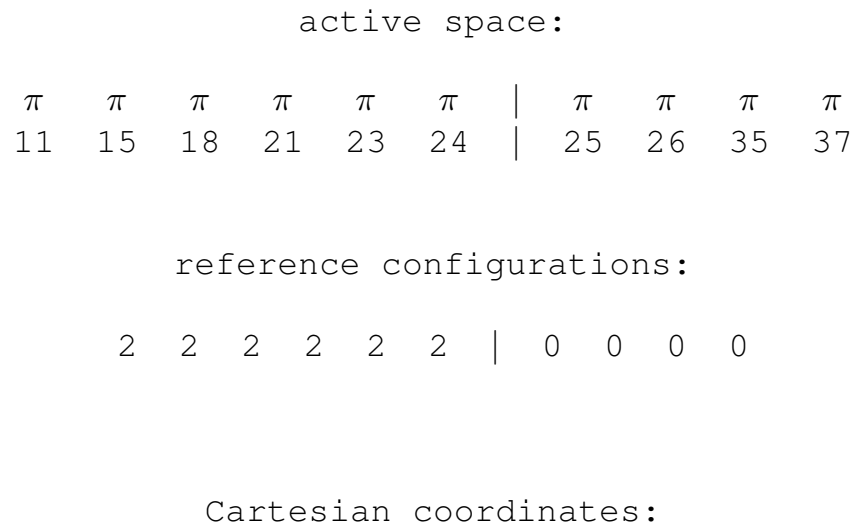


Table S1599 Employed active space, reference configurations, and Cartesian coordinates of the OM3/MRCISD-optimized excited-state equilibrium geometry of the $2{ }^{1} A\left({ }^{1} \pi \pi^{*}\right)$ state of $m$-methoxyphenol (isomer 4) (excited-state energy: $-1668.15629 \mathrm{eV})$.

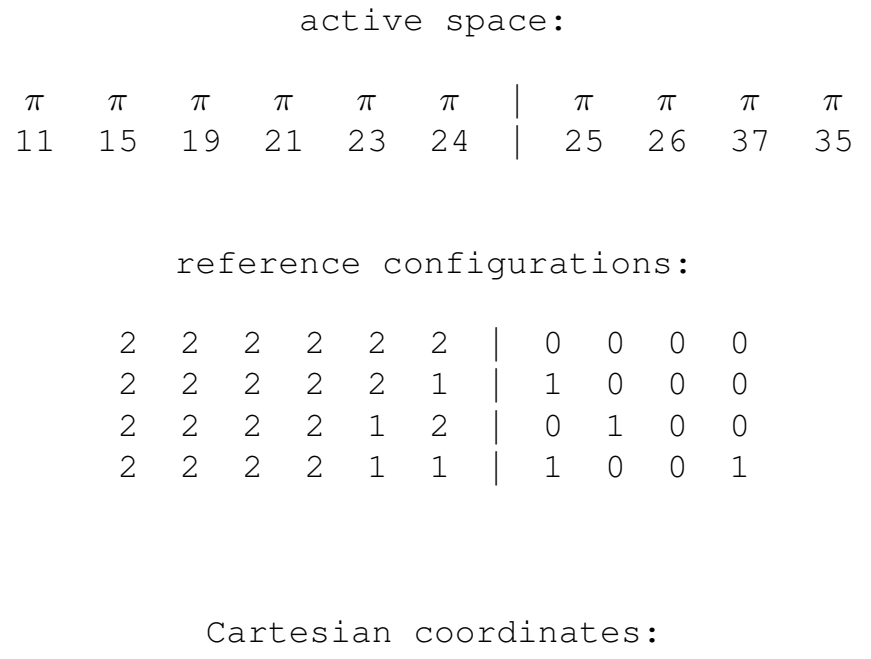

17

$\begin{array}{lrrr}\mathrm{C} & 0.00000 & 0.00000 & 0.00000 \\ \mathrm{C} & 1.41812 & 0.00000 & 0.00000 \\ \mathrm{C} & 2.12638 & 1.23667 & 0.00000 \\ \mathrm{C} & 1.39729 & 2.47140 & 0.00001 \\ \mathrm{C} & -0.03172 & 2.51607 & 0.00000 \\ \mathrm{C} & -0.70166 & 1.24970 & -0.00001 \\ \mathrm{O} & 2.22829 & 3.57677 & 0.00001 \\ \mathrm{C} & 1.59170 & 4.85027 & -0.00002 \\ \mathrm{O} & -2.07091 & 1.32351 & -0.00002 \\ \mathrm{H} & -0.60998 & 3.43780 & -0.00001 \\ \mathrm{H} & -0.56611 & -0.94230 & 0.00000 \\ \mathrm{H} & 1.97114 & -0.94759 & -0.00001 \\ \mathrm{H} & 3.22195 & 1.26392 & -0.00000 \\ \mathrm{H} & 2.42863 & 5.58834 & -0.00001 \\ \mathrm{H} & 0.96861 & 5.01272 & -0.91379 \\ \mathrm{H} & 0.96853 & 5.01276 & 0.91370 \\ \mathrm{H} & -2.46439 & 0.38336 & -0.00002\end{array}$


Table S1600 Employed active space, reference configurations, and Cartesian coordinates of the OM3/MRCISD-optimized ground-state equilibrium geometry of trans-p-methoxyphenol (ground-state energy: $-1672.15318 \mathrm{eV}$ ).

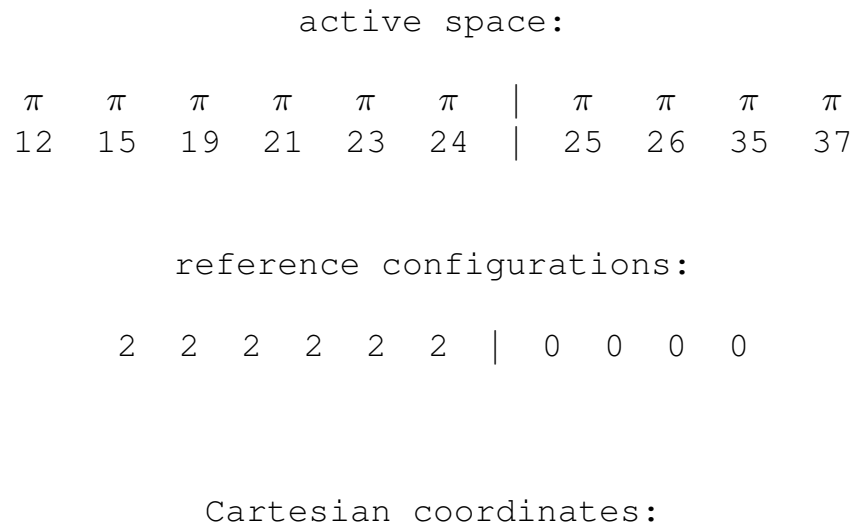


Table S1601 Employed active space, reference configurations, and Cartesian coordinates of the OM3/MRCISD-optimized excited-state equilibrium geometry of the $2{ }^{1} A^{\prime}\left({ }^{1} \pi \pi^{*}\right)$ state of trans-p-methoxyphenol (excited-state energy: $-1668.23629 \mathrm{eV})$.

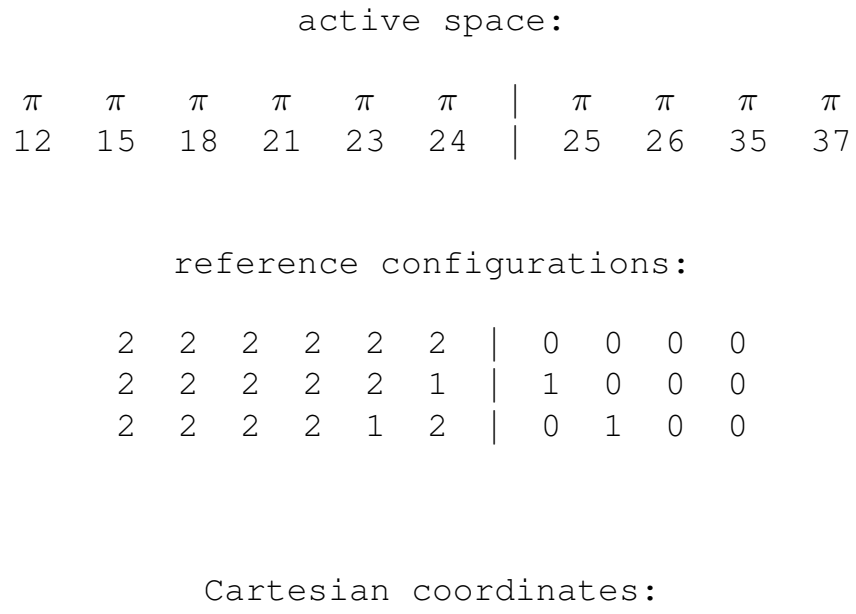


Table S1602 Employed active space, reference configurations, and Cartesian coordinates of the OM3/MRCISD-optimized ground-state equilibrium geometry of cis-p-methoxyphenol (ground-state energy: -1672.14826 eV).

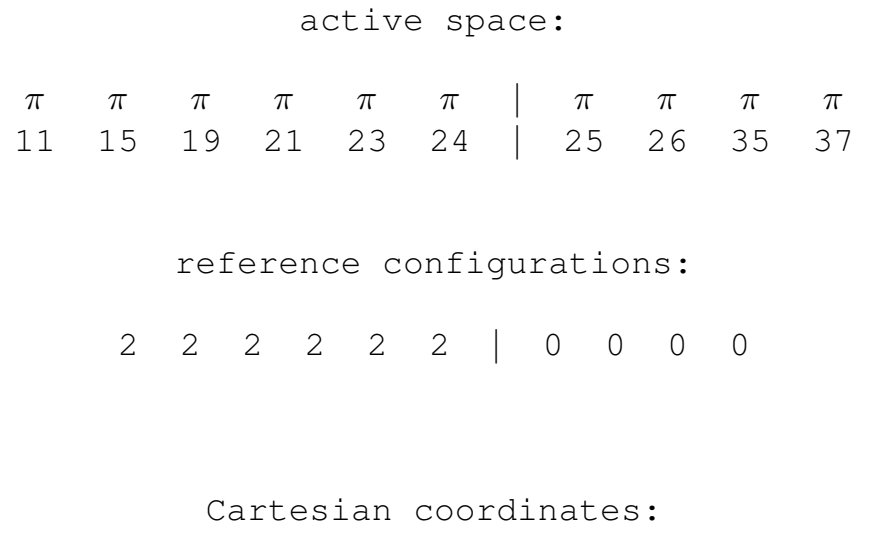

17

$\begin{array}{lrrr}\mathrm{C} & 0.00000 & 0.00000 & 0.00000 \\ \mathrm{C} & 1.40694 & 0.00000 & 0.00000 \\ \mathrm{C} & 2.14879 & 1.20581 & 0.00000 \\ \mathrm{C} & 1.47429 & 2.42586 & 0.00000 \\ \mathrm{C} & 0.06098 & 2.42421 & 0.00000 \\ \mathrm{C} & -0.67778 & 1.22557 & 0.00000 \\ \mathrm{O} & 2.21941 & -1.13566 & -0.00000 \\ \mathrm{C} & 1.54998 & -2.38793 & -0.00000 \\ \mathrm{O} & -0.53691 & 3.67208 & 0.00000 \\ \mathrm{H} & -0.56795 & -0.93801 & -0.00000 \\ \mathrm{H} & 3.24432 & 1.15884 & 0.00000 \\ \mathrm{H} & 2.01034 & 3.38162 & 0.00000 \\ \mathrm{H} & -1.77662 & 1.25346 & 0.00000 \\ \mathrm{H} & 2.36396 & -3.15008 & -0.00000 \\ \mathrm{H} & 0.91450 & -2.53762 & 0.91355 \\ \mathrm{H} & 0.91450 & -2.53762 & -0.91355 \\ \mathrm{H} & -1.54832 & 3.56184 & 0.00000\end{array}$


Table S1603 Employed active space, reference configurations, and Cartesian coordinates of the OM3/MRCISD-optimized excited-state equilibrium geometry of the $2^{1} A^{\prime}\left({ }^{1} \pi \pi^{*}\right)$ state of cis-p-methoxyphenol (excited-state energy: $-1668.22462 \mathrm{eV})$.

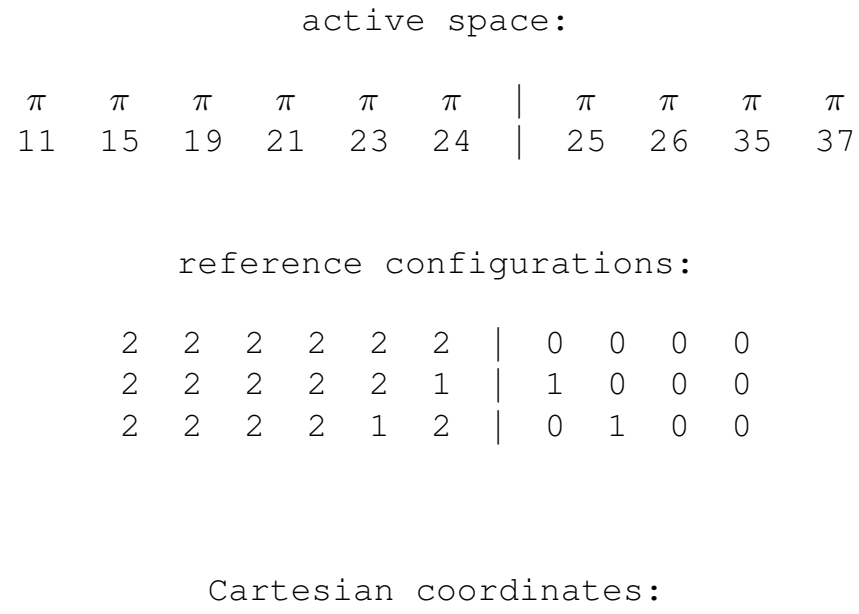


Table S1604 Employed active space, reference configurations, and Cartesian coordinates of the OM3/MRCISD-optimized ground-state equilibrium geometry of cis-o-fluorophenol (ground-state energy: $-1631.31465 \mathrm{eV}$ ).

\begin{tabular}{lcccc|ccc} 
active space \\
$\pi$ & $\pi$ & $\pi$ & $\pi$ & $\pi$ & $\pi$ & $\pi$ & $\pi$ \\
10 & 14 & 17 & 20 & 21 & 22 & 23 & 31
\end{tabular}

reference configurations:

\begin{tabular}{lllll|lll}
2 & 2 & 2 & 2 & 2 & 0 & 0 & 0
\end{tabular}

Cartesian coordinates:

13

$\begin{array}{lrrr}\mathrm{C} & 0.00000 & 0.00000 & 0.00000 \\ \mathrm{C} & 1.40186 & 0.00000 & 0.00000 \\ \mathrm{C} & 2.07168 & 1.23034 & 0.00000 \\ \mathrm{C} & 1.36783 & 2.46224 & 0.00000 \\ \mathrm{C} & -0.04128 & 2.44164 & 0.00000 \\ \mathrm{C} & -0.71073 & 1.21114 & 0.00000 \\ \mathrm{~F} & 3.41227 & 1.25560 & 0.00000 \\ \mathrm{O} & 1.96809 & 3.70256 & 0.00000 \\ \mathrm{H} & -0.58355 & 3.39373 & 0.00000 \\ \mathrm{H} & -1.81183 & 1.19661 & 0.00000 \\ \mathrm{H} & -0.54224 & -0.95720 & -0.00000 \\ \mathrm{H} & 1.98056 & -0.92987 & -0.00000 \\ \mathrm{H} & 2.97972 & 3.59276 & 0.00000\end{array}$


Table S1605 Employed active space, reference configurations, and Cartesian coordinates of the OM3/MRCISD-optimized excited-state equilibrium geometry of the $2{ }^{1} A\left({ }^{1} \pi \pi^{*}\right)$ state of cis-o-fluorophenol (excited-state energy: $-1627.23784 \mathrm{eV})$.

\begin{tabular}{lcccc|ccc} 
active space \\
$\pi$ & $\pi$ & $\pi$ & $\pi$ & $\pi$ & $\pi$ & $\pi$ & $\pi$ \\
9 & 13 & 17 & 20 & 21 & 22 & 23 & 30
\end{tabular}

reference configurations:

\begin{tabular}{lllll|lll}
2 & 2 & 2 & 2 & 2 & 0 & 0 & 0
\end{tabular}

\begin{tabular}{lllll|lll}
2 & 2 & 2 & 2 & 1 & 1 & 0 & 0
\end{tabular}

\begin{tabular}{lllll|lll}
2 & 2 & 2 & 1 & 2 & 0 & 1 & 0
\end{tabular}

Cartesian coordinates:

13

$\begin{array}{llll}\text { C } & 0.00000 & 0.00000 & 0.00000\end{array}$

C $\quad 1.42016 \quad 0.00000 \quad 0.00000$

$\begin{array}{llll}\text { C } & 2.08297 & 1.26169 & 0.00000\end{array}$

$\begin{array}{llll}\text { C } & 1.34859 & 2.50511 & 0.00000\end{array}$

$\begin{array}{llll}\text { C } & -0.08680 & 2.47687 & 0.00001\end{array}$

$\begin{array}{lrrr}\text { C } & -0.74023 & 1.21526 & 0.00001\end{array}$

F $\quad 3.41512 \quad 1.31353-0.00000$

o $\quad 1.92938 \quad 3.73695 \quad 0.00000$

$\mathrm{H} \quad-0.63807 \quad 3.42056 \quad 0.00001$

$\begin{array}{llll}\mathrm{H} & -1.83926 & 1.18071 & 0.00001\end{array}$

$\mathrm{H} \quad-0.53518 \quad-0.96273 \quad-0.00001$

$\mathrm{H} \quad 2.00549 \quad-0.92175 \quad-0.00001$

$\begin{array}{llll}\mathrm{H} & 2.94544 & 3.64729 & 0.00002\end{array}$ 
Table S1606 Employed active space, reference configurations, and Cartesian coordinates of the OM3/MRCISD-optimized ground-state equilibrium geometry of trans-o-fluorophenol (ground-state energy: -1631.25794 eV).

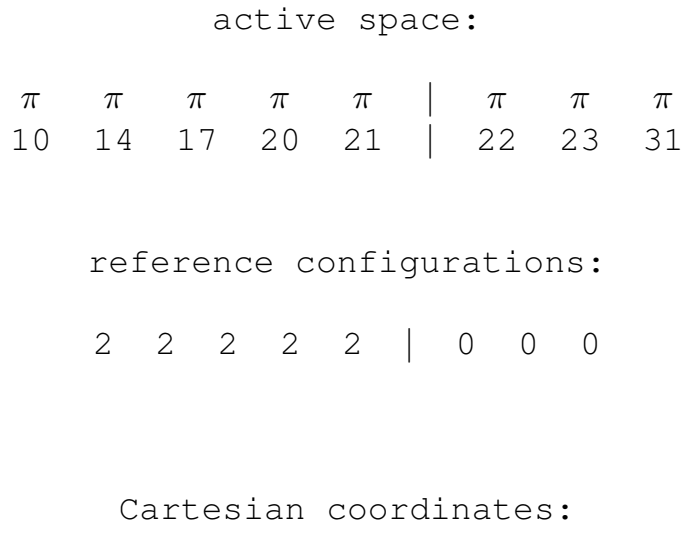

13

$\begin{array}{lrrr}\text { C } & 0.00000 & 0.00000 & 0.00000 \\ \text { C } & 1.40223 & 0.00000 & 0.00000 \\ \text { C } & 2.13159 & 1.21983 & 0.00000 \\ \text { C } & 1.42957 & 2.43964 & 0.00000 \\ \text { C } & 0.02779 & 2.43416 & 0.00000 \\ \text { C } & -0.68309 & 1.22477 & 0.00000 \\ \text { F } & 2.05475 & -1.15979 & -0.00000 \\ \text { O } & 3.50525 & 1.12644 & 0.00000 \\ \text { H } & 1.98818 & 3.38594 & 0.00000 \\ \text { H } & -0.51706 & 3.39080 & 0.00000 \\ \text { H } & -1.78321 & 1.23379 & 0.00000 \\ \text { H } & -0.53599 & -0.95542 & -0.00000 \\ \text { H } & 3.90725 & 2.06276 & 0.00000\end{array}$


Table S1607 Employed active space, reference configurations, and Cartesian coordinates of the OM3/MRCISD-optimized excited-state equilibrium geometry of the $2^{1} A^{\prime}\left({ }^{1} \pi \pi^{*}\right)$ state of trans-o-fluorophenol (excited-state energy: $-1627.19840 \mathrm{eV})$.

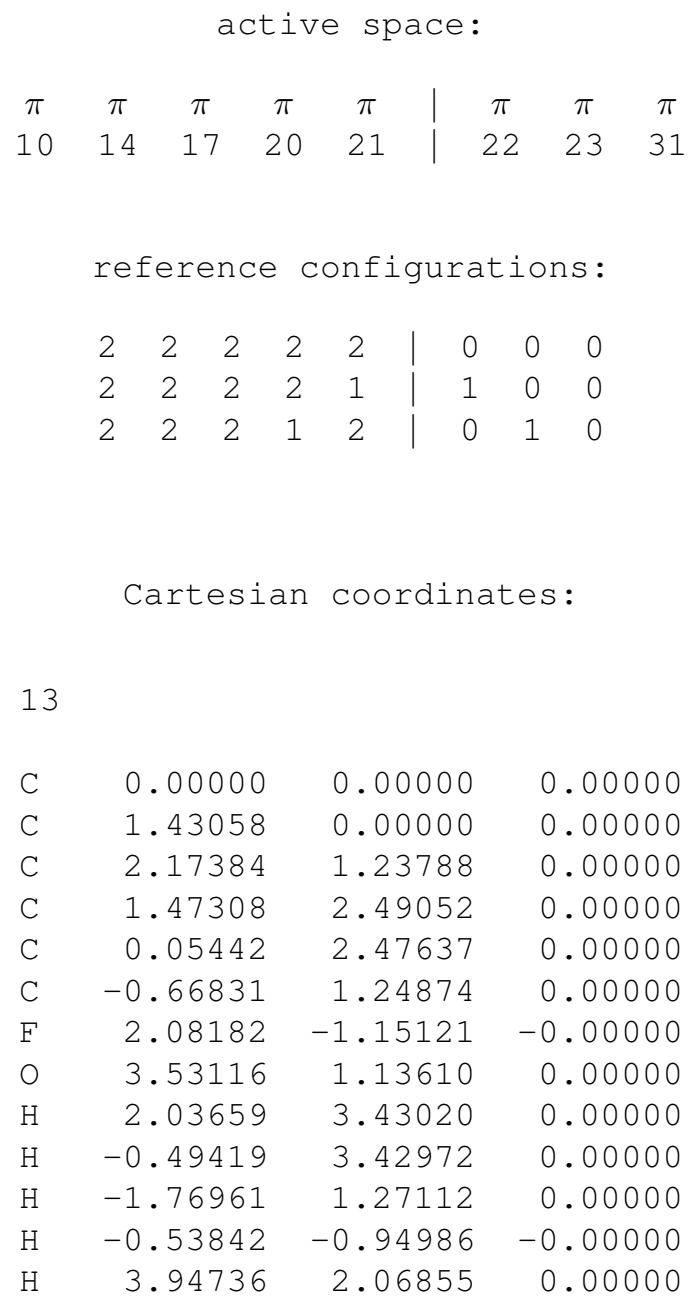


Table S1608 Employed active space, reference configurations, and Cartesian coordinates of the OM3/MRCISD-optimized ground-state equilibrium geometry of $c i s-m$-fluorophenol (ground-state energy: $-1631.42195 \mathrm{eV}$ ).

\begin{tabular}{ccccc|ccc} 
active space : \\
$\pi$ & $\pi$ & $\pi$ & $\pi$ & $\pi$ & $\pi$ & $\pi$ & $\pi$ \\
10 & 14 & 17 & 20 & 21 & 22 & 23 & 30
\end{tabular}

reference configurations:

\begin{tabular}{lllll|lll}
2 & 2 & 2 & 2 & 2 & 0 & 0 & 0
\end{tabular}

Cartesian coordinates:

13

$\begin{array}{lrrr}\text { C } & 0.00000 & 0.00000 & 0.00000 \\ \text { C } & 1.40561 & 0.00000 & 0.00000 \\ \text { C } & 2.17404 & 1.17498 & 0.00000 \\ \text { C } & 1.46839 & 2.39321 & 0.00000 \\ \text { C } & 0.05597 & 2.43719 & 0.00000 \\ \text { C } & -0.66260 & 1.23600 & 0.00000 \\ \text { F } & 2.04802 & -1.17291 & -0.00000 \\ \text { O } & 2.10398 & 3.61864 & 0.00000 \\ \text { H } & -0.44610 & 3.40982 & 0.00000 \\ \text { H } & -1.76413 & 1.26110 & 0.00000 \\ \text { H } & -0.54553 & -0.94748 & -0.00000 \\ \text { H } & 3.26412 & 1.13174 & 0.00000 \\ \text { H } & 3.11243 & 3.48258 & 0.00000\end{array}$


Table S1609 Employed active space, reference configurations, and Cartesian coordinates of the OM3/MRCISD-optimized excited-state equilibrium geometry of the $2{ }^{1} A\left({ }^{1} \pi \pi^{*}\right)$ state of cis- $m$-fluorophenol (excited-state energy: $-1627.31285 \mathrm{eV})$.

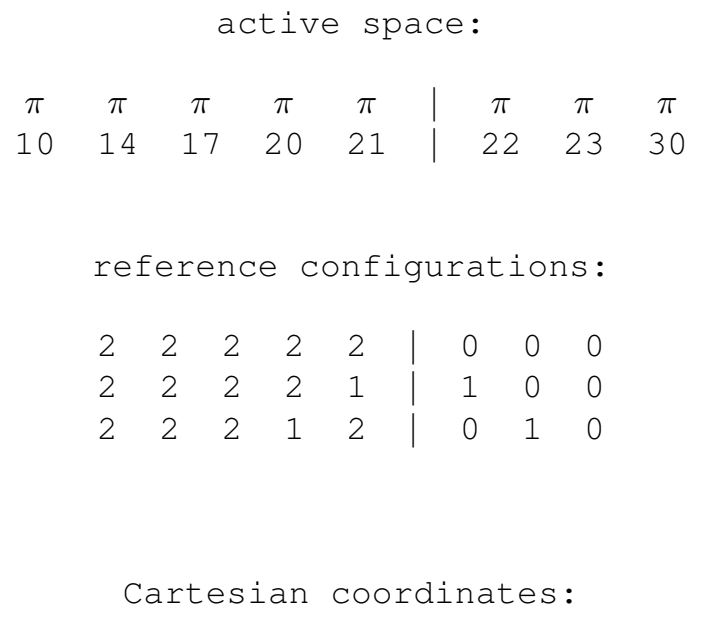

13

$\begin{array}{lrrr}\text { C } & 0.00000 & 0.00000 & 0.00000 \\ \mathrm{C} & 1.42917 & 0.00000 & 0.00000 \\ \mathrm{C} & 2.21620 & 1.18837 & 0.00000 \\ \mathrm{C} & 1.48213 & 2.41731 & 0.00017 \\ \mathrm{C} & 0.04722 & 2.46256 & 0.00017 \\ \mathrm{C} & -0.68689 & 1.24142 & 0.00010 \\ \mathrm{~F} & 2.06069 & -1.17200 & -0.00003 \\ \mathrm{O} & 2.10109 & 3.63596 & 0.00028 \\ \mathrm{H} & -0.45405 & 3.43573 & 0.00022 \\ \mathrm{H} & -1.78371 & 1.26380 & 0.00011 \\ \mathrm{H} & -0.53755 & -0.95459 & -0.00004 \\ \mathrm{H} & 3.30427 & 1.15099 & 0.00000 \\ \mathrm{H} & 3.11326 & 3.51407 & 0.00026\end{array}$


Table S1610 Employed active space, reference configurations, and Cartesian coordinates of the OM3/MRCISD-optimized ground-state equilibrium geometry of trans- $m$-fluorophenol (ground-state energy: $-1631.41810 \mathrm{eV}$ ).

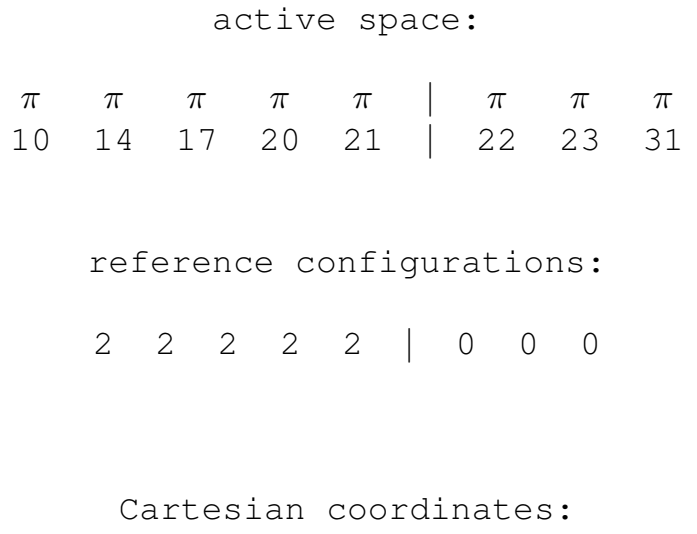

13

$\begin{array}{rrrr}\text { C } & 0.00000 & 0.00000 & 0.00000 \\ \text { C } & 1.40243 & 0.00000 & 0.00000 \\ \text { C } & 2.03274 & 1.26011 & 0.00000 \\ \text { C } & 1.29312 & 2.46264 & 0.00000 \\ \text { C } & -0.10687 & 2.40779 & 0.00000 \\ \text { C } & -0.77352 & 1.17573 & 0.00000 \\ \text { O } & 3.41294 & 1.23130 & 0.00000 \\ \text { F } & -0.63318 & -1.17773 & -0.00000 \\ \text { H } & 1.81648 & 3.42753 & 0.00000 \\ \text { H } & -0.68928 & 3.34294 & 0.00000 \\ \text { H } & -1.86499 & 1.11169 & 0.00000 \\ \text { H } & 1.98169 & -0.92202 & -0.00000 \\ \text { H } & 3.77318 & 2.18320 & 0.00000\end{array}$


Table S1611 Employed active space, reference configurations, and Cartesian coordinates of the OM3/MRCISD-optimized excited-state equilibrium geometry of the $2^{1} A^{\prime}\left({ }^{1} \pi \pi^{*}\right)$ state of trans- $m$-fluorophenol (excited-state energy: $-1627.29934 \mathrm{eV})$.

\begin{tabular}{lcccc|ccc} 
active space : \\
$\pi$ & $\pi$ & $\pi$ & $\pi$ & $\pi$ & $\pi$ & $\pi$ & $\pi$ \\
9 & 14 & 17 & 20 & 21 & 22 & 23 & 30
\end{tabular}

reference configurations:

\begin{tabular}{lllll|lll}
2 & 2 & 2 & 2 & 2 & 0 & 0 & 0
\end{tabular}

\begin{tabular}{lllll|lll}
2 & 2 & 2 & 2 & 1 & 1 & 0 & 0
\end{tabular}

\begin{tabular}{lllll|lll}
2 & 2 & 2 & 1 & 2 & 0 & 1 & 0
\end{tabular}

Cartesian coordinates:

13

$\begin{array}{lrrr}\text { C } & 0.00000 & 0.00000 & 0.00000 \\ \mathrm{C} & 1.42668 & 0.00000 & 0.00000 \\ \mathrm{C} & 2.04717 & 1.29002 & 0.00000 \\ \mathrm{C} & 1.29033 & 2.51092 & 0.00000 \\ \mathrm{C} & -0.13053 & 2.45075 & 0.00000 \\ \mathrm{C} & -0.78793 & 1.19081 & 0.00000 \\ \mathrm{O} & 3.41369 & 1.27553 & 0.00000 \\ \mathrm{~F} & -0.63068 & -1.17254 & -0.00000 \\ \mathrm{H} & 1.81275 & 3.47630 & 0.00000 \\ \mathrm{H} & -0.71866 & 3.37747 & 0.00000 \\ \mathrm{H} & -1.88076 & 1.11693 & 0.00000 \\ \mathrm{H} & 2.01199 & -0.91628 & -0.00000 \\ \mathrm{H} & 3.77178 & 2.23050 & 0.00000\end{array}$


Table S1612 Employed active space, reference configurations, and Cartesian coordinates of the OM3/MRCISD-optimized ground-state equilibrium geometry of $p$-fluorophenol (ground-state energy: $-1631.39991 \mathrm{eV}$ ).

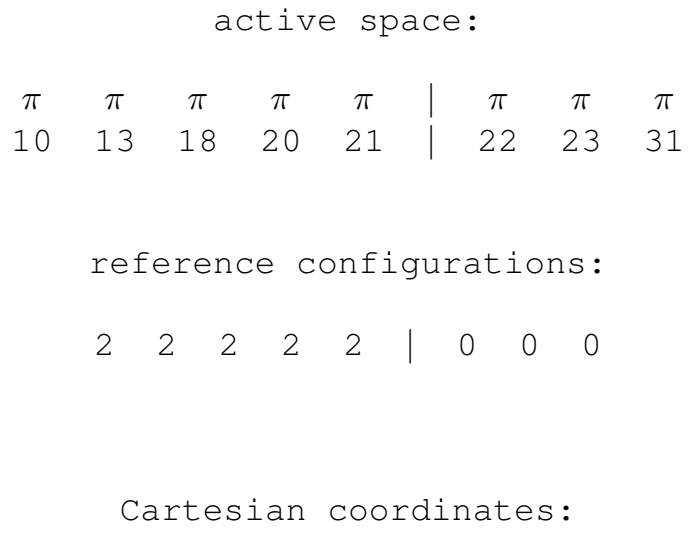

13

$\begin{array}{lrrr}\text { C } & 0.00000 & 0.00000 & 0.00000 \\ \mathrm{C} & 1.39671 & 0.00000 & 0.00000 \\ \mathrm{C} & 2.07894 & 1.23760 & 0.00000 \\ \mathrm{C} & 1.39491 & 2.47171 & 0.00000 \\ \mathrm{C} & -0.00351 & 2.47441 & 0.00000 \\ \mathrm{C} & -0.67127 & 1.23715 & 0.00000 \\ \mathrm{O} & 3.45846 & 1.15133 & 0.00000 \\ \mathrm{~F} & -2.00999 & 1.23726 & 0.00000 \\ \mathrm{H} & 1.95663 & 3.41575 & 0.00000 \\ \mathrm{H} & -0.57608 & 3.40764 & 0.00000 \\ \mathrm{H} & -0.57309 & -0.93319 & -0.00000 \\ \mathrm{H} & 1.97337 & -0.93133 & -0.00000 \\ \mathrm{H} & 3.85794 & 2.08720 & 0.00000\end{array}$


Table S1613 Employed active space, reference configurations, and Cartesian coordinates of the OM3/MRCISD-optimized excited-state equilibrium geometry of the $2^{1} A^{\prime}\left({ }^{1} \pi \pi^{*}\right)$ state of $p$-fluorophenol (excited-state energy: $-1627.37058 \mathrm{eV})$.

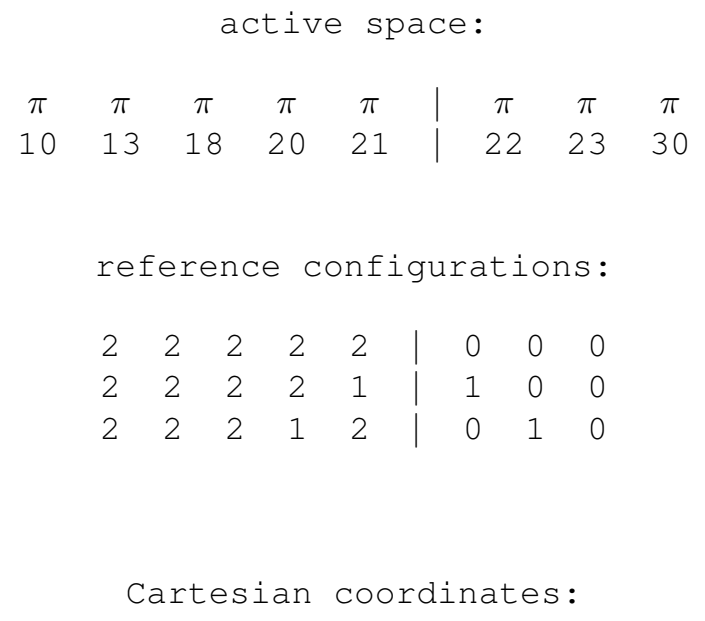

13

$\begin{array}{lrrr}\mathrm{C} & 0.00000 & 0.00000 & 0.00000 \\ \mathrm{C} & 1.41862 & 0.00000 & 0.00000 \\ \mathrm{C} & 2.09730 & 1.26463 & 0.00000 \\ \mathrm{C} & 1.41493 & 2.52872 & 0.00000 \\ \mathrm{C} & -0.00143 & 2.52802 & 0.00000 \\ \mathrm{C} & -0.66416 & 1.26187 & 0.00000 \\ \mathrm{O} & 3.45984 & 1.18283 & 0.00000 \\ \mathrm{~F} & -1.99485 & 1.26508 & 0.00000 \\ \mathrm{H} & 1.98212 & 3.46627 & 0.00000 \\ \mathrm{H} & -0.58066 & 3.45362 & 0.00000 \\ \mathrm{H} & -0.57566 & -0.92740 & -0.00000 \\ \mathrm{H} & 1.99915 & -0.92589 & -0.00000 \\ \mathrm{H} & 3.86846 & 2.11750 & 0.00000\end{array}$


Table S1614 Employed active space, reference configurations, and Cartesian coordinates of the OM3/MRCISD-optimized ground-state equilibrium geometry of cis-o-cresol (ground-state energy: $-1333.78931 \mathrm{eV}$ ).

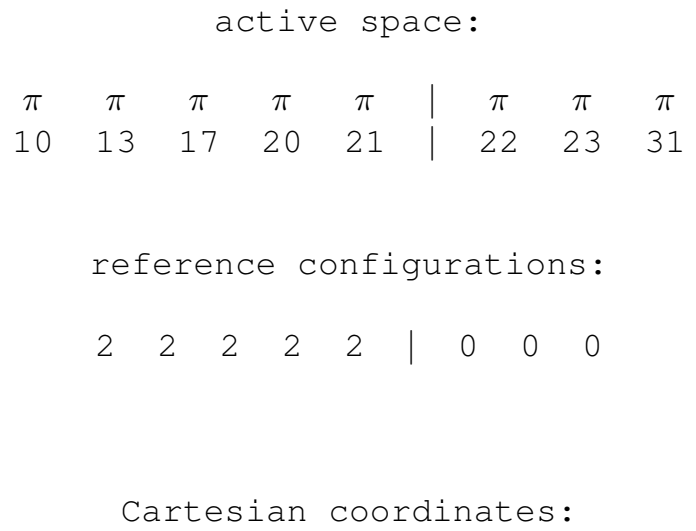


Table S1615 Employed active space, reference configurations, and Cartesian coordinates of the OM3/MRCISD-optimized excited-state equilibrium geometry of the $2{ }^{1} A\left({ }^{1} \pi \pi^{*}\right)$ state of cis-o-cresol (excited-state energy: $-1329.51811 \mathrm{eV}$ ).

\begin{tabular}{|c|c|c|c|c|c|c|c|c|}
\hline \multirow{2}{*}{$\begin{array}{c}\pi \\
11\end{array}$} & $\pi$ & $\pi$ & $\pi$ & $\pi$ & \multicolumn{2}{|c|}{$\pi$} & $\pi$ & $\pi$ \\
\hline & 12 & 17 & 20 & 21 & & 2 & 23 & 31 \\
\hline \multicolumn{9}{|c|}{ ceference c } \\
\hline & 2 & 22 & 2 & 2 & 0 & 0 & 0 & \\
\hline & 2 & 22 & 2 & 1 & 1 & 0 & 0 & \\
\hline & 2 & 22 & 1 & 2 & 0 & 1 & 0 & \\
\hline & 2 & 22 & 2 & 1 & 0 & 1 & 0 & \\
\hline \multicolumn{9}{|c|}{ Cartesian coordinates: } \\
\hline 16 & & & & & & & & \\
\hline$C$ & & .00000 & & 0.00 & & & .00 & 000 \\
\hline C & & .44183 & & 0.00 & & & .00 & 000 \\
\hline C & & .11581 & & 1.25 & & & .00 & 000 \\
\hline C & & .37567 & & 2.47 & & & .00 & 025 \\
\hline C & -0 & 04821 & & 2.47 & & & .00 & 065 \\
\hline C & -0 & .76935 & & 1.22 & & & .00 & 062 \\
\hline C & -2 & .26076 & & 1.25 & & & .00 & 031 \\
\hline O & -0 & .55824 & & -1.25 & & & .00 & 013 \\
\hline $\mathrm{H}$ & & .21527 & & 1.27 & & & .00 & 019 \\
\hline $\mathrm{H}$ & & .91276 & & 3.43 & & & .00 & 031 \\
\hline $\mathrm{H}$ & -0 & .60040 & & 3.42 & & & .00 & 091 \\
\hline $\mathrm{H}$ & -2 & .60383 & & 1.72 & & & .90 & 344 \\
\hline $\mathrm{H}$ & -2 & .60424 & & 1.72 & & & .90 & 310 \\
\hline $\mathrm{H}$ & -2 & .68298 & & 0.21 & & & .00 & 140 \\
\hline $\mathrm{H}$ & -1 & .57655 & & -1.18 & & & .00 & 013 \\
\hline $\mathrm{H}$ & & .97505 & & -0.95 & & & .00 & 004 \\
\hline
\end{tabular}


Table S1616 Employed active space, reference configurations, and Cartesian coordinates of the OM3/MRCISD-optimized ground-state equilibrium geometry of trans-o-cresol (ground-state energy: $-1333.86130 \mathrm{eV}$ ).

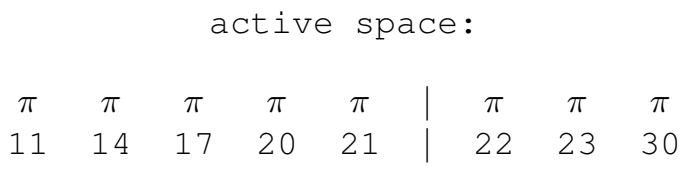

reference configurations:

$\begin{array}{lllllllll}2 & 2 & 2 & 2 & 2 & 0 & 0 & 0\end{array}$

Cartesian coordinates:

$\begin{array}{lrrr}16 & & & \\ & & & \\ \text { C } & 0.00000 & 0.00000 & 0.00000 \\ \text { C } & 1.40364 & 0.00000 & 0.00000 \\ \text { C } & 2.12615 & 1.20597 & 0.00000 \\ \text { C } & 1.38631 & 2.41918 & 0.00000 \\ \text { C } & -0.02132 & 2.43001 & 0.00000 \\ \text { C } & -0.70959 & 1.20979 & 0.00000 \\ \text { C } & 3.62233 & 1.24484 & 0.00000 \\ \text { O } & 2.13756 & 3.58259 & 0.00000 \\ \text { H } & -0.56478 & 3.38543 & 0.00000 \\ \text { H } & -1.81137 & 1.20467 & 0.00000 \\ \text { H } & -0.54721 & -0.95506 & -0.00000 \\ \text { H } & 1.95301 & -0.95602 & -0.00000 \\ \text { H } & 1.51236 & 4.38495 & 0.00000 \\ \text { H } & 4.04471 & 0.22064 & 0.00000 \\ \text { H } & 3.97719 & 1.77160 & -0.89614 \\ \text { H } & 3.97719 & 1.77160 & 0.89614\end{array}$


Table S1617 Employed active space, reference configurations, and Cartesian coordinates of the OM3/MRCISD-optimized excited-state equilibrium geometry of the $2{ }^{1} A\left({ }^{1} \pi \pi^{*}\right)$ state of trans-o-cresol (excited-state energy: $-1329.68307 \mathrm{eV})$.

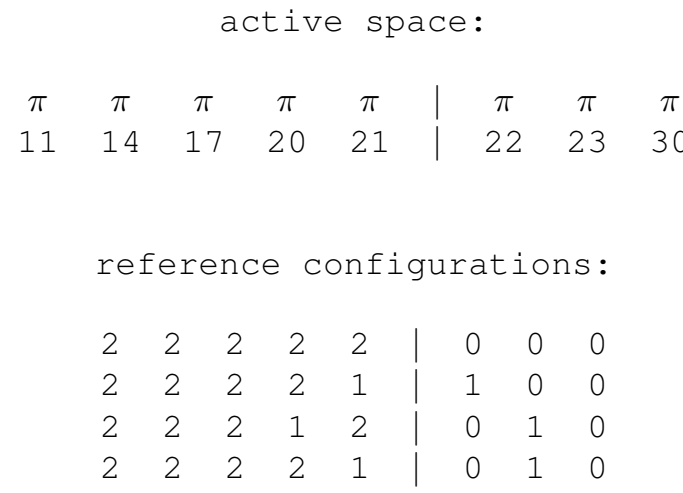

Cartesian coordinates:

16

$\begin{array}{lrrr}\mathrm{C} & 0.00000 & 0.00000 & 0.00000 \\ \mathrm{C} & 1.42060 & 0.00000 & 0.00000 \\ \mathrm{C} & 2.13515 & 1.24478 & 0.00000 \\ \mathrm{C} & 1.36220 & 2.46517 & 0.00007 \\ \mathrm{C} & -0.07642 & 2.46845 & 0.00007 \\ \mathrm{C} & -0.74380 & 1.21636 & 0.00003 \\ \mathrm{C} & 3.62446 & 1.31899 & 0.00005 \\ \mathrm{O} & 2.09371 & 3.62399 & 0.00011 \\ \mathrm{H} & -0.62698 & 3.41630 & 0.00010 \\ \mathrm{H} & -1.84397 & 1.18598 & 0.00003 \\ \mathrm{H} & -0.54144 & -0.96024 & -0.00002 \\ \mathrm{H} & 1.97997 & -0.94531 & -0.00001 \\ \mathrm{H} & 1.46339 & 4.42437 & 0.00015 \\ \mathrm{H} & 4.06405 & 0.30070 & -0.00020 \\ \mathrm{H} & 3.95546 & 1.83787 & -0.89774 \\ \mathrm{H} & 3.95540 & 1.83743 & 0.89809\end{array}$


Table S1618 Employed active space, reference configurations, and Cartesian coordinates of the OM3/MRCISD-optimized ground-state equilibrium geometry of cis- $m$-cresol (ground-state energy: $-1333.84688 \mathrm{eV}$ ).

\begin{tabular}{ccccc|ccc}
\multicolumn{10}{c}{ active space: } \\
$\pi$ & $\pi$ & $\pi$ & $\pi$ & $\pi$ & $\pi$ & $\pi$ & $\pi$ \\
11 & 13 & 17 & 20 & 21 & 22 & 23 & 31
\end{tabular}

reference configurations:

\begin{tabular}{lllll|lll}
2 & 2 & 2 & 2 & 2 & 0 & 0 & 0
\end{tabular}

Cartesian coordinates:

16

$\begin{array}{lrrr}\mathrm{C} & 0.00000 & 0.00000 & 0.00000 \\ \mathrm{C} & 1.39912 & 0.00000 & 0.00000 \\ \mathrm{C} & 2.11689 & 1.20648 & 0.00000 \\ \mathrm{C} & 1.43800 & 2.44196 & 0.00000 \\ \mathrm{C} & 0.03005 & 2.46491 & 0.00000 \\ \mathrm{C} & -0.66980 & 1.24271 & 0.00000 \\ \mathrm{C} & 2.19994 & 3.73570 & 0.00000 \\ \mathrm{O} & -2.05257 & 1.17811 & 0.00000 \\ \mathrm{H} & -0.58313 & -0.92680 & -0.00000 \\ \mathrm{H} & 1.94220 & -0.95927 & -0.00000 \\ \mathrm{H} & 3.21784 & 1.19023 & 0.00000 \\ \mathrm{H} & -0.51943 & 3.41623 & 0.00000 \\ \mathrm{H} & -2.43336 & 2.12148 & 0.00000 \\ \mathrm{H} & 1.95563 & 4.31446 & 0.89991 \\ \mathrm{H} & 3.29107 & 3.54739 & 0.00000 \\ \mathrm{H} & 1.95563 & 4.31446 & -0.89991\end{array}$


Table S1619 Employed active space, reference configurations, and Cartesian coordinates of the OM3/MRCISD-optimized excited-state equilibrium geometry of the $2{ }^{1} A\left({ }^{1} \pi \pi^{*}\right)$ state of $c i s$ - $m$-cresol (excited-state energy: $\left.-1329.59135 \mathrm{eV}\right)$.

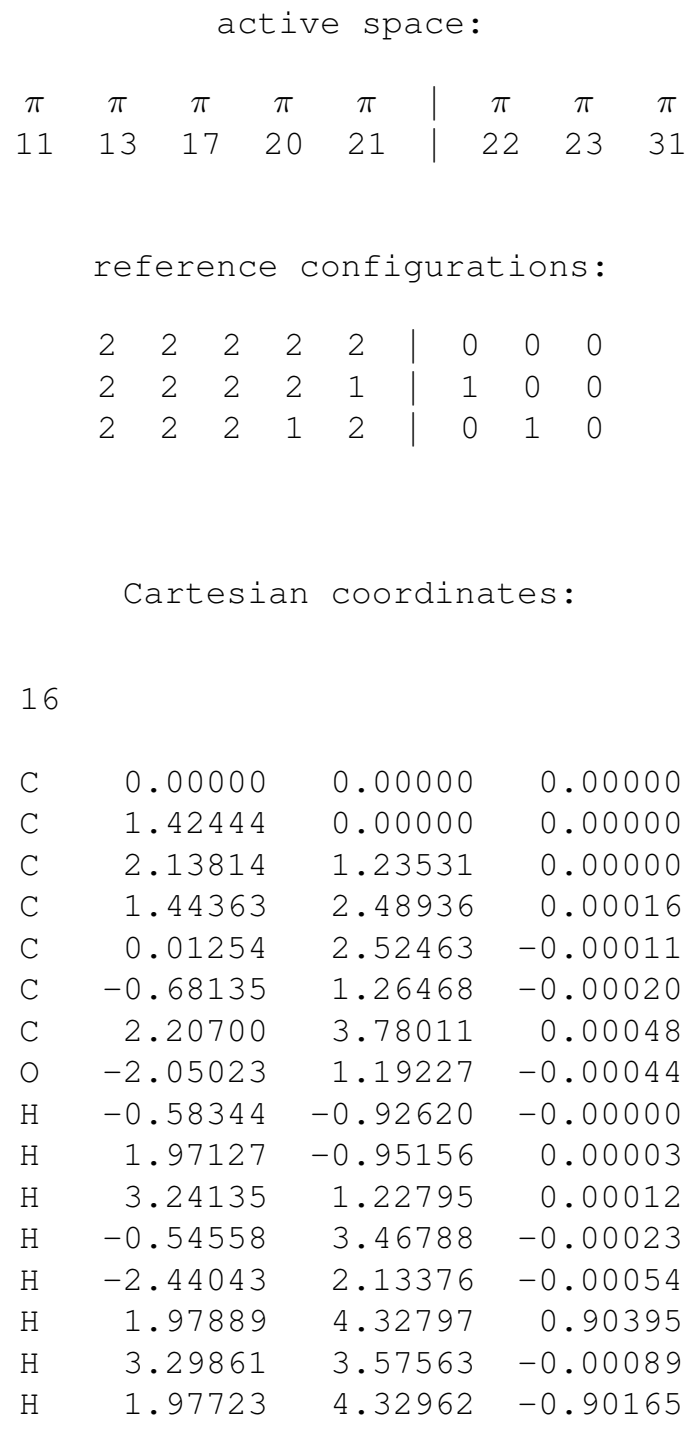


Table S1620 Employed active space, reference configurations, and Cartesian coordinates of the OM3/MRCISD-optimized ground-state equilibrium geometry of trans-m-cresol (ground-state energy: $-1333.85420 \mathrm{eV}$ ).

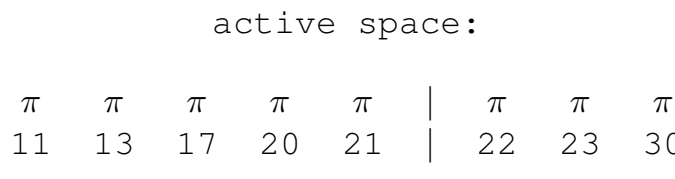

reference configurations:

$\begin{array}{lllllllll}2 & 2 & 2 & 2 & 2 & 0 & 0 & 0\end{array}$

Cartesian coordinates:

16

$\begin{array}{llll}\text { C } & 0.00000 & 0.00000 & 0.00000\end{array}$

$\begin{array}{llll}\text { C } & 1.40007 & 0.00000 & 0.00000\end{array}$

C $\quad 2.11140 \quad 1.20864 \quad 0.00000$

C $\quad 1.42616 \quad 2.44221 \quad 0.00000$

C $\quad 0.01922 \quad 2.46452 \quad 0.00000$

$\begin{array}{llll}\text { C } & -0.67531 & 1.23821 & 0.00000\end{array}$

$\begin{array}{llll}\text { C } & 2.18514 & 3.73804 & 0.00000\end{array}$

$\begin{array}{lrrr}0 & -2.05639 & 1.33351 & 0.00000\end{array}$

$\mathrm{H} \quad-0.56790 \quad-0.94029-0.00000$

$\mathrm{H} \quad 1.94477-0.95826-0.00000$

$\begin{array}{llll}\mathrm{H} & 3.21237 & 1.19597 & 0.00000\end{array}$

$\begin{array}{llll}\mathrm{H} & -0.54527 & 3.40282 & 0.00000\end{array}$

$\begin{array}{llll}\mathrm{H} & -2.45690 & 0.39839 & 0.00000\end{array}$

$\begin{array}{llll}\mathrm{H} & 1.93684 & 4.31611 & 0.89939\end{array}$

$\begin{array}{llll}\mathrm{H} & 3.27717 & 3.55476 & 0.00000\end{array}$

$\mathrm{H} \quad \begin{array}{llll}\text { H } & 1.93684 & 4.31611 & -0.89939\end{array}$ 
Table S1621 Employed active space, reference configurations, and Cartesian coordinates of the OM3/MRCISD-optimized excited-state equilibrium geometry of the $2^{1} A^{\prime}\left({ }^{1} \pi \pi^{*}\right)$ state of trans-m-cresol (excited-state energy: $-1329.60314 \mathrm{eV})$.

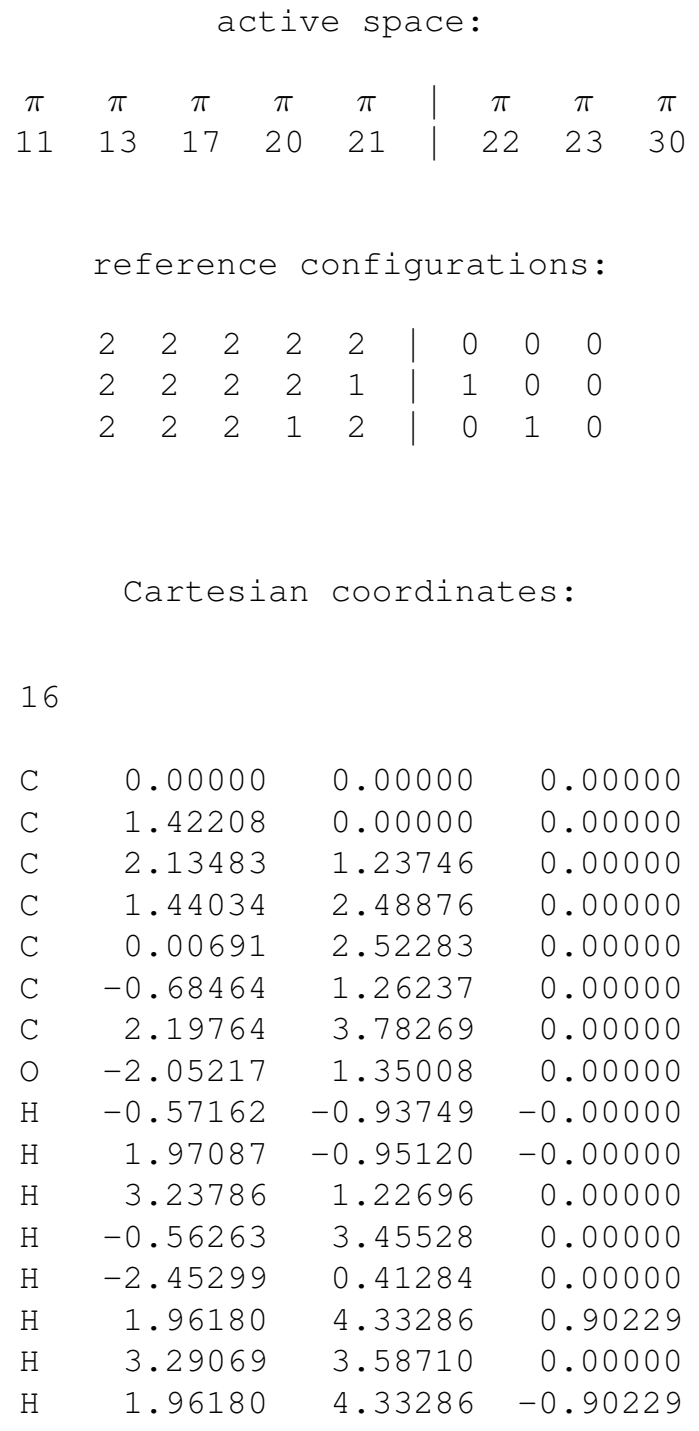


Table S1622 Employed active space, reference configurations, and Cartesian coordinates of the OM3/MRCISD-optimized ground-state equilibrium geometry of $p$-cresol (ground-state energy: $-1333.84511 \mathrm{eV}$ ).

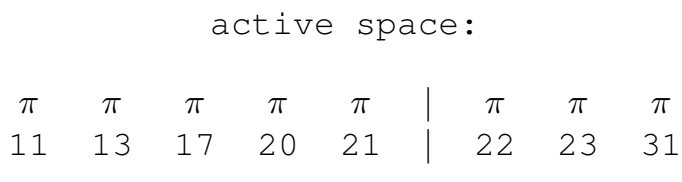

reference configurations:

$\begin{array}{lllllllll}2 & 2 & 2 & 2 & 2 & 0 & 0 & 0\end{array}$

Cartesian coordinates:

$\begin{array}{lrrr}16 & & & \\ & & & \\ \mathrm{C} & 0.00000 & 0.00000 & 0.00000 \\ \mathrm{C} & 1.41276 & 0.00000 & 0.00000 \\ \mathrm{C} & 2.12775 & 1.20073 & 0.00000 \\ \mathrm{C} & 1.40760 & 2.41497 & 0.00000 \\ \mathrm{C} & -0.00166 & 2.43933 & 0.00000 \\ \mathrm{C} & -0.69762 & 1.22509 & 0.00000 \\ \mathrm{O} & 2.17454 & 3.56718 & 0.00000 \\ \mathrm{C} & -0.74590 & -1.30210 & -0.00000 \\ \mathrm{H} & -0.53794 & 3.39847 & 0.00000 \\ \mathrm{H} & -1.80036 & 1.22822 & 0.00000 \\ \mathrm{H} & 1.95839 & -0.95775 & -0.00000 \\ \mathrm{H} & 3.22294 & 1.22496 & 0.00000 \\ \mathrm{H} & 1.56090 & 4.37860 & 0.00000 \\ \mathrm{H} & -0.49286 & -1.87911 & 0.89982 \\ \mathrm{H} & -0.49286 & -1.87911 & -0.89982 \\ \mathrm{H} & -1.84014 & -1.13021 & -0.00000\end{array}$


Table S1623 Employed active space, reference configurations, and Cartesian coordinates of the OM3/MRCISD-optimized excited-state equilibrium geometry of the $2{ }^{1} A\left({ }^{1} \pi \pi^{*}\right)$ state of $p$-cresol (excited-state energy: $-1329.65060 \mathrm{eV}$ ).

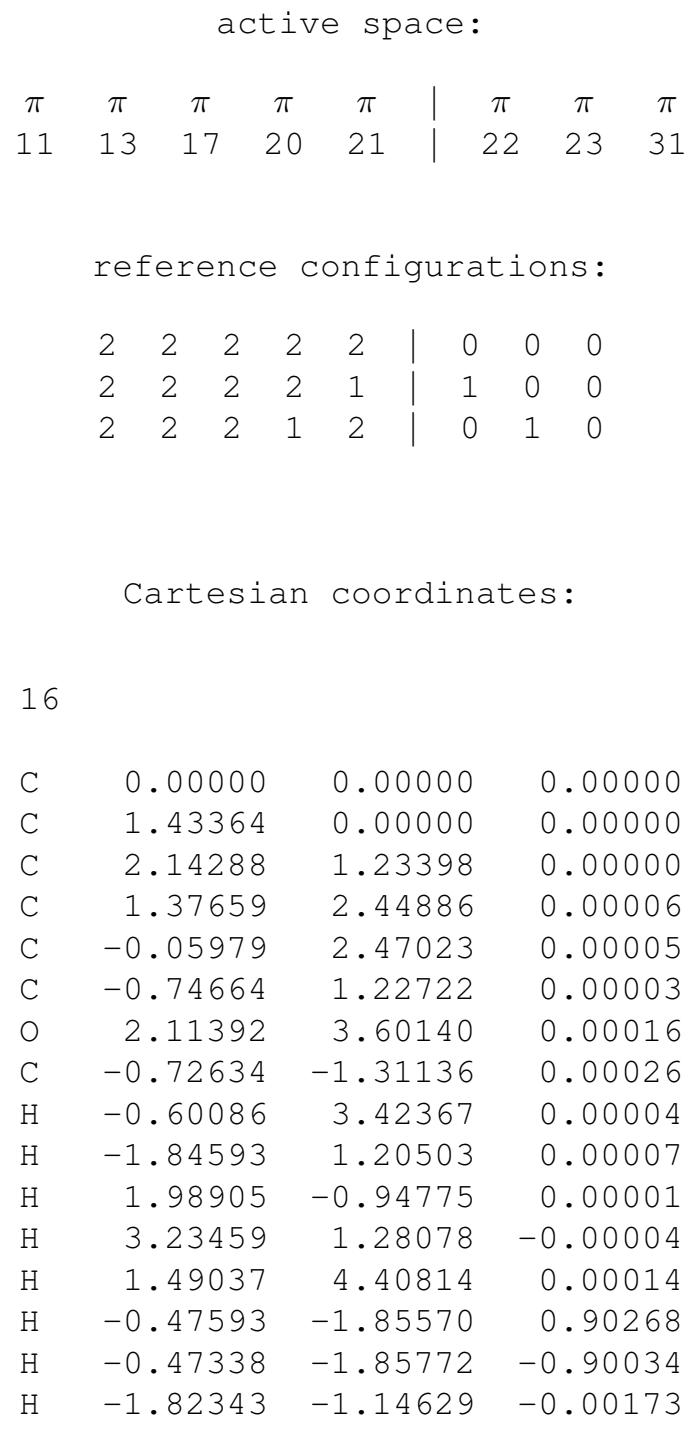

